\title{
Review of Excess Weapons Plutonium Disposition LLNL Contract Work in Russia
}

\author{
L. J. Jardine and G. B. Borisov
}

\section{July 11, 2002}

U.S. Department of Energy

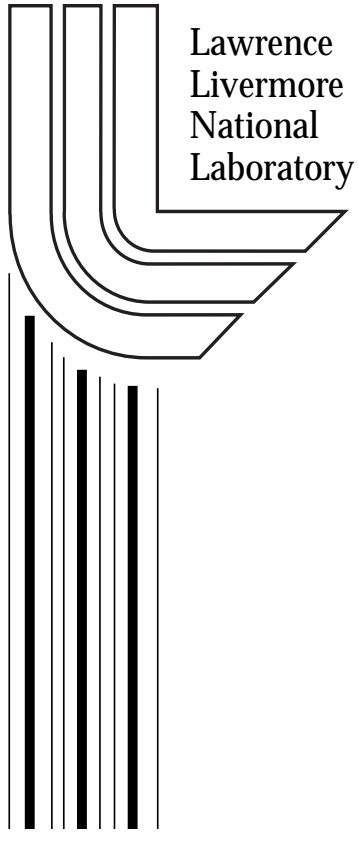




\section{DISCLAIMER}

This document was prepared as an account of work sponsored by an agency of the United States Government. Neither the United States Government nor the University of California nor any of their employees, makes any warranty, express or implied, or assumes any legal liability or responsibility for the accuracy, completeness, or usefulness of any information, apparatus, product, or process disclosed, or represents that its use would not infringe privately owned rights. Reference herein to any specific commercial product, process, or service by trade name, trademark, manufacturer, or otherwise, does not necessarily constitute or imply its endorsement, recommendation, or favoring by the United States Government or the University of California. The views and opinions of authors expressed herein do not necessarily state or reflect those of the United States Government or the University of California, and shall not be used for advertising or product endorsement purposes.

This work was performed under the auspices of the U. S. Department of Energy by the University of California, Lawrence Livermore National Laboratory under Contract No. W-7405-Eng-48.

This report has been reproduced directly from the best available copy.

Available electronically at http://www.doc.gov/bridge

Available for a processing fee to U.S. Department of Energy

And its contractors in paper from

U.S. Department of Energy

Office of Scientific and Technical Information

P.O. Box 62

Oak Ridge, TN 37831-0062

Telephone: (865) 576-8401

Facsimile: (865) 576-5728

E-mail: reports@adonis.osti.gov

Available for the sale to the public from

U.S. Department of Commerce

National Technical Information Service

5285 Port Royal Road

Springfield, VA 22161

Telephone: (800) 553-6847

Facsimile: (703) 605-6900

E-mail: orders@ntis.fedworld.gov

Online ordering: http://www.ntis.gov/ordering.htm

University of California

Lawrence Livermore National Laboratory

Technical Information Department

Livermore, CA 94551 


\title{
Review of Excess Weapons Plutonium Disposition LLNL Contract Work in Russia - in English
}

\author{
L. J. Jardine, LLNL \\ G. B. Borisov, VNIINM \\ U.S. and Russia Co-chairs for Joint \\ Working Group for Plutonium Disposition
}

\section{Proceedings of the 3rd Annual Meeting for Coordination and Review of LLNL Contract Work, State Regional Education Center \\ St. Petersburg, Russia, January 14-18, 2002}

Manuscript date: July 11, 2002

The following photomontage pages are dedicated to the memory of five former Russian colleagues, who made major contributions to our activities over the years:

Yury Sorokin

Victor V. Kushnikov, Leonid M. Borisov

Anna K. Nardova and Yuri F. Volkov 


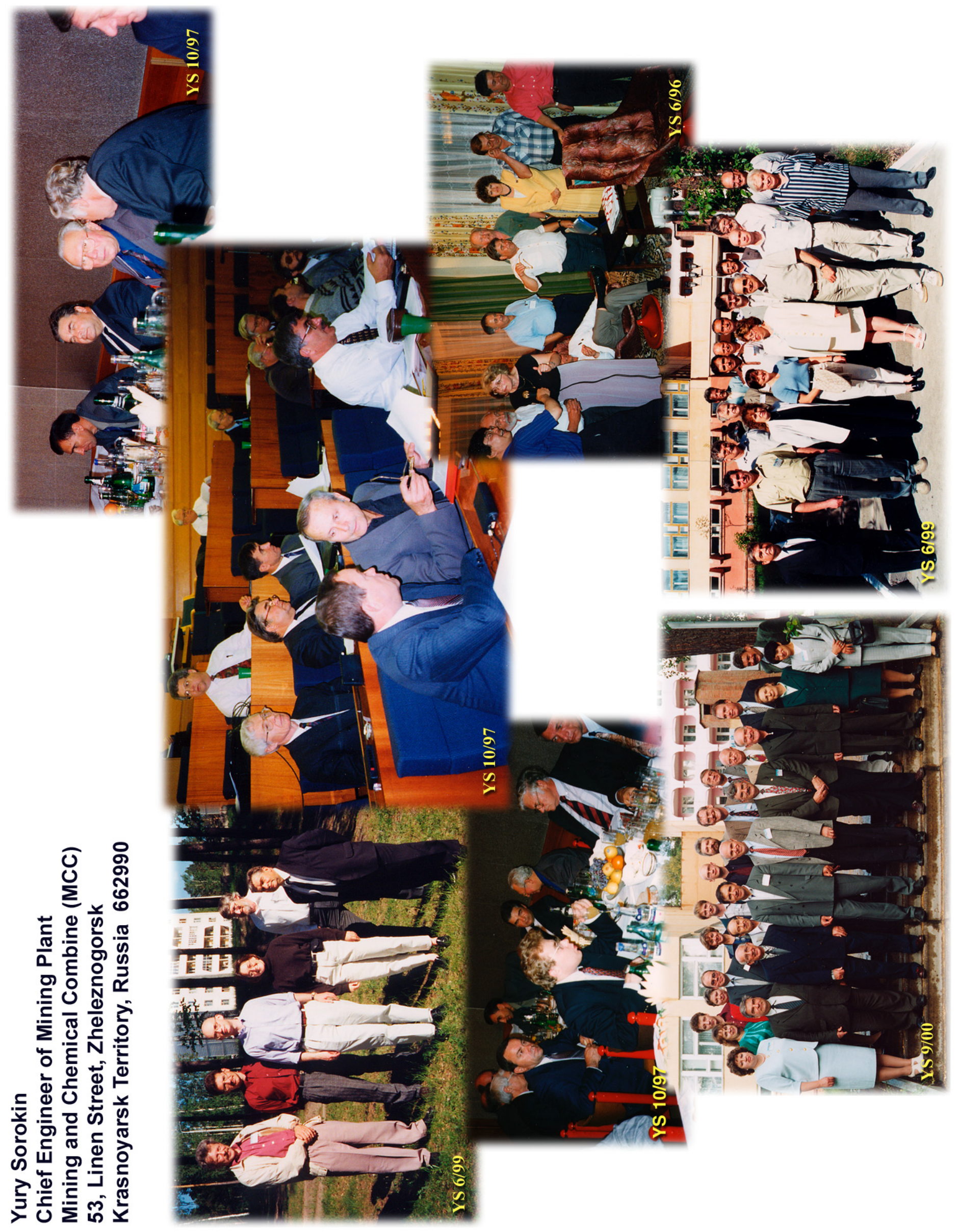




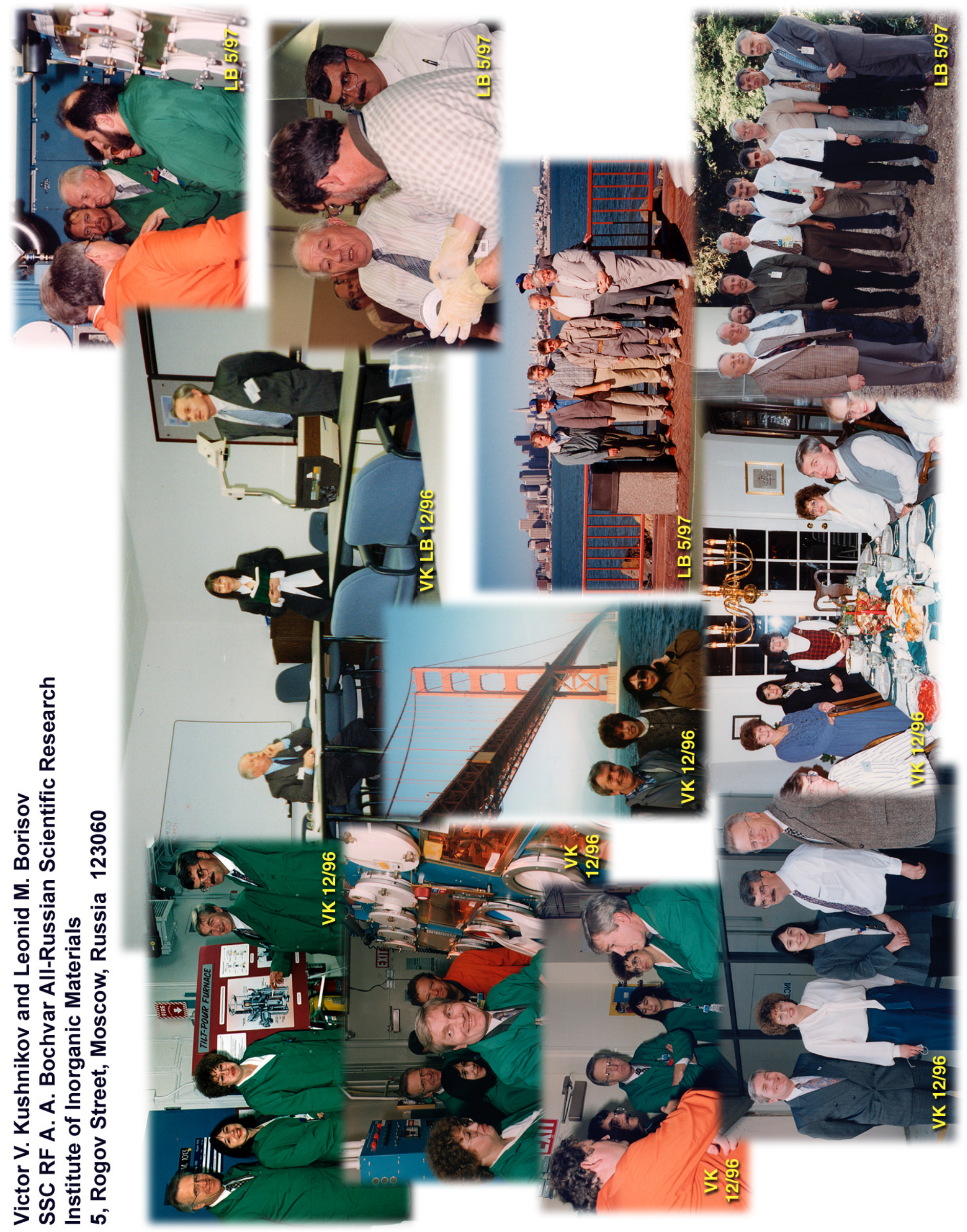




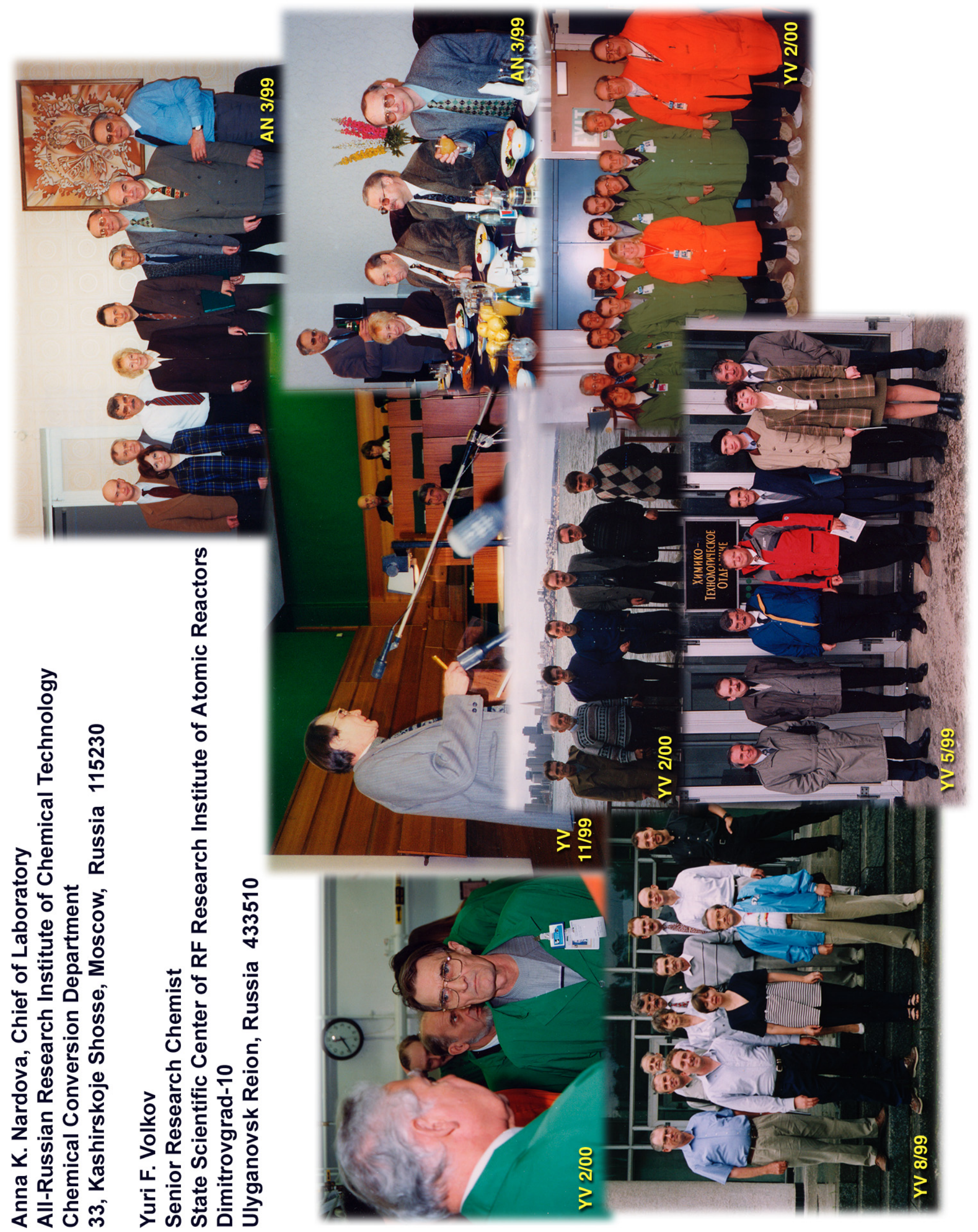




\section{Contents}

\section{Opening Session}

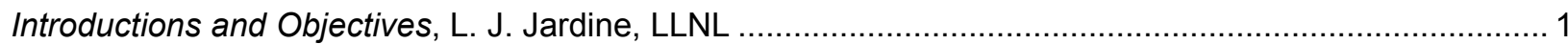

Immobilization of Surplus Weapons Plutonium in Russia, A. Orlova, NNGU, L. J. Jardine, LLNL .............. 3

Pu-containing Materials Management in Russia: Status and Perspectives, G. B. Borisov, VNIINM ........... 7

Packaging, Storage and Transportation of Plutonium, Plutonium Oxide and Unirradiated MOX Fuel

(B512166), A. V. Afanasyev, A. L. Lazarev, A. I. Tokarenko, VNIPIET.

Issues of Storage, Packing and Transportation of Weapons-grade Plutonium Dioxide for the Mayak Conversion Facility, E.M. Glagovsky, VNIINM.

Progress of an Engineering Cost Study on Utilizing the Existing Minatom RF Infrastructure for Storage of Irradiated MOX Fuel (B506234), O. P. Anisimov, N. S. Tikhonov, V. V. Spichev,

N. P. Shafrova, VNIPIET.

Materials Science Challenges in Long-term Wet and Dry Storage of RBMK-1000 Spent

Nuclear Fuel, M. I. Solonin, A. G. Ioltukhovskiy, V. P. Velioukhanov. I. M. Kadarmetov, VNIINM;

L. P. Sinel'nikov, A. N. Timokhin, O. A. Golosov, V. P. Kuznetsov, VNIPIET; V. A. Tsykhanov,

S. V. Pavlov, D. V. Markov, V. P. Smirnov, NIIAR.

The LLNL-Russian Pu Disposition Projects Web Site (B512152), P. E. Burkhanov, A. S. Aloy, E. Yu.

Ivanov, M. M. Moshkov, KRI

\section{Engineering and Industrial Site Plutonium Disposition Contract Activities: K-26, Mayak, RIAR}

\section{II.1. MCC K-26 Site Activities: Justification of Investment Engineering Studies of Weapons Plutonium Sludge Immobilization and MOX Fuel Fabrication Plutonium Immobilization}

Outlook for MCC-LLNL Collaboration in Nuclear Materials Management within the of RF-US Agreements (B347376, B506210, B506233), K. G. Kudinov, A. A. Tretyakov, MCC K-26 ........ 39

On JOI "Immobilization of Plutonium-containing Materials at the MCC" (B506233), V. M. Simanovskiy, O. P. Anisimov, A. A. Shvedov, V. T. Sorokin, A. V. Demin, V. V. Kashcheev, V. V. Iliin,

T. A. Feofanova, HI "VNIPIET".

Technological Aspects of MChC Pu-containing Sludges Vitrification Using MW Melter (B506233),

K. G. Kudinov, A. A. Tretyakov, Yu. P. Sorokin, V. V. Bondin, L. F. Manakova, MCC K-26... 51

Plutonium-containing Wastes Immobilization Activities Accomplished Under LLNL's Contracts at Krasnoyarsk-26 (B506233), K. G. Kudinov, A. A. Tretyakov, V. V. Bondin,

Yu. P. Sorokin, L. F. Manakova, MCC K-26. .55

Overview of the Development of Phosphate Glasses for Immobilizing Pu-containing Sludge at MCC, (B506233), G. B. Borisov, A. V. Nazarov, O. A. Mansourov, Y. Y. Volchok, A. A. Balashov, VNIINM ...... 65 
Borosilicate Glass Matrix Technology Developed for MCC (B506233), A. S. Aloy, B. S. Kuznetsov, E. Yu. Ivanov, A. V. Trofimenko, KRI..

Ecological and Economic Substantiation of Technological Decisions and Comparative Analysis of MCC Glasses for Long-term Storage of Immobilized Sludges in Underground Tanks for JOI (B506233),

T. A. Gupalo, V. P. Beygul, S. L. Speshilov, VNIPIPT

Status of Near Contour Zones of Underground Construction for Long Underground Isolation of Plutonium containing Pulps of MCC (B506233), V.S. Gupalo, VNIPIPT.

\section{II.2. Mayak Site Technical and Economic Engineering Feasilbility Studies of Weapons Plutonium Sludge Immobilization and MOX Fuel Fabrication Plutonium Wastes}

Process Optimization for Plutonium-containing Sludge Reprocessing at RT-1 Plant (B506201), S. I. Rovny, A.S. Skobtsov, PA "Mayak". .93

ECS for Immobilization of Plutonium-containing Materials at the PO Mayak (B506208), V. M.

Simanovskiy, B. V. Gusakov, A. A. Shvedov, V. T. Sorokin, A. V. Demin, V. V. Kashcheyev, S. V. Romanov, V. V. Ilyin, VNIPIET

Technological Control of Bottom Sediment Conditions in Storage Reservoirs (B506201),

S .I. Rovny, G. M. Medvedev, N. M. Emelianov, FSUE PA "Mayak", Ozersk 105

The Technology of Alpha-active Materials Incorporation in Glassy and Mineral Matrixes (B506201),

S. I. Rovny, V. I. Guzhavin, FSUE "Mayak" PA".

Immobilization of Pu-containing Sludge Using Ceramic Melters: Experiments in Support of the EFS at PA "Mayak" (B506206), G. B. Borisov, O. A. Mansourov, A. V. Nazarov, A. V. Balashov Y. Y. Volcho, VNIINM

The KRI Approach for Developing Pu Immobilization Technology for PA Mayak (B506209)

A. S. Aloy, E. B. Anderson, I. V. Smirnov, B. E. Burakov, KRI, St. Petersburg ...

Immobilization of AM-241 Formed under Plutonium Metal Conversion into Monazite-type

Ceramic (B506209), A. S. Aloy, E. N. Kovarskaya, T.I . Koltsova, S. E.Samoylov, KRI; S. I. Rovnyi,

G. M. Medvedev, PA "Mayak", L. J. Jardine, LLNL

Immobilization of Weapon Plutonium-containing Materials and Waste of MOX Fuel Fabrication in the Russian Federation (B506240), V. L. Rozhkov, E. I. Tyurin, Yu. F. Kolotilov, GSPI.

Sverdniichimmash Experience and Capabilities for Participation in the Contract on Weapons-grade Plutonium Immobilization Generated from MOX Fuel Fabrication, R.S. Karimov,

OSC "Sverdniichimmash".

\section{II.3. RIAR Site Studies}

Experience on Russian Military Origin Plutonium Conversion into Fast Reactor Nuclear Fuel, A. Grachev, O. Skiba, A. Bychkov, A. Mayorshin, V. Kisly, D. Bobrov, A. Osipenco, L. Babikov, V. Mishenev, RIAR 
Preliminary Technical and Economic Justification for Treatment and Immobilization of Pyrochemical Residual and Waste Containing Plutonium in RIAR (B506204), L. G. Babikov,

A. V. Bychkov, A. N. Lukinyh, RIAR.

\section{Research and Development for Plutonium Immobilization and Other Related Activities}

\section{III.1. Plutonium Glasses for Immobilization}

Development of Matrix Compositions and Process of Immobilization for Hard-to-Process Pu-containing

Sluges of "Mayak" (B506228), Yu. I. Matyunin, VNIINM

Development and Study of Borosilicate Glasses for Immobilization of Pu-containing Sludges and Industrial Waste of MOX Fuel Mix (B512152, B506216), A. S. Aloy, V. S.Belov, O. A. Iskhakova, T. I.

Koltsova, A. V. Trofimenko, KRI....

Immobilization of Am-containing Waste: Status of Implementing the Work for Agreement B514481, G. B. Borisov, O. A. Mansourov, V. F. Gorbunov, V. S. llyashenko, VNIINM; A. A. Minaev, D. G. Kuznetsov, IPC RAS.

Melting Diagrams of High-temperature Insoluble Compounds as Matrices for Immobilization of REE and Am Radionuclides (B514481), D. G. Kuznetsov, A. A. Minaev, IPC RAS;

G. B. Borisov, O. A. Mansourov, VNIINM

\section{III.2. Plutonium Ceramics for Immobilization}

Summary of Pu Ceramics Developed for Pu Immobilization (B506216, B512161),

B.E. Burakov, E.B. Anderson, KRI

\section{III.3. Accelerated Radiation Damage Studies of the US Pu Ceramic Formulation}

Investigation of Radiation and Chemical Stability of Titanate Ceramics Intended for Actinides Disposal (B501111), A. N. Lukinykh, S. V. Tomilin, A. A. Lizin, A. V. Bychkov, RIAR

Investigation of Accelerated Radiation Damages of the US-specified Pyrochlore-like Ceramics Containing ${ }^{238} \mathrm{Pu}$ (B506205), I. A. Kulikov, A. A. Vashman, V. M. Filine, T. N. Ananina, V. E. Samsonov, O. Y. Panov, VNIINM ....

KRI Radiation Damage Studies of the US Pu Ceramics (B506203), B. E. Burakov,

E. B. Anderson, KRI

\section{III.4. Geologic Disposal for Immobilization of Weapons Plutonium and Other Pu-containing Materials}

Development of an RF Geologic Repository, L. J. Jardine, LLNL 303

Perspectives When Using New Technology for Pu-containing Wastes Management in the Nuclear Industry, V. D. Safutin, I. L. Rybalchenko, VNIPIET 
Work Organization for Creation of RW Geological Storage in Russia (B512155),

T. A. Gupalo, VNIPIPT

Plan for Geological Repositories for Disposal Of Immobilized Plutonium-Containing Materials and other Radioactive Materials at Russian Industrial Sites (B512154), E. B. Anderson,

V. G. Savonenkov, S. I. Shabalev, KRI

Problems of Underground Disposal of High-level Radioactive Waste and Long-term Storage of Spent Nuclear Fuel in Russia: Status Assessment and Position of the IGEM RAS, V. I. Velichkin, V. A. Petrov,

N. N. Tarasov, IGEM-RAS

Complex Program of Engineering and Geologic Surveys for Designing RW Storage Construction in Hard

Rock Massifs (B512155), T. A. Gupalo, V. L. Milovidov, O. A. Prokopova, VNIPIPT

Basic Provisions of the Plan for Creation of Underground Radwaste Isolation Facility in MCC and PA

"Mayak" Areas (B512155), T. A. Gupalo, O. A. Prokopova, V. L. Milovidov, V. P. Beygul, VNIPIPT ....... 351

Preliminary Results of Research on Plutonium and Uranium Migration in Bentonite Buffer and Biotite Gneisses in the Form of Complex Radioactive Contamination (B512134), T. A. Gupalo, S. L. Speshilov,

VNIPIPT; A. A. Tretyakov, I. V. Shramko, S. A. Manakov, MCC

\section{III.5. Other Invited Papers}

State Regulation of Nuclear and Radiation Safety for Activities Related to Immobilization of Surplus Russian Weapons-Grade Plutonium, G. B. Borisov, O. O. Manturov, VNIINM, V. M. Iriushkin, A. A. Stroganov, GAN.

Features of Micro- and Macrostructural Characteristics of Ferrosilicate Fusion Cakes, V. N. Muzgin, A. A. Pupyshev, Ural State Technical University; S. I. Rovny, V. I. Guzhavin, PA “Mayak”... 383

Comparative Radiation Resistance of Cubic Fluorite Structure Phases for Actinides Immobilization, S. V. Stefanovsky, O. I. Kirjanova, RADON; S. V.Yudintsev, IGEM RAS; J. Lian, R.C. Ewing, University of Michigan

Chemical Restrictions to Formation of the Actinide Hosts with Pyrochlore Structure, S.V. Yudintsev, IGEM

RAS, S.V. Stefanovsky, Radon, R.C. Ewing, University of Michigan

Investigation of Plutonium, Americium and Curium Phosphates as a Basis for Inclusion into Kosnaritetype Ceramic Waste Forms, A. I. Orlova, A. A. Charlamova, Nizhni Novgorod State University; Yu. F. Volkov, RIAR

Alkaline Tank Chemistry of Plutonium: Speciations, Redox Reactions and Structural

Transformations, I. G. Tananaev, B. F. Myasoedov, RAS-GEOKHI

Long-lived Radionuclides Immobilization into Mineral-type Matrices by a Self-propagating High-

Temperature Synthesis (SHS), E. M. Glagovsky, A. V. Kouprin, VNIINM; S. V. Yudintsev, IGEM-RAS, L.P. Pelevin, VNIINM, E. E. Konovalov, State Scientific Center of Russian Federation "A. I. Leipunskiy Institute of Physics and Power Engineering" 
Scientific Investigation of Spent Nuclear Fuel Encapsulated into Glass-Ceramic Protective Forms, S. Yu. Sayenko, I. M. Neklyudov, G. A. Kholomeyev, A. P. Pilipenko, A. E. Surkov, B. A. Shilyayev, R. V.

Tarasov, S. V. Gabelkov, E. P. Shevyakova, V. I. Zmiy, Zh. S. Azhazha, NSC-KIPT.

\section{Closing Session and Summary}

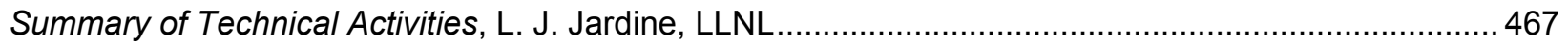

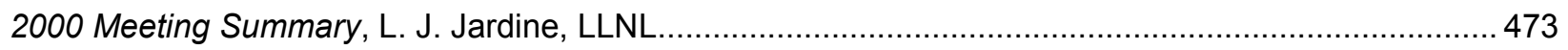

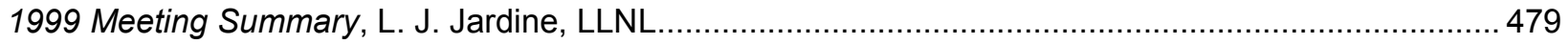

\section{Appendices}

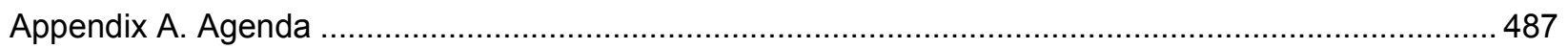

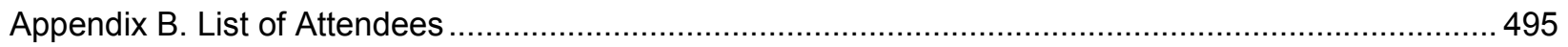

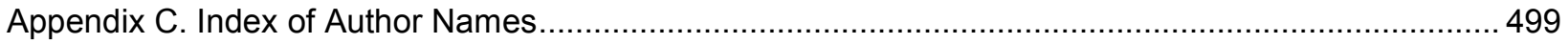




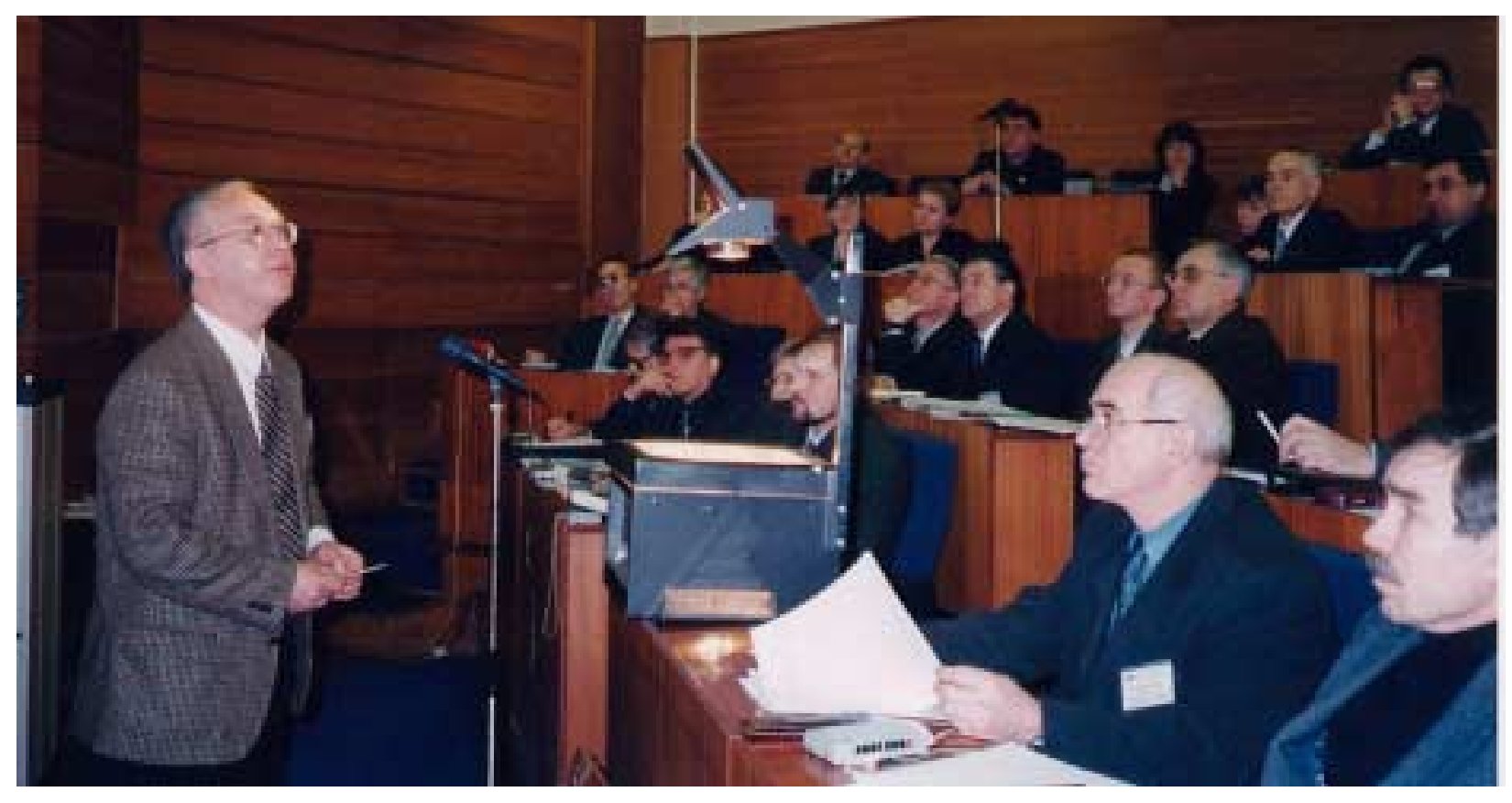




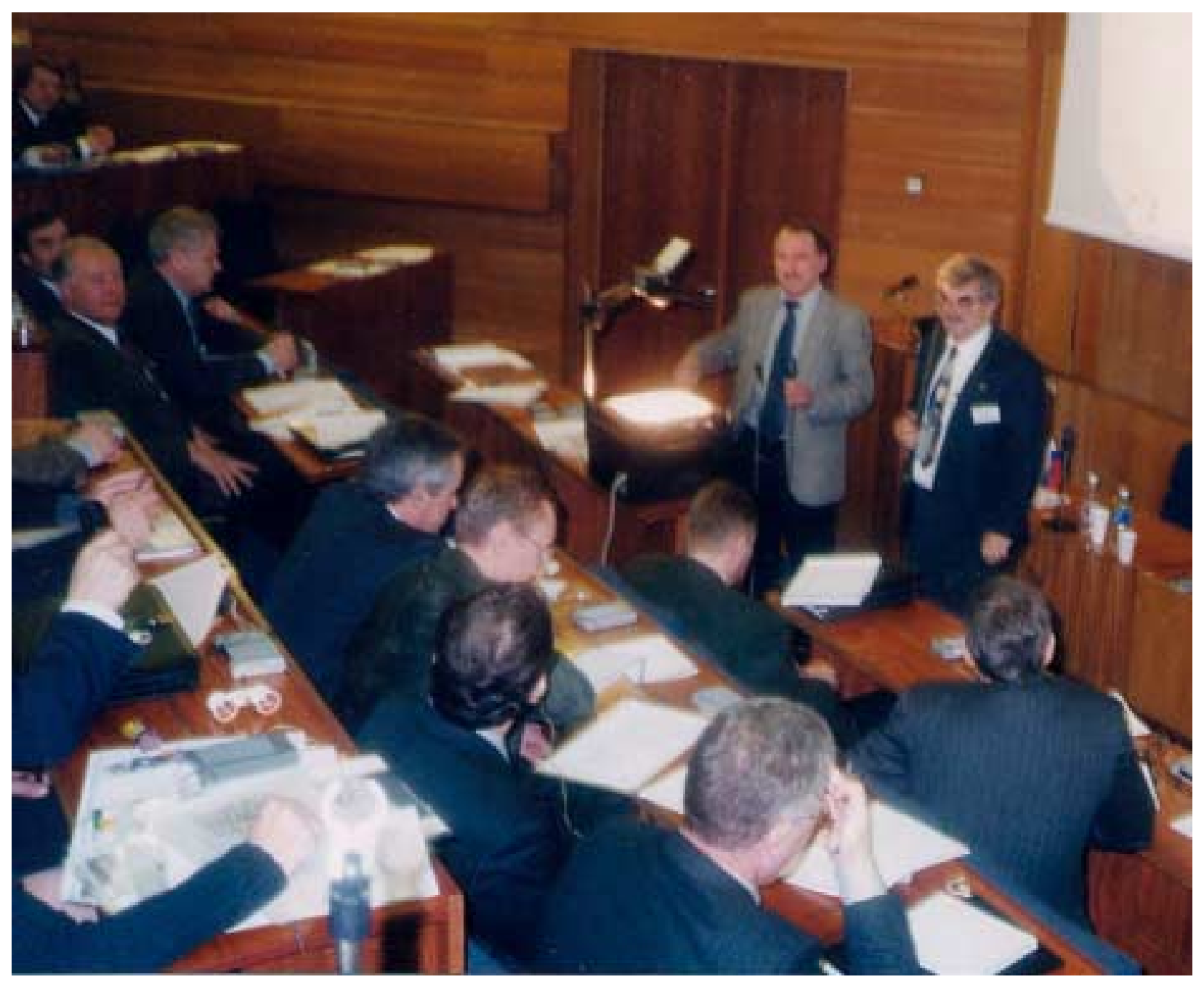

I. Opening Session 

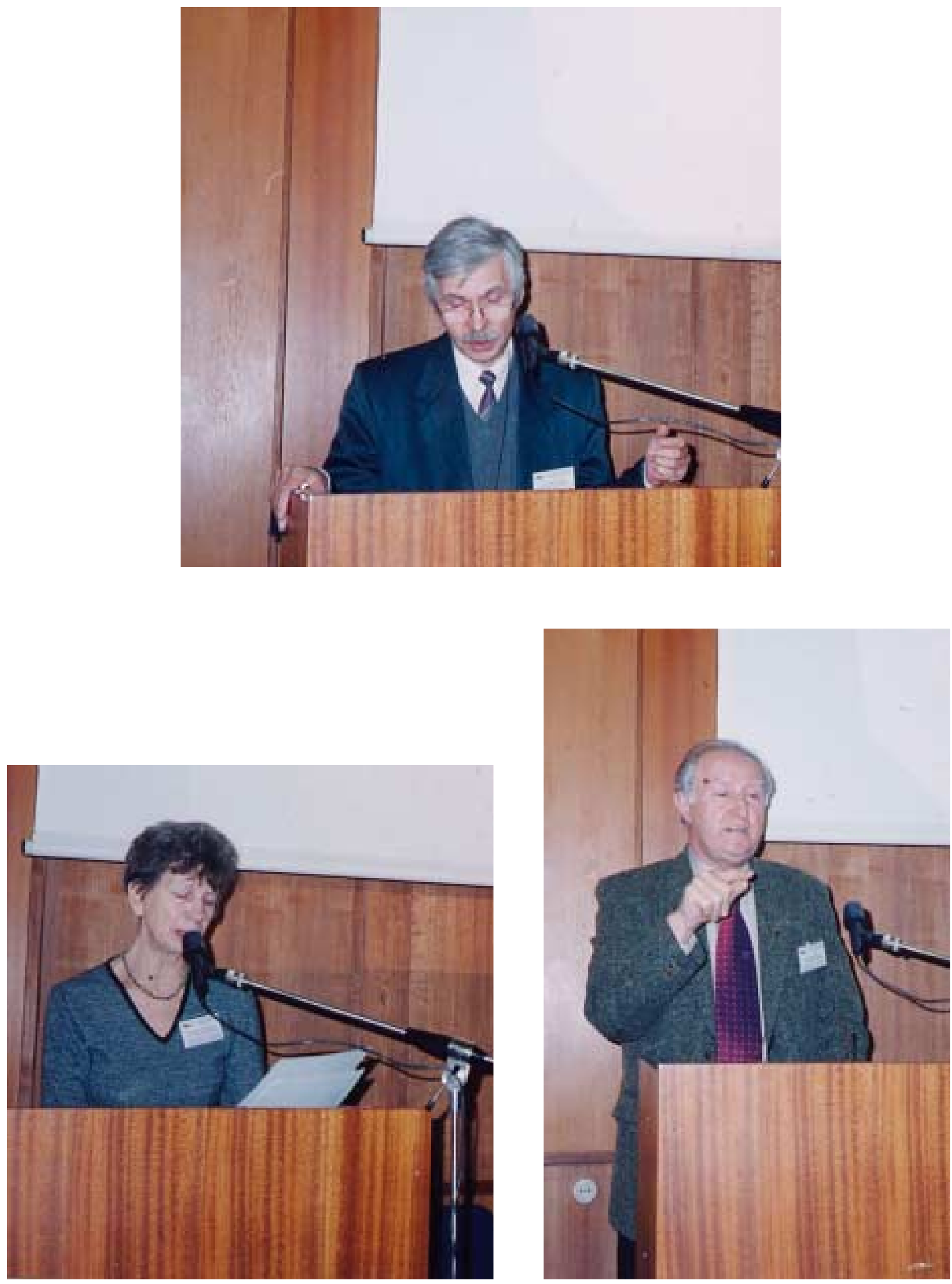


\section{Introductions and Objectives}

\section{J. Jardine, LLNL}

Two previous successful Russian Plutonium Immobilization Projects Review meetings have been held during November 1999 and November 2000 in St. Petersburg. Those meetings summarized the technical work completed and the implementation status of the Russian Pu Immobilization Program contracts. To date, over forty-nine Lawrence Livermore National Laboratory (LLNL) contracts totaling over \$7M involving plutonium immobilization; spent fuel storage and transportation; and plutonium storage, packaging, and transportation have been issued to numerous Russian scientific and design institutes and industrial sites. Over two hundred deliverables and technical reports have been generated by eight Russian organizations, including A. A. Bochvar All-Russia Scientific Research Institute of Inorganic Materials (VNIINM), AllRussian Research and Design Institute of Production Engineering (VNIPIPT), V.G. Khlopin Radium Institute (KRI), Scientific Research Institute of Atomic Reactors (RIAR), All-Russian Design Research Institute of Complex Power Technology (VNIPIET), State Specialized Design Institute (GSPI), and the Krasnoyarsk-26 (K-26) and Mayak Industrial Sites. Two technical proceedings of these previous meetings have been published $[1,2]$.

This third meeting of the recently completed and ongoing Russian plutonium immobilization contract work was held at the State Education Center (SEC) in St. Petersburg on January 14-18, 2002. The meeting agenda is reprinted here as Appendix $A$ and the attendance list as Appendix $B$. The meeting had 58 Russian participants from 21 Russian organizations, including the industrial sites (Mayak, Krasonayarsk26, Tomsk), scientific institutes (VNIINM, KRI, VNIPIPT, RIAR), design organizations (VNIPIET and GSPI), universities (Nyzhny Novgorod, Urals Technical), Russian Academy of Sciences (Institute of Physical Chemistry or IPhCh, Institute of Ore-Deposit Geology, Petrography, Mineralogy, and Geochemistry or IGEM), Radon-Moscow, S\&TC Podol'osk, Kharkov-Ukraine, GAN-SEC-NRS and SNIIChM, the RF Ministry of Atomic Energy (Minatom) and Gosatomnadzor (GAN). This volume, published by LLNL, documents this third annual meeting. Forty-nine technical papers were presented by the Russian participants, and nearly all of these have been collected in this Proceedings.

The two objectives for the meeting were to:

1. Bring together the Russian organizations, experts, and managers performing this contract work into one place for four days to review and discuss their work amongst each other.

2. Publish a meeting summary and proceedings of all the excellent Russian plutonium immobilization and other plutonium disposition contract work in one document so that the wide extent of the Russian immobilization activities are documented, referencable and available for others to use, as were the Proceedings of the two previous meetings [1,2].

Attendees gave talks describing their LLNL contract work and submitted written papers documenting their contract work (in English and Russian), in both hard copy and on computer disks. Simultaneous translation into Russian and English was used for presentations made at the State Region Educational Center (SEC). 


\section{List of Organizations and Facilities Represented by Meeting Attendees}

\begin{tabular}{|c|c|}
\hline $\mathrm{KRI}$ & V. G. Khlopin Radium Institute - St. Petersburg \\
\hline VNIPIET & $\begin{array}{l}\text { All Russian Designing and Scientific Research Institute of Complex Power Technology - St. } \\
\text { Petersburg }\end{array}$ \\
\hline GSPI & Ministry of Atomic Energy of Russian Federation State Specialized Design Institute - Moscow \\
\hline RADON & Scientific and Industrial Association - Moscow \\
\hline RAS-GEOKHI & Vernadsky Institute of Geochemistry and Analytical Chemistry - Moscow \\
\hline RAS-ICP & Russian Academy of Sciences, Institute of Physical Chemistry - Moscow \\
\hline RAS-IGEM & $\begin{array}{l}\text { Russian Academy of Sciences, Institute of Geology of Ore Deposits, Petrography, Mineralogy } \\
\text { and Geochemistry - Moscow }\end{array}$ \\
\hline VNIINM & A. A. Bochvar All-Russian Research Institute of Inorganic Materials - Moscow \\
\hline VNIPIPT & All Russian Research and Design Institute of Production Engineering -Moscow \\
\hline GAN & Federal Nuclear and Radiation Safety Authority of Russia, Gosatomnadzor of Russia \\
\hline GAN-SEC-NRS & Engineering Center for Nuclear and Radiation Safety \\
\hline $\mathrm{K}-26$ & Ministry of Atomic Energy Mining Chemical Combine - Krasnoyarsk-26, Zheleznogorsk \\
\hline KHARKOV & National Science Center Kharkov Institute of Physics and Technology - Ukraine \\
\hline MINATOM & Ministry of the Russian Federation for Atomic Energy \\
\hline RIAR & Research Institute of Atomic Reactors - Dimitrovgrad \\
\hline SChC & $\begin{array}{l}\text { Russian Federation Ministry of Atomic Energy, Siberian Group of Chemical Enterprises - } \\
\text { Tomsk, Serversk }\end{array}$ \\
\hline SNIIChM & Sverd Engineering and Fabrication, Inc. - Yekaterinburg \\
\hline USTU-UPI & Urals Technology University - Yekaterinburg \\
\hline S\&TC & Lutch Ceramics Podol'osk \\
\hline UNG & Nyzhny Novgorod State University \\
\hline
\end{tabular}

\section{References}

1. L. J. Jardine, G. B. Borisov, Excess Weapons Plutonium Immobilization in Russia: A Review of LLNL Contract Work, Lawrence Livermore National Laboratory, Livermore, CA, UCRL-ID-138361, 2000.

2. L. J. Jardine, G. B. Borisov, Immobilization of Excess Weapons Plutonium in Russia: A Review of LLNL Contract Work: Lawrence Livermore National Laboratory, Livermore, CA, UCRL-JC-143846, 2001. 


\section{Immobilization of Surplus Weapons Plutonium in Russia: Opening Remarks ${ }^{1}$}

\section{A. Orlova, Nyzhny Novgorod State University, L. J. Jardine, LLNL}

The work in the area of immobilization of waste from weapons plutonium in Russia, performed by Russian specialists under contracts signed between Russian organizations and the Lawrence Livermore National Laboratory, has been traditionally discussed in scientific Seminars. For two years, these Seminars have been regularly (annually) held in St. Petersburg, Russia. The present Seminar is the third one. The results of this Seminar can be assessed more thoroughly if one sets forth from the results published for the two preceding seminars in 1999 and 2000. [1,2]

Russian-American cooperation in the area of surplus weapons plutonium was begun after President Clinton's speech at the UN in 1993 and the subsequent Clinton-Yeltsin summit in Moscow in January of 1994. Thereafter, the activities of the convened technical groups were concentrated on the needs of the Russian enterprises engaged in the production of plutonium (NPO "Mayak", the Mining Chemical Combine K-26, and the Tomsk Combine).

The collaboration between LLNL and the Russian organizations in the Russian program of plutonium immobilization started in May of 1997. In July of 1998, an agreement was signed between the Governments of the RF and the US on scientific and technical cooperation in the area of disposition of plutonium removed from military nuclear programs. At the same time, some 20 contracts were signed with LLNL related to this same issue.

As we can see, once begun, the scientific and technical collaboration has been expanding as witnessed by this seminar's presentations on the results of ongoing studies. In our opinion, the format used for the annual discussion of the results has been justified and was very beneficial to the Russian attendees

Today, one would like to look back and comment on the past seminars of 1999 and 2000 as the precursors of today's activities. It should be noted that in working on the problems, we have encountered a number of difficulties. However, positive results stand out as predominant.

The major portion of the contracts is being performed at such industrial enterprises as the NPO "Mayak" and the MCC K-26, along with collaborative work with leading scientific and research institutes of the Ministry for Atomic Energy of the RF. This work is in the area of immobilization of Pu-containing pulps and sludges that have been accumulating in the course of the production of plutonium. Added to this are the issues associated with the anticipated waste from planned production of mixed oxide (MOX) uraniumplutonium fuel. Engineering cost studies have shown that direct immobilization at the MCC of the plutonium pulps containing $600 \mathrm{~kg}$ of plutonium is economically more profitable and ecologically more advantageous than would be the extraction of this plutonium. To resolve this problem, and to minimize costs and schedules, use is made of radiochemical systems available at the MCC-26 and the existing infrastructures. Several versions of waste vitrification facilities are being developed. At the MCC, a laboratory ultra-high frequency (UHF) or microwave heating facility has been set up in a hot cell to develop techniques for vitrification of Pu-containing pulps; a technological process has been proposed for the solidification of pulps (the volume of which is some $6,000 \mathrm{~m}^{3}$ ).

\footnotetext{
${ }^{1}$ Based on previous seminars in 1999 and 2000 and the published proceedings [1,2].
} 
The following technical, environmental, and economic aspects of alternative approaches to plutonium imobilization work at the PO "Mayak"are being studied:

1. Extraction of plutonium from pulp waste;

2. Direct immobilization of pulps (the project envisions reprocessing of waste, containing approximately one ton of weapons plutonium, jointly and by using technologies of the KRI and VNIINM. Methods and solutions developed together with GSPI and VNIPIET that are applicable to waste from commercial production of MOX fuel are also being considered if the facility should be situated at the "Mayak" rather than at the MCC. Under consideration, also, is the approach of using a building in which both wastes would be treated and immobilized, i.e., wastes from MOX fuel production, and stored Pu-containing pulps. Experiments are being carried out to find solutions to a number of chemical problems associated with the technology of immobilization of highly active Pu-containing suspensions stored at the PO "Mayak".

At the RIAR site, the following techniques have been validated for the handling of $\mathrm{Pu}(\mathrm{and} \mathrm{U})$ wastes from pyrochemical technology of spent fuel regeneration and plutonium conversion:

1. Reprocessing and concentration based on reprocessing experience of fuel for reactors;

2. Concentration of the wastes for solidification, for example, by conversion into crystalline phosphate precipitates followed by inclusion into glass or ceramic matrices.

Engineering studies of geologic repository sites are in progress at the enterprises K-26 and the PO "Mayak", and at the VNIPIPT. The property of rock formations to adsorb radionuclides during the deterioration of glasses, including glasses containing plutonium, is being studied. Laboratory studies have been expanded to include the migration of radionuclides into actual rocks. The results of these studies are followed with interest by specialists outside of Russia. An on-site geologic repository approachat the MCC K-26 for solidified pulp wastes using bentonite is being explored. This approach may significantly lower the cost of immobilization and disposal of plutonium pulps. The Nizhnekansky granite massif is being studied as a geologic formation suitable for Russian waste disposal. At the PO Mayak, engineering studies are about to begin, as is engineering work on the creation of an underground laboratory.

To select an optimal method for reprocessing or treating Pu-containing waste, and to create pilot- and production-scale facilities for immobilization of this waste, materials are being investigated, along with methods for producing them, which would ensure a high chemical safety barrier against release and migration of plutonium and other radioactive waste contained in the facilities.

VNIINM and the NPO KRI presented the results of their studies and work under contracts on the synthesis of glass-like, glass-crystalline, and mineral-like ceramic materials containing plutonium and other actinides. Also being studied are chemical and phase compositions of the materials, the uniformity of the distribution of radionuclides within them, and their chemical stability. Research and Development $(R \& D)$ work is in progress on the optimization of the vitrification process of Pu-containing pulps at the Mayak and K-26 sites.

Throughout the entire period covered by existing contracts, RIAR, VNIINM, and the NPO KRI have been investigating US-developed ceramics suitable for the immobilization of plutonium. Samples of perovskitepyrochlore titanate ceramic have been synthesized with plutonium-238 to accelerate the radiation damage, crystallographically characterized (at RIAR), and delivered to VNIINM and NPO KRI for studying radiation damage. Threshold dose values were found for internal damage by alpha particles resulting in 
total amorphization of crystalline phases. Change in chemical composition of the product and in its leaching behavior was detected.

Also presented at the seminars were reports, not supported by contracts, on the results of investigations of new materials for plutonium immobilization, and new approaches to synthesizing such materials.

Under one of these contracts, the RIAR transferred to LLNL a device for washing out aqueous solutions of salts from $\mathrm{PuO}_{2}$. The American side expressed its satisfaction with such forms of collaboration.

In conclusion, we present some statistics of our previous seminars:

- In 1999 and 2000, 44 Russian specialists participated in each of the seminars from 15 and 14 different Russian organizations, respectively; Twenty-seven persons participated in the work of both seminars, and 13 organizations were represented by their experts at both seminars. Eight of the organizations are engaged in LLNL contract work.

- In 1999, the number of contract-related reports was 35. In 2000, it was 36. In 1999, there were two initial reports of new work, whereas in 2000 this number went up to six. It should be noted that, counting this year's program, the number of reports of this kind keeps growing from year to year. This reflects the fact that the stature of the seminar is growing: from a working meeting it has advanced to a scientific conference. It would be good to request the organizers to invite American scientists to participate at these seminars with their scientific reports as well.

Brief publications of the contents and proceedings of the 1999 and 2000 Seminars on the Immobilization of Pu Wastes in Russia appeared in the periodicals "Radiokhimia" RAN $(2000,2001)$ and "Voprosy radiacionnoi bezopasnosti " (Issues of Radiation Safety) of the PO "Mayak" (2001). This helps the Russian specialists in obtaining information on the cooperative efforts of Russia and the USA in this field. Moreover, since the periodical "Radiokhimia" is published abroad (in English), this information rapidly reaches specialists in other countries. We express our appreciation to the editorial staffs of these periodicals for having given our Seminar this opportunity.

Our thanks also to all the presenters who participated in the preparation of seminar materials for publishing. This was an outstanding seminar with many excellent technical presentations.

\section{References}

1. L.J. Jardine, G.B. Borisov, Excess Weapons Plutonium Immobilization in Russia: A Review of LLNL Contract Work, Lawrence Livermore National Laboratory, Livermore, CA, UCRL-ID-138361, April 15, 2000.

2. L.J. Jardine, G.B. Borisov, Immobilization of Excess Weapons Plutonium in Russia: A Review of LLNL Contract Work, Lawrence Livermore National Laboratory, Livermore, CA, UCRL-JC-143846, April 2001. 


\section{Pu-containing Materials Management in Russia: Status and Perspectives}

\section{G. B. Borisov, VNIINM}

The US decision not to include the immobilization efforts in Russia in the framework of the US-Russia Agreement in the field of weapons-grade plutonium management signed in 2000 puts those activities in Russia in an uncertain state.

This verdict, based upon a few experts' opinion in the US, is a thoughtless decision (E. Leman in the article "The future of immobilization under the US-Russia Agreement on excess weapons plutonium, Indiana Wells, 2001). Then, the US next decided in 2001 to halt any immobilization activities in the US and to dismantle a demonstration facility for Pu-containing ceramics fabrication at LLNL.

Therefore, the US made a crucial turn away from the appeal of "global" immobilization of plutonium to a complete cancellation of the alternatives for plutonium disposition. The situation is also complicated by the absence of any records about waste treatments generated from plutonium metal conversion and MOX fuel fabrication in the 2000 Agreement. This omission is flawed because the waste treatment operations are one of the most important elements of the conversion and MOX fuel fabrication processes that must be addressed early. For example, output values and environmental impacts are dependent upon what kind of waste treatment process is selected.

At present, the US nevertheless is flexible to fund only waste treatment from the conversion and MOX fuel fabrication operations. But, hereby one needs to mention about various different approaches in plutonium processing practices in the US and Russia. At Russian industrial sites, the practice is still active of deep recovery of weapons-grade plutonium from the liquid wastes (up to 40-100 $\mu \mathrm{g} / \mathrm{l}$ ) prior to borehole disposal as is accepted practice at the Mining and Chemical Combine (MCC) or near-surface pond dumping at Karachay lake near PA "Mayak". As a result of sludge recovery and eventual processing, the recovered surplus plutonium might be accumulated at MCC and PA "Mayak" storage vaults and might be out of declaration requirements of any international agreements on fissile materials control. The PA "Mayak" will subsequently accumulate and recover more than 1.5 Mt excess weapons-grade plutonium; meanwhile the MCC will amass about $600 \mathrm{~kg}$ of weapons material. All recovery techniques are available at these sites for such operations. Thus, the accumulation of excess weapons-grade plutonium should be a point of concern of the international community because it is not under any international agreement.

On the other hand, if the plutonium remains stored in the tanks of the MCC and PA "Mayak", it will cause a serious environmental impact due to the potential danger of waste leaking from the aging tanks that are approaching their design life. The immobilization of the sludge without plutonium recovery would resolve both the environmental problems of the Russian enterprises and resolve non-proliferation issues of weapons plutonium.

Some of results generated during the work made between the LLNL and Russian entities, as well perspectives of future cooperation, are presented in Figs. 1 and 2.

Figure 1 gives flow-sheets in the main processes of Pu-containing materials treatment at MCC, PA "Mayak", RIAR, and SCC. The schemes specifically include some wastes (aqueous tails) generated from 
the Chemical-Metallurgical Productions at PA "Mayak" and SCC. This could be a good example of combined treatment of wastes from the conversion and MOX fuel fabrication processes and sludges.

At the MCC, experimental and design efforts are at the justification of investment (JOI) stage of completion, including the treatment and immobilization of the Pu-containing sludge and its eventual geologic disposal. At the PA "Mayak", work is in progress of comparative assessments of the Pucontaining sludge either with or without plutonium recovery. It is anticipated that this engineering technical feasibility study will be finished at the beginning of 2002. Herewith, it is necessary to point out that the phase or stage of the justification of investments at the PA "Mayak" needs to be implemented also.

The final goal of work related to sludge recovery and immobilization is to design and build either demonstration or full-scale waste treatment facilities at the MCC and PA "Mayak". Realization of this would make it possible to relieve the concern of excess weapons-grade Pu accumulation in Russia.

It is well known that a plutonium conversion demonstration facility is now under development and design at PA "Mayak". A modified facility "Paket" for MOX fuel fabrication for reactor BN-600 is also being created. All our future immobilization activities will likely shift to treatment of Pu- and Am-containing wastes generated from conversion operations (including oxalate and pyroelectrochemical precipitation) at the PA "Mayak", as well as from MOX fuel fabrication at either PA "Mayak" or MCC industrial sites (Figs. 1, 2).

While developing these waste treatment processes, it is necessary to assess the Am-containing wastes from the oxalate precipitation metal conversions operations. High-level wastes from spent nuclear fuel (SNF) reprocessing at RT-1 are immobilized in the ceramic melter EP-500. This melter would allow the vitrification of the toxic and hard-to-deal with Am-containing wastes and deal with the problem of corrosion of fluorides contained in the previously mentioned conversion wastes.

The MOX fuel fabrication wastes are specifically varied in type and have no substantial volumes. An exception is metal wastes, such as metal tackling, balls, molybdenum trays, etc. To compact the metal wastes, induction-slag remelting in a "cold" crucible could be proposed. This technique would also be used for processing glass fibers and lab glassware.

Due to the US cancellation of continuing the Russian plutonium immobilization efforts, we have encountered a so-called "vacuum" that needs to be filled up by new alternative routes. One such alternative is to build up an infrastructure of all activities associated with functioning of the plutonium conversion and MOX fuel fabrication facilities. The infrastructure would include the development of the system of loading/unloading $\mathrm{PuO}_{2}$ from available old-design containers at MCC and SCC to new ones produced in Russia with financial assistance from the US.

In Fig. 2, a summary reflects the up-coming activities on creating a dry storage facility in Russia and transportation means for shipping fresh and irradiated MOX fuel over the Russian territory.

However, those efforts cannot be a full alternative to the immobilization of Pu-containing materials in Russia. Herewith, we encourage the possibility of Germany taking part in funding to some extent the immobilization efforts in Russia. But, at the same time, we are dubious that the German initiative would completely substitute for the US as both an immobilization technology developer and as an investor.

If any trilateral Agreement is signed, some reliable results in this field could be achieved. The US may continue the support of R\&D activities of immobilizing the wastes at the MCC, PA "Mayak", and SCC pursuant to the Agreement on scientific-technical cooperation signed in 1998 should it choose. 


\begin{tabular}{|c|c|c|c|}
\hline Site & Waste type & Treatment & Storage and disposal \\
\hline
\end{tabular}

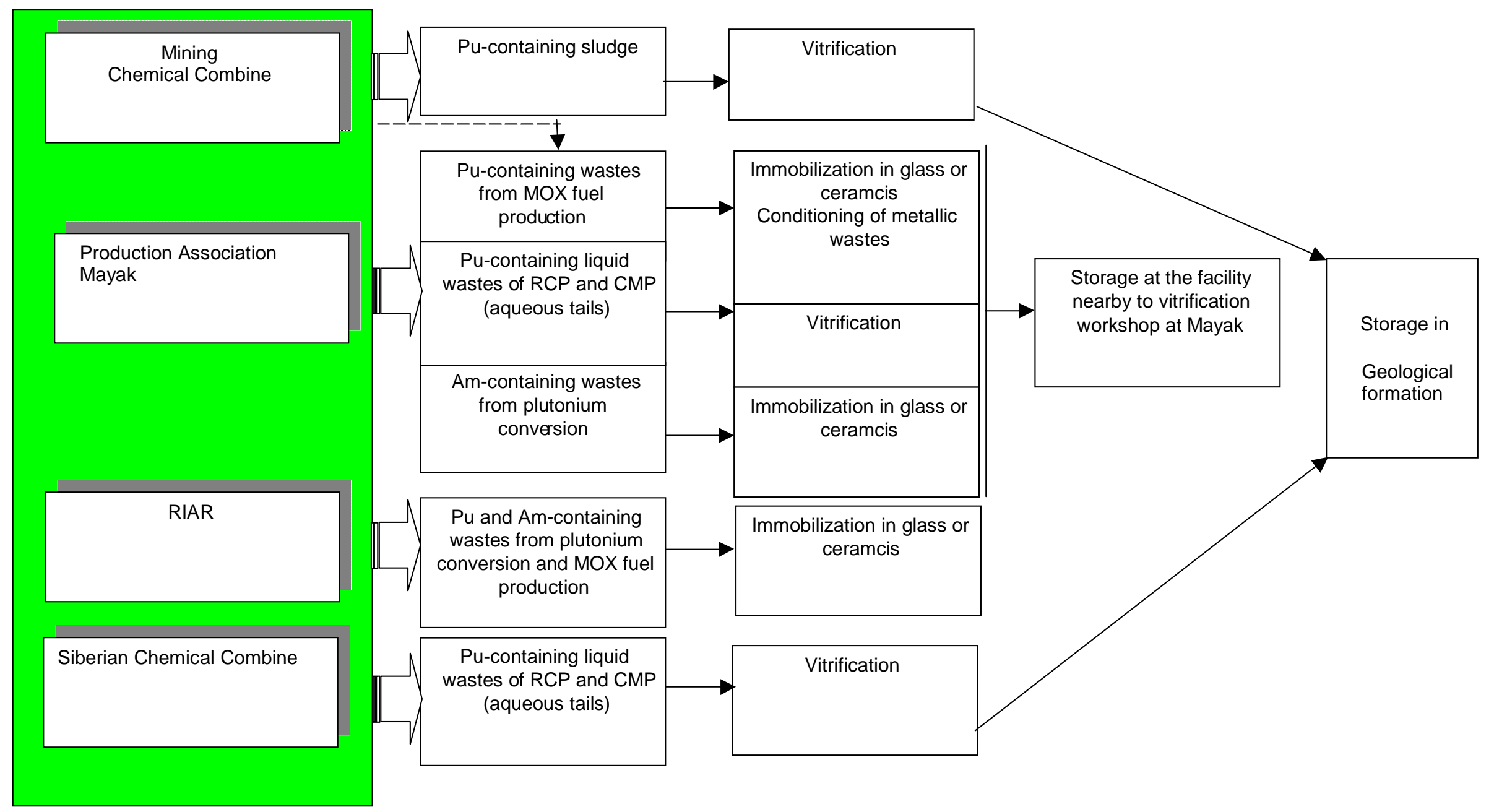

Fig. 1. Treatment of Pu-and Am-containing wastes at MINATOM sites. 
The USA-Russia agreement about scientifictechnologic cooperation in the field of excess weapons-grade plutonium management signed in 1998

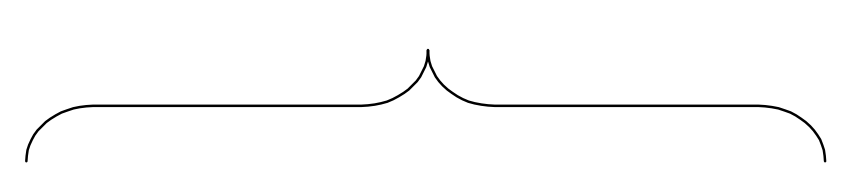

Development of immobilization technologies, matrices and physicochemical properties of immobilized forms during storage and disposal

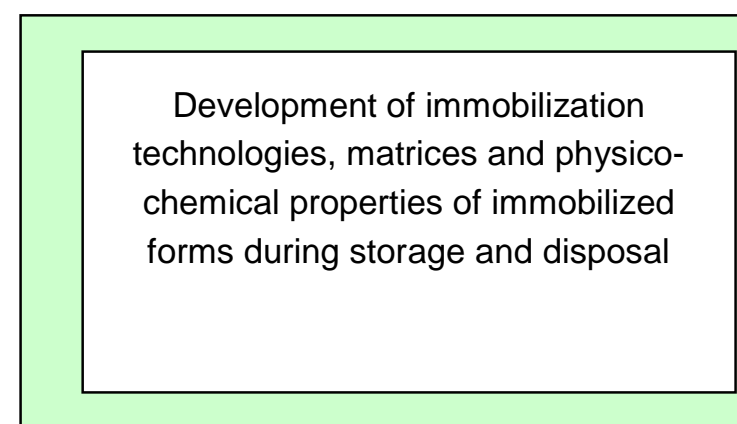

The USA-Russia agreement on weaponsgrade plutonium management withdrawn from defense nuclear programs signed in 2000.
Any intergovernmental agreement on collaboration in the field of Pu-containing wastes treatment (Russia, USA, Germany).
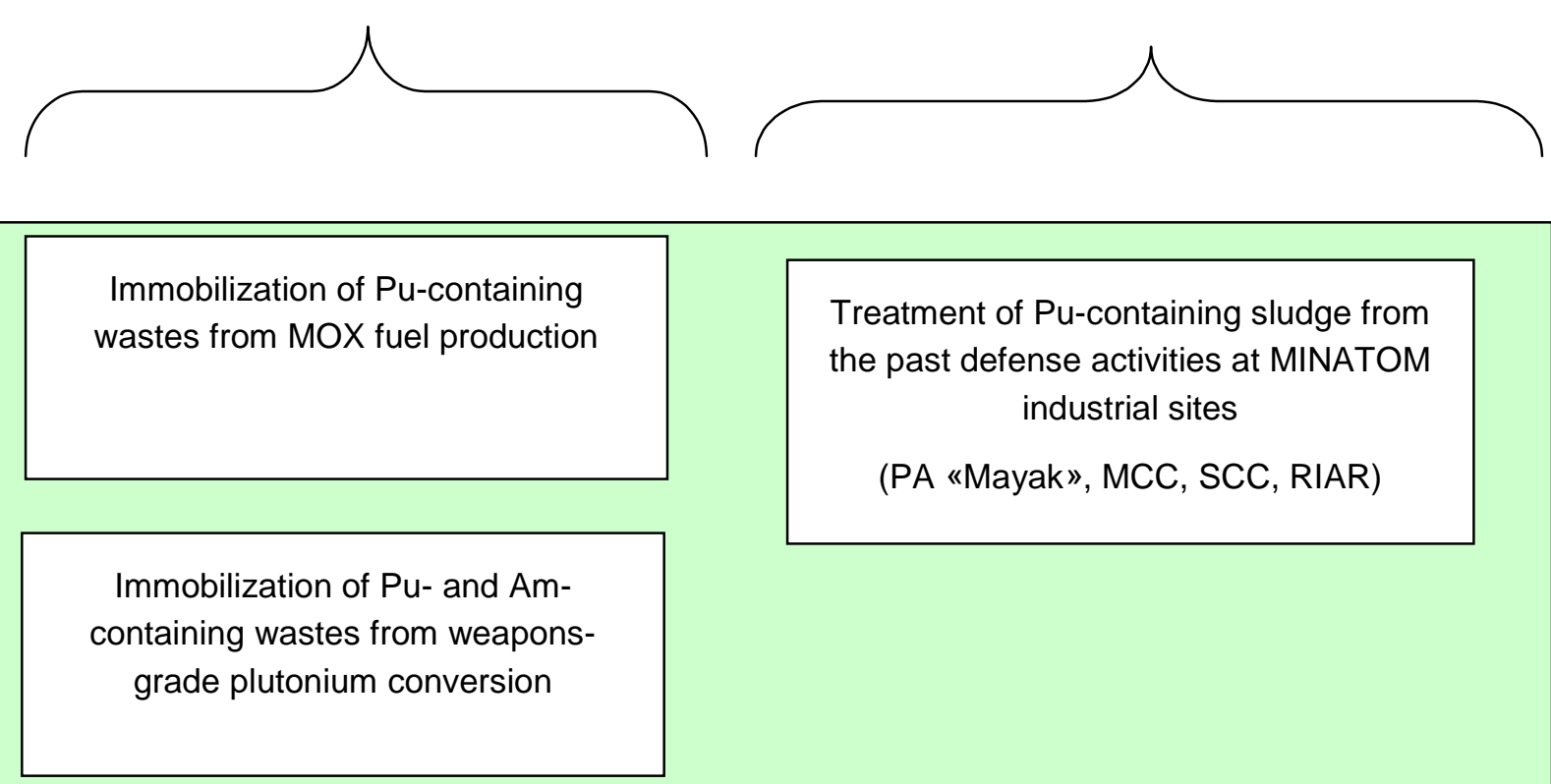

Treatment of Pu-containing sludge from the past defense activities at MINATOM industrial sites

(PA «Mayak», MCC, SCC, RIAR)

Activities in the support of dry storage packaging and transportation for $\mathrm{PuO}_{2}$,

$\mathrm{Pu}$, fresh and irradiated MOX fuel

Fig. 2. Treatment (immobilization) of Pu-containing materials in Russia: status and perspectives. 


\title{
Packaging, Storage and Transportation of Plutonium, Plutonium Oxide and Unirradiated MOX Fuel (B512166)
}

\author{
A. V. Afanasyev, A. L. Lazarev, A. I. Tokarenko, VNIPIET
}

\section{General provisions}

The use of mixed uraniumfresh -plutonium fuel in power reactors requires assurance of safe transportation of components of mixed oxide (MOX) fuel, fuel elements, and fuel assembles. For research on the safety aspects, it is necessary to take into account that the thermal and radiating characteristics and also the fissile properties of MOX fuel are higher than the characteristics of fuel based on uranium dioxide. These factors require additional measures for development of technology of transportation and design of packages for MOX fuel.

According to Russian "Basic rules of safety and physical protection of transportation of nuclear materials" (OPBZ-83), "Regulations of the safe transportation of radioactive materials" by IAEA and other normative documents, the safety of transportation should be ensured by design of transport packages.

VNIPIET has carried out preliminary technical and economic studies of transportation of various kinds of MOX fuel, which aimed to determine the direction of work on use of existing transport containers and development of new ones.

Most probably, the transition to MOX fuel will require essential re-design of available transport packages or creation of new ones. This work will require considerable financial expense and take a long time; therefore, it should already be underway, so that there would not be a delay of deliveries of experimental and serial MOX fuel assemblies to nuclear power plants (NPPs). Besides, it is necessary to elaborate the variants of the transport and technological schemes of manipulation with MOX fuel at the NPP for the VVER-1000 and BN-600 reactors.

\section{Contents of Work under the Contract with LNLL}

The contract (B512166) stipulates the complex engineering study be performed of existing methods of packaging, interim storage, and transportation of plutonium, depleted uranium and mixed oxide fuel, and also includes the scope of needs for packaging, storage, and transportation of fresh MOX fuel and its components.

As a result of the work, methods and technologies of packaging, storage and transportation of fresh MOX fuel, available in Russia, and necessary new or refined methods and technologies should be determined and proved by documents. New methods and technologies should include an estimation of existing design of packages and equipment and various ways of transportation by railway and road, and also the possible routes that can be used. In addition, one of the results of the work should be a list of research and development work for realization of a program of handling fresh MOX fuel. It is necessary also to determine and formalize by documents the needs of changes or additions to federal norms and regulations and an order of receiving the permissions for transportation.

Five tasks are included in the scope of work under the contract: 
Task 1. Packaging, storage and transportation of metallic plutonium, plutonium dioxide and other reactorgrade plutonium mixtures from a storage place to a factory of chemical conversion and reprocessing for manufacture of MOX fuel.

It is necessary to estimate and formalize by documents the available technologies and methods of packaging, storage and transportation of 25 tons of metallic plutonium of weapon quality within the Russian Federation. It is necessary to estimate existing methods used for transportation of metallic plutonium from the storehouse for fissile materials at "Mayak" to a factory for chemical conversion and reprocessing. It is necessary also to estimate existing methods used for transportation of metallic plutonium from Tomsk to the RIAR chemical conversion and reprocessing factory.

It is necessary to estimate available methods and technologies of packaging, storage and transportation of 9 tons of $\mathrm{PuO}_{2}$ either from Tomsk or from MCC to a factory of chemical conversion and reprocessing. It is necessary to estimate the use of a universal design of packages for metallic plutonium and plutonium dioxide.

It is necessary to estimate and formalize by documents the available methods of packaging, storage and transportation of approximately 4 tons of oxides or other kinds of reactor plutonium for mixing with plutonium of weapons quality at a chemical and conversion processing factory.

Task 2: Packaging, storage and transportation of depleted $\mathrm{U}_{3} \mathrm{O}_{8}$ or other kinds of uranium to a factory for manufacturing MOX fuel.

It is necessary to estimate and formalize by documents the available methods and technologies of packaging, storage and transportation of 50 tons (annually) of various kinds of uranium, which can be used as initial material for a new factory for manufacturing of MOX fuel.

Task 3: Packaging, storage and transportation of $\mathrm{PuO}_{2}$ to the plant of $\mathrm{MOX}$ fuel manufacturing.

It is necessary to estimate and formalize by documents the available methods and technologies of packaging, storage and transportation of 38 tons of $\mathrm{PuO}_{2}$ from a factory of chemical conversion and reprocessing to the new plant for manufacturing of MOX fuel (MCC or "Mayak).

Task 4. Packaging, storage and transportation of MOX fuel rods and assemblies to reactors of the Russian Federation.

It is necessary to estimate and formalize by documents the available methods and technologies of packaging, storage and transportation of non-irradiated fuel assembles of fresh MOX fuel, and also the routes from MCC or "Mayak" to reactors, presumably VVER-1000 and BN-600. In addition, the estimate should include methods used for packaging, storage and transportation of fresh fuel assembles from RIAR to reactors BOR-60 and BN-600, and also fresh fuel elements of MOX fuel from "PAKET" ("Mayak"), and MSZ (Electrostal) to the reactor BN-600.

Task 5. Plan of work and proposals for further work.

Development of the plan of further work on packaging, storage and transportation of metallic plutonium, plutonium oxide, and fresh MOX fuel.

It is necessary to carry out the plan of work containing a description of the work required for realization of the concepts stated in the first four tasks. Except technical-economical substantiation, the plan can include other technical work, research work development, and tests. At the center of work assumed in the future, there should be concepts of technology of packing, storage and transportation of metallic 
plutonium, plutonium oxide, and fresh MOX fuel using available and future Russian methods of packing, storage and transportation developed under the given contract.

\section{State of work under the contract.}

The contract was signed by LLNL and VNIPIET in September, 2001. According to the order of export control of Minatom of Russia, the contract was directed to the Export Council of Minatom for approval. At the present time, positive conclusions from departments of Minatom have been received. Within January, 2002, the conclusions should be considered by the Export Council and put in order as the final approval of the work. After receiving this approval and solution of financial issues with LLNL, the work under the contract will be officially started.

January 11,2002 


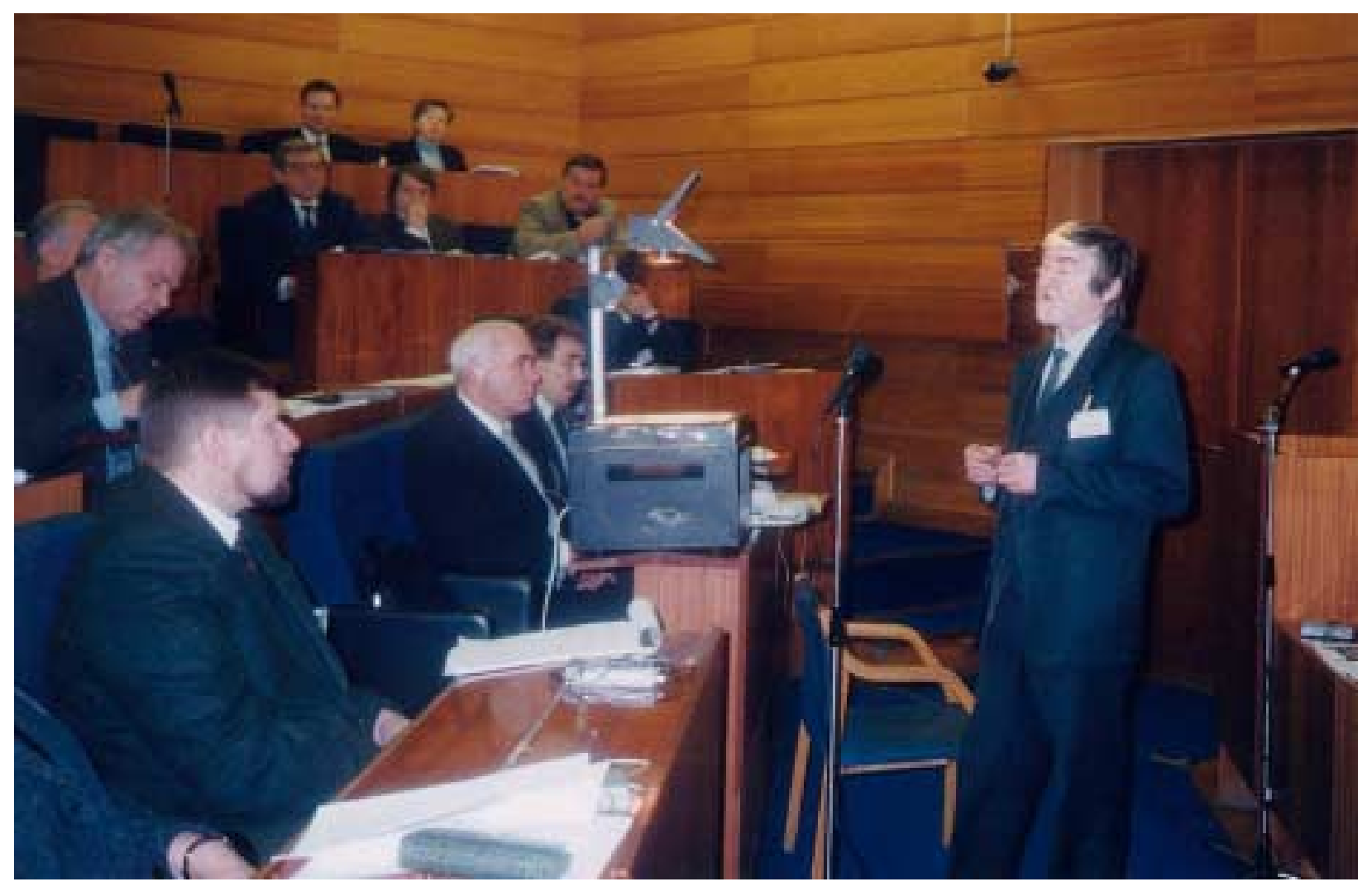




\section{Issues of Storage, Packaging and Transportation of Weapons-grade Plutonium Dioxide for the Mayak Conversion Facility}

\section{E. M. Glagovsky, VNIINM}

Initially, here are a few thoughts related to the problem of storage and transportation of plutonium dioxide. As it is well known, of the $34 \mathrm{Mt}$ to be disposed of under the scope of the USA/Russia Intergovernmental Agreements on excess weapons-grade plutonium management, $25 \mathrm{Mt}$ is plutonium metal, and about $9 \mathrm{Mt}$ is $\mathrm{PuO}_{2}$. Surplus plutonium metal is presently stored at $\mathrm{PA}$ "Mayak", whereas $\mathrm{PuO}_{2}$ is stored at $\mathrm{MCC}$, Zheleznogorsk, and SCC, formerly Tomsk-7.

Herewith it is necessary to mention that $\mathrm{PuO}_{2}$ is placed in containers that do not meet up-to-date safety requirements for storage and transportation. Moreover, the design of the containers requires that every 3 years, the gaskets are to be replaced. Due to radiation processes taking place in the plutonium oxide, the containers are overpressurized; therefore, the replacement procedure seems to be complicated and environmentally dangerous.

The MCC and SCC storage depots are overfilled and the whole $\mathrm{PuO}_{2}$ must be finally packed into new containers and sent to PA "Mayak" for eventual conversion. Before that, the shipped $\mathrm{PuO}_{2}$ must be tested and certified. At PA "Mayak" the containers with the stuff are to be passed through so-called entry surveillance by means of analogue procedures and instrumentation units as it is done at MCC and SCC prior to shipment. Obviously, both the containers and packing and uploading units must be unified or certified at all the enterprises, which will take part in the weapons-grade plutonium disposal programs, including ones that will produce MOX fuel. Hereby, definite issues related to storage and transportation of $\mathrm{PuO}_{2}$ are as follows:

1. Certification of container AT-400R intended for storage and transportation of plutonium dioxide.

2. Development and manufacturing of typical facilities intended for packing, loading and unloading of $\mathrm{PuO}_{2}$ at (a) MCC and SCC storage depots; (b) conversion facility; and (c) MOX fuel production facilities.

3. Adaptation of the theNDA system being currently developed for the conversion facility for its usage at MCC and SCC storage depots, as well as the MOX fuel production workshops.

4. Assessment of the possibility of using fissile materials storage facility at PA "Mayak" for intermediate storage of $\mathrm{PuO}_{2}$ delivered from MCC and SCC for the conversion complex. At present, the storage facility is designed for holding plutonium metal in the protection container AT-400R.

5. Definition of issues related to safe transportation of $\mathrm{PuO}_{2}$ from the conversion complex to $\mathrm{MOX}$ fuel production plants. The dioxide will be equaled and averaged in composition and differentiated from weapons-grade plutonium because at one of the conversion stages, commercial and weapons-grade plutonium will be blended according to the signed plutonium disposition Agreement. That would change the requirements imposed on storage and transportation of the original weapons plutonium material. 


\section{Conclusions}

What kind of specific issues should be resolved with regards to storage, packing, and transportation of $\mathrm{PuO}_{2}$ for the conversion facility in near future?

- What are alterations occurred in physico-chemical properties of $\mathrm{PuO}_{2}$ in the process of long-term storage? Such tests are currently in progress under the framework of the Agreement with a Los Alamos National Laboratory (LANL) contract.

- How do the pressure and composition of gaseous medium change in a sealed container used for $\mathrm{PuO}_{2}$ storage and transportation?

Besides, the following essential things should be also carried out:

- Development of the scientific-technical bases and a design of an internal cask of smaller dimensions and compatible with the protection container AT-400R made in cooperation between the US and Russia.

- Development of the NDA system of plutonium metal and dioxide being delivered to the conversion complex and then to the MOX fuel production plants. 


\section{Progress of an Engineering Cost Study on Utilizing the Existing Minatom RF Infrastructure for Storage of Irradiated MOX Fuel (B506234)}

\section{O. P. Anisimov, N. S. Tikhonov, V. V. Spichev, N. P. Shafrova, VNIPIET}

Since our last meeting concerning the establishment of a dry storage facility for anticipated MOX spent nuclear fuel (SNF), the LI "VNIPIET" (All-Russian Design Research Institute of Complex Power Technology ) has received approval from the Export Council of the Minatom RF (Resolution \# 02-6188 of 12/04/2001) to conduct an engineering cost study (ECS) on the feasibility of utilizing the existing infrastructure of the Russian Federation for storage of MOX SNF. The Resolution, approved by the Chairman of the Export Council and First Deputy of the Minister of Atomic Energy of the Russian Federation, V.B. Ivanov, contains general comments which reflect the position of Russia with regard to the handling of surplus weapons plutonium no longer needed for defense purposes in accordance with the Intergovernmental Agreement between the United States and Russian Federation. These comments do not change the overall content of the work planned under the LLNL Contract B506234. It should be stated, however, that, in conformance with the comments of the Export Council of the Minatom RF, we have excluded from our discussions the SNF of the reactors of the RBMK-1000 and VVER-440 type, since this type of fuel is not part of the project of generation and handling/storing of the MOX. This issue may be discussed by us separately, outside the framework of the Contract B506234, and within the context of the aforementioned Intergovernmental Agreement.

Given the nature of the problem, a detailed discussion of the scope of the Statement of Work (SOW) for the ECS is in order.

\section{Description of Planned Actions Agreed Upon in the Statement of Work for the Conduct of the ECS}

\section{Introduction}

The SOW for recovery of fissile materials defines the work to be performed based upon:

- The Agreement on Scientific and Technical Cooperation in the disposition of plutonium removed from the nuclear military program signed on July 24, 1998 by the Governments of the USA and the RF;

- Jointly defined principles for the treatment and recovery of plutonium no longer needed for defense purposes signed by W. Clinton and B, Yeltsin on August 20, 1996, and

- A Joint Declaration of August 20, 1996, signed by the US Department of Energy Head, Hazel O'Leary and the Minister of the Minatom of the RF, Victor Mikhailov.

In August of 1999, a preliminary discussion took place concerning the problems associated with the storage in Russia of SNF from power generation and of MOX fuel that will be produced in the future from surplus weapons plutonium and that will have to be stored in Russia for several decades. This discussion led to the conclusion that, if one of the mentioned approaches for the storage of future MOX SNF were to utilize as fully as possible the existing infrastructure for nuclear power-generation fuels, a detailed ECS would have to be performed to obtain a detailed description, assessment, and comparison of existing 
technological capabilities for the storage of SNF in Russia. The ECS would be the first step towards a detailed assessment of the possibilities in the area of nuclear technology and the beginning of a formal description of a nuclear storage facility in the RF, the selection of a construction solution that could be recommended for the construction of a storage facility for MOX spent fuel. Before the development of an Engineering Cost Justification (ECJ or JOI), a preliminary evaluation (i.e., the ECS or TEF) should be made of the following:

- The existing capabilities/methods of storage of MOX spent fuels and of nuclear fuels used in power generation;

- Existing and anticipated quantities of SNF from power-generating reactors in Russia;

- Existing and planned storage facilities for the MOX-SNF and methods in use in Russia;

- Existing and planned methods of transporting nuclear fuel throughout Russia in order to unify the SNF storage among various production plants (with locations in the RF where power-generation nuclear fuels are stored).

The ECS will correlate the data on nuclear fuels used for power generation in the Russian Federation with different technical variations in its storage and transportation. This will facilitate the start of the ECJ work and will provide a good documentation base for its subsequent completion.

The results of this ECS will shorten the data collection stage required for the following ECJ and, during the subsequent stage of the ECJ, will eliminate doubts regarding the quality of the collected data by providing descriptions of all possible options for SNF storage and transportation in Russia, together with a selection of preferred storage options for MOX spent fuel.

In accordance with the signed LLNL contract B506234, the LI "VNIPIET" is the primary contractor for this ECS and, as such, may recruit other Russian enterprises at its own discretion.

In summary, one may state that the results of this ECS will be used as initial data for the next, more thorough development stage of the ECJ.

A description of the future work.

The objective of this work (the ECS) is to verify and document the estimates for the following:

- The current and anticipated quantity of MOX spent fuel;

- The existing technologies for the storage and transportation of MOX spent fuel;

- The existing RF infrastructure to assess the capabilities for safe and economical storage and transportation of future quantities of MOX spent fuel obtained from the recovery of surplus weapon plutonium no longer needed for defense purposes.

Based upon the SOW under consideration, the VNIPIET shall prepare an ECS that will:

1. Describe the specifications for existing and future storage systems for MOX SNF in the RF;

2. Analyze possible alternate versions of dry and wet storage;

3. Evaluate the options for the transportation of MOX SNF in Russia;

4. Analyze as thoroughly as possible the existing infrastructures in the RF used for the storage and transportation of SNF;

5. Identify the options for future methods of storage and transportation of MOX spent fuel. 
The ECS is to collect all the data, according to the type of reactor, pertaining to:

- The storage and transportation of SNF from all Russian nuclear power plants (NPP) whose facilities are to be used for storage of SNF;

- Existing and projected quantities of SNF up to the year 2040;

- Existing and planned options for the storage of MOX SNF together with uranium fuel of standard enrichment.

The ECS will summarize and confirm by documentation the existing and predicted quantities of SNF intended for wet storage, capacities and capabilities of existing VVER-1000 reactors and of the BN-600 reactor, both of which are viewed in Russia as suitable for burning the MOX fuel. The ECS is to include a survey of methods of handling the spent fuel from power-generating reactors at the MCC and the PO "Mayak".

This ECS will define and document the characteristics of the storage facilities for the SNF, and will consider the possibility of storing the future MOX spent fuel together with the existing regular types of SNF. The ECS will also evaluate the concepts of high-density storage in holding pools and will assess the decisions on the adoption of new methods of dry storage at various reactor sites and industrial plants in the RF.

It is proposed that the ECS include a comparative evaluation of possible versions of transporting the entire future volume of MOX spent fuel to new dry-storage SNF facilities designed to accommodate the total volume of SNF of NPP in the Russian Federation, for example, the two industrial sites: the MCC and the PO "Mayak". The ECS will also compare the alternative of locating the facility on one storage site outside of the reactor (or possibly on two construction sites, such as at the MCC and the PO "Mayak", where the new, dry-storage technology would be used) with several storage sites at the NPP. The reason being that combining the MOX spent fuel with uranium SNF of the NPP may significantly reduce the costs of storage and simplify the issue of non-proliferation.

The forthcoming effort will make it possible to make recommendations on preferred methods of storage and transportation of MOX-SNF for the subsequent ECJ stage. The study group will work in close contact with the management of the plants involved.

The work is to consist of five basic stages (tasks):

Task 1. A survey and assessment of the status of storage and transportation of SNF from NPP in the RF.

Task 2. An assessment of different versions of long-term storage of power-generation SNF in storage facilities of NPP with VVER and BN reactors in storage facilities of the MCC and the PO "Mayak".

Task 3. An assessment of different versions of transportation for MOX spent fuel.

Task 4. Preparation of a combined report that includes results, recommendations, and conclusions based on the evaluations of the preliminary ECJ.

Task 5. Development of a work plan (road map) for the subsequent ECJ for the storage and transportation of MOX-SNF in the RF.

In addition, it is planned to include in the ECS a general work plan (road map) for the development of the future ECJ (Tasks 6a and 6b). 
A more detailed description of the tasks is shown below:

Task 1. A survey and status assessment of storage and transportation of SNF from NPP in the RF

The LI "VNIPIET" will prepare a technical report containing documented verification of the status of storage and transportation of SNF in the RF.

The report shall contain the following:

1.1 The locations of various NPP using different types of reactors (VVER-1000, BN-600) and the availability of roads and railroad lines.

1.2 General maps (overall plan diagram) of the sites, description of storage methods for regular spent fuel, handling methods, overall quantity of accumulated fuel, and the capacity of the storage facilities at each of the NPP sites under consideration.

1.3 Location diagrams, description of storage methods for regular SNF, its accumulated quantity, and the capacity of the storage facilities at the MCC and the PO "Mayak".

1.4 Diagrams for the transportation of SNF, including the availability of roads and railroad lines at storage facilities and between potential storage facilities at NPP and other storage facilities and reprocessing plants, as well as diagrams of shipping containers.

The objective of Task 1 is to confirm and document the following:

- Existing quantities of SNF;

- $\quad$ Storage and transportation methods for SNF from NPP;

- The existing infrastructure in the RF that would be used for subsequent tasks.

A preliminary technical report will be furnished in two months.

Task 2. An assessment of different versions of long-term storage of power-generation SNF in nuclear power plants with VVER, RBMK, and BN reactors in storage facilities of the MCC K-26 and the PO "Mayak".

The $\mathrm{LI}$ «VNIPIET» is planning to prepare a technical report containing an evaluation of different versions of long-term storage facilities for SNF of reactors VVER-1000 and BN-600.

The report shall contain the following:

2.1. Overall quantity of SNF being received for storage up to the year 2040, including MOX fuel.

2.2. An analysis of feasibility and plans for increasing the capacity of wet storage of SNF in NPP in the $\mathrm{RF}$, at the MCC, and at the PO "Mayak".

2.3. An assessment of future dry-storage technologies for SNF (designs, capacities, problems related to storage and a set of standards).

2.4. An evaluation of the transportation requirements for the SNF, including MOX spent fuel.

Russian storage technologies for SNF (dry and wet storage) are to be analyzed making use of publicly available sources. 
The evaluation will take into account the possibility of using American dry-storage technology so as to evaluate and possibly include one of its versions as an option in the forthcoming ECS on the storage of MOX spent fuel assemblies (SFA).

Within the framework of the present ECS, to assist the LI «VNIPIET», the LLNL will organize conferences for technical information exchange, will furnish written documentation, and will arrange visits to US drystorage facilities to facilitate familiarization with technical details and technical problems of control. This will permit, within the scope of the ECS, to assess the possibility of using American dry technologies in the RF and to take these alternatives into account for future ECJ work.

Since a need for expanded capacities is likely to arise, alternate methods must be explored to increase the storage density of existing holding pools in NPP using VVER-1000 and BN-600 reactors, and of (storage) sites external to the reactors.

The objective of Task 2 is to assess the requirements for different versions of long-term storage and transportation of the SNF, including the MOX fuel.

A preliminary technical report will be furnished in four months.

\section{Task 3. An assessment of different versions of storage of future MOX fuel.}

The LI "VNIPIET" is planning to prepare a technical report containing an evaluation of different versions of storage and transportation of future MOX-SNF.

The report shall contain the following:

3.1. A specific description of the MOX-SNF storage facilities including details and the specifications for the recommended versions.

3.2. A description of transportation methods and specifications for the recommended versions.

In making a selection among the different versions, the capabilities of the infrastructure of the Minatom will be used as much as possible for both storage and transportation of the MOX-SNF.

The objective of Task 3 is the selection of the appropriate versions for storage and transportation of the MOX-SFA.

A preliminary technical report will be furnished in four months.

\section{Task 4. Preparation of a combined report with results and conclusions of the ECS.}

The LI "VNIPIET" will prepare a final technical report comprising the assessments, findings and conclusions of the three preliminary technical reports for Tasks 1, 2, and 3 . The final report will provide recommendations for the subsequent ECJ on the selection of the alternatives for storage and transportation of the SFA, including possible alternatives for the application of American dry-storage technology in the RF. The report will list technical problems and problems related to control aspects that will have to be resolved in the course of the ECJ work.

The final technical report will be furnished in seven months. It will combine the findings and conclusions of the three preliminary technical reports on Tasks 1,2 , and 3. 
Task 5. Preparation of a work plan for future ECJ for storage and transportation of SNF.

A description of all work necessary for complete engineering-cost justification will be prepared. The work plan will include data on the cost, scope of work, dates when reports are due, and justification for the participants in the ECJ effort. Primary emphasis is to be placed on those options of storage and transportation of future MOX-SNF that use existing Russian and prospective versions of storage and transportation of MOX spent fuel. The work plan (road map) will show how to perform a cost estimate of the American technologies, defined in Task 2, in the ECJ. Contained in the plan will be various proposals for the implementation of long-term dry storage of MOX-SNF, including estimates of the costs and work schedules for the future. This document will provide the foundation for the financing of subsequent work.

The work plan will be provided seven months after the signing of the Agreement and will consider in detail the scope of the work by the LI "VNIPIET" in preparing the ECJ.

Task 6. Description and work schedules for the generation of a road map for various options of storage of spent fuel from power reactors in Russia.

\subsection{Objective}

The objective of the work is to define and document the participation of Russian organizations in the effort, to adopt key decisions and calculate the necessary financing and optimal work schedule. This would involve the following: (1) site selection, (2) selection of the storage technology, and (3) the use of a technology that would ensure safe and reliable storage of SNF in Russia, specifically from NPP with VVER-440 and BN-600 reactors. Maximum use should be made of dry-storage technologies being developed in Russia, since that will facilitate creating an optimized work schedule for long-term storage of SNF. As for intermediate, short-term storage, different wet-storage options must be evaluated together with [other] alternatives, including the reconfiguration of the racks in holding pools at the reactors and industrial sites of K-26 and the Mayak.

\subsection{Scope of work}

The LI "VNIPIET" will provide a documented description of the work with an integrated work schedule for it, and an estimate of the needed resources and effort required for:

(1) the selection of a site, (2) the selection of a dry-storage technology for the SNF, and (3) the implementation of the chosen technology on a selected work site in Russia (the construction, licensing, and startup and operation). Specifications for and description of the transportation systems for the SFA will also be developed.

Some American managers view documents of this type as "laying down the tracks" for a nuclear project. To produce an optimal work plan for long-term storage of SNF, maximum use must be made of the earlier engineering studies performed in Russia on the selection of a storage site for it. New technologies for dry storage may be evaluated from the point of view of an optimal work progression. The use of holding pools that exist [now] at the reactors or on industrial sites should be viewed as an opportunity to create intermediate storage facilities quickly, before the implementation of long-term ones. Preliminary estimates must be generated and documented for the fundamental decisions that need to be made, for resources required, and for time needed for work to be performed by Russian organizations whose participation is essential in the area of storage and transportation of SNF. 
During the work, the LI "VNIPIET" shall meet with representatives of the LLNL and other representatives of the Department of Energy of the US (DOE) to discuss the activities description and the task-completion schedules (integrated work schedule) which constitute the "road map". These descriptions and the integrated work schedule must contain data on project stages, participating organizations, and dates for the completion of Russian tasks. The finished documentation must comply with the RF requirements for standards and site selection approval, the establishment of intermediate and long-term SNF storage facilities, and a transportation system.

The draft of the report with the integrated work schedule and other documentation must be prepared within three months after the signing of the Agreement for the performance of the task.. The final report must be included in the final report for Task 4 . A meeting should be convened to discuss the progress of the work and the preparation of the project report.

\section{Deliverables}

Task 6a. The project report will be furnished 3 months after the signing of the Agreement. It will contain the final results of the collection and evaluation of data, including the computation of the schedules, basic decisions, Russian organizations, a glossary of terms, and other types of work within the scope of Task 6 .

Task $6 \mathbf{b}$. The final report will be furnished 7 months after the signing of the Agreement and will be included in the final report on Task 4, which will contain the final detailed results of data collected and an estimate for the participants (including the[ir] cost and the listing), the major [project] stages, a glossary of terms, and other items pertaining to the activities of Task $6 a$.

\section{Structure of the reports}

Each report will have an introduction with a brief summary of its content and a detailed list of references regarding the position of the present contract. Each report will contain information on the tasks corresponding to present statement of work.

The documents of the ECS (the reports) will be furnished to the Customer in English in the form of a hard copy and on electronic medium. 


\title{
Materials Science Challenges in Long-term Wet and Dry Storage of RBMK-1000 Spent Nuclear Fuel
}

\author{
M. I. Solonin, A. G. Ioltukhovskiy, V. P. Velioukhanov. I. M. Kadarmetov, VNIINM; \\ L. P. Sinel'nikov, A. N. Timokhin, O. A. Golosov, V. P. Kuznetsov, VNIPIET; \\ V. A. Tsykhanov, S. V. Pavlov, D. V. Markov, V. P. Smirnov, RIAR
}

\begin{abstract}
By now, in Russia more than 70000 fuel assemblies (FA) have operated their service life at NPPs with RBMK-1000 (11 units); the discharged FAs have been stored in water of cooling ponds and in spent nuclear fuels (SNF) storage facilities for 25 years.

During their operation, more than 3000 spent fuel assemblies became leaky while claddings of some integer fuel rods were subjected to a substantial wear (fretting corrosion up to $400 \mu \mathrm{m}$ in depth, nodular corrosion to a depth of $380 \mu \mathrm{m}$; both kinds of wear proceeded under spacer grids). In this case, the claddings revealed a higher hydrogen uptake and degradation of mechanical properties. During the wet storage in cans, spent FAs show general corrosion proceeding at the rate of 3-5 $\mu \mathrm{m}$ per year.
\end{abstract}

The paper points out that as applied to a long-term (up to 50 years) dry storage of RBMK-1000 spent nuclear fuel, consideration is to be given to the following degradation mechanisms proceeding in stainless steel fuel rod claddings and FA components:

- Oxidation of $\mathrm{Zr}-1 \% \mathrm{Nb}$ alloy (fuel rod claddings, central channel) in the atmosphere of storage (air, nitrogen, argon and impurities).

- Creep of fuel rod cladding material.

- Delayed hydride cracking (DHC) of a fuel rod cladding.

- Corrosion induced cracking of FA components fabricated from stainless steel.

The first results obtained in assessing the creep rate of standard fuel claddings affected by the internal pressure are under consideration.

\section{Introduction}

Currently, NPPs with RBMK-1000 have accumulated large amounts of spent nuclear fuel (more than 70000 SFAs). At the current rate of SFA accumulation, the available SNF storage facilities will be filled up after several years.

SNF is stored in cooling ponds at NPPs and in SFA storage facilities in water at NPP sites. This type of storage results in a noticeable degradation of a fuel rod cladding material (general and nodular corrosion, hydrogen uptake, embrittlement, etc.). In some instances, the properties of the materials of the central channel, spacer grids and other components of spent FA were substantially changed. These changes may involve problems in removing SFAs from storage facilities for subsequent reprocessing and increase radionuclide releases in water and the environment. Taking into account the above, it is important to determine exactly the tolerable time of "wet" storage, particularly of leaky SFAs. 
Subsequent storage is more preferable in "dry" storage facilities which, according to world-wide experience, are cheaper than cooling ponds (with all other conditions being equal: capacity, shipping and storage time, etc.).

However, to transfer SFA to dry storage (e.g., a container), the information has to be available on the condition of structural materials of SFA components after operation and long-term storage in water. One has also to assess the likely mechanisms of the material degradation in the process of dry storage. The combined knowledge of all these factors will promote the choice of the RBMK SFA dry storage conditions (temperature, environment, likely environmental impurities, etc.) and determine the ultimate storage time.

\section{Condition of RBMK-1000 Operated Fuel Assemblies}

RBMK-1000 spent fuel assemblies are supposed to be dry stored in concrete metal containers, TYK-104 and TYK-109. Fifty-seven SFAs may be accommodated in TYK-104 and 72 SFAs in TYK-109. The spent fuel assemblies are divided for dry storage into subassemblies for a total of 114 or 144 SFA subassemblies, respectively.

SFAs are planned to be transferred for dry storage after a 10-year holding period in water cooling ponds.

The power density of 114 SFA subassemblies makes some $5 \mathrm{~kW}$ (for 144 SFA subassemblies, it equals $6.3 \mathrm{~kW})$. The claddings temperature of ultimately heated fuel rods during storage is $180^{\circ} \mathrm{C}(\mathrm{TYK}-104)$ and $300^{\circ} \mathrm{C}$ (TYK-109).

Fuel discharged from a reactor contains several tens of radionuclides, many of which are highly aggressive to structural materials. Table 1 lists characteristics of some fission products contained in spent fuel of RBMK. Radiation characteristics of spent fuel are defined by enrichment, burn-up and holding time after a discharge from a reactor.

The residual power density of RBMK irradiated SFA is $\sim 0.1 \mathrm{~kW} / \mathrm{SFA}$ at $2 \%$ fuel enrichment and 10-year holding period in water (at the mean burn-up rate of $20.5 \mathrm{MW}$.day $/ \mathrm{kgU}$ ).

Some 4.5\% RBMK spent fuel assemblies are leaky.

The investigations implemented by SSC RF RIAR reveal that today the basic mechanism of fuel rod damage involves the interaction between fuel rod claddings and debris.

Table 1. Some characteristics of fission products of $2 \%$ enriched fuel held for 10 years.

\begin{tabular}{|l|c|c|c|}
\hline \multicolumn{1}{|c|}{ Radionuclide } & Half-life & $\begin{array}{c}\text { Activity, } \\
\text { decay/s,t }\end{array}$ & $\begin{array}{c}\text { Mass, } \\
\text { g/t }\end{array}$ \\
\hline Cesium-137 & 30,2 years & $2,3.10^{+15}$ & 713 \\
\hline Cesium-134 & 2,06 years & $1,4.10^{+14}$ & 2,8 \\
\hline Cesium-135 & $2,3.10^{+6}$ years & $9,3.10^{+9}$ & 218 \\
\hline Strontium-90 & 28,5 years & $1,5.10^{+15}$ & 300 \\
\hline lodine-129 & $1,6.10^{+7}$ years & $9,25.10^{+8}$ & 140 \\
\hline Cadmium-133 & 13,6 years & $3,7.10^{+11}$ & $4,3.10^{-2}$ \\
\hline Cerium-144 & 284,3 days & $3,4.10^{+12}$ & $2,8.10^{-2}$ \\
\hline Indium-116 & $5,0.10^{+15}$ years & $2,8.10^{+2}$ & 1,2 \\
\hline
\end{tabular}




\section{Generalization of Data on Post-Irradiation Examinations of RBMK-1000 Type Spent Fuel Assemblies}

During the past 18 years, we have been engaged in studying fuel rod fragments of more than 30 RBMK operated fuel assemblies that reached different burn-up rates.

The corrosion behavior of the fuel cladding material was judged from the thickness of a uniform oxide coat, the nodular corrosion depth and the remaining cross-section of a cladding determined from metallographic sections by optical metallography.

The rate of the cladding uniform corrosion on a wet storage varies within 3-5 $\mu \mathrm{m} / \mathrm{year}$, depending on the burn-up and storage time.

We reviewed the condition of fuel claddings mostly damaged after operation and water storage. Table 2 tabulates the results of corrosion studies.

The investigations evidence that at the burn-up of $1.3 \mathrm{MW}$.day $/ \mathrm{kgU}$, the surface of the RBMK-1000 fuel rod develops nodules up to $40 \mu \mathrm{m}$ depth and up to $150 \mu \mathrm{m}$ deep under spacer grids. At the fuel burn-up growth rate of up to $19.3 \mathrm{MW}$.day $/ \mathrm{kgU}$, these values increase to 130 and $380 \mu \mathrm{m}$, respectively. Within spacer grids, some fuel claddings demonstrate local thinning caused by fretting-corrosion. The thinning reaches $400 \mu \mathrm{m}$ in depth. The appearance of these fuel rods under spacer grids is shown in Fig. 1.

The given nodules depth and fretting-wear (reaching $400 \mu \mathrm{m}$ ) may substantially limit the mechanical strength of a cladding.

The hydrogen uptake by the fuel cladding material was insignificant (3-15) $\cdot 10^{-3} \%$ mass and caused by fuel cladding wear.
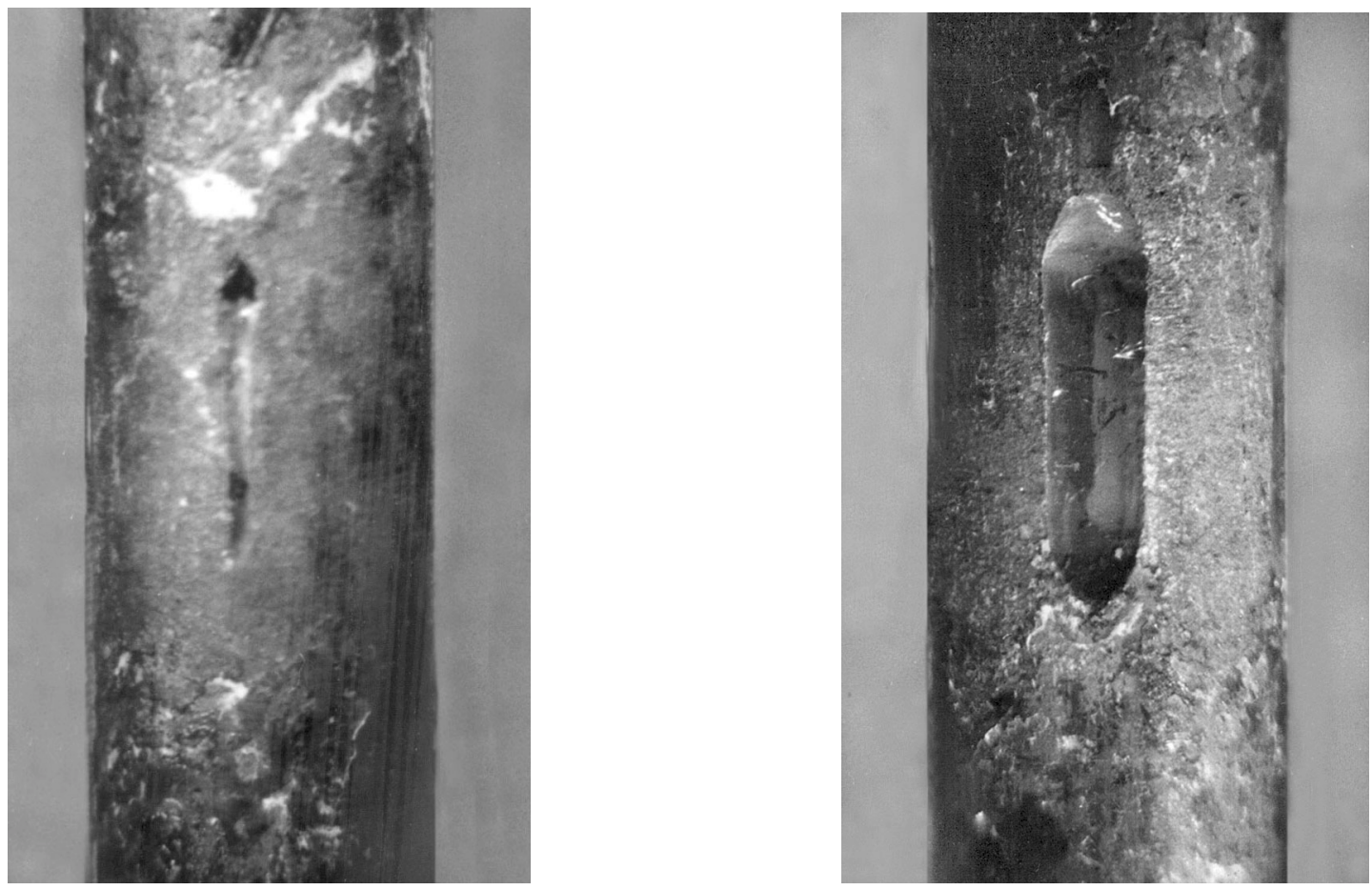

Fig. 1. Appearance of fuel rods under spacer grids. 
Table 2. Summary of data on corrosion properties of fuel claddings and content of hydrogen.

\begin{tabular}{|c|c|c|c|c|c|c|c|}
\hline \multirow[t]{2}{*}{$\mathbf{N}$} & \multirow{2}{*}{$\begin{array}{c}\text { B, } \\
\frac{\text { MW.day }}{\text { kg U }}\end{array}$} & \multicolumn{2}{|c|}{ Outside SG } & \multicolumn{4}{|c|}{ Under SG } \\
\hline & & $\begin{array}{c}\text { Thickness } \\
\text { of oxide } \\
\text { coat, } \mu\end{array}$ & $\begin{array}{c}\text { Depth of } \\
\text { nodules, } \mu\end{array}$ & $\begin{array}{c}\text { Thickness } \\
\text { of oxide } \\
\text { coat, } \mu\end{array}$ & $\begin{array}{c}\text { Depth of } \\
\text { nodules, } \mu\end{array}$ & $\begin{array}{l}\text { Depth of } \\
\text { fretting } \\
\text { area, } \mu\end{array}$ & $\begin{array}{c}\text { Hydrogen } \\
\text { content, } 10^{-3} \% \\
\text { mass }\end{array}$ \\
\hline 1 & 1,3 & $10-20$ & $0-40$ & - & $0-150$ & 0 & $3-6$ \\
\hline 2 & 19,3 & $15-20$ & $0-130$ & - & $0-380$ & 0 & $3-15$ \\
\hline 3 & 19,5 & $60-140$ & - & $90-250$ & $0-180$ & $300-400$ & $9-15$ \\
\hline 4 & 22,7 & $9-65$ & $0-90$ & $30-140$ & $0-190$ & 0 & $3-5$ \\
\hline
\end{tabular}

The tensile tests of fuel rod claddings were implemented at 20 and $350^{\circ} \mathrm{C}$ using annular samples $3 \mathrm{~mm}$ high and a tensile-testing machine at the cross-head speed of $3 \mathrm{~mm} / \mathrm{min}$. To calculate the strength properties, use was made of the nominal thickness of a fuel cladding equal to $0.9 \mathrm{~mm}$. The testing results are summarized in Table 3.

The data presented show that in spite of the significant wear of fuel claddings, their strength and plastic properties remain at a rather high level.

Table 3. Short-term tensile properties of fuel claddings.

\begin{tabular}{|c|c|c|c|c|c|c|c|c|c|}
\hline \multirow[t]{2}{*}{$\begin{array}{l}\text { \# of } \\
\text { SFA }\end{array}$} & \multirow[t]{2}{*}{$\mathrm{T}^{\circ} \mathrm{C}$} & \multicolumn{2}{|c|}{$\begin{array}{c}\sigma_{\mathrm{u}} \\
\mathrm{MPa}\end{array}$} & \multicolumn{2}{|c|}{$\begin{array}{c}\sigma_{0,2} \\
\mathrm{MPa}\end{array}$} & \multicolumn{2}{|c|}{$\delta_{\text {un }} \%$} & \multicolumn{2}{|c|}{$\delta_{\text {tot }} \%$} \\
\hline & & $\min$ & $\max$ & $\min$ & $\max$ & $\min$ & $\max$ & $\min$ & $\max$ \\
\hline \multirow[t]{2}{*}{1} & 20 & 598 & 608 & 567 & 581 & 1,0 & 3,5 & 14 & 22 \\
\hline & 350 & 369 & 429 & 347 & 414 & 1,3 & 4,9 & 17 & 20 \\
\hline \multirow[t]{2}{*}{2} & 20 & 573 & 608 & 507 & 566 & 2,3 & 3,2 & 11,7 & 12,8 \\
\hline & 350 & 414 & 425 & 350 & 402 & 2,2 & 3,2 & 14,9 & 17,6 \\
\hline \multirow[t]{2}{*}{3} & 20 & 563 & 592 & 540 & 562 & 1,5 & 4,0 & 13,1 & 14,2 \\
\hline & 350 & 355 & 397 & 283 & 359 & 2,9 & 5,0 & 13,8 & 16,7 \\
\hline
\end{tabular}

The following degradation mechanisms proceeding in fuel rod claddings and other SFA components in dry storage conditions are under consideration:

1. Interaction of fuel claddings and other components of spent fuel assemblies with the impurities $(O, N$, $\mathrm{H}, \mathrm{C}$, etc.) of possible gas environments (argon, nitrogen, air).

2. Thermal creep of fuel claddings induced by the internal pressure of transmutation gases and helium, allowing for corrosion damage of the outer cladding surface.

3. Delayed hydride cracking of fuel rod claddings and other components fabricated from zirconium alloys with a temperature gradient along the length of a product and thermal cycling being present.

4. Corrosion cracking of spacer grids and other components of spent fuel assemblies fabricated from austenitic chrome-nickel steels in the gas environment with corrosive impurities (O, I, Cs, etc.) and under tensile stresses.

All degradation mechanisms proceeding in SFA structural materials are studied in hot cells and on dummy fuel rods.

Let's consider the first results obtained in assessing the creep rate of standard fuel claddings effected by the internal pressure. Tests were carried out on refabricated fuel rods at $320-600^{\circ} \mathrm{C}$ in helium for $24 \mathrm{~h}$ or until the loss of tightness by a fuel rod. 
The refabricated fuel rods were manufactured from the full-scale fuel rods of RBMK spent fuel assemblies that had been in operation in Unit 2 of the Leningrad NPP to reach a fuel burn-up close to the design burn-up ( 20 MW.day/kg U) and stored in a cooling pond for 12 years, respectively. The condition of the claddings taken for the tests is typical of RBMK fuels that reached the design burn-up.

The refabricated fuel rods may be hypothetically subdivided into two groups; namely, the ones internally pressurized to 0.5 and $1.0 \mathrm{MPa}$ and the ones pressurized to 3.0 and $5.0 \mathrm{MPa}$. The pressure of $0.5 \mathrm{MPa}$ is typical of RBMK spent fuel. To conservatively assess the storage safety, the fuel rods with pressures of 3.0 and 5.0 MPa were tested. These pressures were chosen assuming all fission gas products to be released by the fuel under the cladding during the storage time.

Aside from this, to reach a conservative assessment, fuel rods were chosen subject to high fretting wear resulting from the interaction between spacer grids and cladding.

The main results of short-term testing of the refabricated fuel rods may be summed up as follows.

1. The tests at 320 and $400^{\circ} \mathrm{C}$ evidence no noticeable creep of the cladding material, also under spacer grids.

2. At the heating temperature of $500^{\circ} \mathrm{C}$, neither shape changes nor thermal creep of the cladding of a fuel rod internally pressurized to $0.5 \mathrm{MPa}$ were recorded; the fuel rod retained its tightness. Upon $2 \mathrm{~h}$ heating at the above temperature, the fuel rod internally pressurized to $5.0 \mathrm{MPa}$ revealed the significant ( $16 \mathrm{~mm}$ in diameter) bulging of the cladding in the top plug area (Fig. 2).

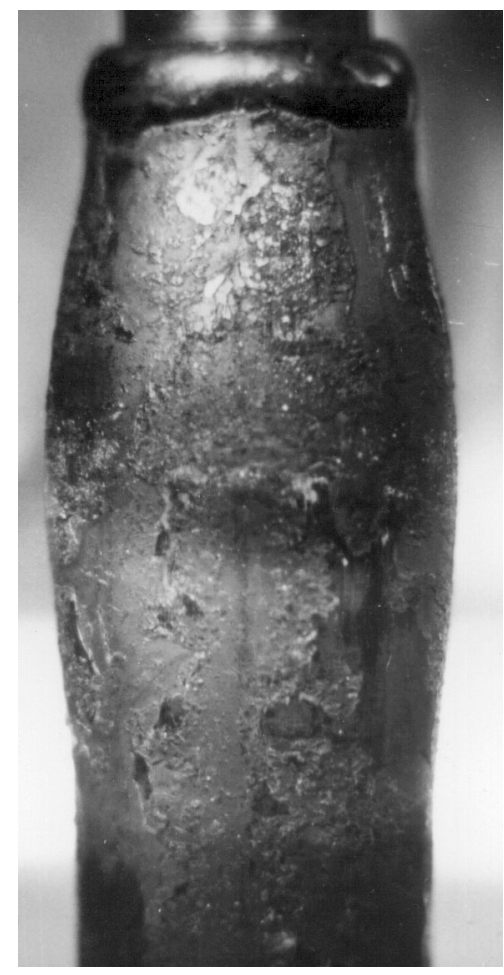

Fig. 2. Appearance of fuel rod near top end.

3. At the heating temperature of $600^{\circ} \mathrm{C}$, the fuel rods internally pressurized to 0.5 and $1.0 \mathrm{MPa}$ demonstrated the classic process of the steady-state creep proceeding at the rates of $(8.9 \pm 0.8) \times 10^{-}$ ${ }^{4} \mathrm{~h}^{-1}$ and $(1.7 \pm 0.3) \times 10^{-3} \mathrm{~h}^{-1}$, respectively. 
Figure 3 illustrates the variation in the tangential strain of this fuel rod. During the whole testing time $(24 \mathrm{~h})$, the fuel rod remained leak-tight. After heating for $13.5 \mathrm{~h}$, the fuel rod internally pressurized to 1.0 MPa lost its tightness due to a strong local bulging under the spacer grid where substantial fretting wear induced by the interaction between the spacer grid dimples and the cladding was identified (Fig. 4).

The heating of the fuel rods internally pressurized to 3.0 and $5.0 \mathrm{MPa}$ at a temperature of $500^{\circ} \mathrm{C}$ resulted in substantial bulges developed by the cladding in the area of the bottom plug (the first fuel rod) and in the area of the bottom and top plugs (the second fuel rod). After heating for $1.5 \mathrm{~h}$, the fuel rod pressurized to 5.0 MPa lost its tightness.

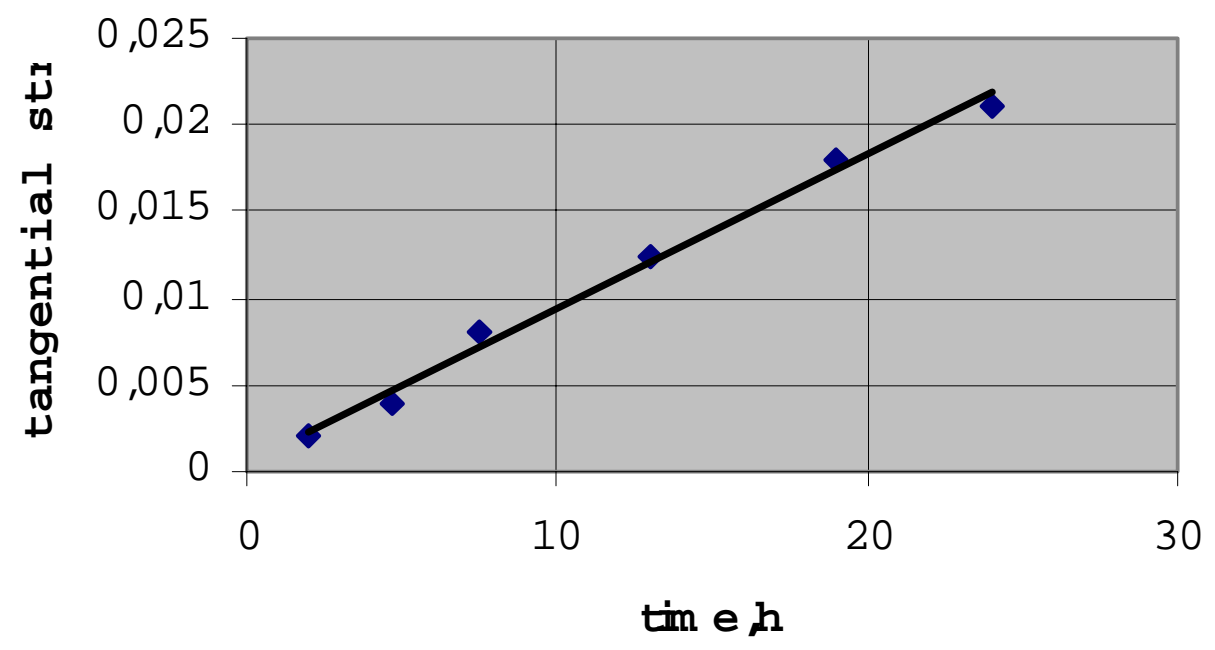

Fig. 3. Variation in cladding tangential strain $\varepsilon_{\varphi}$ 


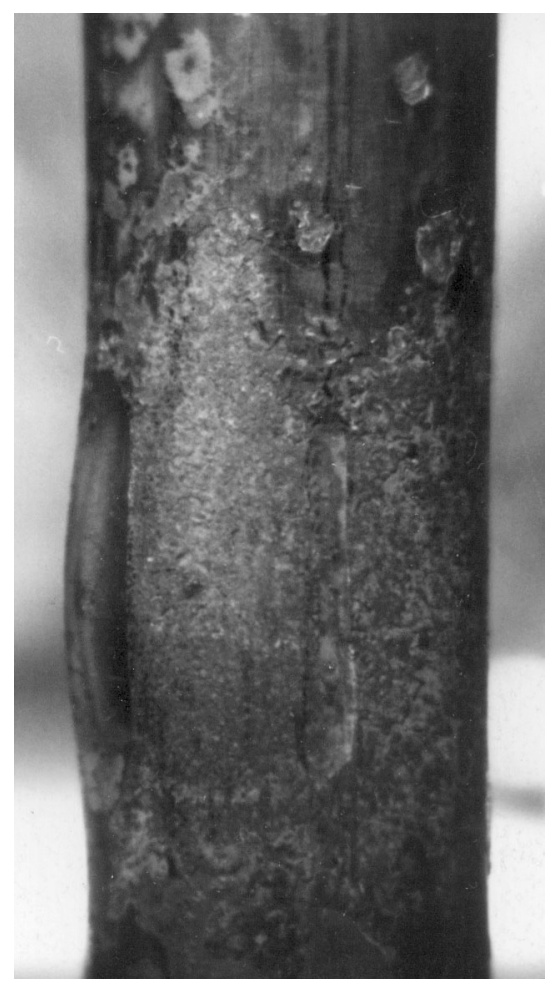

a

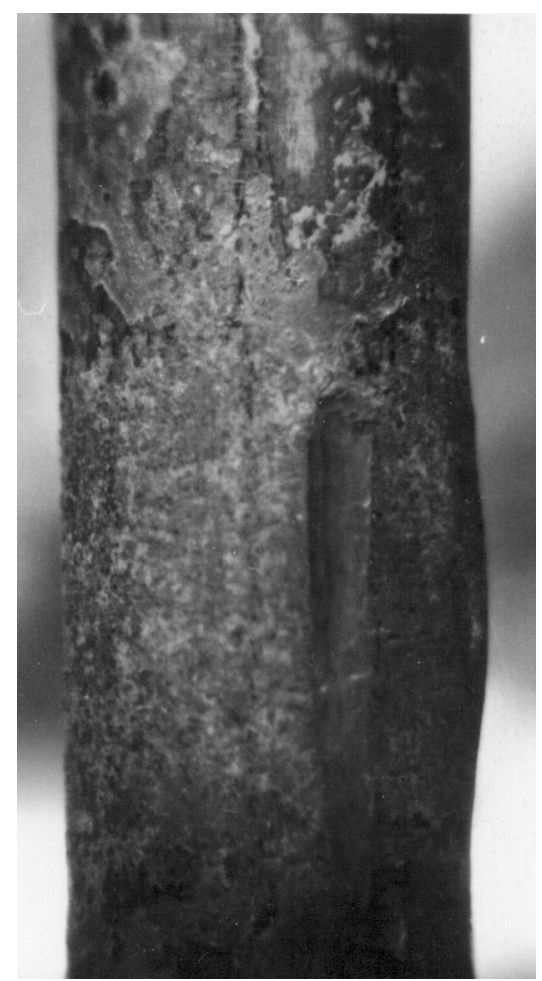

b

Fig. 4. Appearance of fuel rod under spacer grid.

\section{Conclusions}

1. Spent fuel assemblies have been tested following a 3- to 15-year holding period in cooling ponds. It allows us to draw the conclusion that storage may influence changes in SFA material properties:

- Long-term stored fuel rods were established to be in an adequate state similar to that after their operation;

- During wet storage of spent FAs in cans, their general corrosion is observed to proceed at the rate of 3-5 $\mu \mathrm{m} /$ year;

2. The primary investigations of thermal creep rates of short-term tested RBMK-1000 refabricated fuels reveal the following:

- The most susceptible sections of RBMK fuel claddings are locations under spacer grids;

- For transient and emergency conditions, the ultimately tolerable temperature of dry inert gas storage is in the range of $450-550^{\circ} \mathrm{C}$;

- To assess the thermal creep rate at lower temperatures $\left(300-400^{\circ} \mathrm{C}\right)$, longer-time (several months) tests have to be implemented. 


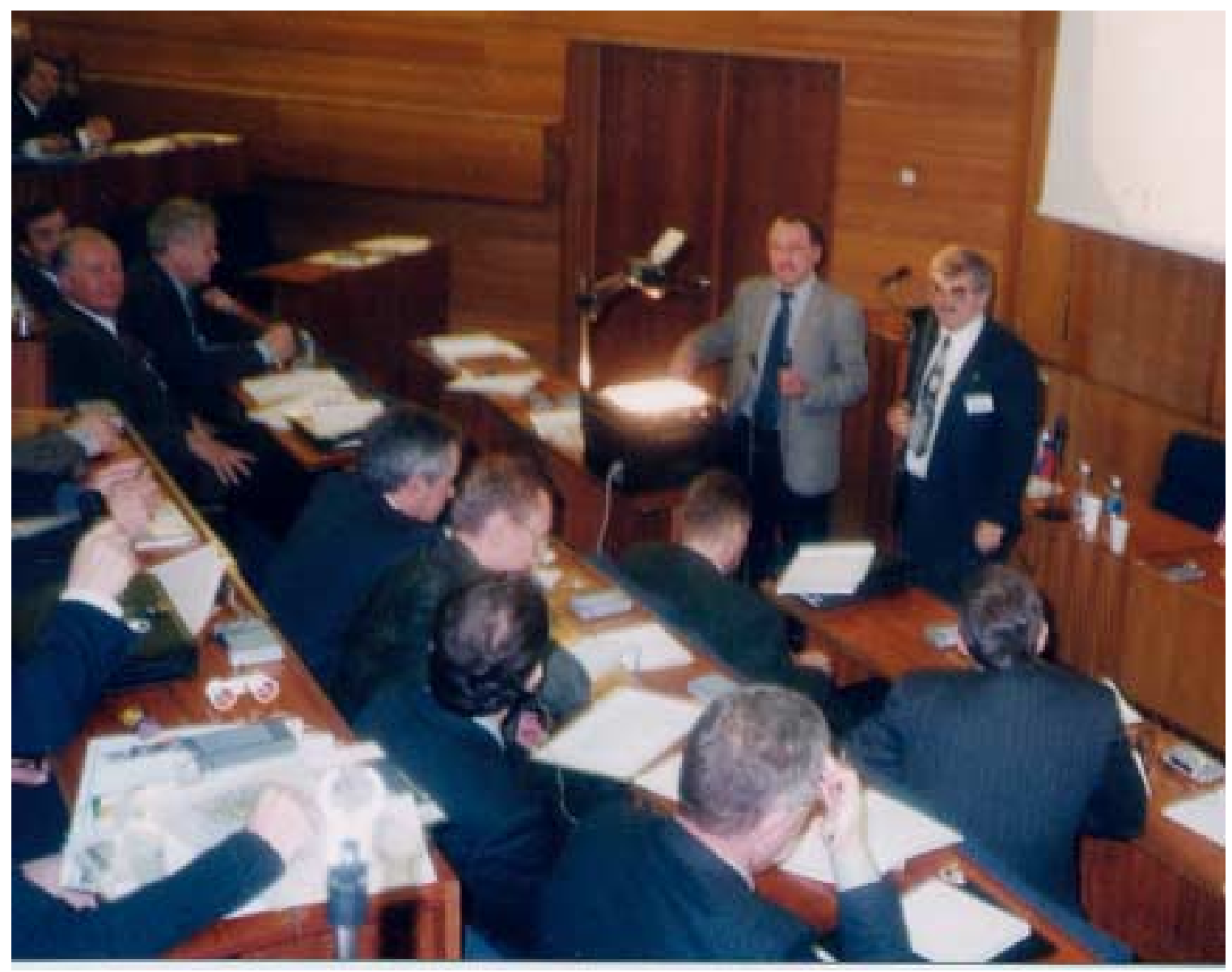




\title{
The LLNL-Russian Pu Disposition Projects Web Site (B512152)
}

\author{
P. E. Burkhanov, A. S. Aloy, E. Yu. Ivanov, M. M. Moshkov, KRI \\ Introduction
}

Lawrence Livermore National Laboratory has or has had 47 LLNL contracts with two Russian plutonium industrial sites at Mayak and MCC K-26 and with six supporting scientific and design Minatom institutes in Moscow, St. Petersburg, and Dimitrovgrad.

It was decided to develop a LLNL-Russian disposition projects web site to summarize the completed work as a subtask in LLNL contract B512152.

The contracts involve excess weapons plutonium storage, packaging and transportation, Pu-containing wastes stabilization, immobilization, geologic disposal, and spent MOX fuel storage and transportation.

\section{An Information-Structuring Concept at the Site and Site Navigation System}

The site has three main sections:

- Plutonium (i.e., Pu-containing materials) storage, packaging and transportation;

- Spent fuel storage and transportation;

- Plutonium immobilization (i.e., immobilization of Pu-containing wastes).

The start page of each section has an interactive map. This map shows the participants' geographical location and enables a transition to pages devoted to them.

The menu system displays at the upper part of a whole primary page and allows a transition between its items by one click. The menu system involves the following items: "Participants", "Programs", "Objectives", "Strategy", "Site map" and a link to the site's home page. "Participants" and "Programs" items have drop-down submenus with lists of participants and programs accordingly. The "Participants" submenu enables a quick transition to pages with information about an institute or combine and their projects.

So, "Programs", "Objectives" and "Strategy" pages have general information on the present subject, but project descriptions are placed at participants' pages.

\section{An Information Structuring Concept for Participants' Pages}

A participant's starting page could be divided into five parts: a site direction name, the whole site navigation menu, a logo and participant name, active links to participant's general information, and last, active links to projects information.

\section{Technical Part}

Any web-site has to meet the following requirements:

- The site structure has to be understandable for user;

- The site navigation system has to be useful and clear; 
- Pages must have a minimal volume;

- A site has to display on screen without distortions and variations irrespective of user's software and hardware.

The menu system was made by "Java" programming language. The menu bar has drop-down menus. The menu system with drop-down menus fills a minimum amount of space at the web-page and involves sufficient number of hyperlinks for comfortable and fast site viewing.

One of the main site-developing problems is providing a high download rate. The download rate depends on page volume and connection line capacity between web-server and user's computer. The second problem is solved by choosing a good internet provider, but the volume of pages depends on the site developers.

The developers took the following steps to minimize page volume:

1. The code of pages was written manually. This means that HTML-editors were used as supporting equipment only and web-pages creation techniques like saving of MS Word documents in web-page format were not used because of their non-optimal code generation and large volume of derivable pages.

2. The processing of photographs, flow-sheets, diagrams, graphs, logos, and other graphical objects were made by Ulead Photoimpact 6.0 and Adobe Photoshop 6.0 editors. The objects were saved in "gif" and "jpeg" formats. Ulead Photoimpact 6.0 was used also for interactive map creation and this map hyperlinks coordinates generation.

3. The Cascading style table (CSS) was created. Using of CSS greatly miniaturizes the volume of pages and time for page making.

4. The headers, logos, and other elements of pages animation was made by Macromedia Flash 5.0. In contrast to "gif"-animation, this progressive technology enables creation of animating elements with smaller volume and better quality.

Unfortunately, page volume isn't the only problem in web-site design. About $30 \%$ of this site creation time was spent for compatibility with main types and versions of browsers, because creators of browsers don't adhere to one standard. As a result of work completed, the site is working properly when accessed through the latest versions of MS Explorer or versions 4.xx of Netscape Navigator. It is necessary to note that about $90 \%$ of internet users use these browsers.

\section{Site address}

The current site address is: www.immobilization.narod.ru

\section{Contact information}

\section{Project coordinators:}

Albert S. Aloy - aloy@atom.nw.ru

Mikhail M. Moshkov-moshkov@atom.nw.ru
Site developers:

Peter E. Burkhanov - burhanov@atom.nw.ru

Eugeny Yu. Ivanov 


\section{Conclusion}

This web-site meets technical and operational requirements because it has good structure and design, comfortable navigation system, and screens without distortions and variations irrespective of the user's software and hardware.

\section{References}

1. Homer A., Ullman C. Dynamic HTML. WROX press Itd. 510 p. 1999.

2. Buyens J., Web tricks. Microsoft press. 438 p. 2000.

3. Buykov D. Internet: information search and site promotion. BHV press. 287 p. 2000.

4. Kyle L., Essential Flash 5 for web professionals. PHPTR press. 301 p. 2001.

5. Bradenbaugh J., JavaScript Application Cookbook. O'Reilly press. 414 p. 2000. 


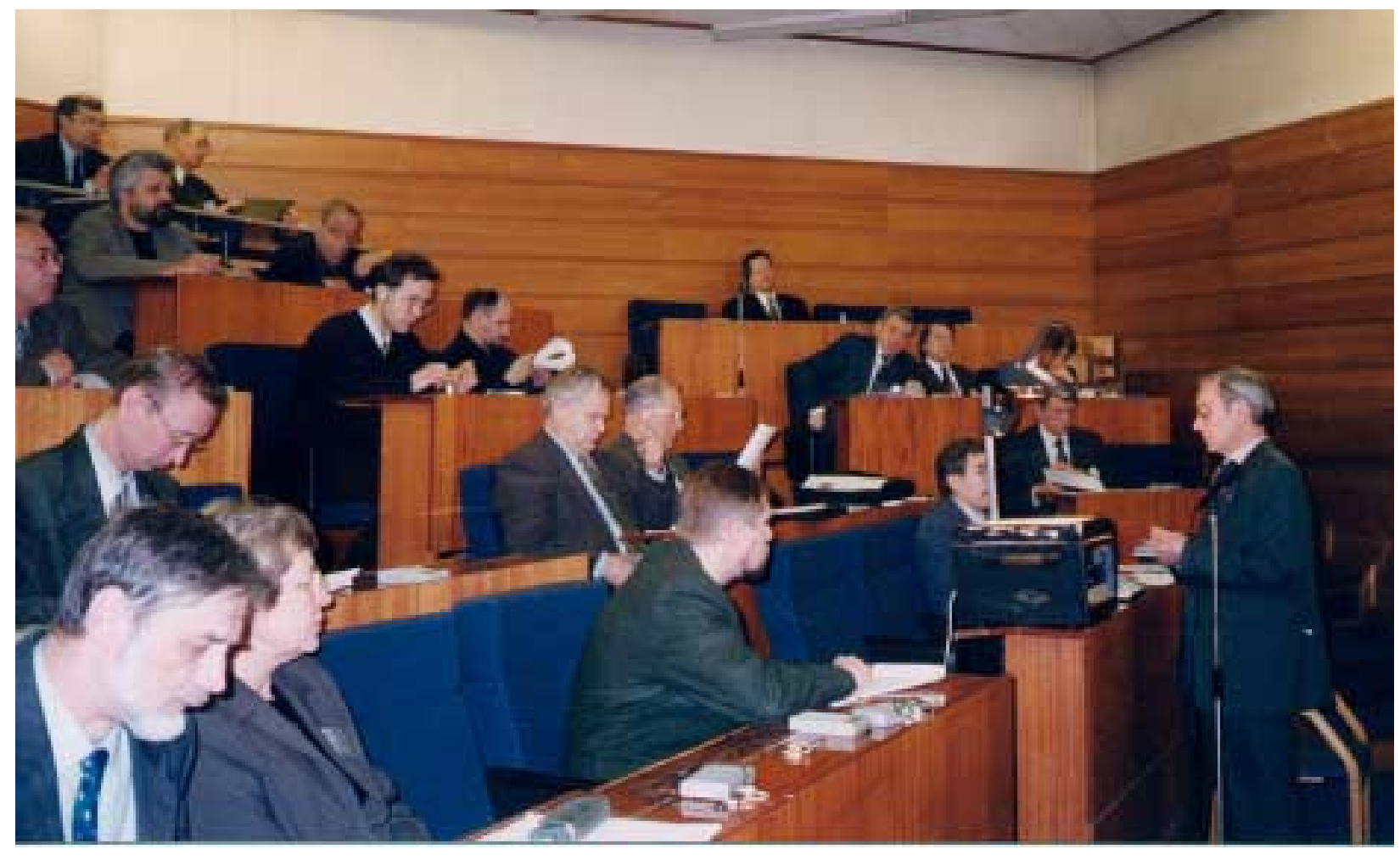




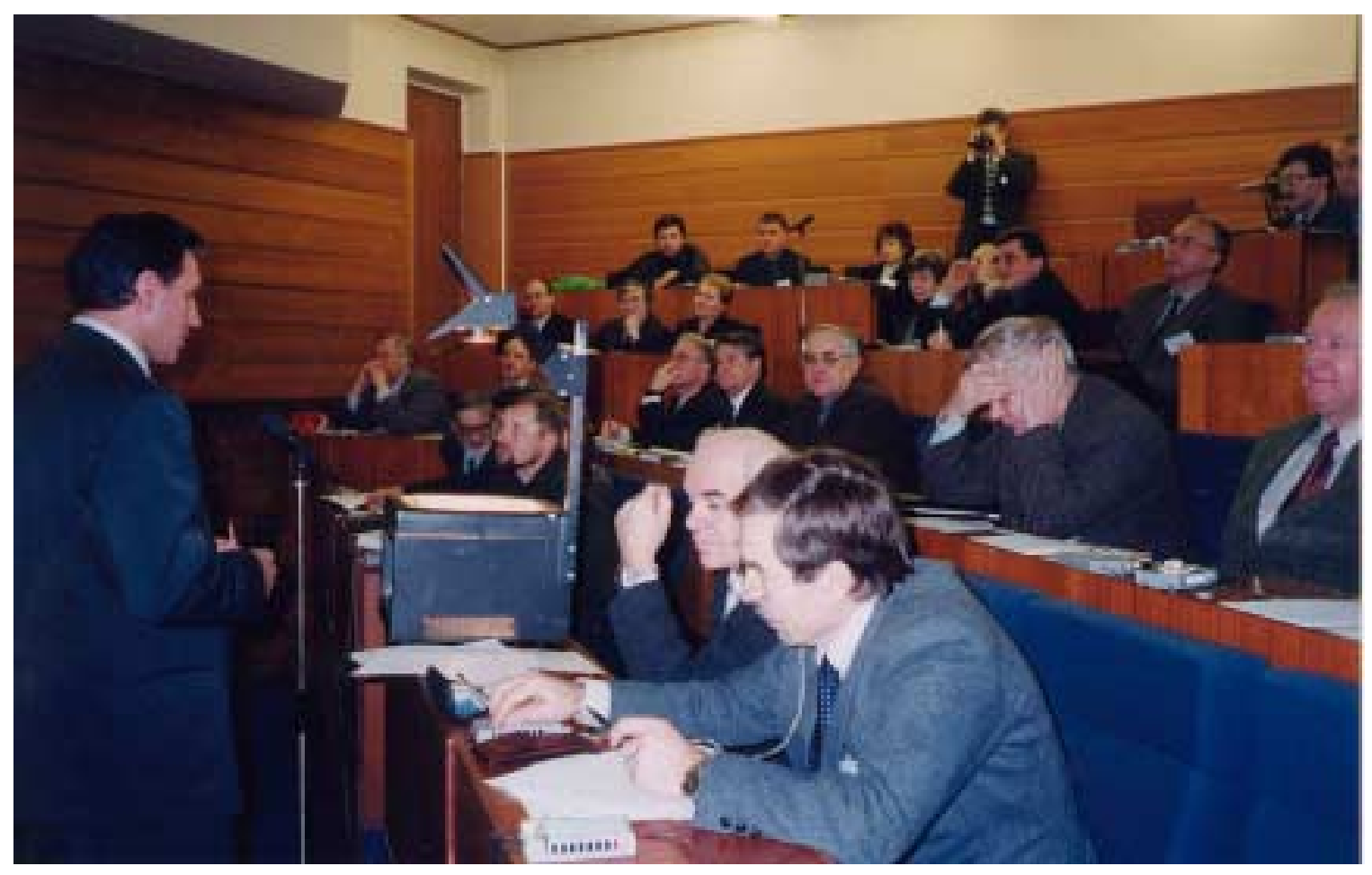

II. Engineering and Industrial Site Plutonium Disposition Contract Activities: K-26, Mayak, RIAR 


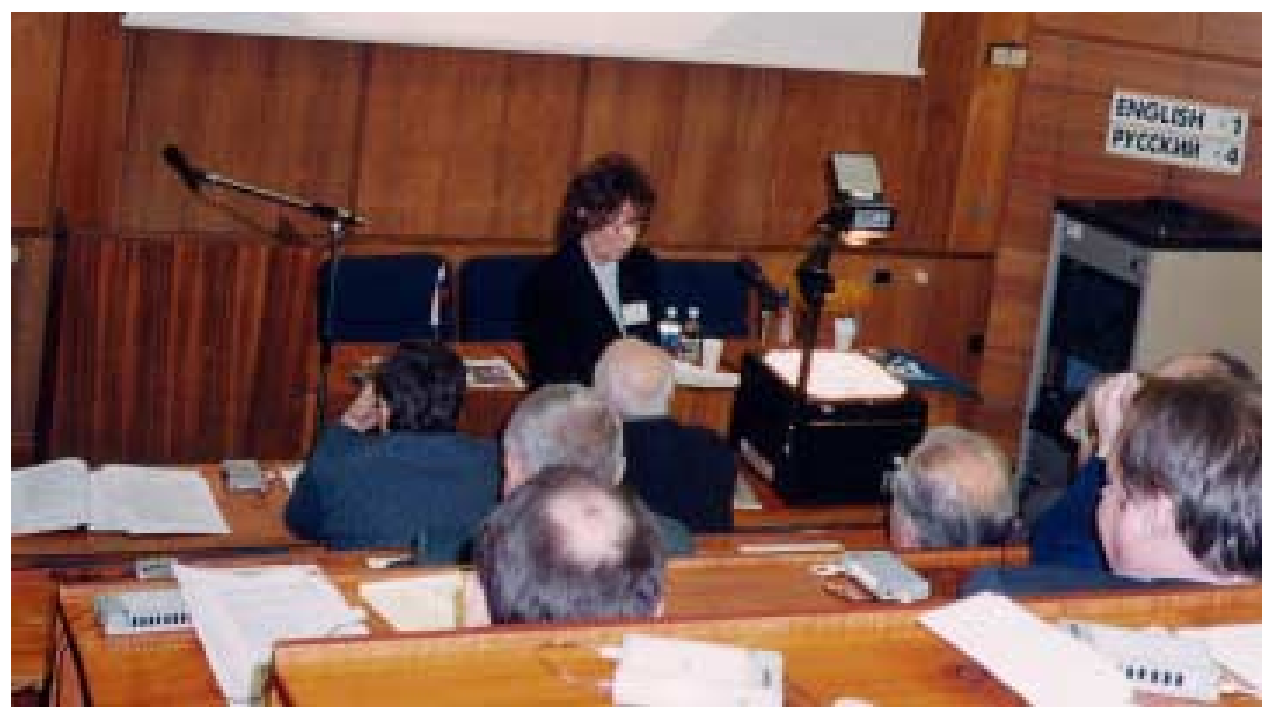

II.1. MCC K-26 Site Activities

Justification of Investment Engineering Studies of Weapons Plutonium Sludge Immobilization and MOX Fuel Fabrication Plutonium Immobilization 


\section{Outlook for MCC-LLNL Collaboration in Nuclear Materials Management within the Framework of RF-US Agreements (B347376, B506210, B506233)}

\section{K. G. Kudinov, A. A. Tretyakov, MCC K-26}

Immediate collaboration between Mining and Chemical Combine (MCC) and Lawrence Livermore National Laboratory (LLNL) started in June 1998 with preparation of feasibility studies (FS or TEF) of plutonium-containing waste immobilization. The objective was to assess the feasibility of immediate immobilization of plutonium-containing sludges into glass and storage of the solidified wastes and compare it to the existing practice, which includes extraction of uranium and plutonium, conditioning liquid radioactive waste (LRW) for disposal in underground strata-collectors, and forwarding recovered plutonium for interim storage (or ready-made product storage). Appropriate research projects were conducted and showed the feasibility of the direct immobilization option. Based on the research, the Declaration of Intent (DOI) was prepared to set out the objectives of specific investments, destination and capacity of Vitrification Facility be implemented. Along with activities associated with preparation of the FS and $\mathrm{DOI}$, the RF Institute and MCC laboratories conducted experiments to solidify radioactive waste simulators in a microwave (MW) facility using microwave ultrahigh frequency (UHF) heating. Work accomplished under the FS and DOI stages made it possible to develop two processes for direct immobilization of plutonium-containing waste and to check effectiveness of the facility to vitrify wastes into phosphate- and borosilicate glass in a laboratory environment. The next stage called for Justification of Investment (JOI) development. The two plutonium-containing waste vitrification technologies, viz., immobilization into phosphate glass and into borosilicate glass, were considered and compared to each other during all stages of JOI preparation work.

Research on chemical stability of glass samples produced, modeling of engineered storage barriers, and distribution of radionuclides are the factors that will determine which one of the two technologies will be selected. Design and engineering modifications of the pilot UHF microwave heating facility are being made to develop engineering specifications for the pilot-scale vitrification facility. The engineering specifications in full are documented in the following papers.

During JOI preparation the Statement of Work was modified to incorporate activities associated with assessment of possible reprocessing wastes from MOX-fuel production if should that be implemented at the facility. For this purpose a preliminary assessment and characterization of plutonium-containing waste streams were carried out and main technological operations were specified. This work was done in view of possible implementation of heat-releasing assemblies of MOX-fuel production facility at MCC.

Experiments carried out with the laboratory UHF microwave heater in a hot cell show the feasibility of vitrifying wastes with applied UHF microwave power. However, some technical problems exist, which could require the due dates of entire project stages be shifted. Therefore, in comparison with employment of the UHF microwave heater, the JOI considers two additional waste vitrification options: utilizing the ceramic melter and cold-crucible induction melter (CCIM). To date, JOI preparation has been completed. The document is undergoing final approvals and agreements. Volume and quality of work accomplished by MCC allow for fulfillment of the next stage, the Pilot-scale Plutonium-containing Sludge Immobilization Facility Design. Main items of work for this facility implementation are provided in Fig. 1. Figures 2 and 3 show main items of work completed under the three LLNL contracts. 
Due to suspension of the US funding of plutonium immobilization programs in 2001, continuation of this MCC work in full is hardly possible. However, we believe that it is necessary to complete the engineering feasibility studies of options to reprocess waste from MOX-fuel production that are already underway. Under this program, it is desirable to continue work for industrial-scale UHF microwave facility implementation per the engineering specifications developed.

The Agreement of September, 2000, on Management of Plutonium that has Been Withdrawn from Nuclear Military Programs, and on the scope of the cooperative activities, calls for determination of packaging, storage, and transportation of various plutonium materials within the RF. Plutonium materials include: plutonium metal, plutonium oxide, MOX-fuel non-irradiated (fresh) assemblies, and irradiated (spent) MOX-fuel assemblies.

MCC produces and stores plutonium dioxide which is to be involved in the MOX-fuel production program. Moreover, pursuant to MinAtom's decision, the industrial-scale facility for MOX-fuel pellets and assemblies production may be located at the Combine. Thus, storage, packaging, and transportation of plutonium dioxide, plutonium mixture, MOX-fuel assemblies are the questions of the day. Resolving the first task, viz., an analysis of the status of these studied issues and planning of future work can be done right now, under a new proposed contract with LLNL.

\section{Proposed Statement of Work}

The following is the Statement of Work proposed for inclusion into the new contract.

Objective: to analyze the existing practices of packaging, storage, and transportation of plutonium, as well as needs for packaging, storage, and transportation of plutonium dioxide, plutonium mixture, and MOXfuel assemblies according to the Agreement of September 2000.

\section{Work Scope}

Work can be divided into four tasks, as follows:

1. Task 1: Packaging, storage of $\mathrm{PuO}_{2}$ at $\mathrm{MCC}$, and transportation of that to the conversion plant for MOX-fuel production.

2. Task 2: Packaging, storage and transportation of the plutonium mixture produced by the conversion plant to the MOX-fuel production facility.

3. Task 3: Packaging, storage and transportation of fresh MOX-fuel assemblies (FAs) to the RF reactors.

4. Task 4: Work plan and proposals for future work.

While the tasks are performed, it is required to analyze and substantiate by appropriate documentation the following:

- Existing Russian methods and technologies for packaging, storage and transportation;

- New or advanced methods and technologies necessary for implementing the Agreement of September 2000 in the RF.

The new or advanced methods and technologies for packaging and storage shall consider the existing canisters, including their configuration. This is necessary to minimize the amount and variety of possible methods of packaging, storage, and transportation. For instance, it is necessary to determine the 
suitability of the existing canisters respecting their external geometry, and suitability of the devices used for transfer of plutonium oxide and plutonium metal, in order to implement the same systems for packaging, storage, and transportation.

Any new methods of packaging, storage, and transportation shall be documented and include R\&D or development of new important elements. It is necessary to determine and document any need for modifications or addenda to RF licensing rules or regulations.

Moreover, it is essential to describe the existing Russian methods and systems for packaging, storage and transportation of plutonium oxide and fresh FAs between RF independent plants. According to the Agreement of September 2000, specific shipments envision transfers of plutonium oxide from MCC to the Conversion Treatment Facility at the Production Association "Mayak", and of the plutonium mixture from PA "Mayak" to MCC, and of fresh fuel assemblies made in Krasnoyarsk to the VVER-1000 and BN-600 reactors. Also, it is required to discuss methods of transportation by rail train and along highways, as well as possible routes. This work will be performed together with All-Russian Design Research Institute of Complex Power Technology (GI VNIPIET) and A. A. Bochvar All-Russia Scientific Research Institute of Inorganic Materials (VNIINM).

The next promising item of future collaboration with LLNL can be implementation of the geologic disposal facility in a granitoid massif. The final stage of the nuclear fuel cycle (with no regard to closeness or openness), is long-term storage of and then geological disposal of radioactive waste or spent nuclear fuel. At present, the most promising site for a radioactive waste geological repository is considered to be the granitoids of the Nizhnekansky massif. One of the largest granitoid massifs in Central Siberia, it is over $1,500 \mathrm{~km}^{2}$ in exposed area. In effecting work, the concept of radioactive waste underground isolation in a safe manner is to be developed in view of the Nizhnekansky massif and of the MCC's underground complex location. Besides, based on mathematical modeling and engineering and geological studies, a comparative assessment of suitability of promising waste isolation sites will be completed, the depth at which the geological storage is to be located will be determined, and basic technological solutions for the engineered barriers will be made. Ecological and economic comparison of the sites and the main technological solutions for the geological storage will be performed using the complex criterion "riskcosts".

The mathematical modeling, comprehensive surface geophysical studies, and data on analogous facilities will minimize expensive geological surveys with deep hole boring, and allow a program for complex investigations in the underground laboratory to be developed. The product of collaboration with the VNIPIPT specialists and specialists of Geological Institutes of Russian Academy of Sciences and other RF geological organizations will be an analysis and summary of data from the available engineering and geological studies, and programs for engineering geological and ecological studies of the promising Nizhnekansky massif sites necessary for implementation of the geological radioactive waste storage.

During geological development, metamorphism almost leveled engineering and geological differences between Nizhnekansky massif granitoids and MCC's gneiss rocks; therefore, processes of water exchange and heat- and mass-transfer in both massifs are to a considerable extent analogous. Therefore results of the studies of MCC's underground environment along with geophysical studies of the promising Nizhnekansky massif sites can minimize expensive geological surveys with deep hole boring. The MCC's underground complex is located in a rock massif whose base is biotite gneiss of high strength. Basic elements of the tectonic pattern at the depth of underground mine locations (over $200 \mathrm{~m}$ ) are fissures and formation fracturing that form two steeply deepening systems at an angle of $60-90^{\circ}$ with each other. Open fissures and tight (closed) fractures contain chlorite, carbonates, iron hydroxides, and clays. The 
underground mines of various design and dimensions were excavated in rocks (gneiss) over 40 years ago. During the underground complex construction, comprehensive data on the geological rocks structure were collected. This database can be used for a preliminary modeling of engineering and geological conditions of the main and alternative sites. It is necessary to collect and systemize the data from the prior on-site and laboratory research on underground water characteristics and chemical composition of rocks at various depths of the Nizhnekansky massif. Today, these materials are contained in independent reports and working documents of various research, geological, and design entities. Besides, it is necessary to collect data from the prior laboratory studies of interaction between radionuclide solutions, and various possible engineered barrier materials, and rocks such as granite and gneiss. All the aforementioned data shall be analyzed for use in preparation of the program for future on-site and laboratory studies of the engineered and natural barriers for the radioactive waste storage.

The items of work suggested should not be considered as the ultimate scope of the MCC-LLNL collaboration. We mentioned only those that were selected in accordance to the interest showed to date by American specialists during working meetings on results of the contractual activities.

In closing, l'd like to hope that the unanimous team formed in performing tasks of the MCC's plutoniumcontaining sludges immobilization contract will continue joint efforts on other tasks to the benefit of both countries.

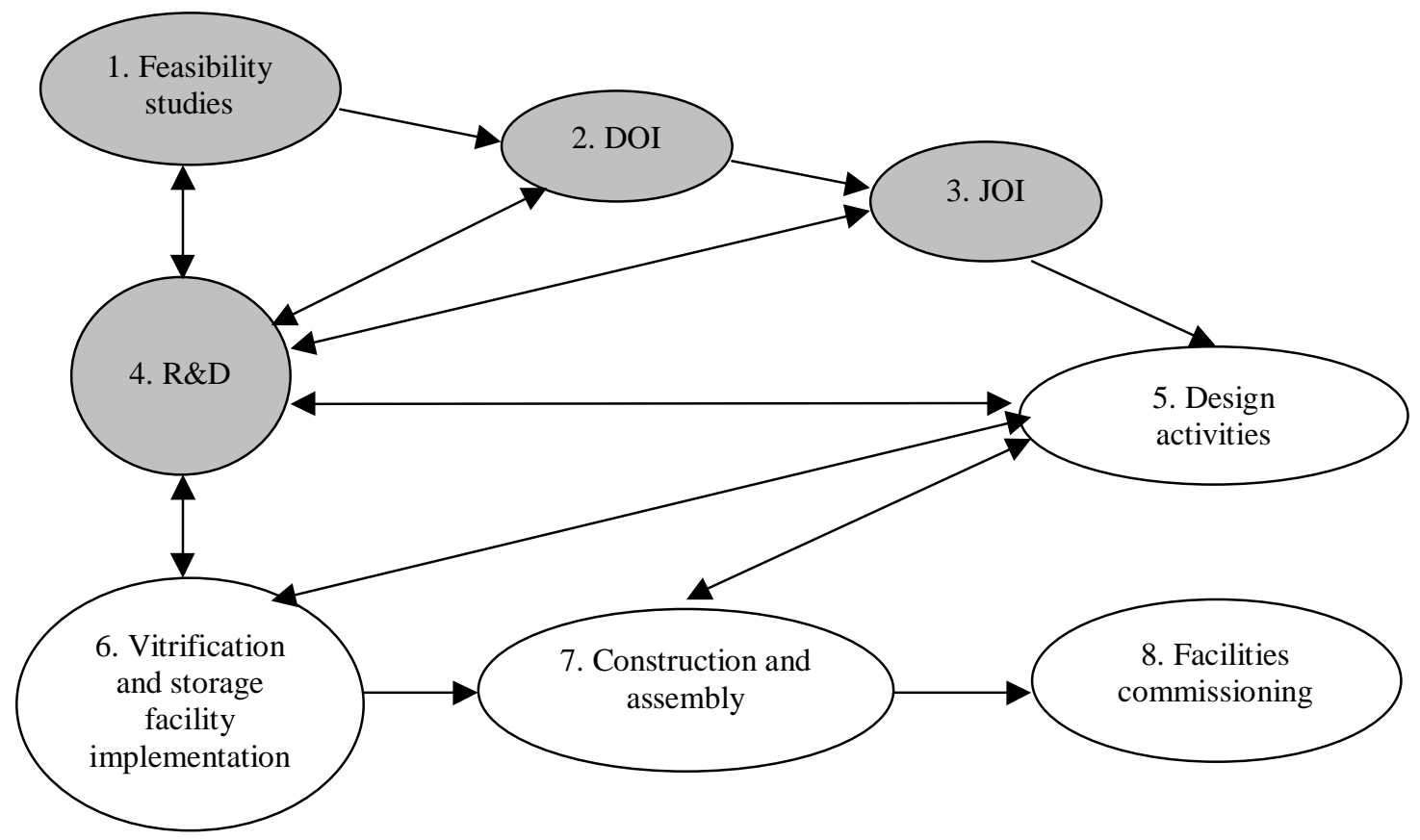

Fig. 1. Illustration of stages 1-8 of the work plan for implementation of Pu-Bearing Sludges Vitrification Facility and Vitrified Waste Storage Facility at MCC. Shaded areas show completed activities. 


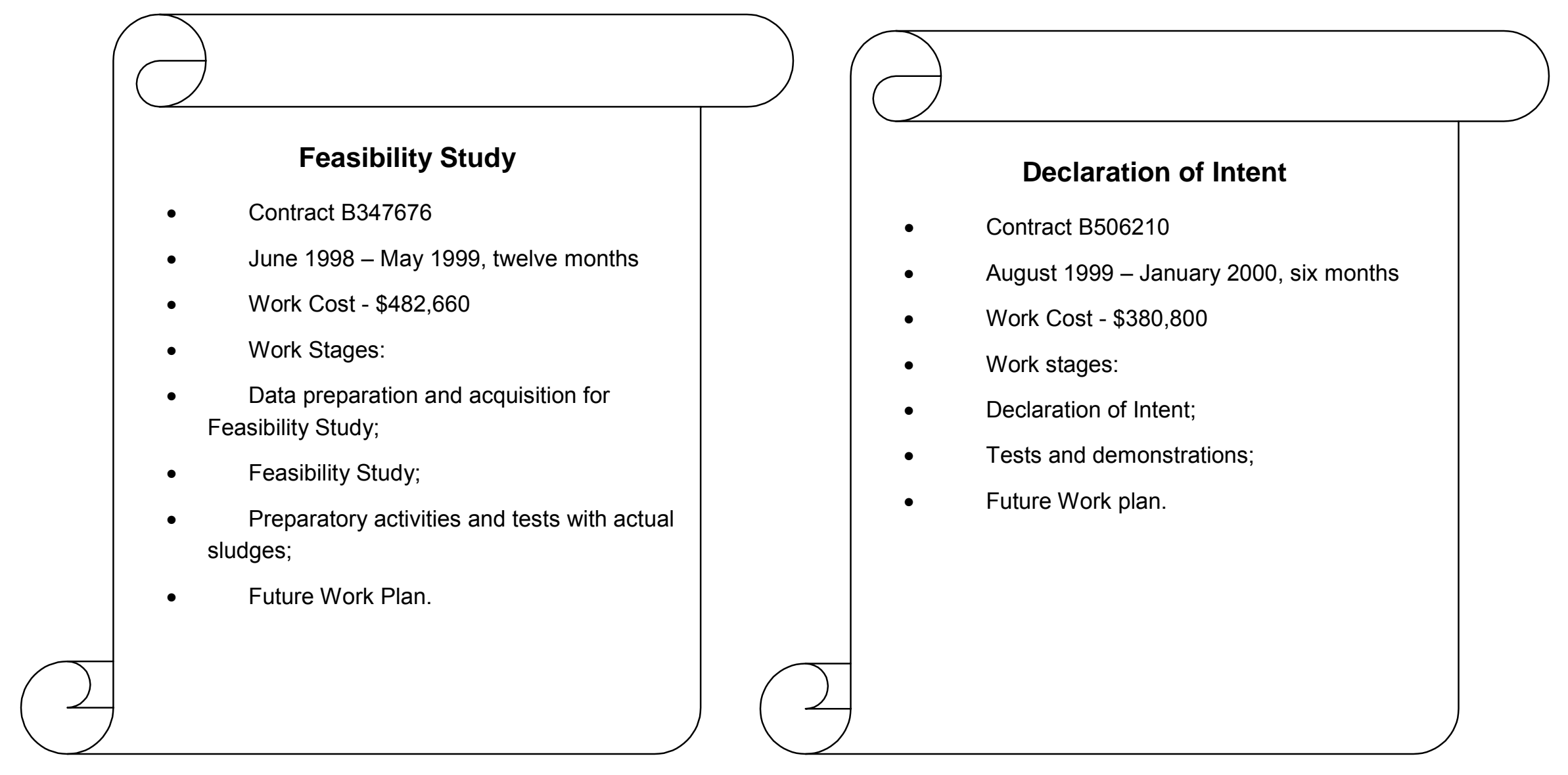

Fig. 2. Main items of work accomplished under FS and DOI preparation contracts for plutonium immobilization. 


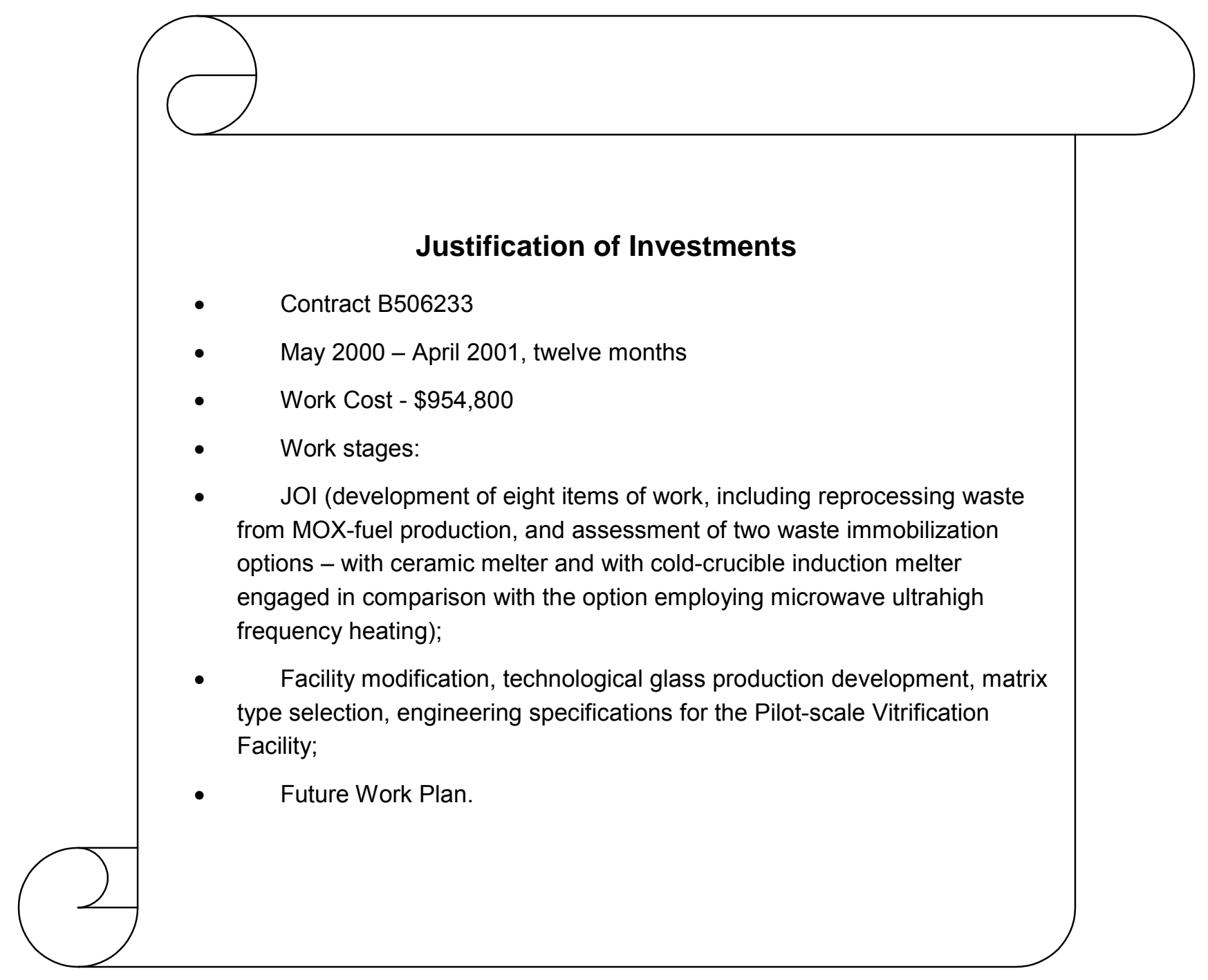

Fig. 3. Main items of work accomplished under JOI preparation contract. 


\title{
On JOI "Immobilization of Plutonium-containing Materials at the MCC" (B506233)
}

\author{
V. M. Simanovskiy, O. P. Anisimov, A. A. Shvedov, V. T. Sorokin, A. V. Demin, V. \\ V. Kashcheev, V. V. Iliin, T. A. Feofanova, HI "VNIPIET"
}

A lot of materials contaminated with both fission products and long-lived nuclides of plutonium have been accumulated at the MCC for the period of the plant's operation.

In accordance with the agreement between LLNL and STC "MCC", the HI "VNIPIET" has carried out the development of the Technical and Economic Feasibility Studies (TEF), DOI, and JOI on the present subject. In addition to the processing of plutonium-containing pulps, which have been accumulated at the plant for the long period of its operation, the problem of processing plutonium-containing radioactive wastes from new MCC MOX-fuel production was added at the JOI stage.

Here is a short description of plutonium-containing materials:

- Plutonium-containing pulps $\left(\sum \mathrm{V}=7260 \mathrm{~m}^{3}, \sum \mathrm{M}_{\mathrm{Pu}}=593,3 \mathrm{~kg}, \mathrm{~A}=1,7-35,2 \mathrm{Ci} / \mathrm{L}\right.$, density med. $\left._{\text {. }}=1,4 \mathrm{~kg} / \mathrm{m}^{3}\right)$;

- Liquid and solid radioactive waste from production of uranium-and-plutonium mixed fuel, MOX-FA fuel elements for WWER-1000 and BN (FN)-600 reactors.

At the $\mathrm{JOI}$ stage, the basic engineering and technical-and-economic appearances of variants were worked out to create plutonium-containing pulp immobilization installations at the existing facilities of the MCC.

Within the framework of the $\mathrm{JOI}$ there were considered two variants of immobilization of plutoniumcontaining pulps accumulated at the MCC, using MW-heating installation or induction smelter with a cold melting pot (ISCM) installation in order to obtain as a solid matrix:

- Variant 1 - borosilicate glass;

- Variant 2 - phosphate glass.

The two-stage solidification process is accepted as a method of liquid HLW immobilization in the variants of pulp vitrification.

The schematic process diagram of pulp vitrification for both variants is represented in Fig. 1.

For the variants of equipment design of the process, a parallel feed evaporator is accepted as an apparatus for the first stage of solidification, and either MW-heating installation with a hot capsule-melting pot (variant A) or installation of induction smelter with a cold melting pot ISCM (variant B) is used for the second stage.

Productivity of any single installation (1 working one and 1 standby) with respect to pulp processed reaches about $77 \mathrm{I} /$ hour (variant 1 ) and $83 \mathrm{I} /$ hour (variant 2).

The quantity (pieces or pi) of capsule melting pots (i.e., canisters) with solidified waste will reach:

- Variant 1: 516 pi./year, and $~ 6800$ pieces for the whole period of processing;

- Variant 2: 900 pi./year, and 11860 pieces for the whole period of processing. 
A capsule-melting pot (or a canister) is a cylindrical metal reservoir having a total volume of $100 \mathrm{I}$ (effective volume: $80 \mathrm{I}$ ).

Capsule-melting pots (or canisters) are to be packed into cases (or larger canisters). Cases are to be covered with metal lids, welded hermetically, and sent for long-term storage to a newly designed repository provided with a forced cooling. The number of cases coming for storage is equal to the number of capsule-melting pots.

The schematic diagram for processing of liquid and solid RW generated during MOX-fuel production is represented in Fig. 2.

Vacuum oil is sent to the solidification unit. Enviro-FlaxTR-20 type hardener is to be applied as an enclosing matrix. Annually, 8 drums, $200 \mathrm{I}$ in volume and filled with solidified oil, will be sent for long-term storage arranged in rehabilitated facilities of the MCC. The rest of the LRW are to be sent for immobilization by the method of underground disposal (disposal into deep water-bearing plates) in accordance with technology accepted at the MCC.

Solid radioactive waste (SRW) generated during the process of MOX-fuel production are subdivided by form and plutonium content:

- Rich waste containing plutonium above $150 \mathrm{mg} / \mathrm{kg}$;

- Poor waste containing plutonium below $150 \mathrm{mg} / \mathrm{kg}$.

SRW with rich plutonium content go to extraction and washing of important components until the plutonium content is no more than $150 \mathrm{mg}$ per $1 \mathrm{~kg}$ of waste.

Solutions containing plutonium go back to a cycle of MOX-fuel production, and washed SRW go to processing installation arranged at one of the MCC facilities, together with waste ineligible for plutonium recovery (i.e., poor waste).

Some solid wastes are sent for preliminary size reduction up to a particle-size dimension no more than 5 $\mathrm{mm}$ in diameter.

Pneumatic transport delivers crushed material to the cyclone situated over the cement mixer for homogenization with a cement mortar. Carbonizing wastes are mixed with cement and liquid radioactive wastes (or water). The mixture of cement mortar with crushed SRW runs into standard drums having an effective volume of $0,2 \mathrm{~m}^{3}$, preliminary filled with scraps of metal and other non-crushable SRW.

About 212 drums with cement compound come annually to long-term storage arranged in rehabilitated facilities of the MCC.

Drums with casehardened waste come for storage to a repository designed for delivery of processed waste during a period of 30 years.

The arrangement of installations for processing of SRW low in plutonium and the repository for cement compound will be specified in detail in the next design stages.

Elaborations of the extent of readiness of technical solutions to processing of plutonium-containing waste generated at MOX-fuel production, which have been carried out at the present stage of work, allow us to make conclusions about the necessity of additional pre-design developmental work over various versions of the given wastes in the TEF stage. 


\section{Economic Comparison}

Recalculation into prices of IV quarter of 2000 is accepted in accordance with Centrobank rate of exchange equal to $\$ 1$ USA $=28$ Rubles.

\begin{tabular}{|l|l|c|c|}
\hline \multicolumn{2}{|c|}{ Economic indexes, Rubles (in costs of IV quarter, 2000): } & Var. 1 & Var. 2 \\
\hline 1 & Investment outlay & 184,0 & 242,9 \\
\hline 2 & Total operational costs of processing and storing of Pu-containing pulps & 786,8 & 1006,4 \\
\hline 3. & Annual operational costs & 59,0 & 75,5 \\
\hline 4 & Comparable total costs of RW management arranging & 1007,7 & 1297,9 \\
\hline 5 & Variation of total costs & $100 \%$ & $129 \%$ \\
\hline
\end{tabular}

Comparison between technical and economic indexes of the variants considered, as well as analyses of technical solutions, has indicated that the inclusion of plutonium-containing pulp into a solid matrix to obtain borosilicate glass (Variant 1 ) is preferable.

Capital investments to construct RW management facilities for MOX-fuel production reach 61,8 million Rubles. Operational costs of MOX-fuel production RW management reach 12,5 million Rubles in costs of IV quarter of 2000.

To allow for continuation of design work on plutonium-containing pulp immobilization (without plutonium recovery) in a solid matrix, the following scientific-research and experimental-designing aspects should be additionally worked out:

- Design implementation and dimension optimization for capsule-melting pots and cases, taking into account process arrangement for solidification, storage and disposal of solidified pulps;

- Design implementation and optimization of arrangement diagram for solidified pulps in repository;

- Precise identification of technological characteristics of pulp solidification processes on the basis of real pulps and simulators, using pilot installations arranged in "hot" cells of the MCC;

- Perfect technology and equipment operation modes, approving of workability of remote control devices for equipment replacement and management of packages of solidified pulps on the basis of experimental and commercial installation;

- Develop design documentation and prototypes for both non-standardized process and transport-andprocess equipment;

- Study of storage and disposal safety for solidified pulps, using the capacities of risk assessment mathematical modeling;

- Develop normative and legal documents providing for regulation in aspects of both storage and disposal of plutonium-containing materials.

The HI VNIPIET has considered remarks and proposals on the JOI carried out by the MCC divisions (Labor protection department, Technical department, CZL), Chief Department of State Fire-fighting Service (GUP PS) under RF IAM, and takes note of the following:

- Given remarks and proposals contain no fundamental features requiring adjustment of the JOI;

- Remarks and proposals will be taken into account at the further stages of design. 


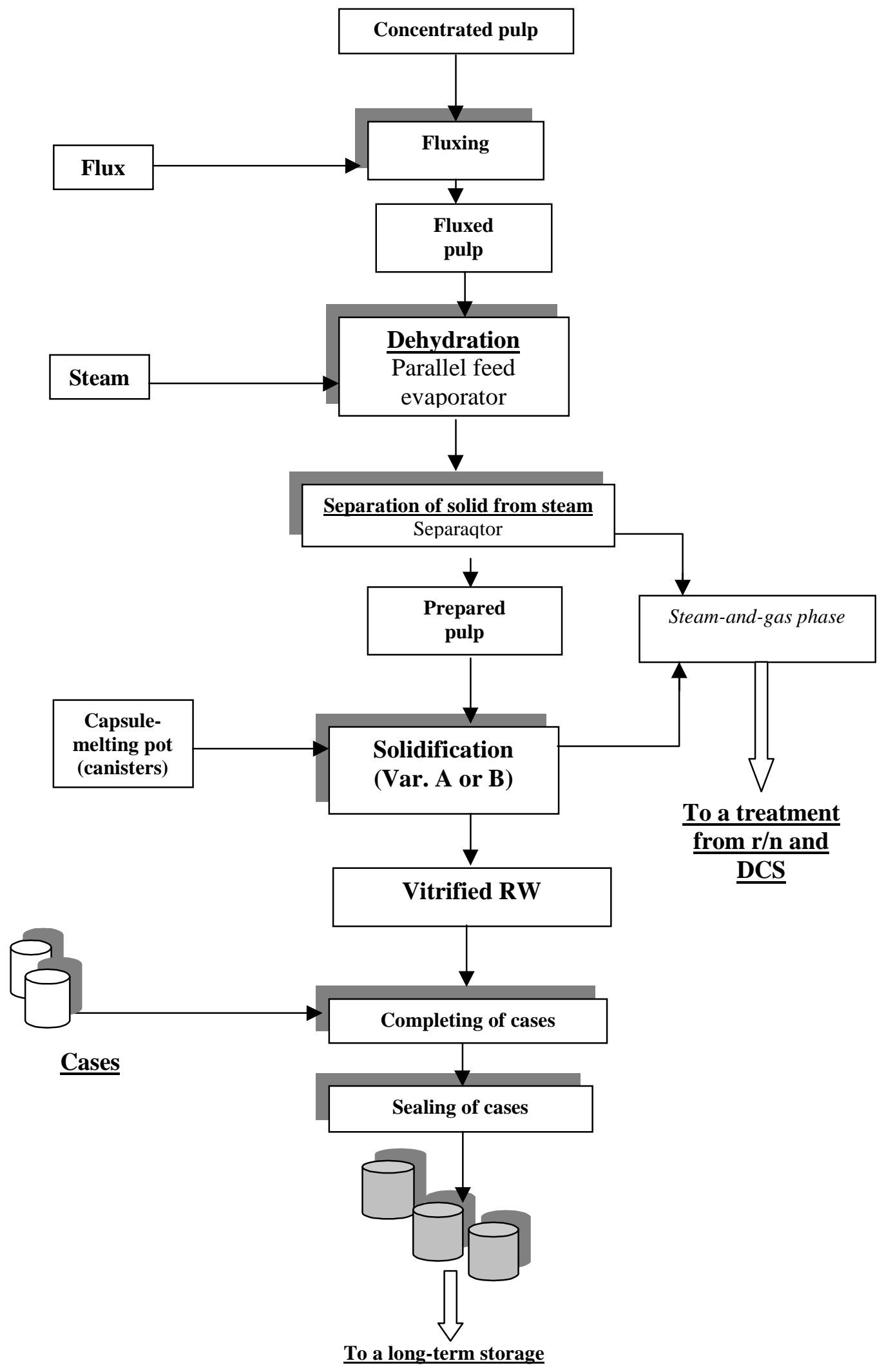

Fig. 1. Schematic process diagram of pulp vitrification by variants 1 and 2. (A or B). 


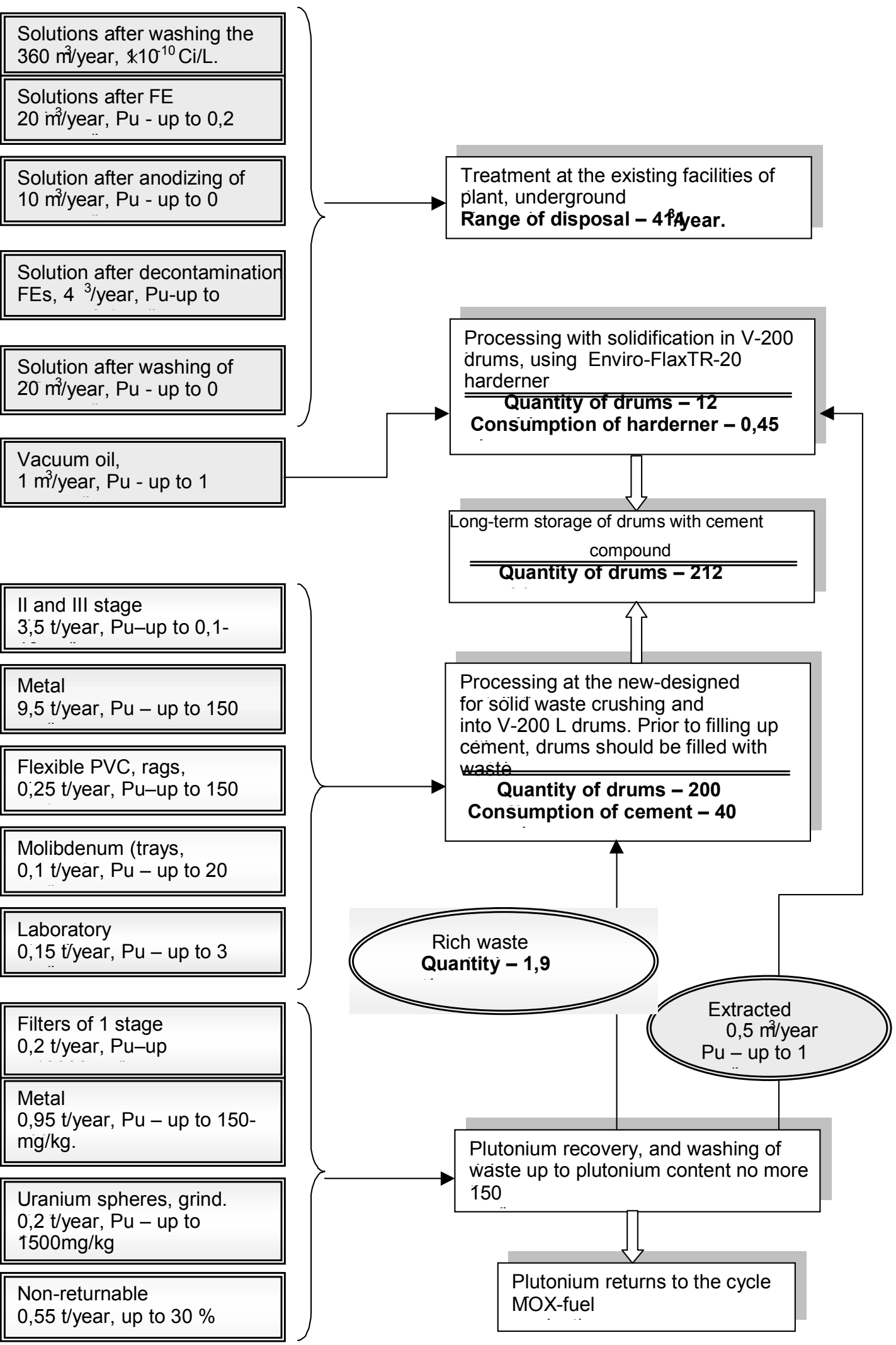

Fig. 2. Schematic diagram of MOX-fuel waste processing. 


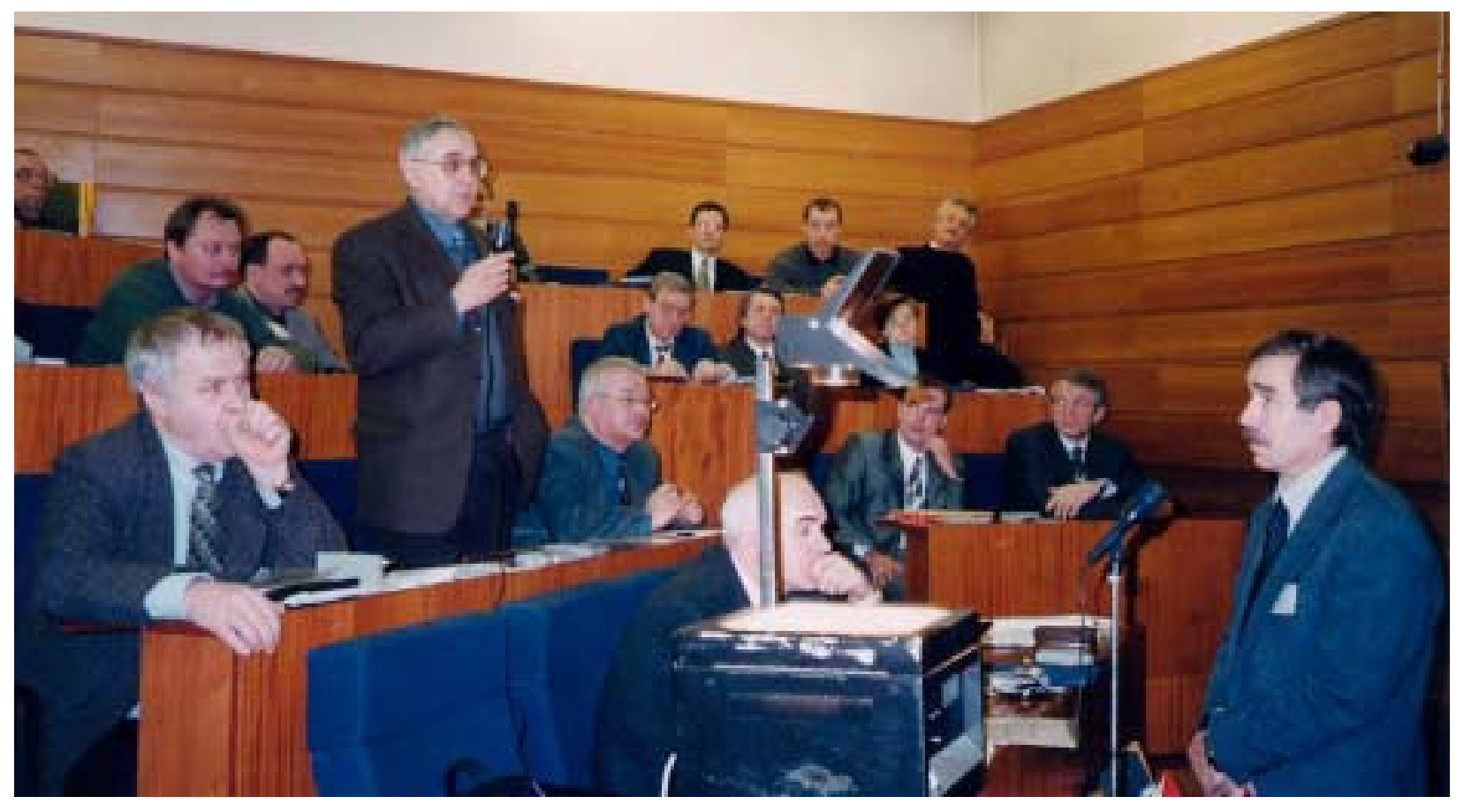




\section{Technological Aspects of MChC Pu-containing Sludges Vitrification, Using MW Melter (B506233)}

\section{K. G. Kudinov, A. A. Tretyakov, Yu. P. Sorokin, V. V. Bondin, L.F. Manakova, MCC $K-26$}

The investigations of one- and two-stage processes for vitrification of the Minatom Siberian Group of Chemical Enterprises (MChC) Pu-containing sludge using a laboratory scale MW heating facility were carried out within the guidelines of joint work between $\mathrm{MChC}$ and Lawrence Livermore National Laboratory (LLNL).

The specifications of the laboratory MW heating facility are as follows:

$\begin{array}{ll}\text { Power supply voltage, } \mathrm{V} & 220 \\ \text { Phase number } & 1 \\ \text { Power supply frequency, } \mathrm{Hz} & 50 \\ \text { Overall power consumption, kWA } & 12 \\ \text { MW radiation power, } \mathrm{kW} & 5,0 \\ \text { MW radiation rate, } \mathrm{MHz} & 2375+-50 \\ \text { Output power control range, } \mathrm{kW} & 0,5:-5,0 \\ \text { Crucible melter filling, \% } & 80 \\ \text { Glass melt temperature, C } & 850-1100 \\ \text { Cooling water consumption, I/min } & 10 \\ \text { Input cooling water temperature, C } & 13 \\ \text { Output cooling water temperature, C } & 40 \\ \text { Compressed air pressure, MPa } & \text { not more than } 0,6 \\ \text { Exhaust rate of emitted gases, } \mathrm{m}^{3} / \mathrm{min} & 1,0\end{array}$

The key process flowsheet for vitrification is given in Fig. 1.

The vitrification process was carried out in melters with various structures and dimensions. The optimum melter structure was stated to be a can melter, with a lid and crucible melter diameter of 300-340 mm and a height of 350-360 mm. The melter is shown in Fig. 2. The crucible melter and the melter lids were connected through flanges, and melting block hermetization at the place of connection was performed using a thermoexpandable graphite lining and mechanical pressing of the crucible melter to the lid. 


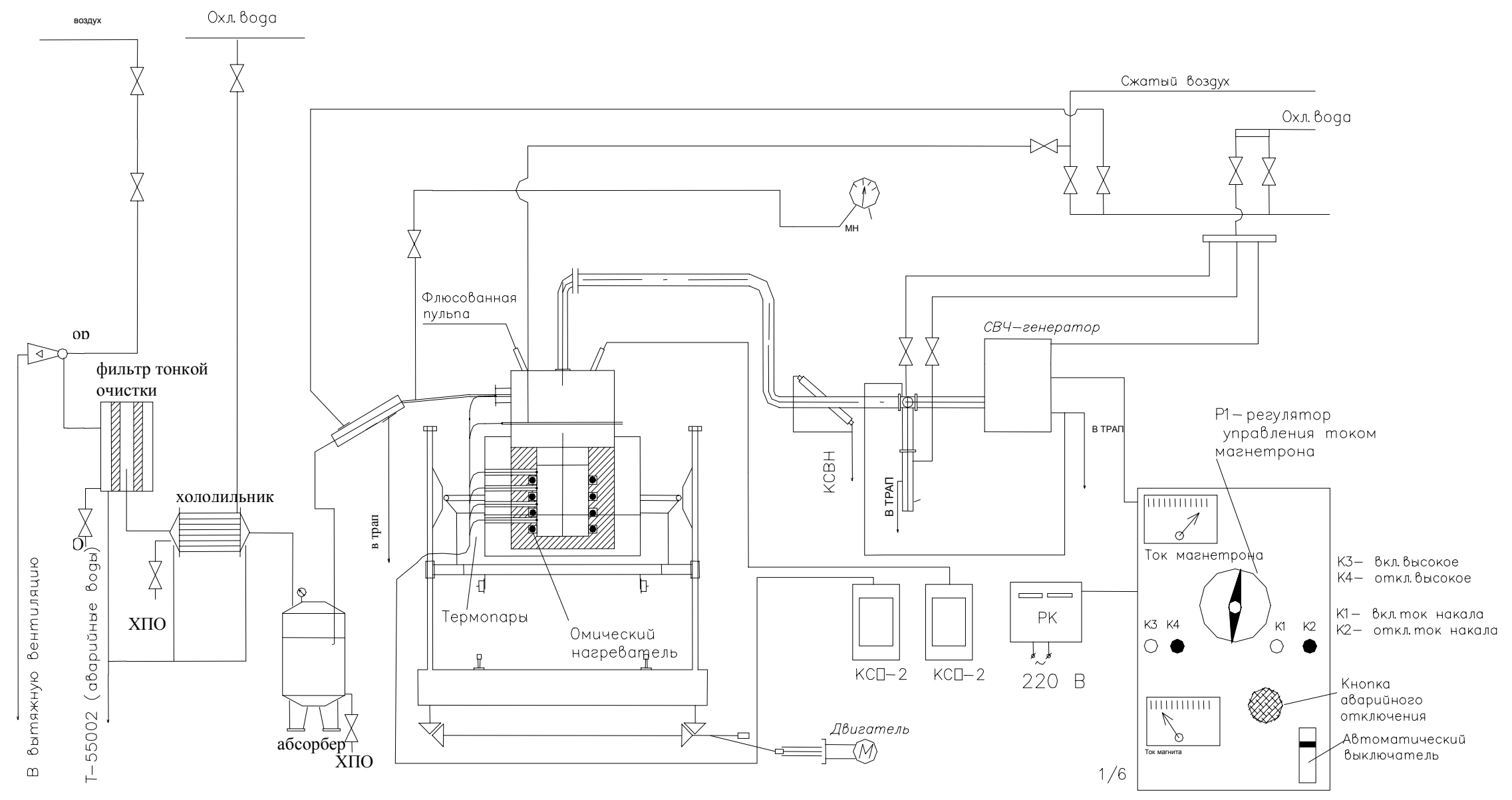

Fig. 1. Принципиальная схема узла остекловывания 1. Compressed air, 2. Cooling water, 3. Injector, 4. fine filter, 5. XПО, 6. Refrigerator, 7. Into exhaust ventilation, 8. Emergency waters, 9. Absorber, 10. Into ТРАП, 11. Fluxed sludge, 12. Thermocouple, 13. Ohmic heater, 14. Engine, 15. KСВН, 16. MW generator, 17. Magnetron current control-PI, 18. Magnetron current, 19. K3-on, high, K4-off, high, 20. On filament current, off filament current, 21. Emergency off button, 22. Automatic switch off, 23. Magnet current. 


\section{Fig. 2. Crucible melter structure.}

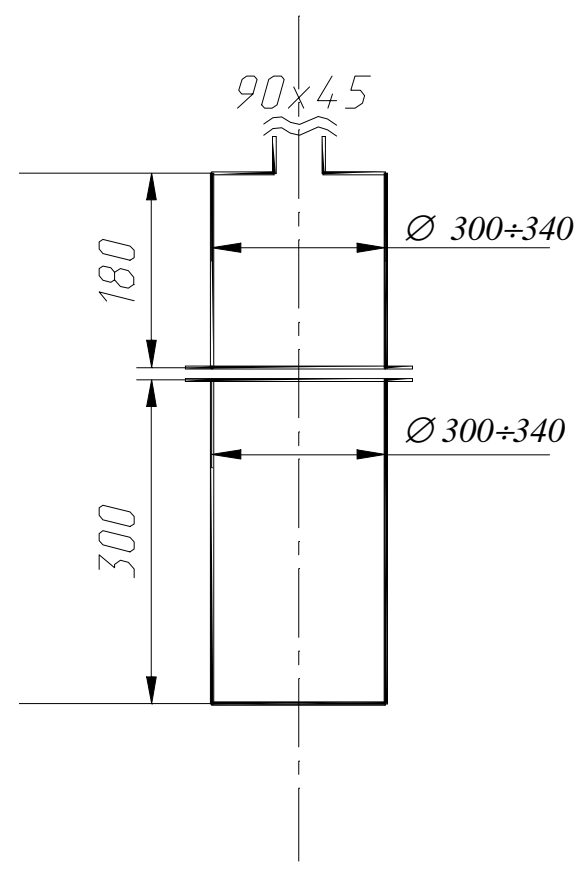

Investigations were carried out both with borophosphate and borosilicate glass-like compositions. It was determined that it is reasonable to use borosilicate compositions as glass-like matrices.

The composition of the vitrified product is given in Table 1.

In the one-stage process, the fluxed sludge was fed by portions in remote mode, using a diaphragm-valve feeder and air lift. In the one-stage regime, flux and Pu-containing sludge calcine are prepared separately, and they are mechanically mixed and fed by portions into the melter.

The volume of one portion, despite differences in crucible melter diameter in the one- and two-stage regimes, was selected to obtain a vitrified product layer of 3-4 $\mathrm{cm}$ in height. A layer of the required height was collected by portions to avoid overfilling the crucible melter with fluxed sludge in the one-stage process, and as the crucible melter was filled with vitrified product, the one portion volume was gradually decreased.

Table 1.-Main components content in borosilicate glass.

\begin{tabular}{|c|c|}
\hline Component & Component content, \% mass \\
\hline $\mathrm{UO}_{3}$ & 20,04 \\
\hline $\mathrm{Fe}_{2} \mathrm{O}_{3}$ & 17,12 \\
\hline $\mathrm{Al}_{2} \mathrm{O}_{3}$ & 4,18 \\
\hline $\mathrm{MnO}_{2}$ & 14,60 \\
\hline $\mathrm{NiO}$ & 2,88 \\
\hline $\mathrm{Cr}_{2} \mathrm{O}_{3}$ & 2,48 \\
\hline $\mathrm{SiO}_{2}$ & 2,30 \\
\hline $\mathrm{Na}_{2} \mathrm{O}$ & 36,4 \\
\hline
\end{tabular}


To increase the efficiency and to obtain a vitrified product that meets the Russian standards for solidified radioactive waste (RAW), it is necessary to use an additional heater outside the crucible melter. The additional heater's optimal power for a melter that is $150 \mathrm{~mm}$ in diameter is $2 \mathrm{kVA}$, and for a melter diameter of $300-340 \mathrm{~mm}$, it is $4 \mathrm{kVA}$.

In the one-stage vitrification, the following results were reached:

- With melter diameter $150 \mathrm{~mm}$, an efficiency of 4 to $4,5 \mathrm{~kg} /$ day of vitrified product;

- With melter diameter $300-340 \mathrm{~mm}$, an efficiency up to $30 \mathrm{~kg} /$ day of vitrified product.

The results of measurements of the chemical stability of the vitrified product are given in Table 2 .

Table 2-Chemical stability of borosilicate glass produced by MW-and ohmic heating.

\begin{tabular}{|c|c|c|c|c|c|c|c|c|c|}
\hline \multirow[t]{2}{*}{ Sample $^{a}$} & \multirow[t]{2}{*}{ pH } & \multicolumn{8}{|c|}{ Leaching rate, $\mathrm{g} / \mathrm{cm}^{2}$.day } \\
\hline & & B $\cdot 10^{-5}$ & $\mathrm{Si} \cdot 10^{-5}$ & $U \cdot 10^{-6}$ & $M n \cdot 10^{-6}$ & $\mathrm{Cr} \cdot 10^{-5}$ & $\mathrm{Fe} \cdot 10^{-3}$ & $\mathrm{Ni} \cdot 10^{-3}$ & $\mathrm{Na} \cdot 10^{-6}$ \\
\hline 1 & 7,91 & 9,33 & 1,69 & 2,52 & 0,90 & 1,32 & 0,12 & 0,70 & 9,20 \\
\hline 2 & 7,50 & 5,83 & 0,64 & 2,30 & 0,90 & 0,78 & 0,12 & 0,70 & 6,20 \\
\hline
\end{tabular}

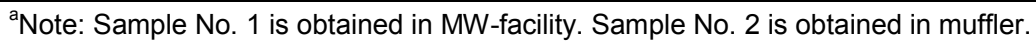

The mechanical compression strength of samples received in the MW facility is $33,0 \mathrm{MPa}$ and in the muffler, it is 18,6 MPa. According to the requirements of State Standard P50926-96, "Wastes, high level solidified," the mechanical compression strength of vitrified wastes must be not less than $9 \mathrm{MPa}$. Thus, all samples meet the requirements of State Standard P50926-96.

In the case of the two-stage process the following results were obtained:

- For melter diameter of $150 \mathrm{~mm}$ the efficiency was up to $7 \mathrm{~kg} / \mathrm{day}$;

- For melter diameter of 300-340 mm it was up to $45 \mathrm{~kg} /$ day for vitrified product.

Data on the chemical stability and mechanical strength of the vitrified product obtained in the two-stage process are comparable with data from the one-stage process.

Therefore, the investigations showed that for immobilization of MChC Pu-containing sludges, both oneand two-stage processes can be used with combined heating, MW and additional ohmic. So, considering that the industrial-scale MW vitrification facility will have 10 times more power, it may be supposed that for full immobilization of the MChC Pu-containing sludges over 13,5 years (as was determined in the JOI) one facility of combined heating would be necessary for the two-stage model, and two facilities would be required for the one-stage regime. 


\title{
Plutonium-containing Wastes Immobilization Activities Accomplished Under LLNL's Contracts at Krasnoyarsk-26 (B506233)
}

\author{
K. G. Kudinov, A. A. Tretyakov, V. V. Bondin, Y. P. Sorokin, L. F. Manakova, \\ MCC K-26
}

Mining and Chemical Combine (MCC) and Lawrence Livermore National Laboratory (LLNL) entered into the Agreement on accomplishment of activities associated with immobilization of plutonium-containing wastes generated from the radiochemical activities at MCC's processing facilities. The contracts entered into by and between MCC and LLNL envisioned development of a technology for plutonium-containing wastes immobilization in order to prevent Pu recovery for military uses. Of various technologies, one for waste vitrification applying microwave ultra-high frequency (UHF) fields was selected as more technologically effective.

The collaboration between MCC and LLNL was possible due to the Intergovernmental Agreement between the RF and the US on Management of Plutonium that has Been Withdrawn from Nuclear Military Programs, and the agreement between the RF and the US Department of Energy (DOE) on the scope of the cooperative activities.

Since May, 1998, three contracts, all entitled "Immobilization of MCC's plutonium-bearing waste" have been signed. Each contract had a particular objective, as described below:

- Contract B347676 - Preparation of feasibility studies of comparison of two technologies to reprocess plutonium-bearing sludges with and without recovery of plutonium (Feasibility Study).

- Contract B506210 - Preparation of Declaration of Intent (DOI) for implementation of Pu-Bearing Sludges Vitrification Facility and Vitrified Waste Storage Facility at MCC.

- Contract B506233 - Development of Justification of Investment (JOI) for implementation of Pilot Plutonium-Bearing Waste Vitrification Facility and Vitrified Waste Storage Facility.

MCC, together with specialists from five RF institutes, All-Russian Design Research Institute of Complex Power Technology (VNIPIET), V.G. Khlopin Radium Institute (KRI-NPO RI), A. A. Bochvar All-Russia Scientific Research Institute of Inorganic Materials (VNIINM), Scientific Research and Design Institute of Installation Technology (VNIKIMT), and All-Russian Research and Design Institute of Production Engineering (VNIPIPT), dealt with the task of how to reliably process and immobilize Pu-bearing sludges generated from radiochemical activities at the processing facilities of MCC.

Preparatory activities, documentation of the work, and some applied research and development have been conducted. Experiments were conducted both in laboratories and in specially equipped "hot cells" that apply microwave ultra-high frequency (UHF) fields to process and vitrify products. Results from the researches have been completed and specified input data stated in baseline documents.

\section{Contract I}

A comparative assessment of the engineering and economical potential has been performed of MCC's two possible Pu-bearing sludge management technologies: (1) reprocessing sludge with plutonium recovery, and (2) direct waste immobilization into a glass matrix using UHF microwave power. 
In three feasibility and contract studies, three Pu-bearing sludge reprocessing options were examined:

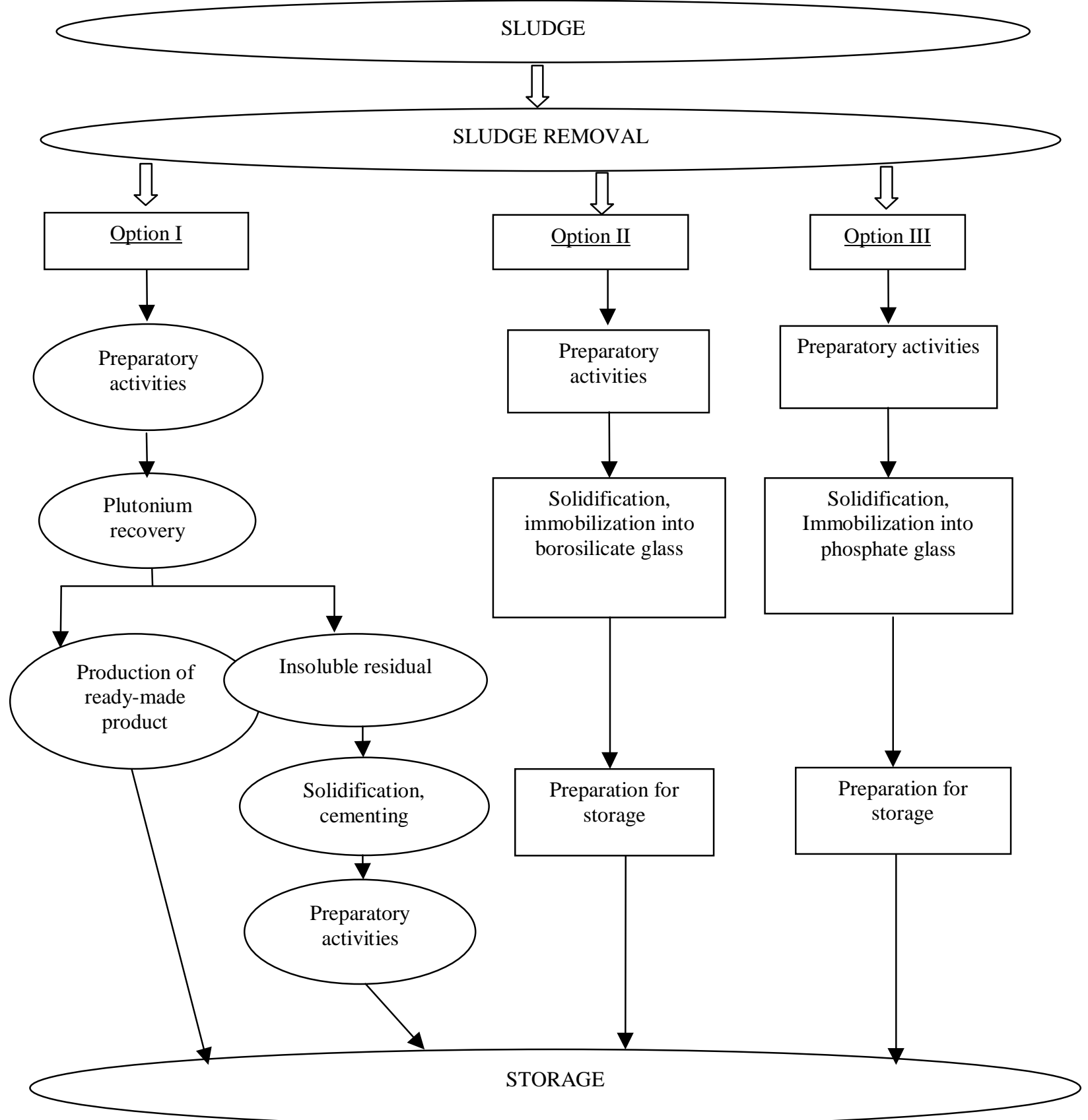

Fig. 1. Schematic diagram of Pu-sludge processing. 
Option 1. Sludge reprocessing with $\mathrm{Pu}$ recovery and purification as $\mathrm{PuO}_{2}$, with cementation of insoluble waste residues-an existing reprocessing procedure at MCC;

Option 2. Microwave sludge solidification without Pu recovery and with borosilicate glass production (the V. Khlopin NPO RI option);

Option 3. Microwave sludge solidification without Pu recovery, and with phosphate glass production (the A. Bochvar VNIINM option).

Option 1 is considered to be the baseline option, and options 2 and 3 are compared to it. Figure 1 shows a schematic diagram for processing sludge as described and assessed in MCC feasibility studies.

The following method for emptying a Pu-sludge storage tank containing radioactive waste is the same for the three options. The MCC wastes are stored in large tanks set in excavations in rock located at process areas underground. Each storage tank is a reinforced concrete reservoir $3200 \mathrm{~m}^{3}$ in volume, $30 \mathrm{~m}$ in height, and $12 \mathrm{~m}$ in diameter, lined inside with sheet stainless steel. Liquid medium-level alkali and nitrate wastes are clarified in the tanks. Then the clarified portion is decanted, and the solid phase, i.e., sludge, is stored in a tank. The heights of the stored residuals in the tanks varies from 4 to $22 \mathrm{~m}$. The sludges differ in composition and density.

Approximately $6,000 \mathrm{~m}^{3}$ of radioactive sludge-like waste has been accumulated in the tanks to date and approximately $600 \mathrm{~kg}$ of Pu was estimated to be in storage at the Technical and Economic Feasibility (TEF) stage.

The MCC plans to decommission the storage tanks in a stage-by-stage manner. One of the difficult tasks is to wash out sediments and treat the recovered sludges in such a way that it would be possible to pump it out for further reprocessing. Special equipment has been developed, manufactured, and tested for sludge discharge at the MCC.

Tank AG-8301/3 has been used as a test facility and reservoir. Height of sludge accumulated in the tank varied from 4 to $4.5 \mathrm{~m}$. To agitate and remove the sludge, special removal equipment was installed in risers of different diameters, which perforated into the tanks.

This tank serves as a large-scale laboratory to obtain reliable sludge composition and density data and to study and improve sludge agitation techniques. Because equipment to agitate and thus obtain a uniform sludge was not installed at the tank beforehand, the sludge composition is non-homogeneous in height. The solid phase concentration varies from $60 \mathrm{~g} / \mathrm{l}$ in the upper layer up to $800 \mathrm{~g} / \mathrm{l}$ in the lower residual layer. Such sludge forms a spatial skeleton and contains almost no free liquid phase.

Owing to a successful combination of engineering decisions and techniques, sludge removal equipment has been continuing to operate in a removal mode. Figure 2 shows the equipment installed in the tank. After removal from the storage tank, the sludge is prepared for further processing. The type of preparation depends on the final reprocessing option.

An insoluble sludge portion that contains no plutonium is solidified in containers $0.2 \mathrm{~m}^{3}$ in volume, employing a cementing facility. In options II and III, plutonium-containing sludge is immobilized either into borosilicate or phosphate glass.

In both vitrification options, the immobilization of Pu-bearing waste into a glass-like matrix is performed in a capsule crucible and afterwards the crucible is installed in a multi-purpose storage canister. The solidified wastes are forwarded for storage in specially equipped storage facilities located in MCC's underground cavities. 


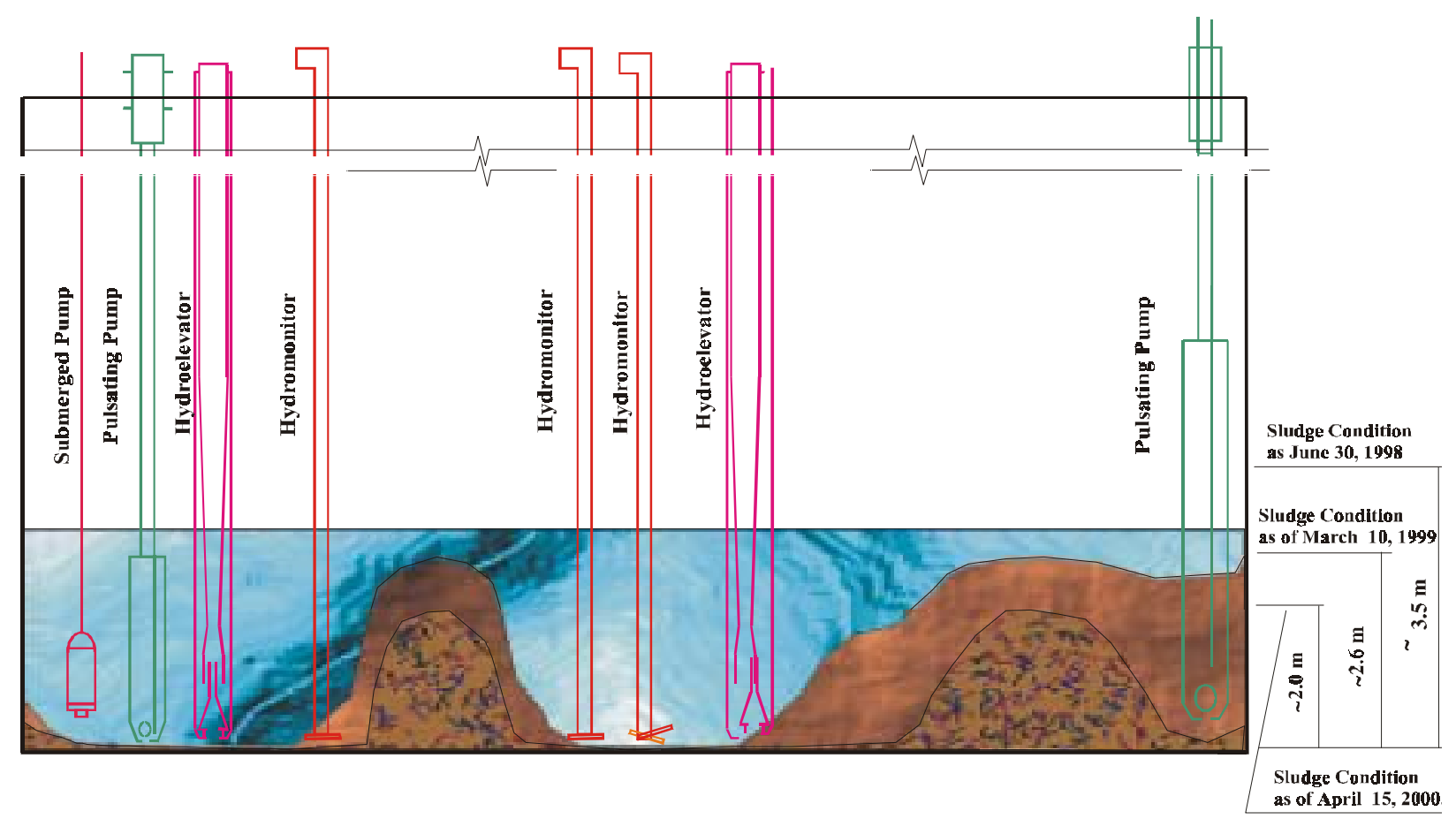

Fig. 2. Illustration of the condition of sludges in a $3200-\mathrm{m}^{3}$ tank as a function of time.

Comparisons of the three options, as estimated at the completion of the feasibility studies, have shown that the immobilization of Pu-bearing sludge into a glass-like matrix is more cost effective than the basic option of sludge processing with Pu recovery.

Results from hot cell experiments in the MCC have persuaded specialists of the efficiency of the 5-kW microwave facility, and that the immobilization of waste into both borosilicate-glass and phosphate-glass matrixes is reproducible and that both vitrification techniques are a viable technology.

Feasibility studies were accomplished in four stages. The last task was preparation of the long-term work plan diagrammed in Fig. 3. The plan ends with implementation of the pilot vitrification and storage facilities in stage 8. All activities listed in the work plan are to be performed over a 4.5-year period, provided that funds are allocated in the amount of \$17.3.M US. The plan consists of eight successive stages.

\section{Contract II}

In the second DOI contract stage, a detailed plan was prepared over a six-month period to set out the objectives of specific investments and technical objectives to be implemented. These were agreed to by the RF Regional Administration, and approved by the RF Ministry of Atomic Energy. Only two options of waste reprocessing were considered, and both options envisaged immediate immobilization of $\mathrm{Pu}$ bearing waste either into borosilicate or phosphate glass matrices. The option of reprocessing the $\mathrm{Pu}$ sludges to recover the Pu was not to be considered further based on the DOI.

Simultaneously, researches to improve waste solidification technologies continued, experience and skills to operate the pilot facility gained, a possibility to remotely control waste solidification process was studied. 


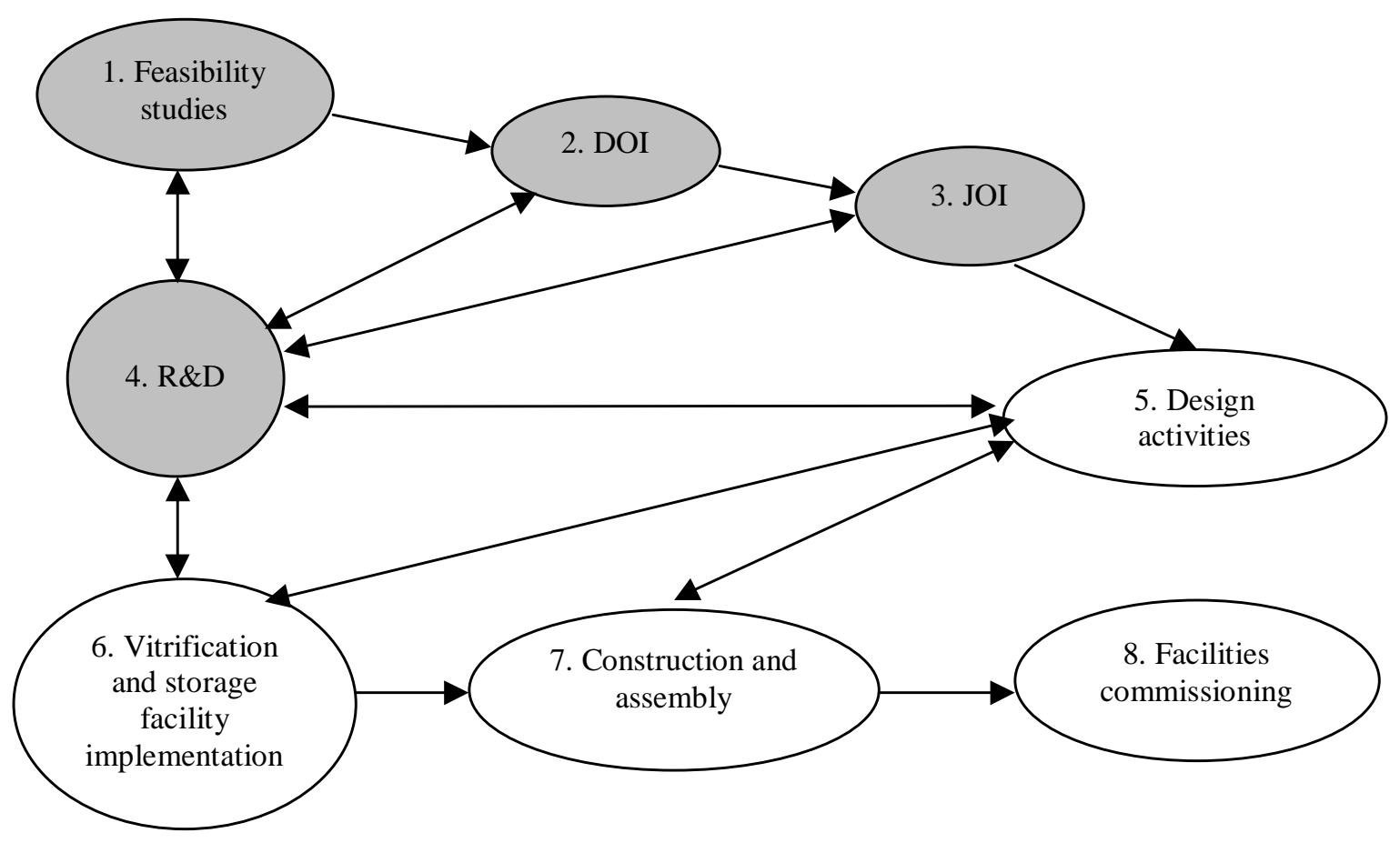

Fig. 3. Illustration of stages 1-8 of the long-term work plan for tasks in RF-US DOE Contracts B347676, B506210, and B506233 (shaded stages-work underway).

\section{Contract III}

At the third stage, the product of the RF-US collaboration became a Task Order development for the JOI.

After intensive discussion of all stages of work and minor modifications and corrections, the third contract, B506233, was signed.

During 2000-2001, MCC together with the RF Institutes developed the JOI for construction of the pilot vitrification facility and the vitrified waste storage facility. The preparation of the JOI was a ladder-type stage, involving the following subtasks:

- Develop Justification of Investment documentation (that is an intensive item of work);

- Conduct hot cell experiments with the actual product;

- Improve particular units of the pilot facility and prepare engineering specifications for the latter in an independent document;

- Determine which one of two technologies to use for waste immobilization into the solid matrix;

- Prepare a future work plan.

The $\mathrm{JOI}$ is the final stage in choosing between the two waste vitrification technologies, according to the terms of the contract. Based on results from experiments, the borosilicate matrix was selected because this vitrification technique, which is based on a heating process with applied UHF-energy, proved to be more technologically effective. In addition, the economic potential determined during feasibility studies also favored the borosilicate glass option. It was shown that placement of the multi-purpose canisters (MPC) of solidified wastes for the borosilicate glass matrixes would require only three storage facilities 
(6800 MPC), while the canisters for phosphate glass matrixes would require five storage facilities (11860 $M P C)$.

Pursuant to the Russian standards, the JOI must show economic necessity, engineering feasibility, and social expediency for the funds invested. The JOI calls for the submission of sufficient information to make the necessary agreements and prepare expert assessments. To meet these requirements, a 12volume document was prepared that contains construction and technical decisions, primary requirements for non-standardized equipment, radiation and nuclear safety decisions, and the environmental impact assessment. Currently, the $\mathrm{JOI}$ is under review for agreement according to Russian policies and requirements.

Five applied research and development $(R \& D)$ reports have been completed that describe the large amount of vitrification work that has been accomplished. Many R\&D issues have been resolved, including:

- Basic principles to control the vitrification process have been determined;

- Plasma formation in the air space of the melter has been mitigated, and the magnetron has been protected against plasma;

- Chemical stability of vitrified waste has been studied with a specially installed facility;

- Auxiliary ohmic crucible heating has been applied that makes it possible to obtain a glass block of proper quality and without crust formation;

- The actual sludge, which contains up to $450 \mathrm{mg}$ of plutonium per $1 \mathrm{~kg}$ of glass and fragmentation radionuclides, has been immobilized into borosilicate glass and melted. According to results from tests on chemical stability and mechanical strength, the product meets Russian standards requirements.

After activities under Contract III were accomplished, the future work plan based on pre-project and research results was developed.

\section{Estimated Sources of Wastes from MOX Fuel Production at the MCC}

During the activities associated with $\mathrm{JOI}$ preparation, there was active discussion of the possible production quantities at MCC of heat-releasing fuel assemblies (FA) based on mixed uranium and plutonium fuel. If FA's are produced only for VVER-1000 and BN-600 reactors, 2.75 MT of weapons plutonium would be used annually. However, if deliveries of MOX-FA's for extra reactors such as Canadian and European are produced, up to $5 \mathrm{MT} / \mathrm{y}$ of weapons-grade plutonium would be used. Commissioning of this extra production would result in the generation of extra Pu-bearing wastes.

The intrinsic wastes from MOX-fuel production, according to the literature, previous work, and results from existing production analyses, are highly varying and complex in composition. However, this waste can be divided into three categories:

1. Defective primary products;

2. Inorganic waste;

3. Organic waste. 
Figure 4 shows plutonium waste categories and sources. It is necessary to point out that organic waste from MOX-fuel production constitute the major portion of total waste. It is obvious that special attention should be paid to treatment of this waste category.

Figure 5 is a block diagram for reprocessing the waste generated from MOX-fuel production. Insoluble waste after incineration or calcination, alkali sintering, and dissolution of sintered products in nitric acid are fluxed and then vitrified.

Having studied the issues of vitrifying Pu-sludge wastes and radiochemical production waste processing, it was timely and useful to consider the possibility of the joint immobilization of such types of waste. One of the scenarios proposed for consideration is combining these two types of wastes in certain ratios at the pre-flux stage. This option is shown with dotted lines in Fig. 5.

The costs to implement Pu-bearing waste reprocessing have been approximately estimated in the JOI and are summarized in Table 1.

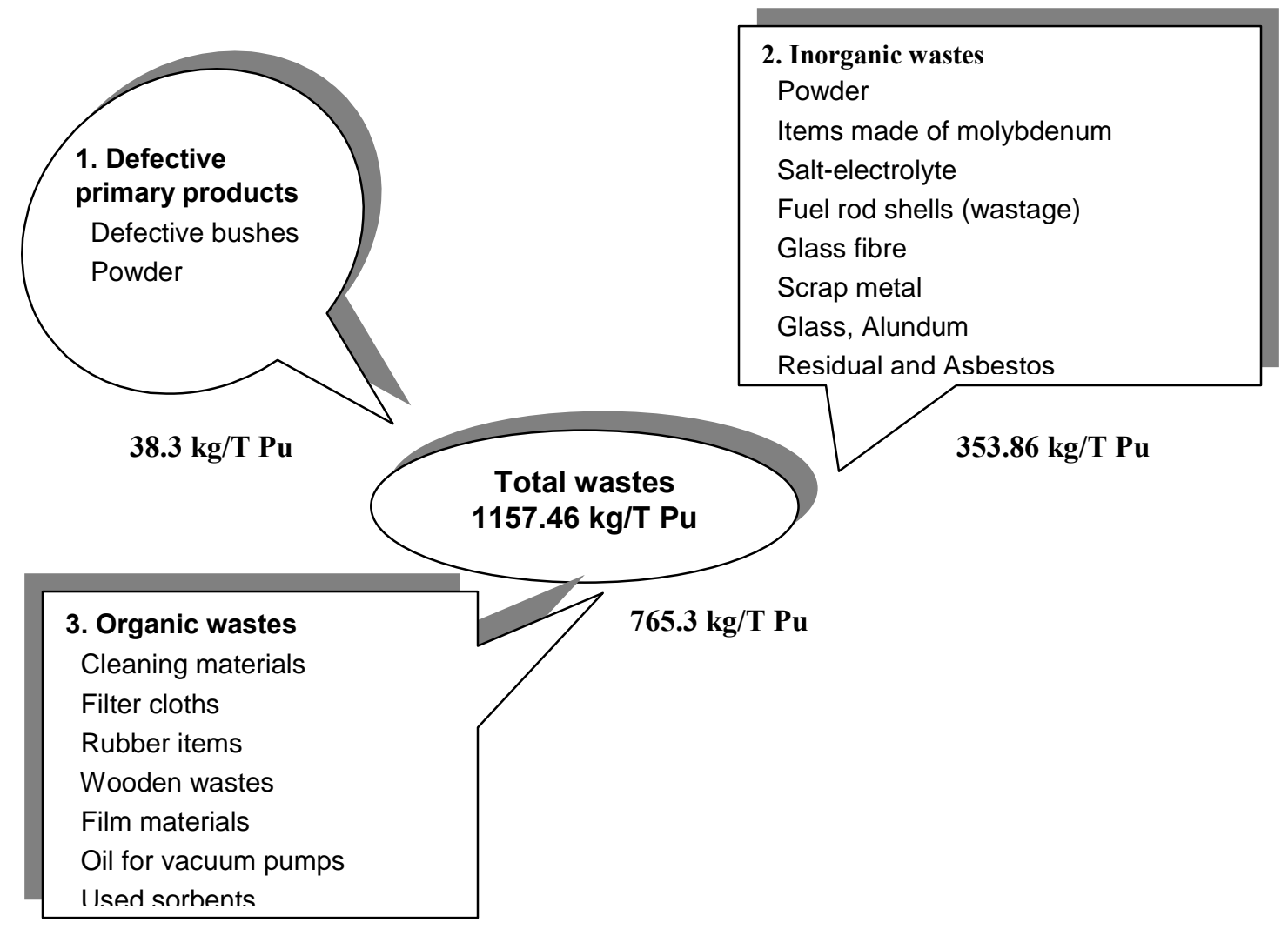

Fig. 4. Plutonium waste categories and sources. 


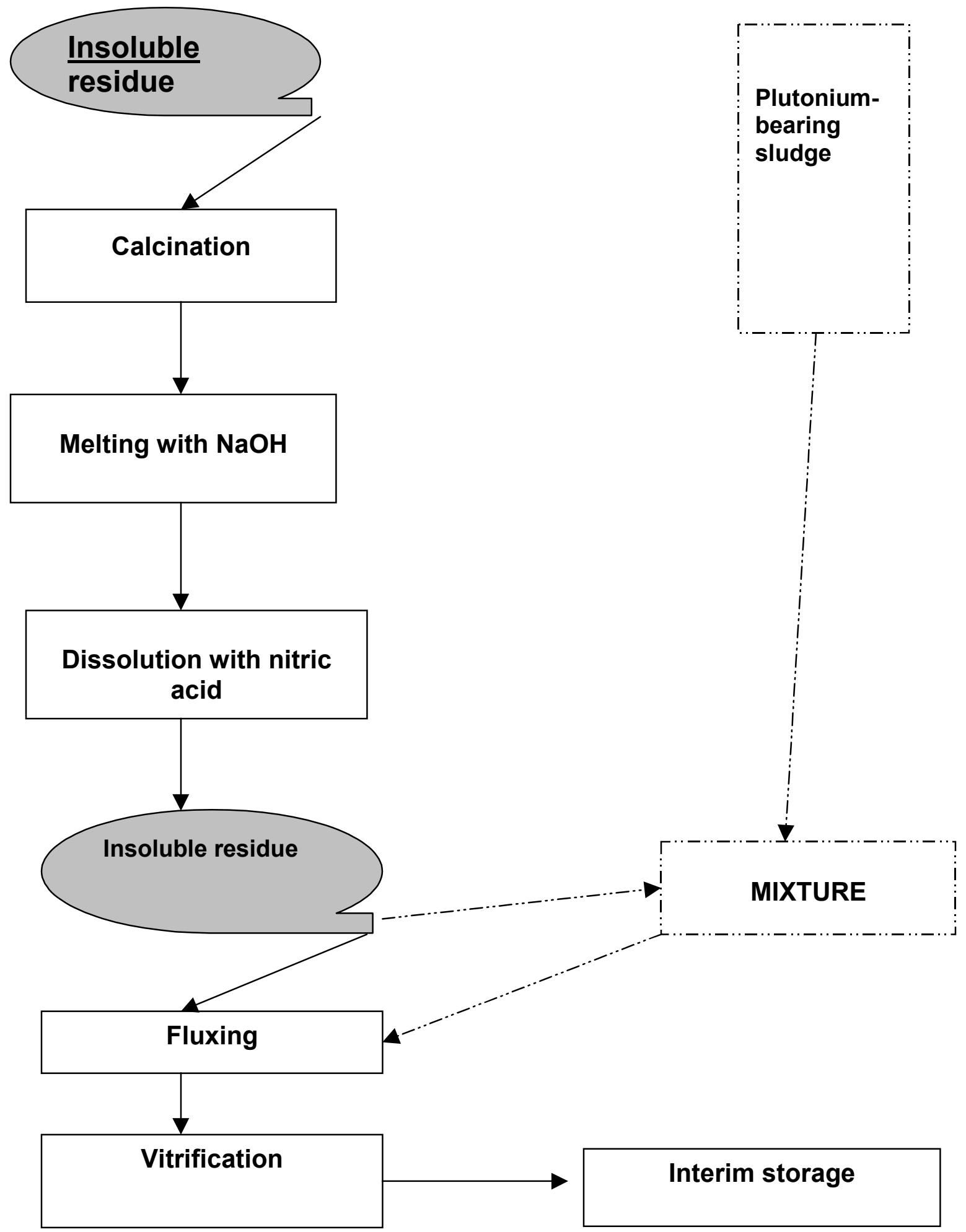

Fig. 5. Block diagram of reprocessing Pu-bearing waste generated from MOX fuel production. 
Table 1. Estimated costs to reprocess Pu-containing wastes.

\begin{tabular}{|l|c|}
\hline Type of waste & Cost \$ M US \\
\hline Radiochemical facility waste: Pu sludges only & 18 \\
\hline MOX-fuel production waste only & $12-14$ \\
\hline Joint reprocessing of both waste types: MOX wastes and Pu sludges & 21 \\
\hline
\end{tabular}

It is evident that joint reprocessing and vitrification of the two waste types is cost effective. A cost difference of $\$ 3$ million compared to $\$ 12-14$ million is attractive and an earnest argument in favor of continuing this work. However, it must be clear that these values presented here are approximate. Therefore, a decision has been made to continue work on a feasibility study of options for processing the Pu-bearing waste generated from MOX-fuel production in order to develop a better cost estimate. This work has become an independent item in a plan to investigate promising trends in waste disposal options for the MCC.

\section{Conclusions}

Results of work accomplished by MCC together with experts from RF Institutes under the contracts are as follows:

- The technology for the plutonium-containing sludges vitrification and immobilization into borosilicate glass with UHF microwave power applied has been developed. Engineering specifications for the pilot vitrification facility have been prepared.

- Joint reprocessing of wastes from MOX-fuel production and radiochemical activities has been assessed.

- Additionally, underground location of MCC's primary facilities, which are engineering barriers with the developed infrastructure and sufficient free underground mined cavities are an opportunity that encourages an RF decision to locate plutonium treatment production, in particular MOX-fuel- and FA's production at this site.

- Use of the existing reservoirs located in MCC's underground cavities for interim storage of solidified wastes has been considered to be a feasible approach.

Handling of radioactive wastes safely is another one of the many problems that are of importance in nuclear facilities. To dispose of waste in an ecologically safe manner is to provide the following conditions:

- Interim storage under permanent control;

- Geological disposal in a repository (solidified radioactive waste).

Both requirements are met by MCC owing to its proximity to the most promising site for a geological repository, the granitoids of the Nizhnekansky massif.

The MCC prefers a technology for the reliable immobilization of plutonium-bearing waste. We believe that continuation of the RF-US collaboration to study these immobilization technologies will be to the benefit of both countries. It is essential to add that the nature of radioactive waste won't allow us to forget to monitor its status whether relations between politicians are good or bad. 


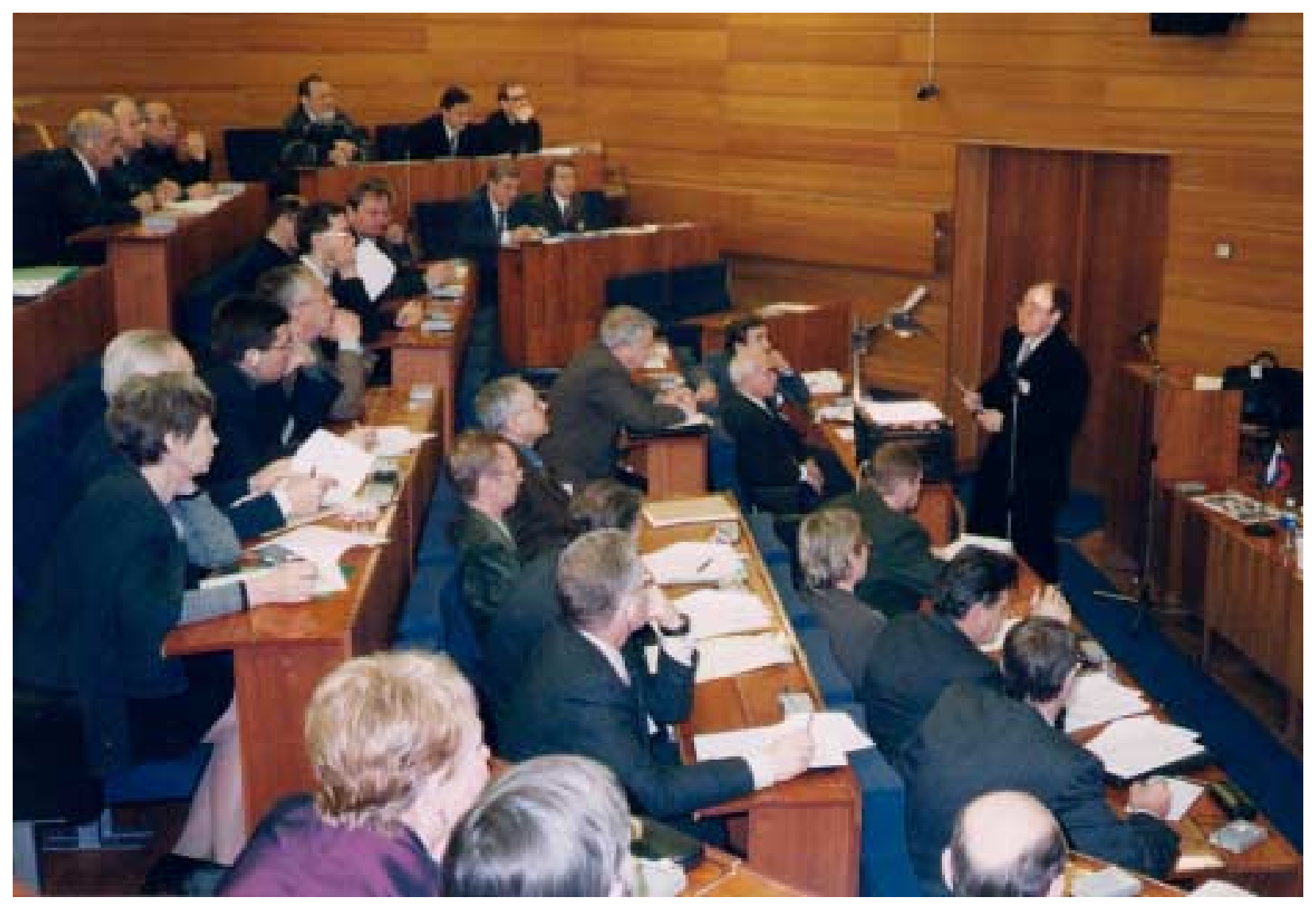




\title{
Overview of the Development of Phosphate Glasses for Immobilizing Pu-containing Sludge at MCC (B506233)
}

\author{
G. B. Borisov, A. V. Nazarov, O. A. Mansourov, Y Y. Volchok, A. A. Balashov, \\ VNIINM
}

For immobilization of Pu-containing sludge being stored in Mining and Chemical Combine (MCC) tanks, lower temperature melting phosphate, boron phosphate, and lead phosphate glasses have been developed at A. A. Bochvar All-Russia Scientific Research Institute of Inorganic Materials (VNIINM). The glasses were considered for use in the technology of vitrifying the sludge by means of microwave heating in an in-situ metallic container.

The glasses demonstrate some advantages in comparison to other vitreous compositions (for example, silicate and borosilicate):

- They are lower melting as it is essentially the process of microwave melting in a stainless-steel noncooled metallic container restricted by the temperature of the wall with up to $850^{\circ} \mathrm{C}$;

- The use of a liquid flux (orthophosphoric acid) that permits an increase in the mobility of the sludge and facilitates its feeding into the container.

The glass compositions and main physico-chemical properties are presented in Table 1.

In addition to glass formulation and characterization measurements, efforts have been implemented to assess the feasibility of immobilizing the MCC's Pu-containing sludge using ceramic melters.

At PA "Mayak," EP-type ceramic melters have been successfully exploited for many years, including EP100 and EP-500 facilities, where 100 and 500 define a specific melter as output of high-level waste $(\mathrm{HLW})$ in liters per hour.

The main advantages of the ceramic melters are as follows:

- Well mastered technology of immobilizing liquid HLW, including ones containing corrosion products and perlite;

- Long-run service life of the melters (6 years for EP-500/1p);

- Simplicity of the technology due to a one-stage vitrification process;

- Low fugacity of radionuclides thanks to relatively low temperatures of melting and molten glass pouring out (e.g., for Cs-137, volatility is less than $1 \%$ at a temperature of $900-1000^{\circ} \mathrm{C}$ ).

However, at the same time, immobilization technology using ceramic melters has a few limitations:

- Huge dimensions of the melters and the lack of automatic systems of remotely dismantling and disposing of the melter equipment once it has reached the end of its operating life;

- Restrictions of the wastes to be treated in chemical composition;

- Molybdenum electrodes are subject to corroding from corrosion-aggressive admixtures, such as iron and nickel oxides and sulfate-ion. 
Table 1. Compositions and some physico-chemical properties of boron phosphate and lead phosphate glasses.

\begin{tabular}{|c|c|c|c|c|}
\hline $\begin{array}{c}\text { Components } \\
\text { wt } \%\end{array}$ & \multicolumn{2}{|c|}{$\begin{array}{l}\text { Phosphate } \\
\text { glasses }\end{array}$} & $\begin{array}{c}\text { Boron phosphate } \\
\text { glasses }\end{array}$ & $\begin{array}{c}\text { Lead phosphate } \\
\text { glasses }\end{array}$ \\
\hline $\mathrm{Na}_{2} \mathrm{O}$ & \multicolumn{2}{|c|}{$18,0-27,0$} & $22,0-27,0$ & $22,0-27,0$ \\
\hline $\mathrm{Al}_{2} \mathrm{O}_{3}$ & \multicolumn{2}{|c|}{$0,1-3,0$} & $0,1-3$ & Up to 3,0 \\
\hline $\mathrm{Fe}_{2} \mathrm{O}_{3}$ & \multicolumn{2}{|r|}{$0,5-8,0$} & $0,5-8,0$ & Up to 10,0 \\
\hline $\mathrm{MnO}_{2}$ & \multicolumn{2}{|r|}{$0,5-5,0$} & $0,5-5,0$ & Up to 5,0 \\
\hline $\mathrm{NiO}$ & \multicolumn{2}{|r|}{$0,1-1,0$} & $0,-1,5$ & Up to 1,5 \\
\hline $\mathrm{Cr}_{2} \mathrm{O}_{3}$ & \multicolumn{2}{|r|}{$0,1-0,5$} & $0,1-0,8$ & Up to 1,0 \\
\hline $\mathrm{SiO}_{2}$ & \multicolumn{2}{|r|}{$0,1-2,0$} & $0,1-3,0$ & Up to 3,0 \\
\hline $\mathrm{CaO}$ & \multicolumn{2}{|c|}{$0,1-1,0$} & $0,1-1,5$ & Up to 1,5 \\
\hline $\mathrm{UO}_{3}$ & \multicolumn{2}{|r|}{$1,0-10,0$} & $1,0-10,0$ & 10,0 \\
\hline $\mathrm{CeO}_{2}\left(\mathrm{PuO}_{2}\right)$ & \multicolumn{2}{|c|}{$0,1-0,5$} & $0,1-0,5$ & $0,1-0,5$ \\
\hline $\mathrm{P}_{2} \mathrm{O}_{5}$ & \multicolumn{2}{|c|}{$50,0-65,0$} & $44,0-52,0$ & $45-52$ \\
\hline $\mathrm{B}_{2} \mathrm{O}_{3}$ & \multicolumn{2}{|c|}{-} & $6,0-12,0$ & - \\
\hline $\mathrm{PbO}$ & \multicolumn{2}{|r|}{-} & - & $6,0-10,0$ \\
\hline Melting temperatures, ${ }^{\circ} \mathrm{C}$ & \multicolumn{2}{|r|}{$850-950$} & $840-870$ & $800-830$ \\
\hline Incorporation rate, wt $\%$ & \multicolumn{2}{|r|}{$30-45$} & $40-47$ & $38-48$ \\
\hline \multirow{2}{*}{$\begin{array}{l}\text { Leaching ability } \\
\mathrm{g} / \mathrm{cm}^{2} \text { day }^{\mathrm{a}}\end{array}$} & $\mathrm{Na}$ & $5 \cdot 10^{-5}-8 \cdot 10^{-6}$ & $(1: 3) \cdot 10^{-6}$ & $(5-7) \cdot 10^{-6}$ \\
\hline & $U$ & $10^{-6}-10^{-7}$ & $10^{-7}$ & $10^{-7}$ \\
\hline \multirow{2}{*}{$\begin{array}{l}\text { Concentration in leachate } \\
\text { after one-day } \\
\text { experimental tests, mg/l }\end{array}$} & $\mathrm{Ce}$ & $0,04-0,06$ & $<<0,03^{a}$ & $<<0,03^{a}$ \\
\hline & $B$ & - & $<<0,03^{a}$ & - \\
\hline
\end{tabular}

${ }^{a}$ Within measurement error by atomic-adsorption method.

The main reason for corrosion of electrodes is the sulfate-ion. Nickel oxide contributes only if the sulfateion is available in the molten glass. For example, if $\mathrm{NiO}$ concentrations are less than $0.4 \mathrm{wt} \%$, a metal phase forms onto the bottom of the melter in the form ingots of nickel sulfides $\mathrm{Ni}_{3} \mathrm{~S}_{2}, \mathrm{NiS}$.

The Pu-containing sludge being stored in MCC's tanks does not contain a plentiful concentration of sulfur. Experimental tests of molybdenum corroding in the molten phosphate glass, containing 6 wt $\% \mathrm{Fe}_{2} \mathrm{O}_{3}$, have shown that the corrosion rate is about $10 \mathrm{~mm} / \mathrm{year}$, which corresponds to a service-life for the electrodes of up to 3 years.

Dimensions of the EP-like melter are defined by the square of a melting zone needed to keep the output at a relevant rate (for example 100 or $500 \mathrm{l} / \mathrm{h}$ ). In the case of immobilizing MCC's sludge, the melter output will be characterized by how quickly one uploads the sludge from the tanks. It is well known from practice that this process runs for a long time; therefore, a small-dimensional facility, for example with an output of 50 or $100 \mathrm{l} / \mathrm{h}$, will be required. A schematic of such a facility is shown in Fig. 1.

The most crucial problem of all Russian melters is the absence of reliable units for feeding and portioning (adding) sludge and solid glass formers. Therefore, in Russia a process of incorporating HLW into borosilicate glass has never been realized. Instead, a technology of vitrifying liquid waste using orthophosphate acid as a glass former has been used. At VNIINM, work is going on to design units for feeding and metering the sludge into the melters. A dosing device with vibro- or airy blending and nonvalve feeding of the sludge into the melter has been developed. 

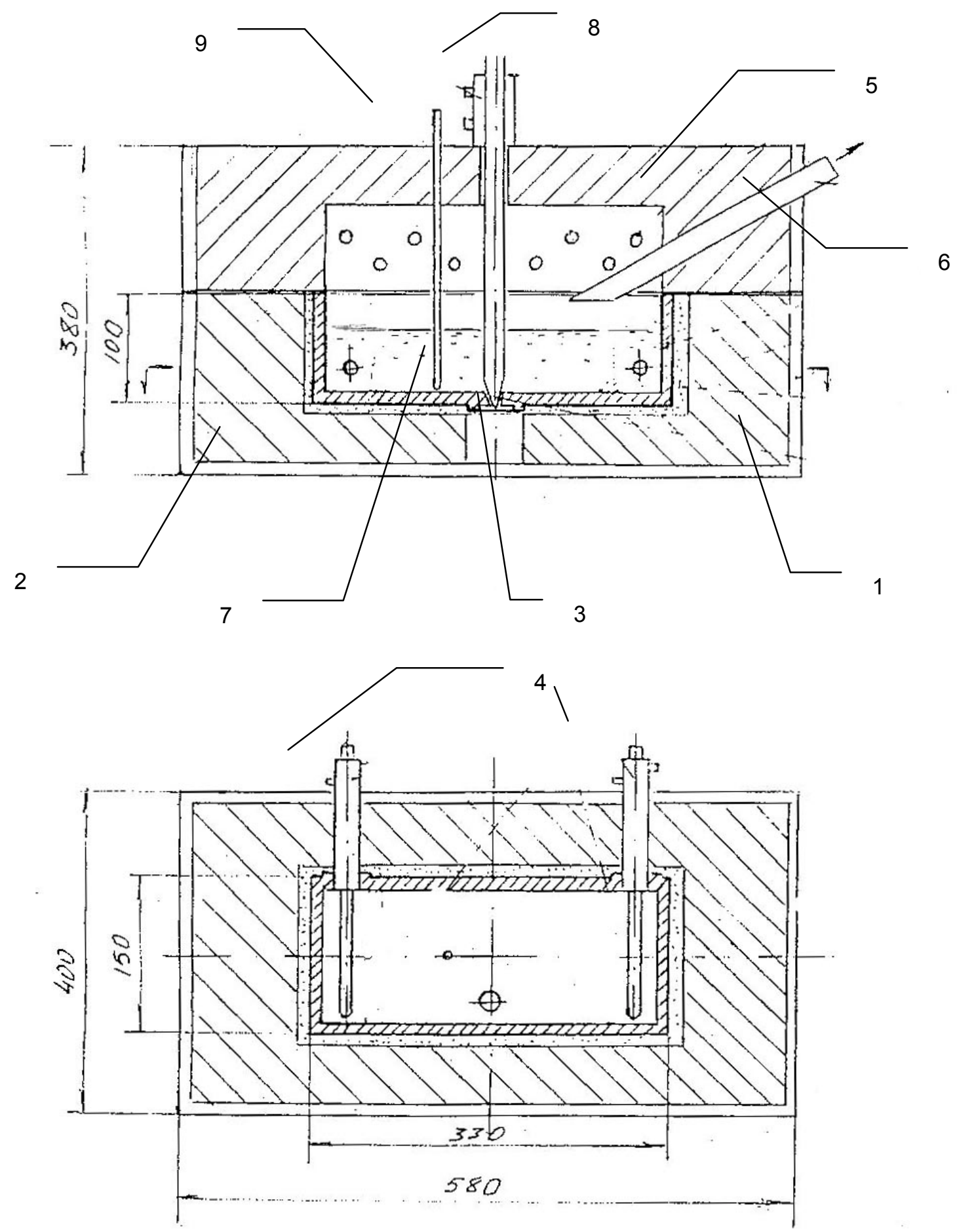

Fig. 1. A general scheme of a small-dimensional facility for treatment of MCC's Pu-containing sludge at MCC: 1 . Melter case; 2. Refractory; 3. Melter basement; 4. Molybdenum electrodes; 5. Melter vault with SiC heaters; 6. Off-gas system; 7. $\mathrm{MoSi}_{2}$ plunger for pouring out the molten glass; 8. Water cooled sludge feeder; 9 . Entries for thermocouple and level measuring unit. 


\section{Conclusions}

- Lower temperature melting, non-crystallizing, stable phosphate, boron phosphate and lead phosphate glasses were developed at VNIINM for treatment of MCC's sludge;

- If difficulties with sludge feeding and metering are resolved, ceramic melters (EP-50 or EP-100) might be applied for immobilization activities at MCC as an alternative to microwave melting. 


\title{
Borosilicate Glass Matrix Technology Developed for MCC (B506233)
}

\author{
A. S. Aloy, B. S. Kuznetsov, E. Yu. Ivanov, A. V. Trofimenko, KRI \\ Introduction
}

In accordance with the requirements specification, investigations were conducted for the development of hot cell solidification technology and the selection of matrix materials.

Borosilicate compositions were developed and synthesized for the solidification of the model sludge, including up to $40 \mathrm{~g} / \mathrm{l}$ sodium. Obtained compositions were analyzed with use of the roentgen-phase ( $\mathrm{x}$ ray) analysis, the differential-thermal analysis and roentgen-spectral microanalysis. Their density, mechanical strength and chemical durability were measured.

The work of equipping the KRI pilot set was conducted in order to investigate the use of MW-energy at different fill levels of the crucible-melter jointly with ARSRICHF specialists.

A device for the core sample extrusion from a tubular drill was developed and made at KRI. The possibility of using a silicone carbide crucible-melter was also investigated.

\section{Development of Two-stage Process of Sludge Solidification}

The investigations on simulated sludge solidification were conducted under a two-stage version of the technology process. For this purpose, preliminary preparation of simulated sludge was conducted for two compositions, which produced dry remains.

Technology development of the simulated sludge, concentrating, and drying was conducted as shown in Fig. 1. The equipment setup consists of:

- Thin-film rotor concentrator (TRC), in which the process of deep concentration by evaporation and drying takes place;

- Pot for receiving the sludge portion and its preparation for feeding into the TRC;

- Feeding device with adjustable volume productivity ranging from 0.5 to $2 \mathrm{l} / \mathrm{h}$;

- Collector for material obtained;

- Refrigerator, bubble-condenser, and off-gas system filter, which is for the vapor condensation and condensate collection;

- Thermostat for feeding of the heat carrier into the TCR jacket at a controlled temperature.

The sludge feeder feeds the sludge into the TRC, where it is concentrated by evaporation to the necessary moisture content, and arrives into the collector by gravity. 


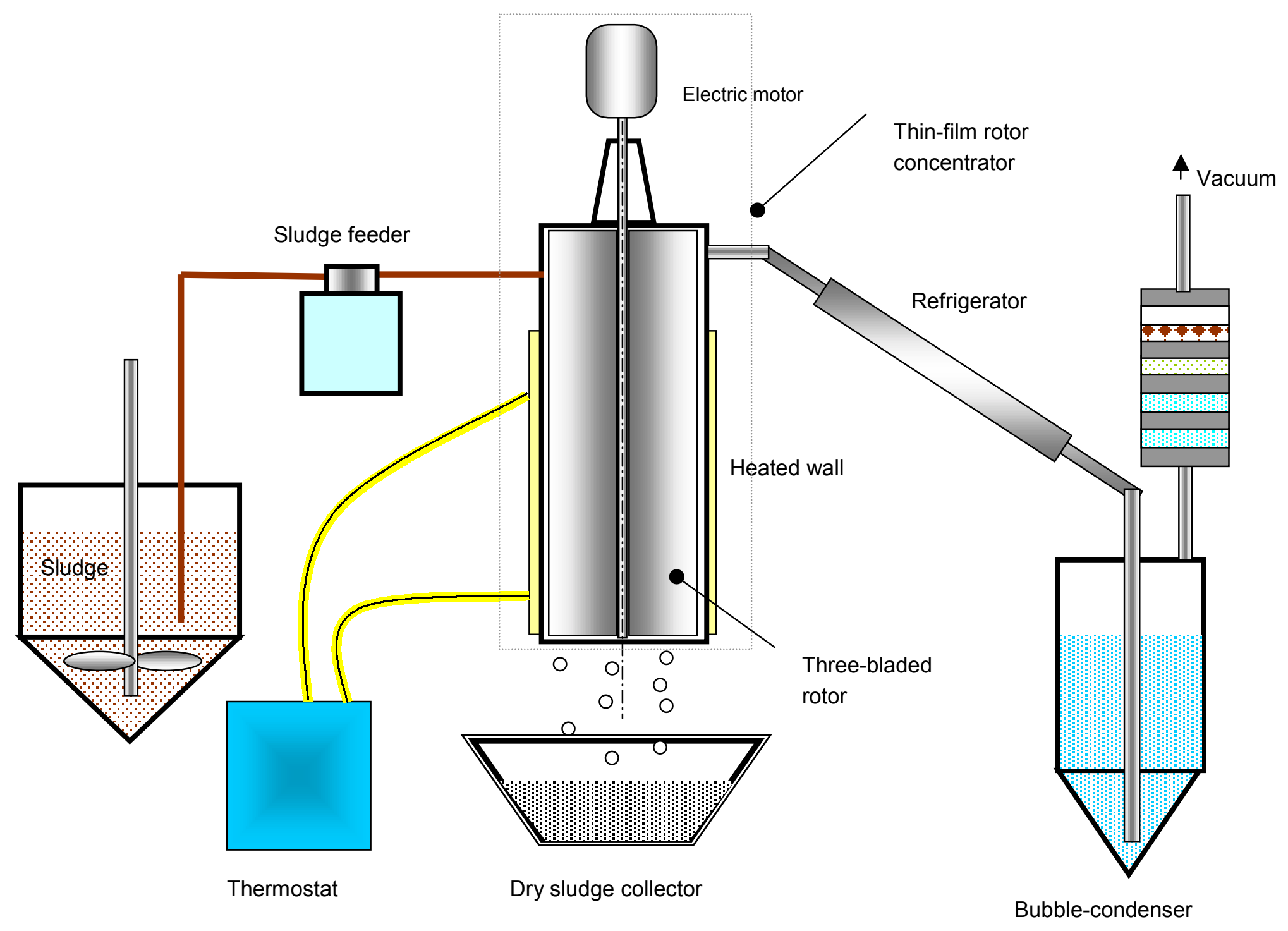

Fig 1. Setup scheme for sludge drying with thin-film rotor concentrator. 
A heat carrier fed into the TCR jacket produces the heat of the reaction surface. Glycerin is used as a heat carrier, which is heated under thermostatic control to the specified temperature.

The TCR is made as a cylinder, which is fixed to the bearing unit by the upper part. The rotor is fixed in the bearing by the console, which passed through the cylinder. Three blades are hard fixed along the rotor. The disk is installed on the rotor above the blades. The gap between the blades and inner part of the cylinder is $\sim 1 \mathrm{~mm}$. There is a jacket on the external surface of the cylinder at the height of the rotor blades, which is used for the heat carrier. The drying sludge collector is located in bottom part of the cylinder.

The rotor is turned by an electric motor using a multiple $V$-belt drive. The frequency of rotor rotation is 750 turn/min.

The sludge is fed into the upper part of cylinder, impacts the disk, splashes on the wall of the cylinder and streams down. The blade of the rotor provides a uniform sludge distribution in the thin film on the heatexchanging surface. The sludge is dispersed, and flows down and falls into the collector. It is possible to obtain a product that ranges from a pasty mass to a friable form depending on selected conditions.

The dry remains of sludge with two compositions used in the work had a moisture content of 35-37 wt\%.

The glass frit (earlier prepared and crushed glass) was selected as flux. This frit composition is shown in Table 1.

Table 1. Compositions of simulated sludge, glass frit, and glass.

\begin{tabular}{|l|c|c|c|c|c|c|}
\hline \multirow{2}{*}{ Component } & \multicolumn{6}{|c|}{ Content, wt\% } \\
\cline { 2 - 7 } & Sludge I & Sludge II & Frit I & Frit II & Glass I & Glass II \\
\hline $\mathrm{SiO}_{2}$ & - & 3.9 & 65.8 & $74-75$ & 32.9 & 39.6 \\
\hline $\mathrm{B}_{2} \mathrm{O}_{3}$ & - & - & 25.7 & - & 12.9 & - \\
\hline $\mathrm{Na}_{2} \mathrm{O}$ & 28.0 & 36.4 & 8.5 & $15-16$ & 18.3 & 26.2 \\
\hline $\mathrm{Al}_{2} \mathrm{O}_{3}$ & 15.6 & 3.9 & - & $0.5-1.0$ & 7.8 & 2.0 \\
\hline $\mathrm{Fe}_{2} \mathrm{O}_{3}$ & 19.6 & 16.5 & - & $0.5-1.0$ & 9.8 & 9.3 \\
\hline $\mathrm{Cr}_{2} \mathrm{O}_{3}$ & 2.6 & 2.3 & - & - & 1.3 & 1.2 \\
\hline $\mathrm{UO}_{2}$ & 20.2 & 19.6 & - & - & 10.1 & 9.8 \\
\hline $\mathrm{MnO}{ }_{2}$ & 14.0 & 13.2 & - & - & 7.0 & 6.6 \\
\hline $\mathrm{CaO}$ & - & - & - & $5-6$ & - & $2.5-3$ \\
\hline $\mathrm{MgO}$ & - & - & - & $3-4$ & - & $1.5-2$ \\
\hline
\end{tabular}

Glass-frit I was produced by melting the charge, which included amorphous silicon oxide, boric acid, and sodium carbon, with exposure for two hours at $1300^{\circ} \mathrm{C}$. After cooling, the glass was crushed and used as flux. Glass-frit II was crushed industrial glass used for packaging.

\section{Development of Technology for Glass Production from Dry Sludge Remains and Glass Frit}

The development of operating procedures for MCC sludge wastes under a two-stage process was conducted in the following manner. The sludge, prepared under MCC's method, was fed into the test equipment for sludge concentration with the thin-film rotor concentrator, and the product was obtained with a moisture content of 35-37 wt\%. The dry remains and glass frit in a 1:1 ratio were fed into the MWset in batches of $250 \mathrm{~g}$. The interval between the batches was $20 \mathrm{~min}$. The productivity of the MW-set 
was $0.4 \mathrm{~kg} / \mathrm{h}$ for melted material under a mean power of $3 \mathrm{~kW}$. The MW equipment was being matched at different infill levels for increasing the efficiency of use of MW energy.

The sampling of glass was periodically conducted with a quartz pipe. The melted material was extracted from the crucible-melter and was dripped onto a metallic slab. The stark drop was analyzed for mass loss under boiling in a Sokslet device for 5 hours. The mass losses consisted of $0.07-0.12 \%$, which corresponds to the mass loses for glass described in previous Progress reports. Therefore, comparatively rapid analyses of glass quality can be conducted at different infill levels of the crucible-melter.

\section{Conclusions}

1. The system of matching MW equipment in different infill levels was developed and tested.

2. Homogeneous glass block was obtained in the crucible $85 \%$ filled without use of active thermoinsulation.

3. Production of the MW equipment increases by a factor of three using the dry remains of sludge.

4. Corrosion of the crucible-melter is not significant at a glass synthesis temperature of $1150^{\circ} \mathrm{C}$. The skull layer is $1 \mathrm{~mm}$ thick.

5. The two-stage process is recommended for the experimental-industrial set of equipment.

\section{Viewgraphs-See following pages}




\section{MW pilot set block-scheme}

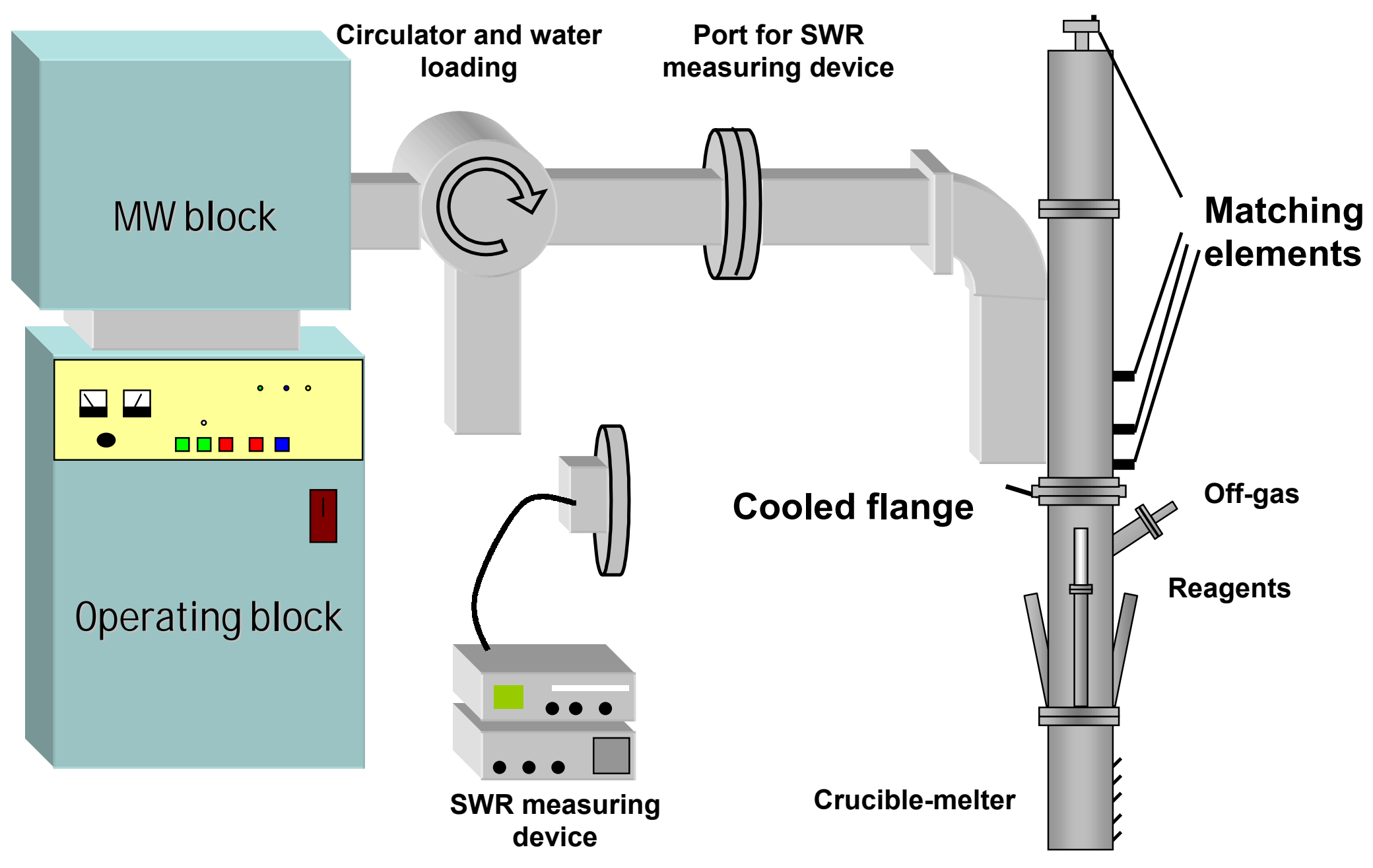




\section{Composition of simulated charge, wt $\%$}

\begin{tabular}{|c|c|c|c|}
\hline $\mathrm{CeO}_{2}$ & 4.5 & $\mathrm{Fe}_{2} \mathrm{O}_{3}$ & 9.0 \\
\hline $\mathrm{Cr}_{2} \mathrm{O}_{3}$ & 0.5 & $\mathrm{NiO}$ & 1.5 \\
\hline $\mathrm{MnO}_{2}$ & 8.0 & $\mathrm{Al}_{2} \mathrm{O}_{3}$ & 7.5 \\
\hline $\mathrm{Cs}_{2} \mathrm{O}$ & 0.4 & $\mathrm{SrO}$ & 2.0 \\
\hline $\mathrm{K}_{2} \mathrm{O}$ & 2.6 & $\mathrm{Na}_{2} \mathrm{O}$ & 19.0 \\
\hline $\mathrm{B}_{2} \mathrm{O}_{3}$ & 9.0 & $\mathrm{SiO}_{2}$ & 36.0 \\
\hline
\end{tabular}

Density, $2,84 \mathrm{~g} / \mathrm{cm}^{3}$

Phase composition: roentgen amorphous phase, $\mathrm{CeO}_{2}$, spinels $\mathrm{Fe}^{+2} \mathrm{Me}^{+3}{ }_{2} \mathrm{O}_{4}$

Mass losses at boiling in distilled water in 5 hours is $0,04 \%$ 


\section{Samples of microphotography}

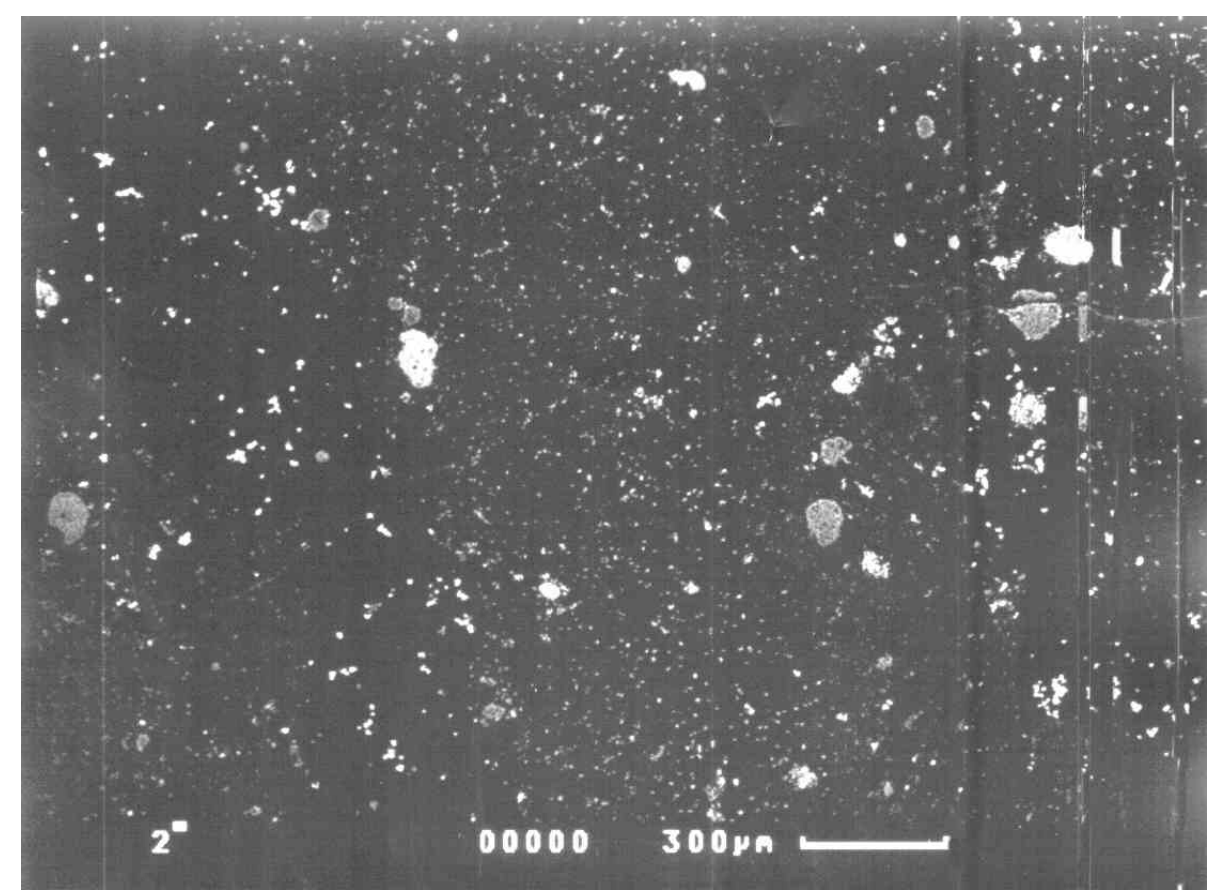

Magnification 48x

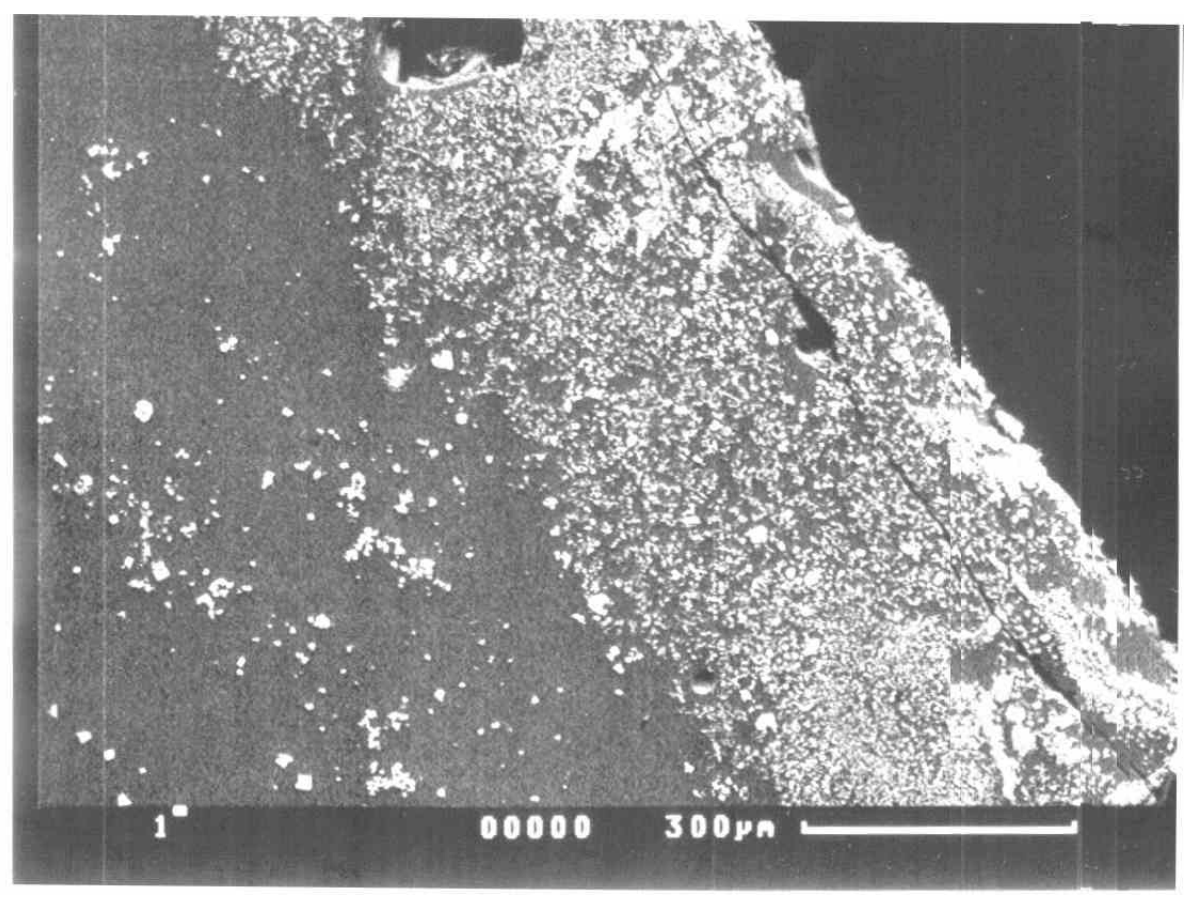

Magnification 92x 


\section{View of crucible with glass}

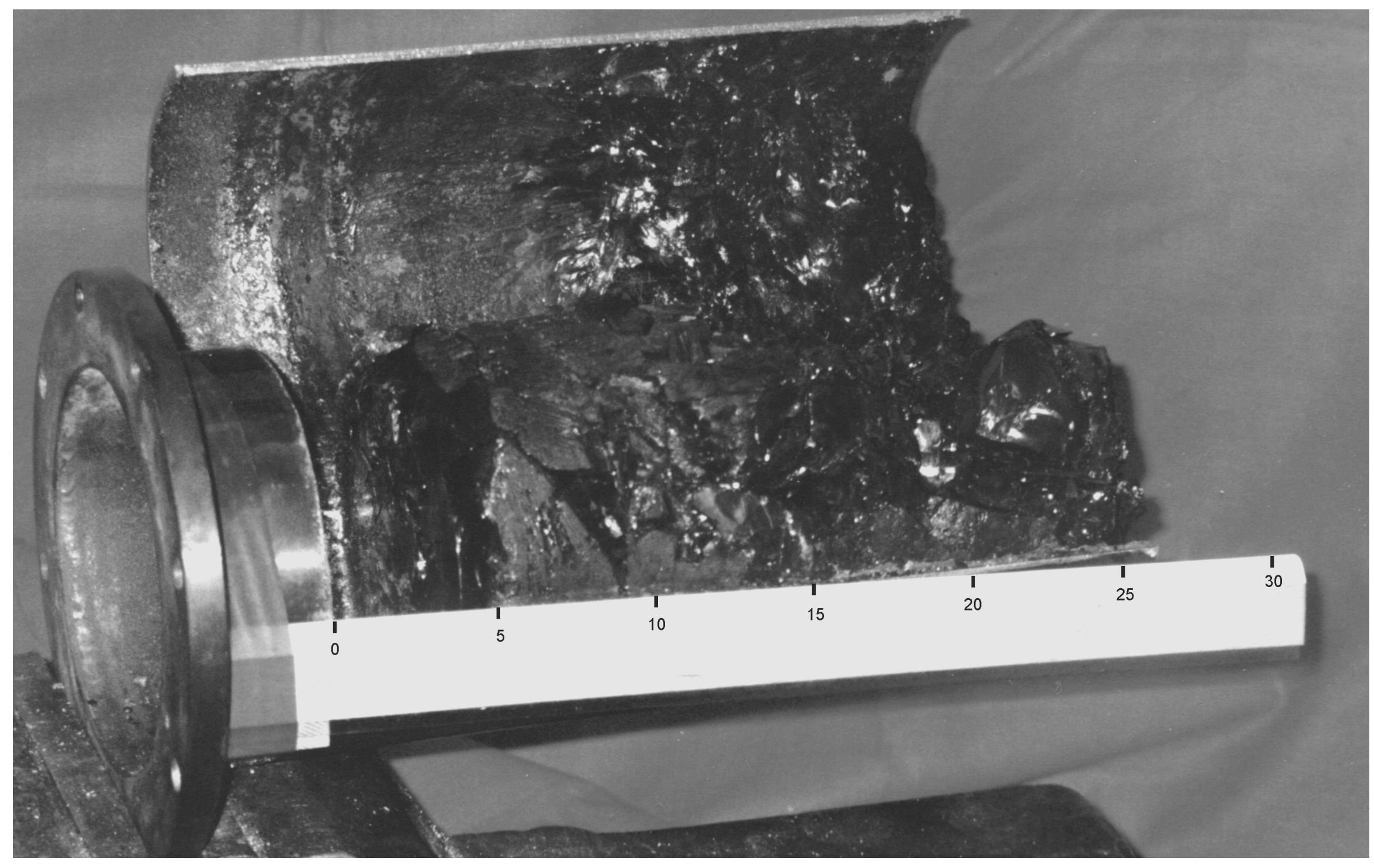




\section{Silicon carbide crucible}

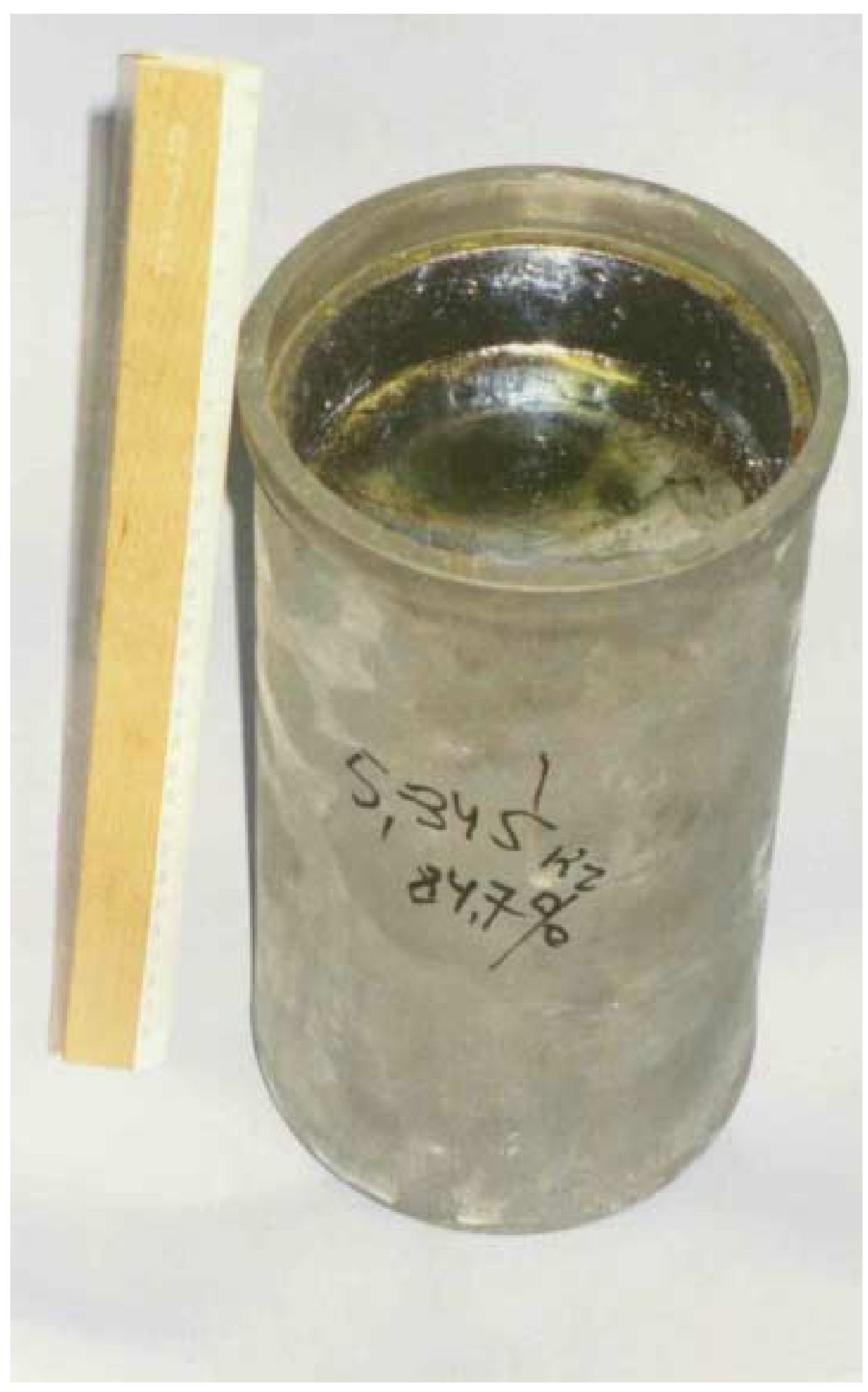




\title{
Leach rate dependence on sodium content in sludge
}

\author{
MCC-1, distilled water, 28 days, $90{ }^{\circ} \mathrm{C}$. \\ Recommended flux composition
}

$\mathrm{SiO}_{2}-80.0 \mathrm{wt} \%, \mathrm{~B}_{2} \mathrm{O}_{3}-20.0 \mathrm{wt} \%$ 


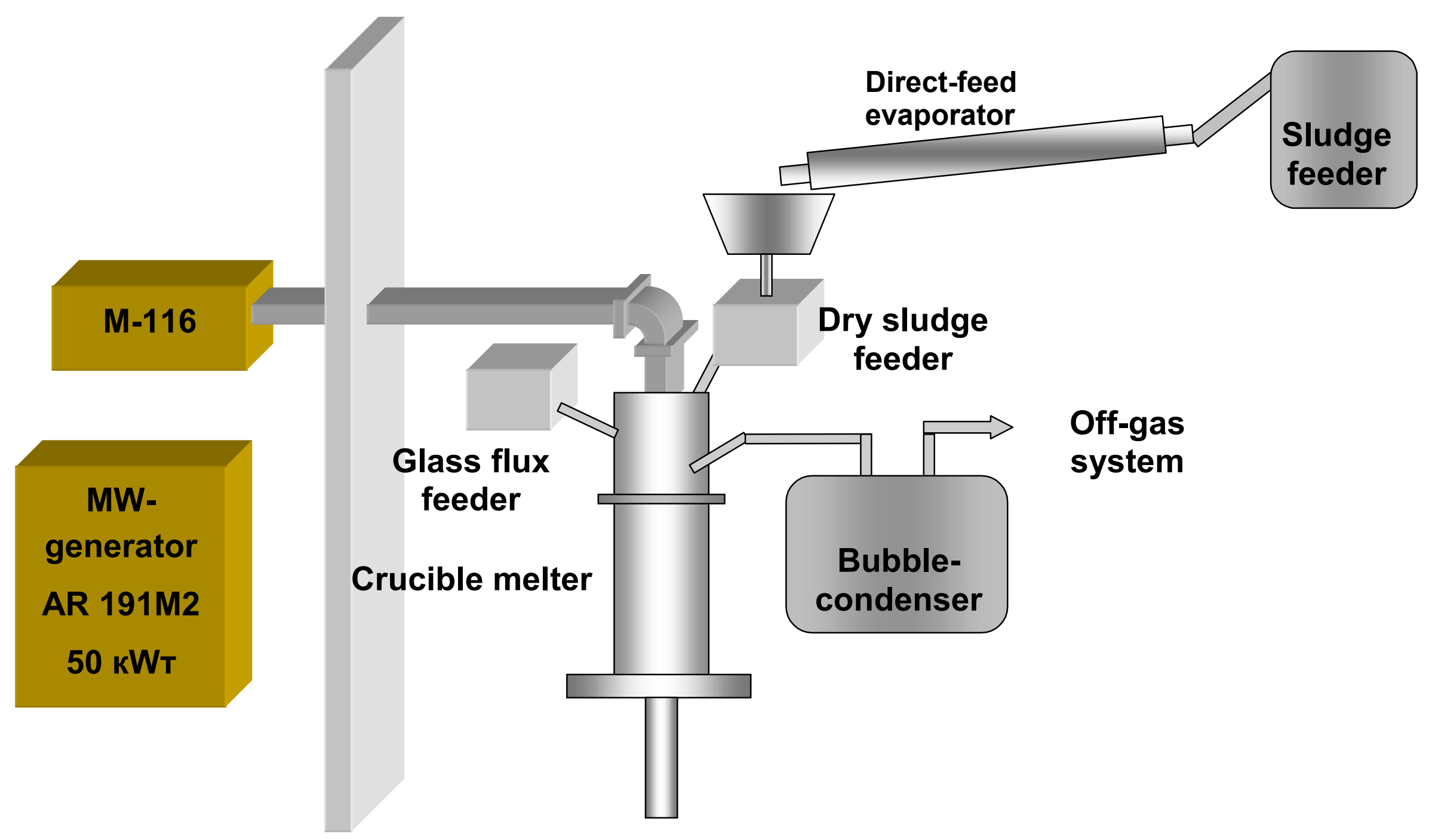

Experimental-industrial set block scheme 


\section{Conclusions}

- The system of matching MW-set in different infill levels was developed and tested.

- The homogeneous glass block was obtained in the crucible filled in $85 \%$ without using of active thermo insulation.

- The productions of MW-set increases in 3 times with using the dry remains of sludge.

- The corrosion of crucible-melter is not significant at the glass synthesis temperature of $1150{ }^{\circ} \mathrm{C}$. The skill layer is $1 \mathrm{~mm}$.

- The two-stage process is recommended for the experimental-industrial set. 


\section{Ecological and Economic Substantiation of Technological Decisions and Comparative Analysis of MCC Glasses for Long-term Storage of Immobilized Sludges in Underground Tanks for JOI (B506233)}

\section{T. A. Gupalo, V. P. Beygul, S. L. Speshilov, VNIPIPT}

Figure 1 presents the sequence of stages of maintenance of plutonium-containing MCC sludges including long-term storage in existing MCC underground facilities and final disposal in the Nizhnekansky granitoid massif.

Based on the analysis of mining-geological and hydro-geological conditions in the rock massif at the storage site, and strength characteristics of tanks $86 \mathrm{a}$, it was established that the length of time of safe storage of solidified sludges in these facilities can be considerably increased; for example, from 30 until 100 years, with minimal additional expense.

As heat release and radiation of containers with solidified sludges is defined basically by short-lived isotopes, the increase of storage time will allow to simplify the further waste maintenance.

Figure 2 presents the scheme of one of the tanks for immobilized sludge storage in facilities 86a with a bentonite barrier. The main difference of this case from the one previously considered in the "Feasibility Study" and "DOI," consists in the following: after the period of forced cooling ventilation, all space between channels, in which containers are emplaced, will be filled with a mixture of bentonite with sand.

All 6800 containers with sludges immobilized in boron silicate glass can be emplaced in two tanks. In each tank, only 148 channels of 172 possible guiding channels will be loaded with containers with waste, 23 rows in height. A layer consisting of $0.5-\mathrm{m}$ of bentonite mixture with sand will be placed on the bottom of each channel. The outer layer of $0.5-\mathrm{m}$ thickness between the external row of channels and internal covering of the tank will be filled with bentonite-sand mixture. The sand is added to bentonite for increase of thermal conductivity. Then loading of containers into all 148 channels is made layer after layer. Judging from the output of installation for immobilization, 515 containers with waste will be prepared during one year that will make up 3.5 rows in height, i.e., one tank will be loaded within 6.5 year.

Containers are cooled using forced ventilation. After the termination of air supply, the entire free space between channels will be filled with a mixture of bentonite and calcinated sand.

To facilitate the thermal regime, 24 channels in the central part of each tank are left free and are not loaded with containers with solidified sludges (Fig. 2). Thus in both tanks there is a place for emplacement of 1104 additional containers in the 48 free channels. Later on it is possible to consider the opportunity to use a part of this free space,; for example, for emplacement of waste from MOX-fuel fabrication.

To justify the engineering solutions for storage design, a number of characteristics are examined using mathematical modeling:

- Modeling of geomechanical processes in near-contour zones of tanks 86a, calculation of underground structure stability for long periods of time, taking into account abnormal situations;

- Modeling dynamics of the thermal regime in the storage in various terms of air supply, variants of layout, and modes of loading containers; 
- Modeling Pu radionuclides migration and sorption processes in the system of engineered barriers at deep disposal.

Modeling of geomechanical processes, predictive calculations of stability of the rock massif and underground structures of facility 86 a have been carried out for normal conditions and abnormal situations, including earthquake and geodynamic shift, in order to account for mining-geological and hydro-geological conditions at the site of the storage location. It was proved that the geomechanical stability of tanks $86 \mathrm{a} / 1$ and $86 \mathrm{a} / 3$, recommended for immobilized sludges emplacement, is ensured for periods of more than 300 years under any seismic effects.

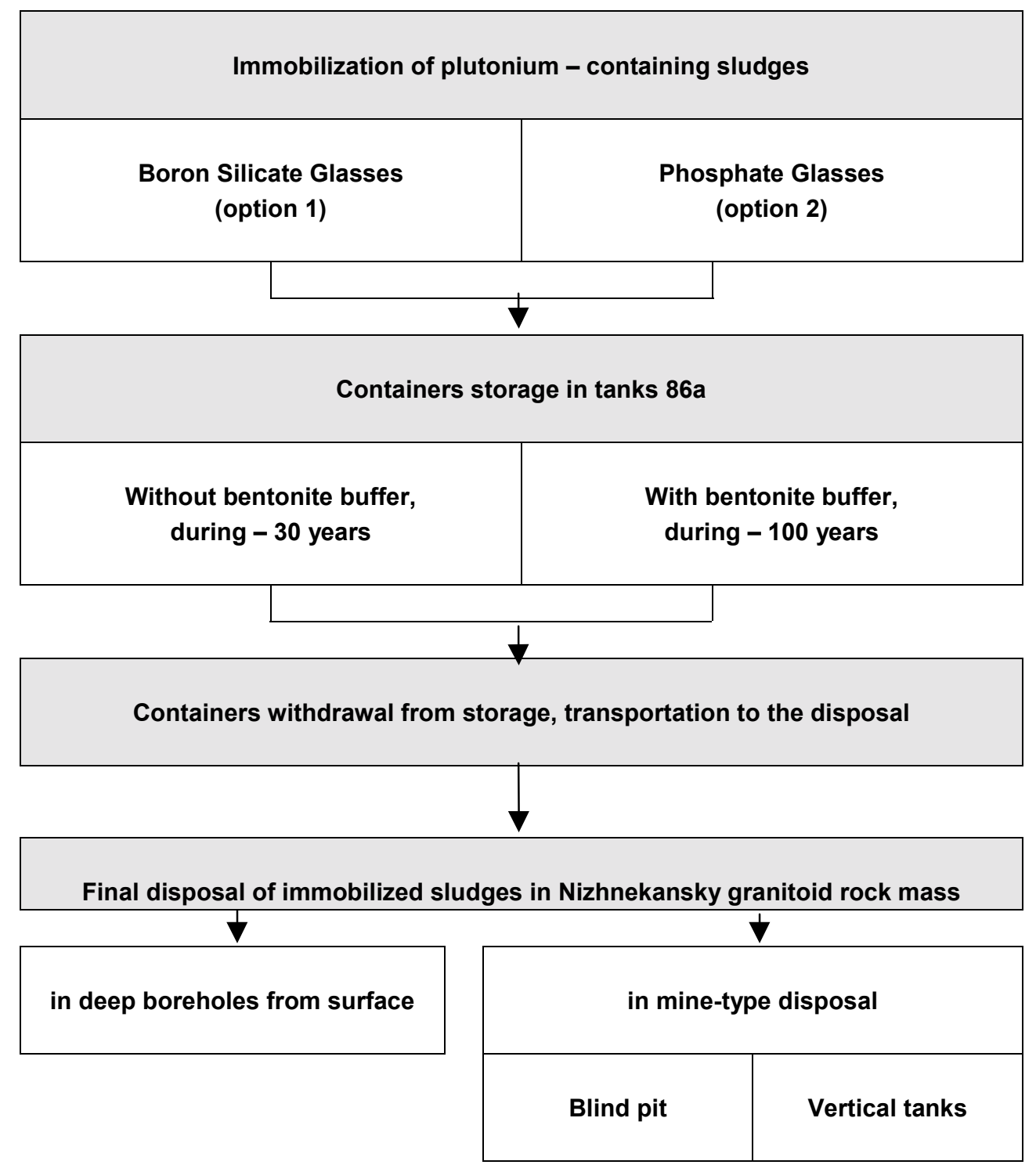

Fig. 1. General description of principal compared option of long-term storage and final disposal of MCC immobilized sludges. 


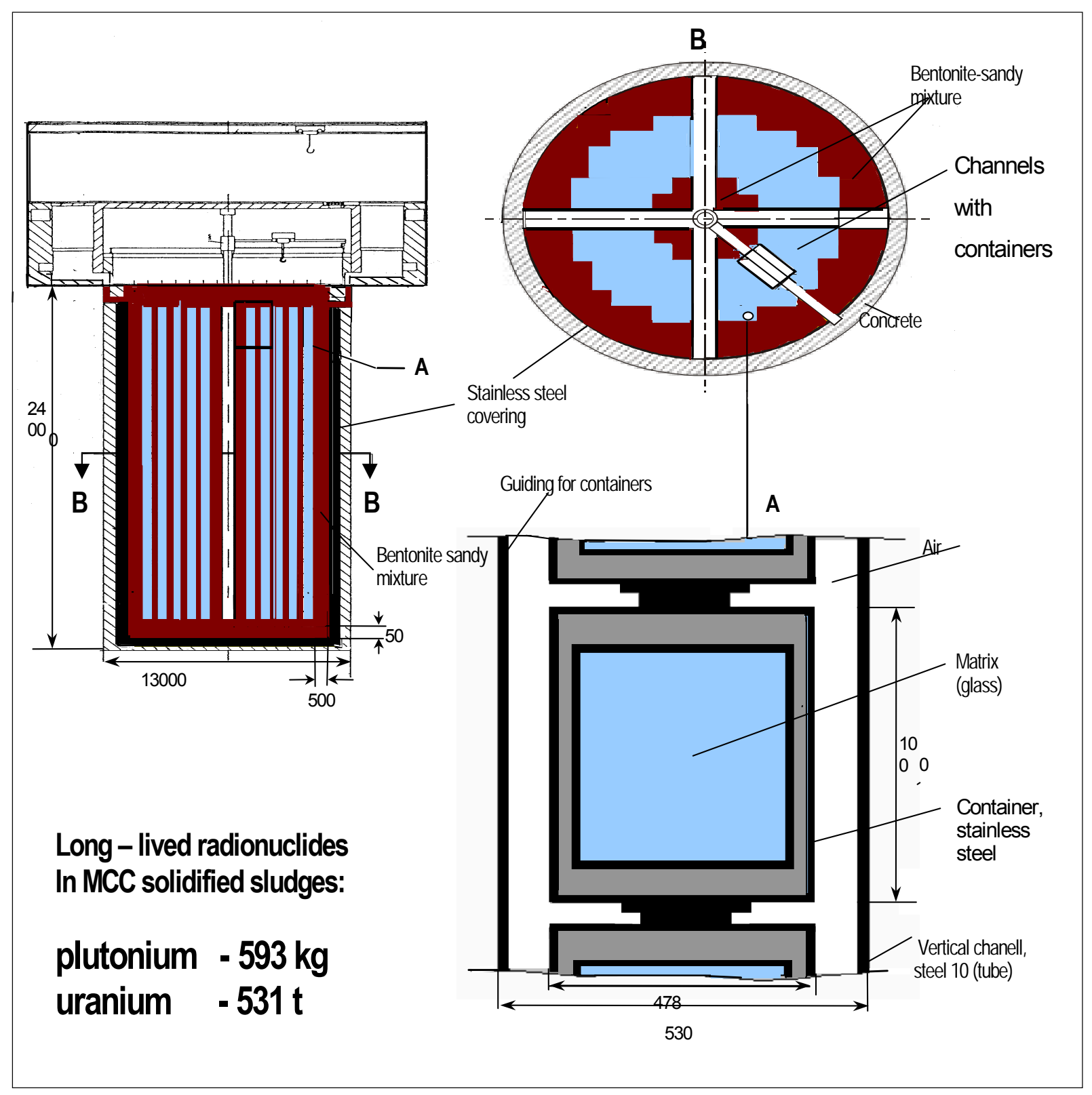

Fig. 2. Scheme of tanks 86 a with bentonite barrier for immobilized sludges (after cooling air supply is stopped).

The system of engineered barriers provides reliable protection of boron silicate matrixes from contact with underground waters for a period of not less than 100 years. For water access inside the containers, it is necessary that a local discontinuity of engineered barriers, shown in Fig. 2, took place one after another. It is obvious that for the period of 100 years, underground waters can not penetrate even to containers.

To estimate a degree of safety of a container within a 100-y period, the depth of local corrosion in a container wall of 8-mm thickness made from stainless steel was calculated. The probability law of Weibular distribution was applied. The results of Japanese researchers and data of VNIPIET show that the corrosion rate of stainless steel in the presence of water vapor can make up $1 \cdot 10^{-3} \mathrm{~mm} / \mathrm{y}$. Average (median) time for corrosion to reach a depth of $2 \mathrm{~mm}$ (that is significantly less than wall thickness of 8 $\mathrm{mm}$ ) amounts to 858 years. The risk of local corrosion to a depth of $2 \mathrm{~mm}$ within 100 years makes up 
$1.2 \cdot 10^{-7} 1 /$ year, that is less than negligible risk of $10^{-6} 1 /$ year. Thus, after 100 years, storage containers withdrawn from the storage will have only insignificant surface corrosion.

The study of thermal regimes inside and outside the tank filled with containers of sludge and surrounded by rock mass was carried out with the help of a computing program specially modified for this purpose and included in the computer code GSTERM, developed in VNIPIPT.

Mathematical modeling of the thermal regime, including loading and forced cooling during 30, 40, 50, 60 or 70 years, then termination of air supply and filling the space between containers with bentonite mixture has been carried out. Estimation of thermal field propagation inside storage and in the adjacent rock massif has been performed for 150-250 years after the beginning of container loading. The result of this calculation is a temperature function in time in all units of a grid covering the investigated area. While performing the calculation, the data used on the dynamics of reduction of containers' heat release were given in the Feasibility Study or TEF developed by VNIPIET.

Table 1 presents the description of calculations performed on modeling the dynamics of heat distribution variation in storage for various types of glasses, diagrams of container loading, schemes of container layout in storage, and terms of air supply.

The optimum term of cooling air supply is 30 years for the final option with emplacement of sludges immobilized in boron silicate glass and placed in two tanks. Due to the fact that the internal part of the tank is free from heat-releasing containers, maximal temperature on channels' walls will not rise above $160^{\circ} \mathrm{C}$. In this case, the channels will be completely immersed into bentonite-sandy mixture that will prevent their bending because of overheating.

In 100 years after loading, the containers will be cooled up to $35^{\circ} \mathrm{C}$ and their radiation will decrease also. This will facilitate further waste maintenance during withdrawal from the storage, transportation and loading into the disposal.

On the basis of the analysis of rock mass properties at the sites of Nizhnekansky granitoid massif suitable for radioactive waste final disposal, the options of final disposal of solidified sludges were considered: in deep boreholes from the surface and in mine drifts.

Most effective is the mine-type variant of disposal with two vertical tanks at depth of 550-580 $\mathrm{m}$ for disposal of sludges immobilized in boron silicate glass. For this variant, the dimensions of the underground part of the disposal are $50 \times 13 \mathrm{~m}$, and the construction cost of the underground facility is $\$ 17$ million dollars.

The option with deep boreholes drilled from the surface requires an underground space of $100 \times 425 \mathrm{~m}$, and the cost of construction is 5.8 times as much.

The appropriate options for phosphate glasses in terms of underground facility construction cost are 1.2 and 10.3 times more expensive than the recommended option for boron silicate glasses.

Radionuclide composition of immobilized sludges is rather favorable for safe disposal, as it contains only $\mathrm{Pu}$ and $\mathrm{U}$ of long-lived radionuclides, which have very low solubility in pore waters in the system of engineered barriers under conditions of deep disposal with a reliable bentonite barrier. Radionuclides of $\mathrm{Pu}$ and $\mathrm{U}$ are well sorbed by bentonite in the presence of iron destruction products, and also on materials - crack fillers in the rock massif enclosing the RW repository. 
Table 1. Characteristics of studied thermal regimes in storage.

\begin{tabular}{|c|c|c|c|c|c|c|}
\hline \multirow[b]{2}{*}{$\begin{array}{l}\text { Type of } \\
\text { glass }\end{array}$} & \multirow[b]{2}{*}{$\begin{array}{l}\text { \# of } \\
\text { loaded } \\
\text { tanks }\end{array}$} & \multicolumn{2}{|c|}{ Number per tanks } & \multirow{2}{*}{$\begin{array}{l}\text { Thickness of } \\
\text { external layer } \\
\text { from bentonite } \\
\text { blocks, m }\end{array}$} & \multirow[b]{2}{*}{$\begin{array}{l}\text { Estimated regime of } \\
\text { containers loading } \\
\text { into tanks }\end{array}$} & \multirow[b]{2}{*}{$\begin{array}{l}\text { Terms of } \\
\text { air supply, } \\
\text { years }\end{array}$} \\
\hline & & $\begin{array}{l}\text { containers } \\
\text { loaded } \\
\text { channels }\end{array}$ & $\begin{array}{l}\text { unloaded } \\
\text { channels in } \\
\text { central part }\end{array}$ & & & \\
\hline \multirow{12}{*}{$\begin{array}{l}\text { Boron } \\
\text { silicate } \\
\text { glass }\end{array}$} & \multirow{4}{*}{3} & \multirow{4}{*}{$\frac{2268}{108}$} & \multirow{4}{*}{-} & \multirow{4}{*}{1.3} & \multirow{4}{*}{$\begin{array}{l}\text { Channel loading begins } \\
\text { from center, } 3 \text { tanks are } \\
\text { loaded }\end{array}$} & 40 \\
\hline & & & & & & $50^{a}$ \\
\hline & & & & & & 60 \\
\hline & & & & & & 70 \\
\hline & \multirow{4}{*}{3} & \multirow{4}{*}{$\frac{2268}{108}$} & \multirow{4}{*}{12} & \multirow{4}{*}{1.0} & \multirow{4}{*}{ same } & $40^{a}$ \\
\hline & & & & & & 50 \\
\hline & & & & & & 60 \\
\hline & & & & & & 70 \\
\hline & \multirow{3}{*}{3} & \multirow{3}{*}{$\frac{2268}{108}$} & \multirow[b]{3}{*}{12} & \multirow[b]{3}{*}{1.0} & \multirow{3}{*}{$\begin{array}{l}\text { All channels of one } \\
\text { tank are loaded from } \\
\text { beneath the tank in } 5 \\
\text { rows, tank are loaded } \\
\text { in turn }\end{array}$} & 40 \\
\hline & & & & & & $45^{a}$ \\
\hline & & & & & & 50 \\
\hline & $\begin{array}{l}2 \\
\text { (final } \\
\text { option) }\end{array}$ & $\frac{3404}{148}$ & 24 & 0.5 & $\begin{array}{l}\text { All channels of one } \\
\text { tank are loaded from } \\
\text { beneath the tank in } 3.5 \\
\text { rows, tank are loaded } \\
\text { in turn }\end{array}$ & $30^{\mathrm{a}, \mathrm{b}}$ \\
\hline \multirow{3}{*}{$\begin{array}{l}\text { Phosphate } \\
\text { glass }\end{array}$} & \multirow[b]{2}{*}{5} & \multirow[b]{2}{*}{$\frac{2436}{116}$} & \multirow[b]{2}{*}{4} & \multirow[b]{2}{*}{1.0} & \multirow{2}{*}{$\begin{array}{l}\text { All channels of one } \\
\text { tank are loaded from } \\
\text { beneath the tank in } 5 \\
\text { rows, tank are loaded } \\
\text { in turn }\end{array}$} & $30^{a}$ \\
\hline & & & & & & 40 \\
\hline & 5 & $\frac{2436}{116}$ & 4 & 1.0 & $\begin{array}{l}\text { All channels of one } \\
\text { tank are loaded from } \\
\text { beneath the tank in } 8 \\
\text { rows, tank are loaded } \\
\text { in turn }\end{array}$ & $30^{a}$ \\
\hline
\end{tabular}

${ }^{a}$ Optimal terms of air supply.

${ }^{\mathrm{b}}$ Term of storage was for 100 years.

Solubility of $\mathrm{Pu}$ and $\mathrm{U}$ radionuclides in a reducing medium at $\mathrm{Eh}<0$ and $\mathrm{pH}=7.5-9.5$ is very small, and the values given in the experimental results of foreign researchers are about $10^{-6}$ and $10^{-4} \mathrm{~g} / \mathrm{l}$.

According to the results of laboratory research performed under the contracts with LLNL in 1999-2000 with experimental samples of boron silicate glasses fabricated in MCC CCL, the concentration of radionuclides leached from matrixes has made up $7.3 \times 10^{-6} \mathrm{~g} / \mathrm{l}$ for $\mathrm{Pu}, 1.1 \times 10^{-3} \mathrm{~g} / \mathrm{l}$ for $\mathrm{U}$. The experiments on $\mathrm{Pu}$ and $\mathrm{U}$ leaching were carried out at $90^{\circ} \mathrm{C}$ with excess of air and without filtration of solutions to separate colloids. In a reducing atmosphere, concentrations of $\mathrm{Pu}$ and $\mathrm{U}$ in ionic form will be considerably lower.

According to NRS-99, the admissible $U$ concentration in water estimated with the account of dose factor and the limit of annual access to human body makes up $8.4 \cdot 10^{-4} \mathrm{~kg} / \mathrm{m}^{3}$. Hence, the content of $U$, even within the limits of the system of engineered barriers before dilution in rock fractured zones, will be close to the admissible level. Therefore, $U$ radionuclides can not be considered in the safety analysis of 
underground isolation as they obviously can not reach the human activity environment with concentrations above admissible values. Mathematical modeling of migration and sorption of $U$ radionuclides in bentonite barrier nevertheless has been carried out. According to the results of modeling, the front of admissible $U$ concentration in pore water in a bentonite barrier can advance $16 \mathrm{~cm}$ within 200,000 years with a thickness of bentonite barrier equal to $150 \mathrm{~cm}$ (Fig. 3).

Admissible concentrations of $\mathrm{Pu}$, which can result in additional individual dose of $1 \mathrm{mSv} / \mathrm{year}$ for population in a zone of storage location according to NRS-99 makes up $1.3 \times 10^{-9} \mathrm{~kg} / \mathrm{m}^{3}$. On the basis of mathematical modeling, it was established that the front of admissible Pu concentration (Fig. 3) can reach a maximal distance of $44 \mathrm{~cm}$ in a bentonite barrier within 75,000 years at total thickness of external bentonite layer of $150 \mathrm{~cm}$, and then a reduction of the radius of $\mathrm{Pu}$ diffusion halo will take place owing to radioactive decay. In 170,000 years the radius of the halo will decrease up to $5 \mathrm{~cm}$.

Based on calculations performed, it is possible to conclude that the risks of $\mathrm{Pu}$ and $\mathrm{U}$ radionuclides release from the repository into a zone of human activity environment are by many orders lower than value of $\times 10^{-6}$ 1/year -a level of negligible risk according to NRS-99, as Pu and $U$ radionuclides will not leave the limits of the system of engineered barriers with concentrations equal to admissible values. 


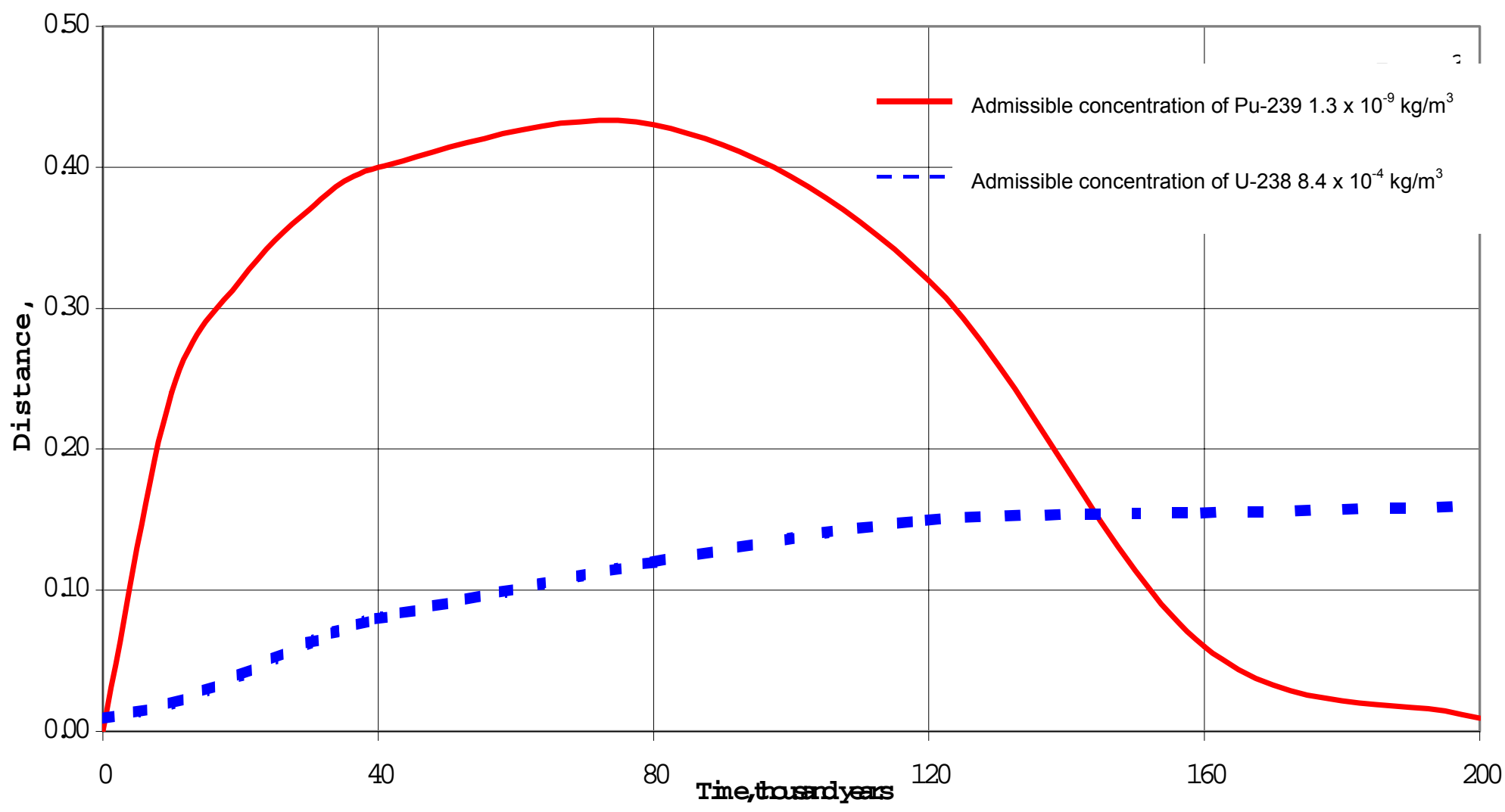

Fig. 3. Dynamics of motion of admissible $\mathrm{Pu}$ and $\mathrm{U}$ concentration front in bentonite barrier of $1,5 \mathrm{~m}$ thick at initial concentration $C_{0}(P u)=1 * 10^{-5} \mathrm{~kg} / \mathrm{m}^{3} ; C_{0}(U)=1 * 10^{-3} \mathrm{~kg} / \mathrm{m}^{3}$. 


\section{Conclusions}

1. The opportunity of safe storage of MCC immobilized sludges in tanks $86 a$ for the term of not less than 100 years has been proved. The increase of storage time from 30 until 100 years with minimal additional expense will considerably facilitate further waste maintenance and improve technical and economic parameters and safety at the disposal phase.

2. Two tanks, $86 \mathrm{a} / 1$ and $86 \mathrm{a} / 3$, are required for storage of 6800 containers with boron silicate glasses. The thickness of external bentonite layer in tanks will make up $0.5 \mathrm{~m}$. Optimum duration of forced cooling of waste in storage is 30 years. The risk of local corrosion of container for $2 \mathrm{~mm}$ depth within 100 years will make up $1.2 \times 10^{-7} 1$ year. After 100 years of storage, containers with a wall thickness of $8 \mathrm{~mm}$ withdrawn from storage will have only insignificant surface corrosion.

3. Mine-type of disposal with vertical tanks proposed by VNIPIPT is 5.8 times more preferable in terms of underground facility construction cost than the option with deep boreholes from the surface.

4. The developed option of immobilized sludge disposal will ensure their ecologically safe isolation for the entire period of potential danger. On the basis of mathematical modeling, it was established that the front of admissible Pu concentration can reach a maximal distance of $44 \mathrm{~cm}$ in a bentonite barrier with a total thickness of external bentonite layer of $150 \mathrm{~cm}$. In 170,000 years, the radius of a halo will decrease up to $5 \mathrm{~cm}$. Risk of $\mathrm{Pu}$ and $\mathrm{U}$ radionuclide release from the repository into a zone of human activity is by many orders of magnitude lower than the negligible risk of $10^{-6}$ per year.

5. Boron silicate glasses are preferred over phosphate glass, for in this case the entire volume of immobilized sludge is emplaced in 6800 containers, while in the case of phosphate glass, emplacement requires 11860 containers. Cost of construction of an underground part of the repository for boron silicate glasses is 1.2 times less than the cost for phosphate glasses. 


\section{Status of Near Contour Zones of Underground Construction for Long Underground Isolation of Plutonium-containing Pulps of MCC (B506233)}

\section{V.S. Gupalo, VNIPIPT}

The placement of radioactive wastes (RAW) in a geological formation for long-term storage provides the use of mountain rocks as a natural barrier that excludes the opportunity for radionuclides to be distributed into the biosphere.

The creation of new underground facilities and the use of present ones for long underground isolation of heat-emitting wastes are connected with technological loads on mountain rocks of the mass. By technogenic loads, we mean the influence of wastes on underground facilities and the containing rock mass. As a result of such influences, the initial characteristics of the rock mass-strength, deformation, isolation etc.-begin to change.

Depending on the rate of change of these characteristics, it is possible or impossible to use the given mass for constructing an object for underground isolation.

The subject of research presented in this report is the influence of thermal load on strength characteristics of the containing rock mass. Numerous laboratory researches have shown that samples of gneisses are, practically speaking, not subject to reduction of strength on heating. An insignificant strength reduction was observed at temperatures above $300^{\circ} \mathrm{C}$. However, this research can not characterize the behavior of a rock mass containing various kinds of fractures with various types of fillers.

As it is known, the mechanism of destruction of a rock mass greatly depends on the strength of connections of the structural elements composing the mass, because they are weaker than the elements themselves and are subject to the influence of temperature to a greater degree.

The mechanism of influence of thermal load on a rock mass can be divided into two components. The first one is associated with the rise of thermal stress in rocks because of non-uniform thermal expansion of minerals. The second mechanism is caused by various physical and thermochemical transformations of rock minerals on heating.

Under increasing temperature, all rock parameters that describe plasticity, creep, and relaxation of stress are increased. Rock viscosity is reduced with heating.

MCC underground structures contain heat exchangers for the nuclear reactor that warmed up the rocks up to $60^{\circ} \mathrm{C}$. To study changes of deformation features of the rock mass under thermal load, we carried out comparisons of geological structure of the containing mass and results of long natural measurements of linear dimensions of the chambers.

The mass which contains the underground constructions is composed of a metamorphic rock complex represented by grey and dark grey biotite-plagioclase gneisses. The basic elements of the tectonic structure of the rock mass are fractures and ruptures containing fillers, such as chlorite, clay, carbonate, and silicium cement.

The analysis of results of measurements has shown that underground structures containing heat sources, and warmed up rocks, undergo wall displacement that has taken the character of non-stop creep. In 
underground structures of similar size and geological conditions but without temperature influence, no displacement of walls is observed.

The comparison of results of tool measurements and geological structure of the rock mass has shown that displacement of walls occurs more intensively on chamber sites where the containing rocks are composed predominantly of clay and chlorite as fracture filler, than on sites filled predominantly with carbonate or silicium cement. Thus, on sites with a prevalence of clay filler, the displacement is $22 \mathrm{~mm}$. The deformation of walls on sites with fillers of carbonate or silicium cement is a backlog of $12 \mathrm{~mm}$.

For a quantitative rating of the change of strength characteristics of the rock mass and to take account of these changes for design purposes, we used data from previous physical modeling performed for other uses.

The results of modeling and researches on natural fracture systems were used to substantiate repository safety.

\section{Conclusions}

Natural research conducted at the sites proposed for long-term storage of solidified wastes has shown the rock mass condition and possible changes of rock properties, depending on fracture systems.

Massifs with fractures filled by silicium cement transfer thermal load practically without change of properties.

The rock massifs with clay filler in fracture systems are subject to the greatest change. Strength characteristics have decreased $15-20 \%$, which can lead to loss of stability in strongly fractured rocks. 


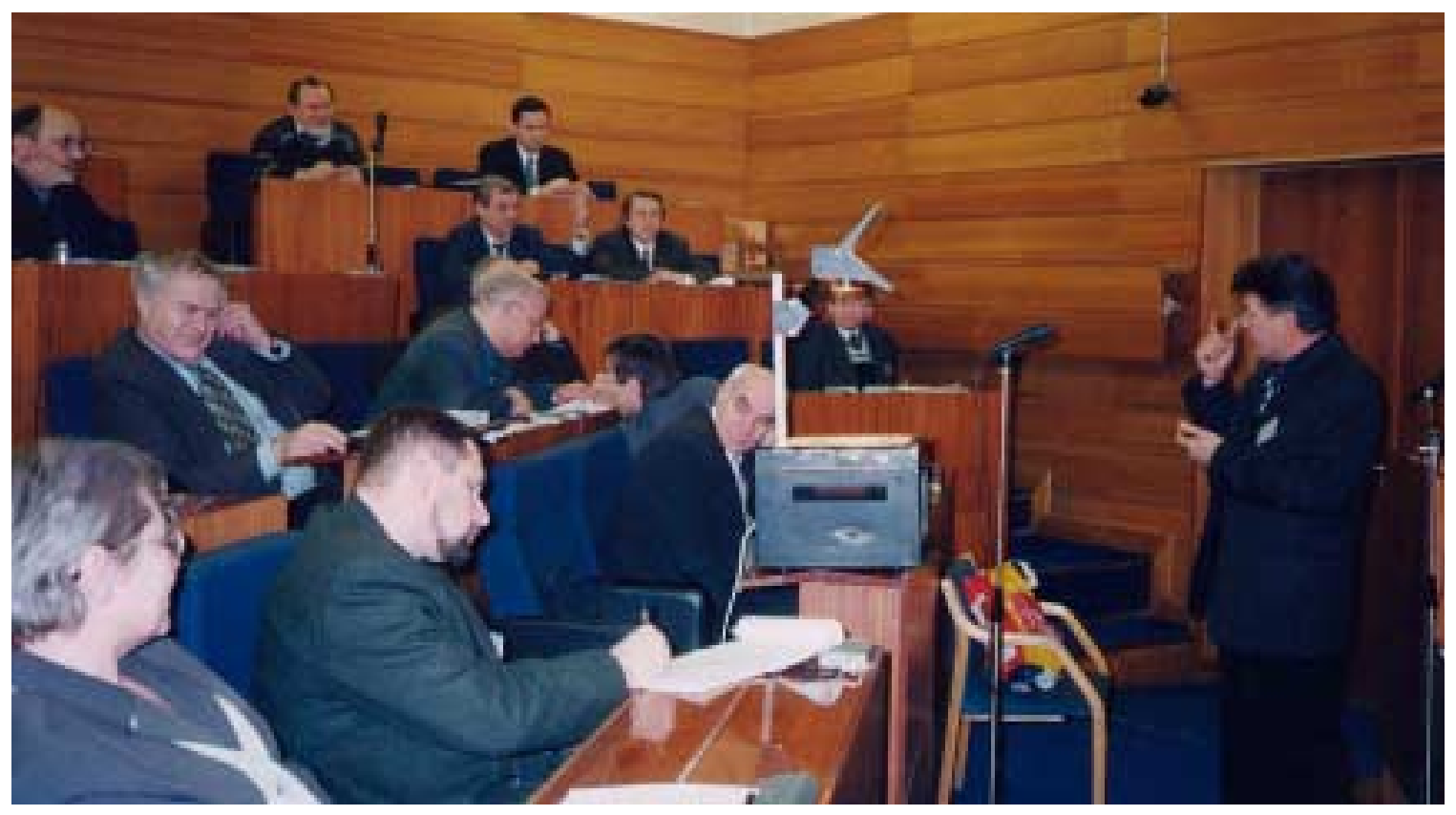

II.2. Mayak Site Technical and Economic Engineering Feasilbility Studies of Weapons Plutonium Sludge Immobilization and MOX Fuel Fabrication Plutonium Wastes 

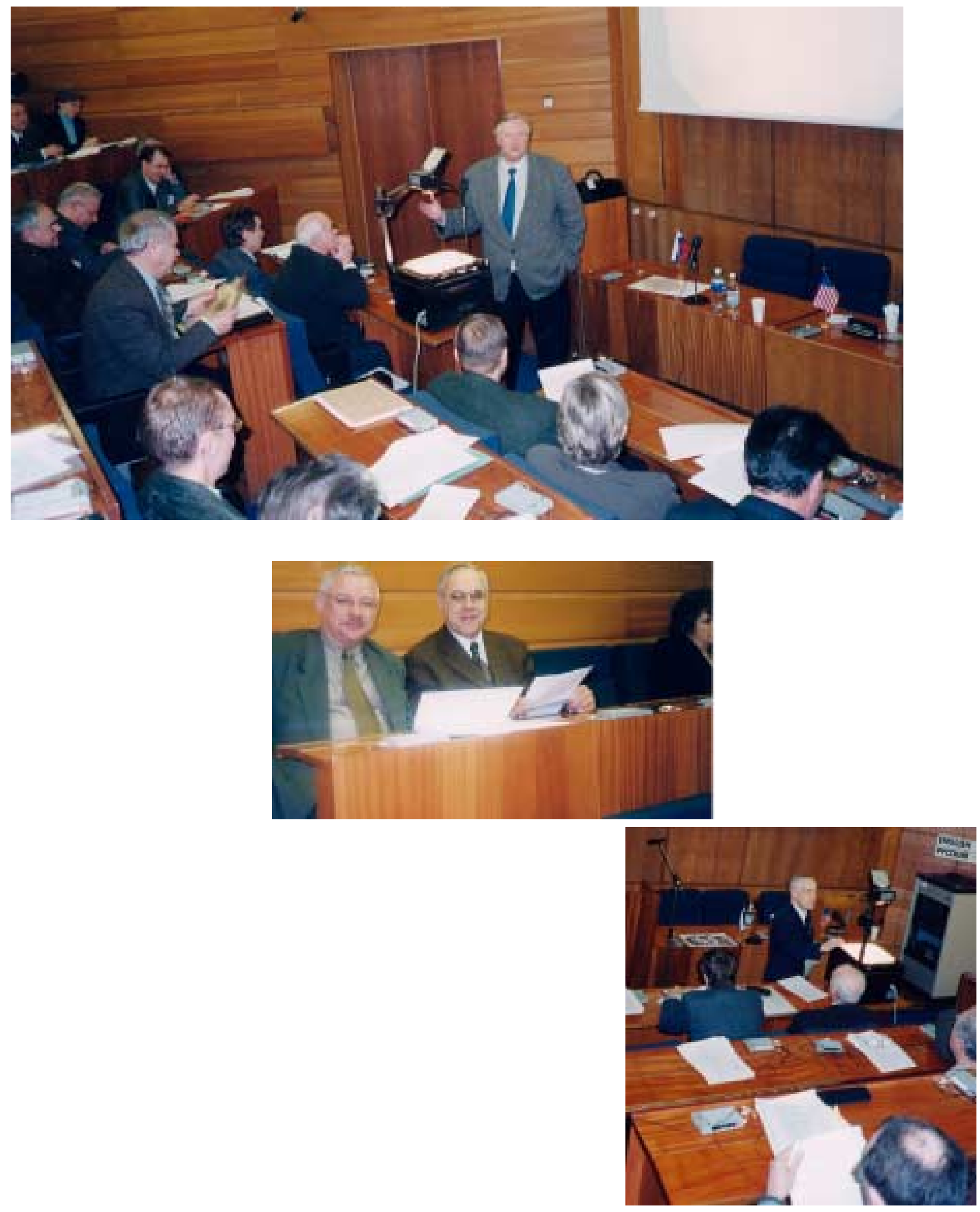


\title{
Process Optimization for Plutonium-containing Sludge Reprocessing at RT-1 Plant (B506201)
}

\author{
S. I. Rovny, A. S. Skobtsov, PA "Mayak"
}

\section{Introduction}

In order to carry out technical-economical studies, the principal layout of the process for plutoniumcontaining sludge reprocessing was proposed. This process considers two options possible for a technical solution of the problem.

In the basic option, it is proposed that solutions will be first dissolved in alkaline, nitric acid, and nitricphosphoric acid solutions. Alkaline solutions are sent for storage and vitrification in the furnace EP-500. Plutonium is extracted from the acidic solutions. The concentrate is solidified in glass-like and mineral-like matrices. Nitric-phosphoric acid solutions are subject to fractionation for further strontium and cesium extraction. The concentrate is solidified at small-scale facilities, and the raffinate is vitrified at EP-500. In the comparison option, after alkaline treatment, the residue is either solidified in the EP-500 furnace or in the cold crucible, or is dissolved in the nitric-acid solution with further solidification at EP-500 or cold crucible.

The proposed options achieve the desired final outcome, which is the transfer of the Pu-containing sludge into a stable inert form that is not suitable for further plutonium extraction. However, technically the processes are rather complex. The first reason for this complexity is that the operations for two-phase systems are used; therefore, radiochemical plant equipment is engaged. The reverse task, which is water solution concentration from alkaline and acid solution treatment, is also rather complex. This process takes place before those solutions are dispatched into the vitrification facility. Based on our calculations, the residues should be compressed by dozens or even hundreds of times, which would be accompanied with residue formation and the need to utilize special equipment.

It seems necessary to test the options proposed before development of the layouts taking into account the factors listed above.

\section{Choice of the Optimum Process for Pu-containing Sludge Reprocessing}

Analysis of the process layout based on the main and comparison options allows us to single out processes considering the factors stated above without changing the technological parameters that had been calculated before. Volumes of the end products and the number of processes were used as the assessment criteria for process optimization.

In the basic and comparison options, three processes with complete residue dissolution can be singled out. As for the first process taken from the comparison option, the residue is first subjected to alkaline treatment and then dissolved in the mixture of nitric and phosphoric acid. Aluminate alkaline solution could be sent for storage or vitrified in the EP-500 furnace. Based on this process, $4.8 \mathrm{~m}^{3}$ of aluminate solution and $0.853 \mathrm{t}$ phosphate glass is generated with $1 \mathrm{~m}^{3}$ of residue.

In the second process, selected from the basic option, after alkaline treatment, the residue is subjected to treatment with nitric acid solution. Plutonium is extracted from this solution and then incorporated into a 
mineral-like matrix. The residue is then further dissolved in the nitric acid and phosphoric acid mixture. Strontium and cesium concentrates are extracted from the acidic solutions with fractionation technology, and later incorporated into boron-silicate glass. The raffinates are sent for vitrification with phosphate glass fabrication. As for the second process, $0.17 \mathrm{Mt}$ of phosphate glass is generated per $1 \mathrm{~m}^{3}$ of the residue, as well $3.2 \mathrm{~kg}$ of ceramics, $1.8 \mathrm{Mt}$ boron-silicate glass with incorporated cesium and strontium, and $0.72 \mathrm{Mt}$ phosphate glass with medium activity level from raffinate reprocessing (a total of $0.89 \mathrm{Mt}$ phosphate glass).

The second process could be simplified with the use of two sub-processes. The first sub-process excludes plutonium extraction, so Pu-containing solution from nitric acid treatment of the reside is fed to ceramics fabrication. Quantitative parameters on the volume of solidified waste coincide with those of the main process.

As for the second sub-process, the plutonium fraction is not extracted, as far as it remains in raffinate after strontium and cesium extraction with its further incorporation into phosphate glass. Per $1 \mathrm{~m}^{3}$ of residue, this process generates $4.8 \mathrm{~m}^{3}$ aluminum, $1.8 \mathrm{~kg}$ boron-silicate glass containing cesium and strontium, and $0.89 \mathrm{t}$ of the medium-level glass is generated. The second process could be used if plutonium extraction is not sufficient after nitric acid treatment of the production residues.

Comparison of two processes without dissolution or partial dissolution of residue shows the following.

The process without residue dissolution utilizes vitrification of the residue after its removal and separation from the decantant in the ceramic melter or in the cold crucible. In this process, $1.17 \mathrm{Mt}$ phosphate glass is generated per $1 \mathrm{~m}^{3}$ of residue.

In the process with partial dissolution, residue is first subjected to treatment with alkaline and then sent for vitrification. The result of this process generates $4.8 \mathrm{~m}^{3}$ of aluminate solution and $0.78 \mathrm{Mt}$ phosphate glass per $1 \mathrm{~m}^{3}$ of residue.

Table 1 gives the parameters of the processes showing the number of the main operations, volumes, and $\beta$ - activity of solidified waste.

Analysis of the data presented in the table shows that the main volume of solidified product generated in the processes with fractionation ( 95\%), represents phosphate glass of a medium level of activity that could be disposed of in the simple disposals. This is true especially for the "Mayak" PA conditions. Only $5 \%$ of the generated waste is the high-level boron-silicate glass that contains cesium and strontium, and ceramics with incorporated plutonium will require specially designed depositories for their disposal. As for the number of operations, the process number four looks like an optimum, especially from the point of view of the end purpose (i.e., impossibility of plutonium extraction).

The volume of waste is significant; plutonium content in the glass is not more than $0,015 \%$. Processes with direct solidification of the residue with partial dissolution in the environment incorporate 2-3 times fewer operations in comparison with fractionation technologies. However, this conclusion is made without taking evaporation techniques into consideration. Those should be used for concentrating solutions after chemical treatments. When the equipment for efficient transfer and even dosage in the two-phase systems (i.e., plutonium-containing sludge) are developed, processes of direct reprocessing of accumulated waste will look more promising. 
Table 1. Characteristics of the processes for plutonium containing sludge reprocessing.

\begin{tabular}{|c|c|c|c|c|}
\hline Reprocessing process & Waste generated in the process & $\begin{array}{c}\text { Waste } \\
\text { generated } \\
\text { per } 1 \mathrm{~m}^{3} \text { of } \\
\text { sludge }\end{array}$ & $\begin{array}{l}\beta \text {-activity } \\
\text { of the } \\
\text { waste, } \mathrm{Ci} / \mathrm{l}\end{array}$ & $\begin{array}{c}\text { \# of } \\
\text { operation } \\
\text { s }\end{array}$ \\
\hline $\begin{array}{l}\text { 1 Residue dissolution- } \\
\text { solidification }\end{array}$ & $\begin{array}{l}\text { Aluminate solution } \\
\text { Phosphate glass }\end{array}$ & $\begin{array}{l}4,8 \mathrm{~m}^{3} \\
0,85 \mathrm{t}\end{array}$ & $\begin{array}{c}0,5-1,4 \\
18,5\end{array}$ & 4 \\
\hline $\begin{array}{l}2 \text { Residue dissolution - Sr, Cs } \\
\text { and Pu extraction- solidification }\end{array}$ & $\begin{array}{l}\text { Aluminate solution } \\
\text { Boron-silicate glass, (Cs, } \mathrm{Sr}) \\
\text { garnet-perovskite ceramics }(\mathrm{Pu}) \\
\text { Phosphate glass }\end{array}$ & $\begin{array}{l}4,8 \mathrm{~m}^{3} \\
1,8 \mathrm{~kg} \\
3,2 \mathrm{~kg} \\
0,89 \mathrm{t}\end{array}$ & $\begin{array}{c}0,5-1,4 \\
10000 \\
<0,2 \\
1,0-2,0\end{array}$ & 8 \\
\hline $\begin{array}{l}3 \text { Residue dissolution - } \\
\text { extraction of } \mathrm{Sr}, \mathrm{Cs} \text { and } \mathrm{Pu}- \\
\text { solidification }\end{array}$ & $\begin{array}{l}\text { Aluminate solution } \\
\text { Boron-silicate glass (Cs, Sr) } \\
\text { Phosphate glass }\end{array}$ & $\begin{array}{l}4,8 \mathrm{~m}^{3} \\
1,8 \mathrm{~kg} \\
0,89 \mathrm{t}\end{array}$ & $\begin{array}{r}0,5-1,4 \\
10000 \\
1,0-2,0\end{array}$ & 7 \\
\hline $\begin{array}{l}4 \text { Residue dissolution - Sr- and } \\
\text { Cs extraction } \mathrm{Sr} \text { and } \mathrm{Cs}- \\
\text { solidification }\end{array}$ & $\begin{array}{l}\text { Aluminate solution } \\
\text { Boron-silicate glass } \\
\text { Phosphate glass }\end{array}$ & $\begin{array}{l}4,8 \mathrm{~m}^{3} \\
1,8 \mathrm{~kg} \\
0,89 \mathrm{t}\end{array}$ & $\begin{array}{c}0,5-1,4 \\
10000 \\
1,0-2,0\end{array}$ & 6 \\
\hline $\begin{array}{l}5 \text { Residue solidification without } \\
\text { dissolution }\end{array}$ & Phosphate glass & $1,17 \mathrm{t}$ & $\leq 25,0$ & 2 \\
\hline $\begin{array}{l}6 \text { Residue dissolution with } \\
\text { alkaline - solidification }\end{array}$ & $\begin{array}{l}\text { Aluminate solution } \\
\text { Phosphate glass }\end{array}$ & $\begin{array}{l}4,8 \mathrm{~m}^{3} \\
0,78 \mathrm{t}\end{array}$ & $\begin{array}{c}0,5-1,4 \\
20,0-25,0 \\
\end{array}$ & 3 \\
\hline
\end{tabular}

\section{Conclusions}

In order to optimize the process of plutonium-containing sludge reprocessing, analysis of the basic and comparison options was carried out for feasibility study performance.

Based on the similarity of the technological processes, six processes were singled out. Those were characterized by the amount solidified waste, their activity, and the number of main operations required.

In order to complete the selection of an option for sludge reprocessing, it was recommended to consider the proposed processes in the course of the performance of the technical economical study. 


\section{Proposals for Future Work}

1. Development of the equipment for transportation and dosing of two-phase systems for waste vitrification facilities.

2. Designing and production of experimental-industrial facilities for fractional waste vitrification.

3. Researches of the matrix generation processes at treatment of the wastes with high sulfur content.

4. Researches of the process for deep concentrating of raffinate from the fractionation facility in order to ensure the assigned degree of radionuclide inclusion in matrix materials.

5. Development of requirements for fractional waste storage and selection of the optimal container parameters for every type of wastes. 


\title{
Engineering Cost Study for Immobilization of Plutonium-containing Materials at the PO Mayak (B506208)
}

\author{
V. M. Simanovskiy, B. V. Gusakov, A. A. Shvedov, V. T. Sorokin, A. V. Demin, V. V. \\ Kashcheyev, S. V. Romanov, V. V. Ilyin, VNIPIET
}

In the process of implementation of military programs on regeneration of spent fuel, large quantities of highly active pulps containing residual amounts of uranium and plutonium have been accumulated at the PO "Mayak".

The pulps are stored in special storage reservoirs in the production areas of the industrial complex for reprocessing of highly active waste (HAW).

With regard to operation and safe storage of waste, the greatest difficulty is presented by Department 57 , which comprises 20 storage reservoirs each with a total capacity of $1,170 \mathrm{~m}^{3}$.

The conversion of highly active solutions into a high-strength, solidified form is being investigated as a means of achieving safe handling of liquid HAW and preventing the proliferation of unrecorded or unmonitored amounts of fissile materials.

This Engineering Cost Study (ECS) contains an engineering and economic assessment of two, alternate versions of technological processes for the immobilization of Pu-containing pulps by using microwave energy for solidification (Fig. 1):

- Version 1, in which the Pu-containing pulp is separated into a concentrate of Pu and $U$, a raffinate of the fission products (FP), and a concentrate of fission radionuclides (cesium, strontium), which are routed to different solidification facilities.

- Version 2, in which direct solidification of the pulp takes place after a preliminary alkaline boil stage.

Operations that are common to all versions of reprocessing of Pu-containing pulps comprise the washing and upraise (or retrieval) stages, a subsequent thickening in the alkaline-boil and sodium rinsing stage, the vaporizing of sodium nitrate, and the storage of its concentrate. Figure 1 summarizes the alternatives assessed in detail in the ECS.

Under Version 1, the precipitate is further treated in Building 951 with nitric acid and, if necessary, a mixture of nitric and phosphoric acids. The acidic solutions are then routed to the UE- 35 facility in Building 802 for fractionation and extractive separation and recovery of uranium and plutonium, using a technology adopted at the PO "Mayak" in Dept. 61, in Bldg. 802 South.

The solidification of the strontium and cesium concentrates is planned to be carried out with the newly installed equipment at the facility "Grom" ['Thunder'] in Bldg. 188, using a technological process that produces borosilicate glass.

The raffinate of the fission products is routed, together with liquid HAW from the reprocessing of nuclear fuel, to Bldg. 120/12 for solidification on the EP-500 facility using a technological process, adopted at the PO "Mayak", that produces aluminophosphate glass. 


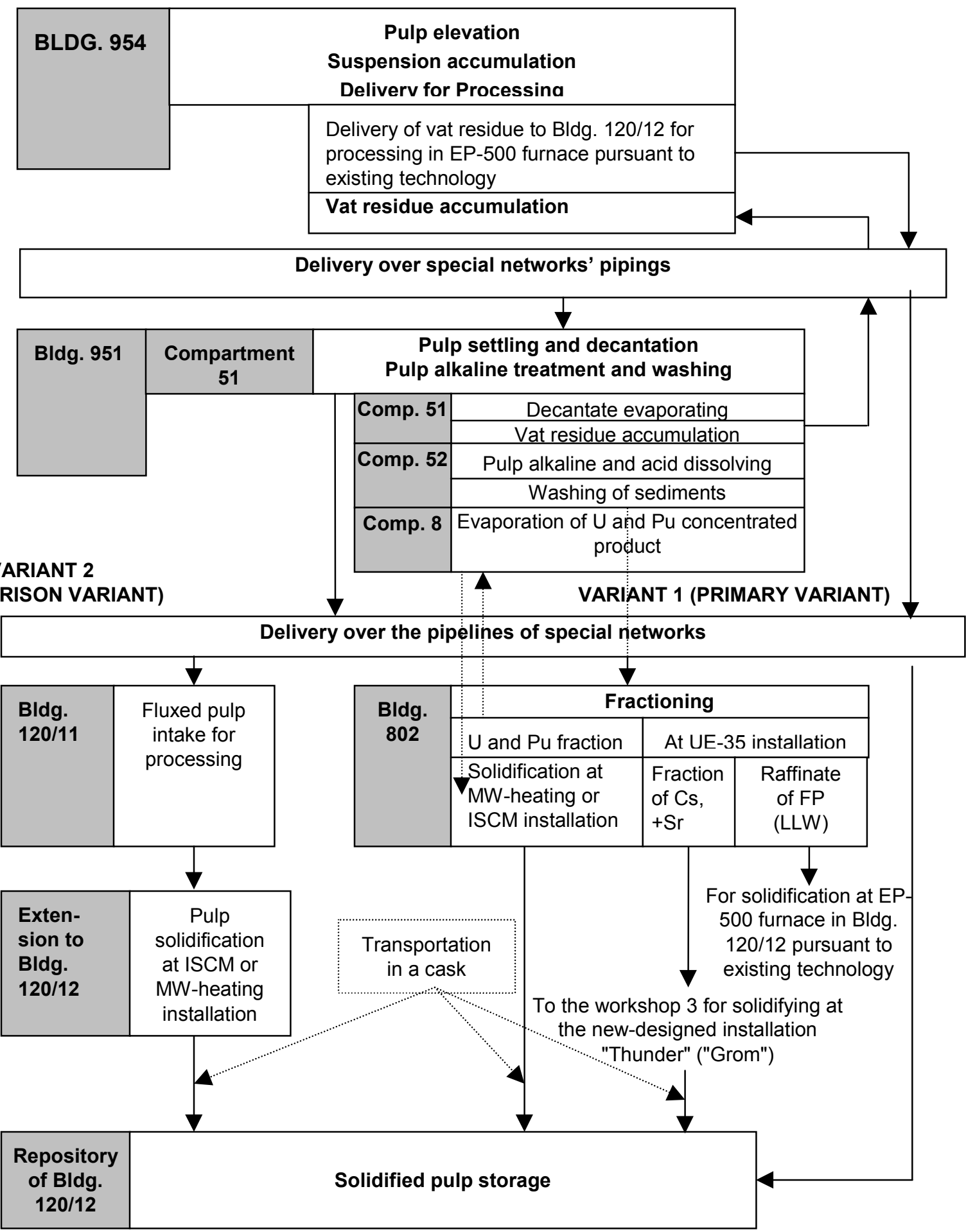

Fig. 1. Production arrangement schematic diagram of Mayak PA plutonium-containing pulp immobilization and recovery assessed in the ECS. 
It is projected that under version 1, the concentrate containing uranium and plutonium will be solidified in a single two-stage facility, the PI-SVCh (IM-UHF) ${ }^{1}$ fiversion 1A), that produces borosilicate glass.

Alternatively, a PI-IPKhT (IM-CCIM) ${ }^{2}$ pversion 1B) will be used in the production of borosilicate glass or a garnet-perovskite ceramic.

The material produced in the solidification facilities is loaded into crucible capsules (or cans) which are then placed into containers in the assembly chamber.

A new chamber is planned as an annex to the storage facility of Bldg. 120/12 for the assembly of containers with crucible-capsules from the IM-UHF facility.

The operation of assembling the containers with cans from the IM-CCIM process is planned for a chamber that was designed earlier for the EP-500/5-10 facility in an annex to Bldg. 120/12.

After checking their exterior for radioactive contamination, using a protective enclosure ('space suit'), the containers are transferred with the aid of a crane into the long-term storage facility for vitrified HAW in Bldg. 120/12.

For Version 2, following the alkali boil stage in Bldg. 951, the Pu-containing pulp is routed to Bldg. 120/12 for vitrification at three work stations (two working units and one spare) using the IM-UHF (Version 2A), which yields borosilicate glass, or the IM-CCIM (version 2B), which produces aluminophosphate glass.

All production operations for the packaging of solidified material into crucible capsules (cans), the loading of the containers and their delivery to the long-term storage facility for vitrified HAW in Bldg. 120/12 are analogous to those of Version 1.

Locating the production facilities for both versions of immobilization of Pu-containing pulps in existing redesigned buildings makes it possible to use the infrastructure and the ancillary production facilities that are currently involved in regular operation of the plants.

The LI "VNIPIET" has published the following materials for this ECS:

- Task 1: Intermediate Report: "Technical Specifications, Results and Plans for Preliminary Data Collection", Inventory \# 00-00149.

- Task 1: $\quad$ Final Report: “Initial Data for the Engineering Cost Study”, Inventory \# 00-00177.

- Task 2: Intermediate Report \#1: "Conceptual Approaches to Reprocessing of Pu-containing Pulps", Inventory \# 00-00023.

- Task 2: Intermediate Report \#2: "Equipment and Engineering Plans and Basic Configuration Solutions", Inventory \# 01-00024.

- Task 2: Final Report: "An Index of Non-standard Equipment and Major Technical Specifications", Inventory \# 01-00038.

- Task 3: Intermediate Report \#1: “Updated Conceptual Approaches and Material Balance. An Index of Basic and Auxiliary Equipment", Inventory \# 01-00050.

- Task 3: Intermediate Report \#2: "Decisions on Production Infrastructure. Consumption of Reagents and Energy. Staffing. Radiation and Nuclear Safety", Inventory \# 01-04826.

\footnotetext{
${ }^{1}$ Induction Melter-UHF.

${ }^{2}$ Induction Melter-Cold Crucible Induction Melter.
} 
- Task 3: Final Report: "Properties of Production Waste. Environmental Protection. Engineering-Cost Estimates. Radiation and Nuclear Safety", Inventory \# 01-04846.

- Task 4: "Proposals for Subsequent Work Plans”, Inventory \# 01-04845.

\section{Economic Estimates}

The estimates of economic indices were done in conformance with Russian requirements for the categorization and production cost accounting (labor, services) and existing regulatory and legislative documents.

The engineering-cost indicators were arrived at by an estimate based on theoretical analyses of major stages in the technological process of pulp-treatment, expert estimates, upward-adjusted proportional cost indicators for similar Russian projects, and on actual economic data from technologies used at the PO "Mayak".

In estimating the investment needed for the implementation of the technical solutions for the different versions of treatment of Pu-containing pulps, the following scope of work was assumed:

\section{Using Version 1}

- Redesign of facilities for dissolving of pulps in alkali or acid and washing the precipitates, and [systems for] the evaporation of uranium and plutonium concentrate.

- Procurement and installation of equipment of the "Grom" facility for the vitrification of $\mathrm{Cs}$ and $\mathrm{Sr}$ concentrate.

- Redesign of Building 188 to provide a total volume of 3,000 $\mathrm{m}^{3}$ for the "Grom" facility.

- Procurement of special vehicles for transportation of containers with crucible-capsules or cans.

- Development of custom equipment.

- Procurement and installation of one vitrification facility, either IM-UHF (version 1A) or IM-CCIM (version 1B), and the renovation of Bldg. 802 to provide the $3,000 \mathrm{~m}^{3}$ needed for its installation.

- Procurement and installation of equipment for one chamber for loading of crucible-capsules into containers and completion of construction work to provide the $2,000 \mathrm{~m}^{3}$ for it in the annex to Bldg. 120/12 (Version 1A).

- Updating of ancillary and service systems (ventilation, air supply, etc.)

\section{Using Version 2}

- Procurement and installation of equipment for three IM-UHF (version 2A) vitrification facilities.

- Procurement and installation of equipment for one chamber in which containers are loaded with crucible-capsules (version 2A).

- Procurement and installation of equipment for three IM-CCIM (version 2B) vitrification facilities.

- Renovation of Bldg. 120/12 (2nd in queue) based on a building volume of $33,000 \mathrm{~m}^{3}$ (version $2 \mathrm{~A}$ ) and $30,000 \mathrm{~m}^{3}$ (version 2B). The required volume of renovation work is $7,000 \mathrm{~m}^{3}$ (version $2 \mathrm{~A}$ ) and 5,000 $\mathrm{m}^{3}$ (version 2B). 
- Development of custom equipment.

- Renovation of ancillary and service systems.

The estimates include all the peripheral expenditures associated with building activities.

Annual operating expenses for handling the Pu-containing pulp are broken out separately according to the following process steps:

- Alkali treatment of pulp in Bldg. 951 (versions 1, 2).

- Dissolving of pulp in acid, boiling down of uranium and plutonium in Bldg. 951 (version 1).

- Extractive separation of the uranium-plutonium fraction in Dept. 61 in

Bldg. 802 South (version 1).

- Fractionation at the UE-35 facility in Bldg. 802 (version 1).

- Solidification of raffinate in Bldg. 120/12 (version 1).

- Solidification in new vitrification facilities (versions 1,2 ).

Expenditures for the solidification of Pu-containing pulp using versions 1 and 2 are determined by direct estimate, which includes the following items:

- Procurement of chemicals and materials including transportation-preparation costs.

- Energy costs.

- Wages of personnel including deductions for social funds.

- Expenditures for worker protection (special meals and protective clothing).

- Depreciation of buildings, structures, and equipment.

- Repair and upkeep of buildings, structures, and equipment.

- Compensation for services from other shops at the PO "Mayak"

- Utilities.

- Deductions for non-budgetary funds for the industry.

The estimates are based on reference data of the PO "Mayak" for the cost of power, special clothing and meals, the wages for various categories of personnel, and the costs of auxiliary services (ref. \# ECJ2115DSP of 11-15-2001).

Depreciation estimates for buildings, structures, and equipment, deductions for various social funds, and non-budgetary industry funds are all made in accordance with governmental regulations of the RF.

The expenditures for enclosures (crucible-capsules, cans, containers) for solidified pulps are based on their mass and dimensional characteristics, material, and estimated quantity.

In view of the upward-adjusted properties of the present estimates for the solidification of pulps, other expenditures were taken at $5 \%$ of the overall estimated costs.

Expenditures for the alkaline and acid treatment of the pulp, the boiling down of uranium and plutonium concentrate, extraction and fractionation, and solidification of the raffinate are all based on the productivity of the primary equipment and technical controls over its use in processing Pu-containing pulp. 
Plans for long-term storage of containers with vitrified pulps call for using the existing storage reservoirs in Bldg. 120/12. To this end, the following facilities will be required:

- $\quad$ Five sections of the storage facility, Vctp. $=107,000 \mathrm{~m}^{3}$ (versions $1,2 \mathrm{~B}$ )

- Four sections of the storage facility, $\mathrm{V}$ стр. $=86,0002 \mathrm{~m}^{3}$ (version $\left.2 \mathrm{~A}\right)$.

Annual operating expenses for long-term storage for solidified pulps were estimated according to cost components based on standardized factors commonly used in estimates dealing with the treatment of Pucontaining materials.

An engineering-cost comparison of alternative versions of immobilization of Pu-containing pulps by microwave solidification was performed using the following summary expenditures:

- Investment expenses to establish the pulp treatment process.

- Operating expenses for pulp reprocessing over a period of 15 years.

- Operating expenses for long-term storage of solidified RAW throughout the estimated period, which would include:

-The period of acceptance of RAW for long-term storage

15 years

-The period of maintaining the temporary storage after fill up

15 years

The results of the comparison of engineering-cost indicators for the alternative versions being considered for the treatment of Pu-containing pulps are shown in Table 1.

The engineering-cost indicators obtained for the alternatives under consideration confirm the desirability of direct solidification of Pu-containing waste at the CCIM facility with resultant production of aluminophosphate glass.

Compared with the borosilicate glass, the alternative of solidification of Pu-containing waste to produce aluminophosphate glass has a [cost] advantage of $5.5 \%$.

The increase in costs of processing the Pu-containing pulp with preliminary separation of uranium and plutonium into concentrate amounts to $23 \%-29 \%$. 
Table 1. Engineering-cost indicators for alternative versions being considered for treatment of Pucontaining pulps.

\begin{tabular}{|c|c|c|c|c|c|}
\hline \multirow[b]{2}{*}{ Expense description } & \multirow{2}{*}{$\begin{array}{l}\text { Unit of } \\
\text { measure }\end{array}$} & \multicolumn{2}{|c|}{ Version 1} & \multicolumn{2}{|c|}{ Version 2} \\
\hline & & $\begin{array}{c}\text { IM-UHF } \\
\text { (version 1A) }\end{array}$ & $\begin{array}{c}\text { IM-CCIM } \\
\text { (version 1B) }\end{array}$ & $\begin{array}{c}\text { IM-UHF } \\
\text { (version 2A) }\end{array}$ & $\begin{array}{c}\text { IM-CCIM } \\
\text { (version 2B) }\end{array}$ \\
\hline \multicolumn{6}{|l|}{$\begin{array}{l}\text { 1. Investment costs for } \\
\text { reprocessing of pulps, }\end{array}$} \\
\hline including & $\$$ & $7,221,300$ & $6,182,000$ & $11,177,900$ & $9,646,500$ \\
\hline solidification facilities & $\$$ & $3,388,600$ & $2,959,600$ & $10,155,500$ & $9,234,400$ \\
\hline \multicolumn{6}{|l|}{$\begin{array}{l}\text { 2. Annual operating costs for } \\
\text { pulp treatment. }\end{array}$} \\
\hline Pulp reprocessing & $\$$ & $3,695,700$ & $3,609,200$ & $2,396,000$ & $2,188,100$ \\
\hline $\begin{array}{l}\text { Acceptance for } \\
\text { long-term storage. }\end{array}$ & $\$$ & 430,900 & 430,900 & 383,200 & 430,900 \\
\hline Long-term storage & $\$$ & 48,800 & 48,800 & 42,800 & 48,800 \\
\hline \multicolumn{6}{|l|}{$\begin{array}{l}\text { 3. Summary operating } \\
\text { expenses for: }\end{array}$} \\
\hline $\begin{array}{l}\text { Reprocessing of pulp over a } \\
\text { period of } 15 \mathrm{yr} \text {. }\end{array}$ & $\$$ & $55,435,500$ & $54,138,000$ & $35,940,000$ & $32,821,500$ \\
\hline $\begin{array}{l}\text { Acceptance for long-term } \\
\text { storage of solidified RAW over } \\
15 \mathrm{yr} \text {. }\end{array}$ & $\$$ & $6,463,500$ & $6,463,500$ & $5,748,000$ & $6,463,500$ \\
\hline $\begin{array}{l}\text { Long-term storage of solidified } \\
\text { RAW over } \\
15 \mathrm{yr} \text {. }\end{array}$ & $\$$ & 732,000 & 732,000 & 642,000 & 732,000 \\
\hline Totals for section 3. & $\$$ & $62,631,000$ & $61,333,500$ & $42,330,000$ & $40,017,000$ \\
\hline $\begin{array}{l}\text { 4. Summary expenditures being } \\
\text { compared. }\end{array}$ & $\$$ & $69,852,300$ & $67,515,500$ & $53,507,900$ & $49,663,500$ \\
\hline $\begin{array}{l}\text { 5. Change in summary } \\
\text { expenditures }\end{array}$ & $\%$ & $100 \%$ & $96.7 \%$ & $76.6 \%$ & $71.1 \%$ \\
\hline
\end{tabular}




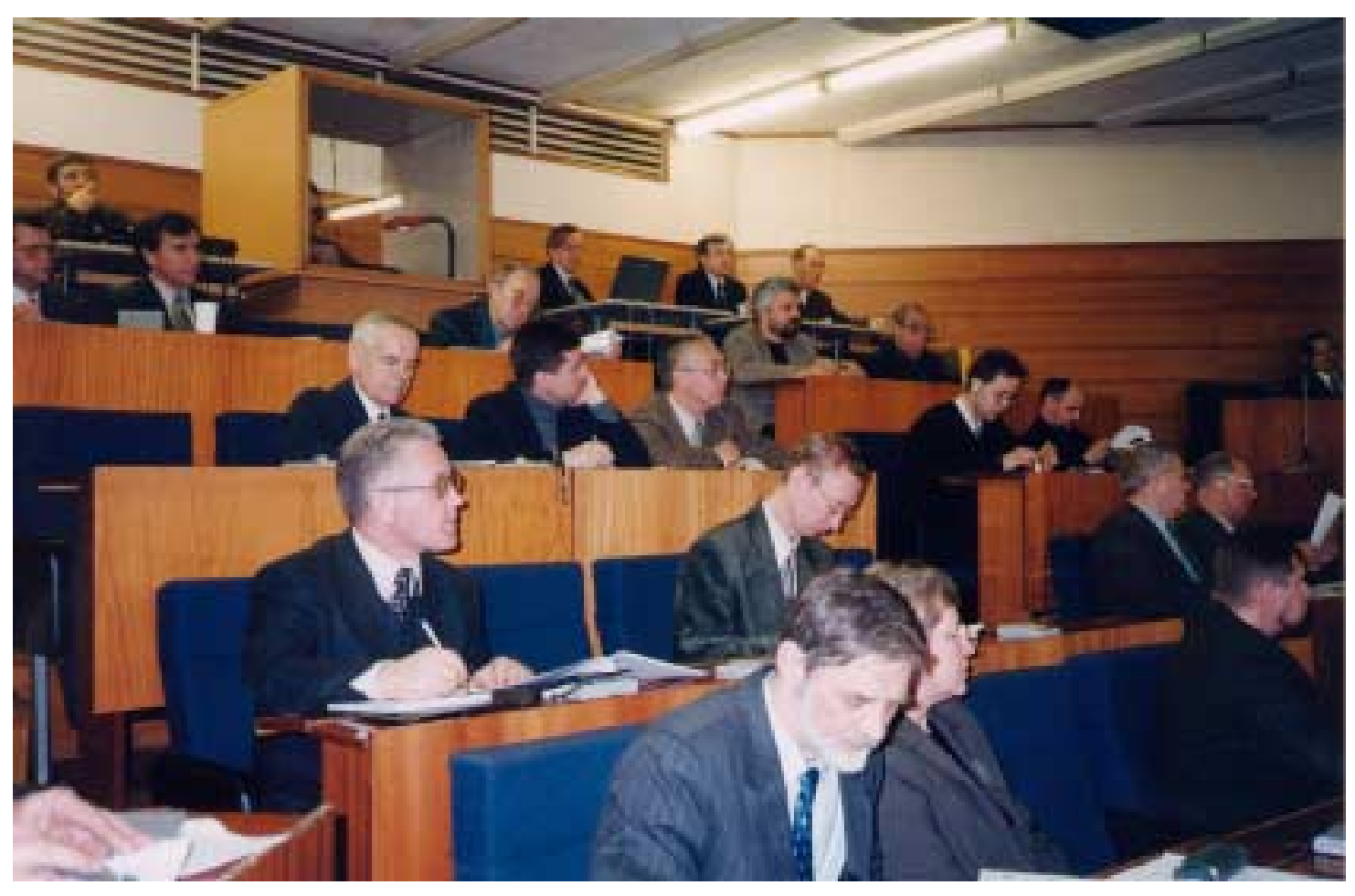




\title{
Technological Control of Bottom Sediment Conditions in Storage Reservoirs (B506201)
}

\author{
S .I. Rovny, G. M. Medvedev, N. M. Emelianov, FSUE PA “Mayak”, Ozersk
}

Accumulation of liquid radioactive wastes of high activity and in large amounts is one of the negative consequences of activity of radiochemical enterprises involved in implementation of defense programs and processing of spent nuclear fuel. A large part of the waste is to be subject to long-term storage at specialized production complexes in order to decrease activity of waste, or up to commissioning of plants for waste processing.

A certain part of radioactive waste containing sizeable quantities of aluminum and other stable impurities is stored in the form of pulps at surface storage reservoirs with rectangular compartments containing a total volume of $1300 \mathrm{~m}^{3}$. Waste stored in the form of pulps allows for significant decrease in volumes of storage reservoirs. About $19,000 \mathrm{~m}^{3}$ of suspensions with a total activity above one hundred million curies contain sizeable amounts of uranium and plutonium. They are stored at the present time in available pulprepositories of the Radiochemical Plant.

Research and practical works are realized at the enterprise in order to define immobilization technology for pulps and the fissile materials contained in them. Independently if an immobilization method is to be accepted, the process of development of technological solutions and their implementation will be very long-term and require comprehensive information on the composition and characteristics of pulps and stored solutions. In this connection, it is necessary to indicate some fields of practical work in the area of the enterprise's activity, which are directly associated with further processing of waste from pulprepositories:

- Permanent technological control of conditions of storage reservoirs in order to provide long-term safe storage of pulps, predict probable deviations, and take steps for their elimination;

- Monitoring physical conditions of bottom sediments, and the degree of pulp concentration, in particular, as this very index will be of the utmost importance when selecting the method of pulp retrieval from storage reservoirs;

- Accumulation and systematization of the data on chemical, physicochemical, and radiochemical characteristics of clarified and pulp phases, as those are of the utmost importance for development and optimization of technological solutions on immobilization of this class of waste.

Determination of chemical and radiochemical characteristics of pulps and solutions is the most laborious and technically complicated task, as no provision is made for any standard system of sampling in storage reservoirs. In addition, examinations of high-level solutions and pulps, in themselves, substances composed of a lot of chemical components, also is a very complicated task. But nevertheless, implementation of this work is of great necessity, as the presence of some impurities, sulphate-ion in particular, can create significant effects on solidification technology.

Both chemical and radiochemical composition of sediments, as well as their structure, are specified for each storage reservoir. Other than the predominant substance of aluminum hydroxide, sediments essentially differ in composition of corrosion products, and also in their stable and radioactive impurities. While the first commissioned storage reservoirs contain hydroxide pulps for the most part, the later ones 
can probably contain ferrocyanide and sulfide pulps. Related changes result from the introduction of new treatment process procedures into basic technology. The possibility of some differences in composition of bottom sediments, depending on the height of pulp's level, should also be taken into account, as reservoirs were filled up to a nominal level of total activity in several procedures conducted over time. Additionally, in response to long-term storage, various processes of sediment aging take place, which result mainly in increasing the chemical inertness of pulps.

Safety assurance for long-term pulp storage requires permanent monitoring of conditions in storage reservoirs. The temperature in storage reservoirs, measured in various areas over the section and height of reservoirs, is the main parameter to be permanently controlled. Temperature distribution over the height of a reservoir depends upon total activity, degree of sediment concentration, and the availability of cooling systems.

The highest temperatures were observed in the bottom part of sediment in uncooled reservoirs as pulp became more concentrated. The temperature of sediments in the bottom part of single reservoirs reached $\sim 140^{\circ} \mathrm{C}$, which resulted in local boiling of the pulp. In order to provide for stabilization of temperature conditions, a periodic water supply was introduced to the bottom part of reservoirs. However, this step was only of a temporary character, because in 10-15 days after suspension's concentration, conditions of the reservoir had already returned to their original state. A more efficient solution to the problem of decreasing temperature in uncooled reservoirs (\#3 - \#8) was reached by means of treatment of suspensions with alkali. This resulted in partial sediment erosion, decrease of sediment thickness, and temperature stabilization within the range of $100^{\circ} \mathrm{C}$ (Fig. 1). Two to three years after alkaline treatment, some growth of sediment volume was observed in many reservoirs; this was evidently caused by formation of secondary sediments, which resulted from processes of aging of aluminate solutions. The following examination of sediment composition at the inter-phase border of reservoir 6 indicated their both specific activity and content of basic elements to be somewhat lower, which, to a certain extent, confirms the secondary nature of their origin.

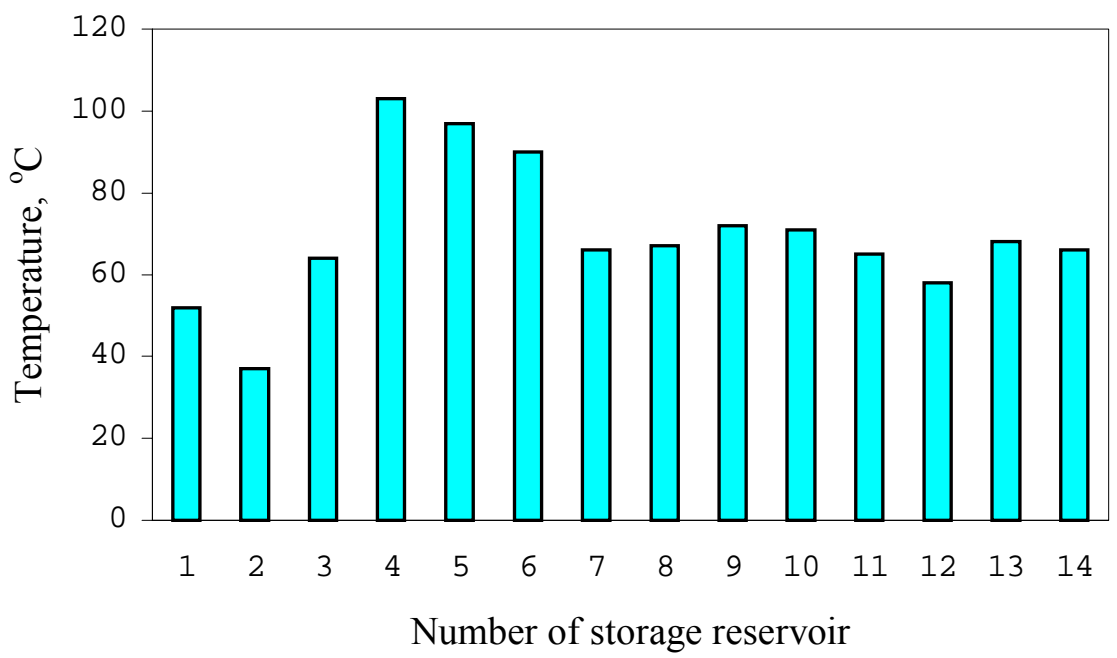

Fig. 1. Average temperature in the bottom part of storage reservoirs. 
Provision is made for periodical monitoring of the chemical and radiochemical composition of the clarified phase in storage reservoirs, which proves to be relatively constant in time. Specific $\beta$-activity of solutions in uncooled reservoirs reaches $1-1,5 \mathrm{Ci} / \mathrm{L}$, and is caused mainly by cesium. The main part of macrocomponent composition of solution substance is represented by sodium and aluminum, whose concentrations are determined by the alkalinity of the solution. When the concentration of alkali falls within the range of $80-120 \mathrm{~g} / \mathrm{L}$, then the concentration of aluminum reaches $5-6,5 \mathrm{~g} / \mathrm{L}$. A substance of iron, chrome, and nickel is observed in rather lower concentrations. Concentrations of uranium and plutonium reach mainly tens and ones, in milligrams per liter, respectively (Table 1).

Table 1. Composition of clarified phase in some pulp repositories.

\begin{tabular}{|c|c|c|c|c|c|}
\hline \multirow{2}{*}{ Component } & \multicolumn{5}{|c|}{ Storage reservoir } \\
\hline & 3 & 4 & 6 & 7 & 8 \\
\hline \multicolumn{6}{|c|}{ Radiochemical composition, Bq/L } \\
\hline$\alpha$-activity & $1,410^{7}$ & $3,610^{7}$ & - & - & - \\
\hline$\beta$-activity & $5,310^{10}$ & $3,510^{10}$ & $5,110^{10}$ & $5,110^{10}$ & $5,710^{10}$ \\
\hline Sr-90 & $7,410^{7}$ & $8,410^{7}$ & - & - & - \\
\hline Cs-137 & $5,210^{10}$ & $3,610^{10}$ & $5,010^{10}$ & $4,010^{10}$ & $4,610^{10}$ \\
\hline Cs-134 & $2,410^{7}$ & $8,810^{7}$ & $1,810^{8}$ & $1,210^{8}$ & $1,410^{8}$ \\
\hline Eu-154 & - & $1,510^{8}$ & - & $1,110^{10}$ & - \\
\hline \multicolumn{6}{|c|}{ Chemical composition, g/L } \\
\hline $\mathrm{U}$ & 0,034 & 0,023 & 0,05 & - & 0,035 \\
\hline $\mathrm{Pu}$ & 0,0006 & 0,0029 & 0,0009 & 0,001 & 0,005 \\
\hline $\mathrm{Am}$ & 0,0002 & 0,0006 & - & - & - \\
\hline Tc & 0,005 & 0,01 & - & - & - \\
\hline $\mathrm{Na}$ & 74,0 & 140,0 & 51,0 & 64,0 & 92,0 \\
\hline $\mathrm{Al}$ & 5,6 & 6,3 & 5,0 & 6,0 & 6,0 \\
\hline $\mathrm{Cr}$ & 0,8 & 1,9 & 0,4 & 2,0 & 0,4 \\
\hline $\mathrm{Ni}$ & 0,2 & 0,3 & 0,01 & 0,01 & 0,02 \\
\hline $\mathrm{Si}$ & 0,3 & 0,2 & - & - & - \\
\hline $\mathrm{Mg}$ & 0,3 & 0,14 & - & - & - \\
\hline $\mathrm{Ca}$ & 2,0 & 1,2 & - & - & - \\
\hline $\mathrm{NaOH}$ & 78,4 & 144,0 & 99,0 & 112,0 & 80,0 \\
\hline $\mathrm{NO}_{3}$ & 82,0 & 114,0 & - & - & - \\
\hline $\mathrm{SO}_{4}{ }^{2-}$ & 14,3 & 3,6 & - & - & - \\
\hline $\mathrm{Cl}^{-}$ & 1,0 & 1,6 & 0,04 & 0,05 & 0,68 \\
\hline
\end{tabular}

An important role in predicting conditions in storage repositories is given to the control of the height level of concentrated pulp in determining, to a significant extent, temperature conditions of the system. Various constantly and periodically controlled characteristics can be applied for evaluation of the height of the pulp layer:

- Temperature distribution over the height of reservoir;

- Dose rate distribution over the height of reservoir;

- Direct measuring of a height of concentrated pulp with the help of a bob (i.e., float). 
The applied methods have demonstrated comparatively well-conforming results, indicating not only the presence of a concentrated phase in storage reservoirs, but also of a suspended phase at the inter-phase border. Thickness of the concentrated part of a pulp in storage reservoirs is represented in Fig. 2.

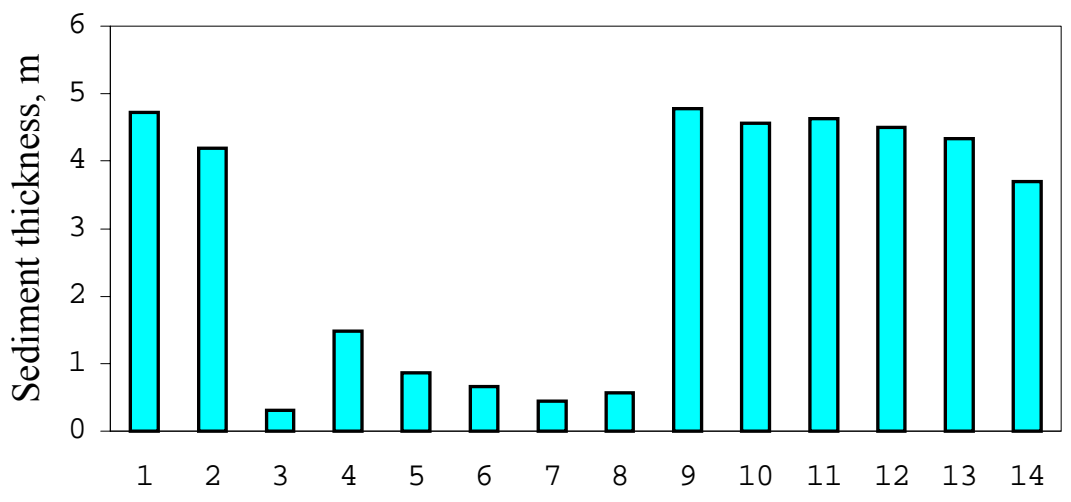

Number of storage reservoir

Fig. 2- Thickness of the concentrated part of a pulp in storage reservoirs.

Analysis of pulp composition in storage reservoirs is necessary for prediction of conditions and assessment of possible effects of the temperature conditions of reservoir, as well as for developmental work on the problems of subsequent technological steps of pulp immobilization. Storage reservoirs are not provided with standard systems for sediment sampling. A special sampling device to perform sediment sampling from various depths was designed and manufactured for this purpose. The device is mounted on rigid beam, with provision for closing of the lid of sampler at the required depth. The sampler is entered into the reservoir through technological cuttings for control-and-measuring devices; then the sampled sediment is reloaded into the special reservoir, and is transported in a protective cask to the laboratory.

Pulp samples from the inter-phase border are of non-stationary, light brown sediment with sizeable amounts of the clarified phase. Samples of concentrated pulp are thick, tough, dark brown sediment. The sediments for analysis were subsequently treated with various reagents $\left(\mathrm{NaOH}, \mathrm{HNO}_{3}-\mathrm{NH}_{4} \mathrm{~F}, \mathrm{HNO}_{3}\right.$ $\mathrm{H}_{3} \mathrm{PO}_{4}$ ) until completely dissolved, and chemical and radiochemical composition of solutions was specified by standard methods. On the basis of analytical results, the composition of the original pulp was calculated. The composition of sediments from single storage reservoirs is presented in Table 2. 
Table 2- Composition of sediments of several pulp repositories.

\begin{tabular}{|c|c|c|c|c|c|}
\hline \multirow{2}{*}{ Component } & \multicolumn{5}{|c|}{ Storage reservoir } \\
\hline & 3 & 4 & 6 & 7 & 8 \\
\hline \multicolumn{6}{|c|}{ Radiochemical composition, $\mathrm{Bq} / \mathrm{L} 10^{-10}$} \\
\hline$\alpha$-activity & 0,25 & 0,07 & - & 0,28 & - \\
\hline$\beta$-activity & 70,1 & 29,4 & 46,4 & 140,0 & 104,0 \\
\hline Sr-90 & 25,2 & 1,8 & - & 27,0 & - \\
\hline Cs-137 & 21,6 & 17,7 & 24,6 & 120,0 & - \\
\hline Cs-134 & 0,015 & 0,08 & 0,15 & 0,05 & 0,04 \\
\hline Eu-154 & 0,23 & 0,11 & 0,12 & 5,7 & - \\
\hline \multicolumn{6}{|c|}{ Chemical composition, g/L } \\
\hline$U$ & 14,9 & 4,9 & 8,1 & - & 6,4 \\
\hline $\mathrm{Pu}$ & 0,115 & 0,228 & 0,36 & 0,44 & 0,18 \\
\hline $\mathrm{Am}$ & 0,012 & 0,003 & - & 0,013 & - \\
\hline Tc & 0,018 & - & - & - & - \\
\hline $\mathrm{Na}$ & 228 & 74,4 & - & 250 & - \\
\hline $\mathrm{Al}$ & 287 & 98,0 & 66,6 & 250 & - \\
\hline $\mathrm{Fe}$ & 68,0 & 43,5 & 48,0 & 25,0 & 32,0 \\
\hline $\mathrm{Cr}$ & 30,0 & - & - & - & - \\
\hline $\mathrm{Ni}$ & 90,0 & 17,5 & 33,3 & - & - \\
\hline $\mathrm{Mn}$ & 24,0 & - & 8,0 & - & 8,0 \\
\hline $\mathrm{Si}$ & 18,0 & - & - & - & - \\
\hline $\mathrm{Mg}$ & 13,2 & 3,4 & 8,0 & - & 8,0 \\
\hline $\mathrm{Ca}$ & 40,5 & - & - & - & - \\
\hline $\mathrm{K}$ & 96,5 & - & - & - & - \\
\hline $\mathrm{SO}_{4}{ }^{2-}$ & 102 & - & - & - & - \\
\hline
\end{tabular}

It should be mentioned that the completed determinations of pulp composition are of single character, and their results are difficult to extrapolate to the whole volume of a pulp in a storage reservoir. Subsequent system investigations of sediment composition should be done with the purpose of selecting the optimal technology, and to determine pulp immobilization parameters. In this connection, the main work to be performed in this direction prior to creation of a pulp processing installation are as follows:

- Improvement of control, and heightening of storage safety;

- Improvement of sampling and analysis methods for solutions and pulps;

- Examination of workability of equipment for pulp evacuation from reservoirs;

- Accumulation of data on chemical and radiochemical composition of pulps. 


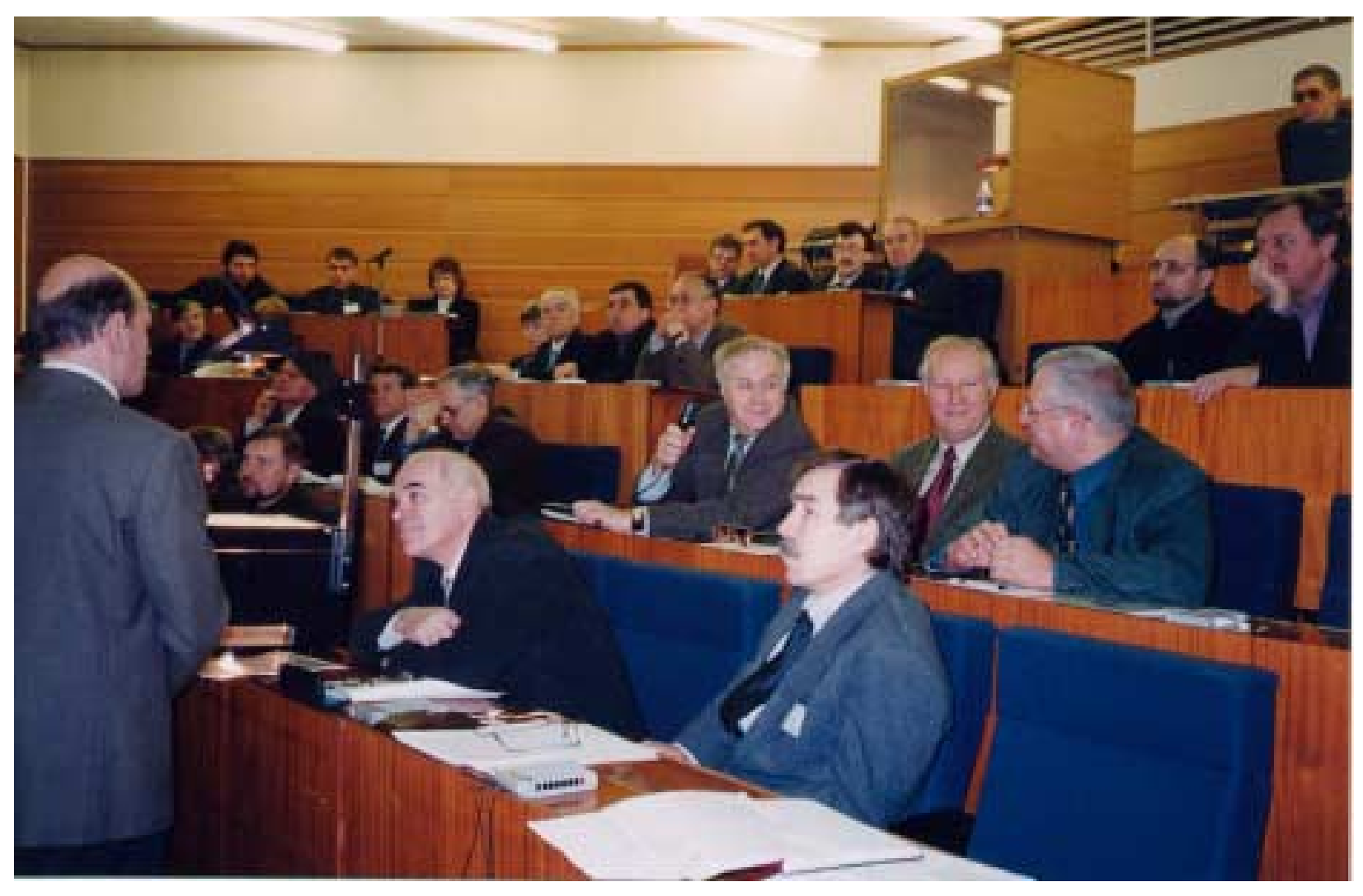




\section{The Technology of Alpha-active Materials Incorporation in Glassy and Mineral Matrixes (B506201)}

\section{S. I. Rovny, V. I. Guzhavin, FSUE "Mayak" PA}

Alpha active solid radioactive wastes (SRW) and liquid radioactive wastes (LRW) from chemicalmetallurgical and radiochemical production of Mayak PA, subject to disposition according to their content of uranium and highly enriched plutonium, are moved to places of temporary storage, namely surface burials and special reservoirs. This practice is inadmissible in respect to the environment.

The analysis of scientific and technical information on the treatment of such wastes showed that methods of bituminization, cementation, incorporation of radioactive wastes into metal matrixes, ceramics on a base of titanium-containing materials, kaolin, bentonite, corundum, and so forth are unacceptable for such long-lived radionuclides as plutonium.

In our opinion, the most acceptable method of solving the problem is the transformation of alpha active waste into a monolithic matrix at high temperatures, and, in particular, using the cold crucible induction melter (CCIM) and the production of glass and mineral matrixes on a base of garnet and basalt.

However, the process of incorporation of real alpha active SRW from "Mayak" PA into a monolithic matrix was not tested, even in a laboratory environment, by any Russian research organization.

In 1997, in cooperation with A. A. Bochvar All-Russian Research Institute of Inorganic Materials (VNIINM) personnel including T.V. Smelova and others, we constructed the CCIM facility, which is still operating successfully. During this period, more than 150 fusions were performed with charge mixtures of different composition.

Nominal operational modes of the high-frequency oscillator $6-60 / 1,76$ at mixture fusion (SRW $=66.6 \%$ of mass) are given in Table 1. For $1 \mathrm{~kg}$ of SRW, we added $158 \mathrm{~g}$ of $\mathrm{F}_{2} \mathrm{O}_{3}$ powder, $200 \mathrm{~g}$ of $\mathrm{SiO}_{2}$, and $140 \mathrm{~g}$ of melted ferric oxide in the form of fragments as the starting material for producing a ferrosilicate matrix in the end.

Averaged chemical composition of the melts is presented in Table 2. The first 12 oxides (boldface) correspond to alpha active SRW and all 19 to a mixture of alpha active SRW and radiochemical production waste. In addition, the physical, chemical, and mineralogical characteristics of the melts were studied.

Table 1. Mode of charge mixture melting.

\begin{tabular}{|l|l|l|l|l|}
\hline Name of operation & $\begin{array}{l}\mathbf{U}, \mathbf{k V}, \text { anode } \\
\text { voltage }\end{array}$ & $\mathbf{I}_{\mathbf{a}}, \mathbf{A}$, anode current & $\begin{array}{l}\mathbf{I}_{\mathbf{c}}, \mathbf{A}, \text { frame } \\
\mathbf{c u r r e n t}\end{array}$ & $\mathbf{T},{ }^{\mathbf{0}} \mathbf{C}$ \\
\hline $\begin{array}{l}\text { Heating of charge mixture and } \\
\text { starting material }\end{array}$ & 7 & 3.5 & 0.7 & Up to 1330 \\
\hline $\begin{array}{l}\text { Melting of starting charge } \\
\text { mixture }\end{array}$ & 5.5 & $3.5-3.8$ & 0.7 & 1450 \\
\hline Fusion & $4-4.5$ & 3.5 & 0.6 & $1400-1500$ \\
\hline Melt hold & $4.5-4.75$ & 3.7 & 0.65 & $1450-1500$ \\
\hline $\begin{array}{l}\text { Heating of the melt before } \\
\text { discharging }\end{array}$ & 5.0 & 3.7 & 0.7 & $1700-1800$ \\
\hline
\end{tabular}


Table 2. Composition of ferrosilicate melt.

\begin{tabular}{|c|c||c|c|}
\hline Oxide & $\begin{array}{c}\text { Oxide content, } \\
\text { \% of mass }\end{array}$ & Oxide & $\begin{array}{c}\text { Oxide content, } \\
\text { \% of mass }\end{array}$ \\
\hline $\mathrm{Fe}_{2} \mathrm{O}_{3}$ & $19-27$ & $\mathrm{TiO}_{2}$ & $0,2-0,3$ \\
\hline $\mathbf{M g O}$ & $9,2-18,1$ & $\mathrm{Na}_{2} \mathrm{O}$ & $0,1-0,2$ \\
\hline $\mathrm{SiO}_{2}$ & $12-17,7$ & $\mathrm{CeO}_{2}$ & $1,0-4,0$ \\
\hline $\mathrm{CaO}$ & $5,1-11,3$ & $\mathrm{ZrO}_{2}$ & $0,5-2,6$ \\
\hline $\mathrm{Al}_{2} \mathrm{O}_{3}$ & $5,2-7,6$ & $\mathrm{La}_{2} \mathrm{O}_{3}$ & $0,5-3,0$ \\
\hline $\mathrm{BaO}$ & $5,0-6,5$ & $\mathrm{SrO}$ & $1,0-6,0$ \\
\hline $\mathrm{Cr}_{2} \mathrm{O}_{3}$ & $2,0-3,0$ & $\mathrm{Cs}_{2} \mathrm{O}$ & $1,0-5,5$ \\
\hline $\mathrm{MnO}_{2}$ & $1,0-1,3$ & $\mathrm{Y}_{2} \mathrm{O}_{3}$ & $0,5-3,0$ \\
\hline $\mathbf{N i O}$ & $0,9-1,3$ & $\mathrm{Nd}_{2} \mathrm{O}_{3}$ & $0,5-3,0$ \\
\hline $\mathrm{PbO}_{2}$ & $0,1-0,3$ & & \\
\hline
\end{tabular}

Over 100 operations of transformation into monolithic product were performed with simultaneous incorporation into the matrix of $66.6 \%$ mass of model calcified high level waste (HLW) and SRW from weapons-grade plutonium production.

The process of melting proceeded calmly, without splashing, using the following temperature modes:

$$
\begin{aligned}
& t_{\text {melter }} \sim 1500{ }^{\circ} \mathrm{C} \\
& t_{\text {melt }} \sim 1650-1670^{\circ} \mathrm{C}
\end{aligned}
$$

The thickness of the skull layer was $10-12 \mathrm{~mm}$.

\section{Production of Basalt Composition}

In the following two operations, the initial charge mixture contained:

- Operation 101-500 g SRW $+500 \mathrm{~g} \mathrm{SiO}_{2}+160 \mathrm{~g} \mathrm{MgO}+79 \mathrm{~g} \mathrm{Fe}_{2} \mathrm{O}_{3}$ with consequent adding of $120 \mathrm{~g}$ of melted $\mathrm{Fe}_{2} \mathrm{O}_{3}$.

- Operation 102-500 g SRW (without $\mathrm{Fe}_{2} \mathrm{O}_{3}$ ) $+500 \mathrm{~g} \mathrm{SiO}_{2}+160 \mathrm{~g} \mathrm{MgO}+108 \mathrm{~g} \mathrm{Fe}_{2} \mathrm{O}_{3}$ also with adding of $120 \mathrm{~g}$ of melted $\mathrm{Fe}_{2} \mathrm{O}_{3}$. SRW mass in charge mixture of operation 15 was $34,8 \%$ of mass, and in operation 16 was $36 \%$ of mass.

Fusion was performed in the optimal operational mode of the oscillator, holding the melt in the crucible for about 2 hours. In operation 101, the melt flowed down into the mold easily and without splashing; in operation 102, it hardly flowed down due to high viscosity, and thickened immediately. The melts produced had a density of $3.1 \mathrm{~g} / \mathrm{cm}^{3}$ and $3.2 \mathrm{~g} / \mathrm{cm}^{3}$, correspondingly, they had internal interstices and were more crystalline in appearance. Maximum incorporation of SRW in such a matrix didn't exceed $36 \%$ of mass. No fusion was performed with a higher value than this one (operation 103).

In operation 101, the composition of the produced melt contained (\% of mass): $\mathrm{SiO}_{2}=36,6 ; \mathrm{Fe}_{2} \mathrm{O}_{3}=19,2$; $\mathrm{MgO}=17,4$; the rest $(26,8)$ were SRW components.

In operation 102 (\% mass.), the product contained $\mathrm{SiO}_{2}=37,8 ; \mathrm{Fe}_{2} \mathrm{O}_{3}=16,4 ; \mathrm{MgO}=18,0$ and the rest $(27,8)$ were SRW components.

The produced compositions are close to natural basalt in their content of oxides of silicon, ion, and magnesium. 


\section{Production of Phosphate, Borosilicate and Borophosphate Glasses with SRW Incorporation}

The objective was unification of "Mayak" PA waste solidification technology (HLW, MLW and alpha active SRW) at the CCIM facility.

The research was conducted with the incorporation of SRW into phosphate borosilicate and borophosphate glasses. At the first stage, the research was done in a laboratory environment in a heated resistance furnace in an alundum crucible, and at the second stage, it was performed at the CCIM facility.

Tables 3-5 show the results of laboratory research on the production of glassy matrices.

Table 3. Laboratory tests on alloying of SRW with phosphate glass, $t=1100-1150{ }^{\circ} \mathrm{C}, \tau=1 \mathrm{~h}$.

\begin{tabular}{|c|c|c|c|c|c|c|}
\hline \multicolumn{5}{|c|}{ Charge mixture composition before melting, $\%$ mass. } & \multirow{2}{*}{ Mass ratio } & \multirow{2}{*}{ Appearance of produced glass } \\
\hline SRW & $\mathrm{Na}_{2} \mathrm{O}$ & $\mathrm{P}_{2} \mathrm{O}_{5}$ & $\mathrm{Al}_{2} \mathrm{O}_{3}$ & $\mathrm{SiO}_{2}$ & & \\
\hline 10,0 & 20,0 & & 21,0 & 4 & 0,444 & Black, bright, without holes \\
\hline 17,90 & 19,6 & 45,4 & 17,1 & - & 0,432 & Dark-brown, dull, without holes \\
\hline 20,0 & 15,0 & 40,0 & 21,0 & 4 & 0,375 & Black, bright, without holes \\
\hline 24,60 & 18,0 & 41,7 & 15,7 & - & 0,432 & The same \\
\hline 30,0 & 15,0 & 40,0 & 11,0 & 4 & 0,375 & $-"-$ \\
\hline 30,0 & 20,0 & 40,0 & 10,0 & - & 0,500 & $-"-$ \\
\hline 30,36 & 16,6 & 38,5 & 14,5 & - & 0,431 & Dark-brown, dull, with holes \\
\hline
\end{tabular}

Table 4. Laboratory tests on alloying of SRW with borosillicate glass, $t=1100-1120{ }^{\circ} \mathrm{C}, \tau=1 \mathrm{~h}$.

\begin{tabular}{|c|c|c|c|c|c|}
\hline \multicolumn{4}{|c|}{ Charge mixture composition before melting, $\%$ mass. } & \multirow{2}{*}{ Mass ratio } & \multirow{2}{*}{ Appearance of produced glass } \\
\hline SRW & $\mathrm{B}_{2} \mathrm{O}_{3}$ & $\mathrm{SiO}_{2}$ & $\mathrm{Na}_{2} \mathrm{O}$ & & \\
\hline 30,27 & 27,73 & 25,20 & 16,80 & 1,10 & Black, bright \\
\hline 38,06 & 24,62 & 22,39 & 14,93 & 1,10 & The same \\
\hline 43,92 & 22,30 & 20,27 & 13,51 & 1,10 & $-1 "-$ \\
\hline 50,60 & 19,64 & 17,86 & 11,90 & 1,10 & $-"-$ \\
\hline 58,30 & 16,60 & 15,00 & 10,10 & 1,10 & $\begin{array}{l}\text { Black, with holes, leached in distilled } \\
\text { water }\end{array}$ \\
\hline 65,00 & 13,92 & 12,66 & 8,44 & 1,10 & The same \\
\hline
\end{tabular}

The given data show that test meltings should be performed at the CCIM facility with incorporation of $\sim 30 \%$ by mass of SRW into phosphate glass, and $\sim 50 \%$ of mass into borosilicate and borophosphate glass with the addition of cerium oxide as a plutonium imitator in every initial charge mixture. 
Table 5. Laboratory tests on alloying of SRW with borophosphate glass, $t=1150-1200{ }^{\circ} \mathrm{C} ; \tau=1 \mathrm{~h}$.

\begin{tabular}{|c|c|c|c|c|c|c|c|}
\hline \multicolumn{6}{|c|}{ Charge mixture composition before melting, \% mass. } & \multirow[b]{2}{*}{ Mass ratio } & \multirow[b]{2}{*}{ Appearance of produced glass } \\
\hline SRW & $\mathrm{B}_{2} \mathrm{O}_{3}$ & $\mathrm{P}_{2} \mathrm{O}_{5}$ & $\mathrm{Al}_{2} \mathrm{O}_{3}$ & $\mathrm{Na}_{2} \mathrm{O}$ & $\mathrm{SiO}_{2}$ & & \\
\hline 16,11 & 9,14 & 29,57 & 18,30 & 13,44 & 13,44 & 0,31 & Black, bright, without holes \\
\hline 24,27 & 8,25 & 26,70 & 16,50 & 12,14 & 12,14 & 0,31 & The same \\
\hline 32,47 & 7,36 & 23,81 & 14,72 & 10,82 & 10,82 & 0,31 & $-"-$ \\
\hline 40,23 & 6,51 & 21,07 & 13,03 & 9,58 & 9,58 & 0,31 & $-"$ \\
\hline 49,84 & 5,47 & 17,68 & 10,93 & 8,04 & 8,04 & 0,31 & Black, bright, there are some holes \\
\hline
\end{tabular}

\section{Production of Borophosphate Matrices at CCIM}

The attempt to produce a borophosphate matrix at CCIM was made in two operations $(104,105)$. It should be noted that previously such matrices were produced only in resistance furnaces. The composition of the charge mixture is presented in Table 6. The SRW composition is the same; $50 \mathrm{~g}$ of silicon oxide, $25 \mathrm{~g}$ of cerium dioxide, and $120 \mathrm{~g}$ of melted ferric oxide as a starting material were added per each kilogram of the charge mixture.

Table 6. Composition of borophosphate charge mixture.

\begin{tabular}{|l|c|}
\hline Oxides & Oxide and SRW, \% mass. \\
\hline $\mathrm{B}_{2} \mathrm{O}_{3}$ & 7,3 \\
\hline $\mathrm{Al}_{2} \mathrm{O}_{3}$ & 6,0 \\
\hline $\mathrm{Na}_{2} \mathrm{O}_{3}$ & 11,0 \\
\hline $\mathrm{SiO}_{2}$ & 11,1 \\
\hline $\mathrm{P}_{2} \mathrm{O}_{5}$ & 14,6 \\
\hline $\mathrm{SRW}$ mixture & 50 \\
\hline
\end{tabular}

Unlike SRW melting, when at first ferric oxide is melted and only then the mixture itself, the melting of the given charge mixture is "diametrically opposite". At the boundaries of heated up ferric oxide, the charge mixture itself begins to melt without melting of ferric oxide fragments at the following oscillator operation parameters: $U_{A}=5 \mathrm{kV}, \mathrm{I}_{\mathrm{A}}=3,0 \ldots 3,2 \mathrm{~A}, \mathrm{I}_{\mathrm{C}}=0,6-0,65 \mathrm{~A}$. At a temperature of $\sim 950^{\circ} \mathrm{C}$, the start of the emission of white-colored gasses was noticed. The temperature at the beginning of charge mixture melting is $1050-1100^{\circ} \mathrm{C}$; at that point, the anode voltage was decreased to $3,5 \mathrm{kV}$. The melt boils up calmly without ejection of separate small parts from the crucible. The diameter of the crust, floating on the top of the melt, is equal to approximately half of the crucible. Temperature of the crust is $1100-1150^{\circ} \mathrm{C}$, and temperature of the melt surface is $\sim 1200^{\circ} \mathrm{C}$. Abundant gas emission of white color starts at the temperature of $\sim 1100^{\circ} \mathrm{C}$. The melt flows down into the mold easily without splashing. The melt is fluid. None of the initial iron fragments are found in the crucible after discharge.

$\begin{array}{ccc} & \begin{array}{c}\text { Charge mixture } \\ \text { mass, } \mathrm{g}\end{array} & \text { Melt mass, g } \\ \text { Operation 104 } & 1195 & 1060 \\ \text { Operation 105 } & 1295 & 1020\end{array}$

The thickness of the skull layer is $5-7 \mathrm{~mm}$. The temperature of the melt, moved to the heated hot plate, is $740-760^{\circ} \mathrm{C}$. There are no cracks. 


\section{Production of Borosilicate Matrix at CCIM}

Operation 106. Charge mixture composition is presented in Table 7; the composition of SRW is the same; $904 \mathrm{~g}$ of mixture of oxides $\mathrm{Na}_{2} \mathrm{O}, \mathrm{B}_{2} \mathrm{O}_{3}$ and $\mathrm{SiO}_{2}, 926 \mathrm{~g}$ of $\mathrm{SRW}$ mixture, $50 \mathrm{~g}$ of cerium dioxide and $120 \mathrm{~g}$ of melted ferric oxide as a start material were taken for melting.

Table 7. Borosilicate charge composition.

\begin{tabular}{|c|c|}
\hline Oxides & Oxide and SRW content, \% mass. \\
\hline $\mathrm{Na}_{2} \mathrm{O}_{3}$ & 13,0 \\
\hline $\mathrm{B}_{2} \mathrm{O}_{3}$ & 21,4 \\
\hline $\mathrm{SiO}_{2}$ & 15,0 \\
\hline $\mathrm{SRW}$ mixture & 50,6 \\
\hline
\end{tabular}

As in operations 104 and 105, this charge mixture began to melt at the edges of iron fragments at the following operational parameters of the oscillator: $U_{A}=5 \mathrm{kV}, \mathrm{I}_{\mathrm{A}}=3,0 \mathrm{~A}, \mathrm{I}_{\mathrm{C}}=0,6 \mathrm{~A}$. At a temperature of $900^{\circ} \mathrm{C}$, emission of gasses ranging in color from yellow-green with brown tint to dark brown was noticed.

The temperature at the beginning of charge mixture melting was $1050-1100^{\circ} \mathrm{C}$. After that, the anode voltage was decreased to $4 \mathrm{kV}$ and then to $3,5 \mathrm{kV}$. The melt boiled up calmly without violent splashes and ejection.

Crust temperature $\sim 1200^{\circ} \mathrm{C}$, melt surface temperature $1300^{\circ} \mathrm{C}$. During melting, abundant emission of brown gas was taking place. During discharge, the melt was very fluid and flowed mainly down the side of the crucible. Temperature of the melt, moved to heated hot plate, was $740^{\circ} \mathrm{C}$. There were no cracks after cooling.

The produced melt was not totally removed from the mold. Part of the melt stuck to the bottom of the mold.

Operation 106: Charge mixture mass, $2000 \mathrm{~g}$, Melt mass, $1505 \mathrm{~g}$

\section{Production of Phosphate Matrix at CCIM}

Operation 107. Composition of the charge mixture used is presented in Table 8, and composition of SRW is the same; $990 \mathrm{~g}$ of mixture of oxides $\mathrm{Na}_{2} \mathrm{O}, \mathrm{P}_{2} \mathrm{O}_{5}, \mathrm{Al}_{2} \mathrm{O}_{3}$ and $\mathrm{SiO}_{2}, 297 \mathrm{~g}$ of SRW mixture, $50 \mathrm{~g}$ of cerium dioxide and $120 \mathrm{~g}$ of melted ferric oxide as a start material were taken for melting.

Table 8. Phosphate charge mixture composition.

\begin{tabular}{|c|c|}
\hline Oxides & Oxide and SRW content, \% mass. \\
\hline $\mathrm{Na}_{2} \mathrm{O}$ & 16,6 \\
\hline $\mathrm{P}_{2} \mathrm{O}_{5}$ & 44,0 \\
\hline $\mathrm{Al}_{2} \mathrm{O}_{3}$ & 7,5 \\
\hline $\mathrm{SiO}_{2}$ & 1,9 \\
\hline $\mathrm{SRW}$ mixture & 30,0 \\
\hline
\end{tabular}

This charge mixture began to melt at the edges of iron fragments at the following operational parameters of the oscillator: $U_{A}=5 \mathrm{kB}, \mathrm{I}_{\mathrm{A}}=3,5 \mathrm{~A}, \mathrm{I}_{\mathrm{C}}=0,7 \mathrm{~A}$. These parameters were not changed during the entire melting process. Temperature of the melt surface was $1400^{\circ} \mathrm{C}$. Melting was calm, without ejections and formation of crust on the surface. A small amount of white gas was emitted, approximately as during SRW melting. 
The melt was very thick during discharge. Initial fragments of ferric oxide were found at the bottom of the crucible. The temperature of the melt, moved to a heated hot plate, was $\sim 660^{\circ} \mathrm{C}$.

After cooling a crack was found from side-to-side at the center of the mold.

Operation 107: Charge mixture mass , $1207 \mathrm{~g}$; melt mass, $980 \mathrm{~g}$

Among all the glassy and mineral matrixes produced containing all main components of calcified HLW and sludges, accumulated during weapons-grade plutonium production at radiochemical plant, and SRW from chemical-metallurgical production, ferro-silicate matrixes proved to be the best prospective products as they incorporate about $66 \%$ of the SRW mass.

The characteristics of these matrices are presented in the report of V.N. Muzgin (in the next to last section of this Proceedings); the rest we studied at "Mayak" PA.

\section{Microhardness of the Produced Fusion Cake}

The research on microhardness were performed on two samples with prepared and polished surfaces. Microhardness was determined according to State Standard GOST 9450-94 at microhardness measuring device PMT-3 and was calculated with the formula:

$$
H=\frac{1854 P}{c^{2}},\left(\frac{\mathrm{kg}}{\mathrm{mm}^{2}}\right),
$$

$\begin{array}{rll}\text { where } H & = & \text { Number of hardness, } \\ P & = & \text { load in grams }(100 \mathrm{~g}), \\ C & = & \text { diagonal of the print in microns. }\end{array}$

Microhardness of light phase was $H_{100}=1542 \pm 42 \mathrm{~kg} / \mathrm{mm}^{2}$, which corresponds to the microhardness of steel U10 (for samples $\mathrm{H}_{100}=1550 ; 1580 ; 1490 \mathrm{~kg} / \mathrm{mm}^{2}$ ).

Microhardness of gray phase was $H_{100}=857 \pm 60 \mathrm{~kg} / \mathrm{mm}^{2}$, which corresponds to the microhardness of steel $\mathrm{St5}$ (for samples $\mathrm{H}_{100}=890 ; 755 ; 925 \mathrm{~kg} / \mathrm{mm}^{2}$ ).

\section{Electric Conductivity}

Research on electric conductivity was carried out with the digital multimeter instrument Protec 505 with measurement error $0.5 \% \pm 2 \mathrm{~d}$ on three samples in three inter-perpendicular directions. During research performance, it was determined that the value of electric conductivity doesn't depend upon direction of measurement.

According to preliminary data, the average electric conductivity of the mineral matrix is $8.5 \pm 2.5$ (kOhm $\times$ $\mathrm{cm})^{-1}$.

\section{Alpha-radiographic Analysis}

Alpha-radiography of glass was performed to study the uniformity of plutonium distribution for the sodiumsilicate matrix. For this purpose, the sample with a plutonium content of $10 \mathrm{~g} / \mathrm{kg}$ was placed on the photographic plate MP-20 (for nuclear research with emulsion layer thickness of $20 \mu \mathrm{m}$ ) for 5 , 10, and 20 minutes. After that, the plate was kept in developer D-19 for 6 minutes. After washing up in the water, the plate was fixed for 10 minutes. Then, final washing in water for 15 minutes and airing were performed. It should be noted that exposure and developing were conducted in green light. 
From the photos in Fig. 1, one can see that plutonium distribution in glass is rather uniform. It is proved by results of research on plutonium distribution in height. For this purpose, the glass was removed from the crucible, broken, reduced to fragments, dispersed, and equal-sized fractions were treated in boiled solution of nitric ( 8 mole/l) and hydrofluoric $(0.4$ mole) acids mixture for 3 hours at weight-space relation of phases $\mathrm{W}=100 \mathrm{l} / \mathrm{kg}$. The clarified solution was analyzed for plutonium content. Five samples, taken from different parts of the melt sample, showed that the mean Pu content in the glass was $9.95 \pm 0.05 \mathrm{mg} / \mathrm{g}$.

Density was $3.58 \pm 0.03 \mathrm{~g} / \mathrm{cm}^{3}$.

Strength was determined according to the standard GOST 25.503-80 on tearing machine IR5113-100 with reverse. There was no plastic deformation of the samples at all and they were loaded firmly up to total destruction. Temporal resistance at compression $\left(\sigma_{t}{ }^{\text {comp }}\right)$ was $3,53 \pm 0,11 \mathrm{~kg} / \mathrm{mm}^{2}$.

Chemical stability was determined by an IAEA method using mass-spectrometer PLAZMA-PQ or atomic-emission spectrometer PERKIN-ELMER with inductively connected plasma to analyze aqueous extract. Average speed of leaching on the sum of all 19 elements was equal to $(0,75 \pm 0,25) \times 10^{-10}$ $\mathrm{g} / \mathrm{cm}^{2} \times$ day, and that the oxides of $\mathrm{Fe}, \mathrm{Ca}, \mathrm{Al}, \mathrm{Mn}, \mathrm{Ni}, \mathrm{Ti}, \mathrm{Ce}, \mathrm{Zr}, \mathrm{La}, \mathrm{Y}, \mathrm{Nd}$ and $\mathrm{Pu}$ have the lowest leachability $\left(<0,1 \times 10^{-10} \mathrm{~g} / \mathrm{cm}^{2} \times\right.$ day $)$.

Thus, the technology developed at "Mayak" PA, for immobilization of alpha-active wastes with incorporation of $65-67 \%$ of SRW into ceramic matrix, and the physical, chemical, and mineralogical characteristics we obtained, allow us to certify the end product and to prepare a feasibility study for the design of an industrial facility with an output capacity of 50-60 tons of melt per year.

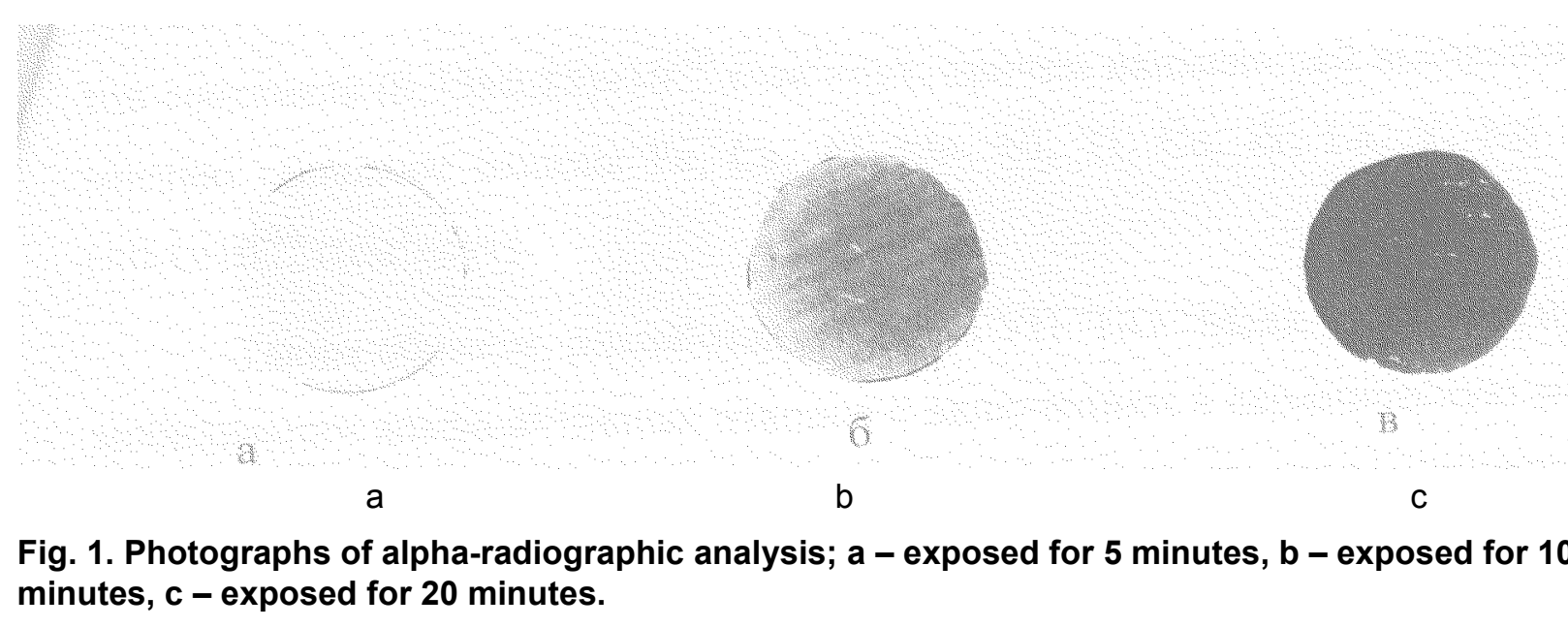

minutes, c - exposed for 20 minutes. 


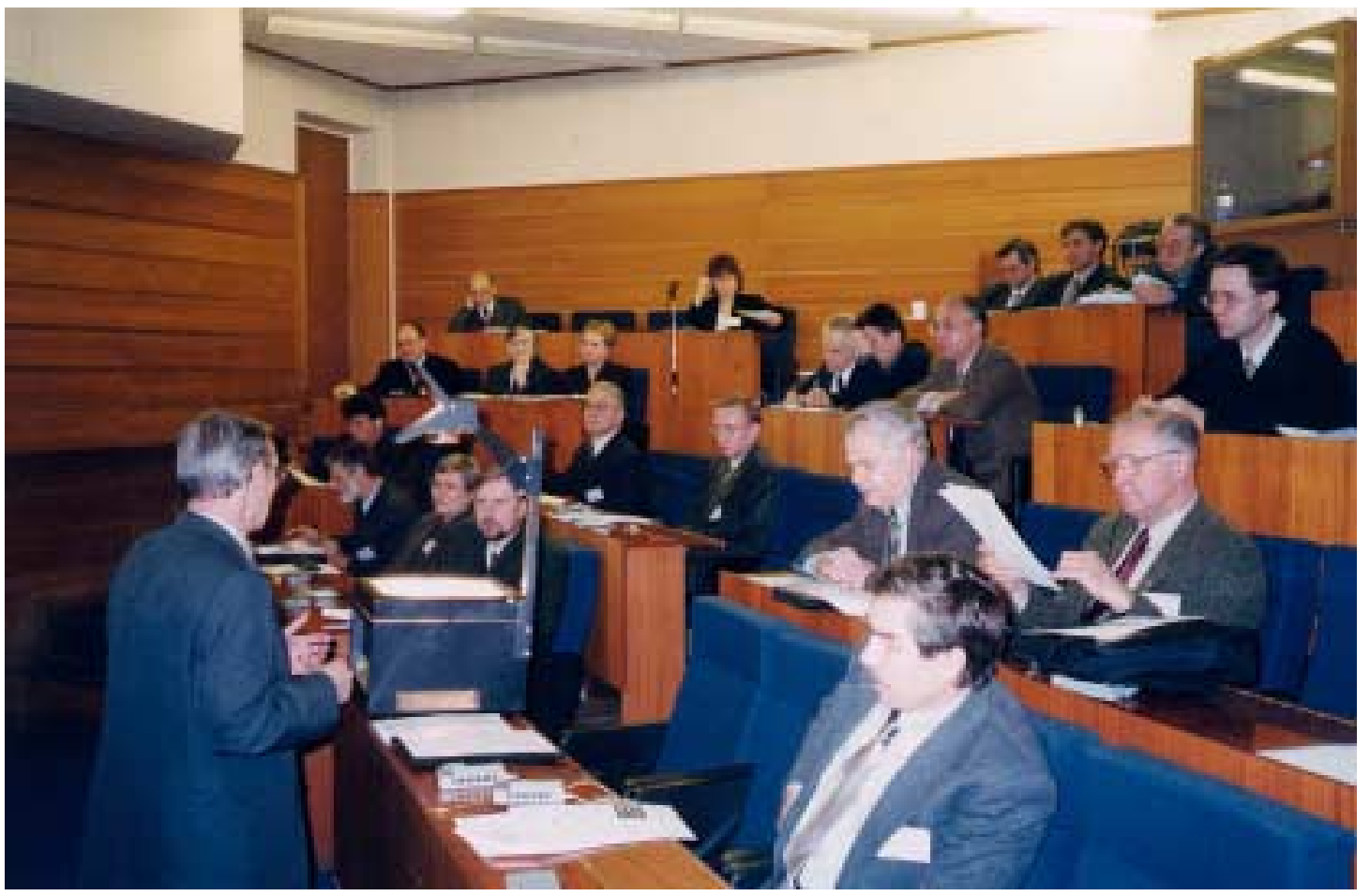




\title{
Immobilization of Pu-containing Sludge Using Ceramic Melters: Experiments in Support of the EFS at PA "Mayak" (B506206)
}

\author{
G.B. Borisov, O.A. Mansourov, A.V. Nazarov, A.V. Balashov, Y.Y. Volcho, VNIINM
}

\begin{abstract}
Management of the plutonium-containing sludge generated as a result of past defense nuclear programs at MINATOM industrial sites is an essential and technologically complicated problem. At present at PA "Mayak", efforts are close to complete in the engineering and feasibility study (EFS) of immobilizing Pucontaining wastes accumulated in Building 954. Various technologic approaches are under assessment, and comparative economic calculations of the effectiveness of using these technologies are being implemented. In support of the EFS at A. A. Bochvar All-Russia Scientific Research Institute of Inorganic Materials (VNIINM) under the scope of Agreement B506206, work was conducted to substantiate or test the possibility of successful operation of EP-type Joule-heated ceramic melters for vitrifying high level wastes. This article conceptually presents a concise look at the application of the mentioned facilities and presents the experimental results obtained.
\end{abstract}

\section{Analysis of Facilities for Immobilization of Liquid and Sludge-type Wastes}

The most widely used facilities for the processing of high-level wastes are described in Fig. 1.

Chronologically, the processing of high-level wastes first employed periodic-type facilities, where wastes are vitrified directly in the containers used for disposal. External electrical resistance elements of internal (microwave heating) heaters were used for solidifying the wastes. Notwithstanding the compactness and simplicity of the this process where evaporation, calcination, and glass melting gecurred simultaneously in

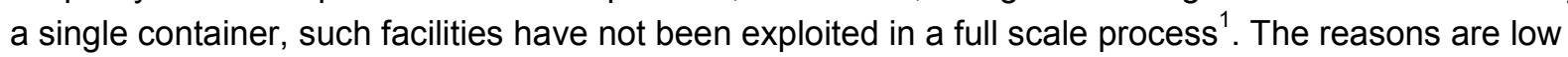
efficiency of the units and the appallingly bad quality of the immobilized form due to its crystallization while cooling.

The second type of facility, put into operation in France in 1978, is operational using medium- and highfrequency induction heating. The two-stage facility integrated a turntable calcination furnace and a medium-frequency induction-type crucible, melting the wastes calcinated in a glass. A substantial disadvantage of the facility is that the crucible has a limited service life that does not exceed a few months. To eliminate this drawback, a high-frequency "cold-crucible" induction melter has been developed. Due to skull present on the crucible's walls, this melter's service life is much longer. In Russia, a pilot cold crucible induction melter (CCIM) facility equipped with a direct-flow evaporator has been operating in test mode for a few years.

\footnotetext{
${ }^{1}$ Mataloni, P., Scoditti, E.. et al. "Laboratory and pilot plant studies for the solidification of aluminum rich HLW produced by EUREX PLANT." Proceedings of the international conference, Nuclear Fuel Reprocessing and Waste Management, France, 1987.

${ }^{2}$ Dyomine, A.V., Poluektov, P.P., Smelova, T.V., Gorn, V.F. et al. "The use of induction melting in cold crucible for the solidification of HLW." Proceedings of the international conference MRS-2000, Australia, 2000.
} 


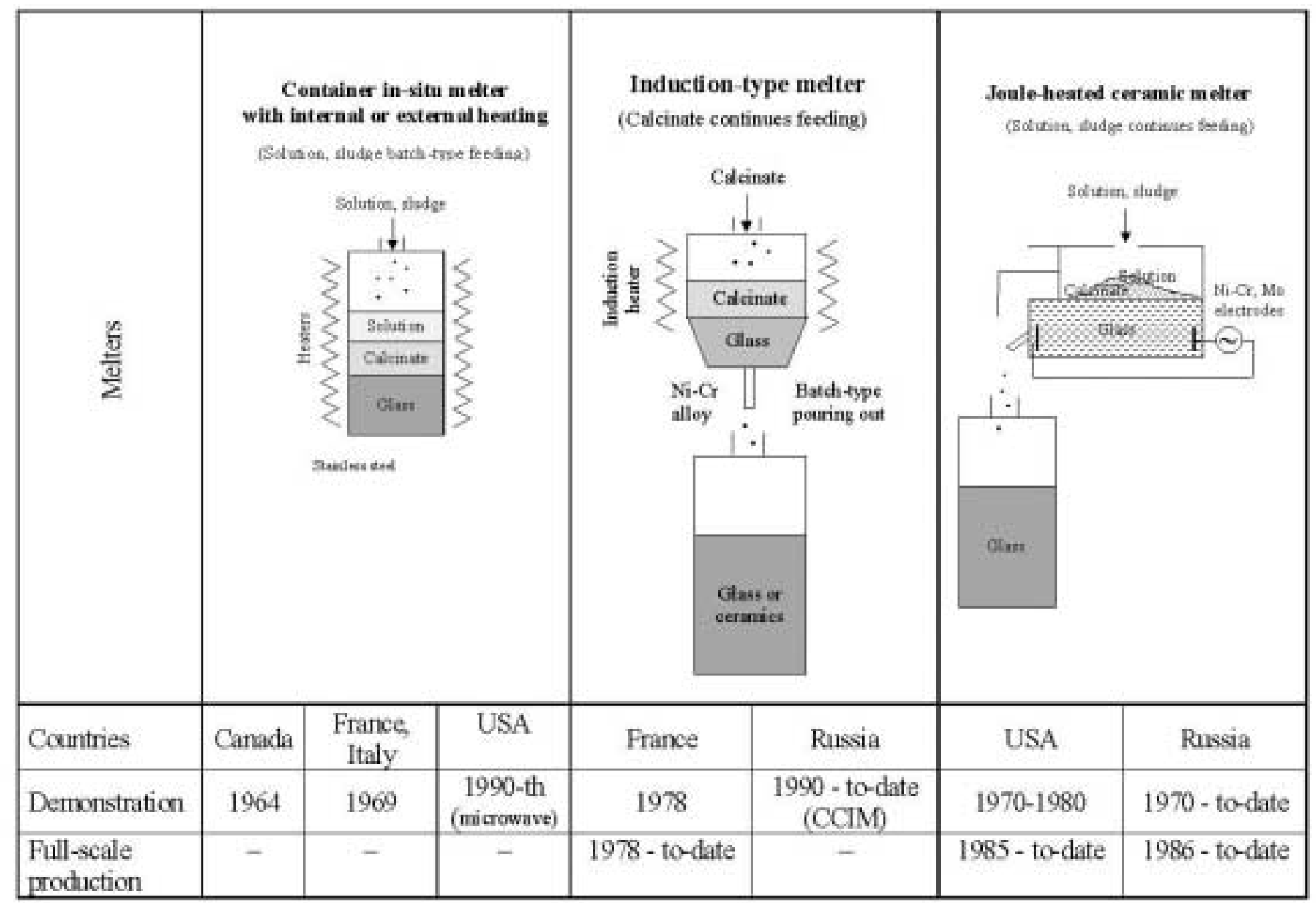

Fig. 1. Melters for the immobilization of liquid high-level wastes. 
The most common facilities for processing high-level waste are Joule-heated ceramic melters. Since 1986, two full-scale ceramic melters at PA "Mayak" have been exploited, one of which is of $500 \mathrm{l} / \mathrm{h}$ output.

In the US at the Savanna-River industrial site, a full-scale ceramic melter of $170 \mathrm{l} / \mathrm{h}$ output was developed. The units have a series of merits, including higher efficiency and operational life of equipment, as well as simplicity of the vitrification process. For instance, the EP-500 Russian ceramic melter was in operation for 6 years. During that timespan, more than $12.000 \mathrm{~m}^{3}$ of high-level wastes were vitrified, and about 2200 tons of a phosphate glass with a total activity of 300 million Ci were obtained.

Under the framework of the excess weapons-grade plutonium disposition program, about $20,000 \mathrm{~m}^{3}$ of high-level Pu-containing sludge at MCC and PA "Mayak" are subject to immobilization in Russia. From the economical and technologic viewpoints, it would be reasonable to immobilize them using one integrated facility, namely in ceramic melters EP-100 or EP-500, which are well developed and functioning on an industrial scale.

Previously, VNIINM experimentally justified the option of immobilizing the Pu-containing sludge at MCC using the EP-100 ceramic melter. Based on PA "Mayak" technical data, the process of immobilizing Pucontaining sludge of building 954 in the ceramic melters EP-100 and EP-500 are presented beneath.

The sludge contains the following major components in $\mathrm{g} / \mathrm{l}$ (averaged values):

$$
\mathrm{Al} \text { - 100; Fe-12; Ni - 10.5; } \mathrm{Cr}-4.3 ; \mathrm{Na}-75 ; \mathrm{S}-5 ; \mathrm{Pu}-0.15^{\mathrm{W}}
$$

The concentration of corrosion-aggressive impurities in the sludge of PA "Mayak" is higher than the respective concentrations in the MCC wastes. This makes some impact on the kind of melter to select for immobilization. In the course of research and exploitation of the EP-500/1p facility, it has been established that if one takes into account the ratio between corrosion-aggressive components in the waste fed for vitrification, it is possible to reduce the corrosion of Mo-electrodes in molten phosphate glass. The corrosion rate of the electrodes is essentially lower when the waste contains as little as possible iron and sulfur. Since aluminum is very concentrated in the PA "Mayak" wastes, it is complicated to immobilize them into borosilicate glasses capable of including as much as $3 \mathrm{wt} \%$ of $\mathrm{Al}_{2} \mathrm{O}_{3}$. Due to a polymorphous carcass-like structure and low viscosity of the melt, the phosphate glasses are prone to dissolve a vast spectrum of waste components at up to $1000^{\circ} \mathrm{C}$. In other words, the phosphate glasses are very active chemically and therefore their use requires that the Mo-electrodes should be protected. In the ceramic melters EP-100 and EP-500, the phosphate glass can incorporate at least $25-27 \mathrm{wt} \%$ of $\mathrm{Al}_{2} \mathrm{O}_{3}$. Thus, using the phosphate glass would reduce the number of containers with immobilized waste sent for eventual disposal by 9-10 times in contrast to the borosilicate glass.

While assessing the option of immobilizing the Pu-containing sludge using the EP-type ceramic melter, the stage of partitioning the sludge has been disregarded for several reasons. First, there is no reliable method of solidifying the concentrate because of Cs-137 fugacity. Second, the Cs batches would need to be intensively cooled in a water pond due to their thermal emission.

According to one of the immobilization options proposed by VNIINM, the sludge is delivered for vitrification without preliminary partitioning. Two processes are under development, including dissolution

\footnotetext{
${ }^{3}$ Borisov, G.B., Moiseenko, N.I., Dzekun, V.I., Polyakov, A.S., Medvedev, G.M., et al. "The experience of exploiting ceramic melter EP-500/1p for vitrifying liquid HLW." Atomic Energy, vol 76, issue 3, 1994.

4 PA "Mayak" initial data for the engineering and feasibility study of immobilizing Pu-containing sludge, 2001.
} 
of the sludge in a mixture of $\mathrm{H}_{3} \mathrm{PO}_{4}, \mathrm{HNO}_{3}$ and its vitrification in the EP-100 melter (Fig. 2) or direct immobilization of the sludge in the EP-500 melter (Fig. 3).

A general problem of all the Russian vitrification facilities is the absence of reliable metering or feeding systems. To feed the Pu-containing sludge into the melter, an apparatus is being developed at VNIINM. It is designed as a closed vessel equipped with systems of pulsation blending and automatic metering.

If a procedure with preliminary dissolution of the sludge prior to its vitrification is accepted, the metering system will be used for blending the solutions. A blending unit consists of a chamber connected with a tank via jets. Liquid is oscillated in the chamber by means of impulse-type feeding and air blow-off. The sludge is fed through the lower hydraulic seal using a cascade-type adjusting system.

A follower regulator stabilizes the pressure inside the vessel, and a leader regulator stabilizes the pressure in the tank linearly. The accuracy of metering is defined by the level measuring unit precision and is within $\pm 2 \%$. The system is composed of serially industrial manufactured units.

\section{Synthesis and Research of Simulated Phosphate and Ultraphosphate Glasses}

To substantiate the feasibility of using EP-type ceramic melters, a number of experiments have been carried out. Phosphate and ultraphosphate glasses of various compositions have been synthesized from simulated solutions (Table 1). A minimum chemical reaction capability providing an acceptable corrosion rate of the Mo-electrodes in molten glass was a criterion while selecting the immobilization matrix. A weight ratio between the sludge and a glass forming agent (orthophosphor acid) has been chosen that minimizes the iron and sulfur in the glass that is produced.

The glasses synthesized were characterized, in particular leaching and the corrosion rate of the electrodes in relevant molten glasses. In addition, crystallization ability and viscosity were also determined.

The viscosity of the glasses was measured by means of a vibration procedure.

The leaching capability of the glasses was identified according to the IAEA procedure, i.e., by means of measuring the concentration of $\mathrm{Na}$ ions in a leachate at room temperature. Results of the experiments are summarized in Table 1.

The crystallization ability of the glasses was defined according to the Glass State Institution's procedure. In essential, glassy samples are placed in a furnace onto special trays and then heated up under different temperatures. The samples are usually kept during 1-2 hours. After taking out from the furnace, the glasses are cooled at room temperature and then observed. The crystallization rate is evaluated by a sixscore scale: 1 - the absence of crystallization; 2 - surface crystallization in the form of a spattered film; 3 - surface crystallization in the form of an uniform thin film; 4 - surface crystallization in the form of an uniform thick film; 5 - partial bulky crystallization (40-60 \%); 6 - full crystallization (60-100 \%).

Research results of the crystallization ability are given in Table 1.

\footnotetext{
${ }^{5}$ Progress report 1. Task 1, stage 1. Agreement B506206. VNIINM. 2001.

${ }^{6}$ Sarkisov P.D., Agarkov A.S. The technical analysis and control of glass fabrication. M.: Stroyizdat. 1976.
} 
"A proposed scheme of the vitrification of Pu-containing sludge at PA "Mayak" into phosphate and ultraphosphate glass es using the EP-100 ceramic melter"

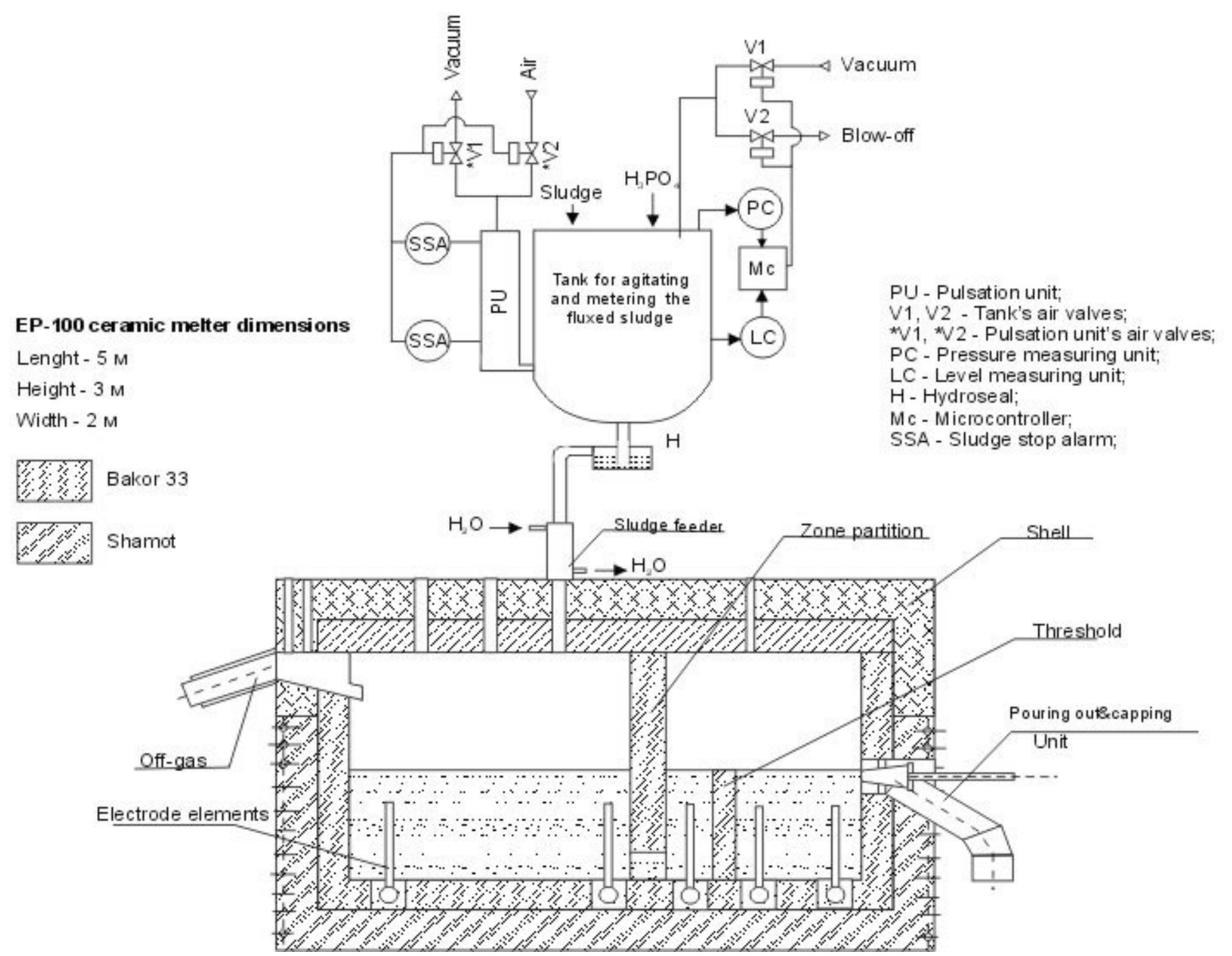

Fig. 2. A proposed scheme of vitrification of Pu-containing sludge at PA "Mayak" into phosphate and ultraphosphate glasses using the EP-100 ceramic melter. 
"A proposed scheme of the vitrification of Pu-containing sludge at PA "Mayak" into phosphate glass es using the EP-500 ceramic melter"

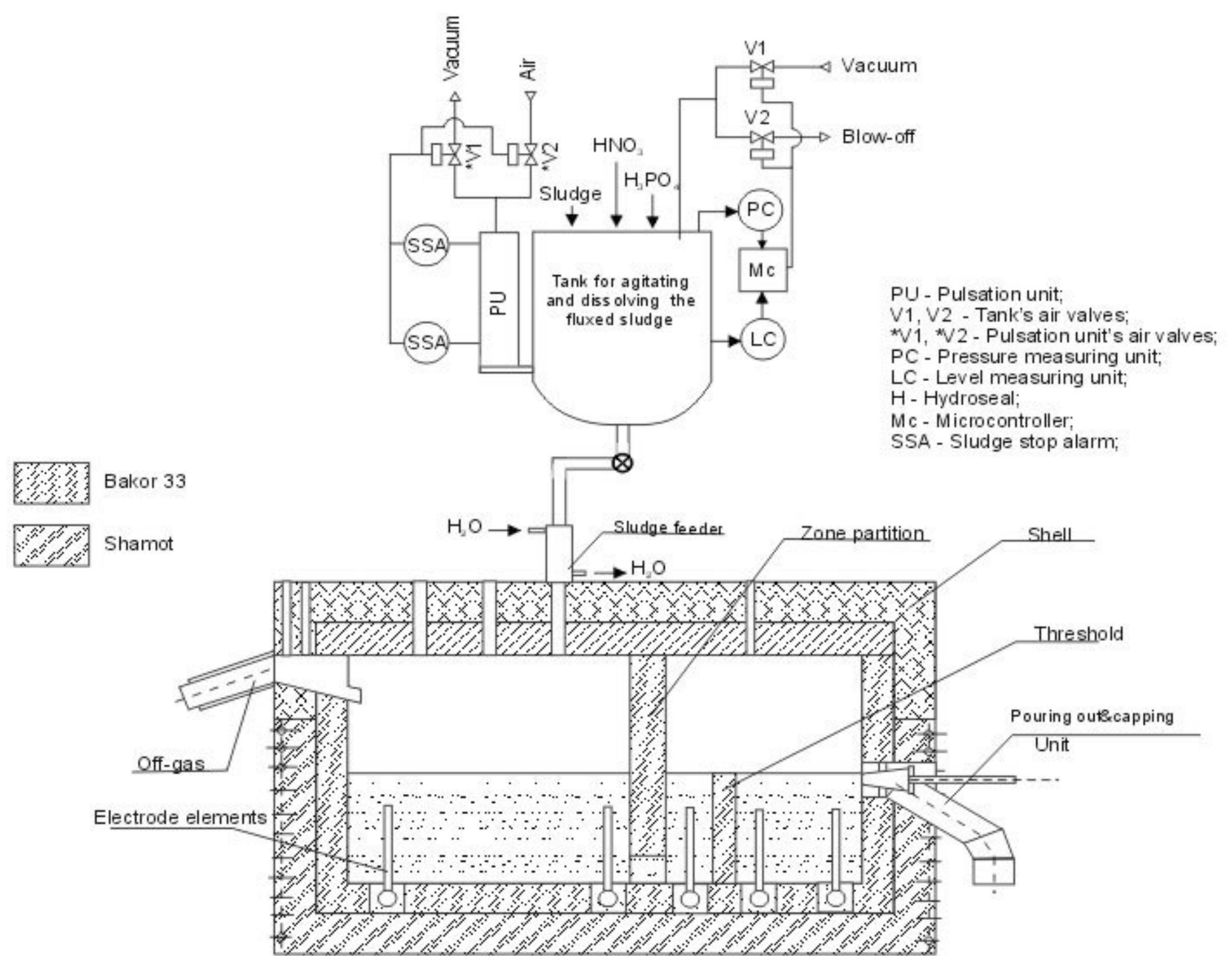

Fig. 3. A proposed scheme of vitrification of Pu-containing sludge at PA "Mayak" into phosphate glass using the EP-500 ceramic melter. 
Table 1. Compositions and basic properties of the synthesized phosphate and ultraphosphate glasses.

\begin{tabular}{|c|c|c|c|c|}
\hline \multirow{2}{*}{$\begin{array}{c}\text { Glass components and } \\
\text { parameters }\end{array}$} & \multicolumn{4}{|c|}{ Calculated glass compositions and properties, wt $\%$} \\
\hline & $\begin{array}{c}\text { Phosphate glass } \\
1\end{array}$ & Phosphate glass 2 & $\begin{array}{l}\text { Ultraphosphate } \\
\text { glass }\end{array}$ & $\begin{array}{l}\text { Borophosphate } \\
\text { glass }\end{array}$ \\
\hline $\mathrm{Na}_{2} \mathrm{O}$ & 26.0 & 25.6 & 26.0 & 26.0 \\
\hline $\mathrm{Al}_{2} \mathrm{O}_{3}$ & 16.4 & 14.0 & 9.90 & 16.0 \\
\hline $\mathrm{Fe}_{2} \mathrm{O}_{3}$ & 1.5 & 4.5 & 0.90 & 1.5 \\
\hline $\mathrm{NiO}$ & 1.16 & 1.7 & 0.70 & 1.2 \\
\hline $\mathrm{SO}_{4}^{2-}$ & 1.43 & 1.25 & 0.86 & 1.2 \\
\hline $\mathrm{P}_{2} \mathrm{O}_{5}$ & 53.51 & 48.85 & 61.64 & 44.0 \\
\hline $\mathrm{CaO}$ & & 0.7 & & 0.7 \\
\hline $\mathrm{U}_{3} \mathrm{O}_{8}$ & & 2.0 & & 2.0 \\
\hline $\mathrm{La}_{2} \mathrm{O}_{3}$ & & 0.5 & & 0.5 \\
\hline $\mathrm{Ce}_{2} \mathrm{O}_{3}$ & & 0.5 & & 0.5 \\
\hline $\mathrm{Cr}_{2} \mathrm{O}_{3}$ & & 0.4 & & 0.4 \\
\hline $\mathrm{B}_{2} \mathrm{O}_{3}$ & & & & 3.0 \\
\hline $\mathrm{Na} / \mathrm{P}$ molar ratio & 1.11 & 1.20 & 0.97 & 1.01 \\
\hline $\begin{array}{l}\text { Glass melting temperature, } \\
{ }^{0} \mathrm{C}\end{array}$ & $950-1000$ & $930-980$ & $900-950$ & $840-870$ \\
\hline $\begin{array}{l}\text { Glass viscosity, ps } \\
1000^{\circ} \mathrm{C} \\
950^{\circ} \mathrm{C} \\
900^{\circ} \mathrm{C} \\
850^{\circ} \mathrm{C} \\
800^{\circ} \mathrm{C} \\
750^{\circ} \mathrm{C} \\
\end{array}$ & $\begin{array}{r}10 \\
7 \\
11 \\
13 \\
22 \\
44 \\
\end{array}$ & $\begin{array}{r}6 \\
9 \\
13 \\
24 \\
34 \\
49 \\
\end{array}$ & $\begin{array}{r}- \\
6 \\
11 \\
16 \\
21 \\
38 \\
\end{array}$ & $\begin{array}{r}- \\
- \\
- \\
5 \\
11 \\
17\end{array}$ \\
\hline $\begin{array}{l}\text { Crystallization ability, scores } \\
950^{\circ} \mathrm{C} \\
900^{\circ} \mathrm{C} \\
800^{\circ} \mathrm{C} \\
700^{\circ} \mathrm{C} \\
600^{\circ} \mathrm{C} \\
500^{\circ} \mathrm{C}\end{array}$ & $\begin{array}{l}1 \\
1 \\
1 \\
1 \\
1 \\
1\end{array}$ & $\begin{array}{l}1 \\
1 \\
1 \\
1 \\
1 \\
1\end{array}$ & $\begin{array}{l}1 \\
2 \\
3 \\
4 \\
5 \\
-\end{array}$ & $\begin{array}{l}- \\
- \\
1 \\
1 \\
1 \\
1\end{array}$ \\
\hline Leaching, $\mathrm{g} / \mathrm{cm}^{2} \times$ day & $1.6^{*} 10^{-5}$ & $2.1 * 10^{-5}$ & $6.0^{*} 10^{-5}$ & $1.3^{*} 10^{-6}$ \\
\hline $\begin{array}{l}\text { The corrosion rate of Mo- } \\
\text { electrodes, mm/year }\end{array}$ & 16.6 & 64.0 & 7.6 & 7.0 \\
\hline
\end{tabular}

Taking into account that one of the decisive issues of using the ceramic melters for the immobilization of Pu-containing sludge is the corrosion resistance of construction materials, a few experiments have been implemented to determine the corrosion rate of the Mo-electrodes in molten phosphate glass. The experimental temperature was as high as $1000^{\circ} \mathrm{C}$ and glassy samples were kept within 100 hours.

The corrosion rate was established by two methods: calculating the weight loss of the Mo-electrodes before and after the experiments, and using an atomic-adsorption method of determining the content of Mo in molten glass.

In the first case, the calculations were made in accordance with the following equation: 


$$
C R=K /\left(\rho_{M 0} \times \tau\right) \times \Delta m / S
$$

where

$$
\begin{aligned}
& K=8.76 \times 10^{4}-\text { a coefficient of transferring the rate per year; } \\
& \tau \text { - time; } \\
& \Delta \mathrm{m} \text { - weight loss of Mo batch; } \\
& \mathrm{S} \text { - surface area of the batch; } \\
& \rho_{\text {Mo }}-\text { Mo density. }
\end{aligned}
$$

Results of the calculations completely correspond with ones obtained by the atomic-adsorption method.

Earlier the corrosion tests had been carried out in alundum crucibles. But during long-term exposure, the crucible $\mathrm{Al}_{2} \mathrm{O}_{3}$ began to dissolve and move into the molten glass, and thus considerably misrepresents the experimental results. To exclude the effect of the dissolving aluminum oxide on the corrosion rate, molten quartz, which is a very stable material versus the molten phosphate glass, has been used for tests (Figs. 4 and 5).

Based upon the PA "Mayak" engineering and feasibility study initial data, nitric solutions in a range of 3.6$5.9 \mathrm{~m}^{3}$ and nitric-phosphor solutions of $0.8-3.4 \mathrm{~m}^{3}$ (in total $-6.1-14.9 \mathrm{~m}^{3}$ ) are generated in the process of vitrifying $1 \mathrm{~m}^{3}$ feeding sludge. According to the procedure proposed by VNIINM, only from 2.67 to $5.25 \mathrm{~m}^{3}$ waste will be produced, i.e. about 0.36 to $0.73 \mathrm{~m}^{3}$ of phosphate glass. The overall bulk of the phosphate glass will be 2666,3200 , and $5300 \mathrm{~m}^{3}$, respectively.

In Russia, the liquid high-level wastes are immobilized in phosphate glasses at a relatively low temperature of $870-1000^{\circ} \mathrm{C}$. As it follows from Table 1, there is a dependency between the amount of the glass-former fed into the sludge and the corrosion rate of the Mo-electrodes in the molten glass.

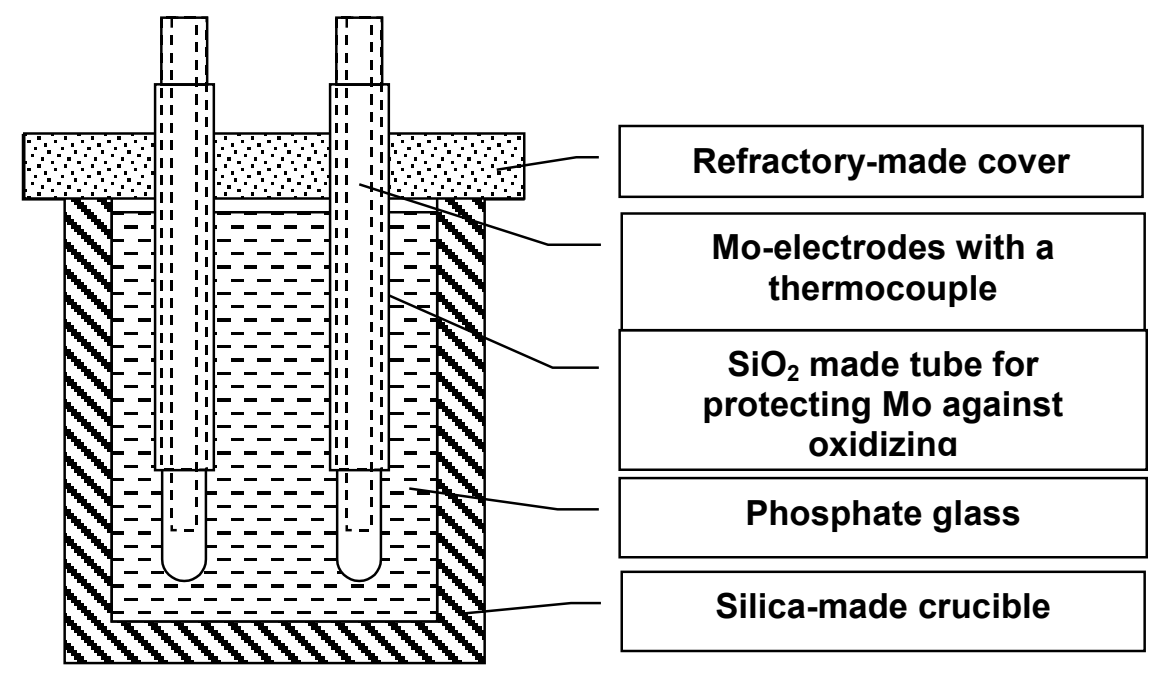

Fig. 4. Experimental cell for measuring the corrosion rate of Mo-electrodes in molten phosphate glass. 


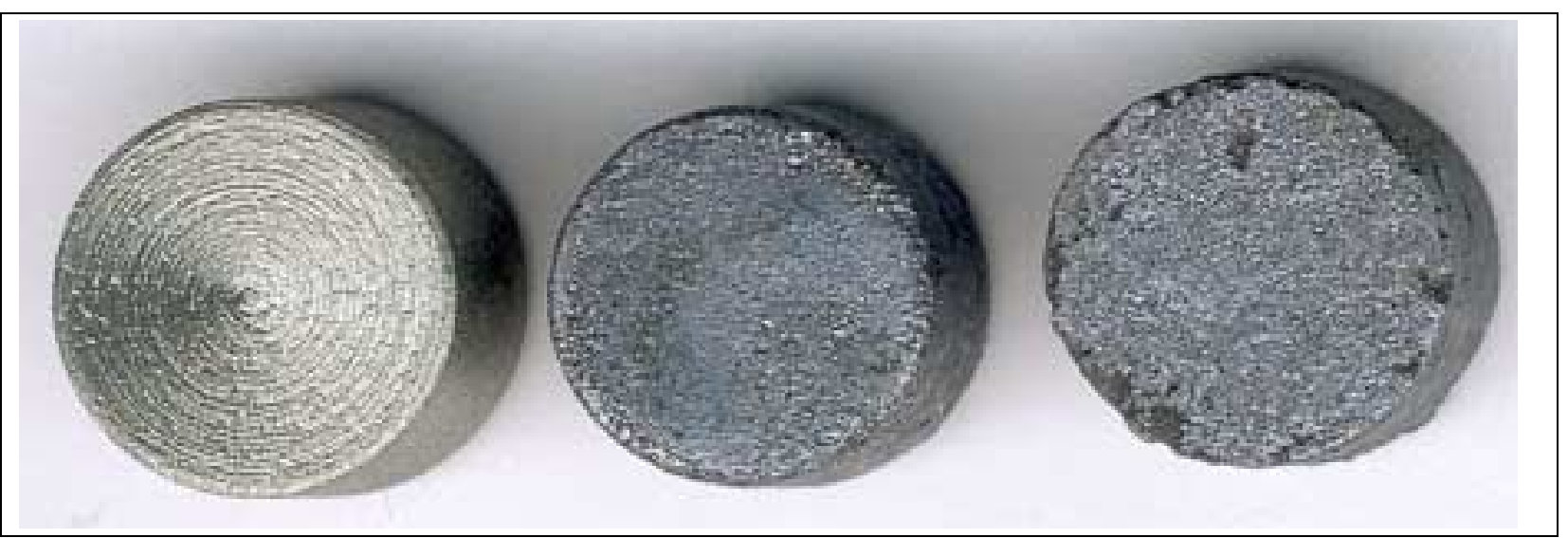

Fig. 5. Images of the Mo batches prior to (1) and after corrosion tests in a phosphate (2) and ultraphosphate (3) glass.

When dissolving the sludge by orthophosphate acid, the concentrations of iron and sulfate ions, which are major corrosion-aggressive impurities, are lower. The lowest corrosion rate of the Mo-electrodes is in the molten glass, where the concentrations of $\mathrm{Fe}_{2} \mathrm{O}_{3}$ and sulfates are within 0.9-1.5 wt\%, and 0.86-1.43 wt\%, respectively. One needs to refer to that though the concentration of sulfates in the glass is higher than the one in the EP-500 operational regime (0.5-0.6 wt\%), due to a decrease in the content of $\mathrm{Fe}_{2} \mathrm{O}_{3}$, the corrosion rate of the Mo batches does not exceed 8-17 mm/year. The corrosion rate of the Mo-batches in glass \# 2 is dramatically high, reaching $64 \mathrm{~mm} /$ year. At such a rate, the Mo-electrodes $60-80 \mathrm{~mm}$ in diameter will completely corrode in a year.

As it is obvious from the table, thanks to lower melting temperature, in the ultraphosphate glass the corrosion rate of Mo-electrodes does not exceed the value of $8 \mathrm{~mm} /$ year. However, the glass is apt to crystallize. Near the devitrification temperature, the crystallization rate of the glass is almost 5 magnitudes greater, which by all means would increase the leaching of major components from the material in the disposal. Therefore, they have been excluded from our eventual research activities.

In addition, borophosphate glasses containing up to $3 w t \% \mathrm{~B}_{2} \mathrm{O}_{3}$ were under investigation. The technology of melting the glasses from solutions has been widely tested in melter EP-40. Physicochemical properties of the glasses were characterized (Table 1). Due to reducing the melting temperature to $870^{\circ} \mathrm{C}$, the corrosion rate of the Mo-electrodes goes down to $7 \mathrm{~mm} /$ year. Adding $\mathrm{B}_{2} \mathrm{O}_{3}$ would eliminate crystallization of the glass during its cooling.

In comparison to borosilicate glasses, the phosphate glasses can incorporate in 3 to 5 times more aluminum oxide (up to $10-16 \mathrm{wt} \%$ ).

Therefore, for immobilizing the Pu-containing sludge using the ceramic melters EP-100 and EP-500, the phosphate glass has been selected and synthesized as follows, \%wt:

$\begin{array}{llll} & & \mathrm{NiO} & 0.7-1.16 \\ \mathrm{Na}_{2} \mathrm{O} & 26.0 & \mathrm{SO}_{4}{ }^{2-} & 0.86-1.43 \\ \mathrm{Al}_{2} \mathrm{O}_{3} & 9.9-16.4 & \mathrm{P}_{2} \mathrm{O}_{5} & 53.51-61.4 \\ \mathrm{Fe}_{2} \mathrm{O}_{3} & 0.9-1.5 & \mathrm{~B}_{2} \mathrm{O}_{3} & 0-3.0\end{array}$

The concentration of $\mathrm{Na}_{2} \mathrm{O}$ might change in a range of $25-27 \mathrm{wt} \%$. Since the amount of $\mathrm{Fe}_{2} \mathrm{O}_{3}$ is less than usual, the glasses developed are not so aggressively corrosive against the Mo-electrodes. 
The phosphate glass has low viscosity in a range of $800-1000^{\circ} \mathrm{C}(22-10 \mathrm{ps})$ and does not crystallize.

One can conclude that challenging data regarding the option of the immobilization of the Pu-sludge in building 954 using the EP-type ceramic melters have been obtained.

\section{Conclusions}

1. The option of using melters, being developed and exploited, for the immobilization of liquid and sludge-type wastes has been assessed. It was shown that Joule-heated ceramic melters are preferable since they are well applied in a full-scale process (US, Russia), and have better outputs (up to $500 \mathrm{l} / \mathrm{h}$ ) and longer operational lives (3-6 years). Besides, the units make it possible to realize all vitrification processes in one stage.

2. Based on simulated experimental research, phosphate and borophosphate glasses have been developed. The compositions are fairly corrosive-gentle to melter Mo-electrodes while immobilizing the Pu-containing sludge. Besides, the glasses do not crystallize due to the presence of a boron additive. The research has shown that due to reducing the concentration of iron oxide in the molten phosphate glass and low melting temperature, the corrosion rate of the Mo-electrodes is within 7-17 $\mathrm{mm} /$ year. This permits us to consider that the melter will have a long-term service life, as long as 6 years. Major technologic and physico-chemical properties (melting temperature, viscosity, crystallization ability, leaching stability) for the phosphate and borophosphate glasses recommended for immobilizing the Pu-containing sludge have been defined. The temperature of synthesizing the glass $\left(900-1000^{\circ} \mathrm{C}\right)$ would allow us to apply equipment of EP-type melters, such as an off-gas treatment system, pouring out units, measuring gauges, etc.

3. Two options of immobilizing the Pu-containing sludge at PA "Mayak" without partitioning are under development:

- Vitrifying the sludge in the phosphate glass using the EP-100 ceramic melter;

- Vitrifying the sludge preliminarily dissolved in phosphate or ultraphosphate glasses using the EP-500 ceramic melter.

4. Work has started to develop the metering (feeding) system of the Pu-containing sludge immobilization using pulsation-type units. 


\title{
The KRI Approach for Developing Pu Immobilization Technology for PA Mayak (B506209)
}

\author{
A. S. Aloy, E. B. Anderson, I. V. Smirnov, B. E. Burakov, KRI, St. Petersburg
}

In accordance with the Statement of Work in B506209, the preliminary feasibility study of the developed processes for solidifying the plutonium-containing sludges from "Mayak" PA section 954 into vitreous or ceramic matrices was continued.

As to the first variant of this process, consideration is given to solidification of the whole sludge volume without any operations of fractionation in vitreous or glass-crystalline borosilicate matrices by means of microwave heating. According to this variant, the sludge, partly washed of soluble salts and mixed with borosilicate flux, is passed to solidification.

According to the second variant, it is proposed to conduct acidic dissolving of sludge. Filtrate upon dissolution is directed to selective extraction of cesium and strontium by cobalt dicarbollyde with subsequent oxalate precipitation of plutonium and rare-earth elements. Insoluble residues of sludge and concentrates of plutonium and rare-earths are solidified in monazite or garnet-perovskite ceramics.

According to the third variant, the sludge, partly washed-off of soluble salts, is solidified into garnetperovskite ceramics.

A comprehensive description of the work performed is presented below.

\section{Variant 2b. Direct Sludge Vitrification after Alkaline Treatment and Washing}

According to this variant (variant $2 \mathrm{~b}$, VNIPIET classification), the ultimated residue (UR) is formed after alkaline treatment of sludge in the tank to dissolve the main part of aluminium oxides and to demolish the dense layer. After settling and washing, this UR contains more than $95 \%$ of $\mathrm{Pu}$ and $\mathrm{Sr}$ as well practically all Fe and Ni. Calculated amount of whole UR, its composition and frit parameters, are shown in Table 1.

Glass-like frit in form of a small beads, which are easy to feed periodically into the melter, will be purchased from the glass industry. The calculated amount of borosilicate glass with density $2.65 \mathrm{~g} / \mathrm{cm}^{3}$ needed to vitrify the whole UR is equal to $4464 \mathrm{mt}$ or $1024 \mathrm{~m}^{3}$. The process is outlined in Fig. 1 .

The available equipment and new apparatuses needed to be developed were described earlier (task 3 , delivery 2).

\section{Variant 1. Partitioning of Dissolved Sludges with Subsequent Inclusion of $\mathrm{Pu}$, REE and An into a Ceramic Matrix}

This variant of solidifying Pu-containing sludges envisages their complete acidic dissolution. The filtrate upon dissolving is passed to selective extraction of $\mathrm{Cs}$ and $\mathrm{Sr}$ by cobalt dicarbollyde with subsequent precipitation of Pu and REE oxalates. Oxalate precipitate is dissolved and directed to solidification in monazite or garnet-perovskite ceramics.

All these operations have been already implemented at "Mayak" PA. Acidic dissolution of sludge completely conforms with the basic option. There are data on compositions and amounts of resultant products. Extraction technology of $\mathrm{Cs}$ and $\mathrm{Sr}$ recovery was checked on a pilot-industrial scale; material balances of this process are available. Operation for oxalate precipitation of $\mathrm{Pu}, \mathrm{An}$ and REE was realized 
Table 1. Amount of glass fabrication and UR composition.

\begin{tabular}{|c|c|c|}
\hline \multirow{2}{*}{ Component } & \multicolumn{2}{|c|}{ Content of components in vitrified waste } \\
\hline & Oxide mass, $\mathrm{mt}$. & Oxide content, wt $\%$ \\
\hline $\mathrm{Al}_{2} \mathrm{O}_{3}$ & 784.4 & 17.58 \\
\hline $\mathrm{Fe}_{2} \mathrm{O}_{3}$ & 117.3 & 2.63 \\
\hline $\mathrm{NiO}$ & 96.0 & 2.15 \\
\hline $\mathrm{Cr}_{2} \mathrm{O}_{3}$ & 21.9 & 0.49 \\
\hline $\mathrm{MnO}_{2}$ & 14.5 & 0.32 \\
\hline $\mathrm{SiO}_{2}$ & 14.1 & 0.31 \\
\hline $\mathrm{Na}_{2} \mathrm{O}$ & 235.0 & 5.27 \\
\hline $\mathrm{SO}_{3}$ & 45.0 & 1.00 \\
\hline $\mathrm{UO}_{2}$ & 9.13 & 0.21 \\
\hline $\mathrm{PuO}_{2}$ & 1.06 & 0.027 \\
\hline \multicolumn{3}{|c|}{ Frit } \\
\hline $\mathrm{SiO}_{2}$ & 1695.4 & 38 \\
\hline $\mathrm{B}_{2} \mathrm{O}_{3}$ & 624.6 & 14 \\
\hline $\mathrm{ZnO}$ & 245.4 & 5.5 \\
\hline $\mathrm{Na}_{2} \mathrm{O}$ & 557.7 & 12.5 \\
\hline Total glass mass & 4464 & \\
\hline
\end{tabular}

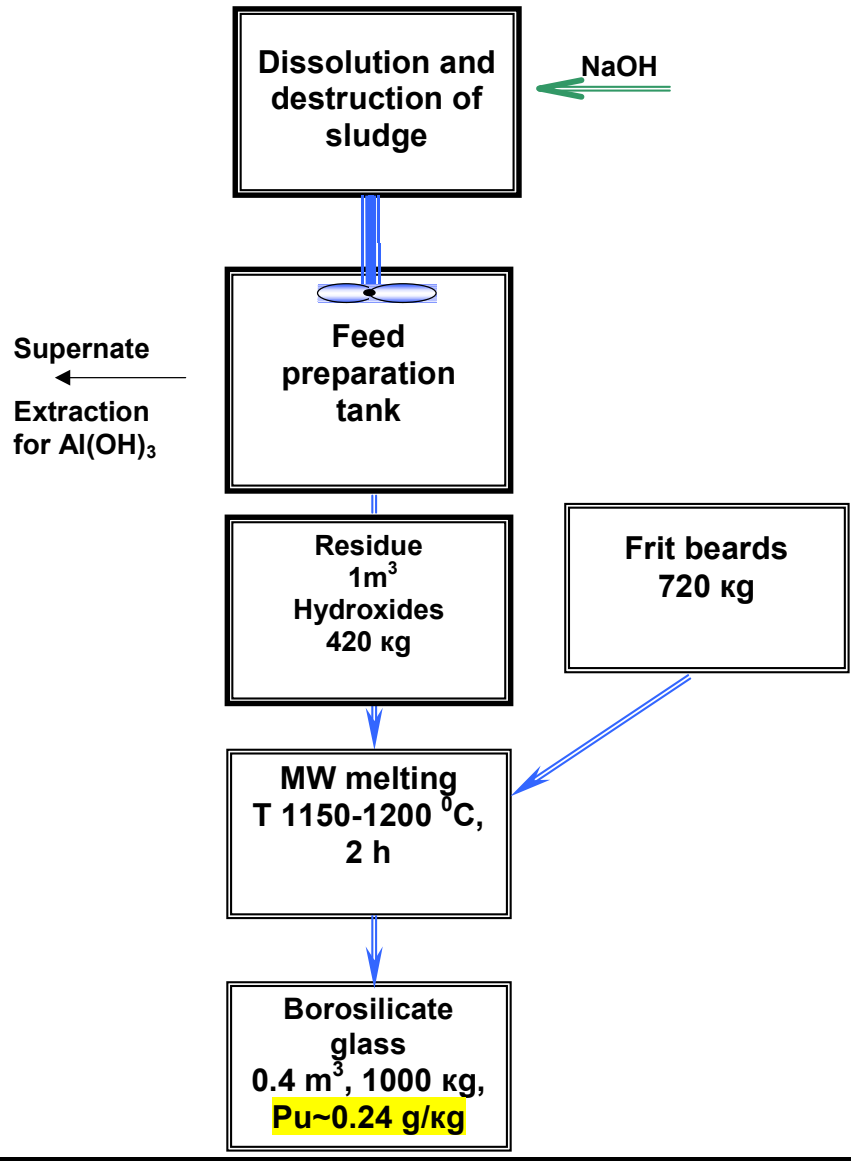

Fig. 1. Block-diagram for sludge reprocessing according to variant $2 \mathrm{~b}$. 
at "Mayak" PA on a pilot-industrial scale. The main indices of the process are known. The specialists of "Mayak" PA prepared "The initial data for feasibility study on immobilization of plutonium-containing waste storaged in building 954" [1] with a detailed description of the basic variant for sludge solidification.

Based on these data and the results of studies in accordance with tasks 1 and 2, we elaborated the key process flowsheet, determined the compositions and amounts of resulting products, prepared material balances and the data on plutonium and radioactivity distribution over process products, and the proposals about process equipment layout.

The proposed variant transfers combined $\mathrm{HNO}_{3}$ solution to a partitioning facility for $\mathrm{Cs}$ and $\mathrm{Sr}$ recovery. $\mathrm{Pu}, \mathrm{Am}$, and REE oxalates are precipitated from raffinate. Low-level decantate and oxalate precipitate are produced; upon dissolving, the latter is passed to solidification. As opposed to the basic option, $\mathrm{HNO}_{3}$ solution is purified of most of the radionuclides and transformed into category of low-level waste.

\section{Key flowsheet for reprocessing of precipitates from storage tanks of building 954 (Variant 1)}

On the basis of available data and results of conducted experiments, the following key flowsheet for waste reprocessing can be proposed:

1. Mechanical washout and delivery of sludge to apparati of section 51 ; precipitate settling in apparati, decanting of clarified phase and its re-use as process liquid for precipitate washout.

2. Alkaline treatment of precipitate on stirring and heating; precipitate settling, alkaline solution decanting.

3. Washing of precipitate by condensate on stirring and heating; precipitate settling, decanting of clarified phase, its combination with alkaline decantate and delivery of combined alkaline solutions to evaporation and subsequent vitrification in a furnace of EP-500 type.

4. Precipitate treatment by $\mathrm{HNO}_{3}$ on stirring and heating; precipitate settling; decanting $\mathrm{HNO}_{3}$ solution.

5. Washing of precipitate by condensate on stirring and heating; decanting of clarified phase; combination of decantates and delivery of combined $\mathrm{HNO}_{3}$ solutions to partitioning facility for $\mathrm{Cs}$ and Sr extraction recovery.

6. If needed, the final dissolution of precipitate in mixture of nitric and phosphoric acids and the transfer of the solutions to partitioning facility for $\mathrm{Cs}$ and $\mathrm{Sr}$ extraction recovery.

7. Partial neutralization of nitric acidic raffinate from partitioning facility, precipitation of Pu and REE oxalates, precipitate settling, decanting of clarified phase and delivery of low-level decantate.

8. Delivery of intermediate-level nitric-phosphoric acidic raffinate to evaporation and solidification in furnaces of mircowave or IPChT type and transfer of vitrified product to storage facility 120/12.

9. Dissolution of oxalate precipitates in nitric acid and delivery of plutonium concentrate to solidification in monazite or garnet-perowskite ceramics.

10. Solidification of Sr-Cs concentrates in accordance with "GROM" facility flowsheet to produce borosilicate glass, transportation of glass blocks to storage facility $120 / 2$.

Figure 2 presents the block-diagram for reprocessing of plutonium-containing sludges in accordance with Variant 1. Process operations and products other than in the basic option are shaded. 
The proposed variant is seen to differ from the basic one by provision of additional operations 7 and 9 and by some changes in operation 5 . Description of these operations is given below.

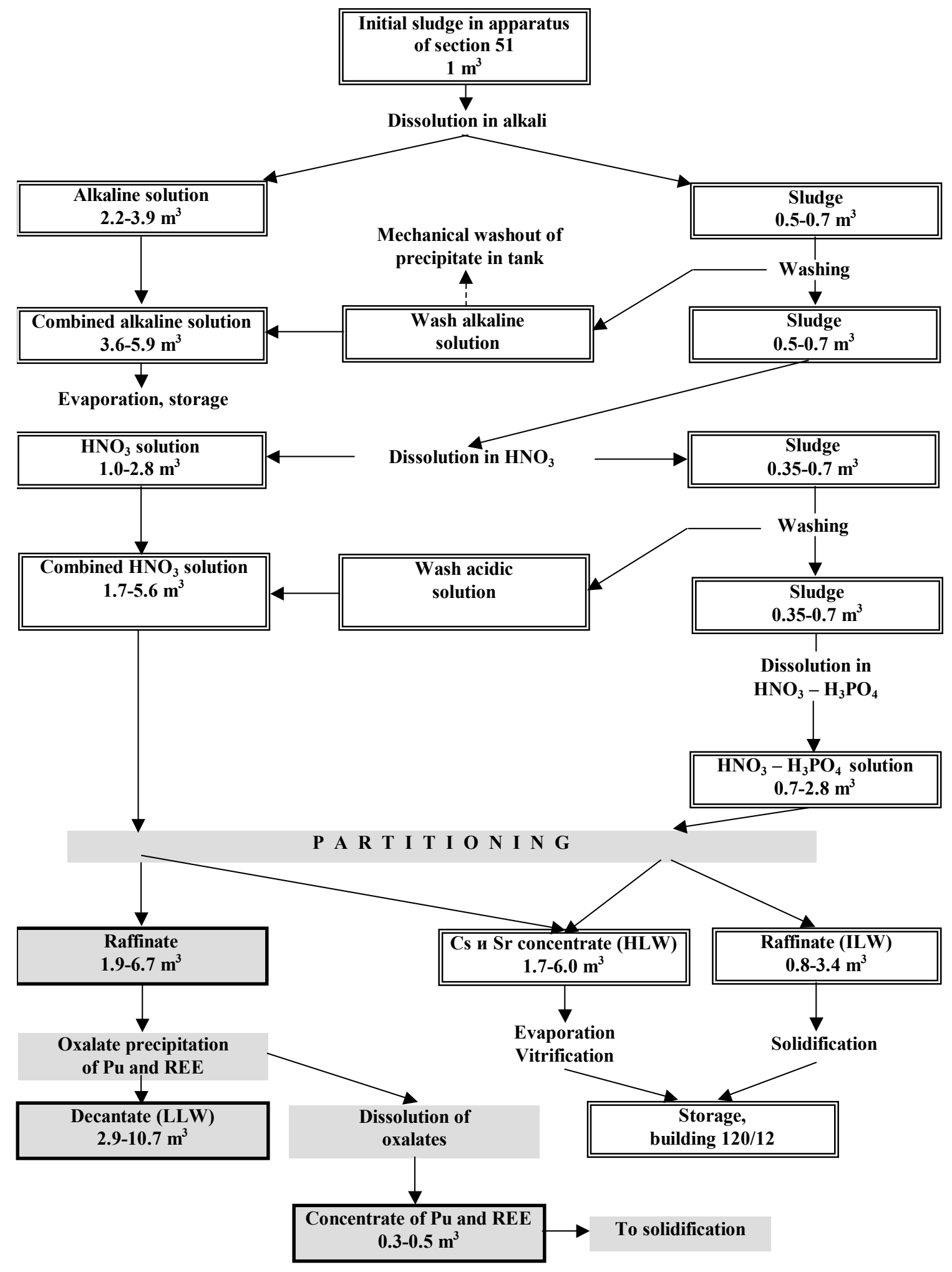

Fig. 2. Block-diagram of precipitate reprocessing by Variant 1. 


\section{Extraction recovery of $\mathrm{Cs}$ and $\mathrm{Sr}$ from combined $\mathrm{HNO}_{3}$ solutions at partitioning facility UE-35}

All acidic decantates and wash solutions are combined and directed for extraction recovery of $\mathrm{Cs}$ and $\mathrm{Sr}$ by the "Mayak" PA technology to the operating facility UE-35. The total volume of $\mathrm{HNO}_{3}$ solutions arising from reprocessing of $1 \mathrm{~m}^{3}$ sludge feed should be within $1.7 \mathrm{~m}^{3}-5.6 \mathrm{~m}^{3}$ (on the average $3.65 \mathrm{~m}^{3}$ ). The mean concentration of nitric acid varies in the range from 2 to $3 \mathrm{M} / \mathrm{l}$. The reference compositions of $\mathrm{HNO}_{3}$ solution directed to partitioning are presented in Table 5 of "Initial data".

Upon reprocessing of $\mathrm{HNO}_{3}$ solutions at UE-35 facility, the resulting raffinates $\left(1.9-6.7 \mathrm{~m}^{3}\right.$, on the average $-4.8 \mathrm{~m}^{3}$ per $1 \mathrm{~m}^{3}$ of sludge feed) are passed to oxalate precipitation of Pu and REE. The obtained Sr-Cs concentrates (1.2-4.0 $\mathrm{m}^{3}$, on the average $-2.7 \mathrm{~m}^{3}$ per $1 \mathrm{~m}^{3}$ of sludge feed) are delivered to evaporation and vitrification in accordance with the GROM facility flowsheet. The compositions of produced solutions are shown in Table 2.

When estimating the expenses on partitioning, one can be guided by "Initial data" (About 20 thousand roubles per $1 \mathrm{~m}^{3}$ of reprocessed waste). Partial neutralization of $\mathrm{HNO}_{3}$ raffinate from partitioning facility, precipitation of $\mathrm{Pu}$ and REE oxalates, precipitate settling, decanting of clarified phase.

The raffinates from UE-35 facility produced after $\mathrm{Cs}$ and Sr recovery should be neutralized up to 0.25-0.3 $\mathrm{M} \mathrm{HNO}_{3}$ for affording the complete precipitation of $\mathrm{Pu}$ and REE oxalates. The neutralizing operation is conducted by "Mayak" PA technology with the use of $14 \mathrm{M} / \mathrm{l}$ caustic soda solution. To neutralize $1 \mathrm{~m}^{3}$ of raffinate, about $150 \mathrm{I}$ of alkali are needed. Duration of the operation is 1-2 $\mathrm{h}$.

Upon the partial neutralization, the Pu and REE oxalates are precipitated in accordance with the "Mayak" PA technology by using $0.7 \mathrm{M} \mathrm{H}_{2} \mathrm{C}_{2} \mathrm{O}_{4}$. Due to the negligible precipitate volume, the precipitation process is carried out batch wise, delivering each new portion of feed solution to precipitate and mother liquor resulting from the preceding precipitation. To precipitate the oxalates from $1 \mathrm{~m}^{3}$ of raffinate, $470 \mathrm{I}$ of $\mathrm{H}_{2} \mathrm{C}_{2} \mathrm{O}_{4}$ solution are needed. After each batch the system is settled for $36 \mathrm{~h}$ and the solution is decanted. 20-30 I of oxalate precipitate are formed from $1 \mathrm{~m}^{3}$ of raffinate.

Table 2. Composition of concentrates and raffinates generated by partitioning technology.

\begin{tabular}{|c|c|c|c|}
\hline \multirow{2}{*}{ Component } & \multirow{2}{*}{ Unit of measurement, g/l } & \multicolumn{2}{|c|}{ Component content } \\
\hline & & Raffinate & Concentrate \\
\hline $\mathrm{Al}$ & $\mathrm{g} / \mathrm{l}$ & up to 8,7 & up to 0,05 \\
\hline $\mathrm{Fe}$ & $\mathrm{g} / \mathrm{l}$ & up to 1,5 & up to 0,15 \\
\hline $\mathrm{Cr}$ & $\mathrm{g} / \mathrm{l}$ & up to 0,5 & up to 0,05 \\
\hline $\mathrm{Ni}$ & $g / l$ & up to 1,0 & up to 0,05 \\
\hline $\mathrm{Mn}$ & $\mathrm{g} / \mathrm{l}$ & up to 1,7 & up to 0,01 \\
\hline $\mathrm{Na}$ & $\mathrm{g} / \mathrm{l}$ & up to 5,5 & up to 0,1 \\
\hline REE total & $\mathrm{g} / \mathrm{l}$ & up to 1,5 & up to 0,01 \\
\hline$U$ & $\mathrm{~g} / \mathrm{l}$ & up to 0,2 & up to 0,005 \\
\hline $\mathrm{Pu}$ & $\mathrm{mg} / \mathrm{l}$ & up to 40 & up to 0,01 \\
\hline$\alpha$-activity & $\mathrm{Bq} / \mathrm{l}$ & up to $5,7 \cdot 10^{8}$ & up to $1 \cdot 10^{5}$ \\
\hline Cs-137 & $\mathrm{Bq} / \mathrm{l}$ & up to $1 \cdot 10^{8}$ & up to $2,8 \cdot 10^{10}$ \\
\hline Sr-90 & $\mathrm{Bq} / \mathrm{l}$ & up to $0,6 \cdot 10^{8}$ & up to $1,4 \cdot 10^{10}$ \\
\hline$\beta$-activity & $\mathrm{Ci} / \mathrm{l}$ & up to 0,1 & up to 1,5 \\
\hline $\mathrm{HNO}_{3}$ & $g / l$ & up to 150 & up to 380 \\
\hline
\end{tabular}


Decantate volume therewith increases by a factor of 1.5-1.6 as compared with raffinate feed volume. The total volume of decantate arising from reprocessing of $1 \mathrm{~m}^{3}$ sludge feed should be within $2.9-10.7 \mathrm{~m}^{3}$ (on the average $7.5 \mathrm{~m}^{3}$ ). The reference composition of decantate is given in Table 3 .

According to the classification now in force in Russia, the decantate may be assigned for the most part to the category of low-level waste and thus be reprocessed by the technology accepted at "Mayak" PA for such waste. If the decantate after some operations of oxalate precipitation meets the requirements on intermediate level waste, it can be directed to solidification in furnaces of IPChT or EP-500 type by the "Mayak" PA technology.

\section{Dissolution of oxalate precipitation in $\mathrm{HNO}_{3}$ and delivery of $\mathrm{Pu}$-concentrate to solidification in monazine or garnet-perowskite ceramics}

Oxalate precipitates of each operation (6-8 batches) are dissolved in $\mathrm{HNO}_{3}$ on heating by live steam up to $93-95^{\circ} \mathrm{C}$. Around $6 \mathrm{~m}^{3}$ of $7 \mathrm{M} \mathrm{HNO}_{3}$ are delivered to $\sim 3,5 \mathrm{~m}^{3}$ of sludge precipitate. The mixture is heated on stirring for $\sim 6 \mathrm{~h}$ up to its complete dissolving. The total volume of Pu-REE concentrate arising from reprocessing of $1 \mathrm{~m}^{3}$ of sludge feed should be in the range of $0,3-0,5 \mathrm{~m}^{3}$ (on the average $0,4 \mathrm{~m}^{3}$ ). The concentrate composition is presented in Table 4.

The Pu-REE concentrate solution is directed to solidification in monazine or garnet-perowskite ceramics.

In Table 5, data are given on distribution of plutonium and radioactivity over products of sludge reprocessing flowsheet.

It is evident that the main amount of plutonium is separated from sludge in the course of dissolving in $\mathrm{HNO}_{3}$. Plutonium losses with $\mathrm{HNO}_{3}-\mathrm{H}_{3} \mathrm{PO}_{4}$ solution and decantate arising from oxalate precipitation do not exceed $10 \%$ of initial amount.

For realizing the variant of Pu-containing sludge reprocessing, it is expected to use only the equipment at "Mayak" PA.

Table 3. Composition of decantate arising from precipitation of $\mathrm{Pu}$ and REE oxalates.

\begin{tabular}{|l|l|l|}
\hline \multicolumn{1}{|c|}{ Component } & \multicolumn{1}{c|}{ Unit of measurement, $g / l$} & \multicolumn{1}{c|}{ Component content } \\
\hline $\mathrm{Al}$ & $\mathrm{g} / \mathrm{l}$ & up to 5,5 \\
\hline $\mathrm{Fe}$ & $\mathrm{g} / \mathrm{l}$ & up to 1,0 \\
\hline $\mathrm{Cr}$ & $\mathrm{g} / \mathrm{l}$ & up to 0,3 \\
\hline $\mathrm{Ni}$ & $\mathrm{g} / \mathrm{l}$ & up to 0,7 \\
\hline $\mathrm{Mn}$ & $\mathrm{g} / \mathrm{l}$ & up to 0,1 \\
\hline $\mathrm{Na}$ & $\mathrm{g} / \mathrm{l}$ & up to 40 \\
\hline $\mathrm{REE}$ total & $\mathrm{g} / \mathrm{l}$ & up to 0,05 \\
\hline $\mathrm{U}$ & $\mathrm{g} / \mathrm{l}$ & up to 0,15 \\
\hline $\mathrm{Pu}$ & $\mathrm{mg} / \mathrm{l}$ & up to 2 \\
\hline$\alpha$-activity & $\mathrm{Bq} / \mathrm{l}$ & up to $2 \cdot 10^{8}$ \\
\hline $\mathrm{Cs}-137$ & $\mathrm{Bk} / \mathrm{l}$ & up to $0,6 \cdot 10^{8}$ \\
\hline $\mathrm{Sr}-90$ & $\mathrm{Bk} / \mathrm{l}$ & up to $0,4 \cdot 10^{8}$ \\
\hline$\beta$-activity & $\mathrm{Ci} / \mathrm{l}$ & up to 0,03 \\
\hline $\mathrm{HNO}_{3}$ & $\mathrm{~g} / \mathrm{l}$ & about 15 \\
\hline $\mathrm{H}_{2} \mathrm{C}_{2} \mathrm{O}_{4}$ & $\mathrm{~g} / \mathrm{l}$ & about 25 \\
\hline & & \\
\hline
\end{tabular}


Table 4. Composition of Pu-REE concentrate.

\begin{tabular}{|l|l|l|}
\hline \multicolumn{1}{|c|}{ Component } & \multicolumn{1}{|c|}{ Unit of measurement, g/l } & \multicolumn{1}{c|}{ Component content } \\
\hline $\mathrm{Al}$ & $\mathrm{g} / \mathrm{l}$ & 0,5 \\
\hline $\mathrm{Fe}$ & $\mathrm{g} / \mathrm{l}$ & 0,3 \\
\hline $\mathrm{Cr}$ & $\mathrm{g} / \mathrm{l}$ & 0,1 \\
\hline $\mathrm{Ni}$ & $\mathrm{g} / \mathrm{l}$ & 0,1 \\
\hline $\mathrm{Na}$ & $\mathrm{g} / \mathrm{l}$ & up to 2,5 \\
\hline $\mathrm{REE}$ total & $\mathrm{g} / \mathrm{l}$ & up to 12 \\
\hline $\mathrm{U}$ & $\mathrm{g} / \mathrm{l}$ & up to 0,2 \\
\hline $\mathrm{Pu}$ & $\mathrm{mg} / \mathrm{l}$ & $\begin{array}{l}370-630, \\
\text { medium }-500\end{array}$ \\
\hline$\alpha$-activity & & up to $6,6 \cdot 10^{9}$ \\
\hline$\beta$-activity & $\mathrm{Bq} / \mathrm{l}$ & up to 1,2 \\
\hline $\mathrm{HNO}_{3}$ & $\mathrm{Ci} / \mathrm{l}$ & about 180 \\
\hline $\mathrm{H}_{2} \mathrm{C}_{2} \mathrm{O}_{4}$ & $\mathrm{~g} / \mathrm{l}$ & up to 0,2 \\
\hline
\end{tabular}

Table 5. Distribution of plutonium and radioactivity over products of sludge reprocessing.

\begin{tabular}{|c|c|c|c|c|}
\hline \multirow{2}{*}{ Product } & \multirow{2}{*}{ Volume, $\mathrm{m}^{3}$} & \multicolumn{2}{|c|}{ Volume activity, $\mathrm{Bq} / \lambda$} & \multirow{2}{*}{$\begin{array}{l}\text { Concentratio } \\
\mathrm{n} \text { of } \mathrm{Pu}, \mathrm{mg} / \lambda\end{array}$} \\
\hline & & $\alpha$ & $\beta$ & \\
\hline Initial sludge & 1 & $(2,5-7,5) \cdot 10^{9}$ & $(1,4-13,3) \cdot 10^{11}$ & $115-440$ \\
\hline Dissolution in alkali & $2,2-3,9$ & to $2,5 \cdot 10^{7}$ & to $5,18 \cdot 10^{10}$ & to 2 \\
\hline Sludge after alkaline dissolution & $0,5-0,7$ & $(4,6-5,7) \cdot 10^{9}$ & $(5,0-5,2) \cdot 10^{11}$ & $208-284$ \\
\hline Alkali washings & $1,4-2,0$ & to $2,0 \cdot 10^{7}$ & to $1,85 \cdot 10^{10}$ & to 1 \\
\hline $\begin{array}{l}\text { Sludge after alkaline dissolution and } \\
\text { washing }\end{array}$ & $0,5-0,7$ & $(4,5-5,6) \cdot 10^{9}$ & $(4,5-4,6) \cdot 10^{11}$ & $206-280$ \\
\hline $\mathrm{HNO}_{3}$ solutions (dissolution and washing) & $1,7-5,6$ & to $6,8 \cdot 10^{8}$ & to $4,3 \cdot 10^{10}$ & to 46 \\
\hline $\mathrm{HNO}_{3}$ raffinate of partitioning facility & $1,9-6,7$ & to $5,7 \cdot 10^{8}$ & to $3,7 \cdot 10^{9}$ & to 40 \\
\hline $\begin{array}{l}\text { Sludge after dissolution in } \mathrm{HNO}_{3} \text { and } \\
\text { washing }\end{array}$ & $0,35-0,7$ & $(2,9-5,8) \cdot 10^{8}$ & $(3,2-4,6) \cdot 10^{11}$ & $4,9-6,9$ \\
\hline $\mathrm{HNO}_{3}-\mathrm{H}_{3} \mathrm{PO}_{4}$ solution & $0,7-2,8$ & $(1,1-2,2) \cdot 10^{8}$ & $(9,6-19,6) \cdot 10^{10}$ & $1,5-3,0$ \\
\hline $\begin{array}{l}\mathrm{HNO}_{3}-\mathrm{H}_{3} \mathrm{PO}_{4} \text { raffinate of partitioning } \\
\text { facility }\end{array}$ & $0,8-3,4$ & $(0,9-1,8) \cdot 10^{8}$ & $(4,1-10,4) \cdot 10^{9}$ & $1,2-2,5$ \\
\hline Sr-Cs concentrate & $1,7-6,0$ & to $1,1 \cdot 10^{5}$ & $(0,8-1,2) \cdot 10^{11}$ & to 0,01 \\
\hline Decantate after oxalate precipitation & $2,9-10,7$ & to $2 \cdot 10^{7}$ & to $1,1 \cdot 10^{9}$ & to 2 \\
\hline $\mathrm{Pu}$ - REE concentrate & $0,3-0,5$ & to $6,6 \cdot 10^{9}$ & to $4,4 \cdot 10^{10}$ & $370-630$ \\
\hline
\end{tabular}

Nitric acid solution arising from sludge dissolution in apparati in building 951 is directed to partitioning at UE-35 facility by the accepted technology. Concentrate of $\mathrm{Cs}$ and $\mathrm{Sr}$ is passed to solidification in shop 3 and raffinate - to section 61 . Acidity of raffinate, oxalate precipitation of $\mathrm{Pu}, \mathrm{An}$ and REE, washing of precipitate and its dissolution in $\mathrm{HNO}_{3}$ are adjusted in apparati $6103 / 2$ and 6107.1 by the "Mayak" PA technology. $\mathrm{Pu}$ - REE concentrate is passed to solidification in monazite ceramics, using available apparatus for hot unaxial pressing, which was ordinarily utilized to fabricate strontium titanate $\left(\mathrm{SrTiO}_{3}\right)$ dense ceramic in industrial scale. 


\section{Variant 3. Immobilization of Actinide-containing Wastes of "Mayak" in the Form of Garnet/perovskite Ceramic}

The garnet/perovskite ceramic suggested for the immobilization of "Mayak" residue wastes is characterized of the following attractive features:

- Garnet and perovskite are dramatically more durable (chemically, thermally, mechanically and to $\alpha$ radiation damage) actinide host-phases than glass;

- Garnet/perovskite ceramic can be obtained as a glass by melting in air;

- Cold crucible melter can be successfully used for the glass synthesis as well as for the fabrication of garnet/perovskite ceramic;

- Generally, melting temperature of garnet/perovskite ceramic is significantly higher than glass melting point (respectively, $1500-1700^{\circ} \mathrm{C}$ and $1000-1400^{\circ} \mathrm{C}$ ). However, varying the precursor chemical composition allows a decrease in synthesis temperature for garnet/perovskite ceramics to the glass interval $\left(1300-1400^{\circ} \mathrm{C}\right)$;

- Garnet and perovskite are minerals geochemically compatible with bed-rock occurring in the "Mayak" region (for example, with porphirite). Glass is a geochemically strange material for those rocks. Therefore, final disposal of actinide ceramic waste form into deep geological formations in "Mayak" region is definitely more justified and attractive than disposal of actinide-doped glass;

- Garnet/perovskite ceramic is more flexible in accomodating inhomogeneous residue wastes than glass.

Formulation of garnet/perovskite ceramic allows significant variation of chemical composition; in particular, the contents of $\mathrm{Am}$, rare-earths, $\mathrm{Ni}, \mathrm{Cr}, \mathrm{Mn}, \mathrm{Fe}, \mathrm{Al}$. However, it is suggested that 1.0-1.5 wt.\% Pu and 3.0-4.0 wt.\% ( $\mathrm{Na}+\mathrm{K})$ be considered as maximum limits. Higher concentrations of these elements are not optimal for ceramic synthesis, and require more precise stoichiometry of starting precursor.

Two possible options are under consideration for using garnet/perovskite ceramic (Fig. 3):

- Direct immobilization of insoluble part of sludges (containing main part of actinide amounts);

- Immobilization of Pu and REE concentrate obtained as a result of partitioning.

\section{Reference}

1. S.I. Rovnyi, A.S. Skobtsov, G.M. Medvedev, M.V. Logunov. Initial data for feasibility study on immobilization of plutonium-containing waste storaged in building 954. Central Laboratory. Current issue 1564 of 20.04.2001.

\section{Viewgraphs (see following pages)}




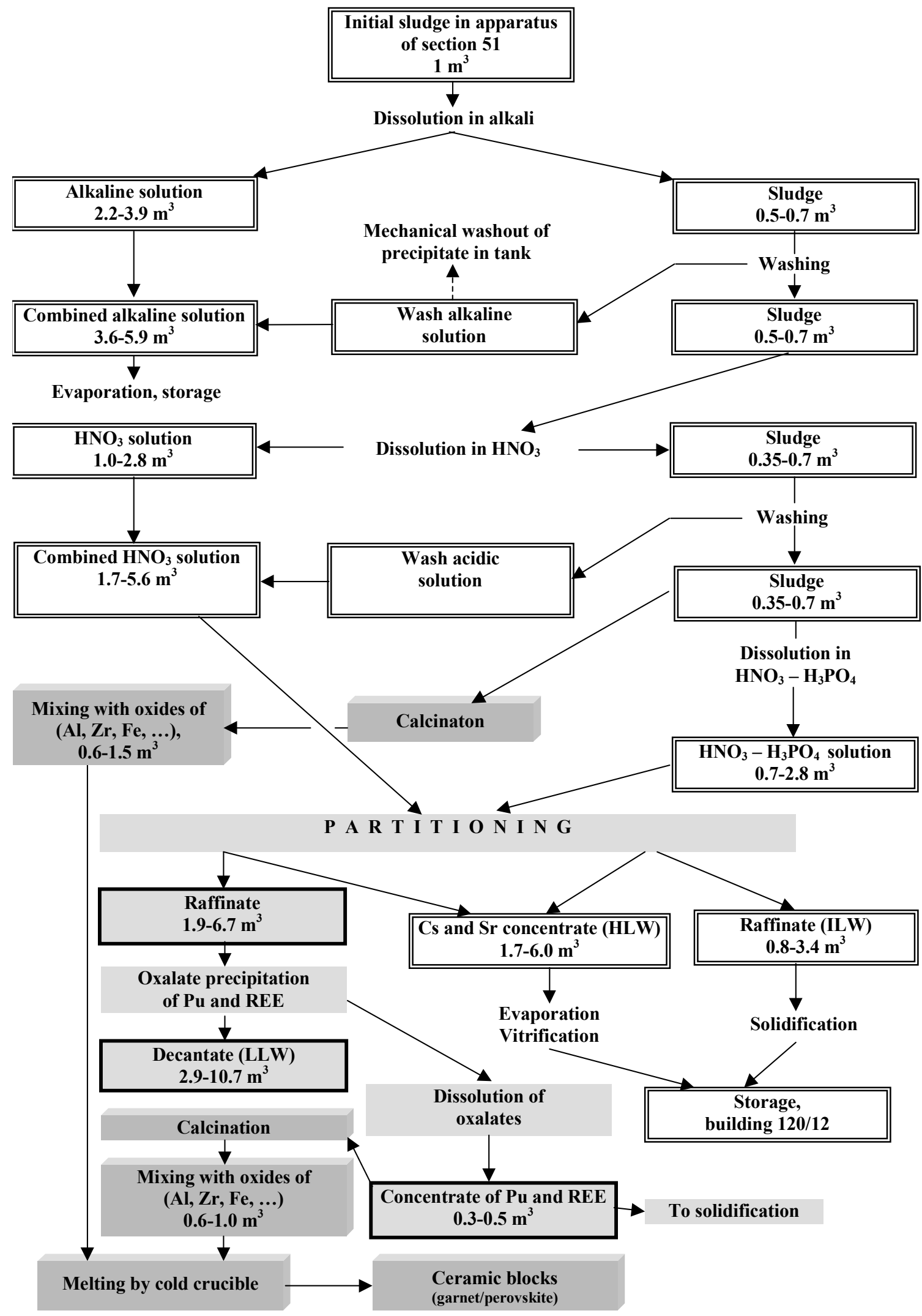

Fig. 3. Block-diagram of sludge immobilization using garnet/perovskite ceramic Variant 3. 


\section{Simulated wastes compositions}

\begin{tabular}{|c|c|c|c|c|c|c|c|c|c|c|c|c|c|c|}
\hline Component & \begin{tabular}{|l} 
PuO \\
2 \\
\end{tabular} & $\mathrm{UO}_{2}$ & $\begin{array}{l}\mathrm{Fe}_{2} \\
\mathrm{O}_{3}\end{array}$ & $\begin{array}{l}\mathrm{Cr}_{2} \\
\mathrm{O}_{3}\end{array}$ & $\mathrm{NiO}$ & $\begin{array}{l}\mathrm{Al}_{2} \\
\mathrm{O}_{3}\end{array}$ & $\begin{array}{l}\mathrm{Mn} \\
\mathrm{O}_{2}\end{array}$ & $\mathrm{SO}_{3}$ & $\mathrm{FeS}$ & $\mathrm{SiO}_{2}$ & SrO & $\begin{array}{l}\mathrm{Cs} \mathbf{s}_{2} \\
0\end{array}$ & $\mathrm{~K}_{2} \mathrm{O}$ & $\begin{array}{l}\mathrm{Na}_{2} \\
\mathrm{O}\end{array}$ \\
\hline BS, wt $\%$ & 0.1 & 0.6 & 8.8 & 1.6 & 7.2 & 58.5 & 1.0 & 3.3 & - & 1.1 & 0.03 & 0.06 & - & 17.7 \\
\hline FBS, wt $\%$ & 0.2 & 0.7 & 6.9 & 1.8 & 7.5 & 55.0 & 1.4 & - & 3.9 & 1.0 & 0.03 & 0.06 & 5.0 & 16.5 \\
\hline
\end{tabular}

Recommended flux composition, wt\%: $\mathrm{Na}_{2} \mathrm{O}-18, \mathrm{SiO}_{2}-54, \mathrm{~B}_{2} \mathrm{O}_{3}-20, \mathrm{ZnO}-8$.

\section{Wastes:Flux}

\begin{tabular}{|l|l|}
\hline 20:80 & Final products are roentgen amorphous without crystalline phase. \\
\hline $30: 70$ & The liquate inhomogeneity and the crystalline phases (spinels) was observed in the samples \\
\hline $40: 60$ & $\begin{array}{l}\text { The phases separation is most significant for sulphate compositions when teardrop-shaped with sulfur } \\
\text { generation is in the samples bulk. In case of sulphide sludge the crystalline phase including sulfur is } \\
\text { observed. }\end{array}$ \\
\hline$>40$ & $\begin{array}{l}\text { In case of fixing more then } 40 \text { wt.\% of wastes inhomogeneity compositions were obtained, creation of } \\
\text { lye (yellow phase) was observed on surface of glass. The main component of lye was sodium sulphate }\end{array}$ \\
\hline
\end{tabular}




\section{Block-diagram for sludge reprocessing}

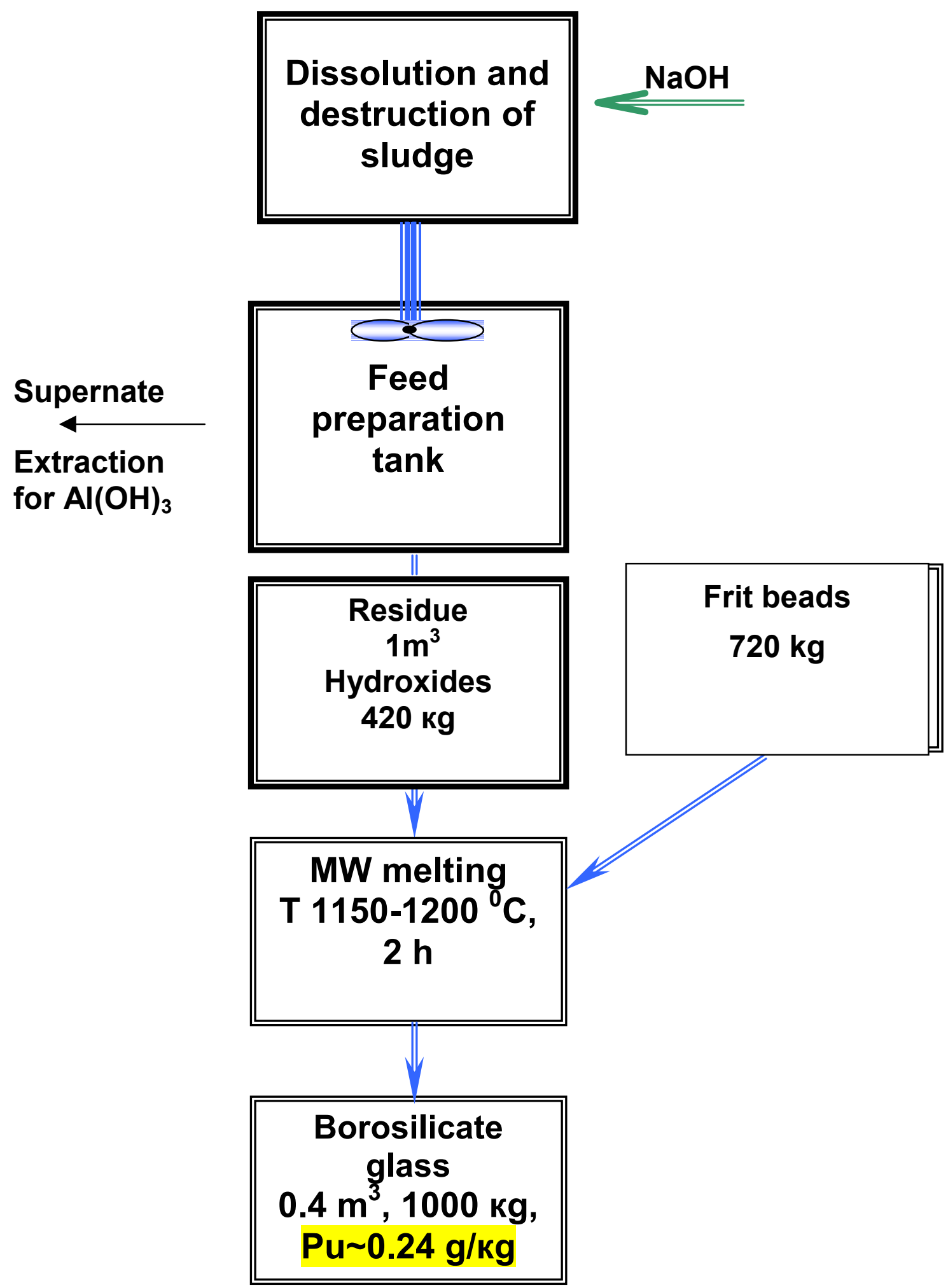

$4500 \mathrm{Mt}$ or $1700 \mathrm{~m}^{3}$ of glass including $1000 \mathrm{~kg}$ of plutonium will be obtained after reprocessing of the whole of ultimated residue. 


\section{Блок-схема опытно-промышленной установки}

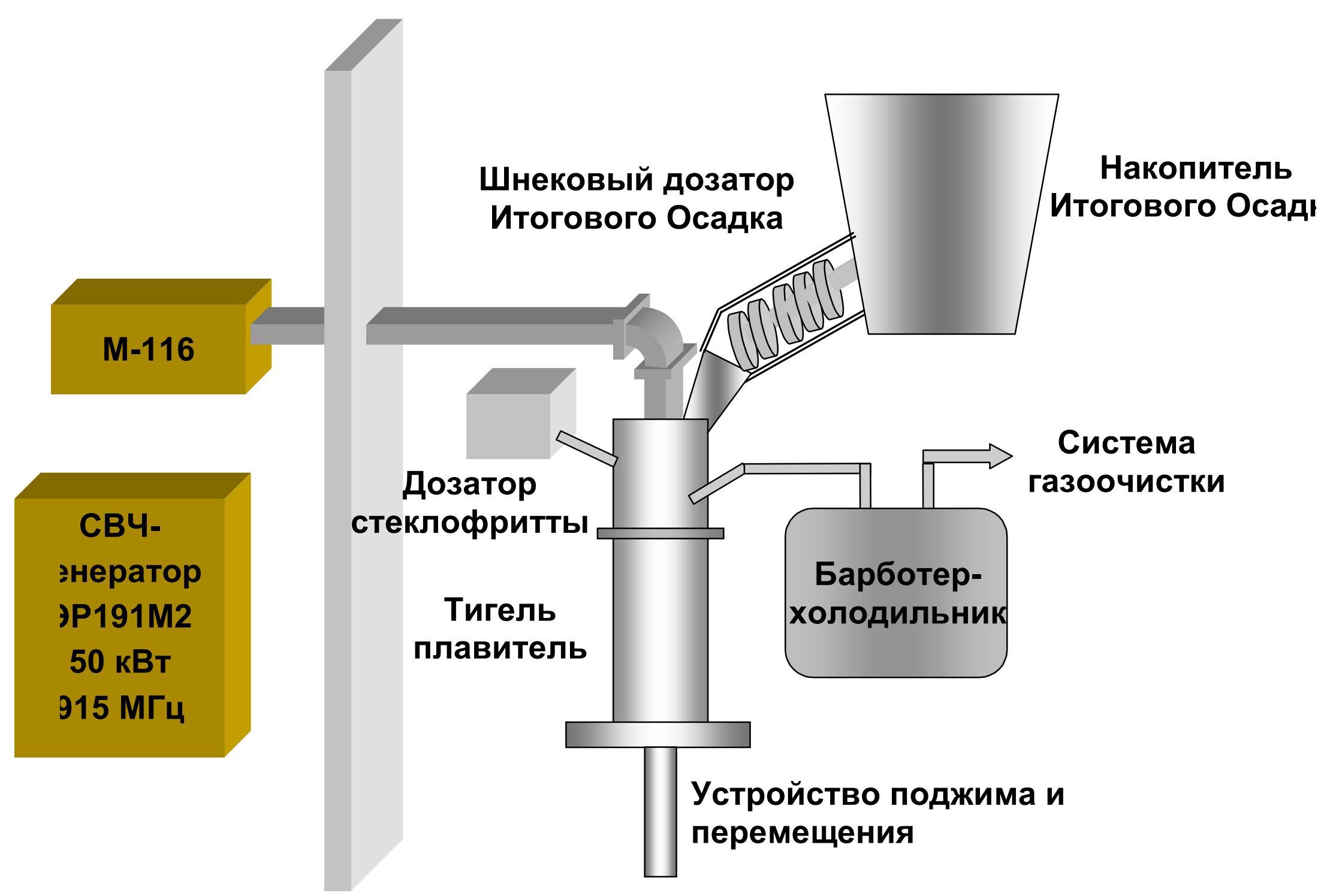




\title{
Immobilization of Am-241 Formed under Plutonium Metal Conversion into Monazite-type Ceramic (B506209)
}

\author{
A. S. Aloy, E. N. Kovarskaya, T.I. Koltsova, S. E. Samoylov, KRI; S. I. Rovnyi, G. \\ M. Medvedev, PA "Mayak", L. J. Jardine, LLNL
}

\begin{abstract}
Lanthanum orthophosphate with the monazite structure was proposed upon examination as a suitable matrix for immobilization of future americium-containing liquid wastes, which could be formed in the conversion of metallic plutonium into oxide at PA "Mayak".

Specimens of monazite non-active ceramics were fabricated from $\mathrm{LaPO}_{4}$ powders obtained using a thinfilm evaporator by either hot pressing or cold pressing and sintering at $900-1300^{\circ} \mathrm{C}$. According to electron microprobe analysis (EMPA), scanning electron microscopy (SEM) and x-ray diffraction (XRD), which were used for characterization of the samples produced, no specimens contained any phase other than the monoclinic monazite phase. Ceramics with the specific activity of Am-241, i.e., $2.13 \times 10^{7} \mathrm{~Bq} / \mathrm{g}$, were prepared by only cold pressing with subsequent sintering at $1300^{\circ} \mathrm{C}$ for 1 hour. The normalized leach rates of lanthanum and americium in distilled water at $90^{\circ} \mathrm{C}$ were less than $1.2 \times 10^{-4}$ and $2.34 \times 10^{-4} \mathrm{~g} / \mathrm{m}^{2} \cdot$ day, respectively.
\end{abstract}

\section{Introduction}

PA "Mayak" in Russia was created at the end of the 1940s to produce weapons-grade plutonium with the aim of reaching nuclear weapons parity.

At the present time, because of great changes in the world and the necessity to promote nonproliferation disposition of excess plutonium, PA "Mayak" will be assigned the new mission of plutonium metal to oxide conversion process.

This process will generate highly active liquid americium-241 waste streams, containing a small amount of impurities such as $\mathrm{Si}, \mathrm{Al}, \mathrm{Gd}$, and others. It is well known [1-3] that lanthanide orthophosphates with monazite structure are a very promising matrix for the immobilization of actinides.

The major advantages of monazite ceramic waste forms are high waste loadings, chemical durability, and radiation stability. The density of monazite ceramic is normally between 4,0 and $5,0 \mathrm{~g} / \mathrm{cm}^{3}$, which significantly reduces the volume of the final product in comparison with borosilicate glass.

Nevertheless, the commonly used technology for fabrication of monazite ceramic waste forms, which is based on the urea precipitation process, has a disadvantage in that it emits substantial quantities of ammonia under radiochemical conditions [3].

To improve the process and adapt it to "Mayak" practice, a thin-film evaporator (TFE) was used to produce lanthanide ceramic precursor powder in a different ratio of $\mathrm{La}_{2} \mathrm{O}_{3}: \mathrm{P}_{2} \mathrm{O}_{5}$. The resulting fine powders were used without milling, mixing, plasticizer addition, and lubrication to produce high-density monazite ceramic specimens both by hot pressing and cold pressing and following sintering. 


\section{Synthesis and Properties of Single-phase Monazite Ceramics}

The single-phase monazite ceramics were produced by the hot pressing method of lanthanum orthophosphate powders obtained with the TFE. Synthesis of monazite ceramics was performed in accordance with two variants, which are present in Fig. 1.

Variant 1

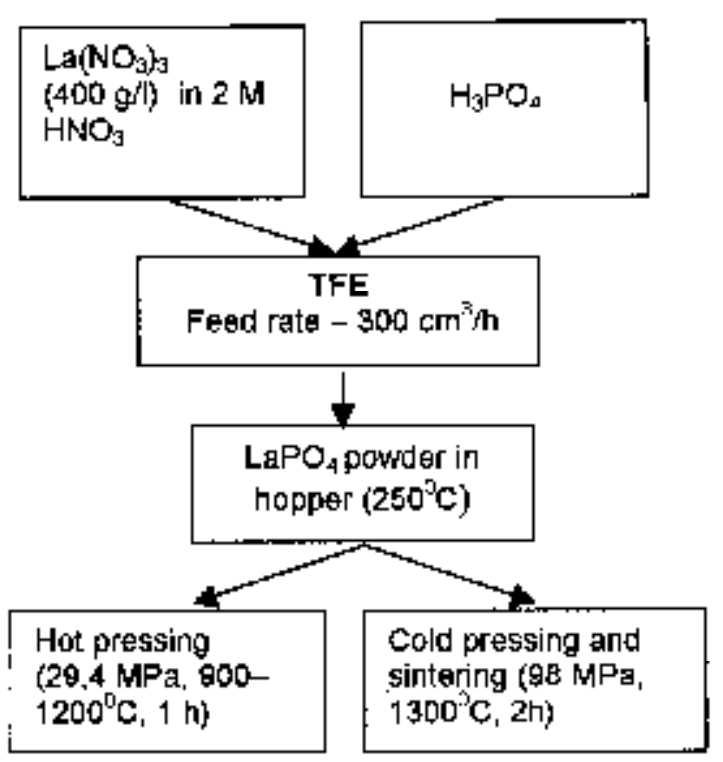

Variant 2

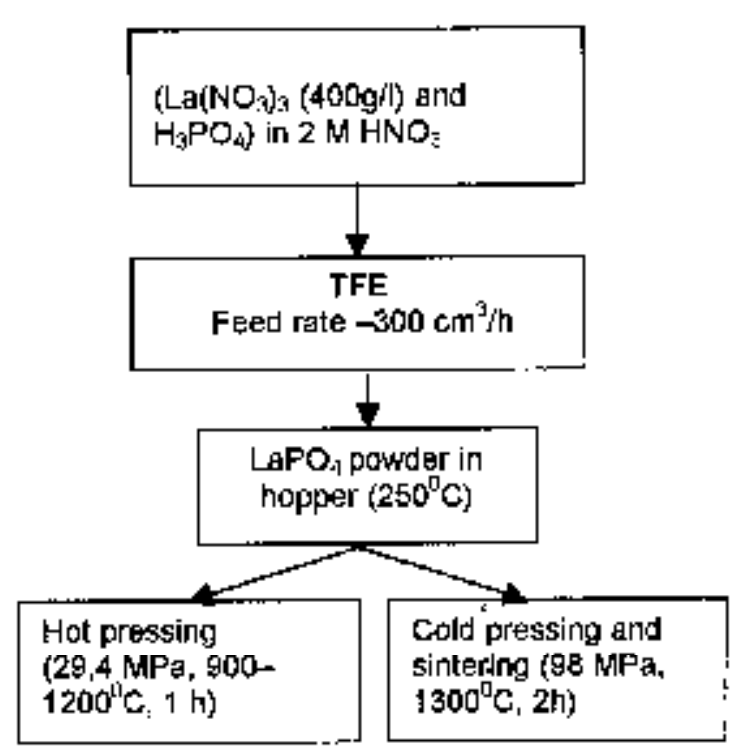

Fig.1. Schemes of synthesis of monazite ceramics.

It is necessary to keep the exact ratio of initial solutions of lanthanum nitrate and orthophosphoric acid to form the lanthanum orthophosphate if the process is conducted according to variant 1 . On the contrary, in variant 2 , it is not necessary because the initial solutions were previously mixed, and the stable colloidal solution was fed to the TFE.

The powder layer formed on the heat-exchanger surface was removed by rotor blades and collected in the hopper heated to $250^{\circ} \mathrm{C}$. To improve TFE operation, a small amount of stripper (polyacrylamide) was added in the initial solution. It was determined by XRD, that the powder obtained represented $\mathrm{LaPO}_{4}$ with the monazite structure and contained an amorphous phase.

Pellets with a weight of $\sim 10 \mathrm{~g}$ and diameter of $20 \mathrm{~mm}$ were formed from the powder by hot pressing in a graphite mold at $29,4 \mathrm{MPa}$ pressure and at temperature $900-1200^{\circ} \mathrm{C}$. Pressing temperature and some properties of the pellets are presented in Table 1.

It can be seen from Table 1 that dense and low-porosity ceramics are formed at relatively moderate temperature $\left(1000^{\circ} \mathrm{C}\right)$. On the XRD data, monazite crystallites have been enlarged from $50 \mathrm{~nm}\left(\right.$ at $\left.250^{\circ} \mathrm{C}\right)$ to $>100 \mathrm{~nm}\left(\right.$ at $1000^{\circ} \mathrm{C}$ ), and the amorphous phase has been crystallized during hot pressing. As a result single-phase ceramics are formed. The uniformity and chemical composition $\left(\mathrm{LaPO}_{4}\right)$ of monazite ceramics were confirmed by EMPA, SEM, and XRD.

SEM photomicrographs of ceramic samples \# 2 and 3 (Figs. 2 and 3) were prepared under similar conditions, both with the addition of stripper and without. These photomicrographs demonstrate the influence of the stripper on the quality of the ceramics. 
Table 1. Pressing temperature and some properties of $\mathrm{LaPO}_{4}$ pellets.

\begin{tabular}{|l|l|l|l|l|}
\hline № & $\begin{array}{c}\text { Pressing temperature, } \\
\mathbf{~} \mathbf{C}^{-}\end{array}$ & $\begin{array}{c}\text { Apparent density, } \\
\mathbf{g} / \mathbf{c m}^{\mathbf{3}}\end{array}$ & $\begin{array}{c}\text { \% of the theoretical } \\
\text { density }\end{array}$ & $\begin{array}{c}\text { Open porosity, } \\
\text { \% vol. }\end{array}$ \\
\hline 1 & 900 & 4,24 & 83.0 & 9,8 \\
\hline $2^{*}$ & 1000 & 4.90 & 96.0 & 1.0 \\
\hline 3 & 1000 & 4.90 & 96.0 & $<0.1$ \\
\hline 4 & 1100 & 4.90 & 96.0 & $<0.1$ \\
\hline $5^{*}$ & 1150 & 4.67 & 91.0 & $<0.1$ \\
\hline 6 & 1200 & 5.00 & 97.6 & $<0.1$ \\
\hline 7 & 1200 & 4.93 & 96.0 & $<0.1$ \\
\hline
\end{tabular}

*Samples were prepared without stripper additive

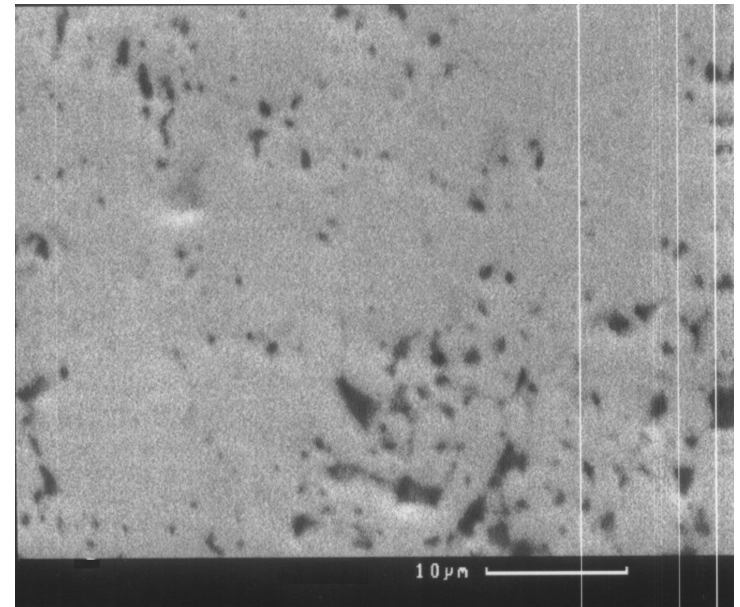

Fig. 2. SEM photomicrograph of monazite ceramic (\#2), without stripper additive.

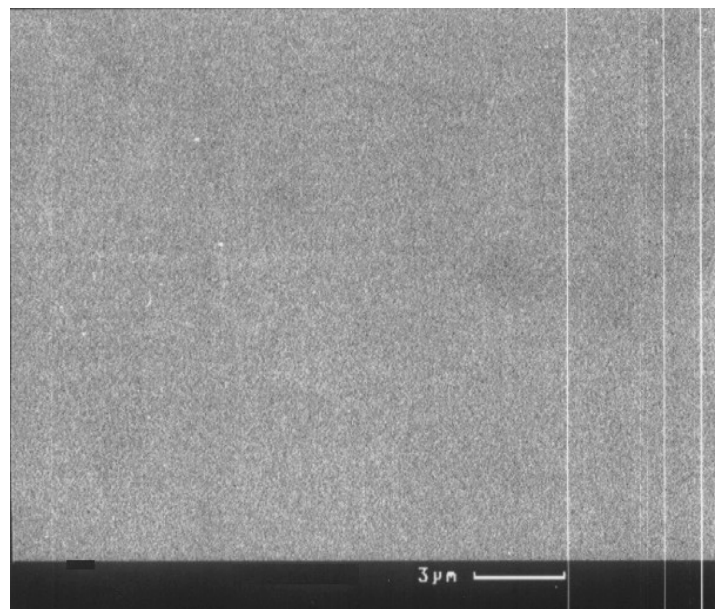

Fig. 3. Photomicrograph of monazite ceramic (\#3), with stripper additive.

As seen in Figs. 2 and 3, the addition of stripper improves the quality of a ceramic owing to the formation of more finely dispersed powder.

The two-phase ceramic is formed at more than 5 mol\% excess of orthophosphoric acid. The phase compositions of ceramics prepared under similar conditions with the different molar ratio $\mathrm{La}_{2} \mathrm{O}_{3}: \mathrm{P}_{2} \mathrm{O}_{5}$ are shown in Table 2. Ten to thirty mol\% orthophosphoric acid excess results in $\mathrm{LaPO}_{4}$ (monazite) and $\mathrm{LaP}_{3} \mathrm{O}_{9}$. The SEM photomicrograph of the two-phase ceramic is shown in Fig. 4.

Table 2. Phase compositions of ceramics with different molar ratio $\mathrm{La}_{2} \mathrm{O}_{3}: \mathrm{P}_{2} \mathrm{O}_{5}$.

\begin{tabular}{|l|l|}
\hline \multicolumn{1}{|c|}{ Molar ratio } & \multicolumn{1}{c|}{ Phase compositions } \\
\hline $1: 1$ & $\mathrm{LaPO}_{4}$ (monazite) \\
\hline $1: 1,05$ & $\mathrm{LaPO}_{4}$ (monazite) \\
\hline $1: 1,1$ & $\mathrm{LaPO}_{4}$ (monazite) $+\mathrm{LaP}_{3} \mathrm{O}_{9}$ \\
\hline $1: 1,3$ & $\mathrm{LaPO}_{4}$ (monazite) $+\mathrm{LaP}_{3} \mathrm{O}_{9}$ \\
\hline
\end{tabular}




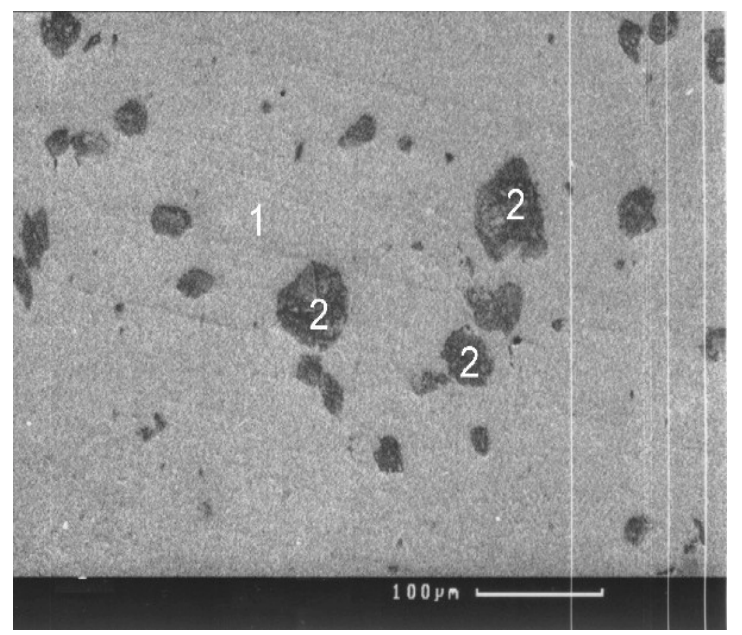

Fig. 4. SEM photomicrograph of two-phase monazite ceramics. (1) $\mathrm{LaPO}_{4} ;$ (2) $\mathrm{LaP}_{3} \mathrm{O}_{9}$.

\section{Monazite Ceramics Doped with Americium-241}

Two ceramic samples were sintered under similar conditions by cold axial pressing ( $98 \mathrm{MPa}$ ) following sintering at $1300^{\circ} \mathrm{C}$ for 2 hours. One of them was doped by Am-241. To prepare the $\mathrm{LaPO}_{4}$ powder, a mixture of $\mathrm{La}\left(\mathrm{NO}_{3}\right)_{3}$ solution in $1 \mathrm{M} \mathrm{HNO}_{3}$ and $\mathrm{H}_{3} \mathrm{PO}_{4}$ with 5 mol\% excess was evaporated to dryness. $\mathrm{Am}\left(\mathrm{NO}_{3}\right)_{3}$ solution in $1 \mathrm{M} \mathrm{HNO}_{3}$ was added to a $\mathrm{La}\left(\mathrm{NO}_{3}\right)_{3}$ solution in $1 \mathrm{M} \mathrm{HNO}_{3}$ to obtain the doped sample. The resulting powder was heated at $700^{\circ} \mathrm{C}$ and ground before pressing. The apparent densities of samples were $\sim 4.3 \mathrm{~g} / \mathrm{cm}^{3}$ which was $\sim 84 \%$ of the calculated value.

Only the undoped sample was studied by EMPA and XRD. These methods showed that the undoped sample contains only one monazite phase. It can be assumed that the doped sample has the same composition because synthesis conditions were identical.

Chemical stability The leaching properties of the fabricated waste forms were investigated by conducting the MCC-1 test [4]. The leaching conditions: leachant- distilled water; leach test vessel material -teflon; $S / V=10 \mathrm{~m}^{-1}$; leach test temperature $-90^{\circ} \mathrm{C}$; the leach test was run for 7 days.

The following ceramic samples were tested:

- Sample \#3 - hot pressed at $1000^{\circ} \mathrm{C}$ (Table 1);

- Sample \#6 - hot pressed at $1200^{\circ} \mathrm{C}$ (Table 1);

- Cold pressed sample $-98 \mathrm{MPa}$, with sintering at $1300^{\circ} \mathrm{C}$ for 2 hours.

The leachates were analyzed by atomic emission spectroscopy with inductively coupled plasma. The results of the leaching tests are summarized in Table 3.

The concentrations of lanthanum in the leachate were below detection level $(0.005 \mathrm{mg} / \mathrm{l})$ in all tests. The values of lanthanum normalized release rates, which was calculated from these concentrations and obviously overestimated, are less than $1.2 \cdot 10^{-4} \mathrm{~g} / \mathrm{m}^{2} /$ day and testify to high chemical stability of ceramics.

The ceramics doped with americium were also tested by the MCC-1 (Table 4). The americium-241 content was determined by gamma spectrometry. Specific activity of Am-241 in the ceramic was $2.13 \cdot 10^{7} \mathrm{~Bq} / \mathrm{g}$. It can be seen from Table 4 , the normalized leach rate of ${ }^{241} \mathrm{Am}$ from the sample studied is low enough and correlates with the data for synthetic monazite [1]. 
Table 3. Results of the MCC-1 test.

\begin{tabular}{|c|c|}
\hline Sample & Concentration La, $\mathbf{m g} / \mathbf{l}$ \\
\hline$\# 3$ & $<0,005$ \\
\hline$\# 6$ & $<0,005$ \\
\hline Cold pressing & $<0,005$ \\
\hline
\end{tabular}

Table 4. Normalized leach rate of ${ }^{241} \mathrm{Am}$ from the $\mathrm{LaPO}_{4}$ monazite ceramic sample.

\begin{tabular}{|c|c|c|}
\hline Sample activity, Bq & Leachate specific activity, $\mathbf{B q} / \mathbf{m l}$ & Normalized leach $\mathbf{r a t e}, \mathbf{g} / \mathbf{m}^{2} \cdot$ day \\
\hline $1.48 \cdot 10^{7}$ & $0.35 \pm 0.10$ & $2.34 \cdot 10^{-4}$ \\
\hline
\end{tabular}

\section{Conclusions}

1. The principle possibility of TFE using for $\mathrm{LaPO}_{4}$ powder production was shown.

2. Addition of polyacrylamide in the amount of $1 \mathrm{wt} \%$ of lanthanum orthophosphate calculated weight improves the TFE operational parameters and quality of the ceramic obtained.

3. Dense low-porosity monazite ceramics (apparent density averages of $96 \%$ of the calculated value) can be prepared by hot pressing at a temperature of $1000^{\circ} \mathrm{C}$.

4. The XRD, EMPA, and SEM showed that the samples are uniform and consist of $\mathrm{LaPO}_{4}$ with monazite structure. With excess $\mathrm{H}_{3} \mathrm{PO}_{4}$, more than $5 \mathrm{~mol} \%$, an additional phase $\mathrm{LaP}_{3} \mathrm{O}_{9}$ is formed along with monazite.

5. The ceramics have high chemical stability; the normalized rates of lanthanum and americium losses are as low as $\sim 10^{-4} \mathrm{~g} / \mathrm{m}^{2}$.day, and are comparable with those for synthetic monazites.

\section{References}

1. Strelnikov A.V., Kovarskya E.N., Kolycheva T.I., 1996. "The method solidification of REE and TUE elements in ceramic", Patent RF, \#2062519, Bul. \#17 Patent.

2. Volkov J.F. 1999, "The compounds with zircon and monazite structures and its availability for radionuclide incorporation", Radiochemistry, Ru, T.41, \#2, p. 161-166.

3. Boatner L.A. and Sales B.C., 1988. "Monazite, Radioactive Waste Forms for the Future". Lutze W., Ewing R.C. Amsterdam: Elservier Science Publishers B.V., p. 495-564.

4. Standard Test Method for Static Leaching of Monolithic Waste Forms for Disposal of Radioactive Waste, ASTM C1220-92, American Society for Testing and Materials, 1916 Race St., Philadelphia, PA, 19103 (MCC-1). 


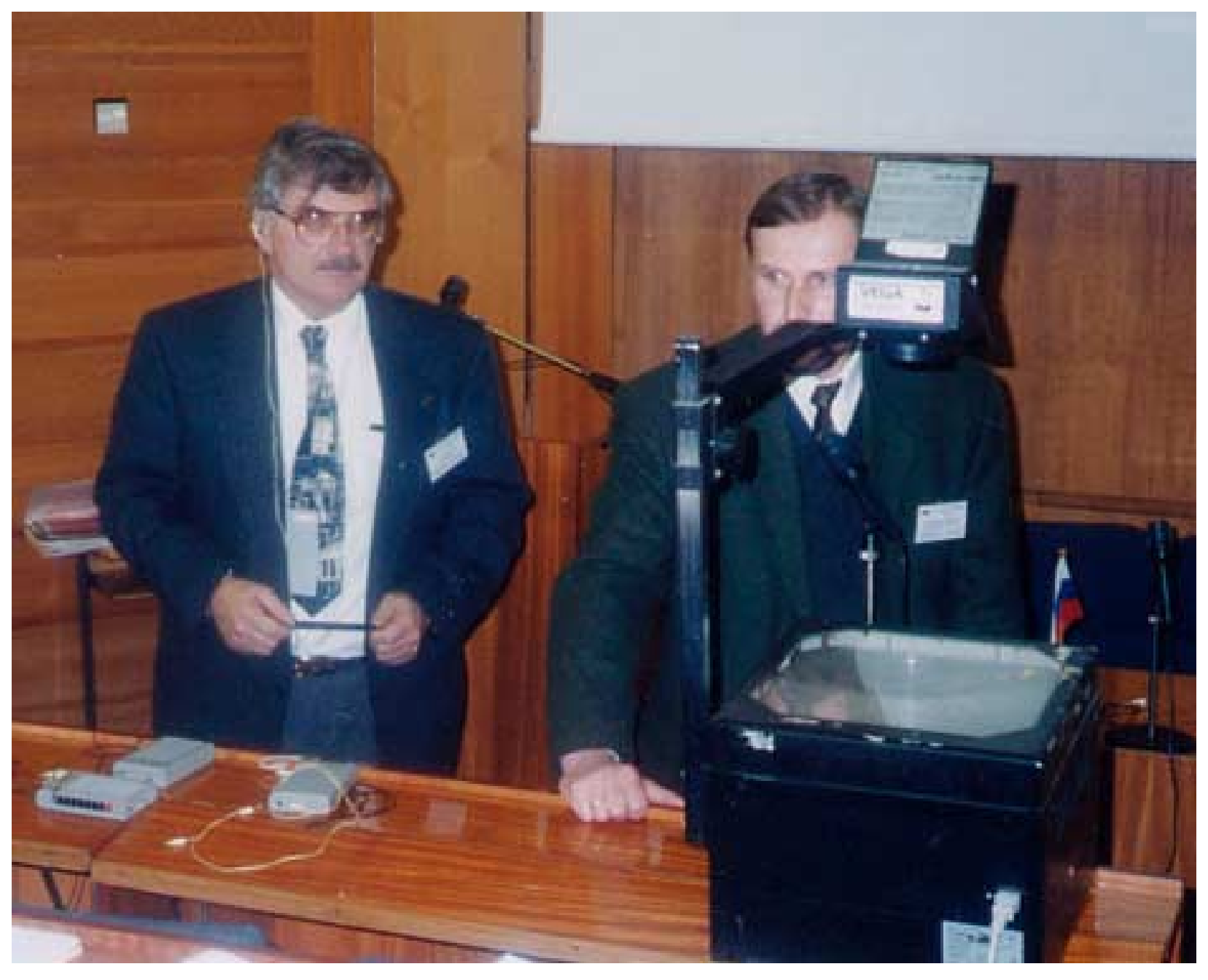




\title{
Immobilization of Weapon Plutonium-containing Materials and Waste of MOX Fuel Fabrication in the Russian Federation: Issue 1. Elaboration and Acquisition of Data for TES (B506240)
}

\author{
V. L. Rozhkov, E. I. Tyurin, Yu. F. Kolotilov, GSPI
}

\section{Summary}

The efforts undertaken by the Minatom State Specialized Design Institute (GSPI) to elaborate and to acquire the data for the technical and economic study (TES), Issue 1 specified in the Statement of Works of B506240 Agreement on Material and Technical Support, have enabled the GSPI to do the following:

1. To forward the request to the Mayak PA for the technical information on the PAKET facility as an analogue of the MOX-fuel commercial fabrication facility.

2. To analyze the technical information submitted by Mayak PA and to include the basic technical data on the PAKET facility in the Progress Report. The PAKET facility technical data encompass the following:

- Range of products and the design capacity;

- Basic processes;

- Basic process equipment;

- Classification and the amount of waste, the procedures of waste treatment and management.

3. To agree upon the technical data on the PAKET facility with the Mayak PA and with other cocontractors.

4. To analyze the results of the previously carried out studies, predesign and design activities and to present in the Progress Report the basic technical data on the MOX-fuel commercial fabrication facility. The technical data on the MOX-fuel commercial fabrication facility cover as follows:

- Range of products and the rated capacity;

- Basic processes;

- Basic process equipment;

- Classification and the amount of waste, the procedures for waste management and treatment.

The basic technical data on the MOX-fuel commercial fabrication facility have been elaborated on the grounds of the basic scenario used for the TES calculation to estimate the costs to implement the Russian Weapon Plutonium Disposal Program.

5. To agree upon the technical data on the MOX-fuel commercial fabrication facility with the Mayak PA and with other co-contractors.

6. To draw up the Issue 1 Progress Report. 


\section{Issue 1. Elaboration and Acquisition of Data for TES}

\section{Range of Products and Design Capacity of the PAKET Facility}

The PAKET facility is designed to fabricate mixed uranium and plutonium fuel elements for the BN-600 reactors. The facility capacity is as follows: fuel elements for 10 fuel assemblies per year.

\section{The PAKET Facility Basic Fabrication Processes}

Two lines are used to fabricate fuel elements:

- Fabrication of reactor core pellets (LT-10 line);

- Charging, assembling and checking of fabricated fuel elements (ST-10 line).

\section{LT-10 Line:}

- Receipt of the feed material - plutonium dioxide and uranium dioxide;

- Uranium and plutonium content determination of the feed material;

- Preparation of master mix of uranium and plutonium dioxides in the given ratio and with addition of the binder (0.1-0.3 wt.\% zinc stearate). The vortex mixer is used for pre-homogenization in the argon atmosphere;

- Homogenization and pressing of fuel compacts;

- Density control of fuel compacts;

- Crushing of fuel compacts, sieving and powder milling to fabricate reactor core pellets;

- Compacting of fuel pellets, geometry and density checking of fuel pellets;

- Sintering of pellets. Pellets are sintered in a sintering blast vertical (SBV) furnace in the argonhydrogen atmosphere under $1450^{\circ} \mathrm{C}-1750^{\circ} \mathrm{C}$ during two hours;

- Sampling for physical and chemical analysis, visual inspection and geometry checking of pellets.

\section{ST10 Line:}

- Checking of the accessories and of the materials, vacuum drying. Vacuum drying is carried out under $200^{\circ} \mathrm{C}-250^{\circ} \mathrm{C}$ temperature and at $1 \times 10^{-1} \mathrm{~mm}$ mercury pressure;

- Charging of the lower shielding section, of the fuel core and of the upper shielding section of fuel elements;

- Mass checking of every of fuel element sections;

- Sealing of fuel elements;

- Pre-decontamination of fuel elements;

- Fuel element failure "cold" detection; 
- Decontamination of fuel elements. The electrochemical ultrasonic treatment facility (EKhUZO) is used to decontaminate fuel elements;

- Checking of fuel element surface cleanliness;

- Fuel element failure "hot" detection. "Hot" detection of fuel element leaktightness is carried out in the furnace retort using a helium leak detector;

- Sorting of the ready-made items;

- Disassembling of rejected fuel elements. 


\section{Waste Categories and Amount Processes to Treat Waste Using the PAKET Facility}

Table 1. Waste categories and amounts to treat waste using the PAKET facility.

\begin{tabular}{|c|c|c|c|c|c|c|c|}
\hline \multirow[b]{2}{*}{ No. } & \multirow[b]{2}{*}{ Equipment, procedures } & \multicolumn{5}{|c|}{ Waste } & \multirow[b]{2}{*}{ Processes to treat waste } \\
\hline & & Waste category & $\begin{array}{c}\text { Units of } \\
\text { measuremen } \\
t\end{array}$ & Amount & $\begin{array}{c}\text { Pu content, } \mathbf{g} / \mathbf{k g} \\
\text { of waste }\end{array}$ & $\begin{array}{c}\text { Specific } \\
\text { activity, } \\
\text { kBq/kg }\left(\mathrm{I} ; \mathrm{m}^{3}\right)\end{array}$ & \\
\hline 1. & $\begin{array}{l}\text { 1-1 glove box } \\
\text { Receipt of feed materials } \\
\left(\mathrm{UO}_{2}, \mathrm{PuO}_{2}\right) \text {, dosing, } \mathrm{U} \\
\text { and Pu content } \\
\text { determination }\end{array}$ & $\begin{array}{l}\text { SRW } \\
\text { Scrap metal } \\
\text { Polyethylene } \\
\text { film } \\
\text { Rubber gloves } \\
\text { Filters } \\
\text { Wiping cloth } \\
\text { LRW } \\
\text { Solutions } \\
\text { resulting from } \\
\text { decontamination }\end{array}$ & $\begin{array}{l}\mathrm{kg} / \text { year } \\
\mathrm{kg} / \text { year } \\
\mathrm{kg} / \text { year } \\
\mathrm{kg} / \text { year } \\
\mathrm{kg} / \text { year } \\
\text { I/year }\end{array}$ & $\begin{array}{l}50 \\
12 \\
15 \\
0.5 \\
2 \\
150\end{array}$ & $\begin{array}{l}0.26 \pm 0.16 \\
0.30 \pm 0.05 \\
1.42 \pm 0.76 \\
3.32 \pm 2.64 \\
7.77 \pm 4.44 \\
<0.05 \mathrm{~g} / \mathrm{l}\end{array}$ & $\begin{array}{l}\text { over } 10^{5} \\
\text { over } 10^{5} \\
\text { over } 10^{5} \\
\text { over } 10^{5} \\
\text { over } 10^{5} \\
\text { over } 10^{5}\end{array}$ & $\begin{array}{l}\text { Nitric acid treatment to obtain } \mathrm{Pu}<200 \\
\mathrm{mg} / \mathrm{kg} \text { content and burial } \\
\text { Similarly } \\
\text { Similarly } \\
\text { Combustion. Ash is supplied to the } \\
\text { operating plant to be treated } \\
\text { Similarly } \\
\text { Accumulation, settling-down. Bottoms are } \\
\text { supplied to the operating plant to be treated. } \\
\text { Solution is supplied for sorption }\end{array}$ \\
\hline 2. & $\begin{array}{l}\text { 1-2 glove box } \\
\text { Homogenization of feed } \\
\text { products }\end{array}$ & $\begin{array}{l}\text { SRW } \\
\text { Scrap metal } \\
\text { Rubber gloves } \\
\text { Filters } \\
\text { Wiping cloth } \\
\text { LRW } \\
\text { Solutions } \\
\text { resulting from } \\
\text { decontamination }\end{array}$ & $\begin{array}{l}\text { kg/year } \\
\text { kg/year } \\
\text { kg/year } \\
\text { kg/year } \\
\text { I/year }\end{array}$ & $\begin{array}{l}10 \\
10 \\
0.5 \\
1 \\
150\end{array}$ & $\begin{array}{l}0.26 \pm 0.16 \\
1.42 \pm 0.76 \\
3.32 \pm 2.64 \\
7.77 \pm 4.44 \\
<0.05 \mathrm{~g} / \mathrm{l}\end{array}$ & $\begin{array}{l}\text { over } 10^{5} \\
\text { over } 10^{5} \\
\text { over } 10^{5} \\
\text { over } 10^{5} \\
\text { over } 10^{5}\end{array}$ & $\begin{array}{l}\text { Nitric acid treatment to obtain } \mathrm{Pu}<200 \\
\mathrm{mg} / \mathrm{kg} \text { content and burial } \\
\text { Similarly } \\
\text { Combustion. Ash is supplied to the } \\
\text { operating plant to be treated } \\
\text { Similarly } \\
\text { Accumulation, settling-down. Bottoms are } \\
\text { supplied to the operating plant to be treated. } \\
\text { Solution is supplied for sorption }\end{array}$ \\
\hline 3. & $\begin{array}{l}\text { 1-3 glove box } \\
\text { Sieving, homogenization of } \\
\text { feed materials }\end{array}$ & $\begin{array}{l}\text { SRW } \\
\text { Scrap metal } \\
\text { Rubber gloves } \\
\text { Filters } \\
\text { Wiping cloth }\end{array}$ & $\begin{array}{l}\text { kg/year } \\
\text { kg/year } \\
\text { kg/year } \\
\text { kg/year }\end{array}$ & $\begin{array}{l}15 \\
10 \\
0.5 \\
2\end{array}$ & $\begin{array}{l}0.26 \pm 0.16 \\
1.42 \pm 0.76 \\
3.32 \pm 2.64 \\
7.77 \pm 4.44\end{array}$ & $\begin{array}{l}\text { over } 10^{5} \\
\text { over } 10^{5} \\
\text { over } 10^{5} \\
\text { over } 10^{5}\end{array}$ & $\begin{array}{l}\text { Nitric acid treatment to obtain } \mathrm{Pu}<200 \\
\mathrm{mg} / \mathrm{kg} \text { content and burial } \\
\text { Similarly } \\
\text { Combustion. Ash is supplied to the } \\
\text { operating plant to be treated } \\
\text { Similarly }\end{array}$ \\
\hline
\end{tabular}


II.2. Mayak Site TEF Studies of Weapons Pu Sludge Immobilization and MOX Fuel Fabrication Plutonium Wastes

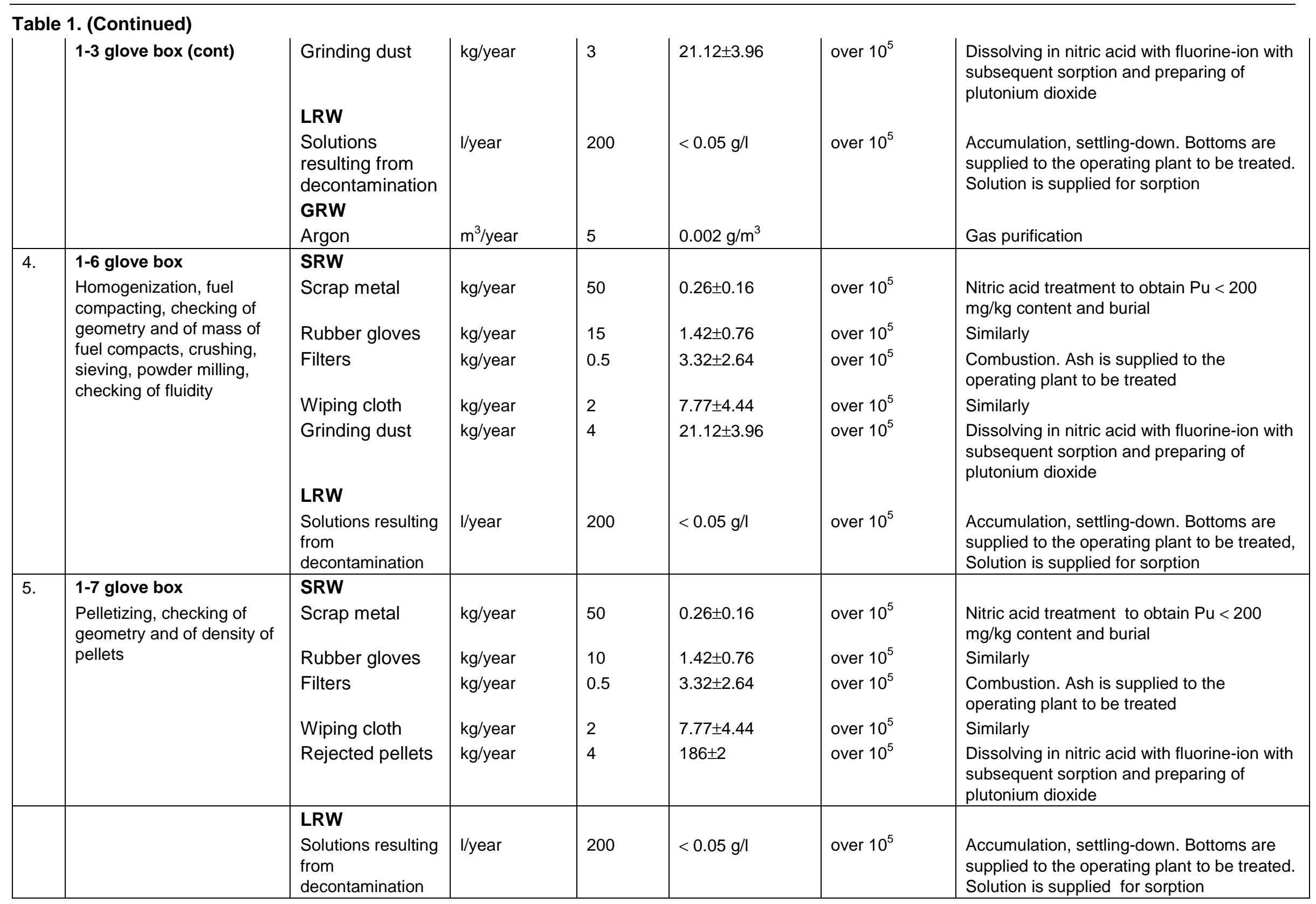


Table 1. (Continued)

\begin{tabular}{|c|c|c|c|c|c|c|c|}
\hline 6. & $\begin{array}{l}\text { 1-8 glove box } \\
\text { Sintering of pellets }\end{array}$ & $\begin{array}{l}\text { SRW } \\
\text { Molybdenum } \\
\text { cans } \\
\text { Rubber gloves } \\
\text { Filters } \\
\text { Wiping cloth } \\
\text { LRW } \\
\text { Solutions } \\
\text { resulting from } \\
\text { decontamination } \\
\text { Vacuum oil } \\
\text { GRW } \\
\text { Argon and } \\
\text { hydrogen } \\
\text { mixture }\end{array}$ & $\begin{array}{l}\text { kg/year } \\
\text { kg/year } \\
\text { kg/year } \\
\text { kg/year } \\
\text { I/year } \\
\text { I/year } \\
\mathrm{m}^{3} / \text { year }\end{array}$ & $\begin{array}{l}5 \\
10 \\
0.5 \\
1 \\
150 \\
20 \\
600\end{array}$ & $\begin{array}{l}0.02 \\
1.42 \pm 0.76 \\
3.32 \pm 2.64 \\
7.77 \pm 4.44 \\
<0.05 \mathrm{~g} / \mathrm{l} \\
<0.0005 \mathrm{~g} / \mathrm{l} \\
0.002 \mathrm{~g} / \mathrm{m}^{3}\end{array}$ & 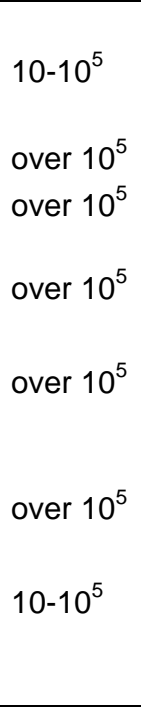 & $\begin{array}{l}\text { Nitric acid treatment to obtain } \mathrm{Pu}<200 \\
\mathrm{mg} / \mathrm{kg} \text { content and burial } \\
\text { Similarly } \\
\text { Combustion. Ash is supplied to the } \\
\text { operating plant to be treated } \\
\text { Similarly } \\
\text { Accumulation, settling-down. Bottoms are } \\
\text { supplied to the operating plant to be treated. } \\
\text { Solution is supplied for sorption. } \\
\text { Accumulation and storing } \\
\text { Gas purification }\end{array}$ \\
\hline 7. & $\begin{array}{l}\text { 2-2 box glove } \\
\text { Sampling to carry out } \\
\text { physical and chemical } \\
\text { analysis, vacuum drying of } \\
\text { pellets }\end{array}$ & $\begin{array}{l}\text { SRW } \\
\text { Scrap metal } \\
\text { Rubber gloves } \\
\text { Filters } \\
\text { Wiping cloth } \\
\text { Laboratory } \\
\text { sample residues } \\
\text { LRW } \\
\text { Solutions } \\
\text { resulting from } \\
\text { decontamination } \\
\text { Vacuum oil }\end{array}$ & $\begin{array}{l}\text { kg/year } \\
\text { kg/year } \\
\text { kg/year } \\
\text { kg/year } \\
\text { kg/year } \\
\text { I/year } \\
\text { I/year }\end{array}$ & $\begin{array}{l}5 \\
10 \\
0.5 \\
1 \\
4.2 \\
150 \\
10\end{array}$ & $\begin{array}{l}0.26 \pm 0.16 \\
1.42 \pm 0.76 \\
3.32 \pm 2.64 \\
7.77 \pm 4.44 \\
186 \pm 2 \\
<0.05 \\
<0.0005 \mathrm{~g} / \mathrm{l}\end{array}$ & $\begin{array}{l}\text { over } 10^{5} \\
\text { over } 10^{5} \\
\text { over } 10^{5} \\
\text { over } 10^{5} \\
\text { over } 10^{5} \\
\text { over } 10^{5} \\
10-10^{5}\end{array}$ & $\begin{array}{l}\text { Nitric acid treatment to obtain } \mathrm{Pu}<200 \\
\mathrm{mg} / \mathrm{kg} \text { content and burial. } \\
\text { Similarly } \\
\text { Combustion. Ash is supplied to the } \\
\text { operating plant to be treated } \\
\text { Similarly } \\
\text { Dissolving in nitric acid with fluorine-ion with } \\
\text { subsequent sorption and preparing of } \\
\text { plutonium dioxide } \\
\text { Accumulation, settling-down. Bottoms are } \\
\text { supplied to the operating plant to be treated. } \\
\text { Solution is supplied for sorption } \\
\text { Accumulation and storing }\end{array}$ \\
\hline 8. & $\begin{array}{l}\text { 2-1-1 glove box } \\
\text { Visual inspection and } \\
\text { geometry checking of } \\
\text { pellets }\end{array}$ & $\begin{array}{l}\text { SRW } \\
\text { Scrap metal } \\
\text { Rubber gloves } \\
\text { Filters }\end{array}$ & $\begin{array}{l}\mathrm{kg} / \text { year } \\
\mathrm{kg} / \text { year } \\
\mathrm{kg} / \text { year }\end{array}$ & $\begin{array}{l}10 \\
15 \\
0.5\end{array}$ & $\begin{array}{l}0.26 \pm 0.16 \\
1.42 \pm 0.76 \\
3.32 \pm 2.64\end{array}$ & $\begin{array}{l}\text { over } 10^{5} \\
\text { over } 10^{5} \\
\text { over } 10^{5}\end{array}$ & $\begin{array}{l}\text { Nitric acid treatment to obtain } \mathrm{Pu}<200 \\
\mathrm{mg} / \mathrm{kg} \text { content and burial } \\
\text { Similarly } \\
\text { Combustion. Ash is supplied to the } \\
\text { operating plant to be treated }\end{array}$ \\
\hline
\end{tabular}


II.2. Mayak Site TEF Studies of Weapons Pu Sludge Immobilization and MOX Fuel Fabrication Plutonium Wastes

\begin{tabular}{|c|c|c|c|c|c|c|c|}
\hline able & 2-1-1 glove box (cont) & $\begin{array}{l}\text { Wiping cloth } \\
\text { Rejected pellets } \\
\text { LRW } \\
\text { Solutions resulting } \\
\text { from } \\
\text { decontamination }\end{array}$ & $\begin{array}{l}\mathrm{kg} / \text { year } \\
\mathrm{kg} / \text { year } \\
\text { I/year }\end{array}$ & $\begin{array}{l}2 \\
55\end{array}$ & $\begin{array}{l}7.77 \pm 4.44 \\
186 \pm 2 \\
<0.05 \mathrm{~g} / \mathrm{l}\end{array}$ & $\begin{array}{l}\text { over } 10^{5} \\
\text { over } 10^{5} \\
\text { over } 10^{5}\end{array}$ & $\begin{array}{l}\text { Similarly } \\
\text { Dissolving in nitric acid with fluorine-ion with } \\
\text { subsequent sorption and preparing of } \\
\text { plutonium dioxide }\end{array}$ \\
\hline 9. & $\begin{array}{l}\text { 2-4 glove box } \\
\text { Charging of lower shielding } \\
\text { section, of fuel core and of } \\
\text { upper shielding section of } \\
\text { fuel elements }\end{array}$ & $\begin{array}{l}\text { SRW } \\
\text { Scrap metal } \\
\text { Rubber gloves } \\
\text { Filters } \\
\text { Wiping cloth } \\
\text { LRW } \\
\text { Solutions } \\
\text { resulting from } \\
\text { decontamination }\end{array}$ & $\begin{array}{l}\mathrm{kg} / \text { year } \\
\mathrm{kg} / \text { year } \\
\mathrm{kg} / \text { year } \\
\mathrm{kg} / \text { year } \\
\text { I/year }\end{array}$ & $\begin{array}{l}5 \\
15 \\
0.5 \\
1 \\
200\end{array}$ & $\begin{array}{l}0.26 \pm 0.16 \\
1.42 \pm 0.76 \\
3.32 \pm 2.64 \\
7.77 \pm 4.44 \\
<0.05 \mathrm{~g} / \mathrm{l}\end{array}$ & $\begin{array}{l}\text { over } 10^{5} \\
\text { over } 10^{5} \\
\text { over } 10^{5} \\
\text { over } 10^{5} \\
\text { over } 10^{5}\end{array}$ & $\begin{array}{l}\text { Nitric acid treatment to obtain } \mathrm{Pu}<200 \\
\mathrm{mg} / \mathrm{kg} \text { content and burial } \\
\text { Similarly } \\
\text { Combustion. Ash is supplied to the } \\
\text { operating plant to be treated } \\
\text { Similarly } \\
\text { Accumulation, settling-down. Bottoms are } \\
\text { supplied to the operating plants to be } \\
\text { treated. Solution is supplied for sorption }\end{array}$ \\
\hline 10. & $\begin{array}{l}\text { 2-4-1 glove box } \\
\text { Checking of the mass of } \\
\text { lower screen, of fuel core } \\
\text { and of upper screen } \\
\text { sections of fuel elements }\end{array}$ & $\begin{array}{l}\text { SRW } \\
\text { Scrap metal } \\
\text { Rubber gloves } \\
\text { Filters } \\
\text { Wiping cloth }\end{array}$ & $\begin{array}{l}\mathrm{kg} / \text { year } \\
\mathrm{kg} / \text { year } \\
\mathrm{kg} / \text { year } \\
\mathrm{kg} / \text { year }\end{array}$ & $\begin{array}{l}5 \\
10 \\
0.5 \\
1\end{array}$ & $\begin{array}{l}0.26 \pm 0.16 \\
1.42 \pm 0.76 \\
3.32 \pm 2.64 \\
7.77 \pm 4.44 \\
\end{array}$ & $\begin{array}{l}\text { over } 10^{5} \\
\text { over } 10^{5} \\
\text { over } 10^{5} \\
\text { over } 10^{5}\end{array}$ & $\begin{array}{l}\text { Nitric acid treatment to obtain } \mathrm{Pu}<200 \\
\mathrm{mg} / \mathrm{kg} \text { content and burial } \\
\text { Similarly } \\
\text { Combustion. Ash is supplied to the } \\
\text { operating plant to be treated } \\
\text { Similarly }\end{array}$ \\
\hline & & $\begin{array}{l}\text { LRW } \\
\text { Solutions } \\
\text { resulting from } \\
\text { decontamination }\end{array}$ & |/year & 150 & $<0.05 \mathrm{~g} / \mathrm{l}$ & over $10^{5}$ & $\begin{array}{l}\text { Accumulation, settling-down. Bottoms are } \\
\text { supplied to the operating plant to be treated. } \\
\text { Solution is supplied for sorption }\end{array}$ \\
\hline 11. & $\begin{array}{l}\text { 2-5 glove box } \\
\text { Placing of a lid. placing of } \\
\text { a latch, vacuum sealing, } \\
\text { helium sealing, setting of } \\
\text { the upper plug in the } \\
\text { cladding, wiping of the } \\
\text { cladding upper end using a } \\
\text { tampon, canning (welding). }\end{array}$ & $\begin{array}{l}\text { SRW } \\
\text { Scrap metal } \\
\text { Rubber gloves } \\
\text { Filters } \\
\text { Wiping cloth }\end{array}$ & $\begin{array}{l}\mathrm{kg} / \text { year } \\
\mathrm{kg} / \text { year } \\
\mathrm{kg} / \text { year } \\
\mathrm{kg} / \text { year }\end{array}$ & $\begin{array}{l}20 \\
20 \\
0.3\end{array}$ & $\begin{array}{l}0.26 \pm 0.16 \\
1.42 \pm 0.76 \\
3.32 \pm 2.64 \\
7.77 \pm 4.44\end{array}$ & $\begin{array}{l}\text { over } 10^{5} \\
\text { over } 10^{5} \\
\text { over } 10^{5} \\
\text { over } 10^{5}\end{array}$ & $\begin{array}{l}\text { Nitric acid treatment to obtain } \mathrm{Pu}<200 \\
\mathrm{mg} / \mathrm{kg} \text { content and burial } \\
\text { Similarly } \\
\text { Combustion. Ash is supplied to the } \\
\text { operating plant to be treated } \\
\text { Similarly }\end{array}$ \\
\hline
\end{tabular}


Immobilization of Weapon Pu-containing Materials and Waste of MOX Fuel Fabrication in the Russian Federation

\begin{tabular}{|c|c|c|c|c|c|c|c|}
\hline & 2-5 glove box (cont) & $\begin{array}{l}\text { LRW } \\
\text { Solutions } \\
\text { resulting from } \\
\text { decontamination } \\
\text { GRW } \\
\text { Argon } \\
\text { Helium }\end{array}$ & $\begin{array}{l}\text { l/year } \\
\mathrm{m}^{3} / \text { year } \\
\mathrm{m}^{3} / \text { year }\end{array}$ & 200 & $\begin{array}{l}<0.05 \mathrm{~g} / \mathrm{l} \\
<0.0002 \mathrm{~g} / \mathrm{m}^{3} \\
<0.0002 \mathrm{~g} / \mathrm{m}^{3}\end{array}$ & over $10^{5}$ & $\begin{array}{l}\text { Accumulation, settling-down. Bottoms are } \\
\text { supplied to the operating plant to be treated. } \\
\text { Solution is supplied for sorption } \\
\text { Gas purification } \\
\text { Similarly }\end{array}$ \\
\hline 12. & $\begin{array}{l}\text { 2-6 glove box } \\
\text { Predecontamination of fuel } \\
\text { elements. }\end{array}$ & $\begin{array}{l}\text { SRW } \\
\text { Scrap metal } \\
\text { Rubber gloves } \\
\text { Filters } \\
\text { Wiping cloth } \\
\text { LRW } \\
\text { Solutions } \\
\text { resulting from } \\
\text { decontamination }\end{array}$ & $\begin{array}{l}\mathrm{kg} / \text { year } \\
\mathrm{kg} / \text { year } \\
\mathrm{kg} / \text { year } \\
\mathrm{kg} / \text { year } \\
\mathrm{I} / \text { year }\end{array}$ & $\begin{array}{l}5 \\
10 \\
0.2 \\
1 \\
200\end{array}$ & $\begin{array}{l}0.26 \pm 0.16 \\
1.42 \pm 0.76 \\
3.32 \pm 2.64 \\
7.77 \pm 4.44 \\
<0.05 \mathrm{~g} / 1\end{array}$ & $\begin{array}{l}\text { over } 10^{5} \\
\text { over } 10^{5} \\
\text { over } 10^{5} \\
\text { over } 10^{5} \\
\text { over } 10^{5}\end{array}$ & $\begin{array}{l}\text { Nitric acid treatment to obtain } \mathrm{Pu}<200 \\
\mathrm{mg} / \mathrm{kg} \text { content and burial } \\
\text { Similarly } \\
\text { Combustion. Ash is supplied to the } \\
\text { operating plant to be treated } \\
\text { Similarly } \\
\text { Accumulation, settling-down. Bottoms are } \\
\text { supplied to the operating plant to be treated. } \\
\text { Solution is supplied for sorption }\end{array}$ \\
\hline 13. & $\begin{array}{l}\text { 2-7 glove box } \\
\text { "Cold" detection of fuel } \\
\text { element failure }\end{array}$ & $\begin{array}{l}\text { SRW } \\
\text { Scrap metal } \\
\text { Rubber gloves } \\
\text { Filters } \\
\text { Wiping cloth } \\
\text { LRW } \\
\text { Solutions resulting } \\
\text { from } \\
\text { decontamination } \\
\text { Vacuum oil }\end{array}$ & $\begin{array}{l}\mathrm{kg} / \text { year } \\
\mathrm{kg} / \text { year } \\
\mathrm{kg} / \text { year } \\
\mathrm{kg} / \text { year } \\
\text { I/year } \\
\text { I/year }\end{array}$ & $\begin{array}{l}5 \\
10 \\
0.3 \\
1 \\
150 \\
10 \\
\end{array}$ & $\begin{array}{l}0.1 \\
0.66 \\
0.68 \\
3.33 \\
<0.05 \mathrm{~g} / \mathrm{l} \\
<0.0005 \mathrm{~g} / \mathrm{l}\end{array}$ & $\begin{array}{l}\text { over } 10^{5} \\
\text { over } 10^{5} \\
\text { over } 10^{5} \\
\text { over } 10^{5} \\
\text { over } 10^{5} \\
10-10^{5}\end{array}$ & $\begin{array}{l}\text { Nitric acid treatment to obtain } \mathrm{Pu}<200 \\
\text { mg/kg content and burial } \\
\text { Similarly } \\
\text { Combustion. Ash is supplied to the } \\
\text { operating plant to be treated } \\
\text { Similarly } \\
\text { Accumulation, settling-down. Bottoms are } \\
\text { supplied to the operating plant to be treated. } \\
\text { Solution is supplied for sorption } \\
\text { Accumulation and storing }\end{array}$ \\
\hline 14. & $\begin{array}{l}\text { EKhUZO facility } \\
\text { Decontamination of fuel } \\
\text { elements }\end{array}$ & $\begin{array}{l}\text { LRW } \\
\text { Solutions resulting } \\
\text { from } \\
\text { decontamination } \\
\end{array}$ & I/year & 1000 & $<0.05 \mathrm{~g} / \mathrm{l}$ & over $10^{5}$ & $\begin{array}{l}\text { Accumulation, settling-down. Bottoms are } \\
\text { supplied to the operating plant to be treated. } \\
\text { Solution is supplied for sorption }\end{array}$ \\
\hline 15. & $\begin{array}{l}\text { Classifying table } \\
\text { Checking of fuel element } \\
\text { surface cleanliness using } \\
\text { smear technique }\end{array}$ & $\begin{array}{l}\text { SRW } \\
\text { Wiping cloth }\end{array}$ & $\mathrm{kg} /$ year & 3 & 3.33 & over $10^{5}$ & $\begin{array}{l}\text { Combustion. Ash is supplied to the } \\
\text { operating plant to be treated }\end{array}$ \\
\hline
\end{tabular}


II.2. Mayak Site TEF Studies of Weapons Pu Sludge Immobilization and MOX Fuel Fabrication Plutonium Wastes

\begin{tabular}{|c|c|c|c|c|c|c|c|}
\hline 16. & $\begin{array}{l}\text { 11/1, 2, } 3 \text { furnaces } \\
\text { "Hot" detection of fuel } \\
\text { element failure }\end{array}$ & $\begin{array}{l}\text { SRW } \\
\text { Filters } \\
\text { LRW } \\
\text { Vacuum oil }\end{array}$ & $\begin{array}{l}\mathrm{kg} / \text { year } \\
\text { l/year }\end{array}$ & $\begin{array}{l}0.2 \\
20 \\
\end{array}$ & $\begin{array}{l}0.68 \\
<0.0005 \mathrm{~g} / 1\end{array}$ & $\begin{array}{l}\text { over } 10^{5} \\
10-10^{5}\end{array}$ & $\begin{array}{l}\text { Combustion. Ash is supplied to the } \\
\text { operating plant to be treated } \\
\text { Accumulation and storing }\end{array}$ \\
\hline 17. & $\begin{array}{l}\text { 2-3-1 glove box } \\
\text { Classifying tables, the } \\
\text { IGLA facility } \\
\text { Detection of fuel element } \\
\text { surface contamination, } \\
\text { visual inspection, checking } \\
\text { of markings, checking of } \\
\text { length of fuel elements, } \\
\text { checking of plane } \\
\text { geometry, checking of } \\
\text { welded joint diameter, } \\
\text { helium content control by } \\
\text { means of the IGLA facility, } \\
\text { checking of fuel core } \\
\text { integrity (uniformity), } \\
\text { testing of welded joints of } \\
\text { ready-made items }\end{array}$ & $\begin{array}{l}\text { SRW } \\
\text { Scrap metal } \\
\text { Rubber gloves } \\
\text { Filters } \\
\text { Wiping cloth } \\
\text { LRW } \\
\text { Solutions resulting } \\
\text { from } \\
\text { decontamination }\end{array}$ & $\begin{array}{l}\mathrm{kg} / \text { year } \\
\mathrm{kg} / \text { year } \\
\mathrm{kg} / \text { year } \\
\mathrm{kg} / \text { year } \\
\mathrm{l} / \text { year }\end{array}$ & $\begin{array}{l}10 \\
10 \\
0.5 \\
1 \\
\\
200\end{array}$ & $\begin{array}{l}0.26 \pm 0.16 \\
1.42 \pm 0.76 \\
3.32 \pm 2.64 \\
7.77 \pm 4.44 \\
<0.05 \mathrm{~g} / \mathrm{l}\end{array}$ & $\begin{array}{l}\text { over } 10^{5} \\
\text { over } 10^{5} \\
\text { over } 10^{5} \\
\text { over } 10^{5} \\
\text { over } 10^{5}\end{array}$ & $\begin{array}{l}\text { Nitric acid treatment to obtain } \mathrm{Pu}<200 \\
\mathrm{mg} / \mathrm{kg} \text { content and burial } \\
\text { Similarly } \\
\text { Combustion. Ash is supplied to the } \\
\text { operating plant to be treated } \\
\text { Similarly } \\
\text { Accumulation, Settling-down. Bottoms are } \\
\text { supplied to the operating plant to be treated. } \\
\text { Solution is supplied to be sorbed }\end{array}$ \\
\hline 18. & $\begin{array}{l}\text { Packing tables } \\
\text { Packing of fuel elements in } \\
\text { containers, storage } \\
\end{array}$ & $\begin{array}{l}\text { SRW } \\
\text { Scrap metal }\end{array}$ & $\mathrm{kg} /$ year & 5 & 0.01 & $10-10^{5}$ & Burial \\
\hline 19. & $\begin{array}{l}\text { B 2-3-1 glove box } \\
\text { Disassembling of rejected } \\
\text { fuel elements }\end{array}$ & $\begin{array}{l}\text { SRW } \\
\text { Scrap metal } \\
\text { Rubber gloves } \\
\text { Filters } \\
\text { Wiping cloth } \\
\text { Rejected } \\
\text { pellets } \\
\text { Grinding dust } \\
\text { LRW }\end{array}$ & $\begin{array}{l}\mathrm{kg} / \text { year } \\
\mathrm{kg} / \text { year } \\
\mathrm{kg} / \text { year } \\
\mathrm{kg} / \text { year } \\
\mathrm{kg} / \text { year } \\
\mathrm{kg} / \text { year }\end{array}$ & $\begin{array}{l}50 \\
\\
15 \\
0.5 \\
\\
1 \\
2.5\end{array}$ & $\begin{array}{l}0.26 \pm 0.16 \\
1.42 \pm 0.76 \\
3.32 \pm 2.64 \\
7.77 \pm 4.44 \\
186 \pm 2 \\
186 \pm 2\end{array}$ & $\begin{array}{l}\text { over } 10^{5} \\
\text { over } 10^{5} \\
\text { over } 10^{5} \\
\text { over } 10^{5} \\
\text { over } 10^{5} \\
\text { over } 10^{5}\end{array}$ & $\begin{array}{l}\text { Nitric acid treatment to obtain } \mathrm{Pu}<200 \\
\mathrm{mg} / \mathrm{kg} \text { content and burial } \\
\text { Similarly } \\
\text { Combustion. Ash is supplied to the } \\
\text { operating plant to be treated. } \\
\text { Similarly } \\
\text { Dissolving in nitric acid with fluorine-ion with } \\
\text { subsequent sorption and preparing of } \\
\text { plutonium dioxide } \\
\text { Similarly }\end{array}$ \\
\hline
\end{tabular}


Immobilization of Weapon Pu-containing Materials and Waste of MOX Fuel Fabrication in the Russian Federation

\begin{tabular}{|c|c|c|c|c|c|c|c|}
\hline \multicolumn{8}{|c|}{ Table 1. (Continued) } \\
\hline & B 2-3-1 glove box (cont) & \begin{tabular}{|l} 
Solutions \\
resulting from \\
decontaminatio \\
n
\end{tabular} & I/year & 200 & $<0.05 \mathrm{~g} / \mathrm{l}$ & over $10^{5}$ & $\begin{array}{l}\text { Accumulation, settling-down. Bottoms are } \\
\text { supplied to the operating plant to be treated. } \\
\text { Solution is supplied to be sorbed }\end{array}$ \\
\hline 20. & \multicolumn{2}{|c|}{ Laboratory solutions (resulting from analyses) } & 1/year & 5 & $2.5 \mathrm{~g} / \mathrm{l}$ & over $10^{5}$ & To be sorbed. \\
\hline 21. & \multicolumn{2}{|c|}{$\begin{array}{l}\text { Solutions resulting from washing of repair bay } \\
\text { floors }\end{array}$} & $\mathrm{m}^{3} /$ year & 21,6 & $<0.003 \mathrm{~g} / \mathrm{l}$ & $10-10^{5}$ & $\begin{array}{l}\text { Accumulation, settling-down. Bottoms are } \\
\text { supplied to the operating plant to be treated } \\
\text { Solution is supplied to be sorbed }\end{array}$ \\
\hline 22. & \multicolumn{2}{|c|}{$\begin{array}{l}\text { Solutions resulting from washing of operator's } \\
\text { area floors }\end{array}$} & $\mathrm{m}^{3} /$ year & 21,6 & $<0.0005 \mathrm{~g} / \mathrm{l}$ & $10-10^{5}$ & $\begin{array}{l}\text { Accumulation, settling-down. Bottoms are } \\
\text { supplied to the operating plant to be treated } \\
\text { Solution is supplied to be sorbed }\end{array}$ \\
\hline
\end{tabular}




\section{Brief Information on MOX-Fuel Commercial Fabrication}

The source information is based on the data on the future MOX-fuel production used by the US-Russian working group for the TES calculations to estimate the costs to implement the basic scenario of the Program to Dispose the Russian Weapon Plutonium.

$\mathrm{U}$ and Pu dioxide powders prepared via nitrate oxalate precipitation are used as the feed material to fabricate fuel assemblies.

The master mix for pelletized fuel are planned to be prepared via mechanical mixing. The COGEMA Company A-MIMAS technology should be followed to prepare the mixed powder, while the process equipment to prepare powder should be similar to that of the Hanau plant.

Charging and canning of fuel elements and gathering of fuel assemblies will follow the Russian technology and the Russian-made equipment will be used.

The fabrication plant to be sited at the Mayak PA includes as follows:

- $\quad 300 \mathrm{~d}$ building designed to fabricate BN-600 and VVER-1000 reactor fuel elements to be constructed at RT-1 plant site;

- Gathering of BN-600 and VVER-1000 reactor fuel assemblies, their packing in transport and packing containers, storing, batching and loading to transport vehicles should be in 281D building to be constructed using building structures of the partially constructed 281 building placed at the extended site of the RI plant.

\section{Range of Products and Design Capacity of MOX-Fuel Commercial Fabrication Facility}

The MOX-fuel commercial fabrication facility is designed to fabricate BN-600 and VVER-1000 reactor fuel elements.

The capacity of the facility is as follows:

- 36685 fuel elements per year to gather 286 BN-600 fuel assemblies (including 363 fuel elements to compensate for process rejects and for destructive acceptance tests of ready-made items);

- 31512 fuel elements per year to gather 100 VVER-1000 fuel assemblies (including 312 fuel elements to compensate for process rejects and for destructive acceptance testing of ready-made items)

\section{Basic Processes of MOX-Fuel Commercial Fabrication Facility}

Procedure to fabricate fuel elements consists of the following basic processes:

- Receipt of feed materials - plutonium dioxide and uranium dioxide;

- Preparing of master-mixture of uranium and plutonium dioxides $\left(\mathrm{PuO}_{2}\right.$ content, 25-30\%) with addition of a binder (zinc stearate);

- Preparing of mixture of plutonium and uranium dioxides for BN-600 reactor fuel elements, mixture homogenization;

- Preparing of mixture of plutonium and uranium dioxides for VVER-1000 reactor fuel elements, mixture homogenization; 
- Pelletizing, check of pellet density;

- Sintering of pellets;

- Grinding of sintered pellets for VVER-1000 reactor fuel elements;

- Sorting of pellets;

- Recycling of rejects;

- Charging of fuel elements, their canning and inspection;

- Recycling of rejected fuel elements;

- Sorting of feed materials, of intermediate and final products. 


\section{Waste Category, Amount and Processes to Treat Waste Using MOX-Fuel Commercial Fabrication Facility}

Table 2. Waste category, amount, and processes to treat waste using MOX fuel commercial fabrication facility.

\begin{tabular}{|c|c|c|c|c|c|c|c|}
\hline \multirow[b]{2}{*}{${ }^{1}$ No } & \multirow[b]{2}{*}{$\begin{array}{l}\text { Equipment, } \\
\text { procedures }\end{array}$} & \multicolumn{5}{|c|}{ Waste } & \multirow[b]{2}{*}{ Processes to treat waste } \\
\hline & & Waste category (type) & $\begin{array}{l}\text { Units of } \\
\text { measure } \\
\text { ment }\end{array}$ & Amount & $\begin{array}{l}\text { Pu } \\
\text { content, } \\
\text { g/kg of } \\
\text { waste }\end{array}$ & $\begin{array}{c}\text { Specific } \\
\text { activity, } \\
\text { kBq/kg }\left(I ; \mathbf{m}^{3}\right)\end{array}$ & \\
\hline 1. & $\begin{array}{l}\text { B-1 glove box } \\
\text { Receipt and } \\
\text { unpacking-repacking } \\
\text { of } \mathrm{UO}_{2}\end{array}$ & $\begin{array}{l}\text { SRW } \\
\text { Scrap metal } \\
\text { Polyethylene film, rubber gloves, } \\
\text { wiping cloth, plastic compound } \\
\text { Filters }\end{array}$ & $\begin{array}{l}\mathrm{kg} / \text { year } \\
\mathrm{kg} / \text { year } \\
\mathrm{kg} / \text { year }\end{array}$ & $\begin{array}{l}6200 \\
150\end{array}$ & $\begin{array}{l}<0.150(\mathrm{U}) \\
<0.150(\mathrm{U}) \\
<10.0(\mathrm{U})\end{array}$ & $\begin{array}{l}\text { under } 10 \\
\text { under } 10 \\
\text { under } 10\end{array}$ & $\begin{array}{l}\text { Empty casks - to be reused } \\
\text { Waste sorting according to waste } \\
\text { categories, packing in special containers, } \\
\text { packing into intraplant containers, } \\
\text { transportation to the waste treatment } \\
\text { facility }\end{array}$ \\
\hline 2. & $\begin{array}{l}\text { B-2 glove box } \\
\text { Receipt of containers } \\
\text { and taking of } \mathrm{PuO}_{2} \\
\text { containing cans out of } \\
\text { containers }\end{array}$ & $\begin{array}{l}\text { SRW } \\
\text { Scrap metal } \\
\text { Polyethylene film, rubber gloves, } \\
\text { wiping cloth, plastic compound } \\
\text { Filters }\end{array}$ & $\begin{array}{l}\mathrm{kg} / \text { year } \\
\mathrm{kg} / \text { year } \\
\text { kg/year }\end{array}$ & $\begin{array}{l}300 \\
150\end{array}$ & $\begin{array}{l}<0.150 \\
<0.150 \\
<10.0\end{array}$ & $\begin{array}{l}\text { over } 10^{5} \\
\text { over } 10^{5} \\
\text { over } 10^{5} \\
\end{array}$ & $\begin{array}{l}\text { Waste sorting according to waste } \\
\text { categories, packing in special containers, } \\
\text { packing in intraplant containers, } \\
\text { transportation to waste treatment facility }\end{array}$ \\
\hline 3. & $\begin{array}{l}\text { B3 glove box } \\
\text { Storing of } \mathrm{PuO}_{2} \\
\text { containing cans }\end{array}$ & $\begin{array}{l}\text { SRW } \\
\text { Scrap metal } \\
\text { Rubber gloves, wiping cloth, plastic } \\
\text { compound } \\
\text { Filters } \\
\text { GRW } \\
\text { Nitrogen }\end{array}$ & $\begin{array}{l}\text { kg/year } \\
\text { kg/year } \\
\text { kg/year } \\
\mathrm{m}^{3} / \text { year }\end{array}$ & $\begin{array}{l}50 \\
50 \\
3 \\
60000\end{array}$ & $\begin{array}{l}<0.150 \\
<0.150 \\
<10.0 \\
<0.0002 \\
\mathrm{~g} / \mathrm{m}^{3}\end{array}$ & $\begin{array}{l}\text { over } 10^{5} \\
\text { over } 10^{5} \\
\text { over } 10^{5}\end{array}$ & $\begin{array}{l}\text { Waste sorting according to waste } \\
\text { categories, packing in special containers, } \\
\text { packing in intraplant containers, } \\
\text { transportation to waste treatment facility } \\
\text { Gas purification }\end{array}$ \\
\hline
\end{tabular}


Immobilization of Weapon Pu-containing Materials and Waste of MOX Fuel Fabrication in the Russian Federation

\begin{tabular}{|c|c|c|c|c|c|c|c|}
\hline 4. & $\begin{array}{l}\text { B4-1,2 glove box } \\
\text { Preparing of master- } \\
\text { mixture }\end{array}$ & $\begin{array}{l}\text { SRW } \\
\text { Scrap metal } \\
\text { Scrap (lids, cans) } \\
\text { Rubber gloves, wiping cloth, plastic } \\
\text { compound } \\
\text { Filters } \\
\text { GRW } \\
\text { Nitrogen }\end{array}$ & $\begin{array}{l}\mathrm{kg} / \text { year } \\
\mathrm{kg} / \text { year } \\
\mathrm{kg} / \text { year } \\
\mathrm{kg} / \text { year } \\
\mathrm{m}^{3} / \text { year }\end{array}$ & $\begin{array}{l}500 \\
600 \\
200 \\
12 \\
120000\end{array}$ & $\begin{array}{l}<0.150 \\
<0.500 \\
<0.150 \\
<10.0 \\
<0.0002 \\
\mathrm{~g} / \mathrm{m}^{3}\end{array}$ & $\begin{array}{l}\text { over } 10^{5} \\
\text { over } 10^{5} \\
\text { over } 10^{5} \\
\text { over } 10^{5}\end{array}$ & $\begin{array}{l}\text { Waste sorting according to waste } \\
\text { categories, packing in special containers, } \\
\text { packing in intraplant containers, } \\
\text { transportation to waste treatment facility } \\
\text { Gas purification }\end{array}$ \\
\hline 5. & $\begin{array}{l}\text { B5 glove box } \\
\text { Storing of master- } \\
\text { mixture }\end{array}$ & $\begin{array}{l}\text { SRW } \\
\text { Scrap metal } \\
\text { Rubber gloves, wiping cloth, plastic } \\
\text { compound } \\
\text { Filters } \\
\text { LRW } \\
\text { Nitrogen }\end{array}$ & $\begin{array}{l}\mathrm{kg} / \text { year } \\
\mathrm{kg} / \text { year } \\
\mathrm{kg} / \text { year } \\
\mathrm{m}^{3} / \text { year }\end{array}$ & $\begin{array}{l}50 \\
50 \\
2 \\
60000\end{array}$ & $\begin{array}{l}<0.150 \\
<0.150 \\
<10.0 \\
<0.0002 \\
\mathrm{~g} / \mathrm{m}^{3}\end{array}$ & $\begin{array}{l}\text { over } 10^{5} \\
\text { over } 10^{5} \\
\text { over } 10^{5}\end{array}$ & $\begin{array}{l}\text { Waste sorting according to waste category } \\
\text { packing in special containers, packing in } \\
\text { intraplant containers, transportation to } \\
\text { waste treatment facility } \\
\text { Gas purification }\end{array}$ \\
\hline 6. & $\begin{array}{l}\text { B6 glove box } \\
\text { Sieving of master- } \\
\text { mixture }\end{array}$ & $\begin{array}{l}\text { SRW } \\
\text { Scrap metal } \\
\text { Rubber gloves, wiping cloth, plastic } \\
\text { compound } \\
\text { Filters } \\
\text { Grinding dust } \\
\text { GRW } \\
\text { Nitrogen }\end{array}$ & $\begin{array}{l}\mathrm{kg} / \text { year } \\
\mathrm{kg} / \text { year } \\
\mathrm{kg} / \text { year } \\
\mathrm{kg} / \text { year } \\
\mathrm{m}^{3} / \text { year }\end{array}$ & $\begin{array}{l}200 \\
100\end{array}$ & $\begin{array}{l}<0.150 \\
<0.150 \\
<10.0 \\
<300.0 \\
\\
<0.0002 \\
\mathrm{~g} / \mathrm{m}^{3}\end{array}$ & $\begin{array}{l}\text { over } 10^{5} \\
\text { over } 10^{5} \\
\text { over } 10^{5} \\
\text { over } 10^{5}\end{array}$ & $\begin{array}{l}\text { Waste sorting according to waste } \\
\text { categories, packing in special containers, } \\
\text { packing in intraplant containers, } \\
\text { transportation to waste treatment facility } \\
\text { Packing in cans, packing of cans in } \\
\text { intraplant container, transportation to waste } \\
\text { treatment facility } \\
\text { Gas purification }\end{array}$ \\
\hline
\end{tabular}


1I.2. Mayak Site TEF Studies of Weapons Pu Sludge Immobilization and MOX Fuel Fabrication Plutonium Wastes

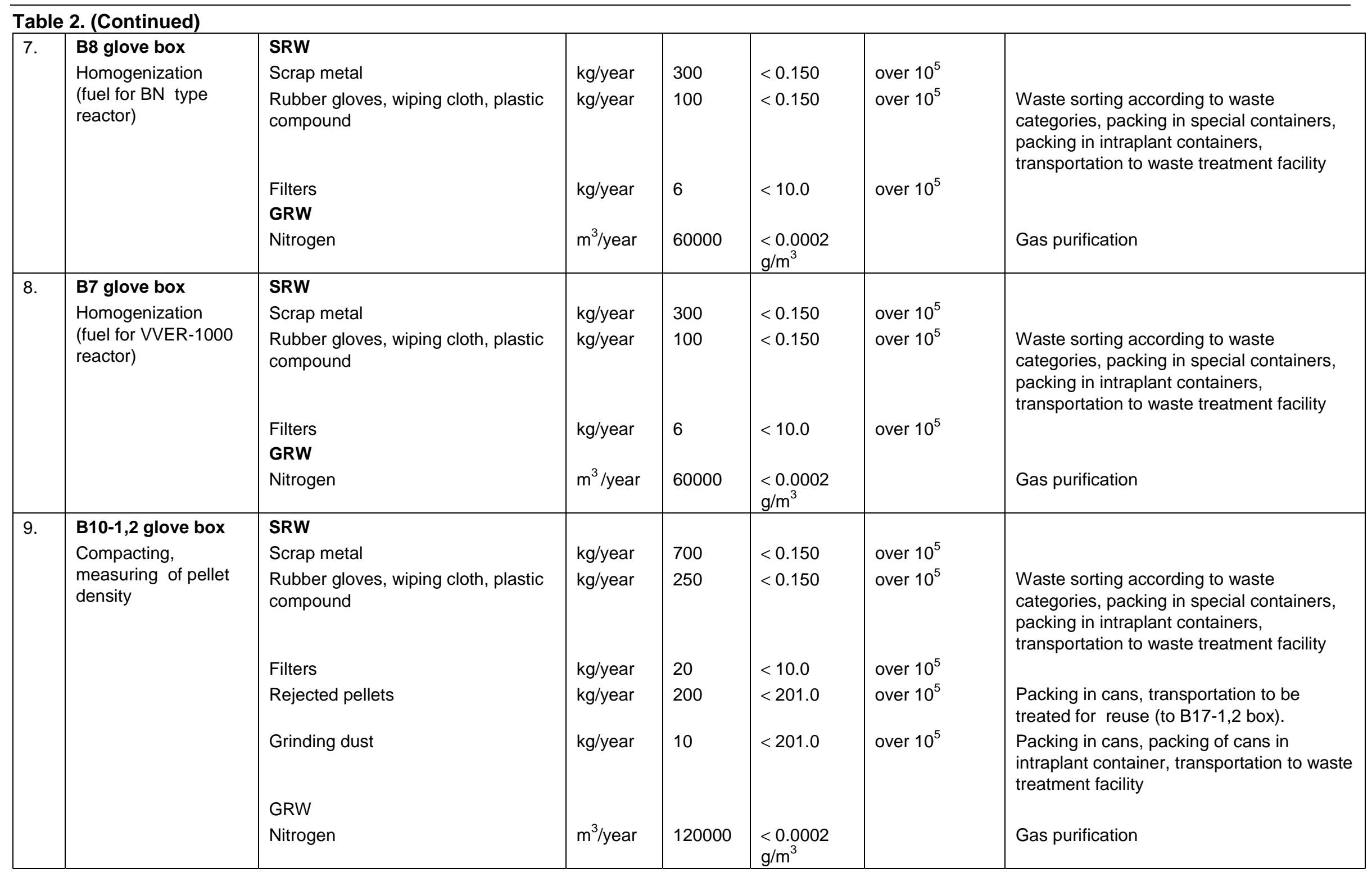


Immobilization of Weapon Pu-containing Materials and Waste of MOX Fuel Fabrication in the Russian Federation

\begin{tabular}{|c|c|c|c|c|c|c|c|}
\hline \multicolumn{8}{|c|}{ able 2. (Continuec } \\
\hline 10. & $\begin{array}{l}\text { B11 glove box } \\
\text { Storing of green } \\
\text { pellets }\end{array}$ & $\begin{array}{l}\text { SRW } \\
\text { Scrap metal } \\
\text { Rubber gloves, wiping cloth, plastic } \\
\text { compound } \\
\text { Filters } \\
\text { GRW } \\
\text { Nitrogen }\end{array}$ & $\begin{array}{l}\mathrm{kg} / \text { year } \\
\mathrm{kg} / \text { year } \\
\mathrm{kg} / \text { year } \\
\mathrm{m}^{3} / \text { year }\end{array}$ & $\begin{array}{l}3 \\
60000\end{array}$ & $\begin{array}{l}<0.150 \\
<0.150 \\
<10.0 \\
<0.0002 \\
\mathrm{~g} / \mathrm{m}^{3}\end{array}$ & $\begin{array}{l}\text { over } 10^{5} \\
\text { over } 10^{5} \\
\text { over } 10^{5}\end{array}$ & $\begin{array}{l}\text { Waste sorting according to waste } \\
\text { categories, packing in special containers, } \\
\text { packing in intraplant containers, } \\
\text { transportation to waste treatment facility } \\
\text { Gas purification }\end{array}$ \\
\hline 11. & $\begin{array}{l}\text { B12-1,2,3 glove box } \\
\text { Sintering of pellets }\end{array}$ & $\begin{array}{l}\text { SRW } \\
\text { Molybdenum pans } \\
\text { Rubber gloves, wiping cloth, plastic } \\
\text { compound } \\
\text { Filters } \\
\text { Ceramics } \\
\text { LRW } \\
\text { Vacuum oil } \\
\text { GRW } \\
\text { Argon and hydrogen mixture }\end{array}$ & $\begin{array}{l}\mathrm{kg} / \text { year } \\
\mathrm{kg} / \text { year } \\
\mathrm{kg} / \text { year } \\
\mathrm{kg} / \text { year } \\
\mathrm{I} / \text { year } \\
\mathrm{m}^{3} / \text { year }\end{array}$ & $\begin{array}{l}12 \\
60 \\
300 \\
140000 \\
\end{array}$ & $\begin{array}{l}<0.02 \\
<0.150 \\
\\
<10.0 \\
<1.50 \\
<0.001 \mathrm{~g} / 1 \\
0.002 \mathrm{~g} / \mathrm{m}^{3}\end{array}$ & $\begin{array}{l}\begin{array}{l}10-10^{5} \\
\text { over } 10^{5}\end{array} \\
\text { over } 10^{5} \\
\text { over } 10^{5} \\
10-10^{5}\end{array}$ & $\begin{array}{l}\text { Waste sorting according to waste } \\
\text { categories, packing in special containers, } \\
\text { packing in intraplant containers, } \\
\text { transportation to waste treatment facility } \\
\text { Packing in special casks, transportation to } \\
\text { waste treatment facility } \\
\text { Gas purification }\end{array}$ \\
\hline 12. & $\begin{array}{l}\text { B14-1,2 glove box } \\
\text { Grinding, crushing of } \\
\text { rejected sintered } \\
\text { pellets (VVER-1000 } \\
\text { pellets) }\end{array}$ & $\begin{array}{l}\text { SRW } \\
\text { Scrap metal } \\
\text { Rubber gloves, wiping cloth, plastic } \\
\text { compound } \\
\text { Filters } \\
\text { Grinding wheels } \\
\text { Rejected pellets } \\
\text { Grinding dust } \\
\text { GRW } \\
\text { Nitrogen }\end{array}$ & $\begin{array}{l}\mathrm{kg} / \text { year } \\
\mathrm{kg} / \text { year } \\
\mathrm{kg} / \text { year } \\
\mathrm{kg} / \text { year } \\
\mathrm{m}^{3} / \text { year }\end{array}$ & \begin{tabular}{|l}
12 \\
50 \\
600 \\
5
\end{tabular} & $\begin{array}{l}<10.0 \\
<1.50 \\
<63.0 \\
<63.0\end{array}$ & $\begin{array}{l}\text { over } 10^{5} \\
\text { over } 10^{5} \\
\text { over } 10^{5} \\
\text { over } 10^{5}\end{array}$ & $\begin{array}{l}\text { Packing in cans, transportation to be } \\
\text { treated for reuse (in B17-1,2 box). } \\
\text { Packing in cans, packing of cans in } \\
\text { intraplant container, transportation to waste } \\
\text { treatment facility } \\
\text { Gas purification }\end{array}$ \\
\hline
\end{tabular}


II.2. Mayak Site TEF Studies of Weapons Pu Sludge Immobilization and MOX Fuel Fabrication Plutonium Wastes

Table 2. (Continued)

\begin{tabular}{|c|c|c|c|c|c|c|c|}
\hline 13. & $\begin{array}{l}\text { B13 glove box } \\
\text { Storing of sorted } \\
\text { pellets } \\
\text { (for VVER-1000 } \\
\text { reactor) }\end{array}$ & $\begin{array}{l}\text { SRW } \\
\text { Scrap metal } \\
\text { Rubber gloves, wiping cloth, plastic } \\
\text { compound } \\
\text { Filters } \\
\text { GRW } \\
\text { Nitrogen }\end{array}$ & $\begin{array}{l}\mathrm{kg} / \text { year } \\
\mathrm{kg} / \text { year } \\
\mathrm{kg} / \text { year } \\
\mathrm{m}^{3} / \text { year }\end{array}$ & $\begin{array}{l}50 \\
50 \\
4 \\
60000\end{array}$ & $\begin{array}{l}<0.150 \\
<0.150 \\
<10.0 \\
<0.0002 \\
\mathrm{~g} / \mathrm{m}^{3}\end{array}$ & $\begin{array}{l}\text { over } 10^{5} \\
\text { over } 10^{5} \\
\text { over } 10^{5}\end{array}$ & $\begin{array}{l}\text { Waste sorting according to waste } \\
\text { categories, packing in special containers, } \\
\text { packing in intraplant containers, transition } \\
\text { to waste treatment facility } \\
\text { Gas purification }\end{array}$ \\
\hline 14. & $\begin{array}{l}\text { B15-1.2 glove box } \\
\text { Sorting of pellets } \\
\text { (for BN type reactor) }\end{array}$ & $\begin{array}{l}\text { SRW } \\
\text { Scrap metal } \\
\text { Rubber gloves, wiping cloth, plastic } \\
\text { compound } \\
\text { Filters } \\
\text { Rejected pellets } \\
\text { GRW } \\
\text { Nitrogen }\end{array}$ & $\begin{array}{l}\mathrm{kg} / \text { year } \\
\mathrm{kg} / \text { year } \\
\mathrm{kg} / \text { year } \\
\mathrm{kg} / \text { year } \\
\mathrm{m}^{3} / \text { year }\end{array}$ & $\begin{array}{l}100 \\
50 \\
4 \\
30 \\
120000\end{array}$ & $\begin{array}{l}<0.150 \\
<0.150 \\
<10.0 \\
<201.0 \\
<0.0002 \\
\mathrm{~g} / \mathrm{m}^{3}\end{array}$ & $\begin{array}{l}\text { over } 10^{5} \\
\text { over } 10^{5} \\
\text { over } 10^{5} \\
\text { over } 10^{5}\end{array}$ & $\begin{array}{l}\text { Waste sorting according to waste } \\
\text { categories, packing in special containers, } \\
\text { packing in intraplant containers, } \\
\text { transportation to waste treatment facility } \\
\text { Packing in cans, transportation to be } \\
\text { treated for reuse (in B17-1,2 box). } \\
\text { Gas purification }\end{array}$ \\
\hline 15. & $\begin{array}{l}\text { B16-1,2 glove box } \\
\text { Storing of pellets (for } \\
\text { BN reactor) }\end{array}$ & $\begin{array}{l}\text { SRW } \\
\text { Scrap metal } \\
\text { Rubber gloves, wiping cloth, plastic } \\
\text { compound } \\
\text { Filters } \\
\text { GRW } \\
\text { Nitrogen }\end{array}$ & $\begin{array}{l}\mathrm{kg} / \text { year } \\
\mathrm{m}^{3} / \text { year }\end{array}$ & $\begin{array}{l}4 \\
120000\end{array}$ & $\begin{array}{l}<0.150 \\
<0.150 \\
<10.0 \\
<0.0002 \\
\mathrm{~g} / \mathrm{m}^{3}\end{array}$ & $\begin{array}{l}\text { over } 10^{5} \\
\text { over } 10^{5} \\
\text { over } 10^{5}\end{array}$ & $\begin{array}{l}\text { Waste sorting according to waste } \\
\text { categories, packing in special containers, } \\
\text { packing in intraplant containers, } \\
\text { transportation to waste treatment facility } \\
\text { Gas purification }\end{array}$ \\
\hline
\end{tabular}


Immobilization of Weapon Pu-containing Materials and Waste of MOX Fuel Fabrication in the Russian Federation

\begin{tabular}{|c|c|c|c|c|c|c|c|}
\hline 16. & $\begin{array}{l}\text { B36 glove box } \\
\text { Assembling of core } \\
\text { column, checking of } \\
\text { column length and } \\
\text { mass (for BN reactor) }\end{array}$ & $\begin{array}{l}\text { SRW } \\
\text { Scrap metal } \\
\text { Rubber gloves, wiping cloth, plastic } \\
\text { compound } \\
\text { Filters } \\
\text { GRW } \\
\text { Nitrogen }\end{array}$ & $\begin{array}{l}\mathrm{kg} / \text { year } \\
\mathrm{kg} / \text { year } \\
\mathrm{kg} / \text { year } \\
\mathrm{m}^{3} / \text { year }\end{array}$ & $\begin{array}{l}150 \\
50 \\
4 \\
60000\end{array}$ & $\begin{array}{l}<0.150 \\
<0.150 \\
<10.0 \\
<0.0002 \\
\mathrm{~g} / \mathrm{m}^{3}\end{array}$ & $\begin{array}{l}\text { over } 10^{5} \\
\text { over } 10^{5} \\
\text { over } 10^{5}\end{array}$ & $\begin{array}{l}\text { Waste sorting according to waste } \\
\text { categories, packing in special containers, } \\
\text { packing in intraplant containers, } \\
\text { transportation to waste treatment facility } \\
\text { Gas purification }\end{array}$ \\
\hline 17. & $\begin{array}{l}\text { B39 glove box } \\
\text { Assembling of lower } \\
\text { shield column, } \\
\text { checking of column } \\
\text { length and mass (for } \\
\text { BN type reactor) }\end{array}$ & $\begin{array}{l}\text { SRW } \\
\text { Scrap metal } \\
\text { Rubber gloves, wiping cloth, plastic } \\
\text { compound } \\
\text { Filters } \\
\text { GRW } \\
\text { Nitrogen }\end{array}$ & $\begin{array}{l}\mathrm{kg} / \text { year } \\
\mathrm{kg} / \text { year } \\
\mathrm{kg} / \text { year } \\
\mathrm{m}^{3} / \text { year }\end{array}$ & $\begin{array}{l}150 \\
50 \\
4 \\
60000\end{array}$ & $\begin{array}{l}<0.150 \\
<0.150 \\
<10.0 \\
<0.0002 \\
\mathrm{~g} / \mathrm{m}^{3}\end{array}$ & $\begin{array}{l}\text { over } 10^{5} \\
\text { over } 10^{5} \\
\text { over } 10^{5}\end{array}$ & $\begin{array}{l}\text { Waste sorting according to waste } \\
\text { categories, packing in special containers, } \\
\text { packing in intraplant containers, } \\
\text { transportation to waste treatment facility } \\
\text { Gas purification }\end{array}$ \\
\hline 18. & $\begin{array}{l}\text { B40 glove box } \\
\text { Drying of columns } \\
\text { (for BN type reactor) }\end{array}$ & $\begin{array}{l}\text { SRW } \\
\text { Scrap metal } \\
\text { Rubber gloves, wiping cloth, plastic } \\
\text { compound } \\
\text { Filters } \\
\text { GRW } \\
\text { Nitrogen }\end{array}$ & $\begin{array}{l}\mathrm{kg} / \text { year } \\
\mathrm{kg} / \text { year } \\
\mathrm{kg} / \text { year } \\
\mathrm{m}^{3} / \text { year }\end{array}$ & $\begin{array}{l}6 \\
60000\end{array}$ & $\begin{array}{l}<0.150 \\
<0.150 \\
\\
<10.0 \\
<0.0002 \\
\mathrm{~g} / \mathrm{m}^{3}\end{array}$ & $\begin{array}{l}\text { over } 10^{5} \\
\text { over } 10^{5} \\
\text { over } 10^{5}\end{array}$ & $\begin{array}{l}\text { Waste sorting according to waste } \\
\text { categories, packing in special containers, } \\
\text { packing in intraplant containers, } \\
\text { transportation to waste treatment facility } \\
\text { Gas purification }\end{array}$ \\
\hline
\end{tabular}


II.2. Mayak Site TEF Studies of Weapons Pu Sludge Immobilization and MOX Fuel Fabrication Plutonium Wastes

Table 2. (Continued)

\begin{tabular}{|c|c|c|c|c|c|c|c|}
\hline 19. & $\begin{array}{l}\text { B41 glove box } \\
\text { Charging of fuel } \\
\text { elements } \\
\text { (for BN type reactor) }\end{array}$ & $\begin{array}{l}\text { SRW } \\
\text { Scrap metal } \\
\text { Rubber gloves, wiping cloth, plastic } \\
\text { compound } \\
\text { Filters } \\
\text { GRW } \\
\text { Nitrogen }\end{array}$ & $\begin{array}{l}\mathrm{kg} / \text { year } \\
\mathrm{kg} / \text { year } \\
\mathrm{kg} / \text { year } \\
\mathrm{m}^{3} / \text { year }\end{array}$ & $\begin{array}{l}200 \\
50 \\
4 \\
60000\end{array}$ & $\begin{array}{l}<0.150 \\
<0.150 \\
<10.0 \\
<0.0002 \\
\mathrm{~g} / \mathrm{m}^{3}\end{array}$ & $\begin{array}{l}\text { over } 10^{5} \\
\text { over } 10^{5} \\
\text { over } 10^{5}\end{array}$ & $\begin{array}{l}\text { Waste sorting according to waste } \\
\text { categories, packing in special containers, } \\
\text { packing in intraplant containers, } \\
\text { transportation to waste treatment facility } \\
\text { Gas purification }\end{array}$ \\
\hline 20. & $\begin{array}{l}\text { B42 glove box } \\
\text { Sealing of fuel } \\
\text { elements } \\
\text { (for BN type reactor) }\end{array}$ & $\begin{array}{l}\text { SRW } \\
\text { Scrap metal } \\
\text { Rubber gloves, wiping cloth, plastic } \\
\text { compound } \\
\text { Filters } \\
\text { GRW } \\
\text { Helium }\end{array}$ & $\begin{array}{l}\mathrm{kg} / \text { year } \\
\mathrm{kg} / \text { year } \\
\mathrm{kg} / \text { year } \\
\mathrm{m}^{3} / \text { year }\end{array}$ & $\begin{array}{l}300 \\
50 \\
8 \\
40000\end{array}$ & $\begin{array}{l}<0.150 \\
<0.150 \\
<10.0 \\
<0.0002 \\
\mathrm{~g} / \mathrm{m}^{3}\end{array}$ & $\begin{array}{l}\text { over } 10^{5} \\
\text { over } 10^{5} \\
\text { over } 10^{5}\end{array}$ & $\begin{array}{l}\text { Waste sorting according to waste } \\
\text { categories, packing in special containers, } \\
\text { packing in intraplant containers, } \\
\text { transportation to waste treatment facility } \\
\text { Gas purification }\end{array}$ \\
\hline 21. & $\begin{array}{l}\text { B43 glove box } \\
\text { "Cold" detection of } \\
\text { fuel element failure } \\
\text { (for BN type reactor) }\end{array}$ & $\begin{array}{l}\text { SRW } \\
\text { Scrap metal } \\
\text { Rubber gloves, wiping cloth, plastic } \\
\text { compound } \\
\text { Filters } \\
\text { LRW } \\
\text { Vacuum oil }\end{array}$ & $\begin{array}{l}\mathrm{kg} / \text { year } \\
\mathrm{kg} / \text { year } \\
\mathrm{kg} / \text { year } \\
\text { I/year }\end{array}$ & $\begin{array}{l}100 \\
50 \\
4 \\
300\end{array}$ & $\begin{array}{l}<0.150 \\
<0.150 \\
<10.0 \\
<0.001 \mathrm{~g} / 1\end{array}$ & $\begin{array}{l}\text { over } 10^{5} \\
\text { over } 10^{5} \\
\text { over } 10^{5} \\
10-10^{5}\end{array}$ & $\begin{array}{l}\text { Waste sorting according to waste } \\
\text { categories, packing in special containers, } \\
\text { packing in intraplant containers, } \\
\text { transportation to waste treatment facility } \\
\text { Packing in special casks, transportation to } \\
\text { waste treatment facility }\end{array}$ \\
\hline 22. & $\begin{array}{l}\text { B44 glove box } \\
\text { Decontamination of } \\
\text { fuel elements (for BN } \\
\text { type reactor) }\end{array}$ & $\begin{array}{l}\text { SRW } \\
\text { Scrap metal } \\
\text { Rubber gloves, wiping cloth, plastic } \\
\text { compound } \\
\text { Filters }\end{array}$ & $\begin{array}{l}\text { kg/year } \\
\text { kg/year } \\
\text { kg/year }\end{array}$ & $\begin{array}{l}200 \\
50\end{array}$ & $\begin{array}{l}<0.150 \\
<0.150 \\
<10.0\end{array}$ & $\begin{array}{l}\text { over } 10^{5} \\
\text { over } 10^{5} \\
\text { over } 10^{5}\end{array}$ & $\begin{array}{l}\text { Waste sorting according to waste } \\
\text { categories, packing in special containers, } \\
\text { packing in intraplant containers, } \\
\text { transportation to waste treatment facility }\end{array}$ \\
\hline
\end{tabular}




\begin{tabular}{|c|c|c|c|c|c|c|c|}
\hline Table & $\begin{array}{l}\text { 2. (Continued) } \\
\text { B44 glovebox (cont) }\end{array}$ & $\begin{array}{l}\text { LRW } \\
\text { Solutions resulting from } \\
\text { decontamination }\end{array}$ & $\mathrm{m}^{3} /$ year & 4 & $<0.001 \mathrm{~g} / \mathrm{l}$ & $10-10^{5}$ & $\begin{array}{l}\text { Accumulation of solutions, piping of } \\
\text { solutions to waste treatment facility }\end{array}$ \\
\hline 23. & $\begin{array}{l}\text { B45 glove box } \\
\text { Detecting of fuel } \\
\text { element } \alpha- \\
\text { contamination (for BN } \\
\text { type reactor) }\end{array}$ & $\begin{array}{l}\text { SRW } \\
\text { Rubber gloves wiping cloth, plastic } \\
\text { compound } \\
\text { Filters }\end{array}$ & $\mathrm{kg} / \mathrm{year}$ & 50 & $\begin{array}{l}<0.150 \\
<10.0\end{array}$ & $\begin{array}{l}\text { over } 10^{5} \\
\text { over } 10^{5}\end{array}$ & $\begin{array}{l}\text { Waste sorting according to waste } \\
\text { categories, packing in special containers, } \\
\text { packing in intraplant containers, } \\
\text { transportation to waste treatment facility }\end{array}$ \\
\hline 24. & $\begin{array}{l}\text { B47 glove box } \\
\text { "Hot" detection of fuel } \\
\text { element failure (for } \\
\text { BN type reactor) }\end{array}$ & $\begin{array}{l}\text { SRW } \\
\text { Filters } \\
\text { LRW } \\
\text { Vacuum oil }\end{array}$ & $\mathrm{kg} /$ year & 400 & $\begin{array}{l}<0.150 \\
<0.001 \mathrm{~g} / \mathrm{l}\end{array}$ & $\begin{array}{l}\text { over } 10^{5} \\
10-10^{5}\end{array}$ & $\begin{array}{l}\text { Packing in special containers, packing in } \\
\text { intraplant containers, transportation to } \\
\text { waste treatment facility } \\
\text { Accumulation to special casks, } \\
\text { transportation to waste treatment facility }\end{array}$ \\
\hline 25. & $\begin{array}{l}\text { B51 glove box } \\
\text { Disassembling of } \\
\text { rejected fuel } \\
\text { elements, sorting of } \\
\text { pellets } \\
\text { (for BN type reactor) }\end{array}$ & $\begin{array}{l}\text { SRW } \\
\text { Scrap metal (fuel element claddings) } \\
\text { Scrap metal } \\
\text { Rubber gloves, wiping cloth, plastic } \\
\text { compound } \\
\text { Filters } \\
\text { GRW } \\
\text { Nitrogen }\end{array}$ & $\begin{array}{l}\mathrm{kg} / \text { year } \\
\mathrm{kg} / \text { year } \\
\mathrm{kg} / \text { year } \\
\mathrm{kg} / \text { year } \\
\mathrm{m}^{3} / \text { year }\end{array}$ & $\begin{array}{l}150 \\
60 \\
50 \\
6 \\
60000\end{array}$ & $\begin{array}{l}<0.150 \\
<0.20 \\
<0.150 \\
<10.0 \\
<0.0002 \\
\mathrm{~g} / \mathrm{m}^{3}\end{array}$ & $\begin{array}{l}\text { over } 10^{5} \\
\text { over } 10^{5} \\
\text { over } 10^{5} \\
\text { over } 10^{5}\end{array}$ & $\begin{array}{l}\text { Waste sorting according to waste } \\
\text { categories, packing in special containers, } \\
\text { packing in intraplant containers, } \\
\text { transportation to waste treatment facility. } \\
\text { Gas purification }\end{array}$ \\
\hline 26. & $\begin{array}{l}\text { B17-1,2 glove box } \\
\text { Storing of "rich" } \\
\text { waste } \\
\text { (scrap, chamotte, } \\
\text { etc.) }\end{array}$ & $\begin{array}{l}\text { SRW } \\
\text { Scrap metal } \\
\text { Rubber gloves, wiping cloth, plastic } \\
\text { compound } \\
\text { Filters } \\
\text { GRW } \\
\text { Nitrogen }\end{array}$ & $\begin{array}{l}\mathrm{kg} / \text { year } \\
\mathrm{kg} / \text { year } \\
\mathrm{kg} / \text { year } \\
\mathrm{m}^{3} / \text { year }\end{array}$ & $\begin{array}{l}50 \\
50 \\
3 \\
120000\end{array}$ & $\begin{array}{l}<0.150 \\
<0.150 \\
\\
<10.0 \\
<0.0002 \\
\mathrm{~g} / \mathrm{m}^{3}\end{array}$ & $\begin{array}{l}\text { over } 10^{5} \\
\text { over } 10^{5} \\
\text { over } 10^{5}\end{array}$ & $\begin{array}{l}\text { Waste sorting according to waste } \\
\text { categories, packing in special containers, } \\
\text { packing in intraplant containers, } \\
\text { transportation to waste treatment facility } \\
\text { Gas purification }\end{array}$ \\
\hline
\end{tabular}


II.2. Mayak Site TEF Studies of Weapons Pu Sludge Immobilization and MOX Fuel Fabrication Plutonium Wastes

Table 2. (Continued)

\begin{tabular}{|c|c|c|c|c|c|c|c|}
\hline 27. & $\begin{array}{l}\text { B18-1,2 glove box } \\
\text { Dosing, scrap } \\
\text { crushing }\end{array}$ & $\begin{array}{l}\text { SRW } \\
\text { Scrap metal } \\
\text { Rubber gloves, wiping cloth, plastic } \\
\text { compound } \\
\text { Filters } \\
\text { GRW } \\
\text { Nitrogen }\end{array}$ & $\begin{array}{l}\mathrm{kg} / \text { year } \\
\mathrm{kg} / \text { year } \\
\mathrm{kg} / \text { year } \\
\mathrm{m}^{3} / \text { year }\end{array}$ & $\begin{array}{l}200 \\
50 \\
8 \\
120000\end{array}$ & $\begin{array}{l}<0.150 \\
<0.150 \\
<10.0 \\
<0.0002 \\
\mathrm{~g} / \mathrm{m}^{3}\end{array}$ & $\begin{array}{l}\text { over } 10^{5} \\
\text { over } 10^{5} \\
\text { over } 10^{5}\end{array}$ & $\begin{array}{l}\text { Waste sorting according to waste } \\
\text { categories, packing in special containers, } \\
\text { packing in intraplant containers, } \\
\text { transportation to waste treatment facility } \\
\text { Gas purification }\end{array}$ \\
\hline 28. & $\begin{array}{l}\text { B19 glove box } \\
\text { Scrap } \\
\text { homogenization }\end{array}$ & $\begin{array}{l}\text { SRW } \\
\text { Scrap metal } \\
\text { Rubber gloves, wiping cloth, plastic } \\
\text { compound } \\
\text { Filters } \\
\text { GRW } \\
\text { Nitrogen }\end{array}$ & $\begin{array}{l}\mathrm{kg} / \text { year } \\
\mathrm{kg} / \text { year } \\
\mathrm{kg} / \text { year } \\
\mathrm{m}^{3} / \text { year }\end{array}$ & $\begin{array}{l}150 \\
50 \\
6 \\
60000\end{array}$ & $\begin{array}{l}<0.150 \\
<0.150 \\
<10.0 \\
<0.0002 \\
\mathrm{~g} / \mathrm{m}^{3}\end{array}$ & $\begin{array}{l}\text { over } 10^{5} \\
\text { over } 10^{5} \\
\text { over } 10^{5}\end{array}$ & $\begin{array}{l}\text { Waste sorting according to waste } \\
\text { categories, packing in special containers, } \\
\text { packing in intraplant containers, } \\
\text { transportation to waste treatment facility } \\
\text { Gas purification }\end{array}$ \\
\hline 29. & $\begin{array}{l}\text { B20 glove box } \\
\text { Assembling of fuel } \\
\text { column, checking of } \\
\text { column length and } \\
\text { mass (for VVER type } \\
\text { reactor) }\end{array}$ & $\begin{array}{l}\text { SRW } \\
\text { Scrap metal } \\
\text { Rubber gloves, wiping cloth, plastic } \\
\text { compound } \\
\text { Filters } \\
\text { GRW } \\
\text { Nitrogen }\end{array}$ & $\begin{array}{l}\mathrm{kg} / \text { year } \\
\mathrm{kg} / \text { year } \\
\mathrm{kg} / \text { year } \\
\mathrm{m}^{3} / \text { year }\end{array}$ & $\begin{array}{l}4 \\
60000\end{array}$ & $\begin{array}{l}<0.150 \\
<0.150 \\
\\
<10.0 \\
<0.0002 \\
\mathrm{~g} / \mathrm{m}^{3}\end{array}$ & $\begin{array}{l}\text { over } 10^{5} \\
\text { over } 10^{5} \\
\text { over } 10^{5}\end{array}$ & $\begin{array}{l}\text { Waste sorting according to waste } \\
\text { categories, packing in special containers, } \\
\text { packing in intraplant containers, } \\
\text { transportation to waste treatment facility } \\
\text { Gas purification }\end{array}$ \\
\hline 30. & $\begin{array}{l}\text { B21 glove box } \\
\text { Storing of pans with } \\
\text { fuel columns (for } \\
\text { VVER type reactor) }\end{array}$ & $\begin{array}{l}\text { SRW } \\
\text { Scrap metal } \\
\text { Rubber gloves, wiping cloth, plastic } \\
\text { compound } \\
\text { Filters }\end{array}$ & kg/year & $\begin{array}{l}50 \\
50\end{array}$ & $\begin{array}{l}<0.150 \\
<0.150 \\
<10.0\end{array}$ & $\begin{array}{l}\text { over } 10^{5} \\
\text { over } 10^{5} \\
\text { over } 10^{5}\end{array}$ & $\begin{array}{l}\text { Waste sorting according to waste } \\
\text { categories, packing in special containers, } \\
\text { packing in intraplant containers, } \\
\text { transportation to waste treatment facility }\end{array}$ \\
\hline
\end{tabular}


Immobilization of Weapon Pu-containing Materials and Waste of MOX Fuel Fabrication in the Russian Federation

\begin{tabular}{|c|c|c|c|c|c|c|c|}
\hline Table & $\begin{array}{l}\text { 2. (Continued) } \\
\text { B21 glovebox (cont) }\end{array}$ & $\begin{array}{l}\text { GRW } \\
\text { Nitrogen }\end{array}$ & $\mathrm{m}^{3} /$ year & 60000 & $\begin{array}{l}<0.0002 \\
\mathrm{~g} / \mathrm{m}^{3}\end{array}$ & & Gas purification \\
\hline 31. & $\begin{array}{l}\text { B22 glove box } \\
\text { Drying of fuel } \\
\text { columns } \\
\text { (for VVER type } \\
\text { reactor) }\end{array}$ & $\begin{array}{l}\text { SRW } \\
\text { Scrap metal } \\
\text { Rubber gloves, wiping cloth, plastic } \\
\text { compound } \\
\text { Filters } \\
\text { GRW } \\
\text { Nitrogen }\end{array}$ & $\begin{array}{l}\mathrm{kg} / \text { year } \\
\mathrm{kg} / \text { year } \\
\mathrm{kg} / \text { year } \\
\mathrm{m}^{3} / \text { year }\end{array}$ & $\begin{array}{l}50 \\
50 \\
4 \\
60000\end{array}$ & $\begin{array}{l}<0.150 \\
<0.150 \\
\\
<10.0 \\
<0.0002 \\
\mathrm{~g} / \mathrm{m}^{3}\end{array}$ & $\begin{array}{l}\text { over } 10^{5} \\
\text { over } 10^{5} \\
\text { over } 10^{5}\end{array}$ & $\begin{array}{l}\text { Waste sorting according to waste } \\
\text { categories, packing in special containers, } \\
\text { packing in intraplant containers, } \\
\text { transportation to waste treatment facility. } \\
\text { Gas purification }\end{array}$ \\
\hline 32. & $\begin{array}{l}\text { B23 glove box } \\
\text { Charging of fuel } \\
\text { elements } \\
\text { (for VVER type } \\
\text { reactor) }\end{array}$ & $\begin{array}{l}\text { SRW } \\
\text { Scrap metal } \\
\text { Rubber gloves, wiping cloth, plastic } \\
\text { compound } \\
\text { Filters } \\
\text { GRW } \\
\text { Helium }\end{array}$ & $\begin{array}{l}\mathrm{kg} / \text { year } \\
\mathrm{kg} / \text { year } \\
\mathrm{kg} / \text { year } \\
\mathrm{m}^{3} / \text { year }\end{array}$ & $\begin{array}{l}200 \\
50 \\
6 \\
40000\end{array}$ & $\begin{array}{l}<0.150 \\
<0.150 \\
<10.0 \\
<0.0002 \\
\mathrm{~g} / \mathrm{m}^{3}\end{array}$ & $\begin{array}{l}\text { over } 10^{5} \\
\text { over } 10^{5} \\
\text { over } 10^{5}\end{array}$ & $\begin{array}{l}\text { Waste sorting according to waste } \\
\text { categories, packing in special containers, } \\
\text { packing in intraplant containers, } \\
\text { transportation to waste treatment facility } \\
\text { Gas purification }\end{array}$ \\
\hline 33. & $\begin{array}{l}\text { B24 glove box. } \\
\text { Testing of fuel } \\
\text { element welded joint } \\
\text { (for VVER type } \\
\text { reactor) }\end{array}$ & $\begin{array}{l}\text { SRW } \\
\text { Scrap metal } \\
\text { Rubber gloves, wiping cloth, plastic } \\
\text { compound } \\
\text { Filters }\end{array}$ & $\begin{array}{l}\mathrm{kg} / \text { year } \\
\mathrm{kg} / \text { year } \\
\mathrm{kg} / \text { year }\end{array}$ & $\begin{array}{l}150 \\
50\end{array}$ & $\begin{array}{l}<0.150 \\
<0.150 \\
<10.0 \\
\end{array}$ & $\begin{array}{l}\text { over } 10^{5} \\
\text { over } 10^{5} \\
\text { over } 10^{5}\end{array}$ & $\begin{array}{l}\text { Waste sorting according to waste } \\
\text { categories, packing in special containers, } \\
\text { packing in intraplant containers, } \\
\text { transportation to waste treatment facility }\end{array}$ \\
\hline 34. & $\begin{array}{l}\text { B26 glove box } \\
\text { Facility for etching of } \\
\text { fuel element surface } \\
\text { (for VVER type } \\
\text { reactors) }\end{array}$ & $\begin{array}{l}\text { SRW } \\
\text { Scrap metal } \\
\text { Rubber gloves, wiping cloth, } \\
\text { plastic compound } \\
\text { Filters } \\
\text { LRW }\end{array}$ & $\begin{array}{l}\mathrm{kg} / \text { year } \\
\mathrm{kg} / \text { year } \\
\mathrm{kg} / \text { year }\end{array}$ & $\begin{array}{l}200 \\
50\end{array}$ & $\begin{array}{l}<0.150 \\
<0.150 \\
<10.0\end{array}$ & $\begin{array}{l}\text { over } 10^{5} \\
\text { over } 10^{5} \\
\text { over } 10^{5}\end{array}$ & $\begin{array}{l}\text { Waste sorting according to waste } \\
\text { categories, packing in special containers, } \\
\text { packing in intraplant containers, } \\
\text { transportation to waste treatment facility }\end{array}$ \\
\hline
\end{tabular}


II.2. Mayak Site TEF Studies of Weapons Pu Sludge Immobilization and MOX Fuel Fabrication Plutonium Wastes

\section{Table 2. (Continued)}

\begin{tabular}{|c|c|c|c|c|c|c|c|}
\hline & & Solutions resulting from etching & $\mathrm{kg} /$ year & 10 & $<0.20 \mathrm{mg} / \mathrm{l}$ & $10-10^{5}$ & $\begin{array}{l}\text { Accumulation of solutions, piping of } \\
\text { solutions to waste treatment facility }\end{array}$ \\
\hline 35. & $\begin{array}{l}\text { B33 glove box } \\
\text { Uncanning of rejected } \\
\text { fuel elements (for } \\
\text { VVER type reactor) }\end{array}$ & $\begin{array}{l}\text { SRW } \\
\text { Scrap metal } \\
\text { Scrap metal (fuel element } \\
\text { claddings) } \\
\text { Rubber gloves, wiping cloth, } \\
\text { plastic compound } \\
\text { Filters } \\
\text { GRW } \\
\text { Nitrogen }\end{array}$ & $\begin{array}{l}\text { kg/year } \\
\mathrm{kg} / \text { year } \\
\mathrm{kg} / \text { year } \\
\mathrm{kg} / \text { year } \\
\mathrm{m}^{3} / \text { year }\end{array}$ & $\begin{array}{l}150 \\
200 \\
50 \\
5 \\
60000\end{array}$ & $\begin{array}{l}<0.150 \\
<0.20 \\
<0.150 \\
<10.0 \\
<0.0002 \\
\mathrm{~g} / \mathrm{m}^{3}\end{array}$ & $\begin{array}{l}\text { over } 10^{5} \\
\text { over } 10^{5} \\
\text { over } 10^{5} \\
\text { over } 10^{5}\end{array}$ & $\begin{array}{l}\text { Waste sorting according to waste } \\
\text { categories, packing in special containers, } \\
\text { packing in intraplant containers, } \\
\text { transportation to waste treatment facility } \\
\text { Gas purification. }\end{array}$ \\
\hline 36. & \multicolumn{2}{|c|}{ Filters of other stages of gas purification } & $\mathrm{kg} /$ year & 2150 & $<0.01$ & $10-10^{5}$ & $\begin{array}{l}\text { Packing in containers, transportation to } \\
\text { waste treatment facility }\end{array}$ \\
\hline 37. & \multicolumn{2}{|c|}{ Laboratory glass and porcelain wares } & $\mathrm{kg} / \mathrm{year}$ & 150 & $<0.003$ & $10-10^{5}$ & $\begin{array}{l}\text { Packing in containers, transportation to } \\
\text { waste treatment facility }\end{array}$ \\
\hline 38. & \multicolumn{2}{|c|}{ Solutions resulting from floor washing } & $\mathrm{m}^{3} /$ year & 280 & & $<10$ & $\begin{array}{l}\text { Accumulation of solutions, their piping to } \\
\text { waste treatment facility }\end{array}$ \\
\hline
\end{tabular}




\section{List of Persons Involved in Elaboration of the Report}

GSPI Federal State Unitary Enterprise:

Tyurin E.I.

Kurnaev A.V.

Kolotilov Yu.F.

Dozorov A.V.

Letoshnev S.I.

Spitsyn E.D.

Golentsov V.I.

Ovsyannikova G.A.

Belokurova E.F.

Kozlova N.A.

Nazarova I.G.

Deryabina O.I.

Mayak PA Federal State Unitary Enterprise:

Rovny S.I.

Bobylev A.I.

Ryakov A.V.

VNIINM RF State Research Centre:

Borisov G.B.

Smelova T.V.

Mansurov O.A.

Matyunin Yu.I.

Radium Institute Research and Production Association

Aloy A.S.

Starchenko V.A.

VNIIPIET State Enterprise

Shvedov A.A.

Gusakov B.V.

Rozhdestvensky V.G.

Kozionov A.N.

Ignatiev V.I.

Inform-Atom Close Joint Stock Company:

Fedorov A.B.

Filippov I.Yu.

Romakhov N.Yu.

Storozhenko J.V. 


\section{Sverdniichimmash Experience and Capabilities for Participation in the Contract on Weapons-grade Plutonium Immobilization Generated from MOX Fuel Fabrication}

\section{R. S. Karimov, OSC "Sverdniichimmash"}

Since 1942, the Institute has been involved in the development of non-standard equipment for the needs of chemical-metallurgical productions (i.e., work for the defense of the country). In 1957, the institute was incorporated into the system of the Ministry of Intermediate Machinery (Minatom nowadays). It was involved in R\&D work, development of industrial and test-scale samples of equipment, and started to manufacture the equipment for industrial needs of the nuclear fuel cycle, radioactive waste treatment, and some components of the evaporators, filters, and centrifuges for the national economy.

Requirements for new, perfect prototypes for Minatom techniques created the necessity to accelerate and increase the production potential of the Institute to satisfy scientific needs for sophisticated mechanical facilities, technological lines for fabrication and reprocessing of nuclear fuel, production of fuel assemblies, and waste management. Work in all these directions was conducted simultaneously.

In order to provide a high technical level of work and quality in equipment manufacturing, it was important to have experts with high business potential, scientific qualifications, and experience in machinery techniques.

Therefore, a system of scientific and technical training was organized and scientific seminars were held at the Institute. Post-graduate courses for scientists were organized as well to improve the experts' qualifications.

Minatom gave considerable assistance in material and technical support of the Institute, especially in support of experimental manufacturing, construction of buildings, and social needs institutions.

For performance of R\&D, testing the equipment, its reliability, and stability of work, we constructed two buildings equipped with testing facilities at the required level of engineering support, and employed service personnel with a high level of expertise.

Two facilities at the machinery plant had the potential to manufacture machines of the centrifuge type, such as evaporators, ion exchange columns, tanks, and vessels. All devices were manufactured with consideration of safety aspects.

Institute experts also gained experience in technologies of welding and corrosion-resistant alloys of nickel, titanium, and aluminum.

The institute has following services:

- Quality control;

- Corrosion studies;

- Standardization;

- Metrology.

Such services assist in providing the high quality of all the work performed by the Institute. 
As it has been stated above, for fuel fabrication the Institute developed dissolvers, filters, and centrifuges, ion-exchange columns and extractors, mixers and precipitators, furnaces, absorbers, and auxiliary equipment, including biologically shielded boxes.

For example, the Institute produces sealed cells with a capacity ranging from dozens of liters to $1000 \mathrm{~m}^{3}$ that are equipped with mechanical means ranging from bridge cranes to manipulators inside the cells.

\section{Equipment for MOX-Fuel Fabrication Based on Hydrochemical Technologies: Carbonate, Oxalate, Ammonia, and Sol-gel}

1. A multi-purpose facility "Receipt" was developed that consists of a pressurized tank with solution, centrifuge, furnace, drying furnace, and a system of purification from dust with a reduction capacity up to $5 \mathrm{~kg} /$ hour of uranium oxide, plutonium, and mixed oxide (MOX) fuel.

2. A large scope of research work was carried out jointly with "Mayak" PA and A. A. Bochvar All-Russia Scientific Research Institute of Inorganic Materials (VNIINM) on the development of the pilot facilities "Zhemchug", "Granat", and "Paket" at which experimental MOX-fuel batches and fuel rods were manufactured. Those were tested in power reactors. Results from testing are positive.

3. Special recognition is accorded to the results from the "Mayak" PA and VNIINM joint work on development and manufacturing of the technological lines for MOX fuel fabrication with the "Sol-gel" method and fuel rod fabrication in building 300. These include evaporators for concentration of plutonium nitric acid solutions, gel-forming facility with a chemical dry area, microsphere separator, recovery furnace, plastified mixer, presses, drying furnace, and sintering furnace for pellets and equipment for fuel rod fabrication.

4. When MOX fuel is fabricated with oxide blending, it is possible to operate with the plutonium affinage (i.e., refining) equipment at "Mayak" PA that consists of the mixer-precipitator, filtration facility, and dust purification.

A contract was signed with China to ship equipment for plutonium refining.

In order to gain experience with MOX-fuel production for BN and VVER reactors, "Mayak" PA will develop both a pilot facility and demonstration facility, CHEMOX and DEMOX.

\section{Equipment for MOX Fuel Fabrication with Dry Technologies}

Equipment used for dry techniques for MOX fuel fabrication is more compact, consumes less metal, is more universal, and allows working with fuel of any burnup and hold time. It also requires fewer technological operations in comparison with equipment for hydrochemical technologies. Dry techniques also reduce the volume of liquid radioactive waste.

\section{Gas fluoride technique}

Significant experience in development and fabrication of the equipment for gas-fluoride techniques has been developed. This expertise was used in Czechoslovakia to build the facilities "2GF" and "FREGAT".

Taking into account the experience from the joint work with The Russian Federation State Scientific Center Scientific and Research Institute of Atomic Reactors (RIAR), VNNICHT, VNIINM, IAE, All Russian Designing and Scientific Research Institute of Complex Power Technology (VNIPIET), and joint work with France, a project was initiated for development of the industrial-scale French-Russian facility for 
regeneration of the highly irradiated $\mathrm{BN}$ reactor fuel by the gas-fluoride method with a production capacity up to $70 \mathrm{t} / \mathrm{h}$.

In the design of such a plant, the following were considered:

- Furnace for spent nuclear fuel (SNF) extraction;

- Oxidizer-separator for fuel separation from fuel rod cladding;

- Fluorizers of the "DS" and "KS" types for gasification of uranium, plutonium oxides and fission products;

- Condensers;

- Facilities equipped with thermostatic absorbers for purification and selective separation of uranium and plutonium;

- Facilities equipped with rectification columns for purification and selective separation of uranium and plutonium;

- Devices for uranium hexaftoride pyrohydrolysis;

- Dissolvers-oxidizers;

- Rectification columns for separation of the gas flows;

- Auxiliary equipment including dust purification for main technique devices.

As for the devices listed, the most attractive is the dissolver-oxidizer (electrolizer) where it is possible to perform several technological operations with different initial materials for final fabrication of uranium, plutonium oxides, and MOX fuel.

\section{Pyroelectric technologies (electrolizer)}

Development of pyroelectric technologies and nuclear fuel reprocessing including MOX fuel fabrication for power reactors was carried out jointly with RIAR, VNIPIET, UGTU, and others.

We developed several devices ("ERA") in which an electrolizer was the main node.

After performing some R\&D work, we generalized the data obtained, and we able to offer a design for the dissolver-electrolizer. That apparatus had loading-offloading nodes, gas supply valves, isolated heating nodes, and coolant. In addition, it included the equipment for acceptance and loading of the initial material, devices for conditioning of residue with chemical-mechanical and thermal methods based on granulometric composition, and other auxiliary equipment.

The product manufactured in this line met the standards on nuclear fuel for power reactors. Later, this device was improved.

For more than a year, MOX fuel containing weapons-grade plutonium has been used in reactor BOR-60.

\section{Granulated MOX fuel fabrication techniques and documentation}

At present, the Institute is working jointly with RIAR on the contract with Japan on development of the documentation on improvement of the granulated MOX fuel fabrication lines to correspond with radiation safety standards as well as on development of the equipment for fuel assemblies and rod fabrication. 


\section{BREST-OD-300 fuel fabrication equipment}

Concurrently, the manufacture of technological and auxiliary equipment for uranium-plutonium fuel fabrication for the power reactor "BREST-OD-300" is in progress.

\section{Equipment for Radioactive Waste Management}

Development of equipment for radioactive waste management started along with development of the main technologies on fuel fabrication at the radiochemical plants.

\section{Concentration of activity from radioactive waste}

Facilities equipped with evaporators for concentrating release water radioactivity for radiochemical plants and nuclear power plants were later improved and foam separators were later added to them.

This equipment has been used for dozens of years. It manifests a stable high level of radioactive water purification and is considered for inclusion in many projects for new radiochemical plants and nuclear power plant (NPP) construction.

In the Institute, we developed original ion exchange columns for a high level of purification from activity that also allows us to minimize the volume of wash through water and volume of sludge sent for solidification and storage.

In order to purify gases, adsorbers with an isothermal zone were developed that capture radioactive powder and aerosols with high efficiency.

Extractors for chemical technologies developed at the Institute are being used successfully at plant facilities.

When we change TBF to a new synthesized chemical compound, then we can perform selective extraction of $\mathrm{Cs}$ and $\mathrm{Sr}$ from release water, which allows the transfer of waste from high level waste (HLW) to intermediate level waste (ILW).

It is necessary to discuss the details of facilities for burning solid radioactive waste (SRW) and liquid radioactive waste (LRW). Many of these facilities are being successfully operated at radiochemical plants and NPPs. The main part in these facilities is a furnace with burnup zone, final burnup zone equipped with a device for catalytical processing of furnace gases, and systems for dry, wet, and combined gas purification. Ash from the furnaces is sent for cementation and temporary storage in sealed barrels.

\section{Radioactive waste solidification}

For LRW and SRW solidification, several design options for cementation facilities were developed. A cement compound with incorporated activity is loaded into metal barrels, which are sealed and then transported for long-term storage.

A large amount of R\&D work was done on vitrification of the HLW and ILW. Some industrial scale pilot facilities were developed and tested.

Currently, "Mayak" PA is operating a facility for HLW vitrification, the direct-heat electric melter EP500. This facility includes auxiliary equipment ranging from equipment for initial solution preparation to the complex system of gas purification. The glass mass in sealed metal barrels is transported to long term storage facilities for radioactive waste. 


\section{Solid radioactive waste conditioning}

Facilities equipped with presses or superpresses for mechanical compression of the metal and non-metal solid waste are of great practical interest.

These facilities allow up to a 10-times reduction in volume of the initial solid radioactive waste. They are equipped with nodes for sorting initial material for pressing. Pressed material is transported to the radioactive waste storage facilities.

For many years, the institute has worked on development of facilities for decontamination, chopping, and melting of the metallic items when radiochemical plants and NPP equipment is dismantled.

Facilities for purification of the water from storage pools and sludge storage facilities have been developed. As a rule, they are equipped with a TV station, pump, filters, centrifuge, ion-exchange columns, and evaporators. They are also equipped with a sludge transportation mechanism, ion exchange raisins, and perlite for solidification facilities.

For many years, a special vehicle for radioactive waste transportation has been operated. This vehicle is equipped with mechanical means for loading-offloading, and has a biological shield.

The pipeline system used to transfer the fragments of fuel assembly cladding material from the SNF dissolution facility to the solid radioactive waste storage facility is operating reliably. This system was developed jointly by "Mayak" PA and Novosibirsk experts.

Based on the contract with Bechtel, SVERDNIICHIMMASH is working on development of the equipment for a fissile material storage facility.

Currently, we are working on manufacturing and shipment of the equipment for:

- $\quad$ LRW disposal (cementation);

- $\quad$ SRW reprocessing (pressing).

For the "Busher" NPP in Iran, we are working on development of a cementation facility with further manufacturing of equipment and shipment to the site.

We have started the pre-contract work on development of equipment for a radioactive waste handling facility for the Kundakulam NPP in India.

At the moment, SVERDNIICHIMMASH is a large production complex in the Urals with technically equipped resources and experts with a high level of expertise. It has the capability to develop and manufacture equipment for RF MINATOM, ranging from centrifuges and sealed pumps to any radiation safety equipment. The company operates in accordance with the RF legislation. 


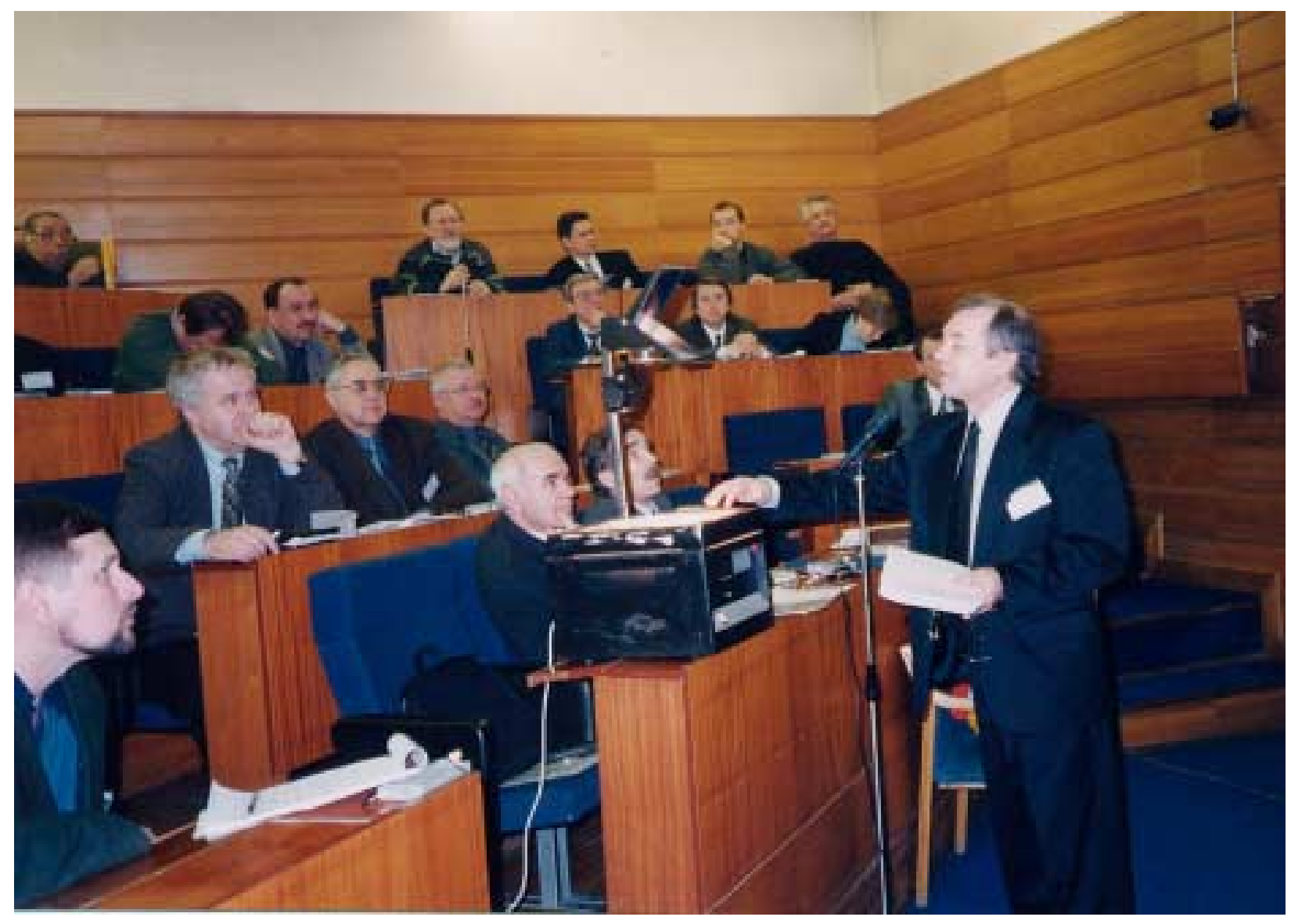




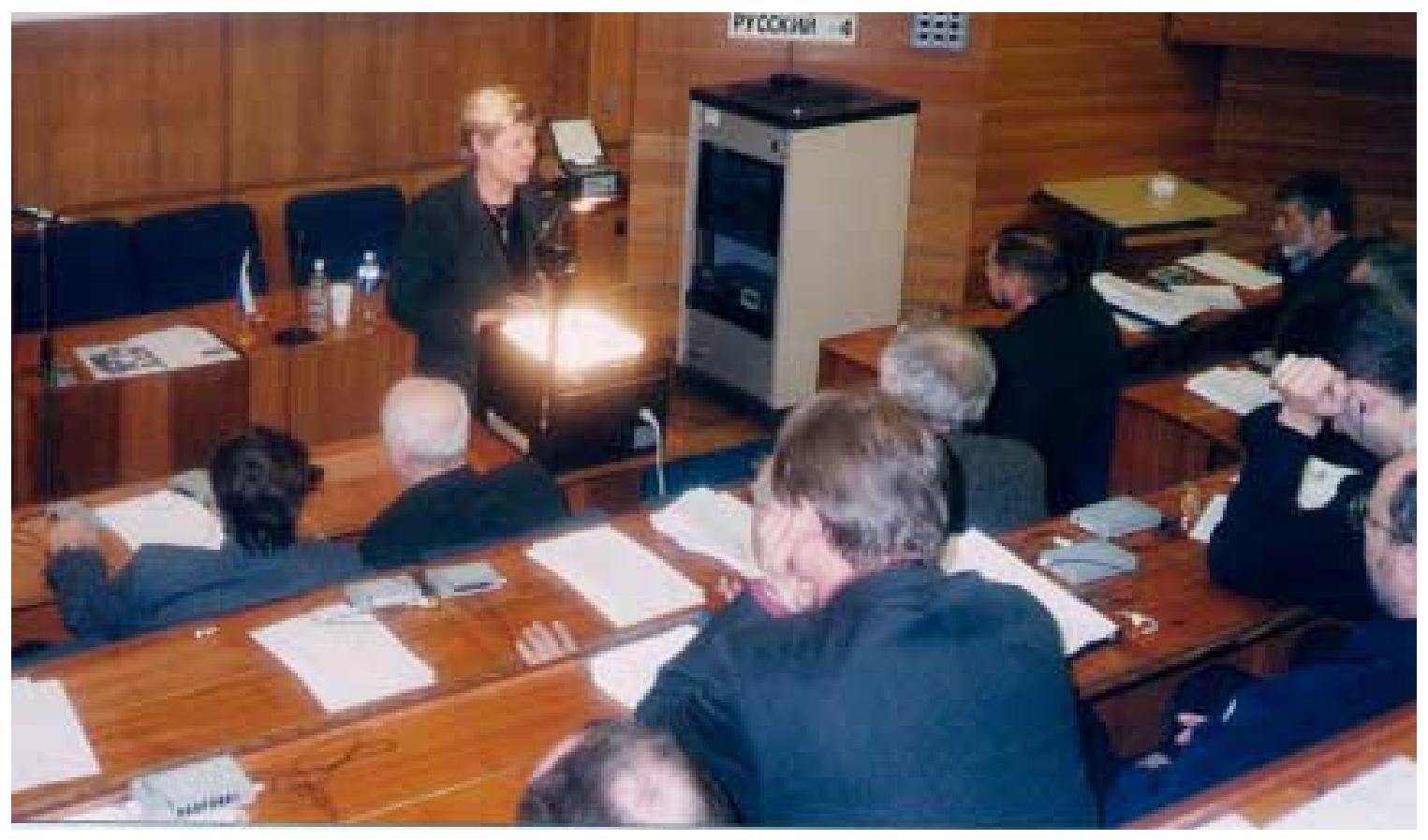

II.3 RIAR Site Studies 


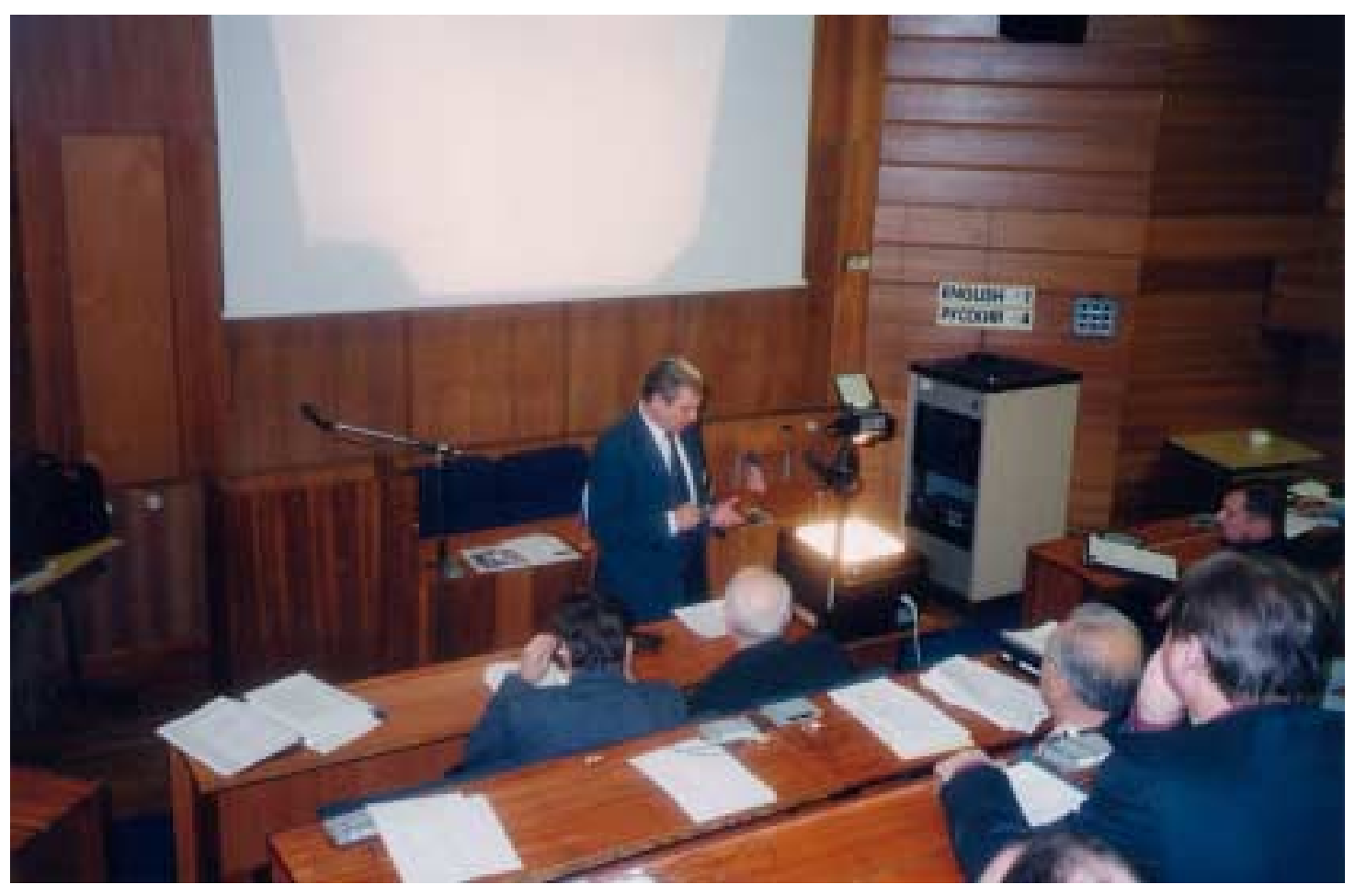




\section{Experience on Russian Military Origin Plutonium Conversion into Fast Reactor Nuclear Fuel*}

Grachev A., Skiba O., Bychkov A., Mayorshin A., Kisly V., Bobrov D., Osipenco A., Babikov L., Mishenev V.

\section{State Scientific Center of Russian Federation "Research Institute on Atomic reactors"}

*No paper was available. The author, A. Bychkov, kindly provided these viewgraphs used for his presentation. 


\section{State Scientific Center of Russian Federation} "Research Institute on Atomic reactors" (Dimitrovgrad)

started at 1998 the program on technological demonstration of conversion of metallic weapon origin plutonium into mixed oxide fuel for fast reactors

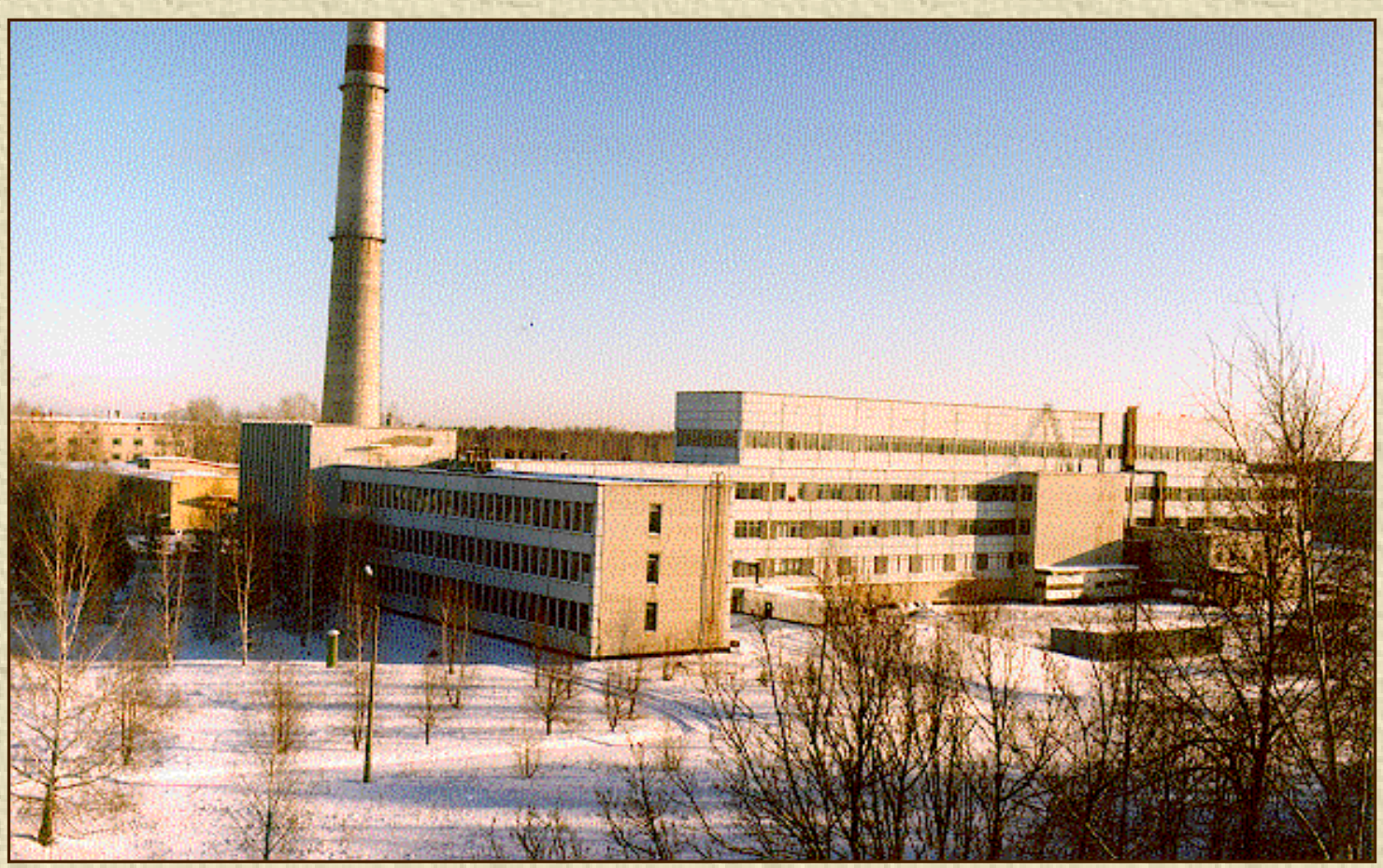


Basis of work

\section{Trends of activity}

\section{Goals of program}

- MINATOM Conception on utilization of military plutonium

- Agreements between Governments of USA and Russia on military plutonium disposition (1998 an 2000)

- International collaboration and support by USA, France, Germany, Japan
- Conversion of weapon metallic plutonium into MOX-fuel

- Production of MOX fuel assemblies for BOR-60 and $B N-600$ reactors

- Preparation to BN-600 operation in mode of hybrid core with weapon plutonium based MOX-fuel: technology adopting, reactor irradiation of lead test assemblies, modernization of technological facilities 


\section{Development background}

- Since 70-s RIAR together with other ex-USSR Institutes were developed one of new technological approach for utilization and recycling of plutonium (different quality) in fast reactors

\section{Main applied process}

Two technologies were as basic:

- Pyroelectrochemical technology - for plutonium conversion and MOX fuel production in molten salt

- Viropacking technology - for production of fuel elements for fast reactors 


\section{History and current status}

1994 2. Start of studies on pyrochemical process application for Pu conversion in frame of AIDA-MOX Program (France-Russia). The processes of Pu-Ga alloy dissolution in molten chlorides with followed precipitation of $\mathrm{PuO}_{2}$ were studied

- 1997 2. Start of studies in frame of USA-Russia Program on military plutonium disposition. The some problems were studied: production and licensing of MOXfuel, pyrochemical conversion of Pu-Ga alloy into plutonium dioxide for pellet production, feasibility studies.

- 1998 2. MINATOM with ROSENERGOATOM support gave to RIAR $50 \mathrm{~kg}$ of PU-Ga military alloy for demonstration of its disposition. The 32 FAs for the BOR-60 reactor and $3 \mathrm{FAs}$ for the $\mathrm{BN}-600$ reactor were manufactured

1999 2. The collaboration with JNC (Japan) was started on the program of production and irradiation of 3 LTAs with vibropacked MOX-fuel for the BN-600 reactor, produced from military plutonium. 3 LTAs are irradiated from May 2000.

- 2000 2. RIAR received second batch of Pu-Ga alloy $(100 \mathrm{~kg})$ for continuation of works 


\section{Pyroelectrochemical process}

The plutonium conversion into MOX fuel is carried out under the original technology developed by SSC RIAR. It includes pyroelectrochemical process for production of fuel. The flow sheet of pyrochemical conversion of metallic plutonium alloy into MOX-fuel includes:

- Dissolution of metal plutonium alloy and uranium oxide in molten salt $\mathrm{NaCl}$ $2 \mathrm{CsCl}$,

- Electrolysis of molten salt with regulated codeposition of $\mathrm{PuO}_{2}$ with $\mathrm{UO}_{2}$,

- Treatment of cathodic deposit and reception of granulated fuel.

The produced MOX fuel is purified from alloy additives (Ga) and corresponds to the vibropacked fuel standard for fast reactors. Start materials are Pu-Ga military alloy and uranium as $\mathrm{UO}_{2}$ Ready product is granulated MOX-fuel for the fast reactors - BOR-60 and $\mathrm{BN}-600$ 


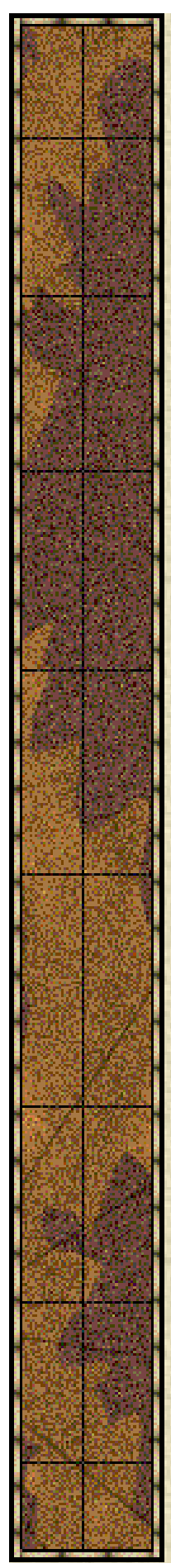

II.3. RIAR Site Studies

Technology development

\section{Technology flow-sheet} of MOX-fuel production from military origin plutonium for fast reactors

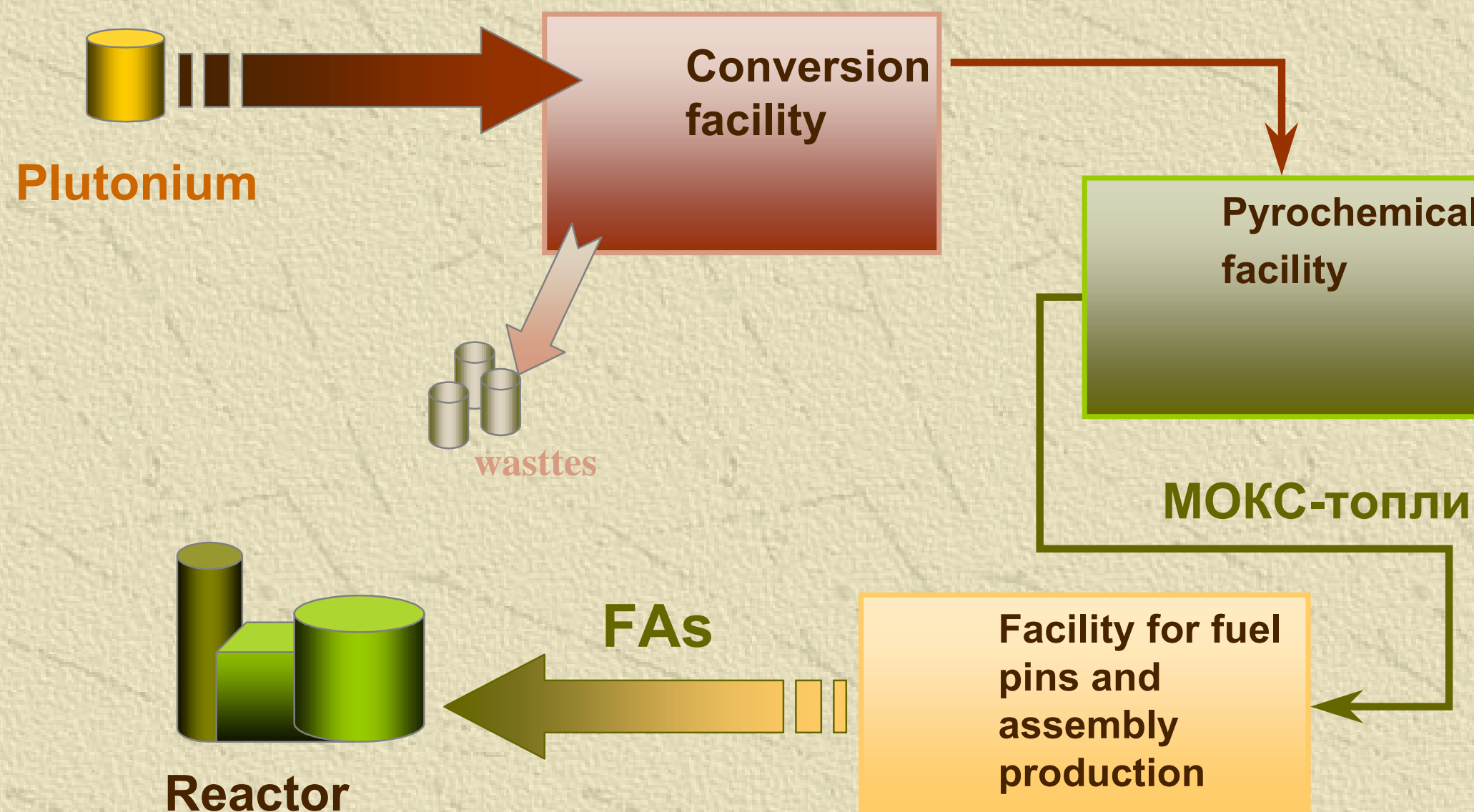




\section{Vibropacking technology}

The fuel pins manufacturing for BOR-60 and BN-600 reactors are carried out by the vibropacking method on a standard procedure, which is used in SSC RIAR more than 20 years.

Some advantages of vibropacking technology and vibropac fuel pins:

- Simplicity and reliability of manufacturing process (less number of steps for production and control, easy automatization and remote controlling)

- Possibility to use granulated fuel with any shapes, as homogeneous and mechanical

- More less thermomechanical stress Fuel-cladding (in comparison with pellet fuel)

For correction of oxygen factor and for elimination of corrosion processes the GETTER added to granulated fuel. 
The results of the demonstration works on conversion of plutonium and MOXfuel production

\section{The BOR-60 Program}

During autumn 1998 and spring 1999 five batches of military origin plutonium were processed.

The production sub-program has main goals: supply of the BOR-60 by new fuel assembly and the followed additional goals:

- Development of processes of metal alloy dissolution in molten salts in kgs-scale

- Determination and optimization of Ga decontamination factor

- Determination of process conditions for future changing of technology procedures and including new procedures to modernization project

The produced MOX fuel was used for manufacturing 32 Fuel assemblies (FAs) and 2 non-complete FAs of the BOR-60 reactor. These FAs are successfully irradiated.

The MOX-fuel is content gallium as $0,001 \ldots 0,09 \%$ 


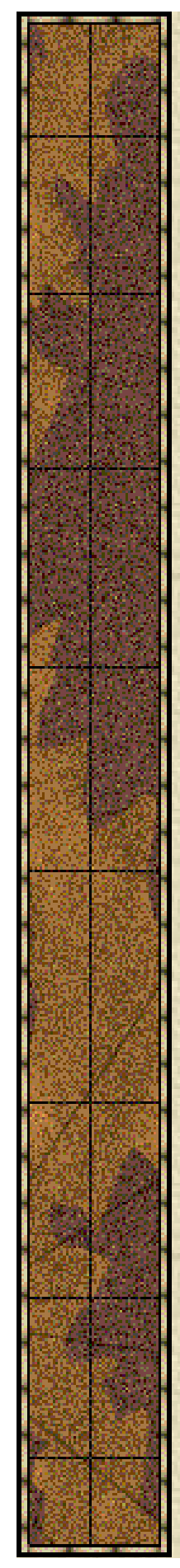

The results of the demonstration works on conversion of plutonium and MOX-fuel production

\section{The BN-600 Program}

On 1999 new batch of MOX-fuel $(90 \mathrm{~kg})$ was produced by revised procedures for the $\mathrm{BN}-600$ reactor with disposition of $22 \mathrm{~kg}$ of $\mathrm{Pu}$.

Gallium content in MOX-fuel is $0,001 \ldots 0,0015 \%$

Three Lead Test Assemblies was produced from that fuel for the BN-600 reactor. These LTAs are irradiated on Beloyarsk NPP since May 2000. 


\section{Pyrochemical production of oxide granulated fuel in RIAR}

Total for September $2001-5424 \mathbf{~ k g}$

II.3. RIAR Site Studies

\begin{tabular}{|c|c|c|c|c|}
\hline Facility type & Fuel & weight, kg & period & reactor \\
\hline \multirow[b]{2}{*}{ Boxes for uranium fuel } & $\mathrm{UO}_{2}$ & 900 & $1976 \ldots 1983$ & BOR-60 \\
\hline & $\mathrm{UO}_{2}$ & 365 & 1983 & BN-350 \\
\hline \multirow{4}{*}{ First hot cell facility for MOX-fuel } & $\mathrm{PuO}_{2}$ & 100 & $1980 \ldots 1982$ & BOR-60 \\
\hline & $(\mathrm{U}, \mathrm{Pu}) \mathrm{O}_{2}$ & 550 & $1983 \ldots 1987$ & BOR-60 \\
\hline & $(\mathrm{U}, \mathrm{Pu}) \mathrm{O}_{2}$ & 75 & 1984 & BN-350 \\
\hline & $(\mathrm{U}, \mathrm{Pu}) \mathrm{O}_{2}$ & 70 & 1987 & BN-600 \\
\hline \multirow{7}{*}{ Semi-Industrial Complex (SIC) } & $\mathrm{UO}_{2}$ & 120 & 1988 & BN-600 \\
\hline & $\mathrm{UO}_{2}$ & 535 & $1988 \ldots 1989$ & RBT-10 \\
\hline & $\mathrm{UO}_{2}$ & 1097 & $1989 \ldots 2000$ & BOR-60 \\
\hline & $\mathrm{UO}_{2}$ & 374 & 1993 & BN-350 \\
\hline & $(\mathrm{U}, \mathrm{Pu}) \mathrm{O}_{2}$ & 325 & $1989 \ldots 1998$ & BOR-60 \\
\hline & $(\mathrm{U}, \mathrm{Pu}) \mathrm{O}_{2}$ & 277 & $1989 \ldots 1992$ & BFS \\
\hline & $(\mathrm{U}, \mathrm{Pu}) \mathrm{O}_{2}$ & 300 & 1990 & BN-600 \\
\hline \multirow[t]{3}{*}{ SIC ( conversion of military Pu) } & & 136 & $1998 \ldots 1999$ & BOR-60 \\
\hline & $(\mathrm{U}, \mathrm{Pu}) \mathrm{O}_{2}$ & 95 & 1999 & BN-600 \\
\hline & & 85 & 2001-2002 & BN-600 \\
\hline \multicolumn{2}{|l|}{ New testing fuels } & 30 & 3. & BOR-60 \\
\hline
\end{tabular}


II.3. RIAR Site Studies

\section{KR-03 Facility}

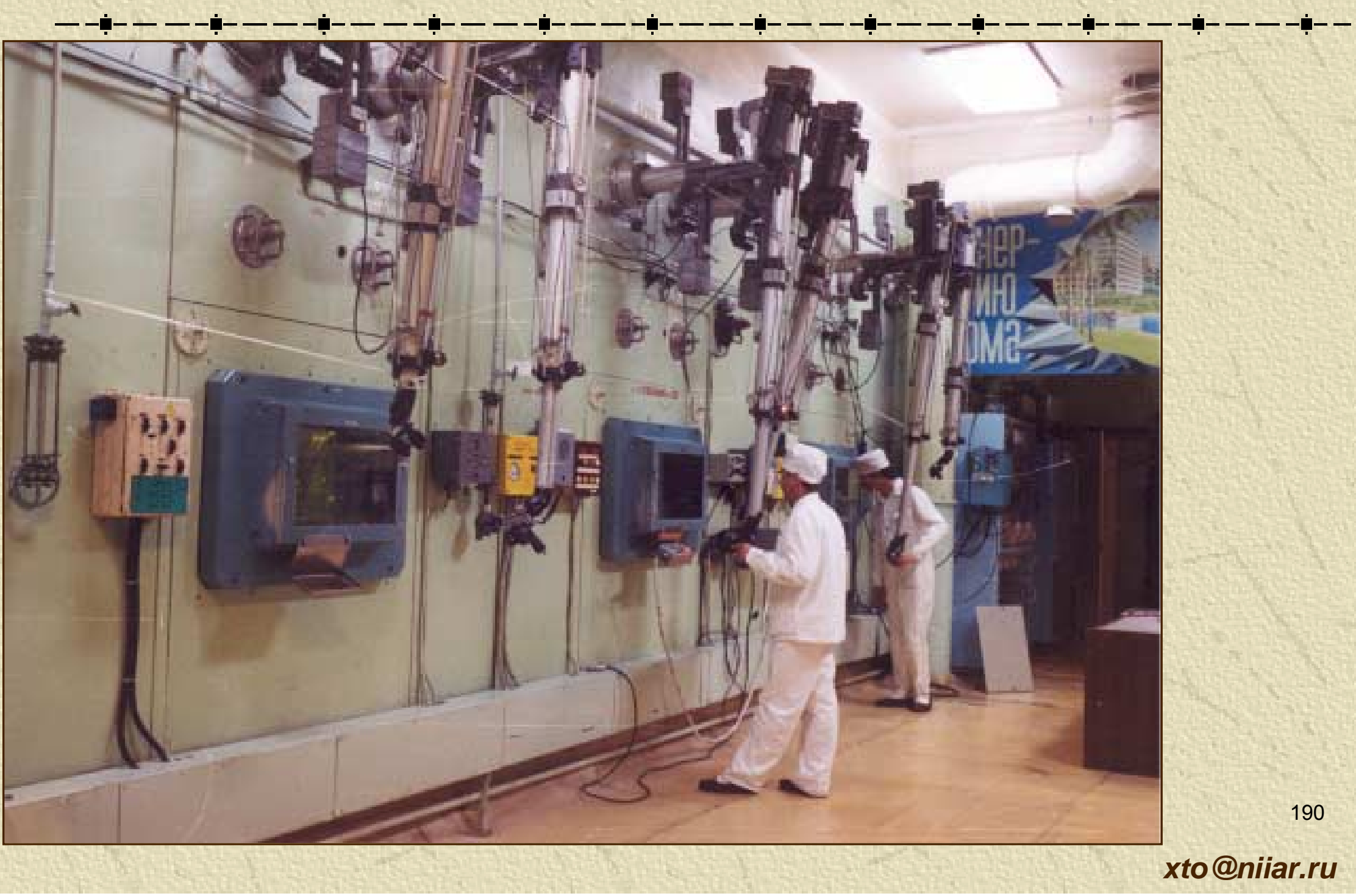


II.3. RIAR Site Studies

\section{BOR-60 Fuel Assemblies from military plutonium}

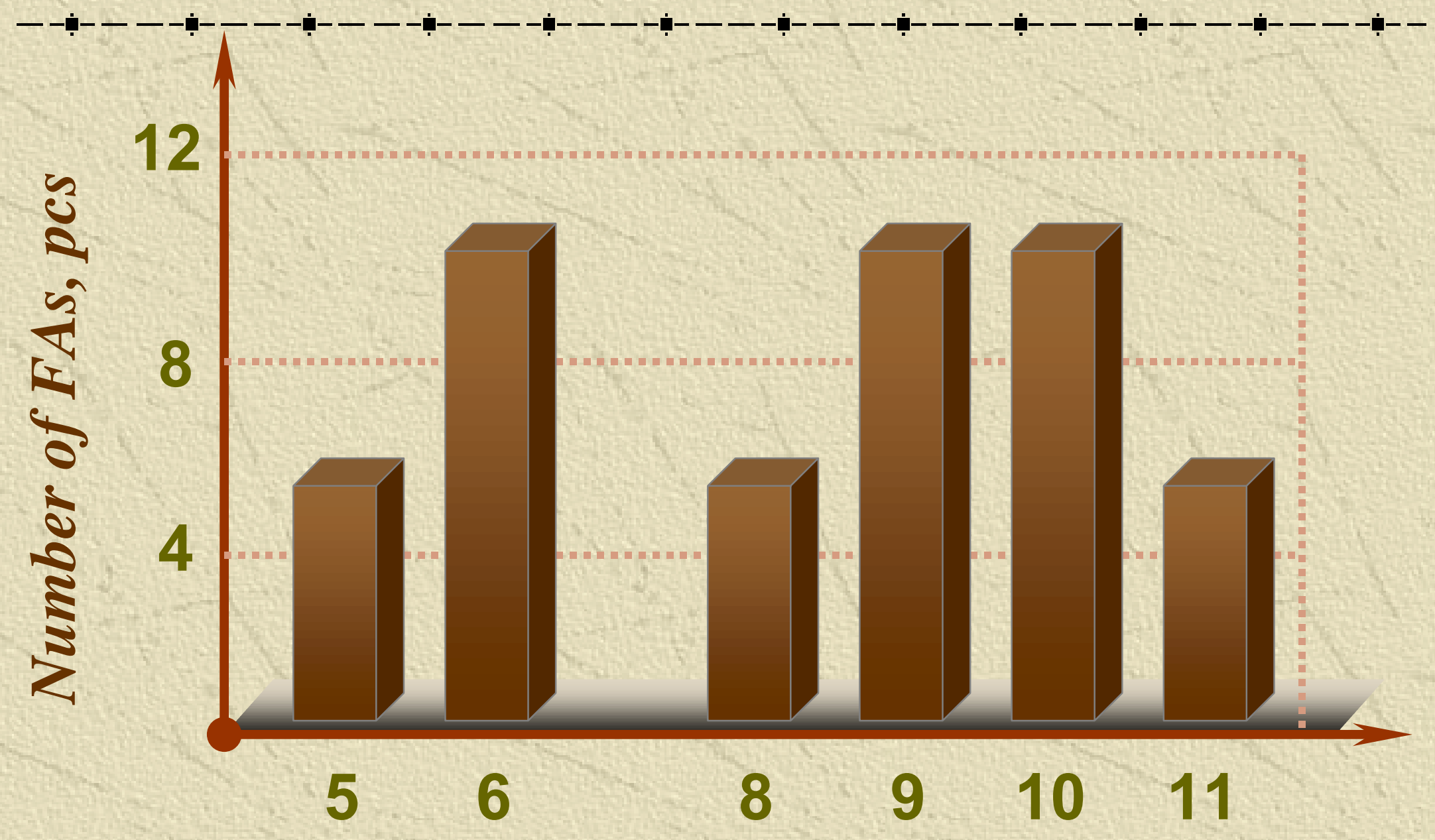

Burn-up, \% h.a. 
II.3. RIAR Site Studies

Fast reactors Fuel assemblies prepared with military plutonium

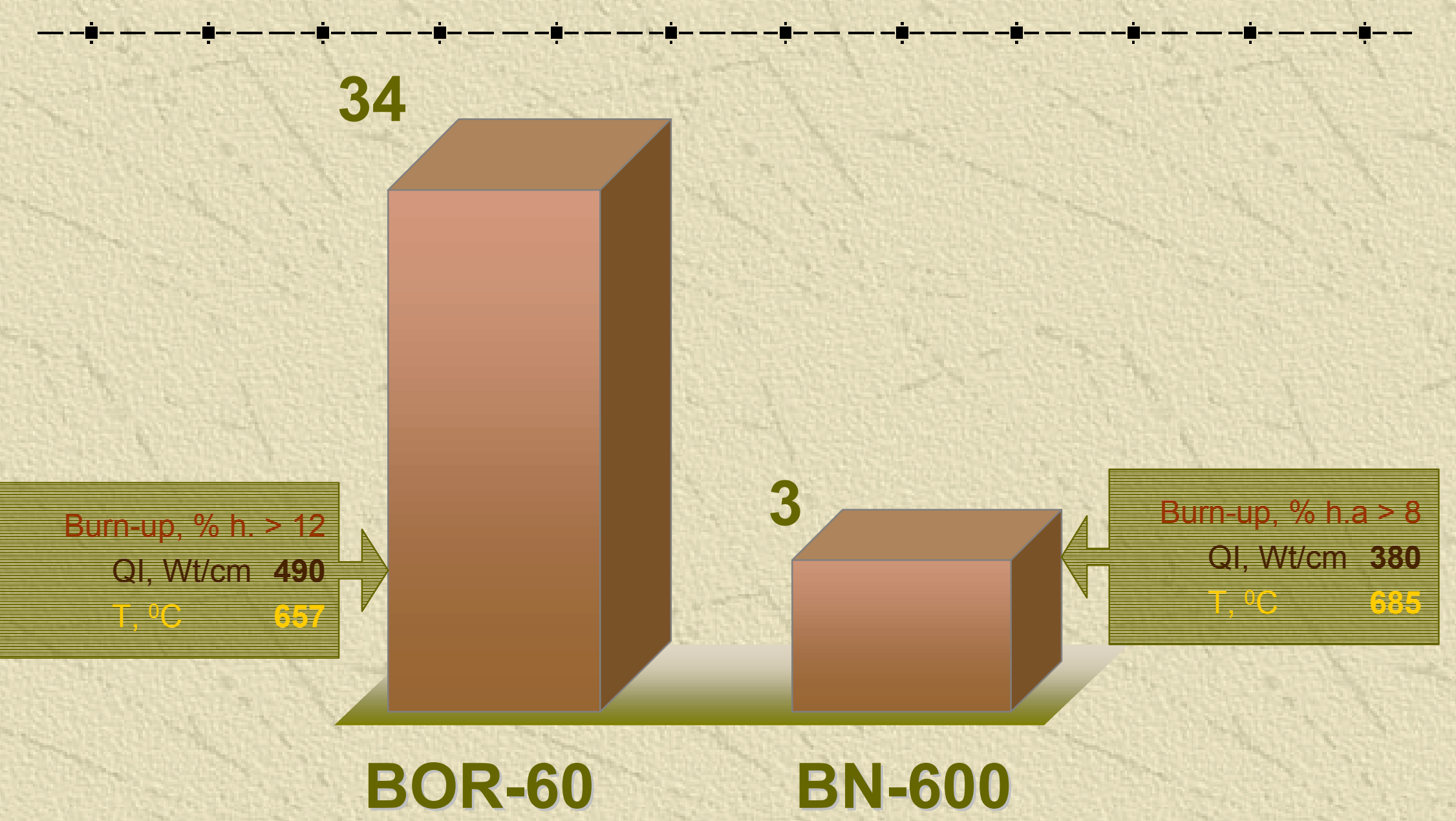


II.3. RIAR Site Studies

\section{Fuel pins and assemblies production with MOX-fuel}

\begin{tabular}{|c|c|c|c|}
\hline Type of fuel & Reactor & Number of assemblies & Number of pins \\
\hline $\mathrm{UPuO}_{2}(\mathrm{~B} \Phi)$ & BOR-60 & 426 & 15762 \\
\hline $\mathrm{UPuO}_{2}(\mathrm{H} \Phi)$ & BOR-60 & $\mathbf{3 2 + 2}$ & $\mathbf{1 2 1 0}$ \\
\hline $\mathrm{UPuO}_{2}(\mathrm{H} \Phi)$ & $\mathrm{BN}-350$ & 2 & 254 \\
\hline $\mathrm{UPuO}_{2}(\mathrm{H} \Phi)$ & $\mathrm{BN}-600$ & $\mathbf{6 + 3}$ & $\mathbf{7 6 2 + 3 8 1}$ \\
\hline $\mathrm{UPuO}_{2}(\mathrm{~B} \Phi)$ & $\mathrm{BN}-600$ & 4 & 508 \\
\hline $\mathrm{UPuO}_{2}(\mathrm{HФ}$, ВФ) & BFS & 8 & 1016 \\
\hline $\mathrm{UO}_{2}+\mathrm{PuO}_{2}$ (Обл.) & BOR-60 & 1 & 6 \\
\hline
\end{tabular}

ВФ. "power grade" Pu;

НФ- "weapon" garde Pu;

Обл.- product after pyrochemical reprocessing 


\section{Plutonium disposition}

In the BN-600 hybrid core

After successful testing of process with first $50 \mathrm{~kg}$ of military $\mathrm{Pu}$, new batch of Pu-Ga alloy $(100 \mathrm{~kg})$ transported to RIAR. This batch of military plutonium are used for testing and fulfillment of the following subjects:

- Isotopic composition correction

- Statistics study for production of MOX-fuel

- Stabilization of manufacture of fuel pins and assemblies 


\section{During nearest years RIAR has possibility to manufacture for the fast reactors:}

- 40 FAs per year for the BOR-60 reactor ( $25 . .35 \mathrm{~kg}$ of $\mathrm{Pu}$ )

- $\sim 10$ FAs per year for the $\mathrm{BN}-600$ reactor ( $60 \mathrm{~kg}$ of $\mathrm{Pu}$ ) 


\section{The Program on Manufacture of 21 LTAs for the BN-600 was started on 2001}

The program on manufacture of fuel and LTAs for the BN-600 has main goals as statistics on production and irradiation vibroMOX-fuel for future introduction in the $B N-600$ hybrid core

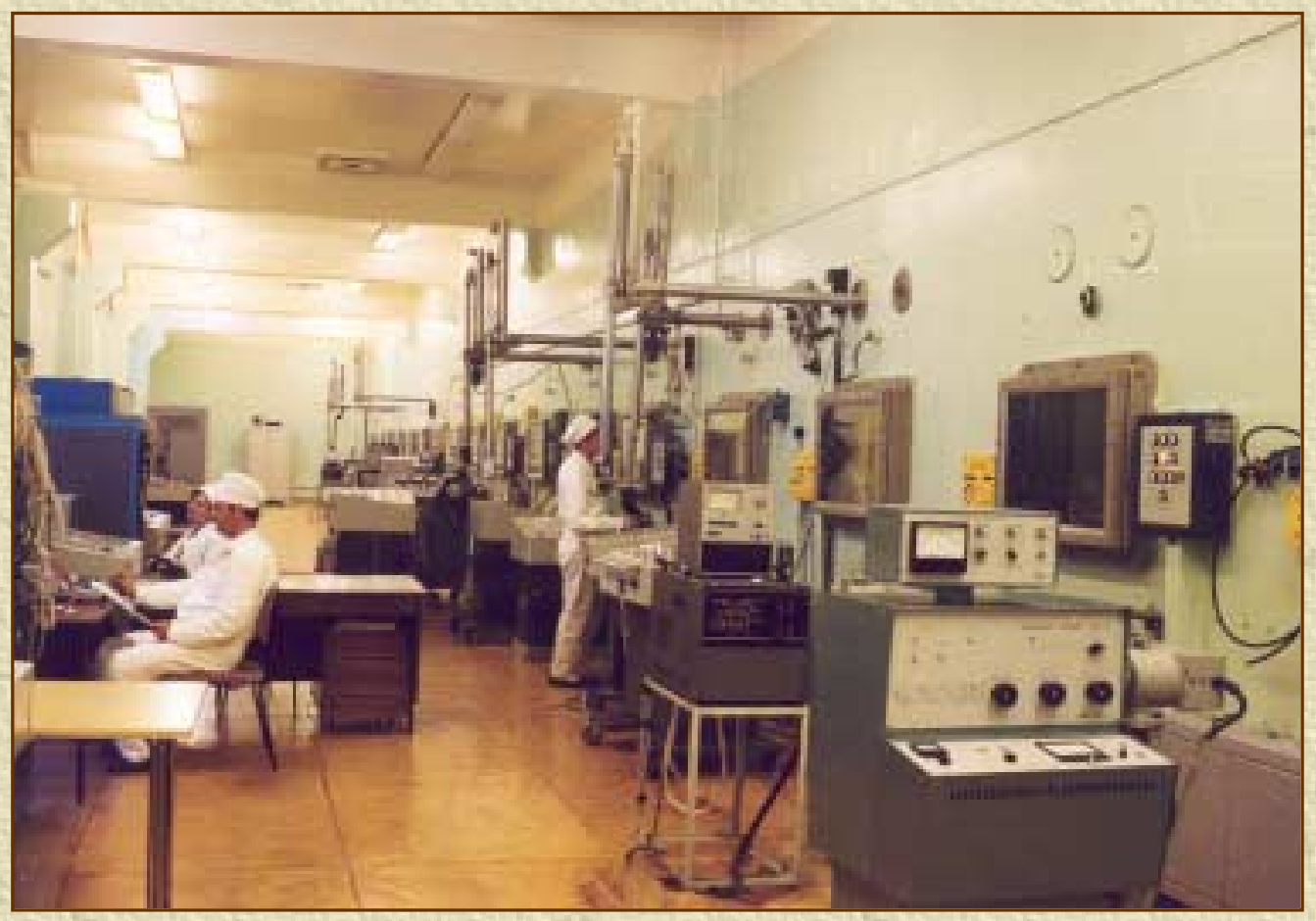


The program on Modernization of Facilities for production of MOX-fuel and fuel pins with military plutonium is started

This program will give to start semi-industrial introduction of the technology for production of FAs for BN-600 hybrid core.

$$
\text { } 50 \text { FAs per year ( } \sim 300 \mathrm{~kg} \text { of } \mathrm{Pu})
$$

The Agreement on fulfilment of this program was signed between

$$
\text { JNC (Japan) and RIAR (Russia). }
$$

Facilities operation start is planned on 2003. 


\section{Other activities on plutonium disposition}

- Military plutonium alloy conversion into dioxide powder with using of molten salt process

- Preparation test facility for production of MOX-pellets and Fuel pins for Lead Test Assemblies for the VVER1000 reactor

- Studies on immobilization processes for wastes contained military plutonium 
Modernization of facilities and production the BN-600 FAs

\section{Basic.problems} and proposed decisions

- Problem of financial support of conversion part (facility) on RIAR site

- Problem of transparency and Pu disposition monitoring by US specialists in frame of USA-Russia Agreement

- It is necessary to fulfill "pillot" project on development, testing of method and equipment for Pu and MOX-fuel at RIAR 


\section{Basic problems} and proposed decisions

- Problem of transportation and storage of source materials and ready Fuel Assemblies

- It is necessary to expand storage, to equip transportation park, to manufacture of containers

- Licensing problems for expanding production capacity up to $300 \mathrm{~kg}$ Pu per year

- It is necessary to develop rules and documents for RIAR activity licensing. RIAR could be considered as "pilot" place for checking of licensing procedures. 


\section{Basic problems}

\section{and proposed decisions}

- Safety problems for high capacity on plutonium conversion (up to $300 \mathrm{~kg}$ annually)

- It is necessary equipped the facilities by new control systems

- It is necessary to re-arrange the RIAR sanitary laboratory for constant control of personal and for biophysical monitoring 


\section{Basic problems} and proposed decisions

- Problem of the $\mathrm{BN}-600$ reactor modernization

- Problem of irradiated MOX-fuel storage

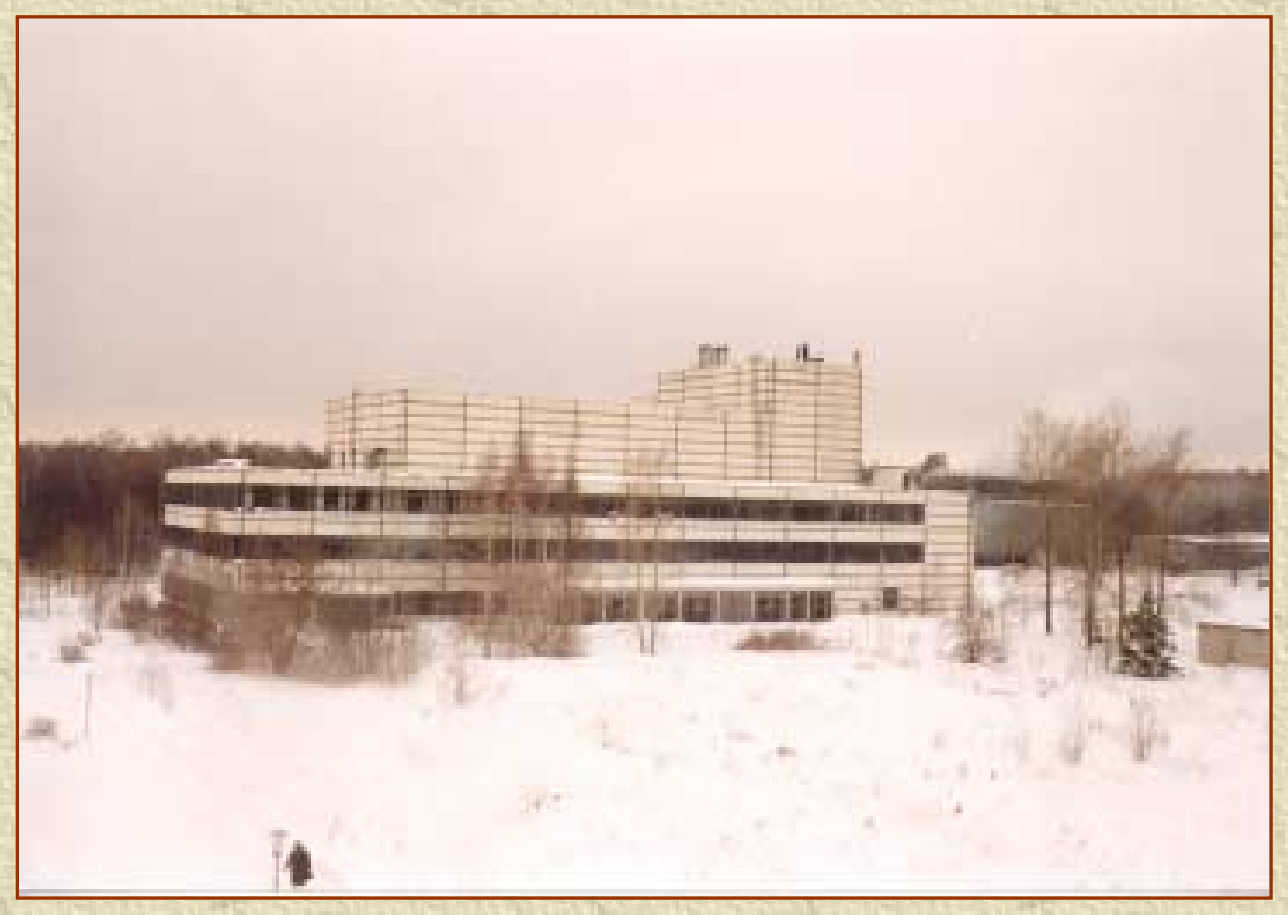




\section{Preliminary Technical and Economic Justification for Treatment and Immobilization of Pyrochemical Residual and Waste Containing Plutonium in RIAR (B506204)}

\section{G. Babikov, A. V. Bychkov, A. N. Lukinyh, RIAR}

Since 1999, the The Russian Federation State Scientific Center Scientific and Research Institute of Atomic Reactors or SSRC RF (hereinafter RIAR) has conducted this work in accordance with Lawrence Livermore National Laboratory (LLNL) Contract B 506204.

The purpose of the work is as follows:

To ascertain the inventory quantity and to realize classification of RIAR's plutonium-containing waste, to conduct preliminary technical and economic justification on direct immobilization into acceptable solid form for consequent storage of flows of RIAR's waste containing plutonium, including the ones generated at conversion of "weapon" plutonium into MOX-fuel.

Provision was made for work realization in 4 stages:

Task 1. Inventory realization for available resources of pyrochemical residua and waste containing plutonium at the site of RIAR.

Task 2. Development of process diagram for direct immobilization of available waste containing plutonium.

Task 3. Process diagram development for direct immobilization of plutonium-containing waste generated in RIAR at conversion of "weapon" plutonium.

Task 4. Development of commercial level process diagram for direct immobilization of plutoniumcontaining waste generated at conversion of "weapon" plutonium at RIAR.

We would like to remind what was implemented under the first three tasks, and then discuss in some more details the results of implementation of the fourth one.

There was conducted inventory and classification of available pyrochemical residua and waste containing plutonium. Very simple diagrams are developed for processing of considered residua and waste for both producing of uranium and plutonium dioxides, and their further application in a fuel of BOR-60 reactor and also for immobilization of waste already available and generated at utilization of plutonium residua. Those diagrams are represented in Figs. 1 and 2.

The variant of phosphate and oxide concentrated product immobilization in a form of alumofluorinephosphate glasses is proposed as the most substantiated one at the present time, and the most suitable for both RIAR and other sites under Minatom.

Evaluation is carried out for inputs to realization of processing of plutonium residua and waste available in RIAR before the start of "weapon" plutonium conversion. The types of processing procedures, and expenses are represented in Table 1. 


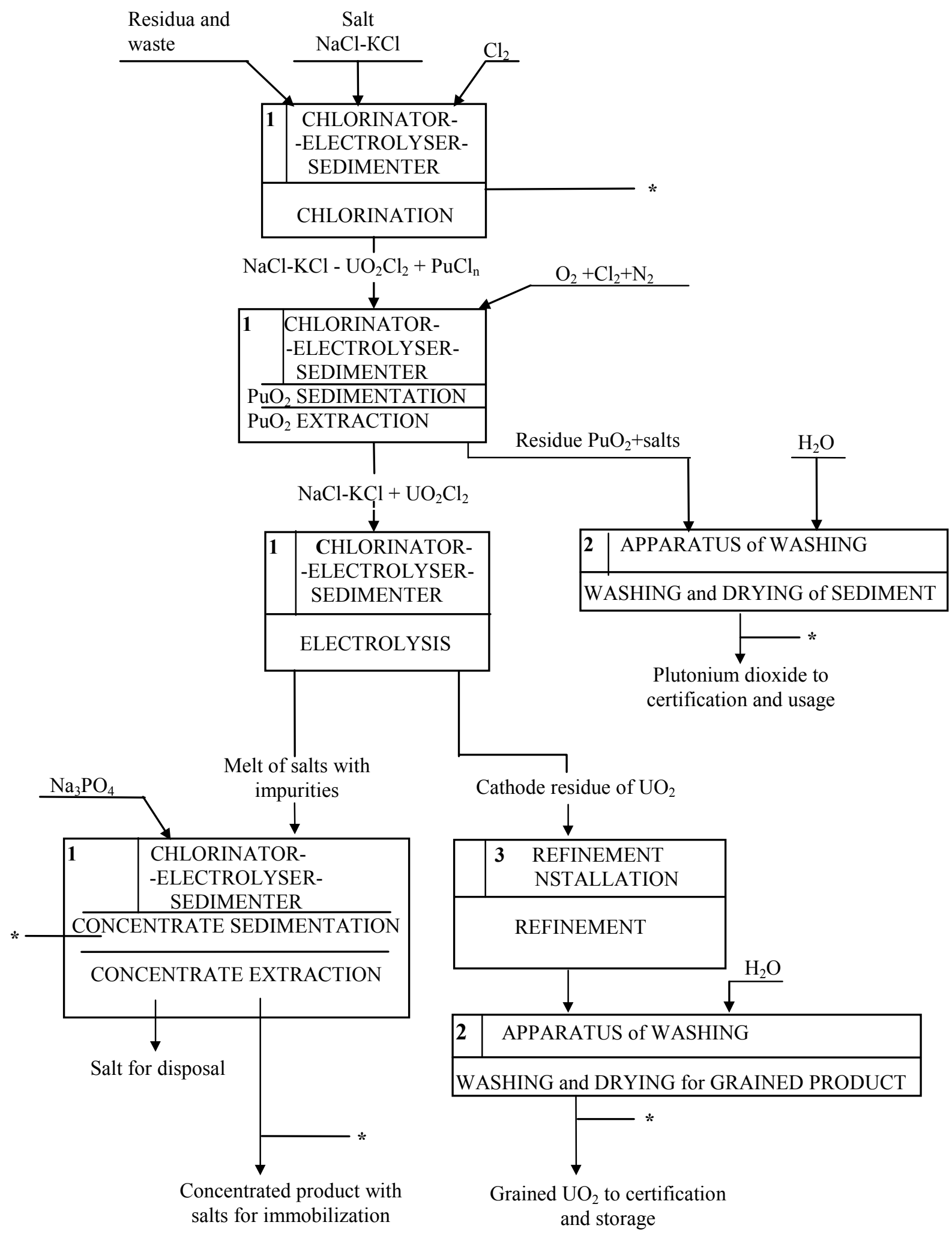

Fig. 1. 


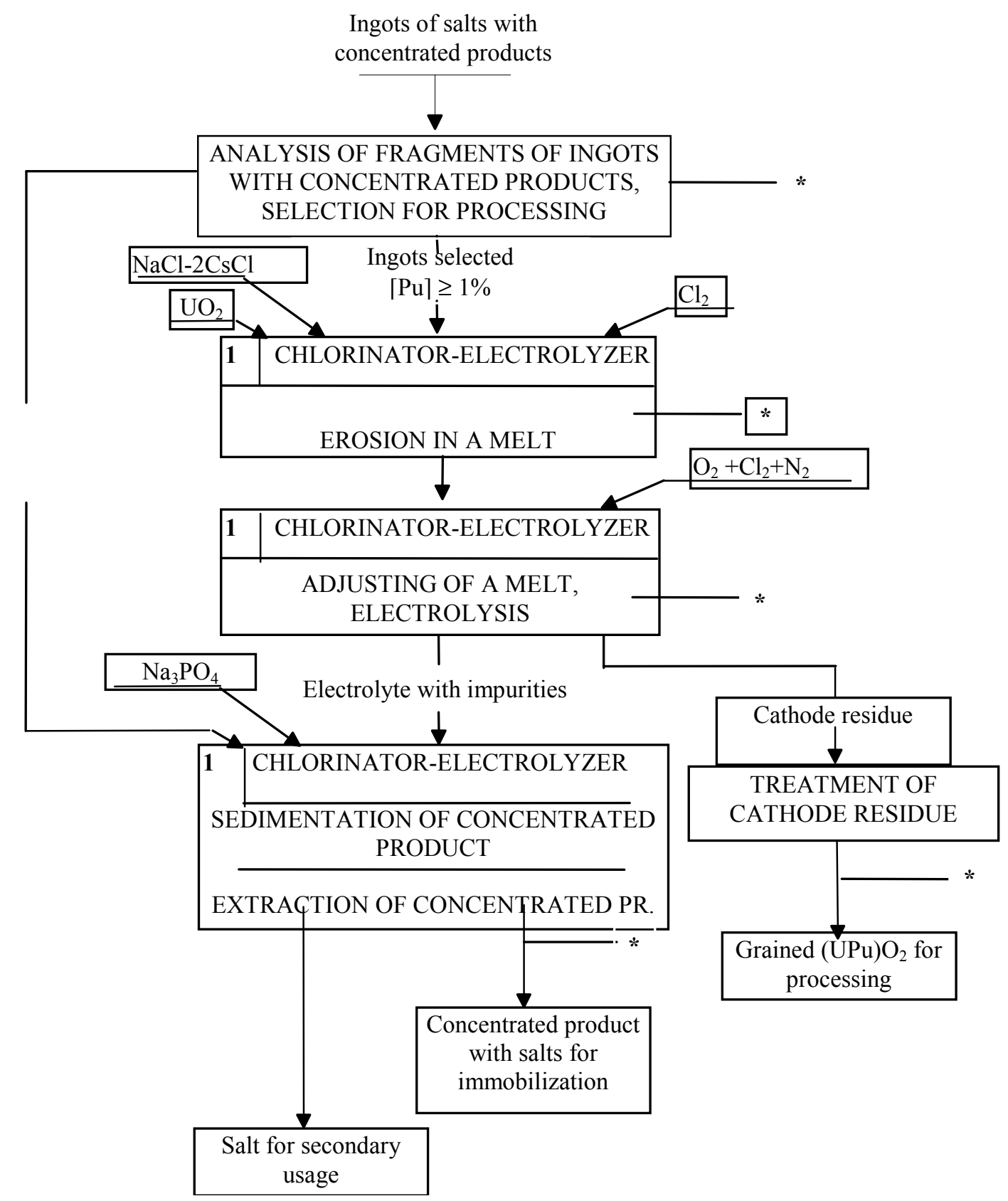

Fig. 2.

In 1998-1999, the RIAR realized conversion of the first delivery $(50 \mathrm{~kg})$ "weapon" plutonium into a fuel for BOR-60 and BN-600 reactors. At that there were generated residua and waste, which were not being considered when working over tasks 1 and 2 . Their inventory and classification is carried out.

Proposal is made for the processing work order in accordance with earlier developed production schemes. 
Table 1. Types, characteristics and values of expenses.

\begin{tabular}{|c|c|c|c|c|c|c|c|}
\hline \multirow{2}{*}{$\begin{array}{c}\text { Pos. } \\
\#\end{array}$} & \multicolumn{3}{|c|}{ Stages of } & \multicolumn{4}{|c|}{ Expense titles (approximate sums, \$ USA) } \\
\hline & $\begin{array}{l}\text { residua and waste } \\
\text { processing }\end{array}$ & Design works & $\begin{array}{c}\text { Equipment } \\
\text { manufacturing }\end{array}$ & Assembly jobs & $\begin{array}{c}\text { Chemical } \\
\text { production, } \\
\text { reagents, utensils }\end{array}$ & $\begin{array}{l}\text { Graphite and } \\
\text { pyrographite } \\
\text { products }\end{array}$ & $\begin{array}{c}\text { Labor expenses } \\
\text { and production } \\
\text { support }\end{array}$ \\
\hline 1 & $\begin{array}{l}\text { Reprocessing of } \\
\text { dead, substandard } \\
\text { fuel, sweepings }\end{array}$ & $\begin{array}{l}\text { Sediment collection } \\
\text { system (SCS), inc. } \\
\text { equipment units, } \\
\text { monitoring and } \\
\text { control circuits } \\
(1500)\end{array}$ & \begin{tabular}{|l} 
SCS, inc. \\
equipment units, \\
monitoring and \\
control circuits \\
$(5000)$
\end{tabular} & $\begin{array}{l}\text { SCS, inc. } \\
\text { equipment units, } \\
\text { monitoring and } \\
\text { control circuits } \\
(1000)\end{array}$ & $\begin{array}{l}\mathrm{Cl}_{2}, \mathrm{Na}_{2} \mathrm{CO}_{3}, \\
\left(\mathrm{NH}_{4}\right)_{2} \mathrm{CO}_{3} \\
(200) \\
\mathrm{Na}_{3} \mathrm{PO}_{4} \\
(25)\end{array}$ & $\begin{array}{l}2 \text { complete sets for } \\
\text { CE } \\
(36000) \\
1 \text { complete for SCS } \\
(8000)\end{array}$ & $(30000)$ \\
\hline 2 & $\begin{array}{l}\text { Processing of } \\
\text { concentrated } \\
\text { products with salts }\end{array}$ & $\begin{array}{l}\text { Sublimate traps } \\
\text { with } \mathrm{PCl}_{3} \\
(500)\end{array}$ & $(200)$ & - & $\begin{array}{l}\mathrm{NaCl}-\mathrm{KCl} ; \mathrm{NaCl}- \\
2 \mathrm{CsCl} \\
(6100) \\
\mathrm{Cl}_{2}, \mathrm{Na}_{2} \mathrm{CO}_{3}, \\
\left(\mathrm{NH}_{4}\right)_{2} \mathrm{CO}_{3} \\
\mathrm{Na}_{3} \mathrm{PO}_{4} \\
(175)\end{array}$ & $\begin{array}{l}1 \text { complete set for } \\
\text { CE } \\
(18000)\end{array}$ & $(15000)$ \\
\hline 3 & $\begin{array}{l}\text { Immobilization of } \\
\text { concentrated } \\
\text { products into } \\
\text { glassy matrixes } \\
\end{array}$ & $\begin{array}{l}\text { Smelter, } \\
\text { monitoring and } \\
\text { control systems } \\
\text { (contractor's work) } \\
(6000)\end{array}$ & $\begin{array}{l}\text { Smelter, monitoring } \\
\text { and control } \\
\text { systems } \\
\text { (contractor's work) } \\
(130000)\end{array}$ & $\begin{array}{l}\text { Smelter, monitoring } \\
\text { and control } \\
\text { systems } \\
\text { (contractor's work) } \\
(20000) \\
\end{array}$ & $\begin{array}{l}\mathrm{NaPO}_{3}, \mathrm{AlF}_{3}, \mathrm{Al}_{2} \mathrm{O}_{3} \\
(1000)\end{array}$ & - & $(5000)$ \\
\hline 4 & $\begin{array}{l}\text { Analytical provision } \\
\text { for the works of all } \\
\text { the sections }\end{array}$ & - & - & - & $\begin{array}{l}\text { Reagents, } \\
\text { chemical and } \\
\text { analytical utensils, } \\
\text { devices, } \\
\text { expendable } \\
\text { materials } \\
(6000)\end{array}$ & - & $\begin{array}{l}\sim 1000 \text { analysis } \\
8800 \text { work.*hour. } \\
(39000)\end{array}$ \\
\hline \multicolumn{2}{|c|}{$\begin{array}{l}\text { TOTAL for all expense } \\
\text { items }\end{array}$} & 8000 & 137000 & 21000 & 13500 & 62000 & 89000 \\
\hline \multicolumn{2}{|c|}{ TOTAL for the Table } & \multicolumn{6}{|l|}{332500} \\
\hline
\end{tabular}


Evaluation of expenses for processing of plutonium residua and waste generated at this stage is carried out. The results are represented in Table 2. Comparison of schemes of processing of plutonium residua and waste is conducted for those developed by the RIAR, and proposed by GSPI for "hypothetic" "weapon" plutonium conversion plant.

It is planned to start in 2005 in the RIAR the operation of "weapon" plutonium conversion complex at production output up to $1500 \mathrm{~kg} \mathrm{MOX-fuel} \mathrm{per} \mathrm{year,} \mathrm{with} \mathrm{further} \mathrm{production} \mathrm{of} \mathrm{fuel} \mathrm{elements} \mathrm{and} \mathrm{FA} \mathrm{(fuel}$ assemblies) for BN-600 reactor. At implementation of the last stage work (Task 4) there was implemented analysis of types of generated wastes and reusable products of this complex, which should be subjected to reprocessing. As well as it was earlier, those types include the following positions: contaminated saltsolvent, washing solutions after preparation of granulated fuel, concentrated phosphates. Combustible products as follows are also additionally included into the circle of reusable products: pyrographite and graphite products, packaging and cleaning materials (polyethylene and polyvinylchloride films, rags, etc.), filtering material of aerosol filters, nozzles from powder filters and sublimation traps. In addition to decrease of their amount, it is supposed to realize secondary recovery of fuel components from all types of waste, reducing a part of irretrievable losses. Discharge gases containing chlorine are included into reusable products. Chlorine will be separated from discharge gases by the way of their step-by-step cooling:

- to $-30^{\circ} \mathrm{C}$ - to decrease humidity of gases;

- to $-100^{\circ} \mathrm{C}$ - to condense chlorine in a liquid phase (basic mass);

- to $-180^{\circ} \mathrm{C}$ - to decrease volume share of chlorine in discharge gases below the permissible limit for working premises (by the means of chlorine condensation in solid state).

After chlorine accumulation in suitable condensers it can be sent for temporary storage and further appropriate usage.

In order to develop a scheme of management of reusable products the possible versions of their transformation were analyzed. For combustible waste, including pyrographite products, it is accepted as advisable to convert their main part into groats or any other free-flowing state, and to burn in a furnace with continuous loading at $\sim 0,1-0,5 \mathrm{~kg} /$ hour productivity. A part of reusable combustible waste can be converted into free-flowing state by embrittlement and crushing at cooling to low temperature. Some more part of combustible waste can be subject to degradation in boiling concentrated sulphuric acid. Material of FPP and FPA filters can be pressed in briquettes (with addition of acetone), and delivered to a furnace for burning.

Gases discharged under waste burning should be set free of dangerous burn products (phosgene, dioxins, chlorous and hypochlorous hydrogen), and of excess moisture. For this purpose they should first be passed through the nozzle heated to $\sim 850^{\circ} \mathrm{C}-1000^{\circ} \mathrm{C}$ to reach destruction of phosgene and dioxins, and then cooled to condense water, $\mathrm{HCl}$ and $\mathrm{HOCl}$, and to set free from radioactive aerosols by the means of filters with FPP material, and only after all those procedures they can be released into atmosphere.

Schematic diagram of procedures of reusable materials' preparation to burning, and procedures of burning is represented in Fig. 3. 
Table 2. Types, characteristics and values of expenses.

\begin{tabular}{|c|c|c|c|c|}
\hline \multirow{2}{*}{$\begin{array}{l}\text { Pos. } \\
\text { numb } \\
\text { er }\end{array}$} & \multirow{2}{*}{$\begin{array}{l}\text { Stages of } \\
\text { residua and waste processing }\end{array}$} & \multicolumn{3}{|c|}{ Expense titles (approximate sums, \$ USA) } \\
\hline & & $\begin{array}{c}\text { Chemical production, reagents, } \\
\text { utensils }\end{array}$ & $\begin{array}{c}\text { Graphite and pyrographite } \\
\text { products }\end{array}$ & $\begin{array}{l}\text { Labor expenses and } \\
\text { production support }\end{array}$ \\
\hline 1 & Processing of salt ingots & $\begin{array}{l}\mathrm{Cl}_{2}, \mathrm{Na}_{2} \mathrm{CO}_{3},\left(\mathrm{NH}_{4}\right)_{2} \mathrm{CO}_{3} \\
(30) \\
\mathrm{Na}_{3} \mathrm{PO}_{4} \\
(70)\end{array}$ & $\begin{array}{l}\text { One complete set for CE } \\
(18000) \\
\text { One complete for SCS* } \\
(2000)\end{array}$ & $\begin{array}{l}\text { Pay of employers and } \\
\text { auxiliary production services } \\
(5000)\end{array}$ \\
\hline 2 & $\begin{array}{l}\text { Immobilization of concentrated } \\
\text { products into glassy matrixes }\end{array}$ & $\begin{array}{l}\mathrm{NaPO}_{3}, \mathrm{AlF}_{3}, \mathrm{Al}_{2} \mathrm{O}_{3} \\
(180)\end{array}$ & - & $(2000)$ \\
\hline 3 & $\begin{array}{l}\text { Analytical provision for the works of } \\
\text { all the sections }\end{array}$ & $\begin{array}{l}\text { REAGENTS, CHEMICAL AND } \\
\text { ANALYTICAL UTENSILS, DEVICES, } \\
\text { EXPENDABLE MATERIALS } \\
(1220)\end{array}$ & - & $(5000)$ \\
\hline & TOTAL for all expense items & $(1500)$ & $(20000)$ & $(12000)$ \\
\hline & TOTAL for the Table & 33500 & & \\
\hline
\end{tabular}

* - Residue collection system. 


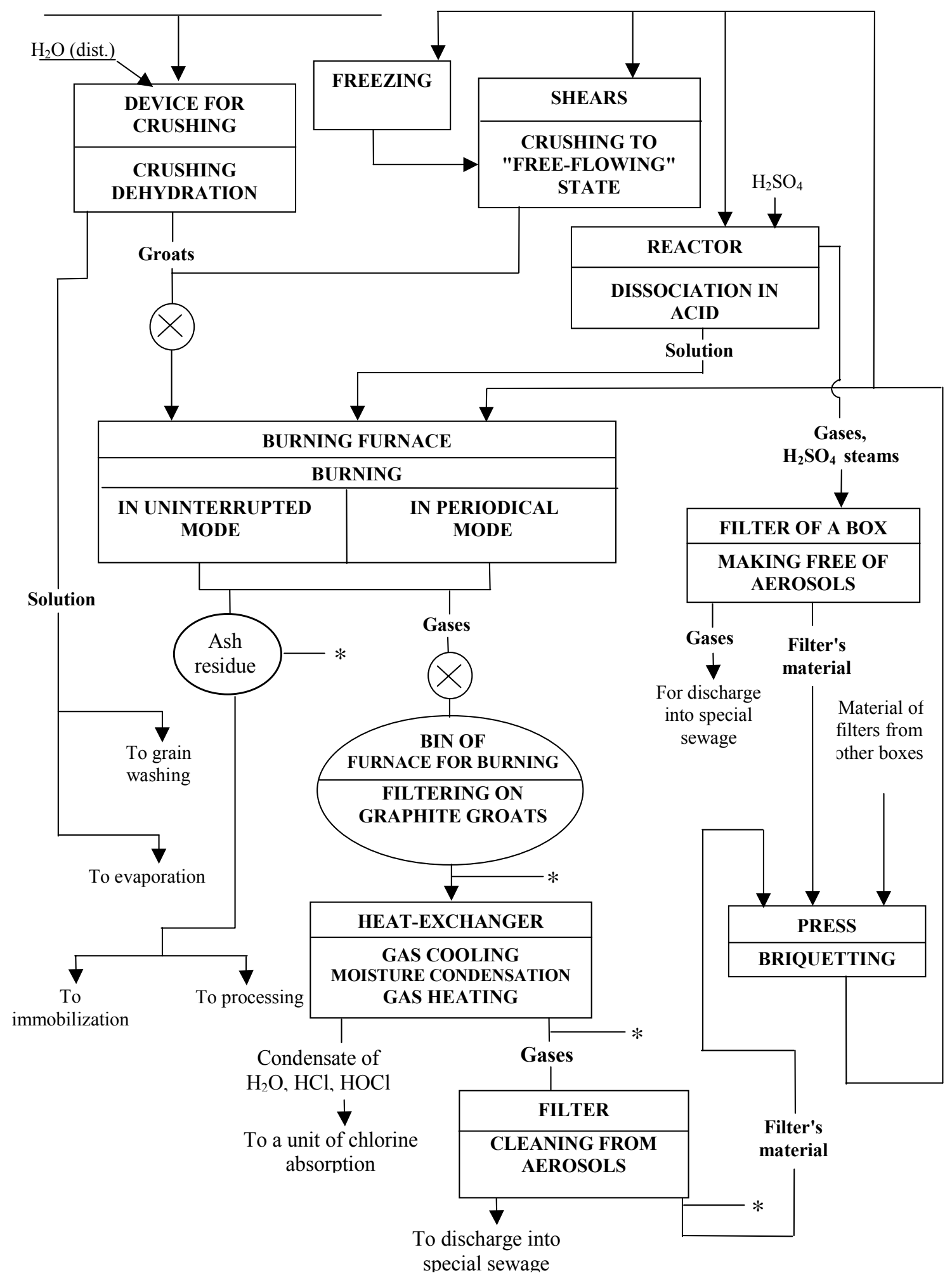

Fig. 3. Schematic diagram of the procedure of reusable material preparation to reprocessing or immobilization.

* Points to control content of radionuclides and high-dangerous combustion products. 
Eventually after burning of any types of combustible waste there will be generated ash residua containing dioxides of uranium, plutonium and americium. Depending on their mass share, ash residua should be sent to secondary salt regeneration for secondary extraction of valued components (according to the diagram of Fig.1), or together with concentrated product of phosphates - to direct immobilization.

It is assumed to test at least in the course of pilot operation of the complex two variants of waste immobilization: confining of concentrated phosphates in ceramics, and their vitrification.

Concentrated product of phosphates represents according to its original characteristics a powdery material - a mixture of phosphates insoluble in melts of salts and in water, and characterized by high thermal and chemical stability. In reality, the only feature discriminating concentrated product of phosphates from other materials suitable for long-term storage and disposal is its developed surface, as it is available in powdery state. It can be compactly formed, if pressed by temperate force (up to 1500 $\mathrm{kg} / \mathrm{cm}^{2}$ ) into briquettes (blocks, discs), and then caked by calcinations at moderate temperature $\left(850^{\circ} \mathrm{C}\right.$, to realize enclosing in ceramics).

The possible variant is to make blocks of oxide ceramics, as well as concentrating of impurities of reusable electrolytes in a form of oxides. Ash residua after burning of combustible waste can also be additionally burned into a mixture of oxide concentrated products. Naturally, it is required to study additionally the possibility of caked concentrated product to include into itself and to retain plutonium and other actinides at a long-term storage. Advantages of inclusion in ceramics against vitrification consist in an absence of necessity to dilute waste with additional components (components of glassy frit), and also in possibility to apply more common and compact equipment.

Nevertheless, in order to make a correct choice of immobilization method for phosphate concentrated product, containing plutonium, it is stipulated to test the method of phosphate concentrate vitrification in a course of pilot operation of the site, as the most substantiated variant by the present moment.

We consider the methods of casehardening and bituminisation to be unacceptable for the RIAR conditions.

So the basic items of the circuit of management of waste (reusable products) from the installation of MOX-fuel production are considered.

Schematic diagram of the basic technological process, provided with some fragments of the system of management of basic reusable products is represented in Fig. 4. Another fragments of general circuit of waste management are given in Figs. 5-7. 


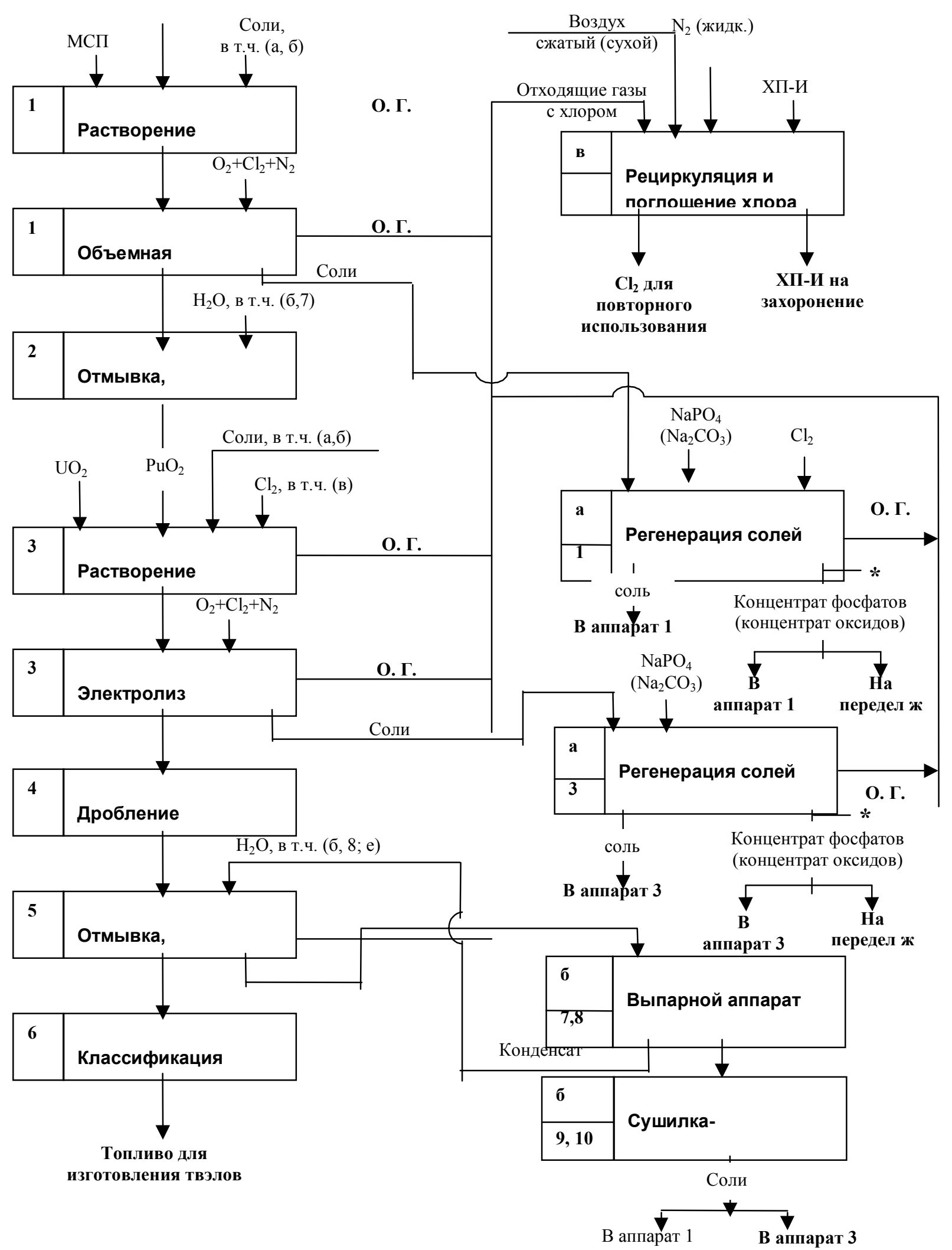

Fig. 4. Diagram of technological process on the basis of modernized installation of MOX-fuel production 


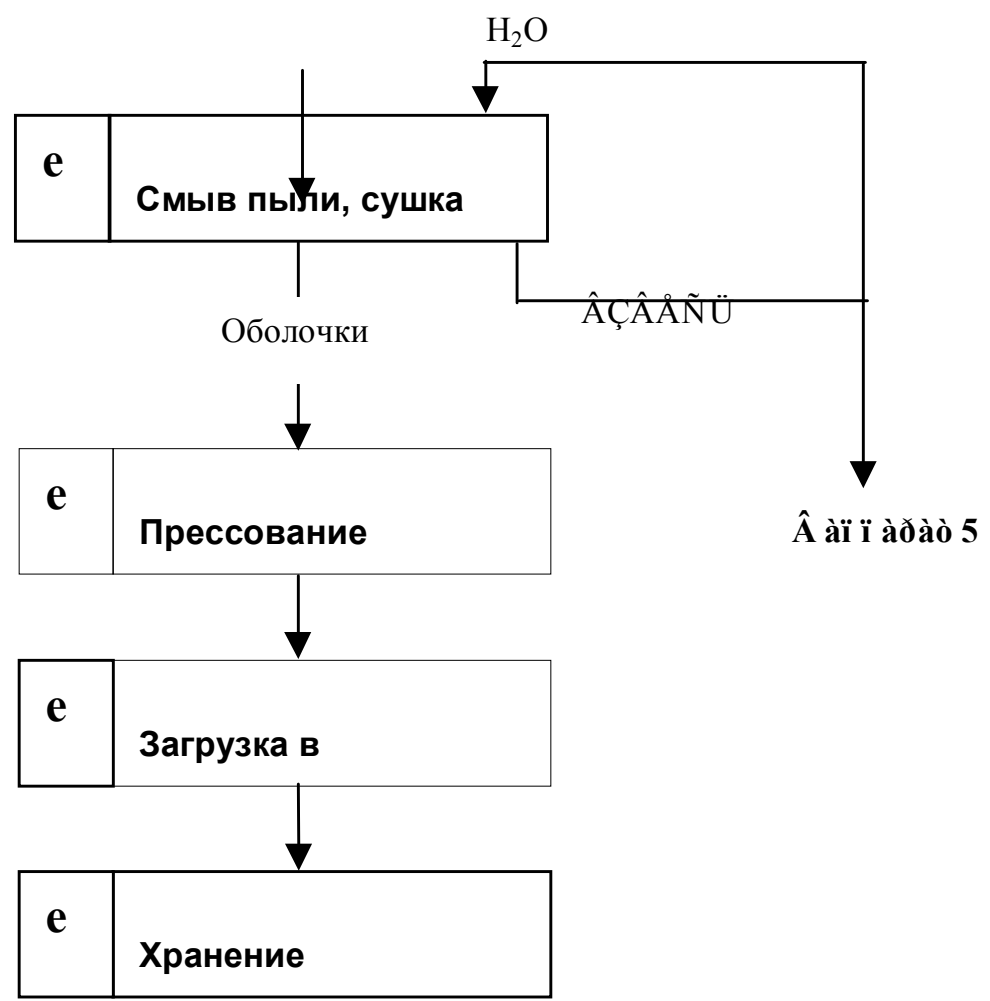

Fig. 5. Treatment circuit for envelops of defective fuel elements.

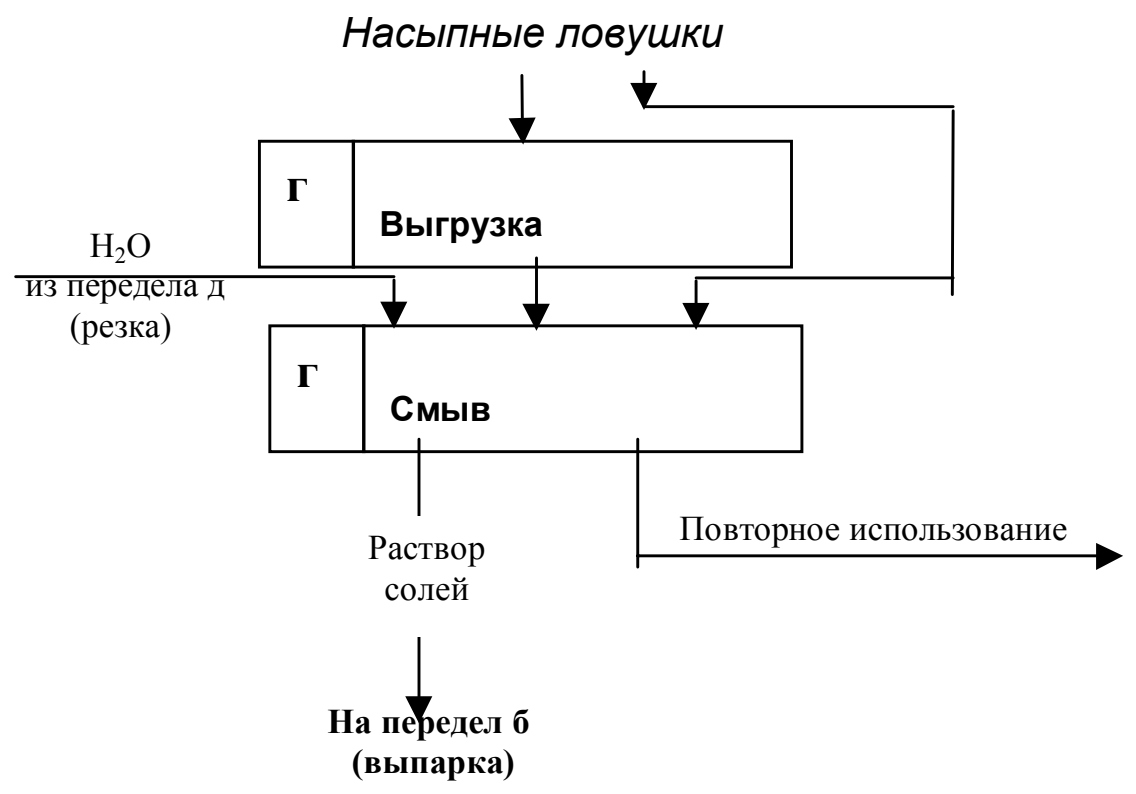

Fig. 6. Treatment circuit for bulk filters and traps of sublimates. 


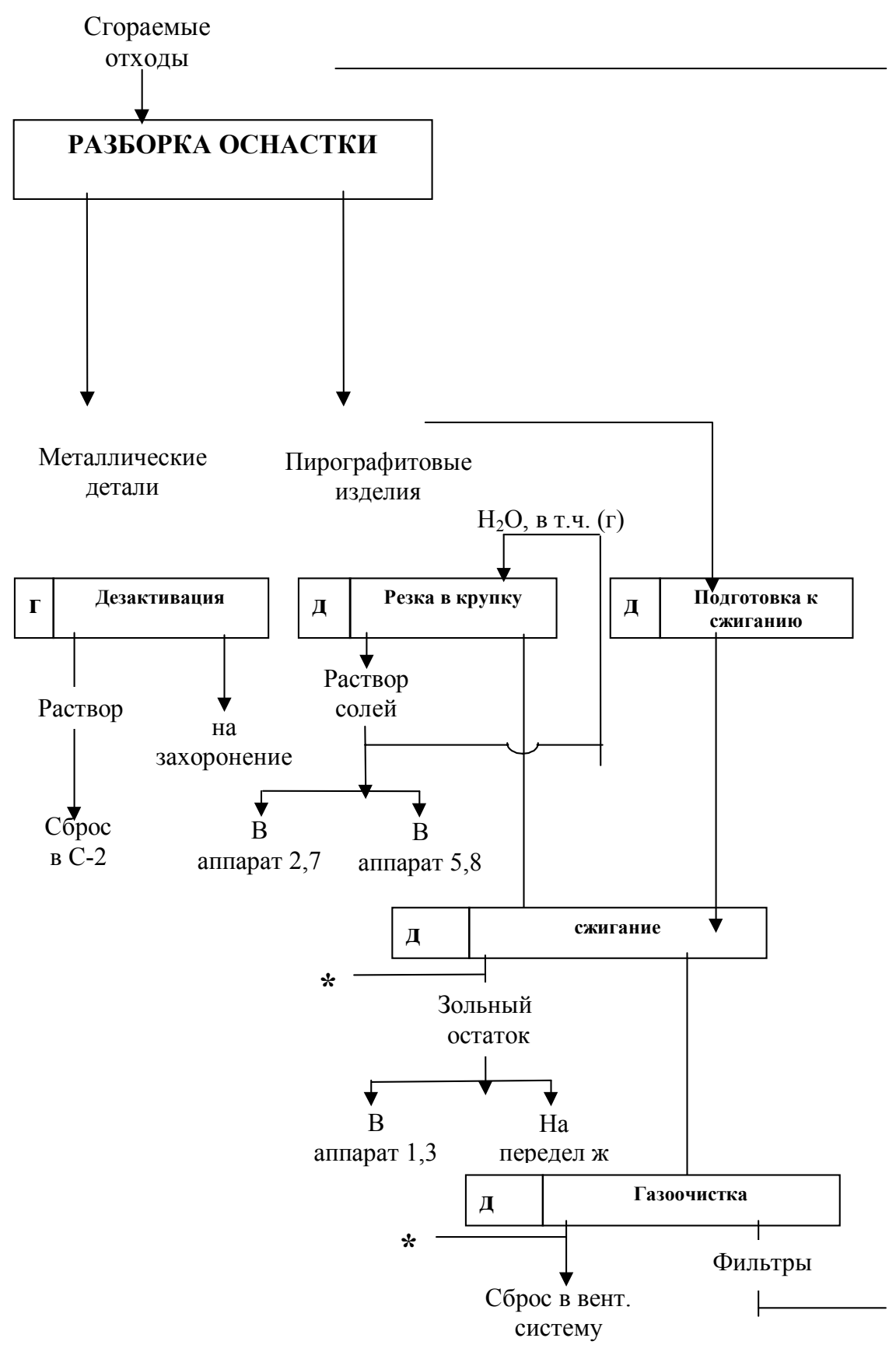

Fig. 7. The circuit of combustible waste processing. 


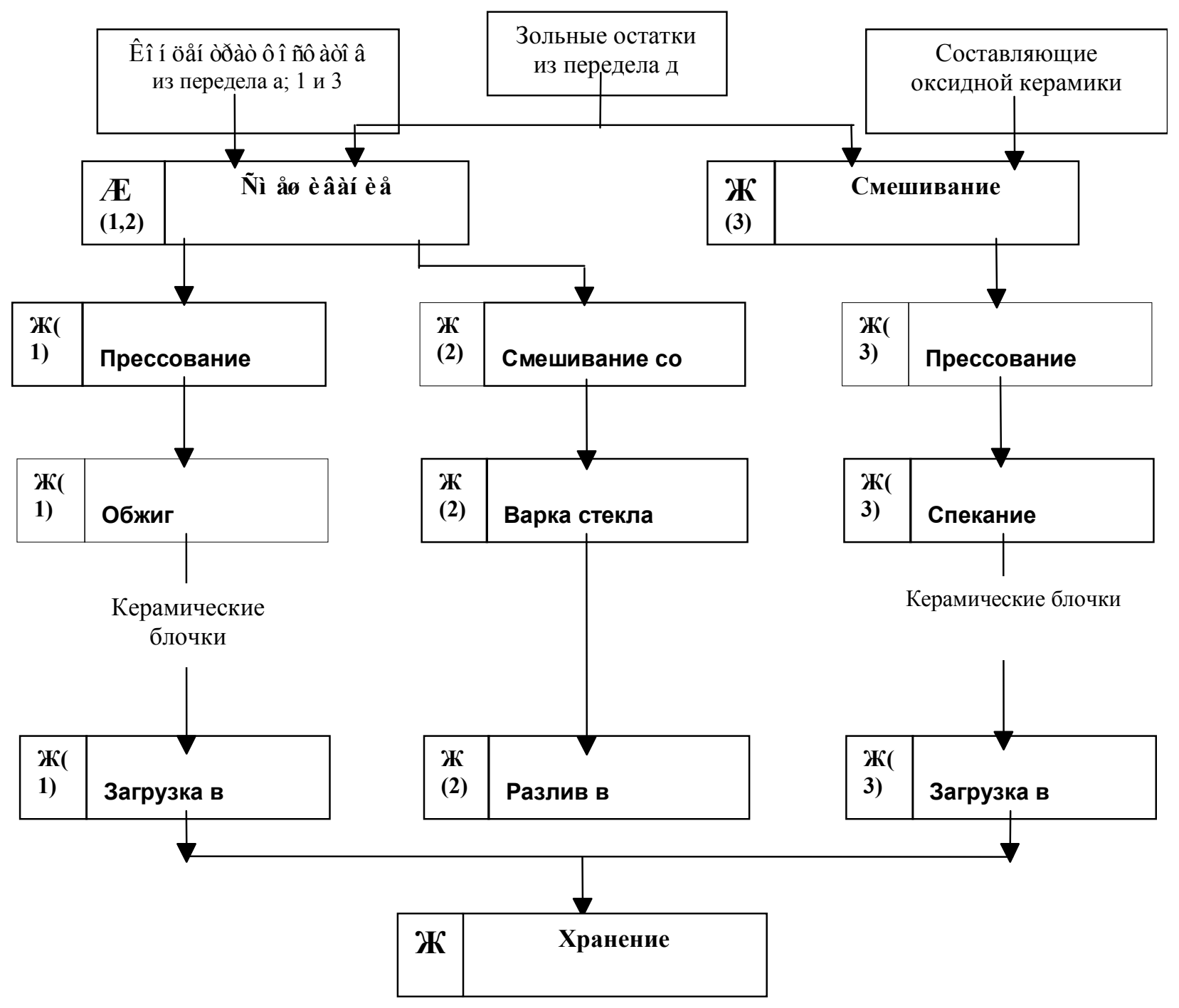

Fig. 8. Diagram of realization of waste immobilization into stable matrixes. 
In the end of run MOX-fuel production waste (and they consist of concentrated product of phosphates or oxides of impurities generated under the process of salt regeneration, and ash residua from processing of combustible spent materials) should be included into solid stable forms, suitable for long-term safe storage and disposal. The variants of immobilization methods are represented in the diagram of Fig. 8.

The processes of processing of waste from MOX-fuel production installation are assumed for realization at the adjoining section (revisions e and $\mathrm{g}$, and probably $\mathrm{d}$ and $\mathrm{f}$ ).

Block representation of waste processing with the proposed equipment arrangement over the boxes is given in Fig. 9.

There are available proposals on the structure of apparatuses for realization of real procedures.Technical and economic evaluations are carried out for the circuit of waste management.

The following technical and economic indexes are accepted for substantiation of expenses of equipment creation for the section of waste processing:

\section{Productivity of the section of waste processing:}

- On burning of pyrographite products. $\sim 350 \mathrm{~kg} /$ year;

- Immobilization of concentrated phosphates and oxides..... $\quad \sim 30 \mathrm{~kg} / \mathrm{year}$.

\section{Waste volume, including:}

- vent. air

- solid high-level

- liquid
4000 thous. $\mathrm{m}^{3} /$ year;

$$
2,5 \mathrm{~m}^{3} \text { / year; }
$$

$3000 \mathrm{~m}^{3} /$ year.

Expenses are summarized in Table 3. Expenses of creation of the section include:

- Work on designing and development of working documentation;

- Production of non-standard equipment, and purchase of ordered products;

- Assembly and start-adjusting jobs;

- Testing operation. 


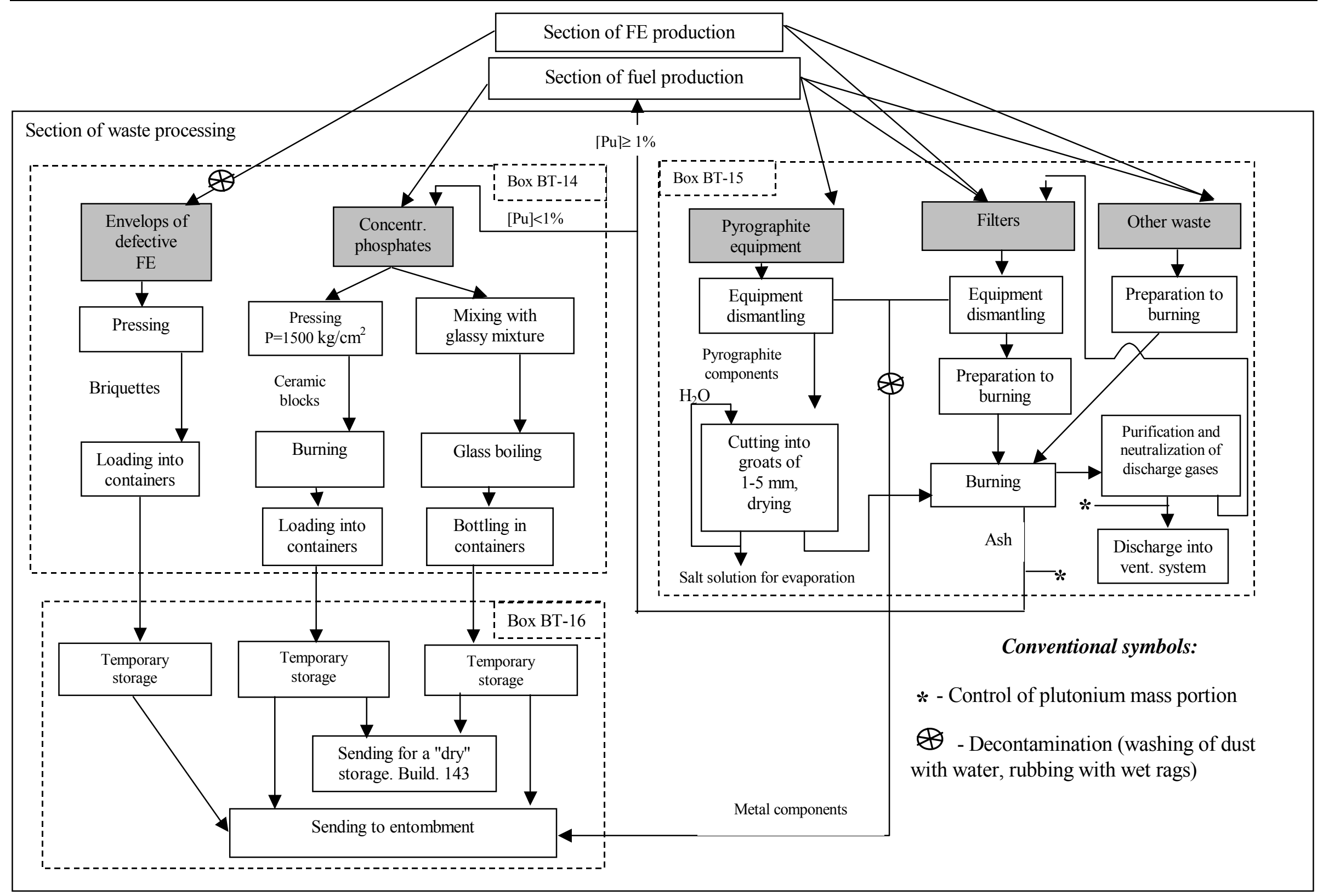

Fig. 9. Block diagram of waste processing. 
Table 3. Equipment expenses for MOX-fuel production installation waste processing.

\begin{tabular}{|c|c|c|c|c|c|}
\hline Pos. \# & Name of equipment & $\begin{array}{l}\text { Quantity } \\
\text { of units }\end{array}$ & $\begin{array}{l}\text { Price, thous. } \\
\text { \$USA }\end{array}$ & $\begin{array}{l}\text { Cost, thous. } \\
\text { \$ USA }\end{array}$ & Notes \\
\hline 1 & Chlorine-recycling unit & 1 & 150 & 150 & $\begin{array}{l}\text { Included into basic } \\
\text { circuit }\end{array}$ \\
\hline 2 & Chlorinator-electrolyzer & $2 / 5$ & 53 & 21 & $\begin{array}{l}\text { Included into basic } \\
\text { circuit }\end{array}$ \\
\hline 3 & Pilot smelter & 1 & 136 & 156 & $\begin{array}{l}\text { Supposed to purchase } \\
\text { at VNIINM }\end{array}$ \\
\hline 4 & $\begin{array}{l}\text { Unit for crushing of } \\
\text { pyrographite products, and } \\
\text { preparation of groats }\end{array}$ & 1 & 34 & 34 & \\
\hline 5 & $\begin{array}{l}\text { Furnace for burning of } \\
\text { pyrographite groats }\end{array}$ & 1 & 30 & 30 & \\
\hline 6 & $\begin{array}{l}\text { Gas purification system } \\
\text { (burning products) }\end{array}$ & 1 & 25 & 25 & \\
\hline 7 & $\begin{array}{l}\text { Press for production of blocks } \\
\text { of concentrated phosphates }\end{array}$ & 1 & 20 & 20 & \\
\hline 8 & Furnace for caking & 1 & 30 & 30 & \\
\hline 9 & Protective boxes & 3 & 13 & 39 & \\
\hline 10 & $\begin{array}{l}\text { Other process } \\
\text { Devices }\end{array}$ & & & 45 & \\
\hline \multicolumn{3}{|l|}{ TOTAL: } & & 550,0 & \\
\hline
\end{tabular}

The table contains chlorine-recycling unit (pos.1), while in reality it is arranged within the limits of "basic" installation of fuel production. The unit is included into the structure of equipment for waste processing, as thanks to it chlorine turns from amount of spent reagents into a group of reusable ones. Chlorinatorelectrolyzer (pos. 2) is included into the structure of waste processing equipment, as it is to be used for regeneration of contaminated salt-diluter, turning it also into a group of reusable products. The value 2/5 means that 2 chlorinators-electrolyzers are to be used in the process, and averagely after each fifth cycle salt-diluter should be subject to regeneration. At that appears the main kind of waste of MOX-fuel production installation, that is the concentrated product of phosphates, containing impurities and sizeable quantity of ${ }^{241} \mathrm{Am}$ isotope.

Preliminary values of capital expenses for creation of modernized complex for MOX-fuel production and manufacturing of vibroconcentrated FE for BN-600 reactor (for fuel output of $1500 \mathrm{~kg}$ per year) valued in 4,3 million \$US, including expenses of creation of the installation itself, accepted as 1.2 million \$US, and waste processing section - 0,4 million $\$$ US. At the present time these values should be reconsidered for a number of reasons. Firstly, in accordance with the "Agreement on modernization of installations for production of granulated MOX-fuel, vibroconcentrated FE and FA of BN-600 reactor with a purpose to study the possibility to apply this installation for conversion of excess weapon plutonium" between Japan Nuclear Cycle Development Institute (JNC) and RF SSC RIAR on 27.07.2001, it is required to arrange additionally both work places and equipment for conversion of metal plutonium alloy (MPA) into plutonium dioxide, and also to create the system of physical protection, and the system of account and control of nuclear materials at the complex. Secondary, with account of requirements of RF Gosatomnadzor, in modernized installation there will be applied boxes provided with additional protection, meeting those 
requirements. The boxes should be equipped with "mechanical arms" (as it is supposed - A-202, manufactured by HWM, Germany).

At the present time designing of the complex is ongoing; at its completion total evaluation of expenses for both creation of the complex in whole and its separate sections will be carried out.

Operational costs of the section of waste processing are determined on the basis of labor expenditures of procedures on waste processing, need of chemical reagents, pyrographite products, auxiliary materials, services of a workshop of waste management, services of analytical laboratory.

Operational costs of the section of waste processing are represented in Table 4. Participation of analytical laboratory is taken into account in columns 2 and 4 of Table 4.

Estimate of purchase expenditures for reagents, materials and pyrographite products is carried out on the basis of the data summarized in Table 5. Estimate of wage fund for the personnel of waste processing section is carried out on the basis of the data given in Table 6 .

Table 4. Operational costs of the section of waste processing.

\begin{tabular}{|c|c|c|}
\hline $\begin{array}{l}\text { Pos. } \\
\text { num. }\end{array}$ & Name of expense items & $\begin{array}{l}\text { Sum } \\
\text { \$US/year }\end{array}$ \\
\hline 1 & Auxiliary materials (materials and reagents, pyrographite products) & 31585 \\
\hline 2 & Wage fund (WF) & 29160 \\
\hline 3 & Charges on pay-roll & 10500 \\
\hline 4 & Other direct expenses & 13575 \\
\hline 5 & $\begin{array}{l}\text { Services of a workshop of waste management, } \\
\text { including treatment of: } \\
\text { - high-level solid waste, } \\
\text { - liquid waste, } \\
\text { - high-level gaseous waste, } \\
\text { - ventilation air }\end{array}$ & $\begin{array}{r}268940 \\
2500 \\
11500 \\
4940 \\
250000\end{array}$ \\
\hline 6 & $\begin{array}{l}\text { Workshop's expenditures, } \\
\text { including: } \\
\text { - WF of administration and management personnel (AMP), } \\
\text { - charges on pay-roll, } \\
\text { - charges on labor protection, } \\
\text { - maintenance and repair of buildings and structures, } \\
\text { - equipment maintenance and operation, } \\
\text { - power inputs, } \\
\text { - other expenditures }\end{array}$ & $\begin{array}{r}52500 \\
2600 \\
950 \\
10500 \\
5000 \\
22450 \\
9500 \\
1500\end{array}$ \\
\hline 7 & $\begin{array}{l}\text { Common institutional expenditures (running cost of maintenance, guard, transport, } \\
\text { AMP) }\end{array}$ & 43740 \\
\hline \multicolumn{2}{|c|}{ TOTAL: } & $450000^{*}$ \\
\hline
\end{tabular}

* - Predicted operational costs of the installation of fuel production in whole reach 1,5 million \$ USA per year. 
Thus, during the works on the Contract B 506204 there were implemented all the given tasks, as well as carried out evaluations of both available amount of plutonium residua a waste, generated at the RIAR in the course of development and mastering of pyroelectrochemical technique of fuel production for atomic reactors, and also predicted costs of their processing and immobilization. Wide search for possible versions of processing and immobilization of waste, including those generated at conversion of "weapon" plutonium into fast neutron reactor fuel, as well as their analysis is carried out; the choice is made, and substantiation of circuits of residua and waste processing, waste immobilization into stable solid matrixes for a long-term safe storage is realized. Real proposals are given on both conduction of waste processing procedure, and also on development of equipment required. Technical and economic evaluations are implemented for prospective costs of creation and operation of the section of reusable waste processing and waste immobilization, included into the complex of fuel and FE production, having productivity of $1500 \mathrm{~kg} /$ year.

Table 5. Material demand.

\begin{tabular}{|l|l|l|l|}
\hline \multicolumn{1}{|c|}{ Material name } & \multicolumn{1}{c|}{$\begin{array}{c}\text { Quantity, } \\
\text { kg/year }\end{array}$} & \multicolumn{1}{c|}{$\begin{array}{c}\text { Price, Rub./kg } \\
\text { (for compl.) }\end{array}$} & \multicolumn{1}{c|}{ Sum, Rub./year } \\
\hline $\mathrm{Na}_{2} \mathrm{CO}_{3}$, sodium carbonate & $5500^{*}$ & 3,58 & 19690 \\
\hline$\left(\mathrm{NH}_{4}\right)_{2} \mathrm{CO}_{3}$, ammonia carbonate & $3000^{*}$ & 8,28 & 24840 \\
\hline $\mathrm{Na}_{3} \mathrm{PO}_{4}$, sodium ortophosphate & 60 & 15 & 900 \\
\hline $\mathrm{AlF}_{3}$, aluminum fluoride & 18 & 62 & 1116 \\
\hline $\mathrm{Al}_{2} \mathrm{O}_{3}$, aluminum oxide & 4,5 & 36 & 162 \\
\hline $\mathrm{NaPO}_{3}$, sodium metaphosphate & 67 & 73 & 4891 \\
\hline $\mathrm{Cl}{ }_{2}$,balloon chlorine & $3500^{*}$ & 5,7 & 19950 \\
\hline Pyrographite products & 2 compl.. & 438000 & 876000 \\
\hline TOTAL: & \multicolumn{2}{|l|}{$947549\left(31585\right.$ \$USA/year ${ }^{* *}$} \\
\hline
\end{tabular}

* - While the unit of chlorine recycling and absorption is not put into operation. ** - The rate of American Dollar is accepted approximately as equal to 30 Rub./\$ USA. 
Table 6. Wage fund estimate for processing of reusable materials and waste.

\begin{tabular}{|c|c|c|c|c|c|c|c|}
\hline $\begin{array}{l}\text { Pos. } \\
\text { N }\end{array}$ & Procedure's name & $\begin{array}{l}\text { Material volume, } \\
\text { Kg/year }\end{array}$ & $\begin{array}{l}\text { Procedure } \\
\text { duration, } \\
\text { hours }\end{array}$ & $\begin{array}{l}\text { Periodicity, } \\
\text { year }^{-1}\end{array}$ & $\begin{array}{l}\text { Personnel } \\
\text { quantity, } \\
\text { work. }\end{array}$ & $\begin{array}{l}\text { Labor } \\
\text { expense, } \\
\text { work./year }\end{array}$ & $\begin{array}{l}\text { WF, \$ } \\
\text { USA/year }\end{array}$ \\
\hline 1 & $\begin{array}{l}\text { Separation of metal devices from } \\
\text { pyrographite products }\end{array}$ & 350 & 6 & 10 & 1 & 60 & 300 \\
\hline 2 & $\begin{array}{l}\text { Crushing of pyrographite products into } \\
\text { groats }\end{array}$ & 350 & 24 & 10 & 1 & 240 & 1200 \\
\hline 3 & Washing and drying of groats & 350 & 24 & 10 & 1 & 240 & 1200 \\
\hline 4 & Burning of pyrographite groats & 350 & 350 & 10 & 1 & 3500 & 17500 \\
\hline 5 & Other waste preparation to burning & 35 & 10 & 10 & 1 & 100 & 500 \\
\hline 6 & Other waste burning & 35 & 6 & 70 & 1 & 420 & 2100 \\
\hline 7 & $\begin{array}{l}\text { Pressing of blocks of concentrated product } \\
\text { of phosphates }\end{array}$ & $23+5$ (ash) & 12 & 50 & 1 & 600 & 3000 \\
\hline 8 & Caking of blocks of concentrated phosphates & $23+5$ (ash) & 12 & 50 & 1 & 600 & 3000 \\
\hline 9 & Vitrification & $\begin{array}{l}23+5 \text { (ash) } \\
+100 \text { (glassy fritta) }\end{array}$ & 12 & 3 & 2 & 72 & 360 \\
\hline \multicolumn{2}{|c|}{ TOTAL: } & & & & & & 29160 \\
\hline
\end{tabular}




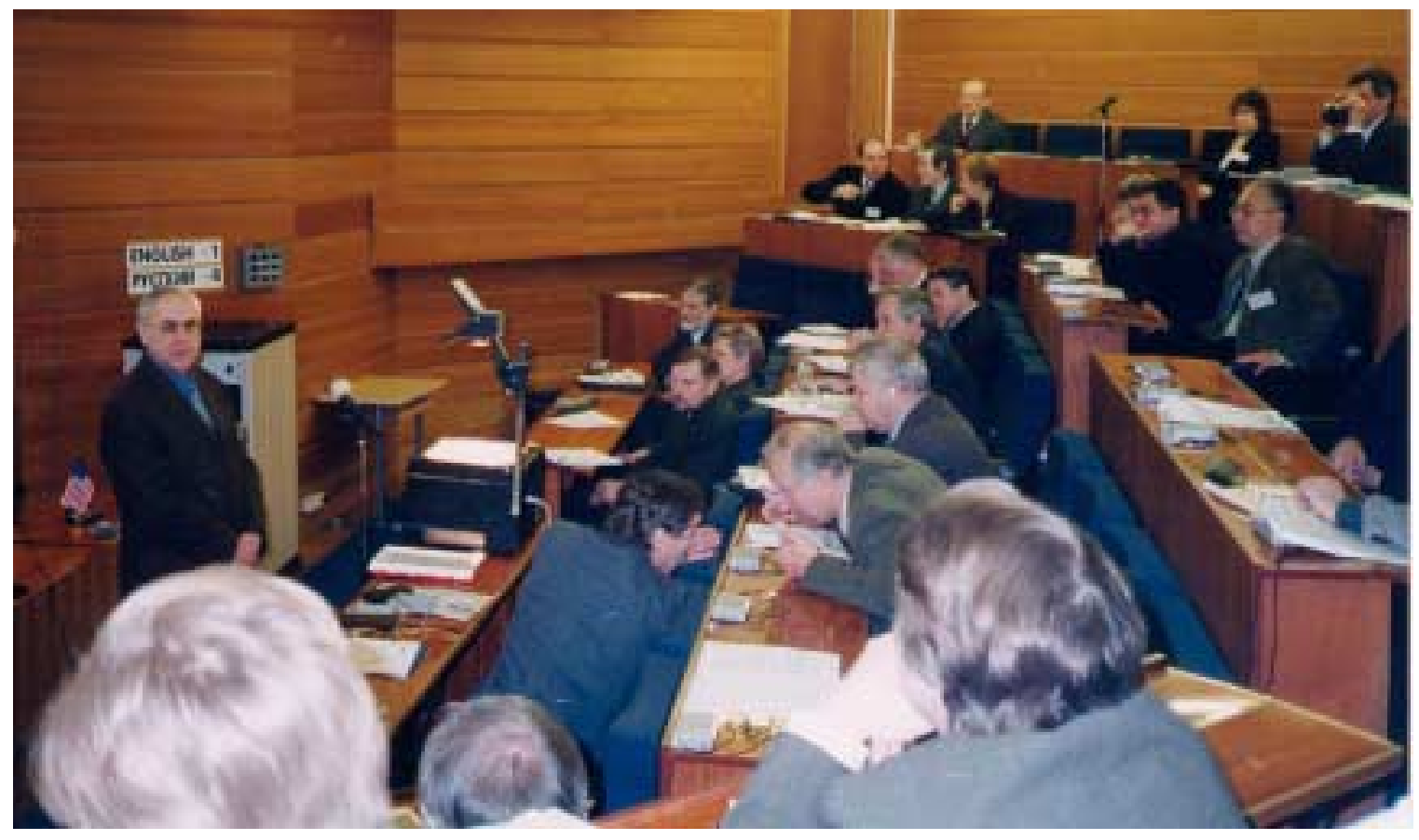

III. Research and Development for Plutonium Immobilization and Other Related Activities 

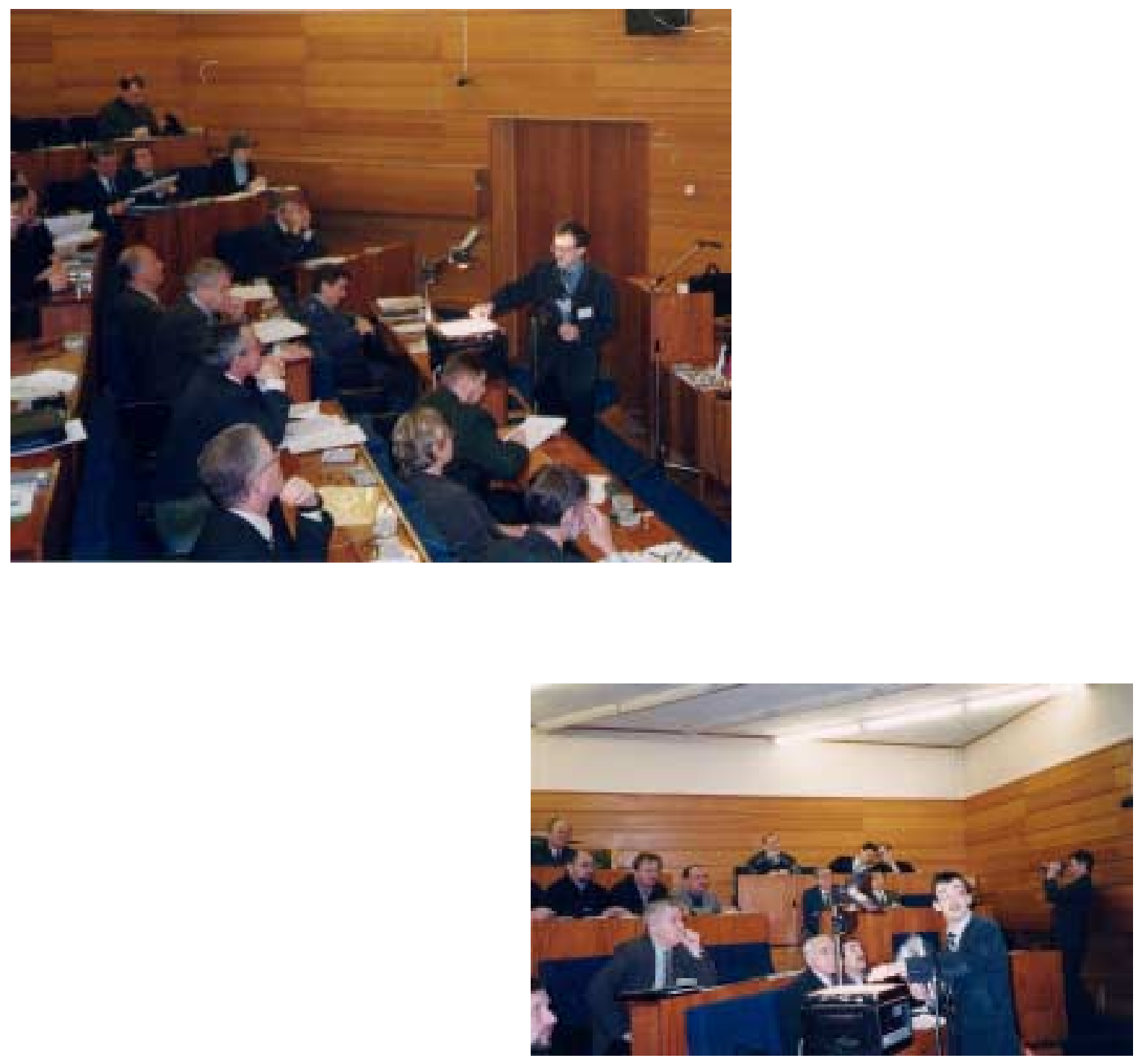

III.1. Plutonium Glasses for Immobilization 


\title{
Development of Matrix Compositions and Process of Immobilization for Hard-to-Process Pu-containing Sludges of "Mayak" (B506228)
}

\author{
Yurii I. Matyunin, VNIINM
}

\section{Introduction}

The immobilization of hard-to-process plutonium-containing sludges with the manufacturing of stable solid compositions is one of the problems that should be solved in the disposal of radioactive wastes.

Work on the selection of materials, preparation with the use of Joule-heated furnaces and the cold crucible induction melter (CCIM) technologies, and investigation of materials that are most suitable for immobilizing Pu-containing sludges have been carried out at the All-Russian Scientific Research Institute of Inorganic Materials (VNIINM) and the Institute of the Geology of Ore Deposits, Petrography, Mineralogy, and Geochemistry (IGEM), Russian Academy of Sciences in the framework of the agreement with Lawrence Livermore National Laboratory (LLNL) on the material and technical support.

This paper presents the data on the synthesis of materials based on phosphate and silicate compositions, which contain simulated wastes of "Mayak" storage tanks, in the muffle furnace and by using the "cold crucible" induction melting (CCIM) method.

\section{Synthesis and Characterization Measurements of Matrices}

At the first stage of the work, we selected materials based on the analysis of waste compositions of PA "Mayak" pyroxene $(\mathrm{Na}, \mathrm{Ca}, \mathrm{Mn})(\mathrm{Mg}, \mathrm{Fe})(\mathrm{Si}, \mathrm{Al})_{2} \mathrm{O}_{6}$ and garnet $(\mathrm{Na}, \mathrm{Ca}, \mathrm{REE})_{3}(\mathrm{Fe}, \mathrm{Cr}, \mathrm{Al}, \mathrm{Zr})_{2}(\mathrm{Si}, \mathrm{Al}, \mathrm{Fe})_{3} \mathrm{O}_{12}$ matrices, as well as phosphate glass used for vitrification of HLW.

Using the lab-scale CCIM facility and muffle furnace, we synthesized phosphate glasses and pyroxenegarnet-like compositions.

\section{Glass Ceramics with Pyroxene Stoichiometry}

Table 1 gives the calculated compositions of synthesized pyroxene-like matrices.

Samples 1 and 2 (Table 1) consist of a glass. In transmitted light, the glass is homogeneous with a brown hue. In crossed nicols, the glass is isotropic. The diffraction patterns do not show any reflections of crystalline phases; i.e., the sample is x-ray amorphous. Waste components are uniformly dissolved in the glass without formation of crystalline phases.

Sample 3 is close in composition to samples 1 and 2. The given ratio between components in a batch guides the formation of a pyroxene group mineral. According to data from transmission microscopy, the sample consists of glass and crystalline phases. Further examination with a "JSM-5300+Link ISIS" scanning electron microscope showed that the sample is composed of the glass and crystalline phases (Fig. 1). The glass accounts for $80-85 \%$ of the sample, and is homogeneous in structure and composition (Fig. 1, Table 2). The glass composition includes $\mathrm{SrO}$ (about 5\%), $\mathrm{Cs}_{2} \mathrm{O}$ (about 6.5\%), and $\mathrm{Ce}_{2} \mathrm{O}_{3}$ (about $7 \%$ ). 
The crystalline phases, represented by forsterite, are cerianite (Fig. 1). Forsterite (with the theoretical formula $\mathrm{Mg}_{2} \mathrm{SiO}_{4}$ ) belongs to the olivine group. Two forsterite generations differing in their crystal shape are formed in the sample. Forsterite- 1 is observed in the form of elongated prismatic crystals, and forsterite-2 forms characteristic, short prismatic crystals often with a skeleton shape. In cross section, the crystals have the form of elongated hexagons.

Table 1. Calculated compositions glass ceramics with pyroxene stoichiometry.

\begin{tabular}{|c|c|c|c|c|c|c|c|c|}
\hline \multirow{2}{*}{$\begin{array}{c}\text { Oxide, wt } \\
\%\end{array}$} & \multicolumn{8}{|c|}{ Sample no. } \\
\hline & 1 & 2 & 3 & 4 & 5 & 6 & 7 & 8 \\
\hline $\mathrm{Na}_{2} \mathrm{O}$ & 9.8 & 9.2 & 9.3 & 13.4 & 13.4 & 15.1 & 10.6 & 29.7 \\
\hline $\mathrm{TiO}_{2}$ & - & - & - & & & 0.51 & 0.5 & 0.5 \\
\hline $\mathrm{Al}_{2} \mathrm{O}_{3}$ & - & - & 2.6 & & & 12.1 & 10.9 & 11.0 \\
\hline $\mathrm{Fe}_{2} \mathrm{O}_{3}$ & 27.9 & 24.5 & 6.0 & 18.3 & \begin{tabular}{|l|}
17.6 \\
\end{tabular} & 20.8 & 30.1 & 7.2 \\
\hline $\mathrm{Cr}_{2} \mathrm{O}_{3}$ & - & - & - & 4.2 & 4.0 & 3.0 & 3.9 & 2.1 \\
\hline $\mathrm{NiO}$ & - & - & - & 2.8 & 2.7 & 1.0 & 1.1 & 0.6 \\
\hline $\mathrm{SiO}_{2}$ & 48.0 & 48.0 & 52.8 & 50.0 & 50.0 & 28.7 & 19.2 & 27.9 \\
\hline $\mathrm{MgO}$ & - & - & 16.2 & - & - & 3.7 & 4.6 & 3.1 \\
\hline $\mathrm{CaO}_{2}$ & - & - & - & - & - & 8.4 & 8.4 & 6.9 \\
\hline $\mathrm{MnO}_{2}$ & - & - & - & - & - & 1.25 & 1.47 & 1.2 \\
\hline $\mathrm{Cs}_{2} \mathrm{O}$ & 6.1 & 7.8 & 5.0 & - & - & - & - & - \\
\hline $\mathrm{SrO}$ & 3.6 & 4.6 & 3.0 & - & - & - & - & - \\
\hline $\mathrm{BaO}$ & 3.6 & 4.6 & - & & & 5.4 & 9.3 & 6.6 \\
\hline $\mathrm{PbO}$ & 1.0 & 1.3 & - & - & - & $0.56^{*}$ & $0.96^{*}$ & $0.38^{*}$ \\
\hline $\mathrm{Ce}_{2} \mathrm{O}_{3}$ & - & - & 5.0 & - & - & - & - & - \\
\hline $\mathrm{Nb}_{2} \mathrm{O}_{3}$ & - & - & - & 1.3 & 0.7 & - & - & - \\
\hline $\mathrm{PdO}$ & - & - & - & 2.7 & 1.4 & - & - & - \\
\hline $\mathrm{Rh}_{2} \mathrm{O}_{3}$ & - & - & - & 1.1 & 0.6 & - & - & - \\
\hline $\mathrm{RuO}_{2}$ & - & - & - & 6.2 & 3.1 & - & - & - \\
\hline $\mathrm{MoO}_{3}$ & - & - & I & - & 6.5 & - & - & - \\
\hline
\end{tabular}
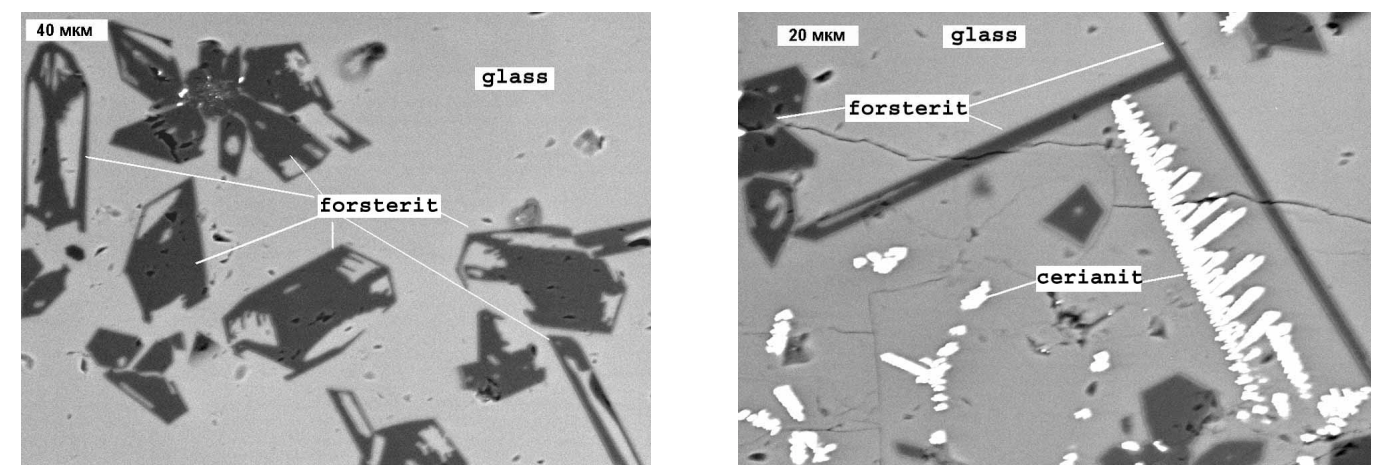

Fig. 1. SEM images (reflected electrons) of sample 3 with pyroxene stoichiometry. 
Table 2. Chemical composition of glass (SEM/EDS analysis).

\begin{tabular}{|l|c|r|r|r|c|}
\hline \multirow{2}{*}{ Oxide } & \multicolumn{5}{|c|}{ Oxide content, wt \% } \\
\cline { 2 - 6 } & $\mathbf{1}$ & $\mathbf{2}$ & $\mathbf{3}$ & $\mathbf{4}$ & Mean \\
\hline $\mathrm{Na}_{2} \mathrm{O}$ & 10.73 & 10.75 & 10.99 & 11.24 & 10.94 \\
\hline $\mathrm{MgO}$ & 6.17 & 6.49 & 6.14 & 5.77 & 6.14 \\
\hline $\mathrm{Al}_{2} \mathrm{O}_{3}$ & 2.68 & 2.45 & 2.21 & 2.76 & 2.52 \\
\hline $\mathrm{SiO}_{2}$ & 55.69 & 54.74 & 55.29 & 53.75 & 54.87 \\
\hline $\mathrm{FeO}$ & 6.78 & 7.11 & 7.62 & 7.43 & 7.24 \\
\hline $\mathrm{SrO}$ & 4.57 & 5.18 & 5.01 & 5.64 & 5.10 \\
\hline $\mathrm{Cs}_{2} \mathrm{O}$ & 6.32 & 6.99 & 6.50 & 6.14 & 6.49 \\
\hline $\mathrm{Ce}_{2} \mathrm{O}_{3}$ & 7.07 & 6.28 & 6.24 & 7.28 & 6.72 \\
\hline
\end{tabular}

The chemical compositions of forsterite-1 and forsterite-2 are identical (Table 3). In addition to the main components, $\mathrm{FeO}$ (approximately $1 \mathrm{wt} \%$ ) and $\mathrm{SrO}$ (approximately $1 \mathrm{wt} \%$ ) as permanent impurities enter into the forsterite composition. Cerianite $\left(\mathrm{CeO}_{2}\right)$ forms small grains several microns in size and skeleton crystals, and virtually is free of impurities.

The examination of transparent sections of samples 4 and 5 under a microscope in transmitted light demonstrated that the samples are composed of glass (80-60\%) and crystalline phases (40-20\%). The glass is transparent with a yellow hue.

The glass constitutes the major portion of the sample. The glass composition is constant in different parts of the samples. The iron content in the glass is increased only in the regions characterized by the growth of $\mathrm{Fe}_{2} \mathrm{O}_{3}$ crystals (Table 4, Fig. 2c).

Table 3. Chemical composition of forsterite.

\begin{tabular}{|l|c|c|c|c|c|c|c|}
\hline \multirow{3}{*}{ Oxide } & \multicolumn{7}{|c|}{ Oxide content, wt \% } \\
\cline { 2 - 8 } & \multicolumn{2}{|c|}{ Forsterite-1 } & \multicolumn{7}{c|}{ Forsterite-2 } \\
\cline { 2 - 8 } & $\mathbf{1}$ & $\mathbf{2}$ & $\mathbf{1}$ & $\mathbf{2}$ & $\mathbf{3}$ & $\mathbf{4}$ & $\mathbf{5}$ \\
\hline $\mathrm{MgO}$ & 55.26 & 54.80 & 55.41 & 55.64 & 54.43 & 54.99 & 55.23 \\
\hline $\mathrm{SiO}_{2}$ & 42.45 & 42.96 & 43.19 & 42.65 & 43.00 & 42.33 & 43.48 \\
\hline $\mathrm{FeO}$ & 1.41 & 1.12 & 0.98 & 1.07 & 1.34 & 1.08 & 0.68 \\
\hline $\mathrm{SrO}$ & 0.88 & 1.13 & 0.42 & 0.64 & 1.23 & 1.60 & 0.61 \\
\hline
\end{tabular}

Table 4. Glass compositions according to SEM/EDS analysis of select area (wt\%).

\begin{tabular}{|l|r|r|r|r|}
\hline \multirow{2}{*}{ Oxide } & \multicolumn{2}{|c|}{ Glass-1 } & \multicolumn{2}{c|}{ Glass-2 } \\
\cline { 2 - 5 } & $\mathbf{1}$ & $\mathbf{2}$ & $\mathbf{1}$ & $\mathbf{2}$ \\
\hline $\mathrm{Na}_{2} \mathrm{O}$ & 11.73 & 11.86 & 15.77 & 15.51 \\
\hline $\mathrm{SiO}_{2}$ & 64.32 & 64.24 & 50.43 & 49.76 \\
\hline $\mathrm{Cr}_{2} \mathrm{O}_{3}$ & 0.46 & 0.25 & 0.68 & 0.31 \\
\hline $\mathrm{Fe}_{2} \mathrm{O}_{3}$ & 19.15 & 19.35 & 30.46 & 31.51 \\
\hline $\mathrm{NiO}^{\mathrm{Ni}}$ & 2.90 & 2.63 & 0.36 & 0.69 \\
\hline $\mathrm{Nb}_{2} \mathrm{O}_{3}$ & 1.44 & 1.68 & 2.30 & 2.22 \\
\hline
\end{tabular}




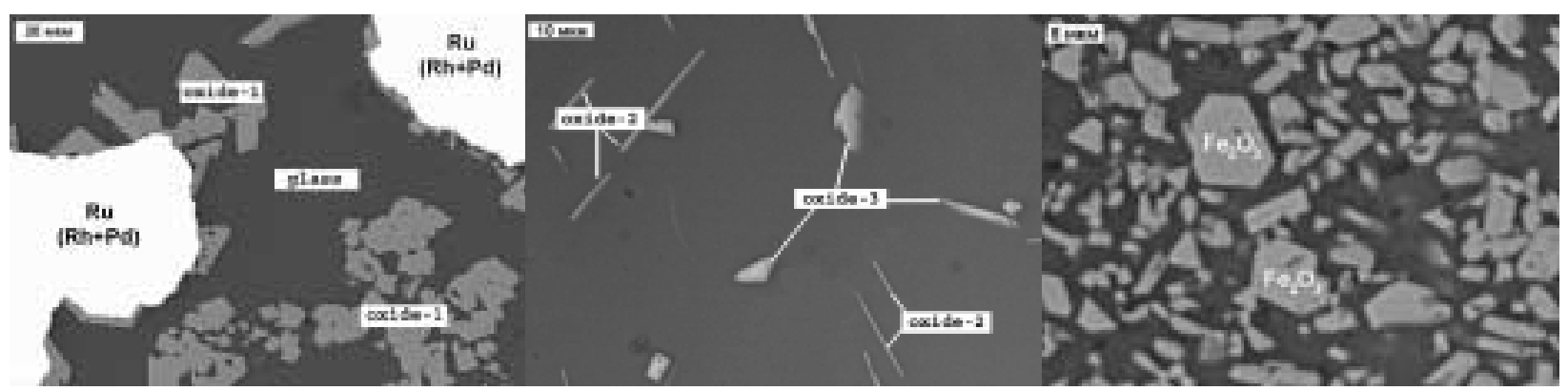

a

b

C

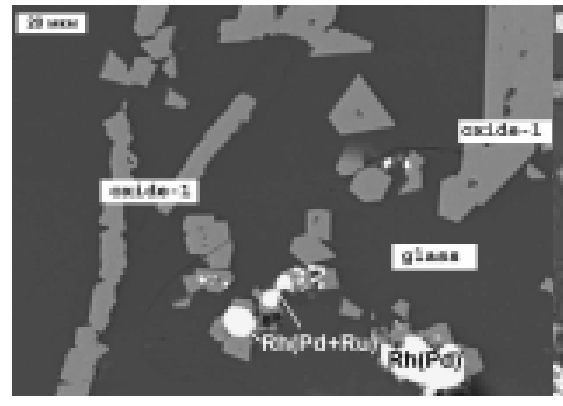

d

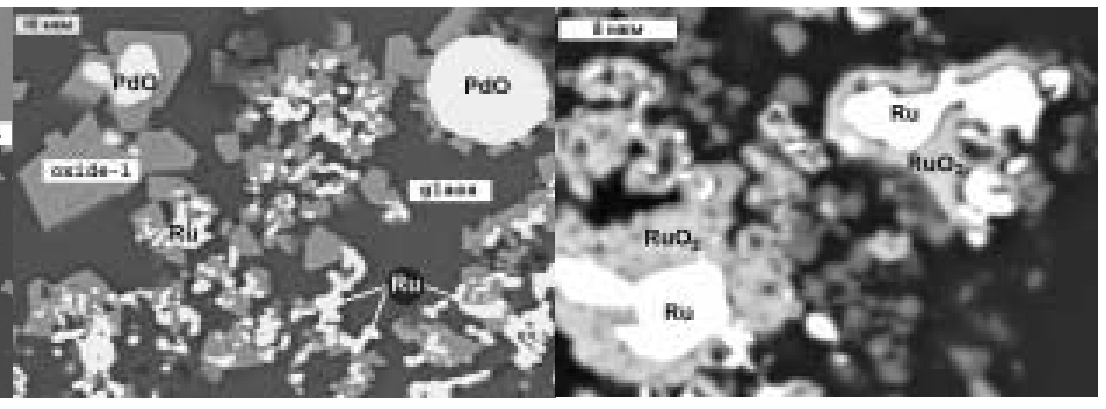

e

Fig. 2. SEM images of glass ceramics structure (sample 4).

Three oxides $(\mathrm{Ni}, \mathrm{Fe}, \mathrm{Cr}, \mathrm{Ru}) \mathrm{O}_{2-x}$ differing in composition and crystal shape, metallic ruthenium and rhodium, palladium oxide $\mathrm{PdO}$, ruthenium oxide $\mathrm{RuO}_{2}$, and iron oxide $\mathrm{Fe}_{2} \mathrm{O}_{3}$ are clearly identified in the glass in the backscattered electron images.

The oxides (Ni, Fe, Cr, Ru) are represented by crystals of three morphological types (Figs. 2a, 2b, 2d). In the section plane, octahedral crystals of oxide- 1 are characterized by different sections. Oxide- 2 is observed in the form of elongated prismatic crystals. Oxide- 3 forms flat hexagonal crystals less than 1 micron in thickness. The oxide compositions are listed in Table 5.

Certain amounts of sodium and silica were identified in some determinations (especially, for oxide-2 and oxide-3). This is explained by the fact that an electron probe covers surrounding glass.

The glass constitutes only $5-20 \%$ of samples 6,7 , and 8 . The diffraction pattern shows the reflections characterized by the interplanar distances that correspond to crystalline phases with the structure of magnetite and nepheline.

Table 5. Oxide compositions (wt\%).

\begin{tabular}{|l|c|c|c|c|c|c|c|c|c|}
\hline \multicolumn{1}{|c|}{ Oxide } & \multicolumn{3}{|c|}{ Oxide-1 } & \multicolumn{2}{c|}{ Oxide-2 } & \multicolumn{4}{c|}{ Oxide-3 } \\
\hline $\mathrm{Na}_{2} \mathrm{O}$ & - & - & - & 0.83 & - & 0.65 & 0.57 & 0.55 & 0.34 \\
\hline $\mathrm{SiO}_{2}$ & 0.92 & 0.82 & 1.06 & 7.15 & 2.70 & 3.27 & 1.59 & 1.37 & 2.21 \\
\hline $\mathrm{Cr}_{2} \mathrm{O}_{3}$ & 22.20 & 27.55 & 28.90 & 33.97 & 31.02 & 65.64 & 64.64 & 64.91 & 64.41 \\
\hline $\mathrm{Fe}_{2} \mathrm{O}_{3}$ & 63.76 & 58.60 & 57.78 & 32.36 & 35.79 & 16.33 & 15.83 & 14.08 & 16.05 \\
\hline $\mathrm{NiO}^{2}$ & 11.73 & 11.49 & 11.15 & 23.57 & 28.98 & 4.20 & 3.61 & 4.28 & 3.93 \\
\hline $\mathrm{Ru}_{2} \mathrm{O}_{3}$ & 1.39 & 1.53 & 1.11 & 2.13 & 1.51 & 9.90 & 13.76 & 14.81 & 13.06 \\
\hline
\end{tabular}




\section{Glass Ceramics with Garnet Stoichiometry}

Calculated compositions of synthesized garnet-like matrices are shown in Table 6.

Table 6. Calculated compositions glass ceramics with garnet stoichiometry.

\begin{tabular}{|l|c|c|c|c|}
\hline \multicolumn{1}{c}{$\begin{array}{c}\text { Oxide, } \\
\text { wt \% }\end{array}$} & \multicolumn{4}{c|}{ Sample no. } \\
\cline { 2 - 5 } & $\mathbf{1}$ & $\mathbf{2}$ & $\mathbf{3}$ & $\mathbf{4}$ \\
\hline $\mathrm{Fe}_{2} \mathrm{O}_{3}$ & 36.0 & 12.0 & 15.4 & 25.8 \\
\hline $\mathrm{Fe}_{2} \mathrm{O}_{3}$ & 36.0 & 12.0 & 15.4 & 25.8 \\
\hline $\mathrm{SiO}_{2}$ & 42.5 & 31.0 & 31.4 & 29.1 \\
\hline $\mathrm{CaO}_{2}$ & 8.5 & 29.0 & 29.3 & 27.1 \\
\hline $\mathrm{Cs}_{2} \mathrm{O}$ & 5.0 & - & - & - \\
\hline $\mathrm{SrO}$ & 3.0 & - & - & - \\
\hline $\mathrm{Ce}_{2} \mathrm{O}_{3}$ & 5.0 & - & - & 8.0 \\
\hline $\mathrm{ZrO}_{2}$ & - & 6.9 & 5.9 & - \\
\hline $\mathrm{CeO}_{2}$ & - & 5.9 & 5.0 & - \\
\hline $\mathrm{La}_{2} \mathrm{O}_{3}$ & - & 3.5 & 3.0 & - \\
\hline $\mathrm{Eu}_{2} \mathrm{O}_{3}$ & - & 2.3 & 3.0 & - \\
\hline $\mathrm{Nd}_{2} \mathrm{O}_{3}$ & - & 9.4 & 8.0 & - \\
\hline $\mathrm{Gd}_{2} \mathrm{O}_{3}$ & - & - & - & 10.0 \\
\hline
\end{tabular}

Sample 1 is x-ray amorphous, all the components are dissolved in the glass, and excessive iron crystallizes in the form of spinel. Samples 2 and 3 have virtually the same composition and structure. They are composed of a grey microcrystalline aggregate and a black homogeneous glass with a conchoidal fracture. The boundary between the glass and the crystallized part of the sample is sharp. The crystallized part of ceramics in the backscattered electron images exhibits a microcrystalline structure and consists of synthetic TR-Ca silicate with the apatite structure (depicted by white color), wollastonite (black), and synthetic garnet (grey) close to andradite (Fig. 3). The phase ratios are as follows: garnet, $70 \%$; wollastonite, $10 \%$; TR-Ca silicate (britholite), $15 \%$; and glass, $~ 5 \%$. The phase compositions are presented in Tables 7 and 8.

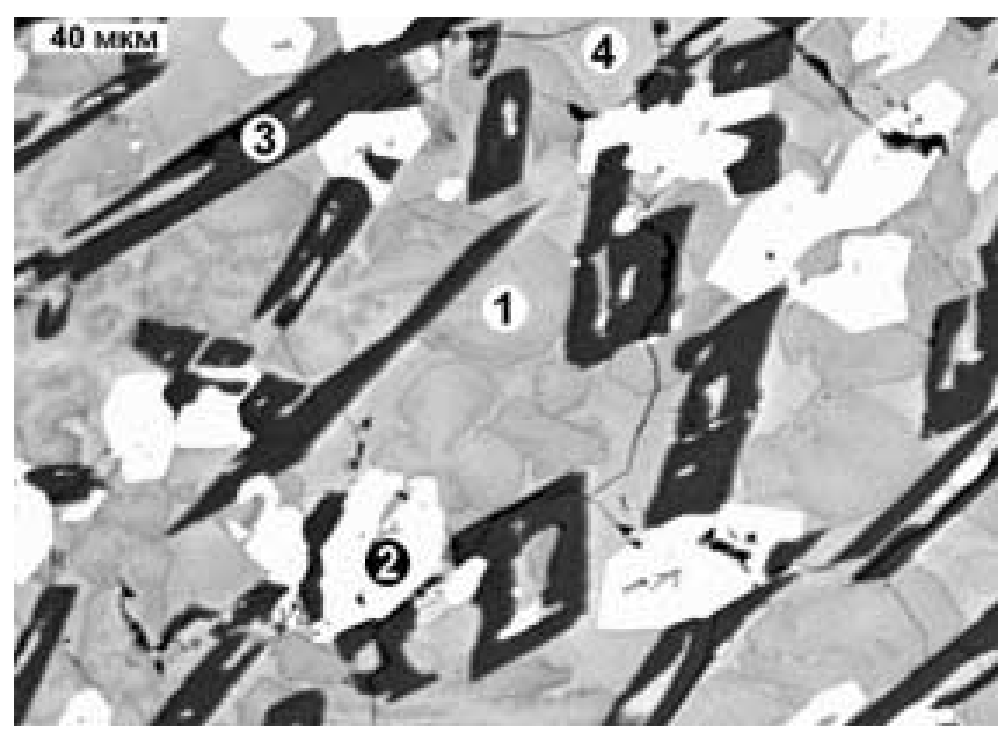

Fig. 3. SEM image, crystallized part of sample no. 3: 1-garnet, 2-britholite, 3-wollastonite, 4-glass. 
Table 7. Compositions and formulas of wollastonite, garnet and glass.

\begin{tabular}{|l|c|c|c|c|c|c|}
\hline \multirow{2}{*}{$\begin{array}{c}\text { Oxide, } \\
\text { wt \% }\end{array}$} & \multicolumn{2}{c|}{ Wollastonite } & \multicolumn{2}{c|}{ Garnet } & \multicolumn{2}{c|}{ Glass } \\
\cline { 2 - 7 } & $\mathbf{1}$ & $\mathbf{2}$ & $\mathbf{1}$ & $\mathbf{2}$ & $\mathbf{1}$ & $\mathbf{2}$ \\
\hline $\mathrm{SiO}_{2}$ & 49.62 & 49.69 & 24.10 & 24.00 & 37.26 & 38.31 \\
\hline $\mathrm{CaO}$ & 48.55 & 48.16 & 25.91 & 25.02 & 13.85 & 13.66 \\
\hline $\mathrm{FeO}$ & 1.83 & 2.15 & - & - & - & - \\
\hline $\mathrm{Fe}_{2} \mathrm{O}_{3}{ }^{*}$ & - & - & 32.42 & 31.44 & 25.88 & 24.67 \\
\hline $\mathrm{ZrO}_{2}$ & - & - & 9.93 & 10.01 & 6.42 & 6.06 \\
\hline $\mathrm{La}_{2} \mathrm{O}_{3}$ & - & - & 0.50 & 0.50 & 5.00 & 5.63 \\
\hline $\mathrm{Ce}_{2} \mathrm{O}_{3}$ & - & - & 1.19 & 1.73 & 5.01 & 5.51 \\
\hline $\mathrm{Nd}_{2} \mathrm{O}_{3}$ & - & - & 4.85 & 4.46 & 4.83 & 5.68 \\
\hline $\mathrm{EuO}$ & - & - & 1.09 & 2.84 & 1.78 & 0.47 \\
\hline$\sum \mathrm{TR}$ & & & 7.63 & 9.53 & 16.62 & 17.29 \\
\hline
\end{tabular}

*Iron is given as $\mathrm{FeO}$ in wollastonite and britholite and as $\mathrm{Fe}_{2} \mathrm{O}_{3}$ in garnet and glass.

Table 8. Compositions and formulas of britholite and glass.

\begin{tabular}{|l|c|c|c|c|c|c|c|c|}
\hline \multirow{2}{*}{$\begin{array}{c}\text { Oxide, } \\
\text { wt \% }\end{array}$} & \multicolumn{3}{|c|}{ "Britholite"a $^{\mathbf{a}}$} & \multicolumn{3}{c|}{ Dark-grey glass $^{\mathbf{b}}$} & \multicolumn{2}{c|}{ Grey glass $^{\text {c) }}$} \\
\cline { 2 - 10 } & $\mathbf{1}$ & $\mathbf{2}$ & $\mathbf{3}$ & $\mathbf{1}$ & $\mathbf{2}$ & $\mathbf{3}$ & $\mathbf{1}$ & $\mathbf{2}$ \\
\hline $\mathrm{SiO}_{2}$ & 21.66 & 21.76 & 23.98 & 35.57 & 37.42 & 35.85 & 32.84 & 32.48 \\
\hline $\mathrm{CaO}$ & 14.24 & 14.45 & 17.56 & 32.52 & 28.22 & 27.02 & 22.58 & 32.65 \\
\hline $\mathrm{FeO}$ & 0.58 & 0.90 & 2.66 & - & - & - & - & - \\
\hline $\mathrm{Fe}_{2} \mathrm{O}_{3}$ & - & - & - & 21.11 & 24.46 & 23.35 & 19.77 & 17.51 \\
\hline $\mathrm{ZrO}_{2}$ & 5.06 & 4.41 & 5.02 & 5.73 & 5.67 & 5.61 & 5.37 & 5.77 \\
\hline $\mathrm{La}_{2} \mathrm{O}_{3}$ & 10.91 & 10.15 & 8.57 & 1.23 & 0.70 & 2.26 & 3.06 & 4.35 \\
\hline $\mathrm{Ce}_{2} \mathrm{O}_{3}$ & 16.78 & 17.63 & 14.08 & 1.56 & 1.18 & 1.86 & 4.73 & 5.39 \\
\hline $\mathrm{Nd}_{2} \mathrm{O}_{3}$ & 24.11 & 23.73 & 21.54 & 1.88 & 1.72 & 3.63 & 8.88 & 8.46 \\
\hline $\mathrm{EuO}$ & 6.67 & 6.96 & 6.59 & 0.40 & 0.63 & 0.37 & 2.77 & 2.40 \\
\hline$\sum \mathrm{TR}_{2} \mathrm{O}_{3}$ & 58.47 & 48.47 & 50.78 & 5.16 & 4.23 & 8.16 & 19.44 & 20.60 \\
\hline
\end{tabular}

${ }^{a}$ Silicate with the apatite (britholite) structure: 1 and 2 - samples taken from the crystalline part and 3 - sample taken from glass-rich region. ${ }^{b}$ Glass near britholite. ${ }^{\circ}$ Glass far from britholite.

\section{Properties of Phosphate Glasses Synthesized by CCIM Method}

The blocks of phosphate glasses whose compositions are listed in Tables 9 and 10 were obtained in the CCIM (1.76 and $5.28 \mathrm{MHz})$ under the chosen melting conditions $\left(1200^{\circ} \mathrm{C}, 1 \mathrm{~h}\right)$ and in alundum crucibles (for comparison).

The glass samples were withdrawn from different regions of the obtained glass blocks. Then, these samples were tested for the iron, chromium, and nickel content (Tables 11, 12).

The results given in the tables show that the distribution of $\mathrm{Fe}_{2} \mathrm{O}_{3}, \mathrm{Cr}_{2} \mathrm{O}_{3}$, and $\mathrm{NiO}$ over the block height is uniform for any used methods of producing phosphate glasses in the CCIM with a high content of $\mathrm{Fe}, \mathrm{Cr}$, and $\mathrm{Ni}$ in the final glass blocks. Moreover, the prepared glasses are homogeneous at $\Sigma \mathrm{CP} \sim 3 \%$. The formation of heterogeneous phase $1-5 \mu \mathrm{m}$ across and conglomerations formed by these dispersed particles was found in materials with $\Sigma C P=10 \%$. However, most likely, owing to the electrodynamic stirring of a melt, the accumulation of the heterogeneous phase was not observed in any zone of the cold crucible. 
Table 9. Compositions of glasses obtained in the CCIM by remelting of preliminarily prepared glass frit.

\begin{tabular}{|c|c|c|c|}
\hline \multirow[t]{2}{*}{ Oxide } & \multicolumn{3}{|c|}{ Compositions of prepared materials, wt \% } \\
\hline & $\begin{array}{c}\text { Glass composition of } \\
\text { EP furnace }\end{array}$ & $\Sigma C P_{\text {ox }}=3 \%$ & $\Sigma C P_{\text {ox }}=10 \%$ \\
\hline $\mathrm{Na}_{2} \mathrm{O}$ & 23.40 & 22.60 & 21.30 \\
\hline $\mathrm{Cs}_{2} \mathrm{O}$ & 0.36 & 0.35 & 0.32 \\
\hline $\mathrm{K}_{2} \mathrm{O}$ & 0.24 & 0.23 & 0.21 \\
\hline$\Sigma \mathrm{Me}_{2} \mathrm{O}$ & 24.00 & 23.18 & 21.83 \\
\hline $\mathrm{Al}_{2} \mathrm{O}_{3}$ & 18.60 & 18.00 & 16.90 \\
\hline $\mathrm{Fe}_{2} \mathrm{O}_{3}$ & 0.08 & 2.26 & 7.35 \\
\hline $\mathrm{Cr}_{2} \mathrm{O}_{3}$ & 0.02 & 0.57 & 1.84 \\
\hline $\mathrm{NiO}$ & 0.01 & 0.28 & 0.92 \\
\hline $\mathrm{MnO}$ & 0.03 & 0.03 & 0.03 \\
\hline $\mathrm{CaO}$ & 0.02 & 0.02 & 0.02 \\
\hline $\mathrm{SrO}$ & 0.16 & 0.15 & 0.14 \\
\hline $\mathrm{BaO}$ & 0.16 & 0.15 & 0.14 \\
\hline $\mathrm{ZrO}_{2}$ & 0.47 & 0.45 & 0.43 \\
\hline $\mathrm{MoO}_{3}$ & 0.31 & 0.30 & 0.28 \\
\hline $\mathrm{La}_{2} \mathrm{O}_{3}$ & 0.14 & 0.13 & 0.13 \\
\hline $\mathrm{CeO}_{2}$ & 0.51 & 0.49 & 0.46 \\
\hline $\mathrm{Nd}_{2} \mathrm{O}_{3}$ & 0.43 & 0.42 & 0.39 \\
\hline $\mathrm{Sm}_{2} \mathrm{O}_{3}$ & 0.06 & 0.06 & 0.05 \\
\hline$\Sigma \mathrm{Me}_{\mathrm{x}} \mathrm{O}_{\mathrm{y}}$ & 21.0 & 23.33 & 27.99 \\
\hline$\Sigma(\mathrm{Fe}, \mathrm{Cr}, \mathrm{Ni})_{\mathrm{x}} \mathrm{O}_{\mathrm{y}}$ & 0.11 & 3.11 & 10.11 \\
\hline $\mathrm{SO}_{4}{ }^{2-}$ & 0.20 & 0.19 & 0.18 \\
\hline $\mathrm{P}_{2} \mathrm{O}_{5}$ & 55.0 & 53.3 & 50.0 \\
\hline
\end{tabular}

Table 10. Calculated compositions of phosphate glasses produced by melting of calcinates into glass melt (wt\%).

\begin{tabular}{|c|c|c|}
\hline \multirow[t]{2}{*}{ Oxide } & \multicolumn{2}{|c|}{ Compositions of prepared materials } \\
\hline & $3 \% \Sigma C P_{\text {ox }}$ & $10 \% \Sigma C P_{o x}$ \\
\hline $\mathrm{Na}_{2} \mathrm{O}$ & 23.2 & 21.6 \\
\hline $\mathrm{Al}_{2} \mathrm{O}_{3}$ & 20.4 & 18.9 \\
\hline $\mathrm{Fe}_{2} \mathrm{O}_{3}$ & 2.18 & 7.27 \\
\hline $\mathrm{Cr}_{2} \mathrm{O}_{3}$ & 0.55 & 1.82 \\
\hline $\mathrm{NiO}$ & 0.27 & 0.91 \\
\hline$\Sigma \mathrm{CP}_{\text {ox }}$ & 3.00 & 10.00 \\
\hline $\mathrm{P}_{2} \mathrm{O}_{5}$ & 53.3 & 49.5 \\
\hline
\end{tabular}


Table 11. Distribution of $\mathrm{Fe}, \mathrm{Cr}$, and $\mathrm{Ni}$ over the height of glass blocks obtained in $\mathrm{CCIM}$ and muffle furnace by feeding calcinate with a high CP content onto glass melt $\left(1200^{\circ} \mathrm{C}, 1 \mathrm{~h}\right)(\mathrm{wt} \%)$. ${ }^{*}$

\begin{tabular}{|c|c|c|c|c|c|}
\hline \multirow{2}{*}{$\begin{array}{c}\text { Composition, preparation } \\
\text { technique }\end{array}$} & \multirow{2}{*}{$\begin{array}{l}\text { Glass } \\
\text { sample }\end{array}$} & \multicolumn{3}{|c|}{ Component content } & \multirow[t]{2}{*}{ Note } \\
\hline & & $\mathrm{Fe}_{2} \mathrm{O}_{3}$ & $\mathrm{Cr}_{2} \mathrm{O}_{3}$ & $\mathrm{NiO}$ & \\
\hline $\begin{array}{l}\Sigma \mathrm{CP} 1.5 \%+6.0 \% \\
\text { CCIM5.28 MHz }\end{array}$ & \begin{tabular}{|l}
15 min \\
30 min \\
45 min \\
top \\
bulk \\
bottom \\
slag lining
\end{tabular} & $\begin{array}{l}2.60 \\
2.12 \\
2.24 \\
2.54 \\
2.16 \\
2.20 \\
1.60\end{array}$ & $\begin{array}{l}0.55 \\
0.48 \\
0.51 \\
0.59 \\
0.57 \\
0.54 \\
0.34\end{array}$ & $\begin{array}{l}0.29 \\
0.25 \\
0.27 \\
0.29 \\
0.26 \\
0.27 \\
0.24\end{array}$ & $\begin{array}{l}\text { Homogeneous } \\
\text { Homogeneous } \\
\text { Homogeneous } \\
\text { Homogeneous } \\
\text { Homogeneous } \\
\text { Homogeneous } \\
\text { Opacified }\end{array}$ \\
\hline Calculated content & & 2.18 & 0.55 & 0.27 & \\
\hline $\begin{array}{l}\Sigma \mathrm{CP} 1.5 \%+6.0 \% \\
\text { Muffle furnace }\end{array}$ & \begin{tabular}{|l} 
top \\
bottom
\end{tabular} & $\begin{array}{l}2.43 \\
1.92 \\
\end{array}$ & $\begin{array}{l}0.66 \\
0.36 \\
\end{array}$ & $\begin{array}{l}0.39 \\
0.20 \\
\end{array}$ & $\begin{array}{l}\text { Homogeneous } \\
\text { Homogeneous }\end{array}$ \\
\hline $\begin{array}{l}\Sigma \mathrm{CP} 1.5 \%+27.0 \% \\
\text { CСIM5.28 МГц }\end{array}$ & \begin{tabular}{|l|}
$15 \mathrm{~min}$ \\
$30 \mathrm{~min}$ \\
$45 \mathrm{~min}$ \\
top \\
bulk \\
bottom \\
slag lining
\end{tabular} & $\begin{array}{l}7.22 \\
7.17 \\
7.73 \\
7.27 \\
7.19 \\
7.39 \\
2.06 \\
\end{array}$ & $\begin{array}{l}1.63 \\
1.68 \\
1.83 \\
1.55 \\
1.79 \\
1.85 \\
0.43 \\
\end{array}$ & $\begin{array}{l}0.91 \\
0.90 \\
0.96 \\
0.90 \\
0.86 \\
0.94 \\
0.34 \\
\end{array}$ & $\begin{array}{l}\text { Dispersed particles }(1-5 \mu \mathrm{m}) \text { and } \\
\text { their conglomerates are uniformly } \\
\text { distributed in glass } \\
\text { Opacified }\end{array}$ \\
\hline Calculated content & & 7.27 & 1.83 & 0.90 & \\
\hline $\begin{array}{l}\Sigma \mathrm{CP} 1.5 \%+27.0 \% \\
\text { Muffle furnace }\end{array}$ & $\begin{array}{l}\text { top } \\
\text { bottom }\end{array}$ & $\begin{array}{l}9.45 \\
5.67 \\
\end{array}$ & $\begin{array}{l}0.66 \\
0.36 \\
\end{array}$ & $\begin{array}{l}0.39 \\
0.20 \\
\end{array}$ & $\begin{array}{l}\text { Multiphase system with dispersed } \\
\text { particles }(1-5 \mu \mathrm{m}) \text { and conglomerates }\end{array}$ \\
\hline
\end{tabular}

${ }^{*}$ X-ray fluorescence analysis.

Table 12. Distribution of Fe, $\mathrm{Cr}$, and $\mathrm{Ni}$ over the height of glass blocks obtained in $\mathrm{CCIM}$ and muffle furnace by remelting of phosphate glasses with $\Sigma C P=3$ and $10 \%\left(1200^{\circ} \mathrm{C}, 1 \mathrm{~h}\right)(\mathrm{wt} \%){ }^{*}$

\begin{tabular}{|c|c|c|c|c|c|}
\hline \multirow{2}{*}{$\begin{array}{c}\text { Composition, preparation } \\
\text { technique }\end{array}$} & \multirow{2}{*}{$\begin{array}{c}\text { Glass } \\
\text { sample }\end{array}$} & \multicolumn{3}{|c|}{ Component content } & \multirow[t]{2}{*}{ Note } \\
\hline & & $\mathrm{Fe}_{2} \mathrm{O}_{3}$ & $\mathrm{Cr}_{2} \mathrm{O}_{3}$ & $\mathrm{NiO}$ & \\
\hline$\Sigma \mathrm{LCP} 3 \%$ & $15 \min$ & 2.26 & 0.68 & 0.27 & Homogeneous \\
\hline CCIM & $30 \mathrm{~min}$ & 2.44 & 0.64 & 0.23 & Homogeneous \\
\hline 1.76 MHz & $45 \min$ & 2.39 & 0.64 & 0.27 & Homogeneous \\
\hline & & 2.51 & 0.71 & 0.24 & Homogeneous \\
\hline & bulk & 2.53 & 0.70 & 0.27 & Homogeneous \\
\hline & bottom & - & - & - & Homogeneous \\
\hline & slag lining & - & - & - & Opacified \\
\hline \multicolumn{2}{|l|}{ Calculated content } & 2.26 & 0.57 & 0.28 & \\
\hline$\Sigma$ CP $3 \%$ & top & 2.52 & 0.58 & 0.27 & Homogeneous \\
\hline Muffle furnace & bottom & 2.54 & 0.58 & 0.29 & Homogeneous \\
\hline$\Sigma \mathrm{CP} 10 \%$ & $15 \min$ & 6.94 & 0.98 & 0.84 & \\
\hline CCIM & $30 \mathrm{~min}$ & 6.83 & 0.82 & 0.82 & Pispersed particles $(1-5 \mu \mathrm{m})$ and \\
\hline $1.76 \mathrm{MHz}$ & $45 \min$ & 6.86 & 0.80 & 0.80 & their conglomerates are uniformly \\
\hline & top & 6.91 & 0.72 & 0.72 & distributed in glass \\
\hline & bulk & 6.71 & 0.73 & 0.73 & \\
\hline & bottom & 7.47 & 0.73 & 0.73 & \\
\hline & slag lining & - & - & - & Opacified \\
\hline \multicolumn{2}{|l|}{ Calculated content } & 7.34 & 1.84 & 0.92 & \\
\hline$\Sigma \mathrm{CP} 10 \%$ & top & 6.67 & 0.62 & 0.76 & Multiphase system with dispersed \\
\hline Muffle furnace & bottom & 6.94 & 0.64 & 0.98 & $\begin{array}{l}\text { particles }(1-5 \mu \mathrm{m}) \text { and their } \\
\text { conglomerates }\end{array}$ \\
\hline
\end{tabular}

${ }^{*}$ Atomic adsorption analysis of $\mathrm{Fe}^{3+}, \mathrm{Cr}^{3+}$, and $\mathrm{Ni}^{3+}$ after the dissolution of glass samples. 


\section{Immobilization of Simulated Waste}

After completing data on waste composition in the PA "Mayak" tanks, and analyzing base and comparative options of processing waste, we selected the simulated composition presented in Table 13. In experimental tests, we used the waste composition prepared by dissolution of relevant salts and subsequent evaporation of solutions in a direct-flow evaporator. The simulated waste composition was obtained as a result of this work. Its residual humidity is about $34 \%$, evaporation temperature $-134^{\circ} \mathrm{C}$.

The main criteria for choosing matrix compositions for waste immobilization are as follows:

- The maximum possible incorporation of waste components into the matrix compositions;

- The minimum addition of fluxes while synthesizing matrices.

The following matrix compositions could be used as base vitreous systems:

1. Phosphate glass $(\mathrm{Ph})$ used for $\mathrm{HLW}$ vitrification at PA "Mayak" (\%, wt): $\mathrm{Na}_{2} \mathrm{O}-21-24 ; \mathrm{Al}_{2} \mathrm{O}_{3}-10-23$; $\mathrm{P}_{2} \mathrm{O}_{5}-51-57$, fission and corrosion-origin components - up to 5 .

2. Silicate matrices like sherlit (Sh) (\%, wt): $\mathrm{Na}_{2} \mathrm{O}-6 ; \mathrm{Al}_{2} \mathrm{O}_{3}-18 ; \mathrm{Fe}_{2} \mathrm{O}_{3}-10 ; \mathrm{SiO}_{2}-44 ; \mathrm{MgO}_{2}-7$;

3. $\mathrm{MnO}_{2}-3,5 ; \mathrm{Cr}_{2} \mathrm{O}_{3}-3,5 ; \mathrm{B}_{2} \mathrm{O}_{3}-8$; and jadheite (\%, wt): $\mathrm{Na}_{2} \mathrm{O}-15,3 \mathrm{Al}_{2} \mathrm{O}_{3}-25,6 ; \mathrm{SiO}_{2}-59,1$.

Simulated phosphate glasses were synthesized in alundum crucibles from the precursor fluxed with phosphate acid and containing plutonium and uranium.

Simulated silicate glasses were synthesized in alundum crucibles from the precursors fluxed with silica and containing uranium. Calculated compositions of phosphate and silicate glasses synthesized in the muffle furnace are given in Table 14.

The synthesis of phosphate and silicate glasses containing simulated waste components was done in a CCIM-Pu facility installed in a glove box.

The precursor (precursor was obtained at up to $450^{\circ} \mathrm{C}$ while heating the relevant simulated waste composition, fluxes, uranium and plutonium solutions in quartz vessels followed by its grinding) was fed onto the surface of molten $\mathrm{Ph}$ (Jad or Sh) glass frit. The melt was heated within 1 hour and then cooled in a crucible. Calculated compositions of the phosphate and silicate glasses synthesized in the CCIM are given in Table 15.

Table 13. Simulated waste composition used in experiments.

\begin{tabular}{|l|c|c|c|}
\hline \multirow{2}{*}{ Components } & \multicolumn{3}{|c|}{ Content } \\
\cline { 2 - 4 } & Solids, $\mathbf{g} / \mathbf{l}$ & Oxides, $\mathbf{g} / \mathbf{l}$ & Oxides, \% wt \\
\hline $\mathrm{Al}\left(\mathrm{NO}_{3}\right)_{3} \times 9 \mathrm{H}_{2} \mathrm{O}$ & 230 & 62.6 & 57.1 \\
\hline $\mathrm{Fe}\left(\mathrm{NO}_{3}\right)_{3} \times \mathrm{nH}_{2} \mathrm{O}$ & 30.5 & 5.7 & 5.2 \\
\hline $\mathrm{Cr}\left(\mathrm{NO}_{3}\right)_{3} \times 9 \mathrm{H}_{2} \mathrm{O}$ & 10 & 2.2 & 2.0 \\
\hline $\mathrm{Ni}\left(\mathrm{NO}_{3}\right)_{2} \times 6 \mathrm{H}_{2} \mathrm{O}$ & 18 & 4.6 & 4.2 \\
\hline $\mathrm{Mn}\left(\mathrm{NO}_{3}\right)_{2} \times \mathrm{nH}_{2} \mathrm{O}$ & 4 & 0.9 & 0.8 \\
\hline $\mathrm{NaNO}$ & 92 & 33.6 & 30.7 \\
\hline$\Sigma$ & 384.5 & 109.6 & 100 \\
\hline
\end{tabular}


Table 14. Calculated compositions of phosphate and silicate glasses synthesized in the muffle furnace.

\begin{tabular}{|l|r|r|r|r|r|r|r|}
\hline Oxides & \multicolumn{7}{|c|}{ Content, \% wt } \\
\hline & \multicolumn{1}{|c|}{$\mathbf{P h}$} & $\mathbf{P h}-\mathbf{U}$ & \multicolumn{1}{c|}{ Ph-U-Pu } & Jad & \multicolumn{1}{c|}{ Jad-U } & Sh & Sh-U \\
\hline $\mathrm{Al}_{2} \mathrm{O}_{3}$ & 21,61 & 21,39 & 21,37 & 24,10 & 23,83 & 19,04 & 18,87 \\
\hline $\mathrm{Fe}_{2} \mathrm{O}_{3}$ & 1,97 & 1,95 & 1,95 & 2,20 & 2,17 & 9,04 & 8,97 \\
\hline $\mathrm{Cr}_{2} \mathrm{O}_{3}$ & 0,76 & 0,74 & 0,74 & 0,84 & 0,82 & 0,67 & 0,65 \\
\hline $\mathrm{NiO}$ & 1,58 & 1,58 & 1,58 & 1,77 & 1,76 & 1,40 & 1,39 \\
\hline $\mathrm{MnO}_{2}$ & 0,31 & 0,31 & 0,31 & 0,35 & 0,34 & 0,27 & 0,27 \\
\hline $\mathrm{Na}_{2} \mathrm{O}$ & 21,97 & 21,76 & 21,74 & 12,95 & 12,81 & 10,23 & 10,14 \\
\hline $\mathrm{P}_{2} \mathrm{O}_{5}$ & 51,80 & 51,31 & 51,26 & 0 & 0 & 0 & 0 \\
\hline $\mathrm{SiO}_{2}$ & 0 & 0 & 0 & 57,79 & 57,19 & 45,66 & 45,28 \\
\hline $\mathrm{MgO}$ & 0 & 0 & 0 & 0 & 0 & 6,39 & 6,34 \\
\hline $\mathrm{B}_{2} \mathrm{O}_{3}$ & 0 & 0 & 0 & 0 & 0 & 7,30 & 7,24 \\
\hline $\mathrm{U}_{3} \mathrm{O}_{8}$ & 0 & 0,96 & 0,96 & 0 & 1,08 & 0 & 0,85 \\
\hline $\mathrm{PuO}_{2}$ & 0 & 0 & 0,09 & 0 & 0 & 0 & 0 \\
\hline
\end{tabular}

Table 15. Calculated compositions of the phosphate and silicate glasses synthesized in the CCIM.

\begin{tabular}{|l|c|c|c|}
\hline \multirow{2}{*}{ Oxides } & \multicolumn{3}{|c|}{ Content, \% wt } \\
\cline { 2 - 4 } & Ph-U-Pu (CCIM) & Jad-U-Pu (CCIM) & Sh-U-Pu (CCIM) \\
\hline $\mathrm{Al}_{2} \mathrm{O}_{3}$ & 21.37 & 23.83 & 19.33 \\
\hline $\mathrm{Fe}_{2} \mathrm{O}_{3}$ & 1.95 & 2.17 & 6.55 \\
\hline $\mathrm{Cr}_{2} \mathrm{O}_{3}$ & 0.75 & 0.83 & 0.68 \\
\hline $\mathrm{NiO}$ & 1.57 & 1.75 & 1.42 \\
\hline $\mathrm{MnO}$ & 0.31 & 0.35 & 0.28 \\
\hline $\mathrm{Na}_{2} \mathrm{O}$ & 21.73 & 12.80 & 10.39 \\
\hline $\mathrm{P}_{2} \mathrm{O}_{5}$ & 51.24 & 0 & 0 \\
\hline $\mathrm{SiO}_{2}$ & 0 & 57.14 & 46.37 \\
\hline $\mathrm{MgO}$ & 0 & 0 & 6.49 \\
\hline $\mathrm{B}_{2} \mathrm{O}_{3}$ & 0 & 0 & 7.42 \\
\hline $\mathrm{U}_{3} \mathrm{O}_{8}$ & 0.97 & 1.01 & 0.96 \\
\hline $\mathrm{PuO}_{2}$ & 0.11 & 0.12 & 0.11 \\
\hline$\Sigma$ & 100 & 100 & 100 \\
\hline
\end{tabular}

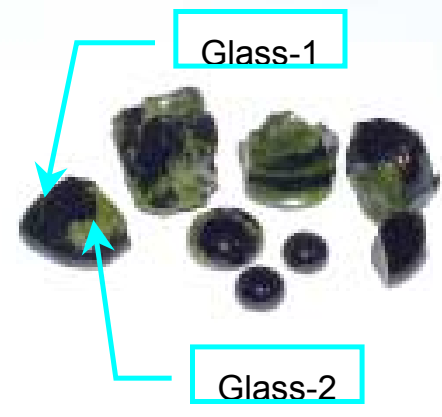

a

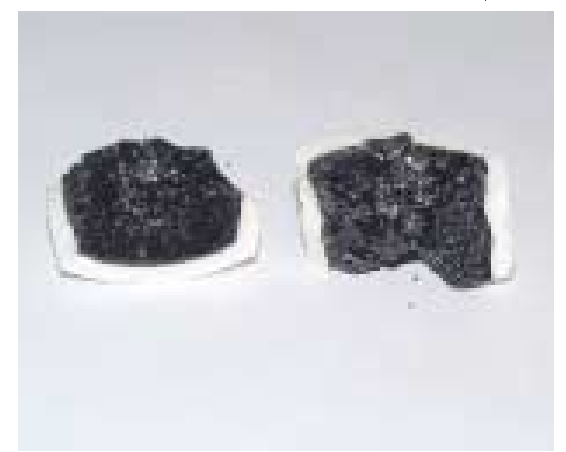

b

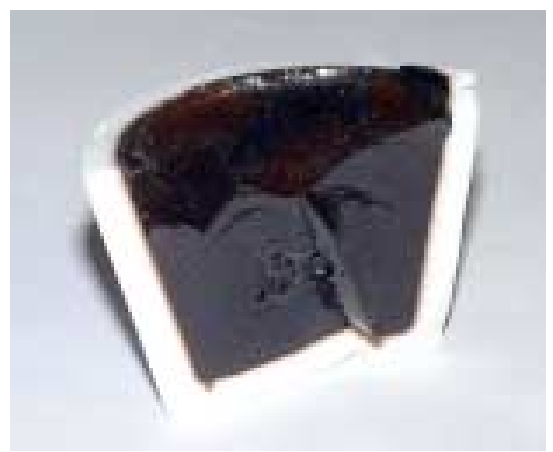

C

Fig. 4. Samples of phosphate and silicate glasses synthesized in the muffle furnace: a-Phosphate glass (Ph), b-Jadheite (Jad), c--Sherlit (Sh).

The Ph sample consists of two different vitreous phases. Glass 1 is transparent and of dark-green color

(Fig. 4a). Glass 2 is not transparent, dim, and of light-green color. From the SEM data, glass 1 is 
homogeneous with even composition through various sample regions (Fig. 5a, Table 16). Glass 2, as is seen from the reflected electron image, is non-homogeneous. Their compositions are equal (Table 16).

Glass 2 is composed of light-grey zones with obvious dendrite-type exclusions of a darker color. The difference in color is caused by their diverse compositions.

Sample Jad (jadheite) consists of transparent glass of brown color with a bubble-like texture (Fig. 4b). It is homogeneous and has even composition through various sample regions (Fig. 6, Table 17).

Sample Sh (Sherlit) is composed of opaque glass of black color (Fig. 4c). In the reflected electron image, one can see a crystal phase in the form of submicron skeletal crystals (Fig. 7).

According to the SEM data, the glass contains less nickel, iron, and chrome (Table 18). The composition of the crystal phase cannot be correctly detected due to minute dimensions less than the electron beam diameter. The data given in Table 18 point out the presence of the crystal phase and vitreous zones. Going from the morphology of skeletal crystals, namely cubic singony and higher concentrations of nickel, iron, and chrome, one can consider that the mentioned crystal phase exhibits spinel-like cubic oxide structure. Results of leaching tests of the samples synthesized in the muffle furnace are presented in Table 19. Preliminary research on the materials produced in the CCIM has shown that uranium and plutonium distribution is homogenous all over the volume of the glass batch central zone.

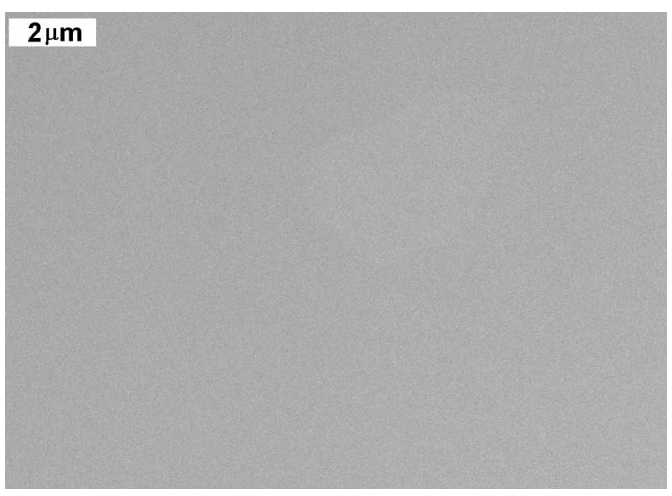

a

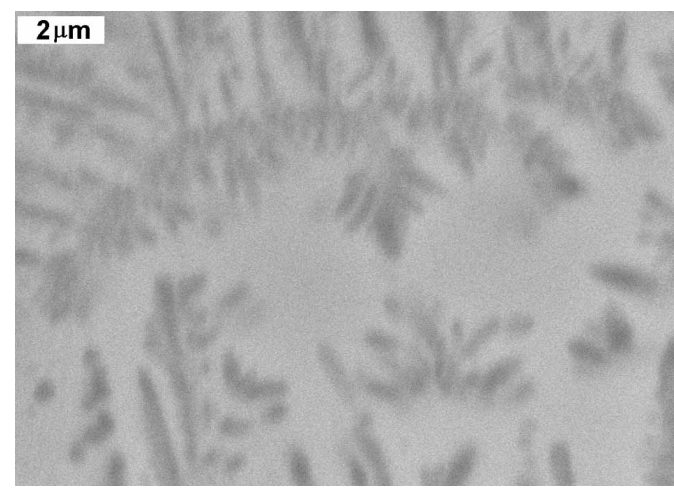

b

Fig. 5. SEM images of sample "Ph" glass: a-Glass-1; b-Glass-2.

Table 16. Composition of precursor and Ph-glass sample (SEM/EDS).

\begin{tabular}{|c|c|c|c|c|c|c|c|c|c|c|}
\hline \multirow[t]{3}{*}{ Oxides } & \multirow{3}{*}{$\begin{array}{c}\text { Precurso } \\
r\end{array}$} & \multicolumn{4}{|c|}{ Glass-1 } & \multicolumn{5}{|c|}{ Glass-2 } \\
\hline & & \multicolumn{3}{|c|}{ Scan $50 \times 50 \mu \mathrm{m}$} & \multirow[t]{2}{*}{ Average } & \multicolumn{2}{|c|}{ Scan $50 \times 50 \mu \mathrm{m}$} & \multirow[t]{2}{*}{ Average } & \multirow{2}{*}{$\begin{array}{c}\text { Light } \\
\text { grey } \\
\text { glass }\end{array}$} & \multirow{2}{*}{$\begin{array}{l}\text { Gray } \\
\text { glass }\end{array}$} \\
\hline & & 1 & 2 & 3 & & 1 & 2 & & & \\
\hline $\mathrm{Na}_{2} \mathrm{O}$ & 21.97 & 24.88 & 25.34 & 25.15 & 25.12 & 23.94 & 24.75 & 24.35 & 22.07 & 16.47 \\
\hline $\mathrm{Al}_{2} \mathrm{O}_{3}$ & 21.61 & 15.00 & 15.02 & 14.97 & 15.00 & 15.24 & 15.48 & 15.36 & 12.55 & 17.84 \\
\hline $\mathrm{P}_{2} \mathrm{O}_{5}$ & 51.80 & 54.44 & 54.05 & 54.47 & 54.34 & 55.46 & 54.15 & 54.80 & 58.94 & 59.51 \\
\hline $\mathrm{Cr}_{2} \mathrm{O}_{3}$ & 0.76 & 0.84 & 0.91 & 0.77 & 0.84 & 0.76 & 0.63 & 0.69 & 0.78 & 0.88 \\
\hline $\mathrm{MnO}$ & 0.31 & 0.43 & 0.35 & 0.25 & 0.34 & 0.30 & 0.35 & 0.32 & 0.50 & 0.55 \\
\hline $\mathrm{Fe}_{2} \mathrm{O}_{3}$ & 1.97 & 2.11 & 2.14 & 2.23 & 2.16 & 2.39 & 2.33 & 2.36 & 2.52 & 2.36 \\
\hline $\mathrm{NiO}$ & 1.58 & 2.30 & 2.18 & 2.16 & 2.21 & 1.92 & 2.32 & 2.12 & 2.63 & 2.40 \\
\hline
\end{tabular}




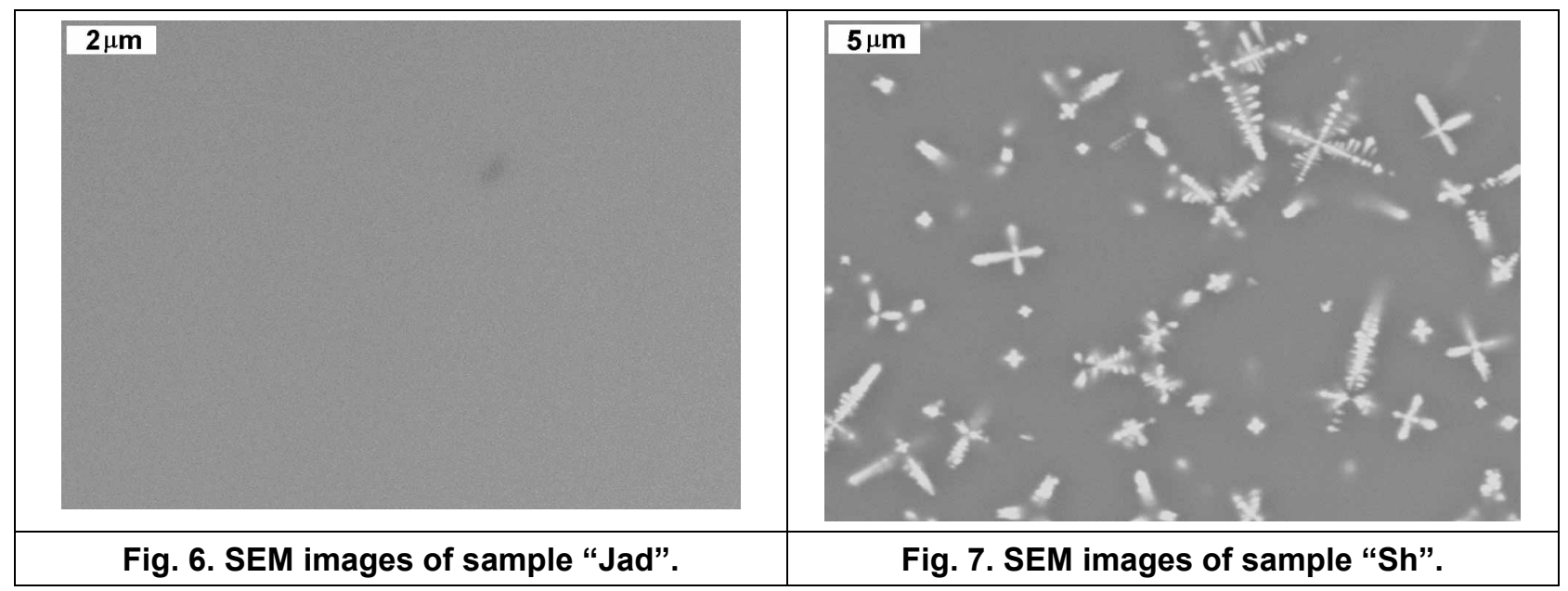

Table 17. Composition of precursor and Jad sample (SEM/EDS).

\begin{tabular}{|l|r|r|r|r|r|}
\hline \multirow{2}{*}{ Oxide } & Precursor & \multicolumn{3}{|c|}{ Scan 50x50 $\boldsymbol{~}$} & \multirow{2}{*}{ Average } \\
\cline { 3 - 5 } & & $\mathbf{1}$ & $\mathbf{2}$ & $\mathbf{3}$ & \\
\hline $\mathrm{Na}_{2} \mathrm{O}$ & 12.46 & 16.37 & 16.41 & 16.58 & 16.45 \\
\hline $\mathrm{Al}_{2} \mathrm{O}_{3}$ & 23.20 & 13.11 & 13.41 & 13.50 & 13.34 \\
\hline $\mathrm{SiO}_{2}$ & 59.35 & 64.27 & 64.15 & 63.68 & 64.03 \\
\hline $\mathrm{Cr}_{2} \mathrm{O}_{3}$ & 0.82 & 0.81 & 0.64 & 0.94 & 0.80 \\
\hline $\mathrm{MnO}$ & 0.34 & 0.53 & 0.42 & 0.36 & 0.44 \\
\hline $\mathrm{Fe}_{2} \mathrm{O}_{3}$ & 2.12 & 2.82 & 3.00 & 2.70 & 2.84 \\
\hline $\mathrm{NiO}$ & 1.71 & 2.10 & 1.97 & 2.25 & 2.10 \\
\hline$\Sigma$ & 100.00 & 100.00 & 100.00 & 100.00 & 100.00 \\
\hline
\end{tabular}

Table 18. Composition of precursor, Sh sample, glass and crystal phase (SEM/EDS).

\begin{tabular}{|c|c|c|c|c|c|}
\hline \multirow[t]{2}{*}{ Oxide } & \multirow[t]{2}{*}{ Precursor } & \multirow{2}{*}{$\begin{array}{c}\text { Scan } \\
50 \times 50 \mu \mathrm{m}\end{array}$} & \multirow[t]{2}{*}{ Glass } & \multicolumn{2}{|c|}{ Crystal phase with significant capture of glass } \\
\hline & & & & 1 & 2 \\
\hline $\mathrm{Na}_{2} \mathrm{O}$ & 10.23 & 13.27 & 10.80 & 7.78 & 11.66 \\
\hline $\mathrm{MgO}$ & 6.39 & 5.69 & 6.04 & 6.53 & 7.43 \\
\hline $\mathrm{Al}_{2} \mathrm{O}_{3}$ & 19.04 & 10.84 & 11.22 & 8.71 & 9.52 \\
\hline $\mathrm{SiO}_{2}$ & 45.66 & 50.97 & 55.12 & 37.11 & 42.96 \\
\hline $\mathrm{Cr}_{2} \mathrm{O}_{3}$ & 0.67 & 0.52 & 0.15 & 2.14 & 2.32 \\
\hline $\mathrm{MnO}$ & 0.27 & 0.45 & 0.22 & 0.35 & 0.39 \\
\hline $\mathrm{Fe}_{2} \mathrm{O}_{3}$ & 9.04 & 9.70 & 6.88 & 28.35 & 26.74 \\
\hline $\mathrm{NiO}$ & 1.40 & 1.26 & 0.48 & 9.01 & 6.78 \\
\hline $\mathrm{B}_{2} \mathrm{O}_{3}$ & 7.30 & $7.30^{*}$ & $7.30^{*}$ & - & - \\
\hline$\Sigma$ & 100.00 & 100.00 & 98.21 & 99.98 & 107.80 \\
\hline
\end{tabular}

${ }^{*} \mathrm{~B}_{2} \mathrm{O}_{3}$ content as $\mathrm{B}_{2} \mathrm{O}_{3}$ content in precursor. 
Table 19. Chemical durability synthesized compositions.

\begin{tabular}{|l|c|c|c|c|}
\hline \multirow{2}{*}{ Materials } & \multicolumn{4}{|c|}{ Leaching rate of sodium, $\mathbf{g} / \mathbf{s m}^{\mathbf{2}} \times \mathbf{d a y} \times \mathbf{1 0}^{\mathbf{- 6}}$} \\
\cline { 2 - 5 } & $\mathbf{1}$ day & $\mathbf{3}$ day & $\mathbf{7}$ day & $\mathbf{1 4}$ day \\
\hline Ph & 8,40 & 1,30 & 0,63 & 1,17 \\
\hline Jad & 15,53 & 3,14 & 0,43 & 2,53 \\
\hline Sh & 4,68 & 1,84 & 0,64 & 1,68 \\
\hline
\end{tabular}

\section{Conclusions}

1. The structural features of glass-ceramic matrices with additions of individual waste components (cesium, strontium, rare-earth elements, zirconium, and platinum group elements) and model simplified waste compositions were considered. The pyroxene- and garnet-based materials make it possible to incorporate $25-50 \mathrm{wt} \%$ of waste oxides with the maximum participation of the main nonactive $\mathrm{HLW}$ components ( $\mathrm{Fe}, \mathrm{Al}, \mathrm{Mg}, \mathrm{Mn}, \mathrm{Cr}$, and $\mathrm{Ni}$ ) in the formation of mineral-like phases. It was established that the main crystalline phases - the concentrators of individual HLW elements - are as follows: garnets ( $\mathrm{Fe}, \mathrm{Al}, \mathrm{REE}$, and $\mathrm{Zr}$ ), pyroxenes ( $\mathrm{Sr}, \mathrm{Fe}$, and $\mathrm{Al}$ ), spinellides ( $\mathrm{Ni}, \mathrm{Cr}$, and platinum group elements), and britholite (REE and $\mathrm{Zr}$ ).

2. Upon introduction of $\mathrm{CP}$ oxides in amounts of 3 and $10 \mathrm{wt} \%$ into the $\mathrm{Na}_{2} \mathrm{O}-\mathrm{Al}_{2} \mathrm{O}_{3}-\mathrm{P}_{2} \mathrm{O}_{5}$ ternary glass melt, the obtained glass blocks are characterized by a uniform distribution of these oxides over the block height. In this case, the produced glasses are homogeneous at an oxide content of $3 \mathrm{wt} \%$, and the heterogeneous phase uniformly distributed throughout the glass block volume is formed in the materials with an oxide content of $10 \mathrm{wt} \%$.

3. The introduction of 3 and $10 \mathrm{wt} \%$ of $\mathrm{CP}$ oxides into phosphate glass melt of operating composition also leads to the formation of the uniform distribution of these oxides over the glass block volume.

4. The uniform distribution of $\mathrm{CP}$ oxides above their chemical solubility in melts of phosphate glasses synthesized by the CCIM method is associated with the melt circulation due to the interaction between inductive currents and magnetic field, which results in the appearance of electrodynamic forces. Depending on the current frequency of an HF generator, the linear velocity of melt circulation is as high as $10-12 \mathrm{~mm} / \mathrm{s}$.

5. The use of the CCIM technology makes it possible to produce the homogeneous melts of phosphate glasses with a high content of $\mathrm{Fe}, \mathrm{Cr}$, and $\mathrm{Ni}$, which provides a way of reprocessing liquid $\mathrm{HLW}$ virtually without limitations on their composition and considerably increases the percentage of incorporation of certain HLW components.

6. Results of leaching tests (14 day) of the samples synthesized in the muffle furnace are $(1-2) \times 10^{-6}$ $\mathrm{g} \times \mathrm{sm}^{2} \times \mathrm{day}^{-1}(\mathrm{Na})$.

7. Preliminary research of the materials produced in CCIM has shown that uranium and plutonium distribution is homogenous all over the volume of the glass batch central zone. 


\title{
Development and Study of Borosilicate Glasses for Immobilization of Pu-containing Sludges and Industrial Waste of MOX Fuel Mix (B512152, B506216)
}

\author{
A. S. Aloy, V. S. Belov, O. A. Iskhakova, T. I. Koltsova, A. V. Trofimenko, KRI \\ Introduction
}

Borosilicate glass-like and glass-crystalline compositions were developed by the science production association "The Radium Institute" (KRI) within the framework of the research performed under the contracts of 1998-2000 for the purposes of vitrifying plutonium-containing sludge, which had been accumulated in the course of work of the Mining Chemical Combine in Zheleznogorsk [1].

The range of compositions of glass matrices was based on the calculations of thermodynamic functions of complex oxide systems in a wide temperature interval [2] and experimental testing aimed to investigate the impact of the chemical composition of the sludge upon the processes of glass formation in the triple system $\mathrm{Na}_{2} \mathrm{O}-\mathrm{B}_{2} \mathrm{O}_{3}-\mathrm{SiO}_{2}$. These glass matrices were suggested for the immobilization of the MCC sludge and they provide the necessary degree of reliability of radionuclide fixation.

The final products in the form of glasses, incorporating up to $50 \mathrm{wt} \%$ of the oxides of the solid sludge residues were obtained as a result of test melting performed in a microwave heating installation both under testbed conditions of the Radium Institute and in the hot cells of the MCC alike [3].

New types of plutonium-containing wastes, which require the creation of some new ways of handling, are formed in the course of work when metallic plutonium is turned into an oxide and in the production of the mixed uranium-plutonium fuel (MOX fuel).

The purpose of this work is the development and synthesis of the compositions, which permit us to jointly vitrify plutonium-containing sludge and wastes formed in the production process of mixed oxide fuel.

As a result of experimental melts, borosilicate glasses were obtained that contain 30,40 and $50 \mathrm{wt} \%$ of the oxide mix consisting of MOX-fuel waste and MCC sludges, with ratios of MOX waste to MCC pulp equal to $10: 90 ; 20: 80$, and $30: 70$.

The principal vitrification method of the mix of MOX-fuel and plutonium-containing MCC pulps and the development of technological solutions and synthesis conditions for final immobilized forms were demonstrated.

The results of studying the physical-chemical properties of the obtained compositions for the purpose of selecting the optimal compositions that meet conditions for further storage were presented in the paper.

By means of electron microprobe analysis (EMPA), x-ray diffraction (XRD), and scanning electron microscopy (SEM) methods, the compounds of borosilicate compositions were identified, their uniformity was estimated, and the compounds of crystalline inclusions, their size and amount were also identified.

Chemical stability tests were conducted on samples MCC- 1 at 25 and $90^{\circ} \mathrm{C}$ and MCC-5 at $90^{\circ} \mathrm{C}$, both in deionised and in mineralised water. 


\section{Synthesis of Borosilicate Glasses}

Preparation of possible wastes from MOX-fuel production was made in accordance with Minatom State Specialized Design Institute (GSPI) listing №639 issued 12.28.2000 and "The Radium Institute and MCC Proposals on Handling Wastes Coming from MOX-fuel Production".

The technology of handling MOX wastes is based on a well-known ability of alpha-nuclides to coprecipitate on iron hydroxide from alkaline solutions when $\mathrm{pH}$ is 10-11; and the decontamination factor for plutonium may reach $10^{3}[4]$.

At the present stage, attention was mostly directed to reprocessing the solutions from etching, anodizing, and decontamination of fuel elements. The compositions of these wastes are presented in Table 1.

Table 1. The compositions of wastes from MOX-fuel fabrication.

\begin{tabular}{|c|c|c|c|c|}
\hline \multirow{2}{*}{ Type of liquid radioactive wastes } & \multirow{2}{*}{$\begin{array}{c}\text { LRW } \\
\left(\mathrm{m}^{3} / \text { year }\right)\end{array}$} & \multicolumn{2}{|c|}{ Chemical composition } & \multirow{2}{*}{$\begin{array}{c}\text { Plutonium } \\
\text { contents, } \\
\text { mg/l }\end{array}$} \\
\hline & & Component & g/l & \\
\hline Solutions from etching the fuel elements & 20 & $\begin{array}{l}\mathrm{HNO}_{3} \\
\mathrm{HF} \\
\mathrm{ZrF}_{4} \\
(\mathrm{U}, \mathrm{Pu}) \mathrm{O}_{2}\end{array}$ & $\begin{array}{r}360 \\
45 \\
90 \\
7.5^{*} 10^{-4}\end{array}$ & 0,2 \\
\hline Solutions from anodizing the fuel elements & 10 & $\mathrm{NaOH}$ & 60 & traces \\
\hline $\begin{array}{l}\text { Solutions from fuel elements } \\
\text { decontamination }\end{array}$ & 4 & $\begin{array}{l}\mathrm{HNO}_{3} \\
(\mathrm{U}, \mathrm{Pu}) \mathrm{O}_{2}\end{array}$ & $\begin{array}{l}25 \\
4 * 10^{-4}\end{array}$ & 0,1 \\
\hline
\end{tabular}

Alkaline precipitation of zirconium and plutonium hydroxides with addition of ions of three-valence iron was used as a general scheme of preparation of these solutions jointly with the sludge for vitrification. $\mathrm{XRD}$ analysis of the powder obtained showed the presence of sodium nitrate and an amorphous phase.

The content of sodium, uranium, zirconium, and iron in the obtained sludge was analyzed by atomemission analysis with inductive-coupled plasma. It was found that sludge contains (wt\%): $\mathrm{Zr}-35,00 ; \mathrm{Na}$ 15,00; Fe - 2,10; and U - 0,13. The composition of the MCC sludge and MOX-fuel production wastes prepared for vitrification are presented in Table 2.

Table 2. Content of components in solid residues of MCC sludge and MOX-fuel wastes.

\begin{tabular}{|l|c|l|c|}
\hline \multicolumn{2}{|c|}{ MCC Sludge } & \multicolumn{1}{c|}{ MOX-fuel Wastes } \\
\hline \multicolumn{1}{|c|}{ Component } & Content (wt\%) & Component & Content (wt \%) \\
\hline $\mathrm{PuO}_{2}$ & 0,20 & $\mathrm{UO}_{2}$ & \\
$\mathrm{UO}_{2}$ & 22,68 & & 0,21 \\
$\mathrm{Fe}_{2} \mathrm{O}_{3}$ & 20,20 & $\mathrm{Fe}_{2} \mathrm{O}_{3}$ & \\
$\mathrm{Cr}_{2} \mathrm{O}_{3}$ & 2,80 & & 4,24 \\
$\mathrm{NiO}$ & 3,20 & $\mathrm{ZrO}_{2}$ & \\
$\mathrm{MnO}_{2}$ & 12,16 & $\mathrm{Na}_{2} \mathrm{O}$ & 66,94 \\
$\mathrm{Al}_{2} \mathrm{O}_{3}$ & 4,20 & & 28,61 \\
$\mathrm{SiO}_{2}$ & 1,36 & & \\
$\mathrm{Cs}_{2} \mathrm{O}$ & 1,00 & \\
$\mathrm{SrO}$ & 3,00 & & \\
$\mathrm{Na}_{2} \mathrm{O}$ & 29,20 & & \\
\hline
\end{tabular}


The high concentration of zirconium in MOX-fuel wastes, reaching $67 \mathrm{wt} \%$ for oxide, may considerably affect the time-and-temperature conditions of the vitrification process as a result of considerable refractability of zirconium compounds.

For that reason, the prepared residues of MOX-fuel wastes and $\mathrm{MCC}$ sludge were mixed in the following proportions (wt\% for oxides): MOX-10=10:90; MOX-20=20:80; MOX-30=30:70.

Frit components in the form of amorphous silica, boron acid and sodium or lithium salts were added to the obtained combined wastes, the amount of glass-forming oxides in the final product calculated as 70,60 and $50 \mathrm{wt} \%$. The following frits were prepared to mix with combined waste streams: Frit 2- $\mathrm{SiO}_{2}-64,0$; $\mathrm{B}_{2} \mathrm{O}_{3}-20,8 ; \mathrm{Na}_{2} \mathrm{O}-9,2 ; \mathrm{Li}_{2} \mathrm{O}-6,0$.

The synthesis of borosilicate compositions was performed in an electric resistance oven of the "SNOL $16 / 12$ " type at the melting temperature of $1200-1250^{\circ} \mathrm{C}$. The compositions of the synthesized compounds, and the conditions of melting are presented in Table 3.

Table 3. Compositions of borosilicate glasses for the immobilization of the mixture of MCC sludge and MOX-fuel waste (wt\%).

\begin{tabular}{|c|c|c|c|c|c|c|c|c|c|}
\hline \multirow{2}{*}{$\begin{array}{c}\begin{array}{c}\text { Ratio MOX } \\
\text { waste: MCC } \\
\text { sludge }\end{array} \\
\text { \# composition }\end{array}$} & \multicolumn{3}{|c|}{$10: 90$} & \multicolumn{3}{|c|}{$20: 80$} & \multicolumn{3}{|c|}{$30: 70$} \\
\hline & 1 & 2 & 3 & 4 & 5 & 6 & 7 & 8 & 9 \\
\hline $\begin{array}{c}\text { Code } \\
\text { composition }\end{array}$ & $\begin{array}{l}\text { MOX- } \\
10 / 30\end{array}$ & $\begin{array}{l}\text { MOX- } \\
10 / 40\end{array}$ & $\begin{array}{l}\text { MOX- } \\
10 / 50\end{array}$ & $\begin{array}{l}\text { MOX- } \\
20 / 30\end{array}$ & $\begin{array}{l}\text { MOX- } \\
20 / 40\end{array}$ & $\begin{array}{l}\text { MOX- } \\
20 / 50\end{array}$ & $\begin{array}{l}\text { MOX- } \\
30 / 30\end{array}$ & $\begin{array}{l}\text { MOX- } \\
30 / 40\end{array}$ & $\begin{array}{l}\text { MOX } \\
30 / 50\end{array}$ \\
\hline $\begin{array}{l}\text { Goncentration } \\
\text { of waste } \\
\text { Component }\end{array}$ & 30 & 40 & 50 & 30 & 40 & 50 & 30 & 40 & 50 \\
\hline $\mathrm{PuO}_{2}$ & 0,06 & 0,08 & 0,10 & 0,05 & 0,07 & 0,09 & 0,05 & 0,06 & 0,08 \\
\hline $\mathrm{UO}_{2}$ & 6,13 & 8,17 & 10,22 & 5,45 & 7,27 & 9,09 & 4,78 & 6,38 & 7,97 \\
\hline $\mathrm{Fe}_{2} \mathrm{O}_{3}$ & 5,58 & 7,44 & 9,30 & 5,10 & 6,80 & 8,50 & 4,62 & 6,16 & 7,70 \\
\hline $\mathrm{Cr}_{2} \mathrm{O}_{3}$ & 0,76 & 1,01 & 1,26 & 0,67 & 0,90 & 1,12 & 0,59 & 0,78 & 0,98 \\
\hline $\mathrm{NiO}$ & 0,86 & 1,15 & 1,44 & 0,77 & 1,02 & 1,28 & 0,67 & 0,90 & 1,12 \\
\hline $\mathrm{MnO}_{2}$ & 3,28 & 4,38 & 5,47 & 2,92 & 3,89 & 4,87 & 2,55 & 3,40 & 4,26 \\
\hline $\mathrm{Al}_{2} \mathrm{O}_{3}$ & 1,13 & 1,51 & 1,89 & 1,01 & 1,34 & 1,68 & 0,88 & 1,18 & 1,47 \\
\hline $\mathrm{Cs}_{2} \mathrm{O}$ & 0,27 & 0,36 & 0,45 & 0,24 & 0,32 & 0,40 & 0,21 & 0,28 & 0,35 \\
\hline SrO & 0,81 & 1,08 & 1,35 & 0,72 & 0,96 & 1,20 & 0,63 & 0,84 & 1,05 \\
\hline $\mathrm{Na}_{2} \mathrm{O}$ & 15,18 & 17,18 & 19,17 & 15,16 & 17,15 & 19,14 & 15,35 & 17,14 & 19,11 \\
\hline $\mathrm{Li}_{2} \mathrm{O}$ & 4,20 & 3,60 & 3,00 & 4,20 & 3,60 & 3,00 & 4,20 & 3,60 & 3,00 \\
\hline $\mathrm{B}_{2} \mathrm{O}_{3}$ & 14,56 & 12,48 & 10,40 & 14,56 & 12,48 & 10,40 & 14,56 & 12,48 & 10,40 \\
\hline $\mathrm{SiO}_{2}$ & 45,17 & 38,89 & 32,61 & 45,13 & 38,84 & 32,55 & 45,09 & 38,78 & 32,48 \\
\hline $\mathrm{ZrO}_{2}$ & 2,01 & 2,68 & 3,35 & 4,01 & 5,35 & 6,69 & 6,02 & 8,03 & 10,04 \\
\hline $\begin{array}{l}\text { Synthesis } \\
\text { conditions, }{ }^{0} \mathrm{C} \text {, } \\
\text { hours,, }\end{array}$ & \multicolumn{9}{|c|}{$\begin{array}{l}1200-1250 \\
3,0\end{array}$} \\
\hline
\end{tabular}




\section{Study of Borosilicate Glasses by EMPA, XRD and SEM Methods}

All synthesized borosilicate glasses were studied by the XRD method. Phase compositions of borosilicate glasses are shown in Table 4.

Table 4. Phase compositions of borosilicate glasses.

\begin{tabular}{|c|c|c|c|c|}
\hline$\#$ & $\begin{array}{l}\text { MOX waste } \\
\text { :sludge MCC }\end{array}$ & $\begin{array}{c}\text { Waste content, } \\
\text { wt } \%\end{array}$ & $\begin{array}{c}\text { Composition } \\
\text { index }\end{array}$ & Phase composition \\
\hline 1 & \multirow{3}{*}{$10: 90$} & 30 & MOX-10/30 & Amorphous phase, spinel \\
\hline 2 & & 40 & MOX-10/40 & Amorphous phase \\
\hline 3 & & 50 & MOX-10/50 & Amorphous phase, spinel \\
\hline 4 & \multirow{3}{*}{$20: 80$} & 30 & MOX-20/30 & Amorphous phase, spinel \\
\hline 5 & & 40 & MOX-20/40 & Amorphous phase \\
\hline 6 & & 50 & MOX-20/50 & Amorphous phase, spinel, baddeleyite \\
\hline 7 & \multirow{3}{*}{$30: 70$} & 30 & MOX-30/30 & Amorphous phase \\
\hline 8 & & 40 & MOX-30/40 & Amorphous phase, spine, baddeleyite \\
\hline 9 & & 50 & MOX-30/50 & Amorphous phase, spinel, baddeleyite \\
\hline
\end{tabular}

From XRD data, the samples 2, 5, and 7 are amorphous. The samples 1, 3, 4, and 8 contain inclusions of spinel $\left(\mathrm{Me}^{+2} \mathrm{Me}_{2}^{+3} \mathrm{O}_{4}\right)$, where $\mathrm{Me}^{+2}-\mathrm{Fe}, \mathrm{Mn}, \mathrm{Ni} ; \mathrm{Me}^{+3}-\mathrm{Fe}, \mathrm{Cr}$, Al, along with an amorphous phase. Samples 6 and 9 contain spinel and baddeleyite $\left(\mathrm{ZrO}_{2}\right)$ inclusions.

Both EMPA and SEM methods were used to estimate the homogeneity of glasses 1-3, 5, and 9, and their calculated compositions and averaged analytical compositions are shown in Table 5. The corrections in glass composition were done by taking into account the corrosion of the crucible and volatilization of boron oxide. Both the calculated and the averaged analytical compositions of glasses are practically equal.

Table 5. The calculated compositions and the averaged analytical compositions of glasses.

\begin{tabular}{|c|c|c|c|c|c|c|c|c|c|c|}
\hline \multirow{3}{*}{$\begin{array}{l}\text { index. } \\
\text { oxide }\end{array}$} & \multicolumn{2}{|c|}{ MOX-10/30 } & \multicolumn{2}{|c|}{ MOX-10/40 } & \multicolumn{2}{|c|}{ MOX-10/50 } & \multicolumn{2}{|c|}{ MOX-20/40 } & \multicolumn{2}{|c|}{ MOX-30/50 } \\
\hline & \multicolumn{10}{|c|}{ Content (wt\%) } \\
\hline & Calc & Anal & Calc & Anal & Calc & Anal & Calc & Anal & Calc & Anal \\
\hline $\mathrm{SiO}_{2}$ & 43,98 & 45,28 & 37,87 & 38,64 & 32,11 & 32,95 & 37,04 & 37,80 & 28,66 & 29,59 \\
\hline $\mathrm{Al}_{2} \mathrm{O}_{3}$ & 8,61 & 8,87 & 8,96 & 9,23 & 8,31 & 8,45 & 10,70 & 11,29 & 17,37 & 19,08 \\
\hline $\mathrm{Na}_{2} \mathrm{O}$ & 14,78 & 12,86 & 16,73 & 15,25 & 18,88 & 17,99 & 16,35 & 16,38 & 16,86 & 15,28 \\
\hline $\mathrm{MnO}_{2}$ & 3,19 & 3,39 & 4,26 & 4,25 & 5,39 & 5,44 & 3,71 & 3,39 & 3,76 & 3,65 \\
\hline $\mathrm{NiO}$ & 0,84 & 0,74 & 1,12 & 0,87 & 1,42 & 1,10 & 0,97 & 0,85 & 0,99 & 0,68 \\
\hline $\mathrm{Cr}_{2} \mathrm{O}_{3}$ & 0,74 & 0,83 & 0,98 & 0,83 & 1,24 & 1,14 & 0,86 & 0,62 & 0,86 & 0,26 \\
\hline $\mathrm{Fe}_{2} \mathrm{O}_{3}$ & 5,43 & 5,21 & 7,24 & 6,75 & 9,16 & 8,35 & 6,48 & 5,92 & 6,79 & 5,99 \\
\hline $\mathrm{ZrO}_{2}$ & 1,96 & 1,90 & 2,61 & 2,87 & 3,30 & 3,30 & 5,10 & 4,88 & 8,86 & 7,75 \\
\hline $\mathrm{SrO}$ & 0,79 & 0,72 & 1,05 & 1,00 & 1,33 & 0,96 & 0,92 & 0,49 & 0,93 & 0,89 \\
\hline $\mathrm{Cs}_{2} \mathrm{O}$ & 0,26 & 0,34 & 0,35 & 0,25 & 0,44 & 0,45 & 0,31 & 0,11 & 0,31 & 0,11 \\
\hline $\mathrm{UO}_{2}$ & 6,03 & 6,44 & 8,03 & 9,27 & 10,16 & 11,60 & 7,00 & 7,71 & 7,19 & 9,32 \\
\hline $\mathrm{Li}_{2} \mathrm{O}$ & 4,09 & $\mathrm{~N} / \mathrm{d}$ & 3,51 & $\mathrm{~N} / \mathrm{d}$ & 2,95 & $\mathrm{~N} / \mathrm{d}$ & 3,43 & $\mathrm{~N} / \mathrm{d}$ & 2,65 & $\mathrm{~N} / \mathrm{d}$ \\
\hline $\mathrm{B}_{2} \mathrm{O}_{3}$ & 9,31 & $\mathrm{~N} / \mathrm{d}$ & 7,28 & $\mathrm{~N} / \mathrm{d}$ & 5,32 & $\mathrm{~N} / \mathrm{d}$ & 7,13 & $\mathrm{~N} / \mathrm{d}$ & 4,76 & $\mathrm{~N} / \mathrm{d}$ \\
\hline Total & 100,00 & 86,60 & 100,00 & 89,21 & 100,00 & 91,73 & 100,00 & 89,44 & 100,00 & 92,59 \\
\hline
\end{tabular}




\section{Glass \#2. MOX-10/40}

The diffraction pattern of MOX-10/40 glass is shown in Fig. 1.

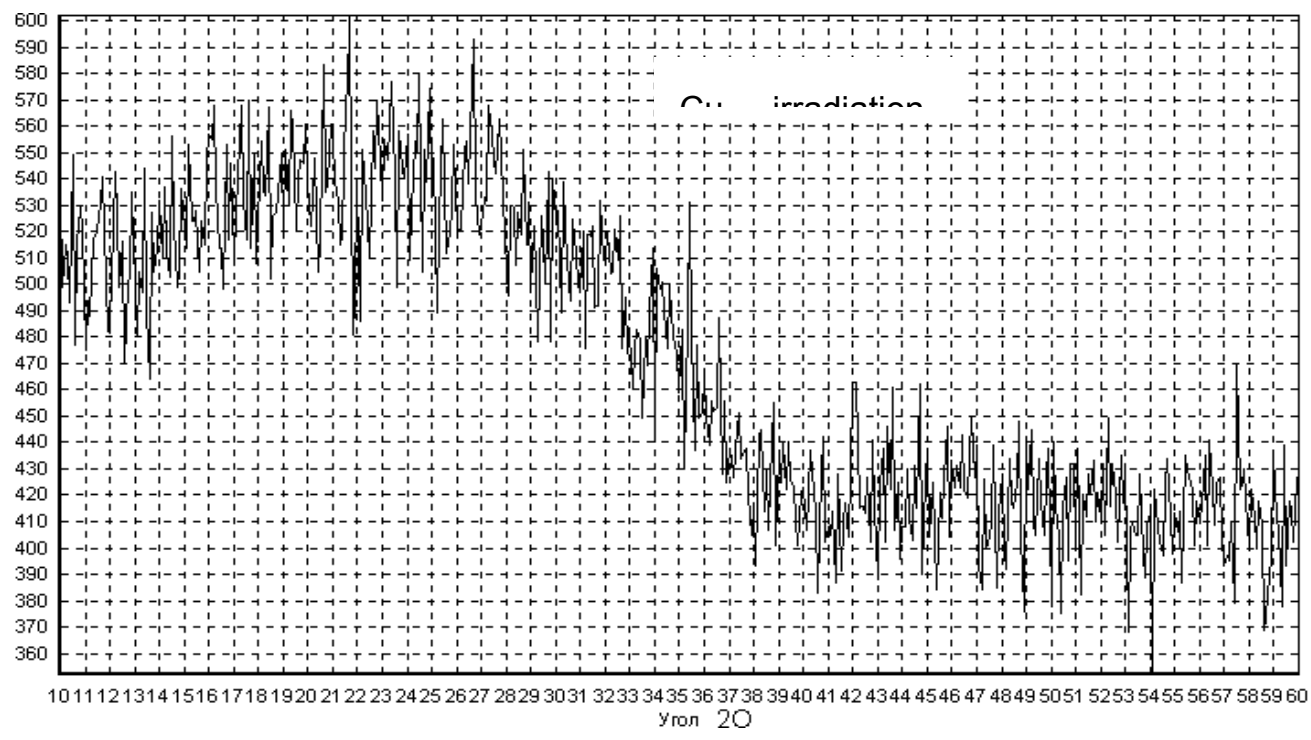

Fig 1. Diffraction pattern of MOX-10/40 glass.

The glass is practically amorphous. The electron microphotographs of MOX-10/40 glass, which was obtained at a different magnification, are given in Figs. 2 and 3. The dark liquation zone is enriched by Al and is depleted by $U$. The white liquation zone is enriched by $\mathrm{U}$. Compositions of liquation zones are given in Table 6. The spinel quantity is equal to $1 \mathrm{wt} \%$. The chemical formula of spinel is $\mathrm{Mn}_{0,55} \mathrm{Ni}_{0,45}$ $\left(\mathrm{Al}_{0,2} \mathrm{Cr}_{1,2} \mathrm{Fe}_{0,5}\right) \mathrm{O}_{4}$.

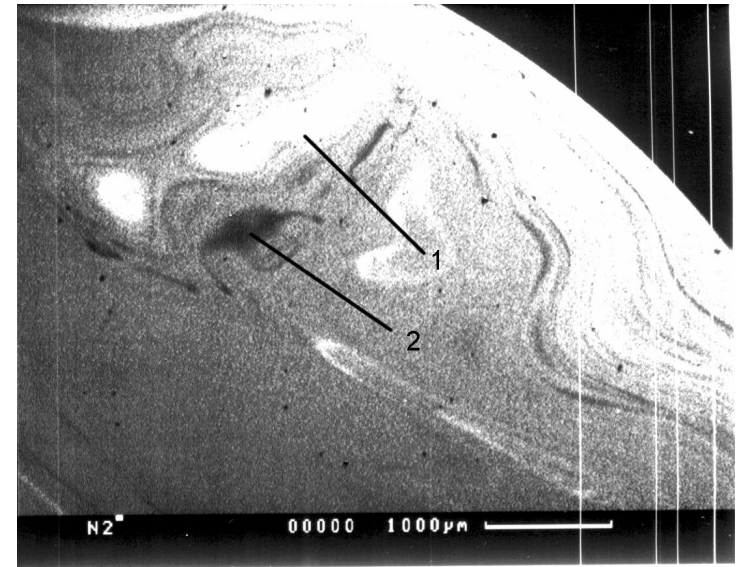

Fig. 2. (1)- white liquation zone; (2)- dark liquation zone.

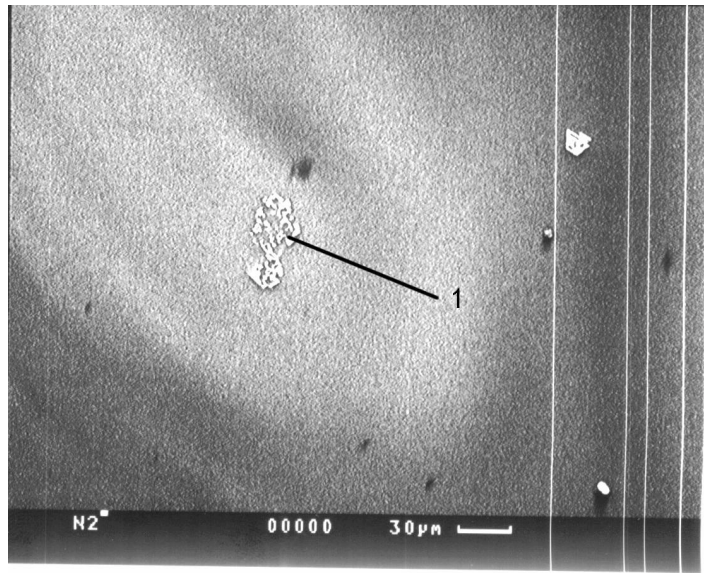

Fig. 3. (1)- the spinel inclusions. 
Table 6. The white and dark liquation zones compositions.

\begin{tabular}{|l|c|c|c|}
\hline \multicolumn{1}{|c|}{ Oxide } & Analytical & White zone & Black zone \\
\hline $\mathrm{SiO}_{2}$ & 38,64 & 34,50 & 36,18 \\
\hline $\mathrm{Al}_{2} \mathrm{O}_{3}$ & 9,23 & 11,24 & 16,89 \\
\hline $\mathrm{Na}_{2} \mathrm{O}$ & 15,25 & 14,72 & 16,20 \\
\hline $\mathrm{MnO}_{2}$ & 4,25 & 3,87 & 3,29 \\
\hline $\mathrm{NiO}$ & 0,87 & 0,66 & 0,69 \\
\hline $\mathrm{Cr}_{2} \mathrm{O}_{3}$ & 0,83 & 0,82 & 0,56 \\
\hline $\mathrm{Fe}_{2} \mathrm{O}_{3}$ & 6,75 & 6,58 & 5,08 \\
\hline $\mathrm{ZrO}_{2}$ & 2,87 & 2,43 & 2,55 \\
\hline $\mathrm{SrO}_{\mathrm{Cs}} \mathrm{O}$ & 1,00 & 1,33 & 0,96 \\
\hline $\mathrm{UO}_{2}$ & 0,25 & 0,24 & 0,00 \\
\hline $\mathrm{Total}$ & 9,27 & 12,81 & 6,79 \\
\hline
\end{tabular}

\section{Glass \#5. MOX-20/40}

The glass is amorphous. The electron microphotographs of the glass, which were obtained at a different magnification, are given in Figs. 4 and 5.

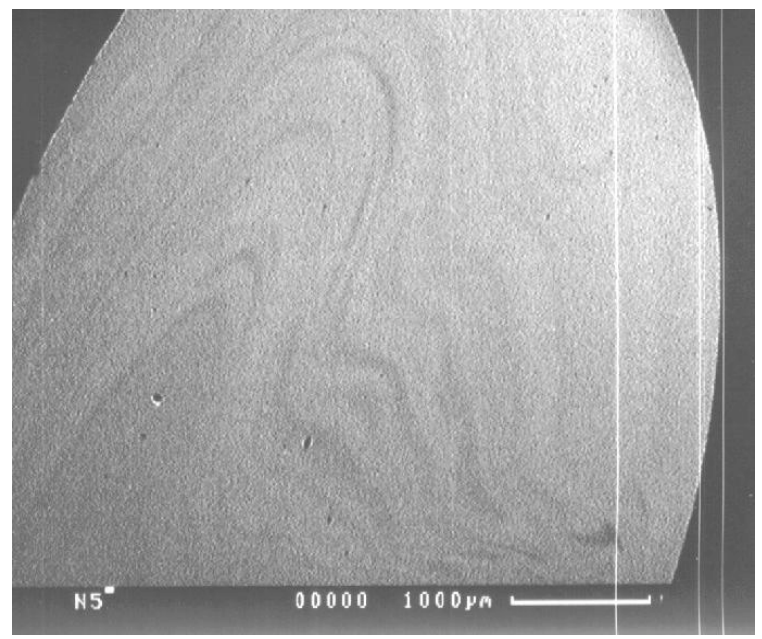

Fig. 4. Liquation zone.

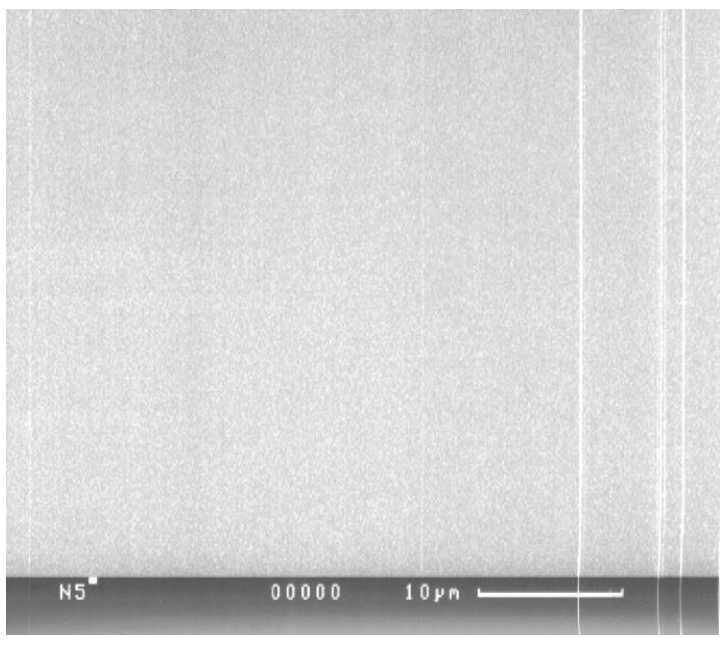

Fig. 5. The homogeneous part of the sample.

The chart in Fig. 6 shows the changes in oxide concentrations of the basic elements in the sample volume. Standard and relative deviations of the content of oxides in glass are presented in Table 7.

It appears from this data that there is a sufficiently uniform distribution of all basic elements in the sample volume. 


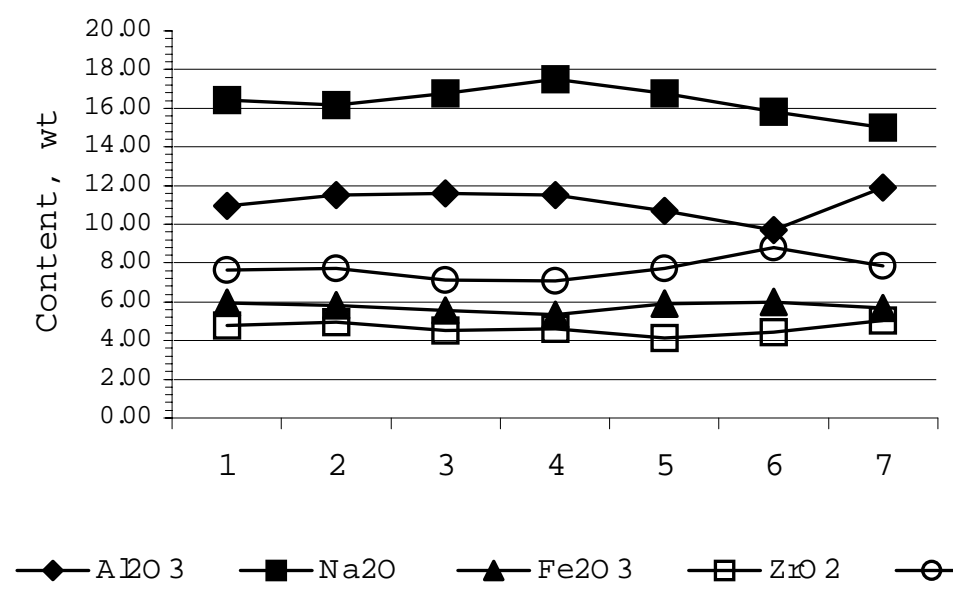

Fig. 6. The concentration change of the basic elements oxides in the sample volume.

Table 7. Standard and relative deviations of oxides content in glass MOX-20/40.

\begin{tabular}{|l|c|c|}
\hline \multicolumn{1}{|c|}{ Oxide } & Standard deviation & Relative deviation, $\pm \mathbf{\Delta} \%$ \\
\hline $\mathrm{Al}_{2} \mathrm{O}_{3}$ & 0,74 & 7 \\
\hline $\mathrm{Na}_{2} \mathrm{O}$ & 0,79 & 5 \\
\hline $\mathrm{Fe}_{2} \mathrm{O}_{3}$ & 0,23 & 4 \\
\hline $\mathrm{ZrO}_{2}$ & 0,30 & 7 \\
\hline $\mathrm{UO}_{2}$ & 0,58 & 8 \\
\hline
\end{tabular}

\section{Glass \#9. MOX-30/50}

The diffraction pattern of MOX-30/50 glass is shown in Fig. 7 where it can be seen the glass contains spinels and baddeleyite $\left(\mathrm{ZrO}_{2}\right)$ inclusions along with an amorphous phase. The electron microphotographs of MOX-30/50 glass are given in Figs. 8 and 9.

The glass has no liquation zones. The distribution of the basic elements oxides in the sample volume is shown in Fig. 10. Standard and relative deviations of oxides content in glass are presented in Table 8.

The values of a relative deviation for sodium, iron and zirconium are higher than allowable limits $(5 \%)$. $U$ and $\mathrm{Al}$ are uniformly distributed in the glass matrix. The compositions of crystalline inclusions are:

$$
\begin{aligned}
& \text { Spinel - } \mathrm{Mn}_{0,4} \mathrm{Ni}_{0,6}\left(\mathrm{Al}_{0,6} \mathrm{Cr}_{0,3} \mathrm{Fe}_{1,1}\right) \mathrm{O}_{4}, \mathrm{Mn}_{0,4} \mathrm{Ni}_{0,6}\left(\mathrm{Al}_{0,4} \mathrm{Cr}_{0,7} \mathrm{Fe}\right) \mathrm{O}_{4} \text { (3-4 wt\%); } \\
& \text { Baddeleyite - } \mathrm{Zr}_{1,96} \mathrm{U}_{0,04} \mathrm{O}_{2}(3 \mathrm{wt} \% \text { ). }
\end{aligned}
$$




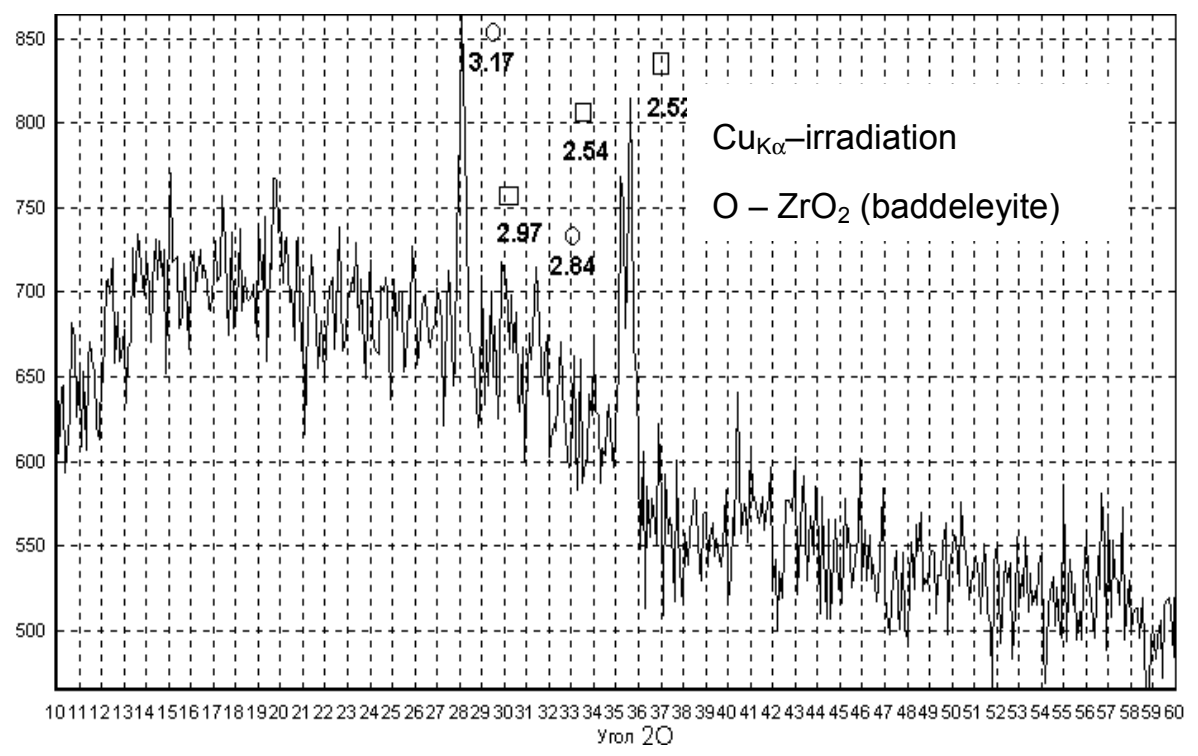

Fig. 7. Diffraction pattern of MOX-30/50 glass.

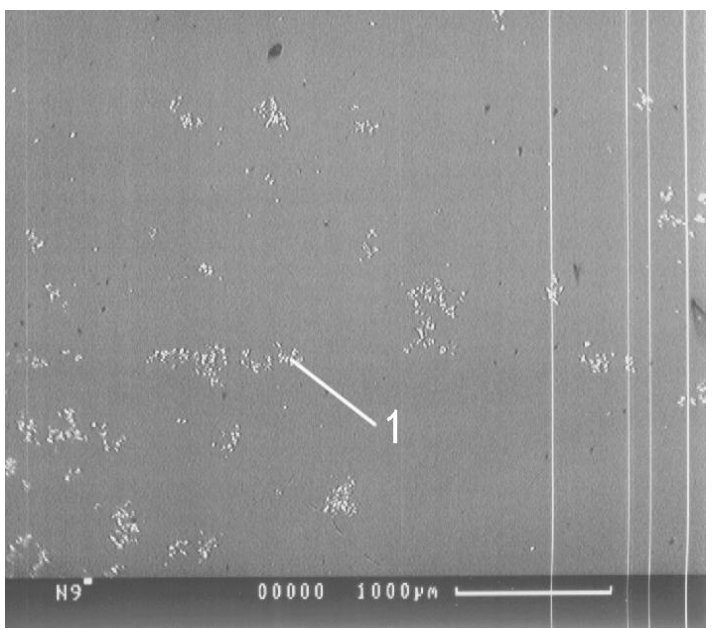

Fig. 8. (1) Inclusions of crystalline phase.

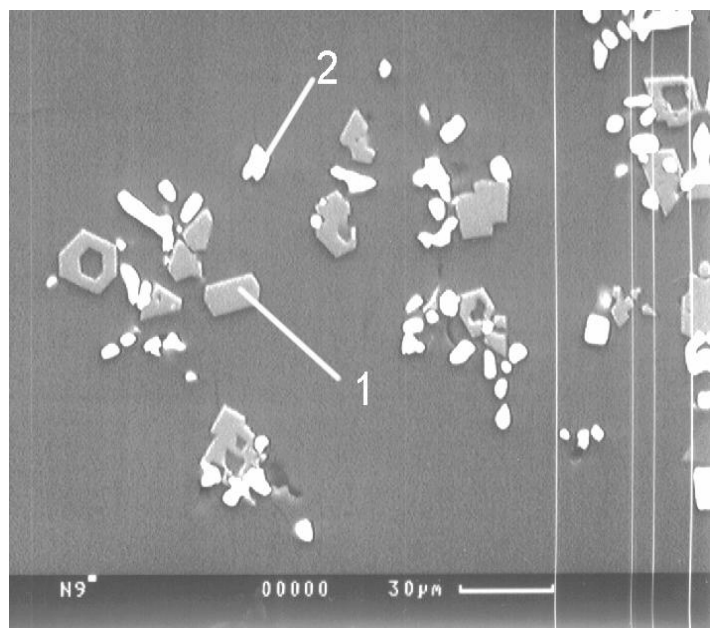

Fig. 9. (1) Crystals of spinel; (2) Crystals of baddeleyite $\left(\mathrm{ZrO}_{2}\right)$. 


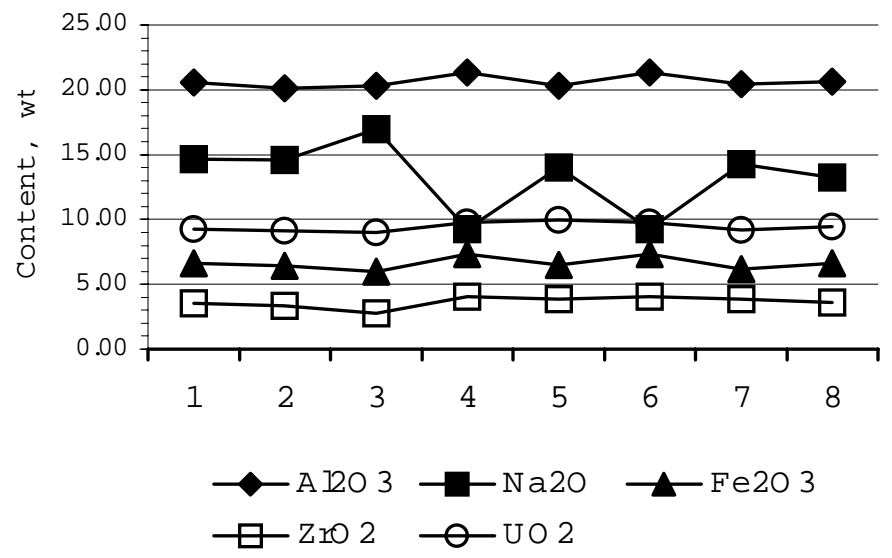

Fig. 10. The concentration change of the basic elements oxides in the sample volume.

Table 8. Standard and relative deviation of oxides content in MOX-30/50 glass.

\begin{tabular}{|l|c|c|}
\hline \multicolumn{1}{|c|}{ Oxide } & Standard deviation & $\begin{array}{c}\text { Relative deviation, } \\
\pm \boldsymbol{\Delta} \%\end{array}$ \\
\hline $\mathrm{Al}_{2} \mathrm{O}_{3}$ & 0,48 & 3 \\
\hline $\mathrm{Na}_{2} \mathrm{O}$ & 2,71 & 24 \\
\hline $\mathrm{Fe}_{2} \mathrm{O}_{3}$ & 0,50 & 9 \\
\hline $\mathrm{ZrO}_{2}$ & 0,42 & 13 \\
\hline $\mathrm{UO}_{2}$ & 0,36 & 4 \\
\hline
\end{tabular}

\section{Chemical Stability Study of Glass Compositions}

Selection of the above compositions has been made based on the results of studying glasses by XRD, EMPA, and SEM methods, as well as on the results of estimating the mass losses of synthesized glass samples after having been boiled for 5 hours in distilled water.

Glass samples MOX-10/40 and MOX-20/40 were subjected to leaching tests by MCC-1 and MCC-5 methods, the tested samples containing $40 \mathrm{wt} \%$ of waste where the ratio of MOX waste to MCC sludge comprised 10:90 and 20:80 accordingly (Table 5).

Chemical stability tests were conducted by MCC- 1 and MCC- 5 methods using deionized (pure) and mineralized water. Pure water parameters correspond to deeply demineralized water or to 30 -fold distillate, $\mathrm{pH}-5,6[5]$.

With the use of pure water, synthetic mineralized water has been prepared, simulating the surface waters of a presumed site of immobilized waste disposal in Krasnoyarsk [6]. Total salt content of mineralized water comprised $0,4 \mathrm{~g} / \mathrm{l}, \mathrm{pH}=7,3$, and its composition is presented in Table 9.

Table 9. Composition of simulated mineralized water.

\begin{tabular}{|c|c|c|c|c|c|c|}
\hline Element & $\mathrm{Na}^{+}$ & $\mathbf{M g}^{++}$ & $\mathrm{Ca}^{++}$ & $\mathbf{C l}^{+}$ & $\mathbf{S O}_{4}{ }^{--}$ & $\mathbf{H C O}_{3}{ }^{-}$ \\
\hline Content, $\mathrm{g} / \mathrm{l}$ & 140 & 1.7 & 3.0 & 133.7 & 12.0 & 134.2 \\
\hline
\end{tabular}




\section{Leaching in static conditions (MCC-1)}

The tests were conducted for 28 days at temperatures of 25 and $90^{\circ} \mathrm{C}$ in deionized and mineralized water, with the ratio $S A / V=0,1 \mathrm{~m}^{-1}$.

Glass samples were leached for $3,7,14$, and 28 days, with a leach sample taken at the end of each period and analyzed for the content of basic glass components and $\mathrm{pH}$. Leach samples were analyzed by atomic-emission method with inductive-bonded plasma, as well as by the mass-spectrometry method with inductive-bonded plasma, with the concentration estimating error not exceeding 10 relative \%.

Table 10 presents the normalized mass losses of elements $\left(\mathrm{NL}_{\mathrm{i}}\right)$ in deionized and mineralized water in static conditions at $25^{\circ} \mathrm{C}$ (MCC-1) on the 28 th day.

Table 10. Normalized mass losses of elements in deionized and mineralized water at $25^{\circ} \mathrm{C}(\mathrm{MCC}-1)$ on 28th day.

\begin{tabular}{|l|c|c|c|c|}
\hline \multirow{2}{*}{ Element } & \multicolumn{3}{|c|}{ Normalized mass losses of elements $\left(\mathbf{g} / \mathbf{m}^{\mathbf{2}} \mathbf{~}\right.$} \\
\cline { 2 - 5 } & \multicolumn{2}{|c|}{ Deionized water } & \multicolumn{2}{c|}{ Mineralized water } \\
\cline { 2 - 5 } & MOX-10/40 & MOX-20/40 & MOX-10/40 & MOX-20/40 \\
\hline $\mathrm{U}$ & 0,0044 & 0,0042 & 0,0593 & 0,0697 \\
\hline $\mathrm{Na}$ & 1,11 & 1,35 & 1,6 & 1,94 \\
\hline $\mathrm{B}$ & 0,158 & 0,214 & 0,701 & 0,634 \\
\hline $\mathrm{Si}$ & 0,092 & 0,077 & 0,159 & 0,139 \\
\hline $\mathrm{Zr}$ & $<0,02$ & $<0,02$ & $<0,02$ & $<0,02$ \\
\hline $\mathrm{Fe}$ & $<0,01$ & $<0,01$ & $<0,01$ & $<0,01$ \\
\hline $\mathrm{Li}$ & 0,124 & 0,103 & 0,232 & 0,213 \\
\hline
\end{tabular}

At $25^{\circ} \mathrm{C}$ for MOX-10/40 and MOX-20/40 compounds, the NL values of the corresponding elements in deionized water are close. The emission of elements into mineralized water is $2-5$ times higher than into deionized water, and for uranium the corresponding NL value increase is by an order of magnitude. The $\mathrm{pH}$ of the deionized water was equal to 5,5 , moderately changing during the test period (by less than 0,5 ). Simulated mineralized water had a higher $\mathrm{pH}=7,3$ and in this water, the leaching process had been proceeding more deeply during the 28 days over which the tests were conducted. The ratio of leaching speeds of mobile ion and silicon did not depend on the leaching medium and fitted in the sequence: $\mathrm{Na}>\mathrm{B}>\mathrm{S}$.

Temperature increase results in a decrease in chemical stability by an order or magnitude and more by separate elements of borosilicate glasses, which has been quoted in a number of studies $[7,8]$.

Table 11 presents normalized mass losses of elements in deionized and mineralized water at $90^{\circ} \mathrm{C}$ (MCC-1) on the 28th day.

Analysis of Table 11 has shown that the mass losses of the same glass elements into deionized water for MOX-10/40 and MOX-20/40 have like values. Normalized mass losses of the elements of MOX-10/40 composition in mineralized water are higher than the corresponding values for MOX-20/40 composition.

Uranium release into mineralized water is 1,5 times higher than into deionized one. In deionized water, the behavior of uranium is caused by formation of a changed layer and by reprecipitation, on the glass surface, of slightly soluble uranium compound with the formula of $\mathrm{UO}_{3} \cdot 2 \mathrm{H}_{2} \mathrm{O}$ [9]. 
Table 11. Normalized mass losses of the elements in deionized and mineralized water at $90^{\circ} \mathrm{C}$ (MCC-1) on 28th day.

\begin{tabular}{|l|c|c|c|c|}
\hline \multirow{2}{*}{ Element } & \multicolumn{3}{|c|}{ Normalized mass losses of the elements $\left(\mathbf{g} / \mathbf{m}^{\mathbf{2}}\right)$} \\
\cline { 2 - 5 } & \multicolumn{2}{|c|}{ Deionized water } & \multicolumn{2}{c|}{ Mineralized water } \\
\cline { 2 - 5 } & MOX-10/40 & MOX-20/40 & MOX-10/40 & MOX-20/40 \\
\hline $\mathrm{U}$ & 0,363 & 0,387 & 0,640 & 0,521 \\
\hline $\mathrm{Na}$ & 20,12 & 22,29 & 17,50 & 14,42 \\
\hline $\mathrm{B}$ & 22,32 & 22,31 & 20,21 & 16,34 \\
\hline $\mathrm{Si}$ & 16,27 & 17,50 & 10,60 & 7,15 \\
\hline $\mathrm{Fe}$ & 0,128 & 0,109 & 0,120 & 0,106 \\
\hline $\mathrm{Zr}$ & $<0,04$ & $<0,04$ & $<0,04$ & $<0,04$ \\
\hline $\mathrm{Li}$ & 16,62 & 15,83 & 11,83 & 10,38 \\
\hline
\end{tabular}

Uranium concentration in leaches is higher in mineralized water, due to formation of stable soluble complex compounds with carbonate- and chloride-ions [10]. Attention is drawn to the low values of the normalized losses of silicon masses, especially in mineralized water, as in this case we observe formation of $\mathrm{FeSiO}_{2}$ colloids or $\mathrm{FeSi}_{3}(\mathrm{OH})_{3}$ complex, which agrees with the data [11]. It is well known that the minimum changes of glass occur in the solutions with $\mathrm{pH}$ within 6-8,5 ranges, where the process is controlled by the diffusion of the components. This $\mathrm{pH}$ range corresponds to the maximum stability of $\mathrm{SiO}_{2}$ whose solubility at $\mathrm{pH}=5-8$ comprises $1.8 \times 10^{-3}$ mole/l, and at $\mathrm{pH}=9,10,11$ it already comprises $(2,1$, $4,4$ and 31,6$) 10^{-3}$ mole/l accordingly.

The $\mathrm{pH}$ values for all the leaches, after 28 days of tests performed in static conditions at $90{ }^{\circ} \mathrm{C}$ in deionized water, were found within 5,6-7,8 range, and in mineralized water the range was $7,3-8,4$. NL values for sodium, boron and silicon-the elements characterizing the glass corrosion-differ by less than an order of magnitude, which reflects the prevailing contribution of glass lattice dissolution mechanism to the process of glass destruction.

Normalized losses of zirconium mass have low values and are practically independent of temperature, duration of tests, and leaching medium composition. MOX-20/40 and MOX-10/40 compounds have pretty much the same chemical stability in deionized water, while the MOX-20/40 compound is more stable in mineralized water.

\section{Leaching in dynamic conditions}

Testing of MOX-20/40 glasses has been performed by the MCC-5 method in deionized and mineralized water at $90{ }^{\circ} \mathrm{C}$ during 14 days in a lab-scale plant of dynamic leaching. The volume of the teflon working cell was $8 \mathrm{~cm}^{3}$, the summary geometrical surface of the tested samples was equal to $20 \mathrm{~cm}^{2}$, and the mass comprised $8.32830 \mathrm{~g}$ for leaching in deionized water and $8.36355 \mathrm{~g}$ for the tests in mineralized water (at $\mathrm{S} / \mathrm{A}=20 \mathrm{~cm}^{3}$ ). Average water flow speeds comprised 0.123 and $0.157 \mathrm{ml} / \mathrm{min}$, respectively.

Control sampling of daily leaches took place during the 3rd, 7th, and 14th days. In addition, the leaches were studied that were obtained during 1-2, 4-6, and 8-13 days. In order to correlate the test data of MCC- 5 with MCC-1, the normalized losses of the glass element mass by MCC- 5 have been calculated for 1-7 and 1-14 days.

Table 12 presents the normalized losses of MPC-2-5-40 glass element mass for 7 and 14 days for dynamic conditions of testing in deionized and mineralized water. 
For dynamic tests, the uranium release into mineralized water is an order of magnitude higher than into deionized water; this result is connected with the $\mathrm{pH}$ solution effect.

A decrease of silicon release correlated with the formation of $\mathrm{H}_{2} \mathrm{SiO}_{3}$ compound and its lower solubility in mineralized water.

Mobile alkaline elements ( $\mathrm{Li}$ and $\mathrm{Na}$ ) are poorly retained in glass and in both mineralized and deionized water.

Thermodynamic calculations have shown that the glass of the given compound has highly detectable amounts of excessive boric anhydride that exceed the amounts of $\mathrm{B}_{2} \mathrm{O}_{3}$ required for the formation of borosilicate glass framework. This explains the high leaching of boron [2]. The summary yield of $\mathrm{Na}+\mathrm{Li}+\mathrm{B}$ elements into deionized water is higher than into demineralized water.

The high leaching degree of borosilicate glass elements containing up to $30 \%$ of highly active waste is also pointed out by G. G. Wicks and Robnett [12], who correlate this fact with the lower solubility of silica in mineralized water.

The conducting of dynamic tests has revealed the increase of the glass mass loss as compared to the static test data. The summary mass loss for the samples tested in deionized water exceeded by 1,5 times the similar value for mineralized water (Table 13). For comparative analysis of the behavior of MOX-20/40 and MOX-10/40 glass compounds in contact with water, the summary mass losses are given for the test conducting periods: $3,7,14$, and 28 days for static conditions and 14 days for dynamic conditions (Table 13). The summary mass losses of the samples have been smoothly increasing from the 3rd to 28th day for both glass compounds, with the sample mass losses in deionized water exceeding the corresponding values in mineralized water. The most significant mass losses of glass samples took place in dynamic conditions in deionized water, where they exceeded by 2.2 times the glass mass loss in static test conditions for 14 days.

Table 12. Normalized losses of MOX-20/40 glass element mass for 7 and 14 days in deionized and mineralized water in dynamic conditions of leaching at $90{ }^{\circ} \mathrm{C}$.

\begin{tabular}{|l|r|c|c|}
\hline \multirow{3}{*}{ Element } & \multirow{2}{*}{$\begin{array}{c}\text { Time } \\
\text { (day) }\end{array}$} & \multicolumn{2}{|c|}{ Normalized mass losses $\left.\mathbf{~} \mathbf{g} / \mathbf{m}^{\mathbf{2}}\right)$} \\
\cline { 2 - 4 } & 7 & Deionized water & Mineralized water \\
\cline { 2 - 4 } & 14 & 0,073 & 0,985 \\
\hline \multirow{2}{*}{$\mathrm{Na}$} & 7 & 0,122 & 1,849 \\
\cline { 2 - 4 } & 14 & 21,13 & 14,51 \\
\hline $\mathrm{Li}$ & 7 & 29,12 & 19,69 \\
\cline { 2 - 4 } & 14 & 12,59 & 8,45 \\
\hline \multirow{3}{*}{$\mathrm{B}$} & 7 & 26,01 & 17,52 \\
\cline { 2 - 4 } & 14 & 20,21 & 13,92 \\
\hline $\mathrm{Si}$ & 7 & 32,63 & 23,51 \\
\cline { 2 - 4 } & 14 & 10,68 & 7,36 \\
\hline
\end{tabular}


Table 13. Losses of MOX-20/40 glass element mass for 3, 7,14 and 28 days in deionized and mineralized water in static, dynamic conditions of leaching at $90{ }^{\circ} \mathrm{C}(\mathrm{wt} \%)$.

\begin{tabular}{|l|l|l|l|l|l|l|}
\hline \multirow{4}{*}{ Time } & \multicolumn{2}{|c|}{ MOX-10/40 } & \multicolumn{3}{c|}{ MOX-20/40 } \\
\cline { 2 - 7 } & \multicolumn{2}{|c|}{ MCC-1 } & \multicolumn{2}{c|}{ MCC-1 } & \multicolumn{2}{c|}{ MCC-5 } \\
\cline { 2 - 7 } & $\begin{array}{c}\text { Deionized } \\
\text { water }\end{array}$ & $\begin{array}{c}\text { Mineralized } \\
\text { water }\end{array}$ & $\begin{array}{c}\text { Deionized } \\
\text { water }\end{array}$ & $\begin{array}{c}\text { Mineralized } \\
\text { water }\end{array}$ & $\begin{array}{c}\text { Deionized } \\
\text { water }\end{array}$ & $\begin{array}{c}\text { Mineralized } \\
\text { water }\end{array}$ \\
\hline 3 & 0,103 & 0,093 & 0,095 & 0,099 & & \\
\hline 7 & 0,190 & 0,091 & 0,133 & 0,123 & & \\
\hline 14 & 0,222 & 0,154 & 0,186 & 0,124 & 0,420 & 0,270 \\
\hline 28 & 0,249 & 0,165 & 0,277 & 0,145 & & \\
\hline
\end{tabular}

\section{Conclusion}

We have shown the principal possibility of vitrification of the mix of MOX fuel and plutonium-containing MCC sludges and the development of technological solutions and synthesis conditions for final immobilized forms.

\section{References}

1. Aloy A.S., Belov V.Z., Iskhakova O.A., Trofimenko A.V., 1999, "Characterization of Borosilicate Composition Developed for the Immobilization of Plutonium-containing Sludges at MCC," A Review of LLNL Contract Work. Proceedings of the Meeting for Coordination and Review of Work Held in St. Petersburg, Russia, Lawrence Livermore National Laboratory, UCRL-ID-138361, November 1-4, pp. 153-166.

2. B.A. Shakhmatkin, N.M. Vedishcheva, A.S. Aloy, M.J. Plodinec, S.L. Marra. Materials Research Society Symposium Proceedings. Scientific Basis for Nuclear Waste Management XX. Vol. 465, Boston, Massachusetts, US, December 2-6, 1996. pp. 181-187.

3. Aloy A.S., Iskhakova O.A., Koltsova T.I., Kusnetsov B.S., Trofimenko A.V., 2000. "Development and Characterization of Borosilicate Glasses for Immobilization of Plutonium-containing Alkaline Sludges" MRS 2000, $24^{\text {th }}$ International Symposium on The Scientific Basis for Nuclear Waste Management, August 27-31, Sydney, Australia.

4. A.S. Aloy, B.S. Kuznetsov, E.Yu. Ivanov, A.V. Trofimenko, Initial Data for the Engineering Feasibility Study on Pu-Bearing Radioactive Waste Immobilization at MCC in [2], p.29-35.

5. Patent (USSR) \# 477945 CCCP, MKI 02 in 1182 B010 13/02. "Method of water purification". L.N. Moskvin, N.N. Kalinin, P.A. Godon. Discovery and invention. 1974. №27

6. L.J. Jardine, T.A. Gupalo, A.A. Tretyakov, S.A. Manakov, "Comparison of the immobilizing properties of Russian phosphate and borosilicate glasses ensuring the safe options for long term underground storage and final disposal of weapon plutonium production waste," WM'00 Conference, February 27March 2, 2000, Tucson, AZ.

7. Kamizono H. "Durability of HLW glass in groundwater," J. Amer. Chem. Soc. 1991, Vol. 74, №9 p 2234-2241.

8. Wicks G.G, Robnett B.M., Rankin W.D., Chemical durability of glass containing SRP-wasteleachability characteristics, protective layer formation, and repository system interactions, Ibid. p. 1524.

9. A.S. Aloy, O.A. Iskhakova, A.V. Trofimenko, V.S. Belov, S.I. Rovnyi, G.M Medvedev. L.J. Jardine, "Characterization of Borosilicate Compositions for Immobilization of Pu-Containing Sludges," 8th 
International Conference on Radioactive Waste Management and Environmental Remediation, September 30-October 4, 2001, Bruges, Belgium

10. Radiochemistry and chemistry of nuclear processes (Editor. A.N. Murin), 1960, pp.510. (Rus.)

11. Wicks G.G., Mosley W.C., Whitkop P.G., Saturday K.A., "Durability of simulated waste glass-effects of pressure and formation of surface layers," J. Non-Cryst. Solids. 1982. Vol. 49 p. 413-428.

12. Wicks G.G., Robnett B.M., Rankin W.D. "Chemical durability of glass containing SRP-Wasteleachability characteristics, protective layer formation, and repository system interactions," lbid. pp. $15-24$. 


\title{
Immobilization of Am-containing Waste: Status of Implementing the Work for Agreement B514481
}

\author{
G. B. Borisov, O. A. Mansourov, V. F. Gorbunov, V. S. Ilyashenko, VNIINM; \\ A. A. Minaev, D. G. Kuznetsov, IPC RAS
}

\begin{abstract}
Disposition of excess weapons-grade plutonium starts with the conversion of plutonium metal into plutonium dioxide acceptable for MOX fuel fabrication. At present, a few conversion options are under assessment: using aqueous technologies, for instance oxalate or ammonia precipitation, and pyroelectrochemical treatment. Conversion operations will inevitably result in the generation of substantial amounts of wastes, of them the most hazardous are highly radioactive Am-containing residues. The given paper discusses experimental tests started and in progress for the development of processes and matrices for immobilizing Am-containing wastes implemented under the framework of Agreement B514481 with Lawrence Livermore National Laboratory (LLNL).
\end{abstract}

\section{Introduction}

Selection of the method of immobilization and of a matrix for Am-containing wastes from conversion of metallic plutonium was based on the following premises.

At PA "Mayak", industrial-scale ceramic melters, EP-500, for vitrifying liquid high-radioactive wastes into sodium-alumina-phosphate glass have been exploited for many years. The glass may contain up to 25-27 wt $\% \mathrm{Al}_{2} \mathrm{O}_{3}$. Phosphate glass incorporates six times more alumina. It follows from this fact that the Amcontaining wastes could be incorporated into phosphate glasses. Due to the similarity of chemical compositions, both the liquid Am-containing wastes from conversion operations and high-level wastes from spent nuclear fuel reprocessing could be jointly solidified using the ceramic melter.

According to Minatom State Specialized Design Institute (GSPI) calculations carried out in the engineering and feasibility study, the conversion of $2.7 \mathrm{t}$ of weapons-grade plutonium by means of oxalate precipitation generated about $2500 \mathrm{~kg}$ of Am-containing wastes. The wastes are in the form of rafinates and washing solutions from plutonium dissolution and purification operations. The total volume of Amcontaining solutions is 50 liters per $1 \mathrm{~kg} \mathrm{Pu}$. It is composed of the following constituents $(\mathrm{g} / \mathrm{l})$ : $\mathrm{Al}-150, \mathrm{HF}$ $-4, \mathrm{HNO}_{3}-190-250, \mathrm{Am}-50-200 \mathrm{mg} / \mathrm{l}, \mathrm{Pu}-2-10 \mathrm{mg} / \mathrm{l}$. After calcination of the wastes at $500^{\circ} \mathrm{C}$, the residues generated are as follows $(\mathrm{kg}): \mathrm{Al}_{2} \mathrm{O}_{3}-2495 ; \mathrm{F}^{-}-332 ; \mathrm{PuO}_{2}-0.3 ; \mathrm{AmO}_{2}-23$. In percent, the residues have the following composition (wt\%): $\mathrm{Al}_{2} \mathrm{O}_{3}-87,54 ; \mathrm{F}^{-}-11,65$ (in the form $\mathrm{AlF}_{3}$ ); $\mathrm{PuO}_{2}-0,01$; $\mathrm{AmO}_{2}-0,8$.

As it is clear from the data presented, the concentration of $\mathrm{PuO}_{2}$ in the wastes is minor; therefore, in the development of an immobilization technique, it is necessary to consider chiefly the incorporation of $\mathrm{AmO}_{2}$. The problem of gallium in the wastes from conversion operations (about $1 \mathrm{wt} \%$ in plutonium) could also be resolved. Its properties are similar to those of aluminum and therefore could be united with americium waste streams.

In Russia at PA "Mayak", americium recovered from spent nuclear fuel is stored in specially-designed tanks and, to date, there is no technology for its stabilization. 
At the Savanna River industrial site in the US, americium and curium are included into the $\mathrm{La}_{2} \mathrm{O}_{3}-\mathrm{B}_{2} \mathrm{O}_{3}$ $\mathrm{SiO}_{2}$ system following transportation and possible usage at the Oak Ridge site. However, this matrix composition cannot be used properly for immobilizing the Am-containing wastes from oxalate precipitation conversion operations due to a high melting temperature (up to $1500^{\circ} \mathrm{C}$ ) of the glass and difficulty of incorporating large amounts of alumina. Because the wastes contain almost $90 \mathrm{wt} \% \mathrm{Al}_{2} \mathrm{O}_{3}$, it would be reasonable to use a three-component system based on $\mathrm{Na}_{2} \mathrm{O}-\mathrm{P}_{2} \mathrm{O}_{5}-\mathrm{Al}_{2} \mathrm{O}_{3}$. The Am-containing wastes are in solutions; therefore, the use of a liquid flux in the form of phosphoric acid is preferable. Besides, the application of this matrix would permit the use of industrial-scale equipment, for example, EP-type ceramic melters.

The presence of fugative $\mathrm{AlF}_{3}$ in the wastes also limits the selection of the matrix. Vapor pressures of $\mathrm{AlF}_{3}$ at $900^{\circ} \mathrm{C}$ is $0.6 \mathrm{~mm}$.m.c.; at $1000^{\circ} \mathrm{C}-4 \mathrm{~mm}$.m.c.; and at $1100^{\circ} \mathrm{C}-45 \mathrm{~mm}$.m.c. As it is obvious, the usage of high-temperature techniques would result in fugacity of $\mathrm{AlF}_{3}$ while the glass is melting.

Based on the data afore mentioned, it is advisable to select a glass matrix that contains at least $20 \mathrm{wt} \%$ $\mathrm{Al}_{2} \mathrm{O}_{3}$, so as not to exceed a melting temperature of $1000^{\circ} \mathrm{C}$.

Another challenging option for immobilizing the Am-containing wastes could be, from our point of view, a low-temperature ceramic material. It is proposed to use industrially-produced alumina-chrome-phosphate binder (ACPB) or alumina-boron-phosphate binder (ABPB). The composition of the binders is as follows, wt\%: $\mathrm{Al}_{2} \mathrm{O}_{3}-6,5-9,0 ; \mathrm{Cr}_{2} \mathrm{O}_{3}-3,5-4,5 ; \mathrm{P}_{2} \mathrm{O}_{5}-35-39 ; \mathrm{H}_{2} \mathrm{O}-47-55$, and $\mathrm{Al}_{2} \mathrm{O}_{3}-8,0-9,0 ; \mathrm{B}_{2} \mathrm{O}_{3}-1,3-1,2$; $\mathrm{P}_{2} \mathrm{O}_{5}-36-39 ; \mathrm{H}_{2} \mathrm{O}-47-55$, respectively. The higher rate of incorporation, the possibility of using microwave heating for treatment of a americium powder fed for vitrification, in junction with the good quality of the compound obtained make these materials good prospects for immobilizing the Am wastes.

\section{Experimental Tests}

The calculated content of $\mathrm{AmO}_{2}$ in the glass is $0.2 \mathrm{wt} \%$. Such a low concentration would provide for less radiation and thermal charge on the glass, while the chemical stability is achieved by $\mathrm{Al}_{2} \mathrm{O}_{3}$.

Preliminarily, the procedure of the synthesis of the Am-containing wastes was tested by simulators. Lanthanum oxide was selected for this purpose due to its analogue crystallographic and chemical properties.

The glasses were synthesized from the glass frit and relevant lanthanum and americium-containing solutions. The last ones were prepared going from the content of $\mathrm{La}_{2} \mathrm{O}_{3}$ and $\mathrm{AmO}_{2}$ in the glass at the concentrations of 0.1 and $0.2 \mathrm{wt} \% . \mathrm{La}_{2} \mathrm{O}_{3}$ powder was dissolved in 6-7 $\mathrm{M} \mathrm{HNO}_{3}$ obtaining a solution of 5$10 \mathrm{mg} / \mathrm{l}$.

To prepare the Am-containing solutions, ${ }^{241} \mathrm{Am}$ isotope was used. A portion of $\mathrm{AmO}_{2}$ in the amount of $160 \mathrm{mg}$ was taken from the ampoule and dissolved in 6-7 $\mathrm{M} \mathrm{HNO}_{3}$. The obtained nitric-acid solution of americium was filtered and washed off by $0.5 \mathrm{M} \mathrm{HNO}_{3}$ and evaporated. The content of ${ }^{241} \mathrm{Am}$ in the solution was determined using gamma-spectrometric analysis.

The glass was melted from preliminarily prepared glass frit by adding lanthanum nitrate at two concentrations: 5 and $10 \mathrm{mg} / \mathrm{ml}$ ( 0.1 and $0.2 \mathrm{wt} \%$, respectively). To establish the influence of lanthanum distribution upon the volume of the glass frit powder, one experiment was carried out without blending.

The glasses were synthesized in corundum crucibles at $900-950^{\circ} \mathrm{C}$ for one hour. The velocity of heating in the furnace was $10-15 \mathrm{grad} / \mathrm{min}$. After holding the samples, they were taken off and cooled to $750^{\circ} \mathrm{C}$. 
As a result, transparent glasses with no crystallization signs were obtained (Fig. 1). The homogeneity of distribution of major components was determined by the atomic-adsorption method.

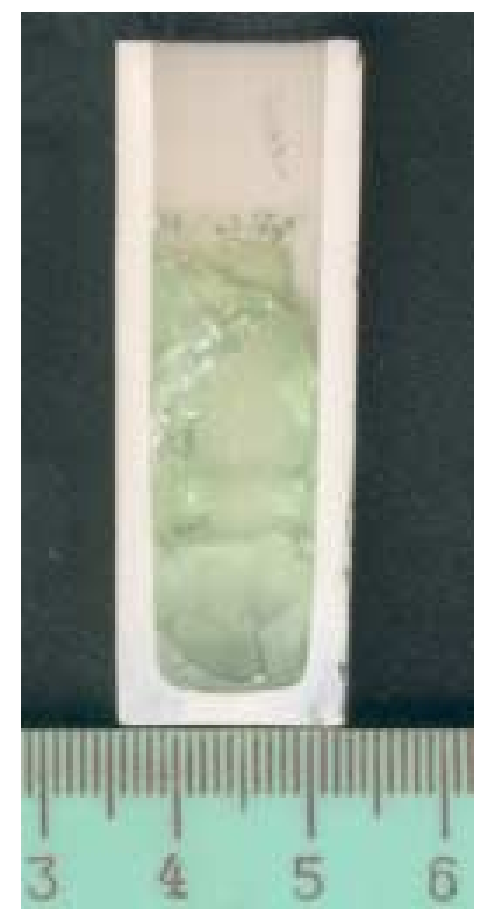

\section{Fig. 1. Image of simulated La-containing glass.}

Am-containing glasses were melted in accordance with the procedure tested while synthesizing simulated glasses. The portion of the glass frit ( $10 \mathrm{~g}$ ) was put into the glass and dissolved by $2 \mathrm{ml} \mathrm{HNO}_{3}$, containing $10 \mathrm{mg} / \mathrm{ml}^{241} \mathrm{Am}$. Then, the obtained product was mixed to get a powder-type state and dried at $100-$ $150^{\circ} \mathrm{C}$ for $0.5-1$ hour. The glasses were synthesized in corundum crucibles, holding them for a duration of 1 hour, at about $900-950^{\circ} \mathrm{C}$. The velocity of heating the samples was $10-15 \mathrm{grad} / \mathrm{min}$. As a result, transparent glasses were obtained with the following composition (wt\%): $\mathrm{Na}_{2} \mathrm{O}-26 ; \mathrm{P}_{2} \mathrm{O}_{5}-50 ; \mathrm{Al}_{2} \mathrm{O}_{3}-$ 20; $\mathrm{AlF}_{3}-4 ; \mathrm{AmO}_{2}$ (over 100\%) - 0.2.

The distribution of ${ }^{241} \mathrm{Am}$ upon the height of the glass frit and glass batch was measured by gammaspectrometry (Fig. 2).

One can see from Fig. 2, in the batches of $5 \mathrm{~cm}$ distance, americium is distributed evenly and then its concentration goes up slightly. Besides, some contamination of the crucible walls occurred while filling up with the glass frit. It is obvious from the data that the crucibles are filled up to $8 \mathrm{~cm}$. From the figure one can see that a glass melt takes about $3 \mathrm{~cm}$ and americium is distributed there equally. 


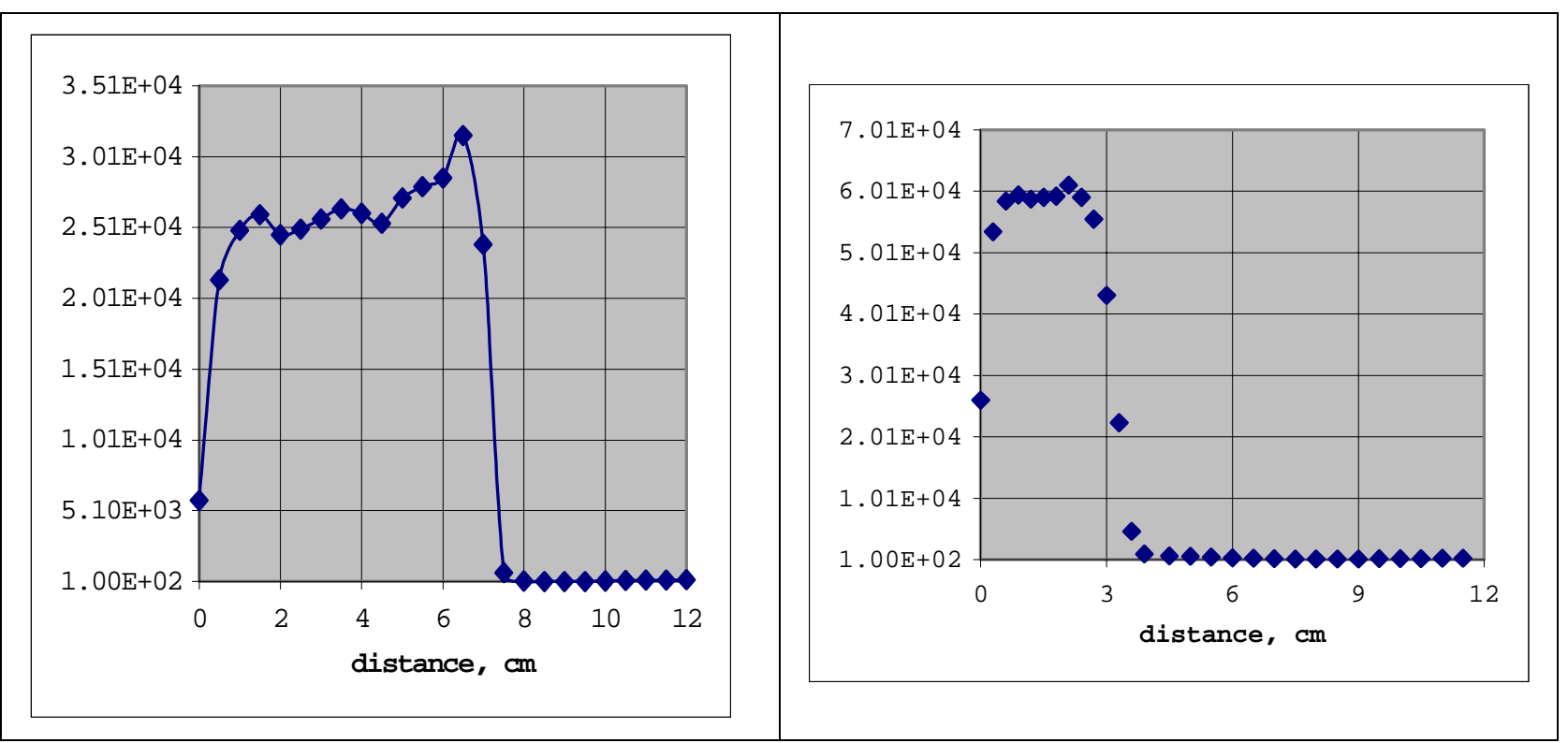

Fig. 2. Americium distribution in the glass frit (1) and melt (2).

The synthesis and investigation of vitreous matrices are to be continued. We to perform experiments for the inclusion of americium into borophosphate glass, determine the chemical stability of materials obtained at 20 and $900^{\circ} \mathrm{C}$, and make XRD and SEM/EDS analyses.

In addition to the research on phosphate glasses, we have initiated some work connected with the development of low-temperature ceramics. First, simulated specimens of ACPB of the following composition were synthesized (wt\%): $\mathrm{Al}_{2} \mathrm{O}_{3}-51,3 ; \mathrm{AlF}_{3}-6,0 ; \mathrm{Nd}_{2} \mathrm{O}_{3}-0,7 ; \mathrm{ACPB}-42$, under the following conditions: treatment temperature $-700^{\circ} \mathrm{C}$, density - up to $2 \mathrm{~g} / \mathrm{cm}^{3}$, open porosity - up to $30 \%$., hydrolytic stability upon aluminum $-2.7^{*} 10^{-6} \mathrm{~g} / \mathrm{cm}^{2 *}$ day, strength for pressure $-65 \mathrm{MPa}$.

\section{Conclusions}

1. Simulated La-containing phosphate glasses were obtained (wt\%): $\mathrm{Na}_{2} \mathrm{O}-26 ; \mathrm{P}_{2} \mathrm{O}_{5}-50 ; \mathrm{Al}_{2} \mathrm{O}_{3}-20$; $\mathrm{AlF}_{3}-4 ; \mathrm{La}_{2} \mathrm{O}_{3}$ (over $100 \%$ ) $-0.1,0.2$. The glasses were melted in corundum crucibles at $900-950^{\circ} \mathrm{C}$. The material is without signs of crystallization. Research was started to define the homogeneity of the distribution of basic components using atomic-adsorption and SEM/EDS analyses.

2. Based on the experiments with the simulated glasses, Am-containing phosphate matrices were synthesized of the following composition: $\mathrm{Na}_{2} \mathrm{O}-26 ; \mathrm{P}_{2} \mathrm{O}_{5}-50 ; \mathrm{Al}_{2} \mathrm{O}_{3}-20 ; \mathrm{AlF}_{3}-4 ; \mathrm{AmO}_{2}$ (over $100 \%)-0.2$. The glasses were melted in corundum crucibles at $900-950^{\circ} \mathrm{C}$. The material is without crystallization signs. Research has started to define the homogeneity of distribution of basic components using gamma-spectrometric and SEM/EDS analyses. At present, the work is ongoing, and, in addition, Am-containing borophosphate glass will be synthesized and characterized.

3. Initial investigation activities have been started to produce low-temperature ceramics based on aluminum phosphates. The compositions are as follows (wt\%): $\mathrm{Al}_{2} \mathrm{O}_{3}-51,3 ; \mathrm{AlF}_{3}-6,0 ; \mathrm{Nd}_{2} \mathrm{O}_{3}-0,7$; binders -42 . Treatment temperature is $-700^{\circ} \mathrm{C}$ or higher. It is anticipated to proceed with this research using other treatment regimes and to characterize them more in detail. 


\title{
Melting Diagrams of High-temperature Insoluble Compounds as Matrices for Immobilization of REE and Am Radionuclides (B514481)
}

\author{
D. G. Kuznetsov, A. A. Minaev, IPC RAS; G. B. Borisov, O. A. Mansourov, VNIINM
}

\section{Summary}

This activity is dedicated to analysis of the diagrams of fusibility of high melting compounds of rare earth elements (REE) that are difficult to dissolve in water, e.g., chromite, niobates, aluminates, and their mixtures. The data on dependence of compact article density on temperature are given, also. The melting point of different compounds and their mixtures is a relevant parameter, because the temperature at which the powders of these compounds and their mixtures is compacted depends on melting point. The obtained data can be utilized at mining of immobilization methods of the radioactive REE-fraction of the $\mathrm{RW}$, and also Am-inclusive waste (as an americium clone of many REE). In addition, these data are of definite scientific interest for analysis of REE and americium compounds.

The series of Am and REE compounds (including neodymium as representative of REE) was selected and analyzed from the literature. The formulas and main properties of some of the best prospective compounds of $\mathrm{Am}$ and $\mathrm{Nd}$ are given in Table 1.

Table 1. Some physicochemical properties of americium and neodymium compounds.

\begin{tabular}{|l|l|l|c|c|l|l|}
\hline № & \multicolumn{1}{|c|}{ Compound } & \multicolumn{1}{|c|}{ Formula } & $\mathbf{T}_{\mathbf{m}},{ }^{\circ} \mathbf{C}$ & $\boldsymbol{\rho}_{\text {roen. }} \mathbf{g} / \mathbf{c m}^{3}$ & Content wt\% & $\begin{array}{c}\text { Dissolubility } \\
\text { in water }\end{array}$ \\
\hline 1 & Am monoaluminate & $\mathrm{AmAlO}_{3}$ & & 9,40 & $\mathrm{Am}, 76,42$ & \\
\hline 2 & $\mathrm{Nd}$ oxide & $\mathrm{Nd}_{2} \mathrm{O}_{3}$ & 1900 & 7,24 & $\mathrm{Nd}, 85,75$ & hydrolyzed \\
\hline 3 & $\mathrm{Nd}$ orthoniobate & $\mathrm{NdNbO}_{4}$ & & & $\mathrm{Nd}, 47,9$ & indissoluble \\
\hline 4 & $\mathrm{Nd}$ niobate & $3 \mathrm{Nd}_{2} \mathrm{O}_{3} \cdot \mathrm{Nb}_{2} \mathrm{O}_{5}$ & & & $\mathrm{Nd}, 67,86$ & indissoluble \\
\hline 5 & $\mathrm{Nd}$ monoaluminate & $\mathrm{NdAlO}_{3}$ & 1947 & 6,99 & $\mathrm{Nd}, 65,81$ & indissoluble \\
\hline 6 & Nd chromite & $\mathrm{NdCrO}_{3}$ & 2417 & 6,91 & $\mathrm{Nd}, 59,06$ & indissoluble \\
\hline
\end{tabular}

Niobates, chromite, and aluminate of $\mathrm{Nd}$ ( $\mathrm{Nd}$ is selected as representative of $\mathrm{REE}$ ) are best prospects among REE compounds for this research. All these compounds are similar in their properties: high melting temperature- $1900-2400^{\circ} \mathrm{C}$, large specific weight $-6-7 \mathrm{~g} / \mathrm{cm}^{3}$, percentage of neodymium - 50-60 wt $\%$, slightly soluble in water, and potentially are suitable (except for $\mathrm{Nd}_{2} \mathrm{O}_{3}$ ) as the matrix material for fixing radioactive $R E E$.

Synthesizing neodymium niobates from $\mathrm{Nd}$ and $\mathrm{Nb}$ oxide powders was studied. The data are shown in Fig. 1. In order to synthesize $100 \%$ of the oxides, calcination at $\sim 1500^{\circ} \mathrm{C}$ is required.

A lower synthesizing temperature of neodymium niobates was reached due to a liquid-phase method of synthesizing based on joint drying and calcination of neodymium and niobium solutions mixtures (i.e., mixtures in which the initial components are blended at close to the molecular level). To create such mixtures, it is necessary to match the solutions of neodymium and niobium compounds. The difficulty of such a combination is that in an alkaline condition, the neodymium hydroxide precipitates, and in an 
acidic condition, niobium acid, as a rule, settles out. It was necessary to find such a niobium compound, which would be stable in acidic conditions. Most eligible Nb inclusive compounds have appeared as a malate water-soluble complex $\mathrm{Nb}$ with hydroxy-butanedionic acid.

In Fig. 2, the plot of neodymium niobates synthesized by drying and calcinating of the mated solutions of $\mathrm{Nd}\left(\mathrm{NO}_{3}\right)_{3}$ and malate complexes of $\mathrm{Nb}$ vs. synthesizing temperature is shown, $\mathrm{t}=4$ hours. Curve $\mathrm{I}-$ $\mathrm{Nd}_{2} \mathrm{O}_{3}: \mathrm{Nb}_{2} \mathrm{O}_{5}=1: 1$; curve $2--\mathrm{Nd}_{2} \mathrm{O}_{3}: \mathrm{Nb}_{2} \mathrm{O}_{5}=3: 1$

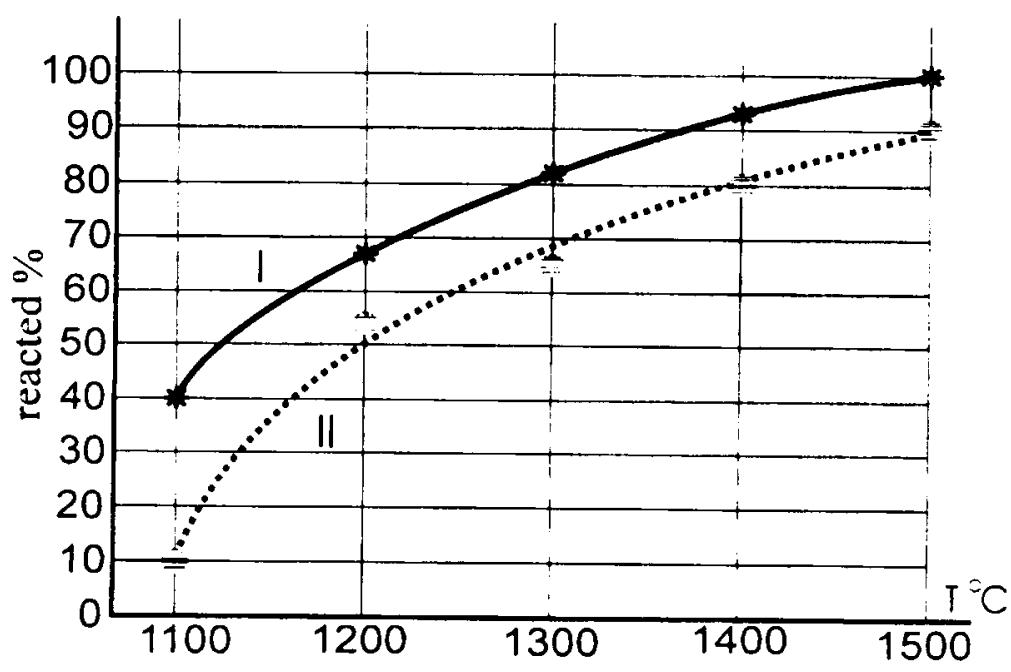

Fig. 1. Plots of neodymium niobates synthesizing from a mixture of oxides vs. synthesizing temperature, $t=4$ hours. Curve $\mathrm{I}-\mathrm{Nd}_{2} \mathrm{O}_{3}: \mathrm{Nb}_{2} \mathrm{O}_{5}=1: 1$; curve $2-\mathrm{Nd}_{2} \mathrm{O}_{3}: \mathrm{Nb}_{2} \mathrm{O}_{5}=3: 1$.

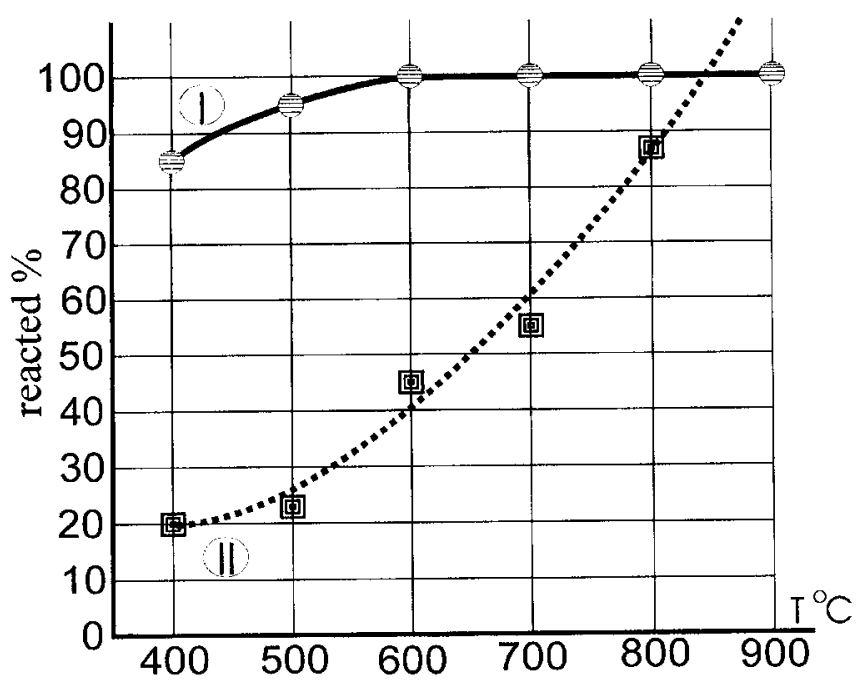

Fig. 2. Plots of neodymium niobates synthesizing by drying and calcination of the combined solutions $\mathrm{Nd}\left(\mathrm{NO}_{3}\right)_{3}$ and malate $\mathrm{Nb}$ complexes vs. temperature of synthesizing, $\mathrm{t}=4$ hours. Curve I - $\mathrm{Nd}_{2} \mathrm{O}_{3}: \mathrm{Nb}_{2} \mathrm{O}_{5}=1: 1$; curve $2-\mathrm{Nd}_{2} \mathrm{O}_{3}: \mathrm{Nb}_{2} \mathrm{O}_{5}=3: 1$. 
Similarly, we studied the liquid-phase synthesis of neodymium chromite, aluminate, and niobate powders and their different mixtures by drying of mated solutions of neodymium and chromium nitrates and a complex compound of niobium with hydroxy-butanedionic acid, with subsequent calcination of the powders. It was shown that drying of solutions and calcination of powders can be mated in one vehicle (e.g., in the atomizer-radiation drying room, such as "Beam", or in the plasmochemical reactor) using finely divided sub-synthesizing powders that are well pressed and cake.

After identifying the optimal conditions for synthesis of neodymium chromite, aluminates, and niobates, we studied the properties of compounds, and also the properties of their mixtures.

\section{Research of Fusibility Diagrams of Aluminate-niobate and Chromite-niobate Systems on the Basis of Nd}

From the technological point of view, one of the most important properties of powders based on $\mathrm{Nd}$ is the initial melting temperature because the sintering temperature and condition of manufacturing of compact articles depends on it. Therefore, we studied the fusibility diagrams of aluminate-niobate and chromiteniobate systems on the basis of $\mathrm{Nd}$.

The fusibility diagrams were studied by accepted methods of high temperature microscopy and isothermal exposure. Thus, the samples were prepared by direct synthesizing from solutions in order to avoid diffusive difficulties and to facilitate achieving equilibrium in the system.

The solutions, with initial components, were dried under an infrared (IR) lamp, the calcinates were heated at $1200^{\circ} \mathrm{C}$ for one hour, and then tablets were made by applying as flow bundle $3 \mathrm{wt} \%$ of paraffin at a pressure of $4 \mathrm{t} / \mathrm{cm}^{2}$. The tablets were sintered in air at $1450^{\circ} \mathrm{C}$. Their density was determined by Archimedes' principle, and on the geometrical sizes, and also loss of weight was determined at the time of sintering. For construction of the fusibility diagram, samples were subjected to isothermal endurance in a vacuum oven over a temperature range of $1700-2150^{\circ} \mathrm{C}$ at intervals of $50^{\circ} \mathrm{C}$. Tantalum and iridium were used as a substrate. The temperature was measured by the absorption pyrometer with accuracy $\pm 25^{\circ} \mathrm{C}$.

The experimental data for the fusibility diagrams (with iridium substrate) are given in Fig. 3 (a)-(d). The system in Fig. 3 (a) is eutectic (eutectic point $\sim 45 \mathrm{wt} \%$ of $\mathrm{NdAlO}_{3}$ ) with presence of the perytectic (perytectic point $\sim 12 \mathrm{wt} \%$ of $\mathrm{NdAlO}_{3}$ ). The sharp fracture of the liquidus curve and loss of weight curve depending on the composition shown in Fig. 4 testifies to the presence of the perytectic.

The systems in Fig. 3 (b) and (d) are eutectic with the presence of a chemical compound. In the system $\mathrm{NdAlO}_{3}-\mathrm{Nd}_{3} \mathrm{NbO}_{7}$, the chemical compound corresponds to the contents of $\mathrm{Nd}_{3} \mathrm{NbO}_{7}-30$ wt\%, and eutectic with the contents of $\mathrm{Nd}_{3} \mathrm{NbO}_{7} \sim 18 \mathrm{wt} \%$ and $\sim 87 \mathrm{wt} \%$. In the system $\mathrm{NdCrO}_{3}-\mathrm{Nd}_{3} \mathrm{NbO}_{7}$ in Fig. 3 (d), the chemical compound corresponds, probably, to the contents of $\mathrm{Nd}_{3} \mathrm{NbO}_{7} \sim 50 \mathrm{w} \%$, and eutectic with the contents of $\mathrm{Nd}_{3} \mathrm{NbO}_{7} \sim 37 \mathrm{w} \%$ and $\sim 75 \mathrm{w} \%$. The eutectic line in this system is at $2050^{\circ} \mathrm{C}$.

In Fig. 4, the research data on weight loss are shown depending on the composition for system of $\mathrm{NdAlO}_{3}$ - $\mathrm{NdNbO}_{4}$ at calcinated temperature $1450^{\circ} \mathrm{C}$ over 4 hours. It was shown that the loss of weight in a mixture of up to $90 \%$ of $\mathrm{NdNbO}_{4}$ was $\sim 0 \%$, and for $\mathrm{NdNbO}_{4}$, less than $2 \%$.

In addition, research was conducted on manufacturing of compact units by cold pressing with subsequent sintering, and also by ardent pressing. The principal technological scheme of manufacturing of ceramic units is given in Fig. 6 . 


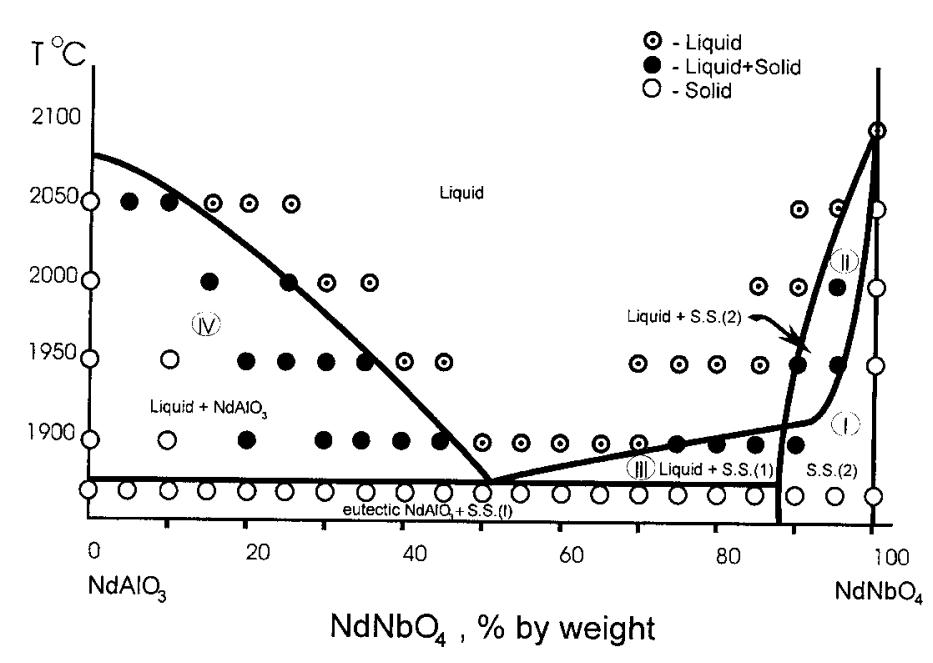

(a)

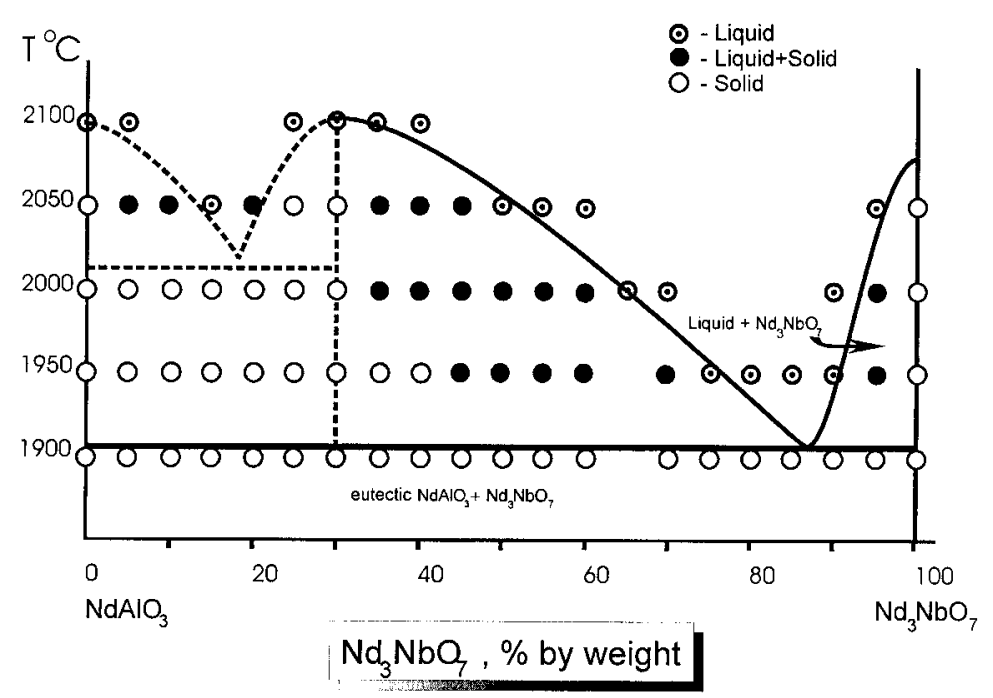

(b)

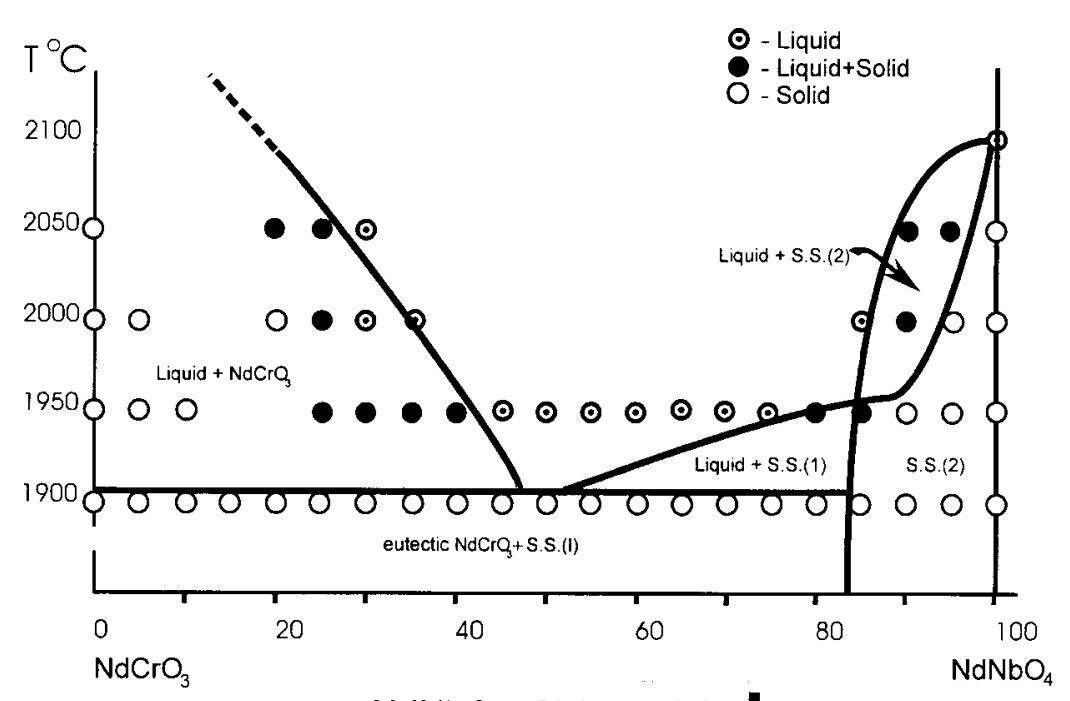

$\mathrm{NdNbO}_{4}, \%$ by weight

(c)

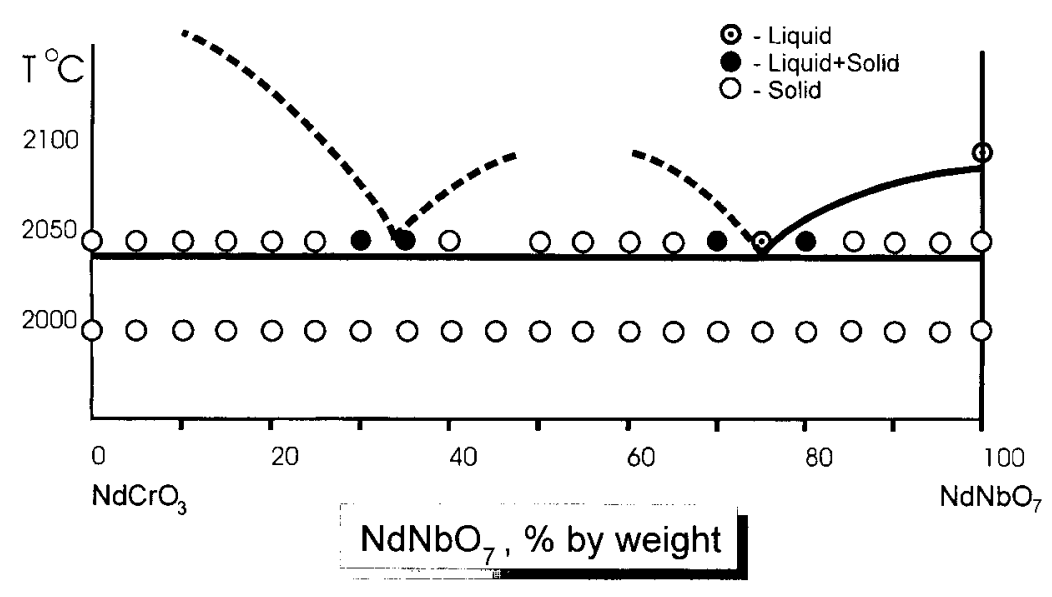

(d)

Fig. 3. The diagrams of fusibility of systems: (a) $\mathrm{NdAlO}_{3}-\mathrm{NdNbO}_{4}$, (b) $\mathrm{NdAlO}_{3}-\mathrm{Nd}_{3} \mathrm{NbO}_{7}$, (c) $\mathrm{NdCrO}_{3}-\mathrm{NdNbO}_{4}$, (d) $\mathrm{NdCrO}_{3}-\mathrm{Nd}_{3} \mathrm{NbO}_{7}($ on the iridium substrate). 


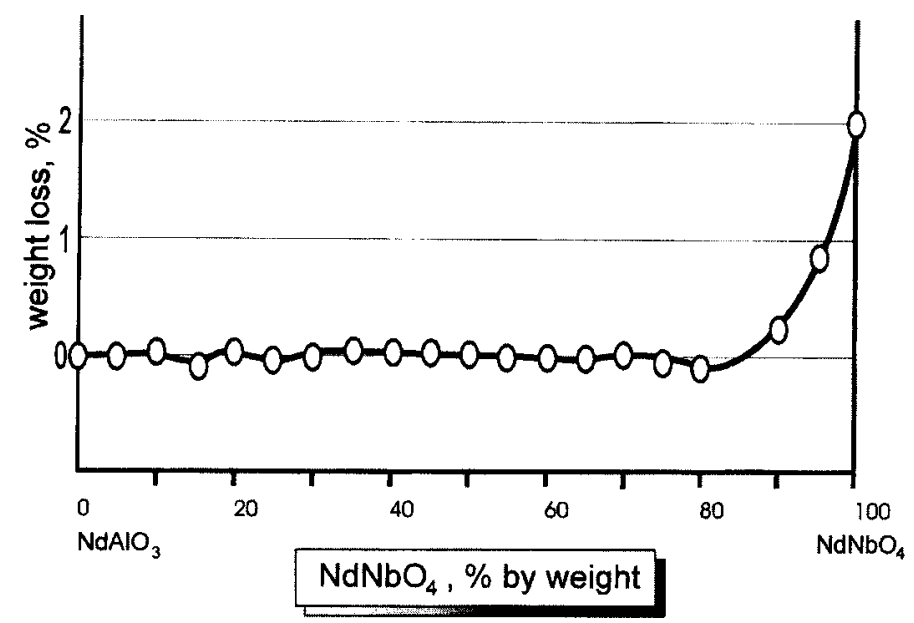

Fig. 4. Loss of weight depending on the composition for the system $\mathrm{NdAlO}_{3}-\mathrm{NdNbO}_{4}$ at calcinated temperature $1450^{\circ} \mathrm{C}$ for 4 hours.

As indicated above, the analysis of the fusibility diagrams was made for competent selection of compositions with the purpose of obtaining articles of maximum density. Clean components are melted with difficulty, and the articles from them have low density after sintering. In Fig. 5 (a) and (b), the dependence of density on the composition for above-stated systems are given. In all cases, the pressure was $4 \mathrm{t} / \mathrm{cm}^{2}$ ( $3 \mathrm{wt} \%$ of paraffin as connection), and the sintering was done at $1450^{\circ} \mathrm{C}, \mathrm{t}=3$ hours.

From Fig. 5, we see that the best density of the compact articles is reached near the eutectic points, and decreases sharply near to compounds. The selection of the optimal composition was made at the rate of the best content of neodymium oxide in $1 \mathrm{~cm}^{3}$ of the material. In the system, $\mathrm{NdAlO}_{3}-\mathrm{NdNbO}_{4}$ [Fig. 5(a)], the composition including 25-50 $\mathrm{w} \%$ of $\mathrm{NdNbO}_{4}$ is optimal, and in the composition $\mathrm{NdAlO}_{3}-\mathrm{Nd}_{3} \mathrm{NbO}_{7}$ [Fig. 5(b)], it is $90 \mathrm{w} \%$ of $\mathrm{Nd}_{3} \mathrm{NbO}_{7}$. Besides clean $\mathrm{Nd}_{3} \mathrm{NbO}_{7}$ is not heat stable; it does not maintain even one air temperature change at $1450^{\circ}-25^{\circ} \mathrm{C}$. Attachment in $\mathrm{Nd}_{3} \mathrm{NbO}_{7} 10 \mathrm{w} \%$ of $\mathrm{NdAlO}_{3}$ increases the thermostability of the article sharply. Owing to the high temperature melting point of the $\mathrm{NdCrO}_{3}-\mathrm{NdNbO}_{4}$ and $\mathrm{NdCrO}_{3}-\mathrm{Nd}_{3} \mathrm{NbO}_{7}$ systems, their compositions can be recommended for production of matrix units. However, special mining technology is required to obtain dense articles from the already synthesized powders in this case. To create such technology is more difficult, owing to the high sintering temperature of such compositions. Thus, as a matter of record, it is possible to recommend optimal compositions for the matrix material on the basis of chemical, thermal, and processing properties that satisfy all the requirements. One advisable composition contains $90 \mathrm{wt} \%$ of $\mathrm{Nd}_{3} \mathrm{NbO}_{7}$ and $10 \mathrm{wt} \% \mathrm{NdAlO}_{3}$.

Isostructural compounds of chromites and aluminates of a number of actinides and lanthanides, and also isostructural compounds of $\mathrm{Me}_{2} \mathrm{O}_{3} \cdot 3 \mathrm{Nb}_{2} \mathrm{O}_{5}$, where $\mathrm{Me}=\mathrm{Am}$, La-Nd are described in [1].

In our view, practically all radionuclides of HLW actinides and lanthanides can be included in any ratio and in great amounts up to $40-60 \mathrm{wt} \%$ in mixed chromite-niobate (aluminate) matrices on the basis of REE. This is certainly more than is permissible (10-20\%) for other parameters; for example, heat release, self-irradiation, etc. 


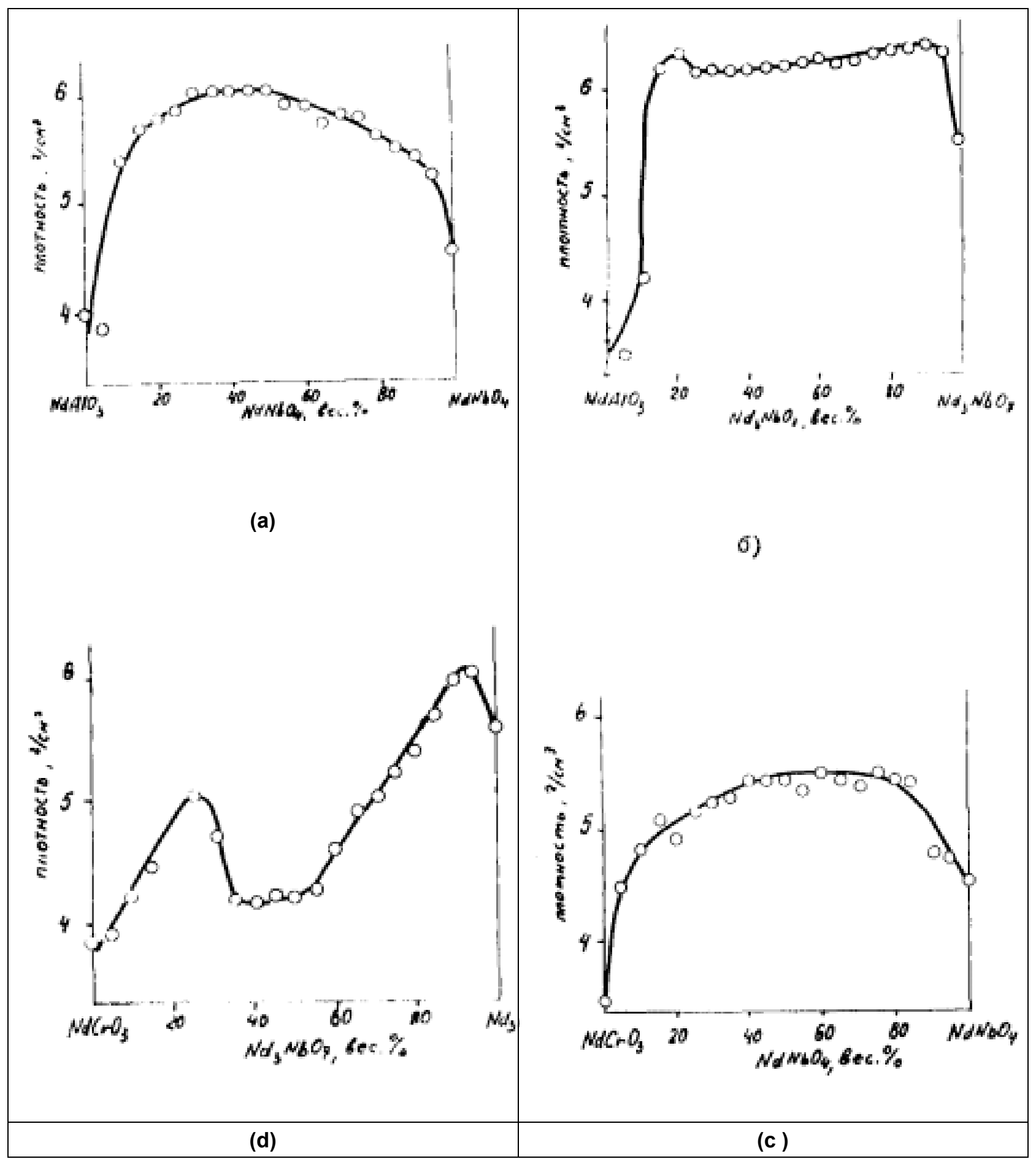

Fig. 5. Density vs. composition for systems: (a) $\mathrm{NdAlO}_{3}-\mathrm{NdNbO}_{4}$, (b) $\mathrm{NdAIO}_{3}-\mathrm{Nd}_{3} \mathrm{NbO}_{7}$, (c) $\mathrm{NdCrO}_{3}-\mathrm{NdNbO}_{4}$, (d) $\mathrm{NdCrO}_{3}-\mathrm{Nd}_{3} \mathrm{NbO}_{7}$, sintering temperature $1450^{\circ} \mathrm{C}, \mathrm{T}=3$ hours, $\mathrm{p}_{\mathrm{s} .}=4 \mathrm{t} / \mathrm{cm}^{2}$.

The principal technological scheme for manufacturing ceramic units from chromite-niobate compositions including REE and Am is shown in Fig. 6. 


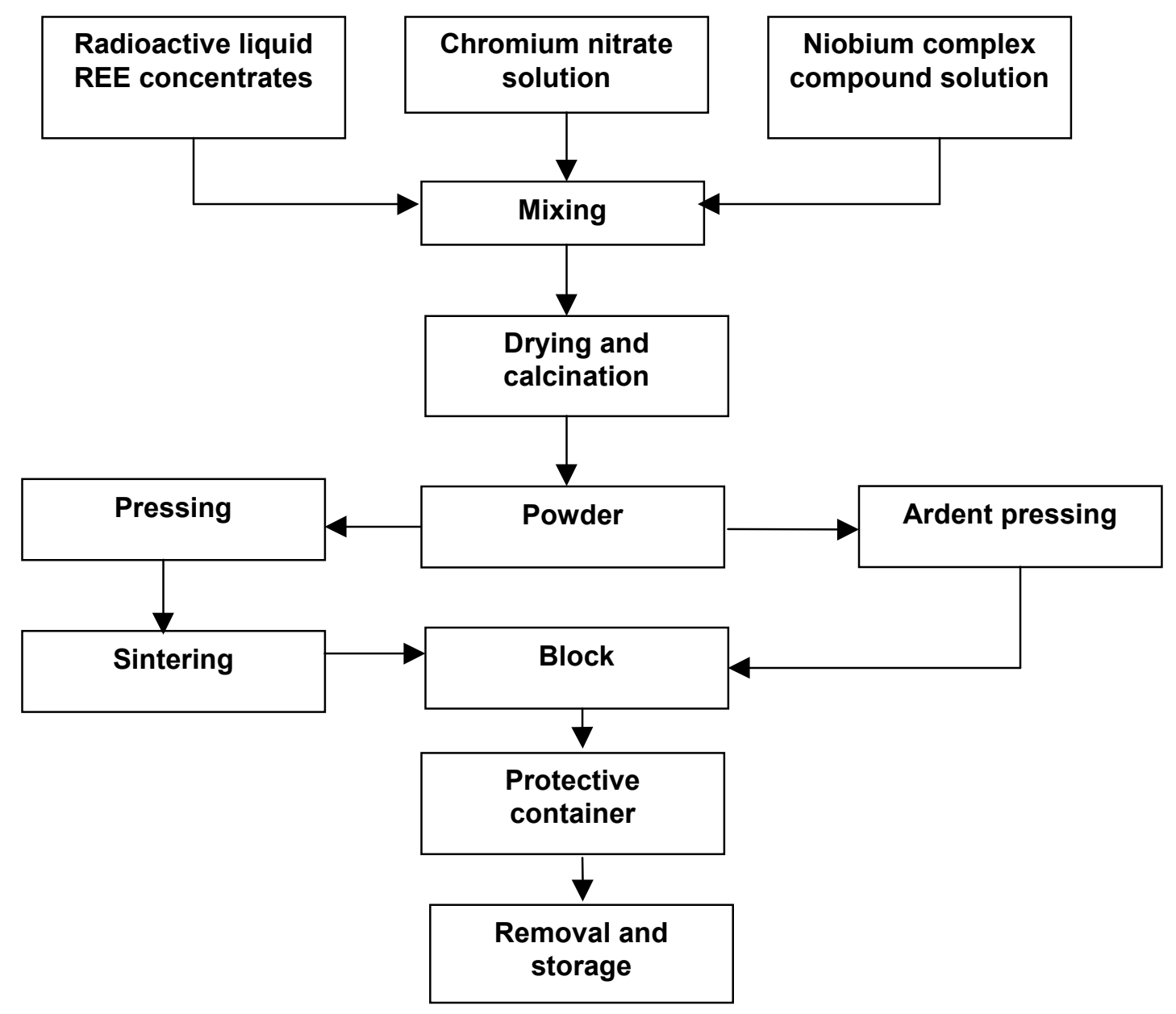

Fig. 6. The principal technological scheme of manufacturing of ceramic blocks from chromiteniobate compositions that include REE and Am. 


\section{Summary}

1. The fusibility diagrams of $\mathrm{NdAlO}_{3}-\mathrm{NdNbO}_{4}, \mathrm{NdAlO}_{3}-\mathrm{Nd}_{3} \mathrm{NbO}_{7}, \mathrm{NdCrO}_{3}-\mathrm{NdNbO}_{4}, \mathrm{NdCrO}_{3}-$ $\mathrm{Nd}_{3} \mathrm{NbO}_{7}$ systems were studied. It was shown that the most high-melting systems are chromites ones. It was established that $\mathrm{NaNbO}_{4}$ is decomposed at temperatures above $1400^{\circ} \mathrm{C}$ with loss of oxygen, and attachment in the system of neodymium chromite or aluminate stabilized it.

2. The attachment of neodymium aluminate to $\mathrm{Nd}_{3} \mathrm{NbO}_{7}$ increases the thermostability of articles formed from it and augments its sintering, which facilitates obtaining dense articles.

3. It is recommended that a composition ranging from $90 \mathrm{wt} \%$ of $\mathrm{Nd}_{3} \mathrm{NbO}_{7}$ and $10 \mathrm{wt} \%$ of $\mathrm{NdAlO}_{3}$ be used as one of the matrix materials.

4. Some main physicochemical, thermal, and operational properties of $\mathrm{Nd}$ chromite, aluminate, niobates and their mixtures have been studied for their use as matrix materials.

5. Nd chromite can theoretically include up to $50 \mathrm{wt} \% \mathrm{Am}$ and /or TPE, in Nd orthoniobate - up to 40 wt $\%$ Am and/or TPE, in $\mathrm{Nd}_{3} \mathrm{NbO}_{7}$ up to $60 \%$ Am and/or TPE, in mixture - 40-60 wt\% Am and/or TPE, though introducing the maximum 15-20 \% Am and /or TPE substantially can be demanded.

6. Some main properties of Am and REE chromites, aluminates, niobates have been analyzed, i.e., the compounds in the form in which one HAW radionuclides and their simulations are in matrix composition. These are chemically, thermally, and radiation-stable chemical compounds, inclusive in the matrix in either a separate phase or as a solid solution when the crystal lattices of phases of chromites, niobates (U, Th, Pu, Am, La-Nd), and aluminates (Pu, Am, La-Nd) are isostructural.

\section{Reference}

1. C. Keller, J. Inorg. Nucl. Chem., Vol. 27, pp. 1233-1246. 


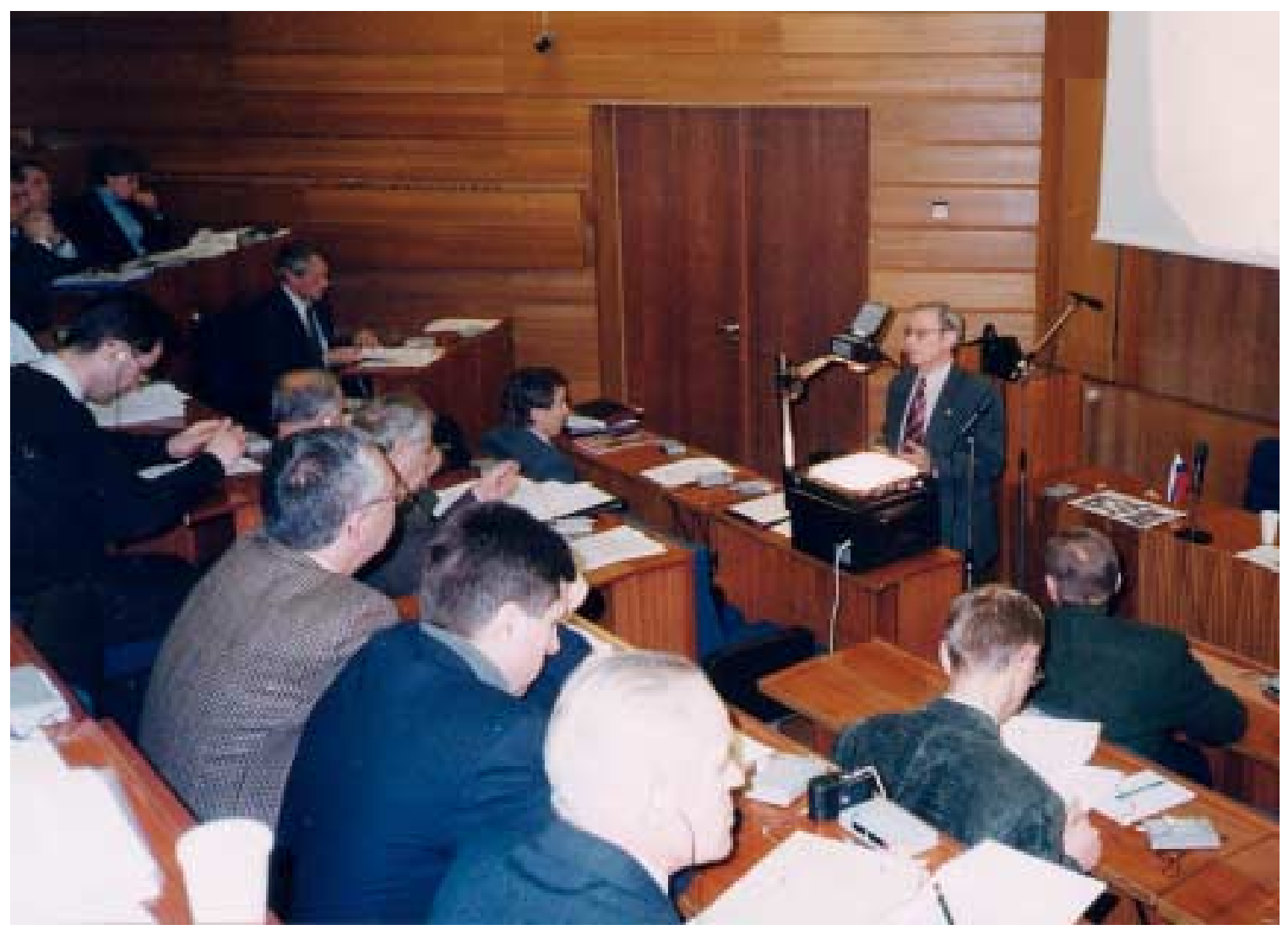

III.2. Plutonium Ceramics for Immobilization 


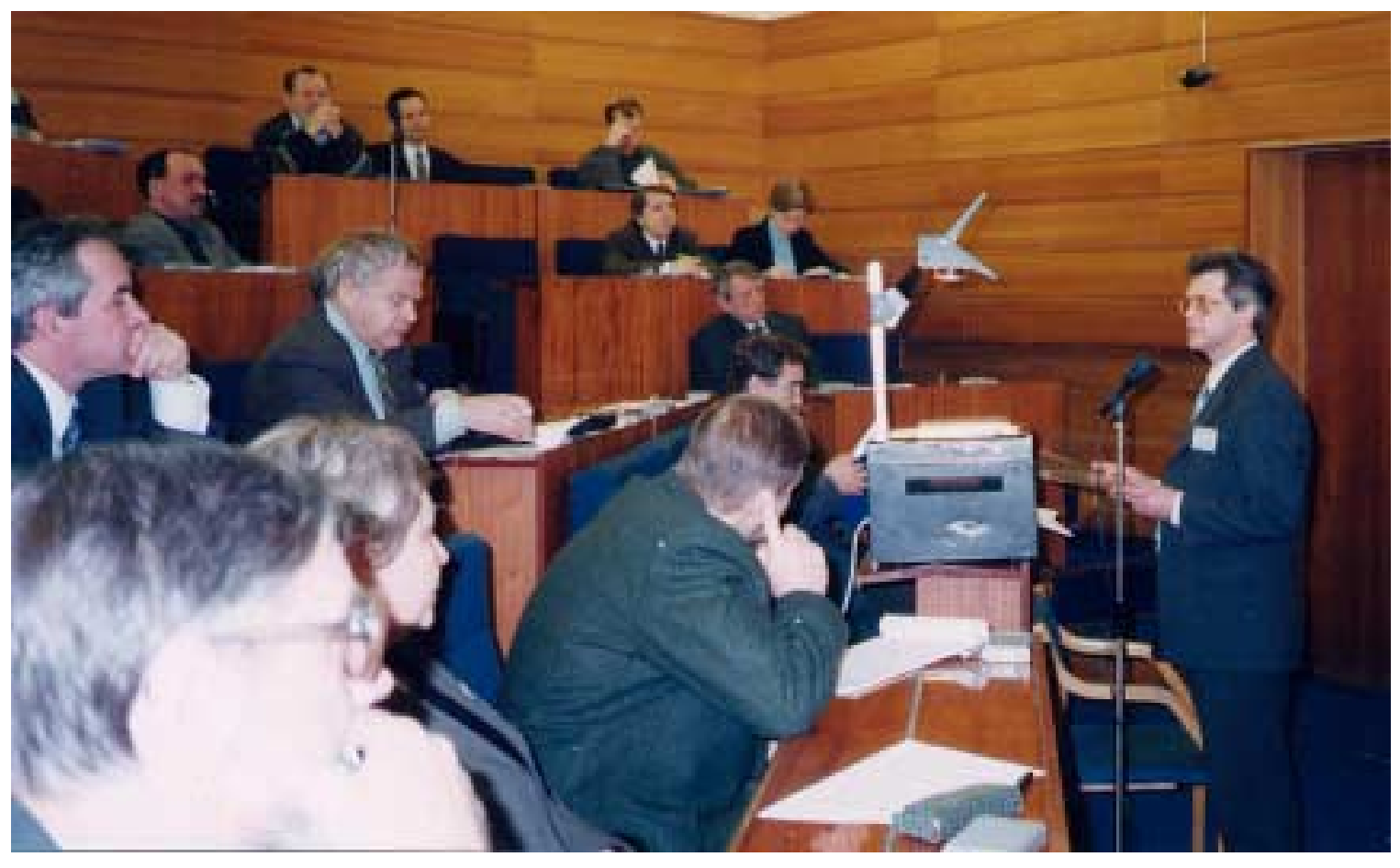




\title{
Summary of Pu Ceramics Developed for Pu Immobilization (B506216, B512161)
}

\author{
B. E. Burakov, E.B. Anderson, KRI
}

\section{Introduction}

Ceramics are the most durable actinide waste forms; however, they are still not generally adopted by the nuclear industry in Russia. Since 1990, the Laboratory of Applied Mineralogy and Radiogeochemistry of the V.G. Khlopin Radium Institute (KRI) has developed several different types of crystalline host-phases acceptable for the economically feasible and environmentally safe immobilization of actinide wastes. We propose that only ceramics, which are based on host-phases similar to naturally occurring accessory minerals including zircon, $(\mathrm{Zr}, \mathrm{Hf}, \mathrm{An}, \ldots) \mathrm{SiO}_{4}$; hafnon, $(\mathrm{Hf}, \mathrm{Zr}, \mathrm{An}, \ldots) \mathrm{SiO}_{4}$; baddeleyite, $(\mathrm{Zr}, \mathrm{Hf}, \mathrm{An}, \ldots) \mathrm{O}_{2}$; tazheranite (cubic zirconia), $(\mathrm{Zr}, \mathrm{Hf}, \mathrm{Ca}, \mathrm{Ti}, \mathrm{An}, \ldots) \mathrm{O}_{2}$; garnet, $(\mathrm{Ca}, \mathrm{Fe}, \mathrm{Gd}, \mathrm{An}, \ldots)_{3}(\mathrm{Al}, \mathrm{Fe}, \mathrm{Si}, \mathrm{An}, \ldots)_{5} \mathrm{O}_{12}$ and perovskite, $(\mathrm{Ca}, \mathrm{Gd}, \mathrm{An}, \ldots)(\mathrm{Al}, \mathrm{Fe}, \mathrm{Ti}, \ldots) \mathrm{O}_{3}$, etc. are the most efficient materials for actinide immobilization in deep geological formations. The use of such ceramic waste forms helps to preserve geochemical equilibrium under geological conditions in a repository, and these materials can be considered as creating an additional barrier to radionuclide migration out of the repository site. A solid solution of $\mathrm{Pu}$ in zirconia, $(\mathrm{Zr}, \mathrm{Pu}) \mathrm{O}_{2}$ could be used as a ceramic nuclear fuel that is competitive with mixed oxide fuel (MOX), $(\mathrm{U}, \mathrm{Pu}) \mathrm{O}_{2}$. It is proposed that spent ceramic fuel, unlike spent MOX fuel, can be finally disposed of without reprocessing directly into geological formations.

The main goals of Agreements B506216 and B512161 were: (1) to obtain different Pu-ceramic samples using the simplest possible synthesis methods; (2) to compare the durability of these ceramics in aqueous solutions, acceptance for industrial-scale use, capacity of Pu and other actinide loading, etc.

Studies of ceramic based on Ti- and Zr-pyrochlore were considered as the most important for the US immobilization program, and the investigation of garnet/perovskite ceramics suggested final adoption of this ceramic at the Mayak site in Russia for immobilization of Pu-Am-containing sludges.

\section{Brief Summary of Results}

In the framework of Agreements B506216 and B512161, the KRI fabricated and studied samples of different Pu-ceramics, including Ti-pyrochlore-based ceramic as the US candidate waste form for Pu immobilization (Table 1).

It was demonstrated at laboratory scale that Pu ceramics could be fabricated by economically sensible methods such as sintering or melting in air. For example, many samples of zirconia-based ceramic doped with 10 wt\% Pu were successfully synthesized avoiding formation of separate Pu-phases [Fig. 1(a)]. Also, garnet and perovskite materials of the following estimated formulas were obtained by melting in air:

Garnet:

- $\left(\mathrm{Y}_{1.6} \mathrm{Gd}_{1.3} \mathrm{Ce}_{0.1}\right)\left(\mathrm{Al}_{4.4} \mathrm{Ga}_{0.5} \mathrm{Cr}_{0.1} \mathrm{U}_{0.01}\right) \mathrm{O}_{12}$;

- $\mathrm{Ca}_{3}\left(\mathrm{Nb}_{1.0} \mathrm{Zr}_{0.9} \mathrm{Pu}_{0.1}\right)\left(\mathrm{Ga}_{1.7} \mathrm{Al}_{1.3}\right) \mathrm{O}_{12}$

- $\mathrm{Ca}_{3}\left(\mathrm{Nb}_{1.60} \mathrm{Zr}_{0.35} \mathrm{Pu}_{0.05}\right)\left(\mathrm{Ga}_{1.5} \mathrm{Al}_{1.5}\right) \mathrm{O}_{12}$; 
- $\left(\mathrm{Ca}_{2} \mathrm{Gd}\right)\left(\mathrm{Gd}_{0.20} \mathrm{Ce}_{0.24} \mathrm{Sn}_{0.01} \mathrm{Pu}_{0.04} \mathrm{Ga}_{1.60} \mathrm{Al}_{2.90}\right) \mathrm{O}_{11.7}$;

- $\mathrm{Na}_{2}\left(\mathrm{Gd}_{1.40} \mathrm{Ce}_{0.08} \mathrm{Zr}_{0.08} \mathrm{Pu}_{0.03}\right)\left(\mathrm{Ga}_{2.3} \mathrm{Al}_{2.6}\right) \mathrm{O}_{11.4}$ (Fig. 1b);

- $\left.\mathrm{Gd}_{1.6} \mathrm{Ce}_{0.3} \mathrm{Ca}_{1.0} \mathrm{Pu}_{0.1}\right)\left(\mathrm{Al}_{3.8} \mathrm{Ga}_{1.2}\right) \mathrm{O}_{12}$;

- $\left(\mathrm{Gd}_{2.1} \mathrm{Ce}_{0.2} \mathrm{Ca}_{0.6} \mathrm{Pu}_{0.1}\right)\left(\mathrm{Al}_{3.8} \mathrm{Ga}_{1.2}\right) \mathrm{O}_{12}$;

Perovskite:

- $\left(\mathrm{Ca}_{0.11} \mathrm{Gd}_{1.70} \mathrm{Ce}_{0.13} \mathrm{Pu}_{0.04}\right)\left(\mathrm{Ga}_{0.04} \mathrm{Al}_{0.96}\right) \mathrm{O}_{3}$;

- $\left(\mathrm{Gd}_{0.8} \mathrm{Ce}_{0.1} \mathrm{Pu}_{0.1}\right) \mathrm{AlO}_{3}$;

- $\left(\mathrm{Gd}_{0.85} \mathrm{Ca}_{0.10} \mathrm{Pu}_{0.05}\right) \mathrm{AlO}_{3}$.

In order to demonstrate the possibility of obtaining large blocks (from 1.0 to $2.5 \mathrm{~kg}$ each) of garnet-based ceramic even at very high temperature through melting in the cold crucible melter, four blocks (of weight 1.5-2.5 kg each) of pure undoped polycrystalline yttrium-aluminum garnet were synthesized (Fig. 2) using the following procedure:

Table 1. Main features of ceramic formulation and fabrication at KRI.

\begin{tabular}{|c|c|c|c|c|}
\hline Ceramic type & Precursor type & $\begin{array}{l}{ }^{239} \mathrm{Pu}\left({ }^{241} \mathrm{Am}\right) \\
\text { content, wt } \%\end{array}$ & $\begin{array}{l}\text { Synthesis } \\
\text { method }\end{array}$ & $\begin{array}{l}\text { Synthesis } \\
\text { conditions }\end{array}$ \\
\hline Zirconia, $(\mathrm{Zr}, \mathrm{Gd}, \mathrm{Pu}) \mathrm{O}_{2}$ & $\begin{array}{l}\text { Co-precipitated } \\
\text { calcined oxalates }\end{array}$ & $10(0.1)$ & \multirow{3}{*}{$\begin{array}{l}\text { Cold pressing } \\
\text { followed by } \\
\text { sintering in air }\end{array}$} & $1450-1500^{\circ} \mathrm{C}$ \\
\hline Zircon, $(\mathrm{Zr}, \mathrm{Pu}) \mathrm{SiO}_{4}$ & Sol-gel & $5-6(0.05)$ & & for 7 hours \\
\hline $\begin{array}{l}\text { US pyrochlore, } \\
(\mathrm{Ca}, \mathrm{Gd}, \mathrm{Pu}, \mathrm{U}, \mathrm{Hf})_{2} \mathrm{Ti}_{2} \mathrm{O}_{7}\end{array}$ & \multirow{2}{*}{$\begin{array}{l}\text { Milled oxide } \\
\text { powders }\end{array}$} & $10(0.1)$ & & $\begin{array}{l}1350^{\circ} \mathrm{C} \\
\text { for } 4 \text { hours }\end{array}$ \\
\hline $\begin{array}{l}\text { Garnet+Perovskite, } \\
(\mathrm{Ca}, \mathrm{Na}, \mathrm{Gd}, \ldots)_{3}(\mathrm{Al}, \mathrm{Ga}, \mathrm{Pu} \ldots)_{5} \mathrm{O}_{12}+ \\
(\mathrm{Ca}, \mathrm{Gd}, \mathrm{Pu}, \ldots)(\mathrm{Al}, \mathrm{Ga}, \ldots) \mathrm{O}_{3}\end{array}$ & & $1-6(0.001-0.05)$ & Melting in air & $\begin{array}{l}1300-1600^{\circ} \mathrm{C} \\
\text { for } 30 \mathrm{~min}\end{array}$ \\
\hline
\end{tabular}

a)

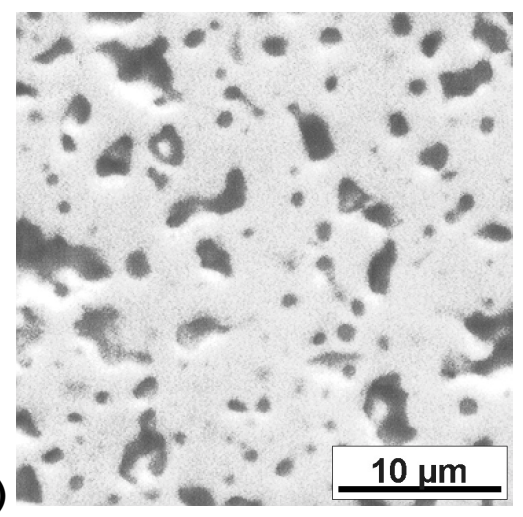

b)

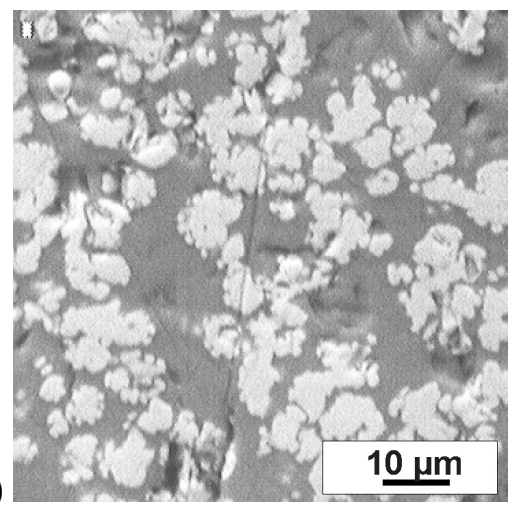

Fig. 1. Back-scattered SEM photomicrographs of ceramics based on: (a) Pu-doped cubic zirconia, $(\mathrm{Zr}, \mathrm{Gd}, \mathrm{Pu}) \mathrm{O}_{2}$, containing $10.3 \mathrm{wt} \% \mathrm{Pu}$ and $20.9 \mathrm{wt} \% \mathrm{Gd}$. There is no separated Pu-phases in ceramic matrix; (b) Two Pu host-phases: dark matrix - garnet, $\mathrm{Na}_{2}(\mathrm{Gd}, \mathrm{Ce}, \mathrm{Zr}, \mathrm{Pu})(\mathrm{Ga}, \mathrm{Al})_{5} \mathrm{O}_{12}$, containing $0.7 \mathrm{wt} . \% \mathrm{Pu}$ and $6.6 \mathrm{wt} \% \mathrm{Na}$; and light inclusions - zirconia, $(\mathrm{Zr}, \mathrm{Gd}, \mathrm{Pu}) \mathrm{O}_{2}$, containing 16.4 wt. $\%$ Pu and 18.3 wt.\% Gd. 
(a)

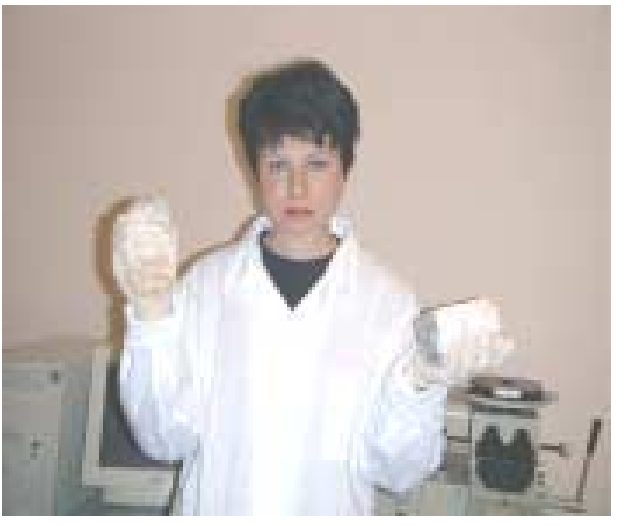

(b)

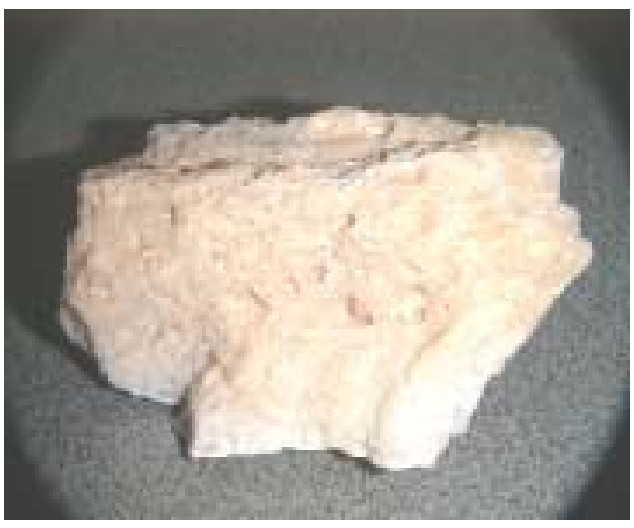

Fig. 2. Samples of pure undoped garnet-based ceramic $\left(\mathrm{Y}_{3} \mathrm{Al}_{5} \mathrm{O}_{12}\right)$ obtained by cold crucible method at temperature $2000^{\circ} \mathrm{C}$ in air: (a) General view of ceramic blocks (1.5-2.0 kg each); (b) Detailed image of selected piece $(10 \mathrm{~cm}$ length).

- Blend of garnet stoichiometry (15 kg) based on metallic $\mathrm{Al}$ and calcined at $1200^{\circ} \mathrm{C}$ oxides: $\mathrm{Al}_{2} \mathrm{O}_{3}$ and $\mathrm{Y}_{2} \mathrm{O}_{3}$, was prepared by dry ball milling for 1 hour;

- Obtained precursor was roughly cold pressed into two blocks and then they were melted at $2000^{\circ} \mathrm{C}$ for 30 minutes.

Most of the Pu-ceramic samples obtained so far have been studied by the MCC-1 leach test (Fig. 3, Table 2). According to the modified MCC-1 procedure used at the Radium Institute for leaching experiments, specimens of known composition and surface area are placed on the bottom of Teflon ${ }^{\mathrm{TM}}$ test vessels with deionized water and then set in an oven at the desired temperature. The ratio of surface area to water volume is maintained at $1: 10$. The test duration is 28 days. The leaching solution is removed and replaced with fresh deionized water after 3 and 14 days after the start of the test. The specimens are cleaned by rinsing with high purity water and ethanol. Analysis of ${ }^{239} \mathrm{Pu}$ in the leaching solution is carried out after 3,14 and 28 days. The measurements of $\mathrm{Pu}$ absorbed on the walls of test vessels is carried out after 28 days. Then a correction for the activity of Pu and Am released on the $3^{\text {rd }}$ and 14th days is provided, assuming a straight-line relation for the absorbed amount of Pu with time for the first 14 days. Only data from the 14th and 28th days are used for interpretation. Normalized Pu mass loss $(\mathrm{NL})$ is calculated as follows:

$\mathrm{NL}=\mathrm{A} \times \mathrm{W}_{0} \times \mathrm{A}_{0}^{-1} \times \mathrm{S}^{-1}$, where:

$\mathrm{A}$ - total activity of $\mathrm{Pu}$ in the water solution after leaching, Bq;

$\mathrm{A}_{0}$ - the initial activity of $\mathrm{Pu}$ in the specimen, Bq;

$\mathrm{W}_{0}$ - the initial mass of the specimen, gram;

$\mathrm{S}$ - specimen surface area without correction on ceramic porosity, $\mathrm{m}^{2}$.

The highest Pu mass loss was observed for zircon-based ceramics obtained from precursor containing $10 \mathrm{wt} \% \mathrm{Pu}$. This was due to the presence of separated inclusions of $\mathrm{PuO}_{2}$ and the high porosity of the sample compared with other ceramic samples. At the same time, the highest leach rate observed for this sample shows that saturation of the leach solution with Pu did not take place for other ceramic samples and it did not decrease Pu release from their matrices. 
Table 2. Normalized Pu mass loss (in $\mathrm{g} / \mathrm{m}^{2}$ ) from ceramic matrices after MCC-1 leach test done in deionized water at 25 and $90^{\circ} \mathrm{C}$. The correction for ceramic porosity was not estimated.

\begin{tabular}{|c|c|c|c|c|}
\hline \multirow{2}{*}{ Sample } & \multicolumn{2}{|c|}{ At $25^{\circ} \mathrm{C}$} & \multicolumn{2}{|c|}{ At $90^{\circ} \mathrm{C}$} \\
\hline & 14 days & 28 days & 14 days & 28 days \\
\hline $\begin{array}{l}\text { Zircon based ceramic doped with } \\
10 \text { wt. \% Pu }\end{array}$ & 0.030 & 0.040 & 0.200 & 0.200 \\
\hline $\begin{array}{l}\text { Zircon based ceramic doped with } \\
\text { 5-6 wt. \% Pu }\end{array}$ & 0.001 & 0.001 & 0.007 & 0.008 \\
\hline $\begin{array}{l}\text { Zirconia based ceramic doped } \\
\text { with } 10 \text { wt. } \% \mathrm{Pu}\end{array}$ & 0.008 & 0.009 & 0.020 & 0.040 \\
\hline $\begin{array}{l}\text { Pyroclore based ceramic doped } \\
\text { with } 10 \text { wt.\% Pu }\end{array}$ & 0.002 & 0.003 & 0.001 & 0.001 \\
\hline
\end{tabular}
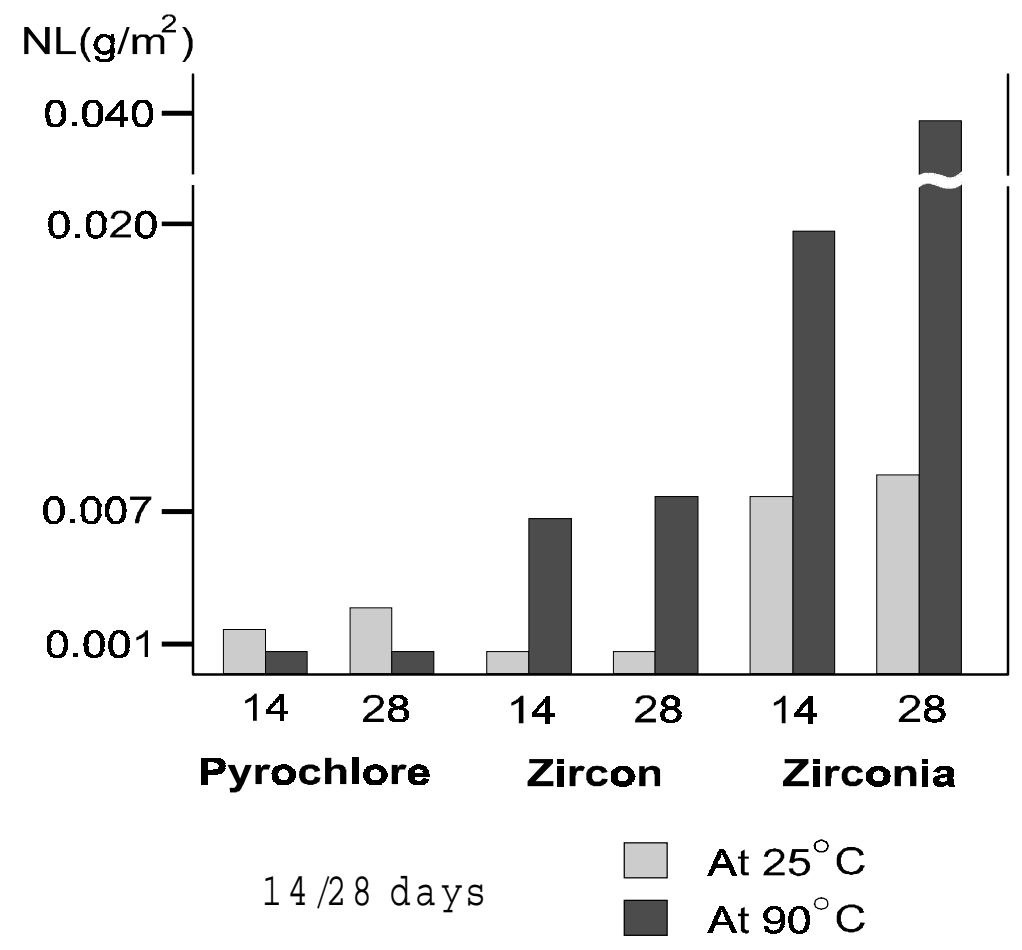

Fig. 3. Diagram of normalized Pu mass loss (in $\mathrm{g} / \mathrm{m}^{2}$ ) from ceramics based on pyrochlore (doped with 10 wt $\%{ }^{239} \mathrm{Pu}$ ), zircon (doped with 5-6 wt\% ${ }^{239} \mathrm{Pu}$ ) and zirconia (doped with $10 \mathrm{wt} \%{ }^{239} \mathrm{Pu}$ ) after MCC-1 leach test carried out in deionized water at 25 and $90^{\circ} \mathrm{C}$ for 14 and 28 days. The correction for ceramic porosity was not estimated.

Results obtained so far allow us to conclude that ceramics based on pyrochlore and zirconia doped with $10 \mathrm{wt} \% \mathrm{Pu}$ and zircon doped with 5-6 wt\% Pu are characterized by similar chemical resistance to leaching in deionized water.

However, data obtained for the MCC-1 leach test are characterized by significant uncertainty because the surface area of each sample was determined without correction for ceramic porosity. Preliminary estimations show that this correction can decrease the value for Pu mass loss more by than 2 orders of magnitude. Taking into account that ceramic porosity increases in the "pyrochlore-zirconia-zircon", real 
Pu mass loss from zircon and zirconia might be significantly lower and, possibly, even less than from pyrochlore.

Behavior of Am during the leach test requires additional investigation. The following preliminary results of normalized Am mass loss (in $\mathrm{g} / \mathrm{m}^{2}$ ) from ceramic matrices (after MCC-1 leach test done in deionized water at $90^{\circ} \mathrm{C}$ for 28 days) were measured: zircon (doped with 5-6 wt.\% Pu)- 0.18; zirconia - 0.33;

pyrochlore -0.04 .

Study of ceramics based on Ti-pyrochlore, Zr-pyrochlore, and garnet/perovskite is continuing at the present time. New samples were recently synthesized (Tables 3,4 ). All these samples will be examined in comparison using XRD, SEM, and EPMA methods and the MCC-1 leach test.

Table 3. Features of pyrochlore-based ceramic samples (obtained in the framework of B512161).

\begin{tabular}{|l|l|l|l|l|}
\hline $\begin{array}{c}\text { Pellet } \\
\text { number }\end{array}$ & $\begin{array}{c}\text { Diameter } \\
\mathbf{m m}\end{array}$ & $\begin{array}{c}\text { Height } \\
\mathbf{m m}\end{array}$ & Weight/g & $\begin{array}{c}\text { Density } \\
\mathbf{g} / \mathbf{c m}^{3}\end{array}$ \\
\hline Pu-doped Ti-pyrochlore (US-type) \\
\hline 1 & 6.9 & 2.6 & 0.439 & 4.5 \\
\hline 2 & 6.9 & 3.5 & 0.618 & 4.7 \\
\hline 3 & 3.5 & 3.2 & 0.144 & 4.7 \\
\hline 4 & 3.5 & 2.6 & 0.118 & 4.7 \\
\hline 5 & 3.5 & 3.6 & 0.159 & 4.6 \\
\hline Undoped Zr-pyrochlore, $\mathrm{Gd}_{2} \mathrm{Zr}_{2} \mathrm{O}_{7}$ \\
\hline 1 & 7.9 & 3.8 & 0.976 & 5.3 \\
\hline 2 & 7.9 & 4.1 & 1.055 & 5.3 \\
\hline Pu-doped Zr-pyrochlore, $\mathrm{Gd}_{2} \mathrm{Zr}_{1.2} \mathrm{U}_{0.5} \mathrm{Pu}_{0.3} \mathrm{O}_{7}$ \\
\hline 1 & 9.4 & 3.4 & 1.221 & 5.2 \\
\hline 2 & 9.4 & 1.0 & 0.371 & 5.6 \\
\hline 3 & 3.5 & 2.5 & 0.141 & 5.9 \\
\hline
\end{tabular}

Table 4. Features of garnet-based ceramic samples (obtained in the framework of B512161).

\begin{tabular}{|l|l|l|l|l|}
\hline $\begin{array}{c}\text { Pellet } \\
\text { number }\end{array}$ & $\begin{array}{c}\text { Diameter } \\
\mathbf{m m}\end{array}$ & $\begin{array}{c}\text { Height } \\
\mathbf{m m}\end{array}$ & Weightg & $\begin{array}{c}\text { Density } \\
\mathbf{g} / \mathbf{c m}^{3}\end{array}$ \\
\hline \multicolumn{5}{|l|}{ Garnet, $(\mathrm{Y}, \mathrm{Pu})_{3} \mathrm{Al}_{5} \mathrm{O}_{12}$} \\
\hline 1 & 7.4 & 1.7 & 0.241 & 3.3 \\
\hline 2 & 7.4 & 2.7 & 0.358 & 3.1 \\
\hline 3 & 7.4 & 2.2 & 0.286 & 3.0 \\
\hline Garnet, $(\mathrm{Gd}, \mathrm{Pu})_{3} \mathrm{Ga}_{5} \mathrm{O}_{12}$ \\
\hline 1 & 8.9 & 1.1 & 0.342 & 5.0 \\
\hline 2 & 7.0 & 1.3 & 0.180 & 3.6 \\
\hline 3 & 7.0 & 2.3 & 0.434 & 4.9 \\
\hline
\end{tabular}




\section{Conclusions}

1. Samples of ${ }^{239} \mathrm{Pu}$-doped ceramics, which are based on Ti-pyrochlore, $(\mathrm{Ca}, \mathrm{Gd}, \mathrm{Pu}, \mathrm{U}, \mathrm{Hf})_{2} \mathrm{Ti}_{2} \mathrm{O}_{7}$; zircon, $(\mathrm{Zr}, \mathrm{Pu}) \mathrm{SiO}_{4}$; cubic zirconia, $(\mathrm{Zr}, \mathrm{Gd}, \mathrm{Pu}) \mathrm{O}_{2}$; garnet, $(\mathrm{Ca}, \mathrm{Na}, \mathrm{Gd}, \mathrm{Pu}, \ldots)_{3}(\mathrm{Al}, \mathrm{Ga}, \mathrm{Pu}, \ldots)_{5} \mathrm{O}_{12}$, and perovskite, $(\mathrm{Ca}, \mathrm{Gd}, \mathrm{Pu}, \ldots)(\mathrm{Al}, \mathrm{Ga}, \ldots) \mathrm{O}_{3}$, were successfully synthesized in air under laboratory conditions using economically attractive synthesis methods: sintering or melting.

2. Taking into account the correction for ceramic porosity, zircon doped with 5-6 wt\% Pu might be considered as the most chemically resistant Pu host-phase to alteration in deionized water. However, optimal Pu loading for zircon-based ceramic is two times less than the one for zirconia- or pyrochlorebased ceramics.

3. Results obtained in our Laboratory on ceramic synthesis might be applied for development of industrial-scale technology of actinide underground disposal using ceramic waste forms that are thermodynamically compatible with geological repository conditions. 


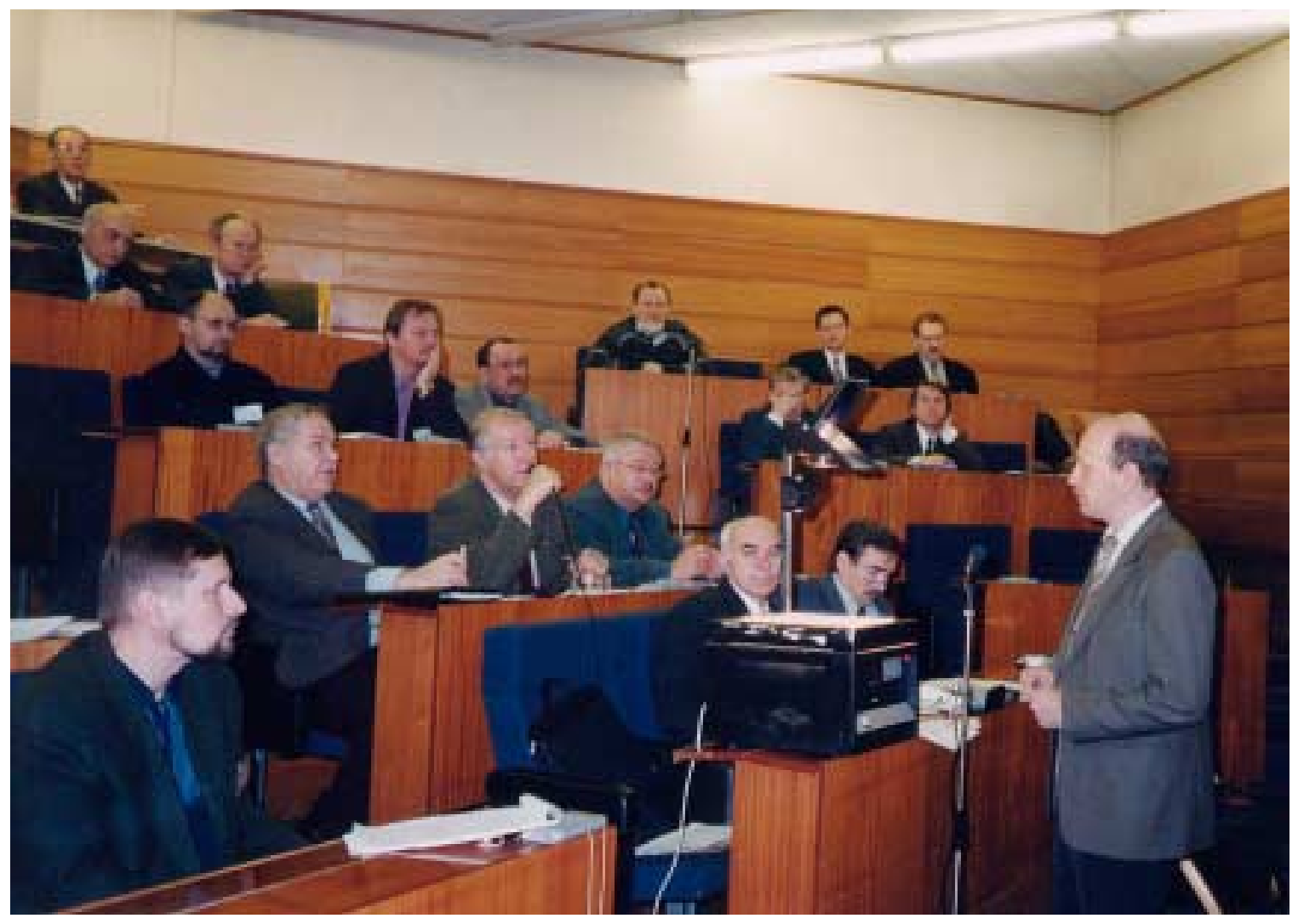

III.3. Accelerated Radiation Damage Studies of the US Plutonium Ceramic Formulation 



\title{
Investigation of Radiation and Chemical Stability of Titanate Ceramics Intended for Actinides Disposal (B501111)
}

\author{
A. N. Lukinykh, S. V. Tomilin, A. A. Lizin, A. V. Bychkov, RIAR
}

\begin{abstract}
The results of $\mathrm{x}$-ray analysis of titanate ceramics containing $8.7 \%$ of ${ }^{238} \mathrm{Pu}$ after a 490 -day exposure after the synthesis are presented. Over this period the accumulated dose accounted for $2.4^{*} 10^{18} \alpha-$ decay/g. The ceramics is noted to be in a state close to the roentgen-amorphous one, and only one or two low intensive reflexes of the main pyrochlore phase are vividly seen in the roentgenograms. The results of the interim investigation of the ceramic chemical stability are presented.
\end{abstract}

\section{Introduction}

The present paper is a progress report among those describing studies on radiation damages of titanate ceramics intended for plutonium immobilization. The work is carried out at the Russian Federation Scientific Research Institute of Atomic Reactors (SSC RF RIAR) together with the Lawrence Livermore National Laboratory (LLNL) under Contract B501111 (Amendment 2).

The previous results [1] were obtained after a year of observations on titanate ceramics behavior affected by the inner $\alpha$-irradiation of ${ }^{238} \mathrm{Pu}$ isotope. The ceramic was noted to have undergone essential changes towards deep amorphization with the dose of $\sim 1.9^{*} 10^{18} \alpha$-decay/g accumulated over this period. Nevertheless, several low intensive diffraction lines of the main pyrochlore crystalline phase were observed testifying to the presence of residual crystalline material. The most dynamic change of the pattern took place within the first six months of the observations, when micro-swelling of the crystalline cell could be x-rayed. In so doing, the gain in cubic lattice volume of the main pyrochlore phase was equal to $1.2-1.6 \%$ of the original one. Later on, the crystalline structure worsened so greatly that more or less precise determination of the crystalline cell parameters became impossible. At the same time, the macroswelling recorded by geometric measurements was observed within the first year of exposure and accounted for $\sim 3-4 \%$ of the original volume.

Along with the structural changes, it is also very important to trace such ceramics properties as chemical (hydrolytic) stability, i.e., capability to capture chemical matrix components including plutonium in dynamics depending on the accumulated dose. Therefore, in July-August, 2001, we additionally carried out tests on damaged ceramics leaching with the accumulated dose of $\sim 2.4^{*} 10^{18} \alpha$-decay along with the $x$-ray analysis. The x-ray data are presented as of the beginning of August, 2001, i.e., one year and four months after the ceramics synthesis.

\section{Experiment}

At present, six ceramic specimens of $\mathrm{Hf}_{-}^{238} \mathrm{Pu}-\mathrm{U}$ type in the form of compact pellets are being investigated. Three of the pellets (Nos. 1, 16, 18) are investigated using x-ray analysis, and one of them (No. 5) is investigated for hydrolytic stability. For investigations in the Debye-Sherrer cell using the photomethod, three $x$-ray specimens were fabricated in the form of sealed quartz capillaries with pellets No.1, 16, and 18 powder. Pellet No.1 is also investigated by the diffractometer, and pellet No.18 is 
additionally investigated as a thin section in the Debye-Sherrer cell. The procedure for specimen fabrication and x-ray technique are presented in reports [2-3] in detail and also in the Proceedings of the previous seminars. Over the period of April-August, 2001, roentgenograms were taken once a month.

Chemical stability was tested by the MCC- 1 technique [4] in distilled water at a temperature of $90^{\circ} \mathrm{C}$ on the $7^{\text {th }}, 14^{\text {th }}$, and $28^{\text {th }}$ days within the period from 18 July to 15 August, 2001 (i.e., in $\sim 16$ months after the ceramics production). Specimen No.5 in the form of a pellet (diameter $-10.88 \mathrm{~mm}$, height $-2.46 \mathrm{~mm}$, mass $-1.013 \mathrm{~g}$, density $-4.49 \mathrm{~g} / \mathrm{cm}^{3}$, surface area $-2.66 \mathrm{~cm}^{2}$ ) was tested. The specimen was placed into a Teflon container with a lid preliminary prepared and washed up using the MCC-1 procedure. Then, the container was filled in with $27 \mathrm{ml}$ of bidistilled water corresponding to the relation between the specimen surface area and the liquid volume equal to $10 \mathrm{~cm}^{-1}$. The specimen was suspended in the center of the liquid volume in the container on a thin platinum wire. The Teflon container with the specimen was placed into a thermostat with the temperature held at $90^{\circ} \mathrm{C}$ during the entire testing. On the $7^{\text {th }}, 14^{\text {th }}$, and $28^{\text {th }}$ days, the solutions were removed and the container walls were washed off by nitric acidulous solution $\left(\left[\mathrm{HNO}_{3}\right]\right)$. Then the acidulous solution $(27 \mathrm{ml})$ was filled in and kept at $90^{\circ} \mathrm{C}$ over 12 hours.

The following elements were determined in the wash-outs and solutions after leaching:

- Uranium - by spectrophotometric method after ion-exchange separation from hafnium and plutonium with an error of $10-12 \%$;

- Plutonium - by $\alpha$-spectrometric method with the error of $10-12 \%$;

- Calcium, titanium, and gadolinium - by spectral method with an error of $20-25 \%$;

- Hydrogen ions - by potentiometric method.

Moreover, the original bidistillate was preliminarily analyzed for content of ceramic elements, such as calcium, gadolinium, and titanium.

The results of the analysis of the bidistilled water, the solutions after leaching and the wash-outs were used for calculations of the normalized losses of cation leaching masses and rates from formulas (1) and (2).

The following formulae was used for calculations of the normalized loss of cation mass:

$(\mathrm{NL}) \mathrm{i}=\mathrm{mi} /(\mathrm{fi} \cdot \mathrm{S})$

where $(\mathrm{NL})_{\mathrm{i}}$ - the normalized loss of i element mass, $\mathrm{g} / \mathrm{m}^{2}$;

$\mathrm{m}_{\mathrm{i}}$ - i element mass in the solution after leaching, $\mathrm{g}$;

$f_{i}$ - i element mass fraction in the starting specimen;

$\mathrm{S}$ - an open "geometric" surface of the specimen, $\mathrm{m}^{2}$;

The nuclide leaching rate was calculated from the formulae:

$R_{i}=(N L)_{i} / t_{n}$

where $R_{i}$ - i element leaching rate, $g /\left(m^{2 *}\right.$ day $)$;

$t_{n}$ - duration of leaching period $n$, day. 


\section{Results}

\section{X-ray analysis}

Photomethod. Visual comparative examination of the diffraction pattern of ceramic specimens made of pellets No.1, 16, and 18 revealed further weakening and blurring of the diffraction peaks of the main pyrochlore phase. Thus, in the roentgenogram of specimen No.1, only one widened low intensive line with hkl 222 indexes (the strongest line of the pyrochlore phase) is vividly seen. The lines with indexes 400, 440 , and 622 observed previously (in a year after the synthesis) almost disappeared. Moreover, in the roentgenogram of specimen No.1, one more very weak line of an unknown phase with the inter-plane distance $\mathrm{d}=1.626 \AA$ is observed.

In the roentgenogram of specimen No.16 one line of the pyrochlore phase with indexes 222 , as well as the lines with indexes 400 and 440 having a track intensity are also observed easily. Along with them four lines of the fluorite-type minor phase with the inter-plane distance $d=3.16 ; 2.73 ; 1.93$ and $1.64 \AA$ are vividly seen. A relative strengthening of the fluorite phase lines along with weakening of the pyrochlore phase diffraction pattern must be noted. Thus, in the roentgenogram taken on 5 April, 2001, the intensity of the strongest lines of the fluorite phase was almost the same. Four months later in the roentgenogram taken on 8 August, 2001, the strongest line of the fluorite phase with index hkl 111 exceeded the strongest line of the pyrochlore phase with index hkl 222 in intensity. Moreover, one more very weak line of an unknown phase with the inter-plane distance $d=2.03 \AA$ is observed.

In general, the diffraction pattern of specimen No.18 coincided with that of specimen No.16 and suffered the same changes. The minor difference is in comparability of the strongest reflexes of the pyrochlore and fluorite phases in their intensity.

The Debye-Sherrer roentgenogram of the thin section of specimen No.18 shows four lines of the pyrochlore phase. The line with index $\mathrm{hkl} 222$ is easily observed, but it is essentially widened and weakened. Lines 400, 440, and 622 are weak and vanishing. The lines of $\mathrm{CaTiO}_{3}$-type orthorhombic phase (or that structurally close to it) become apparent in time. It must be noted that at the previous stage of the observations (in $\sim 1$ year after the ceramics synthesis), the $\mathrm{CaTiO}_{3}$-type lines were not observed in the roentgenograms of the thin section of specimen No.18.

Diffractometry. Within the period of April-August, 2001, four diffractograms of specimen No.1 were taken. Just as did the Debye-Sherrer roentgenograms taken using the photomethod, the diffractograms testify to the weakening of the diffraction pattern in time. In a year of observations, five reflexes of the main pyrochlore phase were observed, while in four months (on the $490^{\text {th }}$ day) only three low intensive reflexes were noted in the diffractogram (Fig. 1). A somewhat unexpected pattern is observed, i.e., the strongest peak with index 222 almost disappeared. Reflexes 400 and 440 have a vanishing intensity. The strongest peak from the observed pyrochlore peaks have indexes 331, although its intensity does not exceed $3 \%$ of the intensity of the strongest metal beryllium line $(2 \theta=51.08$ degrees $)$. Recall that a metal beryllium foil serving as a working intensity reference standard is used for sealing of the dish. In our opinion, such an abnormal distribution of the intensity among the reflexes of the main pyrochlore phase is unlikely to reflect the actual state of affairs and can have the following explanation: some minor phase seems to contribute to the intensity of reflex 331. The value of the contribution depends rather on the intensity of reflex 331 of the pyrochlore phase, than on time. The fact that the angular position of reflex 331 insignificantly changed in time in comparison with other reflexes (Table 1) testifies to this assumption. 
Table 1. Dynamics of the angular position of some of the pyrochlore phase reflexes in time.

\begin{tabular}{|l|c|c|c|r|r|}
\hline \multirow{2}{*}{ Date } & \multicolumn{5}{|c|}{ Diffraction angles 20, grades, for reflexes hkl } \\
\cline { 2 - 6 } & $\mathbf{3 1 1}$ & $\mathbf{2 2 2}$ & $\mathbf{4 0 0}$ & $\mathbf{3 3 1}$ & $\mathbf{6 2 2}$ \\
\hline April 2000 & 29,26 & 30,60 & 35,46 & 38,74 & 60,58 \\
\hline July 2000 & 29,08 & 30,44 & 35,28 & 38,68 & 60,28 \\
\hline October 2000 & 28,92 & 30,30 & 35,14 & 38,72 & 60,10 \\
\hline January 2001 & - & 30,22 & 35,04 & 38,72 & 59,88 \\
\hline April 2001 & - & 30,22 & 35,02 & 38,72 & 59,70 \\
\hline August 2001 & - & - & 35,00 & 38,72 & - \\
\hline
\end{tabular}

At the same time, radiation swelling of the cubic lattice a priori must be isotropic and observed for all reflexes.

In addition to the above pyrochlore reflexes, fifteen very weak reflexes of additional minor phases, whose interpretation is difficult due to their low intensity, are observed in the diffractogram.

\section{Leaching tests}

The results of the solutions analysis after the specimen leaching showed that the content of the matrix components lies within the limits of sensitivity of the analytical methods applied, but for gadolinium it is out of the sensitivity limits. For this reason, the values of the normalized mass loss and leaching rate for gadolinium must be considered as the maximum possible. The $\mathrm{pH}$ value of the starting bidistilled water is equal to 5.95, and in solutions after leaching it is 5.20. Calcium and titanium are discovered in trace amounts in the starting bidistilled water with their concentrations equal to 0.1 and $0.003 \mathrm{mg} / \mathrm{l}$, respectively. The content of these cations, both in the solutions after leaching and the wash-outs, were taken into account when calculating the normalized mass loss and leaching rate.

The calculation results of the normalized mass loss and leaching rates of ceramic components in comparison with the previously obtained data are presented in Tables 2 and 3 . The graphic dependencies of the normalized mass loss and leaching rate in 16 months after the ceramics production are presented in Figs .2 and 3. Comparative plots of leaching results for fresh and damaged ceramics are given in Figs. 4 and 5. 
III.3. Accelerated Radiation Damage Studies of the US Pu Ceramic Formulation
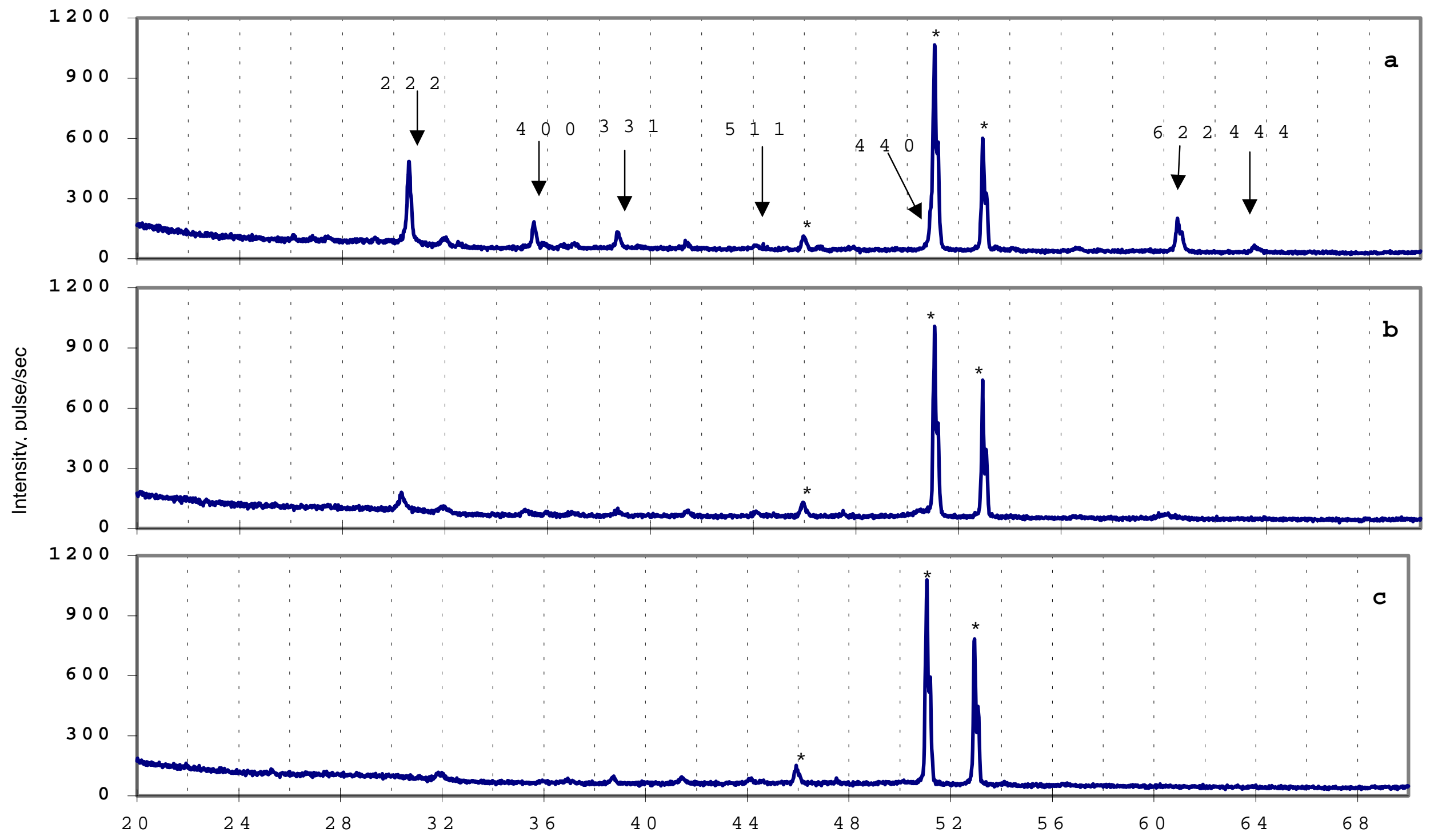

Anale 20. dearee

Fig.1. Diffractograms of $\mathrm{Hf}_{-}^{238} \mathrm{Pu}-\mathrm{U}$ ceramics (specimen No.1) within the angle range of $2 \theta=20-70$ degrees depending on exposure period: $a$ - in 10 days, $b$ - in 192 days ; $c$ - in 490 days after synthesis. The reflexes with indexes hkl are related to the pyrochlore phase, * - Be peaks. 


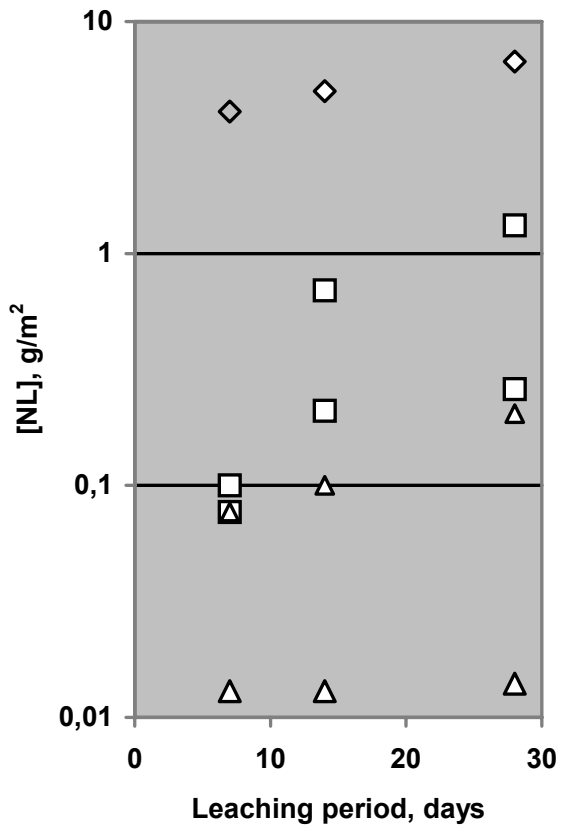

Fig.2. The dependence of the normalized mass losses on the leaching period

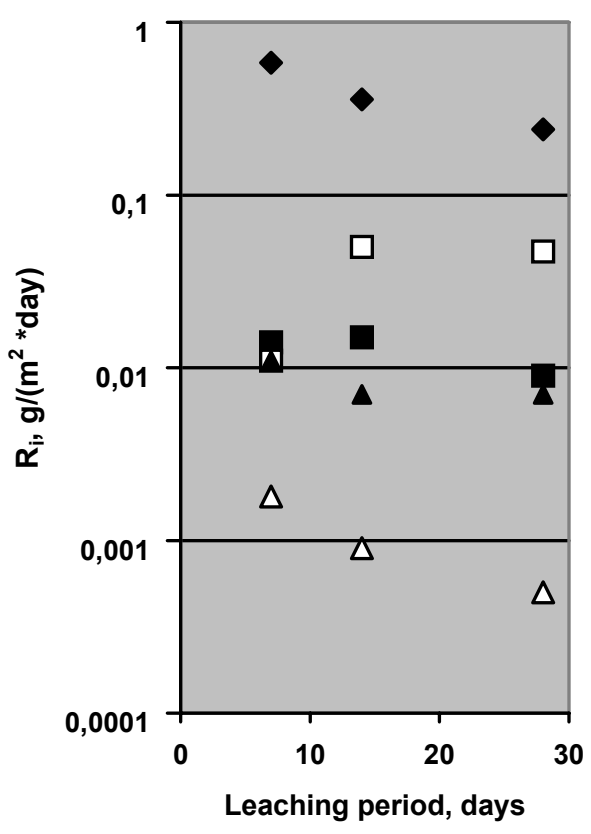

Fig.3. The time dependence of leaching rates 
$\mathrm{Ca}$

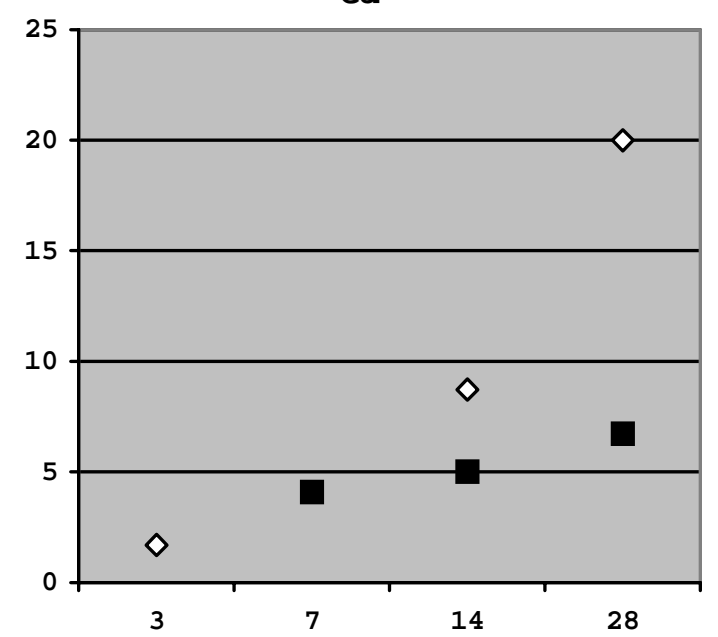

Ti
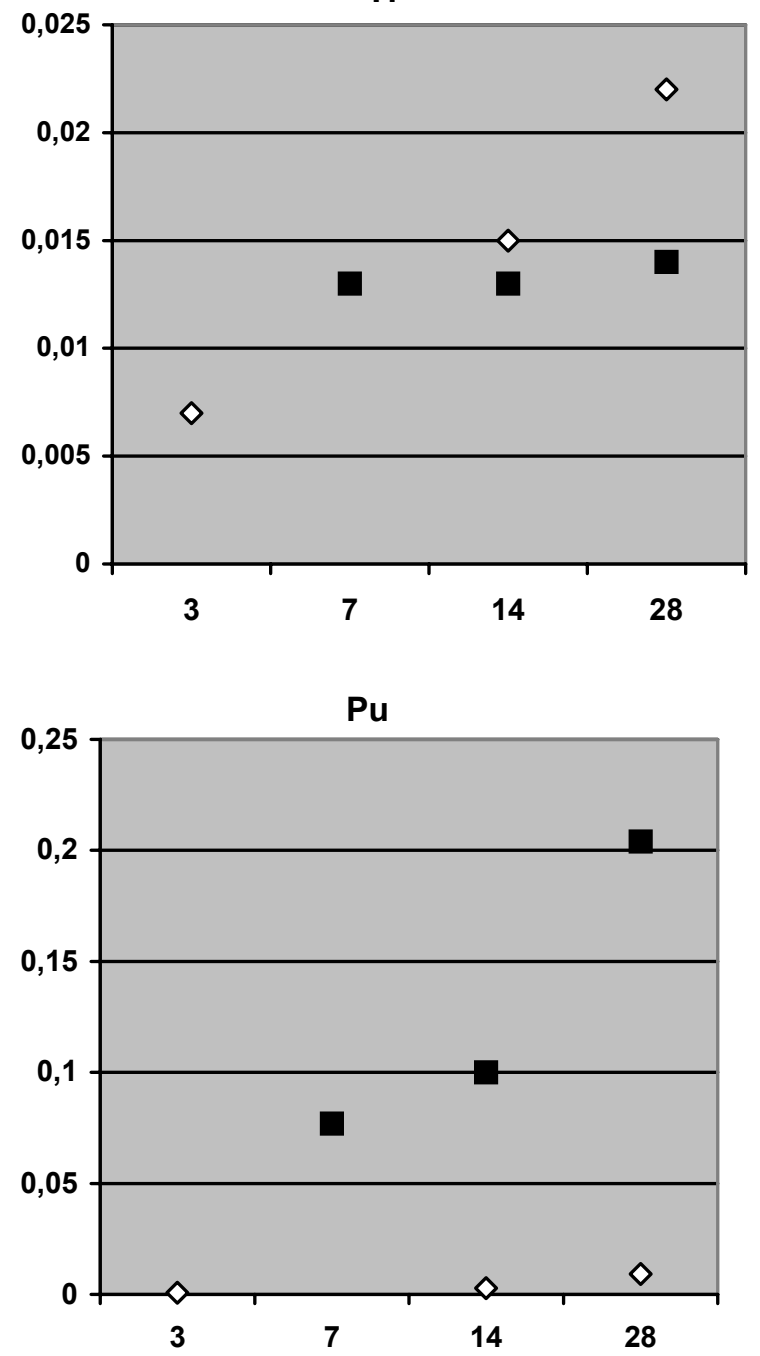

Gd

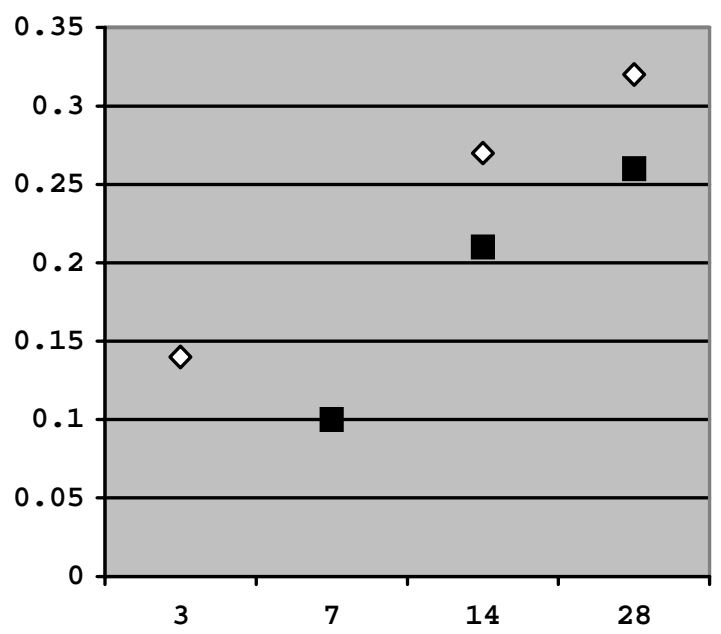

U

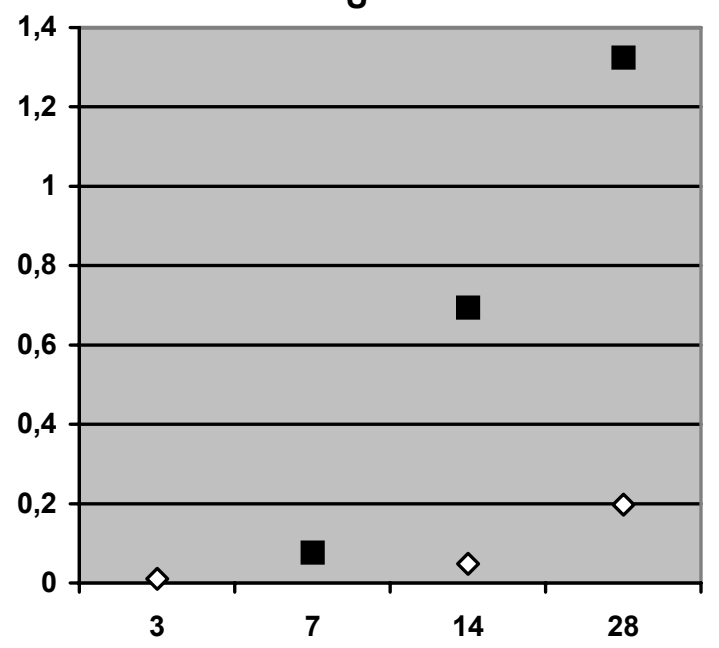

Fig. 4. The normalized mass losses of ceramic components after production $(\diamond)$ and in 16 months of

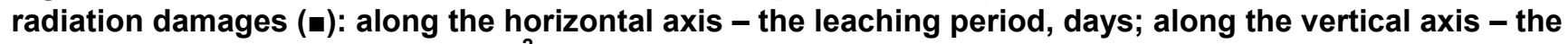
normalized mass losses $\left(\mathrm{NL}_{\mathrm{i}}\right), \mathrm{g} / \mathrm{m}^{2}$. 

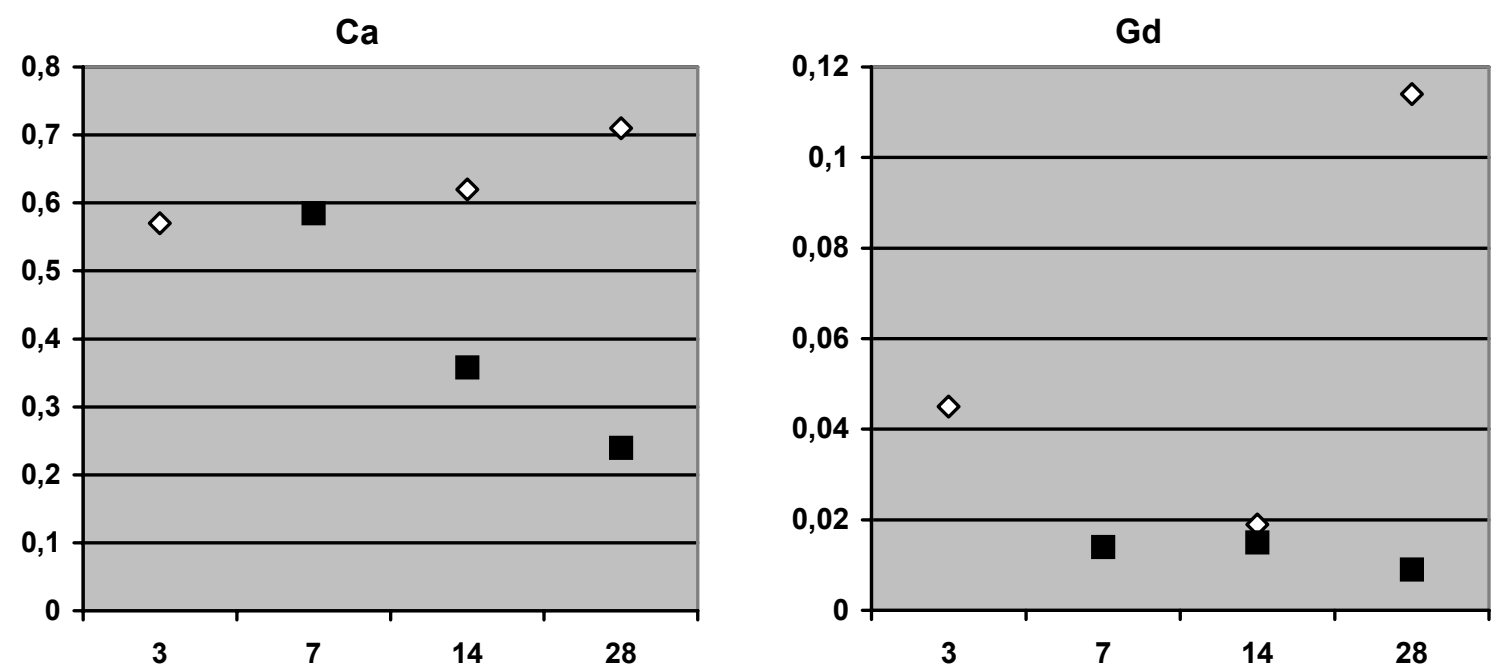

Ti
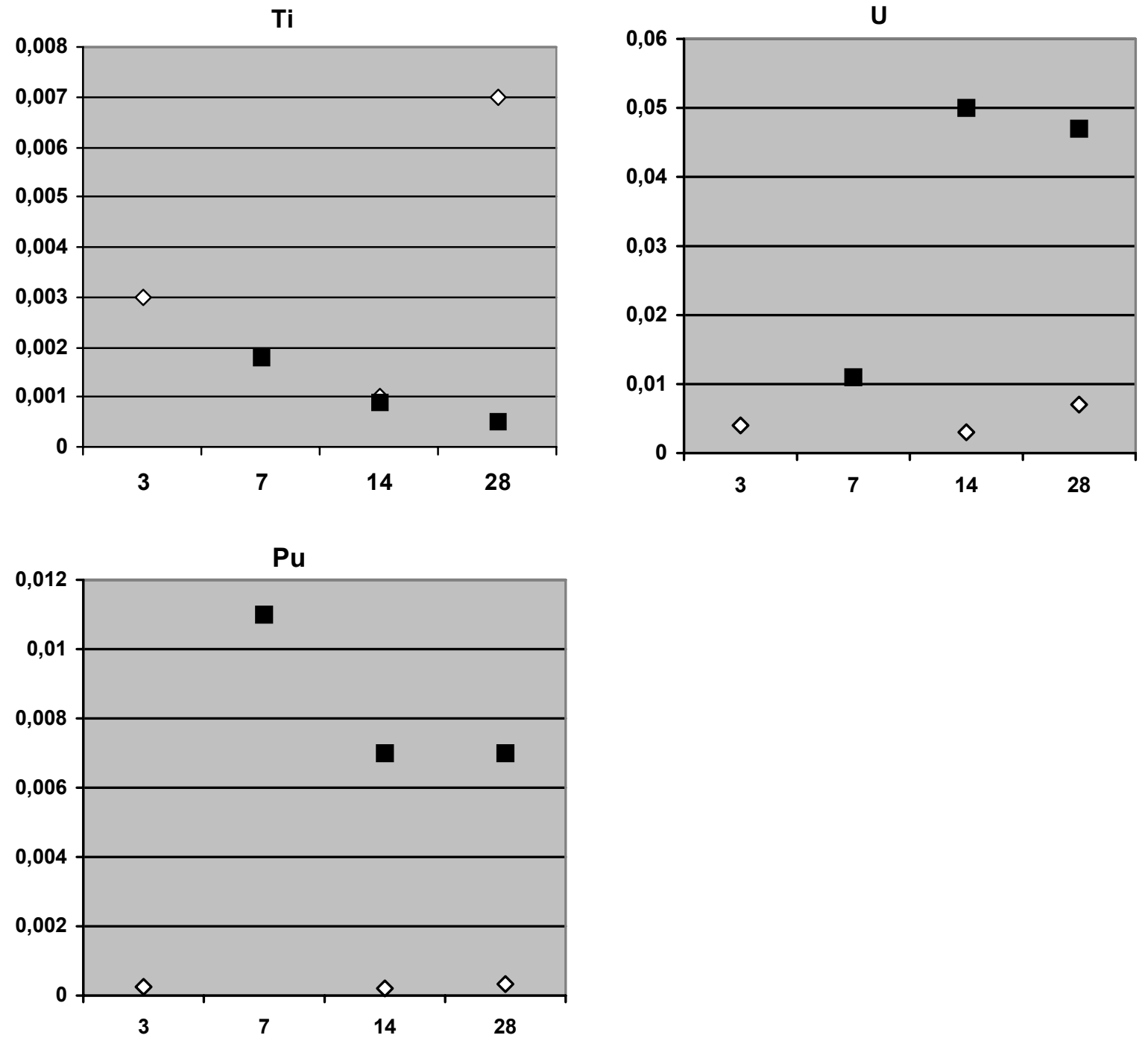

Fig. 5. Leaching rates after ceramic production $(\diamond)$ and in 16 months of radiation damages ( $\square$ ): along horizontal axis, leaching period, days; along vertical axis, leaching rate, $R_{i}, g /\left(m^{2}{ }^{*}\right.$ day). 
Table 2 The normalized mass losses of ceramic components due to leaching.

\begin{tabular}{|c|c|c|c|c|c|c|c|c|c|c|}
\hline \multirow{3}{*}{$\begin{array}{l}\text { Leaching } \\
\text { period, days }\end{array}$} & \multicolumn{10}{|c|}{$N L_{i}, g / m^{2}$} \\
\hline & \multicolumn{2}{|c|}{$\mathrm{Ca}$} & \multicolumn{2}{|c|}{ Gd } & \multicolumn{2}{|c|}{$\overline{T i}$} & \multicolumn{2}{|c|}{$\mathbf{U}$} & \multicolumn{2}{|c|}{$\mathrm{Pu}$} \\
\hline & $\begin{array}{c}\text { after } \\
\text { production }\end{array}$ & $\begin{array}{c}\text { in } 16 \\
\text { months }\end{array}$ & $\begin{array}{c}\text { after } \\
\text { production }\end{array}$ & $\begin{array}{c}\text { in } 16 \\
\text { months }\end{array}$ & $\begin{array}{c}\text { after } \\
\text { production }\end{array}$ & in 16 months & $\begin{array}{c}\text { after } \\
\text { production }\end{array}$ & $\begin{array}{c}\text { in } 16 \\
\text { months }\end{array}$ & $\begin{array}{c}\text { after } \\
\text { production }\end{array}$ & $\begin{array}{c}\text { in } 16 \\
\text { months }\end{array}$ \\
\hline 3 & 1,7 & & 0,14 & & 0,007 & & 0,011 & & 0,0007 & \\
\hline 7 & & 4,09 & & 0,10 & & 0,013 & & 0,077 & & 0,077 \\
\hline 14 & 8,73 & 5,01 & 0,27 & 0,21 & 0,015 & 0,013 & 0,048 & 0,694 & 0,0029 & 0,100 \\
\hline 28 & 20 & 6,72 & 0,32 & 0,26 & 0,022 & 0,014 & 0,197 & 1,324 & 0,0092 & 0,204 \\
\hline
\end{tabular}

Table 3. Leaching rates of ceramic components.

\begin{tabular}{|c|c|c|c|c|c|c|c|c|c|c|}
\hline \multirow{3}{*}{$\begin{array}{l}\text { Leaching } \\
\text { period, days }\end{array}$} & \multicolumn{10}{|c|}{$R_{i}, g /\left(m^{2 *}\right.$ day $)$} \\
\hline & \multicolumn{2}{|c|}{$\mathrm{Ca}$} & \multicolumn{2}{|c|}{ Gd } & \multicolumn{2}{|c|}{$\mathrm{Ti}$} & \multicolumn{2}{|c|}{$\mathbf{U}$} & \multicolumn{2}{|c|}{$\mathrm{Pu}$} \\
\hline & $\begin{array}{c}\text { after } \\
\text { production }\end{array}$ & $\begin{array}{c}\text { in } 16 \\
\text { months }\end{array}$ & $\begin{array}{c}\text { after } \\
\text { production }\end{array}$ & $\begin{array}{c}\text { in } 16 \\
\text { months }\end{array}$ & $\begin{array}{c}\text { after } \\
\text { production }\end{array}$ & $\begin{array}{c}\text { in } 16 \\
\text { months }\end{array}$ & $\begin{array}{c}\text { after } \\
\text { production }\end{array}$ & $\begin{array}{c}\text { in } 16 \\
\text { months }\end{array}$ & $\begin{array}{c}\text { after } \\
\text { production }\end{array}$ & $\begin{array}{c}\text { in } 16 \\
\text { months }\end{array}$ \\
\hline 3 & 0,570 & & 0,045 & & 0,003 & & 0,004 & & 0,00025 & \\
\hline 7 & & 0,585 & & 0,014 & & 0,0018 & & 0,011 & & 0,011 \\
\hline 14 & 0,620 & 0,358 & 0,019 & 0,015 & 0,001 & 0,0009 & 0,003 & 0,050 & 0,00021 & 0,007 \\
\hline 28 & 0,710 & 0,240 & 0,114 & 0,009 & 0,007 & 0,0005 & 0,007 & 0,047 & 0,00033 & 0,007 \\
\hline
\end{tabular}




\section{Discussion}

The experimental data of x-ray analysis obtained at the last stage of the investigations (April-August, 2001) allow us to characterize the main peculiarities of the ceramic current state.

The diffractometric investigations testify that the ceramics is close to absolute roentgen-amorphous state. However, the data obtained by photomethod show that the process of amorphization is not over yet, although it is close to its completion. It seems that the minor difference in evaluation of the ceramics state by these two methods can be explained by methodical causes. One of them can be related to the degree of $x$-ray weakening coming through the seal coverings of the specimens. High sensitivity of the photomethod can be caused by lower degree of $x$-rays absorbing by the quartz capillary (the wall thickness is $15-20 \mu \mathrm{m}$ ) as compared to absorbing in the covering of the diffractometric dish (Be foil + Teflon film). However, we can admit that dispersion of the ceramic material may have certain impact. The ceramic specimens prepared by thorough crushing to powder for the photomethod may receive a somewhat lower dose during self-irradiation as a result. Due to that, the rate of their amorphization may slow down.

The previous data [1] gave a crude evaluation of the absolute amorphization ( 600 days) based on the curve showing the decrease of the integral intensity of the pyrochlore $x$-ray reflex with indexes 222 . With respect to recent data (over $\sim 500$ days after the ceramics synthesis), it should be expected that this evaluation seems to be close to the reality, or the period of absolute roentgen-amorphization will be a little bit less.

The data analysis characterizing chemical stability of the investigated ceramics show that, for the majority of the components, the natural increase of the normalized mass losses and decrease of leaching rate are observed with the increase of the leaching period. The matrix components in 16 months after the ceramics production on the $28^{\text {th }}$ day of leaching can be ordered according to their descending leaching rates and the normalized mass losses: $\mathrm{Ca}, \mathrm{U}, \mathrm{Gd}, \mathrm{Pu}, \mathrm{Ti}$. For the order above, the values of the normalized mass losses were in the range of $6.72-0.0144 \mathrm{~g} / \mathrm{m}^{2}$, and the leaching rate values covered the ranges of $0.24-0.000515 \mathrm{~g} /\left(\mathrm{m}^{2 *}\right.$ day $)$, respectively.

The comparison with the previously obtained data on the same ceramics show a 20-30-fold increase of the normalized mass loss and leaching rate for the radionuclides (uranium and plutonium) over the corresponding leaching periods. At the same time, the contrary tendency is noted for the stable matrix components, i.e., the values of the normalized mass loss and leaching rate are less by $2-10$. This fact seems to be conditioned by the influence of the radiation damages. Alpha-particles and recoil nucleuses gradually deform the crystalline lattice resulting in weakening of actinide capture and increase in the leaching rates.

The values of the normalized mass loss and leaching rate lead to the conclusion about the decrease of the ceramic chemical stability, and primarily it concerns uranium and plutonium. However, the values still allow speaking about reliable Pu immobilization into the given matrix. Even in the state close to the amorphous one, the Pu leaching rate does not exceed $0.012 \mathrm{~g} /\left(\mathrm{m}^{2}\right.$ *day) on the $7^{\text {th }}$ day and decreases with the increase of the leaching period. 


\section{Conclusion}

The impact of Pu-238 alpha-decay on the crystalline structure and chemical stability of the deeply damaged titanate ceramics intended for $\mathrm{Pu}$ immobilization was investigated by $\mathrm{x}$-ray analysis and using the chemical stability tests. The ceramic was found to be in the state close to the roentgen-amorphous one under the integral self-irradiation dose of $2 \cdot 4^{*} 10^{18}$ alpha-decay $/ g$.

Weakening of the capability of the ceramic to capture $\mathrm{Pu}$ and $\mathrm{U}$ with the accumulated radiation damage was found. However, the values of the normalized mass loss and leaching rate allow speaking about high enough chemical stability of the ceramic material under the specified self-irradiation dose.

\section{References}

1. Radiation Damage Studies of Plutonium Ceramics. Contract B501111. Investigation of $\mathrm{Hf}^{238} \mathrm{Pu}-\mathrm{U}$ ceramic specimens amorphization and swelling. SSC RIAR, Dimitrovgrad. Progress report (14), 2000, $12 \mathrm{p.}$

2. Radiation Damage Studies of Plutonium Ceramics. Contract B501111. Production of $\mathrm{Hf}_{-}{ }^{238} \mathrm{Pu}-\mathrm{U}$ ceramic specimens. SSC RIAR, Dimitrovgrad. Report (04), 2000, 13 p.

3. Radiation Damage Studies of Plutonium Ceramics. Contract B501111. Investigation of $\mathrm{Hf}^{238} \mathrm{Pu}-\mathrm{U}$ ceramic specimens. SSC RIAR, Dimitrovgrad. Final report (07), 2000, 18 p.

4. American Society for Testing and Materials (ASTM) C 1220. 1998. Standard Method for Static Leaching Of Monolithic Waste Forms for Disposal of Radioactive Waste, C 1220 - 98, West Conshohoken, Pennsylvania. 


\title{
Investigation of Accelerated Radiation Damages of the US-specified Pyrochlore-like Ceramics Containing ${ }^{238} \mathrm{Pu}$ (B506205)
}

\author{
I. A. Kulikov, A. A. Vashman, V. M. Filine, T. N. Ananina, V. E. Samsonov, \\ O. Y. Panov, VNIINM
}

\begin{abstract}
Accelerated radiation damage of the US-specific pyrochlore-like ceramics containing plutonium, and its physico-chemical properties have been investigated using $\mathrm{x}$-ray diffraction (XRD) spectroscopy, positron annihilation, and electron spin resonance (ESR), scanning electron microscopy (SEM), and microprobe characterization measurements. A wide range of research activities using characterization analyses, including leach rate definitions, density, and microstrength measurements of the ceramics have shown that the properties of the material that contains ${ }^{239} \mathrm{Pu}$ are unchanged 600 days after its fabrication.

Whereas, the ceramics that contain ${ }^{238} \mathrm{Pu}$ demonstrate reduced chemical stability, and the leach rate of Pu goes up 5 to 7 fold.
\end{abstract}

\section{Introduction}

The work was started at A. A. Bochvar All-Russian Research Institute of Inorganic Materials (VNIINM) under the scope of Agreement B506205, which is a part of the mutual collaboration between Lawrence Livermore National Laboratory (LLNL) in the US and Russian scientific institutions to investigate accelerated radiation damages in the US-specific pyrochlore-like ceramics intended for the immobilization of excess weapons plutonium.

\section{Experimental Tests}

The ceramics were synthesized at the Scientific Research Institute of Atomic Reactors (RIAR) in 2000 and delivered to VNIINM for eventual characterization measurements. The composition of the ceramics is given in Table 1. Ceramic samples were pellets of 5 and $10 \mathrm{~mm}$ in diameter, and 1.5-3.0 mm in thickness.

Table 1. The composition of the ceramic pellets.

\begin{tabular}{|l|c|c|c|c|c|c|}
\hline \multirow{2}{*}{ Ceramics } & \multicolumn{7}{|c|}{ Composition, \% mole. } \\
\cline { 2 - 7 } & $\mathbf{C a O}$ & $\mathrm{TiO}_{2}$ & $\mathbf{H f O}_{2}$ & $\mathbf{G d}_{2} \mathbf{O}_{3}$ & $\mathbf{P u O}_{2}$ & $\mathrm{UO}_{2}$ \\
\hline Ceramics, containing ${ }^{238} \mathrm{Pu}$ & 9,9 & 35,5 & 10,6 & 7,9 & 12,6 & 23,5 \\
\hline Ceramics, containing ${ }^{239} \mathrm{Pu}$ & 12,0 & 36,0 & 11,0 & 7,6 & 12,3 & 20,8 \\
\hline
\end{tabular}

Radiation alterations in the crystal structure were researched by means of XRD spectroscopy, positron annihilation, and ESR characterization methods. Morphology and element distribution upon the material were revealed using SEM and microprobe analysis. Chemical stability was characterized by definition of leach rates of ceramic major components pursuant to the MCC-1 procedure at 20 and $90^{\circ} \mathrm{C}$ in deionized water with $\mathrm{pH} \sim 5.8-6.7$. 


\section{Results}

\section{Positron annihilation measurements}

As shown earlier, the main structural components of the matrix being observed by positron annihilation are external electrons of oxygen ions, oxygen vacancies, as well as pores inside the lattice.

Parameters of the spectra calculated by means of the PATFIT-88 program are shown in Table 2, where $T_{1}, T_{2}$ and $T_{3}$ are life-time parameters and $I_{1}, I_{2}, I_{3}$ are their intensities. The errors of those parameters are pointed out in brackets. $T_{1 / 2}$ is a life-time parameter measured at a half of the peak's height; $T_{f}$ is a positron life-time specified for each the material and calculated from values $T_{i}$ and $I_{i}$.

As is evident from Table 2, positron life-time spectra are composed of three components. A portion of positrons annihilate at $150-180$ ps on the external electrons of oxygen ions. The timely component at $280-$ $310 \mathrm{ps}$ is characterized by positron annihilation in regular voids or in oxygen vacancies. The third component at 8.3 ps points out the formation of a positronium in pores or vacancies boundless. According to the current assessments of the link between positronium life times and pore sizes those characteristics are adherent to the pore radius of $2 \mathrm{~nm}$.

It is clear from Table 2 that immediately after annealing there is no a timely component related to pores. However, with the growth of irradiation dose rate, the concentration of pores goes up (see column $\mathrm{I}_{3}$ ).

Table 2. Life-time parameters of positrons in ${ }^{238} \mathrm{Pu}$-containing ceramics.

\begin{tabular}{|c|c|c|c|c|c|c|}
\hline Sample & $T_{1}, p s$ & $T_{2}, p s$ & $\mathrm{~T}_{3}, \mathrm{~ns}$ & $I_{1}, \%$ & $I_{2}, \%$ & $I_{3}, \%$ \\
\hline $\begin{array}{l}\text { №2*№4 annealed at } 1200 \\
{ }^{\circ} \mathrm{C} \text {, during } 4 \mathrm{~h}, 1 \text { day }\end{array}$ & $154(3)$ & $281(5)$ & & $38,3(4,3)$ & $61,7(4,3)$ & 0 \\
\hline №2*№4 48 days & $175(8)$ & $307(5)$ & $8,30(F)$ & $35,0(4,5)$ & $65,0(4,5)$ & $0,03(0,02)$ \\
\hline №2*№4 216 days & $167(10)$ & $290(5)$ & $8,30(F)$ & $32,9(4,7)$ & $66,9(4,7)$ & $0,21(0,01)$ \\
\hline
\end{tabular}

The experimental dependency of average life-time parameters in annealed samples from the time since ceramics fabrication is presented in Fig. 1. The average life-time of positrons has been calculated by the following formula: $T_{a v}=T_{1}{ }^{*} I_{1}+T_{2}{ }^{*} l_{2}$. The positron annihilation method allows us to define the transferring from the crystal state to amorphous one and back. It is obvious that the average life-time starts to increase rapidly and reaches the maximum close to 50 days and then gradually slows down. Such a type of dependency could be explained by accumulation of elementary defects in the oxide lattice (mostly interstitial oxygen ions and oxygen vacancies) and amorphous fields during self-irradiation. The increasing part of the graph demonstrates a quick accumulation of defects, and the slumping one shows ceramics amorphization. The effective time of amorphization (130 \pm 56 days) is correlated to the time ( $135 \pm 13$ days) determined at RIAR by means of XRD diffractometry. 


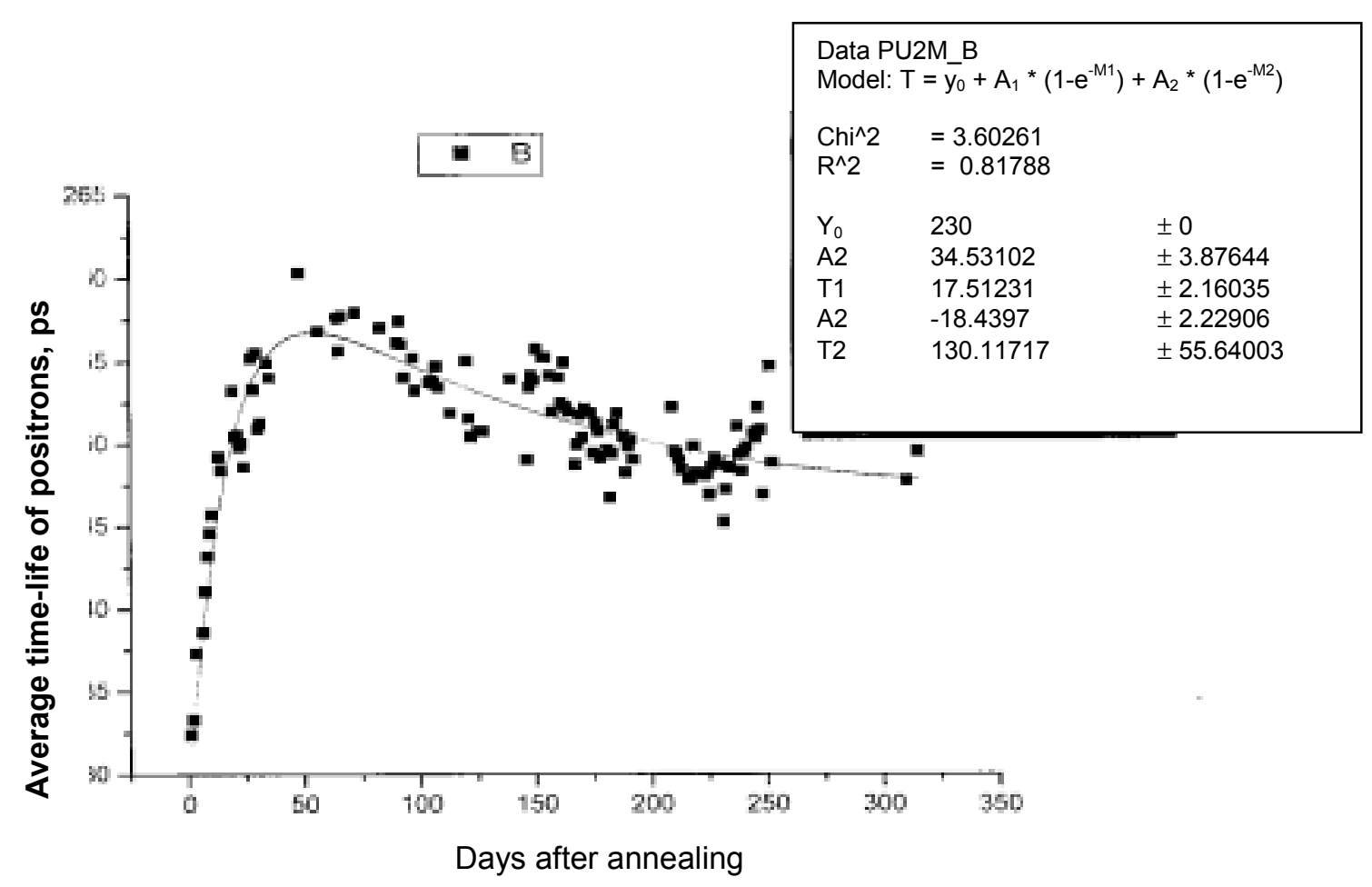

Fig. 1. The dependency of life-time of positrons in annealed ceramic samples from the time of fabrication.

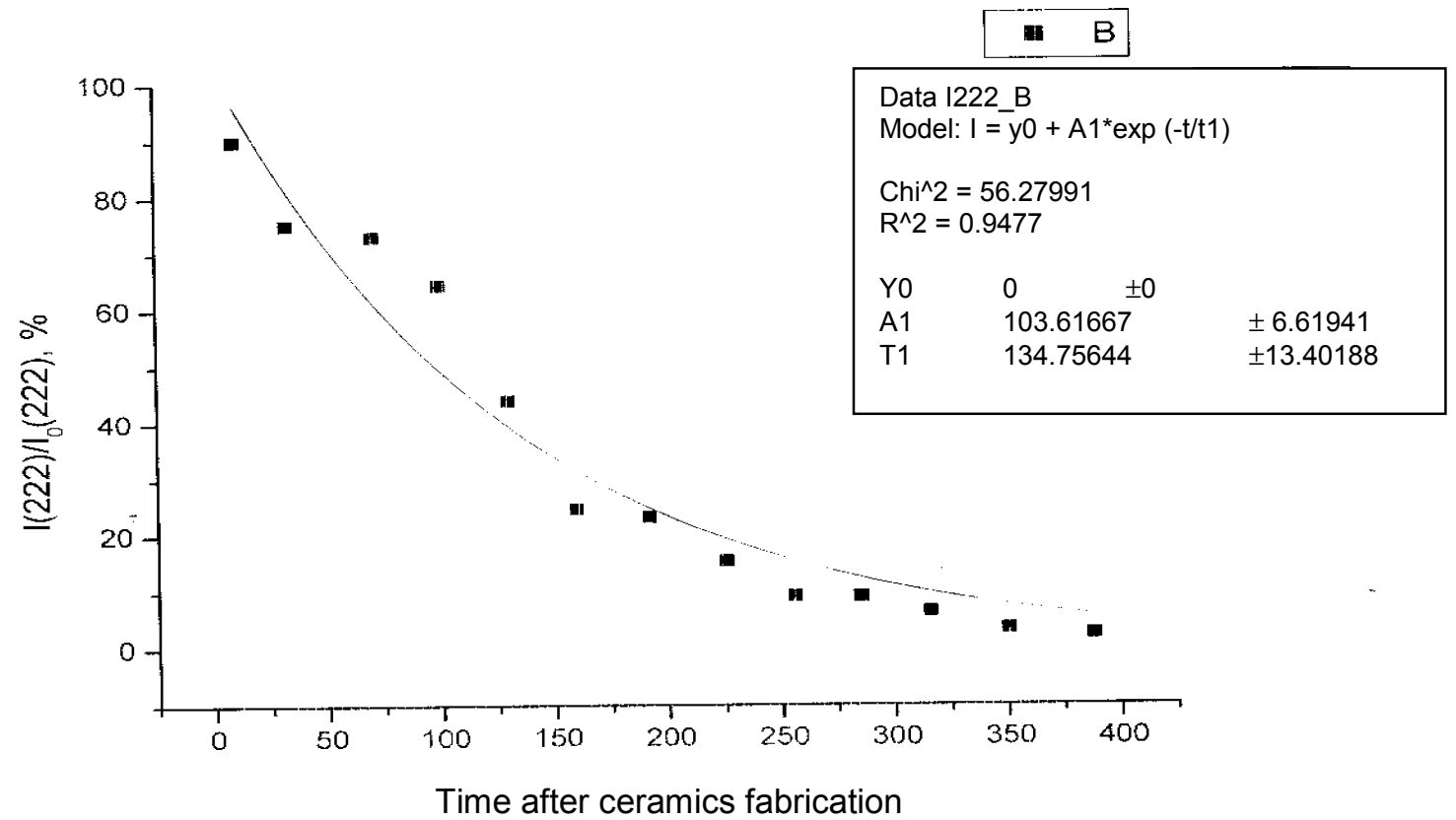

Fig. 2. The alteration of the intensity of reflex 222 of the pyrochlore phase depending upon the time since ceramics fabrication. 


\section{Electron spin resonance measurements}

The electron spin resonance method permits us to identify paramagnetic centers or inorganic free radicals, being formed in ceramic-type materials during self-irradiation. Besides, the method helps to define the influence of self-irradiation on the ceramics structure- in particular, the alteration of the nearby surrounding of paramagnetic ions included into the ceramics or available there in the form of admixtures.

With the pyrochlore ceramics studied a priori, one should expect signals being observable due to the presence of $\mathrm{Gd}$ (III) ions being in the basic state of ${ }^{8} \mathrm{~S}_{7 / 2}$ and having 7 unpaired electrons on 4-f orbital, as well as signals of stable paramagnetic centers generated from ${ }^{238} \mathrm{Pu}$ irradiation. Therewith, the substantial concentration of gadolinium will inevitably result in the exchange of unpaired electrons between neighbor ions. This widens the ESR signal making it uninformative.

In Fig. $3,^{1}$ there is an ESR spectrum of Pu-containing ceramics irradiated at $9 \cdot 10^{8} \mathrm{~Gy}$. The spectrum is caused from paramagnetic ions of Gd (III) and could be interpreted as a superposition of two broadening lines being differentiated by intensities and $\mathrm{g}$-factor. Besides, the most intensive line is characterized by $\mathrm{g}$ $=2.09$ and the less intensive one by $g=6.2$. Since they are too wide, there are no fine or ultrafine structures.

At the same time, if we look at the central part of the ESR spectrum more attentively (Fig. 3), one can identify the field with a slightly observable multiplication, which is composed of two duplets of the ultrafine structure $\left(A_{1}=13 \mathrm{Gs}, g_{1}=2.028\right.$ and $A_{2}=53 \mathrm{Gs}, g_{2}=2.024$, respectively $)$ and one singlet with $g_{3}=2.016$ (Fig. 4). ${ }^{1}$

The value $g_{3}$ and the widening of the singlet $(\sim 1.7 \mathrm{Gs})$ make it possible to associate it with unpaired delocalized electrons captured in vacancies or defects being generated as a result of the ceramics selfirradiation. Meanwhile, the two duplets correspond to inorganic free radicals of nuclei with spin $1 / 2$. In particular, those nuclei could be ${ }^{239} \mathrm{Pu}$ and ${ }^{3} \mathrm{He}$ or admixture atoms.

In general, the fact that we have determined the two duplets and the singlet on the ESR spectrum of the ceramics is very essential since in literature about radiochemistry of solids there are not any references regarding the origin and type of paramagnetic centers. The more profound scrutiny of ESR measurements of the ceramics would permit us to obtain important data about the mechanism of radiation damage of the ceramic materials.

\section{Electron scanning microscopy and microprobe analyses (SEM/EDS)}

It is well known that in the pyrochlore-like ceramics, the oxides are constituted as follows: $\mathrm{CaTiO}_{3}, \mathrm{HfTiO}_{4}$, $\mathrm{CdTi}_{2} \mathrm{O}_{8}, \mathrm{CaHfTi}_{2} \mathrm{O}_{7}, \mathrm{CaCeTi}_{2} \mathrm{O}_{7}, \mathrm{CaTiO}_{3}, \mathrm{HfTiO}_{4}, \mathrm{CdTi}_{2} \mathrm{O}_{8}, \mathrm{CaHfTi}_{2} \mathrm{O}_{7}, \mathrm{PuTi}_{2} \mathrm{O}_{6}, \mathrm{UTi}_{2} \mathrm{O}_{6}$. The average atomic ratio of metals to titanates was as $\mathrm{Ca} / \mathrm{Ti}-0.47,2 \mathrm{Cd} / \mathrm{Ti}-0.18, \mathrm{Hf} / \mathrm{Ti}-0.11,2 \mathrm{U} / \mathrm{Ti}-0.34,2 \mathrm{Pu} / \mathrm{Ti}-$ 0.20 . Therefore, $\Sigma \mathrm{Me} / \mathrm{Ti}=1.3$ indicates some lack of titanium oxide for linking all the oxides into titanates. Actually, XRD analysis of the Pu-containing ceramics has shown that there is no titanium oxide in the material (Fig. 5).

\footnotetext{
${ }^{1}$ Figures 3 and 4 were not supplied.
} 


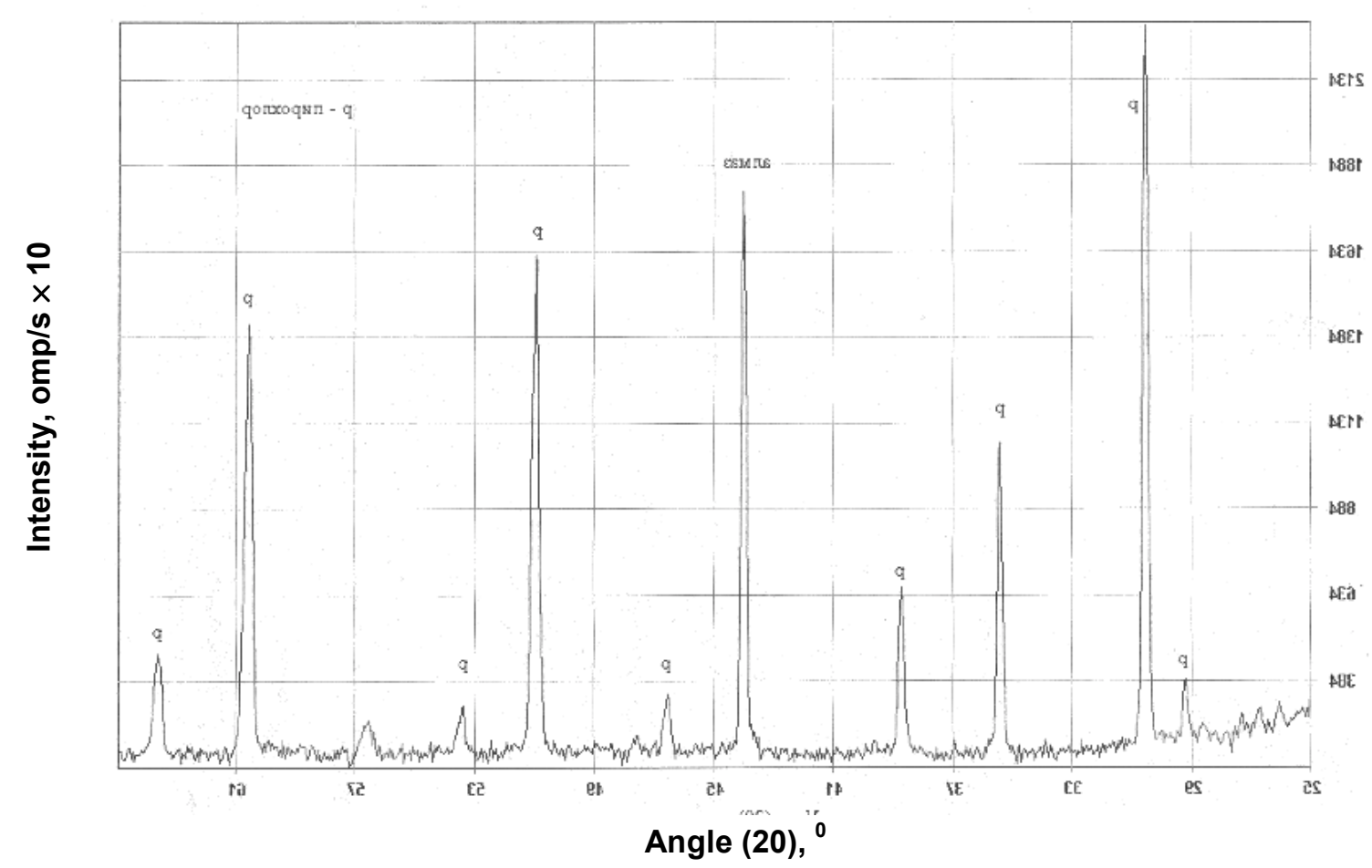

Fig. 5. Diffractogram of original Pu-containing ceramics.

Two different surfaces of $50 \times 50$ square were characterized in the emission of $\mathrm{Ti}, \mathrm{Ca}, \mathrm{Hf}, \mathrm{Cd}, \mathrm{Pu}$, and $\mathrm{U}$. Zones for scanning were selected occasionally.

Images of the ceramics in Ti characteristic emission show that the material contains microzones both enriched (more light) and with no $\mathrm{Ti}$ (more dark). Zones enriched with $\mathrm{Ti}$ are prolonged, uneven bundles 7-15 microns in width. Zones without Ti are also uneven with $5-10 \times 5-10$ microns. It is necessary to mention that enriched zones are situated nearby ones with poor Ti, however this fact is occurred not so usually.

Images of the same surfaces in the $\mathrm{Ca}, \mathrm{Pu}, \mathrm{U}, \mathrm{Gd}$, $\mathrm{Hf}$ characteristic emission show that the zones enriched with $\mathrm{Ti}$ are, at the same time, depleted in other elements $(\mathrm{Ca}, \mathrm{Gd}, \mathrm{Pu}, \mathrm{U})$. In general, one needs to note that the ceramic zones are characterized by uneven distribution of elements.

Table 3 gives the oxide content in the microzones.

One can see from Table 3 that all the elements are distributed upon the sample unevenly. For example, the concentration of uranium changes from 1.2\% (Field 1, zone 1) to $79 \%$ (Field 2, zone 1). This field is expectedly composed of uranium dioxide (i.e., depleted of Ti, which is only $0.2 \mathrm{wt} \%$ ). Those results are correlated with data presented in Fig. 6.

Therefore, it has been demonstrated that the structure of the ceramics is uneven.

One needs to mention that we failed to prepare a ceramic sample, containing ${ }^{238} \mathrm{Pu}$, for SEM/EDS analyses due to its unsatisfactory state. 


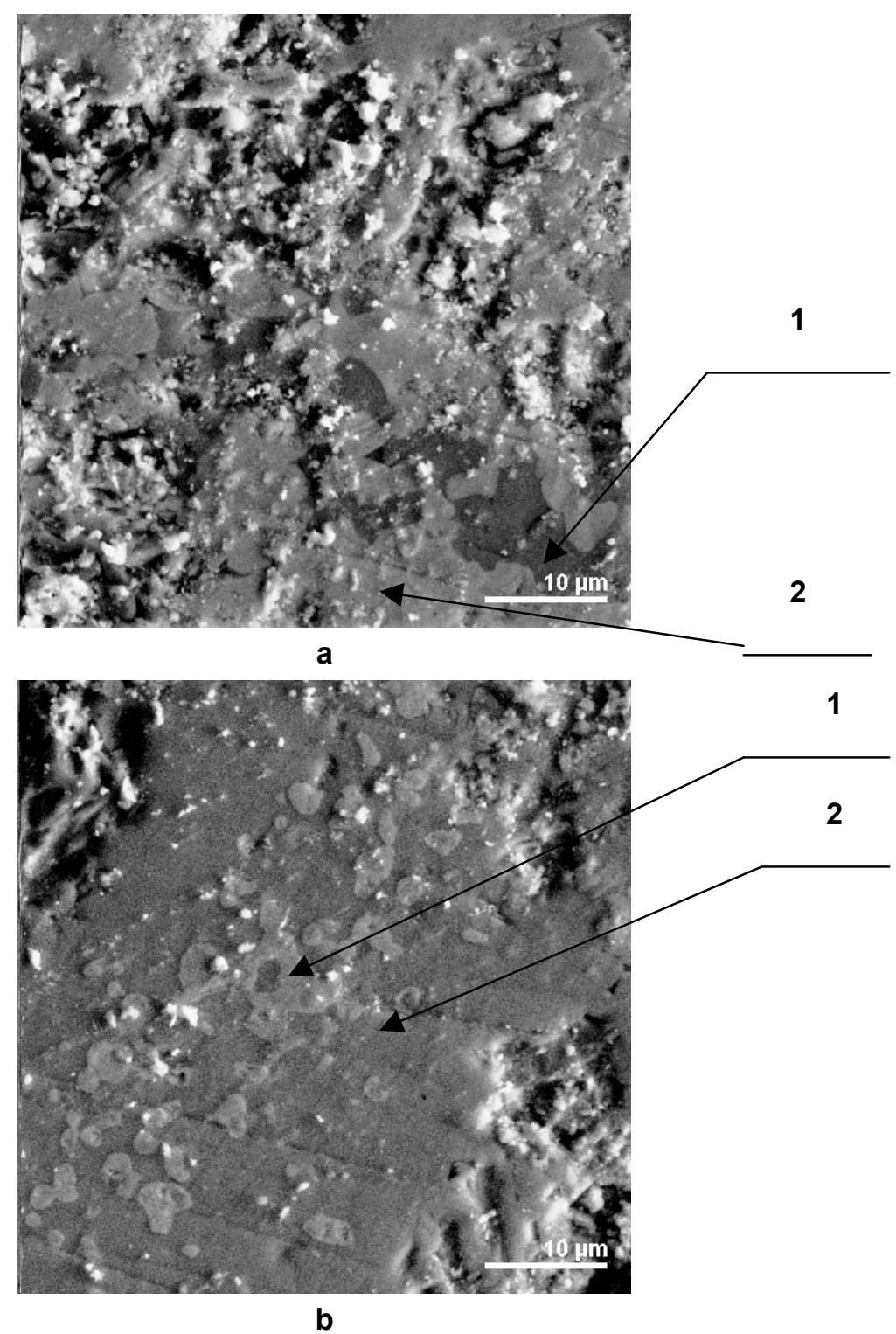

Fig. 6. Image of surface of the ceramics with ${ }^{239} \mathrm{Pu}$ in secondary electrons (a) zone 1; (b) zone 2.

Table 3. The concentrations of elements in the ceramics (wt) characterized by SEM/EDS analysis.

\begin{tabular}{|l|c|r|r|r|}
\hline & \multicolumn{2}{|c|}{ Field 1 (Fig 6, a) } & \multicolumn{2}{c|}{ Field 2 (Fig 6, b) } \\
\hline & Zone 1 & Zone 2 & Zone 1 & Zone 2 \\
\hline $\mathrm{Ca}$ & 0.33 & 7.5 & 0.6 & 8.2 \\
\hline $\mathrm{Cd}$ & 0.2 & 8.2 & 1.6 & 2.8 \\
\hline $\mathrm{Hf}$ & 15.3 & 8.5 & 0.17 & 7.5 \\
\hline $\mathrm{U}$ & 1.2 & 25.6 & 79.0 & 36.0 \\
\hline $\mathrm{Pu}$ & 0.2 & 8.4 & 6.2 & 4.6 \\
\hline $\mathrm{Ti}$ & 48 & 18.9 & 0.2 & 11.0 \\
\hline $\mathrm{O}$ & 34.8 & 22.9 & 12.2 & 29.9 \\
\hline
\end{tabular}




\section{Leaching tests}

The chemical stability of the Pu-containing ceramics was measured by defining leach rates of Pu and some other structural components $(\mathrm{U}, \mathrm{Ca})$ at 20 and $90^{\circ} \mathrm{C}$ pursuant to the MCC-1 procedure. Table 4 presents leach rate values of $\mathrm{Pu}, \mathrm{U}$, and $\mathrm{Ca}\left(\mathrm{g} / \mathrm{m}^{2} \cdot \mathrm{s}\right)$ after 3 days experimental testing. The tests were carried out in the period of up to 515 days after ceramics fabrication. One needs to say that while performing experimental tests, it is important to bear in mind not only $\mathrm{Pu}$ in the leachant, but also $\mathrm{Pu}$ strips (adsorbed onto container walls). As is clear from Table 4, depending upon the plutonium isotope and leaching temperature, the concentration of $\mathrm{Pu}$ in the leachant changes from 17.5 to $75 \%$, and ranges in the strips from 25 to $84.6 \%$. Leaching tends to be most rapid at $90^{\circ} \mathrm{C}$. This is well illustrated by experimental data of Pu leaching given in Figs. 7 and 8.

At the initial stage of storage (up to 250 days), one can observe that the leach rate of Pu goes up slightly with the growth of time of storage. At the same time, if the ceramics are kept for a period of more than 250 days, the leach rate of ${ }^{238} \mathrm{Pu}$ boosts up rapidly versus that of ${ }^{239} \mathrm{Pu}$. Based on the work implemented at RIAR, the ceramics seemingly deteriorate after 200 days due to self-irradiation and amorphization.

This, in turn, results in increasing leach rates of $\mathrm{Pu}$.

Table 4. Leach rates of Plutonium from the ceramics.

\begin{tabular}{|c|c|c|c|c|c|}
\hline \multirow{2}{*}{$\begin{array}{l}\text { Leaching } \\
\text { temperature }\end{array}$} & \multirow{2}{*}{$\begin{array}{l}\text { Plutonium } \\
\text { isotope }\end{array}$} & \multirow{2}{*}{$\begin{array}{c}\text { Storage time, } \\
\text { days }\end{array}$} & \multirow{2}{*}{$\begin{array}{l}\text { Leach rate, } \\
\text { g/m } / \mathrm{m}^{2 *} \text { day. }\end{array}$} & \multicolumn{2}{|c|}{ Content, \% } \\
\hline & & & & In leachant & In strips \\
\hline \multirow[t]{7}{*}{$20^{\circ} \mathrm{C}$} & \multirow[t]{3}{*}{ Pu-239 } & 180 & $4,0 * 10^{-5}$ & 75,0 & 25,0 \\
\hline & & 270 & $5,3^{*} 10^{-5}$ & - & - \\
\hline & & 515 & $7,4^{*} 10^{-5}$ & 68,0 & 32,0 \\
\hline & \multirow[t]{4}{*}{ Pu-238 } & 180 & $6,5^{*} 10^{-4}$ & - & - \\
\hline & & 270 & $8,0^{*} 10^{-4}$ & 71,3 & 28,7 \\
\hline & & 365 & $1,0^{*} 10^{-3}$ & 69,1 & 29,9 \\
\hline & & 515 & $1,4^{*} 10^{-3}$ & 65,0 & 35,0 \\
\hline \multirow[t]{7}{*}{$90^{\circ} \mathrm{C}$} & \multirow[t]{3}{*}{ Pu-239 } & 270 & $1,2 * 10^{-3}$ & 15,4 & 84,6 \\
\hline & & 365 & $1,4^{*} 10^{-3}$ & 25,0 & 75,0 \\
\hline & & 515 & $1,7^{*} 10^{-3}$ & 29,4 & 70,6 \\
\hline & \multirow[t]{4}{*}{ Pu-238 } & 180 & $1,8^{*} 10^{-3}$ & 33,3 & 66,7 \\
\hline & & 270 & $2,0 * 10^{-3}$ & 27,5 & 72,5 \\
\hline & & 365 & $2,7^{*} 10^{-3}$ & 22,3 & 77,7 \\
\hline & & 515 & $5,6^{*} 10^{-3}$ & 17,5 & 82,5 \\
\hline
\end{tabular}



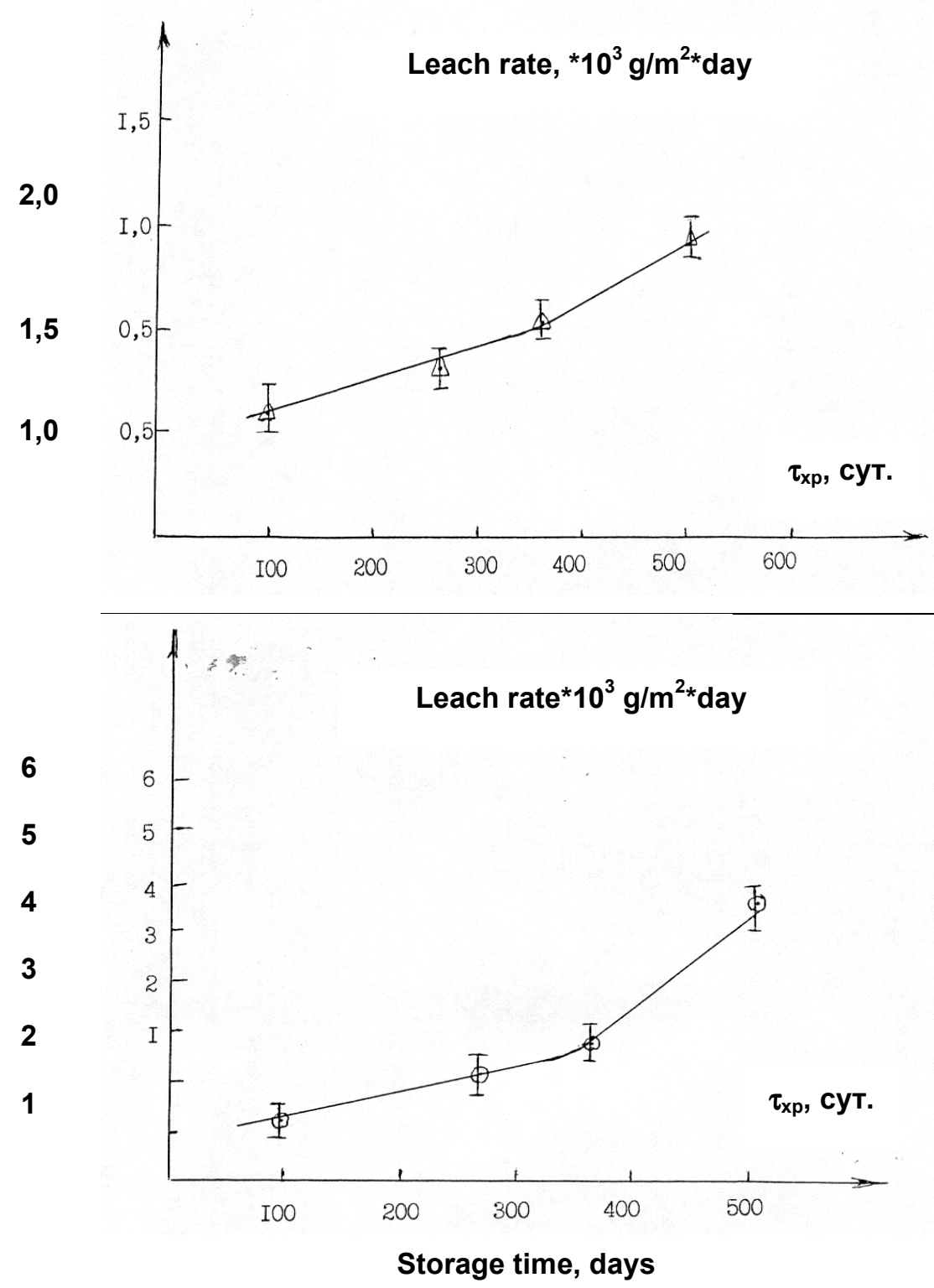

Fig. 7. Leach rates of ${ }^{238} \mathrm{Pu}$ depending upon storage time and leaching temperature (a) at $20^{\circ} \mathrm{C}$ and (b) at $90^{\circ} \mathrm{C}$. 

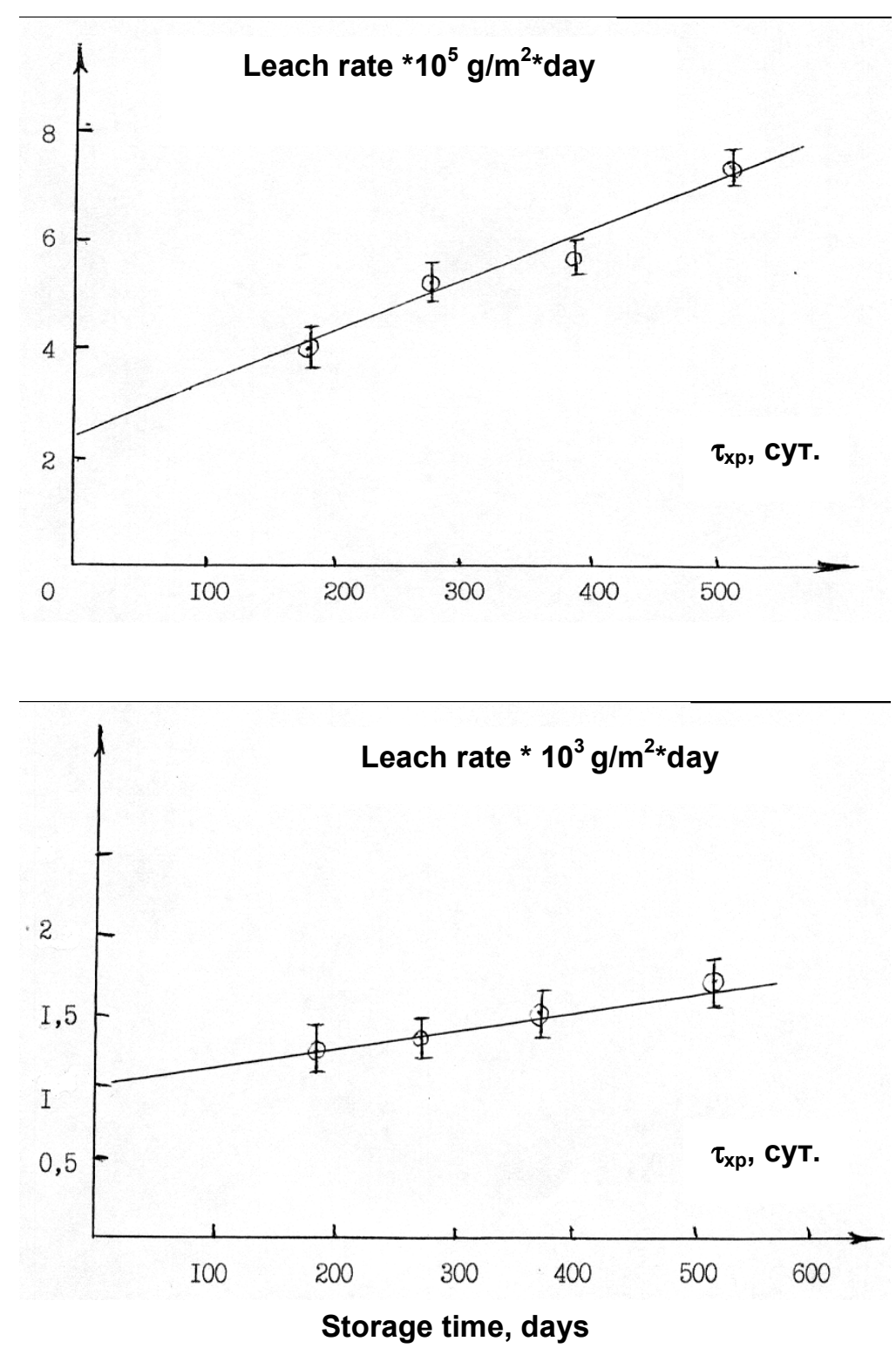

Fig. 8. Leach rates of ${ }^{239} \mathrm{Pu}$ depending upon storage time and leaching temperature (a) at $20^{\circ} \mathrm{C}$ and (b) at $90^{\circ} \mathrm{C}$. 


\section{Conclusions}

By means of XRD diffraction, positron annihilation, electron spin resonance, and SEM/EDS, the influence of self-irradiation on physico-chemical properties of the US-specified pyrochlore ceramics has been investigated.

It was shown that the structure of the material is uneven. The distribution of elements in the ceramic is also unequal. A suggestion has been made that there is a zone enriched totally with uranium.

The formation of vacancies and pores in the crystal lattice and interstitial shift of oxygen ions were established for the ceramics that contain ${ }^{238} \mathrm{Pu}$ after annealing at $1200^{\circ} \mathrm{C}$ using positron annihilation analysis. It was demonstrated that with the growth of the irradiation dose rate, defects and amorphous zones are prone to accumulate in the matrix. After 150 days storage time, the ceramics starts to become amorphous.

The leach rate of ${ }^{238} \mathrm{Pu}$ measured according to the MCC-1 procedure goes up substantially at $90^{\circ} \mathrm{C}$ starting 250 days after ceramics fabrication. 


\section{KRI Radiation Damage Studies of the US Pu Ceramics (B506203)}

\section{B. E. Burakov, E. B. Anderson, KRI}

\section{Introduction and Brief Summary of Previous Results}

In the framework of Agreement B506203, the V.G. Khlopin Radium Institute (KRI) has received samples of ${ }^{238-9} \mathrm{Pu}$-doped pyrochlore ceramic fabricated at the Research Institute of Atomic Reactors (RIAR) under Agreement B501111. At the end of July 2000, these samples arrived in St. Petersburg and were taken off containers. At present they are still used for radiation damage study experiments. The first results of ceramic examinations were obtained 4 months after ceramic fabrication.

All samples of pyrochlore ceramics doped with ${ }^{238} \mathrm{Pu}$ and ${ }^{239} \mathrm{Pu}$ were prepared under the same conditions using a Lawrence Livermore National Laboratory (LLNL) procedure. Although this procedure requires the following typical chemical composition (in wt\%):

$$
\mathrm{TiO}_{2}-35.86 ; \mathrm{HfO}_{2}-10.65 ; \mathrm{Gd}_{2} \mathbf{O}_{3}-7.95 ; \mathrm{CaO}-9.95 ; \mathrm{PuO}_{2}-11.90 ; \mathbf{U O}_{2}-23.69 \text {, }
$$

the $\mathrm{Pu}$ and $\mathrm{U}$ contents were slightly changed in order to increase the concentration of the ${ }^{238} \mathrm{Pu}$ isotope to $8.7 \mathrm{wt} \%$ (total content of $\mathrm{PuO}_{2}-12.6 \mathrm{wt} \%$ ).

The main features of these samples are shown in Tables 1 and 2.

Table 1. Features of pyrochlore ceramic samples doped with ${ }^{238} \mathrm{Pu}$ (average density $4.8 \mathrm{~g} / \mathrm{cm}^{3}$ ).

\begin{tabular}{|c|c|c|c|}
\hline Sample \# & Pellet weight, gram & Diameter, $\mathbf{~ m m}$ & Height, $\mathbf{~ m m}$ \\
\hline 19 & 0.1846 & 5.14 & 1.83 \\
\hline 20 & 0.1845 & 5.15 & 1.83 \\
\hline 21 & 0.1940 & 5.14 & 1.91 \\
\hline 3 & 0.7206 & 10.61 & 1.74 \\
\hline 8 & 0.6742 & 10.65 & 1.58 \\
\hline
\end{tabular}

Table 2. Features of pyrochlore ceramic samples doped with ${ }^{239} \mathrm{Pu}$ (average density $4.9 \mathrm{~g} / \mathrm{cm}^{3}$ ).

\begin{tabular}{|c|c|c|c|}
\hline Sample \# & Pellet weight, gram & Diameter, $\mathbf{~ m m}$ & Height, $\mathbf{m m}$ \\
\hline 13 & 0.5991 & 10.66 & 1.33 \\
\hline 18 & 0.5828 & 10.68 & 1.29 \\
\hline 2 & 0.2733 & 5.15 & 2.75 \\
\hline 9 & 0.2803 & 5.25 & 2.75 \\
\hline 14 & 0.2496 & 5.15 & 2.47 \\
\hline
\end{tabular}

Two small (5-mm diam) ceramic pellets doped with ${ }^{238} \mathrm{Pu}$ and ${ }^{239} \mathrm{Pu}$ were placed into the same microprobe specimen holder (with acrylic resin matrix) and polished. This specimen was studied by optical microscopy (in reflection light) and then, after Al-coating, examined by SEM and microprobe analysis. For qualitative microprobe analysis, we used the "Camebax" SEM equipped with WDS and cathodoluminescence spectrometers.

The only visible difference between samples when examined in the optical microscope (4 months after ceramic fabrication, at cumulative dose $26 \times 10^{23}$ alpha decays $/ \mathrm{m}^{3}$ for ceramic doped with ${ }^{238} \mathrm{Pu}$ ) is the 
presence of micro and macro-cracks in the matrix of ${ }^{238} \mathrm{Pu}$-doped ceramic. Both ceramics contain inclusions of rutile.

Numerous inclusions of newly formed U-oxide phase (without significant Pu admixture) were observed by SEM method in the matrix of the ${ }^{238} \mathrm{Pu}$-doped sample. Interpretation of $\mathrm{x}$-ray diffraction (XRD) results in combination with SEM data allowed us to identify this $\mathrm{U}$-oxide phase as $\mathrm{U}_{2} \mathrm{O}_{5}$.

Two big (10 mm diam) ceramic pellets doped with ${ }^{238} \mathrm{Pu}$ and ${ }^{239} \mathrm{Pu}$ were placed into a special sample handler with a Be-window, which was developed in $\mathrm{KRI}$ for XRD analysis of highly radioactive materials.

Precision XRD analysis was carried out under the following conditions: $\mathrm{CoK}_{\alpha}$ irradiation; current- $40 \mathrm{~mA}$; tube voltage- $30 \mathrm{kV}$, scan speed $2 \%$ min., step sampling- $0.01^{\circ}$. Calibration of the XRD detector is carried out using a polycrystalline alumina standard - reflections (104) and (1010). The equipment is calibrated immediately after it is repaired or cleaned, or the anode is replaced, etc.

One 5-mm diam pellet of each sample, one doped with ${ }^{238} \mathrm{Pu}$ and the other with ${ }^{239} \mathrm{Pu}$, were placed on the bottom of Teflon ${ }^{\mathrm{TM}}$ test vessels with distilled water and then set at $90^{\circ} \mathrm{C}$ in the oven. The ratio of surface area to water volume was 1:10. The test duration for these two samples was 28 days without breaking stages. Plutonium and $U$ absorbed on the vessel walls were washed by nitric acid, analyzed separately, and then included in the final calculations. Normalized Pu and U mass loss (NL) are calculated as follows:

$$
\mathrm{NL}=\mathrm{A} \times \mathrm{W}_{0} / \mathrm{A}_{0} \times \mathrm{S},
$$

where: $A$ - total activity of $\mathrm{Pu}$ or $\mathrm{U}$ in the water solution after leaching,

Bq; $A_{0}$ - the initial activity of Pu or $U$ in the specimen,

$\mathrm{Bq} ; W_{0}-$ the initial mass of the specimen, gram;

$S$ - specimen surface area without correction on ceramic porosity, $\mathrm{m}^{2}$.

The first results of the MCC-1 leach tests have shown that $\mathrm{Pu}$ and $\mathrm{U}$ releases from ceramic doped with

${ }^{238} \mathrm{Pu}$ is two or three orders of magnitude higher in comparison with that of the sample doped with ${ }^{239} \mathrm{Pu}$.

This paper summarizes all the results obtained during the two years of research.

\section{XRD analysis}

The intensity of ${ }^{238} \mathrm{Pu}$-doped pyrochlore $\mathrm{x}$-ray reflections decreased and the full width of peak half maximum (FWHM) increased depending on cumulative dose (Table 3, Fig. 1). No significant changes of XRD spectra over time were observed for ceramic doped with ${ }^{239} \mathrm{Pu}$.

Table 3. Main features of $\mathrm{x}$-ray diffraction peaks of pyrochlore ceramic doped with ${ }^{238} \mathrm{Pu}$ after the following cumulative doses (in alpha decays $/ \mathrm{m}^{3} \times 10^{23}$ ): (1) 26 ; (2) 43 ; (3) 57 ; (4) 82 ; (5) 110 .

\begin{tabular}{|l|c|l|c|c|c|c|}
\hline$\#$ & $\begin{array}{c}\text { Intensity } \\
\mathbf{( 3 1 1 )}\end{array}$ & $\begin{array}{c}\text { FWHM } \\
\mathbf{( 3 1 1 )}\end{array}$ & $\begin{array}{c}\text { Intensity } \\
\mathbf{( 2 2 2}\end{array}$ & $\begin{array}{c}\text { FWHM } \\
\mathbf{( 2 2 2}\end{array}$ & $\begin{array}{c}\text { Intensity } \\
\mathbf{( 4 0 0 )}\end{array}$ & $\begin{array}{c}\text { FWHM } \\
\mathbf{( 4 0 0 )}\end{array}$ \\
\hline$(1)$ & 108 & 0.420 & 1535 & 0.475 & 492 & 0.465 \\
\hline$(2)$ & 75 & 0.435 & 1017 & 0.495 & 307 & 0.495 \\
\hline$(3)$ & 53 & No data & 643 & 0.540 & 174 & 0.510 \\
\hline$(4)$ & No data & No data & 373 & 0.690 & 86 & 0.780 \\
\hline$(5)$ & No data & No data & 110 & No data & No data & No data \\
\hline
\end{tabular}




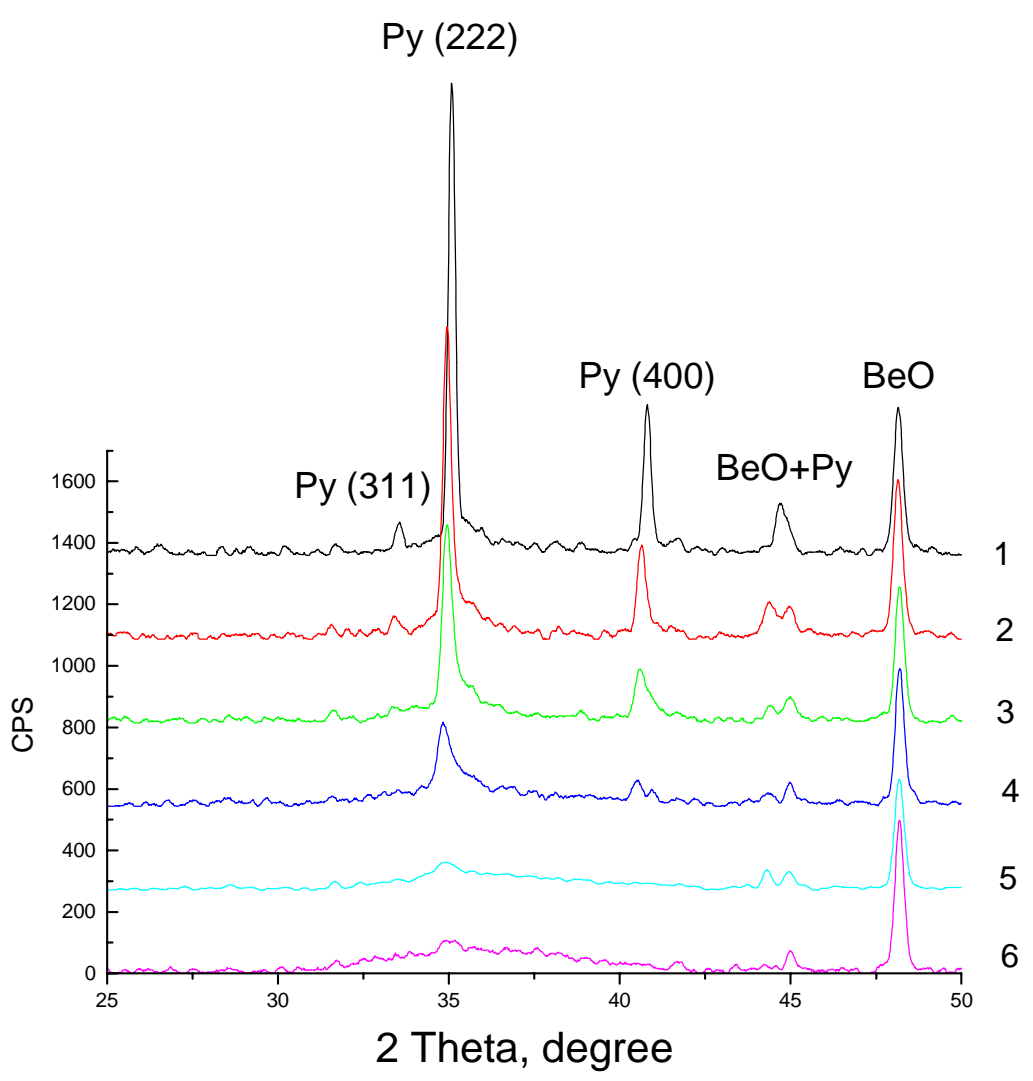

Fig. 1. XRD spectra of pyrochlore-based ceramics doped with ${ }^{238} \mathrm{Pu}$ after the following cumulative doses (in alpha decays $/ \mathrm{m}^{3} \times \mathbf{1 0}^{23}$ ): (1) 26; (2) 43; (3) 57; (4) 82; (5) 110; (6) 130.

$\mathbf{P y}$ - peaks of pyrochlore and $\mathbf{B e O}$ - reflections from Be-window that covered ceramic sample. There is a clear tendency for the main pyrochlore peak (222) to shift to a low-angle direction (position of pure $\left.\mathrm{Gd}_{2} \mathrm{Ti}_{2} \mathrm{O}_{7}\right)$.

\section{SEM and Microprobe Analyses}

For the first time, a sample of pyrochlore-based ceramic doped with ${ }^{238} \mathrm{Pu}$ was studied by SEM and EPMA methods. It was confirmed that under self-irradiation, the matrix of ${ }^{238} \mathrm{Pu}$-doped pyrochlore became inhomogeneous. It was not clearly proved that inclusions of $\mathrm{UO}_{x}$ in ${ }^{238} \mathrm{Pu}$-doped ceramic (Table 4, Fig. 2a,b) is a result of pyrochlore destruction due to radiation damage. However, these inclusions are typical only for the ${ }^{238} \mathrm{Pu}$-doped sample and visually their number significantly increased 1 year after the ceramic was examined for the first time by the SEM method. Also, the inclusions of Hf-Ti-Ca-O phase were identified (Table 5, Fig. $2 \mathrm{c}, \mathrm{d}$ ) in ceramic doped with ${ }^{238} \mathrm{Pu}$ only after a cumulative dose of $110 \times 10^{23}$ alpha decays $/ \mathrm{m}^{3}$. Their formation was observed in the most inhomogeneous areas of the pyrochlore (Table 5). In order to prove that the formation of $\mathrm{U}$-oxide and $\mathrm{Hf}-\mathrm{Ti}-\mathrm{Ca}-\mathrm{O}$ phases took place not only at the surface layer of ${ }^{238} \mathrm{Pu}$-doped ceramic because of reaction with radiolysis products of air medium, this sample was studied again by the SEM method immediately after re-polishing. 
Although the chemical composition of pyrochlore in different areas of ${ }^{239} \mathrm{Pu}$-doped sample is varied also (Table 6), this inhomogeneity is not visible by the SEM method in absorbed electrons (as for the ${ }^{238} \mathrm{Pu}$ doped sample in Fig. 2), and it is not accompanied by precipitation of newly formed phases.

Table 4. Normalized data (in wt\% El) of microprobe analysis of $\mathrm{UO}_{\mathrm{x}}$ inclusions in pyrochlore ceramics doped with ${ }^{238} \mathrm{Pu}$ after the following cumulative doses (in alpha decays $/ \mathrm{m}^{3} \times 10^{23}$ ): (3) 57 ; (4) 82 ; (5) 110.

\begin{tabular}{|c|c|c|c|c|c|c|c|}
\hline \# & $\mathbf{H f}$ & $\mathbf{T i}$ & $\mathbf{C a}$ & $\mathbf{P u}$ & $\mathbf{U}$ & $\mathbf{G d}$ & $\mathbf{0}$ \\
\hline \multirow{3}{*}{$(3)$} & - & 0.1 & 0.7 & 2.0 & 84.2 & 0.9 & 12.1 \\
\cline { 2 - 8 } & - & 0.2 & - & 70.7 & 17.3 & 0.1 & 11.7 \\
\cline { 2 - 8 } & - & 0.2 & 0.7 & 4.9 & 78.9 & 3.1 & 12.1 \\
\hline \multirow{3}{*}{$(4)$} & 0.3 & 1.0 & 0.4 & 13.8 & 68.5 & 3.3 & 12.6 \\
\hline \multirow{3}{*}{$(5)$} & - & 4.4 & 1.9 & 8.7 & 71.1 & 2.2 & 14.3 \\
\cline { 2 - 8 } & - & 4.7 & 1.9 & 8.6 & 62.2 & 8.6 & 14.5 \\
\cline { 2 - 8 } & - & 2.1 & 0.7 & 7.8 & 64.2 & 12.9 & 13.4 \\
\hline
\end{tabular}
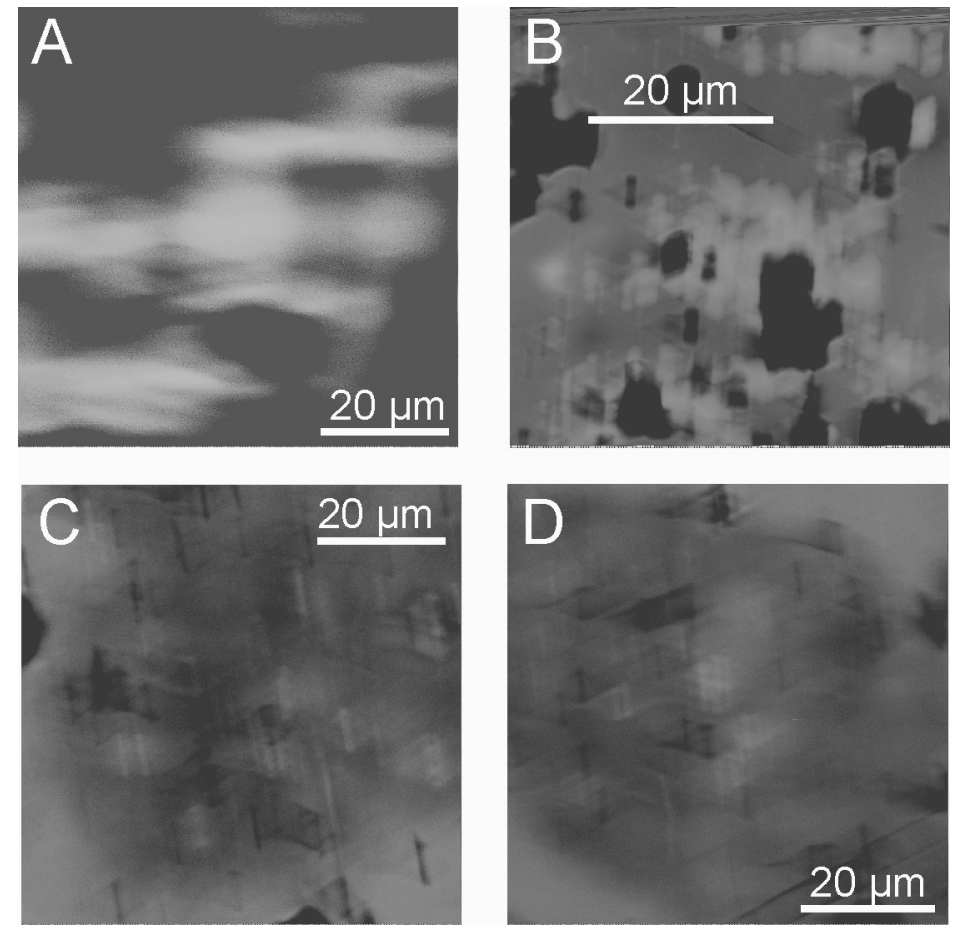

Fig. 2. SEM images (in absorbed electrons) of ${ }^{238} \mathrm{Pu}$-doped pyrochlore ceramics after 15 months since ceramic fabrication: $A$ and $B-$ typical inclusions of $U_{x}$ phase (light) in pyrochlore matrix (gray). Also, some pores (black) can be observed on image $B ; C$ and $D$ - inhomogeneous areas (dark) in pyrochlore matrix (gray). In these areas there are inclusions of $\mathrm{TiO}_{x}$ phase (black) and $\mathrm{Hf}-$ Ti-Ca-O phase (light dots). 
Table 5. Normalized data (in wt\% El) of microprobe analysis of pyrochlore phase (in the different pellet areas) in ceramics doped with ${ }^{238} \mathrm{Pu}$ after the following cumulative doses (in alpha decays $/ \mathrm{m}^{3} \times 10^{23}$ ): 3) 57 ; 4) 82; 5) 110 .

\begin{tabular}{|c|c|c|c|c|c|c|c|}
\hline Pellet area & $\mathrm{Hf}$ & $\mathrm{Ti}$ & $\mathrm{Ca}$ & $\mathrm{Pu}$ & $U$ & Gd & 0 \\
\hline \multirow{2}{*}{ (3) peripheral } & 3.6 & 20.4 & 7.9 & 3.4 & 32.9 & 7.2 & 24.5 \\
\hline & 6.7 & 20.1 & 7.3 & 12.1 & 21.1 & 8.3 & 24.3 \\
\hline \multirow{2}{*}{ (3) central } & 4.2 & 21.5 & 7.3 & 21.6 & 15.7 & 4.8 & 24.8 \\
\hline & 4.2 & 20.7 & 6.7 & 26.8 & 13.2 & 4.0 & 24.3 \\
\hline \multirow{2}{*}{ (3) peripheral } & 4.7 & 20.3 & 7.2 & 14.6 & 21.7 & 7.2 & 24.3 \\
\hline & 7.1 & 19.9 & 7.7 & 9.5 & 23.1 & 8.3 & 24.3 \\
\hline (4) peripheral & 4.3 & 18.9 & 8.5 & 15.1 & 21.5 & 8.3 & 23.4 \\
\hline \multirow{2}{*}{$\begin{array}{l}\text { (5), near uranium oxide } \\
\text { inclusions }\end{array}$} & 7.5 & 18.6 & 5.2 & 10.7 & 22.4 & 10.1 & 22.3 \\
\hline & 7.4 & 14.1 & 4.9 & 10.7 & 26.6 & 13.5 & 20.5 \\
\hline \multirow{3}{*}{$\begin{array}{l}\text { (5), near inclusions of Hf- } \\
\text { Ti-Ca-O phase }\end{array}$} & 6.6 & 19.4 & 6.7 & 10.3 & 28.9 & 7.0 & 23.5 \\
\hline & 6.5 & 19.9 & 6.5 & 11.6 & 28.4 & 4.4 & 23.2 \\
\hline & 6.5 & 19.5 & 6.5 & 14.7 & 27.3 & 5.1 & 23.5 \\
\hline \multirow{4}{*}{$\begin{array}{l}\text { (5), inclusions of } \mathrm{Hf}-\mathrm{Ti}- \\
\text { Ca-O phase }\end{array}$} & 28.6 & 21.1 & 7.1 & 5.3 & 8.5 & 3.9 & 24.6 \\
\hline & 28.6 & 19.9 & 7.2 & 10.0 & 1.7 & 4.9 & 23.8 \\
\hline & 37.7 & 17.0 & 5.8 & 2.1 & 10.8 & 1.7 & 22.6 \\
\hline & 66.6 & 5.8 & 4.2 & 2.3 & - & 1.0 & 18.0 \\
\hline
\end{tabular}

Table 6. Normalized data (in wt\% El) of microprobe analysis of pyrochlore phase (in the different pellet areas) in ceramics doped with ${ }^{239} \mathrm{Pu}$.

\begin{tabular}{|l|c|c|c|c|c|c|c|}
\hline \multicolumn{1}{|c|}{ Pellet area } & $\mathbf{H f}$ & $\mathbf{T i}$ & $\mathbf{C a}$ & $\mathbf{P u}$ & $\mathbf{U}$ & $\mathbf{G d}$ & $\mathbf{O}$ \\
\hline \multirow{4}{*}{ peripheral } & 5.5 & 19.1 & 6.6 & 16.9 & 26.1 & 2.2 & 23.5 \\
\cline { 2 - 8 } & 6.4 & 19.4 & 6.6 & 18.3 & 20.0 & 5.6 & 23.7 \\
\cline { 2 - 8 } & 6.1 & 19.7 & 6.7 & 20.8 & 19.7 & 3.1 & 23.8 \\
\cline { 2 - 8 } & 6.4 & 20.3 & 6.9 & 21.3 & 18.0 & 2.9 & 24.2 \\
\hline \multirow{2}{*}{ central } & 7.2 & 22.0 & 7.7 & 6.2 & 27.4 & 7.1 & 24.3 \\
\cline { 2 - 8 } & 6.6 & 20.1 & 7.4 & 6.7 & 27.1 & 7.8 & 24.3 \\
\hline \multirow{2}{*}{ peripheral } & 5.6 & 21.1 & 7.7 & 1.8 & 27.5 & 11.4 & 24.9 \\
\hline \multirow{2}{*}{ peripheral } & 6.4 & 17.6 & 4.1 & 15.8 & 29.8 & 5.5 & 20.7 \\
\cline { 2 - 8 } & 5.2 & 19.9 & 6.1 & 8.8 & 29.0 & 8.3 & 24.3 \\
\hline
\end{tabular}

\section{Geometric density measurement}

Measurement of geometrical density was carried out for single pellet of ${ }^{238} \mathrm{Pu}$-doped pyrochlore, which was stored in a glove-box in air as an uncontrolled medium since the ceramic samples were received from RIAR. Fifteen months after ceramic fabrication (at cumulative dose $110 \times 10^{23}$ alpha-decays $/ \mathrm{m}^{3}$ ), the density had changed from 4.8 to $4.3 \mathrm{~g} / \mathrm{cm}^{3}$ (approximately $10 \%$ ).

\section{MCC-1 leach test}

The results of one-stage MCC-1 leach tests are summarized in Table 7. It was shown that after 380 years of storage, the ceramic waste form based on Ti-pyrochlore containing $10 \mathrm{wt} \%{ }^{239} \mathrm{Pu}$ will become completely amorphous and this may cause the increase of Pu release in deionized water by approximately 1000 times and $U$ release by 30 times in comparison with initial levels. 
Table 7. Normalized Pu mass loss (without correction on ceramic porosity) from pyrochlore-based ceramics doped with ${ }^{238} \mathrm{Pu}\left({ }^{239} \mathrm{Pu}\right)$ after MCC-1 leach test (at $90^{\circ} \mathrm{C}$ in deionized water for 28 days) depending on cumulative doses. Ceramic density $-4.9 \mathrm{~g} / \mathrm{cm}^{3}$.

\begin{tabular}{|c|c|c|c|}
\hline $\begin{array}{c}\text { Cumulative doses in } \\
\text { ceramics doped with } \\
\mathrm{x} 10^{23} \mathrm{Pu} \\
\text { alpha decays } / \mathrm{m}^{3}\end{array}$ & \multicolumn{2}{|c|}{$\begin{array}{c}\text { Normalized Pu mass loss, } \mathrm{g} / \mathrm{m}^{2}, \text { at } 90^{\circ} \mathrm{C} \text {, after 28- } \\
\text { day test in deionized water for sample doped with } \\
{ }^{238} \mathrm{Pu}\left({ }^{239} \mathrm{Pu}\right)\end{array}$} & $\begin{array}{c}\text { Equal years of storage } \\
\text { estimated for ceramics } \\
\text { doped with } 10 \text { wt\% }{ }^{239} \mathrm{Pu}\end{array}$ \\
\hline & $\mathbf{U}$ & $\mathbf{P u}$ & 80 \\
\hline 29 & $0.03(0.01)$ & $0.22(0.0003)$ & 140 \\
\hline 49 & $2.30(1.78)$ & $0.28(0.004)$ & 280 \\
\hline 100 & $0.48(0.06)$ & $0.84(0.003)$ & 380 \\
\hline 133 & $0.97(0.03)$ & $1.93(0.002)$ & \\
\hline
\end{tabular}

\section{Conclusions}

1. Radiation damage of pyrochlore phase causes its chemical inhomogeneity and, as assumed, precipitation of separate $\mathrm{U}$-oxide and $\mathrm{Hf}-\mathrm{Ti}-\mathrm{Ca}-\mathrm{O}$ phases. It was found that, depending on cumulative dose, there is a clear tendency of the pyrochlore peak to shift to the direction of low angles, i.e., to the position of pure pyrochlore, $\mathrm{Gd}_{2} \mathrm{Ti}_{2} \mathrm{O}_{7}$. This is additional evidence that under self-irradiation the amorphization of pyrochlore structure could be accompanied with precipitation of impurities such as $\mathrm{Pu}, \mathrm{U}$, etc., into separate phases.

2. Fifteen months after ceramic fabrication (at cumulative dose $110 \times 10^{23}$ alpha-decays $/ \mathrm{m}^{3}$ ), the geometrical density (measured for single pellet stored in air in uncontrolled medium) was changed from 4.8 to $4.3 \mathrm{~g} / \mathrm{cm}^{3}$ (approximately $10 \%$ ).

3. Normalized Pu mass loss increases depend on cumulative dose. It is estimated that after 380 years of storage, the ceramic waste form based on Ti-pyrochlore containing $10 \mathrm{wt} \%{ }^{239} \mathrm{Pu}$ will become completely amorphous and this may cause the increase of Pu release in deionized water by approximately 1000 times and that of $U$ by 30 times in comparison with initial levels. 


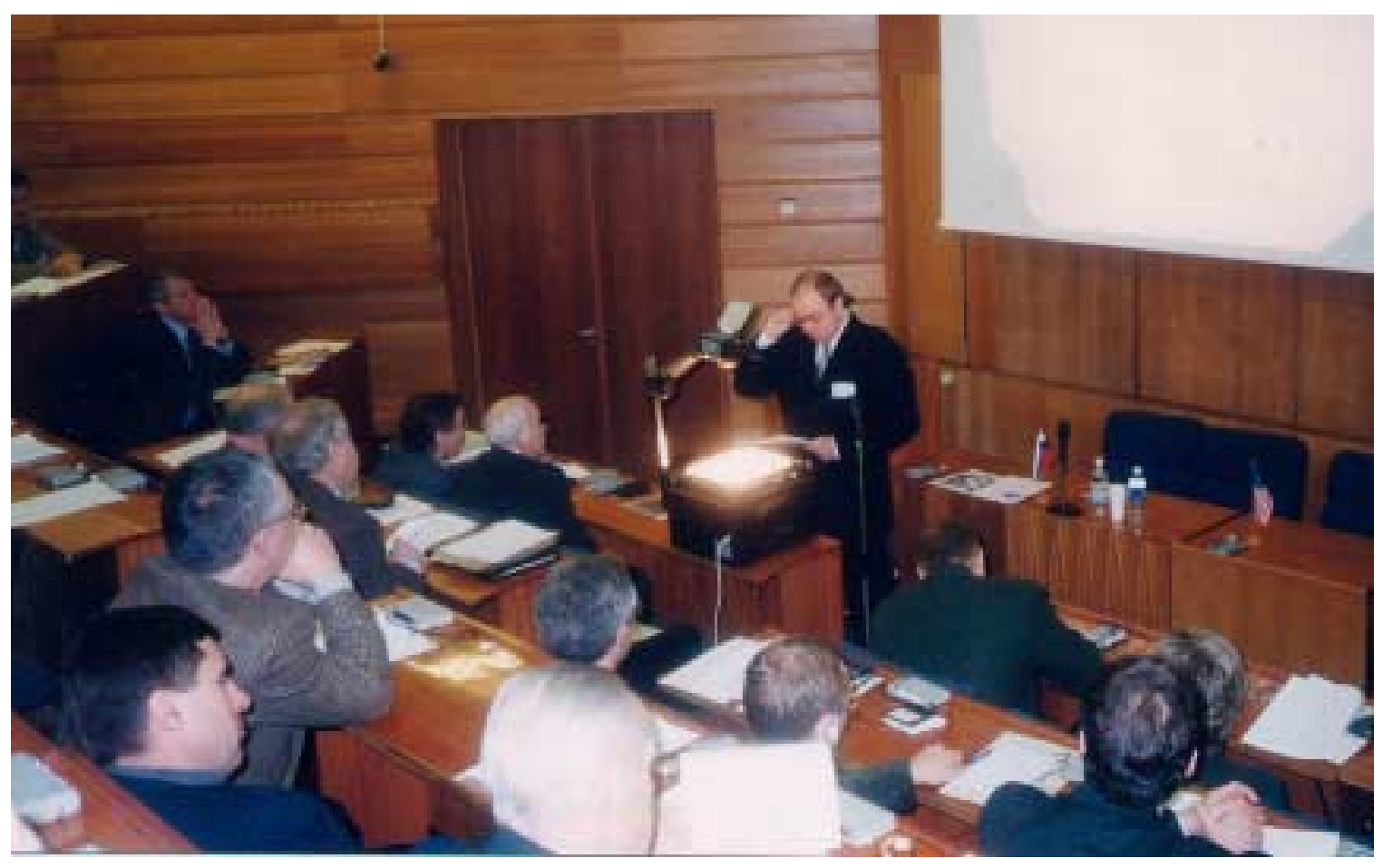

III.4. Geologic Disposal for Plutonium Immobilization and Other Pu-containing Materials 


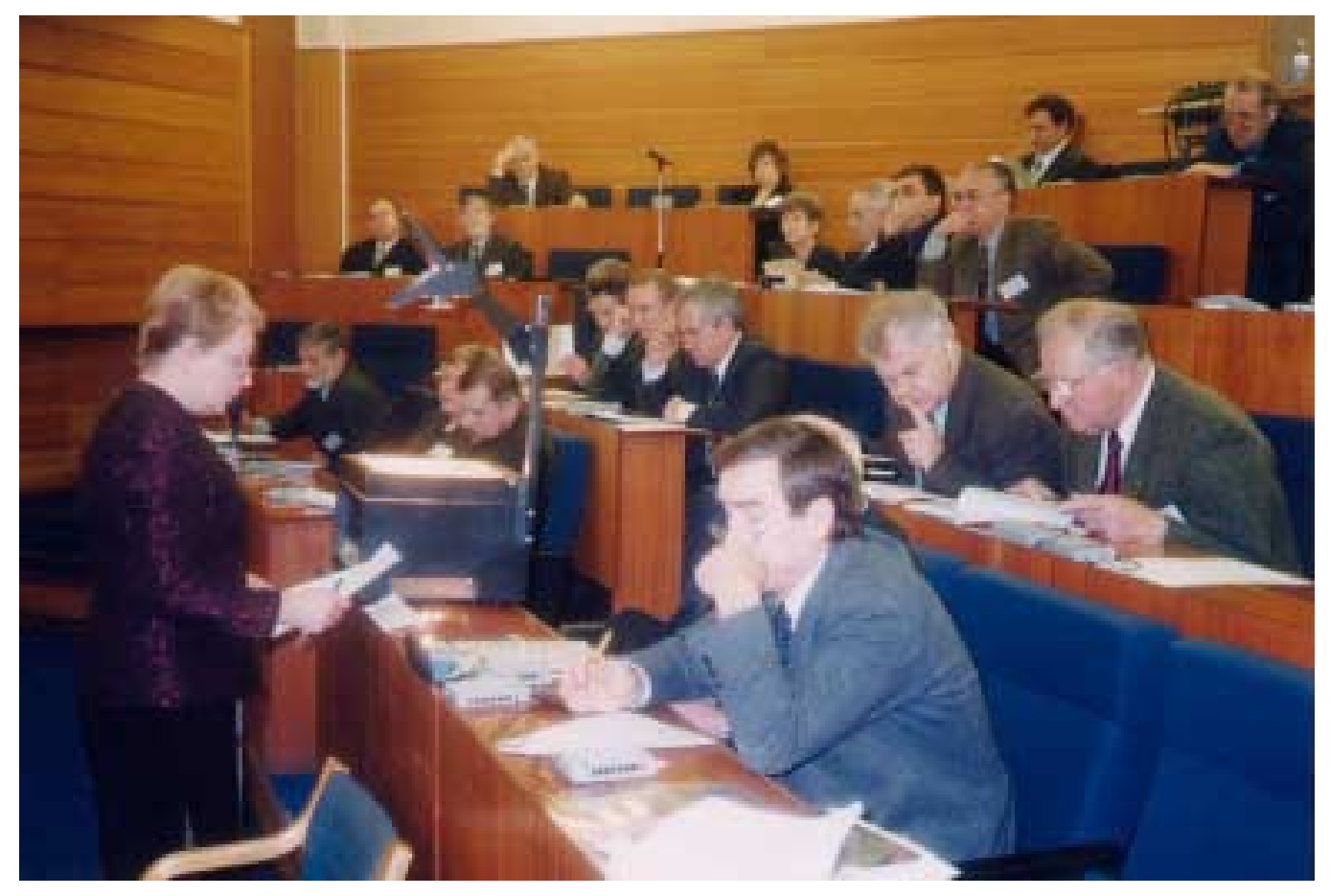




\section{Development of an RF Geologic Repository}

\section{J. Jardine, LLNL}

Lawrence Livermore National Laboratory (LLNL) has been actively and continuously engaged in Russian geologic disposal activities since 1995. The first joint US-Russian meeting on Disposition of Excess Weapons Plutonium was held in January 1995 at Los Alamos National Laboratory (LANL). The meeting resulted in the appointment of Dr. L. J. Jardine from LLNL and Dr. T. A. Gupalo from the All-Russian Research and Design Institute of Production Engineering (VNIPIPT) as the US-Russian Federation (RF) joint co-chairs for geologic disposal of plutonium-containing materials, respectively. The initial joint studies focused on the geologic disposal of plutonium-containing materials and immobilized plutonium waste forms. The first joint work of LLNL and VNIPIPT was documented in the October 1996 Paris P8 Nuclear Experts Meeting [1].

These initial cooperative studies led to the definition of Russian geologic repository activities of mutual interest. A series of four contracts between LLNL and VNIPIPT were issued, starting in 1996 and continuing through 2001. The contracts (UTA-96-0331, \$100K; B338273, \$150K; B501120, \$160K; B512134: \$92K) studied experimentally various geologic disposal aspects of plutonium migration and other radionuclide migration and engineered barrier materials, and developed computer models. The most recent experimental contract work, B512134, performed about 1-cubic-meter scale, radionuclide and plutonium migration tests in actual underground rocks at the underground RF Mining Chemical Combine's (MCC) K-26 facilities, using plutonium-containing glasses with fission products as sources of radionuclides from a simulated engineered barrier system [2].

Another LLNL contract with VNIPIPT, B512155, produced an integrated plan of how to develop a geologic repository at the two sites near K-26 and Mayak [3]. A separate LLNL contract with the V.G. Khlopin Radium Institute (KRI), B512154, developed geotechnical portions of the plan for the K-26 site [4]. These two final reports were approved by Minatom as LLNL deliverables; they represent a current approved Minatom plan for developing geologic repositories in Russia at these two sites [3].

In addition to the contracts with VNIPIPT and KRI, LLNL negotiated and completed contracts B347676, B506210, and B506233 with the MCC K-26 site and contracts B506201 and B506208 with the Mayak site for engineering studies that developed geologic disposal concepts for their immobilized plutoniumcontaining materials. The engineering reports focused on immobilization of plutonium-containing sludges but also addressed preliminary geologic disposal concepts, including costs, for the geologic disposal of immobilized plutonium-containing waste forms $[5,6]$.

In addition to these joint US-Russian plutonium disposition studies funded by LLNL with US Department of Energy (DOE) funds, LLNL participated in four key International Science and Technology Center (ISTC) projects that focus on Russian geologic disposal activities near the K-26 site. The first was ISTC Project 307B: L. Jardine helped T. Gupalo, VNIPIPT, develop the proposal and secure ISTC funding in 1997, and he served as the US Collaborator. The final project report for Project 307B documents over 40 years of heat effects from underground nuclear reactor steam generators and other heated components on the gneiss rocks of the underground MCC K-26 facility. In addition to heat effects, the project assessed thermal-mechanical, hydrology, and geochemistry effects in the gneiss rock masses [7]. 
L. Jardine also served as the US Collaborator for ISTC Project 255 and ISTC Project 1491. These two projects, managed by $\mathrm{E}$. Anderson, KRI, developed various site characterization activities for the repository site near K-26 for the Ninzenkansky granite massif [8,9]. In 2001, L. Jardine assisted DOE-RW and VNIPIPT to define and fund a new DOE-RW project, ISTC Partner Project 2377. Project 2377 will implement portions of the current Minatom plan for the K-26 site and will, after three years, provide valuable data for updating and revising the Minatom plan for the K-26 site [10].

LLNL is also assisting DOE-RW to manage a small effort by the Institute of Ore Deposits Geology, Petrology, Mineralogy and Geochemistry-Russian Academy of Sciences (IGEM-RAS) to develop geotechnical inputs to a scientific plan for developing the granite massif site K-26. The IGEM-RAS Plan will provide inputs to the new ISTC Project 2377 activities. Similarly, the engineering studies performed by VNIPIET and MCC for geologic disposal for immobilized plutonium forms will be valuable inputs to ISTC Project $2377[5,6]$.

In summary, LLNL has been actively involved since 1995 in developing and defining the current Russian geologic disposal program activities near the Mayak and MCC K-26 sites. Figure 1 illustrates how these various LLNL activities have been integrated and focused on developing geologic disposal in Russia. The various LLNL contracts are shown in the figure with the specific LLNL contract number. Reference 11 provides another recent summary of the status of the Russian repository program activities for the K-26 and Mayak sites.

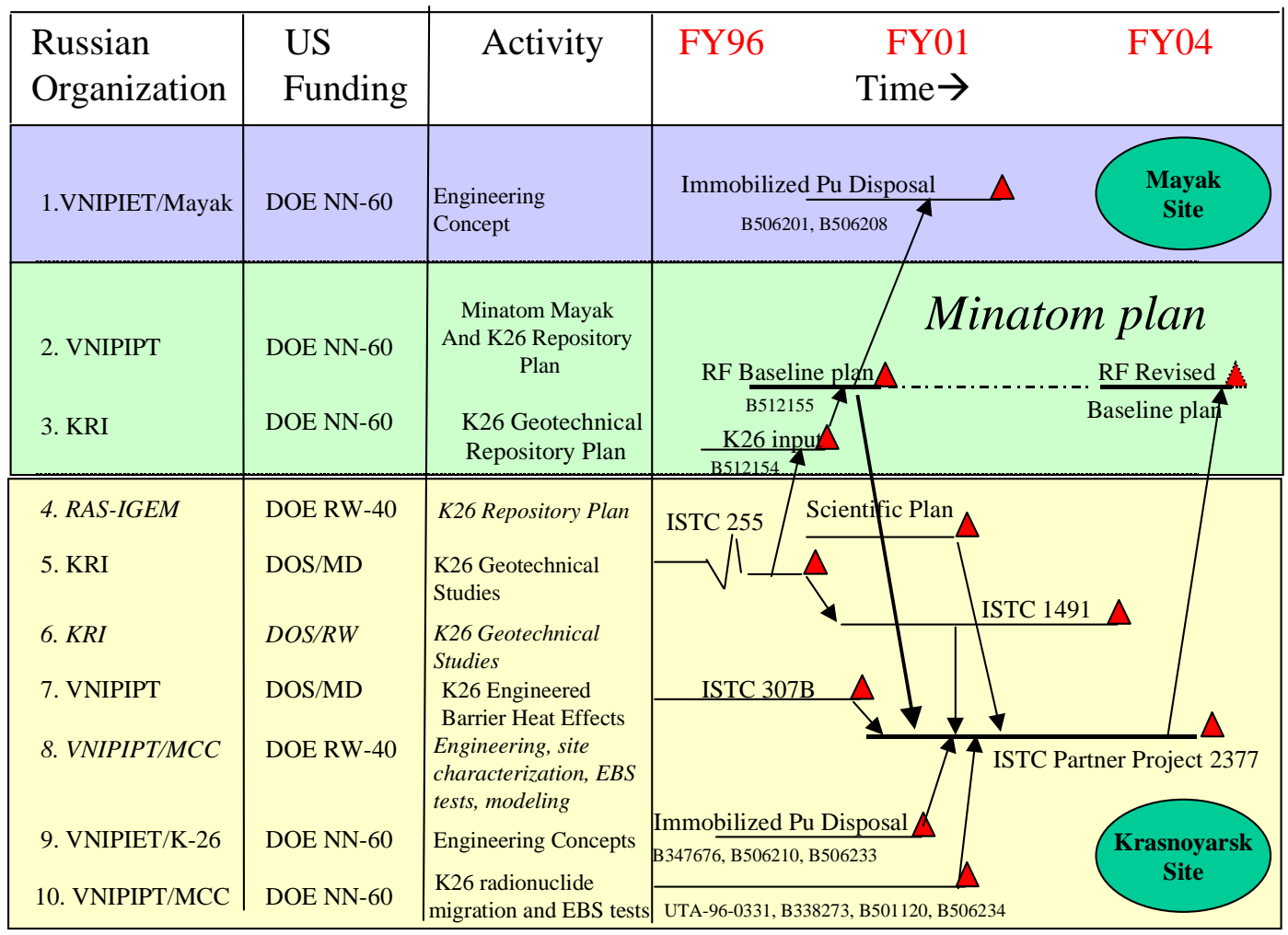

Fig. 1. LLNL has been continiously engaged in RF geologic disposal activities near the Mayak and K-26 sites since 1995. 
LLNL has recently published a report summarizing and referencing the RF activities [12]. The report separates various publications into one of three types: (1) policy and overview, (2) MCC K26 site, and (3) Mayak site.

\section{References}

1. L. J. Jardine, T. A. Gupalo, et al., US-Russian Joint Study Report of Geologic Disposition Options for Excess Plutonium from Dismantled Nuclear Weapons (Combined Report), September 30, 1996 (UCRL-ID-124711); Joint United States Russian Plutonium Disposition Study Summary Report, September 1996.

2. T. A. Gupalo, Large-Scale Laboratory and In-Situ Investigations of Pu and U Radionuclides Migration in the Multi-Barrier System for Underground Isolation of the MCC Vitrified Waste, Final Report, RP \#215, (B512134), December, 2001.

3. L. J. Jardine, T. A. Gupalo, Geologic Repository Plan for Disposal of Plutonium-Containing and Other Radioactive Materials in Russian Industrial Sites, (B512155), UCRL-CR-146982, Lawrence Livermore National Laboratory, Livermore, CA, February 2002.

4. E. Anderson, Plan for Geologic Repositories for Disposal of Immobilized Plutonium-containing Materials and Other Radioactive Materials at Russain Industrial Sites, final report RP-188 (B512154), August, 2001.

5. MCC Engineering Reports, RP-95, RP-118, and RP-231.

6. Mayak Engineering Report, RP-232.

7. Development of Quantitative Criteria for Suitability of Rock Mass for Safe Long-term Storage of Waste from Weapons Plutonium Production, Project 307B Final Report (RP-227).

8. E. Anderson, Project 255 Final Report, RP-226.

9. Work Plan 1491, RP-229.

10. Project 2377 Work Plan, RP-228.

11. T. A. Gupalo, E. B. Anderson and L. J. Jardine, Status of Geologic Disposal and Isolation in Russia for Plutonium-Containing Wastes of Military Programs, DISTEC 2000 Conference, Berlin, Germany, September 4-6, 2000 (UCRL-JC-138202).

12. L. J. Jardine, Russian Geologic Repository Technical Papers and Reports, Lawrence Livermore National Laboratory, Livermore, CA, UCRL-ID-147699, Feb. 18, 2002. 


\title{
Perspectives When Using New Technology for Pu-containing Wastes Management in the Nuclear Industry
}

\author{
V. D. Safutin, I. L. Rybalchenko, VNIPIET
}

\begin{abstract}
This work encompasses Pu-containing waste materials in the nuclear industry; international concepts of spent nuclear fuel (SNF) and high-level waste (HLW) management; the status and perspectives on the immobilization of HLW materials including glass, ceramics, and synroc; and a demonstration of new immobilization technologies. The direction of our research and development work includes a review of SNF and HLW storage in various regions; review of the criteria for immobilized material characterization and for geologic disposal; evaluation of possible life times of immobilized waste forms with different matrices; engineering comparison of various immobilization technology and equipment, and selection of a prospective scheme. Ultimately these will be followed by development of a demonstration facility for HLW immobilization, and development of a concept for a Regional repository.
\end{abstract}

\section{Plutonium-containing Waste Materials}

Plutonium-containing waste materials in the nuclear industry are formed at various stages of the nuclear fuel cycle (e.g., reprocessing of irradiated fissile materials, manufacturing of mixed oxide U-Pu fuel). Some quantities of wastes containing weapons-grade plutonium are stored as residues of nuclear military programs in Russia. It is imperative that we solve the problems of immobilization of such waste materials for disposal.

At the same time, one should understand, that significantly larger quantities of Pu were accumulated in some other countries as a result of military nuclear activities and nuclear energy production. Sooner or later this Pu will be used for energy production at commercial nuclear power plants (NPPs). Some quantities of that Pu will be left as a waste material.

Even more fissile materials $(\mathrm{U}$ and $\mathrm{Pu}$ ) are contained in spent nuclear fuel at commercial NPPs. Significant amounts of plutonium were recovered from SNF at plant reprocessing facilities.

Unfortunately, the management of these waste materials and their final disposal, including SNF if it is not to be reprocessed, remains a problem not yet solved at the technical, organizational, or social levels.

The concept of final disposal is not considered to be acceptable by the society in many countries, due to lack of confidence in the technology proposed and poor information on the possibility of reliable solutions.

This problem should have a final solution, especially the disposal of HLW; otherwise nuclear power programs have no prospects to grow or even remain at present levels.

\section{International Concepts of SNF and HLW Management}

At present, the principal concepts of SNF and HLW management and basic safety requirements have been developed at the international level. 
Deep geologic formations have been suggested for isolation of radioactive wastes with a multibarrier system to prevent radionuclide releases into the environment.

Some examples of geologic repositories of this type are underground facilities in Finland (Olkiluoto), Sweden (Clab), and the US (WIPP). The Yucca-Mountain repository also can be considered as a good example of a future repository for SNF for all the US nuclear power plants. Developing a deep repository for long-lived wastes requires significant resources. International cooperation can become a driving force for some countries.

The International Atomic Energy Agency (IAEA) attempted to suggest an international mechanism for Spent Fuel Storage and Plutonium Storage some 20 years ago. No actual results have emerged from this. But at present, the political situation has radically changed. And such international cooperation can be revived.

The IAEA "Joint Convention of Safety of Spent Fuel Management and on Safety of Radioactive Wastes Management" can become a basic document on the safety aspects and institutional arrangements. The IAEA is also proposing set of safety rules and requirements as well as a mechanism of effective implementation for such standards.

However, before such an international project can become feasible, some practical confirmation on a national level is desirable. The US and Russia could make available a demonstration facility for the treatment, storage, and disposal of HLW and SNF.

\section{Immobilization of HLW materials: Status and Perspectives}

A key question in submitting a license application for a facility for the immobilization of HLW materials, including glass, ceramics, and synroc, is whether the long-term safety of the facility can be guaranteed.

Up to now only industrial-scale vitrification has been developed with the storage of vitrified radioactive waste (RW) in special surface repositories. The long-term fate of such surface repositories is not clarified (but must be in operation for hundreds of years). A final disposal solution has not been proposed by the technical community so far. Due to this uncertainty, the position of some "Green" nuclear opponents is understandable.

However, new matrices for immobilization of long-lived nuclides are well known (ceramics and synroc). The Australian synroc group has already proposed various approaches to immobilization of $\mathrm{Pu}$ and other high-level reprocessing wastes.

A laboratory-scale facility was created, and the technology confirmed on non-radioactive materials. A demonstration facility for immobilizing weapons plutonium with no fission products in a pyrochlor ceramic matrix with a $10 \%$ weight Pu concentration was constructed and operated at a small scale in 1999-2000 at Lawrence Livermore National Laboratory (LLNL) in the US.

Unfortunately no ceramic demonstration facilities have been constructed to date that operate with real HLW solutions.

As for the disposal of SNF, commercial repositories exist in Finland and Sweden. Design concepts for retrievable storage and disposal facilities were developed, but their practical implementation has been postponed due to various economic, political and other reasons. 


\section{Demonstration of a New Technology for SNF and HLW Disposal}

A new technology is urgently needed for SNF and HLW disposal. It is difficult to expect that it can be done soon; it requires time. But real programs for development and construction of demonstration facilities should be prepared.

This is especially important when the danger of possible terrorist attacks on nuclear facilities is a reality. Any existing spent fuel storage facility anywhere in the world may become a target for such a terrorist action that could result in contaminating a large territory.

A special large regional repository can easily withstand attempts of terrorist actions, providing additional safety and non-proliferation guarantees.

A number of principal questions should be solved at a demonstration stage:

- Selection of proper matrices for immobilization of wastes that may be stable for the whole period of potential danger of a specific nuclide;

- Comparison of various immobilized radioactive materials resistance to environmental conditions for a long-term perspective;

- Engineering comparison of various proposed immobilization technologies and equipment with the selection of procedures and principal equipment;

- Development of a regulatory baseline for controlled storage and final disposal of PU- and HLWcontaining materials at national and international levels.

A variety of work being done by the Russian organizations and laboratories under the excess plutonium disposition agreement provides a good basis to create a demonstration facility for Pu and HLW immobilization into stable matrices.

At the All Russian Designing and Scientific Research Institute of Complex Power Technology (VNIPIET), some work on engineering evaluation of a process and equipment for immobilization on long-lived radionuclides into mineral-like matrices is being done. Emphasis is given to the selection of principal equipment for melting, with different heating devices, comparison with pressing technology, etc.

It is considered that the primary goal at present should be directed to the design and engineering work, with construction of a demonstration facility first and then later a full-scale pilot plant at the industrial site.

The final goal is to demonstrate effectiveness of new HLW immobilization and disposal technologies and to convince regulatory authorities, political structures, and the public of their reliability and technical and economic feasibility.

\section{References}

1. IAEA Bulletin. 2000. Vol. 42, N 3.

2. Safety of Radioactive Waste Management // Proc. of Int. Conf. (13-17 March, 2000 Cordoba). Vienna: IAEA, 2000.

3. Joint Convention on Safety of Spent Fuel Management and on Safety of Radioactive Waste Management // INFCIRC/546. IAEA, 2000.

4. ANSTO Annual Report. 1999-2000.

5. Jardine L. Russia Excess Weapons Plutonium Disposition. LLNL Project Review 2000: Meeting summary. January 2002. 


\section{Work Organization for Creation of RW Geological Storage in Russia (B512155)}

\section{T. A. Gupalo, VNIPIPT}

Studies of areas and mountain rock masses have been carried out since the beginning of the 1970 s with the purpose of creating geological storage for the underground isolation of Minatom radioactive waste. Most goal-directed research was carried out within the PA "Mayak" site in the Chelyabinsk region and the Mining and Chemical Combine (MCC) site in the Krasnoyarsk region.

Despite the long time and level of detail of the research, the necessary complex of geological and geophysical studies for the initial phases of design and exploration work has not been carried out. Thus, only the M-2 site in the buffer zone of PA "Mayak" and the northeast part of the Verkhne-Itatsky site have been studied.

In 2002, a new problem was included in the list of RF Minatom primary goals. It is the "Underground isolation of waste from processed spent nuclear fuel (SNF) at the enterprises of the Department of the Nuclear Fuel Cycle," which should result in justification and selection of geological sites, optimization of design decisions on underground isolation of solidified waste from processed irradiated nuclear fuel (SNF), including defense programs waste.

Taking into account the versatility and complexity of research required for performance of this task, the highly skilled experts from design and research institutes of Minatom of Russia, Russian Academy of Sciences, and specialized geological organizations of the Urals and the Siberian region will participate in this effort.

Figure 1 presents the organizational structure for the basic direction of research that should be carried out for selection and justification of sites for geological storage construction, optimization of the design decisions on underground isolation of radwaste from SNF processing and defense programs, and ensure the safety of the underground facilities created for long periods of time.

All-Russian Research and Design Institute of Production Engineering (VNIPIPT) should provide methodical guidance and participate directly in all studies, ensuring uniform direction of work, and immediate use of data from geological prospecting and scientific research for development of pre-design and design decisions according to the Construction Rules and Regulations and GOSTs (i.e., RF State Standards) for design work. 
D irections of research on RW undergound isolation

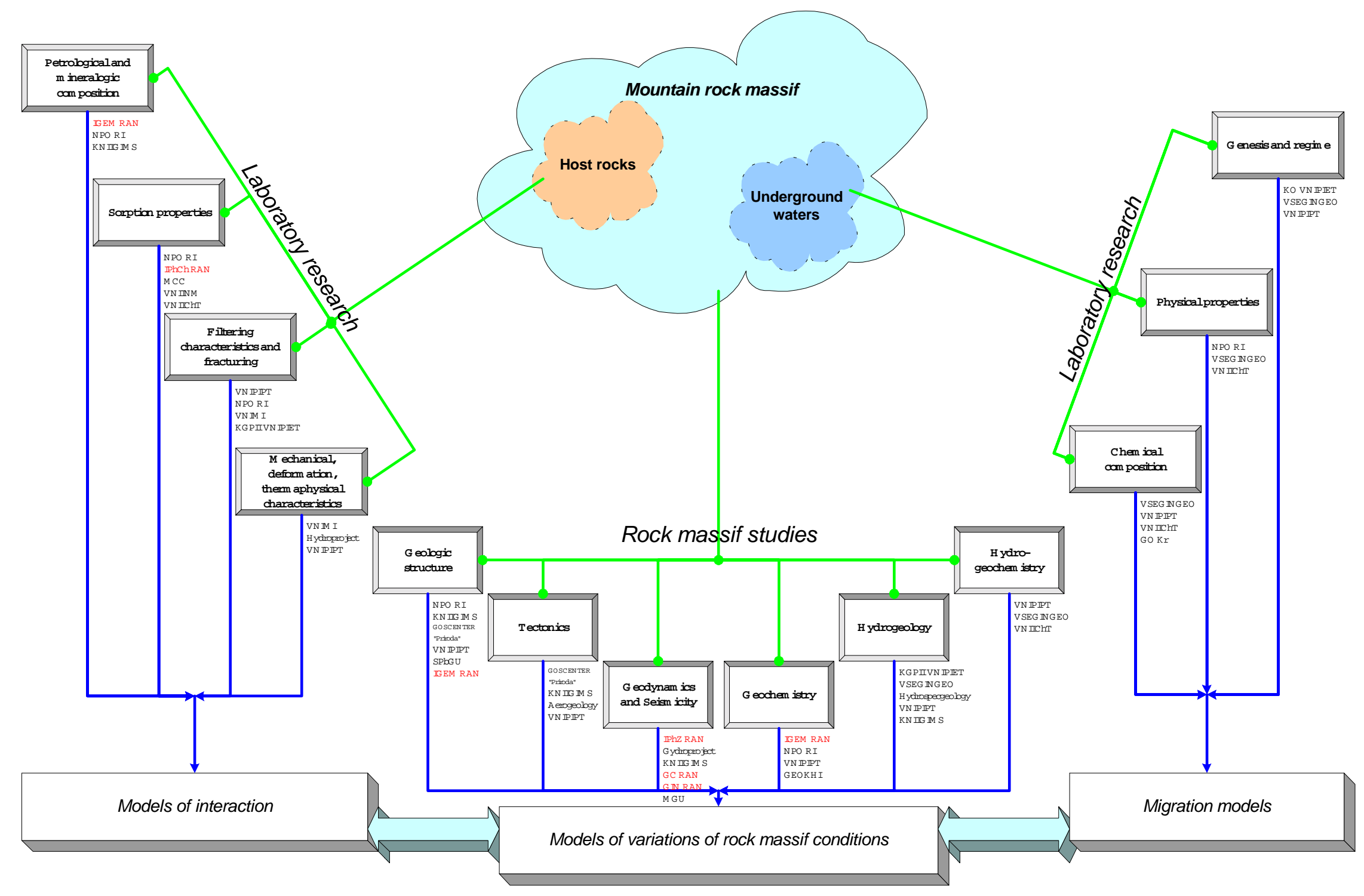

Fig. 1. Direction of research on RW underground isolation. 
In order to perform the Nizhnekansky massif repository program in a more efficient and purposeful manner, we plan to establish a Russian Science and Technology Center for Development of Technologies for RW Disposal in Geologic Formations (Fig. 2).

The Center shall bring together and coordinate laboratories, institutions, companies, and organizations that work on the Russian underground isolation site program. The Center will analyze Russian waste management scenarios and develop plans, review completed work, and coordinate development of research and engineering projects. The center would also share technologies with foreign laboratories and organizations involved in geologic repository development. Another function of the Center will be system analysis of program requirements and activities. This will provide a basis for activity prioritization and decision-making that will be independent from any other organization.

A branch of this Center will be created at MCC that will enable us to coordinate and perform engineering explorations in order to investigate rock mass properties in the MCC area.

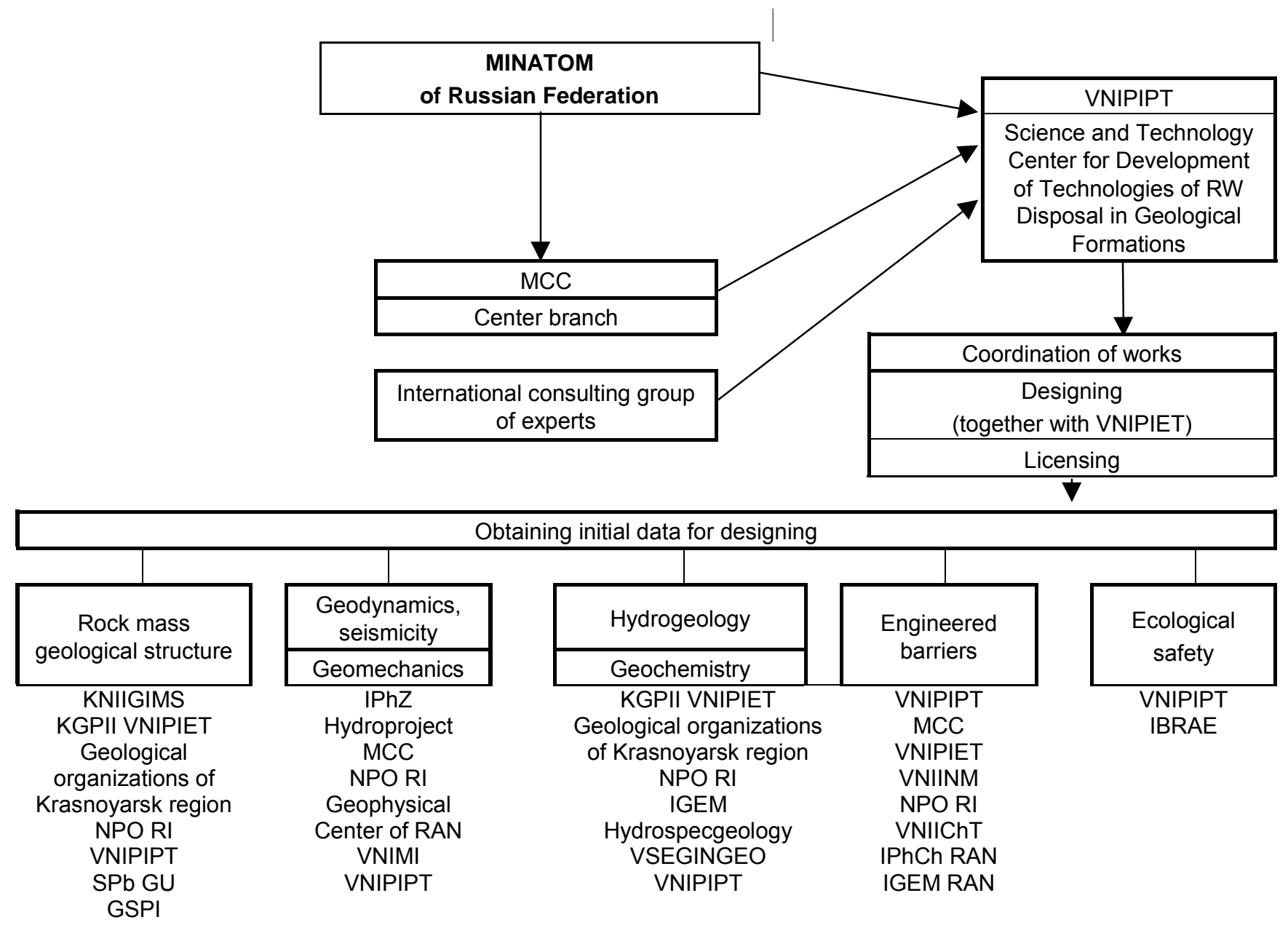

Fig. 2. Scheme of work organization for creating $\mathrm{RW}$ underground isolation facility at Nizhnekansky rock massif. 


\section{Conclusions}

1. The analysis of work carried out since the 1970 s for creation of a radioactive waste geological storage in Minatom confirms the necessity of uniform coordination and methodical guidance of research and design and exploration work in the given direction.

2. In order to improve this work, the new problem "Underground isolation of waste from processed SNF at the enterprises of the Department of Nuclear Fuel Cycle" was included in the list of Minatom primary tasks in 2002. The management of work under this task is assigned to VNIPIPT, the lead organization on designing the underground facilities of Minatom of Russia.

3. In order to perform the work on the program to create Russian underground isolation sites in a more efficient and purposeful manner, we plan to establish a Russian Science and Technology Center for Development of Technologies for RW Disposal in Geological Formations. This Center will carry out its own development, and involve highly skilled experts of Minatom of Russia and the Russian Academy of Sciences and specialized organizations of the Ural and the Siberian region in works on the given subject. This will provide coordination during performance of scientific and technological projects and facilitate interaction with foreign collaborators. 


\title{
Plan for Geological Repositories for Disposal Of Immobilized Plutonium-Containing Materials and other Radioactive Materials at Russian Industrial Sites (B512154)
}

\author{
E. B. Anderson, V. G. Savonenkov, S. I. Shabalev, KRI
}

\section{Introduction}

The V. G. Khlopin Radium Institute (KRI), together with experts from Russian Academy of Sciences and other organizations, conducts scientific work on different aspects of the geologic disposal in the Krasnoyarsk region. $\mathrm{KRI}$ has assembled an experienced staff of researchers and technical specialists to perform this work on the disposal of immobilized plutonium-containing materials and other solid radioactive wastes.

Within the framework of the "Material Support Agreement B512154," KRI took part in a project to develop a detailed plan of activities for developing and operating geologic repositories sites near both the PA "Mayak" and the Mining-Chemical Combine (MCC) Krasnoyarsk-26 industrial sites. KRI participation was limited to delivery of materials (data) on the Krasnoyarsk region. These data include planned activity on site selection and research and, also, R\&D work in an underground research laboratory.

According to the Statement of Work, All-Russian Research and Design Institute of Production Engineering (VNIPIPT) prepared and developed the single integrated plan. KRI has provided VNIPIPT with specific inputs to the plan, as requested by VNIPIPT. This paper presents in brief the report of the Radium Institute prepared for Agreement B512154.

\section{Content and Results of Scientific and Research Work Needed to Create the System of HLW and SNF Underground Isolation in the Territory of the Nizhnekansky Granitoid Massif}

The safe disposal of high-level radioactive wastes (HLW) and/or of spent nuclear fuel (SNF) is possible if a block can be found within the rock strata with properties that exclude release of radionuclides to the biosphere in a quantity presenting any danger to the population or to the biosphere as a whole.

The problem posed on the choice of a site suitable for repository construction should be solved step-bystep, serially enlarging the scale, the level of detail, and safety substantiation for the sites proposed. The problems to be solved can be divided into three general sets of activities (see the abridged version in Table 1):

- Section 1. In-the-field (expeditionary) searches and explorations;

- Section 2. Office and laboratory activities within the walls of different organizations;

- Section 3. Experimental research in underground laboratory.

This type of division of work was implemented at the request of VNIPIPT. However, in many respects it is conditional, as the problems solved within the framework of the different sections are interdependent, and are often being solved at the same time. The tasks are described in Table 1, accompanied with brief comments. 
Table 1. Contents and results of scientific and research works required to create the system of HLW and SNF underground isolation in the territory of the Nizhnekansky granitoid massif (abridged version).

\section{Short description of scientific studies}

\section{In-the-field (expeditionary) work, including preparation and primary results processing}

Tasks of Section 1 are initiated after task 2.1-2.3 are completed

\subsection{Implementation of reconnaissance} expeditionary works (geological, geomorphological, geophysical) within the limits of prospective region of the massif

1.2. Preparation and implementation of integrated geophysical works (resistivity, seismic, gravitation, magnetic prospecting and surveying) at the promising sites of the massif at a scale of $1: 50,000-1: 20,000(1: 25,000)$

1.3. Geologic mapping and sampling works in the territory of the massif and sites of geophysical studies

1.4. Carrying out of geomorphological studies for the selected part of massif and its surroundings

1.5. Hydrological, hydrogeological and geochemical field studies of the massif and its single parts

1.6-1.7. Deep drilling, working up and realization of the program for bore-hole studying and testing and integrated detailed engineering and geological surveying of the selected site (scales of mapping materials are 1:10,000 and more)
Correction of the available cartographical data describing the structure and geomorphological features of the massif

Assessment of structure and internal pattern of the massif and its single sites, including selection of the sites with the lowest rock permeability

The data for geological mapping at a scale 1:50,000 $-1: 20,000$; geological and mineralogical, petrographical, radiogeochemical and other characterization of rocks of massif surface

Structural and tectonic mapping, detection of active fractures and zones of their dynamic effect

Special mapping, determination of hydrogeological conditions and groundwater characteristics, detection of jointing active zones by geochemical methods

Volumetric model of the site for underground construction with the basic parameters of geological surroundings
12

24 for works at a scale of $1: 50,000$ and 36 for scale $1: 20,000$

12-24 (excluding office work)

12

36 


\begin{tabular}{|l|l|c|}
\hline \multicolumn{1}{|c|}{ Short description of scientific studies } & \multicolumn{1}{|c|}{ Results (input data for designing) } & $\begin{array}{c}\text { Duration, } \\
\text { months }\end{array}$ \\
\hline $\begin{array}{l}\text { 1.8. Choice and substantiation of construction } \\
\text { option for underground laboratory, statement of } \\
\text { tasks and drawing up of the program of complex } \\
\text { research works }\end{array}$ & $\begin{array}{l}\text { General data for designing of scientific-research and } \\
\text { demonstration underground laboratory }\end{array}$ & 12 \\
\hline 2. Laboratory and office studies & $\begin{array}{l}\text { Substantiation of regional geological structure } \\
\text { selected }\end{array}$ & 12 \\
\hline $\begin{array}{l}\text { 2.1. Comparative analyzing of large-scale } \\
\text { lithosphere structures in order to select the } \\
\text { prospective region }\end{array}$ & Initial data on geological formation, rock massif & 12 \\
\hline $\begin{array}{l}\text { 2.2. Analyzing of the selected structure with the } \\
\text { purpose to find the most prospective geological } \\
\text { formations, rock massif (the data of geological } \\
\text { and geophysical surveys, decoding of aerospace } \\
\text { materials, etc.) }\end{array}$ & & 6 \\
\hline $\begin{array}{l}\text { 2.3. Analyzing of geological conception and } \\
\text { geological criteria in order to range and optimize } \\
\text { them, as applied to the special conditions of the } \\
\text { massif }\end{array}$ & $\begin{array}{l}\text { Requirements for selection of specific formation and } \\
\text { rock massive }\end{array}$ & \\
\hline $\begin{array}{l}\text { 2.4. Analyzing of the results of integrated field } \\
\text { works to make a preliminary assessment of } \\
\text { massif fitness, and to develop additional criteria of } \\
\text { site selection }\end{array}$ & Substantiation of rock massif selection & \\
\hline $\begin{array}{l}\text { 2.5. Mineralogical and petrographical, } \\
\text { petrophysical, geochemical, isotopic- } \\
\text { geochronological, isotope-geochibical and other } \\
\text { studies of rock samples. }\end{array}$ & $\begin{array}{l}\text { Characterization of matter composition and main } \\
\text { properties of enclosing geological medium }\end{array}$ & $\begin{array}{c}\text { on duration } \\
\text { of in-the- } \\
\text { field work) }\end{array}$ \\
\hline
\end{tabular}




\begin{tabular}{|l|l|c|}
\hline \multicolumn{1}{|c|}{ Short description of scientific studies } & \multicolumn{1}{|c|}{ Results (input data for designing) } & $\begin{array}{c}\text { Duration, } \\
\text { months }\end{array}$ \\
\hline $\begin{array}{l}\text { 2.6. Radiogeochemical studies of rocks and } \\
\text { minerals, including study of retaining properties } \\
\text { relatively to basic radionuclides to be disposed, } \\
\text { and determination of basic mechanisms of } \\
\text { radionuclide migration }\end{array}$ & $\begin{array}{l}\text { Obtaining of integrated radiogeochemical parameters } \\
\text { for evaluation of rock barrier properties, and probable } \\
\text { radionuclide migration. Input data for the modeling of } \\
\text { migration processes, and for engineered barriers } \\
\text { system designing }\end{array}$ & 60 \\
\hline $\begin{array}{l}\text { 2.7. Computer simulation of conditions for } \\
\text { radionuclide localization and migration in } \\
\text { granitoids of fracture and block structure }\end{array}$ & $\begin{array}{l}\text { Evaluation of migration conditions and space-and- } \\
\text { time haloes of radionuclide dispersion for the massif } \\
\text { specific conditions }\end{array}$ & 36 \\
\hline $\begin{array}{l}\text { 2.8. Analyzing of results of integrated geological } \\
\text { and geophysical, structural and tectonic, } \\
\text { hydrogeochemical studies to determine the } \\
\text { migration and barrier properties of jointing zones, } \\
\text { zones of increased granitoid permeability, and } \\
\text { weathering zones }\end{array}$ & $\begin{array}{l}\text { Evaluation of migration conditions out of frames of } \\
\text { working block (facility allotment) }\end{array}$ & 36 \\
\hline $\begin{array}{l}\text { 2.9. Registration of radioactive materials to be } \\
\text { stored and disposed, including characteristics of } \\
\text { packages' parameters, thermal flux, radiation } \\
\text { loads }\end{array}$ & $\begin{array}{l}\text { Evaluation of lithosphere space volumes with the } \\
\text { properties required for underground complex } \\
\text { construction }\end{array}$ & 24 \\
\hline $\begin{array}{l}\text { 2.10. Selection of phases-carriers and matrixes } \\
\text { for long-lived radionuclides immobilization; } \\
\text { evaluation of compatibility between RAW to be } \\
\text { disposed, and enclosing granitoids }\end{array}$ & $\begin{array}{l}\text { Safety assurance for a long-term storage (disposal) } \\
\text { of long-lived radionuclides }\end{array}$ & 36 \\
\hline $\begin{array}{l}\text { 2.11. Prediction for tectonic development, } \\
\text { alteration of climate and relief, and assessment of } \\
\text { dynamic impact of active fractures to } \\
\text { hydrogeological conditions and migration } \\
\text { processes }\end{array}$ & $\begin{array}{l}\text { Prediction of conditions of a long-term isolation of } \\
\text { radionuclides (repository arrangement on sufficient } \\
\text { depth excluding effects of external processes and } \\
\text { impacts) }\end{array}$ & 36 \\
\hline
\end{tabular}




\begin{tabular}{|c|c|c|}
\hline Short description of scientific studies & Results (input data for designing) & $\begin{array}{c}\text { Duration, } \\
\text { months }\end{array}$ \\
\hline $\begin{array}{l}\text { 2.12. Preparing and analyzing of the } \\
\text { geoinformation system (GIS) in order to adjust } \\
\text { and to use it as initial complex database for sites } \\
\text { and construction region (geology, hydrogeology, } \\
\text { fractures, relief, hydraulic net, land using, } \\
\text { anomalies, guarded areas, etc.) }\end{array}$ & $\begin{array}{l}\text { Descriptions of initial conditions of the region for } \\
\text { construction of ecologically dangerous plant }\end{array}$ & 24 \\
\hline \multicolumn{3}{|c|}{$\begin{array}{l}\text { Note: the results of studies on items 2.9-2.12 are included into materials of input data for Justification of Investment (JOI) } \\
\text { and Environmental Impact Assessment (EIA) }\end{array}$} \\
\hline \multicolumn{3}{|l|}{ 3. In underground research laboratory } \\
\hline $\begin{array}{l}\text { 3.1. Designing of laboratory's underground } \\
\text { openings, to provide for representability of the } \\
\text { studied zones and implemented sampling }\end{array}$ & Assurance of input data representability & 12 \\
\hline $\begin{array}{l}\text { 3.2. Mining-sinking, geomechanical, physical and } \\
\text { chemical tests for devices, methods and } \\
\text { technique intended to create underground } \\
\text { openings and specialized bore-holes }\end{array}$ & $\begin{array}{l}\text { Provision for the minimum effect from mining jobs to } \\
\text { the isolating properties of mountain massif }\end{array}$ & 36 \\
\hline $\begin{array}{l}\text { 3.3. Structural and microstructural analysis } \\
\text { carrying out for the block of rocks within the } \\
\text { ranges of mining openings }\end{array}$ & $\begin{array}{l}\text { Determination of structure and nature of rock block } \\
\text { anisotropy }\end{array}$ & 60 \\
\hline $\begin{array}{l}\text { 3.4. Hydrogeological studies of composition, } \\
\text { distribution and conditions of intraformation } \\
\text { groundwater; evaluation of possibilities and } \\
\text { methods to control water flows in permeable } \\
\text { zones }\end{array}$ & $\begin{array}{l}\text { Parameters of the main migration mechanism - i.e. } \\
\text { radionuclide transferring with water flows }\end{array}$ & 84 \\
\hline $\begin{array}{l}\text { 3.5. Integrated field studies carrying out: } \\
\text { geomechanical, radiation and thermal, etc. }\end{array}$ & $\begin{array}{l}\text { Determination of the effect of short-range zone rock } \\
\text { changes to conditions of HLW and SNF isolation }\end{array}$ & 48 \\
\hline
\end{tabular}




\begin{tabular}{|c|c|c|}
\hline Short description of scientific studies & Results (input data for designing) & $\begin{array}{l}\text { Duration, } \\
\text { months }\end{array}$ \\
\hline $\begin{array}{l}\text { 3.6. Development of engineered barriers } \\
\text { groupings, and carrying out of field tests for } \\
\text { imitated and real packages of RAW }\end{array}$ & $\begin{array}{l}\text { Evaluation of engineered barriers within the overall } \\
\text { system of SNF and RAW storage and disposal } \\
\text { (structure, arrangement, life-time, operation, etc.) }\end{array}$ & 36 \\
\hline $\begin{array}{l}\text { 3.7. Gas survey carrying out in laboratory } \\
\text { openings; tracer gas tests }\left(\mathrm{He}, \mathrm{H}, \mathrm{CH}_{4}\right)\end{array}$ & $\begin{array}{l}\text { Definition of gas permeability for rocks, jointing } \\
\text { zones, contact zones, etc. }\end{array}$ & 36 \\
\hline $\begin{array}{l}\text { 3.8. Planning and creation of the system of force } \\
\text { and sampling bore-holes (from the earth surface } \\
\text { and from underground laboratory openings) to } \\
\text { carry out tracer tests. Tracer tests for a large rock } \\
\text { block with a use of standard xanthane colorants } \\
\text { and single isotopes }(\mathrm{H}, \mathrm{He}, \mathrm{Br}, \mathrm{l} \text {, et al.) }\end{array}$ & $\begin{array}{l}\text { Determination of the main parameters of migration } \\
\text { system }\end{array}$ & 60 \\
\hline $\begin{array}{l}\text { 3.9. Modeling of structural and geochemical } \\
\text { migration areas within the ranges of lithosphere } \\
\text { space of the underground laboratory. Validity } \\
\text { appraisal for the results of field tests with real } \\
\text { conditions of prospective site for construction of } \\
\text { underground complex for HLW and SNF isolation }\end{array}$ & $\begin{array}{l}\text { Quantitative assessments of dimensions of buffering } \\
\text { zone, facility allotment zone (controlled), place and } \\
\text { depth for arranging of repositories, HLW disposal } \\
\text { bore-holes, etc.) }\end{array}$ & $\begin{array}{l}36 \\
\text { (after com- } \\
\text { pletion of } \\
\text { experiments } \\
\text { in situ) }\end{array}$ \\
\hline $\begin{array}{l}\text { 3.10. Statement and carrying out of the studies on } \\
\text { definition of rock deformation caused by } \\
\text { spontaneous and cyclic processes (earthquake, } \\
\text { tidal friction, etc.) }\end{array}$ & $\begin{array}{l}\text { Safety prediction for radioactive materials isolation in } \\
\text { blocks of the granitoid massif }\end{array}$ & 36 \\
\hline $\begin{array}{l}\text { 3.11. Substantiation and selection of the } \\
\text { integrated monitoring system for geotechnological } \\
\text { system of HLW and SNF isolation in the Northern } \\
\text { part of the Nizhnekansky granitoid massif }\end{array}$ & $\begin{array}{l}\text { Parameters of monitoring system for ecologically } \\
\text { dangerous subject }\end{array}$ & $\begin{array}{l}12 \text { (after } \\
\text { completion } \\
\text { of } \\
\text { experiments } \\
\text { in situ) }\end{array}$ \\
\hline
\end{tabular}




\begin{tabular}{|l|l|c|}
\hline \multicolumn{1}{|c|}{ Short description of scientific studies } & \multicolumn{1}{c|}{ Results (input data for designing) } & $\begin{array}{c}\text { Duration, } \\
\text { months }\end{array}$ \\
\hline $\begin{array}{l}\text { 3.12. Selection substantiation for engineering } \\
\text { arrangements and technical means called to } \\
\text { restrict and eliminate negative processes } \\
\text { development in controlled system of radioactive } \\
\text { materials isolation }\end{array}$ & $\begin{array}{l}\text { Safety assurance for radioactive materials } \\
\text { underground storage }\end{array}$ & $\begin{array}{c}24-36 \text { (after } \\
\text { completion } \\
\text { of } \\
\text { experiments } \\
\text { in situ) }\end{array}$ \\
\hline $\begin{array}{l}\text { 3.13. Integrated analysis and scientific } \\
\text { substantiation of safety of the complex for HLW } \\
\text { and SNF underground isolation in blocks of the } \\
\text { Nizhnekansky granitoid massif }\end{array}$ & $\begin{array}{l}\text { Input data for carrying out the ecological expertise for } \\
\text { the HLW and SNF underground isolation complex } \\
\text { construction }\end{array}$ & $\begin{array}{c}36 \text { (after } \\
\text { completion } \\
\text { of } \\
\text { experiments } \\
\text { in situ) }\end{array}$ \\
\hline $\begin{array}{l}\text { 3.14. Subject selection and forming the program } \\
\text { intended to demonstrate in underground } \\
\text { laboratory the safety of HLW and SNF isolation } \\
\text { conditions }\end{array}$ & Demonstration arrangements for the local \\
administration and population & $\begin{array}{c}12 \text { (after } \\
\text { completion } \\
\text { of } \\
\text { experiments } \\
\text { in situ) }\end{array}$ \\
\hline
\end{tabular}




\section{References}

1. Criteria for Underground Disposal of Solid Radioactive Wastes, IAEA Safety Series No 60, IAEA, Vienna, 1983.

2. Safety Principles and Technical Criteria for the Underground Disposal of High-level Radioactive Wastes, Safety Series No. 99, IAEA, Vienna, 1989.

3. Siting, Design and Construction of deep geological repository for the disposal of high level alpha bearing wastes - IAEA-TECDOC-563, IAEA, Vienna, 1990.

4. Report on Radioactive Waste Disposal, IAEA Technical Reports Series No 349, IAEA, Vienna, 1993.

5. Experience in selection and characterization of sites for geological disposal of radioactive waste IAEA-TECDOC-991, IAEA, Vienna, 1997.

6. E. B. Anderson, V. G. Savonenkov, S. I. Shabalev, et al. Complex Geological Investigations to Select a Site for High-Level Waste Disposal in the Krasnoyarsk Region (Russia). "Defense Nuclear Waste Disposal in Russia: International Perspective" NATO ASI 1/18. Ed. by M. J. Stenhouse and V. I. Kirko. Kluwer Academic Publishers, Dordrecht, the Netherlands, 1998, pp. 135-141.

7. E. B. Anderson, S. I. Shabalev, V. G. Savonenkov, E. F. Lyubtseva. Investigations of the Nizhnekansky granitoid massif (Middle Siberia, Russia) as a promising site for deep geological disposal of HLW. Proceedings of the International Conference on Radioactive Waste Disposal "Disposal Technologies and Concepts 1998 (DisTec'98)" held in Hamburg, Germany, September 9-11, 1998, pp. 105-110 (1998). Published by Kontec Gesellschaft für technische Kommunikation mbH.

8. Investigations of granitoids of the Nizhnekansky massif for radwaste disposal (Materials of the Coordination Scientific-Technical Council meeting held in Zheleznogorsk, March 16-22, 1998). Ed. E. F. Lyubtseva, St. Petersburg, 1999, 182 p. (in Russian)

9. E. B. Anderson, S. I. Shabalev, V. G. Savonenkov, et al. Investigations of the Nizhnekansky Granitoid Massif (Middle Siberia, Russia) as a Promising Territory for Deep Geological Disposal of HLW: Results of Pre-exploration Stages of the Work. "Scientific Basis for Nuclear Waste Management XXII”, Mat. Res. Soc. Symp. Proc., Vol. 556, pp. 543-550 (1999).

10. E. B. Anderson, V. G. Savonenkov, S. I. Shabalev, et al. The Proposed Geologic Repository Site at the Nizhnekansky Granitoid Massif near Krasnoyarsk, Russia, Proc. of the $9^{\text {th }}$ International High-level Radioactive Waste Management Conference (IHLRWM), Las Vegas, NV, USA, 2001, on CD - ISBN: 0-89448-654-3.

11. S. I. Shabalev, E. B. Anderson, V. G. Savonenkov, et al. Laboratory Characterization of Rock Specimens from the Nizhnekansky Massif (Potential Site for HLW Final Disposal in Middle Siberia, Russia), Proc. of the $9^{\text {th }}$ International High-level Radioactive Waste Management Conference (IHLRWM), Las Vegas, NV, USA, 2001, on CD - ISBN: 0-89448-654-3. 


\title{
Problems of Underground Disposal of High-level Radioactive Waste and Long-term Storage of Spent Nuclear Fuel in Russia: Status Assessment and Position of the IGEM RAS
}

\author{
V. I. Velichkin, V. A. Petrov, N. N. Tarasov, IGEM-RAS
}

\section{Russian State Policy with Regard to Isolation of RAW and SNF}

The policy of Russian governmental agencies with regard to the final stages of handling of radioactive waste (RAW) and spent nuclear fuel (SNF) for the period of 2000 through 2006 is concisely stated in the Unified Federal Objectives Program (UFOP), the "Nuclear and Radiation Safety of Russia". This program was established by the government of the Russian Federation by decree № 149 of February 22, 2000. The Ministry for Atomic Energy of the Russian Federation (Minatom) is the primary coordinating agency responsible for its implementation.

The program states that Russia's strategic objective in the area of reliable isolation of RAW and SNF involves the creation of experimental-industrial regional storage facilities for RAW on the archipelago of Novaya Zemlya, at the PA "Mayak", in the region of the Krasnoyarsk MChC, and in the Far East Region. It also includes the building of temporary storage facilities for SNF in the regions indicated and on the territories of operational nuclear power plants (NPP). The modest financial resources provided for the execution of the program tasks are clearly insufficient to achieve significant breakthroughs towards the practical solution of this grandiose problem.

The program also provides relatively modest sums for research aimed at perfecting the means and methods of conditioning the RAW, for the transportation of various nuclear materials, selection of geological environment and sites for the storage and disposal of RAW and SNF.

Along with work performed under government programs in the period between 1995 and 2000, research was conducted on the collection and analysis of materials pertinent to the selection of a storage and disposal site for RAW and SNF on the Kola peninsula. This effort was supported financially by the TACIS program in concert with Minatom.

Initiated by Minatom and Russian Academy of Sciences Institute of Geology of Ore Deposits, Petrography, Mineralogy and Geochemistry (IGEM RAS), and currently being considered, are proposals to use the territory of the Priargunskoe Production Mining Chemical Association (PPMChA, East Transbaikal area) for the storage of SNF and other nuclear materials.

RAS specialists are preparing proposals for resolving the issues of isolation of the RAW and SNF in the Far East region.

On the whole, the Russian Academy of Sciences is supportive of the Minatom strategy of creating regional facilities for the safe isolation of radioactive materials; however, the specialists from the RAS and the Minatom have differing approaches to the solution of some specific problems. 


\section{Status and Assessment of Current Efforts in Russia with Regard to Isolation of RAW and SNF: Results of the IGEM RAS Studies}

In keeping with the goal of establishing regional experimental-industrial disposal sites for RAW and storage facilities for SNF in Russia, work on the selection and investigation of geologic media and suitable sites, and on other factors essential for reliable isolation of the disposal sites, is currently underway, or in planning stages, in areas where radiochemical concerns of the Minatom RF are situated, or where atomic fleet vessels are based (Fig. 1).

IGEM RAS participates in all these efforts by performing comprehensive studies to provide geologic solutions to problems of underground high-level waste (HLW) disposal and SNF storage [9] (Laverov et al., 2000). These studies, together with associated theoretical and experimental investigations, are carried out by the highly qualified scientific personnel of the Institute using the latest high-precision analytical equipment.

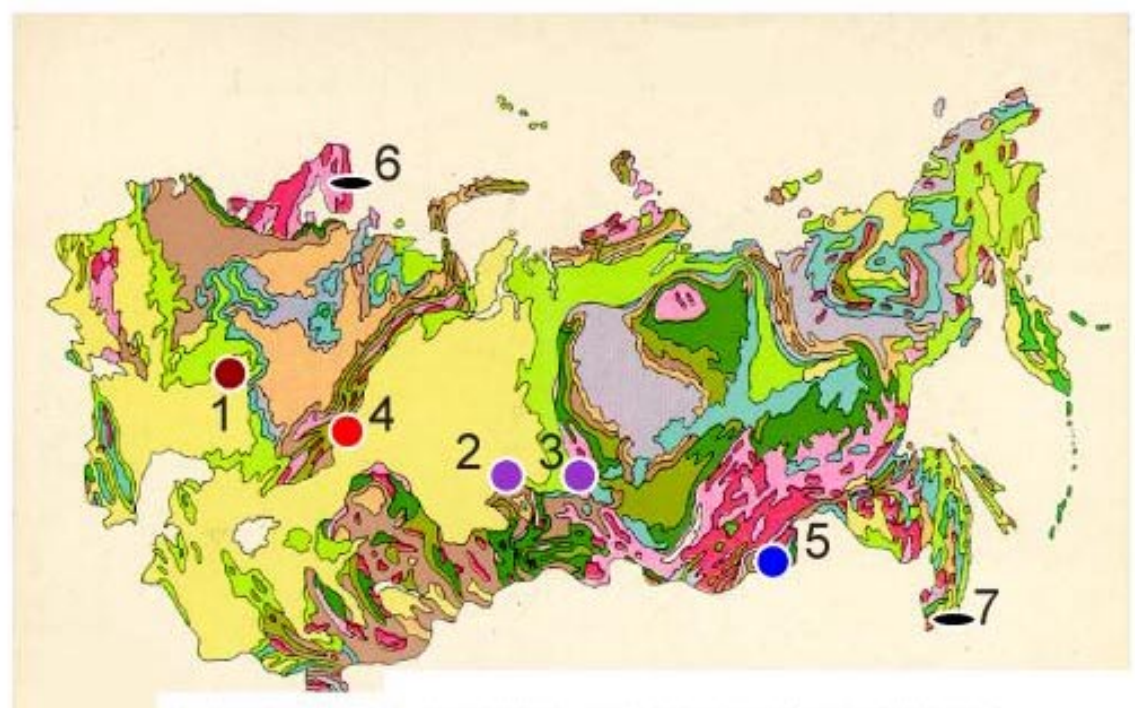

1- Dimitrovgrad; 2 - Tomsk 7; 3 - Krasnoyarsk 26; 4 - PA "Mayak"

5 - Krasnokamensk; 6- Kola Peninsula region; 7 - Far East region

Fig. 1. Radioactive hazardous territories and facilities have been studied by specialists of the IGEM RAS.

Basic research is conducted at the IGEM RAS in the following areas:

- The behavior of intrinsic and natural radionuclides in natural and industrial landscapes.

- The geological and hydrogeochemical conditions for migration and concentration of radionuclides and other toxic substances in ground water in crystalline (magmatic and metamorphic) rock massifs and stratified sedimentary systems.

- The physical and geochemical isolation properties of rock with respect to various isotopes, and changes in such properties at elevated temperatures and pressures. 
- Tectonic disturbance in promising geologic blocks and their stress-strain state at present and in the past periods of tectonic deformation, the object being to arrive at an estimate of the time limit for reliable isolation of radioactive materials over the potentially hazardous life interval of such materials.

- The peculiarities of convectional heat mass transfer as a function of heat emission from the buried $\mathrm{HLW}$ in order to use predictive modeling for the migration of contaminated ground water in cases when the hermetic sealing of HLW repositories has been compromised.

- Physical and chemical principles of synthesizing various matrix-preservers of HLW, their mineralchemical composition and capacity, their chemical, thermal, and radiation stability, forms of occurrence and the patterns of radionuclide distribution.

Presented below is the status of the work, either in progress or in planning stages, performed in areas of prospective regional facilities for storage-burial of RAW and SNF. Also shown are the results of the IGEM RAS studies conducted on the range of problems under consideration.

\subsection{Research in the northwestern region of Russia}

During the 1990s, under the direction of the All-Russian Research and Design Institute of Production Engineering (VNIPIPT) and with the participation of their experts, prospecting and geological-engineering work was carried out on the southern island of the Novaya Zemlya archipelago, in the area of the Bashmachnaya Guba. As a result, a 6 sq. km area was chosen on a territory of carbonate rock of Paleozoic age, frozen for many years, in which RAW would be buried at a [relatively] shallow level [8] (Krone et al., 2000).

The assumption is that low- and medium-activity waste would be buried here, i.e., waste generated by the operation and, subsequently, the salvaging of naval and civilian vessels of the Northern Atomic Fleet. Currently under discussion is the development of a working design for a start-up system of this experimental-industrial facility.

Unfortunately, the version of RAW burial under discussion here does not solve the main radiation problem of the Northwest region, namely, reliable isolation of HLW and SNF resulting from the scrapping of atomic submarines (AS) and generated by the operation of the Kola NPP. The project also has a number of other drawbacks.

In the period between 1995 and 2000, studies were conducted in the northwest region involving the collection, evaluation and integration of geological and geophysical information with the aim of selecting a site for the storage of SNF and burial of RAW, including HLW. A decision was made that the future site will be equipped with appropriate engineering systems suitable not only for the isolation of spent nuclear materials from the nuclear fleet and its supporting enterprises, but also for the Kola NPP. The storage of the SNF is envisioned as being in aboveground structures, whereas the burial of the RAW is to be in an underground repository.

The work was performed in coordination with the Minatom RF and with financial support from the TACIS program. The lead organization in this effort was the Mining Institute KNC RAS. A group of IGEM RAS personnel participated in the work.

The actiivities of collection, evaluation, and integration of geological and geophysical data, and the selection of the site were based on a process of elimination, in which unsuitable sites would be gradually discarded. In the first stage-evaluation of regional candidate site-the following areas were eliminated from 
consideration: sites that contained, or were likely to contain, mineral resources; seismically unstable zones; large regional fault lines with their adjoining disturbed areas; and heavily populated territories.

In the second stage, out of the remaining surface area (which by now had shrunk to no more than $15 \%$ of the initial area), 25 promising sites were selected based on an evaluation of the materials of the Murmansk and Archangelsk geologic funds. Of these, 16 are on the Kola Peninsula (Figs. 2, 3).

In the third stage, in accordance with the regional and local criteria for site selection, a comparative analysis and assessment of geologic characteristics of the above 25 sites was made, which resulted in seven sites being selected for further study. The final determination of their suitability for underground disposal was made with the aid of quantitative ratings. These were obtained on the basis of experts' estimates of the degree of conformance of the characteristics of each site to ranking criteria, including environmental factors that would ensure the safety of the future facilities, and social and economic conditions. Finally, two sites were chosen for further study: Dalniye Zenetsy and Kuzruka, located, respectively, on the northern and southern shores of the Kola Peninsula. Both are composed of massive plagiomicrocline granites with low porosity and little fracturing.

In the next stage, involving drilling, geophysical studies, geological surveying, and special hydrogeological, petrochemical, petrophysical, and geodynamic investigations, both sites will have to be investigated in detail and a construction site suitable for the underground laboratory will be chosen.

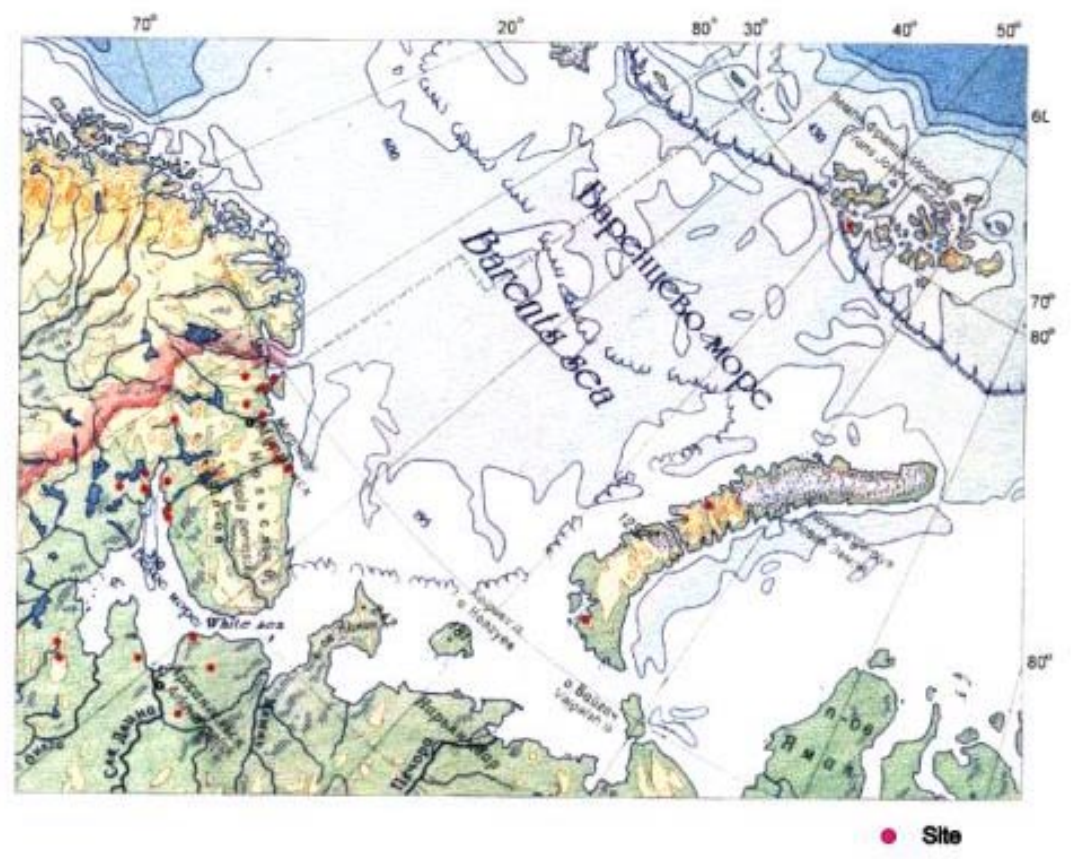

Fig. 2. Location of the sites within the Kola region (after Improvement..., 1996). 


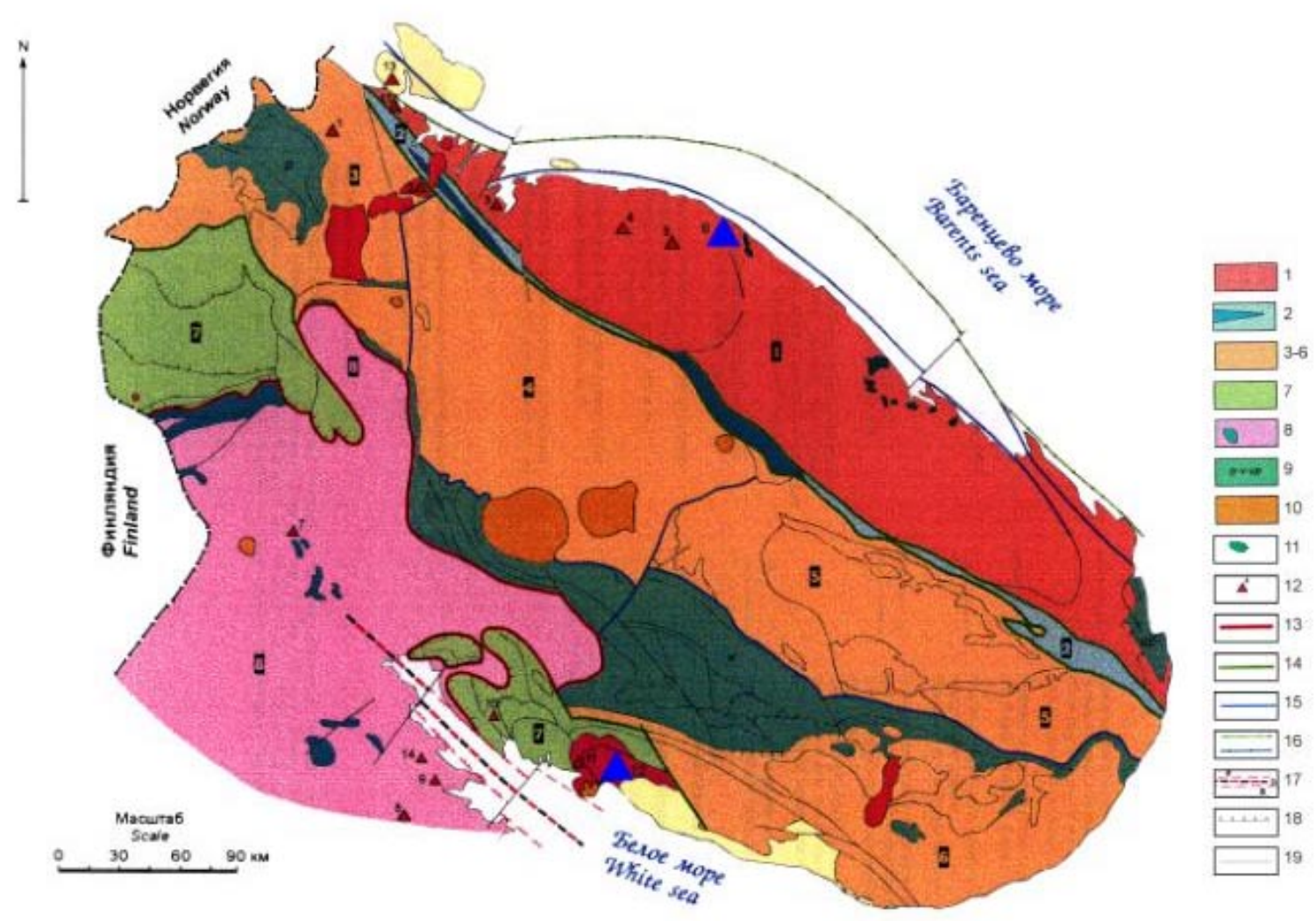

Fig. 3

Fig. 3. Structural map of the Kola region (Rev., 1996).

1- Murmansk block; 2- Uraguba-Severo-Keivskaya paleocollision zone with the Archean greenstone belt relict; 3-6Kola block and domains - western (3), central (4), Keyvsky (5) and south-east (6); 7- Laplandian-Kolvitsa zone of granulite; 8- Belomorian geoblock; green stone belt relicts of the Archean age; 9- Rifting and collision zone of the Early Proterozoic age (Pechenga - Varzuga - Ust-Ponoy); 10- Paleozoic massifs of alkaline rocks; 11- Sedimentary rocks of Upper Proterozoic age; 12- Riphean dolerite; 13- Early Proterozoic granitoid complexes: Litsky-Aragubsky (a), Umba (b); 14- Sites location: 1 - Kirikovan, 2 - Valsejavr, 3 - Kutovoi, 4 - Kiijavr, 5 - Odjavr, 6 - Dalny Zelentsy, 7 - Yona, 8 - Vinga, 9 - Poyakonda-Nigrozero, 10 - Porya, 11 - Sormozero, 12 - Kuzreka, 13 - Sredny, 14 Kyulya, 15 - Titovka; 15- Boundary between Kola-Mezen and Belomorian geoblocks;16-Boundary of second range structure; 17- Boundary of next range domain; 18- System of fault with kinematic changes at time "fault-reverse fault" with periodical recent seismic activity; 19-Kandalaksha graben (a) and its axis (b); 20- Thrust fault; 21- Other tectonic or geological boundary.

\subsection{The area of the PA "Mayak"}

Stored at this facility are more than $2000 \mathrm{t}$ of highly active aluminophosphate glass, with a total activity of some 300 million $\mathrm{Ci}$, that was produced in the course of solidification of previously accumulated liquid HLW [5] Glagolenko et al., 1997). After an appropriate holding period in temporary storage, this solid waste is to be sent to geologic disposal. Additional quantities of solidified HLW are being produced regularly in the process of vitrification of liquid $\mathrm{HLW}$, which are generated during the current reprocessing of the SNF. Future plans envision the removal from all types of liquid HLW the independent actinide and the Cs-Sr fractions, which, after having been immobilized in appropriate matrices, will also be added to the existing HLW awaiting burial. 
Thus, the necessity becomes quite evident for creating an experimental-industrial facility in the area of the PA "Mayak" for underground disposal of solidified HLW.

Work related to the selection of a site suitable for the construction of a burial site for HLW was first undertaken on the territory of the PA "Mayak" by VNIPIPT specialists in late "80s. At the "Mars-2" site ( $0.25 \mathrm{sq} . \mathrm{km}$ area), which is located within the confines of the health-protection zone (HPZ), several boreholes were drilled to a depth of 1,200 $\mathrm{m}$ and geophysical studies were performed [7] (Kedrovsky et al., 1991). Unfortunately, these efforts were not followed up. Nonetheless, the core samples from the boreholes and the results of geophysical mapping are to this day the only reliable, factual materials characterizing the depth structure of the central portion of the HPZ of the PA "Mayak". These data are particularly important because, for social reasons, nuclear materials may be buried at the PA "Mayak" only within the confines of the HPZ.

The geologic structure of the HPZ territory and adjacent areas studied by the specialists of the IGEM RAS is shown in the attached geologic map (Fig. 4). It has been determined that the volcanic rock of andesitebasalt composition, a constituent of the central block of the HPZ, is a geological medium that is capable of providing reliable isolation of the HLW [12] (Velichkin et al., 1997).

In undisturbed and non-eroded state, the andesite-basalts possess high density, elasticity, and strength characteristics in conjunction with low values of effective porosity and permeability (Fig. 5). The filtration rate of ground water in these rocks is estimated at about $5 \times 10^{-5} \mathrm{~m} / \mathrm{yr}$.

At the same time, the territory of the PA "Mayak" is characterized by an intense yet nonuniform tectonic disturbance. It is "broken up" by extensive (many km) and massive (many hundreds of meters) zones of regional faults of different orientation into separate blocks, which, in turn, are often dissected by smaller intrablock faults of second order, as well as by separate zones of disturbed rock of third and higher orders (small zones of schist formation and convergence cracking). Within the faults of the first and second orders, the rocks are subject to intense schist formation, fragmentation, and include tectonic seams frequently made complex by shoves of postquatenary age. The tectonic elements listed above possess high permeability and, in the case of mountain andesite-basalts, are the primary transport medium for ground water [11] (Petrov et al., 2000). In connection with the high water permeability and the likelihood of "rejuvenation" by the most recent tectonic moves, the fault zones are considered unsuitable for engineering structures for a prospective disposal site. However, situated among them are blocks that have been disturbed to a considerably lesser degree. From this group of blocks, contained within the contour of the HPZ and composed of basic volcanic rock with highly efficient isolation properties, two sites (Fig. 6) were recommended for further detailed study to determine their suitability for the construction of an underground laboratory. The limited dimensions of both sites (1.5 sq. km and $3 \mathrm{sq} . \mathrm{km})$ will apparently preclude situating the laboratory in a mining shaft. In this situation it would be advisable to consider the possibility of creating the underground laboratory and the future burial site in special boreholes drilled from above.

Since the solidified HLW that have been made ready to be buried contain significant quantities of longlived radioisotopes whose toxicity persists for many millennia, the planned HLW disposal site must be situated in a geological environment that will guarantee reliable isolation of the buried waste from the ecosphere for a time period commensurate with the geologic time scale. Consequently, the upcoming detailed investigation of prospective sites must not only select a site of minimal tectonic disturbance and 


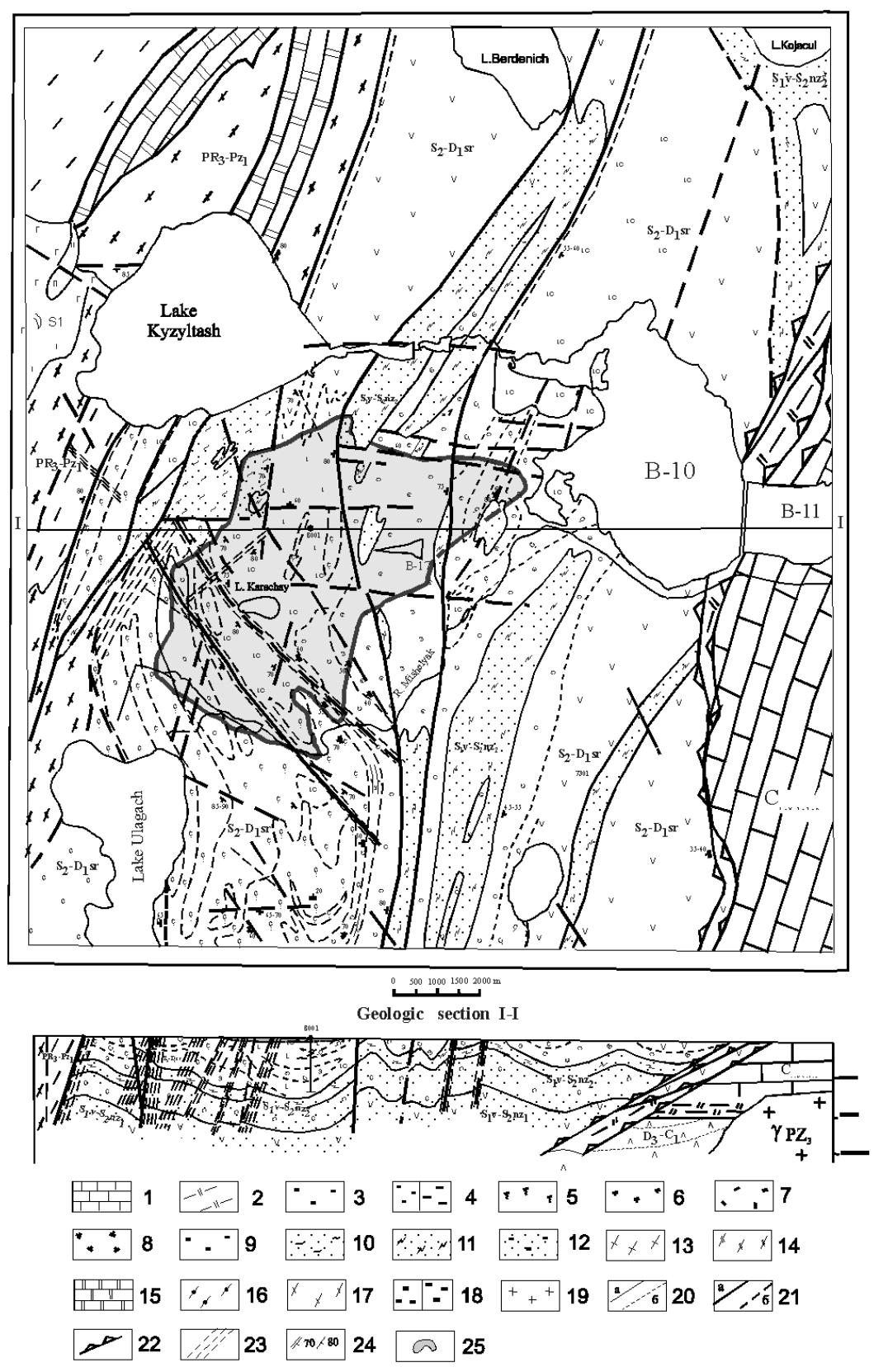

Fig. 4. Geologic map of the PA "Mayak" territory. 1-13- Paleozoic structural-formation complex: 1- limestone, marbled limestone $\mathrm{C}_{1-2} ; 2$ - polymict sandstones, calcareous clayey shale; 3- non-articulated volcanogenic-sedimentary rocks of $D_{3}-C_{1} ; 4-9$ - volcanogenic and volcanogenic-sedimentary rocks of $S_{2}-D_{1}$ : lavas and tuffs-lavas of pyroxene-plagioclasic porphyrites of andesite-basaltic composition (4); coarse-psephite tuffs up to agglomerate-blocky (5); fine- psephitic tuffs (6); tuffs aleuro-psammitic and psammitic (7); tuffs-aleurolites, tuffssandstones, siliceous tuffites with elements of lamination (8); non-articulated volcanogenic and volcanogenicsedimentary rocks $S_{2}-D_{1}(9) ; 10-12$ - volcanogenic and volcanogenic-sedimentary rocks of $S_{1}-S_{2}$ : chlorite-sericitic schists (10); siliceous and coaly-siliceous schists (11); non- articulated volcanogenic-sedimentary rocks $S_{1}-S_{2}(12)$; 13-quartz-albite-chlorite-sericitic schists; 14-17- metamorphic rocks of $\mathrm{PR}_{3}-\mathrm{PZ}_{1}$ structural-formation complex: chlorite schists (14); marbled limestone, marbled (15); biotite, biotite-amphibole schists and gneisses (16); amphibolites (17); 18-19-intrusive rocks: gabbro, gabbro-pyroxenites (18); granite, granodiorite (19); 20- stratigraphic (a) and rock facies (b) boundaries; 21- established (a) and proposed (b) faults; 22- Argajaschskyi overthrust; 23- shear zones; 24lamination (a) and faults (b); 25- outline of underground water contamination aureole spreading from the Lake Karachay. 


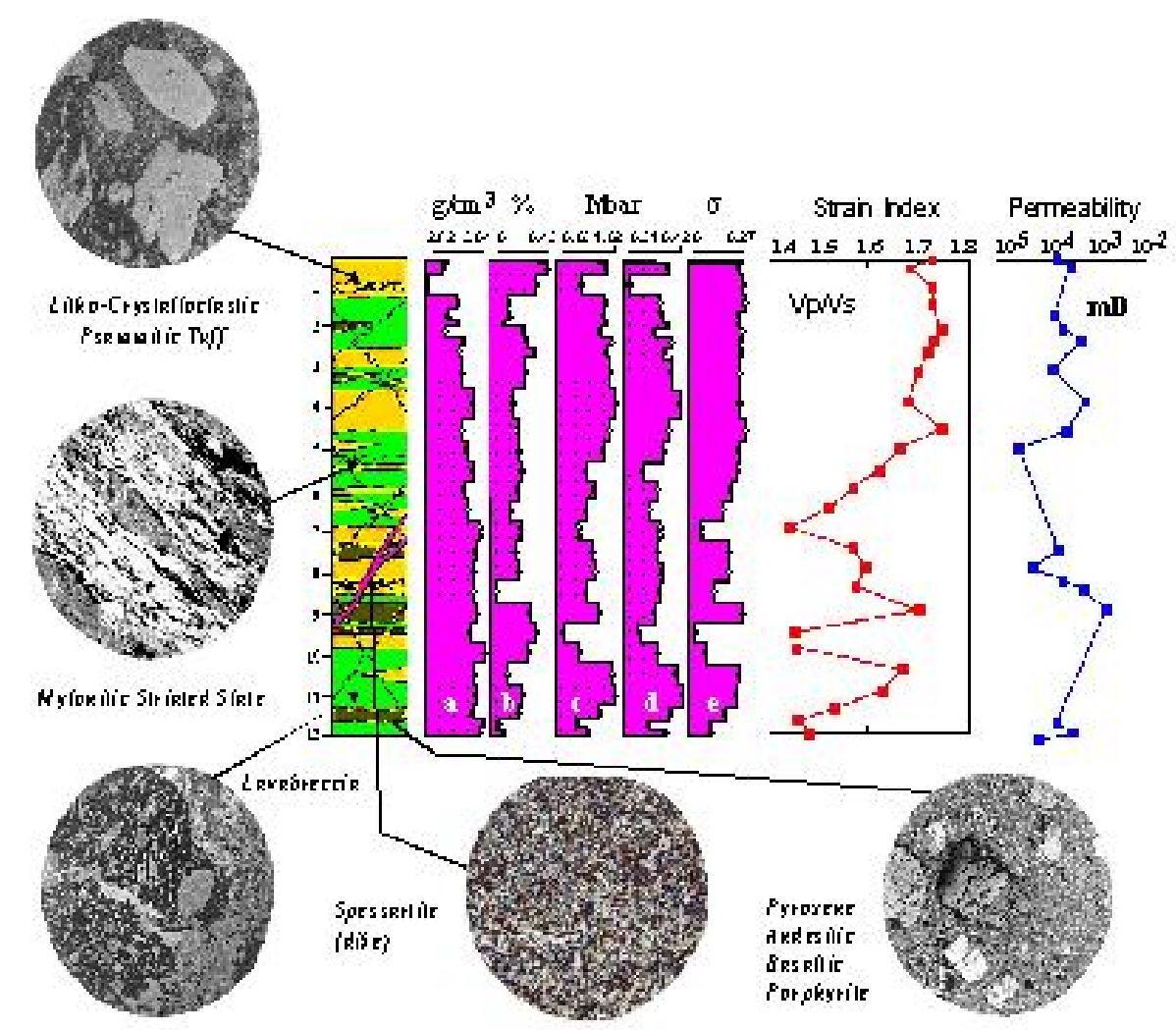

Notice: a - density, b - effective porasity, c - Yaung's modulus, d'- shear modulus, e - Poissor's atio

\section{Fig. 5. Variation of the main petrophysical parameters of metavolcanic rocks as a function of depth in the boreholes 8001-8002, Mars-2 site of the PA "Mayak" territory.}

low levels of stress in the geologic medium, but must also incorporate special studies to evaluate the prospects of preserving the isolation capabilities of the chosen site throughout the radiobiologically dangerous life span of the long-lived radionuclides contained in the buried HLW.

The activities of studying the chosen areas, and the selection from among them of the actual site for the construction of the underground laboratory, should include drilling operations, geophysical, geostructural, engineering-geological, hydrogeological, hydrogeochemical, as well as special petrophysical, petrochemical, and geodynamic investigations.

The most complex and critical of the issues that need to be resolved in the process of selecting a site is estimating the preservation of isolation capabilities of the chosen site on a geologic time scale. The initial information for such an estimate is based on data reflecting the current stress-strain state of the geologic medium at the site and of the blocks adjacent to it, and on information on the geodynamic conditions that existed in the area in the preceding geological epochs. In the case when the dynamic conditions and the orientation of tectonic stresses for the current time period coincide with those of the preceding geological epochs, and the time lapse between them is in millions of years, one may claim with sufficient correctness that the geodynamic conditions of the territory being studied remained stable over the noted time interval and were preserved for the time needed for the isolation of long-lived isotopes. 


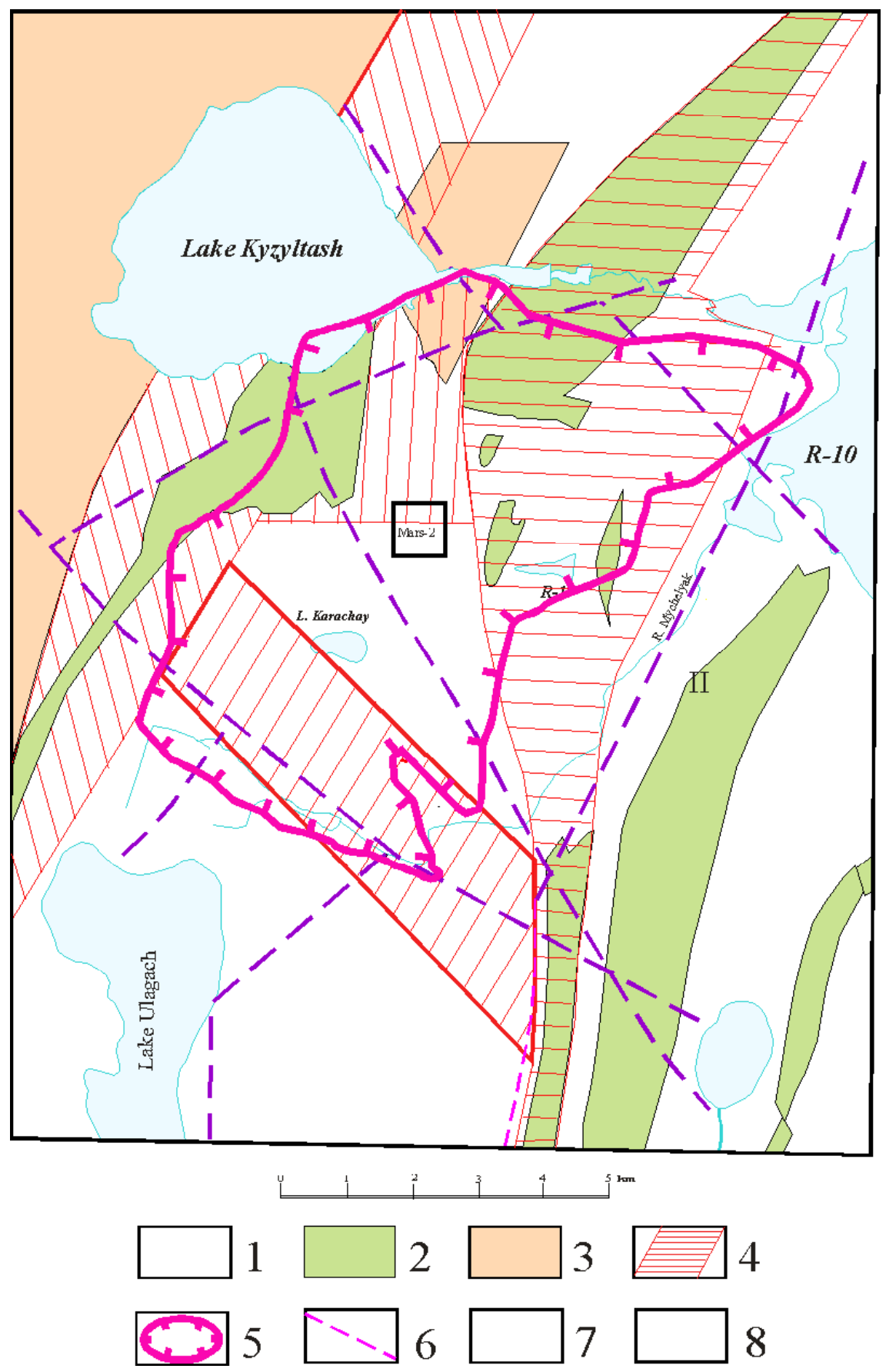

Fig. 6. Tectonic zoning of the "Mayak" operations area. 1-13- structural blocks: upper suite (1), lower suite (2), metamorphic complex (3); 4- blocks of intensive tectonic disturbance; 5- outline of contamination aureole; 6- rupture disturbances selected on geomorphological data; 7- outline of areas potentially favorable for HLW underground disposal; 8- boundary of sanitary-protective zone.

The contemporary geodynamic situation in the vicinity of the PA "Mayak" is defined by the location of the indicated territory in the Ural Mountains system, and in a section of it that is under pressure from the Ufa 
ledge of the early Russian platform (Fig. 7). It has been established that the geologic medium of the PA "Mayak" territory is subjected to sublatitudinal regional compression whose main tectonic-stress vector has an absolute value of 20-50 MPa at a depth of $300 \mathrm{~m}$ from the surface [2] (Aleynikov et al., 1988). In this situation, the numerous, differently oriented tectonic disturbances of the PA "Mayak" territory are characterized by nonuniform dynamic and hydrodynamic parameters. Faults with submeridional orientation are in a "compressed" state, which produces a drastic reduction in their permeability. The tectonic elements with north-east and north-west orientation are in intermediate conditions, which predetermined their variable water permeability. The sublatitudinal fracture disturbances are in a state of transverse relative tension, which promotes a heightened permeability in such disturbances as compared with those having different orientations. This description of the geodynamic conditions is confirmed by the first results of studies that were initiated in the area of the PA "Mayak" for purposes of monitoring the relaxation processes [1] (Adushkin et al., 1997).

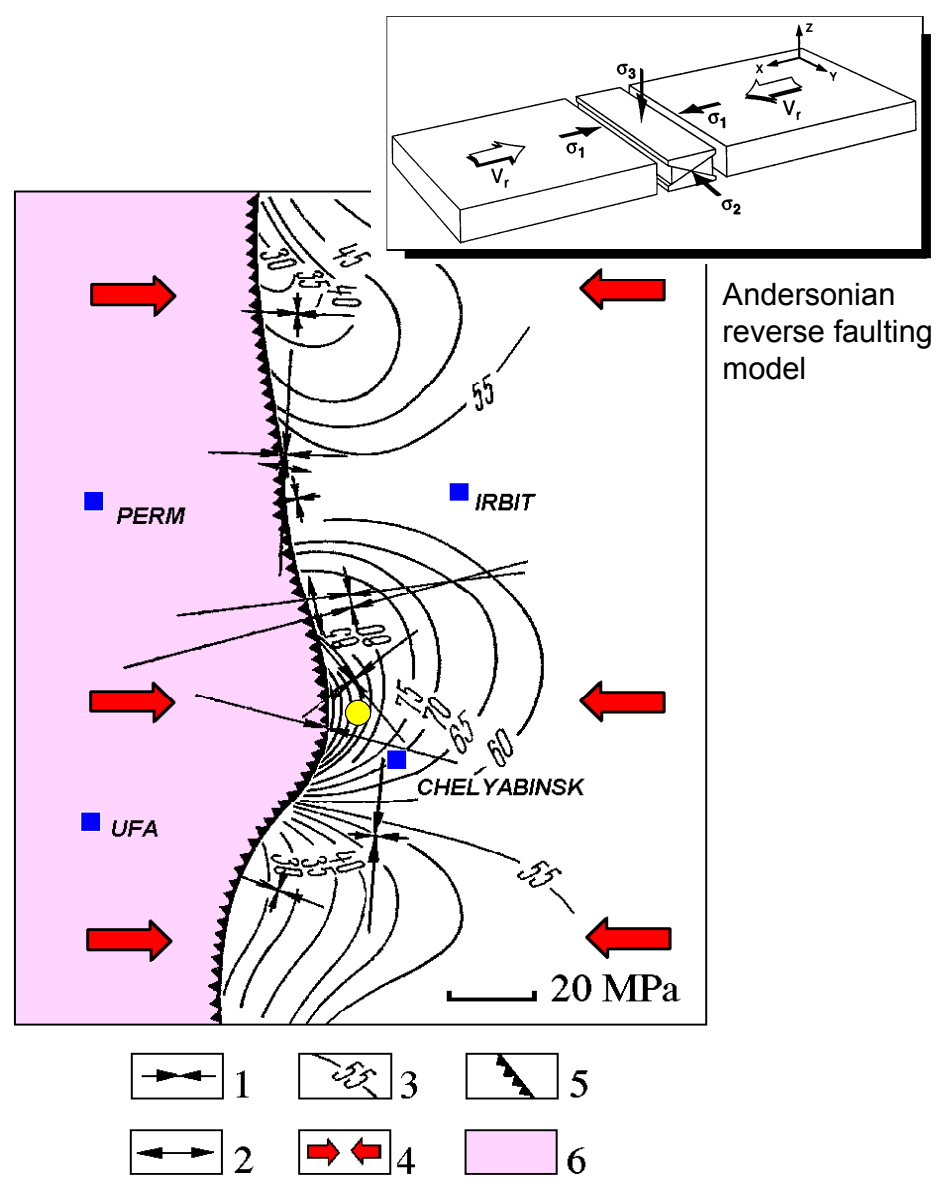

Fig. 7. Simulation of regional stress field and deformation pattern. These conditions have remained in the Urals at least since the Holocene period (after Aleinikov at al., 1988). 1 compression $(\sigma 1), 2$ - tension $(\sigma 3), 3$ - value of deviatoric shear stress $(\tau), 4$ - direction of regional shortening, 5 - boundary between the folded belt and sub-platform, 6 - solid-state punch of the model.

Special studies of morphology, internal structure, bedding conditions, and spatial-temporal correlation of differently oriented fractured structures of the bedrock on the PA "Mayak" territory made it possible to reconstruct the map of the late Cenozoic tectonic deformations, and to show its similarity to the present- 
day geodynamic conditions (Fig. 8). These data make it possible to make a preliminary conclusion regarding the probability of preserving the indicated conditions over the period of potential danger from the long-lived radionuclides.

Thus, in the course of the last few years, comprehensive studies have been carried out in the area of the PA "Mayak". These studies should be viewed as an important, albeit initial, stage in the efforts to find a solution to the problem of reliable isolation of HLW that has been accumulating and is being produced [now] at the enterprise.

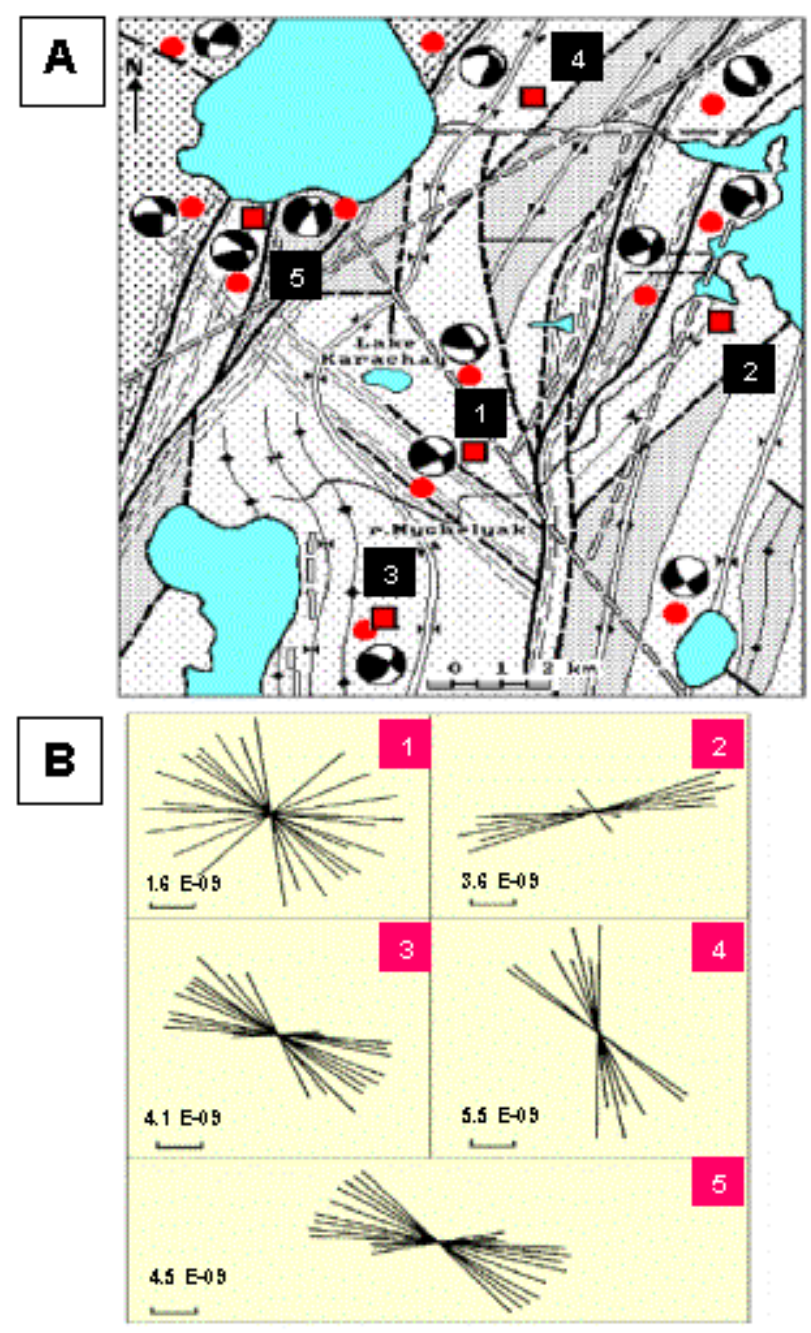

Fig. 8. A comparison of stress regime and rock mass state of the PA "Mayak" territory during Cenozoic (A) and modern (B) episodes of deformation. Orientation of the major principal stress $\sigma 1$ (black sections) reproduced for the latest displacements during Cenozoic period. Current distribution, orientation and value of strain are derived (after Adushkin et al., 1997) from monitoring of seismorelaxation process intensity in the blocks.

During the next phase of work, the character and degree of tectonic disturbance, and the present stressstrain state of the geologic medium of both prospective sites need to be studied in greater detail. It is also desirable to continue the research into the reconstruction of the tectodynamic conditions of the geological past. And in the final phase of the work, a geodynamic model of the area around the PA "Mayak" should 
be developed so as to be able to evaluate the likely progression of the tectonic processes over an interval of time needed for long-term isolation of buried HLW. Once these and all other data have been taken into account, the site for the underground laboratory will be selected.

\subsection{The MChC Area (Krasnoyarsk-26)}

The MChC area is intended to serve as the place of burial of the solidified HLW made from liquid waste from the reprocessing of SNF. A new reprocessing plant, the PT-2, is planned. Once it becomes operational, the amount of HLW subject to burial will increase sharply. Simultaneous work is planned on both the HLW burial and the creation of required conditions for the storing of SNF.

During the 1992-1998 period, various organizations of the Minatom, the Ministry of the Environment, the Krasnoyarsk region, and the RAS in the region of the MChC, conducted comprehensive studies to identify prospective sites for the burial of the HLW and the storage of SNF. The Radium Institute of the Minatom RF was the lead institute in this effort.

During the first stage of the project, data from [published] literature were used to analyze regional geologic materials that characterized a wide expanse of the area around the $\mathrm{MChC}$, and 20 parcels were selected for further consideration. After a comparative analysis during the second stage, two parcels were selected for [further] geological and geophysical investigations. These were conducted during the third stage of the work. The result of this effort was the selection of two areas (the Itatsky and Kamenny) in the Nizhnekansky granitoid massif (Fig. 9) on which, after a detailed study, a site for the construction of the underground laboratory would be chosen [3] (Anderson et al., 1998).

It should be noted that the field work done in the course of the above investigations suffers from a serious methodological flaw. In view of the very substantial volume of geophysical work performed (which is fully justified, given the natural environment of the region being studied), the geological investigations were obviously insufficient, and lacked a clear-cut systematical basis. This gives rise to the possibility of an inadequate geological interpretation of the results of geophysical observations, and may result in a dearth of representative rock [samples] for a statistically reliable characterization of the basic parameters of the geologic environment.

The Nizhnekansky granitoid massif, which includes the prospective sites, has an area in excess of 3,500 sq. $\mathrm{km}$. It is composed of biotite granites and, to a lesser degree, leucogranites, plagiogranites, granodiorites, quartz diorites, diorites, and their analogs with elevated alkalinity. The diorite-granodiorites belong to the first (the early) phase, whereas the biotite granites and leucogranites belong to the second phase of the Nizhnekansky granitoid complex [4] (Datsenko, 1995).

The Nizhnekansky massif is dissected by a series of faults with northwest and submeridional orientation along which are found translated loose deposits of quaternary age [10] (Lukina, 1999). This information must be taken into account when investigating the tectonic disturbance and evaluating the geodynamic processes during the creation of territories that appear promising for burial [purposes].

In 1999, one borehole was drilled on each of the two promising sites: a $500 \mathrm{~m}$ borehole (1-I) on the Itatsky site, and a $700 \mathrm{~m}$ borehole $(1-\mathrm{K})$ on the Kamenny. Core samples from these boreholes are the subject of comprehensive research by specialists of the IGEM RAS. 


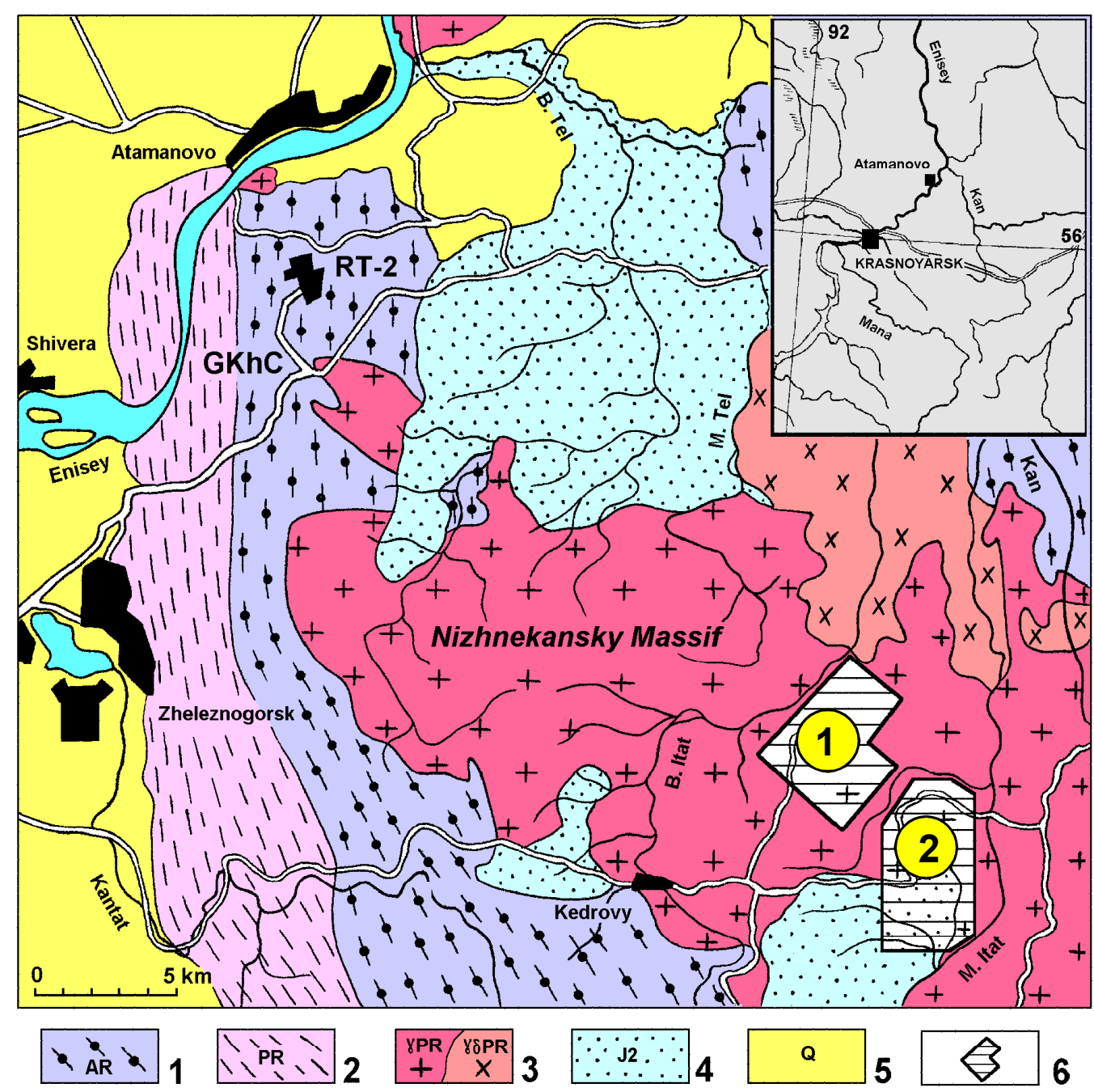

Fig. 9. Geographical situation and geological scheme of the Nizhnekansky granitoid massif with location of the "Itatsky" (1) and "Kamenny" (2) potential sites (after Anderson et al., 1998). 1 gneissic complex (AR), 2 - mica-gneissic complex with amphibolite, marble and quartzite (PR), 3 granitoid complex (PR): granites-leucogranites (left) and diorites-granodiorites (right), 4 volcano-sedimentary complex (J), 5 - sediments $(Q), 6$ - sites selected for further more detailed investigation.

A detailed geological documentation of core samples from both boreholes was performed. This included the recording and description of all petrographic variants of granitoids that have been exposed by drilling, a record of how they manifest themselves, their morphological forms, and the extent of dispersion of deformational, metamorphic and hydrothermal-metasomatic transformations. A representative quantity of samples was selected to determine the mineral-chemical composition and petrophysical properties of the various types of granitoids that had remained unaltered, as well as of those that had been modified to various extent. 
An investigation of the core material revealed significant differences in the core sections with respect to the extent of dispersal of facial varieties of rocks, the extent of their transformation, and their petrochemical properties.

In the upper portion of the 1-I borehole cross-section (the "Itatsky" site) (Fig. 10) and down to a depth of $170 \mathrm{~m}$, frequent alternation is observed between granites and granodiorites with tonalites and diorites. The central portion of the section appears as an extended interval of quartz diorites, diorites and tonalites. Below the $310 \mathrm{~m}$ mark, and to the end of the borehole section, alternation of relatively constant (up to 50 $\mathrm{m}$ ) intervals of granites, adamellites, quartz diorites and diorites is observed. The quartz diorites and the diorites are connected by gradual transitions. Veined and diked formations were recorded in the lower portion of the section at a depth of $400 \mathrm{~m}$. The dispersion of the facial varieties of rock within the boundaries of the given section is as follows (in \%): granites, including granites and adamellites proper (27), granodiorites (10), tonalites (10), quartz diorites (5), diorites (16), lamprophyres and diorite porphyrites (2).

In the section of the 1-K borehole (the "Kamenny" site) (Fig. 11) down to a depth of $500 \mathrm{~m}$ leucocratic granitoids are distinctly predominant (granites, adamellites, leucogranites and alaskites). Among them, at a depth range of $100-220 \mathrm{~m}$, interlayers of granodiorites are observed. The section interval of $220-460$ is represented primarily by leucogranites and alaskites, while below the $500 \mathrm{~m}$ mark, and down to the end of the borehole section, granodiorites, tonalites, and quartz diorites are clearly predominant.

The dispersion of the facial varieties of rocks with respect to the total volume of rock in the section is as follows (in \%): leucogranites (28), alaskites (7), granites, including granites and adamellites proper (21), granodiorites (18), tonalites (16), quartz diorites (5), and lamprophyres and diorite porphyrites (5).

In comparing two sections one fact stands out: the most prevalent $(50 \%)$ and the most constant in thickness at the "Kamenny" site are the granites and leucogranites, whereas at the "Itatsky" site, it is quartz diorites and diorites. Moreover, diorites are absent in the section from the "Kamenny" site, and leucogranites and alaskites are absent in the section from the "Itatsky" site.

The surrounding rock [formations] are subject to early postmagmatic, hydrothermal-metasomatic and syngenetic and epigenetic deformational transformations.

Early postmagmatic transformations are observed in sections of both boreholes (recrystallization and new formations of quartz, feldspar, and dark-colored minerals leading to the formation of gneiss-like and deformational textures (cataclasites-I and mylonites-I). In the 1-I borehole, they are developed in the upper and lower parts of the sections (in intervals of 20-150 m and 460-500 m), whereas in the case of the $1-\mathrm{K}$ borehole, they are seen only in the lower section (in the 500-600 m interval).

The more recent, conjugate, low-temperature, hydrothermal-metasomatic (new formations of hydromica, carbonate, chlorite, montmorillonite) and deformational changes (cataclasites-II and mylonites-II) are frequently combined spatially with zones of early transformations, however, often they can develop independently of them. In this case, the halos of the metasomatites are accompanied by zones of microbrecciated rock and a dense network of microveins.

Both geologic sections studied are characterized by nonuniform tectonic disturbance. The fracture disturbances are represented by the following: 1) ptygmatic plication, blastomylonite seams, and zones of blastocataclasites that were formed during the early stages of the formation of the massif. 2) Zones of brecciation, crushing, and mylonitization that are accompanied by hydrothermal-metasomatic changes in 


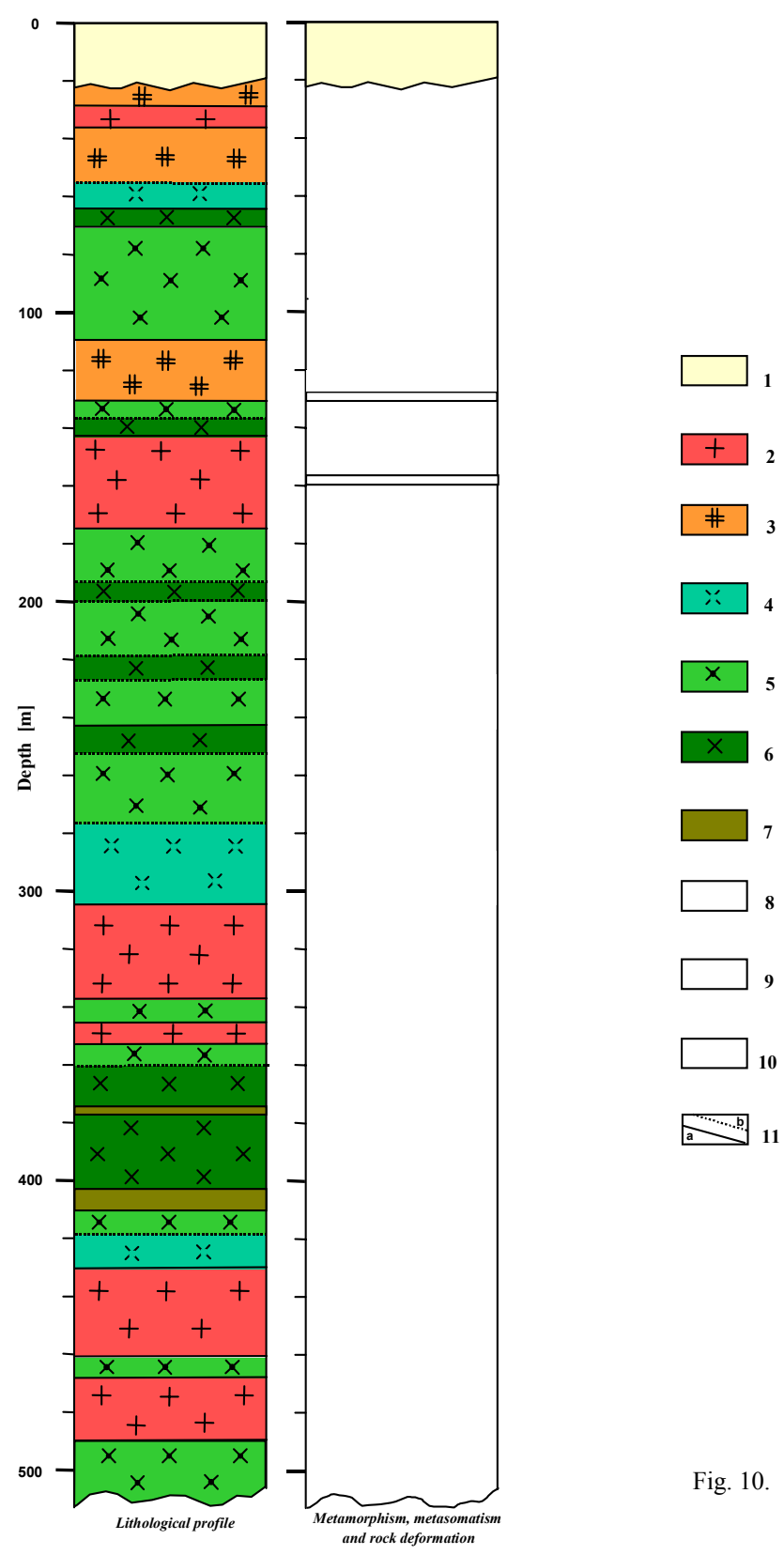

Fig. 10. Lithology and deformation for the borehole 1-I («ltatsky» site). 1 - Quaternary and Jurassic deposits, 2 - granites, adamellites, quartz monzonites, 3 - granodiorites, quartz monzodiorites, 4 tonalites, plagiogranites, 5 - quartz diorites, monzodiorites, 6 - diorites, 7 - dikes of lamprophyres, microdiorites and diorite porphyrites, 8 - moderate metasomatic and metamorphic transformations with veinlets and spotted mineralization, narrow sutures of cataclasites and mylonites I and II types, 9 - intense metasomatic and metamorphic transformations, cataclasites and mylonites I and II types with blastesis, 10 - zones of intense disintegration of rock, 11- clearly defined (a) and proposed (b) boundaries between facies of rocks. 


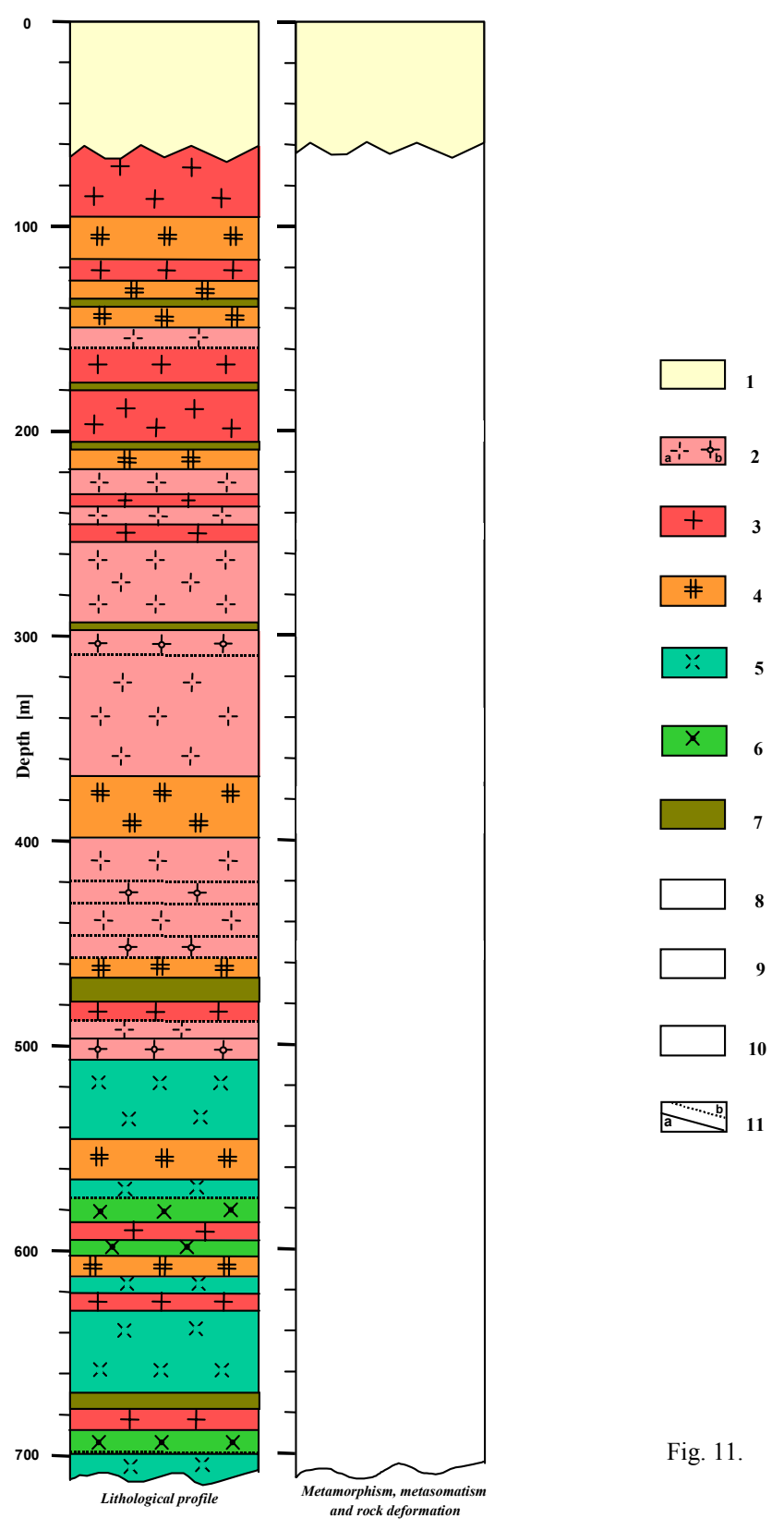

Fig. 11. Lithology and deformation for the borehole 1-K («Kamenny» site). 1 - Quaternary and Jurassic deposits, 2 - leucocratic granites (a) and alaskites (b), 3 - granites, adamellites, quartz monzonites, 4 - granodiorites, quartz monzodiorites, 5 - tonalites, plagiogranites, 6 - quartz diorites, monzodiorites, 7 - dikes of lamprophyres, microdiorites and diorite porphyrites, 8 moderate metasomatic and metamorphic transformations with veinlets and spotted mineralization, narrow geosutures of cataclasites and mylonites I and II types, 9 - intense metasomatic and metamorphic transformations, cataclasites and mylonites I and II types with blastesis, 10 - zones of intense disintegration of rock, 11- clearly defined (a) and proposed (b) boundaries between facies of rocks. 
the rock. 3) Zones of densely packed veins and veinlets of various compositions. 4) Systems of nonmineralized fissures.

In the borehole 1-I tectonic zones were observed in the intervals of 39.7-54.0, 121.5-131.1, and 416.0$440.0 \mathrm{~m}$. Their central portions are represented by mylonite and cataclasite seams, which are accompanied by hydrothermal-metasomatic transformations of the rock and a network of veinlets of various compositions. Also observed here were leaching voids.

In the $1-\mathrm{K}$ borehole, the largest tectonic zones related to the interfaces of diverse rock types and to dikes are seen on intervals 209.2, 380.0-398.0, 464.0-466.7, and $679.0 \mathrm{~m}$. In the central portions of the zones, cataclasts, fragmentation and mylonites are also very noticeable, whereas the zones themselves are accompanied by haloes of the hydrothermal-metasomatic transformations in the rock, a network of veinlets of various compositions, and leaching voids.

Investigation of the petrophysical properties of the core material from the boreholes demonstrated the following. The density and the specific gravity of the rock increase with increasing alkalinity from leucogranites and granites $\left(2.61-2.63 \mathrm{~g} / \mathrm{cm}^{3}\right)$ to quartz diorites and tonalites $\left(2.74-2.79 \mathrm{~g} / \mathrm{cm}^{3}\right)$. Granodiorites, granite-gneiss, and gneiss are characterized by having intermediate values (2.65-2.75 $\mathrm{g} / \mathrm{cm}^{3}$ ). The density and the specific gravity of the rock increase with depth in the 1-K borehole, and in the 1-I borehole, they reach maximal values in the central portions of the section.

The effective porosity of the rock varies from $0.14 \%$ to $0.95 \%$. Exceptions to this are the specimens of the supergenetically modified granites (up to $1.31 \%$ ) and the metasomatically modified lamprophyres (5.6\%). The rock samples from the 1-I borehole are less porous, however. In the 1-K borehole, the porosity diminishes with depth.

A comparison of the data on effective porosity, permeability, the dynamics of water saturation, and the structure of the porous zones has demonstrated that, in the rock samples studied, secondary porosity is preponderant, and that the primary channels for fluid filtration in the rock matrix are systems of connected microcracks. The values of the filtration parameters of the core sample from the 1-K borehole are considerably higher than in the sample from the 1-I borehole, which is primarily related to the peculiarities of the structure of the porous space of the rock.

The elastic properties of the rock in the core samples also differ from each other. Mean velocity of Pwaves along the axis of the $1-\mathrm{K}$ core sample is $4.794 \mathrm{~km} / \mathrm{s}$, whereas it is $5.589 \mathrm{~km} / \mathrm{s}$ in the case of the 1-I [sample] (Fig. 12). The velocities of the P-waves in the borehole 1-K range from $2.460 \mathrm{~km} / \mathrm{s}$ to $5.670 \mathrm{~km} / \mathrm{s}$ as compared with $5.000 \mathrm{~km} / \mathrm{s}$ to $5.875 \mathrm{~km} / \mathrm{s}$ in the case of $1-I$. Therefore, the anisotropy of P-waves differs drastically between the sections. In the $1-\mathrm{K}$ borehole, the anisotropy coefficient may reach a value of $35 \%$, whereas in the case of the $1-1$ borehole, it does not exceed $8 \%$.

Altogether, based on the array of petrophysical parameters studied, the rock of the 1-K borehole exhibits a comparatively greater degree of nonuniformity in physical characteristics than that of the 1-I borehole.

Thus, the geologic sections revealed by the 1-K and 1-I boreholes differ sharply from each other in the facial composition of rock, the degree of their tectonic disturbance, the intensity of the development of early postmagmatic, hydrothermal-metasomatic and deformational transformations and, as a result, petrophysical properties of the rock. Therefore, it is essential that the comparative studies of the anisotropy of the mineral-chemical, petrostructural and petrophysical characteristics be continued with the goal of developing the most likely scenarios of the changes in their state under the action of tectonic, thermal and radiation effects. 

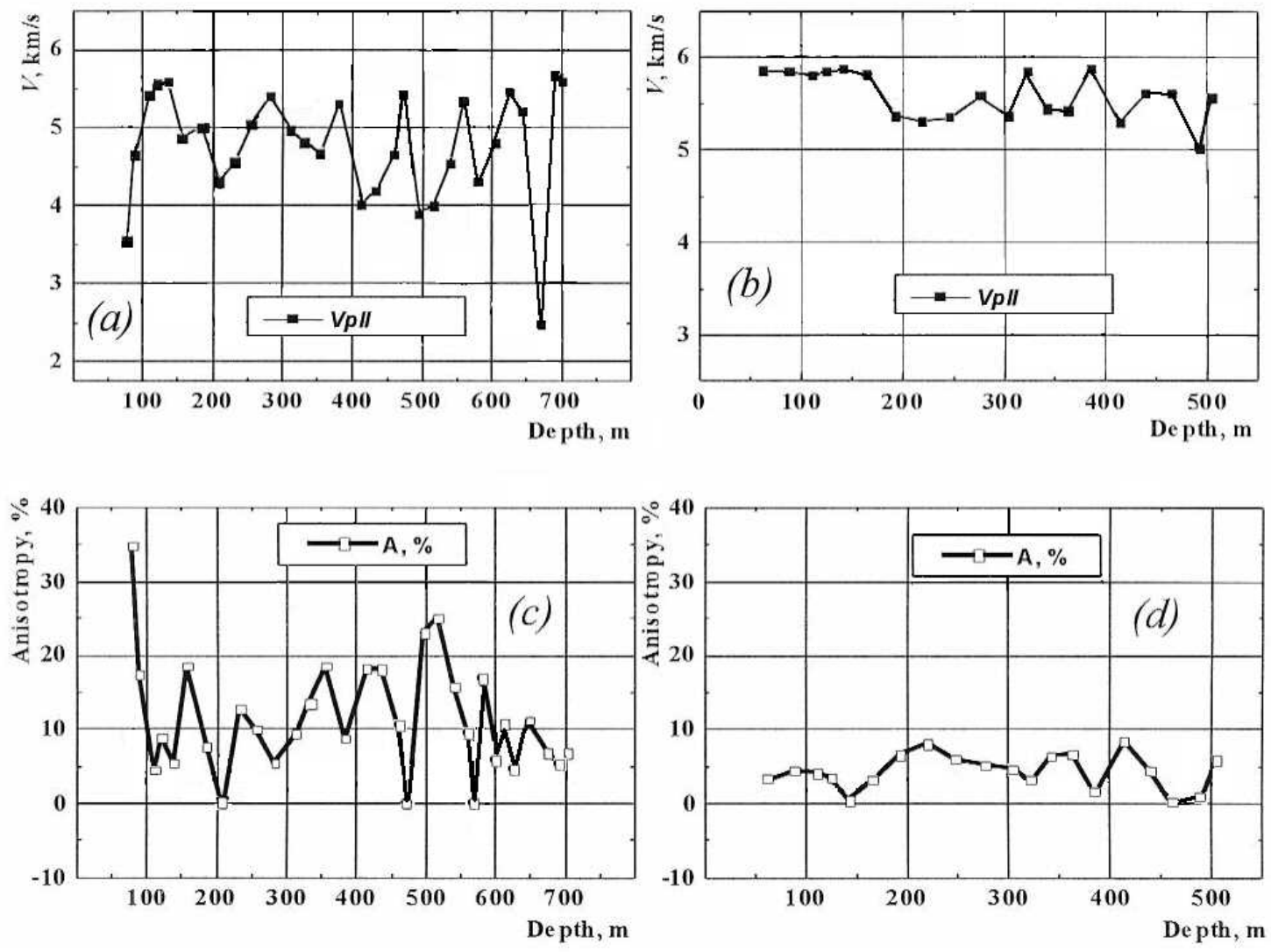

Fig. 12. Variations of elastic wave propagation and anisotropy of $P$-waves as a function of depth for the "Kamenny" (a, c) and "Itatsky" (b, d) sites.

In the course of performing the above petrochemical, petrophysical, and petrostructural studies, important results were obtained, which are significant not only for an assessment of the properties and the state of the geologic environment of the Nizhnekansk region, but ought to be viewed as an inseparable part of the methodology of selecting sites for deep geological repositories for HLW in tectonically disturbed crystalline rock formations.

\subsection{Area of the Priargunsk Mining-Chemical Production Association (Eastern Transbaikal region)}

Achieving safe isolation of accumulated and continually arriving SNF is one of the serious radioecological problems in Russia. At present, SNF storage facilities at NPP and in ports where nuclear powered vessels are based either have been filled to their design capacities or are close to it. For this reason, selecting sites for and building additional dry-storage facilities for SNF belong to some of most pressing problems for the Minatom of Russia. Additional impetus to expedite the finding of a practical solution to this problem was provided by the passage last year of legislation allowing the import and storage of foreign SNF in Russia, with all the associated economic advantages.

Very attractive in this regard, owing to its geographic location, environmental conditions, and economic and technological capabilities, is the territory of the Priargunsk Mining-Chemical Production Association (PMCPA). The only enterprise in Russia for mining and processing of uranium ores, it is located in the Eastern Transbaikal region (in the southeastern part of the Chitinsk Oblast, Krasnokamsky Region). In 
over 30 years of operation, the PMCPA specialists have acquired a great deal of expertise in the area of handling of radioactive materials and in performing various tunneling and mining operations. The PMCPA has a highly professional work force and an extensive and multifaceted industrial and power-generation infrastructure. The location of the facility is far enough removed from large population and industrial centers, yet it is well connected with other regions of Russia by a railroad line and by highways. Its territory is characterized by weak seismicity and by having been thoroughly explored geologically. Underground water is found at elevations of $+500-700 \mathrm{~m}$. The climate is arid, typical for plains, with annual precipitation slightly exceeding $300 \mathrm{~mm}$. The surface topography of the PMCPA is rugged, with low mountains, the elevation ranging from +500 to $+900-1000 \mathrm{~m}$.

The territory of the PMCPA comprises two rock systems. The upper system consists of volcanogenic and terrigenous-carbonaceous sedimentary Mesozoic rock. The volcanogenic rock comprises the Tulukevsk volcano-tectonic structure that includes the main portion of the worked uranium deposits. These rock systems have been intensively tectonically disturbed, modified by hydrothermal processes, and are insufficiently stable mechanically. For this reason, they are not suitable for geological disposal. The sedimentary Mesozoic rock systems are classified in the same category. They are insufficiently stable mechanically, are situated in the lower sections of the topography, and are subject to water encroachment.

The lower rock system is composed of gneisses, crystalline shale, gneiss-like and normal pre-Paleozoic and mid-Paleozoic granites. In undisturbed state, this is dense, massive rock with high strength characteristics. As a rule, it comprises the elevated portions of the topography. The proposal is to find in this rock massif a sufficiently undisturbed block of appropriate dimensions and situate in it all the necessary communication systems for an underground repository for SNF.

In view of the surface topography, and for environmental, engineering and economic reasons, an underground repository in the form of a tunnel is seen as the most suitable approach for reliable isolation of SNF at the PMCPA. Performed by specialists of the PMCPA and IGEM RAS, preliminary studies of the various alternatives for the location of the future storage facility produced four suitable plots in the area of the lower system (Fig. 13). All of them would permit implementing the proposed tunnel version for the storage facility. The most suitable site appears to be plot \# 1 (Fig. 14) which is composed of lightly disturbed granite-gneisses and is the closest to the railroad tracks. To characterize the properties of this site, samples of rock were collected for further studies, which have already begun.

The tunnel approach for the construction of the SNF storage facility is additionally supported by a number of considerations:

- The repository location is in a rock massif, and the fact that there is above it a protective, untouched rock layer around $300 \mathrm{~m}$ thick, excludes the possibility of external action upon the stored materials.

- The high strength, low permeability, and low level of disturbance of the crystalline rock housing the repository drastically reduce the danger of it being destroyed and of water penetrating into it.

- Compared with the shaft method of storage, the tunnel approach avoids the need to construct lifting mechanisms and stationary water pumping systems and also simplifies the ventilation systems.

It is also important to note that the majority of the working population of the PMCPA area is employed in enterprises that are linked, directly or indirectly, to uranium and are not prone to radiationphobia. 


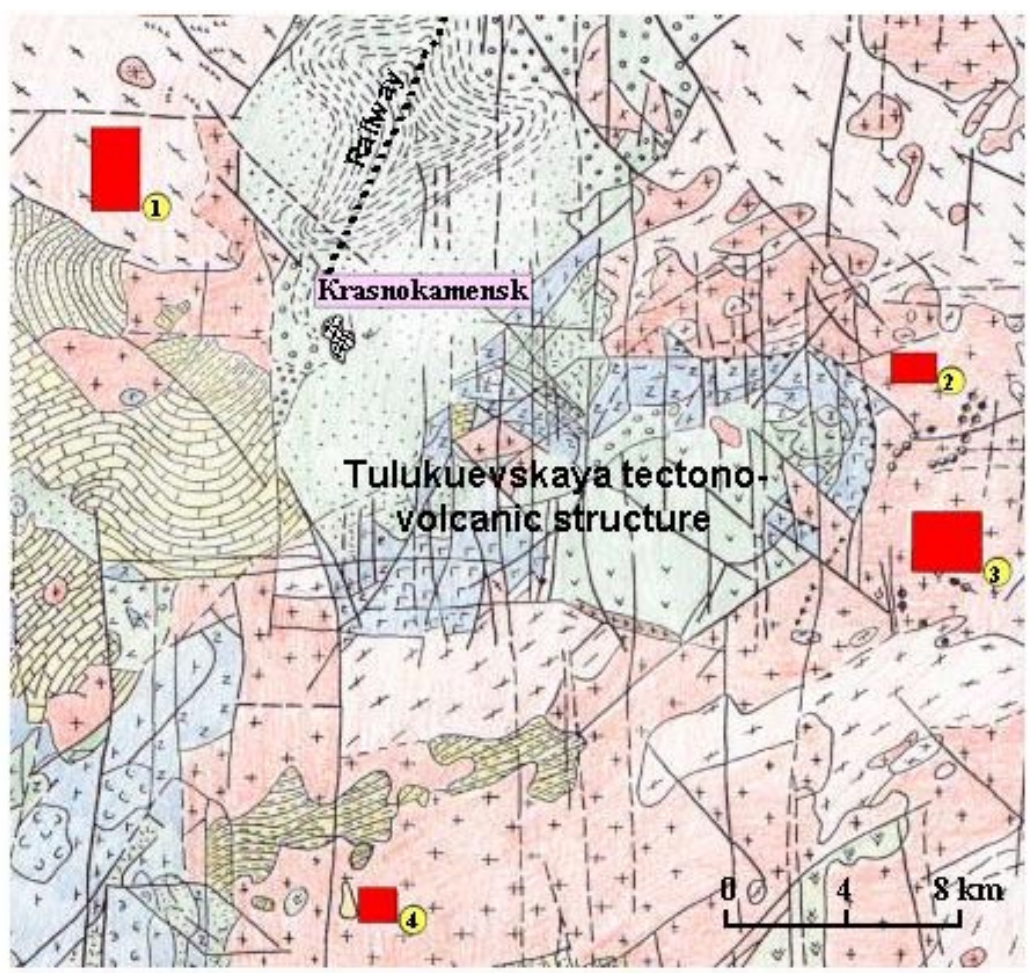

Fig. 13. Geological scheme of the PPGKhO operation area with location of the potential sites in the granitic frame of the Tulukuevskaya tectono-volcanic structure.

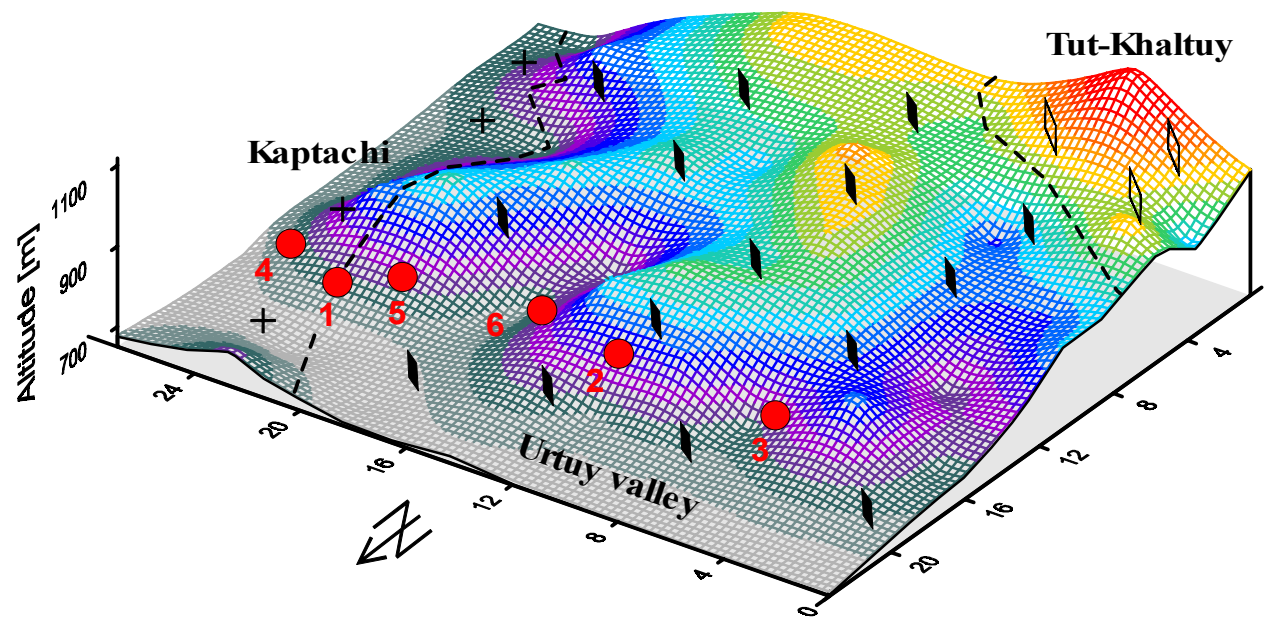

Fig. 14. 3D image of granitic potential site No. 1 in the vicinity of the PPGKhO operation area. Porphyry granites (cross), granito-gneisses (black rhomb), quartzites (white rhomb) and sampling points are shown. 


\subsection{The Far East region}

A tense situation has developed in connection with radiation fears in areas where the Pacific atomic submarine fleet is stationed and in places where enterprises are located that service the AS, or are involved in scrapping decommissioned AS. Reasons for this include a severe shortage of special structures required to isolate the SNF, the reactor compartments, and other materials that are generated in the course of scrapping the AS, as well as the lack of ability to reliably isolate the accumulated and the newly-produced RAW.

Earlier, there were suggestions about readying sites for facilities for handling the RAW and SNF on the coast of the Sea of Okhotks on rock systems in the permafrost zone. Specialists from the DVGI RAS and IGEM RAS are proposing as a promising candidate for such installations the Artemovsk area, a few dozen kilometers northeast of the city of Vladivostok and north of the AS bases and their service facilities on the coast of the Sea of Japan.

The area is composed of Neogen terrigenous, argillaceous-sandy sediments covered by massive (up to $300 \mathrm{~m}$ thick) plateau basalts (Fig. 15). These are pierced by volcanic alkaline basalt structures up to 4 million years old. The sedimentary rock is dissected by variously oriented fractures, many of which do not penetrate the plateau basalts. This is an indication that after the establishment of the plateau basalts, the tectonic activity in the area under study has decreased sharply. In view of the data presented, and given the high strength and low porosity of the plateau basalts, these rock systems may be considered suitable for underground storage facilities for radioactive materials.

The rugged topography of the region, with elevation differences ranging from $+600-650 \mathrm{~m}$ to $+900-$ $1200 \mathrm{~m}$, justifies considering the possibility of building a shaft-type storage facility in it (see Fig. 15).
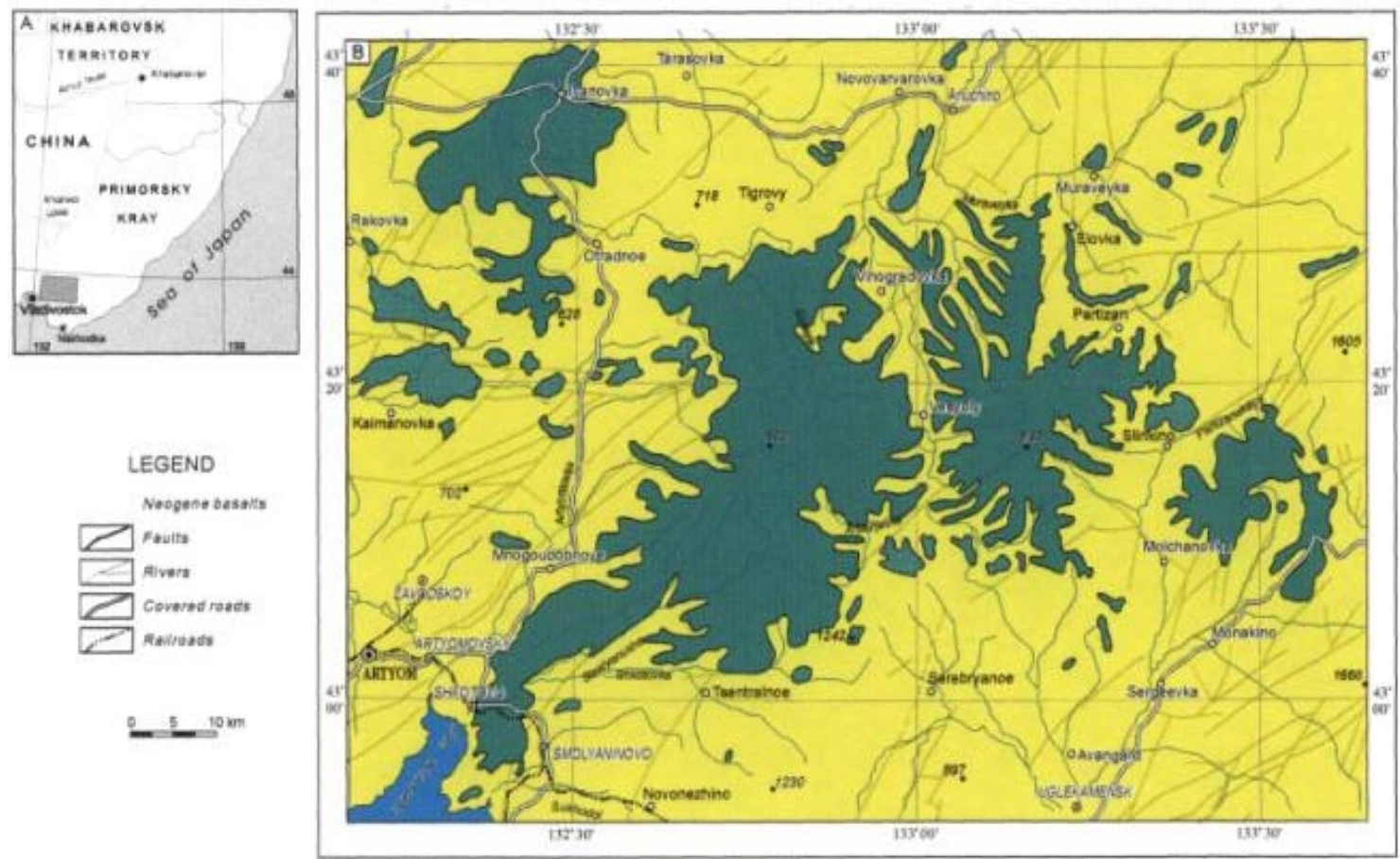

Fig. 15. Geologic scheme of the Artemiyev Region in South Primoriye (after DVGI RAS, 1998). 


\section{Some Strategic Aspects of Isolating the RAW and SNF in Russia}

A sharp increase in the threat of terrorist acts directed at nuclear facilities, heightened danger of uncontrolled proliferation of nuclear weapons and weapons-grade nuclear materials, insufficient technological maturity and ambiguity in engineering cost effectiveness of a number of operations in the final phases of RAW handling cycle, all these make it imperative that corrections be introduced into Russia's strategy for safe isolation of radioactive materials. Thus, it is advisable to consider the following proposals:

- Generate all necessary technical and engineering prerequisites and conditions for expeditious and unconditional elimination of the practice of storing high-level waste in liquid form.

- Within the range of technologies used in handling liquid HLW, provisions should be made to fractionate HLW segregating into individual groups the short- medium- and long-lived radioisotopes. Research should be intensified to improve the technologies for deep fractionation of HLW and improve their cost effectiveness.

- The extracted fractions of liquid HLW, and liquid HLW that for some reason have not been fractionated, must be solidified without fail. Accordingly, it will be necessary to embark upon a wide scope of research to find, develop, and implement, on industrial scale, new types of glass-like, glasscrystalline, and crystalline matrices-preservers that would be capable of ensuring reliable isolation of non-separated liquid HLW, separated fractions of liquid HLW, and, if necessary, individual radionuclides for the duration of their radiologically hazardous period.

- It is advisable to continue and expand comprehensive research to develop techniques for segregated disposal of HLW fractions containing short- medium- and long-lived radioisotopes, and to select geologic environments for the location of these fractions. Also, existing plans for the implementation of safe underground disposal of the currently accumulated solid and solidified HLW should be refined, expanded, and made more specific.

- The method practiced in Russia for disposition of liquid short- and medium-lived RAW with a low level of activity in deep sedimentary layers with slow water-exchange rates and under strict adherence to technological regimes and existing restrictions is an environmentally safe and very effective method that can be recommended for further use in regions with appropriate geological structure and hydrogeological conditions.

- Steps must be taken without delay to drastically reduce the risk of any external action on storage facilities, whether existing ones or under construction, for SNF and other nuclear materials. The security of the facilities should be substantially tightened, and a decision about locating such facilities underground must be made, which will sharply reduce uncontrolled access to stored nuclear materials. Natural conditions permitting, tunnel-type storage facilities should be recommended as the most economical and technically acceptable method for reliable isolation of nuclear materials.

- The contemporary concept for handling of HLW and SNF must be flexible, dynamic, and subject to appropriate corrections as new engineering and technological improvements are developed. Since engineering and technological uncertainties, disputed issues, and ambiguous evaluations exist in the area of reprocessing of SNF, the most rational method of isolating SNF in the near future may be the protracted, controlled storage of it coupled with the ability of withdrawing it [if necessary]. The time gained as a result of postponing the final decision date for the SNF issue will make it possible to work through the problems that are still unresolved in a comprehensive manner and to create a concept for 
the final handling stages of the SNF that would be optimal for the present and the future generations of people.

\section{References}

1. Adushkin, V.V., Loktev, D.N., Spivak, A.A. 1997. Diagnostics of the rock massif on the territory of PA "Mayak" based on the results of monitoring of relaxation processes.// Issues in Radiation Safety, 1. pp. 18-30.

2. Aleynikov, A.L., Bellavin, O.V., Bulashevich, Yu.P. et al., 1988. Horizontal Stresses and Tectogenesis of Ural. // Plutonic Formation of Ural and Adjacent Regions. Sverdlovsk: Publ. House UrO AN SSSR. pp. 106-113.

3. Anderson, E.B., Shabalev, S.I., Savonenkov, V.G., Lyubtseva, E.F. 1998. Investigations of Nizhnekanskiy granitoid massif (Middle Siberia, Russia) as a promising site for deep geological disposal of HLW. Proc. of the Int. Conf. on Rad. Waste Disp. Hamburg, Germany. pp. 105-110.

4. Datsenko, V.M., 1995. Nizhnekanskiy Massif - the standard for the Nizhnekanskiy granitoid complex (Yenisey Range). Novosiibirsk. p. 123.

5. Glagolenko, Yu.V., Dzekun, E.G., Rovny, S.I. et al., 1997. Reprocessing of spent nuclear fuel at the PT-1 plant: history, problems, prospects // Issues in Radiation Safety, 2. pp. 3-12.

6. Improvement of the Safety of Radioactive Waste Management in the North-West Region of Russia. Tacis Project No. R4.10/95. NUCRUS 95410, 1996.

7. Kedrovsky, O.L., Shischitz, I.Yu., Gupalo, T.A. 1991. Substantiation of high-level radioactive waste and spent nuclear fuel conditions of localization in geologic formations // Journ. of Atomic Energy, 70, 5. pp. 294-297 (in Russian).

8. Krone, J., Baker, A., Ahlstrom, P.E. et al. 2000. Feasibility investigations into a nuclear repository on Novaya Zemlya in Russia. Proc. of the Int. Conf. on Rad. Waste Disp., Berlin, Germany. pp. 43-48.

9. Laverov, N.P., Velichkin, V.I., Omelyanenko, B.I. et al., 2000. New Approaches to Underground Disposal of High-Level Waste in Russia // Geoecology, 1. pp. 3-12.

10. Lukina, N.V., 1999. Nizhnekansky Granitoid Massif: The Latest and Contemporary Tectonic Shifts, Morphology and Kinematics of Active Breaks and Megafractures, Current Stress Field. Possibilities for Safe Disposal of HLW // Research of Granitoids of Nizhnekansky Massif for Deep Disposal of RAW, SPb. pp. 49-59.

11. Petrov, V.A., Velichkin, V.I., Poluektov, V.V., Tarasov, N.N. 2000. Integration of geological, geomechanical and petrophysical data for the preliminary prediction of the HLW disposal system behaviour at the Southern Urals, Russia. Proc. of the Int. Conf. on Rad. Waste Disp., Berlin, Germany. pp. 218-223.

12. Velichkin, V.I., Omelianenko, B.I., Tarasov, N.N. et al. 1997. Geological aspects of solidified highlevel radioactive waste (HLW) disposal problem at PA "Mayak", Chelyabinsk district, Russia. Proc. of the 6th Int. Conf. on Rad. Waste Man. and Envir. Remed., Singapore. pp. 399-402. 


\section{Complex Program of Engineering and Geologic Surveys for Designing RW Storage Construction in Hard Rock Massifs (B512155)}

\section{T. A. Gupalo, V. L. Milovidov, O. A. Prokopova, VNIPIPT}

A number of primary research objectives were established according to the regional approach, adopted by the Russian Federation, to settling the problem of RW isolation.

Areas under investigation include the district of the Nizhnekansky rock massif located close to the Mining Chemical Combine (MCC) area and the territory of PA "Mayak". Construction of underground laboratories and radioactive waste $(\mathrm{RW})$ storage is considered quite possible at these sites.

In the design and construction of these facilities, a special role is given to surveys, whose structure and volume are specified by the features of designed complex, and to the complexity and extent of knowledge of the geological conditions of the sites and facilities. In all cases, work is carried out stage-by-stage both in structure and volume, sufficient for the basic tasks of designing.

The complex program of engineering surveys for various stages of designing RW storage and an underground laboratory at the territory of the Nizhnekansky rock massif is described in the remainder of this paper.

The complex research that should be carried out during design and construction of RW storage for PA "Mayak" is similar to that described for the Nizhnekansky massif. However, the extent of knowledge in terms of hydrogeology of the MCC territory is much higher, which would allow a reduction in the volume of research required, and in the time and cost of construction work, accordingly.

\section{Complex Research at the DOI Stage}

Engineering surveys for the "Declaration of Intent" (DOI) should be aimed at complex study of the natural and anthropogenic conditions of the region that will allow the definition of areas and sites for the further investigation.

Table 1. Complex of research at the DOI stage.

\begin{tabular}{|c|c|c|}
\hline Engineering and geologic surveys & Scientific support & Design and construction work \\
\hline $\begin{array}{l}\text { Analysis of physical and chemical, } \\
\text { geologic- hydrogeologic materials } \\
\text { Engineering-geologic } \\
\text { reconnaissance } \\
\text { Aerogeophysical or surface } \\
\text { geophysical research } \\
\text { Seismic assessment of the territory } \\
\text { Assessment of the ecological state } \\
\text { at the territory }\end{array}$ & $\begin{array}{l}\text { Zoning of the territory according to } \\
\text { the degree of suitability of geologic } \\
\text { formations for underground RW } \\
\text { disposal } \\
\text { Study of rock masses and } \\
\text { underground waters } \\
\text { Preliminary assessment of the } \\
\text { reliability of engineered and } \\
\text { geochemical barriers } \\
\text { Definition of main directions of } \\
\text { investigations in the underground } \\
\text { laboratory }\end{array}$ & $\begin{array}{l}\text { Justification and choice of the site } \\
\text { (sites) for further investigations } \\
\text { Pre-design and construction } \\
\text { solutions for underground laboratory } \\
\text { and RW repository Extensive } \\
\text { assessment of the ecological safety } \\
\text { of underground RW isolation } \\
\text { Elaboration, expert's review and } \\
\text { approval of DOl documentation }\end{array}$ \\
\hline
\end{tabular}




\section{Complex Research at the JOI Stage}

The engineering survey for preparation of the "Justification of Investment into construction" (JOI) involves getting the required data sufficient to substantiate the suitability of the site, and within the site, the suitability of the construction subsite(s). Subsites are required for making principle layout and design decisions and the assessment of the environmental effects of repository construction and operation.

Table 2. Complex of research for JOI.

\begin{tabular}{|c|c|c|}
\hline $\begin{array}{c}\text { Engineering surveys } \\
\text { (scale } 1: 100000-1: 50000)\end{array}$ & Scientific support & Design and construction works \\
\hline $\begin{array}{l}\text { Engineering and geodetic, } \\
\text { hydrometeorological, ecological } \\
\text { surveys, engineering-geologic } \\
\text { survey, } \\
\text { Surface geophysical studies } \\
\text { Hydrogeologic surveys; } \\
\text { Drilling and study of } 1-2 \text { prospecting } \\
\text { boreholes of } 1000-1500 \text { m deep }\end{array}$ & $\begin{array}{l}\text { Development of preliminary } \\
\text { engineering-geologic models. } \\
\text { Assessment of geodynamic } \\
\text { processes. } \\
\text { Study of geochemical rock } \\
\text { properties. } \\
\text { Assessment of solidified RW } \\
\text { compositions, reliability of matrices } \\
\text { and containers. } \\
\text { Computer modeling of heat and } \\
\text { mass transfer processes in rock } \\
\text { massif; }\end{array}$ & $\begin{array}{l}\text { Selection and justification of the } \\
\text { alternative sites for construction. } \\
\text { Development of principle layout and } \\
\text { design solutions for the underground } \\
\text { laboratory and RW repository } \\
\text { facility. } \\
\text { Assessment of technogenic impact } \\
\text { on the environment of future RW } \\
\text { repository facility (EIA). }\end{array}$ \\
\hline
\end{tabular}

\section{Underground Laboratory}

Validity of geologic data on rock mass structure and processes occurring in it under natural and technogenic factors can be proved under natural conditions. This is possible only as a result of implementation of a research program in a special underground laboratory, constructed at the site of the proposed repository.

The design of the underground laboratory at the Nizhnekansky rock massif specifies two vertical shafts and horizontal drifts. Horizontal drifts may be located at depths of 600 and more meters.

Engineering surveys for underground laboratory draft design, followed by the "Technical and Economic Justification" (TEO) for the RW repository, should provide for acquisition of required materials and data on natural and technogenic conditions for development of design solutions. The surveys serve as a basis for construction decisions, making design and volume and layout solutions, compiling general plans for facility design, development of engineering security, and activities for natural environment protection.

Table 3. Complex of research for underground laboratory.

\begin{tabular}{|c|c|c|}
\hline $\begin{array}{c}\text { Engineering surveys } \\
\text { (scale 1:50000-1:5000) }\end{array}$ & Scientific support & Design and construction works \\
\hline $\begin{array}{l}\text { Engineering and geologic survey. } \\
\text { Surface-based geophysical studies. } \\
\text { Hydrogeological and emanation } \\
\text { surveys. } \\
\text { Borehole drilling at least of } 9 \text { wells of } \\
800-1500 \text { m deep } \\
\text { Microseismic zoning } \\
\text { Geodynamic studies at special test- } \\
\text { sites. } \\
\text { Hydrometeorological surveys. }\end{array}$ & $\begin{array}{l}\text { Study of rocks stress and strained } \\
\text { state. } \\
\text { Development of geomonitoring } \\
\text { system. } \\
\text { Development of geologic- } \\
\text { hydrogeologic, thermal and } \\
\text { physical and migration models. } \\
\text { Modeling stability of matrix } \\
\text { materials to environmental } \\
\text { impacts. }\end{array}$ & $\begin{array}{l}\text { Selection of the site for construction. } \\
\text { Detailed study of surface and } \\
\text { underground facilities design and } \\
\text { layout. } \\
\text { Mechanization know-how of driving } \\
\text { works. } \\
\text { Evaluation of seismic effect of } \\
\text { underground facilities. } \\
\text { Activities for environmental protection. } \\
\text { State expert's examination of design } \\
\text { documentation. }\end{array}$ \\
\hline
\end{tabular}




\section{Construction and Operation of Underground Laboratory}

The types of engineering and geologic surveys and scientific research to be done during construction and operation of the underground laboratory are given in Table 4.

The engineering and geological research includes drilling work; geological, geophysical, and hydrogeological research at driving mine trunks and mountain workings; and the study of geodynamic and other processes.

Table 4. Complex research for construction and operation of the underground laboratory.

\begin{tabular}{|c|c|}
\hline Engineering and geologic surveys & Scientific research \\
\hline \multicolumn{2}{|l|}{ Construction } \\
\hline $\begin{array}{l}\text { Drilling of two boreholes of up to } 1000 \text { m deep } \\
\text { Geologic, geophysical and hydrogeological investigations } \\
\text { in boreholes. } \\
\text { Geological mapping during driving of mountain workings. } \\
\text { Test-filtration works in workings and boreholes. } \\
\text { Study of stress and strained rock mass state. }\end{array}$ & $\begin{array}{l}\text { Development the system of surface and underground } \\
\text { geomonitoring. } \\
\text { Elaboration of detailed engineering and geological } \\
\text { models of the rock mass. } \\
\text { Modeling of processes in system "waste-engineered } \\
\text { barriers-rock mass". }\end{array}$ \\
\hline \multicolumn{2}{|l|}{ Operation } \\
\hline $\begin{array}{l}\text { Detailed study of geologic structure, hydrogeologic, } \\
\text { geodynamic, geomechanical and other processes. } \\
\text { Geomonitoring. }\end{array}$ & $\begin{array}{l}\text { Study of engineering loading impact on the rock mass } \\
\text { and engineered barriers. } \\
\text { Modification of civil work technology and safe radioactive } \\
\text { waste management. } \\
\text { Evaluation of reliability and safety of RW isolation. }\end{array}$ \\
\hline
\end{tabular}

After research is completed, state experts will review the results of the host rock massif studies. And after obtaining data demonstrating suitability of the subsite selected for RW isolation and expert approval of the results of completed experiments, the TEO for construction will be developed.

\section{Complex of Research at the TEO Stage}

Background for development of the TEO are the geological, geophysical, and hydrogeological investigations performed during building and operation of the underground laboratory and the results of the comprehensive research and development work carried out in in-situ conditions.

In addition, surveys to support design decisions for the RW repository project and measures for environmental protection will be carried out. The research activities for the TEO are presented in Table 5.

Table 5. Complex of researches at the TEO stage.

\begin{tabular}{|c|c|c|}
\hline $\begin{array}{l}\text { Engineering surveys } \\
\text { (scale } 1: 5000-1: 2000)\end{array}$ & Scientific support & Design and construction works \\
\hline $\begin{array}{l}\text { Engineering-geological } \\
\text { survey. } \\
\text { Site geophysical studies. } \\
\text { Emanation surveys. } \\
\text { Drilling of } 6 \text { boreholes of } 800 \text { - } \\
1200 \text { m deep } \\
\text { Studies on geodynamic test } \\
\text { sites. }\end{array}$ & $\begin{array}{l}\text { Development of mathematical model of } \\
\text { engineering-geological conditions for } \\
\text { near and distant field of RW repository } \\
\text { Development of models of climate } \\
\text { variations, geological and } \\
\text { hydrogeological conditions. } \\
\text { Assessment of economical safety of the } \\
\text { future repository. }\end{array}$ & $\begin{array}{l}\text { Detailed studies on layout of surface and } \\
\text { underground facilities complex. } \\
\text { Elaboration of RW management } \\
\text { procedure. Elaboration of radiation safety } \\
\text { system. } \\
\text { Recommendations on nature } \\
\text { management. } \\
\text { Development of design documentation, } \\
\text { state examination and TEO approval. }\end{array}$ \\
\hline
\end{tabular}




\section{Complex Research at the Working Documentation Stage}

Engineering surveys for development of the "Working Documentation" are aimed at detailing the conditions of interaction of RW repositories with host rocks and the environment. They should provide data required for calculations of surface buildings and underground facility structures and their engineering security, and to specify design decisions for individual issues raised during TEO development. The list of the basic work at this stage is given in Table 6 .

Table 6. Complex of research at the working documentation stage.

\begin{tabular}{|c|c|c|}
\hline $\begin{array}{c}\text { Engineering surveys } \\
\text { (scale } 1: 2000-1: 1000)\end{array}$ & Scientific support & Design and construction works \\
\hline $\begin{array}{l}\text { Engineering-geological survey. } \\
\text { Surface geophysical studies. } \\
\text { Borehole drilling of } 6 \text { boreholes } \\
\text { of } 800-1000 \text { m deep. } \\
\text { Geodynamic studies on special } \\
\text { test sites. }\end{array}$ & $\begin{array}{l}\text { Modeling of radionuclide migration from } \\
\text { RW repository. } \\
\text { Forecast of scenarios for development } \\
\text { of natural and technogenic events. } \\
\text { Development of geomechanical model } \\
\text { Probability analysis of RW safe } \\
\text { disposal. }\end{array}$ & $\begin{array}{l}\text { Substantiation of volume and layout } \\
\text { scheme of repository. } \\
\text { Unit detail drawing of facilities' } \\
\text { structures. } \\
\text { Development of driving technique. } \\
\text { Development of activities for } \\
\text { environment protection and its } \\
\text { rehabilitation. }\end{array}$ \\
\hline
\end{tabular}

\section{Complex of Research During Construction, Operation, and Sealing of Repository}

The complex of research activities during construction, operation, and closure of the RW storage are listed in Table 7. They should provide acquisition of data on the state and variations in separate components of the geological environment at the area of the facility. They are carried out to increase the reliability and operational suitability of the surface-based and underground complex of facilities, safety of work execution, and protection of the environment and the health of people.

Table 7. Complex of research at construction, operation, and sealing of repository.

\begin{tabular}{|c|c|}
\hline Engineering and geologic and scientific research & Design and construction works \\
\hline \multicolumn{2}{|l|}{ Construction } \\
\hline $\begin{array}{l}\text { Engineering and geologic surveys. } \\
\text { Examination of technical condition of drifts by methods of } \\
\text { geophysics. } \\
\text { Study of mining and geological, hydrogeological, } \\
\text { radiation and other processes in the rock mass. }\end{array}$ & $\begin{array}{l}\text { Development of geomonitoring system. } \\
\text { Modification of design and construction solutions. } \\
\text { Development of recommendations to improve driving } \\
\text { technique and drift supports. }\end{array}$ \\
\hline \multicolumn{2}{|l|}{ Operation } \\
\hline $\begin{array}{l}\text { Drilling and examination of technological boreholes. } \\
\text { Surface-based and underground geomonitoring. } \\
\text { Evaluation and forecasting of mining and geologic } \\
\text { conditions' variations during RW repository operation. }\end{array}$ & $\begin{array}{l}\text { Development of recommendations for change of } \\
\text { technological procedure and regime of repository } \\
\text { operation, improvement of methods of engineering } \\
\text { protection. }\end{array}$ \\
\hline \multicolumn{2}{|l|}{ Sealing } \\
\hline $\begin{array}{l}\text { Specialized investigations of geoecological state of RW } \\
\text { repository site. }\end{array}$ & $\begin{array}{l}\text { Development of activity plan for rehabilitation of the } \\
\text { territory of RW repository. }\end{array}$ \\
\hline
\end{tabular}




\title{
Basic Provisions of the Plan for Creation of Underground Radwaste Isolation Facility in MCC and PA "Mayak" Areas (B512155)
}

\author{
T. A. Gupalo, O. A. Prokopova, V. L. Milovidov, V. P. Beygul, VNIPIPT
}

A huge amount of radioactive waste (RW) has been accumulated in the Russian Federation during implementation of the defense and energy programs, and industrial and research activity connected with nuclear materials. Ninety-nine percent of waste by volume and total activity are concentrated on the radiochemical enterprises of Minatom.

At present, the most justifiable and technically feasible technology of isolation of solidified RW is its disposition in weakly permeable geological formations in specially created underground facilities (e.g., mountain drifts, deep boreholes).

The All-Russian Research and Design Institute of Production Engineering (VNIPIPT) has been engaged in the problem of site selection for construction of regional RW storage in the territory of the former USSR, and then in the Russian Federation during the last 30 years. These activities involve:

1. Development of suitability criteria for the main types of geological formations and zoning in the USSR territory;

2. Preparation of "Sketch map of geological formations, perspective for disposition of solidified radioactive waste in the European part of Russian Federation";

3. Development of general technical requirements for underground solidified RW repositories in weakly permeable geological formations (GTT-92);

4. Geological research aimed at site selection for an underground repository at the facilities in Krasnoyarsk, Chelyabinsk, Novaya Zemlya, Tomsk, in territory of Kolsky peninsula, Far East, the Ukraine, Byelorussia, the Republic of Cuba;

5. Similar research aimed at settling the problem of the Navy and sea shipping radwaste isolation;

6. Selection of areas for location of regional repositories for radwaste from nuclear power stations;

7. Development of design decisions for construction of borehole and mined types of RW storage.

Currently, VNIPIPT participates in research on selection and justification of a site for underground RW storage at the Nizhnekansky granitoid massif (for MCC) and in the buffer zone of PA "Mayak".

It is necessary to note that the construction of underground RW storage facilities differs from the construction of other industrial and energy-producing facilities because of the following:

- Increased ecological hazard;

- Long life of mountain drifts requires constant observation while in service;

- Large depth of underground facilities.

These facilities are specified as multibarrier systems to ensure reliable RW isolation due to engineered and natural barriers. The role of engineered barriers, as a rule, is limited in time; whereas, a natural barrier (enclosing geological environment) keeps its properties for hundreds of thousands of years. 
Therefore, optimal engineering and economic solutions for designing underground RW storage can be developed only on the basis of the comprehensive study of geologic and hydrogeological, geodynamic, and seismic conditions in the area of its location.

In this connection, engineering-geological and scientific research should be directed toward obtaining detailed and authentic data ensuring reliable design solutions.

The construction stages for RW storage, reflected in a model of its life cycle, are submitted in Fig. 1. The initial stage of a model includes selection of an underground isolation strategy, including definition of the radwaste inventory and volume and development of the isolation concept.

The concept of underground isolation, in turn, defines the kind of waste, place, and conditions of storage and requirements (i.e., criteria) for the underground isolation site.

Then, the area, site, and subsite for construction are selected according to accepted geological criteria. For this purpose, the engineering-geological research is conducted for the pre-design stages (DOI, JOI) Results of this work and subsequent engineering research should ensure an opportunity for designing and construction of an underground laboratory, and the experiments carried out in it will become a basis for development of the Feasibility Study, and the "Working Documentation for Storage." The life cycle of $\mathrm{RW}$ storage is completed when it is sealed.

While performing engineering and geological research for designing underground RW storage, it is necessary to take into account the entire legislative base concerning radwaste management in the Russian Federation and existing normative documents used in construction of facilities for various purposes.

The legislative and normative basis for RW underground storage construction is represented by the following interconnected groups of documents:

- Documents for activity regulation at the federal level;

- Normative documents to determine activity at all stages of research, design, construction, and operation of the storage facility (Fig. 2).

There is no experience in construction and operation of underground RW storage at deep locations in the Russian Federation now. Nor are there the normative documents for regulation of the kinds and volumes of exploration and research for justification of design works for underground storage.

Therefore, the normative base, which at present regulates all surveys for construction of enterprises, buildings, and structures for different purposes, including underground mountain drifts, requires certain alterations.

When performing an engineering-geological survey, it is necessary to observe strictly all stages of research. This allows different levels of detail at different stages of design.

The most critical decision is the choice of a site for storage location. This includes searching a suitable area with subsequent allocation of the site and promising subsites. 


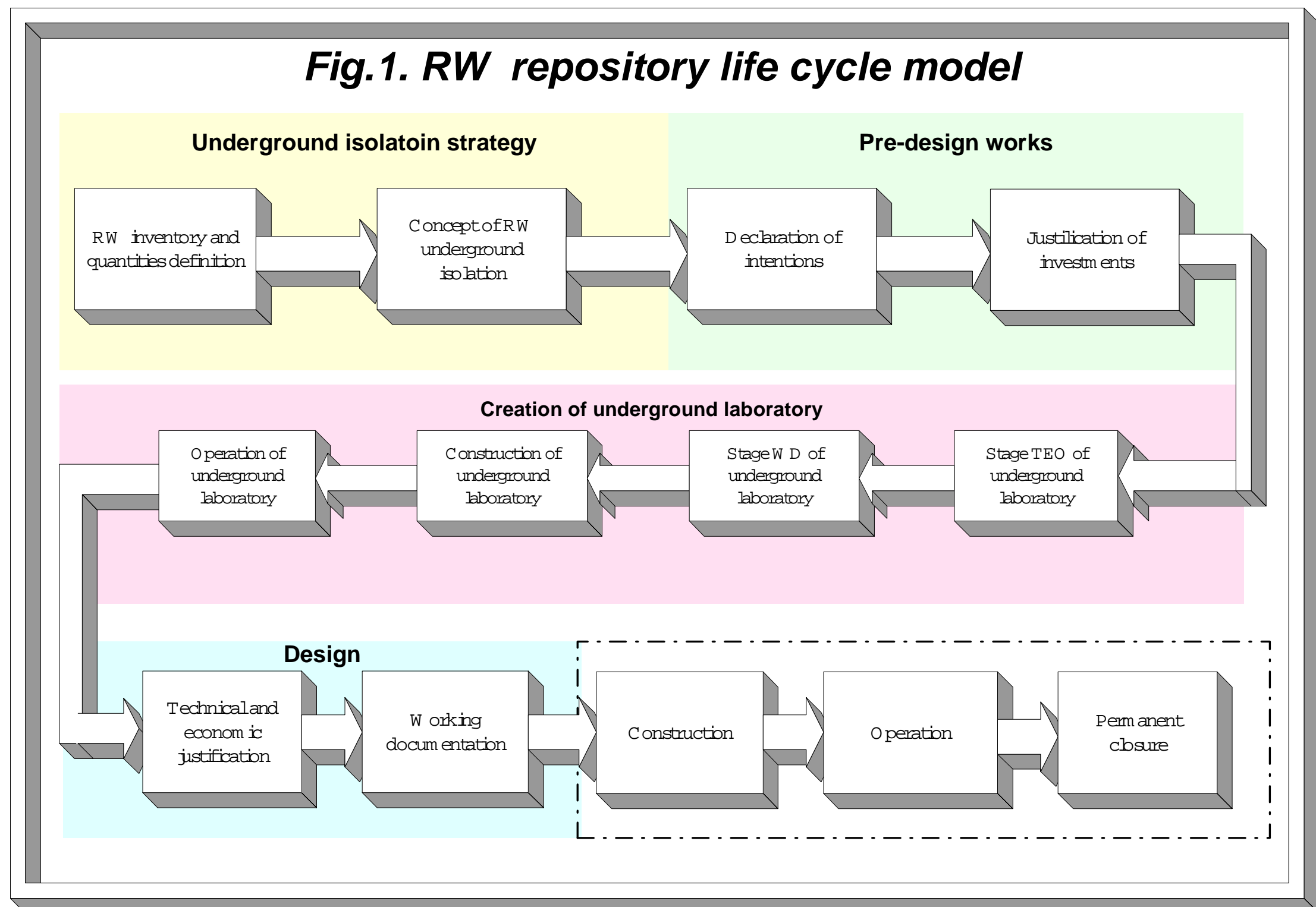




\section{Fig.2 Hierarchic structure of legislative and normative basis on RW management in the Russian Federation}

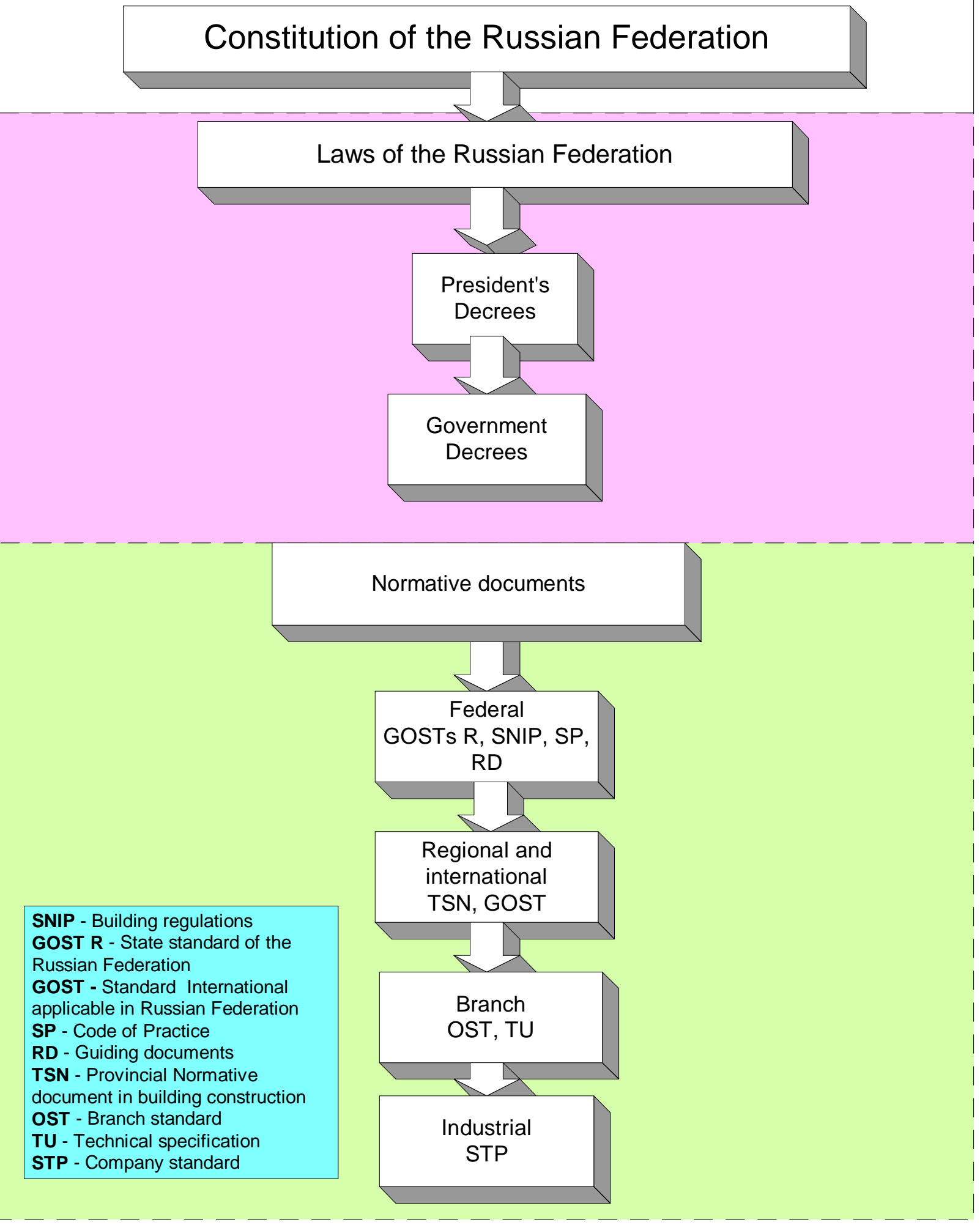


One scheme for choosing a promising site (Fig. 3) has the following characteristics.

When exploring the area, the analysis of its geological and hydrogeological conditions is performed, paying special attention to tectonic activity, seismiscity, and hydro-geological conditions. As a result, the sites potentially suitable for RW storage construction are allocated, analyzed, compared, and the most promising ones (i.e., with the best characteristics, and meeting the required criteria) are chosen. This work is carried out at the DOI stage.

Later on, at the $\mathrm{JOI}$ stage, the study of the sites allocated in the DOI stage results in selection of one or two of the most promising sites. Analysis of their geologic and hydrogeological conditions enables the selection of several promising subsites within these sites, and compare them to select the best prospect.

The final evaluation of constructing RW storage at the chosen site requires building an underground laboratory to conduct research of various processes occurring in the mountain rock mass during RW storage (disposal), and to define the optimum conditions of waste disposition, methods of isolation, etc.

It should be emphasized once again that all kinds of engineering and geologic work is carried out under the programs developed on the basis of technical projects, made up according to the requirements of existing normative documents and standards.

Of special importance are maximum information and reliability of initial geologic and hydrogeological data at the first stages of work, since this allows for optimal design solutions and the exclusion of unforeseen increases in construction cost by revealing engineering and geological complications at an early stage. 


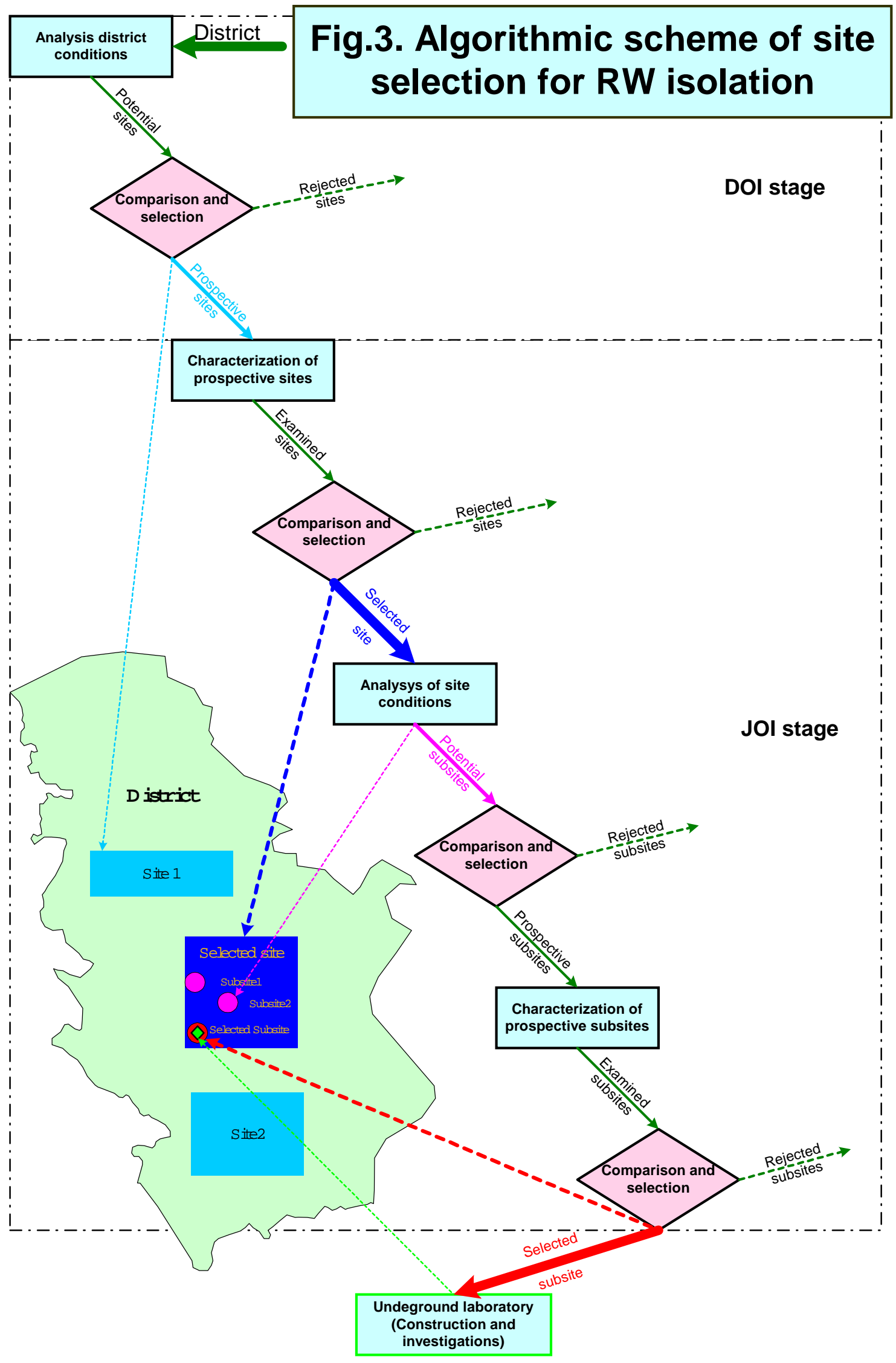




\section{Preliminary Results of Research on Plutonium and Uranium Migration in Bentonite Buffer and Biotite Gneisses in the Form of Complex Radioactive Contamination (B512134)}

\section{T. A. Gupalo, S. L. Speshilov, VNIPIPT; A. A. Tretyakov, I. V. Shramko, S. A. Manakov, MCC}

Findings of the comprehensive studies aimed at analysis of uranium and plutonium migration in a multibarrier system of radioactive waste underground isolation at Krasnoyarsk Mining and Chemical Combine (MCC) are presented here.

Velocities of leaching radionuclides from samples of boron phosphate and boron silicate matrices by free water and bound water of bentonite buffer in natural conditions have been defined. Values of key parameters of uranium and plutonium diffusion mass transfer have been counted on the basis of a numerical mathematical model taking into account heterogeneous interaction. A field experiment on analysis of migration of complex radioactive contamination in fissured biotite gneisses has been carried out.

Scientific research was devoted to problems of analysis of $\mathrm{U}^{238}$ and $\mathrm{Pu}^{239}$ radionuclides' migration in the form of complex radioactive contamination formed during leaching real vitrified radioactive waste at the Krasnoyarsk MCC. These operations are an extension of the studies that had been carried out under contract B501120 with matrix materials and materials of engineered barriers. They are aimed at obtaining experimental results under conditions similar to those of storage created in the MCC workings (and, probably, in radwaste burial subsequently) for vitrified high-level sludges.

Work includes complex laboratory and in-situ experiments on analysis of isotope leaching by free fracture-vein water and by water saturating a porous space of buffer material; on definition of parameters of radionuclide diffusion mass transfer; and on physical simulation of mass transfer with convective flow in fractured zones of the MCC rock massif.

The report presents findings only of the initial stage of work that is stipulated by the long duration of processes of radionuclide leaching from glass matrices and their low velocity of diffusion mass transfer in materials of engineered barrier. The total duration of these experiments will be as long as 3 to 5 years.

To perform predictive calculations of isotope migration in rocks of the engineered barrier during isolation of vitrified high level waste (HLW), first it was necessary to study the physical and chemical processes of the interaction of a complex radioactive solution with a solid phase of buffer material. During the laboratory experiments, values of the sorptive capacity of bentonite clays, distribution coefficients, and kinetic constants of heterogeneous interaction for uranium and plutonium were obtained.

Studies were carried out with natural fracture-vein water selected in workings planned for the creation of MCC vitrified sludge storage. In the course of the experiments, complex technological solutions from the radiochemical plant, which will be solidified into boron silicate or boron phosphate matrices, were added to samples of water. Radioactive water contained plutonium $\left(\mathrm{Pu}^{239}\right)$, uranium $\left(\mathrm{U}^{238}\right)$, cesium $\left(\mathrm{Cs}^{134,137}\right)$, ruthenium $\left(\mathrm{Ru}^{106}\right)$, cerium $\left(\mathrm{Ce}^{144}\right)$, europium $\left(\mathrm{Eu}^{154,155}\right)$, strontium $\left(\mathrm{Sr}^{90}\right)$, and other isotopes.

Experiments to study diffusion mass transfer of complex radioactive contamination in materials of engineered antimigration barriers were carried out with two types of bentonite clays: natural bentonite 
from a field in Khakassya and sodium bentonite obtained from the same natural bentonite by special treatment with sodium salts under plant conditions.

It is these clay materials that are considered as basic material for creation of a stable, long-term prospective antimigration engineered barrier while constructing storage (and, probably, subsequent burial) for vitrified radioactive sludges of Krasnoyarsk MCC.

Results of these experiments on the study of sorption-capacity properties of bentonites are summarized in Table 1.

Table 1. Sorption-capacitive properties of natural and sodium bentonites of the Khakassya deposit as against KMSS real complex solutions.

\begin{tabular}{|c|c|c|}
\hline \multirow[t]{2}{*}{ Parameter } & \multicolumn{2}{|c|}{ Experimental value } \\
\hline & Natural bentonite & Activated bentonite \\
\hline $\begin{array}{l}\text { Specific sorption capacity: } \\
\text { on } \mathrm{Pu}^{239}(\mathrm{Mк} / / \Gamma) \\
\text { on } \mathrm{U}^{238}(\mathrm{M} / / \Gamma)\end{array}$ & $\begin{array}{l}93,80 \\
41,12 \\
\end{array}$ & $\begin{array}{l}92,68 \\
40,54 \\
\end{array}$ \\
\hline $\begin{array}{l}\text { Specific distribution } \\
\text { coefficient: } \\
\text { on } \mathrm{Pu}^{239}(\mathrm{Mл} / \mathrm{r}) \\
\text { on } \mathrm{U}^{238}(\mathrm{Mл} / \mathrm{r})\end{array}$ & $\begin{array}{r}103,65 \\
65,79 \\
\end{array}$ & $\begin{array}{l}99,37 \\
64,86\end{array}$ \\
\hline $\begin{array}{l}\text { "Sorption" velocity constant: } \\
\text { on } \mathrm{Pu}^{239}(1 / \text { ( } а \mathrm{ac}) \\
\text { on } \mathrm{U}^{238}(1 / \mathrm{4ac})\end{array}$ & $\begin{array}{l}1,02 \\
0,78\end{array}$ & $\begin{array}{l}1,50 \\
0,48\end{array}$ \\
\hline
\end{tabular}

Rather large values of sorption-capacity properties are explained by data of mineralogical studies of bentonites of the Khakassya field: the part of the minerals that are active sorbents equals approximately $75 \%$ of the mass. These minerals are represented by montmorillonite $(55 \%)$, hydromica $(12 \%)$, kaolinite (6\%), and chlorite (2\%).

As follows from Table 1, natural and activated bentonites possess close values of sorptive-capacity properties; the characteristic property of sodium bentonite is its large swelling value (3.7 times as large as natural bentonite).

Experiments on analysis of migration of uranium and plutonium leached from boron phosphate and boron silicate glasses were carried out at laboratory diffusion devices and on the experimental test stand installed in naturally water-saturated pit-hole, drifted in a working at a depth of $260 \mathrm{~m}$. The general view of the experimental stand is given in Fig. 1.

Both in diffusion devices and in the test stand, samples of vitrified radioactive waste contacted natural and sodium bentonites with mass moisture content of $65-180 \%$, porosity $-65-71 \%$, and volume mass $1,23-1,98 \mathrm{~g} / \mathrm{cm}^{3}$.

The composition of actual MCC radioactive waste solidified in boron phosphate and boron silicate matrices used in the experiments are shown in the descriptions of glasses in Tables 2 and 3. 


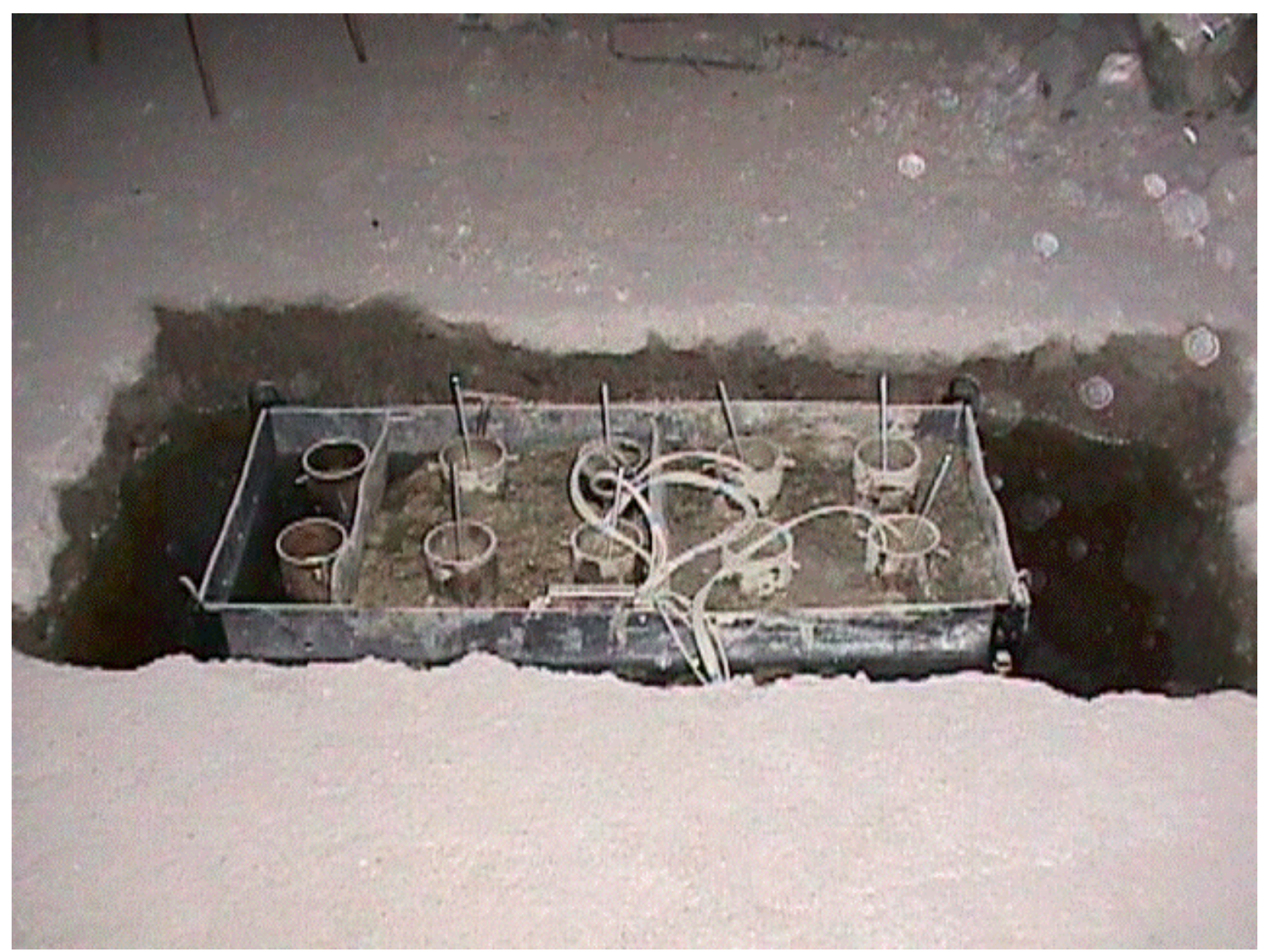

Fig. 1. General view of the test stand for large-scale experiments in drifts.

Data of calculations of average velocities of leaching uranium and plutonium during 120 days by pore (bound) water of the bentonite buffer are given in Table 4; the velocities of leaching uranium by free fracture-vein water are given here for comparison.

Table 2. Composition and activity of boron silicate glass.

\begin{tabular}{|l|c|l|c|l|r|l|c|}
\hline \multicolumn{7}{|c|}{ Glass composition: } \\
\hline $\mathrm{UO}_{3}$ & $10,5 \%$ & $\mathrm{Na}_{2} \mathrm{O}$ & $17,5 \%$ & $\mathrm{FeO}_{3}$ & $7,6 \%$ & $\mathrm{SiO}_{2}$ & $41 \%$ \\
\hline $\mathrm{Al}_{2} \mathrm{O}_{3}$ & $2,6 \%$ & $\mathrm{~B}_{2} \mathrm{O}_{3}$ & $10 \%$ & $\mathrm{MnO}_{2}$ & $7 \%$ & $\mathrm{Li}_{2} \mathrm{O}$ & $1 \%$ \\
\hline $\mathrm{NiO}$ & $1,5 \%$ & $\mathrm{PuO}_{2}$ & $0,05 \%$ & $\mathrm{Gr}_{2} \mathrm{O}_{3}$ & $1,3 \%$ & - & - \\
\hline \multicolumn{7}{|c|}{ Isotopic composition and activity of fission products. } \\
\hline & $\mathrm{Cs}^{137}$ & $\mathrm{Ce}^{144}$ & $\mathrm{Eu}^{154}$ & $\mathrm{Cs}^{134}$ & $\mathrm{Co}^{60}$ & $\mathrm{Ru}^{106}$ & $\mathrm{Sb}^{125}$ \\
\hline $\begin{array}{l}\text { Activity, } \\
\text { Cu/kg }\end{array}$ & $1.7 \cdot 10^{-3}$ & $1.13 \cdot 10^{-4}$ & $1.01 \cdot 10^{-4}$ & $5.5 \cdot 10^{-6}$ & $8.5 \cdot 10^{-6}$ & $6.9 \cdot 10^{-5}$ & $6.6 \cdot 10^{-5}$ \\
\hline
\end{tabular}

Note: $\beta$ act. $-0,037 \mathrm{Cu} / \mathrm{kg} ; \mathrm{Sr}^{90}-0,024 \mathrm{Cu} / \mathrm{kg} ; \mathrm{EDR}-0,12 \mathrm{mkR} / \mathrm{c} \cdot \mathrm{kg}$. 
Table 3. Composition and activity of phosphate glass.

\begin{tabular}{|l|l|l|l|l|l|l|l|}
\hline \multicolumn{7}{|c|}{ Glass composition: } \\
\hline $\mathrm{UO}_{3}$ & $7.5 \%$ & $\mathrm{Na}_{2} \mathrm{O}$ & $15.9 \%$ & $\mathrm{FeO}_{3}$ & $9.0 \%$ & $\mathrm{SiO}_{2}$ & $1.6 \%$ \\
\hline $\mathrm{Al}_{2} \mathrm{O}_{3}$ & $3.5 \%$ & $\mathrm{~B}_{2} \mathrm{O}_{3}$ & $7.6 \%$ & $\mathrm{MnO}_{2}$ & $7.9 \%$ & $\mathrm{Li}_{2} \mathrm{O}$ & $44.2 \%$ \\
\hline $\mathrm{NiO}$ & $1.7 \%$ & $\mathrm{PuO}_{2}$ & $0.05 \%$ & $\mathrm{Gr} 2 \mathrm{O} 3$ & $1.0 \%$ & - & - \\
\hline \multicolumn{7}{|c|}{ Isotopic composition and activity of fission products. } \\
\hline & $\mathrm{Cs}^{137}$ & $\mathrm{Ce}^{144}$ & $\mathrm{Eu}^{154}$ & $\mathrm{Cs}^{134}$ & $\mathrm{Co}^{60}$ & $\mathrm{Ru}^{106}$ & $\mathrm{Sb}^{125}$ \\
\hline $\begin{array}{l}\text { Activity, } \\
\text { Cu/kg }\end{array}$ & $2.09 \cdot 10^{-3}$ & $1.19 \cdot 10^{-4}$ & $1.24 \cdot 10^{-4}$ & $6.76 \cdot 10^{-6}$ & $1.04 \cdot 10^{-6}$ & $8.49 \cdot 10^{-5}$ & $8.12 \cdot 10^{-5}$ \\
\hline
\end{tabular}

Note: $\beta$ act. $-0.04 \mathrm{Cu} / \mathrm{kg} ; \mathrm{SR}^{90}-0.03 \mathrm{CU} / \mathrm{KG} ; \mathrm{EDR}-0,16 \mathrm{mkR} / \mathrm{c} \cdot \mathrm{kg}$.

Table 4. Results of estimation average leaching rates of $\mathrm{U}^{238}$ and $\mathrm{Pu}^{239}$ for 120 days.

\begin{tabular}{|c|c|c|c|c|c|c|}
\hline \multirow[t]{2}{*}{$\begin{array}{l}\text { Type of glass } \\
\text { matrix }\end{array}$} & \multirow[t]{2}{*}{ Buffer material } & \multirow{2}{*}{$\begin{array}{c}\text { Ave. } \\
\text { volume } \\
\text { mass, } \\
\mathrm{g} / \mathrm{cm}^{3}\end{array}$} & \multirow{2}{*}{$\begin{array}{c}\text { Ave. } \\
\text { moisture } \\
\text { content, \% }\end{array}$} & \multicolumn{2}{|c|}{$\begin{array}{l}\text { Rate of leaching with porous } \\
\text { (bound) water, } \mathrm{g} / \mathrm{cm}^{2} \text {.day }\end{array}$} & \multirow{2}{*}{$\begin{array}{c}\text { Rate of } \\
\mathrm{U}^{238} \\
\text { leaching } \\
\text { with free } \\
\text { water, } \\
\mathrm{g} / \mathrm{cm}^{2} \text {.day }\end{array}$} \\
\hline & & & & $\mathrm{Pu}^{239}$ & $U^{238}$ & \\
\hline \multirow{2}{*}{$\begin{array}{l}\text { Boron silicate } \\
\text { glass }\end{array}$} & Natural bentonite & 1,98 & 65 & $1,06 \cdot 10^{-10}$ & $4,66 \cdot 10^{-7}$ & \multirow{2}{*}{$5,87 \cdot 10^{-7}$} \\
\hline & Sodium bentonite & 1,22 & 173 & $9,23 \cdot 10^{-10}$ & $4,75 \cdot 10^{-7}$ & \\
\hline \multirow{2}{*}{$\begin{array}{l}\text { Boron } \\
\text { phosphate } \\
\text { glass }\end{array}$} & Natural bentonite & 1,98 & 63 & $1,26 \cdot 10^{-10}$ & $2,47 \cdot 10^{-7}$ & \multirow{2}{*}{$9,59 \cdot 10^{-7}$} \\
\hline & Sodium bentonite & 1,23 & 172 & $3,90 \cdot 10^{-10}$ & $1,20 \cdot 10^{-7}$ & \\
\hline
\end{tabular}

As follows from the table, velocities of uranium leaching are a factor of $10^{3}$ higher than plutonium leaching. This can be explained mainly by the higher concentration of uranium in glasses (which is also, approximately, by a factor of $10^{3}$ ).

The velocity of leaching plutonium by the pore water of natural bentonite is somewhat lower than by pore water of sodium bentonite. This may be connected, basically, with the greater moisture content and swelling capacity of the latter (i.e., for the same time, sodium bentonite absorbed more natural water); for uranium no such well-defined regularity was marked. Comparison of velocities of leaching uranium by free and pore water leads to an unexpected conclusion: they are values of the same order of magnitude and for boron silicate glasses they are very close, while for boron phosphate glass, the velocity of leaching by free water appeared to be approximately 5 times as large. (However, boron phosphate glass is less stable and it cracks at leaching by free water).

It was established in the experiments that, at leaching, plutonium and uranium with bound water boron phosphate matrix is rather stable: the velocity of leaching uranium from it is lower than for boron silicate matrix (anyway, for 120 days). However, more durable experiments are required to draw reliable and authentic conclusions.

During the experiments, distribution of uranium, plutonium, and other radionuclides per unit of volume of a wet soil was defined and coefficients of diffusion and distribution were calculated. The given work reports the values of migration parameters obtained for the period of 455 days after the beginning of uranium leaching and for 120 days after the beginning of leaching plutonium by bound pore water of bentonites. These values are given in Table 5. 
Table 5. Diffusion coefficients, sorption velocity constants, and uranium and plutonium distribution coefficients obtained in dynamic experiments.

\begin{tabular}{|l|l|l|}
\hline \multicolumn{1}{|c|}{ Parameter } & \multicolumn{2}{|c|}{ Experimental value } \\
\cline { 2 - 3 } & \multicolumn{1}{|c|}{ Natural bentonite } & Sodium bentonite \\
\hline $\begin{array}{l}\text { Coefficient of diffusion, } \mathrm{D}_{0} \text { ( } \mathrm{m}^{2} / \text { year): } \\
\mathrm{U}^{238}\end{array}$ & 0,01 & 0,01 \\
$\mathrm{Pu}^{239}$ & 0,003 & \\
\hline Dimensionless coefficient of distribution, $\beta=\frac{n_{0}}{\gamma K d}$ & 0,48 & 0,47 \\
& 0,03 & \\
$\mathrm{U}^{238}$ & & \\
$\mathrm{Pu}^{239}$ & 4200 & 4200 \\
\hline Velocity constant of heterogeneous reaction, $\alpha$ (1/year) & 13140 & $*$ \\
$\mathrm{U}^{238}$ & $\mathrm{Pu}^{239}$ &
\end{tabular}

The results of small-scale laboratory studies in diffusion devices and large-scale experiments on the experimental stand testify that natural and sodium bentonites have practically identical geomigration performance.

The in-situ experiment consisted of launching actual radioactive solutions into a zone of technogenic fracturing, monitoring the depth of plutonium and uranium propagation, and comparing it to the depth of penetration of the colored neutral indicator.

A site of rock mass composed of biotite gneisses (a main part of the rock mass) with a sufficient degree of fracturing, and dry as a principal condition, was chosen for trial work.

To perform the experiment, three pit-holes of $30 \mathrm{~cm}$ in diameter and of $20 \mathrm{~cm}$ in depth with a smooth bottom were drilled and equipped in a zone of fracturing, as shown in Fig. 2.

At the first stage of experiment in pit-hole No. 1, fracture-vein water taken from the adjoining working was injected. The value of the average filtering coefficient of fissured gneisses amounted to $5.2 \mathrm{~m} / \mathrm{day}$.

At the second stage, 15 liters of actual radioactive solutions prepared from fracture-vein water (composition of solutions is given in Table 6 ) by addition of a fixed amount of technological solutions of the radiochemical plant were supplied to pit-hole No. 1. Specific activities of the solution and individual isotopes and mass concentrations of plutonium and uranium in the indicator solution are presented in Table 7.

The solution injected into pit-hole No. 1 contained a neutral indicator, black ink "Raduga-2," that does not interreact with the rock.

Injection of a radioactive solution was carried out with free lowering of the level; immediately after the level reaches the pit-hole bottom, the latter was drilled out by means of perforator.

Radioactive and colored rock was sampled layer by layer and analyzed in the MCC Central plant laboratory. In the course of drilling-out the depth of ink penetration, we defined the length of the zone of hydrodispersion and continuous colouring. 


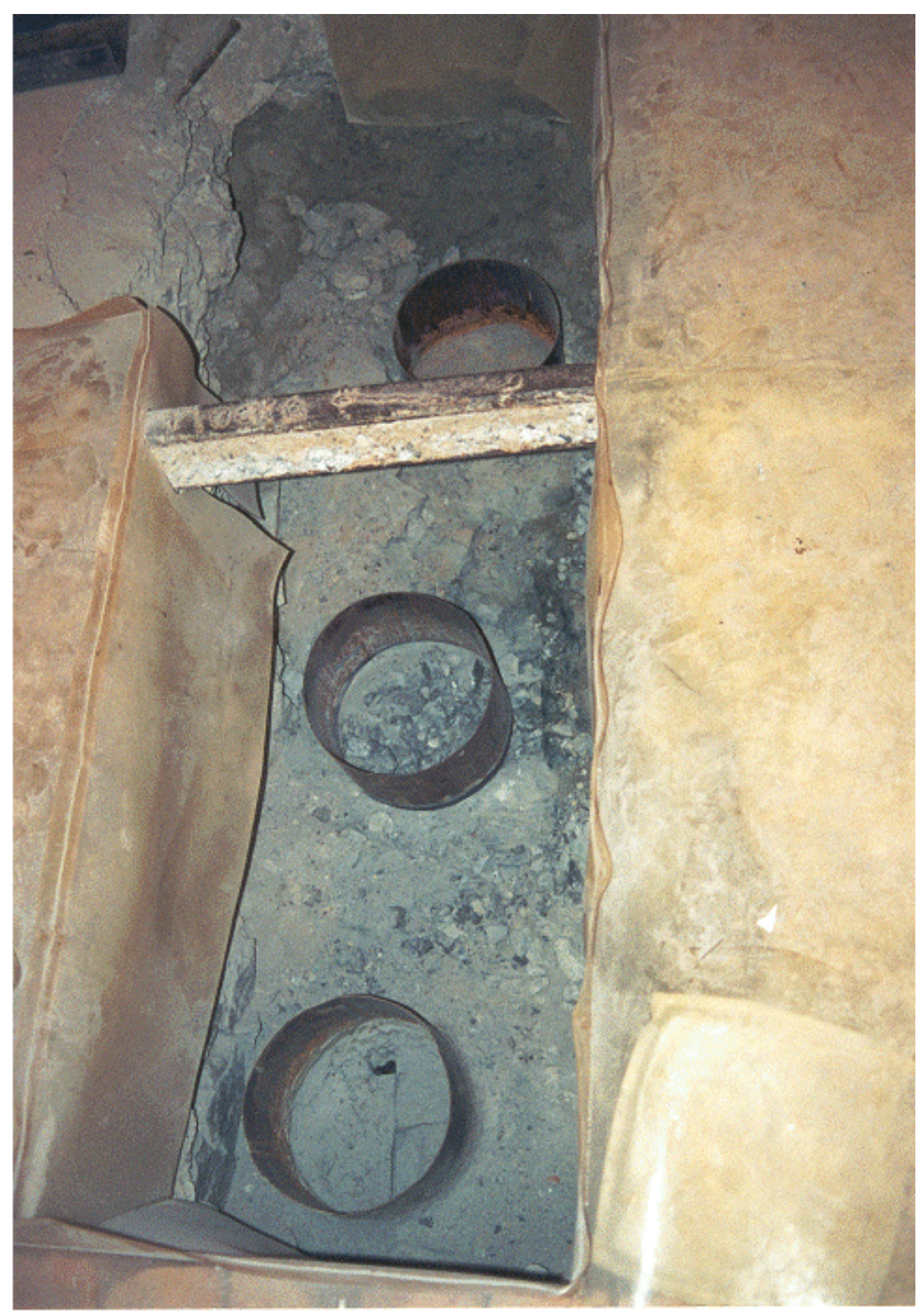

Fig. 2. General view of the test pit.

Fig. 3 presents the scheme of experiment for the definition of rock migration parameters in a zone of fracturing with the help of the neutral indicator. 
Table 6. General chemical composition of indicator liquid supplied to test pit No. 2(total volume 15 I).

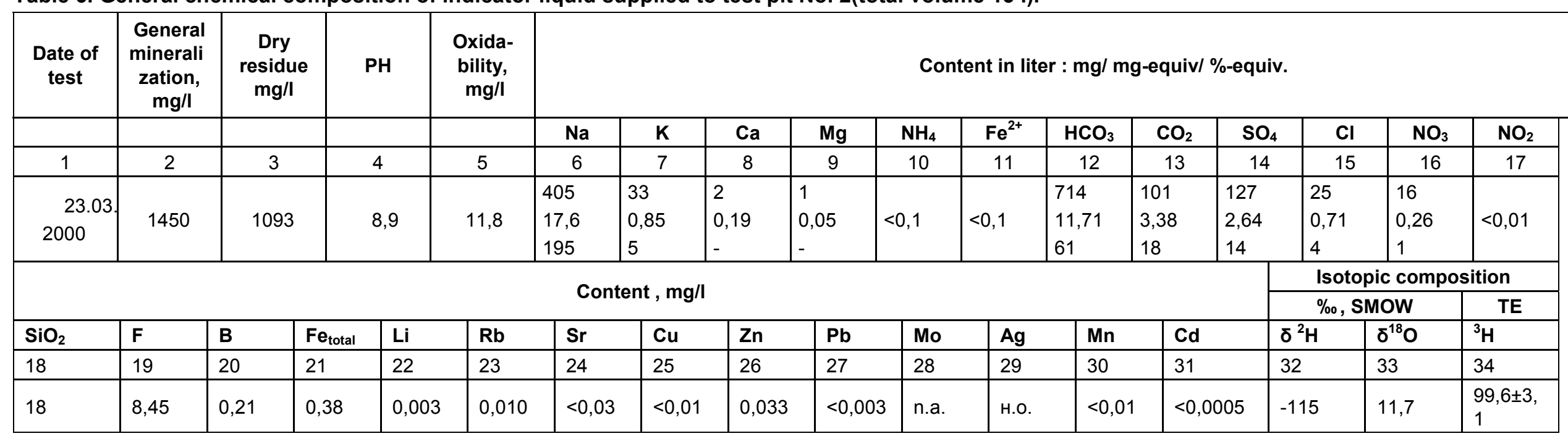

Table 7. Isotope specific activity and mass concentration of $\mathrm{U}$ and $\mathrm{Pu}$ in indicator solution supplied to test pit No 2.

\begin{tabular}{|c|c|c|c|c|c|c|c|}
\hline \multirow{2}{*}{$\begin{array}{c}\text { Total specific } \\
\text { activity of solution, } \\
\text { Cu/l }\end{array}$} & \multicolumn{6}{|c|}{ Isotope specific activity, Bq/l } & \multirow{2}{*}{$\begin{array}{c}\text { Concentration of } \\
\mathrm{U}^{238}, \mathrm{mkg} / \mathrm{l}\end{array}$} \\
\hline & $\mathrm{Cs}^{137}$ & $\mathrm{Cs}^{134}$ & $\mathbf{R u}^{106}$ & $\mathrm{Co}^{60}$ & $\mathrm{Ce}^{144}$ & $\mathrm{Pu}^{239}$ (мкг/л) & \\
\hline 0,011 & $1,6 \cdot 10^{7}$ & $3,8 \cdot 10^{5}$ & $1,4 \cdot 10^{6}$ & $4,8 \cdot 10^{3}$ & $3,2 \cdot 10^{5}$ & $2,7 \cdot 10^{3}(1,17)$ & $1,7 \cdot 10^{3}$ \\
\hline
\end{tabular}




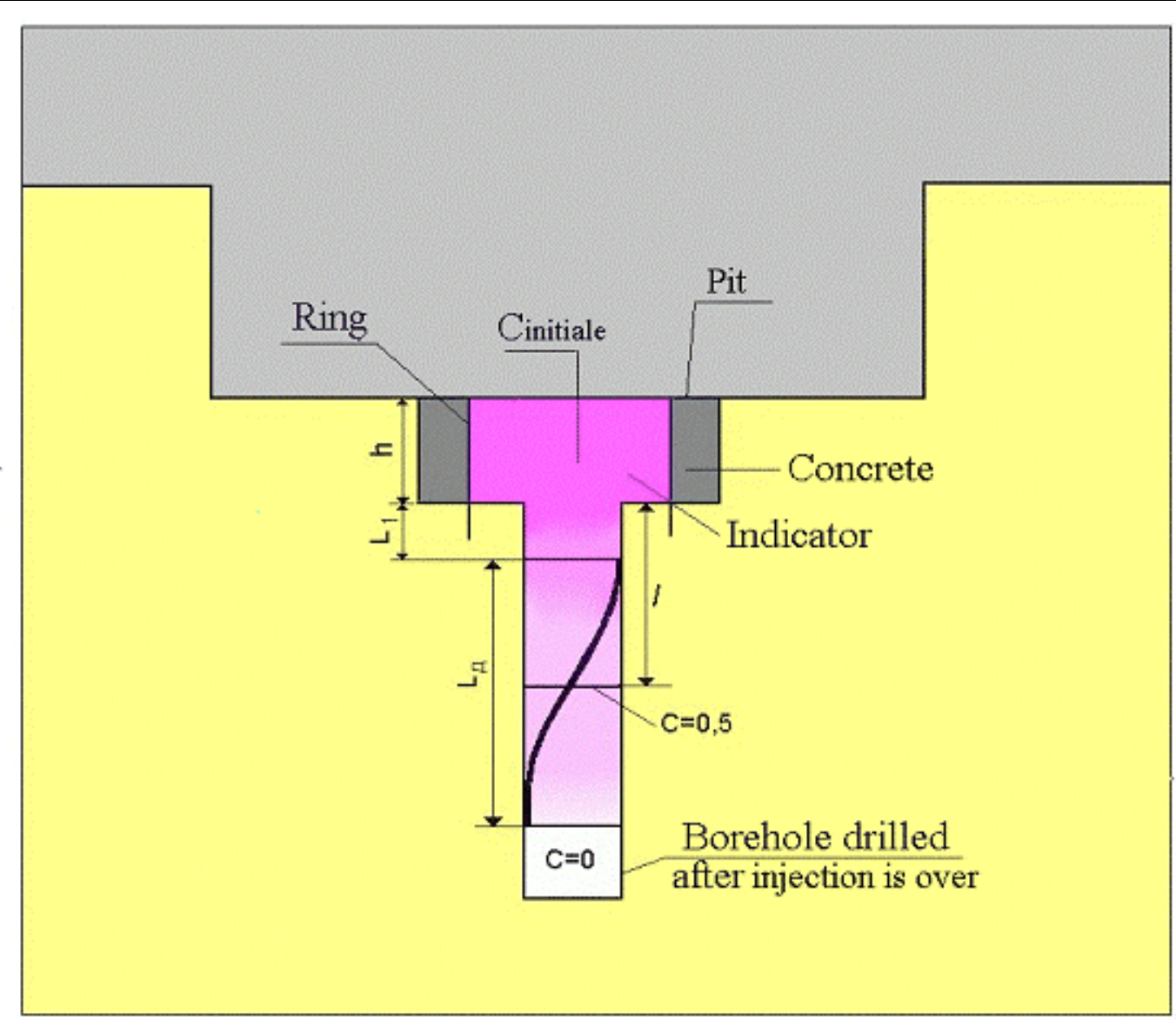

L1- length of zone with indicator initial concentration, m;

$\mathrm{Ld}$ - length of dispersion zone between pointsof relative concentration

$$
\mathrm{C}_{1}=0,99, \quad \mathrm{C}_{2}=0,01, \mathrm{C}=\frac{\mathrm{C}_{1} \mathrm{x}_{1} \mathrm{t}_{1}}{\mathrm{C}_{\text {initial }}}
$$

1 - distance up to the middle of the dispersion zone $(\mathrm{C}=0,5), \mathrm{m}$;

ho - active fracturing $\left(\mathrm{n}=\frac{\mathrm{h}}{1}\right)$;

$$
\mathrm{Ld}=6,6 \sqrt{\frac{\mathrm{Dt}}{\mathrm{n}_{0}}} \text { (asorptive tracer), } \mathrm{m} \text {; }
$$

$\mathrm{D}$ - coefficient of dispersion $\left(\mathrm{m}^{2} /\right.$ day);

$\mathrm{t}$ - indicator seepag time.

Fig. 3. Scheme of experiment to define neutral indicator migration parameters. 
.Table 8 presents the results of hydrodynamic and geomigration calculations.

Table 8. Results of definition of hydrodynamic and geomigration parameters in test pit No.1.

\begin{tabular}{|l|l|}
\hline \multicolumn{1}{|c|}{ Parameter } & \multicolumn{1}{c|}{ Value } \\
\hline Time of infiltration $(\mathrm{t})$ & 0,038 day (55,4 minutes) \\
\hline Hydraulic gradient $(\mathrm{J})$ & $\sim 1$ \\
\hline Filtration velocity $(\mathrm{V})$ & $5,2 \mathrm{~m} /$ day \\
\hline Permeability coefficient $(\mathrm{K})$ & $5,2 \mathrm{~m} /$ day \\
\hline Depth of ink penetration & $2,15 \mathrm{~m}$ \\
\hline Depth of a zone of continuous colouring (L1) & $0,35 \mathrm{~m}$ \\
\hline Half-depth of a zone of hydrodispersion $(\mathrm{l})$ & $1,25 \mathrm{~m}$ \\
\hline Length of a zone of hydrodispersion $(\mathrm{L}$ () & $1,8 \mathrm{~m}$ \\
\hline Active fracturing $\mathrm{n}_{0}$ & 0,16 \\
\hline Coefficient of hydrodispersion $(Д)$ & $0,312 \mathrm{~m}^{2} /$ day \\
\hline Parameter of hydrodispersion $(\lambda)$ & $0,06 \mathrm{~m}$ \\
\hline
\end{tabular}

While drilling out the pit-hole bottom, the geologic description of each layer of rocks was carried out and areas of projections of real fracture surfaces on a plane were measured.

The rock selected layerwise was crushed and pounded up to the required size for conducting radiochemical and $\gamma$-spectrometric analyses and carefully averaged.

We have carried out the calculation of radionuclide specific activity per unit of surface area of real fractures' projection on a plane, and constructed distribution curves of activity or mass concentration of individual radionuclides in depth.

Distribution curves of plutonium specific activity and uranium mass concentration per unit of fracture surface area in depth are shown in Figs. 4 and 5. Distribution coefficients and kinetic sorption constants are reported in Table 9.

Table 9. Coefficients of distribution $(\mathrm{Kd})$ and constant of uranium and plutonium heterogeneous interaction rates $(\alpha)$ in fractured biotite gneisses.

\begin{tabular}{|l|c|c|}
\hline \multicolumn{1}{|c|}{ Isotope } & $\mathbf{K d}, \mathbf{~ m}^{3} / \mathbf{k g}$ & $\boldsymbol{\alpha}$, day \\
\hline $\mathrm{Pu}^{239}$ & $5.9 \cdot 10^{-4}$ & 1800 \\
\hline $\mathrm{U}^{238}$ & $5.2 \cdot 10^{-4}$ & 300 \\
\hline
\end{tabular}




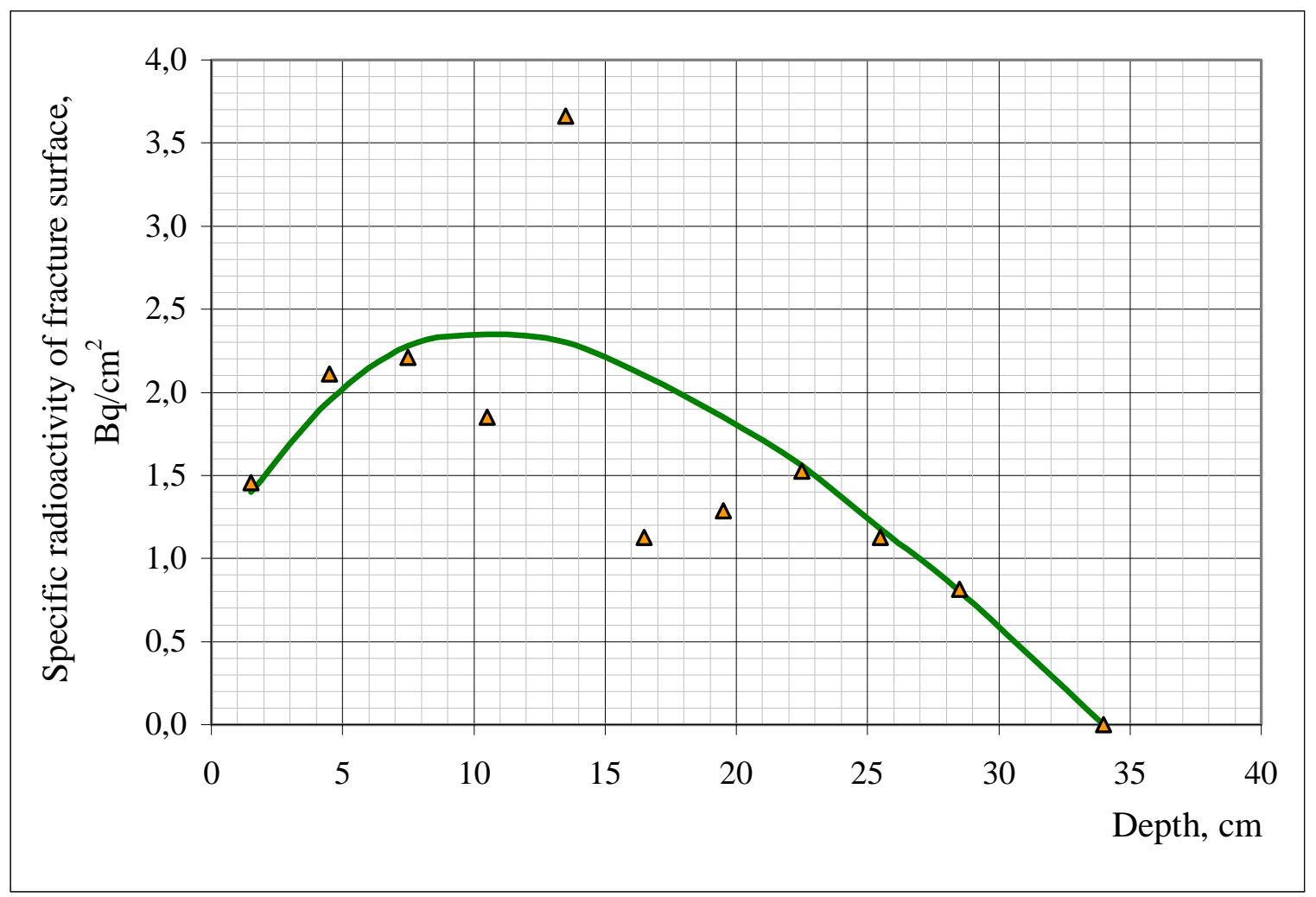

Fig. 4. Curve of $\mathrm{Pu}^{239}$ specific activity distribution in depth.

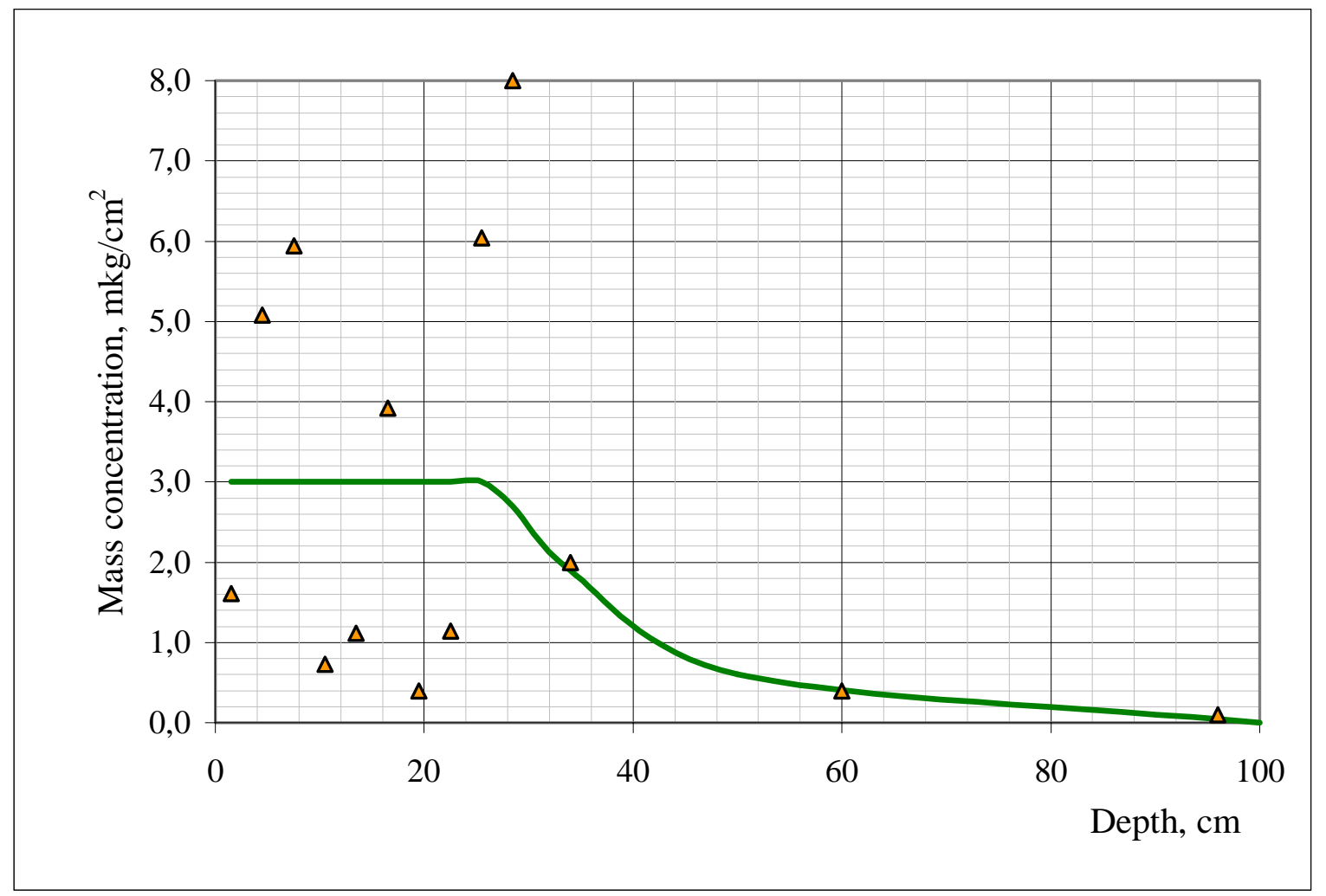

Fig. 5. Curve of $\mathrm{U}^{238}$ mass concentration distribution in depth. 


\section{Conclusions}

1. Bentonite of the field in Khakassya possesses high sorption-capacity properties as to plutonium and uranium in the form of complex radioactive contamination.

2. The data obtained during in-situ experiments testify that interaction of complex radioactive solutions (i.e., solution containing several isotopes with close values of ionic potentials) with rocks and material of engineered barriers differs considerably from interaction of a single component or two-three component solutions. Therefore, to prepare input data for designing HLW burials it is necessary to use only real multicomponent solutions.

3. Velocities of leaching uranium by bound pore water of bentonites are on the order of $n \cdot 10^{-7} \mathrm{~g} / \mathrm{cm}^{2}$ day, and that of plutonium $-\mathrm{n} \cdot 10^{-10} \mathrm{~g} / \mathrm{cm}^{2}$ day both for boron phosphate and for boron silicate matrices; but boron phosphate glass is less stable at interaction with free underground fracture-vein water (and more subject to cracking). As a whole, the boron phosphate matrix strongly retains uranium and plutonium, and is quite competitive in comparison with boron silicate.

4. The zone of artificial fracturing formed during driving of workings by drilling and blasting operations is most hazardous from the point of view of isotope migration because of its high permeability: the coefficient of filtering here reaches $5.2 \mathrm{~m} / \mathrm{day}$. The coefficient of gneiss hydraulic dispersion in a zone of technogenic fracturing makes up $0.312 \mathrm{~m}^{2} /$ day.

5. In local zones of tectonic dislocation (in natural genesis fracturing zones ), the coefficient of filtration on the average makes up only $4^{*} 10^{-4} \mathrm{~m} /$ day.

6. The most favorable factor to prevent the threat of isotope migration for large distance in a zone of technogenic fracturing is high sorptive capacity of undisturbed gneisses: for plutonium, it constitutes $4 \mathrm{~Bq} / \mathrm{cm}^{2}\left(1.75 \cdot 10^{-3} \mathrm{mkg} / \mathrm{cm}^{2}\right)$, and for uranium, $8 \mathrm{mkg} / \mathrm{cm}^{2}$. 


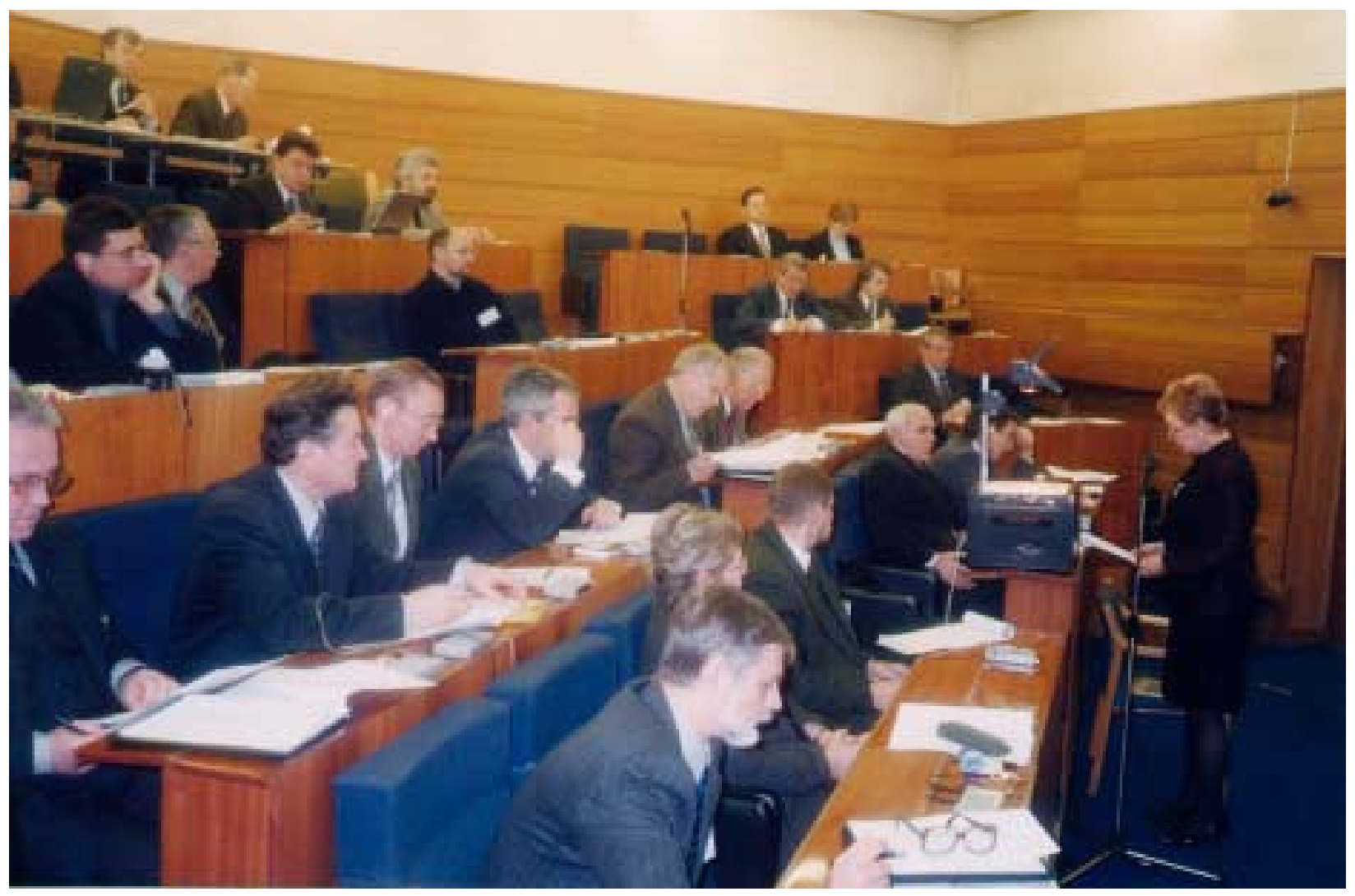

III.5. Other Invited Papers 



\section{State Regulation of Nuclear and Radiation Safety for Activities Related to Immobilization of Surplus Russian Weapons-grade Plutonium}

\section{M. Iriushkin, A. A. Stroganov, GAN SEC-NRS, G. B. Borisov, O. O. Manturov, VNIINM}

\section{Licensing of Activities Related to Utilization of Nuclear Power in the Russian Federation}

The licensing activities related to utilization of nuclear power in the Russian Federation (RF) has two aspects:

1. Required stages of the licensing process for RF Minatom activities related to immobilization of surplus Russian weapons-grade plutonium

2. Normative basis for licensing of the activities related to immobilization of plutonium

The "Provision on licensing of activities related to utilisation of nuclear power" (Decree N 865 of the RF Government) prescribes an order for licensing by the Federal Nuclear and Radiation Safety Authority (Gosatomnadzor) of Russia of any works related to nuclear energy sector.

The list of works related to utilization of nuclear power that require a license, approved by the Government of the Russian Federation, includes:

- Siting, construction, operation, and decommissioning of nuclear plants, radiation sources and radioactive materials and radioactive substances storage places, and radioactive wastes storage facilities.

- Nuclear materials and radioactive substances management, including management during prospecting and mining of uranium ores, during manufacture, use, processing, transportation and storage of nuclear materials and radioactive substances;

- Radioactive wastes management during storage, processing, transportation and utilization;

- Use of nuclear materials and/or radioactive substances in scientific research and experimental design;

- Design and construction of nuclear plants, radiation sources, radioactive wastes storage facilities;

- Design and manufacture of the equipment for nuclear plants, radiation sources, nuclear materials and radioactive substances storage places, radioactive wastes storage facilities;

- Expertise of design, construction, technological documentation and documents, substantiating nuclear and radiation safety measures for nuclear plants, radiation sources, nuclear materials and radioactive substances storage places, radioactive waste depositories, works related to nuclear materials, radioactive substances and radioactive wastes management.

Such breadth of detail in the full licensing process allows us to detect (and to avoid) any deviations from or violations of the safety requirements by an operator or nuclear facility or radiation source at the earliest stage of project implementation. 
Licensing of activities related to utilization of nuclear power in the Russian Federation is the final stage of general decision making.

All pre-licensing stages of the decision making process are completely prescribed by Decree of the Government of the Russian Federation No. 306 "REGULATIONS of the decision making order related to siting and construction of nuclear plants, radiation sources and storage facilities" (March 14, 1997). These Regulations were developed in compliance with the Federal Law of Utilisation of Atomic Energy, and other legislative and statutory legal acts of the Russian Federation.

Item 6 of these Regulations determines the following:

6. To substantiate the decision on installation or construction of an object the following document compilation procedure is established:

(a) Proposals on objects siting construction are put forward by the customer who, on the basis of assessment of the regional needs specified in the programs of development of national industries or the products and services market research, compiles a Declaration of Intentions.

The Declaration of Intentions shall include the required information on the goals, the investment sources and opportunities, the siting options, the periods of object construction and commissioning, the relevant technical and economic indicators, and the radiological and environmental safety of the project;

(b) In case of a positive outcome of the review of the Declaration of Intentions, the Customer shall take the decision to follow the established procedure to develop the applicable investment substantiation package.

(d) To approve the investment substantiation package, the Customer shall use guidance of the state examination authorities, including the environmental examination authority, and the opinions of relevant federal executive authorities, and the decisions taken by the executive authorities of the subjects of the Russian Federation concerning co-ordination of the proposed project's deployment site;

(e) The Customer may use the investment substantiation package to conduct sociological studies, public opinion polls or referendums relevant to project construction feasibility, and to produce a business plan to confirm to the credit supplier or the organisation the relevant credit guarantees and the solvency and the financial stability of the enterprise or any different subject of investment in terms of the possibility for the investor to fulfil the obligations thereof;

(f) Using guidance of the approved investment substantiation package the Customer shall decide on development of a feasibility study, which study shall be the principal design document relevant to project construction;

(g) Feasibility studies related to projects of federal significance shall undergo the required co-ordination and state examination, including environmental examination implemented in compliance with the Federal Law of Environmental Examination, and shall be approved by the relevant federal executive authority. Feasibility studies of regional and/or local significance projects shall undergo applicable co-ordination and examination procedures as well, and shall be approved, accordingly, by the executive authorities of the subjects of the Russian Federation and the Customer;

(h) The primary document required for a nuclear power plant deployment and construction permit to be issued in compliance with the safety requirements shall be the feasibility study of which the initial development phase (project site selection) shall include generation of materials required to substantiate the investment; 
(k) Issue of a license for project construction shall be the function of the Federal Nuclear and Radiological Safety Supervision Authority of Russia guided by a relevant application filed by the project operator and a review of the materials developed in the feasibility study;

(I) Any decisions related to expansion, reconstruction, upgrade or technical retooling of operational projects that do not involve revision of previously allocated land plots shall not require co-ordination with the land management authorities.

The Ordinance \# 306 also determines all formal participants for any stage of the decision-making process.

\section{Supposed stages of licensing process for the RF Minatom activities related to immobilization of surplus Russian weapons-grade plutonium}

The experts of Gosatomnadzor of Russia (GAN) have determined 45 different stages of RF Minatom organizational activities related to immobilization of plutonium which must be approved, permitted, and licensed by different governmental bodies of the Russian Federation, including GAN. A full list of these approvals, permissions, and licenses is presented in Appendix 1.

This list was developed in order to prepare an informal regulatory plan-scheme of the approval/permission/licensing responsibilities of different regulatory bodies. The list was developed in accordance with:

- the Ordinances \#306 and \#865 of the Russian Federation Government;

- Existing preliminary Minatom action plans backed up by contracts between US National Laboratories and Minatom R\&D institution and facilities such as A. A. Bochvar All-Russia Scientific Research Institute of Inorganic Materials (VNIINM), the Khlopin Radium Institute, All Russian Designing and Scientific Research Institute of Complex Power Technology (VNIPIET), State Specialized Project Design Institute (GSPI), "Mayak," etc.

The list in Appendix 1 includes only approval/permission/licensing responsibilities related to safety regulation for immobilization of plutonium, together with long-lived high level radioactive waste to the vitrified form, and temporary storage of immobilized plutonium. It does not include any issues related to final disposal of immobilized plutonium.

The present list and respective materials were developed without a high level political decision made by the RF and the US. Because up to now this political decision has not been made, the developed list has no formal status.

The different specific activities of RF Minatom facilities related to receiving the approvals, permissions, and licenses mentioned above are presented in Appendix 2. In particular, the list in Appendix 2 includes the specific activities that are needed for safety-related equipment and software certification.

\section{Normative basis for licensing of the activities related to Immobilization of plutonium}

The experts of Gosatomnadzor of Russia have determined the needed normative basis for licensing of the activities related to immobilization of plutonium

One specific Regulatory Document "Plutonium Immobilization Facilities. Safety Requirements" is needed. This Regulatory Document shall set up safety requirements for immobilization of plutonium together with long-lived high level radioactive waste to the vitrified form. 
Currently, a document at the federal level regulating requirements for ensuring safety for Plutonium Immobilization Facilities (PIFs) does not exist. Up to now similar technologies have not been implemented in Russia on an industrial scale. On the contrary, plutonium was extracted from high level process radioactive waste (RAW) of radiochemical production before their conditioning (vitrification) to the maximum extent. Therefore, a "mechanical" transfer of regulatory requirements for licensing of high level RAW vitrification facilities to tasks of safety regulation of nuclear facilities for plutonium immobilization is unacceptable. Such a transfer can result in significant reduction of nuclear criticality safety requirements and physical protection requirements for the facility itself during its operation, and for plutonium immobilized in the matrix and intended for the long-term storage.

Analysis of the following issues shall be carried out in a course of development of the Regulatory Document:

- Specific character and existing experience of weapons-grade plutonium and plutonium-containing RAW handling in Russia and abroad;

- Existing technologies for conditioning of high-level RAW containing nuclear materials, including operational practice of installations for the high level process of RAW vitrification at fuel cycle facilities;

- Results of national and foreign studies in the area of immobilization of plutonium in a composition of long-lived, high-level RAW;

- Foreign regulatory documents regulating activities in this area.

GAN's experts have prepared a Statement of Work for development of this regulatory Document. The Regulatory Document shall contain the following main sections :

- $\quad$ List of the Used Abbreviations

- Conventional Signs

- Main Terms and Definitions

1. Purpose and Scope of Applicability

2. Principles, Criteria, and General Provisions on Ensuring Nuclear Safety of Plutonium Immobilization Facilities

3. Requirements for Ensuring Safety Implemented in Design of Plutonium Immobilization Facilities

4. Safety Ensuring in Operation of Plutonium Immobilization Facilities

5. Safety Ensuring in Decommissioning of Plutonium Immobilization Facilities

6. Safety requirements for Plutonium Immobilization Facilities are to be set up in this Regulatory Document. Consistent (at all stages of the lifetime of the facilities) implementation of these safety requirements is necessary to ensure the protection of personnel and general public and environmental protection. 


\section{Appendix 1. Regulatory Activities for Russian Weapons-grade Pu immobilization}

\section{Abbreviations}

TEO - Technical and Economic Analysis for Construction

JOI - Justification of Investment

DOI - Declaration of Intent

TEI - Technical and Economic Investigations for Choice of Immobilization Technology

Table A1. List of supposed regulatory activities for Russian weapons-grade plutonium immobilization process.

\begin{tabular}{|c|c|c|}
\hline Index & $\begin{array}{l}\text { Time, } \\
\text { Month }\end{array}$ & Regulatory activity \\
\hline G1 & $2-3$ & $\begin{array}{l}\text { Expertise of applications, inspections and issues Licenses of GAN for R\&D work related to } \\
\text { the development of technologies of Immobilization of Plutonium, contained in: } \\
\text { Radioactive pulps and sludge, accumulated at GHK ( } 600 \mathrm{~kg}) \text { and at "Mayak" }(800 \mathrm{~kg}) \text { as the } \\
\text { result of former activity } \\
\text { Radioactive wastes from MOX-fuel fabrication used of the Russian weapon Plutonium } \\
\text { Verification technologies are researched, which use: } \\
\text { Microwave foundry with two different kinds of matrix (VNIINM and RIAS) } \\
\text { Induction foundry with cold crucible (for conductive liquid RW contained Pu) } \\
\text { Traditional ceramic foundry (like EP-furnace at "Mayak") } \\
\text { Applicant obtains Sanitary conclusions from Ministry of Health. } \\
\text { Applicants: VNIINM, RIAS, "Mayak", GHK, likely IPPE and Others }\end{array}$ \\
\hline G2 & $1-2$ & $\begin{array}{l}\text { Licenses of Mintorg for Export of Nuclear Technologies } \\
\text { Applicant: VNIINM, RIAS, GHK, Mayak and Others }\end{array}$ \\
\hline G3 & $6-8$ & $\begin{array}{l}\text { JOI Review by MINPRIRODY, Ministry of Health, MVD (in case of siting of immobilization } \\
\text { facility at "Mayak") } \\
\text { Applicant: "Mayak", VNIPIET }\end{array}$ \\
\hline G3a & $6-8$ & $\begin{array}{l}\text { JOI Review by MINPRIRODY, Ministry of Health, MVD (in case of siting of immobilization } \\
\text { facility at GHK) } \\
\text { Applicant: GHK, VNIPIET }\end{array}$ \\
\hline G4 & $3-4$ & $\begin{array}{l}\text { Review and expertise of JOI by GAN (in case of siting of immobilization facility at "Mayak") } \\
\text { Applicant: "Mayak", VNIPIET }\end{array}$ \\
\hline G4a & $3-4$ & $\begin{array}{l}\text { Review and expertise of JOI by GAN (in case of siting of immobilization facility at GHK) } \\
\text { Applicant: GHK, VNIPIET }\end{array}$ \\
\hline G5 & $1-2$ & $\begin{array}{l}\text { Decision of Government and Local Authority (Chelyabinsk region) for Siting and Construction } \\
\text { of immobilization facility at "Mayak" }\end{array}$ \\
\hline G5a & $1-2$ & $\begin{array}{l}\text { Decision of Government and Local Authority (Krasnojarsk region) for Siting and Construction } \\
\text { of immobilization facility at GHK }\end{array}$ \\
\hline
\end{tabular}




\begin{tabular}{|c|c|c|}
\hline G6 & $4-6$ & $\begin{array}{l}\text { Expertise of application, inspection and issues Siting License of GAN for immobilization } \\
\text { facility at "Mayak") } \\
\text { Applicant: Mayak }\end{array}$ \\
\hline G6a & $4-6$ & $\begin{array}{l}\text { Expertise of application, inspection and issues Siting License of GAN for immobilization } \\
\text { facility at GHK } \\
\text { Applicant: GHK }\end{array}$ \\
\hline G7 & $2-3$ & $\begin{array}{l}\text { Review, expertise and Coordination at GAN and Ministry of Health } \\
\text { of Technical Specification (Task Order) for Design o of immobilization facility at "Mayak") } \\
\text { Applicant: VNIPIET }\end{array}$ \\
\hline $\mathrm{G} 7 \mathrm{a}$ & $2-3$ & $\begin{array}{l}\text { Review, expertise and Coordination at GAN and Ministry of Health } \\
\text { of Technical Specification (Task Order) for Design of immobilization facility at GHK } \\
\text { Applicant: VNIPIET }\end{array}$ \\
\hline G8 & $2-3$ & $\begin{array}{l}\text { Expertise of applications, conducting of inspections and issues Licenses of GAN for } \\
\text { Equipment Design } \\
\text { (for immobilization facility at "Mayak",GHK). } \\
\text { Applicant: SVERDNIIHIMMASH, VNIKIMT and Other }\end{array}$ \\
\hline G9 & $1-3$ & $\begin{array}{l}\text { Review and Coordination by GAN of Technical Specifications for Design of Equipment (for } \\
\text { immobilization facility at "Mayak", GHK). } \\
\text { Applicant: SVERDNIIHIMMASH and Other }\end{array}$ \\
\hline G10 & $6-10$ & $\begin{array}{l}\text { TEO Review and expertise by MINPRIRODY, Ministry of Health, MVD (for } \\
\text { immobilization facility at "Mayak") } \\
\text { Applicant: VNIPIET }\end{array}$ \\
\hline G10a & $6-10$ & $\begin{array}{l}\text { TEO Review and expertise by MINPRIRODY, Ministry of Health, MVD (for } \\
\text { immobilization facility at GHK) } \\
\text { Applicant: VNIPIET }\end{array}$ \\
\hline G11 & $6-10$ & $\begin{array}{l}\text { Review and expertise of approved TEO for immobilization facility at "Mayak" by GAN. } \\
\text { Applicant: VNIPIET }\end{array}$ \\
\hline G11a & $6-10$ & $\begin{array}{l}\text { Review and expertise of approved TEO for immobilization facility at GHK by GAN. } \\
\text { Applicant: VNIPIET }\end{array}$ \\
\hline G12 & $3-4$ & $\begin{array}{l}\text { Public Review of short description of TEO of immobilization facility at "Mayak". Public } \\
\text { discussions. } \\
\text { Applicant: VNIPIET }\end{array}$ \\
\hline G12a & $3-4$ & $\begin{array}{l}\text { Public Review of short description of TEO of immobilization facility at GHK. Public } \\
\text { discussions. } \\
\text { Applicant: VNIPIET }\end{array}$ \\
\hline G13 & $1-2$ & $\begin{array}{l}\text { Decision of Local Authority of OZERSK for Construction of immobilization facility at "Mayak" } \\
\text { Applicant: "Mayak" }\end{array}$ \\
\hline G13a & $1-2$ & $\begin{array}{l}\text { Decision of Local Authority of Tomsk-7 for Construction of immobilization facility at GHK. } \\
\text { Applicant: GHK }\end{array}$ \\
\hline
\end{tabular}




\begin{tabular}{|c|c|c|}
\hline G14 & $2-4$ & $\begin{array}{l}\text { Review and Coordination at GAN of Technical Specifications (Task Orders) for Design of } \\
\text { Additional Equipment for immobilization facility at "Mayak" and GHK (equipment for } \\
\text { conditioning of minority RW contaminated by Plutonium) } \\
\text { Applicant: SVERDNIIHIMMASH and Other }\end{array}$ \\
\hline G15 & $4-6$ & $\begin{array}{l}\text { Review and expertise by GAN of Design Documents for Equipment for immobilization } \\
\text { facility Review of Design Documents for radiometric Equipment by Ministry of Health } \\
\text { Applicant: SVERDNIIHIMMASH and Other }\end{array}$ \\
\hline G16 & $2-3$ & $\begin{array}{l}\text { Expertise of application, inspection and issues Licenses of GAN for Equipment } \\
\text { Manufacturing for immobilization facility } \\
\text { Applicant: SVERDNIIHIMMASH and Others producers }\end{array}$ \\
\hline G17 & $6-10$ & $\begin{array}{l}\text { Expertise of application, inspections and issues License of GAN for Construction of } \\
\text { immobilization facility at "Mayak". } \\
\text { Applicant: "Mayak" }\end{array}$ \\
\hline G17a & $6-10$ & $\begin{array}{l}\text { Expertise of application, inspections and issues License of GAN for Construction of } \\
\text { immobilization facility at GHK. } \\
\text { Applicant: GHK }\end{array}$ \\
\hline G18 & $4-6$ & $\begin{array}{l}\text { Review by GAN and expertise of Documentation for Imported Equipment (if it will be } \\
\text { supposed to use at "Mayak"-facility) } \\
\text { Applicant: "Mayak" }\end{array}$ \\
\hline G18a & $4-6$ & $\begin{array}{l}\text { Review by GAN and expertise of Documentation for Imported Equipment (if it will be } \\
\text { supposed to use at GHK-facility) } \\
\text { Applicant: GHK }\end{array}$ \\
\hline G19 & $6-10$ & $\begin{array}{l}\text { Certification of Equipment for immobilization facility by GAN and MINATOM } \\
\text { Applicant: Producers }\end{array}$ \\
\hline G20 & $1-3$ & $\begin{array}{l}\text { Obtaining of permission from Regional Office of Minprirody for immobilization facility } \\
\text { at "Mayak" related to releases and discharges of radioactive substances } \\
\text { Applicant: "Mayak" }\end{array}$ \\
\hline G20a & $1-3$ & $\begin{array}{l}\text { Obtaining of permission from Regional Office of Minprirody for immobilization facility } \\
\text { at GHK related to releases and discharges of radioactive substances } \\
\text { Applicant: GHK }\end{array}$ \\
\hline G21 & $2-4$ & $\begin{array}{l}\text { Obtaining of Sanitary conclusion from Regional Office of Ministry of Health for } \\
\text { immobilization facility at "Mayak" } \\
\text { Applicant: "Mayak" }\end{array}$ \\
\hline G21a & $2-4$ & $\begin{array}{l}\text { Obtaining of Sanitary conclusion from Regional Office of Ministry of Health for } \\
\text { immobilization facility at GHK } \\
\text { Applicant: GHK }\end{array}$ \\
\hline G22 & $1-3$ & $\begin{array}{l}\text { Coordination of Program for disposition of Radioactive materials for immobilization facility at } \\
\text { "Mayak" with Ministry of Health } \\
\text { Applicant: "Mayak" }\end{array}$ \\
\hline G22a & $1-3$ & $\begin{array}{l}\text { Coordination of Program for disposition of Radioactive materials for immobilization facility at } \\
\text { GHK with Ministry of Health } \\
\text { Applicant: GHK }\end{array}$ \\
\hline
\end{tabular}




\begin{tabular}{|c|c|c|}
\hline G23 & $2-4$ & $\begin{array}{l}\text { Permits of GAN to Operating personnel of immobilization facility at "Mayak" } \\
\text { Applicant: "Mayak" }\end{array}$ \\
\hline G23a & $2-4$ & $\begin{array}{l}\text { Permits of GAN to Operating personnel of immobilization facility at GHK } \\
\text { Applicant: GHK }\end{array}$ \\
\hline G24 & $1-2$ & $\begin{array}{l}\text { Inspection for } \\
\text { Preparedness of immobilization facility at "Mayak" to operate. } \\
\text { Participants: GAN, Local Authority, Regional Offices of MVD, Ministry of Health, Minprirody, } \\
\text { Applicant: "Mayak" }\end{array}$ \\
\hline G24a & $1-2$ & $\begin{array}{l}\text { Inspection for } \\
\text { Preparedness of immobilization facility at GHK to operate. } \\
\text { Participants: GAN, Local Authority, Regional Offices of MVD, Ministry of Health, Minprirody, } \\
\text { Applicant: GHK }\end{array}$ \\
\hline G25 & $8-10$ & $\begin{array}{l}\text { Expertise of application, inspection and issues of Operation License of GAN for } \\
\text { immobilization facility at "Mayak" } \\
\text { Applicant: "Mayak" }\end{array}$ \\
\hline G25a & $8-10$ & $\begin{array}{l}\text { Expertise of application, inspection and issues of Operation License of GAN for } \\
\text { immobilization facility at GHK } \\
\text { Applicant: GHK }\end{array}$ \\
\hline G26 & $4-8$ & $\begin{array}{l}\text { Expertise of application, inspection and issues Licenses of GAN for Decommissioning of } \\
\text { immobilization facility at "Mayak". Applicant obtains Sanitary conclusions from Ministry of } \\
\text { Health. } \\
\text { Applicant: "Mayak" }\end{array}$ \\
\hline G26a & $4-8$ & $\begin{array}{l}\text { Expertise of application, inspection and issues Licenses of GAN for Decommissioning of } \\
\text { immobilization facility at GHK. Applicant obtains Sanitary conclusions from Ministry of Health. } \\
\text { Applicant: GHK }\end{array}$ \\
\hline G27 & $9-12$ & $\begin{array}{l}\text { Certification of Software have been used under design of an equipment of immobilization } \\
\text { facility } \\
\text { Applicant: VNIPIET and Other }\end{array}$ \\
\hline
\end{tabular}




\section{Appendix 2. Detailed List of Industrial Activities (including pre-licensing activities) for Russian Weapons-grade Plutonium Immobilization Process}

\begin{tabular}{|c|c|}
\hline Index & Block of Activities \\
\hline$A 7$ & Research and Development in field of Plutonium Immobilization (See 1st Level Dictionary) \\
\hline 1 & 2 \\
\hline I-A1.1 & $\begin{array}{l}\text { Preliminary Research and Development for matrix and process selection. } \\
\text { R\&D work related to the development of technologies of Immobilization of Plutonium, contained in: } \\
\text { Radioactive pulps and sludge, accumulated at GHK }(600 \mathrm{~kg}) \text { and at "Mayak" }(800 \mathrm{~kg}) \text { as the result of } \\
\text { former activity } \\
\text { Radioactive wastes from MOX-fuel fabrication used of the Russian weapon Plutonium } \\
\text { Vitrification technologies are investigated, which use: } \\
\text { Microwave foundry with two different kinds of matrix (VNIINM and RIAS) } \\
\text { Induction foundry with cold crucible (for conductive liquid RW contained Pu) } \\
\text { Traditional ceramic foundry (like EP-furnace at "Mayak") } \\
\text { Responsible: VNIINM, RIAS; } \\
\text { "Mayak", GHK; } \\
\text { likely IPPE and Others }\end{array}$ \\
\hline I-A1.2 & Engineering Feasibility Study (TEI) based on R\&D results \\
\hline I-A1.3 & R\&D in support for TEI \\
\hline A8 & $\begin{array}{l}\text { It is suppose don't construct Immobilization Demo Facility } \\
\text { (See 1st Level Dictionary) }\end{array}$ \\
\hline A9 & $\begin{array}{l}\text { Treatment, immobilization, storage and disposal radwaste resulted from operations of Pu-conversion } \\
\text { facilities, MOX-fuel fabrication facilities, and Pu-radwaste from former "Mayak" and GHK activities } \\
\text { accumulated at sites (See 1st Level Dictionary) }\end{array}$ \\
\hline IA-2.1 & Prepare safety analysis report for different immobilization technologies \\
\hline IA-2.2 & $\begin{array}{l}\text { Compare environmental impacts } \\
\text { for different immobilization technologies }\end{array}$ \\
\hline IA-2.3 & Develop disposal concept \\
\hline IA-2.4 & Compile requirements for equipment (technical specifications) \\
\hline IA-2.5 & Complete TEI \\
\hline IA-2.6 & Coordinate and review TEI \\
\hline IA-2.7 & Additional Analysis Amend TEI \\
\hline I-D2.1 & Is TEl approved? \\
\hline I-A3.1 & Preliminary cost Estimates for immobilization facility at "Mayak" \\
\hline I-A3.1a & Preliminary cost Estimates for immobilization facility at GHK \\
\hline I-A3.2 & Prepare Declaration of Intent (DOI) for immobilization facility at "Mayak" \\
\hline I-A3.2a & Prepare Declaration of Intent (DOI) for immobilization facility at GHK \\
\hline I-A3.3 & Coordinate and review DOI for immobilization facility at "Mayak" \\
\hline I-A3.3a & Coordinate and review DOI for immobilization facility at GHK \\
\hline I-A3.4 & Amend DOI for immobilization facility at "Mayak" \\
\hline I-A3.4a & Amend DOI for immobilization facility at GHK \\
\hline
\end{tabular}




\begin{tabular}{|c|c|}
\hline I-D3.1 & Is DOI for immobilization facility at "Mayak" approved? \\
\hline I-D3.1a & Is DOI for immobilization facility at GHK approved? \\
\hline $\mathrm{I}-\mathrm{A} 4.1$ & Prepare Task Order for Justification of Investment (JOI) for immobilization facility at "Mayak" \\
\hline I-A4.1a & Prepare Task Order for Justification of Investment (JOI) for immobilization facility at GHK \\
\hline $\mathrm{I}-\mathrm{A} 4.2$ & Compile Data for Environmental Impact Assessment (EIA) for immobilization facility at "Mayak" \\
\hline I-A4.2a & Compile Data for Environmental Impact Assessment (EIA) for immobilization facility at GHK \\
\hline I-A4.3 & Prepare JOI for immobilization facility at "Mayak" \\
\hline I-A4.3a & Prepare JOI for immobilization facility at GHK \\
\hline I-A4.4 & Prepare EIA for immobilization facility at "Mayak" \\
\hline I-A4.4a & Prepare EIA for immobilization facility at GHK \\
\hline I-A4.5 & Prepare Safety Analysis Report (SAR) for licensing the immobilization facility siting at "Mayak" \\
\hline I-A4.5a & Prepare Safety Analysis Report (SAR) for licensing the immobilization facility siting at GHK \\
\hline I-A4.6 & State Expert Review of JOI for immobilization facility at "Mayak" \\
\hline I-A4.6a & State Expert Review of JOI for immobilization facility at GHK \\
\hline I-A4.7 & Prepare Site Selection Documentation for immobilization facility at "Mayak" \\
\hline I-A4.7a & Prepare Site Selection Documentation for immobilization facility at GHK \\
\hline I-A4.8 & Coordinate review of the JOI for immobilization facility at "Mayak" \\
\hline I-A4.8a & Coordinate review of the $\mathrm{JOI}$ for immobilization facility at GHK \\
\hline I-D4.1 & Is JOI for immobilization facility at "Mayak"Approved? \\
\hline I-D4.1a & Is JOI for immobilization facility at GHK Approved? \\
\hline I-A5.1 & Secure siting license for immobilization facility at "Mayak" \\
\hline I-A5.1a & Secure siting license for immobilization facility at GHK \\
\hline I-A6.1 & Coordinate and review TEO for immobilization facility at "Mayak" \\
\hline I-A6.1a & Coordinate and review TEO for immobilization facility at GHK \\
\hline I-A6.2 & Prepare TEO Document for immobilization facility at "Mayak" \\
\hline I-A6.2a & Prepare TEO Document for immobilization facility at GHK \\
\hline I-A6.3 & Compile requirements for equipment for immobilization facility at "Mayak" \\
\hline I-A6.3a & Compile requirements for equipment for immobilization facility at GHK \\
\hline I-A6.4 & Prepare preliminary safety analysis report (PSAR) for immobilization facility at "Mayak" \\
\hline I-A6.4a & Prepare preliminary safety analysis report (PSAR) for immobilization facility at GHK \\
\hline I-A6.5 & Justification of Nuclear and Radiation Safety at Design Stage for immobilization facility at "Mayak" \\
\hline I-A6.5a & Justification of Nuclear and Radiation Safety at Design Stage for immobilization facility at GHK \\
\hline I-A6.6 & Modify TEO and carry out additional R\&D for immobilization facility at "Mayak" \\
\hline I-A6.6a & Modify TEO and carry out additional R\&D for immobilization facility at GHK \\
\hline I-A6.7 & Coordinate and review TEO for immobilization facility at "Mayak" \\
\hline I-A6.7a & Coordinate and review TEO for immobilization facility at GHK \\
\hline I-A6.8 & Modify TEO Documentation for immobilization facility at "Mayak" \\
\hline I-A6.8a & Modify TEO Documentation for immobilization facility at GHK \\
\hline I-A6.9 & R\&D in support for TEO for immobilization facility at "Mayak" \\
\hline I-A6.9a & R\&D in support for TEO for immobilization facility at GHK \\
\hline I-A6.10 & Preparation of Task Order for Design for immobilization facility at "Mayak" \\
\hline I-A6.10a & Preparation of Task Order for Design for immobilization facility at GHK \\
\hline I-D6.1 & Is one immobilization technology selected for immobilization facility at "Mayak"? \\
\hline I-D6.1a & Is one immobilization technology selected for immobilization facility at GHK? \\
\hline I-D6.2 & Is TEO approved for immobilization facility at "Mayak"? \\
\hline I-D6.2a & Is TEO approved for immobilization facility at GHK? \\
\hline I-A7.1 & Obtain and compile initial data for facility construction for immobilization facility at " $\mathrm{N}$ \\
\hline
\end{tabular}




\begin{tabular}{|c|c|}
\hline I-A7.1a & Obtain and compile initial data for facility construction for immobilization facility at GHK \\
\hline I-A7.2 & Develop and coordinate working documents for immobilization facility at "Mayak" \\
\hline $\mathrm{I}-\mathrm{A} 7.2 \mathrm{a}$ & Develop and coordinate working documents for immobilization facility at GHK \\
\hline I-A7.3 & Compile requirements for additional equipment for immobilization facility at "Mayak" \\
\hline $\mathrm{I}-\mathrm{A} 7.3 \mathrm{a}$ & Compile requirements for additional equipment for immobilization facility at GHK \\
\hline I-A7.4 & $\begin{array}{l}\text { Develop and Coordinate Design Documents for Custom-made Equipment for immobilization facility at } \\
\text { "Mayak" }\end{array}$ \\
\hline I-A7.4a & $\begin{array}{l}\text { Develop and Coordinate Design Documents for Custom-made Equipment for immobilization facility at } \\
\text { GHK }\end{array}$ \\
\hline I-A7.5 & Identification of nuclear and radiation safety for immobilization facility at "Mayak" \\
\hline $\mathrm{I}-\mathrm{A} 7.5 \mathrm{a}$ & Identification of nuclear and radiation safety for immobilization facility at GHK \\
\hline I-A8.2 & Equipment fabrication and procurement for immobilization facility at "Mayak" \\
\hline I-A8.2a & Equipment fabrication and procurement for immobilization facility at GHK \\
\hline I-A8.1 & Building constructions or modifications for immobilization facility at "Mayak" \\
\hline I-A8.1a & Building constructions or modifications for immobilization facility at GHK \\
\hline I-A8.4 & Install equipment and perform initial run for immobilization facility at "Mayak" \\
\hline $\mathrm{I}-\mathrm{A} 8.4 \mathrm{a}$ & Install equipment and perform initial run for immobilization facility at GHK \\
\hline I-A8.6 & Cold testing on simulators for immobilization facility at "Mayak" \\
\hline I-A8.6a & Cold testing on simulators for immobilization facility at GHK \\
\hline I-A8.7 & Prepare final safety analysis report (FSAR) for operations for immobilization facility at "Mayak" \\
\hline $\mathrm{I}-\mathrm{A} 8.7 \mathrm{a}$ & Prepare final safety analysis report (FSAR) for operations for immobilization facility at GHK \\
\hline I-A8.3 & Prepare, review, and approve operating instructions for immobilization facility at "Mayak" \\
\hline I-A8.3a & Prepare, review, and approve operating instructions for immobilization facility at GHK \\
\hline I-A8.5 & Train and certify personnel for immobilization facility at "Mayak" \\
\hline I-A8.5a & Train and certify personnel for immobilization facility at GHK \\
\hline I-A8.8 & Obtaining of operation license for immobilization facility at "Mayak" \\
\hline I-A8.8a & Obtaining of operation license for immobilization facility at GHK \\
\hline I-D8.1 & Is operating license approved for immobilization facility at "Mayak"? \\
\hline I-D8.1a & Is operating license approved for immobilization facility at GHK? \\
\hline I-A8.9 & Modify operating documents for immobilization facility at "Mayak" \\
\hline I-A8.9a & Modify operating documents for immobilization facility at GHK \\
\hline I-A8.10 & Commission of facility at "Mayak" \\
\hline I-A8.10a & Commission of facility at GHK \\
\hline I-A9.1 & Pu immobilization facility operations at "Mayak" \\
\hline I-A9.1a & Pu immobilization facility operations at GHK \\
\hline I-D9.1 & MOX fuel fabrication location decision \\
\hline I-D9.2 & If GHK is funded for immobilization activities? \\
\hline I-A10.1 & Decommission of Pu immobilization facility at "Mayak" \\
\hline I-A10.1a & Decommission of Pu immobilization facility at GHK \\
\hline
\end{tabular}




\title{
Features of Micro- and Macrostructural Characteristics of Ferrosilicate Fusion Cakes ${ }^{1}$
}

\author{
V. N. Muzgin, A. A. Pupyshev, Ural State Technical University; S. I. Rovny, V. I. \\ Guzhavin, FSUE PA "Mayak"
}

A possible variant of radioactive waste $(\mathrm{RW})$ processing is immobilization into a mineral-like matrix, which allows us to obtain a final product of high mechanical strength and chemical stability. When conducting a process of RW immobilization, it is necessary to provide for minimum radionuclide losses on the stage of high-temperature fusion, as well as sufficiently uniform distribution within the structure of the monolithic product to be obtained. This has been required in the study of microstructural and structural characteristics of compact and powder patterns of various fusion cakes. The original chemical composition of ferrosilicate fusion cakes is represented in Table 1.

Table 1. Original chemical composition of ferro-silicate fusion cakes.

\begin{tabular}{|l|ll|l|ll|}
\hline \multicolumn{1}{|c|}{ Oxide } & \multicolumn{2}{|c|}{$\begin{array}{c}\text { Content, } \\
\text { \% mas. } \mathbf{M}_{\mathbf{M e O}} / \mathbf{M}_{\mathbf{S i O} 2}\end{array}$} & \multicolumn{1}{c|}{ Oxide } & \multicolumn{2}{c|}{$\begin{array}{c}\text { Content, } \\
\text { \% mas. } \mathbf{M}_{\text {Meo }} / \mathbf{M}_{\mathbf{S i O} 2}\end{array}$} \\
\hline $\mathrm{Al}_{2} \mathrm{O}_{3}$ & 7,6 & 0,5 & $\mathrm{Na}_{2} \mathrm{O}$ & 0,1 & 0,011 \\
\hline $\mathrm{BaO}$ & 6,5 & 0,14 & $\mathrm{Nd}_{2} \mathrm{O}_{3}$ & 0,5 & 0,01 \\
\hline $\mathrm{CaO}$ & 11,3 & 0,68 & $\mathrm{NiO}$ & 1,3 & 0,06 \\
\hline $\mathrm{CeO}_{2}$ & 1,0 & 0,02 & $\mathrm{PbO}_{2}$ & 0,3 & 0,0043 \\
\hline $\mathrm{Cr}_{2} \mathrm{O}_{3}$ & 3,0 & 0,13 & $\mathrm{SiO}_{2}$ & 17,7 & 1 \\
\hline $\mathrm{Cs}_{2} \mathrm{O}$ & 1,0 & 0,024 & $\mathrm{SrO}_{2}$ & 1,0 & 0,033 \\
\hline $\mathrm{Fe}_{2} \mathrm{O}_{3}$ & 27,5 & 1,17 & $\mathrm{TiO}_{2}$ & 0,3 & 0,013 \\
\hline $\mathrm{La}_{2} \mathrm{O}_{3}$ & 0,5 & 0,01 & $\mathrm{ZrO}_{2}$ & 0,5 & 0,014 \\
\hline $\mathrm{MgO}$ & 18,1 & 1,52 & $\mathrm{Y}_{2} \mathrm{O}_{3}$ & 0,5 & 0,015 \\
\hline $\mathrm{MnO}_{2}$ & 1,3 & 0,1 & $\mathrm{~K}_{2} \mathrm{O}$ & 0,1 & 0,0012 \\
\hline
\end{tabular}

The phase composition patterns were determined on powders of 53-60 $\mu \mathrm{m}$ coarseness by the method of $\mathrm{x}$-ray polycrystal diffractometry. Measurements were carried out with the use of the automatic powder $\mathrm{x}$ ray diffractometer, STADI-P (by "STOE" Company, Germany), provided with Bragg-Brentano geometry. Phase identification was carried out by accounting for the original phase composition of patterns with the help of powder x-ray data-base PDF-2 (ICDD, USA). Quasi-quantitative identification of phase composition was based on a standard material, corundum.

All the patterns of fusion cakes are practically one-phase ones ( 100\%). Their structural composition corresponds with cubic phases of a type of $\mathrm{MgAl}_{0,6} \mathrm{Fe}_{1,4} \mathrm{O}_{4}, \mathrm{MgAl}_{0.8} \mathrm{Fe}_{1.2} \mathrm{O}_{4}, \mathrm{CaMgSiO}_{4}, \mathrm{Fe}_{3} \mathrm{O}_{4}$ or similar. Therefore, fusion cakes consist of solid solution of oxides of $\mathrm{Me}_{3} \mathrm{O}_{4}$ aggregate composition. No splitting of diffraction maximums, which can indicate the presence of one structural type-solution with various lattice parameters, was detected. No specific showings of amorphous phase substance were detected, according to the $\mathrm{x}$-ray structural analysis data.

\footnotetext{
${ }^{1}$ Figures not provided.
} 
The results of measuring various samples in each of the fusion cakes are similar to one another. Investigations of microstructure were realized on compact samples. The samples were consequently ground and polished by diamond pastes of various coarseness. Etching was not required, as the microstructure is well enough observed on sections not subjected to etching.

Determination of quantitative microphase composition and porosity was made by a petrographic procedure on metallographic microscope NEOPHOT-32 (Germany) provided with a SIAMS-600 commercial system of image adaptation. The section images were transferred from microscope to computer by the means of projecting scanner SCANNEX.

The procedure applied for digital image analysis consisted of the following steps:

- The most characteristic areas were selected in the image of the section;

- Then the image was binarized, i.e., sortied by color map into the selected phase to be analyzed and the last, or sorting into pores and the last. In Fig. 2, the left figure represents the visual image margin; the middle figure shows image binarization with development of the second phase; and the right figure shows image binarization and developing porosity.

- For each of the areas, we measured integral parameters and size distributions);

- Similar characteristics were obtained when averaged over all selected areas of a sample.

On all the samples under reflected light, the background basic phase is a dark brown color, and the second phase development was registered as a light gray color in the form of mash circuits or dendrites.

Patterns of various fusion cakes were studied in detail using the described procedure of quantitative microstructural measurements. The results obtained for various fusion cakes are close to each other.

Scanning electron spectroscopy and x-ray electron microprobe spectral analysis were used to identify microphases existing in a sample, as well as to determine their element composition, including investigation of 20 elements inside the microphases.

Investigations were conducted on the electron scanning microscope-microprobe analyzer "CAMEBAX" by the French Company "Cameca". The device is equipped with crystal diffraction spectrometers provided with various crystals-analyzers. This gives the opportunity to specify the elements from boron up to the end of the Periodic Table of elements on the basis of various series x-ray spectra. Definition under the mode of secondary electrons reaches about $10 \mathrm{~nm}$, and in the mode of characteristic x-ray radiation, up to $1 \mu \mathrm{m}$.

Compact samples to be studied were ground, then washed with acetone, and polished with diamond pastes. Samples were washed in alcohol when changing a paste. In order to provide a sufficient level of electrical conductivity, as well as to prevent local heating of a sample in the point of analyzing, the samples to be studied were covered with a sputtered copper layer to a thickness of about $5,0 \mathrm{~nm}$.

Initially, visual examination of a sample was used to select the most characteristic place for analysis, the so-called field of the sample, with a size of $800 \times 1000 \mu \mathrm{m}^{2}$, and which was representative of the microphase structure of the given sample. This field is large enough to assess the dimensions and forms of phases in the samples to be studied. A sample section of $200 \times 150 \mu \mathrm{m}^{2}$ is marked out on this field with a rectangular contour for further detailed quasi-quantitative and quantitative measurements. The rectangle outlines the basic microphases to be observed in the sample. The further study of quantitative chemical composition of microphases and element distribution inside them is realized in the given 
rectangle. The white straight line running across this rectangle indicates a run of the electron tester, when scanning the given section.

Quantitative element analysis included measurements of the intensity of x-ray characteristic radiation of elements under determination in selected points of patterns to be studied, and on comparison samples, as well as static adaptation of measurement results, registration of a background and dead time of detector, computation of element mass concentrations by special programs taking into account possible matrix effects. The energy of tester's electrons was $25 \mathrm{KeV}$, and current of tester was $0,4 \cdot 10^{-7} \mathrm{~A}$.

Oxides or minerals from the complete set of comparison samples provided by the manufacturer of the device were used in experiments as comparison samples (Table 2).

Табл. 2. Образцы сравнения, использованные в анализе

\begin{tabular}{|c|c|}
\hline $\mathrm{Me}$ & Образец сравнения \\
\hline $\mathrm{Al}$ & $\mathrm{Al}_{2} \mathrm{O}_{3}$, альбит $\mathrm{Na}\left(\mathrm{AlSi}_{3} \mathrm{O}_{8}\right)$ \\
\hline $\mathrm{Ba}$ & Варитин $\mathrm{BaSO}_{4}$ \\
\hline $\mathrm{Ca}$ & $\begin{array}{l}\text { Родонит } \mathrm{CaMn}_{4}\left(\mathrm{Si}_{5} \mathrm{O}_{15}\right) \text {, андрадит } \\
\mathrm{Ca}_{3} \mathrm{Fe}_{2}\left(\mathrm{SiO}_{4}\right)_{3} \text {, волластонит } \mathrm{Ca}_{3} \mathrm{Si}_{3} \mathrm{O}_{9}\end{array}$ \\
\hline $\mathrm{Ce}$ & $\mathrm{CeAl}_{4}$ \\
\hline Cs & $\mathrm{CsF}$ \\
\hline $\mathrm{Cr}$ & $\mathrm{Cr}_{2} \mathrm{O}_{3}$ \\
\hline $\mathrm{Fe}$ & $\mathrm{Fe}_{2} \mathrm{O}_{3}$, андрадит $\mathrm{Ca}_{3} \mathrm{Fe}_{2}\left(\mathrm{SiO}_{4}\right)_{3}$ \\
\hline $\mathrm{K}$ & $\mathrm{K}\left(\mathrm{AISi}_{3} \mathrm{O}_{8}\right)$ \\
\hline $\mathrm{La}$ & $\mathrm{LaF}_{3}$, манганит $\mathrm{La}_{0,6} \mathrm{Sr}_{0,4} \mathrm{MnO}_{3}$ \\
\hline $\mathrm{Mg}$ & Форстерит $\mathrm{Mg}_{2} \mathrm{SiO}_{4}$ \\
\hline $\mathrm{Mn}$ & Родонит $\mathrm{CaMn}_{4}\left(\mathrm{Si}_{5} \mathrm{O}_{15}\right)$ \\
\hline $\mathrm{Na}$ & Альбит $\mathrm{Na}\left(\mathrm{AlSi}_{3} \mathrm{O}_{8}\right)$ \\
\hline $\mathrm{Nd}$ & $\mathrm{Nd}-\mathrm{Fe}-\mathrm{B}$ \\
\hline $\mathrm{Ni}$ & Ni металлический \\
\hline $\mathrm{Pb}$ & $\mathrm{PbS}$ \\
\hline $\mathrm{Si}$ & Форстерит $\mathrm{Mg}_{2} \mathrm{SiO}_{4}$, родонит $\mathrm{CaMn}_{4}\left(\mathrm{Si}_{5} \mathrm{O}_{15}\right)$, волластонит $\mathrm{Ca}_{3} \mathrm{Si}_{3} \mathrm{O}_{9}$ \\
\hline $\mathrm{Sr}$ & Манганит $\mathrm{La}_{0,6} \mathrm{Sr}_{0,4} \mathrm{MnO}_{3}$ \\
\hline $\mathrm{Ti}$ & $\mathrm{TiO}_{2}$ \\
\hline $\mathrm{Zr}, \mathrm{Y}$ & $\mathrm{Y}_{2} \mathrm{O}_{3}-\mathrm{ZrO}_{2}$ \\
\hline $\mathrm{Y}$ & $\mathrm{Y}_{2} \mathrm{O}_{3}-\mathrm{ZrO}_{2}$ \\
\hline
\end{tabular}

The results of quantitative analysis of the given sample in selected points of observation are represented in Table 3. The line "Sum" indicates the total content of oxides, determined in the point of observation. 
Table 3. Results of quantitative analysis, \% mass.

\begin{tabular}{|c|c|c|c|c|c|c|c|}
\hline \multirow[t]{2}{*}{ Oxide } & \multicolumn{6}{|c|}{ Point of observation } & \multirow[t]{2}{*}{ Oxide } \\
\hline & 1 & 2 & 3 & 4 & 5 & 6 & \\
\hline $\mathrm{Al}_{2} \mathrm{O}_{3}$ & 12,30 & 13,36 & 7,38 & 12,68 & 6,81 & 11,47 & $\mathrm{Al}_{2} \mathrm{O}_{3}$ \\
\hline $\mathrm{BaO}$ & 0,00 & 0,00 & 11,59 & 0,00 & 11,66 & 0,00 & $\mathrm{BaO}$ \\
\hline $\mathrm{CaO}$ & 0,00 & 0,00 & 12,28 & 0,00 & 12,18 & 0,00 & $\mathrm{CaO}$ \\
\hline $\mathrm{CeO}_{2}$ & 0,00 & 0,00 & 2,59 & 0,00 & 2,72 & 0,00 & $\mathrm{CeO}_{2}$ \\
\hline $\mathrm{Cr}_{2} \mathrm{O}_{3}$ & 28,82 & 0,27 & 0,02 & 7,01 & 0.03 & 0,12 & $\mathrm{Cr}_{2} \mathrm{O}_{3}$ \\
\hline $\mathrm{Cs}_{2} \mathrm{O}$ & 0,00 & 0,00 & 1,90 & 0,00 & 1,92 & 0,00 & $\mathrm{Cs}_{2} \mathrm{O}$ \\
\hline $\mathrm{Fe}_{2} \mathrm{O}_{3}$ & 37,98 & 63,77 & 8,14 & 59,25 & 8,28 & 66,61 & $\mathrm{Fe}_{2} \mathrm{O}_{3}$ \\
\hline $\mathrm{K}_{2} \mathrm{O}$ & 0,00 & 0,00 & 0,57 & 0,00 & 0,56 & 0,00 & $\mathrm{~K}_{2} \mathrm{O}$ \\
\hline $\mathrm{La}_{2} \mathrm{O}_{3}$ & 0,00 & 0,00 & 0,71 & 0,00 & 0,77 & 0,00 & $\mathrm{La}_{2} \mathrm{O}_{3}$ \\
\hline $\mathrm{MgO}$ & 15,33 & 13,44 & 8,82 & 13,70 & 9,21 & 13,33 & $\mathrm{MgO}$ \\
\hline $\mathrm{MnO}_{2}$ & 1,89 & 3,26 & 1,80 & 2,51 & 1,85 & 2,84 & $\mathrm{MnO}_{2}$ \\
\hline $\mathrm{Na}_{2} \mathrm{O}$ & 0,00 & 0,00 & 4,49 & 0,00 & 4,28 & 0,04 & $\mathrm{Na}_{2} \mathrm{O}$ \\
\hline $\mathrm{Nd}_{2} \mathrm{O}_{3}$ & 0,00 & 0,00 & 1,86 & 0,00 & 1,81 & 0,00 & $\mathrm{Nd}_{2} \mathrm{O}_{3}$ \\
\hline $\mathrm{NiO}$ & 3,26 & 4,26 & 0,12 & 3,88 & 0,13 & 4,13 & $\mathrm{NiO}$ \\
\hline $\mathrm{PbO}_{2}$ & 0,00 & 0,00 & 1,08 & 0,00 & 1,10 & 0,00 & $\mathrm{PbO}_{2}$ \\
\hline $\mathrm{SiO}_{2}$ & 0,00 & 0,00 & 31,30 & 0,00 & 31,72 & 0,00 & $\mathrm{SiO}_{2}$ \\
\hline SrO & 0,00 & 0,00 & 2,20 & 0,00 & 2,20 & 0,00 & $\mathrm{SrO}$ \\
\hline $\mathrm{TiO}_{2}$ & 0,26 & 1,09 & 0,74 & 0,64 & 0,76 & 1,39 & $\mathrm{TiO}_{2}$ \\
\hline $\mathrm{ZrO}_{2}$ & 0,00 & 0,00 & 0,47 & 0,00 & 0,50 & 0,00 & $\mathrm{ZrO}_{2}$ \\
\hline $\mathrm{Y}_{2} \mathrm{O}_{3}$ & 0,00 & 0,00 & 1,10 & 0,00 & 1,10 & 0,00 & $\mathrm{Y}_{2} \mathrm{O}_{3}$ \\
\hline Sum & 99,86 & 99,49 & 99,17 & 99,70 & 99,59 & 99,95 & \\
\hline
\end{tabular}

$E_{e l}=25 \mathrm{KeV} \quad I_{\text {sound }}=0,4 \cdot 10^{-7} \mathrm{~A}$

Figures $7-9^{2}$ represent at the same time images of selected rectangular section of observation of elements (except oxygen) to be determined under secondary electrons and characteristic x-ray radiation. The type of image is indicated under each figure (sec. el. - secondary electrons; Me - element in which characteristic $\mathrm{x}$-ray radiation the given image was obtained).

These images show clearly the distribution of all elements over the sample. In addition, in electron images we discovered an irregularity in second phase composition (polygons), which could not be detected by observation in an optical microscope. This is caused by a difference in average atom number for various sections of the sample, which corresponds with variation of the chemical composition of microphase. Images obtained by characteristic $x$-ray radiation of elements really show the irregularity of their distribution in the second phase (in polygons).

Similar measurements were implemented for each observation point of selected fields in various patterns of fusion cakes.

The basic phase of various patterns of fusion cakes contains all the elements inserted into the fusion cake. Basic phase compositions in samples of fusion cakes are very similar to one another. The second phase, that is microphase (polygons), contains in all samples only $\mathrm{Fe}_{2} \mathrm{O}_{3}, \mathrm{Cr}_{2} \mathrm{O}_{3}, \mathrm{NiO}, \mathrm{MnO}_{2}, \mathrm{TiO}_{2}, \mathrm{MgO}$ and $\mathrm{Al}_{2} \mathrm{O}_{3}$. The quantitative composition of the second microphase in fusion cakes is practically equal. Chrome is usually concentrated in the central part of polygon inclusions. However, there are some

\footnotetext{
${ }^{2}$ Figures not provided.
} 
inclusions of this type that do not contain chrome. Total content of $\mathrm{Fe}_{2} \mathrm{O}_{3}$ and $\mathrm{Cr}_{2} \mathrm{O}_{3}$ in the given inclusions reaches $67 \%$, and the content of $\mathrm{MgO}$ and $\mathrm{Al}_{2} \mathrm{O}_{3}$ is $25-27 \%$.

Samples of some fusion cakes can be characterized by the the presence of special zones in the basic phase, in which there can be a generated microphase with a high content of $\mathrm{MgO}, \mathrm{CaO}$, and $\mathrm{SiO}_{2}$. This microphase takes the form of dendrites. Dimensions of such zones can reach $400 \mu \mathrm{m}$, although the zones of so sizeable dimensions are to be observed very rarely. Mostly, the dimension of these zones does not exceed 50-100 $\mu \mathrm{m}$. The given microphase has a low concentration of rare-earth elements. And vice-versa, the sections of the basic phase between dendrites of this microphase have an essentially heightened concentration of rare-earth elements. Besides that, the diffusion zone is to be observed in the border between the basic phase and this microphase.

The study of thermal behavior of powdery patterns with particle size dimension of 53-60 $\mu \mathrm{m}$ is conducted by the method of thermal analysis. Measurements are performed with the help of an automatic diversion system by F. Paulik, J. Paulik, L. Erdey of MOM Company (Hungary) under conditions of linear controlled temperature increas from room temperature up to $1500^{\circ} \mathrm{C}$ at a heating rate of 15 degrees $/ \mathrm{min}$. in air.

Studying the data from thermal analysis allows us an opportunity to draw the following conclusion. There are to be observed low thermal effects usual for inactive powdery patterns, associated with sorptiondesorption of water and air components. In addition, within the temperature range of $950-1000^{\circ} \mathrm{C}$, no large endoeffect (heat sorption) is registered in DTA curve, which is to be observed for all samples, when their chemical composition is stable. Under cooling of samples in this temperature range, the possible reverse exoeffect (heat release ) does not appear. To all appearances, the endoeffect is of irreversible character, and is related to survival capability of the second microphase.

It seams to be important to complete the study of fusion cakes with methods of high-temperature combinational scattering of light, and x-ray spectroscopy of oxygen in microphases. It is also necessary to conduct a similar investigation on samples subjected to chemical effects (leaching) and also to long-term high-temperature treatment (accelerated aging).

\section{Investigation methods}

1. Optical spectroscopy -NEOPHOT-32 provided with SIAMS-600 system of image adaptation

2. X-ray crystal diffractometry - diffractometer STADI-P of STOE Company (cubic, a type of $\mathrm{MgAl}_{0,6} \mathrm{Fe}_{1,4} \mathrm{O}_{4}, \mathrm{CaMgSiO}_{4}, \mathrm{MgAl}_{0,8} \mathrm{Fe}_{1,2} \mathrm{O}_{4}$ or $\mathrm{Fe}_{3} \mathrm{O}_{4}$, etc.)

3. Electron scanning spectroscopy and X-ray electron microprobing spectrum analysis - microprobing analyzer - CAMEBAX by CAMECA Company

4. Thermal analysis - automatic diversion system by MOM Company 


\title{
Comparative Radiation Resistance of Cubic Fluorite Structure Phases for Actinides Immobilization
}

\author{
S. V. Stefanovsky, O. I. Kirjanova, RADON; S. V. Yudintsev, IGEM RAS; J. Lian, \\ R.C. Ewing, University of Michigan
}

\begin{abstract}
This paper summarizes recent studies on radiation resistance of cubic fluorite-structure phases under irradiation with $1 \mathrm{MeV} \mathrm{Kr}^{+}$ions at room temperature. The phases studied were complex pyrochlore, murataites (three varieties with three-, five- and eight-fold fluorite lattice) as well as brannerite. The critical amorphization doses $\left(D_{c}\right)$ obtained were compared to reference data on fluorite structure zirconia and pyrochlores. The phases were found to be increasingly susceptible to radiation damage in the following order: fluorite $<$ pyrochlore (two-fold fluorite unit cell $=2 \mathrm{C}$ ) $<$ murataite-3C $<$ murataite- $5 \mathrm{C}<$ murataite-8C. For the murataite $3 \mathrm{C}, 5 \mathrm{C}$, and $8 \mathrm{C}$ varieties, the $D_{\mathrm{c}}$ values were found to be $\left(\times 10^{18}\right.$ ions $\left./ \mathrm{m}^{2}\right): 1.85,1.75$, and 1.68 , respectively.

\section{Introduction}

Disposal of the actinide-bearing wastes from nuclear applications as well as residues from conversion of excess weapons plutonium into mixed oxide (MOX) fuel is a priority ecological task. One of the ways of solving this problem is waste immobilization into matrices capable of strong retention of actinides for a long time. The most promising phases for this purpose are titanates with fluorite-type lattice like zirconolite, pyrochlore, and murataite $[1,2]$. These phases are capable of accumulating actinides in the amount of 30-50 wt\% while keeping high chemical durability (leach rates of uranium and plutonium from these phases are $\left.10^{-4}-10^{-6} \mathrm{~g} \cdot \mathrm{m}^{-2} \cdot \mathrm{day}^{-1}\right)[1,2]$.

The above-mentioned values are correct for the phases with non-damaged crystalline structure. Amorphization of their lattices under radioactive decay leads to reduction in fixation capability in the matrix, and the leachability of actinides in water solution increases by orders of magnitude [3]. Leaching of daughter actinide isotopes located in the most strongly damaged areas of the crystalline lattice grows to the greatest extent. Some of them $\left({ }^{241} \mathrm{Am},{ }^{237} \mathrm{~Np}\right)$ are more dangerous as compared to the mother isotopes. This is the reason why a study of the effect of radiation on properties of the phases and their capability to retain radionuclides has great importance in the evaluation of long-term stability of actinide host phases. Numerous data have been collected for zirconolite and pyrochlore, but data are unavailable for murataite.
\end{abstract}

The main goal of our work was to study radiation resistance of murataite and to compare it with data for other actinide-bearing phases.

\section{Synthesis and Brief Characterization of the Samples}

Ceramics were produced by inductive melting in the cold crucible, IMCC, (Samples IMCC, Z-24, F-3) and in a resistive furnace (Samples $M-1 / 7$ and $M-3 / 7$ ). They were examined with $x$-ray diffraction, scanning (SEM) and transmission electron microscopy (TEM). All the samples are composed of one or few phases 
with fluorite-type structure. Preparation conditions, experimental and analytical equipment as well as data on texture and composition of the samples have been described in detail in our previous works [4-7].

Sample IMCC was composed of murataite and minor rutile, crichtonite, and glass. Murataite occurs in three varieties with three- (murataite- $3 \mathrm{C}$ ), five- (murataite-5C), and eight-fold (murataite-8C) repeatability of the elementary fluorite motif. The murataite- $5 \mathrm{C}$ variety is predominant. The characteristic of this phase is zoned distribution of elements within its grains. Uranium and zirconium contents decrease and iron and aluminum contents increase from the core towards the rim. Core and rim compositions correspond to the formulae: $\left(\mathrm{Ca}_{2.15} \mathrm{U}_{0.51} \mathrm{Zr}_{0.61} \mathrm{Mn}_{1.33} \mathrm{Ti}_{6.95} \mathrm{Fe}_{0.66} \mathrm{Al}_{0.80}\right) \mathrm{O}_{21.80}$ and $\left(\mathrm{Ca}_{1.64} \mathrm{U}_{0.21} \mathrm{Zr}_{0.19} \mathrm{Mn}_{1.55} \mathrm{Ti}_{6.49} \mathrm{Fe}_{1.09} \mathrm{Al}_{1.83}\right) \mathrm{O}_{21.35}$, respectively. Rutile $\left(\mathrm{Ti}_{0.95} \mathrm{Zr}_{0.03} \mathrm{Ca}_{0.01} \mathrm{Al}_{0.01} \mathrm{Fe}_{0.01}\right) \mathrm{O}_{2.00}$ and crichtonite $\mathrm{Ca}_{0.1 .45} \mathrm{U}_{0.15} \mathrm{Zr}_{0.13} \mathrm{Mn}_{2.30} \mathrm{Ti}_{13.95}$ $\mathrm{Fe}_{1.85} \mathrm{Al}_{2.02} \mathrm{O}_{38.00}$ compositions are depleted with uranium and no effect on uranium partitioning in the sample has been observed. Glass was formed due to dissolution of the cold crucible protective putty during the melting run. Uranium and zirconium concentrations in glass are lower than the detection limit.

Sample $\mathrm{M}-1 / 7$ has a formulation close to previous sample composition. This is composed of murataite$5 \mathrm{C}$, murataite-8C as well as minor rutile. Compositions of core and rim of murataite- $5 \mathrm{C}$ in this sample are described by formulae: $\mathrm{Ca}_{2.70} \mathrm{U}_{0.51} \mathrm{Zr}_{0.90} \mathrm{Mn}_{1.16} \mathrm{Ti}_{6.69} \mathrm{Fe}_{0.51} \mathrm{Al}_{0.51} \mathrm{O}_{21.59}$ и $\mathrm{Ca}_{2.25} \mathrm{U}_{0.39} \mathrm{Zr}_{0.58} \mathrm{Mn}_{1.36} \mathrm{Ti}_{6.82} \mathrm{Fe}_{0.64}$ $\mathrm{Al}_{0.96} \mathrm{O}_{21.59}$, respectively. Murataite- $8 \mathrm{C}$ and rutile have uniform elements distribution. Their formulae are $\left(\mathrm{Ca}_{2.02} \mathrm{U}_{0.26} \mathrm{Zr}_{0.26} \mathrm{Mn}_{1.62} \mathrm{Ti}_{6.70} \mathrm{Fe}_{0.84} \mathrm{Al}_{1.30}\right) \mathrm{O}_{21.29}$ и $\left(\mathrm{Ti}_{0.93} \mathrm{Zr}_{0.03} \mathrm{U}_{0.01} \mathrm{Mn}_{0.01} \mathrm{Al}_{0.01} \mathrm{Fe}_{0.01}\right) \mathrm{O}_{2.00}$, respectively. The murataite- $5 \mathrm{C}$ rim is close in chemical composition to the murataite- $8 \mathrm{C}$ variety. Volume contents of the phases in Sample M-1/7 were found to be $\sim 70 \%$ murataite-5C, $20 \%$ murataite- $8 \mathrm{C}$, and $10 \%$ rutile.

Composition of Sample M-3/7 differs from the previous two by the addition of $20 \mathrm{wt} \%$ cerium oxide. This sample consists of pyrochlore (60-70\%), murataite $(20-30 \%)$, and crichtonite $(\sim 10 \%)$. Their formulae are $\mathrm{Ca}_{0.64} \mathrm{Mn}_{0.28} \mathrm{Ce}_{0.68} \mathrm{Zr}_{0.16} \mathrm{U}_{0.16} \mathrm{Ti}_{1.96} \mathrm{Fe}_{0.04} \mathrm{Al}_{0.08} \mathrm{O}_{7.0}, \mathrm{Ca}_{1.56} \mathrm{Ce}_{0.78} \mathrm{Zr}_{0.13} \mathrm{U}_{0.13} \mathrm{Mn}_{1.56} \mathrm{Ti}_{6.37} \mathrm{Fe}_{0.91} \mathrm{Al}_{1.56} \mathrm{O}_{21.65}$, and $\mathrm{Ca}_{0.79} \mathrm{Ce}_{0.81} \mathrm{Zr}_{0.36} \mathrm{Mn}_{2.00} \mathrm{Ti}_{12.16} \mathrm{Fe}_{1.95} \mathrm{Al}_{3.92} \mathrm{O}_{38.00}$, respectively. The major uranium-bearing phase is pyrochlore. Murataite occurs as the $8 \mathrm{C}$ variety.

Elemental ratio in Sample Z-24 corresponds to Gd-containing zirconolite. Silica ( 6\%) is present due to crucible putty contamination. In addition to zirconolite, minor zirconia, rutile, and glass were also found. Fine zirconia grains are overgrown by zirconolite crystals. Glass fills interstitials between zirconolite grains and contain rare rutile crystals. Zirconolite composition was found to be $\mathrm{Ca}_{0.4} \mathrm{Gd}_{0.8} \mathrm{ZrTi}_{1.4} \mathrm{Al}_{0.4} \mathrm{O}_{7.0}$.

Sample F-3 consists of pyrochlore ( 60\%), brannerite ( 30\%), and rutile $(\sim 10 \%)$. The pyrochlore and brannerite formulae are $\mathrm{Ca}_{1.25} \mathrm{U}_{0.75} \mathrm{Zr}_{0.12} \mathrm{Ti}_{1.86} \mathrm{Al}_{0.02} \mathrm{O}_{6.67}$ and $\mathrm{Ca}_{0.25} \mathrm{U}_{0.73} \mathrm{Zr}_{0.08} \mathrm{Ti}_{2.02} \mathrm{Al}_{0.05} \mathrm{O}_{6.00}$, respectively.

\section{Radiation Resistance of the Phases}

The $1 \mathrm{MeV} \mathrm{Kr}^{+}$ion irradiation was performed at room temperature using an intermediate voltage electron microscope (IVEM) that is interfaced with a Tandem ion accelerator at Argonne National Laboratory.

Radiation damage was observed by in-situ TEM. Individual grains of each phase were monitored during the irradiation, and the $D_{c}$ values were obtained by averaging the ion doses required for amorphization based on observing the selected area electron diffraction (SAED) patterns of these individual monitored grains. Subsequent high resolution transmission electron microscopy (HRTEM) was used for more detailed studies of the samples that were retained from different dose levels.

The $D_{\mathrm{c}}$ values obtained are given in Table 1 . 
Table 1. The $D_{\mathrm{c}}$ values for the titanate phases.

\begin{tabular}{|c|c|c|c|c|c|c|c|c|c|}
\hline Sample & \multicolumn{2}{|c|}{ IMCC } & \multicolumn{2}{|c|}{$M-1 / 7$} & \multicolumn{2}{|c|}{ M-3/7 } & \multirow{2}{*}{$\begin{array}{c}\mathrm{Z}-24 \\
\mathrm{Z}\end{array}$} & \multicolumn{2}{|c|}{$F-3$} \\
\hline Phase & M-5C & $M-3 C$ & $M-8 C$ & $M-5 C$ & Py & $M-8 C$ & & $\mathbf{P}$ & B \\
\hline $\begin{array}{l}D_{\mathrm{c}}, \mathrm{x} 10^{18} \\
\mathrm{~m}^{-2}\end{array}$ & $\begin{array}{l}1.66 \pm \\
0.06\end{array}$ & $\begin{array}{l}1.85 \pm \\
0.06\end{array}$ & $\begin{array}{l}1.82 \pm \\
0.06\end{array}$ & $\begin{array}{l}1.85 \pm \\
0.06\end{array}$ & $\begin{array}{l}1.75 \pm \\
0.13\end{array}$ & $\begin{array}{l}1.69 \pm \\
0.13\end{array}$ & $\begin{array}{l}2.94 \pm \\
0.06\end{array}$ & $\begin{array}{l}2.36 \pm \\
0.13\end{array}$ & $\begin{array}{l}1.38 \pm \\
0.06\end{array}$ \\
\hline
\end{tabular}

$\mathrm{B}$ - brannerite, $\mathrm{M}$ - murataite, $\mathrm{Py}$ - pyrochlore, $\mathrm{Z}$ - zirconolite

Within the experimental error all three murataite structure varieties have close $D_{\mathrm{c}}$ values. In Sample M-1/7 murataite- $5 \mathrm{C}$ is somewhat more radiation resistant than murataite- $8 \mathrm{C}$. Murataite- $3 \mathrm{C}$ occurring in Sample IMCC is rendered amorphous at slightly higher ion dose than murataite-5C. Resistance of the murataite varieties to ion-irradiation increases in the raw: murataite- $8 \mathrm{C}<$ murataite- $5 \mathrm{C}<$ murataite- $3 \mathrm{C}$, i.e., towards decrease in repeatability of elementary fluorite cell. This is confirmed by experimental data on radiation stability of the pyrochlore structure phases (two-fold fluorite unit cell). As shown in Table 1 in Sample M$3 / 7$, the pyrochlore structure phase is more resistant to ion-irradiation as compared to murataite structure phase with similar chemical composition. Note the significant cerium effect on radiation stability of the pyrochlore and murataite phases (reduction in $D_{\mathrm{c}}$ ).

As shown in Table 1, the Gd-containing zirconolite has the highest radiation resistance among the titanate phases studied in the present work $\left(D_{\mathrm{c}}=(2.94 \pm 0.06) \times 10^{18} \mathrm{ion} / \mathrm{m}^{2}\right)$, which is in good agreement with reference data $[3,8,9]$ obtained under the same conditions. This result demonstrates that change in molecular weight of zirconolite and high neutron absorbers content (occurrence of Gd) doesn't effect the high radiation stability of the zirconolite structure phase. Transformation of zirconolite structure to fluorite structure was observed during ion-irradiation. Similarly, murataite- $8 \mathrm{C} \rightarrow$ murataite- $5 \mathrm{C} \rightarrow$ fluorite $\rightarrow$ amorphous structure transformation occurred during ion-irradiation of murataite (Fig. 1).
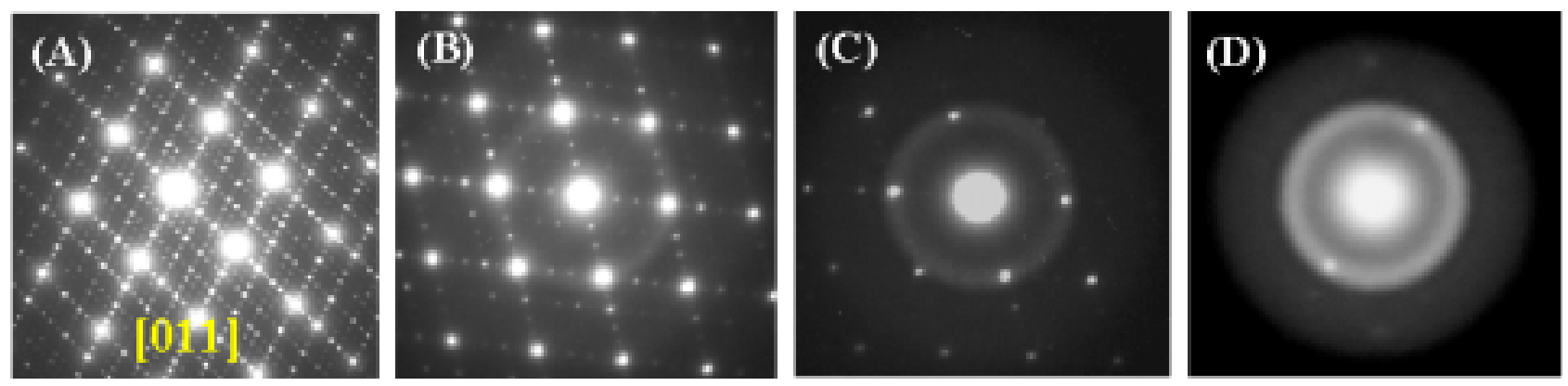

Fig. 1. Diffraction patterns of murataite-8C in $M-1 / 7$ sample before $(A)$ and after irradiation with $1 \mathrm{MeV} \mathrm{Kr}{ }^{+}$at room temperature to doses: (B) $4,38 \cdot 10^{17}$ ions $/ \mathrm{m}^{2}$ (0.04 dpa); (C) $9,39 \cdot 10^{17}$ ions $/ \mathrm{m}^{2}$ $(0.10 \mathrm{dpa})$; (D) $1,56 \cdot 10^{18}$ ions $/ \mathrm{m}^{2}(0.16 \mathrm{dpa})$. Ion irradiation induced order-disordering transformation: murataite-8C (A) $\rightarrow$ murataite-5C (B) $\rightarrow$ fluorite (C) $\rightarrow$ amorphous (D) occurred during amorphization.

In Sample F-3, the uranium-bearing pyrochlore is approximately two times more resistant to ion-irradiation than the brannerite structure phase. The $D_{\mathrm{c}}$ values for the given phases with ideal stoichiometry and with deviations from it are similar. The difference in radiation resistance of the pyrochlore and brannerite phases is due to features of their structures. Three-network structure composed by $\mathrm{TiO}_{6}$ octahedra 
sharing a corner in pyrochlore is more closely connected than the brannerite structure, where the zigzag sheets formed by $\mathrm{TiO}_{6}$ octahedra by sharing common edge connected by the cation of $\mathrm{U}$ or $\mathrm{Ca}$ located at the eight-coordinated inter layer sites. Therefore, the brannerite structure is more easily amorphized than the pyrochlore structure. Moreover, brannerite has heavier mass density than pyrochlore and also a larger nuclear collision cross-section, which leads to larger damage volume resulting from the highly energetic incident ions. This also accounts for the difference in radiation stability between pyrochlore and brannerite structure phases. Ion-induced order-disorder transformation was observed for phases with both pyrochlore and brannerite structures.

In the whole, the $D_{\mathrm{c}}$ value for the murataite structure is lower than for zirconolite, but higher than for brannerite. The latter has the lowest radiation resistance among all the crystalline phases studied. Pyrochlores in Samples M-3/7 and F-3 differ in the $D_{\mathrm{c}}$ value which is probably due to their different chemical compositions.

\section{Comparison of Radiation Stability of Murataite and Other Actinide-bearing Phases}

To date, radiation resistance has been determined for a number of potential actinide host phases by different methods $[3,8]$. For pyrochlores, structurally and chemically similar to murataite type phases, structure damage equivalent to 0.1 displacements per atoms (dpa) is reached under $1 \mathrm{MeV} \mathrm{Kr}^{+}$ion irradiation to ion dose of $\sim 10^{18}$ ions $/ \mathrm{m}^{2}$. Amorphization of the phases studied is supposed to take place at $\sim 0.3 \mathrm{dpa}$ for zirconolite, $\sim 0.2 \mathrm{dpa}$ for pyrochlore and murataite, and $\sim 0.1 \mathrm{dpa}$ for brannerite. A calculation of the number of equivalent alpha-decay events from experimentally obtained dpa values for murataite gave $3 \times 10^{21}$ alpha-events $/ \mathrm{kg}$. This dose corresponds to the time required to amorphize murataite: 600 700 y for $10 \mathrm{wt} \%{ }^{239} \mathrm{Pu}$ and $6,000-7,000$ y for $1 \mathrm{wt} \%{ }^{239} \mathrm{Pu}$ content, respectively. These values are close to data previously obtained for pyrochlore-structured gadolinium titanate [3,8]. If waste will contain actinides with lower half-lives $\left({ }^{240,241} \mathrm{Pu},{ }^{241} \mathrm{Am}\right)$, the amorphization process will require a shorter time. It can be also suggested that due to the zoned structure of the murataite grains, disordering of their structure in the core will happen sooner than in their rim.

The $D_{\mathrm{c}}$ values for murataite phases are closer to values for the most of other potential actinide hosts. In particular, these values range between 0.3 and $0.5 \mathrm{dpa}$ for zirconolite $\mathrm{CaZrTi}_{2} \mathrm{O}_{7}$ and 0.2-0.3 dpa for pyrochlore [3,8,9]. The values of 0.3-0.4 dpa were obtained for zircon $\mathrm{ZrSiO}_{4}$ and britholite $\mathrm{Ca}_{2} \mathrm{La}_{8}\left(\mathrm{SiO}_{4}\right)_{6} \mathrm{O}_{2}$ [8]. Perovskite $\mathrm{CaTiO}_{3}$ has 2-5 times higher radiation resistance as compared to zirconolite [10]. Maximum radiation stability is demonstrated among all the actinide host phases known have cubic zirconia $\mathrm{ZrO}_{2}$ [3] and $\mathrm{Gd}_{2} \mathrm{Zr}_{2} \mathrm{O}_{7}$ pyrochlore [11] as well as monazite $\mathrm{CePO}_{4}[3,8]$. Even irradiation to a dose equivalent to tens of dpa doesn't result in disordering of their lattice. The reason is the high annealing rate of radiation defects in the lattice of these phases [11] even at relatively low temperatures.

On the whole, the $D_{\mathrm{c}}$ value reduces with increase of repeatability of fluorite unit cell in the order, "fluorite" $\left(\mathrm{ZrO}_{2}{ }^{\text {cubic }}\right)>$ titanate "pyrochlore" $>$ murataite- $3 \mathrm{C}>$ murataite-5C $>$ murataite-8C (Table 2$)$ and for each phase - with complication of its chemical composition. This is in agreement with general knowledge on radiation stability of non-metal crystals [3] and connected to the different rates of radiation annealing. 
Table 2. The $D_{c}$ values for fluorite structure phases after $1 \mathrm{MeV} \mathrm{Kr}^{+}$ion irradiation at room temperature.

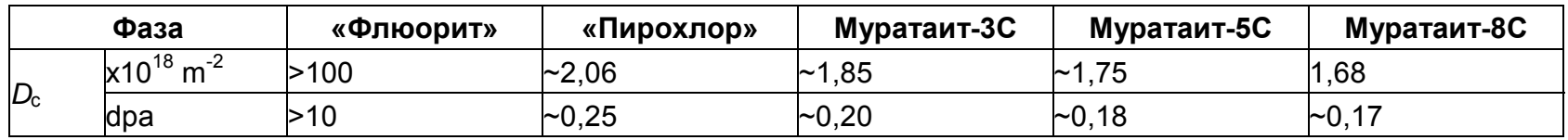

\section{Conclusions}

Critical amorphization doses for titanates at room temperature after irradiation with $1 \mathrm{MeV} \mathrm{Kr}^{+}$ions were found to be $\left(\times 10^{18}\right.$ ion $\left./ \mathrm{m}^{2}\right)$ : $~ 3$ for zirconolite, 1.8-2.4 for pyrochlore, 1.7-1.9 for murataite, and $\sim 1.4$ for brannerite. Murataite varieties with three-, five-, and 8-fold fluorite unit cells have similar radiation resistance. Taking into account these data, time required to fully amorphize murataite is estimated to be $600-700$ y at $10 \mathrm{wt} \%{ }^{239} \mathrm{Pu}$ content and $6,000-7,000$ y at $1 \mathrm{wt} \%{ }^{239} \mathrm{Pu}$ content. These values are close to data for pyrochlore structure titanates proposed for excess weapons plutonium immobilization.

The work was performed under financial support of DOE-CRDF (Project RC0-20002-SC14).

\section{References}

1. A. Jostsons, L. Vance, B. Ebbinghaus, Proc. Int. Conf. On Future Nuclear Systems, Global '99, Jackson Hole, 1999, CD-Rom.

2. S.V. Stefanovsky, S.V. Yudintsev, B.S. Nikonov, et al., Mat. Res. Soc. Symp. Proc. 556 (1999) 121128.

3. R.C. Ewing, W.J. Weber, F.W. Clinard, Progr. Nucl. Energy. 29 [2] (1995) 63-127.

4. S.V. Stefanovsky, O.I. Kirjanova, S.V. Yudintsev, O.A. Knyazev, Waste Management '01. Proc. Int. Symp. Tucson, 2001, CD Rom.

5. O.I. Kirjanova, S.V. Stefanovsky, S.V. Chizhevskaya, Phys. Chem. Mater. Treat. (Russ.) [2] (2000) 18-25.

6. S.V. Stefanovsky, O.I. Kirjanova, S.V. Yudintsev, et. al., Phys. Chem. Mater. Treat. (Russ.) [3] (2000) $72-80$.

7. O.I. Kirjanova, A.V. Ochkin, S.V. Stefanovky, N.E. Cherniavskaya, Adv. Mater.(Russ.) [4] (2001) 1723.

8. R.C. Ewing, W.J. Weber, W. Lutze, Disposal of Weapon Plutonium. E.R. Merz and C.E. Walter (eds). Kluwer Academic Publishers, 1996. P. 65-83.

9. K.L. Smith, N.J. Zaluzec, G.R. Lumpkin, Mat. Res. Soc. Symp. Proc. 506 (1998) 931-932.

10. T.J. White, R.C. Ewing, L.M. Wang, et al., Mat.Res. Soc. Symp. Proc. 353 (1995) 1413-1420.

11. S.X. Wang, B.D. Begg, L.M. Wang, et al., J. Mater. Res., 14 [12] (1999) 4470-4473. 


\title{
Chemical Restrictions to Formation of the Actinide Hosts with Pyrochlore Structure
}

\author{
S.V. Yudintsev, IGEM RAS, S.V. Stefanovsky, Radon, R.C. Ewing, University of \\ Michigan
}

\begin{abstract}
Development and implementation of methods for liquid high-level waste (HLW) partitioning allows the separation of radionuclides into different groups for their further most effective management [1]. It is suggested that the most promising methods of utilization of the dangerous actinides and long-lived fission products are their immobilization into durable crystalline forms for ultimate disposal and burning (transmutation) under neutron flux in a reactor [2]. One of the prospective host phases for both of these routes is pyrochlore-structure compound [3]. New phases have been produced in the systems: $\mathrm{CaO}-$ $\mathrm{CeO}_{2}-\mathrm{TiO}_{2}, \mathrm{CaO}-\mathrm{UO}_{2}\left(\mathrm{ThO}_{2}\right)-\mathrm{ZrO}_{2}, \mathrm{CaO}-\mathrm{UO}_{2}\left(\mathrm{ThO}_{2}\right)-\mathrm{Gd}_{2} \mathrm{O}_{3}-\mathrm{TiO}_{2}-\mathrm{ZrO}_{2}$, and $\mathrm{CaO}-\mathrm{ThO}_{2}-\mathrm{SnO}_{2}$. The precursors were cold pressed at $200-400 \mathrm{MPa}$ and sintered at $1500-1550{ }^{\circ} \mathrm{C}$ for $4-8$ hours in air or at $1300-1350{ }^{\circ} \mathrm{C}$ for $5-50$ hours in oxygen medium (Ce-doped batches only). The products were examined with $x$-ray diffraction (XRD), scanning electron microscopy (SEM/EDS), and transmission electron microscopy (TEM). In the ceramics with bulk compositions $\mathrm{CaCeTi}_{2} \mathrm{O}_{7}, \mathrm{CaThZr}_{2} \mathrm{O}_{7}$, $\left(\mathrm{Ca}_{0.5} \mathrm{GdTh}_{0.5}\right)(\mathrm{TiZr}) \mathrm{O}_{7},\left(\mathrm{Ca}_{0.5} \mathrm{GdU}_{0.5}\right)(\mathrm{TiZr}) \mathrm{O}_{7}$, and $\mathrm{CaThSn}_{2} \mathrm{O}_{7}$, pyrochlore was the major phase, but minor fluorite-type oxide was also present. In the samples with target compositions $\mathrm{CaUZr}_{2} \mathrm{O}_{7}$ and $\left(\mathrm{Ca}_{0.5} \mathrm{GdU}_{0.5}\right) \mathrm{Zr}_{2} \mathrm{O}_{7}$ pyrochlore was not identified, rather a fluorite-structured oxide appeared. Different occurrences of pyrochlore and defect fluorite in the ceramics produced are due to crystal chemical constraints. Three- and four-charged actinides can be incorporated into the eight-coordinated positions of the lattice. Among the other elements that can occupy the octahedral sites of the pyrochlore structure, we note $\mathrm{Zr}^{4+}, \mathrm{Sn}^{4+}$, and $\mathrm{Tc}^{4+}$ with dimensions $0.072,0.069$, and $0.065 \mathrm{~nm}$. Pyrochlore structure phases may also provide a stable host for the immobilization of three of five long-lived fission products: ${ }^{93} \mathrm{Zr}\left(\mathrm{T}_{1 / 2}=1.5\right.$ ${ }^{*} 10^{6}$ years $),{ }^{126} \mathrm{Sn}\left(1.0 * 10^{5}\right.$ years $)$, and ${ }^{99} \mathrm{Tc}\left(2.13 * 10^{5}\right.$ years $)$.
\end{abstract}

\section{Introduction}

A search for optimal HLW immobilization forms has been ongoing in the US, Australia, Russia, France, and UK for more than forty years [4-6]. Actinides are the most troublesome constituent of HLW due to their long half-lives $\left(2.44 \cdot 10^{4}, 2.14 \cdot 10^{6}\right.$ and $4.32 \cdot 10^{2}$ years for ${ }^{239} \mathrm{Pu},{ }^{237} \mathrm{~Np}$ and ${ }^{241} \mathrm{Am}$, respectively), radiation, and chemical toxicity. Actinide separation, as a specific waste stream of HLW for immobilization in a stable form, has been proposed as a way of insuring that these actinides are not released to the biosphere. In Russia this process is under implementation at PA "Mayak" [7]. Moreover, actinide-bearing residues are produced by weapons plutonium conversion and some solid waste incineration.

Promising host phases for plutonium are pyrochlore-structured compounds [8]. In the US, a waste form composed of a pyrochlore-type phase (80-90 vol\%), and 5-10\% each of rutile, and brannerite (ideal formula $\mathrm{UTi}_{2} \mathrm{O}_{6}$ ) has been designed [9]. The pyrochlore phase can accommodate both $\mathrm{Pu}$ and neutron absorbers $(\mathrm{Gd}, \mathrm{Hf})$. One difficulty with the titanate pyrochlore has been a concern for amorphization due to alpha-decay event damage. At typical waste loadings, this material may become amorphous in less than 800 years [10]. 
Numerous investigations were conducted throughout the world to optimize the formulation of Pu-bearing host phases. The gadolinium zirconate pyrochlore, $\mathrm{Gd}_{2} \mathrm{Zr}_{2} \mathrm{O}_{7}$, exhibits a remarkable radiation stability, that is it will not become amorphous for tens of millions of years, and is chemically durable [11]. In order to immobilize the partitioned waste with complex compositions, additional investigations have been required. Host phases for the long-lived fraction of HLW have to be high capable of incorporating both actinides (U, $\mathrm{Np}, \mathrm{Pu}, \mathrm{Am}, \mathrm{Cm}$ ) and zirconium and rare earth elements (REE). In this paper some new crystalline pyrochlore-structured actinide phases have been synthesized and characterized.

The pyrochlore lattice is isometric $(F d 3 m, Z=8)$ with cell parameter, "a", that is twice that of the fluoritestructure phases [12]. One-eight of anions are missing. As a result, the stoichiometry transforms from ${ }^{\mathrm{VIII}} \mathrm{A}_{4}{ }^{\mathrm{IV}} \mathrm{O}_{8}$ (four fluorite molecules) to ${ }^{\mathrm{VIII}} \mathrm{A}_{2}{ }^{\mathrm{VI}} \mathrm{B}_{2}{ }^{\mathrm{IV}} \mathrm{O}_{6}{ }^{\mathrm{IV}} \mathrm{X}$ and coordination numbers $(\mathrm{CN})$ of ions are indicated by the Roman numerals with the following coordination geometries: $\mathrm{AO}_{6} \mathrm{X}_{2}, \mathrm{BO}_{6}, \mathrm{OA}_{2} \mathrm{~B}_{2}$ and $\mathrm{XA}$, where " $A$ " and " $B$ " are cations in two different structural sites and " $X$ " is an additional anion. Oxygen anions are located at vertices of B-site octahedra. They also occupy six of the eight apices of a distorted cubic polyhedron with $\mathrm{CN}=8$. The other two apices of the "cube" are occupied by " $X$ " anions. Due to occurrence of two different cationic positions the pyrochlore structure is ordered, as compared to the disordered A- and B-site in the fluorite structure. To date more than 500 synthetic pyrochlore-structured compositions including actinide-bearing varieties have been synthesized [8,9,11,13-18]. Natural pyrochlores are typically tantalite-niobate varieties and they often contain high $\mathrm{U}, \mathrm{Ti}, \mathrm{Ca}$, and $\mathrm{REE}$ concentrations $[19,20]$.

The structure of pyrochlore depends in a sensitive way on the composition of the A- and B-site [13-18]. Phases with the ideal formula $\mathrm{A}^{3+}{ }_{2} \mathrm{~B}^{4+}{ }_{2} \mathrm{O}_{7}$ have pyrochlore structure if $\mathrm{R}_{\mathrm{A}}: \mathrm{R}_{\mathrm{B}}$ ratio ranges between 1.46 and 1.80 [17] (or by other data $-1.46 \ldots 1.78$ [18]). Here $R_{A}$ and $R_{B}$ are ionic radii in "A" and "B" sites, respectively. On this basis, for any octahedrally coordinated ion in combination with a specific A-site cation $(\mathrm{CN}=8)$ one may predict if the pyrochlore structure will form. Connection between ionic sizes in different sites affects the possible compositional variations of pyrochlores, particularly for pyrochlores that are possible actinide waste hosts. We have studied pyrochlore structure-type phases containing actinides, REEs, and zirconium. Specific attention was focused on compounds with perfect stoichiometry ${ }^{\mathrm{VIII}}\left(\mathrm{CaA}^{4+}\right)^{\mathrm{VI}} \mathrm{B}^{4+}{ }_{2} \mathrm{O}_{7}$, where $\mathrm{A}^{4+}=\mathrm{Ce}$, Th, $\mathrm{U}$; and $\mathrm{B}^{4+}=\mathrm{Ti}$ and/or $\mathrm{Zr}$. It should be noted, that compounds exist for which stability of either pyrochlore or fluorite structure depends on temperature. For example, the pyrochlore to fluorite transformation for $\mathrm{Gd}_{2} \mathrm{Zr}_{2} \mathrm{O}_{7}$ occurs at temperature $1550-1600{ }^{\circ} \mathrm{C}$ [21].

\section{Experimental}

Nine samples with pyrochlore stoichiometries and different schemes for the occupation of the A- and Bsites were synthesized (Table 1). Batches were prepared from oxides milled in an agate mortar to 20-30 $\mu \mathrm{m}$ in size. Powders were compacted under pressure of $200-400 \mathrm{MPa}$ in cylindrical pellets $12-20 \mathrm{~mm}$ in diameter and $4-5 \mathrm{~mm}$ in height. These pellets were placed in alumina-made crucibles and sintered at $1500-1550{ }^{\circ} \mathrm{C}$ for $6-10$ hours. Ce-pyrochlores were synthesized at $1300-1350{ }^{\circ} \mathrm{C}$ for $5-50$ hours in pure oxygen medium. The latter transformed cerium to tetravalent state required to form a pyrochlore. In some cases to get better homogenization once-sintered samples were re-milled and heated again. Evidence for equilibrium in the systems was established by unchanging phase composition of the samples at longer test durations (5...20 hours). The ceramics produced were examined by X-ray diffraction (XRD) using a DRON-4 (Russian model) and X'pert MPD (Phillips Co.) diffractometers (Cu $\mathrm{K}_{\alpha}$ radiation), scanning electron microscopy with energy dispersive system (SEM/EDS) using a JSM-5300+Link ISIS unit, and transmission electron microscopy (TEM) using a JEM-100c unit equipped with a KEVEX-5100 analyzer. 


\section{Results}

In most of the samples pyrochlore was found to be a major phase (Table 1). Pyrochlore is present in all of the titanate ceramics. Brannerite, perovskite, and fluorite-type oxide also occurred in the samples. Zirconate pyrochlore was formed in the samples containing Th only. Substitution of $U$ for Th yielded a fluorite-type oxide rather than pyrochlore. In the mixed titanate-zirconate ceramics, pyrochlore is the major phase and cubic oxide is present as a minor phase (5-30 vol\%).

The measured pyrochlore compositions do not exhibit perfect stoichiometry. This is one explanation for the presence of additional minor phases in the samples (5-10\% each of brannerite, perovskite, and fluorite-structured oxide). A major difference is in the Ca excess (0.03-0.12 formula units) with respect to actinides and cerium (Table 1). The lowest $\mathrm{Ca}:(\mathrm{Ce}, \mathrm{An})$ atomic ratio was found in the Ce-pyrochlore sintered at $1350^{\circ} \mathrm{C}$. The same data on the Ce-pyrochlore composition were published earlier [22]. This ratio reaches maximum value (1.47) in U-doped pyrochlores prepared at $1500-1550^{\circ} \mathrm{C}$. Probably, the occupation of the eight-fold coordinated sites is more preferable for $\mathrm{Ca}^{2+}$ rather than tetravalent actinides or cerium, especially at high temperatures.

Compositions of most pyrochlores, except those produced in the system Ca-U-Ti-O and Ca-Ce-Ti-O, have been established for the first time. Diffraction data on these phases in the PDF database are also absent. The interplanar spacing for $d_{222}$ of the Ce-pyrochlore $\mathrm{Ca}_{1.03} \mathrm{Ce}_{0.99} \mathrm{Ti}_{1.98} \mathrm{O}_{6.98}$ is $0.2930 \mathrm{~nm}$ corresponding to the unit cell dimension of $1.015 \mathrm{~nm}$, which is close to $\mathrm{Tb}_{2} \mathrm{Ti}_{2} \mathrm{O}_{7}$ (JCPDS \#23-565). Other phases have formulae: $\mathrm{Ca}_{0.91} \mathrm{Th}_{0.84} \mathrm{Zr}_{2.25} \mathrm{O}_{7.09},\left(\mathrm{Ca}_{0.44} \mathrm{GdTh}_{0.42}\right) \mathrm{Zr}_{2.13} \mathrm{O}_{7.05}, \mathrm{Ca}_{0.92} \mathrm{Th}_{0.92} \mathrm{Sn}_{2.00} \mathrm{Fe}_{0.08} \mathrm{O}_{6.96}$, $\left(\mathrm{Ca}_{0.47} \mathrm{Gd}_{0.95} \mathrm{Th}_{0.40}\right)\left(\mathrm{Zr}_{1.29} \mathrm{Ti}_{0.89}\right) \mathrm{O}_{7.05}$, and $\left(\mathrm{Ca}_{0.62} \mathrm{Gd}_{0.97} \mathrm{U}_{0.23}\right)\left(\mathrm{Zr}_{0.84} \mathrm{Ti}_{1.34)} \mathrm{O}_{6.90}\right.$. The most typical feature of these samples is the deficiency in A-site cations in the eight-coordinated sites. Probably some fraction of the $\mathrm{Zr}^{4+}$ ions can also occupy this site. Unit cell dimensions for these pyrochlores are 1.23, 1.21, 1.16, 1.04 , and $1.02 \mathrm{~nm}$, respectively, which correlates with the radii of cations that are predominant in their compositions.

Substitution of uranium for thorium in the zirconate phases with formulations $\mathrm{CaThZr}_{2} \mathrm{O}_{7}$ and $\left(\mathrm{Ca}_{0.5} \mathrm{GdTh}_{0.5}\right) \mathrm{Zr}_{2} \mathrm{O}_{7}$ makes the pyrochlore structure unstable. This is probably because the $\mathrm{U}^{4+}$ radius $(0.100 \mathrm{~nm})$ is less than that of $\mathrm{Th}^{4+}(0.105 \mathrm{~nm})$. As a result the ratio of ionic radii for cations in the ${ }^{\mathrm{VIII}}[\mathrm{A}]$ and ${ }^{\mathrm{VI}}[\mathrm{B}]$ sites is lower and outside of the range typical of pyrochlore-type structures. Partial substitution of smaller $\mathrm{Ti}^{4+}(0.061 \mathrm{~nm})$ for $\mathrm{Zr}^{4+}(0.072 \mathrm{~nm})$ in the octahedral sites leads to an increase in this ratio; thus, providing for the stability of the pyrochlore structure for the synthetic phases with ideal stoichiometry: $(\mathrm{CaU})(\mathrm{ZrTi}) \mathrm{O}_{7}$ and $\left(\mathrm{Ca}_{0.5} \mathrm{GdU}_{0.5}\right)(\mathrm{ZrTi}) \mathrm{O}_{7}$. 
Table 1. Chemical and phase compositions of the samples fabricated and calculated pyrochloretype phases formulae.

\begin{tabular}{|c|c|c|}
\hline Target composition & Phases identified & Pyrochlore formulae, SEM/EDS data \\
\hline $\mathrm{CaUTi}_{2} \mathrm{O}_{7}$ & Pyrochlore $>$ brannerite & $\mathrm{Ca}_{1.06} \mathrm{U}_{0.72} \mathrm{Ti}_{2.22} \mathrm{O}_{6.94}$ \\
\hline $\mathrm{CaCeTi}_{2} \mathrm{O}_{7}$ & Pyrochlore $>$ perovskite $>$ FSO & $\mathrm{Ca}_{1.03} \mathrm{Ce}_{0.99} \mathrm{Ti}_{1.98} \mathrm{O}_{6.98}$ \\
\hline $\mathrm{CaThSn}_{2} \mathrm{O}_{7}$ & Pyrochlore >> FSO & $\mathrm{Ca}_{0.92} \mathrm{Th}_{0.92} \mathrm{Sn}_{2.00}\left(\mathrm{Fe}_{0.08}\right)^{*} \mathrm{O}_{6.96}$ \\
\hline $\mathrm{CaThZr}_{2} \mathrm{O}_{7}$ & Pyrochlore $>$ perovskite $\sim$ FSO & $\mathrm{Ca}_{0.91} \mathrm{Th}_{0.84} \mathrm{Zr}_{2.25} \mathrm{O}_{7.09}$ \\
\hline $\mathrm{CaUZr}_{2} \mathrm{O}_{7}$ & FSO & 7 \\
\hline$\left(\mathrm{Ca}_{0.5} \mathrm{GdTh}_{0.5}\right) \mathrm{Zr}_{2} \mathrm{O}_{7}$ & Pyrochlore >> FSO & $\left(\mathrm{Ca}_{0.44} \mathrm{GdTh}_{0.42}\right) \mathrm{Zr}_{2.13} \mathrm{O}_{7.05}$ \\
\hline$\left(\mathrm{Ca}_{0.5} \mathrm{GdU}_{0.5}\right) \mathrm{Zr}_{2} \mathrm{O}_{7}$ & FSO & - \\
\hline$\left(\mathrm{Ca}_{0.5} \mathrm{GdTh}_{0.5}\right)(\mathrm{ZrTi}) \mathrm{O}_{7}$ & Pyrochlore $>>$ FSO & $\left(\mathrm{Ca}_{0.47} \mathrm{Gd}_{0.95} \mathrm{Th}_{0.40}\right)\left(\mathrm{Zr}_{1.29} \mathrm{Ti}_{0.89}\right) \mathrm{O}_{7.05}$ \\
\hline$\left(\mathrm{Ca}_{0.5} \mathrm{GdU}_{0.5}\right)(\mathrm{ZrTi}) \mathrm{O}_{7}$ & Pyrochlore > FSO & $\left(\mathrm{Ca}_{0.62} \mathrm{Gd}_{0.97} \mathrm{U}_{0.23}\right)\left(\mathrm{Zr}_{0.84} \mathrm{Ti}_{1.34}\right) \mathrm{O}_{6.90}$ \\
\hline
\end{tabular}

FSO - fluorite-structure oxide. ${ }^{*}$ - due to contamination of batch with iron during milling.

\section{Discussion}

We have analyzed the conditions that lead to the formation of the pyrochlore structure for the two compositions $\mathrm{A}_{2} \mathrm{~B}_{2} \mathrm{O}_{7}$ and $(\mathrm{CaA}) \mathrm{B}_{2} \mathrm{O}_{7}$, where $\mathrm{A}=$ lanthanides or tri- and tetravalent actinides. Among the titanates, this structure is typical of intermediate and heavy lanthanides with small ionic radii (Table 2). For germanates (radius of ${ }^{\mathrm{Vl}} \mathrm{Ge}^{4+}$ ion is smaller than $\mathrm{Ti}^{4+}$ and equal to $0.053 \mathrm{~nm}$ ) the pyrochlore structure was obtained for the phases of the small REEs (yttrium group) under very high pressure (6 GPa) [23]. Among hafnates and zirconates, the pyrochlore lattice is typical of the largest lanthanides from La to Gd. $\mathrm{Gd}$, Eu, and Sm take up in this row a specific position (Table 2). For these elements, both pyrochlore- and fluorite-type phases with the same composition were obtained [18]. The boundary between these structural forms occurs between 1600 and $2000^{\circ} \mathrm{C}$. Pyrochlore-structured plumbates do not occur at all due to large radius of the ${ }^{\mathrm{VI}} \mathrm{Pb}^{4+}$ ion $(0.078 \mathrm{~nm})$. Oxides with fluorite lattice, where $\mathrm{REE}^{3+}$ and $\mathrm{Pb}^{4+}$ occupy the same sites with $\mathrm{CN}=8$, are formed instead.

Thus, for the lanthanide-bearing pyrochlore-type structures, the ionic radii in the six-coordinated site may range between $0.061 \mathrm{~nm}\left(\mathrm{Ti}^{4+}\right)$ and $0.072 \mathrm{~nm}\left(\mathrm{Zr}^{4+}\right)$. The optimum ionic radius is in the middle part of this range. For example, $\mathrm{Sn}^{4+}$ can form pyrochlore-type phases with almost all of the lanthanides [15]. Size of $\mathrm{Tc}^{4+}(r=0.065 \mathrm{~nm})$ takes an intermediate position between $\mathrm{Ti}^{4+}(r=0.061 \mathrm{~nm})$ and $\mathrm{Sn}^{4+}(r=0.069 \mathrm{~nm})$ and, therefore, can also be accommodated in the octahedral sites of the pyrochlore lattice. This may be important for immobilization of long-lived ${ }^{99} \mathrm{Tc}\left(\mathrm{T}_{1 / 2}=2.13 \cdot 10^{5}\right.$ years $)$, which is an ecologically dangerous product of ${ }^{235} \mathrm{U}$ fission in the nuclear reactor.

Existing data on rare earth pyrochlores provides an evaluation of stability for similar actinide-bearing phases. Ionic radii of $\mathrm{Pu}^{3+}, \mathrm{Am}^{3+}$, and $\mathrm{Cm}^{3+}$ are close to radii of $\mathrm{Nd}^{3+}, \mathrm{Pm}^{3+}$, and $\mathrm{Sm}^{3+}$. Therefore, for actinide compounds with the same ion in the octahedral sites as for the rare earth element (REE) phases, the pyrochlore structure can be formed (Table 2). The occurrence of at least four such compounds for curium and three compounds each of americium and plutonium can be suggested.

Unlike $\mathrm{Cm}^{3+}$-titanate, phases of $\mathrm{Pu}^{3+}$ and $\mathrm{Am}^{3+}$, as well as of the light REEs (from La to $\mathrm{Nd}$ ), form monoclinic $\left(P 2_{1}\right)$ or orthorhombic $\left(P n a 2_{1}\right)$ structures. This is probably due to larger ionic radii of their trivalent cations than is required to form the pyrochlore type lattice. In spite of the absence of a cubic lattice for $\mathrm{Pu}_{2} \mathrm{Ti}_{2} \mathrm{O}_{7}$ and $\mathrm{Am}_{2} \mathrm{Ti}_{2} \mathrm{O}_{7}$, pyrochlore-structured titanates with significant $\mathrm{Pu}$ and $\mathrm{Am}$ contents 
have been previously obtained [24]. In these phases, the lanthanide ions with smaller radii stabilize pyrochlore structure. In particular, $\mathrm{Gd}_{2} \mathrm{Ti}_{2} \mathrm{O}_{7}$-based pyrochlore solid solution has been established to incorporate up to $16 \mathrm{~mol} \% \mathrm{Pu}_{2} \mathrm{Ti}_{2} \mathrm{O}_{7}$. As the radius of the lanthanide ion decreases, the content of the plutonium pyrochlore end-member increases to $22 \mathrm{~mol} \%$ for $\mathrm{Er}_{2} \mathrm{Ti}_{2} \mathrm{O}_{7}$ and $33 \mathrm{~mol} \%$ for $\mathrm{Lu}_{2} \mathrm{Ti}_{2} \mathrm{O}_{7}$.

Americium content in the pyrochlore $(\mathrm{Er}, \mathrm{Am})_{2} \mathrm{Ti}_{2} \mathrm{O}_{7}$ was found to be $61 \mathrm{~mol} \%$ [24]. Replacement of some fraction of $\mathrm{Ti}^{4+}$ ions in the octahedral sites by larger $\mathrm{Hf}^{4+}$ or $\mathrm{Zr}^{4+}$ ions will probably lead to the same result, i.e., stabilization of the pyrochlore structure for the phase with $\mathrm{Pu}_{2}(\mathrm{Ti}, \mathrm{Hf}, \mathrm{Zr})_{2} \mathrm{O}_{7}$ composition [17].

Actinides can be present in crystalline phases in various valence states $[8,16-18,25]$. In the samples produced at $1200-1600^{\circ} \mathrm{C}$ in air or inert argon medium, actinides normally have a formal charge $3+(\mathrm{Cm}$, $\mathrm{Am}$ ) or $4+(\mathrm{U}, \mathrm{Np})$. Plutonium may occur as either $\mathrm{Pu}^{4+}$ (in air) or $\mathrm{Pu}^{3+}$ (in inert or reducing gas media). Similar behavior is characteristic of $\mathrm{Ce}[18,22,26]$. The difference between them is the higher stability of the tetravalent state for $\mathrm{Pu}$ and trivalent state for $\mathrm{Ce}$. A rather small fraction of the total actinide content may be incorporated into the $\left(\mathrm{A}^{3+}\right)_{2}\left(\mathrm{~B}^{4+}\right)_{2} \mathrm{O}_{7}$ pyrochlores in tetravalent form as, for example, in the phase $\left(\mathrm{Pu}^{3+}{ }_{2-x} \mathrm{Pu}^{4+}{ }_{x}\right)(\mathrm{Hf}, \mathrm{Zr})_{2} \mathrm{O}_{7+x}[17]$.

Table 2. Possible pyrochlore-type structure for phases of REEs and actinides (An) with nominal stoichiometry: ${ }^{\mathrm{VIII}} \mathrm{REE}_{2}{ }^{\mathrm{VI}} \mathrm{B}_{2} \mathrm{O}_{7},{ }^{\mathrm{VII}} \mathrm{An}_{2}{ }^{\mathrm{VI}} \mathrm{B}_{2} \mathrm{O}_{7}$, and ${ }^{\mathrm{VIII}}\left(\mathrm{CaAn}^{4+}\right){ }^{\mathrm{VI}} \mathrm{B}^{4+}{ }_{2} \mathrm{O}_{7}$.

\begin{tabular}{|c|c|c|c|c|c|}
\hline$A^{\mathrm{VIII}}=\mathrm{REE}^{3+}$ & $\begin{array}{c}\mathrm{Ti}^{4+} \\
(0,061 \mathrm{~nm})\end{array}$ & $\begin{array}{c}\mathrm{Sn}^{4+} \\
(0,069 \mathrm{~nm})\end{array}$ & $\begin{array}{c}\mathrm{Hf}^{4+} \\
(0,071 \mathrm{~nm})\end{array}$ & $\begin{array}{c}\mathrm{Zr}^{4+} \\
(0,072 \mathrm{~nm})\end{array}$ & $\begin{array}{c}\mathrm{A}^{\mathrm{VIII}}=\mathrm{An}^{3+} \\
\mathrm{Ca}^{2+}+(\mathrm{An}, \mathrm{Ce})^{4+}\end{array}$ \\
\hline $\mathrm{La}^{3+}(0,116 \mathrm{~nm})$ & - & + & + & + & \\
\hline $\mathrm{Ce}^{3+}(0,114 \mathrm{~nm})$ & - & + & + & + & \\
\hline $\operatorname{Pr}^{3+}(0,113 \mathrm{~nm})$ & - & + & + & + & \\
\hline $\mathrm{Nd}^{3+}(0,111 \mathrm{~nm})$ & - & + & + & + & $\mathrm{Pu}^{3+}(0,110)$ \\
\hline $\mathrm{Pm}^{3+}(0,109 \mathrm{~nm})$ & - & + & + & + & $\mathrm{Am}^{3+}(0,109)$ \\
\hline $\mathrm{Sm}^{3+}(0,108 \mathrm{~nm})$ & + & + & + & \pm & $C a+\operatorname{Th}(0,1085)$ \\
\hline $\mathrm{Eu}^{3+}(0,107 \mathrm{~nm})$ & + & + & + & \pm & $\mathrm{Cm}^{3+}(0,108)$ \\
\hline \multirow[t]{2}{*}{$\mathrm{Gd}^{3+}(0,105 \mathrm{~nm})$} & + & + & + & \pm & $\mathrm{Ca}+U(0,106)$ \\
\hline & + & + & + & \pm & $C a+N p(0,105)$ \\
\hline \multirow[t]{2}{*}{$\mathrm{Tb}^{3+}(0,104 \mathrm{~nm})$} & + & + & + & - & $\mathrm{Ca}+\mathrm{Ce}(0,1045)$ \\
\hline & + & + & + & - & $\mathrm{Ca}+\mathrm{Pu}(0,104)$ \\
\hline $\mathrm{Dy}^{3+}(0,103 \mathrm{~nm})$ & + & + & - & - & \\
\hline $\mathrm{Y}^{3+}(0,102 \mathrm{~nm})$ & + & + & - & - & \\
\hline $\mathrm{Ho}^{3+}(0,102 \mathrm{~nm})$ & + & + & - & - & \\
\hline $\mathrm{Er}^{3+}(0,100 \mathrm{~nm})$ & + & + & - & - & \\
\hline $\mathrm{Tm}^{3+}(0,099 \mathrm{~nm})$ & + & + & - & - & \\
\hline $\mathrm{Yb}^{3+}(0,099 \mathrm{~nm})$ & + & + & - & - & \\
\hline $\mathrm{Lu}^{3+}(0,098 \mathrm{~nm})$ & + & - & - & - & \\
\hline
\end{tabular}

$(+)$ - pyrochlore structure is stable, $(-)$ - instable. $( \pm)$ - structure varieties both of pyrochlore and fluorite types are possible. Ionic radii of the cations were taken from ref. [25].

To increase tetravalent actinide solubility in the pyrochlore, simultaneous incorporation of a charge compensating cation, such as $\mathrm{Ca}^{2+}$, is required. This leads to compounds for which trivalent ions are replaced by pair of di- and tetravalent cations according to the scheme: $2 \mathrm{REE}^{3+}=\mathrm{Ca}^{2+}+\mathrm{Ce}^{4+}\left(\mathrm{U}^{4+}, \mathrm{Th}^{4+}\right.$, $\left.\mathrm{Np}^{4+}, \mathrm{Pu}^{4+}\right)$. We suggest, based on a comparison of radii of these ionic pairs and REEs, that several phases of tetravalent actinides and cerium with pyrochlore structure may form. Average radii of pairs 
$\left(\mathrm{Ca}^{2+}+\mathrm{U}^{4+}\right)$ and $\left(\mathrm{Ca}^{2+}+\mathrm{Np}^{4+}\right)$ are close to the ionic radius of $\mathrm{Gd}^{3+}$. For the $\mathrm{Ca}^{2+}-\mathrm{Th}^{4+}$ pair, such an analog is $\mathrm{Pm}^{3+}$ or $\mathrm{Sm}^{3+}$, and for the pairs with $\mathrm{Ce}^{4+}$ or $\mathrm{Pu}^{4+}$, it is $\mathrm{Tb}^{3+}$ (Table 2). Pyrochlores with a composition close to $\mathrm{CaCeTi}_{2} \mathrm{O}_{7}$ and $\mathrm{CaUTi}_{2} \mathrm{O}_{7}$ have been obtained experimentally [22, 27]. Pyrochlores $\mathrm{Ca}(\mathrm{Pu}, \mathrm{U}, \mathrm{Zr}) \mathrm{Ti}_{2} \mathrm{O}_{7}$ and $\mathrm{Ca}(\mathrm{Np}, \mathrm{Zr}) \mathrm{Ti}_{2} \mathrm{O}_{7}$ containing up to $45 \mathrm{wt} \% \mathrm{PuO}_{2}$ and $\mathrm{NpO}_{2}$ have also been synthesized [8]. Stannate pyrochlores with formula $\left(\mathrm{REE}_{2} / \mathrm{Ca}^{2+} \mathrm{An}^{4+}\right) \mathrm{Sn}^{4+}{ }_{2} \mathrm{O}_{7}$ and ratio of ionic radii in two different structural sites ranged between 1.55 and 1.60 are typical of lanthanide [15] and actinide varieties as well. We have also produced Th-pyrochlore (Table 2). Data on the correlation between composition and structure in the pyrochlore group must be carefully considered in designing an actinide host phase, especially in the case of complex waste stream compositions. Radii of ions in the octahedral sites of the pyrochlore structure for the phases with REEs and trivalent actinides may range between 0.055 and $0.075 \mathrm{~nm}$. This condition corresponds to the radii of $\mathrm{Ti}^{4+}, \mathrm{Sn}^{4+}, \mathrm{Hf}^{4+}$, and $\mathrm{Zr}^{4+}$ ions. Tetravalent actinides can occupy the $[\mathrm{A}]^{\mathrm{VIIII}}$-sites at simultaneous incorporation of charge compensators $\left(\mathrm{Ca}^{2+}\right.$ cations).

\section{Conclusions}

New actinide (Th, $U$ ) and cerium pyrochlore phases have been synthesized. As compared with their ideal formulae, Ca excess in amount of 0.03-0.12 formula units with respect to cerium and actinides has been found. The other three- and four-charged actinides can be easily incorporated into the eight-coordinated positions of the lattice. Among the elements that can occupy the octahedral sites of the structure, we note $\mathrm{Zr}^{4+}, \mathrm{Sn}^{4+}$, and $\mathrm{Tc}^{4+}$ with dimensions $0.072,0.069$, and $0.065 \mathrm{~nm}$. Thus, pyrochlore-type phases may also provide a host for long-lived fission products: ${ }^{93} \mathrm{Zr}\left(\mathrm{T}_{1 / 2}=1.5^{*} 10^{6}\right.$ years $),{ }^{126} \mathrm{Sn}\left(1.0{ }^{*} 10^{5}\right.$ years), and ${ }^{99} \mathrm{Tc}$ $\left(2.13 * 10^{5}\right.$ years $)$. The last element is one of the principal contributors to dose in most analyses of radioactive elements release from a nuclear waste repository [1].

\section{Acknowledgements}

Authors thank O.I. Kirjanova (SIA Radon, Russia), M.I. Lapina, A.V. Sivtsov (IGEM RAS, Russia), and S. Chae (KIGAM, Korea) for assistance at synthesis of the samples and their examination. The work was supported by DOE US - CRDF (grant RC0-20002-SC14).

\section{References}

1. Actinide and Fission Product Partitioning and Transmutation. Proc. VI-th Information Exchange Meeting, Madrid, Spain, 11 - 13 Dec. 2000.

2. Journ. Nucl. Materials, 274, N1/2, (1999). (Proc. IV-th Workshop on Inert Matrix Fuel).

3. P.E. Raison and R.G. Haire, Proc. Global'01, Paris, Sept. 09-13, 2001. File \#FP127.pdf.

4. W. Lutze and R.C. Ewing (eds), Radioactive Waste Forms for the Future, NY, (1988).

5. R.C. Ewing, W.J. Weber, W. Lutze, Disposal of Weapon Plutonium, E.R. Merz and C.E. Walter (eds), Kluw. Ac. Publ., Netherlands, 65 (1996).

6. R.C. Ewing, Proc. Nat. Acad. Sci. USA, 96, 3432 (1999).

7. E.G. Dzekun, Y.V. Glagolenko, E.G. Drozhko, "SPECTRUM '96", Seattle, 2138 (1996).

8. E.R. Vance, B.D. Begg, R.A. Day, C.J. Ball, MRS Symp. Proc. 353, 767 (1995).

9. B.B. Ebbinghaus, R.A. VanKonynenburg, F.J. Ryerson et al., "Waste Management '98", Proc Int. Symp., Tuscon, CD-Rom. Rep. N65-04 (1998).

10. W.J. Weber and R.C. Ewing, Science, 289, 2051 (2000). 
11. S.X. Wang, B.D. Begg, L.M. Wang et al., J. Mat. Res., 14 (12), 4470 (1999).

12. N.V. Belov, Miner. Iss. L'vov Geol. Soc. (Russ.), (4), 21 (1950).

13. V.A. Isupov, Crystallogr. (Russ.), 3 (1), 99 (1958).

14. E. Aleshin and R. Roy, J. Am. Cer. Soc., 45, 18 (1962).

15. R.A. McCauley, J. Appl. Phys., 51 (1), 290 (1980).

16. B.C. Chakoumakos and R.C. Ewing, MRS Symp. Proc. 44, 641 (1985).

17. P.E. Raison, R.G. Haire, T. Sato, T. Ogawa, MRS Symp. Proc. 556, 3 (1999).

18. B.D. Begg, N.J. Hess, D. McCready, J. Nucl. Mat., 289, 188 (2001).

19. G. R. Lumpkin, B. C. Chakoumakos, R. C. Ewing, Amer. Miner., 71, 569 (1986).

20. G. R. Lumpkin and R. C. Ewing, Phys. Chem. Miner., 16, 2 (1988).

21. T. Moriga, A. Yoshiasa, F. Kanamaru, et al., Solid State lonics, 31, 319 (1989).

22. H. Xu, Y. Wang, R.L. Putnam et al., MRS Symp. Proc. 608, 461 (2000).

23. G. Bocquillon, C.R. Seances Acad. Sci., Ser. C., 287, 5 (1978).

24. S.S. Shoup and C. E. Bamberger, MRS Symp. Proc. 465, 379 (1997).

25. R.D. Shannon, Acta Cryst., 32 (Pt. A), 751 (1976).

26. B.D. Begg, E.R. Vance, R.A. Day, et al., MRS Symp. Proc. 465, 325 (1997).

27. A.E. Ringwood, Miner. Mag., 49 (2), 159 (1985).

\section{Viewgraphs}




\section{Approach to HLW management was changed in the last decade}

\section{Former determined (non alternative) way, the $70-90$ s of the last century}

- One type of HLW,

- One industrial-scale technology,

- Single waste form,

- One route for utilization.

Previous practice, Scheme \#1:

\begin{tabular}{|c|c|c|}
\hline Type of HLW $\rightarrow$ & Technology of Waste Forms fabrication $\rightarrow$ & Waste Form \\
\hline $\begin{array}{l}\text { Liquid HLW from conventional ("wet") } \\
\text { Spent fuel reprocessing }\end{array}$ & $\begin{array}{l}\text { Through melting, for example in a Joule- } \\
\text { heated furnace EP-500, } \\
\text { Russia, "MAYAK" } \\
\text { radiochemical plant }\end{array}$ & $\begin{array}{l}\text { Vitreous matrix (for example } \\
\text { Na-Al-P } \\
\text { Glass in Russia) } \\
\downarrow\end{array}$ \\
\hline
\end{tabular}

\section{Current situation with alternative ways to HLW utilization:}

- Many types of HLW,

- Various waste forms,

- Different methods for waste form fabrication

- Some routes for wastes utilization

Current situation, Scheme \#2:

\begin{tabular}{|c|c|c|}
\hline Type of HLW $\rightarrow$ & Waste Form & $\begin{array}{l}\leftarrow \text { Technology of Waste } \\
\text { Forms fabrication }\end{array}$ \\
\hline $\begin{array}{l}\text { Liquid HLW from the conventional (PUREX) spent fuel } \\
\text { reprocessing }\end{array}$ & \multirow{5}{*}{$\begin{array}{l}\text { Vitreous form (Glass), } \\
\text { Crystalline form } \\
\text { (ceramic) } \\
\text { Glass-ceramic, et al. } \\
\downarrow\end{array}$} & \multirow{2}{*}{$\begin{array}{l}\text { Through melting: } \\
\text { EP-500 furnace, } \\
\text { Inductive melting in a cold } \\
\text { crucible (IMCC), } \\
\text { Microwave heating }\end{array}$} \\
\hline Groups of partitioned HLW: $\mathrm{Cs}+\mathrm{Sr}, \mathrm{Tc}, \mathrm{An}+\mathrm{REE}$, et al. & & \\
\hline $\begin{array}{l}\text { Pyrochemical wastes from "dry" spent fuel reprocessing } \\
\text { in a molten salt }\end{array}$ & & \multirow{2}{*}{$\begin{array}{l}\text { Solid phase methods: } \\
\text { Hot-pressing (HP), } \\
\text { Cold pressing and sintering } \\
(\mathrm{CP}-\mathrm{S})\end{array}$} \\
\hline HLW after conversion of weapon-grade Pu into MOX fuel & & \\
\hline \multirow[t]{2}{*}{ Excess of weapon usable Pu } & & $\begin{array}{l}\text { Self propagating high- } \\
\text { temperature synthesis (SHS) }\end{array}$ \\
\hline & $\begin{array}{l}\text { Disposal in the Earth, } \\
\text { Burning in reactors }\end{array}$ & \\
\hline
\end{tabular}

Specific feature of the current situation: we may select or design appropriate waste form with optimal properties for every kind of HLW (existed or expected in the future) and use optimal route for their industrial fabrication for further disposal and / or transmutation. 
Radii (nm) for the VI- and VIII-coordinated actinide cations and their likely substitutions in the pyrochlore structure (Chakoumakos, Ewing, 1985).

\begin{tabular}{|c|c|c|c|c|c|c|c|}
\hline $\mathrm{Ac}^{3+}$ & VI & 0.112 & A & $\mathrm{Pu}^{3+}$ & VI & 0.100 & A \\
\hline \multirow[t]{2}{*}{$\mathrm{Th}^{4+}$} & VI & 0.094 & A & \multirow[t]{2}{*}{$\mathrm{Pu}^{4+}$} & VI & 0.086 & A \\
\hline & VIII & 0.105 & A & & VIII & 0.096 & A \\
\hline $\mathrm{Pa}^{3+}$ & $\mathrm{VI}$ & 0.104 & A & $\mathrm{Pu}^{5+}$ & VI & 0.074 & B \\
\hline \multirow[t]{2}{*}{$\mathrm{Pa}^{4+}$} & $\mathrm{VI}$ & 0.090 & $A$ & $\mathrm{Pu}^{6+}$ & $\mathrm{VI}$ & 0.071 & $B$ \\
\hline & VIII & 0.101 & $A$ & $\mathrm{Am}^{2+}$ & VIII & 0.126 & $A$ \\
\hline \multirow[t]{2}{*}{$\mathrm{Pa}^{5+}$} & $\mathrm{VI}$ & 0.078 & $B$ & \multirow[t]{2}{*}{$\mathrm{Am}^{3+}$} & $\mathrm{VI}$ & 0.0975 & $A$ \\
\hline & VIII & 0.091 & $A$ & & VIII & 0.109 & $A$ \\
\hline$U^{3+}$ & $\mathrm{VI}$ & 0.1025 & A & \multirow[t]{2}{*}{$\mathrm{Am}^{4+}$} & VI & 0.085 & $A$ \\
\hline \multirow[t]{2}{*}{$U^{4+}$} & $\mathrm{VI}$ & 0.089 & A & & VIII & 0.095 & A \\
\hline & VIII & 0.100 & A & $\mathrm{Cm}^{3+}$ & VI & 0.097 & $\mathrm{~A}$ \\
\hline$U^{5+}$ & $\mathrm{VI}$ & 0.076 & $B$ & \multirow[t]{2}{*}{$\mathrm{Cm}^{4+}$} & $\mathrm{VI}$ & 0.085 & A \\
\hline \multirow[t]{2}{*}{$\mathrm{U}^{6+}$} & $\mathrm{VI}$ & 0.073 & $B$ & & VIII & 0.095 & $A$ \\
\hline & VIII & 0.086 & $A$ & $\mathrm{Bk}^{3+}$ & $\mathrm{VI}$ & 0.096 & $A$ \\
\hline $\mathrm{Np}^{2+}$ & $\mathrm{VI}$ & 0.110 & $A$ & \multirow[t]{2}{*}{$\mathrm{Bk}^{4+}$} & $\mathrm{VI}$ & 0.083 & A \\
\hline $\mathrm{Np}^{3+}$ & $\mathrm{VI}$ & 0.101 & A & & VIII & 0.093 & A \\
\hline \multirow[t]{2}{*}{$N p^{4+}$} & $\mathrm{VI}$ & 0.087 & A & & & & \\
\hline & VIII & 0.098 & A & & & & \\
\hline $\mathrm{Np}^{5+}$ & $\mathrm{VI}$ & 0.075 & B & & & & \\
\hline $\mathrm{Np}^{6+}$ & $\mathrm{VI}$ & 0.072 & $B$ & & & & \\
\hline $\mathrm{Np}^{7+}$ & $\mathrm{VI}$ & 0.071 & $B$ & & & & \\
\hline
\end{tabular}

Hypothetical formulae of the actinide-doped pyrochlore with accommodation of these elements into two different positions:

$$
\begin{aligned}
& \mathrm{A}^{2+}{ }_{2} \mathrm{~B}^{5+}{ }_{2} \mathrm{O}_{7}, \mathrm{~B}=\mathrm{Pa}, \mathrm{U}, \mathrm{Np}, \mathrm{Pu} \\
& \begin{array}{ll}
\text { I. } \mathrm{A}_{1}{ }^{4+} \mathrm{A}_{2}{ }^{2+} \mathrm{B}^{4+}{ }_{2} \mathrm{O}_{7} ; & \text { II. } \mathrm{A}_{1}{ }^{4+} \mathrm{A}_{2}{ }^{+} \mathrm{B}^{4+}{ }_{2} \mathrm{O}_{6} \mathrm{~F} \\
A_{1}=\mathrm{Th}, \mathrm{Pa}, \mathrm{U}, \mathrm{Np}, \mathrm{Pu}, \mathrm{Am}, \mathrm{Cm} ; \mathrm{A}_{2}=\mathrm{Na}^{+}, \mathrm{Ca}^{2+} ; B=\mathrm{Ti}^{4+} .
\end{array}
\end{aligned}
$$

Actinides in the both structural positions: $\mathrm{Am}^{2+}{ }_{2} \mathrm{U}^{5+}{ }_{2} \mathrm{O}_{7}$ 

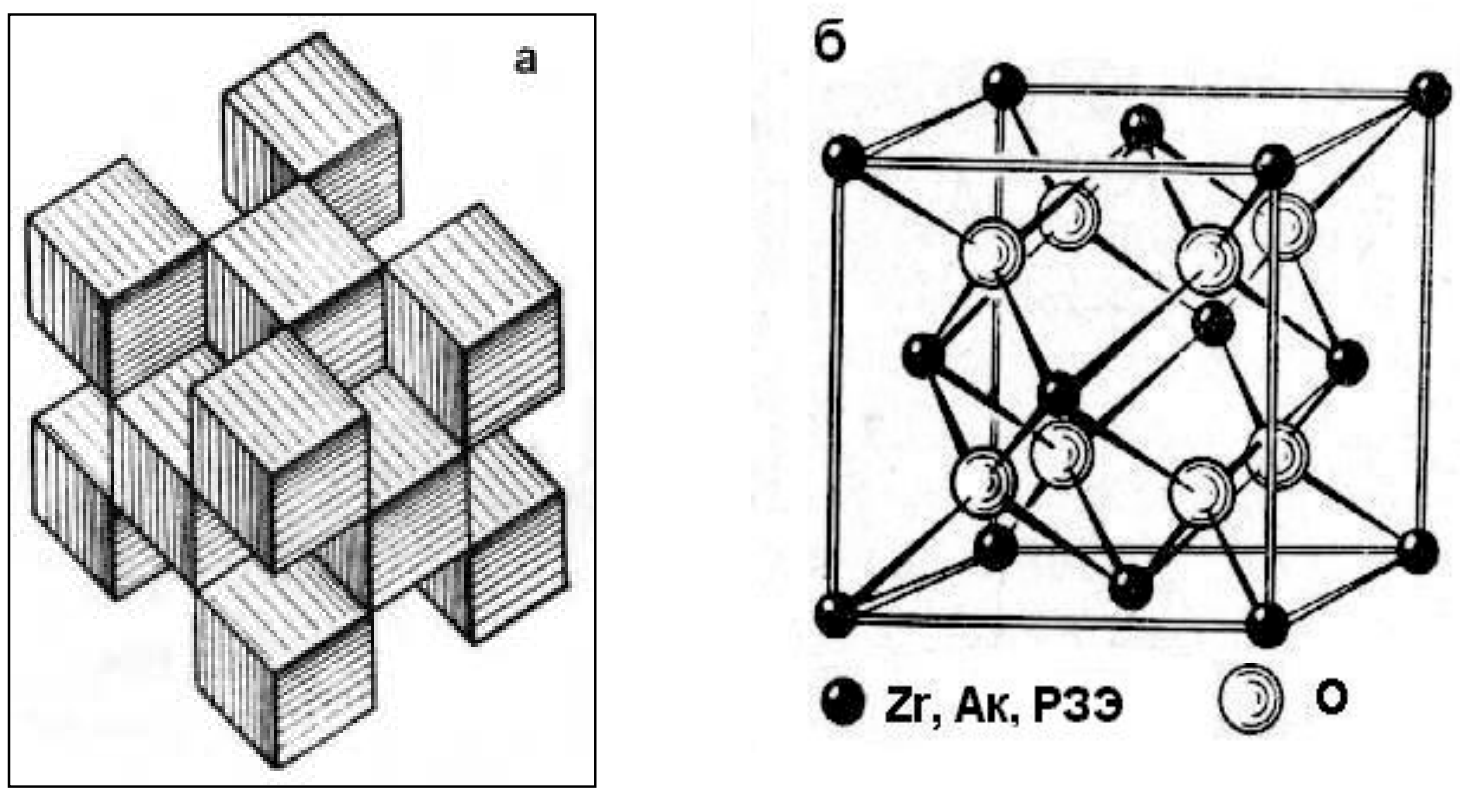

Fig. 1a. General view of the fluorite-type crystal structure in polyhedral (left) and atomic (right) image.
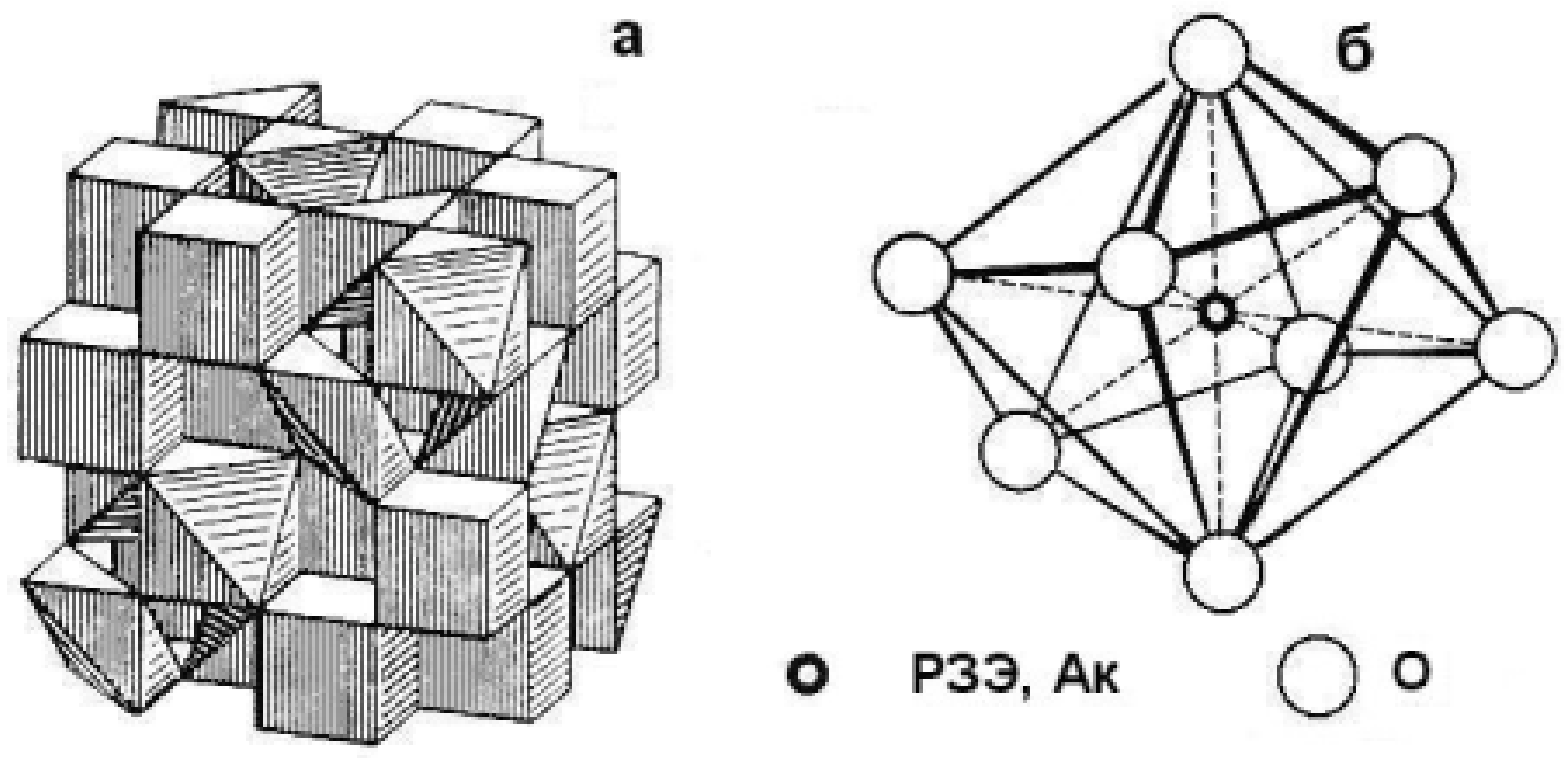

- P3Э, Ак

Fig. 1B. Pyrochlore structure in polyhedral image (left) and arrangement around the eightcoordinated cation (right). 
Fig. 2. SEM images of the ceramics with nominal stoichiometry: $\mathrm{CaUTi}_{2} \mathrm{O}_{7}(\mathrm{~A}), \mathrm{CaCeTi}_{2} \mathrm{O}_{7}(\mathrm{~B})$, $\mathrm{CaThSn}_{2} \mathrm{O}_{7}$ (C), $\mathrm{CaThZr}_{2} \mathrm{O}_{7}$ (D), $\mathrm{CaUZr}_{2} \mathrm{O}_{7}(\mathrm{E}),\left(\mathrm{Ca}_{0.5} \mathrm{GdTh}_{0.5}\right) \mathrm{Zr}_{2} \mathrm{O}_{7}(\mathrm{~F}),\left(\mathrm{Ca}_{0.5} \mathrm{GdTh}_{0.5}\right)\left(\mathrm{ZrTi} \mathrm{O}_{7}(\mathrm{G})\right.$, and $\left(\mathrm{Ca}_{0.5} \mathrm{GdU}_{0.5}\right)\left(\mathrm{ZrTi} \mathrm{O}_{7}(\mathrm{H})\right.$. Scale bars are equal to $50 \mu \mathrm{m}$. Electron diffraction patterns for phases with the pyrochlore-type (top) or fluorite (bottom) lattice are shown at insets.
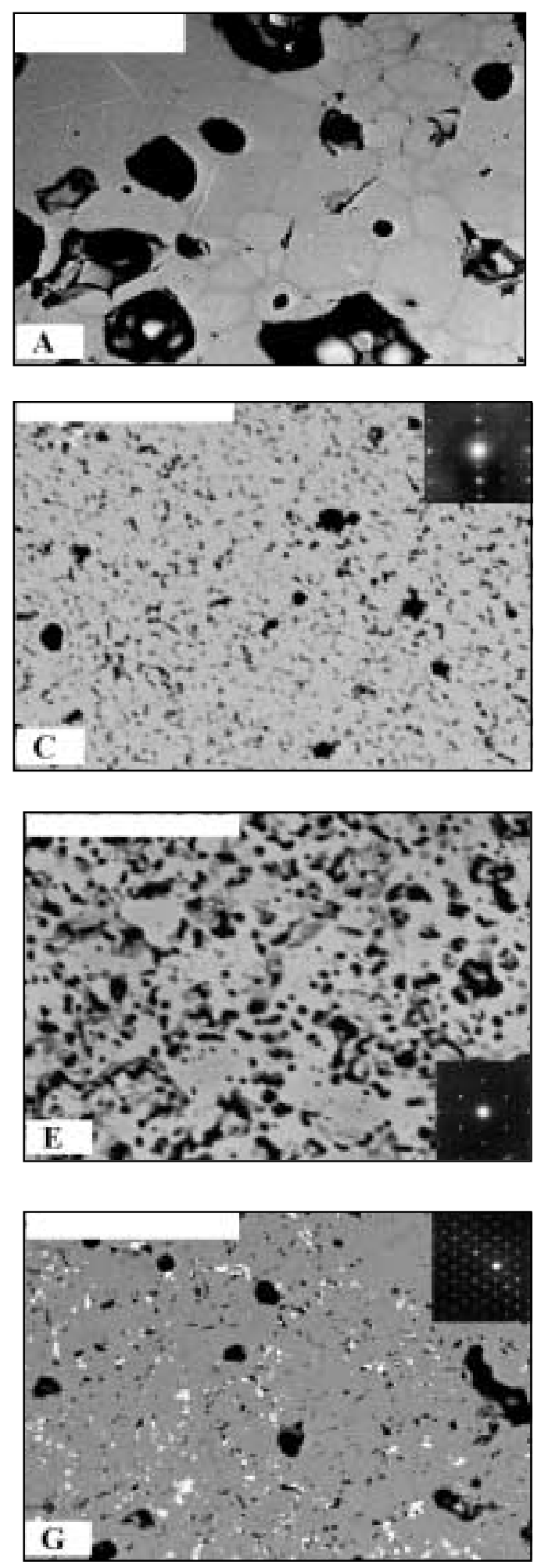
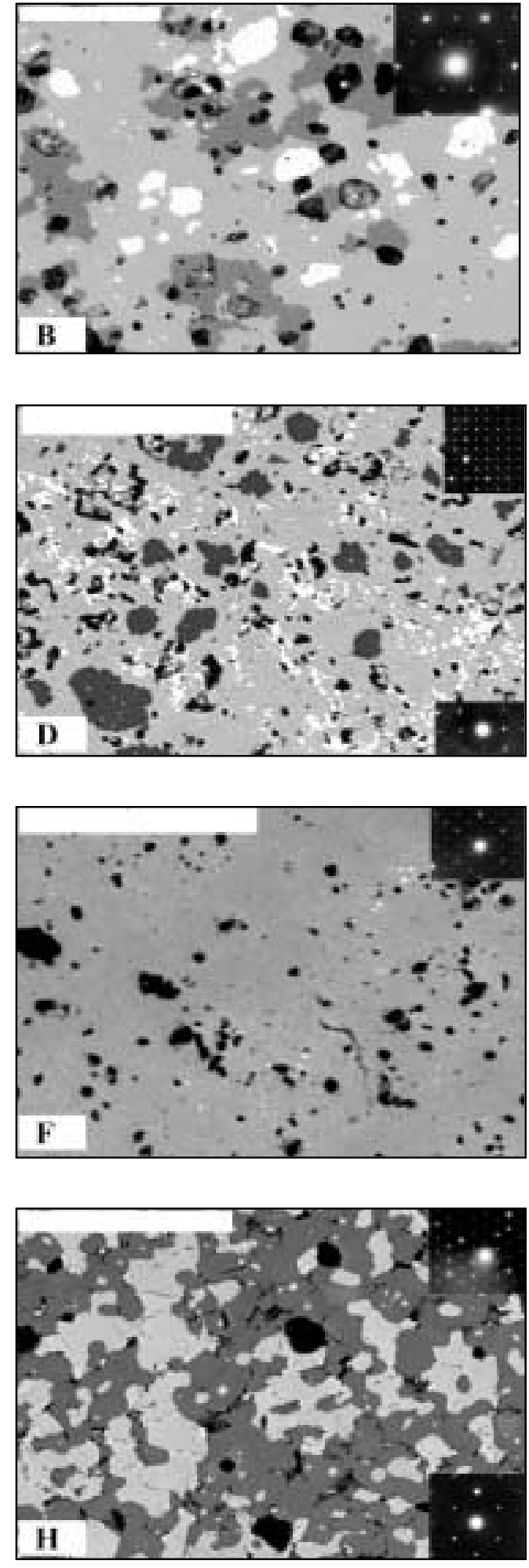


\section{Investigation of Plutonium, Americium and Curium Phosphates as a Basis for Inclusion into Kosnarite-type Ceramic Waste Forms}

\section{A. I. Orlova, A. A. Charlamova, Nizhni Novgorod State University; Yu. F. Volkov, RIAR}

The scientific basis for the proposal that zirconium phosphate ceramics could be used for including of wastes of pyroelectrochemical technology of spent fuel reprocessing has been developed by scientists from Nizhni Novgorod State University and the Research Institute of Atomic Reactors (RIAR). This problem examines the development of a method for regeneration of electrolyte (molten alkaline chloride salts), radioactive and non-radioactive admixtures, concentrating them by phosphate precipitation and moving them into a solid form appropriate for insulation from the biosphere.

This report presents the results of one stage of many in this concept devoted to phosphates of the actinide series (i.e., f- elements): plutonium, americium, curium, and some lanthanides.

The aims of this research project were:

- Concentrating actinides from alkaline chloride melts by phosphate treatment;

- Refining molten salts;

- Immobilizing phosphate sediments into ceramics.

Although directed at the pyroelectrochemical technology of reprocessing of spent nuclear fuel, the results may be also useful for other technologies.

The objects presented:

- $\quad$ - Pu (III), Am (III), Cm (III) and Pu (IV) phosphates and lanthanide (III) phosphates.

- - Actinide (lanthanide) - zirconium phosphate solid solutions

The methods used included x-ray powder diffraction, radiometry, EPR spectroscopy, and infrared (IR) spectroscopy.

The experiments included synthesis of phosphates, crystal chemical investigations, and radiation testing.

\section{Results and Discussion}

\section{Actinide series (III) phosphates, $\mathrm{Na}(\mathrm{K})_{3} \mathrm{An}_{2}\left(\mathrm{PO}_{4}\right)_{3}$ (Slide 1)}

Phosphates of $\mathrm{Na}(\mathrm{K})_{3} \mathrm{An}_{2}\left(\mathrm{PO}_{4}\right)_{3}(\mathrm{An}=\mathrm{Pu}, \mathrm{Am}, \mathrm{Cm})$ compositions were prepared by reaction of precipitation from molten salts $\mathrm{NaCl}-\mathrm{Na}_{3} \mathrm{PO}_{4}$ and $\mathrm{KCl}-\mathrm{K}_{3} \mathrm{PO}_{4}$ at $820-840^{\circ} \mathrm{C}$, and then washing of sediments by acid solutions and drying.

Phosphates of $\mathrm{Na}(\mathrm{K})_{3} \mathrm{Ln}_{2}\left(\mathrm{PO}_{4}\right)_{3}(\mathrm{Ln}=\mathrm{Ce},-\mathrm{Gd})$ compositions were prepared by reaction of precipitation from molten salts $\mathrm{NaCl}-\mathrm{Na}_{3} \mathrm{PO}_{4}$ and $\mathrm{KCl}-\mathrm{K}_{3} \mathrm{PO}_{4}$ at $820-840^{\circ} \mathrm{C}$, and also from water salt solutions at room temperature, followed by further drying and stage-by-stage burning.

The reactions of phosphate forming, x-ray powder diffraction, and IR data of prepared phosphates of $\mathrm{Pu}$ (III), Am (III), Cm (III), and Lanthanide (III) are presented below (Fig. 1). 


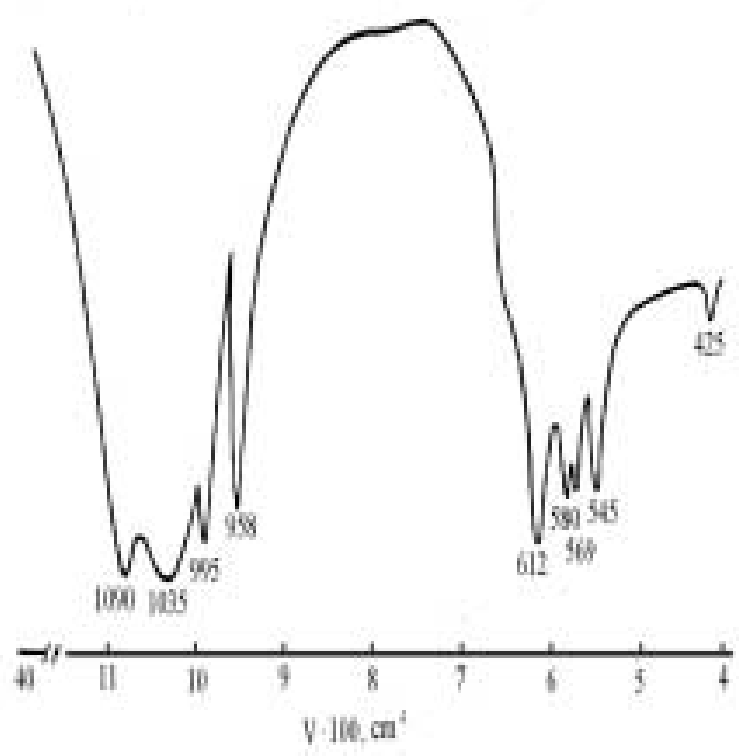

Fig. 1. $\mathrm{Na}_{3} \mathrm{Pu}_{2}\left(\mathrm{PO}_{4}\right)_{3}$

Monazite type, sp. gr. $\mathbf{P} \mathbf{2}_{1} / \mathbf{n}$

$\begin{array}{lcccccl} & \mathrm{a}, \AA & \mathrm{b}, \AA & \mathrm{c}, \AA & \beta^{0} & \mathrm{~V}, \AA^{3} & \mathrm{z} \\ \mathrm{Na}_{3} \mathrm{Pu}_{2}\left(\mathrm{PO}_{4}\right)_{3} & 6,69 & 6,92 & 6,39 & 104,07 & 287 & 1 \\ \mathrm{Na}_{3} \mathrm{Am}_{2}\left(\mathrm{PO}_{4}\right)_{3} & 6,78 & 7,00 & 6,44 & 103,75 & 297 & 1 \\ \mathrm{~K}_{3} \mathrm{Am}_{2}\left(\mathrm{PO}_{4}\right)_{3} & 6,74 & 6,98 & 6,44 & 103,80 & 294 & 1 \\ \mathrm{~K}_{3} \mathrm{Cm}_{2}\left(\mathrm{PO}_{4}\right)_{3} & 6,77 & 7,09 & 6,35 & 104,08 & 312 & 1 \\ & & & \text { Reaction } & \mathbf{R C l}_{3}+\mathbf{A}_{3} \mathbf{P O}_{4} \rightarrow \mathbf{A}_{3} \mathbf{R}_{\mathbf{2}}\left(\mathbf{P O}_{4}\right) \\ & & \boldsymbol{A C l} \text { (melt) }\end{array}$

$\mathrm{Na}(\mathrm{K})_{3} \mathrm{Ln}_{2}\left(\mathrm{PO}_{4}\right)_{3}$

$\mathrm{Ln}=\mathrm{Ce}, \mathrm{Pr}, \mathrm{Nd}, \mathrm{Sm}, \mathrm{Eu}, \mathrm{Gd}$
a, $\AA$
b, $\AA$
$c, \AA \quad \beta,^{\circ}$
6.82-6.73
7.05-6.92
6.38-6.44 103.2-103.3
$\mathrm{Na}_{3} \mathrm{R}\left(\mathrm{PO}_{4}\right)_{2}$

Glaserite type, sp. gr. Pnmm

\begin{tabular}{|c|c|c|c|c|}
\hline a, $\AA$ & $\mathrm{b}, \AA$ & c, $\AA$ & z & \\
\hline $\mathrm{Na}_{3} \mathrm{Pu}\left(\mathrm{PO}_{4}\right)_{2} \quad 18,44$ & 7,00 & 15,86 & 12 & \\
\hline $\mathrm{Na}_{3} \mathrm{Tm}\left(\mathrm{PO}_{4}\right)_{2} \quad 18,43$ & 6,97 & 15,82 & 12 (analog) (Ref.) & \\
\hline
\end{tabular}

The structures of the synthesized f-element (III) phosphates were established:

$\mathrm{Na}_{3} \mathrm{Pu}_{2}\left(\mathrm{PO}_{4}\right)_{3}$ monazite-type, sp. gr. P2 $1 / n$

$\mathrm{Na}_{3} \mathrm{Am}_{2}\left(\mathrm{PO}_{4}\right)_{3}$ monazite-type, sp. gr. P2 $1 / \mathrm{n}$

$\mathrm{K}_{3} \mathrm{Am}_{2}\left(\mathrm{PO}_{4}\right)_{3}$ monazite-type, sp. gr. P2 $1 / \mathrm{n}$

$\mathrm{K}_{3} \mathrm{Cm}_{2}\left(\mathrm{PO}_{4}\right)_{3}$ monazite-type, sp. gr. P2 $1 / n$

$\mathrm{Na}_{3} \mathrm{Pu}\left(\mathrm{PO}_{4}\right)_{2}$ glaserite-type, sp. gr. Pnmm (analog $\mathrm{Na}_{3} \mathrm{Tm}\left(\mathrm{PO}_{4}\right)_{2}$, having $\mathrm{HT}$ sp. gr. $\left.\mathrm{R} \overline{3} \mathrm{c}\right)($ Ref.).

The solid solutions $\mathrm{Na}_{1+x} \mathrm{Zr}_{2-\mathrm{x}} \mathrm{Ln}_{\mathrm{x}}\left(\mathrm{PO}_{4}\right)_{3}$ (the limits from $\mathrm{Yb} \mathrm{x}=1$ up to $\left.\mathrm{Gd} \mathrm{x}=0,2\right)$ were prepared and identified as having kosnarite structure type (type $\mathrm{NaZr}_{2}\left(\mathrm{PO}_{4}\right)_{3}$, NZP), sp. gr. $\mathrm{R} \overline{3} \mathrm{c}$ (ours and ref.). 
II. Plutonium (IV) Phosphate $\mathrm{NaPu}_{2}\left(\mathrm{PO}_{4}\right)_{3}$ (Slide 2)

$\begin{array}{lllll}\mathrm{NaTh}_{2}\left(\mathrm{PO}_{4}\right)_{3} & 17.28 \AA & 6.81 \AA & 8.15 \AA & 101.3^{\circ}(\mathrm{NThP}) \\ \mathrm{NaPu}_{2}\left(\mathrm{PO}_{4}\right)_{3} & 17.14 & 6.65 & 7.99 & 101.1 \\ \mathrm{NaPu}_{2}\left(\mathrm{PO}_{4}\right)_{3} & 9.09 & 23.69 & 3.98 \mathrm{~g} / \mathrm{cm}^{3} \\ \mathrm{NaZr}_{2}\left(\mathrm{PO}_{4}\right)_{3} & 8.80 & 22.78 & 3.20(\mathrm{NZP})\end{array}$

\begin{tabular}{|c|l|l|l|}
\hline \multirow{2}{*}{$\mathbf{M}$} & \multicolumn{3}{|c|}{$\mathbf{x}$} \\
\cline { 2 - 4 } & \multicolumn{1}{|c|}{$\mathbf{N Z P}$} & \multicolumn{1}{|c|}{$\mathbf{N Z P}+\mathbf{M O}_{2}$} & \multicolumn{1}{|c|}{ NaThP } \\
\hline $\mathrm{Np}$ & $0 \leq \mathrm{x} \leq 0.6$ & $0.6<\mathrm{x}<1.8$ & $1.8 \leq \mathrm{x} \leq 2.0$ \\
& $\bullet$ & 0 & $\bullet$ \\
$\mathrm{Pu}$ & $0 \leq \mathrm{x} \leq 0.2$ & $0.2<\mathrm{x}<2.0$ & 2.0 \\
& 0 & 0 & $\bullet$ \\
\hline
\end{tabular}

Solid Solutions $\mathrm{NaZr}_{2-\mathrm{x}} \mathrm{M}_{\mathrm{x}}\left(\mathrm{PO}_{4}\right)_{3} . \quad \mathrm{T}=1000^{\circ} \mathrm{C}$ Supposed limitless solid solutions $\mathrm{Np}-\mathrm{Zr} \quad \mathrm{T}>1350^{\circ} \mathrm{C} \quad 0 \leq \mathrm{x} \leq 2 \quad \mathrm{Pu}-\mathrm{Zr} \quad \mathrm{T}>1050^{\circ} \mathrm{C} \quad 0 \leq \mathrm{x} \leq 2$

The phosphate of the $\mathrm{NaPu}_{2}\left(\mathrm{PO}_{4}\right)_{3}$ composition was prepared by solid state reactions:

$$
\begin{array}{ll}
\mathrm{NaH}_{2} \mathrm{PO}_{4}+\mathrm{PuO}_{2}+\left(\mathrm{B}_{2} \mathrm{O}_{3}\right), & \text { at } \mathrm{T}=1200{ }^{\circ} \mathrm{C} \\
\mathrm{NaH}_{2} \mathrm{PO}_{4}+\mathrm{PuO}_{2}+\mathrm{H}_{3} \mathrm{PO}_{4}, & \text { at } \mathrm{T} \text { up to } 1100{ }^{\circ} \mathrm{C} .
\end{array}
$$

Also the solid state solutions $\mathrm{NaZr}_{2-\mathrm{x}} \mathrm{Pu}_{\mathrm{x}}\left(\mathrm{PO}_{4}\right)_{3}$ and $\mathrm{NaZr}_{2-\mathrm{x}} \mathrm{Np}_{\mathrm{x}}\left(\mathrm{PO}_{4}\right)_{3}$ were synthesized at $1000^{\circ} \mathrm{C}$.

Structure of $\mathrm{NaPu}_{2}\left(\mathrm{PO}_{4}\right)_{3}$ :and of solid solutions $\mathrm{NaZr}_{2-x} \mathrm{M}_{\mathrm{x}}\left(\mathrm{PO}_{4}\right)_{3}(\mathrm{M}-\mathrm{U}, \mathrm{Np}, \mathrm{Pu})$ were established by the structural investigations as two crystalline forms of $\mathrm{NaPu}_{2}\left(\mathrm{PO}_{4}\right)_{3}$ :

- $\mathrm{NaTh}_{2}\left(\mathrm{PO}_{4}\right)_{3}$ (NThP)- type, sp. gr. C2/c

- Kosnarite type (NZP), sp. gr. R $\overline{3} \mathrm{c}$

and also solid solutions of kosnarite type (NZP), sp. gr. R $\overline{3} \mathrm{c}$, for the following compositions:

- $\mathrm{NaZr}_{2-\mathrm{x}} \mathrm{Pu}_{\mathrm{x}}\left(\mathrm{PO}_{4}\right)_{3} \quad 0 \leq \mathrm{x} \leq 0,2 \quad$ (prepared at $1000^{\circ} \mathrm{C}$ )

- $\mathrm{NaZr}_{2-\mathrm{x}} \mathrm{Np}_{\mathrm{x}}\left(\mathrm{PO}_{4}\right)_{3} \quad 0 \leq \mathrm{x} \leq 0,6 \quad$ (prepared at $1000^{\circ} \mathrm{C}$ )

- $\mathrm{KZr}_{2-\mathrm{x}} \mathrm{U}_{\mathrm{x}}\left(\mathrm{PO}_{4}\right)_{3} \quad 0 \leq \mathrm{x} \leq 0,2 \quad$ (prepared at $1100^{\circ} \mathrm{C}$ ) (ref.).III. Realized Structure Types (Slide 3,

Fig. 2).

Among the f-element (III) and (IV) phosphate compositions investigated, the following structure types were realized.

- $\quad$ kosnarite $\left(\mathrm{NaZr}_{2}\left(\mathrm{PO}_{4}\right)_{3}, \mathrm{NZP}\right)$ - type, sp. gr. $\mathrm{R} \overline{3} \mathrm{c}$

- monazite $\left(\mathrm{CePO}_{4}\right)$ - type, sp. gr. $\mathrm{P} 2_{1} / \mathrm{n}$

- $\quad \mathrm{NaTh}_{2}\left(\mathrm{PO}_{4}\right)_{3}(\mathrm{NThP})$ - type, sp. gr. C2/c

In these compounds, the coordination numbers of cations forming the framework are different: 6, 9, 9 . 

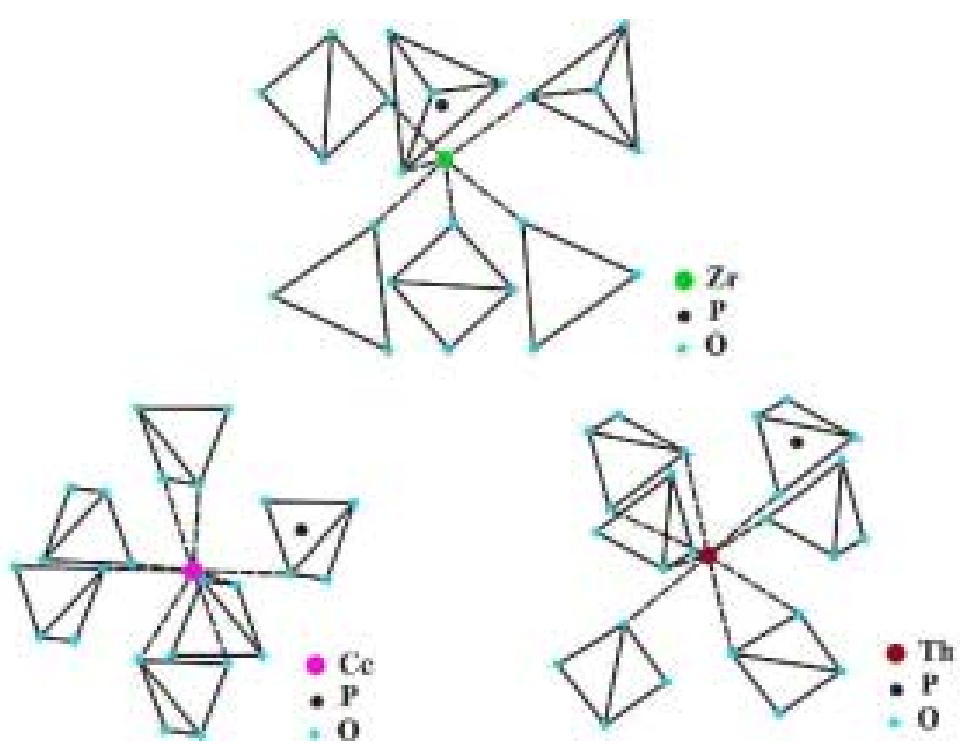

Fig. 2. Realized structure types.

\section{Kosnarite structure-the only single-phase, mineral-like radwaste matrix (Slides 4 and 5)}

It is obvious, that complex phosphates of f-elements (III), (IV) and zirconium may form solid solutions with a kosnarite-type structure. This structure, which is an analog of $\mathrm{NaZr}_{2}\left(\mathrm{PO}_{4}\right)_{3}$, is presented in Fig. 3 .

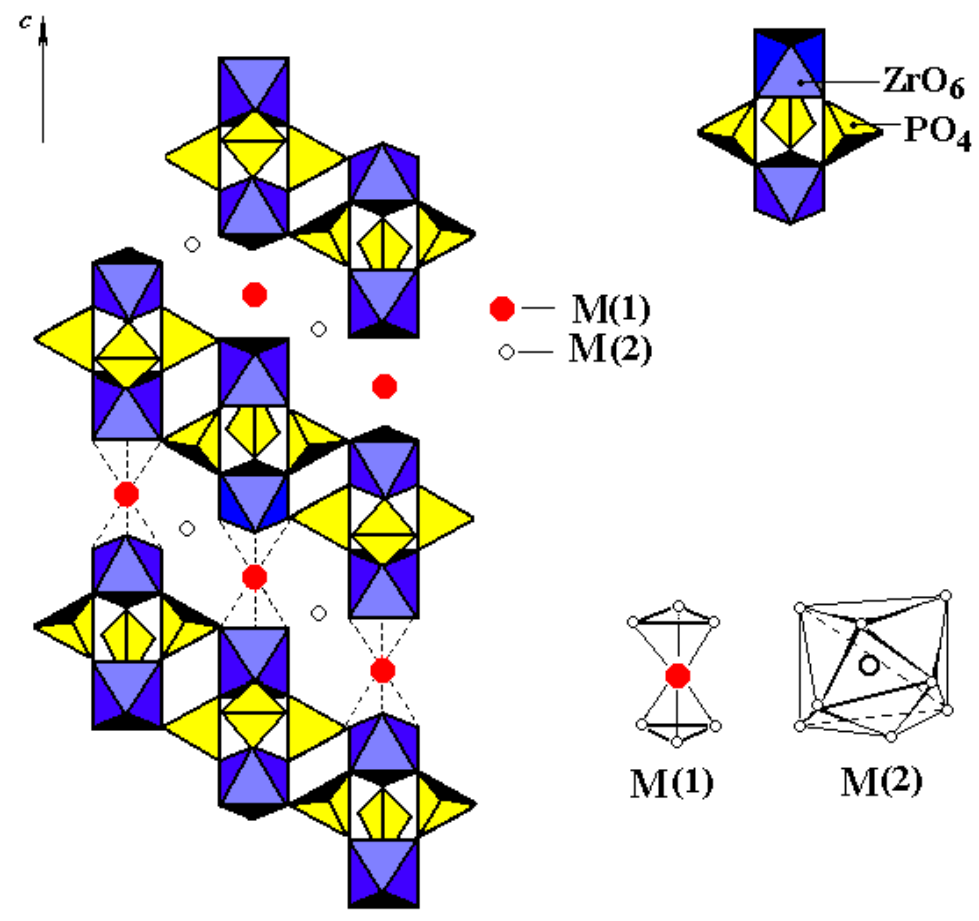

Fig. 3. Kosnarite structure, an analog of $\mathrm{NaZr}_{2}\left(\mathrm{PO}_{4}\right)_{3}$ 


\begin{tabular}{|c|c|}
\hline $\begin{array}{l}\text { M1 position } \\
\mathrm{Li}, \mathrm{Na}, \mathrm{K}, \mathrm{Rb}, \mathrm{Cs} \\
\mathrm{H}, \mathrm{Cu}(\mathrm{I}), \mathrm{Ag} \\
\mathrm{Mg}, \mathrm{Ca}, \mathrm{Sr}, \mathrm{Ba} \\
\mathrm{Mn}, \mathrm{Co}, \mathrm{Ni}, \mathrm{Cu}, \mathrm{Zn}, \mathrm{Cd} \\
\mathrm{Sc}, \mathrm{Fe}, \mathrm{Y}, \mathrm{La}, \mathrm{Ce}-\mathrm{Lu} \\
\mathrm{Zr}, \mathrm{Hf}, \mathrm{Nb}\end{array}$ & $\begin{array}{l}\text { L position } \\
\mathrm{Nb}, \mathrm{Sb}, \mathrm{Ta} \\
\mathrm{Ti}, \mathrm{Zr}, \mathrm{Hf}, \mathrm{Ge}, \mathrm{Sn} \\
\mathrm{U}(\mathrm{IV}), \mathrm{Np} \text { (IV), Pu(IV) } \\
\mathrm{Al}, \mathrm{Cr}, \mathrm{Fe}, \mathrm{Ga}, \mathrm{Sm}-\mathrm{Yb} \\
\mathrm{MG}, \mathrm{CA}, \mathrm{SR} \\
\text { NA, K }\end{array}$ \\
\hline & $\left.\mathrm{OO}_{4}\right)_{3}$ \\
\hline $\begin{array}{l}\text { M2 position } \\
\mathrm{Li}, \mathrm{Na}, \mathrm{K}, \mathrm{Rb} ; \mathrm{Mg}, \mathrm{Ca}, \mathrm{Sr}\end{array}$ & $\begin{array}{l}\text { P position } \\
\mathrm{Si}, \mathrm{P}, \mathrm{V}, \mathrm{As}, \mathrm{S}\end{array}$ \\
\hline
\end{tabular}

Fig. 4. Chemical substitutions in phosphates with $\mathrm{NaZr}_{2}\left(\mathrm{PO}_{4}\right)_{3}$ structure type.

Phosphates of the $\mathrm{NaZr}_{2}\left(\mathrm{PO}_{4}\right)_{3}$ structure type are the only known single-phase host crystalline structure that allows the incorporation of almost all nuclides presented in a typical nuclear waste (Slide 5; Fig. 4).

\section{Radiation testing ( $\alpha$ - and $\gamma$ - irradiation)}

Radiation testing of the phosphates were carried out for $\mathrm{Am}_{2}\left(\mathrm{PO}_{4}\right)_{3} \mathrm{~A}=\mathrm{Li}, \mathrm{Na}, \mathrm{K}, \mathrm{Rb}, \mathrm{Cs}, \mathrm{M}=\mathrm{Ti}, \mathrm{Zr}, \mathrm{Hf}$, and cal-alkaline, $\mathrm{REE}, \mathrm{Nb}$, and other element phosphates were also tested. Testing conditions included:

External irradiation ${ }^{60} \mathrm{Co}$ source -, $\mathrm{Y}$ - radiation

- Accumulated dose $510^{8} \mathrm{~Gy}, 0.5 \mathrm{y}$; Chemical or structural destruction were not established (x-ray, IR) Internal irradiation. $\mathrm{NaPu}_{2}\left(\mathrm{PO}_{4}\right)_{3}$

- $\quad{ }^{239} \mathrm{Pu}$, during 2 years; $9.1 \cdot 10^{13} \mathrm{a}$-particles $/ 1 \mathrm{mg}$ of phosphate and such amounts of recoil atoms were isolated. Chemical or structural destruction were not established (x-ray).

- ${ }^{238} \mathrm{Pu}$ (up to $79 \%$ ) + mixture ${ }^{239} \mathrm{Pu},{ }^{240} \mathrm{Pu} ; 9.3 \cdot 10^{15} \mathrm{\alpha}$-particles/1 mg of phosphate and such amounts of recoil atoms were isolated.

- Phosphate metamictization are appeared after 1 month exposure.

Limit doses of metamictization of Pu-phosphate, Synroc (Ref.) and US Titanate ceramic (Ref.) are compared in Table 1.

Table 1. Radiation testing results.

\begin{tabular}{|l|l|l|}
\hline \multicolumn{1}{|c|}{$\mathrm{NaPu}_{2}\left(\mathrm{PO}_{4}\right)_{3}$} & \multicolumn{1}{|c|}{ Synroc } & \multicolumn{1}{c|}{ US titanate ceramic } \\
\hline $\begin{array}{l}{ }^{239} \mathrm{Pu}, 61 \% \text { mass. During } 2 \text { years } \\
\text { appreciable changes didn't find out } \\
9,1 \cdot 10^{16} \alpha / \mathrm{g}\end{array}$ & - & - \\
\hline $\begin{array}{l}{ }^{238} \mathrm{Pu}, 48 \% \text { (mass.) Metamictization } \\
\text { after } 1 \text { month, } 9,3 \cdot 10^{18} \alpha / \mathrm{g}\end{array}$ & $\begin{array}{l}\text { Metamictization (perovskite, } \\
\text { zirconolite) } 4,7 \cdot 10^{18} \alpha / \mathrm{g}\end{array}$ & $\begin{array}{l}238 \mathrm{Pu}, 8,7 \% \text { (mass.) Metamictization } \\
\text { after } 6 \text { months, } 8,8 \cdot 10^{17} \alpha / \mathrm{g}\end{array}$ \\
\hline $\begin{array}{l}\mathrm{X} \text {-ray changes were appreciable if } \\
1,3-1,5 \cdot 10^{18} \alpha / \mathrm{g} \text { were emancipated }\end{array}$ & & $\begin{array}{l}\mathrm{X} \text {-ray changes were appreciable if } \\
0,5-2,5 \cdot 10^{17} \alpha / \mathrm{g} \text { were emancipated }\end{array}$ \\
\hline
\end{tabular}




\section{Conclusions}

1. Phosphates of Plutonium (III), Americium (III), Curium (III) and Lanthanides (III) Na(K) $)_{3} \mathrm{An}(\mathrm{Ln})_{2}\left(\mathrm{PO}_{4}\right)_{3}$ and Plutonium (IV) $\mathrm{NaPu}_{2}\left(\mathrm{PO}_{4}\right)_{3}$ were prepared. Solid solutions of $\mathrm{Zr}$ and $\mathrm{An}(\mathrm{Np}, \mathrm{Pu}$ ) phosphates were also prepared.

2. The $\mathrm{Pu}, \mathrm{Am}, \mathrm{Cm}$ phosphate structure types were determined: monazite, kosnarite, $\mathrm{NaTh}_{2}\left(\mathrm{PO}_{4}\right)_{3}-$ types. The existence of the f-element and $\mathrm{Zr}$ phosphate solid solutions having kosnarite -type structure was established.

3. The radiation ( $\alpha$ and $\gamma$ ) testing were carried out. The metamictization dose limits of Pu-containing phosphates were determined and were compared with ones for other known Pu-containing materials.

\section{References}

1. A.A. Burnaeva, Yu. F. Volkov, A.I. Krukova. Crystal Structure Peculiarity of Double Plutonium (III) and Sodium Phosphates and some other f-element Phosphates. Radiochemistry. 1994. V. 36. N4. P. 288 $-294$.

2. M. Vlasse, C. Parent, R. Salmon, G. Le Flem, P. Hagenmuller. The Structure of the $\mathrm{Na}_{3} \mathrm{Ln}\left(\mathrm{XO}_{4}\right)_{2}$ phases ( $L n=$ Rare Earth, $X=P, V$, As). Solid State Chem. 1980. V.35. P. $318-324$.

3. A. A. Burnaeva, Yu. F. Volkov, A. I. Krukova, I. A. Korshunov, O. V. Skiba. Dimorphism and Structure of Plutonium Phosphate $\mathrm{NaPu}_{2}\left(\mathrm{PO}_{4}\right)_{3}$. Radiochemistry. 1992. Vol. 34. N 5, P. $12-21$.

4. Y. Miyajima, T. Miyoshi, Y. Tamaki, M. Matsuoka, Y. Yamamoto, Ch. Masquelier, M. Tabuchi, Yu. Saito, H. Kageyama. Solubility Range and lonic Conductivity of large trivalent ion doped $\mathrm{Na}_{1+x} \mathrm{M}_{\mathrm{x}} \mathrm{Zr}_{2-}$ ${ }_{x} \mathrm{P}_{3} \mathrm{O}_{12}$ (M:In, Yb,Er, Y,Dy,Tb,Gd) solid electrolites. Solid State Ionics. 1999. V. 124. P. 201 - 211.

5. A. I. Orlova. Isomorphism in Crystal $\mathrm{NaZr}_{2}\left(\mathrm{PO}_{4}\right)_{3}$-like Phosphates. Radiochemistry. 2002. In press.

6. H. T. Hawkins, D. R. Spearing, D. K. Veirs, J. A. Danis, D. M. Spilde, B. E. Scheetz. Synthesis and Characterization of Uranium (IV)-Bearing Members of the NZP structural Family. Chem. Mater. 1999. N11. P. $2851-2857$.

7. A. I. Krukova, I. A. Kulikov, G. Yu. Artemeva, O. V. Pechenevskaya, V. A. Alferov. Crystal Phosphate $\mathrm{NaZr}_{2}\left(\mathrm{PO}_{4}\right)_{3}$ - family. Radiation stability. Radiochemistry. 1992. V. 34, N6. P. $82-89$.

8. A. I. Orlova, Yu. F. Volkov, R. F. Melkaya, L. Yu. Masterova, I. A. Kulikov, V. A. Alferov. Synthesis and Radiation Stability of NZP-structure Phosphates containing f-elements. Radiochemistry). 1994. V. 36, N4. P. $295-298$.

9. A. A. Charlamova, A. I. Orlova, Yu. F. Volkov. f-element (III) Phosphates (Ce-Cd, Pu, Am, Cm). In Book of Abstracts of 3rd Russian Radiochemistry Conference "Radiochemistry 2000". St. Petersburg. 2000. P. $61-62$.

10. Yu. F. Volkov, A. N. Lukinikh, S. V. Tomilin, A. V. Bychkov. Investigation of U.S. Titanate Ceramics Radiation Damage Due to ${ }^{238} \mathrm{Pu}$ Alpha-Decay. In Book of Proceedings: Immobilization of Excess Weapon Plutonium in Russia. A Review of LLNL Contract Work. 2000 St. Petersburg Meeting. 2001. LLNL.(Ed. L. J. Jardine, G. B. Borisov). P. $197-219$.

11. A.E.Ringwood, S. E. Kesson, K. D. Reeve, D. M. Levins, E. J. Ramm. Synroc. Ch. 4. In Book: Radioactive Waste Forms for the Future. Ed. W. Lutze, R.C. Ewing. 1988. P. 233 - 334. 


\section{Slide 1. $\mathrm{Na}(\mathrm{K})_{3} \mathrm{An}_{2}\left(\mathrm{PO}_{4}\right)_{3}$}

monazite type, sp. gr. $P 2_{1} / n$

$\begin{array}{lllll}a, \AA & b, \AA & c, \AA & \beta^{0} & V, \AA^{3} z\end{array}$

$\begin{array}{lllllll}\mathrm{Na}_{3} \mathrm{Pu}_{2}\left(\mathrm{PO}_{4}\right)_{3} & 6,69 & 6,92 & 6,39 & 104,07 & 287 & 1\end{array}$

$\begin{array}{lllllll}\mathrm{Na}_{3} \mathrm{Am}_{2}\left(\mathrm{PO}_{4}\right)_{3} & 6,78 & 7,00 & 6,44 & 103,75 & 297 & 1\end{array}$

$\begin{array}{lllllll}\mathrm{K}_{3} \mathrm{Am}_{2}\left(\mathrm{PO}_{4}\right)_{3} & 6,74 & 6,98 & 6,44 & 103,80 & 294 & 1\end{array}$

$\begin{array}{lllllll}\mathrm{K}_{3} \mathrm{Cm}_{2}\left(\mathrm{PO}_{4}\right)_{3} & 6,77 & 7,09 & 6,35 & 104,08 & 312 & 1\end{array}$

Reaction $\quad \mathrm{RCl}_{3}+\mathrm{A}_{3} \mathrm{PO}_{4} \rightarrow \mathrm{A}_{3} \mathrm{R}_{2}\left(\mathrm{PO}_{4}\right)$

$\mathrm{ACl}$ (melt)

$\mathrm{Na}(\mathrm{K})_{3} \mathrm{Ln}_{2}\left(\mathrm{PO}_{4}\right)_{3}$

Ln = Ce, Pr, Nd, Sm, Eu, Gd
a, $\AA$
$b, \AA$
c, $\AA$
$\beta, \circ$

$6.82-6.73 \quad 7.05-6.92$

6.38-6.44 103.2-103.3

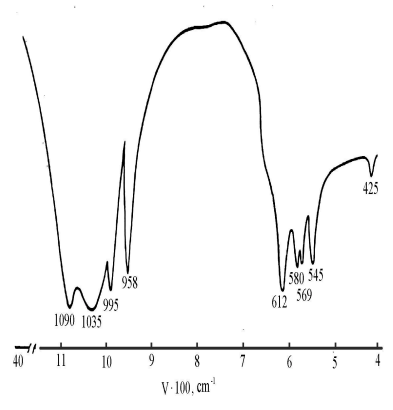

$\mathrm{Na}_{3} \mathrm{Pu}_{2}\left(\mathrm{PO}_{4}\right)_{3}$

$\mathrm{Na}_{3} \mathrm{R}\left(\mathrm{PO}_{4}\right)_{2}$

Glaserite type, sp. gr. Pnmm

a, $\AA \quad b, \AA \quad c, \AA \quad z$

$\mathrm{Na}_{3} \mathrm{Pu}\left(\mathrm{PO}_{4}\right)_{2} \quad 18,44 \quad 7,00 \quad 15,8612$

$\mathrm{Na}_{3} \operatorname{Tm}\left(\mathrm{PO}_{4}\right)_{2} \quad 18,43 \quad 6,97$ 15,82 12 (analog) (Ref.)

Reaction

$\mathrm{PuCl}_{3}+\mathrm{Na}_{3} \mathrm{PO}_{4} \rightarrow \mathrm{Na}_{3} \mathrm{Pu}\left(\mathrm{PO}_{4}\right)_{2} \downarrow$

$\mathrm{NaCl}$ (melt)

The structures of the synthesized f-element (III) phosphates were established:

$\mathrm{Na}_{3} \mathrm{Pu}_{2}\left(\mathrm{PO}_{4}\right)_{3} \quad$ monazite-type, sp. gr. $\mathrm{P} 2_{1} / \mathrm{n}$

$\mathrm{Na}_{3} \mathrm{Am}_{2}\left(\mathrm{PO}_{4}\right)_{3} \quad$ monazite-type, sp. gr. $\mathrm{P}{ }_{1} / \mathbf{n}$

$\mathrm{K}_{3} \mathrm{Am}_{2}\left(\mathrm{PO}_{4}\right)_{3} \quad$ monazite-type, sp. gr. $\mathrm{P}{ }_{1} / \mathrm{n}$

$\mathrm{K}_{3} \mathrm{Cm}_{2}\left(\mathrm{PO}_{4}\right)_{3} \quad$ monazite-type, sp. gr. $\mathrm{P} 2_{1} / \mathrm{n}$

$\mathrm{Na}_{3} \mathrm{Pu}\left(\mathrm{PO}_{4}\right)_{2} \quad$ glaserite-type, sp. gr. Pnmm (analog $\mathrm{Na}_{3} \mathrm{Tm}\left(\mathrm{PO}_{4}\right)_{2}$, having HT sp. gr. $\mathrm{R} \overline{3} \mathrm{c}$ ) (Ref.).

The Solid solutions $\mathrm{Na}_{1+\mathrm{x}} \mathrm{Zr}_{2-\mathrm{x}} \mathrm{Ln}_{\mathrm{x}}\left(\mathrm{PO}_{4}\right)_{3}$ (the limits from $\mathrm{Yb} \mathrm{x}=1$ up to $\left.\mathrm{Gd} \mathrm{x}=0,2\right)$ were prepared and identified as having kosnarite structure type (type $\mathrm{NaZr}_{2}\left(\mathrm{PO}_{4}\right)_{3}, \mathrm{NZP}$ ), sp. gr. $\mathrm{R} \overline{\mathrm{c}} \mathrm{c}$ (our and ref.). 


\section{Slide 2. Phosphate $\mathrm{NaPu}_{2}\left(\mathrm{PO}_{4}\right)_{3}$ and $\mathrm{Zr}$-Pu Phosphate Solid Solutions}
$\mathrm{NaTh}_{2}\left(\mathrm{PO}_{4}\right)_{3} \quad 17.28 \AA \quad 6.81 \AA$
$8.15 \AA \quad 101.3^{\circ}(\mathrm{NThP})$
- $\mathrm{NaPu}_{2}\left(\mathrm{PO}_{4}\right)_{3} \quad 17.14 \quad 6.65$
$7.99 \quad 101.1$
$\mathrm{NaPu}_{2}\left(\mathrm{PO}_{4}\right)_{3} \quad 9.09 \quad 23.69 \quad 3.98 \mathrm{~g} / \mathrm{cm}^{3}$
$\mathrm{NaZr}_{2}\left(\mathrm{PO}_{4}\right)_{3} \quad 8.80 \quad 22.78 \quad 3.20$ (NZP)

\begin{tabular}{|c|c|c|c|}
\hline \multirow{2}{*}{ M } & \multicolumn{3}{|c|}{$\mathbf{x}$} \\
\hline & NZP & $\mathrm{NZP}+\mathrm{MO}_{2}$ & NaThP \\
\hline $\mathrm{Np}$ & $0 \leq x \leq 0.6$ & $\begin{array}{l}0.6<x<1.8 \\
0\end{array}$ & $1.8 \leq x \leq 2.0$ \\
\hline $\mathrm{Pu}$ & $0 \leq x \leq 0.2$ & $\begin{array}{l}0.2<x<2.0 \\
0\end{array}$ & \\
\hline
\end{tabular}

Solid Solutions $\mathrm{NaZr}_{2-\mathrm{x}} \mathrm{M}_{\mathbf{x}}\left(\mathrm{PO}_{4}\right)_{3}$.

$$
\mathrm{T}=1000^{\circ} \mathrm{C}
$$

\section{Supposed limitless solid solutions}

\section{$\mathrm{Np}=\mathrm{Zr} \quad \mathrm{T}>1350^{\circ} \mathrm{C} \quad 0 \leq \mathrm{x} \leq 2 \quad \mathrm{Pu}-\mathrm{Zr} \quad \mathrm{T}>1050^{\circ} \mathrm{C} \quad 0 \leq \mathrm{x} \leq 2$}

The Phosphate of $\mathrm{NaPu}_{2}\left(\mathrm{PO}_{4}\right)_{3}$ composition was prepared by solid state reactions:

- $\mathrm{NaH}_{2} \mathrm{PO}_{4}+\mathrm{PuO}_{2}+\left(\mathrm{B}_{2} \mathrm{O}_{3}\right)$, at $\mathrm{T}=1200{ }^{\circ} \mathrm{C}$

- $\mathrm{NaH}_{2} \mathrm{PO}_{4}+\mathrm{PuO}_{2}+\mathrm{H}_{3} \mathrm{PO}_{4}$, at $\mathrm{T}$ up to $1100{ }^{\circ} \mathrm{C}$.

- Also solid state solutions $\mathrm{NaZr}_{2-\mathrm{x}} \mathrm{Pu}_{\mathrm{x}}\left(\mathrm{PO}_{4}\right)_{3}$ and $\mathrm{NaZr}_{2-\mathrm{x}} \mathrm{Np}_{\mathrm{x}}\left(\mathrm{PO}_{4}\right)_{3}$ were synthesized at $1000{ }^{0} \mathrm{C}$.

Structure of $\mathrm{NaPu}_{2}\left(\mathrm{PO}_{4}\right)_{3}$ :and of solid solutions $\mathrm{NaZr}_{2-\mathrm{x}} \mathrm{M}_{\mathbf{x}}\left(\mathrm{PO}_{4}\right)_{3}(\mathrm{M}-\mathrm{U}, \mathrm{Np}, \mathrm{Pu})$ are presented at Slide 2. The structural investigations allowed to establish two crystalline forms of $\mathrm{NaPu}_{2}\left(\mathrm{PO}_{4}\right)_{3}$ :

$$
\begin{aligned}
& \mathrm{NaTh}_{2}\left(\mathrm{PO}_{4}\right)_{3} \text { (NThP)- type, sp. gr. C2/c } \\
& \text { kosnarite type (NZP), sp. gr. } \mathrm{R} \overline{3} \mathrm{c}
\end{aligned}
$$

and also solid solutions of kosnarite type (NZP), sp. gr. $\mathbf{R} \overline{3}$, for the following compositions:

- $\mathrm{NaZr}_{2-\mathrm{x}} \mathrm{Pu}_{\mathrm{x}}\left(\mathrm{PO}_{4}\right)_{3} \quad 0 \leq \mathrm{x} \leq 0,2 \quad$ (prepared at $1000{ }^{\circ} \mathrm{C}$ )

- $\mathrm{NaZr}_{2-\mathrm{x}} \mathrm{Np}_{\mathrm{x}}\left(\mathrm{PO}_{4}\right)_{3} \quad 0 \leq \mathrm{x} \leq 0,6 \quad$ (prepared at $1000{ }^{\circ} \mathrm{C}$ )

- $\mathrm{KZr}_{2-\mathrm{x}} \mathrm{U}_{\mathrm{x}}\left(\mathrm{PO}_{4}\right)_{3} \quad 0 \leq \mathrm{x} \leq 0,2 \quad$ (prepared at $1100{ }^{\circ} \mathrm{C}$ ) (ref.). 


\section{Slide 3. Realized Structure Types}

Among the f-element (III) and (IV) phosphate compositions investigated, the following structure types realized.
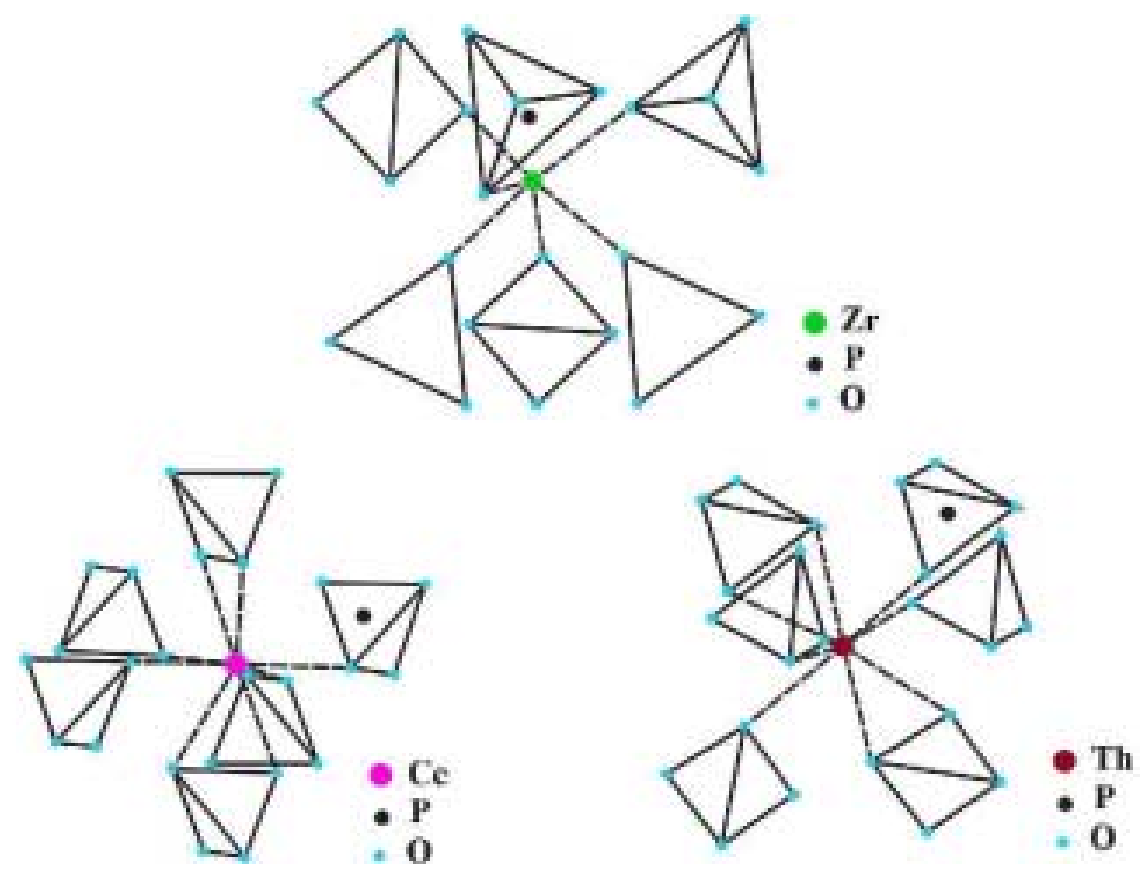

\footnotetext{
kosnarite $\left(\mathrm{NaZr}_{2}\left(\mathrm{PO}_{4}\right)_{3}, \mathrm{NZP}\right)$ - type, sp. gr. $\mathrm{R} \overline{\mathrm{3}} \mathrm{c}$

monazite $\left(\mathrm{CePO}_{4}\right)$ - type, sp. gr. $\mathrm{P}_{1} / \mathrm{n}$

$\mathrm{NaTh}_{2}\left(\mathrm{PO}_{4}\right)_{3}(\mathrm{NThP})$ - type, sp. gr. C2/c
}

In this compounds the coordination numbers of cations forming the framework are different: 6, 9, 9. 


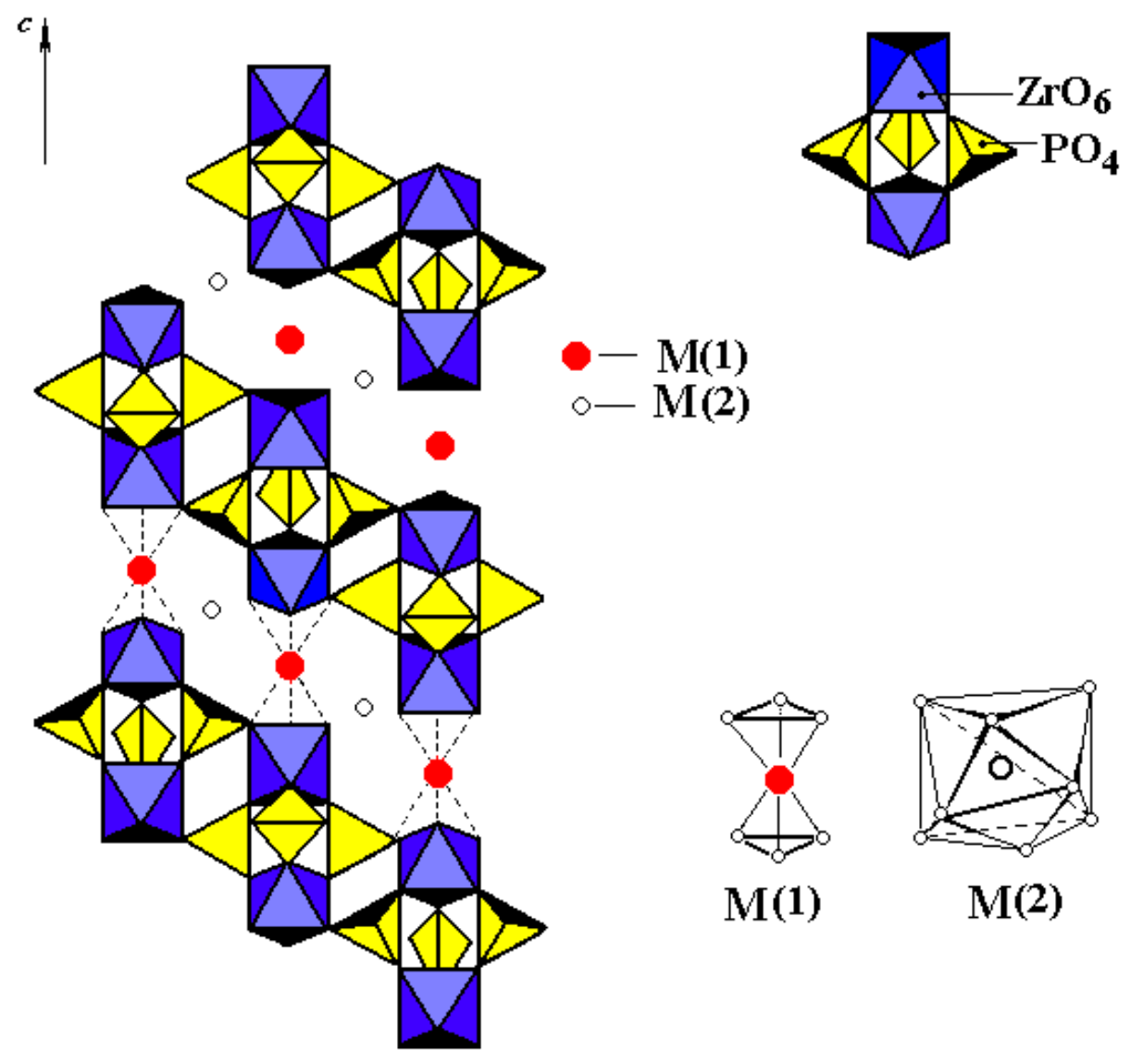


Slide 5. Chemical Substitutions in Phosphates $\mathrm{NaZr}_{2}\left(\mathrm{PO}_{4}\right)_{3}$ Structure Type

\begin{tabular}{|c|c|}
\hline $\begin{array}{l}\text { M1 position } \\
\mathrm{Li}, \mathrm{Na}, \mathrm{K}, \mathrm{Rb}, \mathrm{Cs} \\
\mathrm{H}, \mathrm{Cu}(\mathrm{I}), \mathrm{Ag} \\
\mathrm{Mg}, \mathrm{Ca}, \mathrm{Sr}, \mathrm{Ba} \\
\mathrm{Mn}, \mathrm{Co}, \mathrm{Ni}, \mathrm{Cu}, \mathrm{Zn}, \mathrm{Cd} \\
\mathrm{Sc}, \mathrm{Fe}, \mathrm{Y}, \mathrm{La}, \mathrm{Ce}-\mathrm{Lu} \\
\mathrm{Zr}, \mathrm{Hf}, \mathrm{Nb}\end{array}$ & $\begin{array}{l}\text { L position } \\
\mathrm{Nb}, \mathrm{Sb}, \mathrm{Ta} \\
\mathrm{Ti}, \mathrm{Zr}, \mathrm{Hf}, \mathrm{Ge}, \mathrm{Sn} \\
\mathbf{U}(\mathbf{I V}), \mathbf{N p} \text { (IV), Pu(IV) } \\
\mathrm{Al}, \mathrm{Cr}, \mathrm{Fe}, \mathrm{Ga}, \mathrm{Sm}-\mathrm{Yb} \\
\mathrm{Mg}, \mathrm{Ca}, \mathrm{Sr} \\
\mathrm{Na}, \mathrm{K}\end{array}$ \\
\hline \multicolumn{2}{|c|}{$(\mathrm{M} 1)(\mathrm{M} 2)_{3} \mathrm{~L}_{2}\left(\mathrm{PO}_{4}\right)_{3}$} \\
\hline $\begin{array}{l}\text { M2 position } \\
\mathrm{Li}, \mathrm{Na}, \mathrm{K}, \mathrm{Rb} ; \mathrm{Mg}, \mathrm{Ca}, \mathrm{Sr}\end{array}$ & $\begin{array}{l}\text { P position } \\
\text { Si, } \mathrm{P}, \mathrm{V}, \mathrm{As}, \mathrm{S}\end{array}$ \\
\hline
\end{tabular}




\section{Slide 6. Radiation Testing of Phosphates: Conditions and Results}

External irradiation ${ }^{60} \mathrm{Co}$ source -, $\mathrm{y}$ - radiation

accumulated doses $510^{8}$ Gy 0.5 year

Phosphates $\mathrm{AM}_{2}\left(\mathrm{PO}_{4}\right)_{3} \mathrm{~A}=\mathrm{Li}, \mathrm{Na}, \mathrm{K}, \mathrm{Rb}, \mathrm{Cs}, \mathrm{M}=\mathrm{Ti}, \mathrm{Zr}$, Hf, and cal-alkaline, $\mathrm{REE}, \mathrm{Nb}$ and other element phosphates were tested

Chemical or structural destruction were not established (X-ray, IR)

Internal irradiation. $\mathrm{NaPu}_{2}\left(\mathrm{PO}_{4}\right)_{3}$

${ }^{239} \mathrm{Pu}$, during 2 years

9.1.10 ${ }^{13} \alpha$-particles/1mg of phosphate and such amounts of recoil atoms were isolated Chemical or structural destruction were not established (X-ray).

${ }^{238} \mathrm{Pu}$ (up to $79 \%$ ) + mixture ${ }^{239} \mathrm{Pu},{ }^{240} \mathrm{Pu}$

9.3.10 ${ }^{15} \alpha$-particles/1 $\mathrm{mg}$ of phosphate and such amounts of recoil atoms were isolated Phosphate metamictization are appeared after 1 month exposition

Table 1. Comparison of limit doses of metamictization of Pu phosphate, Synroc, and US Titanate ceramic.

\begin{tabular}{|c|c|c|}
\hline \multicolumn{1}{|c|}{$\mathrm{NaPu}_{2}\left(\mathrm{PO}_{4}\right)_{3}$} & Synroc & US titanate ceramic \\
\hline${ }^{239} \mathrm{Pu}, 61 \%$ mass. & - & - \\
During 2 years appreciable & & \\
Changes didn't find out & & \\
$9,1 \cdot 10^{16} \alpha / \mathrm{g}$ & & ${ }^{138} \mathrm{Pu}, 8,7 \%$ (mass.) \\
& & Metamictization \\
${ }^{238} \mathrm{Pu}, 48 \%$ (mass.) & after 6 months \\
Metamictization & Metamictization & $8,8 \cdot 10^{17} \alpha / \mathrm{g}$ \\
after 1 month & (perovskite, zirconolite) & X-ray changes \\
$9,3 \cdot 10^{18} \alpha / \mathrm{g}$ & $4,7 \cdot 10^{18} \alpha / \mathrm{g}$ & were appreciable if \\
\hline X-ray changes & & $0,5-2,5 \cdot 10^{17} \alpha / \mathrm{g}$ \\
were appreciable if & & were emancipated \\
$1,3-1,5 \cdot 10^{18} \alpha / \mathrm{g}$ & & \\
were emancipated & &
\end{tabular}




\title{
Alkaline Tank Chemistry of Plutonium: Speciations, Redox Reactions and Structural Transformations
}

\author{
I. G. Tananaev, B. F. Myasoedov, RAS-GEOKHI
}

\section{Introduction}

Highly alkaline radioactive wastes exist in aging tanks in many countries. In the US, they are located at Hanford, Savannah River, INEL, West Valley, and Oak Ridge. In Russia, alkaline wastes are situated in the "Mayak" reprocessing plant. These tanks contain the actinide elements uranium, neptunium, plutonium, and americium in quantities of concern for separation, treatment, and disposal $[1,2]$.

Due to their long half lives, these transuranic (TRU) wastes will require disposal in a high-level nuclear repository [3]. Approximately 200,000 curies of actinides (plus technetium) in the US are estimated to be in the waste tanks, including 540-860 kg [1,2] of Pu. Knowledge of actinide elements accumulated in highly alkaline conditions is essential to understand and predict their solubility, sorption, phase distribution in tanks; to determine whether chemical separations are needed for waste treatment, design, and optimization of a rational separations process; and to determine the molecular level compatibility with the vitrification process $[4,5]$.

As we enter this cleanup phase, reduction of immobilized high level waste (HLW) volume has become an increasingly important and desirable outcome. One of the options for HLW cost reductions that both Savannah River and Hanford Sites are pursuing is caustic sludge washing [6-8]. The use of caustic digestion in HLW processing schemes introduces a new concern that TRU solubilities, notably for Pu and Am, will be excessive and therefore require further processing of the low activity waste (LAW) stream. Recent work on the caustic digestion of Hanford sludges has shown a large variability in Pu solubility. In particular, Lumetta et al. [9] found a $6 \%$ loss with a $3 \mathrm{M} \mathrm{NaOH}$ caustic wash at $100^{\circ} \mathrm{C}$ for 5 hours (tank 241-S-104, "Redox" sludge). In contrast, Temmer, et al. [10] found no detectable Pu dissolution under ostensibly identical conditions with identical sludge samples. These results suggest that knowledge of $\mathrm{Pu}$ behavior in basic media is not yet well understood.

The primary goal of our investigation was to obtain data on the composition and properties of plutonium (IV) hydrous oxide compounds formed in alkaline media under different conditions. Such information is important to understand Pu (IV) behavior, and the forms of its existence, in alkaline tank waste sludge. This knowledge then may be applied in assessing plutonium criticality hazards in the storage, retrieval, and treatment of Hanford Site tank wastes.

Studies of $\mathrm{Pu}(\mathrm{VI})$ stability in 1 to $15 \mathrm{M} \mathrm{NaOH}$ revealed that plutonium concentrations decreased with time [11]. A corresponding solid phase increased with time while decreasing alkali concentration accelerated the plutonium precipitation rate. It is presumed that $\mathrm{H}_{2} \mathrm{O}_{2}$ formed in solutions by radiolysis reduced $\mathrm{Pu}(\mathrm{VI})$ to $\mathrm{Pu}(\mathrm{V})$. In these tests, the $\mathrm{Pu}(\mathrm{V})$ subsequently disproportionated.

The second objective of the present work was to study the stability of $\mathrm{Pu}(\mathrm{V})$ over a wide range of $[\mathrm{NaOH}]$ and to assess the rate and extent of the postulated disproportionation reaction. 


\section{Reagents and Equipment}

Most experiments were carried out using $\mathrm{Pu}$ (IV) nitrate solutions with known concentrations of $\mathrm{HNO}_{3}$. Preparation of the solutions was performed by following method: to $\sim 0.05 \mathrm{M}$ plutonium nitrate in $\sim 3 \mathrm{M}$ $\mathrm{HNO}_{3}$, purified by the common anion exchange method, hydrogen peroxide up to $0.05 \mathrm{M}$ was added and the mixture was heated on a boiling water bath to attain a sharp change of solution color from blue to brown, which indicated a full $\mathrm{H}_{2} \mathrm{O}_{2}$ decomposition and a transition of $\mathrm{Pu}$ (III) into $\mathrm{Pu}$ (IV). From this solution $\mathrm{Pu}\left(\mathrm{C}_{2} \mathrm{O}_{4}\right)_{2}{ }^{*} 6 \mathrm{H}_{2} \mathrm{O}$ was precipitated by the slow addition of $\sim 1 \mathrm{M}$ oxalic acid to $\sim 0.1 \mathrm{M}$ excess at slight heating. After coagulation for $\sim 2 \mathrm{~h}$, the compound was separated from the mother solution by centrifugation and washed by $1 \mathrm{M} \mathrm{HNO}_{3}+0.01 \mathrm{M} \mathrm{H}_{2} \mathrm{C}_{2} \mathrm{O}_{4}$. Then $\mathrm{Pu}\left(\mathrm{C}_{2} \mathrm{O}_{4}\right)_{2}{ }^{*} 6 \mathrm{H}_{2} \mathrm{O}$ was dissolved in concentrated $\mathrm{HNO}_{3}$ at boiling and the solution obtained was evaporated to a small volume. After cooling the solution was diluted by $\sim 4$ times by $2 \mathrm{M} \mathrm{HNO}_{3}$ and treated by $\mathrm{H}_{2} \mathrm{O}_{2}$ to stabilize plutonium in the tetravalent state as mentioned above.

By the method described above, two $\mathrm{Pu}$ (IV) stock solutions were prepared with metal concentrations 0.233 and $0.206 \mathrm{M}$, respectively. Free acid concentrations in the solutions were equal to 3.1 and $4.35 \mathrm{M}$. The tetravalent state of plutonium in the test solutions was verified by absorption spectrophotometry using a Shimadzu model UV-3100 PC UV-Vis-NIR spectrophotometer (Japan production). Plutonium concentrations were determined by a weight analysis. For this purpose, $0.1 \mathrm{ml}$ aliquots of $\mathrm{Pu}$ (IV) stock solutions were carefully (without boiling) evaporated to dryness in small Pt crucibles and residues were calcinated at $800^{\circ} \mathrm{C}$ for $\sim 2 \mathrm{~h}$ to $\mathrm{PuO}_{2}$. Free $\mathrm{HNO}_{3}$ contents in $\mathrm{Pu}$ (IV) solutions were determined by direct titration with $0.1 \mathrm{M} \mathrm{NaOH}$ in the presence of phenolphthalein as an indicator. At $\left[\mathrm{H}^{+}\right]$calculations, it was assumed that in the course of the titration 4 moles of sodium hydroxide were spent for the precipitation of 1 mole of $\mathrm{Pu}$ (IV).

Powder x-ray diffraction patterns of solid samples were measured by a 100-mm Guinier camera, model XDC-700, using CuK $\alpha_{1}$-radiation (Philips PW 1140/90/96 generator, Holland) and Si as internal standard. IR spectra were taken using a Specord-M80 instrument (Germany). The thermal analysis of compounds was performed by a Q-1500 D derivatograph (Hungary). Thermostats of U-2 model (Germany) have been used to maintain constant solution temperatures in the range 20 to $80^{\circ} \mathrm{C}$ within $\pm 0.2^{\circ} \mathrm{C}$. The separation of precipitates from supernatant liquids was performed using medical centrifuges (model CLK-1, Russia), provided a separation factor $1,750 \mathrm{~g}$. Weights of $\mathrm{Pu}(\mathrm{IV})$ compounds samples were determined by a Sartorius microbalance with a sensitivity $1 \mu \mathrm{g}$. The $\alpha$-activity of plutonium solutions was measured by a LS-6500 scintillation counter (USA, Beckman).

Working sodium hydroxide solutions were obtained by the dilution of $16.7 \mathrm{M}$ chemical purity grade $\mathrm{NaOH}$. They were stored in polyethylene bottles. Standard solutions of other reagents $\left(\mathrm{NaNO}_{2}, \mathrm{NaNO}_{3}, \mathrm{Na}_{2} \mathrm{SO}_{4}\right.$, $\mathrm{Na}_{3} \mathrm{PO}_{4}$ and so on) were prepared by dissolving corresponding salts in distilled water or by the neutralization of respective acids with $\mathrm{NaOH}$.

\section{Experimental Methods}

\section{Precipitation, coagulation and isolation of $\mathrm{Pu}$ (IV) compounds}

The preparation of $\mathrm{Pu}(\mathrm{IV})$ hydrous oxide samples was carried out by direct or reverse precipitation in polyethylene test-tubes using calculated amounts of $\mathrm{NaOH}$ to obtain a chosen excess of the precipitant. With direct precipitation, a calculated excess of ammonium or sodium hydroxide was added to a measured volume (usually $0.3-0.4 \mathrm{ml}$ ) of a Pu (IV) stock solution diluted 3 to 5 -fold by water. With reverse precipitation, $\mathrm{Pu}(\mathrm{IV})$ stock solution aliquots were poured into polyethylene test tubes containing solution 
with known concentration of $\mathrm{NaOH}$. In both cases, the resulting suspensions were stirred and kept for a controlled period of time at a chosen temperature in a thermostat or in a boiling water bath. The solid phases then were separated by centrifugation and washed 4-5 times with water. The $\mathrm{pH}$ value of the last wash water was not higher than 8 . Washed precipitates were collected in small crucibles and dried in a desiccator over $\mathrm{KOH}$ pellets and converted into powders.

For the preparation of $\mathrm{Pu}$ (IV) hydrous oxide samples under hydrothermal conditions, $\mathrm{NaOH}$ or $\mathrm{NH}_{4} \mathrm{OH}$ in an excess $\sim 0.2 \mathrm{M}$ was added to $0.3-0.4 \mathrm{ml}$ of a $\mathrm{Pu}(\mathrm{IV})$ stock solution and the obtained mixture was diluted to $5-7 \mathrm{ml}$ with water. After stirring and coagulation at room temperature, the precipitate was separated from mother solutions by centrifugation and washed one time with $5 \mathrm{ml}$ of water. Then it was suspended in $1 \mathrm{ml}$ of titrated sodium or ammonium hydroxide and transported into a Teflon test-tube with outer diameter equal to $6 \mathrm{~mm}$. The test-tube was sealed in a glass ampoule and heated for several hours at a controlled temperature in the range of 100 to $200^{\circ} \mathrm{C}$. After cooling and ampoule opening the suspension was replaced into a centrifuge test-tube and then treated as described above.

Similar methods were used to prepare of Pu (IV) hydrous oxides precipitated in the presence of different anions. Known amounts of the anion salts were introduced into aliquots of a $\mathrm{Pu}$ (IV) stock solution (direct precipitation) or into $\mathrm{NaOH}$ solution (reverse precipitation) under conditions otherwise similar to those described in the absence of anions.

\section{Measurements of the IR spectra of $\mathrm{Pu}(\mathrm{IV})$ hydrous oxide compounds}

A compound to be studied was preliminarily dried for 1-3 days in a desiccator over $\mathrm{KOH}$ pellets. Then 2$2.5 \mathrm{mg}$ of the compound was mixed with $200 \mathrm{mg}$ of special purity grade $\mathrm{NaCl}$ or $\mathrm{KBr}$ and ground into powder for 10-20 minutes in an agate mortar. The mixture obtained was converted into a transparent disk $(\mathrm{d}=2 \mathrm{~cm})$ by pressing $\sim 1$ minute at $190 \mathrm{kP} / \mathrm{cm}^{2}$. The disk was installed in a special holder and placed in the compartment of an IR spectrophotometer. IR spectra were registered in the range of $350-4000 \mathrm{~cm}^{-1}$ ( $\mathrm{NaCl}$ matrix) or $250-4000 \mathrm{~cm}^{-1}$ ( $\mathrm{KBr}$ matrix). The measurements at low wave numbers were performed using blank disks for compensation of IR absorption by matrixes. The disks were prepared from pure $\mathrm{NaCl}$ or $\mathrm{KBr}$ in the same way as described above.

\section{Plutonium (V) tests}

Plutonium (primarily ${ }^{239} \mathrm{Pu}$ ) was purified by anion exchange and oxalate precipitation. Plutonium (IV) was stabilized with hydrogen peroxide in $4 \mathrm{M} \mathrm{HNO}_{3}$ solutions at $80^{\circ} \mathrm{C}$, and $\mathrm{Pu}(\mathrm{VI})$ by fuming with perchloric acid. Preparation of alkaline $\mathrm{Pu}(\mathrm{V})$ solutions by chemical or electrochemical reduction of alkaline $\mathrm{Pu}(\mathrm{VI})$ solutions gave products contaminated with $\mathrm{Pu}(\mathrm{IV})$. Crystalline $\mathrm{NH}_{4} \mathrm{PuO}_{2} \mathrm{CO}_{3}$, synthesized by known techniques, was used to prepare $\mathrm{Pu}(\mathrm{V})$ solutions. This compound had a white color, which was not altered by exposure to neutral $\mathrm{H}_{2} \mathrm{O}$ solutions.

Standardized $\mathrm{NaOH}$ solutions were prepared from $17.7 \mathrm{M} \mathrm{NaOH}$ kept sealed in a plastic vessel to prevent carbonate contamination. All reagents were pure and extra pure grade. Solutions were prepared from distilled water purified of $\mathrm{CO}_{2}$ by $\mathrm{pH} 4$ acidification and boiling. Plutonium valence was determined by spectrophotometry (Shimadzu UV-3100 PC, Japan). 


\section{Results and Discussion}

\section{Composition and hygroscopic properties of $\mathrm{PuO}_{2} \cdot \mathrm{xH}_{2} \mathrm{O}$}

The majority of $\mathrm{PuO}_{2}{ }^{*} \mathrm{xH}_{2} \mathrm{O}$ samples were prepared using reverse precipitation by $\mathrm{NaOH}$ solutions. This permitted us to avoid possible $\mathrm{Pu}$ (IV) disproportionation, which can take place at low acid concentration arising as an intermediate step in the course of $\mathrm{NaOH}$ addition to $\mathrm{Pu}$ (IV) stock solution. The probability of such a reaction was quite real, because plutonium concentration in the stock solutions was rather high. To lower the probability of $\mathrm{Pu}$ (IV) disproportionation at $\mathrm{PuO}_{2}{ }^{*} \mathrm{xH}_{2} \mathrm{O}$ precipitation, the aliquots of a $\mathrm{Pu}$ (IV) stock solution were diluted by 3 to 5 times by water and quickly mixed with a chosen amount of $\mathrm{NaOH}$ under intensive stirring.

As it is seen in Table 1, the compositions of $\mathrm{PuO}_{2}{ }^{*} \mathrm{xH}_{2} \mathrm{O}$ kept under comparable drying conditions are practically independent of the precipitation method (direct or reverse). The compositions likewise are not sensitive, at low temperatures, to the amount of $\mathrm{NaOH}$ excess or to coagulation time. At higher temperature, the $x$ value for $\mathrm{PuO}_{2}{ }^{*} \mathrm{xH}_{2} \mathrm{O}$ tends to decrease, especially at higher $\mathrm{NaOH}$ concentrations.

The mean $x$ values for $\mathrm{PuO}_{2}{ }^{*} \mathrm{xH}_{2} \mathrm{O}$ precipitated at 10 to $60^{\circ} \mathrm{C}$ and dried over $\mathrm{KOH}$ pellets and over $25 \%$ $\mathrm{H}_{2} \mathrm{SO}_{4}$ are equal to 1.56 and 2.76 , respectively. The difference $\Delta \mathrm{x}=1.20$ is a reflection of hygroscopic properties of such $\mathrm{PuO}_{2}{ }^{*} \mathrm{xH}_{2} \mathrm{O}$ samples.

Table 1. Composition of $\mathrm{PuO}_{2}{ }^{*} \mathrm{xH}_{2} \mathrm{O}$ samples prepared by $\mathrm{NaOH}$ precipitation at $12-100^{\circ} \mathrm{C}$.

\begin{tabular}{|c|c|c|c|c|c|c|}
\hline \multirow[b]{2}{*}{$\begin{array}{l}\text { Precipitation } \\
\text { type }\end{array}$} & \multicolumn{3}{|c|}{ Conditions of coagulation } & \multicolumn{3}{|c|}{$\mathrm{X}$ values after drying over } \\
\hline & $\underset{M}{[\mathrm{NaOH}]}$, & $\mathrm{t},{ }^{\circ} \mathrm{C}$ & $\mathbf{T}, \mathbf{h}$ & $\mathrm{KOH}$ & $50 \% \mathrm{H}_{2} \mathrm{SO}_{4}$ & $25 \% \mathrm{H}_{2} \mathrm{SO}_{4}$ \\
\hline Reverse & 0.2 & 12 & 0.2 & 1.51 & 2.28 & 2.63 \\
\hline -"- & 0.2 & 12 & 5 & 1.63 & 2.32 & 2.60 \\
\hline -"- & 1 & 12 & 5 & 1.47 & 2.19 & 2.74 \\
\hline -"- & 2.4 & 12 & 96 & 1.46 & 2.22 & 2.59 \\
\hline -"- & 15.4 & 12 & 160 & 1.63 & 2.41 & 2.63 \\
\hline -"- & 2.4 & 60 & & 1.44 & 2.10 & 2.46 \\
\hline -"- & 1 & 100 & 1 & 1.55 & 2.15 & 2.69 \\
\hline -"- & 1 & 100 & 2 & 1.31 & 2.11 & 2.61 \\
\hline -"- & 1 & 100 & 9 & 1.56 & 2.18 & 2.52 \\
\hline -"- & 0.2 & 100 & 9 & 1.50 & 2.00 & 2.56 \\
\hline -"- & 3 & 100 & 9 & 1.17 & 1.72 & 2.33 \\
\hline -"- & 8.1 & 100 & 9 & 1.32 & 1.86 & 2.45 \\
\hline -"- & 15.4 & 100 & 9 & 1.28 & 1.98 & 2.47 \\
\hline Direct & 0.2 & 12 & 0.2 & 1.57 & 2.16 & 2.70 \\
\hline -"- & 1 & 100 & 9 & 1.50 & 2.18 & 2.85 \\
\hline -"- & 1 & 60 & 5 & 1.62 & 2.25 & 2.96 \\
\hline -"- & 2 & 20 & 3 & 1.77 & 2.42 & 3.09 \\
\hline -"- & 0.2 & 20 & 3 & 1.61 & 2.18 & 3.16 \\
\hline -"- & 2 & 100 & 3 & 1.22 & 1.96 & 2.70 \\
\hline -"- & 8 & 100 & 3 & 1.37 & 1.88 & 2.86 \\
\hline
\end{tabular}


The $\mathrm{PuO}_{2}{ }^{*} \mathrm{xH} \mathrm{H}_{2} \mathrm{O}$ compounds coagulated at $100^{\circ} \mathrm{C}$ but dried under the same conditions have mean $\mathrm{x}$ values 1.38 and 2.72 and $\Delta \mathrm{x}=1.34$. For $\mathrm{PuO}_{2}{ }^{*} \mathrm{xH}_{2} \mathrm{O}$ samples obtained under hydrothermal conditions at 180 to $200^{\circ} \mathrm{C}$, the respective values are $\mathrm{x}=0.67$ and 2.47 , and $\Delta \mathrm{x}=1.80$. Thus, with increasing temperature, the number of water molecules in $\mathrm{PuO}_{2}{ }^{*} \mathrm{xH}_{2} \mathrm{O}$ prepared under comparable conditions decreases, but the hygroscopic properties of the compounds slightly increase.

\section{Thermal behavior of plutonium (IV) hydrous oxide}

From the data presented above, it is evident that $\mathrm{PuO}_{2}{ }^{*} \mathrm{xH}_{2} \mathrm{O}$ easily loses a part of its water even at room temperature. The dehydration of $\mathrm{PuO}_{2}{ }^{*} \mathrm{xH}_{2} \mathrm{O}$ at elevated temperatures was studied by thermo-gravimetric and differential thermal techniques. The measurements were conducted with 25 to $50 \mathrm{mg} \mathrm{PuO}_{2}{ }^{*} \times \mathrm{H}_{2} \mathrm{O}$ previously dried in a desiccator over $50 \% \mathrm{H}_{2} \mathrm{SO}_{4}$.

Thermo-gravimetric scans of the various $\mathrm{PuO}_{2}{ }^{*} \mathrm{xH}_{2} \mathrm{O}$ samples were similar regardless of the samples preparation conditions. Selected scans are presented in Fig. 1. In all cases, the mass loss occurred monotonously, in one step, in the range $50-300^{\circ} \mathrm{C}$. All differential thermal analysis curves showed gentle endothermic peaks at about $110^{\circ} \mathrm{C}$ with height being proportional to the $\mathrm{x}$ values of the compounds being investigated. Based on these results, we conclude that water in $\mathrm{PuO}_{2}{ }^{*} \mathrm{xH}_{2} \mathrm{O}$ does not have discrete binding (for example, as a crystalline hydrate). Instead, a continuous range of bonding energy characterizes the associated water. This conclusion agrees well with the hygroscopic properties of $\mathrm{PuO}_{2}{ }^{*} \times \mathrm{H}_{2} \mathrm{O}$ at room temperature.

It was of some interest to study the behavior in the air of products obtained by isothermal heating of $\mathrm{PuO}_{2}{ }^{*} \mathrm{xH}_{2} \mathrm{O}$ samples to elevated temperatures. For this purpose, $\sim 12 \mathrm{mg}$ of $\mathrm{PuO}_{2}{ }^{*} \mathrm{xH}_{2} \mathrm{O}$, prepared by reverse precipitation from $1 \mathrm{M} \mathrm{NaOH}$ and subsequent coagulation at $100^{\circ} \mathrm{C}$ during $9 \mathrm{~h}$, was heated

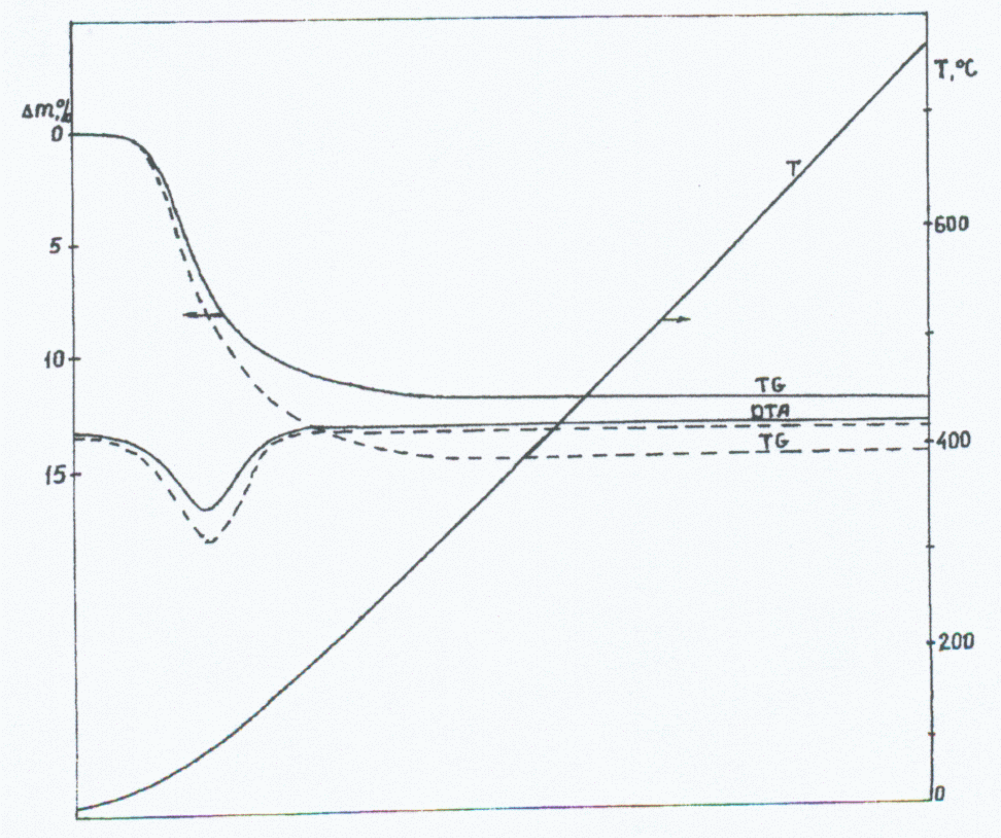

Fig. 1. Thermal analysis of $\mathrm{PuO}_{2}{ }^{\star} \mathrm{xH}_{2} \mathrm{O} ;-\mathrm{PuO}_{2}{ }^{\star} 0.89 \mathrm{H}_{2} \mathrm{O} ; \mathrm{PuO}_{2}{ }^{*} 1.6 \mathrm{H}_{2} \mathrm{O}$. 
stepwise at different temperatures for $1.5 \mathrm{~h}$. After every step of heating, the product was stored for 1 day in desiccators over dry $\mathrm{KOH}$ and then over $25 \%$ sulfuric acid, and its weight was measured. The temperature constancy was maintained with an accuracy of $\pm 3^{\circ} \mathrm{C}$ in the range $20-300^{\circ} \mathrm{C}$ and $\pm 10^{\circ} \mathrm{C}$ at $\mathrm{t}>300^{\circ} \mathrm{C}$.

From the data in Table 2, it is clear that the residual water content of $\mathrm{PuO}_{2}{ }^{*} \mathrm{xH}_{2} \mathrm{O}$ decreases with increasing temperature. However, the ability of the residual compounds to reabsorb water vapor under otherwise comparable conditions is not affected by the temperature of drying over the range of 20 to $350^{\circ} \mathrm{C}$. Remarkably, this ability is observed even at $520^{\circ} \mathrm{C}$. Increasing the time of heating at constant temperature affects the hygroscopic properties of $\mathrm{PuO}_{2}{ }^{*} \mathrm{xH}_{2} \mathrm{O}$ only to a very limited extent.

The low influence of temperature on $\mathrm{PuO}_{2}{ }^{*} \mathrm{xH}_{2} \mathrm{O}$ hygroscopicity can be explained by the rather high thermal stability of primary crystallites. This supposition was confirmed by direct estimations of crystallite size in $\mathrm{PuO}_{2}{ }^{*} \mathrm{xH}_{2} \mathrm{O}$ samples, after their heating to different temperatures, using $\mathrm{x}$-ray powder diffraction methods. The experiments were conducted with $\mathrm{PuO}_{2}{ }^{*} \mathrm{xH}_{2} \mathrm{O}$ samples prepared by precipitation from 0.2 , 1 , and $10 \mathrm{M} \mathrm{NaOH}$ and coagulated for 3 hours at $75^{\circ} \mathrm{C}$. The samples $(6 \pm 1 \mathrm{mg})$ then were heated for 2 hours to various temperatures and examined by $x$-ray powder diffractometry.

Table 2. Hygroscopic behaviour of $\mathrm{PuO}_{2}{ }^{*} \mathrm{xH}_{2} \mathrm{O}$ after isothermal heating for $1.5 \mathrm{~h}$ at different temperatures.

\begin{tabular}{|l|l|l|l|l|l|l|l|}
\hline \multicolumn{1}{|c|}{$\mathbf{t}^{\circ}{ }^{\circ} \mathbf{C}$} & \multicolumn{1}{c|}{$\mathbf{2 0}$} & \multicolumn{1}{c|}{$\mathbf{1 6 5}$} & \multicolumn{1}{c|}{$\mathbf{1 9 5}$} & \multicolumn{1}{c|}{$\mathbf{2 9 5}$} & \multicolumn{1}{c|}{$\mathbf{3 5 0}$} & \multicolumn{1}{c|}{$\mathbf{5 2 0}$} & $\mathbf{8 0 0}$ \\
\hline $\mathrm{x}_{1}$ & 1.50 & 1.23 & 0.97 & 0.74 & 0.37 & 0.04 & 0 \\
\hline $\mathrm{x}_{2}$ & 2.85 & 2.48 & 2.20 & 2.08 & 1.67 & 0.66 & 0 \\
\hline$\Delta \mathrm{x}=\mathrm{x}_{1}-\mathrm{x}_{2}$ & 1.35 & 1.25 & 1.23 & 1.34 & 1.30 & 0.62 & 0 \\
\hline
\end{tabular}

Note: $x_{1}$ and $x_{2}$ are $x$ values after keeping of the product 1 day over $\mathrm{KOH}$ and $25 \% \mathrm{H}_{2} \mathrm{SO}_{4}$, respectively;

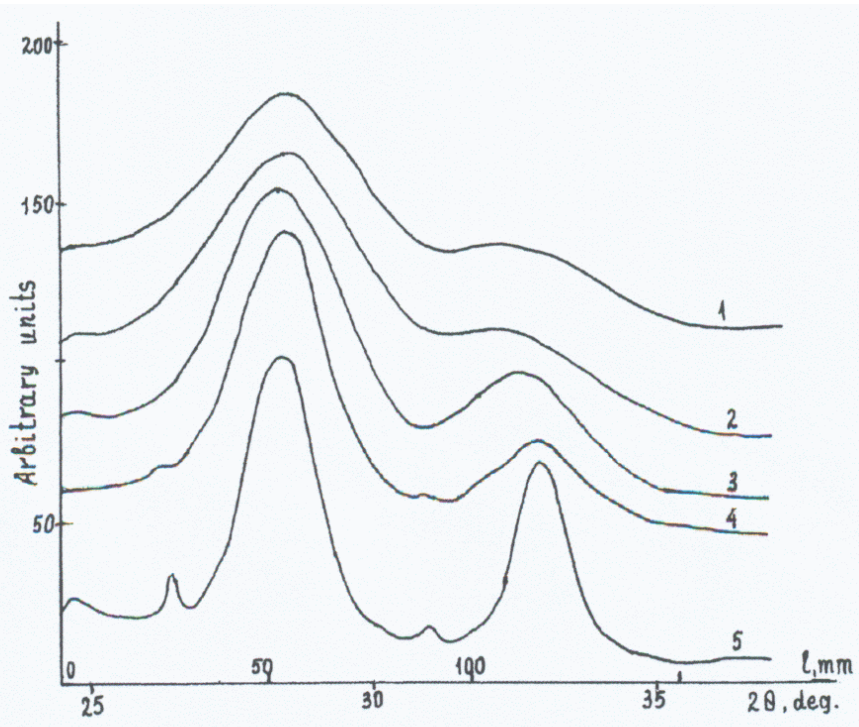

Fig. 2. X-ray diffraction patterns of $\mathrm{PuO}_{2}{ }^{*} \mathrm{xH}_{2} \mathrm{O}$ (the shape of (111) line) as a function of heating temperature (in ${ }^{\circ} \mathrm{C}$ ): $1-75 ; 2-200 ; 3-300 ; 4-400 ; 5-800$. 
It was found that the half-width of the diffraction line smoothly decreases about 3-fold as heating temperature increases from 75 to $800^{\circ} \mathrm{C}$. Nevertheless, the diffraction line half-width remains rather large even after calcination of the $\mathrm{PuO}_{2}{ }^{*} \mathrm{xH}_{2} \mathrm{O}$ samples at $800^{\circ} \mathrm{C}$ for $2 \mathrm{~h}$. Half-width variations as the function of heating time are insignificant (Fig. 2).

The observed changes of the diffraction line half-width correspond to an increase in the $\mathrm{PuO}_{2}{ }^{*} \mathrm{xH}_{2} \mathrm{O}$ crystallite size from $\sim 2.5$ to $7 \mathrm{~nm}$. It is seen that $\mathrm{PuO}_{2}$ obtained by alkaline precipitation of $\mathrm{PuO}_{2}{ }^{*} \mathrm{xH}_{2} \mathrm{O}$ followed by calcinations at $800^{\circ} \mathrm{C}$ consists of very small crystals. However, these crystals are not hygroscopic. Probably, hygroscopic properties of $\mathrm{PuO}_{2}$ depend not only on the size of crystals, but also on their regularity.

\section{Influence of tank waste anions on $\mathrm{PuO}_{2} \bullet \mathrm{xH}_{2} \mathrm{O}$ precipitation}

A special investigation was performed to determine the influence of anions, which are present in tank wastes, on $\mathrm{Pu}(\mathrm{IV})$ precipitation by $\mathrm{NaOH}$. In preliminary experiments $\mathrm{Pu}$ (IV) precipitation was conducted by reverse method at $60^{\circ} \mathrm{C}$ from $1 \mathrm{M} \mathrm{NaOH}$ solutions containing different anions. The experiments showed that the plutonium compounds precipitated in the presence of $2 \mathrm{M} \mathrm{NO}_{2}{ }^{-}, 0.5 \mathrm{M} \mathrm{SO}_{4}{ }^{2-}$ or $\mathrm{CO}_{3}{ }^{2-}, 0.1$ $\mathrm{M} \mathrm{C}_{2} \mathrm{O}_{4}{ }^{2-}$, EDTA, HEDTA, or citrate had, after careful water washing, the same composition and hygroscopic properties as the $\mathrm{PuO}_{2}{ }^{*} \mathrm{xH}_{2} \mathrm{O}$ obtained by $\mathrm{Pu}(\mathrm{IV})$ precipitation from pure $\mathrm{NaOH}$ solutions. The IR spectra of the final products also exhibited no bands, which could be attributed to absorption of the listed anions. However, remarkably, other behavior of $\mathrm{Pu}(\mathrm{IV})$ was observed at the precipitation in the presence of silicate and phosphate anions.

In additional experiments, $\mathrm{Pu}$ (IV) precipitation was performed at room temperature from $0.2 \mathrm{M} \mathrm{NaOH}$ containing different anions. Obtained precipitates were coagulated for 3 hours, carefully washed by water and treated by $5.5 \mathrm{M} \mathrm{NaOH}$ on a boiling water bath for $\sim 1$ hour. Then the precipitates were centrifuged and washed by water. The mother solutions together with wash waters were analyzed for corresponding anions. Determination of nitrite was conducted by a spectrophotometric method with the use of sulfanylic acid and $\alpha$-nafthylamine. EDTA and HEDTA were titrated at $\mathrm{pH} 2$ by a standard $3^{*} 10^{-3} \mathrm{M} \mathrm{Th}\left(\mathrm{NO}_{3}\right)_{4}$ solution. Chromate anions were determined by direct measurement of their light absorption at $372 \mathrm{~nm}$. The spectrophotometric method was also used for the estimation of $\mathrm{Fe}(\mathrm{CN})_{6}{ }^{4-}$ concentration. In this case, optical density was measured at $420 \mathrm{~nm}$ after oxidation of ferrocyanide anions to $\mathrm{Fe}(\mathrm{CN})_{6}{ }^{3-}$ by persulfate. Oxalate was titrated by $\mathrm{KMnO}_{4}$ in $\sim \mathrm{M} \mathrm{H}_{2} \mathrm{SO}_{4}$. To confirm the absence of sulfate, the analyzed solution was neutralized by $\mathrm{HClO}_{4}$ and mixed with $\mathrm{Ba}\left(\mathrm{ClO}_{4}\right)_{2}$. Carbonate anions were determined directly in the washed $\mathrm{Pu}$ (IV) precipitate. The analyzed sample was treated by $\mathrm{HNO}_{3}$ in $\mathrm{Ar}$ flow and the gas was passed through $\mathrm{Ba}(\mathrm{OH})_{2}$ solution.

As seen in Table 3, the capture of anions usually was below the sensitivity of chosen analytic methods. The exceptions took place only in the case of $\mathrm{NO}_{2}{ }^{-}$and $\mathrm{CrO}_{4}{ }^{2-}$ anions. However, the nitrite and chromate contents in the $\mathrm{Pu}$ (IV) precipitates were also low and close to their detection limits.

Table 3. Contents of $\mathrm{PuO}_{2} \bullet \mathrm{xH}_{2} \mathrm{O}$ precipitated from $0.2 \mathrm{M} \mathrm{NaOH}$ at $20^{\circ} \mathrm{C}$ in the presence of anions.

\begin{tabular}{|c|c|c|c|c|c|}
\hline Anion, A & $\begin{array}{l}\text { [A] in } \mathrm{NaOH} \\
\text { solution, } \mathrm{M}\end{array}$ & $\begin{array}{c}\mathrm{Mol}_{0} \% \text { of } \mathrm{A} \text { in } \\
\mathrm{PuO}_{2} \bullet \times \mathrm{H}_{2} \mathrm{O}\end{array}$ & Anion, A & $\begin{array}{l}\text { [A] in } \mathrm{NaOH} \\
\text { solution, } \mathrm{M}\end{array}$ & $\begin{array}{c}\mathrm{Mol} \% \text { of } \mathrm{A} \\
\text { inPuO } \\
\text { in }_{2} \bullet \mathrm{xH}_{2} \mathrm{O}\end{array}$ \\
\hline $\mathrm{NO}_{2}^{-}$ & 2 & 7 & $\mathrm{CrO}_{4}{ }^{2-}$ & 0.2 & 2 \\
\hline EDTA & 0.1 & $<0.4$ & $\mathrm{SO}_{4}^{2-}$ & 0.2 & $<0.01$ \\
\hline HEDTA & 0.1 & 1 & $\mathrm{CO}_{3}{ }^{2-}$ & 0.2 & $<0.1$ \\
\hline $\mathrm{C}_{2} \mathrm{O}_{4}{ }^{2-}$ & 0.1 & $<5$ & $\mathrm{Fe}(\mathrm{CN})_{6}{ }^{4-}$ & 0.2 & $<0.1$ \\
\hline
\end{tabular}


The mentioned anions did not significantly alter the rate or completeness of $\mathrm{PuO}_{2}{ }^{*} \mathrm{xH}_{2} \mathrm{O}$ precipitation. The well-known increase of $\mathrm{PuO}_{2}{ }^{*} \mathrm{xH}_{2} \mathrm{O}$ solubility in the presence of carbonate and ethylenediaminetetraacetate may have been obscured by the rather high concentration of fine plutonium hydrous oxide particles in the mother solutions after centrifugation.

In contrast to the anions considered above, $\mathrm{Pu}$ (IV) precipitation characteristics were remarkably altered for alkaline solutions containing silicate or phosphate. In both cases, change in the color of precipitates from green to greenish-gray was observed. The increase of precipitate specific volumes was also evident. Water washing of the precipitates gave significant peptization. These results prompted further study of $\mathrm{Pu}$ (IV) precipitation in the presence of silicate and phosphate anions.

The IR spectra of products obtained by Pu (IV) alkali precipitation in the presence of silicate ions show a very intense absorption band with maximum at $1000 \mathrm{~cm}^{-1}$ and two shoulders (Fig. 3). This band is attributed to valent vibrations of silicate anions. A shoulder is also observed on a strong absorption band attributed to valent vibration of water. These unique IR spectral features indicate the presence of the large amounts of silicate in the products being examined. This conclusion was confirmed by the results of direct analyses of $\mathrm{Pu}(\mathrm{IV})$ compounds precipitated from alkaline solutions in the presence of silicate.

Based on these results, it is possible to conclude that $\mathrm{PuO}_{2}{ }^{*} \mathrm{xH}_{2} \mathrm{O}$ cannot be present in alkaline tank wastes containing significant silicate concentrations. Under such conditions, $\mathrm{Pu}$ (IV) should exist as various basic silicates according to the waste composition. However, this conclusion requires additional study and may be complicated by the behavior of mixed hydroxides of Pu (IV) and Fe (III) or other elements in alkaline silicate media. This problem, too, requires special investigation.

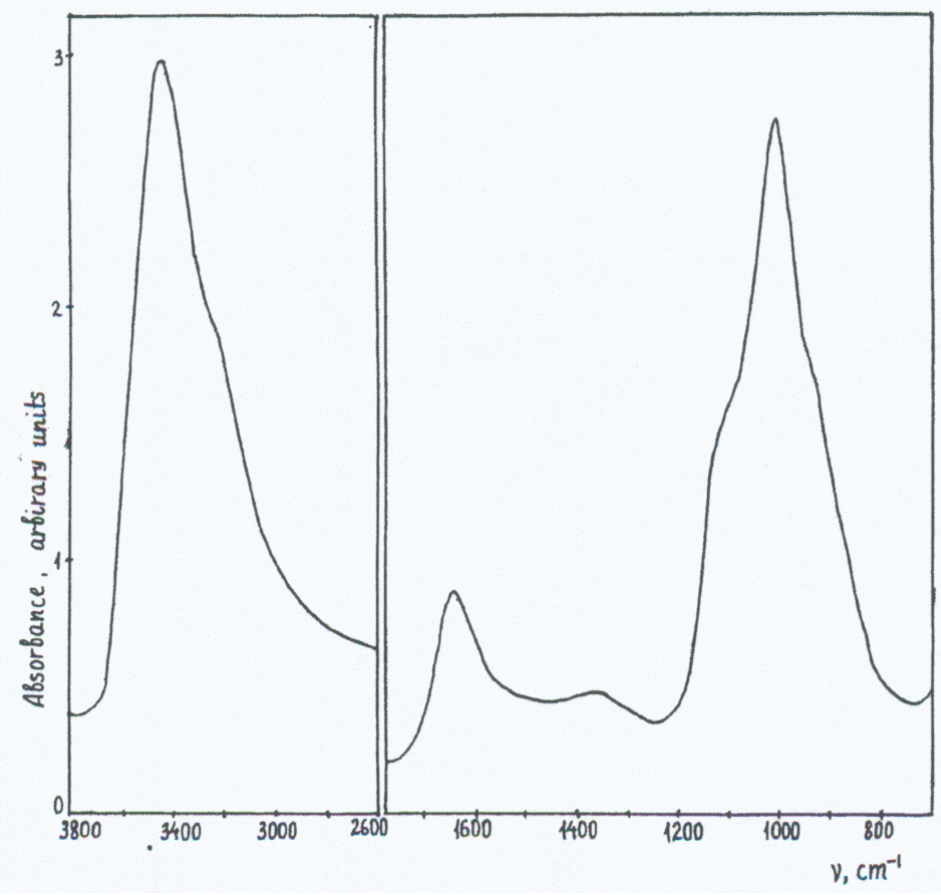

Fig. 3. IR absorption spectrum of $\mathrm{PuO}_{2} \cdot 0.88 \mathrm{SiO}_{2}{ }^{*} \mathrm{xH}_{2} \mathrm{O}$. 
The alkali precipitation behavior of $\mathrm{Pu}$ (IV) is similar in the presence of phosphate and of silicate. However, the degree of anion capture and the stability of the product compounds at high $\mathrm{NaOH}$ concentration for phosphate are significantly lower than for silicate. IR spectra of basic Pu (IV) phosphates have a characteristic strong and complex band with maximum at $1020 \mathrm{~cm}^{-1}$.

The completeness of Pu (IV) precipitation from phosphate- and silicate-bearing alkaline solutions is somewhat lower than from similar pure $\mathrm{NaOH}$. Special difficulties also occur on washing of the precipitate by water or diluted electrolytes because of precipitate peptization.

\section{Investigation of the behavior of plutonium (V) in alkaline media}

Alkaline solutions of $\mathrm{Pu}(\mathrm{V})$ were prepared by dissolving crystalline $\mathrm{NH}_{4} \mathrm{PuO}_{2} \mathrm{CO}_{3}$ in $8 \mathrm{M} \mathrm{NaOH}$. After mixing of $\mathrm{NaOH}$ solution and $\mathrm{NH}_{4} \mathrm{PuO}_{2} \mathrm{CO}_{3}$, the solid phase and mother solution became pink. After 1.5 hours' of equilibration, $\mathrm{Pu}(\mathrm{V})$ concentration was measured by spectrophotometric titration with $\mathrm{Pu}(\mathrm{VII})$ prepared by electrochemical oxidation of $0.01 \mathrm{M} \mathrm{Pu}(\mathrm{VI})$ in $8 \mathrm{M} \mathrm{NaOH}$. The $\mathrm{Pu}(\mathrm{VII})$ concentration was measured by titration with standard $\mathrm{K}_{4}\left[\mathrm{Fe}(\mathrm{CN})_{6}\right]$ solutions The $\mathrm{Pu}(\mathrm{V})$ solubility was estimated as $0.008 \mathrm{M}$ at $12^{\circ} \mathrm{C}$.

For each disproportionation test, 0.027 mmole of $\mathrm{NH}_{4} \mathrm{PuO}_{2} \mathrm{CO}_{3}$ was precipitated in a plastic tube. The white sediment was washed free of occluded salts, $5 \mathrm{~mL}$ of alkali of selected concentration added, and the suspension stirred in a thermostat. Stirring was halted at hourly intervals and solution spectra recorded (1000 to $400 \mathrm{~nm}$ ). The spectra showed absorptions at 620 to $630 \mathrm{~nm}$ inherent to $\mathrm{Pu}(\mathrm{VI})$; and at 500 to $600 \mathrm{~nm}$ and 800 to $810 \mathrm{~nm}$ assigned to $\mathrm{Pu}(\mathrm{V})$. Plutonium (IV) precipitates almost completely in $\mathrm{NaOH}$ solution and no corresponding spectrum is observed. After 5 hours, the spectra became constant and the initial $\mathrm{Pu}(\mathrm{V})$ was judged to be at equilibrium with its disproportionation products. Figure 4 illustrates the spectra as a function of $\mathrm{NaOH}$ concentration. The $\mathrm{Pu}(\mathrm{V})$ and $\mathrm{Pu}(\mathrm{VI})$ concentrations were evaluated based on measured molar extinction coefficients $\varepsilon_{810}^{(\mathrm{V})}=\varepsilon_{630}^{(\mathrm{VI})}=10 \mathrm{~L} /(\mathrm{mol} \mathrm{cm})$.

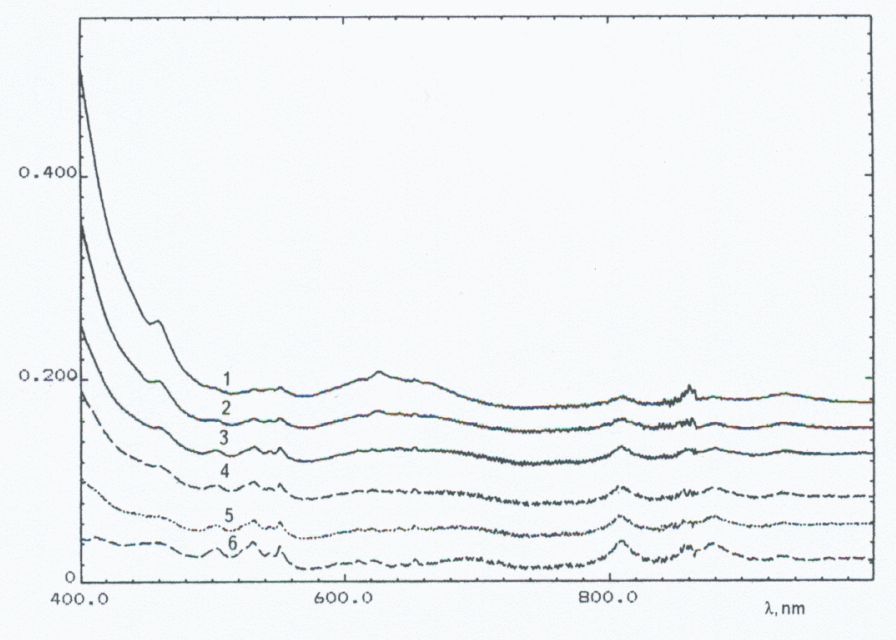

Fig. 4. Electron absorption spectra of equilibrium solutions of $\mathrm{Pu}(\mathrm{V})$ as a function of $[\mathrm{NaOH}]$ at $40^{\circ} \mathrm{C}: 1.5 .65 \mathrm{~mol} / \mathrm{L} ; 2.6 .10 \mathrm{~mol} / \mathrm{L} ; 3.6 .60 \mathrm{~mol} / \mathrm{L} ; 4.7 .00 \mathrm{~mol} / \mathrm{L} ; 5.7 .46 \mathrm{~mol} / \mathrm{L} ; 6.8 .02 \mathrm{~mol} / \mathrm{L}$. 
The total amount of dissolved $\mathrm{Pu}(\mathrm{V})$ and (VI) was calculated and the amount of $\mathrm{Pu}$ in the solid phase analyzed by redissolving to determine material balance.

Using the literature data about the species formations of $\mathrm{Pu}(\mathrm{VI}),(\mathrm{V})$ and (IV) in alkaline solutions [14], the ionic-molecular form disproportionation reaction could be presented as:

- $2 \mathrm{PuO}_{2}(\mathrm{OH})_{4}{ }^{3-}+2 \mathrm{H}_{2} \mathrm{O}=\mathrm{PuO}_{2}(\mathrm{OH})_{4}{ }^{2-}+\mathrm{Pu}(\mathrm{OH})_{5}{ }^{-}+3 \mathrm{OH}^{-}(1)$ or

- $2 \mathrm{PuO}_{2}(\mathrm{OH})_{4}{ }^{3-}+2 \mathrm{H}_{2} \mathrm{O}=\mathrm{PuO}_{2}(\mathrm{OH})_{4}{ }^{2-}+\mathrm{Pu}(\mathrm{OH})_{4}+4 \mathrm{OH}^{-}(2)$.

To calculate the equilibrium constants, one should know the concentrations of all the valence forms of plutonium in solution. In this case, the Pu (IV) concentration is known by the solubility of Pu (IV) hydrous oxide (S) at $26^{\circ} \mathrm{C}$ as a function of $\mathrm{NaOH}$ concentration [14]. The temperature dependence of solubility has not been studied. Assuming that the Pu (IV) hydrous oxide solubility does not change in the interval 26$40^{\circ} \mathrm{C}$, equilibrium constants can be calculated. $\mathrm{Pu}(\mathrm{VI})$ and $\mathrm{Pu}(\mathrm{V})$ concentrations are determined from spectrophotometric measurements. The disproportionation equilibrium constant was calculated as $\mathrm{K}^{\mathrm{d}}=$ $[\mathrm{Pu}(\mathrm{VI})] \mathrm{S}^{\mathrm{S}}\left[\mathrm{OH}^{-}\right]^{\mathrm{n}} /[\mathrm{Pu}(\mathrm{V})]^{2}$ ( $\mathrm{n}=3$ or 4$)$. In 1-4 M NaOH disproportionation equilibrium reaction moves to the right side. $\mathrm{Pu}(\mathrm{V})$ concentration in these solutions was so low that we could not determine $\mathrm{K}^{\mathrm{d}}$ values. In $[\mathrm{NaOH}]$ interval from 4 to $8 \mathrm{~mol} / \mathrm{L}$, the equilibrium constants increase from 3.05 to 0.20 (reaction 1 ) and from 12.22 to 1.64 (reaction 2). In alkaline concentration higher than $8 \mathrm{~mol} / \mathrm{L}$, Plutonium (V) is stable for at least one week in solution. The dependence of $\mathrm{K}_{\mathrm{d}}$ on $\mathrm{NaOH}$ concentration is linear in $\log \left(\mathrm{K}_{\mathrm{d}}\right)-[\mathrm{NaOH}]$ ordinates. The analytic form of this dependence has been calculated as: $\mathrm{K}_{d}=\mathrm{K}_{d}{ }^{0} / \mathrm{e}^{\alpha[\mathrm{NaOH}]}$, when $\mathrm{K}_{d}{ }^{\circ}$ and $\alpha$ are constants.

To investigate $\mathrm{Pu}(\mathrm{VI})$ and $\mathrm{Pu}(\mathrm{IV})$ reproportionation, known amounts of freshly precipitated $\mathrm{Pu}(\mathrm{IV})$ hydrous oxide and $\mathrm{Pu}(\mathrm{VI})$ in $\mathrm{NaOH}$ solution $\left(1\right.$ to $10 \mathrm{M}$ ) were mixed at $40^{\circ} \mathrm{C}$. The recorded absorption spectra stabilized after four hours and the final $\mathrm{Pu}(\mathrm{VI})$ concentrations were determined. The equilibrium constant for the reproportionation, $\mathrm{K}_{r}$, then was calculated by the formula: $\mathrm{K}_{\mathrm{r}}=[\mathrm{Pu}(\mathrm{V})]^{2} /\left(\mathrm{S}[\mathrm{Pu}(\mathrm{VI})] \cdot[\mathrm{OH}]^{\mathrm{n}}\right)$, when $n=3$ or 4 .

The $\mathrm{K}_{\mathrm{d}}$ and $1 / \mathrm{K}_{\mathrm{r}}$ in reactions 1 or 2 are linearly dependent on $\mathrm{NaOH}$ activity but are not identical. This disparity reveals that complex mechanisms exist in the heterogeneous formation of plutonium hydrous oxide and its subsequent interactions with dissolved species. Related studies show sorption of $\mathrm{Pu}(\mathrm{V})$ on $\mathrm{Pu}$ (IV) hydrous oxide occurs in $\mathrm{NaOH}$ solution; $\alpha$-radiolytic reactions also may affect oxidation state at extended contact times.

The dis- and reproportionation data may be used to calculate the potential of the $\mathrm{Pu}(\mathrm{V}) / \mathrm{Pu}(\mathrm{IV})$ electrode pair. The equilibrium constants, $\mathrm{K}_{\mathrm{d}}$ and $\mathrm{K}_{\mathrm{r}}$, can be used to assess the thermodynamic characteristics of the disproportionation and reproportionation processes. The free energy, $G$, is related to the equilibrium constant and electrode potential: $G=-R T I n K^{d}=-n F E_{f}$; where $n$ is the reaction's electron change (one in this case), $F$ is the Faraday constant, and $E_{f}$ is the potential change (in volts). Thus, $E_{f}=(0.000198 \mathrm{~T})$ $\log \mathrm{K}^{\mathrm{d}}$ and $\mathrm{E}_{\mathrm{f}}=(-0.000198 \mathrm{~T}) \log \mathrm{K}^{\dagger}$ for reproportionation data and equilibrium constant. The disproportionation reaction may be expressed as the sum of the respective oxidation and reduction half reactions for $\mathrm{Pu}(\mathrm{V}): \mathrm{Pu}(\mathrm{V})=\mathrm{Pu}(\mathrm{VI})+\mathrm{e}^{-}, \mathrm{E}_{\mathrm{V} / \mathrm{VI}}$; and $\mathrm{Pu}(\mathrm{V})+\mathrm{e}^{-}=\mathrm{Pu}(\mathrm{IV}) ; \mathrm{EV}_{/ \mathrm{VV}}$ to give $2 \mathrm{Pu}(\mathrm{V})=\mathrm{Pu}(\mathrm{VI})+$ $\mathrm{Pu}(\mathrm{IV})$ and $\mathrm{E}_{f}=\mathrm{E}_{\mathrm{V} / \mathrm{VI}}+\mathrm{E}_{\mathrm{V} / \mathrm{V} \text {. }}$. 
Because the $E_{V I V}$ potential (which is the negative of $E_{V / V I}$ ) has been measured in previous research and $E_{f}$ can be determined from $K^{d}$, the $E_{V / I V}$ potential can be calculated and is $E_{f}-E_{V / V I}$ or $E_{f}+E_{V / V V}$. Results for the similarly computed dis- and reproportionation data are listed in Table 4.

The calculated $\mathrm{Pu}(\mathrm{V})$ reduction potentials, $\mathrm{E}_{\mathrm{V} / \mathrm{IV}}$, given in Table 4 (from disproportionate data) indicate that $\mathrm{Pu}(\mathrm{V})$ should disproportionate in the range 4 to $8 \mathrm{M} \mathrm{NaOH}$. This coincides with the experimental observations. The calculated $\mathrm{E}_{\mathrm{V} / \mathrm{V}}$ from reproportionate data contradicts the experimental results and do not well agree between them. But we can draw an important conclusion: The value of the oxidation potential of the pair $\mathrm{Pu}(\mathrm{V}) / \mathrm{Pu}(\mathrm{IV})$ in $4-8 \mathrm{M} \mathrm{NaOH}$ is in the range from 0.15 to $0.29 \mathrm{~V}$. The large uncertainty of determination of $\mathrm{E}_{\mathrm{V} / \mathrm{V}}$ is due to a lot of experimental difficulties of the system studied; especially, there are questions of behavior of $\mathrm{Pu}$ (IV) in alkaline medium. It may be that $\mathrm{Pu}$ (IV) hydrous oxide has higher chemical activity under the experimental conditions in reactions with $\mathrm{Pu}(\mathrm{VI})$ and $\mathrm{Pu}(\mathrm{V})$. The actual $\mathrm{Pu}$ (IV) concentration (which determines the equilibrium) also may be greater than the solubility of $\mathrm{Pu}$ (IV) hydrous oxide exhibited in pure alkaline solutions.

Table 4. Disproportionation/Reproportionation equilibrium constants at $40^{\circ} \mathrm{C}$ and the formal potential of the $\mathrm{Pu}(\mathrm{V}) / \mathrm{Pu}(\mathrm{IV})$ pair.

\begin{tabular}{|c|c|c|c|c|c|c|}
\hline \multicolumn{7}{|c|}{ From Disproportionation Data: $2 \mathrm{Pu}(\mathrm{V})=\mathrm{Pu}(\mathrm{IV})+\mathrm{Pu}(\mathrm{VI}) ; \quad \mathrm{K}^{\mathrm{d}}=[\mathrm{Pu}(\mathrm{VI})][\mathrm{Pu}(\mathrm{IV})][\mathrm{OH}]^{\mathrm{n}} /[\mathrm{Pu}(\mathrm{V})]^{2} ;$} \\
\hline$[\mathrm{NaOH}], \mathrm{M}$ & $\begin{array}{l}{[\mathrm{Pu}(\mathrm{VI})]} \\
\mathrm{M}\end{array}$ & $\begin{array}{l}{[\mathrm{Pu}(\mathrm{V})]} \\
\mathrm{M}\end{array}$ & {$[P u(I V)]=S^{*}, M$} & $\mathrm{E}_{\mathrm{VI} / \mathrm{V}}^{* *}, \mathrm{~V}$ & $\begin{array}{l}E_{V / I V}, V \text { from } \\
\text { reaction (1) } n=3\end{array}$ & $\begin{array}{l}E_{V / I V}, V \text { from } \\
\text { reaction (2) } n=4\end{array}$ \\
\hline 4.00 & $6.0 \times 10^{-3}$ & $2.7 \times 10^{-4}$ & $5.8 \times 10^{-7}$ & 0.22 & 0.26 & 0.29 \\
\hline 6.60 & $1.2 \times 10^{-3}$ & $9.3 \times 10^{-4}$ & $2.8 \times 10^{-6}$ & 0.215 & 0.21 & 0.27 \\
\hline 8.02 & $6.7 \times 10^{-4}$ & $2.4 \times 10^{-3}$ & $3.4 \times 10^{-6}$ & 0.21 & 0.17 & 0.23 \\
\hline
\end{tabular}

\begin{tabular}{|c|c|c|c|c|c|c|}
\hline \multicolumn{5}{|c|}{ From Reproportionation Data: $\mathrm{Pu}(\mathrm{VI})+\mathrm{Pu}(\mathrm{IV})=2 \mathrm{Pu}(\mathrm{V})$} & \multicolumn{2}{|c|}{$\mathrm{K}^{\mathrm{r}}=[\mathrm{Pu}(\mathrm{V})]^{2} /[\mathrm{Pu}(\mathrm{VI})] \cdot[\mathrm{Pu}(\mathrm{IV})] \cdot\left[\mathrm{OH}^{-}\right]^{\mathrm{n}} ;$} \\
\hline$[\mathrm{NaOH}], \mathrm{M}$ & $\begin{array}{l}{[\mathrm{Pu}(\mathrm{VI})]} \\
\mathrm{M}\end{array}$ & $\begin{array}{l}{[\mathrm{Pu}(\mathrm{V})]} \\
\mathrm{M}\end{array}$ & {$[\mathrm{Pu}(\mathrm{IV})]=\mathrm{S}^{*}, \mathrm{M}$} & $E_{V I / V}^{* *}, V$ & $\begin{array}{l}E_{\text {VIIV, }} V \text { from } \\
\text { reaction (1) } n=3\end{array}$ & $\begin{array}{l}E_{V / I V}, V \text { from reaction } \\
\text { (2) } n=4\end{array}$ \\
\hline 4.00 & $1.9 \times 10^{-3}$ & $9.3 \times 10^{-4}$ & $5.8 \times 10^{-7}$ & 0.22 & 0.15 & 0.25 \\
\hline 6.60 & $8.3 \times 10^{-4}$ & $1.2 \times 10^{-3}$ & $2.8 \times 10^{-6}$ & 0.215 & 0.20 & 0.19 \\
\hline 8.02 & $9.0 \times 10^{-4}$ & $1.3 \times 10^{-3}$ & $3.4 \times 10^{-6}$ & 0.21 & 0.22 & 0.27 \\
\hline
\end{tabular}

${ }^{\star}$ Data from [12]; **Data from [13]. 


\section{Conclusions}

From the results of the investigation conducted, a number of general conclusions can be made concerning the composition and properties of $\mathrm{Pu}$ (IV) hydrous oxide compounds precipitated from alkaline solutions of different composition. First of all it is necessary to note that $\mathrm{PuO}_{2}{ }^{*} \mathrm{xH}_{2} \mathrm{O}$ is a rather stable compound and cannot transition easily into crystalline plutonium dioxide during long-term aging. This compound consists of very small $\mathrm{PuO}_{2}$ crystallites and water with continuous range of bonding energy. A part of the water can be easily lost, but this process is reversible. Composition and properties of $\mathrm{PuO}_{2}{ }^{*} \mathrm{xH}_{2} \mathrm{O}$ are not strongly altered at long-term aging, modeled by heating of the compound under hydrothermal conditions.

The absence of the $\mathrm{PuO}_{2}{ }^{*} \mathrm{xH}_{2} \mathrm{O}$ transition into crystalline dioxide at aging is a very important and favorable fact for criticality safety during the retrieval and treatment of alkaline tank waste. The reason is that crystalline $\mathrm{PuO}_{2}$ has high density and, apparently, low ability to agglomerate with the amorphous hydroxide compounds present in alkaline tank waste. In any case, it is quite reasonable to expect that the crystalline $\mathrm{PuO}_{2}$ could more easily concentrate during tank waste treatment than the semi-amorphous $\mathrm{PuO}_{2}{ }^{*} \mathrm{xH}_{2} \mathrm{O}$.

The precipitation of $\mathrm{PuO}_{2}{ }^{*} \mathrm{xH}_{2} \mathrm{O}$ from alkaline media proceeds through the formation of solid phase and very fine particles remaining as a suspension in supernatant solution. As a result, plutonium concentration in mother solutions over $\mathrm{PuO}_{2}{ }^{*} \mathrm{xH}_{2} \mathrm{O}$ after centrifugation is usually about 2 to 3 orders higher than observed for the solubility of the compound. This circumstance should be taken into account for the choice of method for the separation and purification of alkaline liquid tank wastes from plutonium.

$\mathrm{PuO}_{2}{ }^{*} \mathrm{XH}_{2} \mathrm{O}$ has the capability to react in alkaline media with silicate and, to a lesser extent, with phosphate ions. As a result, the $\mathrm{Pu}$ (IV) compounds precipitated from alkaline solution in the presence of $0.05 \mathrm{M} \mathrm{SiO}_{3}{ }^{2-}$ at low $\mathrm{NaOH}$ concentrations are more likely silicates than $\mathrm{PuO}_{2}{ }^{*} \times \mathrm{H}_{2} \mathrm{O}$. Significant amounts of silicate are also present in products obtained at $7 \mathrm{M} \mathrm{NaOH}$. $\mathrm{PuO}_{2}{ }^{*} \mathrm{xH}_{2} \mathrm{O}$ samples prepared separately under hydrothermal conditions do not lose the ability to form silicate-containing products. Thus, it is reasonable to conclude that $\mathrm{PuO}_{2}{ }^{*} \mathrm{xH}_{2} \mathrm{O}$ cannot be present in alkaline tank wastes containing significant silicate concentrations. Under such conditions, $\mathrm{Pu}$ (IV) should exist as various basic silicates, which can give, in contrast to $\mathrm{PuO}_{2}{ }^{*} \mathrm{xH}_{2} \mathrm{O}$, considerable peptization. However, this conclusion may be complicated by the unknown behavior of mixed hydroxides of $\mathrm{Pu}(\mathrm{IV})$ with other elements in alkaline silicate media. This problem requires special investigation.

Based on the disproportionation reaction equilibrium constants, the $\mathrm{Pu}(\mathrm{V}) / \mathrm{Pu}(\mathrm{IV})$ oxidation potential was estimated to be 0.65 volts at $1 \mathrm{M} \mathrm{NaOH}$ and about $0.25 \pm 0.05 \mathrm{~V}$ in $4 \mathrm{M} \mathrm{NaOH}$. Waste tanks contain a wide variety of redox agents capable of oxidizing actinide elements to these higher oxidation states. On the basis of these experiments, the heterogeneous equilibrium system of $\mathrm{Pu}(\mathrm{IV}),(\mathrm{V}),(\mathrm{VI})$ species in alkaline media was proposed. 


\section{Acknowledgments}

Members of the IPC RAS: Prof. N.N. Krot, Drs. A.M. Fedosseev, and A.B. Youssov, collaborated on this work. We also thank Dr. M.S. Grigoriev for x-ray diffraction and V.P. Perminov and L.N. Astafurova for technical support. We are especially grateful to Mr. C. Delegard, Pacific Northwest National Laboratory, for his unceasing attention to our work, and valuable discussions of the experimental results. The work was supported by the U.S. Department of Energy, under the Office of Science and Technology, and the Office of Environmental Management.

\section{References}

1. Gephart, R.E.; Lundgren, R.E. "Hanford Tank Clean up: A Guide to -Understanding the Technical Issues", Pacific Northwest Laboratory, PNL-10773, 1995.

2. Agnew, S.F. "Hanford Defined Wastes: Chemical and Radionuclide Compositions", Los Alamos National Laboratory, LAUR-94-2657 Rev.2, 1995.

3. Congress U.S. "Long-Lived Legacy: Managing High-Level and Transuranic Waste at the DOE Nuclear Weapons Complex. Office of Technology Assessment. IOTA-BP-O-83. 1991.

4. DOE Pacific Northwest Laboratories, DOE/RL-92-61 rev.1

5. Delegard, C.H., Elovich, R.J., Barney, G.S., Peretroukhin, V.F. Chemical factors affecting plutonium compound solubility in Hanford site radioactive tank wastes. Book of Abstracts, 214th ACS National Meeting, Las Vegas, NV, September, 7-11 (1997), NUCL-036.

6. Davis, N.R., Wells, M.N., High-Level Waste System Plan Rev.7 (U). HLW-OVP-96-0083. October 1996.

7. D`Entremont, P.D., Jacobs, R.A., Fowler, J.R., Brown, D.F., McCabe, D.J., Walker, D.D., Gillam, J.M. High-Level Waste System Process Interface Description. WSRC-TR-94-0442, March 1995.

8. Kirkbride, R.A., Allen, G.K., Certa, P.J., Manuel, A.F., Orme, R.M., Shelton, L.W., Slaathaug, R.S., Wittman, R.S., MacLean, G.T., Penwell, D.L. Tank Waste Remediation System Operation and Utilization Plan. HNF-SD-WM-SP-012 Rev. OA. September 1997.

9. Lumetta, G.J.; Burgeson, I.E.; Wagner, M.J.; Liu, J.; Chen, Y.L. Washing and Caustic Leaching of Hanford Tank Sludge: Results of FY 1997 Studied. PNNL-11636, August 1997.

10. Temer, D.J.; Villarreal, R. Sludge Washing and Alkaline Leaching Tests on Actual Hanford Tank Sludge: A Status Report. LAUR-95-2070, August 1995.

11. Delegard, C.H. Solubility of $\mathrm{PuO}_{2}{ }^{*} \mathrm{H}_{2} \mathrm{O}$ in alkaline Hanford high-level waste solutions. Radiochimica Acta, 41 (1), 11-21. 1987.

12. Bourges J. "Preparation et Identification du Plutonium a l'Etat d'Oxydation-V en Mileau Basique." Radiochem. Radioanal. Lett. 12 (1972) 111-117.

13. Shilov V.P. "Oxidation of Neptunium(VI) by Plutonium(VI) in Alkaline Medium." Sov. Radiochem. 18 (1976) 592-593.

14. Peretroukhine V.F., Kryutchkov S.V., Silin V.I., and Tananaev I.G. "Determination of the Solubility of $\mathrm{Np}(\mathrm{IV})-(\mathrm{VI}), \mathrm{Pu}(\mathrm{III})-(\mathrm{VI}), \mathrm{Am}(\mathrm{III})-(\mathrm{VI})$ and Tc(IV),(V) Hydroxo Compounds in 0.5-14 M NaOH Solutions," WHC-EP-0897 (1996), Westinghouse Hanford Company, Richland, Washington, USA. 


\title{
Long-lived Radionuclides Immobilization into Mineral-type Matrices by a Self-propagating High-Temperature Synthesis (SHS)
}

\author{
E. M. Glagovsky, A. V. Kouprin, VNIINM; S. V. Yudintsev, IGEM-RAS, L .P. Pelevin, \\ VNIINM, E. E. Konovalov, State Scientific Center of Russian Federation "A. I. \\ Leipunskiy Institute of Physics and Power Engineering"
}

\begin{abstract}
The immobilization of high-level wastes, primarily long-lived transuranic and transplutonic radionuclides, requires more reliable and stable matrices, which, in contrast to glass, would provide for safe waste disposal for thousands and millions of years. The most challenging are mineral-type crystal matrices, where the radionuclides are safety fixed into the mineral structure.

Ceramics compositions based on pyrochlore and cubic zirconia oxide have been synthesized using the SHS method and then characterized by the authors. The distribution of elements between the ceramics's phases has been scrutinized in detail. The ceramic matrices are proposed for use as a form for immobilizing long-lived radionuclides after high-level waste partitioning.
\end{abstract}

\section{Introduction}

To isolate the environment from various radionuclides in high-level wastes (HLW) such as transuranic and transplutonic elements, and from cesium and strontium, a vitrification method is applied. However, this technology can be assessed only as an intermediate step for long-term storage of high-level wastes. Therefore, work is under way to develop mineral-type matrix materials, which have high stability in the natural aggressive environment and, thus, durability.

A few matrices, where the radionuclides are encapsulated into the crystal phase lattice, have been proposed. Of those, $\mathrm{ZrO}_{2}$ with a cubic fluorite-type lattice [1] as well as titanates and zirconates with a pyrochlore-type structure [2,3]. Those phases are capable of incorporating substantial quantities of actinides and rare-earth elements that accompany them in HLW with radiation and chemical stability. The pyrochlore-type composition $(\mathrm{Ca}, \mathrm{Gd}, \mathrm{U}, \mathrm{Pu})_{2}\left(\mathrm{Hf}, \mathrm{Ti}_{2}\right)_{7} \mathrm{O}_{7}$ has been selected in the $\mathrm{US}$ to immobilize excess weapons-grade plutonium [4].

In Russia and overseas, technologies are under development that utilize the principles of melting, e.g., "Cold" crucible inductive melting and powder metallurgy (SYNROC, Australia).

We also consider that a self-propagating high-temperature synthesis $[5,6]$ is one of the prospective methods of HLW immobilization into mineral-type matrix systems.

\section{Description of SHS Process}

The intent of the process is to construct a combustion wave regime as a result of the local initiation of a compacted mixture of fuel and oxidizer powder. In this regime, chemical transformations are concentrated in a mobile zone of combustion.

The formation of condensed reaction products in the combustion process is a very important and decisive feature of the SHS method, especially for HLW reprocessing. 
The main parameters of the SHS process are:

- Duration of initiation - 0.05-5.0 s;

- Temperature in a combustion wave - 1500-2500 K;

- Combustion velocity $-0.5-20 \mathrm{~mm} / \mathrm{s}$.

The SHS technology has the following essential merits:

- The absence of external heaters, low energy capacity;

- Ecological safety;

- High output;

- Design simplicity of technological equipment;

- The feasibility of remote management without unique technological equipment.

HLW solidification by the SHS process can reprocess different types of radioactive waste under remote and safe management with a gas-off treatment up to valid concentrations. This is very important because in this case secondary wastes are not being generated.

One should note that this technology for waste processing has been developed for the first time. This important work the authors of the paper share with specialists of the Institute of Structural Macrokinetics of the Russian Academy of Sciences [7].

\section{Experimental Research and Results}

The following experimental tests were performed using simulated ("model") calcinates of HLW:

- An analysis of mineralogical data with the purpose of selecting the most prospective minerals as the matrix materials heavily fixing the radionuclides ( $\mathrm{Am}$, Th, Cf, $\mathrm{Cm}, \mathrm{Sr}, \mathrm{Cs}$ ).

- Thermodynamic calculations for forecasting the synthesis of the minerals using the SHS process;

- Lab-scale study of the synthesis using various energy-forming and structure-forming components;

- Development of experimental equipment for force-type SHS compacting;

- Testing of design and technologic parameters of the SHS compacting using the experimental equipment;

- Synthesis of samples from 29 to $100 \mathrm{~mm}$ diameter on the base of perovskite, zirconolite, pyrochlore, Y-Al-granite with incorporation of the simulated calcinate.

Leachibility and a phase composition of the synthesized materials were studied. Leach rate tests were carried out according to the Russian standard GOST 29114-91. As a result of the analysis, it was established that the leach rates of the rare-earth elements are $<10^{-7} \mathrm{~g} / \mathrm{cm}^{2 *}$ day which satisfies considerations about the solubility of these materials.

Lab-scale tests of the materials, and the study of their properties and structures allowed us to select a few prospective compositions of similar elementary composition and crystal structure (such as zirconolite, perovskite, pyrochlore, phergusonite). 
In nature, these minerals are accompanied by uranium, thorium, and rare-earth elements $(\mathrm{Y}, \mathrm{Ce})$. Besides, the study has shown that a composition like Y-Al granite is also of interest for use as the matrix material.

The results of the study have shown that the materials are a multi-phase system, including similar 1-2 phases, as well as metalloid generated by reduction of the oxide-oxidizer. It was demonstrated that the ratio of the mineral-type phases is greatly dependent upon the amount of the calcinate included into the precursors.

The synthesized mineral-type compositions are high-thermodynamically stable under the conditions of the synthesis and after. The experimental tests made it possible to select the optimum regimes of the precursor combustion, and optimum quantities of the calcinates and additions to get the high qualitative mineral-type material.

The synthesis has demonstrated that it is possible to incorporate up to $20-25 \mathrm{wt} \%$ of the simulated calcinate into the matrices. The composition of the simulated calcinate, PC-20, (Table 1) prepared by specialists of A. A. Bochvar All-Russia Scientific Research Institute of Inorganic Materials (VNIINM) at the facility of distributive drying and calcination, imitates the real calcinates, including actinides and rare-earth elements.

The most important technologic problem of the synthesis of materials using the SHS process is to obtain dense, compact materials. This problem and other ones related to the SHS process are summarized and presented in a paper by Academician A. G. Merzhanov [8] concerning different operational methods of the direct synthesis of samples with the definite form, sizes, and properties. The simplest and the most studied option is coaxial pressing, by which, in a special press-mould, a hot product of combustion becomes non-porous. This approach was used in this work.

In the course of the research, three samples of various compositions were synthesized and investigated. The first two were based on zirconium titanate, and the third one on pyrochlore-type yttrium titanate. The starting precursor was prepared from thoroughly powdered basic oxides $\left(\mathrm{Y}_{2} \mathrm{O}_{3}, \mathrm{Gd}_{2} \mathrm{O}_{3}, \mathrm{UO}_{2}, \mathrm{ZrO}_{2}\right)$, oxidizer $\left(\mathrm{MoO}_{3}\right)$ and zirconium and titanium metals (Table 2). As a result of the synthesis, $\mathrm{Zr}$-containing samples (\#14 and \# 47) were a slug-like view, but the Ti-containing one (\# 56) exhibited a massive texture. For characterization, a probe of each sample's central part was taken.

Phase composition was researched using a "Dron-3" x-ray diffraction (XRD) facility (copper anode, $35 \mathrm{kV}$, $20 \mathrm{~mA}$, at $100 \mathrm{impulses} / \mathrm{second}$, velocity $-1^{\circ}$ at $2 \theta$ per minute in a range of angles $\left.6-80^{\circ}\right)$. Chemical composition and high-level waste simulants distribution were determined in two samples (\#47 and \# 56) using a JSM-5300 SEM facility equipped with a "Link ISIS" EDS spectrometer. The SEM/EDS analysis was made at $25 \mathrm{kV}, 1 \mathrm{nA}$, electronic beam diameter of 1-3 microns, within 50 seconds. Pure metals, oxides, and phosphates were used as specimens. The sensitivity limit is $0.2-0.4 \mathrm{wt} \%$, analytical accuracy is $\pm 10 \%$ specific. To examine the phases, an electron diffraction method was applied. The research was done using a "JEM-100c" TEM facility equipped with a KEVEX-5100 EDS spectrometer.

Table 1. Composition of the simulated calcinate PC-20.

\begin{tabular}{|c|c|c|c|c|c|c|}
\hline Oxide & $\mathbf{C e}_{2} \mathbf{O}_{3}$ & $\mathbf{L a}_{\mathbf{2}} \mathbf{O}_{3}$ & $\mathbf{G d}_{\mathbf{2}} \mathbf{O}_{3}$ & $\mathbf{Z r O}_{2}$ & $\mathbf{M n O}_{2}$ & $\mathbf{F e}_{2} \mathbf{O}_{3}$ \\
\hline Content, \% wt & 25,5 & 45,3 & 3,7 & 19,3 & 3,7 & 2,4 \\
\hline
\end{tabular}


Table 2. Starting precursor and product compositions obtained in SHS tests.

\begin{tabular}{|c|l|l|l|}
\hline Sample \# & \multicolumn{1}{|c|}{ Starting precursor } & \multicolumn{1}{c|}{$\begin{array}{c}\text { Base } \\
\text { component }\end{array}$} & \multicolumn{1}{|c|}{ Product phase composition } \\
\hline 14 & $\left(\mathrm{Y}_{2} \mathrm{O}_{3}, \mathrm{Gd}_{2} \mathrm{O}_{3}, \mathrm{UO}_{2}, \mathrm{ZrO}_{2}\right)+\mathrm{MoO}_{3}+\mathrm{Zr}$ & Cubic oxide & Fluorite-type oxide, molybdenum metal \\
\hline 47 & $\left(\mathrm{Y}_{2} \mathrm{O}_{3}, \mathrm{ZrO}_{2}\right)+\mathrm{MoO}_{3}+\mathrm{Zr}$ & Same & Same \\
\hline 56 & $\left(\mathrm{Y}_{2} \mathrm{O}_{3}, \mathrm{TiO}_{2}\right)+\mathrm{MoO}_{3}+\mathrm{Ti}$ & Pyrochlore & Pyrochlore, molybdenum metal \\
\hline
\end{tabular}

Two crystal phases were identified in all samples (Fig. 1). The first one is molybdenum metal without any impurities, which could be identified by SEM/EDS analysis. For a basic reflection, the interplanar distance is $d_{110}=2.22 \mathrm{~A}$; in addition, there are less intensive reflections, including $d_{310}=0.99 \mathrm{~A}$ (according to the JCPDS \#42-1120 data base's standard). In sample \# 4, molybdenum exclusions are of rounded form. Their sizes are usually 10-20 microns. In sample \# 56, molybdenum forms grain aggregates of 1-3 microns [7].

The second predominant component is composed of fluorite (samples \# 14, 47) and pyrochlore (sample \# 56) phases. XRD patterns of the fluorite phase are equal to cubic oxide $\left(\mathrm{Zr}_{0.7} \mathrm{Y}_{0.3}\right) \mathrm{O}_{1.85}$ (according to the JCPDS \#77-2112 data base standard). A main reflection on the diffraction patterns is $d_{111}=2.97-2.98 \mathrm{~A}$ that is similar to the cell's parameter at $5.15 \mathrm{~A}$. Its composition in sample \# 47 could be evaluated as $\left(\mathrm{Zr}_{1-\mathrm{x}} \mathrm{Y}_{\mathrm{x}}\right) \mathrm{O}_{2-0.5 \mathrm{x}}, 0.46<\mathrm{x}<0.52$. The $\mathrm{Y}: \mathrm{Zr}$ ratio varies from 0.85 to 1.08 (in atomic quantities).

Reflections of the pyrochlore in sample \# 56 are identical to ones for (JCPDS \#27-0982). A size $\left(d_{222}=\right.$ $2.91 \mathrm{~A}$ ) of an elementary cell equal to $10.1 \mathrm{~A}$ has been calculated upon its basic reflection. Pyrochlore grains are of quadrant or isometric cross-sections of 10-20 microns. The grains have a non-uniform structure (Fig. 1), which is characterized in the higher concentration of molybdenum and the lower one of yttrium (Table 4) in boundary grains in contrast to the central ones. Since we did not find any separated molybdenum-containing phase in the matrix, one might suggest that the molybdenum is included into the pyrochlore as a structural component.

It can be explained by the assumption that the fraction of molybdenum to be incorporated into the pyrochlore increases at the end of the SHS process, for instance, due to lessening the temperature or by transition of molybdenum metal to a four-valent state.
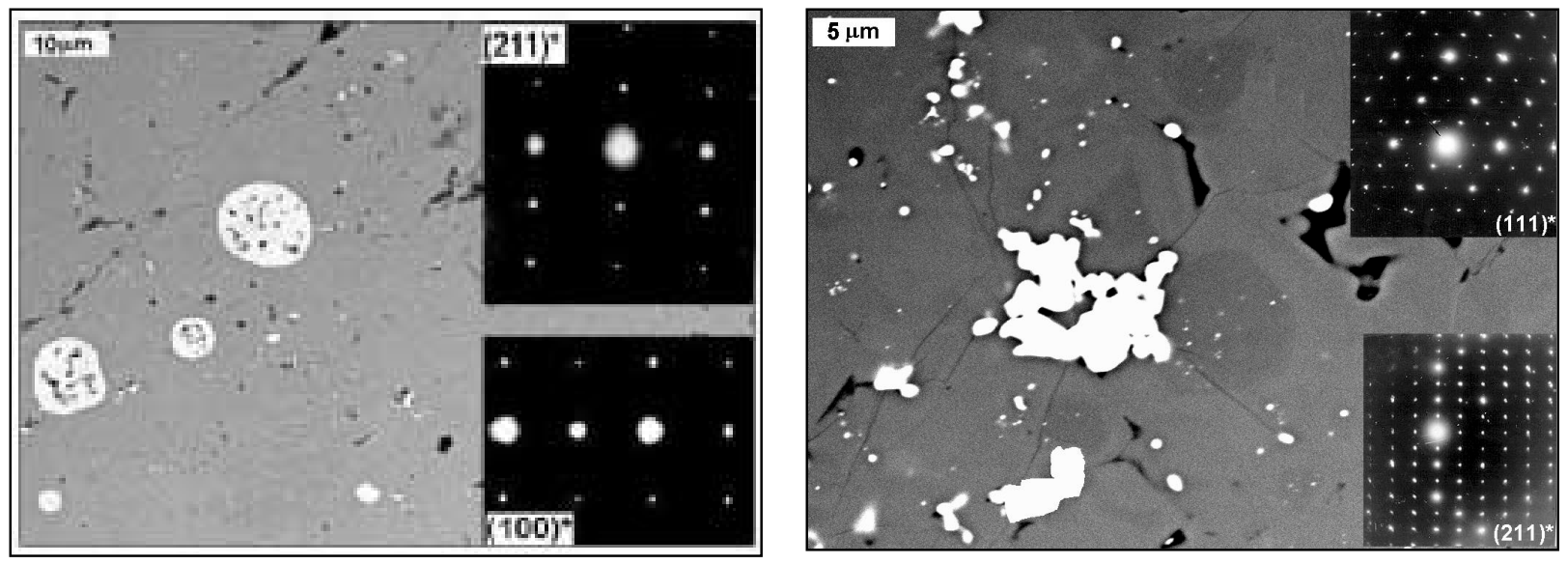

Fig. 1. SEM/EDS view of samples \# 47 (left side) and \# 56 (right side). 
Going from the comparison of charge ratios of elements of the mentioned phase $\left(\mathrm{Y}^{3+}, \mathrm{Ti}^{4+}\right)$, as well as molybdenum's valent states, it is likely that molybdenum in the pyrochlore is in the form $\mathrm{Mo}^{4+}$, substituting $\mathrm{Ti}^{4+}$ ion $\left({ }^{\mathrm{VI}} \mathrm{R}_{\mathrm{Mo}}{ }^{4+}=0.65 \mathrm{~A},{ }^{\mathrm{V} /} \mathrm{R}_{\mathrm{Ti}}{ }^{4+}=0.61 \mathrm{~A}\right)$, similar in charge and equal in size. Meanwhile, bearing in mind the high temperature and low oxidizing potential of the SHS process, we can encounter the presence of other ions such as $\mathrm{Ti}^{3+}(0.67 \mathrm{~A})$ or $\mathrm{Mo}^{3+}(\mathrm{R}=0.69 \mathrm{~A})$. That fact is approved by a deficit of the summary number of cations in the formulation in contrast to stoichiometry (Table 3). The shortage could be leveled assuming that about $5 \%$ of titanium is in the form $\mathrm{Ti}^{3+}$. At the same time, the fluorite-type sample \# 47 does not contain molybdenum. It is presumably resulted from the higher difference of $\mathrm{Zr}^{4+}$ and $\mathrm{Mo}^{4+}$ ion sizes ( 0.84 and $0.65 \mathrm{~A}$, respectively), that obstructs the molybdenum to enter the phase's crystal lattice.

Table 3. The formulation and composition of the fluorite-type oxide in sample \# 47.

\begin{tabular}{|l|c|c|c|c|c|}
\hline \multicolumn{1}{|c|}{ Oxide, \% wt } & $\mathbf{1}$ & $\mathbf{2}$ & $\mathbf{3}$ & $\mathbf{4}$ & $\mathbf{5}$ \\
\hline $\mathrm{Y}_{2} \mathrm{O}_{3}$ & 43.7 & 44.1 & 46.4 & 49.8 & 49.3 \\
\hline $\mathrm{ZrO}_{2}$ & 56.3 & 55.9 & 53.6 & 50.2 & 50.7 \\
\hline $\mathrm{Sum}$ & 100.0 & 100.0 & 100.0 & 100.0 & 100.0 \\
\hline Ion & \multicolumn{2}{|c|}{ The number of atoms in the formulation counted on the sum of cations equal to 1 } \\
\hline $\mathrm{Y}^{3+}$ & 0.46 & 0.46 & 0.49 & 0.52 & 0.52 \\
\hline $\mathrm{Zr}^{4+}$ & 0.54 & 0.54 & 0.51 & 0.48 & 0.48 \\
\hline Sum of cations & 1.0 & 1.0 & 1.0 & 1.0 & 1.0 \\
\hline $\mathrm{O}^{2-}$ & 1.77 & 1.77 & 1.76 & 1.74 & 1.74 \\
\hline
\end{tabular}

Table 4. The formulation and composition of the pyrochlore-type phase in sample \# 56.

\begin{tabular}{|c|c|c|c|c|c|c|}
\hline \multirow{2}{*}{$\begin{array}{c}\text { Oxide, \% } \\
\text { wt }\end{array}$} & \multicolumn{3}{|c|}{ Composition of various grains } & \multicolumn{3}{|c|}{ Zonal crystal } \\
\hline & 1 & 2 & 3 & \multicolumn{2}{|c|}{ Grain center } & Edge \\
\hline $\mathrm{TiO}_{2}$ & 38.9 & 39.6 & 38.4 & 40.5 & 39.7 & 39.2 \\
\hline $\mathrm{Y}_{2} \mathrm{O}_{3}$ & 54.6 & 54.6 & 55.5 & 56.7 & 56.9 & 54.9 \\
\hline $\mathrm{MoO}_{2}$ & 6.5 & 5.8 & 6.1 & 2.8 & 3.4 & 5.9 \\
\hline Sum & 100.0 & 100.0 & 100.0 & 100.0 & 100.0 & 100.0 \\
\hline Ion & \multicolumn{6}{|c|}{ The number of atoms in the formulation counted for $7 \mathrm{O}^{2-}$ ions } \\
\hline $\mathrm{Ti}^{4+}$ & 1.89 & 1.92 & 1.87 & 1.96 & 1.93 & 1.90 \\
\hline $\mathrm{Y}^{3+}$ & 1.88 & 1.87 & 1.92 & 1.94 & 1.96 & 1.89 \\
\hline $\mathrm{Mo}^{4+}$ & 0.20 & 0.17 & 0.19 & 0.08 & 0.10 & 0.18 \\
\hline $\begin{array}{l}\text { Sum of } \\
\text { cations }\end{array}$ & 3.97 & 3.97 & 3.98 & 3.99 & 3.99 & 3.97 \\
\hline $\mathrm{O}^{2-}$ & 7.0 & 7.0 & 7.0 & 7.0 & 7.0 & 7.0 \\
\hline
\end{tabular}

\section{Discussion of Results}

Results of the study have shown that the structure of the phase accommodating the actinides is mostly dependent upon their chemical composition. For say, the $\mathrm{Y}_{2} \mathrm{O}_{3}-\mathrm{ZrO}_{2}$ system is of fluorite, meanwhile $\mathrm{Y}_{2} \mathrm{Ti}_{2} \mathrm{O}_{7}$ is of pyrochlore type. To understand the process of incorporating the actinides into the phases, one needs to look at their structures in more detail.

The fluorite-type lattice (Fm3m group, $Z=4$ ) can be characterized in the form of cubic polyhedrons connected with each other by common edges as if it were a three-dimensional chessboard (Fig. 2). In the 

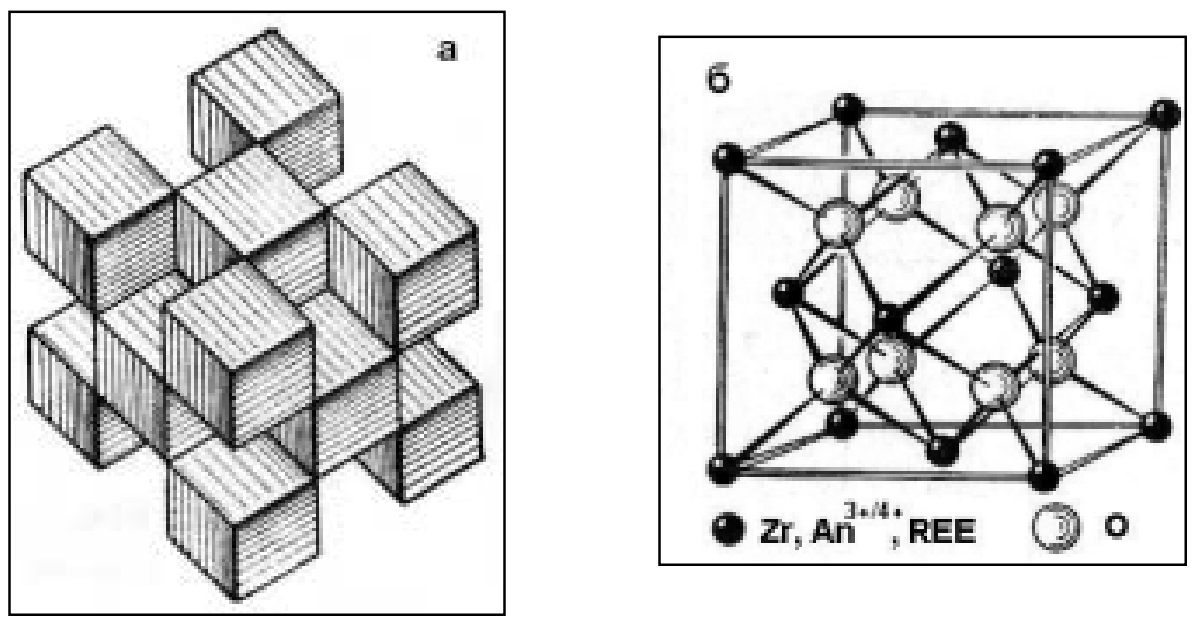

Fig. 2. A fluorite-type lattice in polyhedral (a) and ball (b) views.

cube's center there are cations atop anions. The lattice is inherent to cerium oxide $\left(\mathrm{CeO}_{2}\right)$, as well as to four-valent actinide oxides $\left(\mathrm{ThO}_{2}, \mathrm{UO}_{2}, \mathrm{PuO}_{2}\right)$. For zirconium dioxide there are a few various structures: monoclinic, tetragonal, and cubic [9]. The monoclinic modification transfers to the tetragonal one in a range of from 1050 to $116{ }^{\circ} \mathrm{C}$. The tetragonal goes to the cubic face-centered lattice of fluorite type at $>2300^{\circ} \mathrm{C}$. Some additives $\left(\mathrm{Ca}^{2+}, \mathrm{Y}^{3+}, \mathrm{REE}^{3+}, \mathrm{An}^{3+/ 4+}\right)$ make it possible to establish the transfer with obtaining a stabilized phase of cubic zirconium dioxide at lower temperature. In the $\mathrm{ZrO}_{2}-\mathrm{Y}_{2} \mathrm{O}_{3}$ system in a range of $10-30 \%$ molar $\mathrm{Y}_{2} \mathrm{O}_{3}$ the fluorite-type oxide is formed at below $1000^{\circ} \mathrm{C}$ [9]. Eight-coordinated positions in the lattice can be filled out by three- and four-valent actinides and lanthanides. For providing the electrical neutrality of the lattice, the three-valent actinide ions entering is accompanied by reducing number of oxygen anions and vacancies. The general formulation of that matter is as follows:

VIII $\left[\left(\mathrm{REE}, \mathrm{An}^{3+}\right)_{\mathrm{x}}\left(\mathrm{An}^{4+}, \mathrm{Zr}\right)_{1-\mathrm{x}}\right] \mathrm{O}_{2-0.5 \mathrm{x}}$.

To fix the fluorite structure, the difference in ion sizes should be higher than some specific value. Based upon different data, the ionic radiuses ratio for (REE, An) ${ }^{3+/ 4+}: \mathrm{Zr}^{4+}$, provided that the lattice is in a stable state, is 1.3-1.4. It means that the ion's radius, which reacts with zirconium $\left(\mathrm{Zr}^{4+}=0.84 \mathrm{~A}\right)$ forming the fluorite type oxide, cannot exceed 1.05A.

That requirement is met by ions of heavy rare-earth elements (from terbium to lutetium), yttrium, as well as four-valent cerium and actinides from thorium to plutonium. For all those materials, the existence of fluorite-type oxides and solid solutions has been experimentally recognized. With the increase of the ratio between the sizes of ions incorporated into the structure and zirconium ion, the fluorite lattice goes to an unstable state and transforms to relative, but fairly diverse pyrochlore-type structure.

The pyrochlore structure $(\mathrm{Fd} 3 \mathrm{~m}, \mathrm{Z}=8)$ is of fluorite type. Its elementary cell resembles the double fluorite-type cell (Fig. 3), where a half of polyhedrons in the form of cube do not have two anions located alongside the long diagonal. As a result, an octahedron is formed and the phase's formulation changes from ${ }^{\mathrm{VIII}} \mathrm{A}_{4}{ }^{\mathrm{IV}} \mathrm{X}_{8}$ (the fluorite's quadruple formulation) to ${ }^{\mathrm{VIII}} \mathrm{A}_{2}{ }^{\mathrm{VI}} \mathrm{B}_{2}{ }^{\mathrm{IV}} \mathrm{O}_{6}{ }^{\mathrm{IV}} \mathrm{X}$. A coordination numbers of ions are: $\mathrm{AO}_{6} \mathrm{X}_{2}, \mathrm{BO}_{6}, \mathrm{OA}_{2} \mathrm{~B}_{2} и \mathrm{XA}$, where " $\mathrm{A}$ " and " $\mathrm{B}$ " are cations in two various positions and " $\mathrm{X}$ " is a complementary anion. $\mathrm{O}^{2-}$ anions compose the tops of the polyhedron and take six of the eight tops of the polyhedron; the other two are captured by anions " $\mathrm{X}$ " $\left(\mathrm{O}^{2-}, \mathrm{F}^{-}, \mathrm{Cl}^{-}\right)$anions. The lattice is inherent to natural minerals, basically tantalum-niobates and a great variety of artificial phases $\left(A_{1} A_{2}\right)\left(B_{1} B_{2}\right) O_{7}$, where $A$ and $B$ are cations with a charge from +1 to +4 and from +3 to +5 , respectively [10-12, 3$]$. 

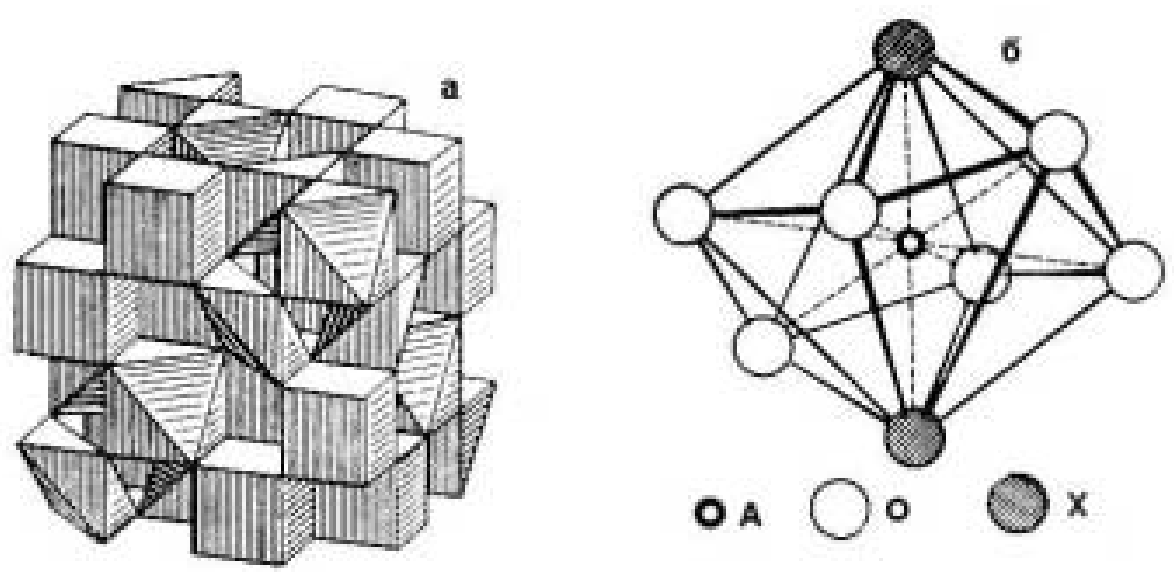

Fig. 3. A pyrochlore structure (Belov, 1950; Isupov, 1958). a - general view, b- $\mathrm{AO}_{6} \mathrm{X}_{2}$ polyhedron structure.

One of the requirements of the pyrochlore lattice's stability is a correlation of cation sizes in two various positions $[13,14,3]$. Based on the structural analysis, a conclusion has been made that the pyrochlore lattice is stable only if the ion radiuses ratio varies from 1.30 to 1.80 (or $1.55 \pm 0.25$ ). It is mostly stable within the range from 1.50 to 1.60 , which resembles minimum free energy matter formation. The alteration of the ratio results in destabilization of the lattice and its decomposition after capping the mentioned critical magnitudes. The option of optimum compositions for fixing the actinide-containing wastes is thereby defined.

The synthesis of rare-earth pyrochlores is realizable provided that octahedral ion radiuses are $0.55-$ $0.75 \mathrm{~A}$. That range covers four-valent ions of titanium $(0.605 \mathrm{~A})$, hafnium $(0.71 \mathrm{~A})$ and zirconium $(0.72 \mathrm{~A})$; for those the existence of pyrochlore-type compositions has been experimentally confirmed. Titanates of lanthanides of an yttrium group (from $\mathrm{Tb}^{3+}$ to $\mathrm{Lu}^{3+}$ ) and zirconates of larger ions of $\mathrm{REE}_{2} \mathrm{Zr}_{2} \mathrm{O}_{7}(\mathrm{REE}=$ $\mathrm{La}^{3+}-\mathrm{Gd}^{3+}$ ) lanthanides are also have the pyrochlore-type structure. The same lattice is likely inherent to three-valent $\mathrm{Pu}^{3+}, \mathrm{Am}^{3+}$ n $\mathrm{Cm}^{3+}$ zirconates, which have similar ionic radiuses with the abovementioned lanthanides.

Among other elements that are capable of demonstrating pyrochlore phases, one can mention technetium $(0.65 \mathrm{~A})$, ruthenium $(0.62 \mathrm{~A})$ and rhodium $(0.60 \mathrm{~A})$. The availability of rare-earth pyrochlores in the last two elements is also corroborated experimentally. Ruthenium, rhodium, and specifically technetium generated in the process of uranium-235 fission are environmentally perilous.

Results obtained for rare-earth pyrochlores make it possible to assess the analogous phases of threevalent actinides. Taking into account the data about ionic radiuses [15], one could suggest the presence of four such materials for curium, and three each for americium and plutonium. Despite the curium, the titanates of three-valent Pu and Am are crystallized in the same monoclinic synergy [16], as the rare-earth element titanates. This can be explained by the availability of three-valent ions of those elements of larger sizes, as is necessary to form the pyrochlore lattice.

Though the cubic lattices for $\mathrm{Pu}_{2} \mathrm{Ti}_{2} \mathrm{O}_{7}$ and $\mathrm{Am}_{2} \mathrm{Ti}_{2} \mathrm{O}_{7}$ are unstable, pyrochlores with higher content of the actinides have been produced [16]. These are solid solutions, where lanthanum ions of fewer radiuses play the role of stabilizers of the pyrochlore lattice. In particular, experimentally, it has been identified that the pyrochlore based on gadolinium titanate $\left(\mathrm{Gd}_{2} \mathrm{Ti}_{2} \mathrm{O}_{7}\right)$ can incorporate up to $16 \%$ molar of $\mathrm{Pu}_{2} \mathrm{Ti}_{2} \mathrm{O}_{7}$. With the decrease of lanthanum ion sizes, the content of the plutonium's final metamict state increases to 
$22 \%$ (for $\mathrm{Er}_{2} \mathrm{Ti}_{2} \mathrm{O}_{7}$ ) and $33 \%$ (for $\mathrm{Lu}_{2} \mathrm{Ti}_{2} \mathrm{O}_{7}$ ). The americium content in the pyrochlore $(\mathrm{Er}, \mathrm{Am})_{2} \mathrm{Ti}_{2} \mathrm{O}_{7}$ is much higher $\left(\sim 61 \%\right.$ molar counted for $\left.\mathrm{Am}_{2} \mathrm{Ti}_{2} \mathrm{O}_{7}\right)$. One can expect that the same result (stabilizing of the pyrochlore lattice) will be got by a half-done substitution of the titanate for hafnium, zirconium, or tin cations with the formation of a solid solution $(\mathrm{Pu} / \mathrm{Am})_{2}(\mathrm{Ti}, \mathrm{Sn}, \mathrm{Hf}, \mathrm{Zr})_{2} \mathrm{O}_{7}$.

Here it is amenable to note that under definite conditions the actinides could be located in the pyrochlore either in three- or four-valent states [3]. The following stochiometric formulation meets that case: $\left(\mathrm{Pu}^{3+}{ }_{2-}\right.$ $\left.{ }_{x} \mathrm{Pu}^{4+}\right)(\mathrm{Hf}, \mathrm{Zr})_{2} \mathrm{O}_{7+x}$. If the fraction of ion +4 increases, the pyrochlore structure gets unstable and transforms into the fluorite-type lattice.

In particular, this occurred due to the oxidizing of plutonium while heating in air at $500-700{ }^{\circ} \mathrm{C}$ with the transition of the $\left(\mathrm{Pu}^{3+}\right)_{2} \mathrm{Zr}_{2} \mathrm{O}_{7}$ pyrochlore-type phase to cubic dioxide $\left(\mathrm{Pu}^{4+}, \mathrm{Zr}\right) \mathrm{O}_{2}$.

The high-level wastes contain the actinides as follows: $\mathrm{U}>\mathrm{Np}, \mathrm{Pu}>\mathrm{Am}, \mathrm{Cm}$. Of those, under SHS conditions (high temperature, reducing conditions), uranium and neptunium will likely be in a four-valent state, but americium and curium in a three-valent one. Plutonium might exhibit simultaneously in both of them. To include the actinides' four-valent ions into the pyrochlore phase, it is necessary to involve the ions of fewer charges (for example, calcium) into the lattice. Herewith, an isomorphous exchange could be achieved $-2 \mathrm{REE}^{3+}=\mathrm{An}^{4+}(\mathrm{Th}, \mathrm{U}, \mathrm{Np}, \mathrm{Pu})+\mathrm{Ca}^{2+}$.

Pyrochlore of the $\mathrm{CaUTi}_{2} \mathrm{O}_{7}$ formulation has been experimentally obtained [17-19].

Besides, $\mathrm{Ca}(\mathrm{Pu}, \mathrm{U}, \mathrm{Zr}) \mathrm{Ti}_{2} \mathrm{O}_{7}$ and $\mathrm{Ca}(\mathrm{Np}, \mathrm{Zr}) \mathrm{Ti}_{2} \mathrm{O}_{7}$ pyrochlores, containing up to $45 \mathrm{wt} \% \mathrm{PuO}_{2}$ and $\mathrm{NpO}_{2}$ were also synthesized [2]. One can suggest the existence of materials where strontium is included instead of calcium for neutralizing the charge. The realization of the procedure would permit the incorporation of both actinides and short-lived isotopes of higher radiotoxicity (Sr-90).

The other matrices capable of incorporating alpha-emitters and $\mathrm{Sr}-90$ are $\mathrm{SrPu} \mathrm{uii}_{4} \mathrm{O}_{12}$ or $\mathrm{SrAm}_{2} \mathrm{Ti}_{4} \mathrm{O}_{12}$ with a perovskite structure [16].

\section{Conclusions}

Matrices based on the fluorite-type phase (samples \#14 and 47) or pyrochlore-type yttrium titanate (sample \# 56), as well as molybdenum metal, have been synthesized using the SHS method. The formation of the fluorite-type and pyrochlore-type materials is correlated with the general rules of the structure's stability. A main factor of their occurrence in the $\mathrm{A}_{2} \mathrm{O}_{3}-\mathrm{BO}_{2}(\mathrm{~A}=\mathrm{TR}, \mathrm{An} ; \mathrm{B}=\mathrm{Ti}, \mathrm{Zr}$ ) system is element ionic radiuses ratio. If the difference in ion sizes is insignificant (for example, yttrium and zirconium), an oxide with a fluorite-type lattice $\left(\mathrm{Zr}_{1-x}, \mathrm{Y}_{\mathrm{x}}\right) \mathrm{O}_{2-0.5 \mathrm{x}}$ is formed. That structure might incorporate other lanthanides, as well as three- and four-valent actinides, including americium $\left(\mathrm{Am}^{3+}=1.09 \mathrm{~A}\right)$, curium $\left(\mathrm{Cm}^{3+}=1.08 \mathrm{~A}\right)$, uranium $\left(\mathrm{U}^{4+}=1.0 \mathrm{~A}\right)$, plutonium $\left(\mathrm{Pu}^{3+}=1.10 \mathrm{~A}, \mathrm{Pu}^{4+}=0.96 \mathrm{~A}\right)$ and neptunium $\left(\mathrm{Np}^{4+}\right.$ $0.98 \mathrm{~A})$.

At substantially larger difference of ionic sizes both for yttrium $\left(\mathrm{Y}^{3+}=1.02 \mathrm{~A}\right)$ and titanium $\left(\mathrm{Ti}^{4+}=0.61 \mathrm{~A}\right)$, cations will be located on eight $\left(\mathrm{Y}^{3+}\right)$ or sixth $\left(\mathrm{Ti}^{4+}\right)$ coordinated positions without overlapping with each other. As a result, the fluorite structure becomes unstable and transforms to the pyrochlore-type lattice.

The structure also contains molybdenum (up to $6 \mathrm{wt} \% \mathrm{MoO}_{2}$ ) that can be explained by the similarity of $\mathrm{Mo}^{4+}(0.65 \mathrm{~A})$ and $\mathrm{Ti}^{4+}(0.61 \mathrm{~A})$ ion sizes. The same behavior for technetium $\left(\mathrm{Tc}^{4+}=0.65 \mathrm{~A}\right)$ makes it possible to immobilize both actinides and Tc-99 $\left(T_{1 / 2}>2 \times 10^{5}\right.$ years) generated in the course of uranium235 fission with a yield of $6 \%$ into the pyrochlore-type matrices. 
To incorporate the four-valent actinides into the pyrochlore-type phase, a few ions of rare-earth elements should be substituted by calcium ions, and likely strontium. The last conclusion needs to be tested additionally. The follow-on efforts will be concentrated on the production of the pyrochlore-type materials by means of the SHS method. They will simultaneously contain actinides, zirconium, and lanthanides, including neutron absorbers ( $\mathrm{Gd}$ or $\mathrm{Sm}$ ), as well as fission products $(\mathrm{Sr}, \mathrm{Tc})$.

\section{References}

1. Gong W.L., Lutze W., Ewing R.C. Zirconia - a ceramic for excess weapons plutonium wastes. In: Sci. Bas. Nucl. Waste Manag.-XXII, 1999, MRS Symp. Proc. 556. P.63-70.

2. Vance E.R., Begg B.D., Day R.A., Ball C.J. Zirconolite-rich ceramics for actinide wastes. In: Sci. Bas. Nucl. Waste Manag.-XVIII. 1995. MRS Symp. Proc. 353. Pt.2. P.767-774.

3. Raison P.E., Haire R.G., Sato T., Ogawa T. Fundamental and technological aspects of actinide oxide pyrochlores: relevance for immobilization matrices. In: Sci. Bas. Nucl. Waste Manag.-XXII, 1999, MRS Symp. Proc. 556. P.3-10.

4. Ebbinghaus B.B., VanKonynenburg R.A., Ryerson F.J. et al. Ceramic formulation for the immobilization of plutonium. In: Proc. Intern. Conf. "HLW, LLW, Mixed Wastes and Environm. Restor. - Working Towards a Cleaner Environment", 1998, Tuscon, AZ, CD-Rom. Sess. 65-04.

5. Merzhanov A.G. A self-propagating high-temperature synthesis. Physical chemistry. Modern problems. M., 1983, p.6-45.

6. Glagovsky E.M., Kuprin A.V., Pelevin L.P. Konovalov E.E. et al. Immobilization of high-level wastes into mineral-type materials by a self-propagating high-temperature synthesis. Atomic Energy. 1999. v.87. issue 1. p.57-61.

7. Merzhanov A.G., Borovinskaya I.P., Makhonin N.S et al. A method to process various radioactive wastes. Patent of Russia \# 2065216 from 18.03.1994.

8. Merzhanov A.G., Scientific bases of achieving and perspectives of evolving the processes of heavyfire combustion. Chemical Series. RAS publishing, 1997, \#1, pp 8-32.

9. Maddrell E. R. Effect of conjoint additions of rare earth element oxides on the phase stability of zirconia. In: Sci. Bas. Nucl. Waste Manag.-XIX. 1996, MRS Symp. Proc. 412. P.353-360.

10. Aleshin E., Roy R. Crystal chemistry of pyrochlore. J. Am. Cer. Soc., 1962, V.45, N1, P.18-25.

11. Chakoumakos B.C. Systematic of the pyrochlore structure type, ideally $A_{2} B_{2} X_{6} Y$. J. Sol. State Chem., 1984, V. 53, P.120-129.

12. Chakoumakos B.C., Ewing R.C. Crystal chemical constraints on the formation of actinide pyrochlores. In: Sci. Bas. Nucl. Waste Manag.-VIII, 1985, MRS Symp. Proc. 44, P.641-646.

13. Belov N.V. Essays about structural mineralogy. The Lvovsky geologic society's mineralogical collection, 1950, \#4, p.21-34.

14. Isupov V.A.Geometric criterion of a pyrochlore-type structure. Crystallography, 1958, v.3, \#1, p.99100.

15. Shannon R.D. Revised effective ionic radii and systematic studies of interatomic distances in halides and chalcogenides. Acta Cryst., 1976, Pt.A, V.32, P.751-767.

16. Shoup S.S., Bambergher C. E. Syntheses of titanate-based hosts for the immobilization of Pu (III) and Am (III). In: Sci. Bas. Nucl. Waste Manag.-XX, MRS Symp. Proc. 465, 1997, P.379-386.

17. Ringwood A.E., Disposal of high-level nuclear wastes: a geological perspective. In: Miner. Mag., 1985, 49, Pt.2, P.159-176. 
18. Solomah A.G., Sridhar T.S., Jones S.C. Immobilization of uranium-rich high-level radioactive liquid waste in Synroc-FA. In: Advances in Ceram. Nucl. Waste Manag., 20. Am. Ceram. Soc., Columbus, Ohio, 1986, P.259-265.

19. Stefanovsky S.V., Yudintsev S.V., Nikonov B.S. et al. Pyrochlore-type phases for actinides and rare earths elements immobilization. In: Sci. Bas. Nucl. Waste Manag. XXII. MRS Symp. Proc. 556, 1999. P.27-34. 


\title{
Scientific Investigation of Spent Nuclear Fuel Encapsulated into Glass-Ceramic Protective Forms
}

\author{
S. Yu. Sayenko, I. M. Neklyudov, G. A. Kholomeyev, A. P. Pilipenko, A. E. Surkov, \\ B. A. Shilyayev, R. V. Tarasov, S. V. Gabelkov, E. P. Shevyakova, V. I. Zmiy, Zh. S. \\ Azhazha, NSC-KIPT
}

\begin{abstract}
To isolate spent nuclear fuel by encapsulation (i.e., containment of waste products in metal canisters with protective buffer filling material), we proposed a number of glass-ceramic compositions as prospective protective materials. This paper describes the investigations carried out at the National Science Center Kharkov Institute of Physics and Technology (NSC KIPT), Kharkov, Ukraine.

NSC KIPT, within the framework of the STCU Project, created protective glass-ceramic materials by hot isostatic pressing (HIP) of powder mixtures based on rocks and minerals, which were preliminarily sintered in air.
\end{abstract}

\section{Introduction}

At present in the field of nuclear power, priority is given to factors concerning new levels of reliability, safety, and ecological admissibility not only for nuclear power plant reactors, but also for accompanying stages of the nuclear fuel cycle. One of the main problems is the solution of the task of reliable and safe isolation of the spent nuclear fuel (SNF).

The open nuclear fuel cycle considers the direct disposal of the spent fuel while the closed cycle considers the disposal of the vitrified wastes (after reprocessing the SNF) in deep geological formations. However, a third approach is also possible-the long-term controlled storage of highly radioactive wastes or SNF before final disposal in geological formations. In recent years, many countries have given the highest priority to the strategy of long-term controlled storage of SNF before disposal or reprocessing.

Preliminary long-term storage requires creation of additional temporary storage facilities. In order to achieve high safety, these facilities require additional protective barriers (e.g., special constructions inside the metal containers, protective filling particulate materials, monolithic containers, etc.) to prevent the possible exit of radionuclides from wastes or spent fuel into the environment within the long period of preliminary storage.

Over the last ten years, there has been increased research attention paid to glass ceramics and ceramic materials used to immobilize highly radioactive wastes and nuclear spent fuel. Chemically stable materials with stable natural analogs attract the most interest.

This STCU project is intended to supply the experimental and calculational grounding for the principle of the long-term, dry method of SNF storage, and its subsequent disposal, by the use of spent nuclear fuel encapsulation into protective glass-ceramic monoliths. This principal would fully comply with safety standards both for SNF storage in the monolithic protective form on the open ground and the subsequent final disposal of fuel without interim overloading. The protective form is created by hot isostatic pressing 
of compositions on the base of rocks and minerals. The material obtained looks like a glass-ceramic composition consisting of crystalline, mineral-like grains tied together by a matrix of the glassy phase.

The main objectives of the project are the creation of the glass-ceramic materials on the base of natural granites and clays, optimization of parameters of sintering and sintering under pressure, determination of the main characteristics of the glass-ceramic materials proposed as protective engineering barriers for SNF encapsulation, the grounding of terms of safe long-term storage and disposal of the SNF contained in the glass-ceramic monolith, and the determination of the maximum duration of use.

\section{Scientific and Technological Basis}

\section{Basis for use of the glass-ceramic composition}

It is generally accepted that to create safe conditions for preliminary storage, non-hermetic spent fuel assemblies to be processed are placed into hermetic cartridges; fuel assemblies intended for further disposal have to be encapsulated into a hermetic protective form. Regarding storage in the cartridges at present, this problem is thoroughly worked out: metal-concrete containers (i.e., casks) are proposed for transportation or dry interim storage. The question concerning fuel assembly encapsulation into the hermetic protective form is still unsolved. In this case, investigations are needed to optimize the composition of materials for the protective form. Interest in mineral like compositions has also increased.

Among the most interesting research, work with the following materials should be noted: glass and glass ceramics based on natural basalt (Czech Republic), ceramic based on clay (Russia, US, Germany), artificial ceramic Synrock (Australia), phosphate ceramics (US), monazite ceramics (US), sphene glass ceramics (Canada), and diopside glass ceramics (Japan).

However, it is noteworthy that all investigations into development of new ceramic compositions are carried out taking into account economic expediency, local raw materials, and specific features of radioactive waste management in the country. Therefore, with due regard for the current economic situation in Ukraine and the natural geological formations in its territory, natural materials, In particular (K-Na)-feldspars with the general formula $\mathrm{K}(\mathrm{Na}) \mathrm{AlSi}_{3} \mathrm{O}_{8}$, are of the most interest as initial components for SNF encapsulation and radioactive waste immobilization into mineral like protective matrices. These natural aluminosilicates are exactly the rock forming minerals of the most widespread bedrock-granite, which is proposed as a geological medium for SNF disposal in Ukraine. The granites are characterized by high stability of structure and composition. Besides, their crystalline structure can include ions of volatile products of fission (e.g., Cs due to isomorphous ion exchange). It should be noted that the main silicate and aluminosilicate minerals (i.e., quartz and its varieties-tridymite and crystabolite, feldspars, kaolin and clay minerals) possess high radioactive resistance. For quartz, for example, the fixed changes in the near surface layers are observed at the irradiation dose $10^{6}-10^{7} \mathrm{~Gy}$. The limit dose for clay minerals of the kaolinite and montmorillonite groups makes up $10^{7} \mathrm{~Gy}$.

It is known that many rocks, mostly consisting of silicates, are polyphase, and they contain both crystalline and glass-forming components (e.g., volcanic glass or partly amorphized mineral phases). They preserve their characteristics after being irradiated by relatively big doses. On the whole, the radiation resistance of the rock depends on correlation and the number of these components [1-3].

As archeological findings show, ceramics based on clay have resisted natural factors over hundreds and thousands of years. These china ceramics look like a glass-ceramic material, whose glass phase based on silicon oxide contains grains of mullite, $\alpha$-quartz and feldspars [4]. Aluminosilicate glass-ceramic materials possess high radiation resistance and maintain their stability at a dose of $\gamma$-radiation $10^{8}$ Gy [5]. 


\section{Proposed technological approach}

The treatment of spent fuel of the RBMK nuclear reactor is of the most interest because of the large quantity of this kind of waste in the Chernobyl region. Also, the method of encapsulating the NSF into stable and chemical durable monoliths in the shape of intact bundles or cut into pieces may be used for other kinds of reactors besides RBMK.

The RBMK spent fuel assembly is divided (by cutting on central metal rod) into three parts: two fuel bundles and one extension rod. The fuel bundle is placed in a stainless-steel capsule (thin-wall, $\sim 4-5 \mathrm{~mm}$ tube with welded lower lid) and filled with protective material (a powder mixture or sintered from powder mixture hole-disks). The capsule is then dried and evacuated with the help of pumping gas out through the metal capillary tube welded into the upper lid. Then the capillary tube is squeezed and finally sealed by welding the tube end. During further treatment by sintering under pressure by HIP $\left(T=1000-1050^{\circ} \mathrm{C}\right.$, $\mathrm{P}=100-150 \mathrm{MPa}$ ) due to the tri-axial action of the high pressure gas, argon, at the selected temperature, the powder in the hermetically sealed capsule is compacted to a completely pore-free body. The compacted glass-ceramic block thus obtained prevents any release of radioactive materials outside the metal capsule. In addition, this solves an important problem of immobilizing volatile fission products, in the first instance, Cs.

After HIP treatment, the capsule is loaded into the stainless-steel can and sealed by capping, vacuuming, and welding. Further two approaches can be realized:

1. Cans are placed in standard canisters sealed by capping, welding ,and inerting (Ar), and the filled canisters housed into concrete vaults or modules for radiological protection for interim storage; or

2. Cans are placed in standard transport containers, which are transported to a repository facility for final disposal.

With this concept, which is based on the multiple barrier principle, absolute isolation of the radioactive waste may be obtained for a very long time. The scheme of the proposed protective multi-barrier system for SNF storage and final deep geological disposal is shown in Fig. 1. 


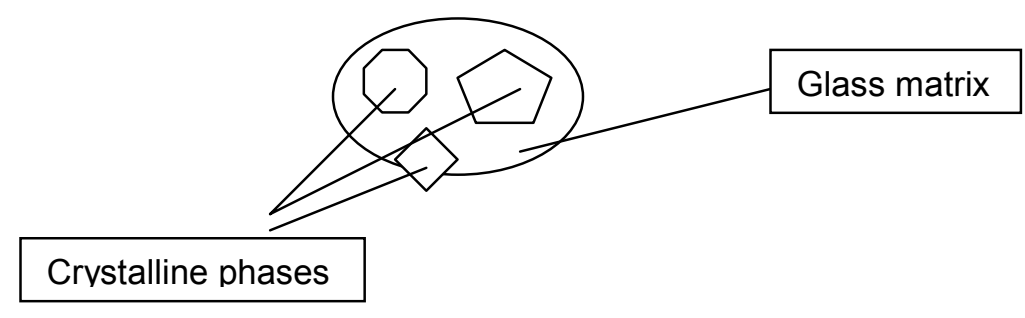

Fig. 1. Repository barrier system for spent nuclear fuel disposal: 1-encapsulation station; 2- bedrock (granite); 3- buffer material; 4- compacted buffer material (bentonite clay); 5- spent fuel waste form; 6- fuel rod; 7- glass-ceramic monolith; 8- metal capsule.

\section{Main Stages of the Project}

The scientific basis for development of the approach to encapsulate the RBMK spent fuel into a monolithic, stable glass-ceramic protective containment will be established and studied under this Project. In this project framework, a wide range of R\&D works will be performed, in particular, the following:

1. Develop a method to synthesize the glass-ceramic compositions for manufacture of protective buffer materials;

2. Conduct short-scale laboratory corrosion and radiation tests and investigate physico-mechanical properties of sintered glass-ceramic materials before and after tests.

3. Substantiate application of the cryogenic thermocompressor for ensuring the environmental cleanliness of the encapsulation process by HIP treatment. The main purpose of this investigation is to work out the thermocompressor working parameters that are a part of the HIP installation to ensure its simultaneous use both as a source of high pressure and as a gas-holder. As noted above, the regime of the gas-holder allows creation of the closed cycle of the working gas and ensures ecological purity of the proposed process of SNF encapsulation.

4. Study the behaviour of uranium and actinides of used $\mathrm{UO}_{2}$-fuel during encapsulating the spent fuel simulator.

5. Study the behavior of simulated fission products of nuclear fuel (e.g., gases, cesium and iodine) during encapsulation of the spent fuel simulator.

6. Conduct experiments to work out the encapsulation parameters using short-scale segments of a simulated spent fuel assembly.

7. Predict long-term performance of the SNF waste form under conditions of deep geological disposal.

8. Develop the design and technical basis at NSC KIPT to demonstrate the proposed encapsulation method.

\section{Preliminary Results}

\section{Sintering the glass-ceramic compositions}

The following powder compositions based on natural components were used as initial materials: granite, albitite, kaolin, and bentonite clays. Parameters of the 2-stage scheme of obtaining dense glass ceramics are optimized: stage 1 is the preliminary sintering in air $\left(970^{\circ} \mathrm{C}, 10\right.$ hours); stage 2 is the following final sintering under pressure by the HIP method $\left(920^{\circ} \mathrm{C}, 100 \mathrm{MPa}, 1\right.$ hour). Parameters were optimized with the purpose of minimizing costs of possible future industrial production of glass-ceramic materials created 
in this work. The glass-ceramic materials obtained (Figs. 2-4) contain (all figures in \% mass): $42-58$ glass phase and such main crystalline phases as mullite (20-50), $\alpha$-quartz (1-16), and feldspars (0-13). In chemical composition, the glass phase consists mainly of oxides of: silicon (79-91), aluminum (7-11), potassium and sodium $(2-11 \%$ mass $)$.

In the course of work, we studied the structural-phase changes that occur in powder compositions based on rocks and minerals during thermal treatment. It was established that during preliminary sintering in air at temperatures of $900-970^{\circ} \mathrm{C}$, the processes that take place include decomposition of kaolinite or montmorillonite into amorphous silica and alumina; forming mullite out of them, and initial and main stages of forming the glass phase during interaction of excess of amorphous silica with feldspars. In the materials preliminarily sintered in air, when sintered under pressure, porosity is eliminated, formation of the glass phase is completed, and the newly formed feldspars are crystallized.

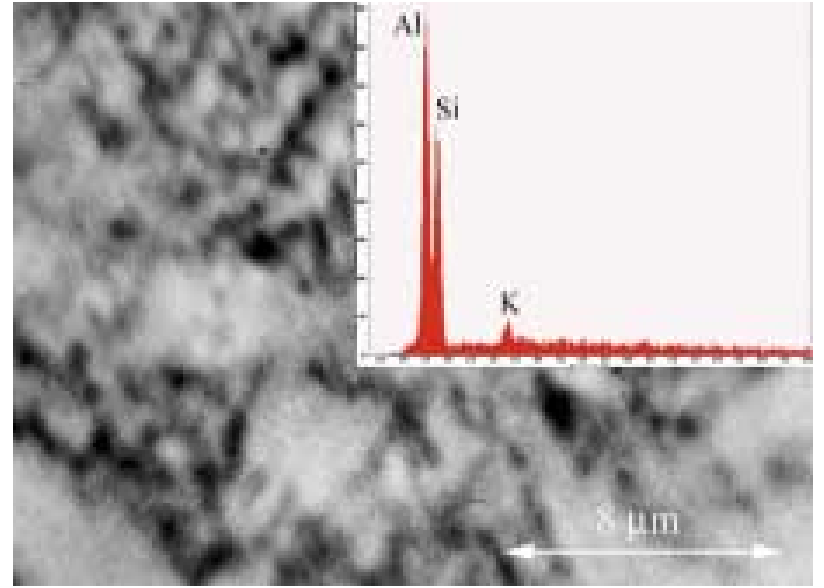

Fig. 2. SEM: fine grain appears (mullite) in glass ceramics with the ratio $\mathrm{Al}: \mathrm{Si}=1,5: 1$.

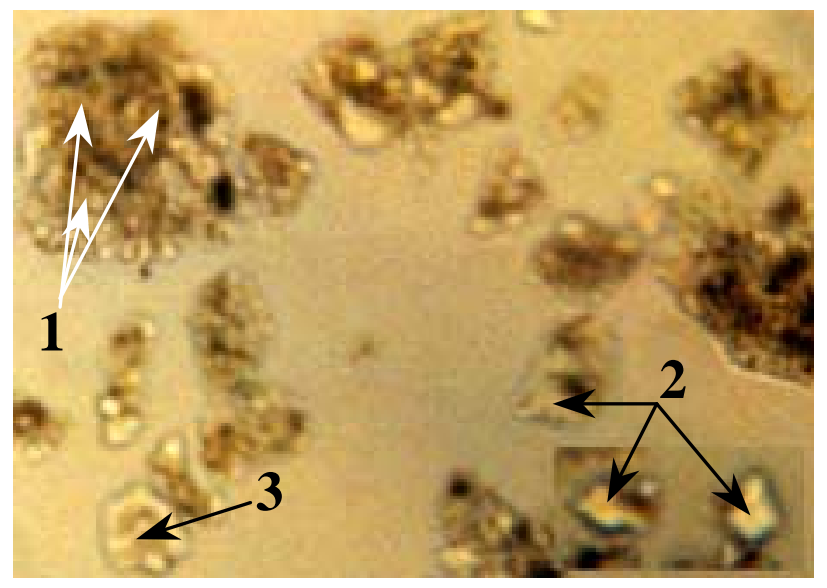

(a)

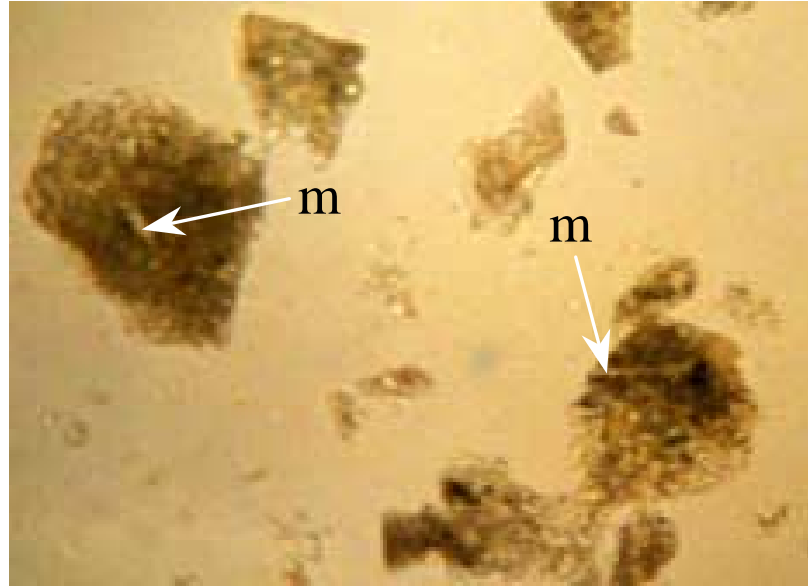

Fig. 3. CO (without the analyzer): glass-like basis with the newly-formed mullite $(m), \times 600$.

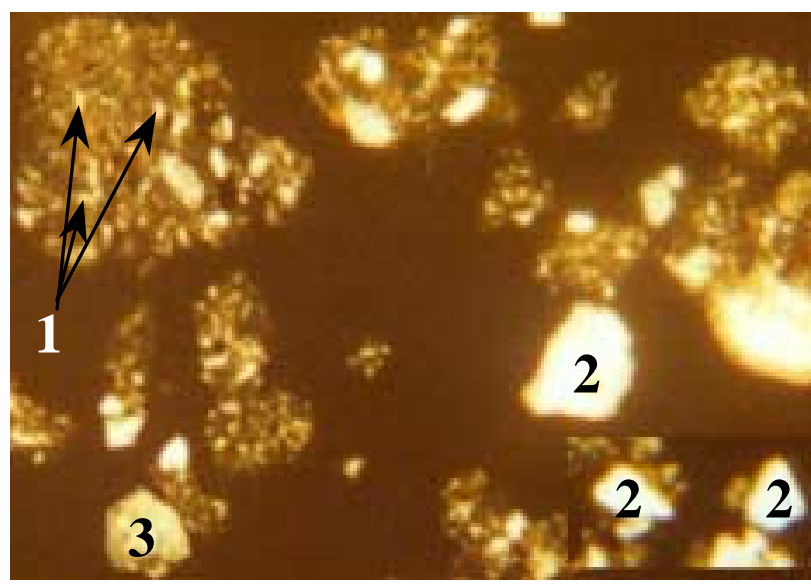

(b)

Fig. 4. CO: aggregates of the eutectic mass with areas of the newly-formed feldspars (1) relict of quartz (2) and feldspar (3); (a) view without analyzer, (b) view with the analyzer, $\times 600$. 


\section{Calculated Estimates of the Use of Glass-ceramic Protective Materials}

\section{Radiation effect of SNF on material of the protective glass-ceramic capsule}

At the present stage, we calculated the radiation characteristics of RBMK SNF (from the moment of unloading out of the reactor core until 10 years has passed, and with an initial enrichment $2 \%$ and depth of burn up $24.9 \mathrm{MW}$.day/ton of $U$ during its storage in the range from 10 to $10^{4}$ years). The following time dependences for RBMK spent fuel were obtained: specific activity, energetic spectrum of $\gamma$-quantums and the rate of their emission, and specific radiation heat emission, as well as calculated estimates of the absorbed dose of irradiation received by the protective glass-ceramic material on the part of fuel. These parameters are necessary for the following forecast of the influence of the encapsulated fuel irradiation on the change of mechanical properties and chemical resistance of the developed glass-ceramic materials.

The calculated specific activity of the products of fission and actinides and summary specific activity of RBMK nuclear spent fuel are given in Fig. 5 .

Proceeding from the fact that in the fuel practically full absorption of low energetic and charged particles occurs, in the absorbed dose calculation, we considered only the penetration of $\gamma$-irradiation with energy more than $60 \mathrm{keV}$. Results of the calculation of power of the irradiation absorbed dose received by the protective material on the part of fuel contained in one fuel bundle, and the values of the absorbed dose on the various depths of the material at exposition from 10 to $10^{4}$ are given in Fig. 6 and Fig. 7 , respectively.

The obtained dependence of the absorbed dose in the glass-ceramic material of the protective capsule on the time of irradiation by spent fuel contained in the spent bundle (Fig. 7) shows that in 200 years of storage, the glass ceramic will receive about $96 \%$, and in 500 years, $\sim 99 \%$ of the full dose, whose value is a total of $\sim 8 \cdot 10^{7} \mathrm{~Gy}$. 

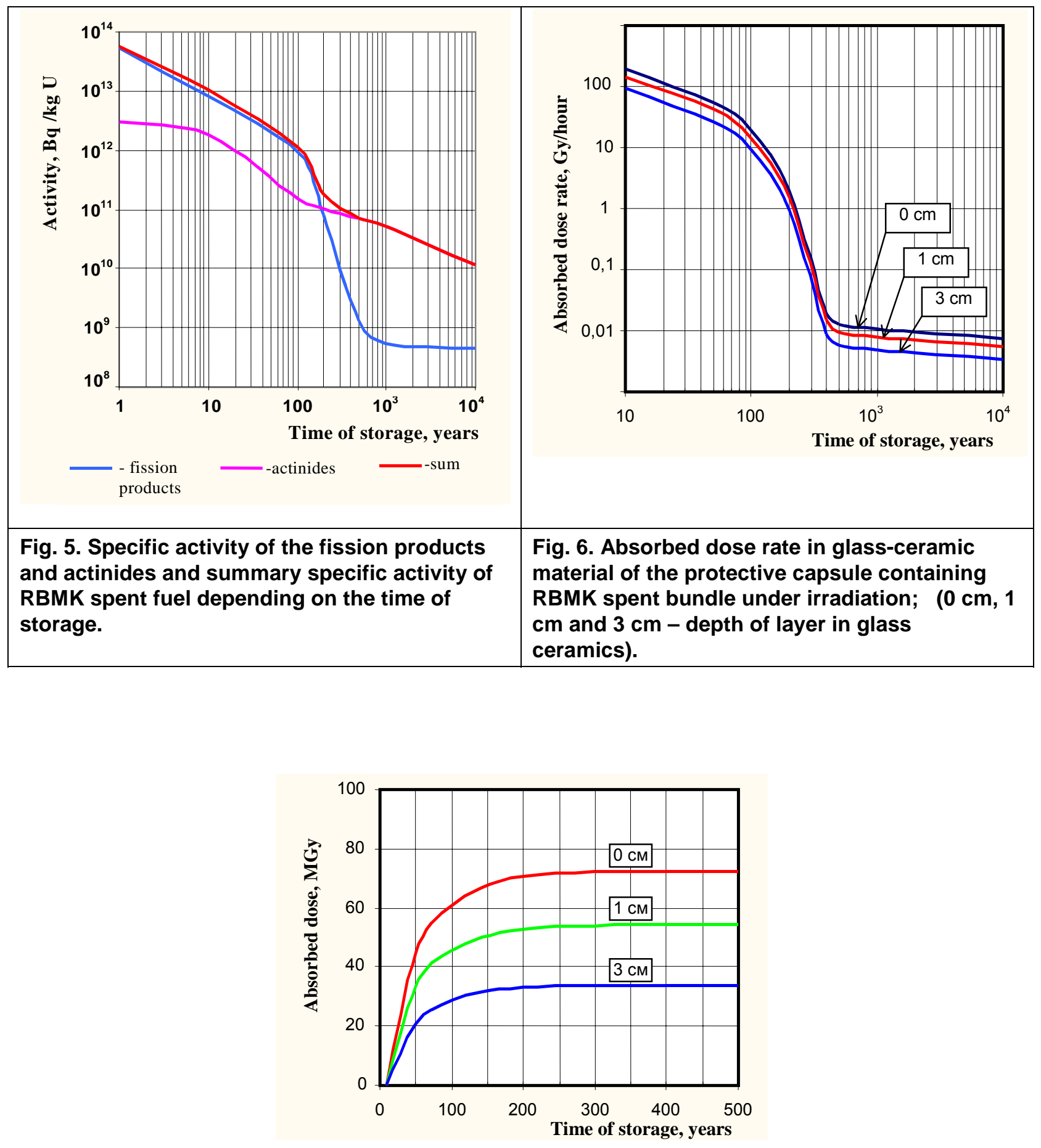

Fig. 7. The absorbed dose of irradiation in the glass ceramic material of the protective capsule of the RBMK spent fuel bundle depending on the time of storage; $(0 \mathrm{~cm}, 1 \mathrm{~cm}, 3 \mathrm{~cm}-$ depth of the layer in glass ceramics). 


\section{Mathematical model of the strain-deformed state of glass ceramics under irradiation and choice of compounds for initial compositions and glass ceramic materials}

The proposed mathematical model of the two-component system "glassy-like matrix-crystalline grains" allows us to determine by the calculation method the regularities of influence of glass-ceramic structural parameters and the value of the absorbed dose on the inside stress which appears in the material under irradiation.

The main proximities and assumptions taken in the model are as follows:

- Glass ceramics is regarded as a two-component system, consisting of crystalline grains identical in size and phase composition and the glass phase compact matrix;

- Glass ceramics look like an aggregate of cells of the spherical form, in the center of each there is a crystalline grain surrounded by the layer of the glass phase;

- The cells touch each other, the space not occupied by the cells is also filled with the glass phase;

- Each cell is in power balance with the neighboring cells.

Increase of the volume of crystalline grains and decrease of the glass phase volume, caused by irradiation, result in deforming the two-component system which causes the stress of its state. Equation of balance in spherical coordinates, added by boundary conditions on the inside and outside surfaces of the glass-phase spherical layer, are solved according to the stresses on the boundary "crystalline grain glass-phase layer". The grain is in the state of all-round compression. The stress in the spherical layer, compressing radial and stretching circles are maximum on the inside surface of the glass-phase layer. For estimate calculation of durability with the brittle character of destructions which is specific for ceramic, the criterion of Moor is recommended, according to which the equivalent stress and, correspondingly, the terms of durability look like that [6].

$$
\begin{aligned}
& \sigma_{e k v}=\sigma_{t}-\left(\sigma_{\mathrm{B}} / \sigma_{-\mathrm{B}}\right) \sigma_{\mathrm{r}}, \\
& \sigma_{\text {ekv }}<\sigma_{\mathrm{B}}
\end{aligned}
$$

where: $\sigma_{t}, \sigma_{r}$ - circular and radial stresses correspondingly;

$\sigma_{\mathrm{B}}, \sigma_{-\mathrm{B}}$ - limit of durability of the material during one axis tension and compression correspondingly (e.g., for quartz glass the ratio $\left.\sigma_{\mathrm{B}} / \sigma_{-\mathrm{B}} \approx 0,05[7]\right)$.

In accordance with the proposed mathematical model, the algorithm of calculation of equivalent stresses on the inside surface of the glass-phase layer depending on the volume share of components at the given value of the absorbed dose is made up. For the absorbed dose $8 \cdot 10^{7} \mathrm{~Gy}$ the dependence of equivalent stresses on the content of glass phases in the two-component glass ceramics, whose crystalline components are quartz, orthoclase or mullite, is calculated. The results of the calculations given in Fig. 8 show that:

- with the growth of the glass-phase state in glass ceramics, the stresses are increasing in accordance with approximately degree law (with index of degree 4.1-4.7);

- $\quad$ with the growth of the crystalline grain sizes and the value of the relative volume changes under irradiation, the equivalent stresses in glass ceramics are increasing. 


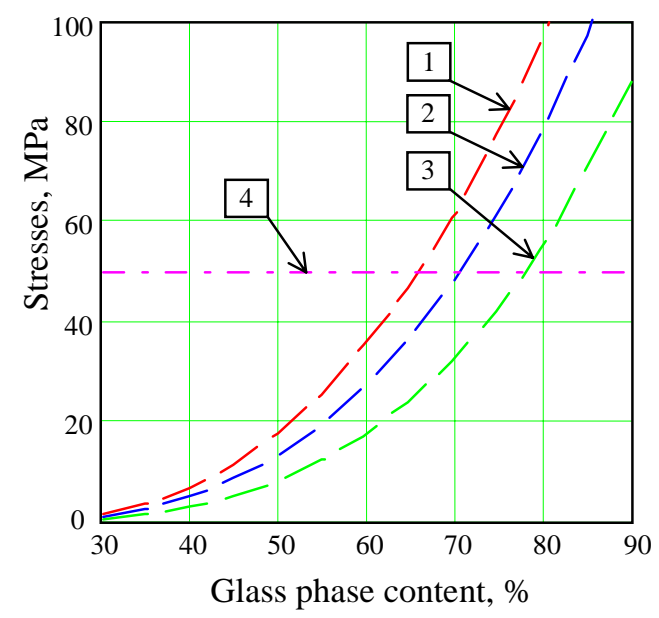

Fig. 8. Dependence of equivalent stresses in the system "glass phase - crystalline grain" under irradiation by the dose $8 \cdot 10^{7} \mathrm{~Gy}$ on the content of the glass phase for various crystalline components of the system: 1-quartz $(35 \mu)$, 2-orthoclase (size $\sim 10 \mu), 3$ - mullite (size $\sim 1 \mu$ ); 4 - glass-ceramic mechanical durability on tension.

To make is clear, the graph of Fig. 8 gives the average durability on tension of the glass-ceramic materials. With the content of the glass phase $40-50 \%$, the stress in glass ceramics reaches $5-25 \mathrm{MPa}$, which makes up not more than a half of the durability limit on tension (about $\sim 55 \mathrm{MPa}$ according to our experimental tests). The content of the glass phase more than $50 \%$ is inadmissible, because the equivalent stress will exceed the limit of durability on glass-ceramic tension.

Analysis of the results obtained showed that:

1. Mathematical model allows the calculation of stresses, caused by irradiation in glass ceramics dependent on the content of glass phase, sizes and relative increase of crystalline grains under irradiation.

2. So, that stresses do not exceed $50 \%$ of limit of glass-ceramic durability on tension, the content of glass phases should not increase $50-55 \%$.

3. Decrease of sizes of crystalline grains results in reducing equivalent stress in glass ceramics under irradiation.

\section{Conclusions}

The compositions of initial materials as powder mixtures based on natural rocks (granite, albitite, kaolin and bentonite clay) are chosen for obtaining glass-ceramic materials. The content of granite in an initial composition makes up from 10 to $70 \%$ and the content of albitite, from 10 to $45 \%$.

Parameters of the two-stage scheme of obtaining dense glass-ceramic materials from the powder compositions (granite + kaolin), (albitite + kaolin), (granite + bentonite clay) are optimized: (1) the preliminary sintering of powder composition on the air; (2) the following final sintering under pressure.

Glass-ceramic materials with the following phase composition are obtained: glass phase (42-58\%) mullite (20-50\%), $\alpha$-quartz (1-16\%), feldspars $(0-13 \%)$, silica glass $(0-10 \%)$, and opaque minerals $(4-12 \%)$. 
By ratio of the initial components, it is possible to change the oxide composition of the glass phase from the eutectic $\left(80 \% \mathrm{SiO}_{2}, 12 \% \mathrm{Al}_{2} \mathrm{O}_{3}, 8 \% \mathrm{~K}_{2} \mathrm{O}+\mathrm{Na}_{2} \mathrm{O}\right)$ to silica one $\left(91 \% \mathrm{SiO}_{2}, 7 \% \mathrm{Al}_{2} \mathrm{O}_{3}, 2 \% \mathrm{~K}_{2} \mathrm{O}+\mathrm{Na}_{2} \mathrm{O}\right)$.

Investigations showed that, by purposefully changing the ratio of rocks and minerals in the initial powder compositions as well as parameters of the preliminary sintering and sintering under pressure, one can control both density and phase composition of the glass ceramics including the composition of its glass phase.

The parameters were optimized with the purpose of minimizing the cost of the possible future industrial production of the glass-ceramic materials obtained in this work. For this:

1. Cheap natural rocks and minerals (granites, albitite and kaolin and bentonite clays) were used;

2. Parameters were chosen for both preliminary sintering $\left(970^{\circ} \mathrm{C}, 10\right.$ hours $)$ and sintering under pressure $\left(920^{\circ} \mathrm{C}, 100 \mathrm{MPa}\right)$ which can be performed on manufactured industrial technological equipment;

3. Taking into consideration the fact that the most expensive stage of obtaining glass-ceramic materials is hot isostatic pressing, the use of pressure up to $100 \mathrm{MPa}$ was grounded.

It was established that during sintering in air and under pressure, the compositions from rocks and minerals undergo the following processes: decomposition of kaolinite and montmorillonite into amorphous silica and alumina, forming mullite out of them; forming the glass phase during interaction of the excess of amorphous silica with feldspars, and crystallizing the newly-formed feldspars from the glass phase.

The dependence of the absorbed dose and the speed of its intake in the glass-ceramic material of the protective capsule on the time of irradiation by the RBMK spent fuel contained in one fuel bundle over the period of 1000 years was calculated. It was shown that in 200 years of storage, glass ceramics will receive about $96 \%$, and in 500 years, $\sim 99 \%$ of the fuel dose, whose value will make up $\sim 8 \cdot 10^{7} \mathrm{~Gy}$.

A mathematical model of the stressed-deformed state of glass ceramic under irradiation was proposed. This model allows us to calculate stresses in glass ceramic caused by irradiation depending on the content of glass phase, sizes and relative increase of volume of crystalline grains under irradiation. To provide the appropriate level of stresses in glass ceramics, the content of the glass phase shouldn't increase to the value $50 \%$. It was shown that reducing sizes of crystalline grains resulted in reducing equivalent stresses. In the framework of the mathematical model, glass ceramic materials can be considered good prospects if they are obtained from the compositions (granite + kaolin) with content of granite from $10-15 \%$ to $70 \%$ and content of albitite from $10-15 \%$ to $45 \%$, because stresses in them will make up not more than $50 \%$ of the durability limit on tension and their high size stability can be expected under irradiation on the part of the nuclear spent fuel. Taking into account only the results of experiments, glass-ceramic materials on the base of compositions with a content of granite up to $70 \%$ can be considered prospective. The compositions of the glass-ceramic materials will be finally chosen after the complex of investigations into the effect of $\gamma$-irradiation on them, corrosion tests (Project stage \#2), studying the behavior of uranium, actinides (stage \#4) and products of fission cesium, strontium, iodine (stage \#4) during the manufacturing of the protective form simulators. 


\section{Acknowledgements}

The work was performed under STCU Project \#1580, and the Foreign Collaborator is Dr. L. J Jardine, Lawrence Livermore National Laboratory in the USA. The Science and Technology Center in Ukraine (STCU) is an international organization located in Kiev to support scientists from Ukraine, and is financed by the USA, Canada, Japan, and the European Union.

\section{References}

1. Investigation of ceramic materials for radioactive waste storage, JOM, 2000, vol. 52, No. 10, p.4.

2. Kedrovskiy O.L., Shishic I.Yu., Gupalo T.A. et al. A substantiation of requirements for localization of a high active wastes and nuclear spent fuel in geological formations. j. Atomic energy, 1991, v.70. No. 5, pp. 294-297 (in Russian).

3. N.J. Weber, F.P. Roberts A review of radiation effects in solid nuclear waste forms, Nucl. Technology, v. $60,1983$.

4. U.D. Kingeri Introduction in ceramics, Edit. lit. on build - , M., 1967 (in Russian).

5. Krylova N.V., Poluektov P.P. Properties of the baked shapes of a high-active wastes as one of barriers of system of a burial, j. Atomic energy, v.78, No. 5, May, 1995 (in Russian).

6. Balickiy A.V. Technology for manufacture of vacuum instrumentation. "ENERGY", M. - L., 1966 (in Russian).

7. A strength of materials to a deforming and fracture, part 1 / Edit by Trochenko V.T.- Kiev, Naukova Dumka, 1993 (in Russian). 
VIEWGRAPHS

\title{
SCIENTIFIC INVESTIGATION OF SPENT NUCLEAR FUEL ENCAPSULATED INTO GLASS-CERAMIC PROTECTIVE FORMS
}

S. Yu. Sayenko, I.M. Neklyudov, G.A. Kholomeyev, A.P. Pilipenko, A.E. Surkov, B.A. Shilyayev, R.V. Tarasov, S.V. Gabelkov, E.P. Shevyakova, V.I. Zmiy, Zh.S. Azhazha, National Science Center Kharkov Institute of Physics and Technology (NSC KIPT), Kharkov, Ukraine

\section{Abstract}

This paper describes the investigations carried out at the NSC KIPT in the frame of STCU Project into creating protective glass-ceramic materials by method of hot isostatic pressing (HIP) of powder mixtures (on the base of rocks and minerals) preliminarily sintered on the air.

\author{
Contents
}

The scientific and technological base

Technological approach

The main stages of project

Preliminary results 


\section{Physical \& Chemical Background}

1. The main mineral of granite rock - feldspar group (aluminosilicates with the general formula $\left.\mathrm{K}(\mathrm{Na}, \mathrm{Ca}) \mathrm{AlSi}_{3} \mathrm{O}_{8}\right)$. According to some recommendations aluminosilicates, namely nepheline (a sodium aluminosilicate $\mathrm{Na}_{3} \mathrm{~K}\left[\mathrm{AISiO}_{4}\right]_{4}$ ), are proposed as the Cs host. Attention should also be paid to other natural minerals, such as sphene ( $\left.\mathrm{CaTiAISiO}{ }_{8}\right)$, and cesium analogue of leucite CsAlSi ${ }_{2} \mathrm{O}_{6}$, which can incorporate $\mathrm{Cs}$ too.

2. At the moment granite deposits of the Ukrainian crystalline shield are considered the most suitable for HLW repository.

3. The principle of phase compatibility keeping the natural balance is observed: the material of protective monolith is similar in composition to that of a bedrock in the place of disposal.

4. But natural granite $\Rightarrow$ some porosity and jointing. It can be regarded as its disadvantage if it is used as a geological barrier for disposal of radioactive wastes.

5. We are paying attention to producing ceramic articles and first of all faience and china ones. The main components for producing these articles are feldspar, quartz (main components of granite) and clay.

6. Radiation-induced volume changes:

- Glass matrix in which crystalline chemical stable phases are distributed.

- To manufacture the engineering protective barrier in nuclear spent waste form $\Rightarrow$ to modify natural granite with the help of grinding up, selecting and adding other components, compacting by cold pressing and heat treatment the green pellets by sintering under pressure.

- The modification of the natural granite $\Rightarrow$ preserve chemical composition of the mineral components (the most radiation and corrosion resistant phases are chosen, e.g. feldspar and quartz), on the other hand, to obtain the material with the fine grain structure and to exclude completely the possible defects of the structure, in particular, cracks and pores.

- As the initial natural minerals, the granites of the Ukrainian crystalline shield were chosen with the content of the main mineral phases: feldspars $\mathrm{K}(\mathrm{Na}, \mathrm{Ca}) \mathrm{AlSi}_{3} \mathrm{O}_{8}$ up to $(65-70) \mathrm{wt} \%$, quartz $\left(\mathrm{SiO}_{2}\right)$ up to $30 \mathrm{wt} \%$, mica minimum. The use of the additions of the clay (kaolinite) rocks will ensure the high plasticity of the synthesized composition. 


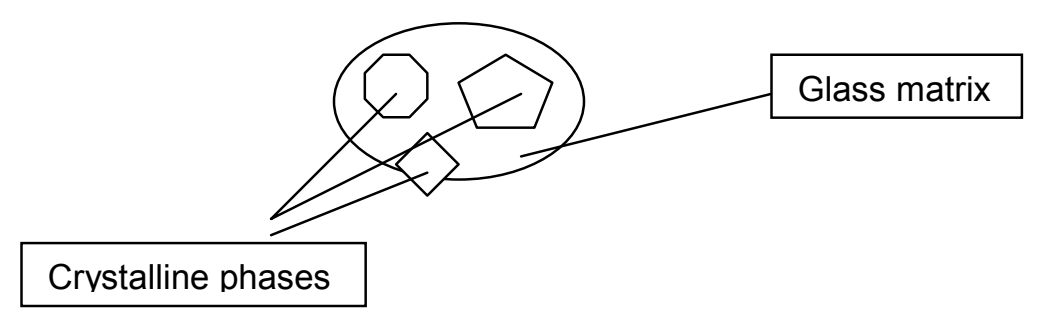

Fig. 2. Glass-ceramic structure.

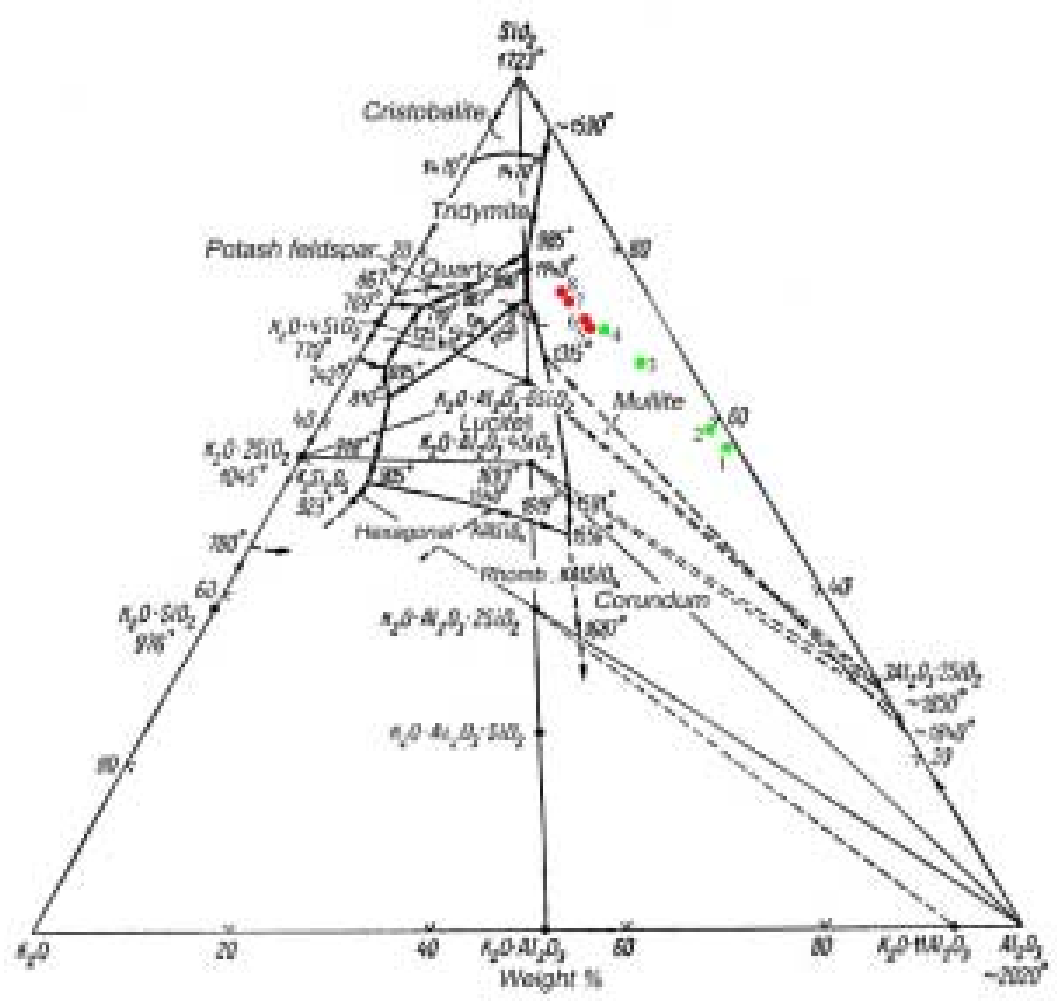

Fig. 6. Phase diagram.

Mullite is the main phase in glass-ceramic composition which allows to secure mechanical stability and corrosion resistance. Just for this reason we are working in the area (65-90) wt\% $\mathrm{SiO}_{2}$. 


\section{The main objectives of the project:}

Creation of the glass-ceramic materials on the base of natural granites and clays. Optimization of parameters of sintering and sintering under pressure.

Determination of the main characteristics of the glass-ceramic materials proposed as protective engineering barriers for spent nuclear fuel encapsulation.

Grounding of terms of safe long-term storage and disposal of the spent nuclear fuel contained in the glass-ceramic monolith, and the determination of the maximum terms of the use. 


\section{Proposed technological approach}
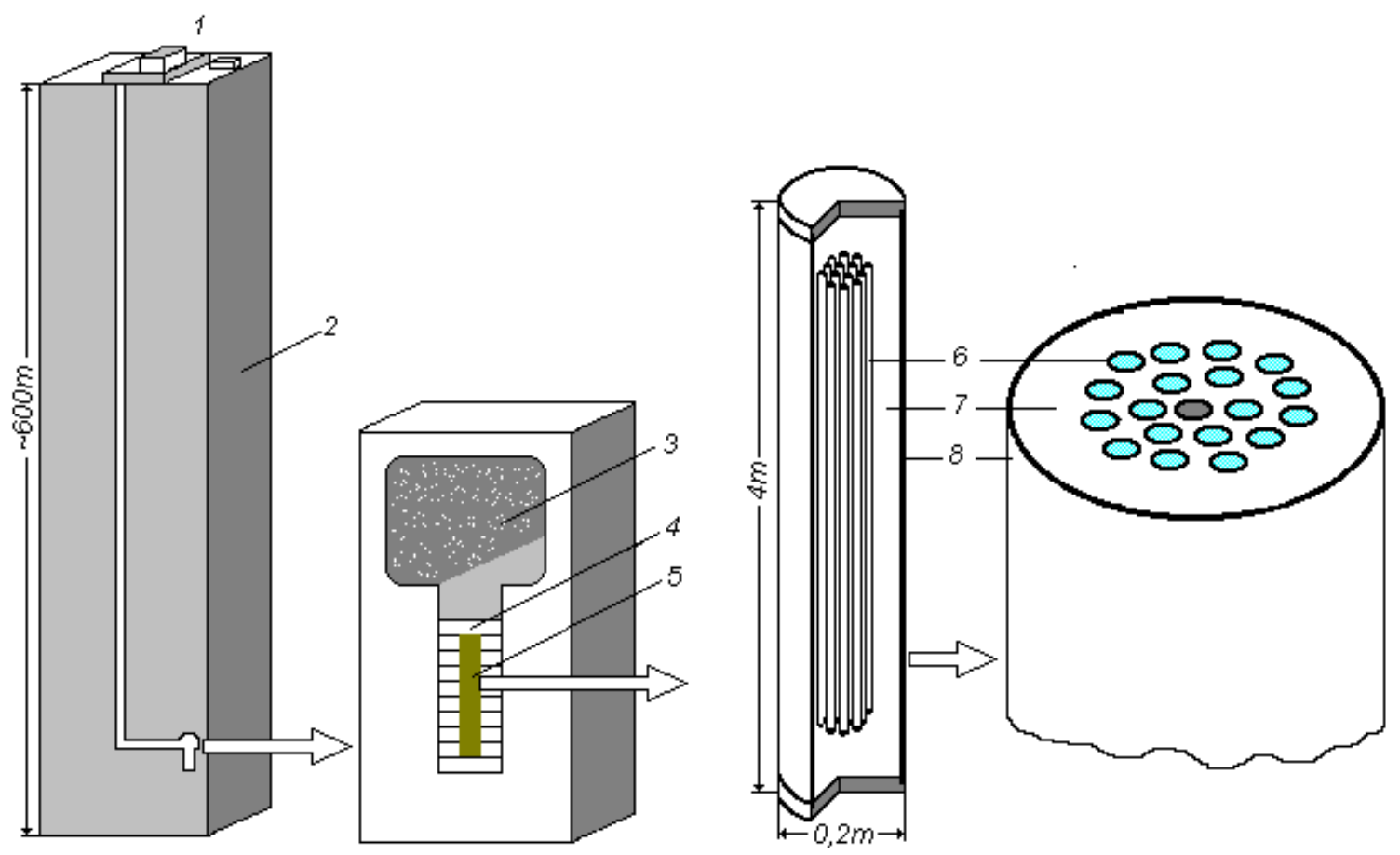

Fig. 1. Repository barrier system for spent nuclear fuel disposal: 1-encapsulation station; 2- bedrock (granite); 3- buffer material; 4- compacted buffer material (bentonite clay); 5- spent fuel waste form; 6- fuel rod; 7- glass-ceramic monolith; 8- metal capsule. 


\section{Project: The main stages}

1. Development of method to synthesize the glass-ceramic compositions for manufacture of protective buffer materials.

2. Carrying out short-scale laboratory corrosion and radiation tests and investigation of physico-mechanical properties of sintered glass-ceramic materials before and after tests.

3. Substantiation of application of the cryogenic thermocompressor for ensuring the environmental cleanness of the encapsulation process by HIP-treatment.

4. Studying the behaviour of uranium and actinides of used $\mathrm{UO}_{2}$-fuel during encapsulating the spent fuel simulator.

5. Studying the behaviour of simulated fission products of nuclear fuel (e.g. gases, cesium Cs and iodine I) during encapsulating the spent fuel simulator.

6. Experimental working out of encapsulation parameters using short-scale segments of simulated spent fuel assembly.

7. The long-term prediction of spent fuel waste form performance under conditions of deep geological disposal.

8. Development at the NSC KIPT of design and technical base to demonstrate the proposed encapsulation method. 


\section{Preliminary Results}

The composition (granite + clay) preliminary sintered on the air at temperatures $1050-1100^{\circ} \mathrm{C}$. It is established, that during further sintering under pressure the dense glass-ceramic material is produced which consists of the crystalline phases (feldspar, quartz, mullite) and the glassy phase.

Mullite and secondary phases were created as a result of processes of crystallizing in the glass phase during sintering under pressure.

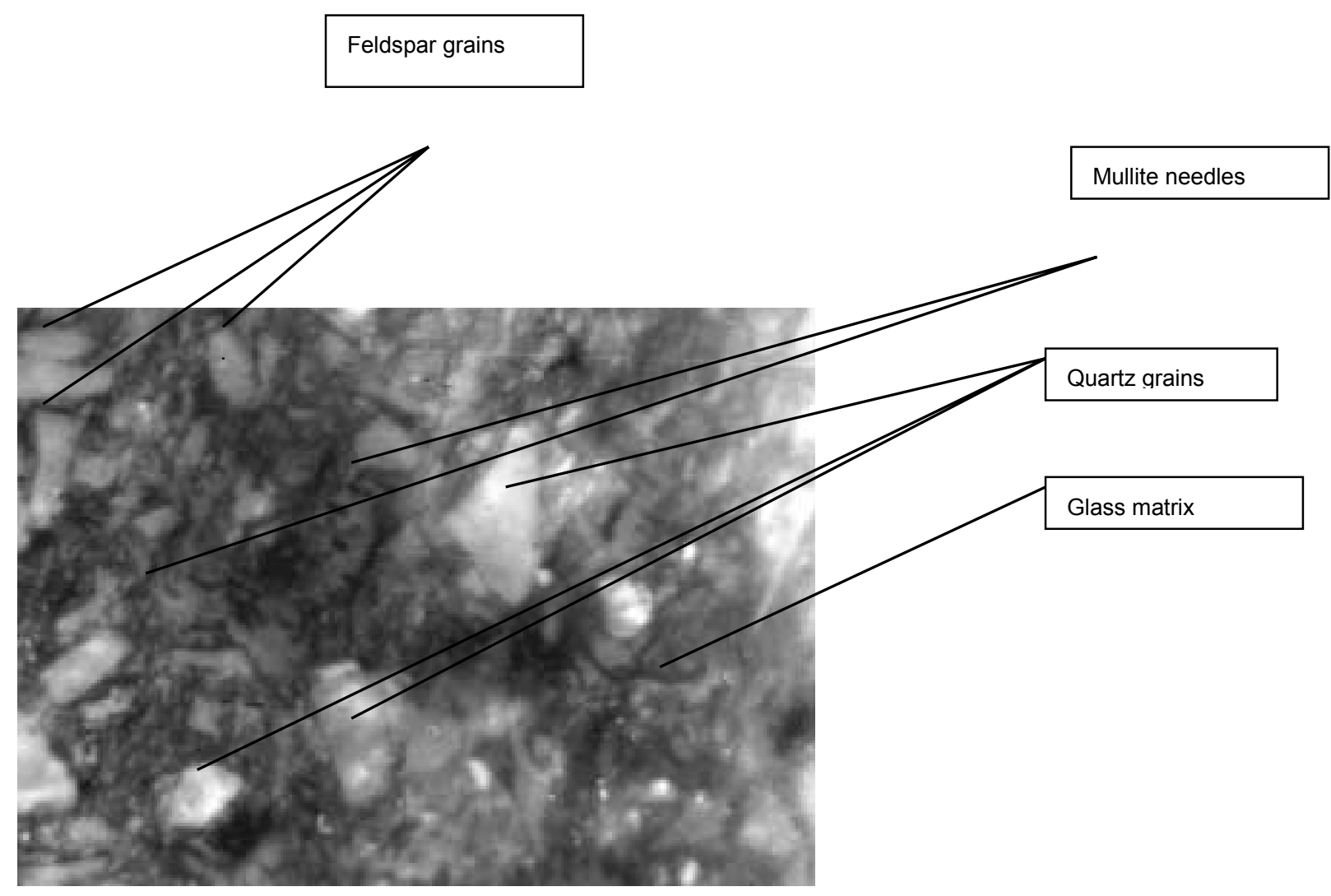

Fig. 7. Photography of structure. 
Irradiation from the RBMK spent fuel bundle, characteristics of thermo and irradiation effecting on protective material are presented in Fig. 3, 4 and 5.

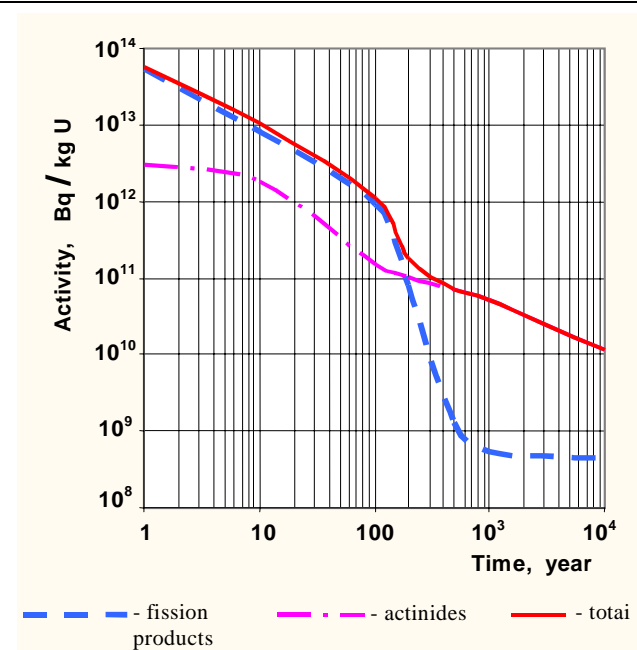

Fig. 3. Activity from RBMK spent fuel assembly.

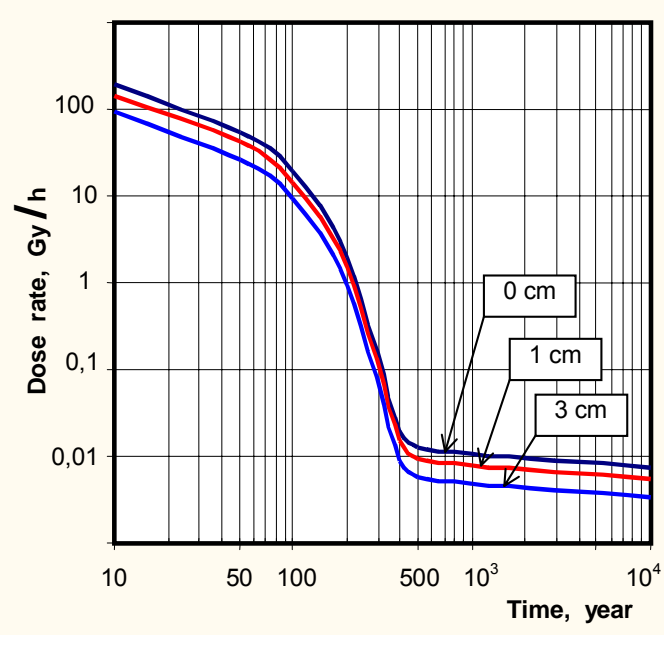

Fig. 4. Dose rate: on distance of 0,1 and $3 \mathrm{~cm}$ from nuclear spent fuel.

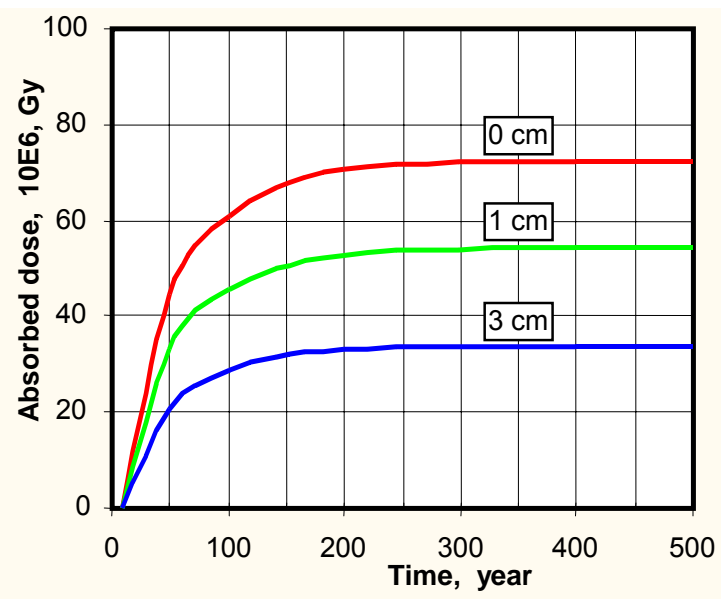

Fig. 5. Absorbed dose in glass-ceramic protective material on distance of 0,1 and $3 \mathrm{~cm}$ from nuclear spent fuel. 


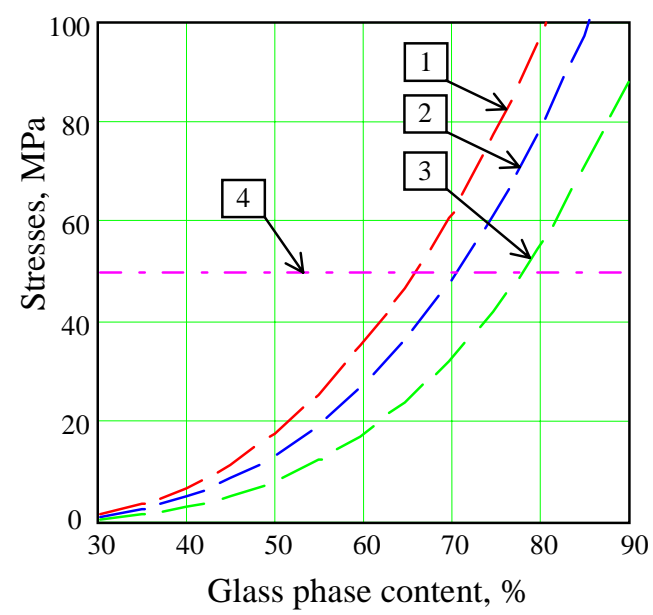

Fig. 8. Dependence of equivalent stresses in the system "glass phase crystalline grain" under irradiation by the dose $8 \cdot 10^{7} \mathrm{~Gy}$ on the content of the glass phase for various crystalline components of the system: 1 - quartz $(35 \mu)$, 2 - orthoclase (size $\sim 10 \mu$ ), 3 - mullite (size $\sim 1 \mu$ ); 4 - glass-ceramic mechanical durability on tension.

1. Mathematical model allows to calculate stresses, caused by irradiation in glass ceramics dependent on the content of glass phase, sizes and relative increase of crystalline grains under irradiation.

2. Stresses do not exceed $50 \%$ of limit of glass-ceramic durability on tension the content of glass phases should not increase $50-55 \%$.

3. Decrease of sizes of crystalline grains results in reducing equivalent stress in glass ceramics under irradiation. 


\section{Conclusion}

- For isolation of nuclear spent fuel with the purpose of the following interim storage and final disposal in geological formations, the way of their enclosing into artificial mineral-like ceramic and glass-ceramic monolith has been proposed. To obtain a material for protective monolith we propose to use powder mixture, as initial material for further treatment by compacting at room temperature, preliminary sintering and final sintering under pressure (HIP-ing).

- The main components for powder mixture in this case are natural granite together with the additions of natural clays.

- In glass-ceramics, the crystalline phases, which existed before or newly formed secondary ones, will provide the wide isomorphism of the alkaline ions $\mathrm{Cs}^{+}$in the cation sub-lattice (e.g. replacing $\mathrm{Na}$ for $\mathrm{Cs}$ in $\mathrm{K}(\mathrm{Na}, \mathrm{Ca}) \mathrm{AlSi}_{3} \mathrm{O}_{8}$ ). At the same time, the glassy multicomponent phase will be capable of dissolving the part of alkaline ions and creating the reliable immobilizing medium to incorporate volatile radionuclides which can release from $\mathrm{UO}_{2}$ during HIP-ing under temperature about $1000^{\circ} \mathrm{C}$.

- Using of the additional glass-ceramic protective barrier (the layer not less than $40 \mathrm{~mm}$ ), at long-term interim storage will allow to provide solution of the problem of the so-called "delayed decision" of managing the nuclear spent fuel for the period minimum $\mathbf{3 0 0}$ years. During this period the variant of the system of the final isolation of the spent fuel can be chosen completely. 


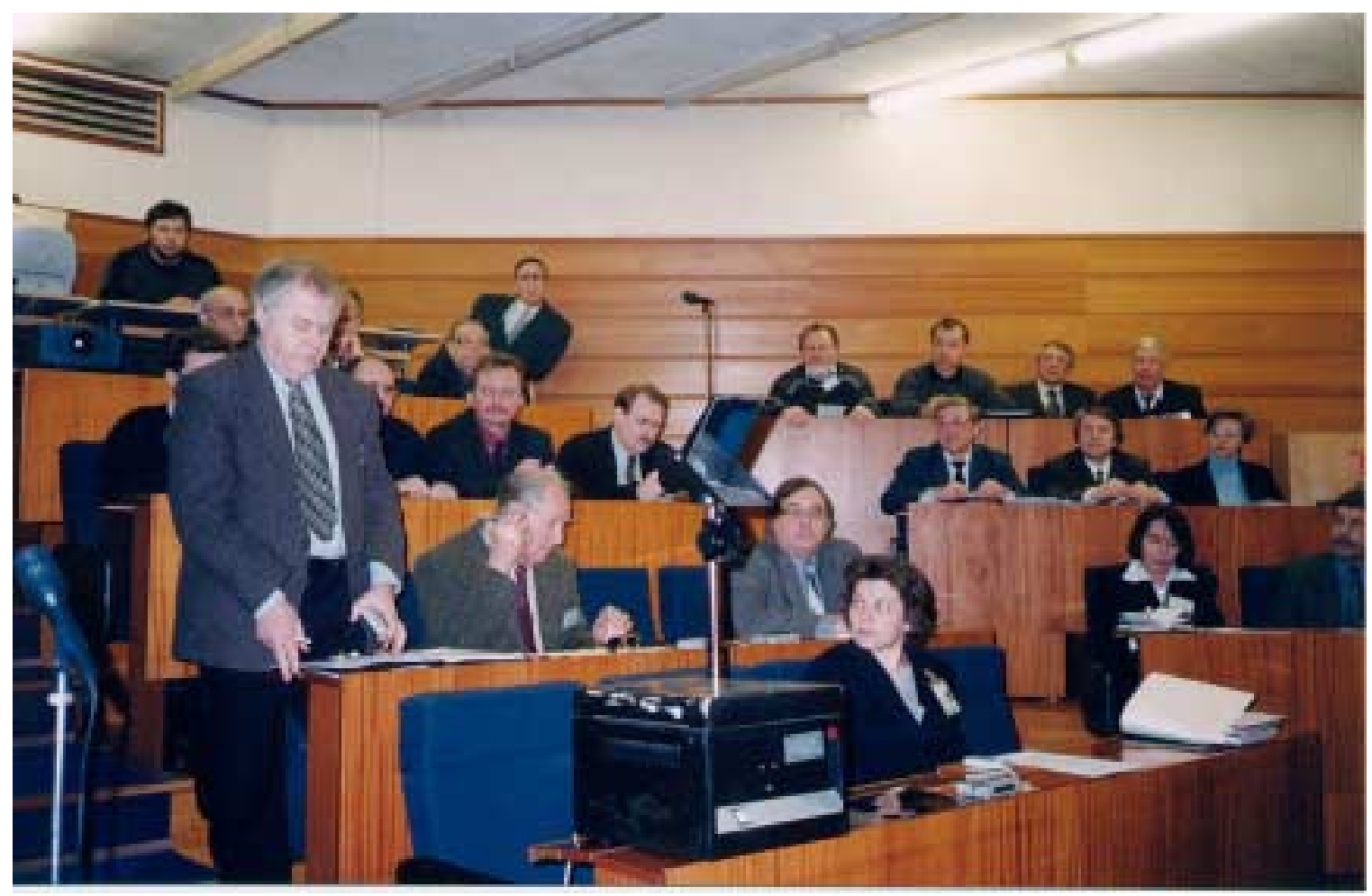

IV. Closing Session and Summary 


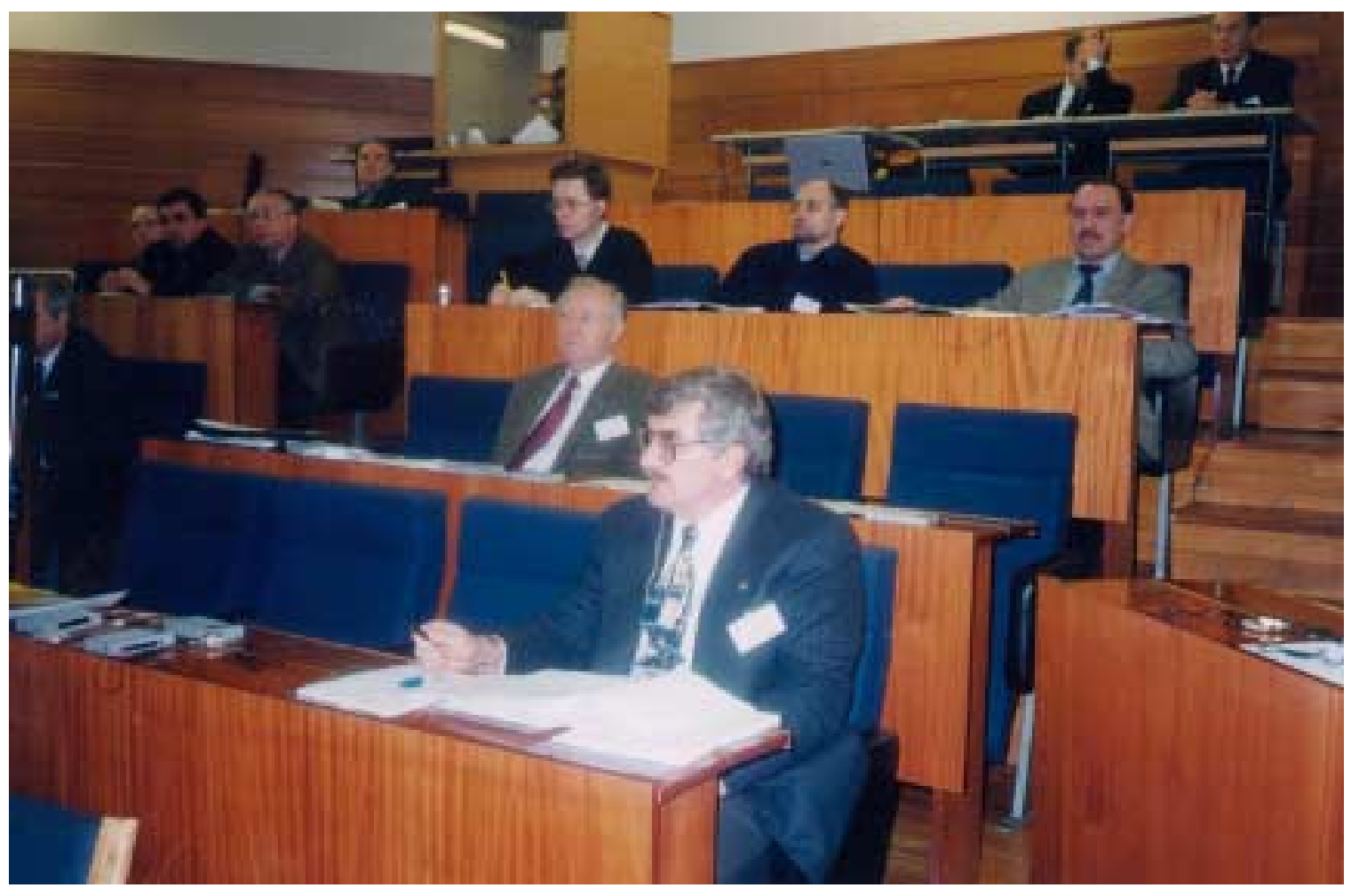




\section{Summary of Technical Activities}

\section{J. Jardine, LLNL}

\section{Russian Plutonium Disposition at LLNL}

Until April 19, 2002, when formally announced in the Federal Register [1], the US was pursuing a dualtrack approach to plutonium disposition: immobilization and irradiation in civilian reactors as MOX fuel. In the April 2002 announcement, the US cancelled its immobilization program due to budgetary constraints and the US is now pursuing only a single-track path of burning weapons plutonium in US reactors as MOX fuel.

Russia, however, has always considered plutonium to be a valuable national resource and was extremely reluctant to even consider the immobilization option. Since 1997, LLNL has had the lead responsibility for developing and defining the immobilization technology alternative with the Russians. As a result of solid technical arguments and LLNL contract work performed by numerous Russian Minatom organizations, in the fall of 1999 Minatom changed its position toward the process, and offered to immobilize a portion (1 Mt) of its weapons-grade plutonium during US-RF negotiations of the September 2000 Plutonium Disposition Agreement. This policy shift was unimaginable in 1997, when the Russian plutonium immobilization activities began. Later in the summer of June 2000, the US rejected the Russian offer to immobilize $1 \mathrm{Mt}$ of weapons plutonium at the Mayak and Krasnoyarsk-26 sites. Instead the US negotiated the replacement of this amount with $1 \mathrm{Mt}$ of plutonium oxide for burning in Russian reactors which is contained in the US-Russian Excess Weapons Plutonium Disposition Bilateral Agreement signed in September 2000 by Gore and Kasyanov.

In the summer of 2001, LLNL was directed to begin the close out of Russian activities performed under contracts with Russian organizations on immobilization topics and to not issue any new immobilization contracts. In 2001, LLNL was then assigned the lead plutonium disposition responsibilities for Russian (1) weapons plutonium storage, packaging and transportation and (2) MOX spent fuel storage and transportation. LLNL is currently discussing with the DOE the need to create an assignment, with LLNL as lead organization, that would be responsible for Russian waste treatment, storage and disposal of the plutonium-containing wastes from plutonium conversion and the MOX fuel fabrication facilities. These activities involve the same Russian organizations that participated in Russian plutonium immobilization; these organizations are critical to the success of the RF program.

With the publication of this Proceedings, three years of successful Russian Plutonium Immobilization Project Review meetings have been held and documented - in November 1999, November 2000, and January 2002. The meetings, all held in St. Petersburg, summarized the Russian technical work completed and the implementation status of the Russian Pu Immobilization Program and other RussiaLLNL contracts. By January 2002, over forty-nine LLNL contracts totaling over $\$ 7 \mathrm{M}$ involving plutonium immobilization, spent fuel storage and transportation, and plutonium storage, packaging and transportation had been issued to six Russian scientific and design institutes and two industrial sites. Over 250 deliverables and technical reports have been generated by these Russian organizations, including VNIINM, VNIPIPT, KRI, RIAR, GSPI, and VNIPIET, and the Krasnoyarsk-26 and Mayak Industrial Sites. Including this document, three technical Proceedings containing over 135 Russian technical papers, written for and presented at these meetings, have now been published [2,3]. 
This third meeting, for review of recently completed and ongoing Russian plutonium immobilization contract work, was held at the State Education Center (SEC) in St. Petersburg on January 14-18, 2002. The meeting was organized by LLNL and the DOE in conjunction with the V. G. Khlopin Radium Institute, and participants represented Russian scientific research centers, planning and design institutes, universities, and various industrial enterprises. Fifty-eight Russian participants attended from 21 Russian organizations, including the industrial sites (Mayak, Krasnoyarsk-26, Tomsk), scientific institutes (VNIINM, KRI, VNIPIPT, RIAR), design organizations (VNIPIET, GSPI), universities (Nyzhny Novgorod, Urals Technical), Russian Academy of Sciences (Institute of Physical Chemistry, IGEM), Radon-Moscow, S\&TC Podol'osk, Kharkov-Ukraine, GAN-SEC-NRS and SNIIChM, Minatom and GAN.

Technical efforts have focused on the needs of the Russian plutonium production industrial sites of Mayak, Krasnoyarsk-26, and Tomsk. A strategy and approach for the working immobilization group had been developed and published [4,5] and was being implemented. However, it is now being closed out. The goal was to establish a full-scale plutonium immobilization facility at a Russian industrial site by 2005. Supporting R\&D projects were carried out at Russian institutes to meet the technical needs of Russian industrial sites to immobilize plutonium-containing materials. Special R\&D on plutonium materials was also being carried out to support plutonium disposition in both Russia and the US, including nonproliferation studies of plutonium recovery from immobilization forms and accelerated radiation damage studies of the US-specified ceramic waste form. This extraordinary cooperation on certain aspects of the weapons plutonium problem progressed remarkably well, and a great deal of work with weapons plutonium has been completed and documented in the past four years.

The joint work has helped Russian specialists gain a better understanding of plutonium supply-anddemand economics. In turn, several Minatom organizations and LLNL reports were published acknowledging that not all Russian plutonium is of sufficient quality for use in a reactor or elsewhere. The Krasnoyarsk-26 and Mayak sites went on record as preferring to immobilize weapons plutonium for disposal rather than to recover the plutonium in some of their tanks for storage and reuse, which is the current Minatom policy and practice. The decisions made by these two sites were based on engineering studies performed by Russian organizations under LLNL contracts with DOE funds based on metrics of costs, schedules, secondary wastes and environmental impacts. Tomsk-7 also wanted to participate in plutonium immobilization engineering studies for their site and a statement of work was negotiated by LLNL to assess $\sim 1 \mathrm{Mt}$ of weapons plutonium. However, the project negotiations were stopped by DOE in April 2001 before a contract was signed. The Russian organizations have performed all their contract immobilization work to the end, in detail and on schedule, knowing it would not be continued with US funds. The sites also stated in these 2002 meetings that they would pursue Minatom funds to continue the development of the immobilization of about $\sim 1.8 \mathrm{MT}$ of Pu at Krasnoyarsk-26 and $1.5 \mathrm{MT}$ of Pu at Mayak, but were not hopeful of getting Russian funds to continue immobilization as an alternative to the required recovery route. This acknowledgment could lead the Russians to agree not to install the processes required to recover plutonium at future plutonium conversion and MOX facilities to levels of past or current practice. Ultimately, this should reduce the costs of the Russian disposition program and lead to faster reductions of Russia's plutonium inventory.

Based on the January 2002 meetings and discussions, we present the following overall summary and select highlights of the major technical papers. The agenda in Appendix A provides a complete list of the papers by session and paper number. 


\section{Session I. Plutonium Storage, Packaging, and Transportation Highlights}

1. Russian regulations are not clear. The 1972-73 IAEA requirements (there are sets of IAEA guidelines dated 1973 or 1985 or 1996 that can be followed) are currently followed for plutonium oxide containers at MCC and Tomsk. There is a need to upgrade the current oxide containers that do not have hermetic seals. New rules went into effect in 2001 so Russian organizations will have to upgrade their containers first before shipping oxide.

2. Oxide is worse than metal for long-term storage in terms of potential pressurization. All 400R containers generated from past US-RF joint work are for plutonium metal. There are no acceptable 400Rs for oxide containers. Oxide containers do not meet current RF storage and transportation requirements and need to be upgraded.

3. A decision is needed to either make more old containers or make new containers to the new requirements of a standard size. Considerations must be given to the fact there are higher radiation levels in blended Pu after the isotopics are adjusted in new container designs.

\section{Session II. Spent Fuel Storage, Packaging, and Transportation Highlights}

1. Options for storage of MOX spent fuel and the number of sites need to be defined, probably for the Mayak and K-26 sites. The state of the spent fuel transportation fleet needs to be documented. The BOR-60 spent fuel storage situation requires an assessment at RIAR.

2. About $4.5 \%$ of the current RBMK spent fuel has leaks in the cladding. The same cladding material is used in RBMKs as in VVERs, but the VVER cladding leak rates are not known.

3. MOX spent fuel produces higher heat loads so spent MOX fuel may need longer water pool storage times, and that could impact the cladding integrity.

\section{Session III. MCC Site Activities Highlights}

1. Stopping at the $\mathrm{JOI}$ for plutonium sludge immobilization is unfortunate, but necessary, given the US position on immobilization. However, if MOX fabrication is selected for the MCC site, maybe matching $\$ 1$ of US and $\$ 1$ of Russian funds can be negotiated to make a MOX fuel fabrication waste treatment facility that can also treat the Pu sludge with its $1 \mathrm{Mt}$ of weapons $\mathrm{Pu}$. The estimated total cost is $\$ 20 \mathrm{M}$ ( $\$ 20,000 / \mathrm{Kg}$ of plutonium immobilized).

2. Oxide at $\mathrm{K}-26$ for the conversion facility must be shipped, but key issues are where to ship the oxide and what containers to use for Pu that has been in extended storage since 1996. The original design TK-30 storage/transport container for plutonium oxide is not acceptable because it has no hermetic seal and the oxide must be repackaged prior to shipment.

3. Oxide returned for MOX fabrication after blending at the conversion site will have higher radiation doses than the original weapons-plutonium oxide. The special methods required for monitoring systems are not known by the MCC at this time.

4. A MOX fabrication waste treatment study was done at $\mathrm{K}-26$, but more work is needed to bring it to a JOI level of detail.

5. KRI and VNIINM summarized the borosilicate and phosphate glasses they developed for the MCC. 
6. VNIPIPT summarized the studies of the storage of vitrified waste and further analyses of heat transfer, radionuclide migration and modeling of the immobilized sludges in the underground MCC facility.

\section{Session IV. Mayak Site Activity Highlights}

1. Mayak reported on the new third melter for the RT-1 high-level radioactive wastes that began operating on October 25, 2001. Eighteen million Ci have been vitrified since the new start up. Characterization of the B954 area sludges continued. The challenges of the high S content and the large $\mathrm{Al}$ and $\mathrm{NO}_{3}$ contents are key unresolved issues for Mayak. It must be decided whether to leave these chemicals in or to take them out because they are not radioactive components, complicate the processing, and increase the volume of the solidified wastes.

2. VNIPIET provided initial cost estimate numbers of $\$ 70 \mathrm{M}$ to $\$ 50 \mathrm{M}$ for a sludge immobilization facility for Mayak to immobilize $1 \mathrm{Mt}$ of plutonium.

3. The MOX fabrication waste engineering feasibility work is progressing.

4. GSPI has summarized the Paket experience and other work to provide waste stream estimates for a commercial-scale MOX fabrication facility making 286 BN-600 assemblies per year and 100 VVER1000 fuel assemblies per year. The effects of German, Russian, and French equipment on the waste streams were also assessed.

5. The history of the SNIIChM equipment design and non-standard equipment company in Yekaterinburg was summarized. There, 400 of 1300 people currently work in research. There were as many as 4000 workers in the past.

6. The LD300 Brest reactor, if it were ever to become a viable technology, would have major equipment problems and today could not be built according to SNIIChM.

\section{Session V. RIAR Site Activities}

1. No license to process and fabricate weapons-grade Pu fuel in Russia has been considered except in a closed city and this is a major issue.

2. The financing of the pilot plutonium metal conversion line for the front end and the waste treatment processes at RIAR are unresolved issues. The LLNL TEF contract has identified the technical issues and initial cost estimates for waste treatment processes.

\section{Session VI. R\&D Support Paper Activities}

1. Several papers addressed Am in the plutonium conversion wastes for Mayak. However, nobody addressed the gallium in the wastes, and this is a serious shortcoming that must be assessed in an integrated way.

2. The pyrochemical conversion process wastes were addressed by RAS-ICP.

3. It was claimed by KRI that $1300-1600^{\circ} \mathrm{C}$ ceramic processing of powders for 30 minutes resulted in no significant volatility problems with $\mathrm{Am}$ and $\mathrm{Ga}$.

4. Papers addressed the borosilicate and phosphate glass R\&D at KRI and VNIINM. 
5. Accelerated radiation damage study results of the US pyrochlor ceramics were reported by RIAR, KRI, and VNIINM.

\section{Session VII. Geologic Repository Activities}

1. Multiple papers were given by VNIPIPT, KRI, and RAS-IGEM for geologic disposal.

2. A key VNIPIET document for the Russian Federation is a draft plan for developing repository sites near the K-26 and Mayak sites.

3. The fastest possible estimated schedule to obtain operational repositories in Russia is 25 years for the $\mathrm{K}-26$ site and 20 years for the Mayak site but the real time is likely to be much longer.

\section{Session VIII.Minatom Representative Comments on the Meeting}

1. Immobilization is not in the Bilateral 2000 Agreement; however, waste treatment problems remain and need to be pursued as part of the Agreement.

2. The US supports the 2000 Agreement, but needs ways to reduce costs and make the program more efficient.

3. New ideas are needed for implementing the Agreement. Technologies must be selected but these technologies must not need dozens of years to implement. The technologies must be safe and provide nonproliferation security. The choices must be economically viable.

4. It must be remembered that when Pu is extracted from wastes, this makes new secondary wastes that then must be treated. Today is different than when in the past plutonium for weapons was the key mission for the RF. Plutonium in wastes is not that valuable as a material now. The main task is to make the plutonium into a very unattractive form for use in weapons.

5. It is extremely necessary to assess existing wastes at the enterprises or industrial sites. The MOX and conversion wastes from implementing the Agreement are insignificant in comparison. Setting up separate facilities only for the Agreement wastes is too extravagant for Russia. We may need to pursue these current existing wastes, plus these new wastes generated from the Agreement in some integrated new facility.

6. The key burden for the technical work must remain on the industrial sites (Mayak, MCC, RIAR). The sites must coordinate and integrate the work of all RF participants performing technical work.

7. Participants are requested to brief Shidlovsky and his department staff, so they will all know the extent of your LLNL work contacts, the work started, the work completed, and your results.

\section{Concluding Remarks}

In summary, this was an excellent meeting and the first objective was achieved. The papers have been collected and once this proceeding is published by LLNL, the second objective will be achieved. It was also remarkable to get more than 21 different Russian organizations and such plutonium experts to attend a single meeting at a single place, and to prepare 49 technical papers. 


\section{References}

1. 19432 Federal Register, Vol. 67, No. 76, Friday, April 19, 2002.

2. L.J. Jardine, G.B. Borisov, Excess Weapons Plutonium Immobilization in Russia: A Review of LLNL Contract Work, Lawrence Livermore National Laboratory, Livermore, CA, UCRL-ID- 138361, April 2000.

3. L.J. Jardine, G.B. Borisov, Immobilization of Excess Weapons Plutonium in Russia: A Review of LLNL Contract Work, Lawrence Livermore National Laboratory, Livermore, CA, UCRL-ID-143846, April 2001.

4. L.J. Jardine, G.B. Borisov, O.A. Mansourov, Stabilization and Immobilization of Excess Weapons Origin Plutonium, Lawrence Livermore National Laboratory, Livermore, CA, UCRL-JC-129790, April 1998.

5. L.J. Jardine, G.B. Borisov, O.A. Mansourov, Immobilization of Excess Weapons Plutonium in Russia, Lawrence Livermore National Laboratory, Livermore, CA UCRL-JC-132920, February 1999. 


\section{Meeting Summary ${ }^{11}$}

\section{J. Jardine, LLNL}

\section{K-26 Site}

Nine presentations described the K-26 site plutonium immobilization activities. The third engineering step, the Justification of Investment ( $\mathrm{JOI}$ ), is progressing on schedule. The $\mathrm{JOI}$ is being built on two previously completed engineering studies and steps, the TEF and the DOI. These two studies determined that recovery and extraction of the $600 \mathrm{~kg}$ of weapons plutonium in the plutonium sludges at the radiochemical plants should not be pursued due to unacceptable economics, environmental impacts of secondary wastes, and radiation exposures when compared to the direct immobilization of Pu-containing sludges. As a result, two direct immobilization options are being developed in the $\mathrm{JOI}$, and work on recovery of plutonium from the sludges has been stopped. The Institutes, KRI and VNIINM, are providing the immobilization technologies to the design team of VNIPIET and MCC K-26.

The total cost estimates developed during the TEF and the DOI for all engineering, design construction, equipment large-scale engineering testing, and start-up testing remain at a cumulative cost of $\$ 17.3 \mathrm{M}$ (Table 1). The hot radioactive startup is scheduled for early 2005. This cost and schedule is possible only because maximum use is being made of existing MCC K-26 radiochemical plant systems and infrastructures. During the JOI, the plutonium immobilization system design is being modified so as to accept Pu-containing wastes from future MOX fabrication should the Russian Federation locate the industrial-scale MOX facility at the MCC K-26 site. This provision requires adding facilities for sorting solid wastes, an incinerator for combustible MOX production wastes, and some modifications and additions to the melter feed preparation areas. Metallic non-combustible wastes also require some additions. The total cost for adding this MOX fabrication waste immobilization capability is $\$ 20.3 \mathrm{M}$, or an increase of $\$ 3 \mathrm{M}$. The construction schedule for the plutonium sludge immobilization has not changed. It is necessary to start the plutonium sludge immobilization before the MOX Pu-containing wastes become available because the operating period for sludge immobilization is 13 years.

Table 1. K-26 Integrated Pu containing waste immobilization building cost estimate.

\begin{tabular}{|l|c|c|}
\hline \multicolumn{1}{|c|}{ Immobilization Facility } & $\begin{array}{c}\text { Capital and Engineering } \\
\text { Costs }\end{array}$ & Delta Costs \\
\hline Only Pu Sludges & $\$ 17.3 \mathrm{M}$ & - \\
\hline $\begin{array}{l}\text { All: Pu Sludges and MOX } \\
\text { Wastes }\end{array}$ & $\$ 20.3 \mathrm{M}$ & $+\$ 6 \mathrm{M}-\$ 8 \mathrm{M}$ \\
\hline Only MOX Wastes & $\$ 12$ to $\$ 14 \mathrm{M}$ & - \\
\hline
\end{tabular}

This contract review meeting, previous deliverables from the MCC K-26 team, and other meetings have identified some technical risks that could impact the overall schedule. These technical risks are being

\footnotetext{
${ }^{1}$ Taken from L. J. Jardine, G.B. Borisov, Immobilization of Excess Weapons Plutonium in Russia: A Review of LLNL Contract Work, Lawrence Livermore National Laboratory, Livermore, CA, UCRL-ID-143846, April 2001.
} 
addressed by various scientific and design institutes. In particular, they include melter development problems such as (1) melter crucible sealing problems; (2) sludge feed pipe plugging; (3) good glass melting in the crucible center but not at the sides; (4) different glass quality or homogeneity in different parts of the glass melt; (5) localized heating and crystallization into an unacceptable glass product; (6) melter feed loading problems; (7) melt level control; (8) melter temperature control problems; (9) volatility problems; (10) plasma formation in melter; and (11) contamination in the waveguides. At this time, these melter development problems are believed solvable but there is a technical risk of a schedule delay.

To help manage the risks, several things are now being undertaken. The JOI will develop two options in addition to the microwave melter: a ceramic melter and a cold crucible melter. VNIPIET will collect the necessary data for the engineering assessments to be sure the JOI design can accommodate a change of melter, if necessary. A meeting at NIKIMT was scheduled for January 15-17, 2001, with Russian microwave melter experts and two of the US experts who developed microwave melting for Pu-containing materials at Rocky Flats. This meeting will help identify specific corrective actions to help develop the microwave melting technology for the MCC K-26 site and to be sure all unresolved technical issues have been identified.

A project review meeting will be held at K-26 in early March 2001 to review progress and the findings of the JOI.

\section{Mayak Site}

Eleven presentations were made about the Mayak site plutonium immobilization activities. The first engineering step, the TEF, for immobilizing about $1 \mathrm{Mt}$ of weapons plutonium in existing radiochemical plant sludges is underway. The TEF will compare the recovery and extraction of the $1 \mathrm{MT}$ of weapons plutonium with direct immobilization options using technologies provided by KRI and VNIINM. Comparisons will be based on economics, environmental impacts of secondary wastes, and radiation exposure.

Another Technical and Economic Feasibility study suffers from a slow start but is also underway. This is the integrated TEF, which considers how to treat and immobilize MOX fabrication waste from the industrial-scale facility, if sited at Mayak and not MCC K-26. GSPI will develop estimates of solid and liquid Pu-containing waste streams based on past Russian MOX fuel fabrication experience at PAKET and granite and past engineering studies. KRI and VNIINM will use these results to provide technologies for immobilizing these Pu- containing wastes. VNIPIET will prepare the integrated TEF designs and perform the comparative assessments.

I expressed a concern that the plutonium conversion immobilization TEF for Mayak has not yet been started. This TEF study is a critical element because Mayak has been selected as the site for the plutonium conversion mission, and it has been recognized that the treatment and immobilization of the Pucontaining conversion wastes is a critical first activity. Delaying assessments of waste treatment and immobilization is not preferred. Information needs to be provided back to the plutonium conversion processes in order to optimize designs for both the main conversion process and for waste treatment.

A single integrated waste treatment and immobilization building is being assessed for the Mayak site. It is envisioned that solid and liquid wastes from the plutonium conversion activities (both pilot and industrial scale) can form the basic requirements for designing the building. It is assumed that, for small incremental costs, the immobilization building functions can be modified to also treat and immobilize both MOX fabrication wastes (from the industrial-scale facility, if sited at Mayak, and the PAKET facility for the BN- 
600 hybrid core) and existing Pu-containing sludges studied in the TEF. The detailed definition of these incremental costs is now underway. Very preliminary estimates have been made for the integrated Pucontaining waste treatment and immobilization building at Mayak for those three types of plutoniumcontaining wastes. The cost is between $\$ 27 \mathrm{M}$ to $\$ 30 \mathrm{M}$. The cost for a similar building at Mayak that could accommodate only the Pu conversion and MOX fabrication wastes but no plutonium sludges is $\$ 16-18 \mathrm{M}$ (Table 2). Thus, the difference or incremental capital cost is $\$ 9$ to $\$ 14 \mathrm{M}$ to immobilize an additional $1 \mathrm{Mt}$ of weapons-grade plutonium at Mayak rather than recover the $1 \mathrm{Mt}$ of plutonium from the sludges and return the plutonium oxide to storage and back to the Russian Federation stockpiles. Mayak and VNIPIET will require a hot cell type construction for the plutonium-conversion wastes so the waste treatment costs are significant.

Table 2. Mayak integrated Pu-containing waste immobilization building cost estimate.

\begin{tabular}{|l|c|c|}
\hline \multicolumn{1}{|c|}{ Immobilization Facility } & $\begin{array}{c}\text { Capital and Engineering } \\
\text { Costs }\end{array}$ & Delta Costs \\
\hline $\begin{array}{l}\text { Only Pu conversion and } \\
\text { MOX wastes }\end{array}$ & $\$ 16 \mathrm{M}$ to $\$ 18 \mathrm{M}$ & Baseline \\
\hline $\begin{array}{l}\text { All: } \mathrm{Pu} \text { conversion, wastes, } \\
\text { MOX wastes, Pu sludges }\end{array}$ & $\$ 27 \mathrm{M}$ to $\$ 30 \mathrm{M}$ & $\$ 9$ to $\$ 14 \mathrm{M}$ \\
\hline Only Pu sludges & $\$ 20 \mathrm{M}$ & $\$-$ \\
\hline
\end{tabular}

These initial cost estimates are necessarily very preliminary and will be defined and refined during the ongoing TEF studies.

It was agreed to meet at Mayak for three days during February 5-16, 2001, to review progress of both the TEF for plutonium sludge immobilization and the TEF for MOX Pu-containing wastes. An MCC K-26 representative will be invited. The prospects for plutonium conversion waste immobilization will also be discussed.

\section{The RIAR Site}

The RIAR site has nearly completed the TEF that identifies the options for immobilizing the Pu-containing pyrochemical wastes. It was agreed to meet in mid-February or early March to discuss the TEF and to define an R\&D study that would develop a suitable waste form for immobilization.

\section{Tomsk Site}

Tomsk did not present a paper. Tomsk has no current contract for plutonium immobilization. It has been agreed to meet at Tomsk January 11 and 12 to discuss plutonium immobilization options at the radiochemical plant for some existing Pu-containing sludges. Draft SOWs have been received from Tomsk, and they will be discussed at the meeting. Minatom determined that the Pu-containing waste materials from the Tomsk CMP could not be discussed or studied at this time. It is possible that a TEF will be undertaken for the Tomsk plutonium sludges as a result of the meeting.

\section{Spent Fuel Storage Work}

The contract for VNIPIET to perform a pre-TEF study has not been signed. VNIPIET staff will visit LLNL November 27-29, 2000, to discuss the pre-TEF and a draft roadmap of the work needed to develop a 
storage capability and transportation capability in Russia for spent MOX fuel. VNIPIET will arrange a visit to the BN-600 site in 2001 to brief the NPP staff on the study and to collect their data and spent fuel storage needs.

\section{R\&D papers, glasses, ceramics and geologic disposal}

\section{Glasses}

Glasses or glass-like matrixes are being developed by KRI and VNIINM for use in immobilization of various Pu-containing materials at the MCC K-26 and Mayak sites. VNIINM is the lead institute for glass development. VNIINM completed a contract identifying various glass compositions for CMP wastes at Mayak and has just started a contract for identifying glass compositions for Mayak MOX Pu-containing wastes. A new contract for VNIINM using the ISTC partnering process to define glass compositions for immobilizing plutonium conversion wastes with high americium and gallium is in process. A new R\&D contract is being drafted for VNIINM to help develop the microwave melter technology together with NIKIMT. KRI has been developing glass compositions for the MCC K-26 and Mayak sites. A new R\&D contract for $\mathrm{KRI}$ is being put in place to continue work on this glass form.

\section{Ceramics}

Ceramics are being developed at a very low level because Russia has no suitable sources of concentrated weapons plutonium that are being considered for immobilization. The low concentration levels of plutonium in Pu-containing materials would be best treated by immobilization in glass matrices rather than ceramics. $\mathrm{KRI}$ is the lead Russian institute for development of ceramic matrices for plutonium immobilization. A small R\&D contract is planned for KRI to continue development of a granite-perovskite matrix for immobilization of Mayak plutonium sludges but the prospect for continuing ceramic work in Russia is limited.

\section{Geologic Disposal}

Geologic disposal is a critical part of the plutonium immobilization activities; it is addressed in all ongoing engineering studies at K-26 and Mayak. Contracts at VNIPIPT have studied behavior of plutonium sorption rock media that are typical of a Russian repository, based on the degradation of Pu-containing glasses made at MCC under simulated geologic disposal conditions. A new VNIPIPT contract continues this work and scales up the experiments. Large $\left(\sim \mathrm{m}^{3}\right)$ migration tests are being conducted in underground rocks using actual radioactive materials under a new LLNL contract. These tests will be looked on by many in the world as leading the collection of critical data needed to validate computer modeling of radionuclide migration in geologic repositories. VNIPIPT also developed an innovative geologic disposal option based on in-site disposal using bentonite for the K-26 JOI; this option dramatically reduces the disposal costs for immobilized sludges from $\$ 60-80 \mathrm{M}$ to $\$ 5 \mathrm{M}$, a savings of $\$ 55 \mathrm{M}$ to $\$ 75 \mathrm{M}$. Further evaluations are being performed to determine if this is a viable option for the JOI at the MCC. Studies of the granite massif at the Nizhnekansky site for a Russian geologic repository were reported by KRI and VNIPIPT. The VNIPIPT data, although limited, estimates projected water infiltration rates (flow time) in the granite massif to be on the order of multiples of 20,000 years to travel $1 \mathrm{~km}$. This time can be thought of as multiple plutonium-239 half-lives.

The geologic disposal work at Mayak was reported by the RAS-IGEM and VNIPIPT. These papers showed that substantial work has been completed for the Mayak site regarding geological and engineering 
studies. The Mayak site is ready to begin detailed, specific site investigations; engineering studies; and development of an underground laboratory required to locate and license a geologic repository at Mayak.

The coupling and integration of immobilization activities with long-term storage and geologic disposal of an immobilized waste form is also recognized. A series of integrated contracts are being developed for VNIPIPT, KRI, VNIINM, RAS-IPC, Mayak, and MCC K-26 to address these complex, coupled issues.

\section{Accelerated Radiation Damage Studies of the US Pu Ceramic for Immobilization}

The three institutes, RIAR, VNIINM, and KRI, are performing a series of measurements on $\mathrm{Pu}^{238}$ and $\mathrm{Pu}^{239}$ ceramic samples with the US-specified ceramic composition. LLNL experts provided the fabrication procedures and non-radioactive ceramic precursors materials. The samples with plutonium were fabricated by RIAR and shipped to VNIINM and KRI for additional measurements. The Russian experts met in October 2000 in the US with US experts to discuss the preliminary results and plans for the future. The Russian measurements can supplement the US data. It was agreed to extend the Russian contracts to April 2002 so that additional accelerated radiation damage measurements can be made. A future decision will be made whether additional $\mathrm{Pu}^{238}$ samples need to be fabricated in Russia.

\section{Other Contracts}

The success of transferring the RIAR salt washer hardware for $\mathrm{PuO}_{2}$ to LLNL can not be understated. It is a remarkable achievement and the first known example of a Russian plutonium processing technological piece of hardware being transferred to the West and the US The US is looking for additional examples of exporting Russian technology to the US and Russian participants are encouraged to make proposals.

Other papers by VNIINM, IGEM, and Radon provided additional information to the Russian experts on various aspects of plutonium immobilization. 


\section{Meeting Summary ${ }^{2}$}

Forty-four experts from 15 Russian organizations took part in the seminar. They represented Russian scientific research institute centers, planning and design institutes, universities and various industrial enterprises: A. A. Bochvar All-Russia Scientific Research Institute of Inorganic Materials (VNIINM), a state science center (SSC); the V. G. Khlopin Radium Institute (KRI), a scientific production association (SPA); the Scientific Research Institute of Atomic Reactors (RIAR), a SSC; VNIPIPT All-Russian Research and Design Institute of Production Engineering (VNIPIPT); the All-Russia Scientific Research Institute of Theoretical Physics; the Institute of Physical Chemistry of the Russian Academy of Sciences (RAS); the Institute of Ore-Deposit Geology, Petrography, Mineralogy, and Geochemistry of the RAS (IGEM); the Nizhnii Novogorod N. I. Lobachevskii State University; All-Russian Design and Research Institute of Complex Power Technology (VNIPIET); the Scientific Research and Design Institute of Installation Technology (NIKIMT); the State Union Design Institute (GSPI); universities, (Nyzhny Novgorod), the Russian Academy of Sciences; Chelybinsk-7- (Sneshinsk); and the industrial sites of Mayak Production Association; the Mining and Chemical Combine at Krasnoyarsk-26 (K-26); and the Tomsk Siberian Chemical Combine. Most of those participating in the seminar were contractors under these contracts. Representatives of the US Department of Energy and the Russian Federation Ministry of Atomic Energy (Minatom) also took part in the seminar.

\section{Remarks}

"The objective is to develop in these Russian projects the engineering and technical bases as well as the Russian approvals needed to conduct industrial scale immobilization of plutonium-containing materials at a Russian industrial site by 2005 . Supporting R\&D projects are carried out at the Russian Institutes that directly support the specific technical needs of the Russian industrial sites to immobilize plutoniumcontaining materials."

Special R\&D on plutonium-containing materials is also carried out to support excess weapons disposition in both Russia and the US. This currently includes nonproliferation studies of plutonium recovery from immobilization forms and accelerated radiation damage studies of the US specified plutonium ceramic for immobilizing plutonium."

—L. Jardine, LLNL

\section{Questions}

John F. Baker, US Department of Energy, provided a response to the following questions:

What is the main thing for your country [is seeking] from the activity of LLNL and Russian Organizations and from American financial support of Russia on the Pu-problem?

These activities contribute to the US-Russian process of irreversible nuclear arms reductions as well as to both ' nonproliferation objectives. Plutonium immobilization provides technical options other than burning MOX fuel in nuclear reactors as a means to disposition excess weapons plutonium.

\footnotetext{
2 Taken from L.J. Jardine, G.B. Borisov, Excess Weapons Plutonium Immobilization in Russia: A Review of LLNL Contract Work, Lawrence Livermore National Laboratory, Livermore, CA, UCRL- ID-138361, April 2000.
} 


\section{What was the direct Program goal that was solved during the Meeting?}

The St. Petersburg Meeting brought Russian plutonium experts together, in many cases for the first time, from 15 different organizations from within Russia to discuss, exchange, and integrate their plutonium technical work results and future plans. These interactions will provide a sound foundation for defining the future technical activities for both the Russian participants and the US.

\section{What new successes would the American side await from Russian -American collaboration in the future?}

The joint goal of the Russian work is to establish a full-scale plutonium immobilization facility at a Russian industrial site by 2005. This work of the meeting and future work will provide the bases for joint decisions, if such a goal can be achieved.

\section{Summary of Scientific Accomplishments}

The reports of LLNL contract work presented at the seminar fall into two groups as documented in the published strategy and approach: Group 1, R\&D on plutonium immobilization and Group 2, the engineering technology and industrial scale immobilization of plutonium (an overview of contracts). Both groups are also united by the common goal of nonproliferation of fissile materials, nuclear materials security, and the solution of the radioecological problems of Russia, and the world as a whole.

The scientific principles of the incorporation of Pu-containing materials and waste in glass and ceramic matrices were discussed in the seminar.

In its reports, the Radium Institute presented the results of R\&D on the development and synthesis of plutonium-containing phosphate and borosilicate glasses and of work on the gathering and generation of raw data for conducting an engineering feasibility studies of the immobilization of plutonium-containing sludges of the Mining and Chemical Combine (K-26) and the Mayak Production Association. A strategic approach to the immobilization of plutonium-containing sludges of the Mayak Production Association was presented. The results of research on the solubility of plutonium in different kinds of glass, their resistance to leaching, and the sedimentation of plutonium from glass melts during prolonged holding production runs were summarized. The approach to the engineering aspects of vitrification is based on the production of borosilicate glass compounds incorporating up to $50 \%$ of the mass of the plutonium sludge waste components while microwave heating is performed. A glass melter pilot plant is housed in the hot cell of the Central Plant Laboratory of the Mining and Chemical Combine (K-26).

At the A. A. Bochvar All-Russia Scientific Research Institute of Inorganic Materials, a range of work related to the immobilization of plutonium-containing materials is now under way. It involves the development of glass-like and mineral-like matrices incorporating up to $10 \mathrm{wt} \%$ plutonium (phosphate and borosilicate glasses; compounds based on pyroxenes, borobasalts, and garnets), and the study of their basic physical and chemical properties, including determination of the homogeneity, chemical resistance, and leachability of the materials produced. Various process flow diagrams for the handling of plutonium-containing materials by using modern science-intensive methods of solidification, such as induction melting in a cold crucible and vitrification by microwave heating, are being developed and studied (both independently and jointly with other scientific organizations such as: NIKIMT, the Radium Institute, and others). Within the framework of this research, engineering feasibility studies are being carried out to determine approaches and to select the most nearly optimal processing method in order to build pilot and industrial scale plants for handling plutonium-containing wastes, residues, and sludge from past military activities at the Ministry of Atomic Energy industrial sites (the Mining and Chemical Combine, the Siberian Chemical Complex, the 
Mayak Production Association, the All-Russia Scientific Research Institute of Theoretical and Experimental Physics, the Scientific Research Institute of Atomic Reactors). Alternatives are being determined and a number of studies are currently under way to solve the problem of handling and immobilizing plutonium-containing wastes from future activities related to the conversion of metallic plutonium and the production of mixed-oxide fuel (MOX) in Russia. Furthermore, preliminary research aimed at studying the behavior of immobilized forms under simulated conditions of temporary storage and geological disposal is under way.

At the Radium Institute research has been conducted on adapting existing experience in ceramic synthesis by using plutonium , and in some cases simulant elements ( $\mathrm{Ce}$, Eu, etc.), for experiments to immobilize plutonium and americium. The optimal compositions and conditions of synthesis of Pucontaining ceramics based on zirconium dioxide, zirconium/zirconium dioxide, and pyrochlore have been determined. It was shown that all the ceramics synthesized had high resistance to plutonium leaching. Data have been obtained on the isomorphic capacity of the ceramic matrices.

There were discussions by IGEM of various kinds of crystalline materials (based on zirconates, titanates, aluminates, phosphates, and silicates, including analogs of natural minerals) as possible matrices for incorporation of actinide-containing waste, various methods of synthesizing them (including fusion methods - crystallization, solid-phase reactions), heating and pressing (molding) conditions, and the composition of the gas atmosphere. Comparisons were made of the leaching characteristics of plutonium, americium, and other radioactive and nonradioactive elements under hydrothermal conditions (the Institute of Ore-Deposit Geology, Petrography, Mineralogy, and Geochemistry of the RAS).

The properties of a ceramic developed at LLNL were investigated by three Russian organizations, KRI, VNIINM and RIAR. This oxide ceramic based on the structure of the mineral pyrochlore has been made the baseline composition for the US concept of immobilization of excess-plutonium. The basic results of the synthesis and crystal-chemical analysis of various ceramic samples containing cerium, uranium, and plutonium-239 were presented. New data have been obtained on the specifics of phase formation as a function of the composition and conditions of synthesis of ceramics. Research is now being conducted on accelerated radiation damage using $\mathrm{Pu}^{238}$ due to alpha decay and on the effect of such damage on the properties of ceramics when immobilized plutonium containing waste is buried (the Scientific Research Institute of Atomic Reactors, a Russian Federation SSC, KRI and VNIINM.

The conditions for producing a ceramic containing plutonium-238 and the conditions for joint accelerated tests of the radiation resistance of the ceramic from LLNL (the Russian Federation Scientific Research Institute of Atomic Reactors, a SSC; the All-Russia Scientific Research Institute of Inorganic Materials, a SSC; and the Radium Institute) have been drawn up and approved by LLNL experts.

The plutonium-containing waste formed at the Scientific Research Institute of Atomic Reactors during the development and experimental adoption of the pyrochemical process of MOX fuel production has been characterized and classified. Schemes and processes have been proposed for processing these plutonium residues into either reactor fuel and into immobilized forms. This includes work at RIAR related to similar forthcoming tasks in the development of corresponding schemes to process of the first arriving shipments of "weapons-grade" plutonium metal that is converted to oxide using molten salt processes (the Scientific Research Institute of Atomic Reactors, a Russian Federation SSC).

The results of laboratory research on physical and chemical processes of the interaction of plutonium solutions with gneiss-type rocks and materials from the breakdown of engineering barriers of geologic repositories at high temperatures and various pressures (up to $30 \mathrm{MPa}$ ) were scrutinized in relation to the 
problem of the geological burial of immobilized plutonium forms. This research simulates, in an accelerated environment, the actual process of the slow breakdown, by ground water, of the elements of the multibarrier system of the geologic repository for immobilized plutonium-containing wastes and the confinement of most of the plutonium leached from the matrices to the neighboring rock zones of the underground repository. Experiments showed that the increase in pressure associated with depth reduces the strength with which the plutonium is bound to the rocks. In addition, the first results were presented from laboratory research on the physical and chemical characteristics of actual plutonium-containing samples of phosphate and borosilicate glasses, produced by plutonium sludge wastes from weaponsgrade plutonium production (the All-Russia Scientific Research Institute of Industrial Technology, the Institute of Physical Chemistry of the Russian Academy of Sciences, and the Mining and Chemical Combine).

Technical and economic feasibility studies have been made of several scenarios at K-26 for the immobilization of plutonium-containing sludges: waste solidification with and without plutonium extraction, with incorporation in matrices of borosilicate or phosphate glass. An underground pilot plant for plutoniumcontaining wastes and fission product solidification using microwave energy has been installed in the hot cell of the Central Plant Laboratory of the Mining and Chemical Combine. The plant has undergone radioactive materials testing. Prototypes of the glasses have been produced, with incorporation of simulated and real production waste recovered from storage tanks. The engineering parameters of the solidification process have been determined. Studies have been made of the chemical stability and industrial strength of the glass product and the leachability of certain radionuclide from it (the Mining and Chemical Combine). The microwave melting system was developed and shipped for waste solidification at the industrial site of the Mining and Chemical Combine by NIKIMT.

Thin-film inorganic sorbents based on zeolites and hard-to-dissolve inorganic compounds that are selective for cesium, strontium, and uranium and that have high capacity as well as thermal, chemical, and radiation resistance have undergone testing at the Central Plant Laboratory of the Mayak Production Association. These heat-treated sorption products are said to be suitable for safe long-term storage (the All-Russia Scientific Research Institute of Theoretical Physics, the Ural'sk State Technical UniversityUral'sk Polytechnic Institute). A method of removing cobalt and uranium from liquid radioactive wastes and natural waters using special brands of asbestos has also been developed. The method has been tested at the Central Plant Laboratory of the Mayak Production Association. A simple and economical method has been developed for removal of uranium from radioactive contaminated lubricants and cooling fluids, using demulsifiers and decontaminants (the All-Russia Scientific Research Institute of Theoretical Physics).

Research has been conducted on the separation and recovery of plutonium from the glass and ceramic immobilization forms of Pu-containing glass and ceramics from the standpoint of nuclear-weapons nonproliferation (the Radium Institute; the All-Russia Scientific Research Institute of Inorganic materials, a RF SSC).

The results were presented from the cooperation between Russian Federation state science centers and LLNL on the purchase by the US party of a device developed at the Scientific Research Institute of Atomic Reactors, a RF SSC, for washing residual chloride salts from $\mathrm{PuO}_{2}$ precipitates so that plutonium oxide can be used directly in the US excess fissile-materials immobilization program processes.

There were discussions of proposals on future contracts between LLNL and Russian organizations in the area of plutonium immobilization in Russia. These included contracts related to work on the technology 
and industrial scale immobilization of plutonium at K-26 and Mayak, as well as R\&D on plutonium immobilization in glass and ceramics and on burial in geological formations of immobilized solid forms.

There also were discussions of questions and proposals for the creation of a unified Russian center for the investigation of the chemical and radiation stability of a glass that contains radioactive waste; questions of americium immobilization from future plutonium conversion activities; changes in the properties of immobilized materials during long-term storage and geologic disposal; and expansion of work at industrial sites (the behavior of waste under burial conditions - the Mayak Production Association and the Mining and Chemical Combine) as being key issues en route to success in solving the environmental plutonium problems and the disposition of excess weapons plutonium.

\section{Conclusion}

In summary, this was an excellent meeting. The first objective-to bring the Russian organizations, experts, and managers together to review and discuss their work with each other-was achieved. It was remarkable to have 15 different Russian organizations attend a single meeting at a single place.

The papers have now been collected, and once a proceedings is prepared by LLNL, the second objective -to publish a meeting summary and proceedings-will also have been met. These proceedings will document the wide extent of the Russian immobilization activities, provide a reference for the work, and make it available to others. 


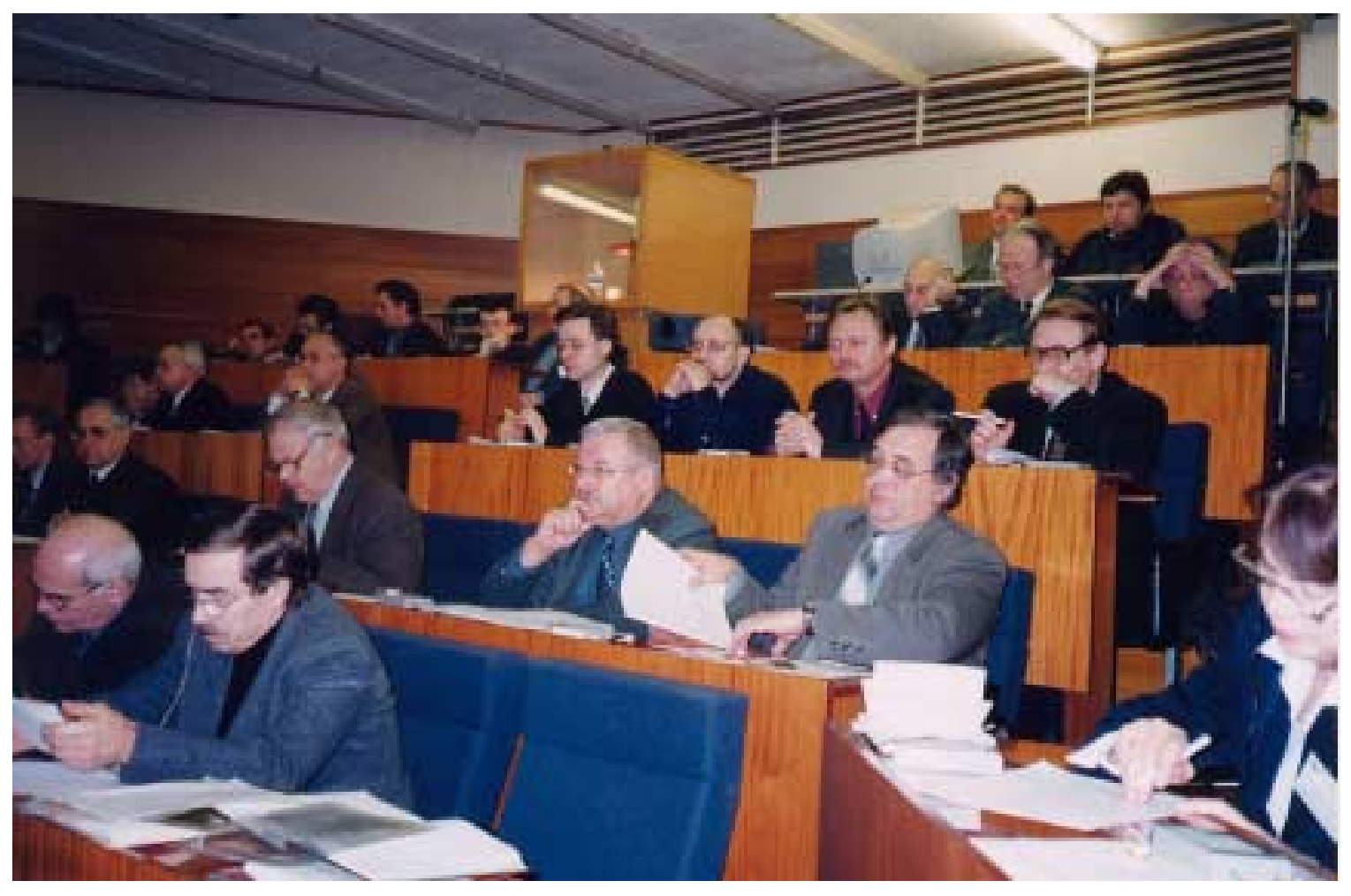

Appendix A. Agenda 



\title{
Appendix A. Agenda
}

\section{Russia Excess Weapons Plutonium Disposition LLNL Projects Review Meeting}

\author{
State Regional Education Center (SEC), St. Petersburg, Russia \\ January 14-18, 2002
}

January 14, 2002

\section{Opening Session}

1. Introductions and Objectives.

LLNL - L. J. Jardine

2. Welcome and Opening Remarks KRI - A. Rimski-Korsakov

3. Observations of Two Previous Meetings 1999 and 2000 LLNL - L. J. Jardine UNG - A. V. Orlova

4. Overview of the LLNL Russian Plutonium Disposition Contracts. LLNL - L. J. Jardine

5. The Immobilization of Pu-containing Materials in Russia: Status and

Perspectives VNIINM - G. B. Borisov

6. Russian Plutonium Metal Oxide Fresh MOX. Fuel Packaging, Storage and Transportation Contract Activities (B512166) VNIPIET - A. Tokarenko

7. Issues of Storage and Transportation of $\mathrm{PuO}_{2}$ for Plutonium Conversion

Facility at Mayak. VNIINM - E. M. Glagowski

8. Russian Spent MOX Fuel Storage and Transportation Contract Activities (B506234). VNIPIET - O. Anisimov

9. The Changes in Structural Construction Characteristics that Occurred during

Dry and In-Pond Storage of Irradiated Spent Fuel Bundles. VNIINM - I. M. Kadarmetov

10. The LLNL Russian Pu Disposition Projects Web Site (B512152)

M. Moshkov, A. Aloy

KRI - P. Burkhanov

January 15, 2002

II. Engineering and Industrial Site Plutonium Disposition Contract Activities: K-26, Mayak, RIAR

1. MCC K-26 Site Activities: Justification of Investment Engineering Studies of Weapons Plutonium Sludge Immobilization and MOX Fuel Fabrication Plutonium Immobilization 
1.1. Perspectives of the Collaboration Between MCC and LLNL in Terms of Nuclear Materials Handling Held in the Frameworks of the Agreements Achieved by Governments of

Russia and USA (B5347376, B506210, B506233) , A. A. Tretyakov. MCC K-26 - V. Bondin

1.2. The Final MCC K-26 JOI Phase Work (B506233) VNIPIET - A. V. Shvedov

1.3. Technological Aspects for the MCC Plutonium-Bearing Waste Solidification using

UHF Melter (B506233) K. G. Kudinov, Y. P. Sorokin, V. V. Bondin,

F. Ludmila, F. Manakova MCC K-26 - A. A. Tretyakov

1.4. Review of the Works Performed in the Frameworks Between MCC and LLNL in Terms of MCC Plutonium-Bearing Waste with MOX Production Induced (B506233)

K. G. Kudinov, A. A. Tretyakov, Y. P. Sorokin, V. V. Bondin. MCC K-26 - L. F. Manakova

1.5. Phosphate Glass Matrixes Developed at VNIINM (B506233)

G. Borisov, Y. Volchok, T. Smelova, A. Balashov. VNIINM - A. V. Nazarov

1.6. Borosilicate Glass Matrix Technology Developed for MCC (B506233) A. Aloy.... KRI - E. Ivanov

1.7. Ecological and Economical Substantiation of the Technological Decisions and Comparative Analysis of MCC Glasses for a Long-Term Storage of Immobilized Sludge in Underground Capacities for the JOI (B506233) T. A. Gupalo....... VNIPIPT - V. P. Beygul

1.8. Study of Condition of Workings Boundary Zones, Prospective for Long-Term Underground Isolation of Immobilized Plutonium Containing Sludge (B506233). VNIPIPT - V. S. Gupalo

\section{Mayak Site Technical and Economic Engineering Feasibility Studies of Weapons Plutonium Sludge Immobilization and MOX Fuel Fabrication Plutonium Wastes}

2.1. Overview and Approach for the Mayak Pu Containing Waste Studies (B506201)

S. I. Rovny, A. S. Skobtsov, G. M. Medvedev, N. M. Emelianov, R. S. Karimov...... Mayak - S. I. Rovny

2.2. The Technical and Economic Feasibility Study for Mayak Pu Sludge Immobilization (B506208) VNIPIET - A. V. Shvedov

2.3. State and Perspectives of Activities Performed at Mayak PA in the Field of Radioactive Waste Handling, including the Vitrification Furnace EP-500 (B506201) S. I. Rovny, A. S. Skobtsov,

G. M. Medvedev, N. M. Emelianov, R S. Karimov. Mayak -A. S. Skobtsov

2.4. Summary of Monitoring and Sampling of 954 Tanks at Mayak (B506201) S. I. Rovny,

A. S. Skobtsov, N. M. Emelianov, R. S. Karimov Mayak - G. M. Medvedev

2.5. Pu-containing Waste Immobilization Technology in Mineral -like Matrices (B506201) S. I. Rovny,

S. Skobtsov, G. M. Medvedev, N. M. Emelianov, R. S. Karimov ..................................Mayak - N. M. Emelianov

2.6. Experimental Tests Made at VNIINM in Support of the Engineering and Feasibility Study of Pu Sludge Immobilization at Mayak (B506206) G. Borisov, O. Mansourov. VNIINM - A. V. Balashov

2.7. The KRI Approach for Developing Pu Immobilization Technology for P O Mayak. KRI - A. S. Aloy

2.8. MOX Fabrication Pu Residues and Wastes for the Mayak Engineering Feasibility Study

(B06237, B506241, B506240, B506239, B506238) A. Dozorov . GSPI - Y. F. Kolotilov 
2.9. Experience and Capabilities of SNIIChM to Implement Contracts in the Field of Weapons

Plutonium Conversion and MOX Fuel Waste Handling SNIIChM - R. S. Karimov

\section{RIAR Site Studies}

3.1. RIAR Activities in the Field of Weapon-Grade Plutonium Disposition RIAR - A.V. Bychkov

3.2. Current Status of B506204 Agreement: Development of Large-Scale Flow Sheet for Immobilization of Pyrochemical Pu-Containing Waste at RIAR (B506204)

A. V. Bychkov, D. A. Bobrov, A. N. Lukinykh RIAR - L. G. Babikov

January 16, 2002

\section{Pu Sludge Research and Development (R\&D) Plutonium Immobilization and Other Related Activities}

\section{Plutonium Glasses for Immobilization}

1.1. The Development of Matrices for the Immobilization of Hard-to Process Sludges of Mayak (B506228) VNIINM - Y. I. Matyunin

1.2. Summary of Pu Glasses and Ceramics Developed for Pu Immobilization at the MCC K-26 and Mayak Site (B512152) $\mathrm{KRI}$ - A.S.Aloy

1.3. The immobilization of Am-containing Waste from Pu Conversion Operations (B514481)

G. Borisov, A. Nazarov, Y. Gorbunov, V. Ilyashenko VNIINM - O.A. Mansourov

1.4. The Immobilization of Pu Conversion Pyrochemical Salt Wastes (B514481) RAS-IPC - A. A. Minaev VNIINM - G. Borisov, O. Mansourov

\section{Plutonium Ceramics for Immobilization}

2.1 Summary of Pu Ceramics Developed for Pu Immobilization (B506216, B512161) KRI - B. E. Burakov

\section{Accelerated Radiation Damage Studies of the US Pu Ceramic Formulation}

3.1. Current Status of B501111 Agreement. Investigation of Radiation and Chemical Stability of Titanate Ceramics Intended for Actinides Disposal (B501111)

S. V. Tomilin, A. A. Lizin, A.V. Bychkov RIAR - A. N. Lukinykh

3.2. Accelerated Radiation Damage Study of the US Specified Ceramics at VNIINM (B506205) VNIINM - I. A. Kulikov

3.3. KRI Studies of the US Pu Ceramics (B506203). KRI - B. E. Burakov

\section{Geologic Disposal for Plutonium Immobilization and Other Pu Containing Materials}

4.1. Overview Comments for Russian Geologic Repository Session LLNL - L. J. Jardine

4.2. Russian Federation Perspectives on Managing Radioactive Wastes from New Pu Processing Production Plants..... VNIPIET - I. Rybalchenko 4.3. A Minatom Geologic Repository Plan (B512155) VNIPIPT - T. A. Gupalo 
4.4. Inputs for the Nizhnekansky Massif to a Minatom Plan for the MCC K-26 Site

(B512154).

KRI - E. B. Anderson

4.5. IGEM RAS Vision of the Issues of SNF Long-term Storage and HLW Geologic

Disposal in Russia

IGEM- V. I. Velichkin

4.6. Primary Positions of RW Underground Isolation Facilities Creation in Areas of MCC Arrangement and PA Mayak (B512155) T. A. Gupalo, O. A. Prokopova, V. P. Beygul.

VNIPIPT- V. I. Milovidov

4.7. Integrated Program of Engineering and Geological Researches of Rock Massifs for RW Underground Isolation.

(B512155) T. A. Gupalo, V. L. Milovidov.....

VNIPIPT - O. A. Prokopova

4.8. Preliminary Results In Situ and Laboratory Researches on Migration of Complex Radioactive Solutions,

Containing Pu and U, in Bentonite Barrier and Fractured Zones (B501120,

B512134) VNIPIPT - T. A. Gupalo, MCC - I. V. Shramko, S. A. Manakov .

VNIPIPT - S. L. Speshilov

\section{January 17, 2002}

\section{Other Invited Papers}

5.1. Minatom Representative Comments....

Minatom - L. I. Petrova

5.2. GAN Representative Comments

GAN - V. I. Iryushkin

5.3. Micro- and Macrostructure Peculiarities of Ferromagnetic Fusion Cakes

Urals Technical University .

USTU-UPI - V. N. Musgin

5.4. Tomsk Site Representatives Comments V. A. Matukha, A. S. Ryabov

Tomsk

5.5. Russian -US Pu Experts Workshop Support (B512167)

KRI - M. M. Moshkov

5.6. Comparative Radiation Resistance of Fluorite Structure Phases for Actinides Immobilization,

Radon - O. Kirjanova, RAS IGEM - S. Yudintsev Radon - S. Stefanovsky

5.7. Chemical Restrictions to Formation of the Actinide Hosts with Pyrochlore Structure,

Radon - S. Stefanovsky RAS IGEM - S. I. Yudintsev

5.8. The Investigation of Plutonium, Americum and Curium Phosphates on the Scientific Basis

of the Immobilization into Ceramic Forms - Y. Ph. Volkov, A. A. Charlamova UNG - A.V. Orlova

5.9. Alkaline Tank Waste Chemistry of Plutonium: Speciation, Redox Reactions and Structural

Transformations, B. Myasoedov

GEOKHI - I. G. Tananaev

5.10. Immobilization of Minor Actinides in Mineral-Type Matrices Using Self-Propagating High-Temperature

Synthesis, E. Glagowski

VNIINM - A. V. Kuprin

5.11. Scientific Investigation of Spent Nuclear Fuel Encapsulated into Glass-Ceramic Protective

Forms

Kharkov - S. Sayenko 
IV. Discussion of Future Direction for LLNL Russian Pu Disposition Projects..... LLNL - L. J. Jardine VNIINM - G. B. Borisov

1. Engineering and Industrial Site Plutonium Disposition Activities at the $K-26$ and Mayak Industrial Sites

2. Other Sites: RIAR, Tomsk

January 18, 2006

3. Research and Development (R\&D) Plutonium Disposition Activities

Pu Glasses for Immobilization

Pu Ceramics for Immobilization

Geologic Disposal for Pu Immobilization

Pu Storage, Packaging and Transportation

Spent MOX fuel Storage and Transportation Dry Storage

Other

V. Summary and Wrap-up LNNL - L. J. Jardine VNIINM - G. B. Borisov 


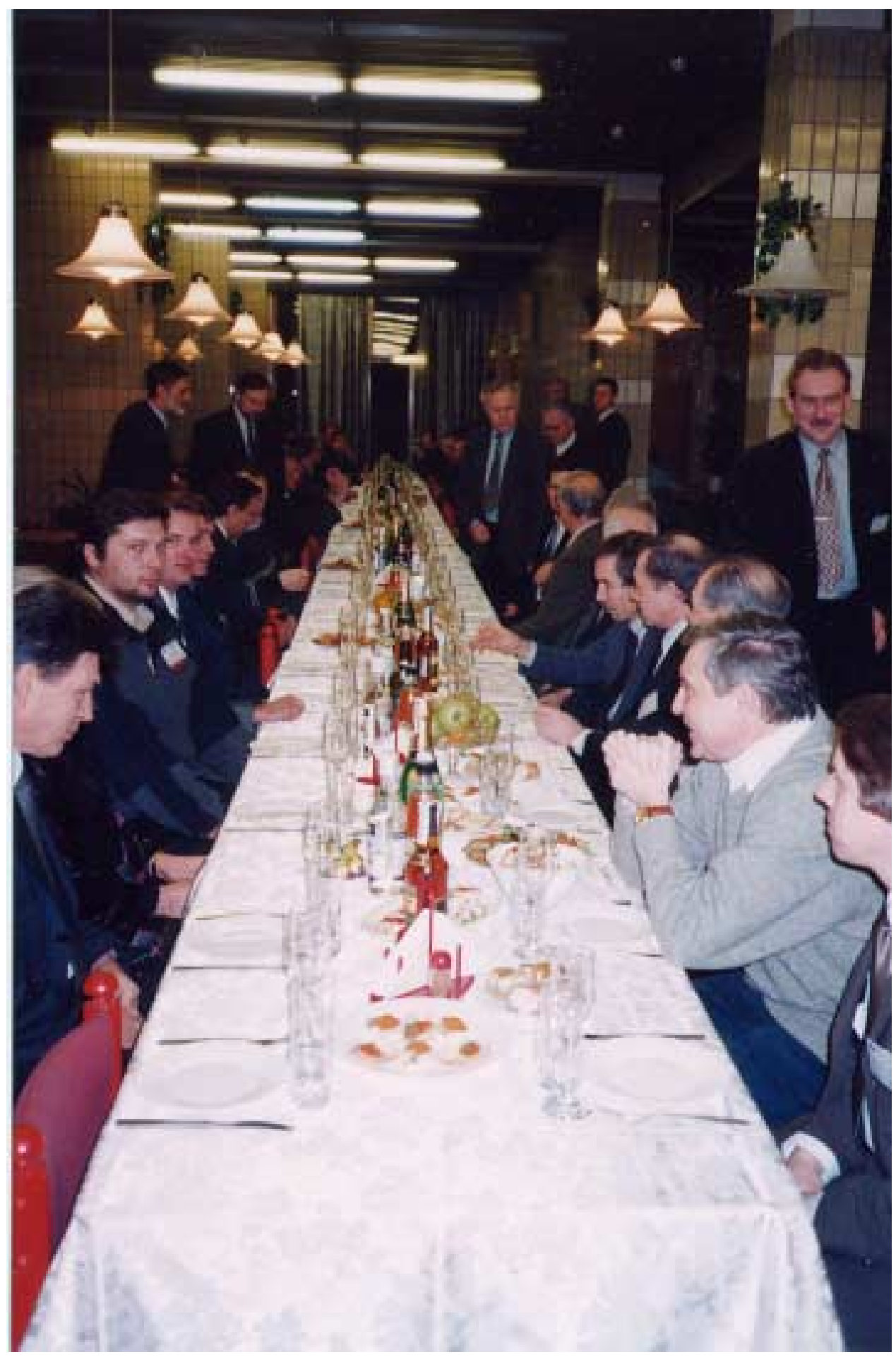

Appendix B. Attendees 



\section{Appendix B. List of Attendees}

\section{Russia Excess Weapons Plutonium Disposition LLNL Projects Review Meeting}

\section{State Regional Education Center (SEC), St. Petersburg, Russia January 14-18, 2002}

\begin{tabular}{|l|l|}
\hline & \\
Name & Institution \\
\hline L.J. Jardine & LLNL, Livermore, CA US \\
\hline L'udmila Petrova & Minatom, Moscow \\
\hline V.L.Milovidov & VNIPIPT, Moscow \\
\hline O.A.Prokopova & VNIPIPT, Moscow \\
\hline S.L.Speshilov & VNIPIPT, Moscow \\
\hline Alexey Minaev & ICP/RAS, Moscow \\
\hline Georg B.Borisov & VNIINM, Moscow \\
\hline Yuri I.Matyunin & VNIINM, Moscow \\
\hline Oleg Mansourov & VNIINM, Moscow \\
\hline A.Balashov & VNIINM, Moscow \\
\hline Igor Kulikov & VNIINM, Moscow \\
\hline I.Kardametov & VNIINM, Moscow \\
\hline E.Glagovsky & VNIINM, Moscow \\
\hline A.V. Kouprin & VNIINM, Moscow \\
\hline I.G.Tananaev & GEOKHI, Moscow \\
\hline Sergey V.Yudintsev & IGEM, Moscow \\
\hline V.I.Velichkin & IGEM, Moscow \\
\hline Vladimir M.Iryushkin & GAN, Moscow \\
\hline A.V.Obruchnikov & STC NRS (GAN), Moscow \\
\hline Yu.Kolotilov & GSPI, Moscow \\
\hline A.Dozorov & GSPI, Moscow \\
\hline Sergey Stefanovski & "Radon", Moscow \\
\hline Albina I.Orlova & State University, Nyzhny Novgorod \\
\hline Alexandre V.Bychkov & RIAR, Samara \\
\hline A.N.Lukinykh & RIAR, Samara \\
\hline L.G.Babikov & RIAR, Samara \\
\hline A.A.Lizin & RIAR, Samara \\
\hline V.V.Bondin & K-26, Zeleznogorsk \\
\hline Alexander A.Tretyakov & K-26, Zeleznogorsk \\
\hline L'udmila F.Manakova & K-26, Zeleznogorsk \\
\hline Sergey I.Rovny & "Mayak", Ozersk \\
\hline A.S.Skobtsov & "Mayak", Ozersk \\
\hline Gennady Medvedev & "Mayak", Ozersk \\
\hline Nikolay Emelyanov & "Mayak", Ozersk \\
\hline V.N.Muzgin & \\
\hline
\end{tabular}




\begin{tabular}{|l|l|}
\hline Ravil S.Karimov & SNIIChM, Ekaterinburg \\
\hline Vladimir A.Matukha & SChC, Seversk \\
\hline Vyachevlav G.Balakhonov & SChC, Seversk \\
\hline Sergey Yu.Saenko & FTI, Khar'kov, Ukraine \\
\hline Alexander A.Rimski-Korsakov & KRI, St.Petersburg \\
\hline Evgeny B.Anderson & KRI, St.Petersburg \\
\hline Albert S.Aloy & KRI, St.Petersburg \\
\hline Mikhail M.Moshkov & KRI, St.Petersburg \\
\hline Boris E.Burakov & KRI, St.Petersburg \\
\hline S.I.Shabalev & KRI, St.Petersburg \\
\hline Sergey A.Bartenev & KRI, St.Petersburg \\
\hline Ol'ga A.Iskhakova & KRI, St.Petersburg \\
\hline Petr E.Burkhanov & KRI, St.Petersburg \\
\hline E.Yu.Ivanov & KRI, St.Petersburg \\
\hline A.Trofimenko & KRI, St.Petersburg \\
\hline AnastasiyaV.Pankratova & KRI, St.Petersburg \\
\hline L'udmila G.Tsaritsina & KRI, St.Petersburg \\
\hline Alexander A.Shvedov & VNIPIET, St.Petersburg \\
\hline V. V. Il'in & VNIPIET, St.Petersburg \\
\hline A.L.Lazarev & VNIPIET, St.Petersburg \\
\hline O.Anisimov & VNIPIET, St.Petersburg \\
\hline I.Rybal'chenko & VNIPIET, St.Petersburg \\
\hline Gennady I.Babaynits & S\&TC "Lutch-Ceranics", Podol'sk (Dir.) \\
\hline Konstantin G.Babaynits & S\&TC "Lutch-Ceranics", Podol'sk (Production \\
\hline & Manager) \\
\hline
\end{tabular}




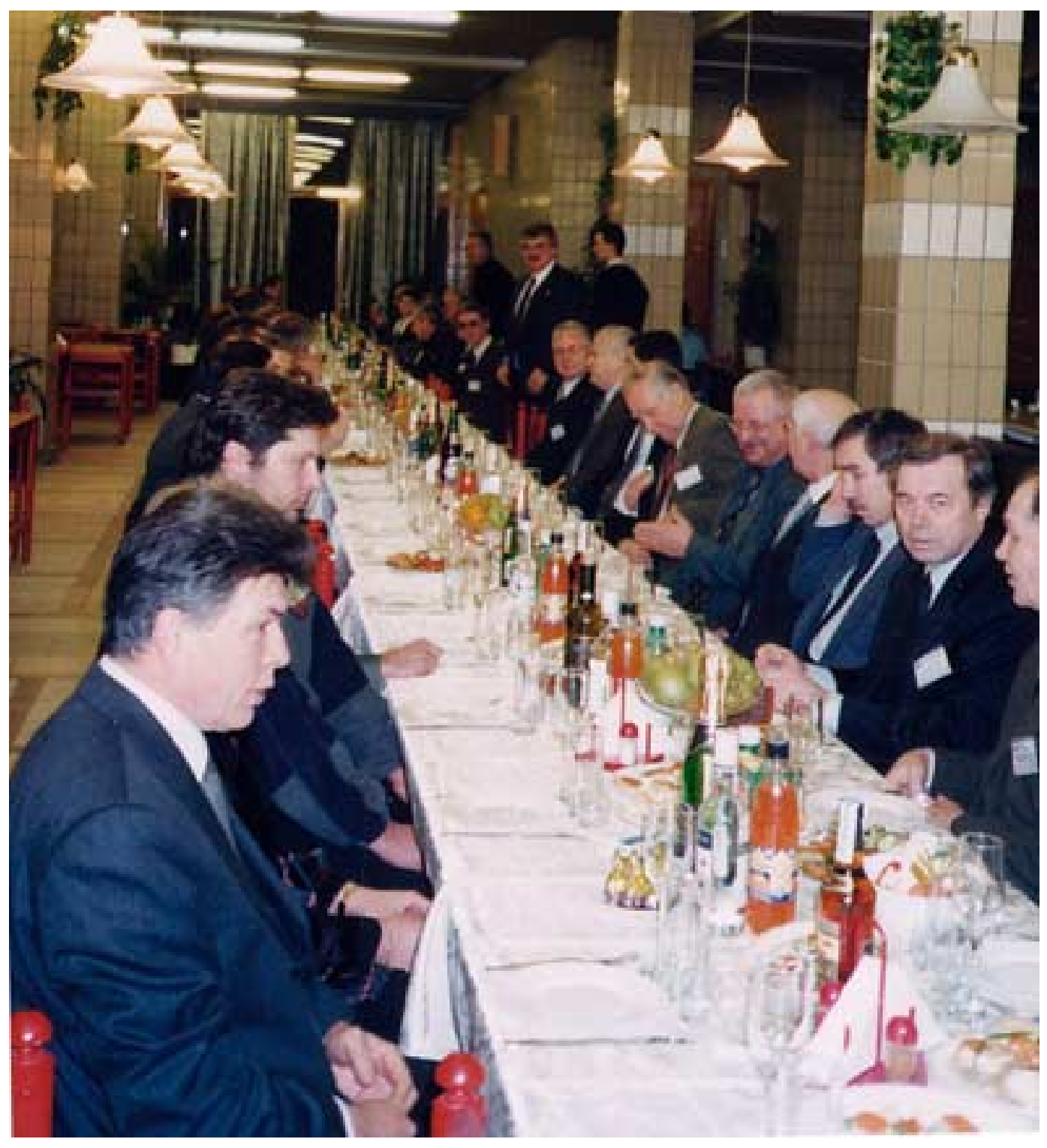

Appendix C. Index of Author Names 

Afanasyev, A. V. 11

Aloy, A. S. 33, 69, 129, 141, 237

Ananina, T. N. 285

Anderson, E. B. 129, 265, 295, 315

Anisimov, O. P. 17, 45

Azhazha, Zh. S. 443

Babikov, L. G. 179, 203

Balashov, A. 65, 119

Belov, V. S. 237

Beygul, V. P. 81, 351

Bobrov, D. 179

Bondin, V. V. 51, 55

Borisov, G. B. 7, 65, 119, 251, 255, 371

Burakov, B. E. 129, 265, 295

Burkhanov, P. E. 33

Bychkov, A. V. 179, 203, 273

Charlamova, A. A. 407

Demin, A. V. 45, 97

Emelianov, N. M. 105

Ewing, R.C. 389, 395

Feofanova, T. A. 45

Filine, V. M. 285

Gabelkov, S. V. 443

Glagovsky, E. M., 15, 433

Golosov, O. A. 25

Gorbunov, V. F. 251

Grachev, A. 179

Gupalo, T. A. 81, 311, 347, 351, 357

Gupalo, V. S. 89

Gusakov, B. V. 97

Guzhavin, V. I. 111, 383

Iliin, V. V. 45, 97

Ilyashenko, V. S.251

loltukhovskiy, A. G. 25

Iriushkin, V. M. 371

Iskhakova, O. A.237

Ivanov, E. Yu. 33, 69

Jardine, L. J. 1, 3, 141, 303, 467,

473, 479

Kadarmetov, I. M. 25

Karimov, R.S. 171

Kashcheev, V. V. 45, 97

Kholomeyev, G. A. 443

Kirjanova; O. I. 389

Kisly, V. 179

Kolotilov, Yu. F.147

Koltsova, T. I. 14, 237

Konovalov, E. E.433
Kouprin; A. V. 433

Kovarskaya, E. N. 141

Kudinov, K. G. 39, 51, 55

Kulikov, I. A. 285

Kuznetsov, B. S. 69

Kuznetsov, D. G. 251, 255

Kuznetsov, V. P. 25

Lazarev, A. L. 11

Lian, J. 389

Lizin, A. A.273

Lukinyh, A. N. 203, 273

Manakov, S. A. 357

Manakova, L. F. 51, 55

Mansourov, O. A. 65, 119, 251, 255

Manturov, O. O. 371

Markov, D. V. 25

Matyunin, Yu. I. 223

Mayorshin, A. 179

Medvedev, G. M. 93, 105, 141

Milovidov, V. L. 347, 351

Minaev, A. A. 251, 255

Mishenev, V. 179

Moshkov, M. M. 33

Myasoedov, B. F. 419

Nazarov, A. V. 65, 119

Neklyudov, I. M. 443

Orlova, A. I. 3, 407

Osipenco, A. 179

Panov, O. Y. 285

Pavlov, S. V. 25

Pelevin, L.P. 433

Petrov, V. A. 323

Pilipenko, A. P. 443

Prokopova, O. A. 347, 351

Pupyshev, A. A. 383

Romanov, S. V. 97

Rovny, S. I. 93, 105, 111, 141, 383

Rozhkov, V. L. 147

Rybalchenko, I. L. 307

Safutin, V. D. 307

Samoylov, S. E.141

Samsonov, V. E. 285

Savonenkov, V. G. 315

Sayenko, S. Yu. 443

Shabalev, S. I. 315

Shafrova, N. P. 17

Shevyakova, E. P. 443

Shilyayev, B. A.443

Shramko, I. V. 357
Shvedov, A. A. 45, 97

Simanovskiy, V. M. 45, 97

Sinel'nikov, L. P. 25

Skiba, O. 179

Skobtsov, A. S. 93

Smirnov, I. V. 129

Smirnov, V. P. 25

Solonin, M. I. 25

Sorokin, V. T. 45, 97

Sorokin, Yu. P. 51, 55

Speshilov, S. L. 81, 357

Spichev, V. V. 17

Stefanovsky, S. V. 389, 395

Stroganov, A. A. 371

Surkov, A. E. 443

Tananaev, I. G. 419

Tarasov, N. N. 323

Tarasov, R. V. 443

Tikhonov, N. S. 17

Timokhin, A. N. 25

Tokarenko, A. I. 11

Tomilin, S. V. 273

Tretyakov, A. A. 39, 51, 55, 357

Trofimenko, A. V. 69, 237

Tsykhanov, V. A. 25

Tyurin, E. I. 147

Vashman, A. A. 285

Velichkin, V. I. 323

Velioukhanov, V. P. 25

Volchok, Y. Y. 65, 119

Volkov, Yu. F. 407

Yudintsev, S. V. 389, 395, 433

Zmiy, V. I. 443 


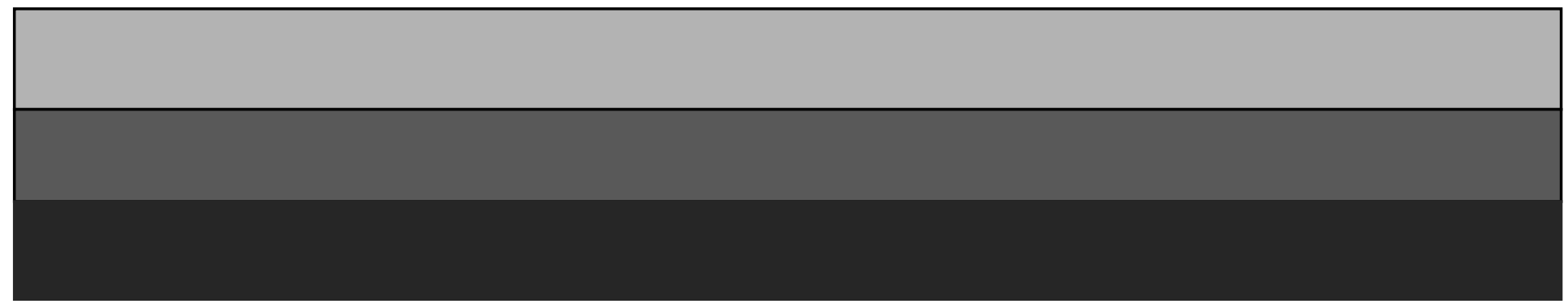

